Fall 2006

\title{
2006 Miracle Yearbook
}

Cedarville University

Follow this and additional works at: https://digitalcommons.cedarville.edu/yearbooks

Part of the Higher Education Commons, Organizational Communication Commons, and the Public Relations and Advertising Commons

\section{Recommended Citation}

Cedarville University, "2006 Miracle Yearbook" (2006). Yearbooks. 19.

https://digitalcommons.cedarville.edu/yearbooks/19

This Book is brought to you for free and open access by DigitalCommons@Cedarville, a service of the Centennial Library. It has been accepted for inclusion in Yearbooks by an authorized administrator of DigitalCommons@Cedarville. For more information, please contact digitalcommons@cedarville.edu. 


\section{MIRACLE}

CEDARVILLE UNIVERSITY

2006

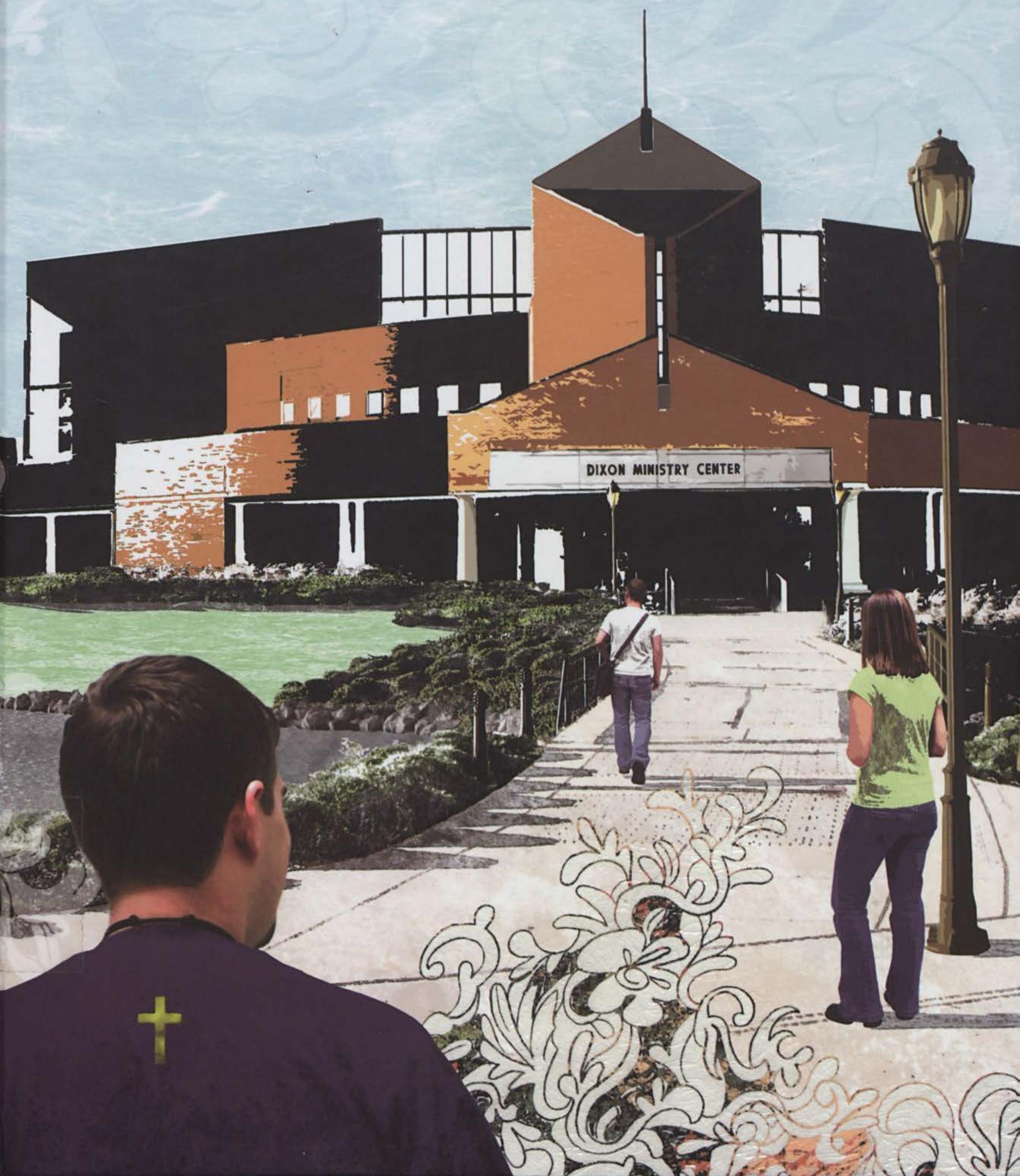




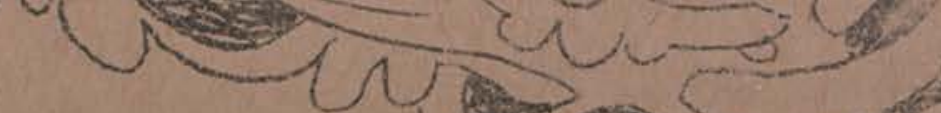






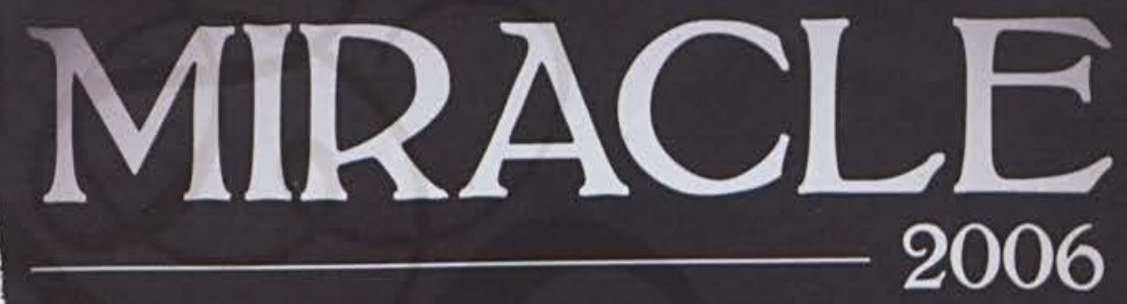

CEDARVILLE UNIVERSITY

251 N. MAIN STREET

CEDARVILLE, OH 45314

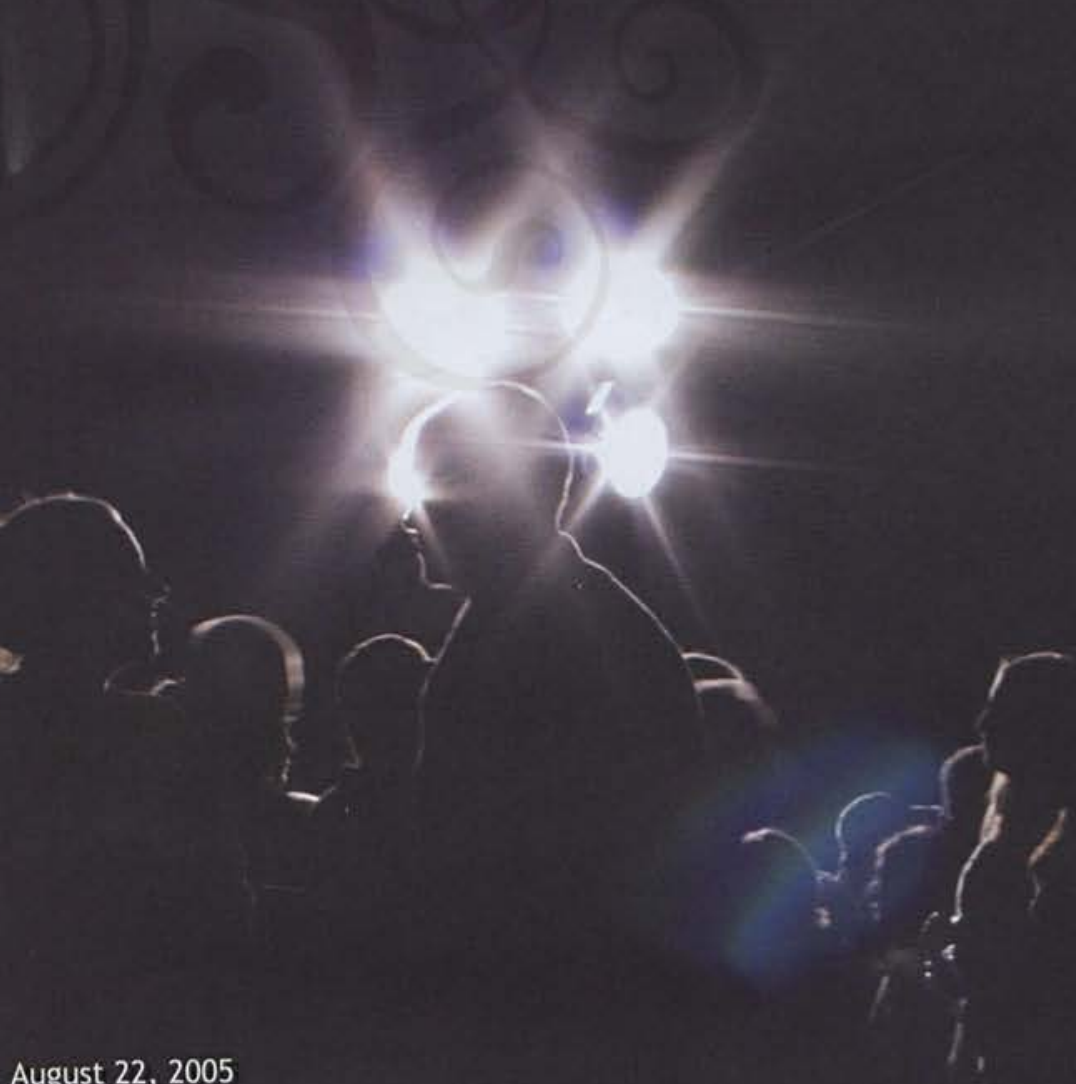

This year holds so much potential and so much excitement, but already seems so different. This is evident in the changes that have taken place around campus, like the new sidewalk in front of the ENS that was built overtop the student-made pathway in the grass, or the addition of freshman plinko in SGA chapel to get Matt Shiraki a date, or the replacement of Stalkernet with Facebook in the Cedarville community. However, the important things always seem to remain the same. We still have all-in checks, Sunday-night pizza at Chuck's, and Pastor Rohm's famous "Giants in the Land" speech. Despite all the adjustments, Cedarville is still the place where friendships are formed, preparations for the future are made, and memories that will last a lifetime are created. I am so excited to see what God will accomplish within the next nine months! 

The new business trading room which opened in the fall, widens the business students' horizons and gives them an opportunity to apply what they are learning in the classroom, exemplifying Cedarville University's commitment to excellence.

\section{VLS}

\section{$97-0.70$}

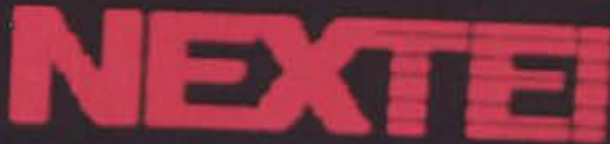

1

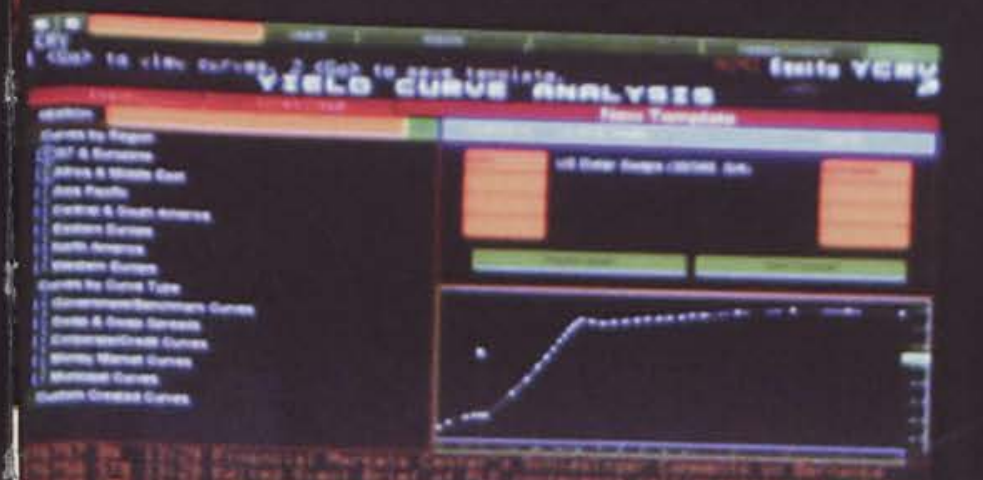

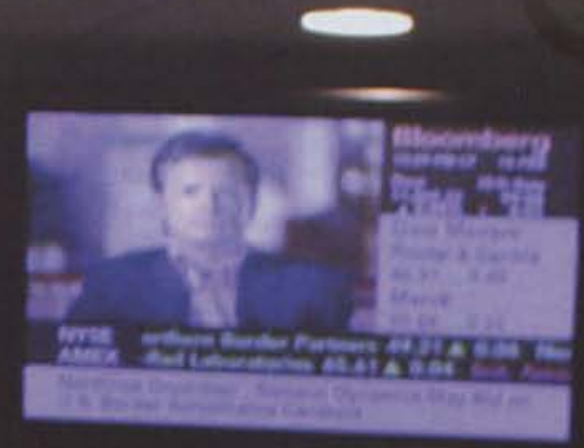
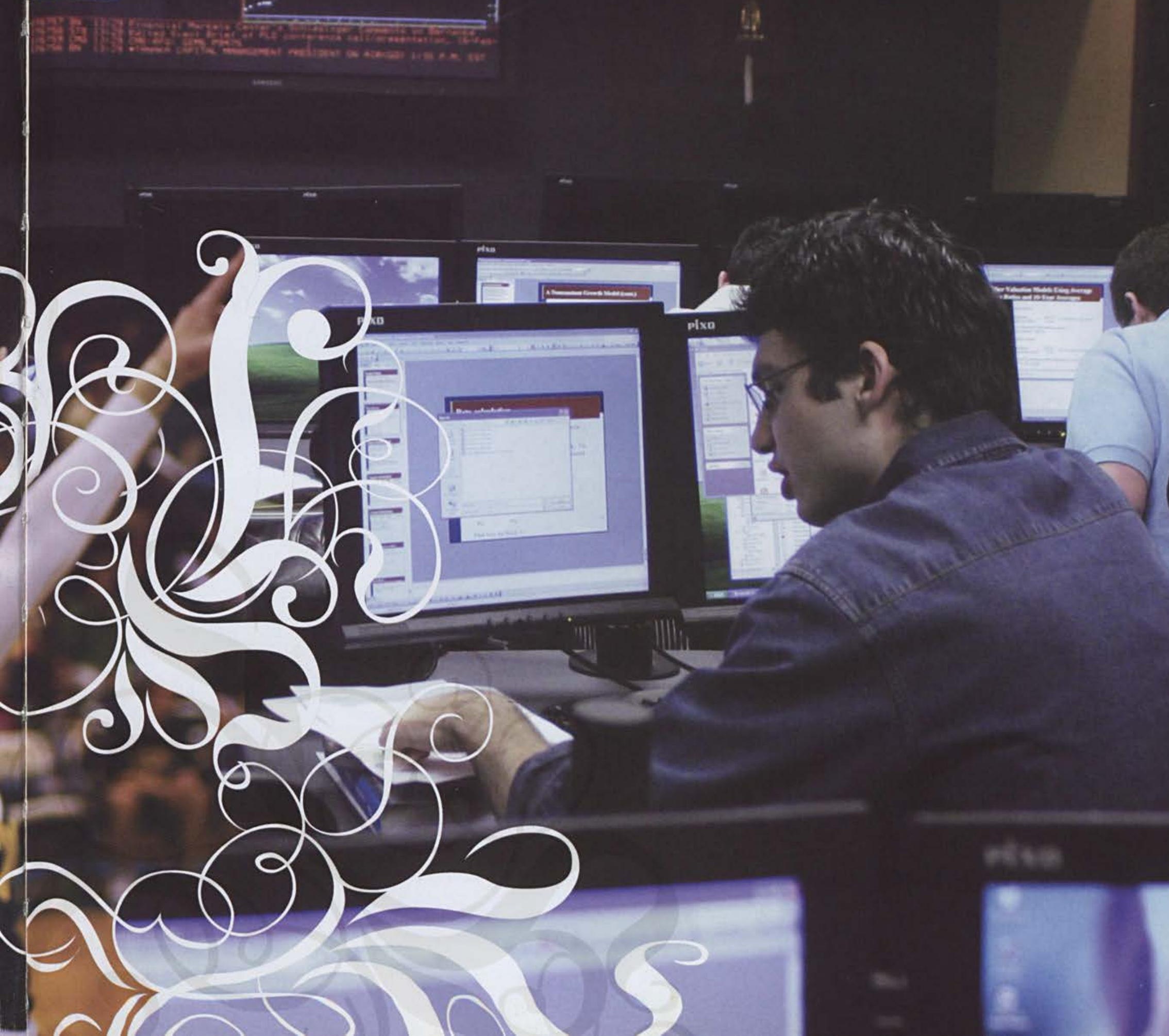


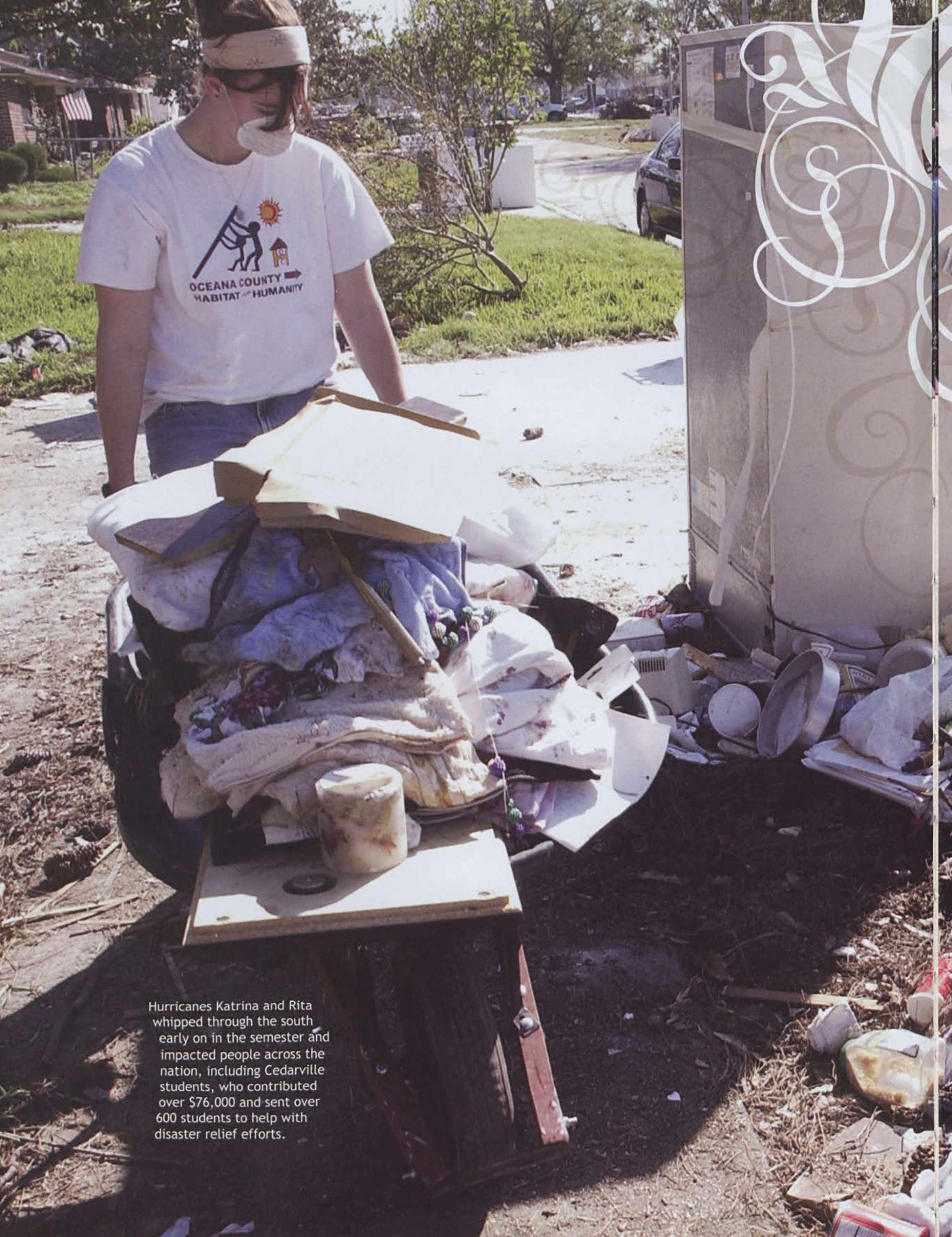




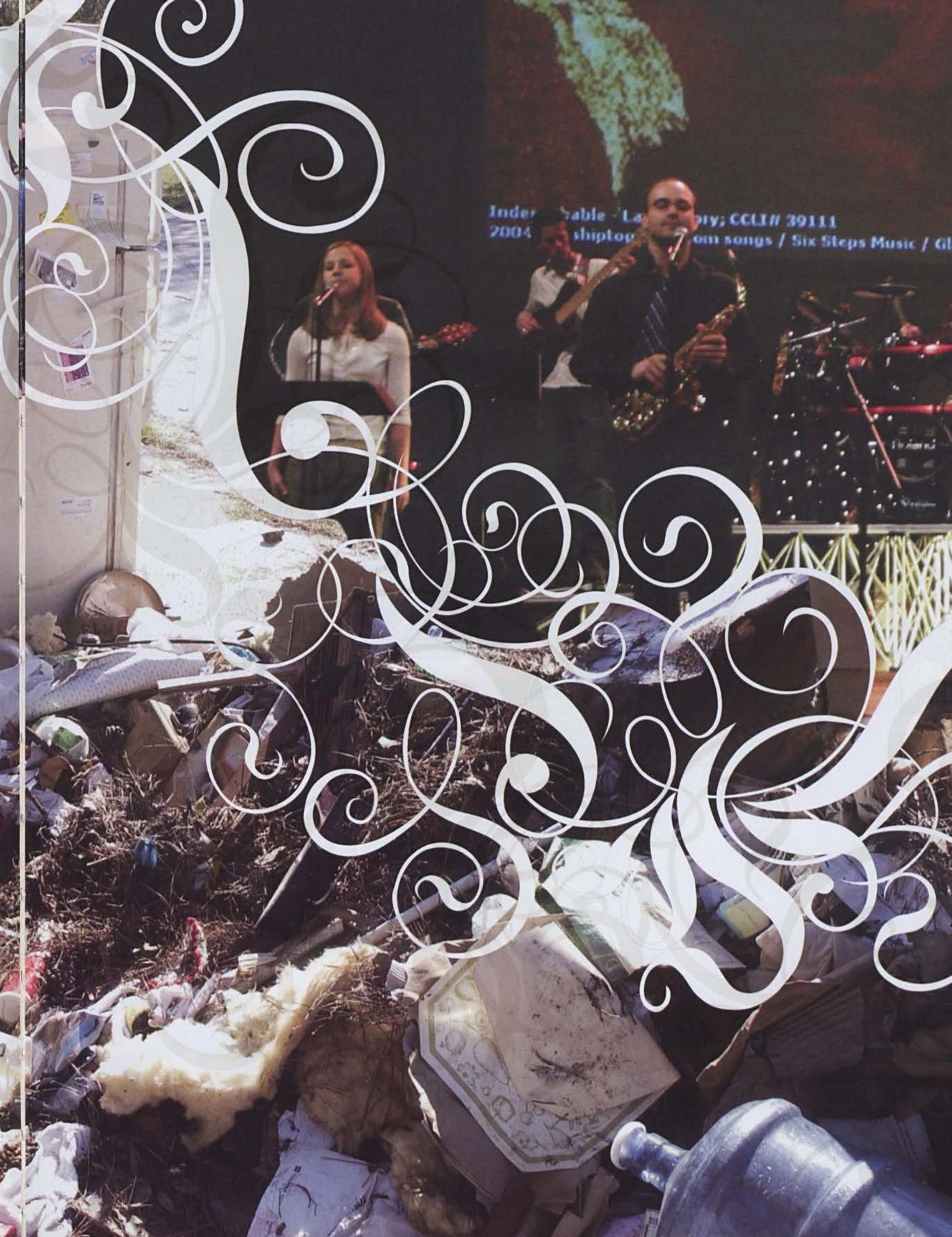


A chapter in the university book closed as the last AD7

fellowship took place in December. After 36 years, Pastor

Rohm decided it was time to draw these services to a close, and a final fellowship worship service ended the tradition.

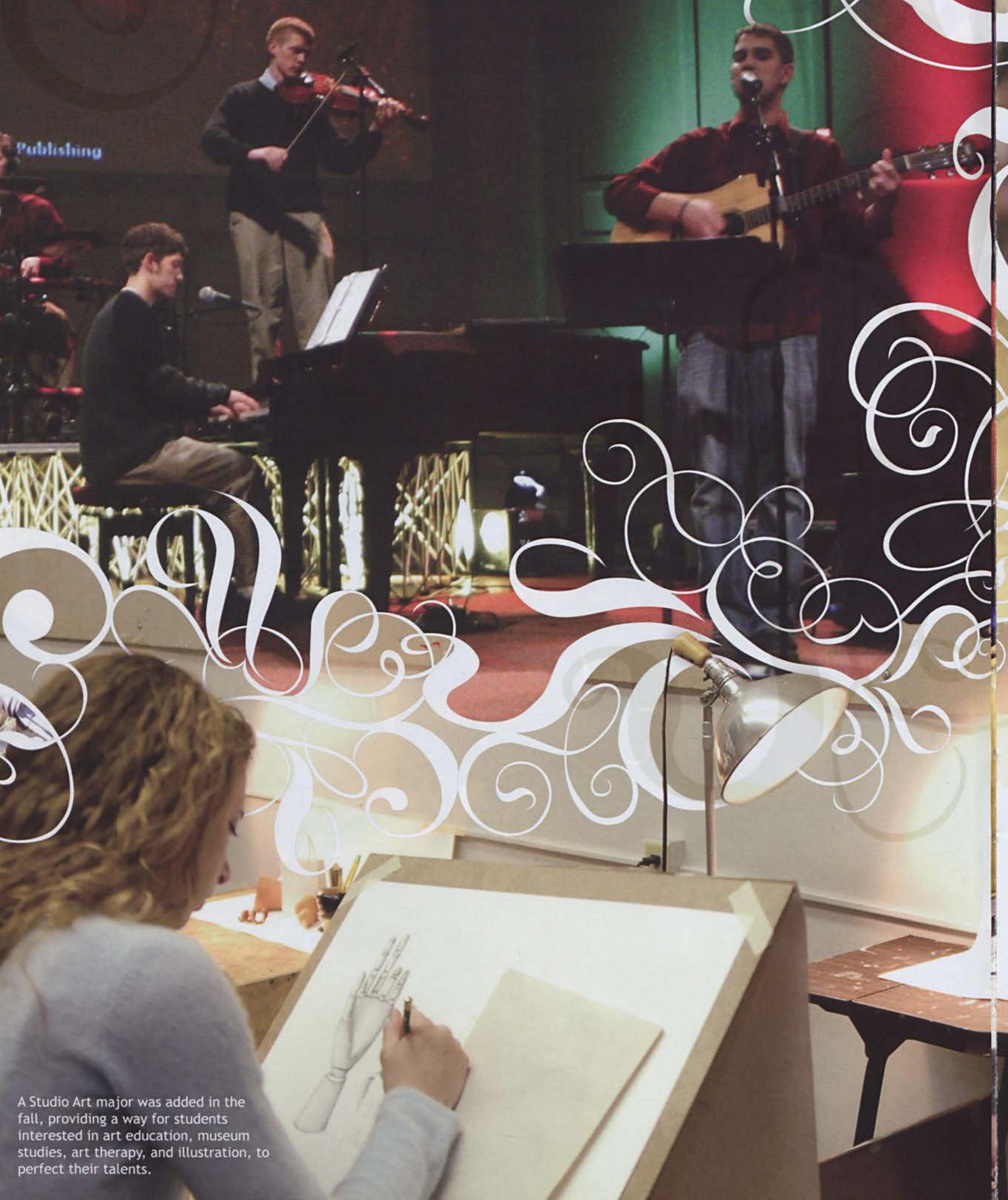




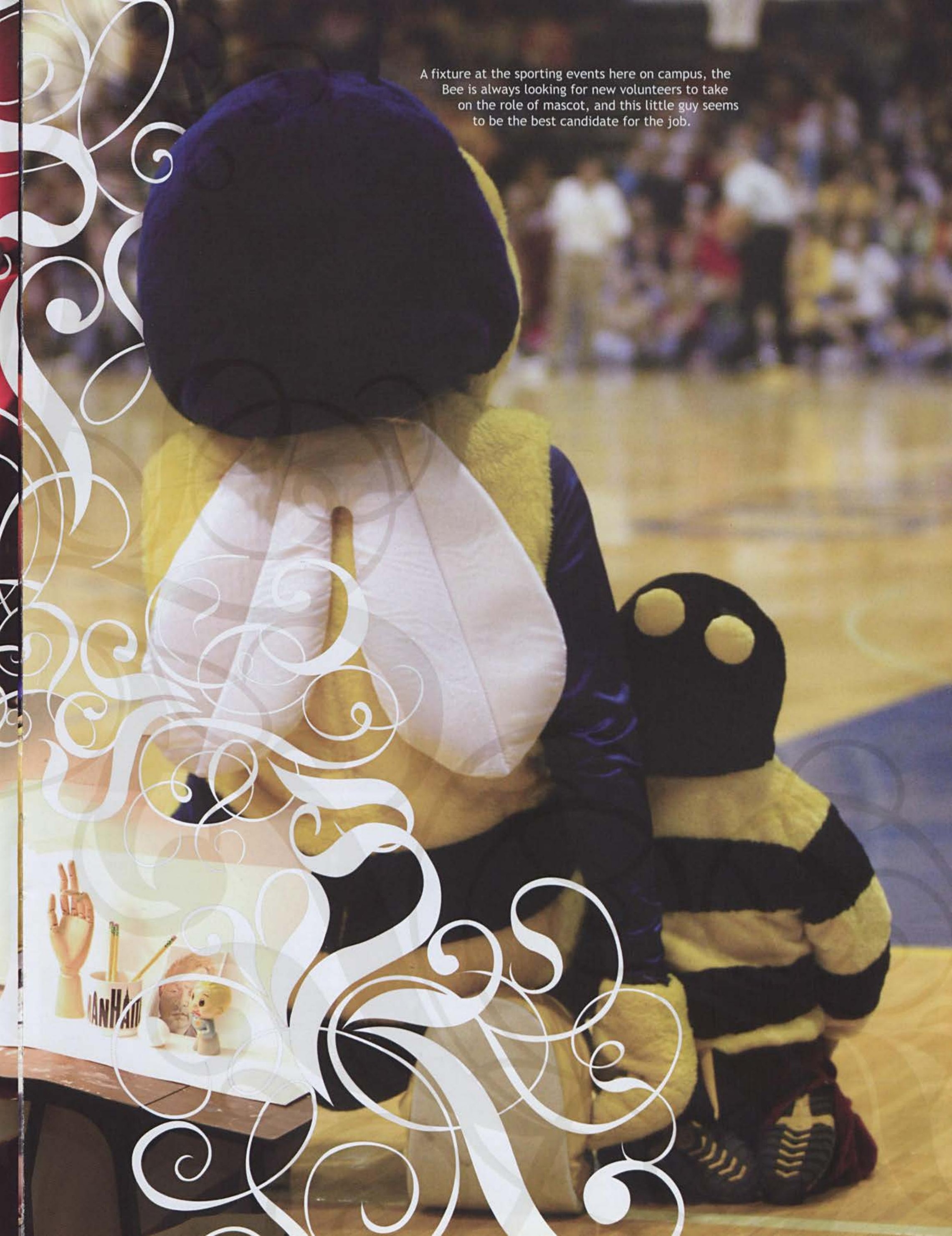


- Dr. Ager sits behind his desk, surrounded by the many papers and books that are commonly found in the office of a college professor.

$\checkmark$ The 1998 MIS team to Hungary spent the summer teaching English and spreading the Gospel. Dr. Ager has led these teams for the past nine years and has built a relationship with Pastor Geza Basci, who has had a great impact on Dr. Ager's life.

A Dr. Ager's three grandchildren, John-Paul, Abigail, and Jacob, celebrate Homecoming 2003 with their grandpa.

Fr. Ager loves his Harley-Davidson bike and enjoys taking it out on the weekends for a spin with his wife, Ruth, sitting behind him.

* Dr. Ager was the 2003 recipient of the Distinguished Educator Award, which is given to faculty members who exhibit truly exceptional performance in the classroom and have contributed greatly to both the intellectual and spiritual development of the students.
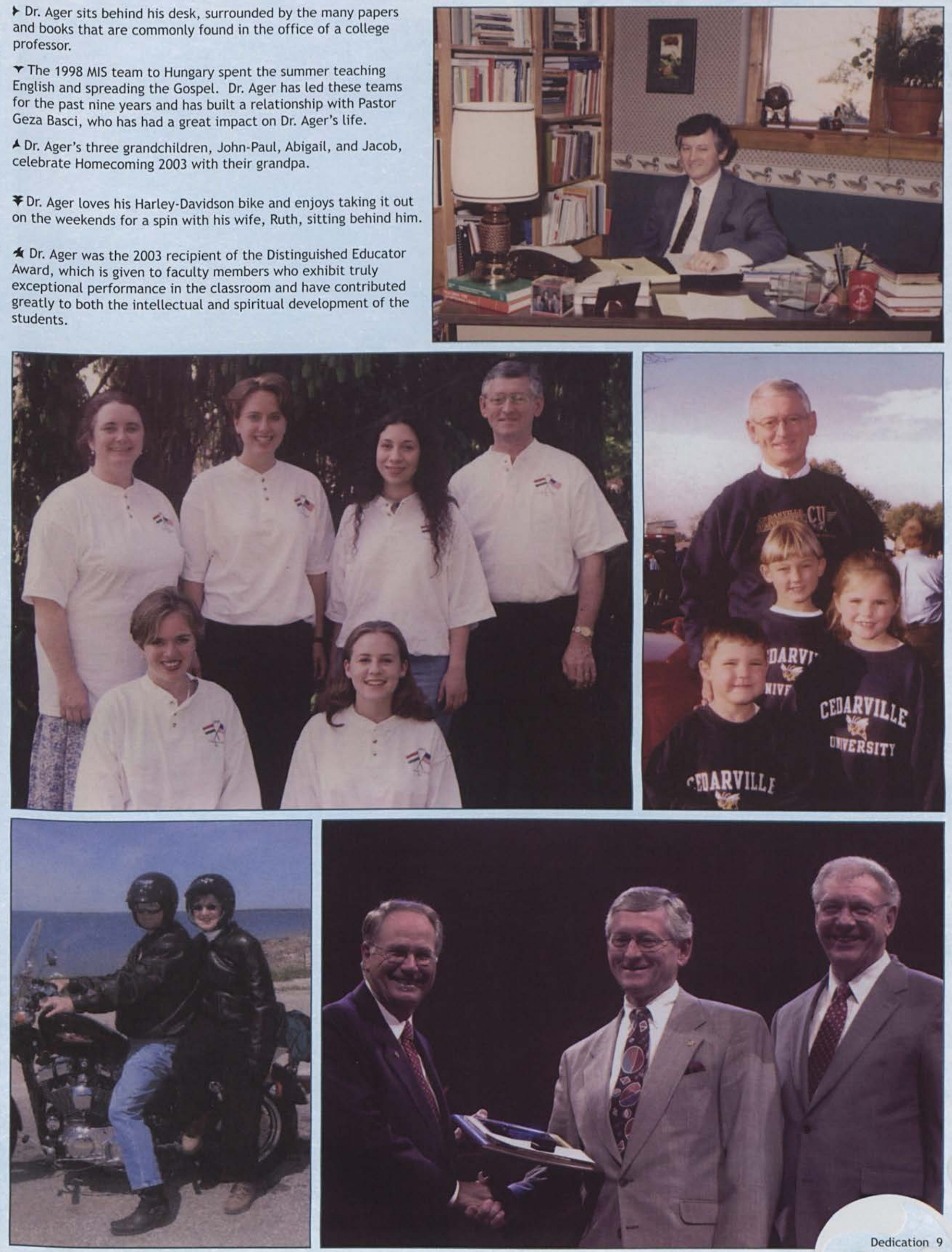


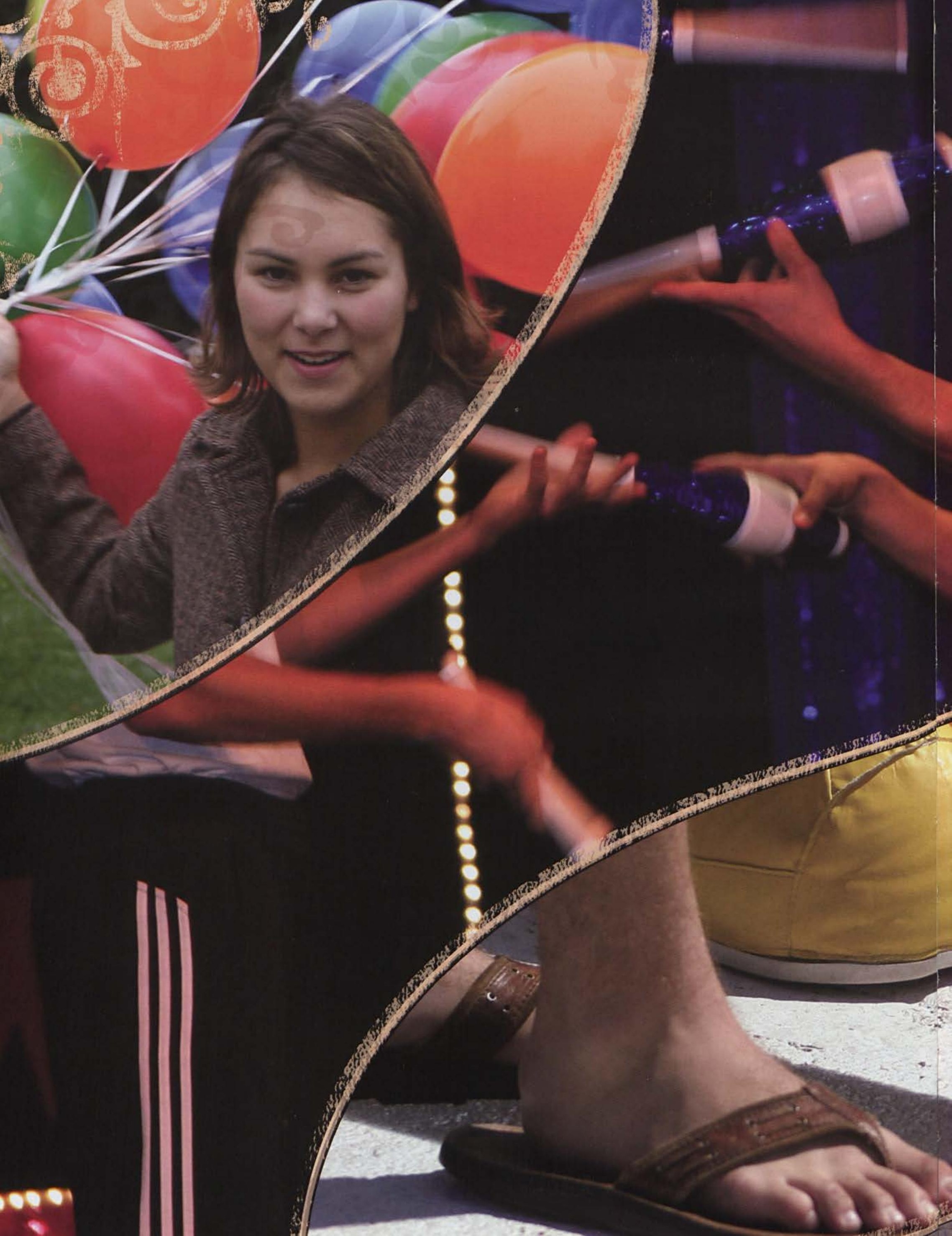





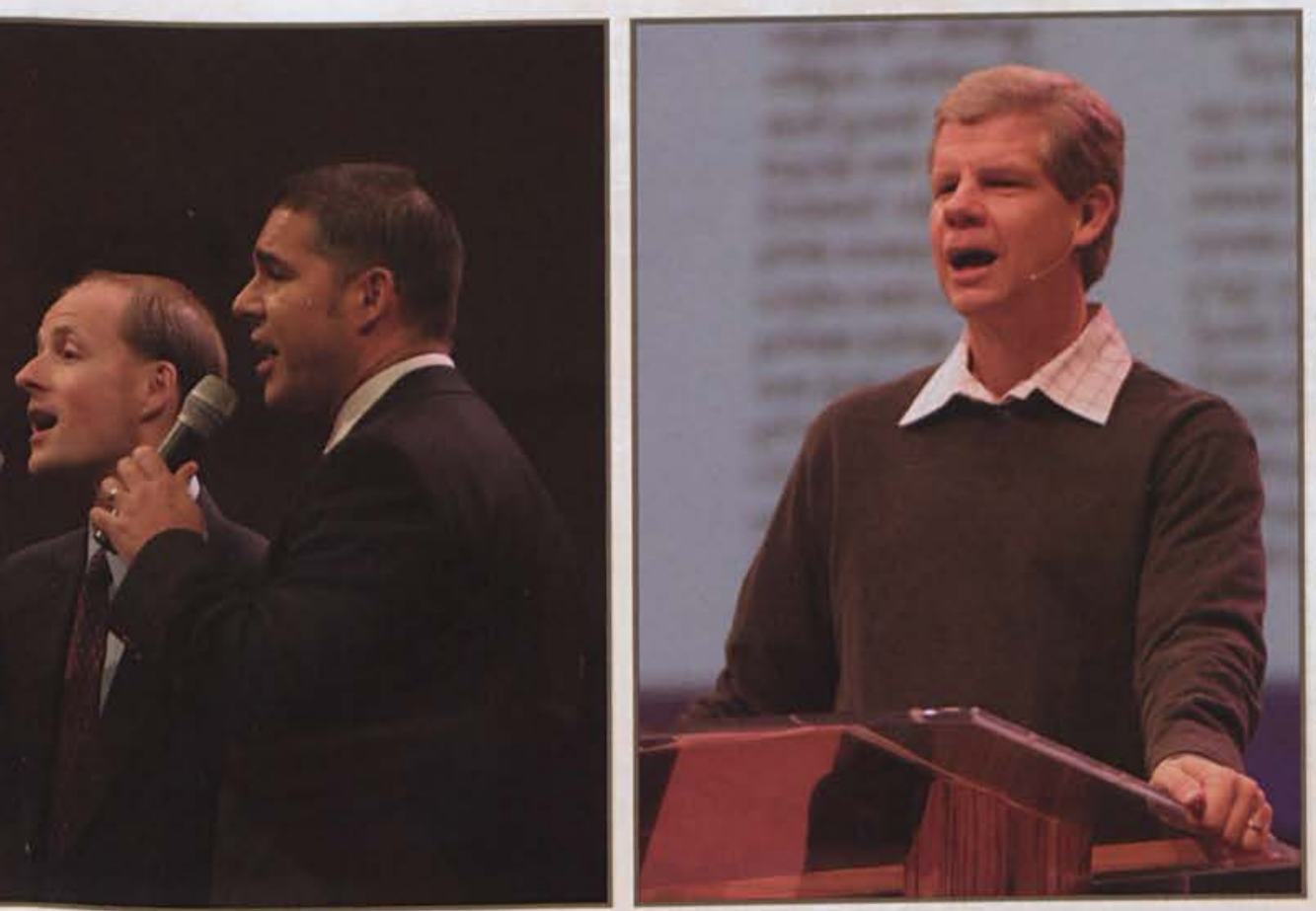

* Andrew Rodriguez, Dave Hoecke, Jeff Beste, and Mike DiCuirci [not pictured] sing a deceptively effortless verse of "Palms of Victory," but as Dave Hoecke remembers, you still have to "remember the right words and hit the right pitches!"

$\checkmark$ Dr. Brown delivers his key address the first night of the conference, touching many students, including junior Christopher Guarnera, who said, "I began to realize a theme that God has been trying to make clearer to me: it's all about Him."

A To show our appreciation for his time and excellent messages, Cedarville presents Dr. MacDonald with a few "gifts," including a university blanket. Dr. MacDonald finds his own way to show his appreciation, at least for the blanket.

$\checkmark$ In addition to the usual singers and instruments, members of the university brass choir help lead worship with an unparalleled power.

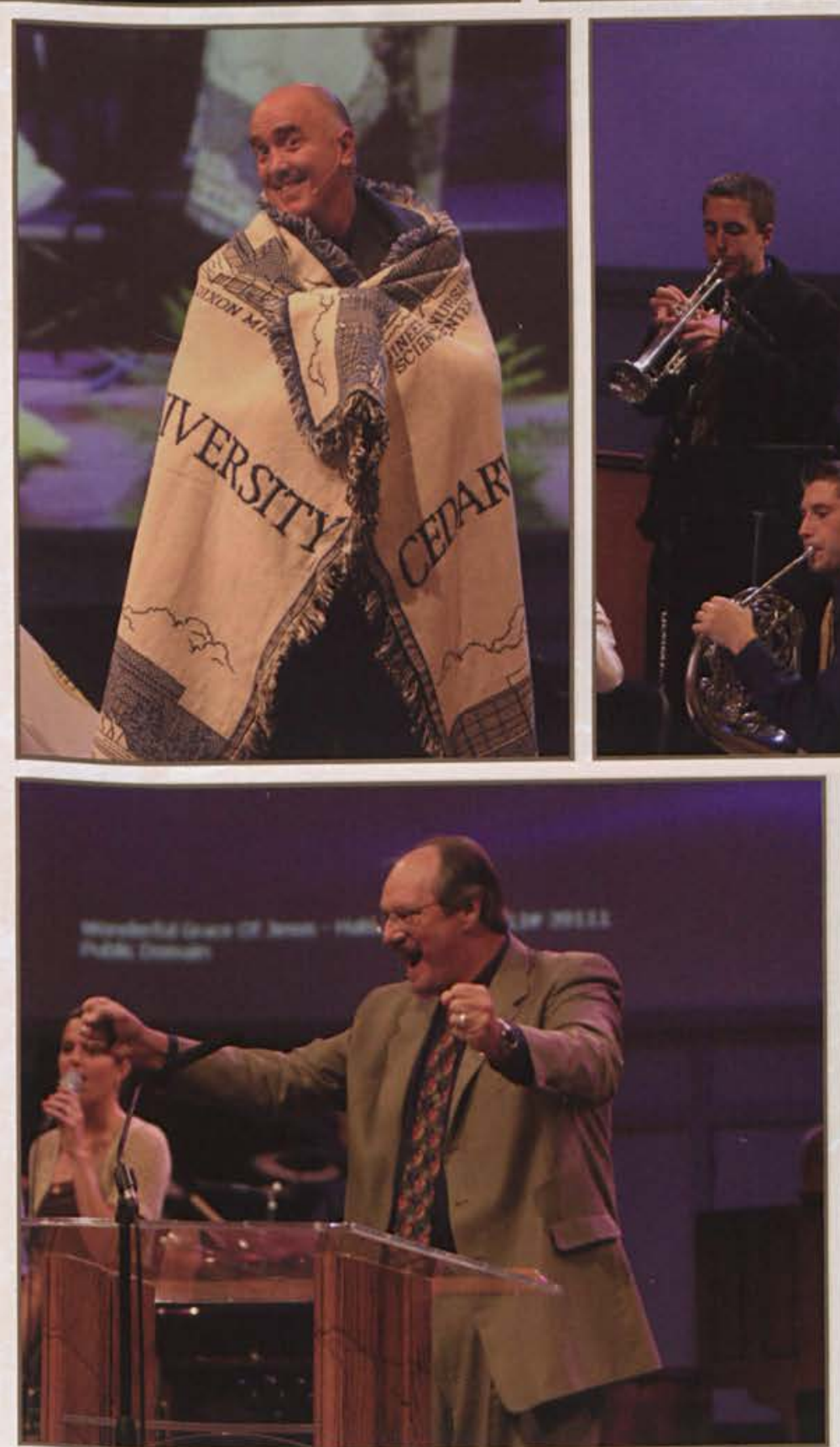

^raditions abound during the Bible Conference each fall, but perhaps none so powerful as when Mr. Michael DiCuirci, professor of music, leads the student body in singing the "Lion of Judah."

Sisters Katelyn and Rachel Talbott weave beautiful harmony into the age-old hymn, "As I Survey."
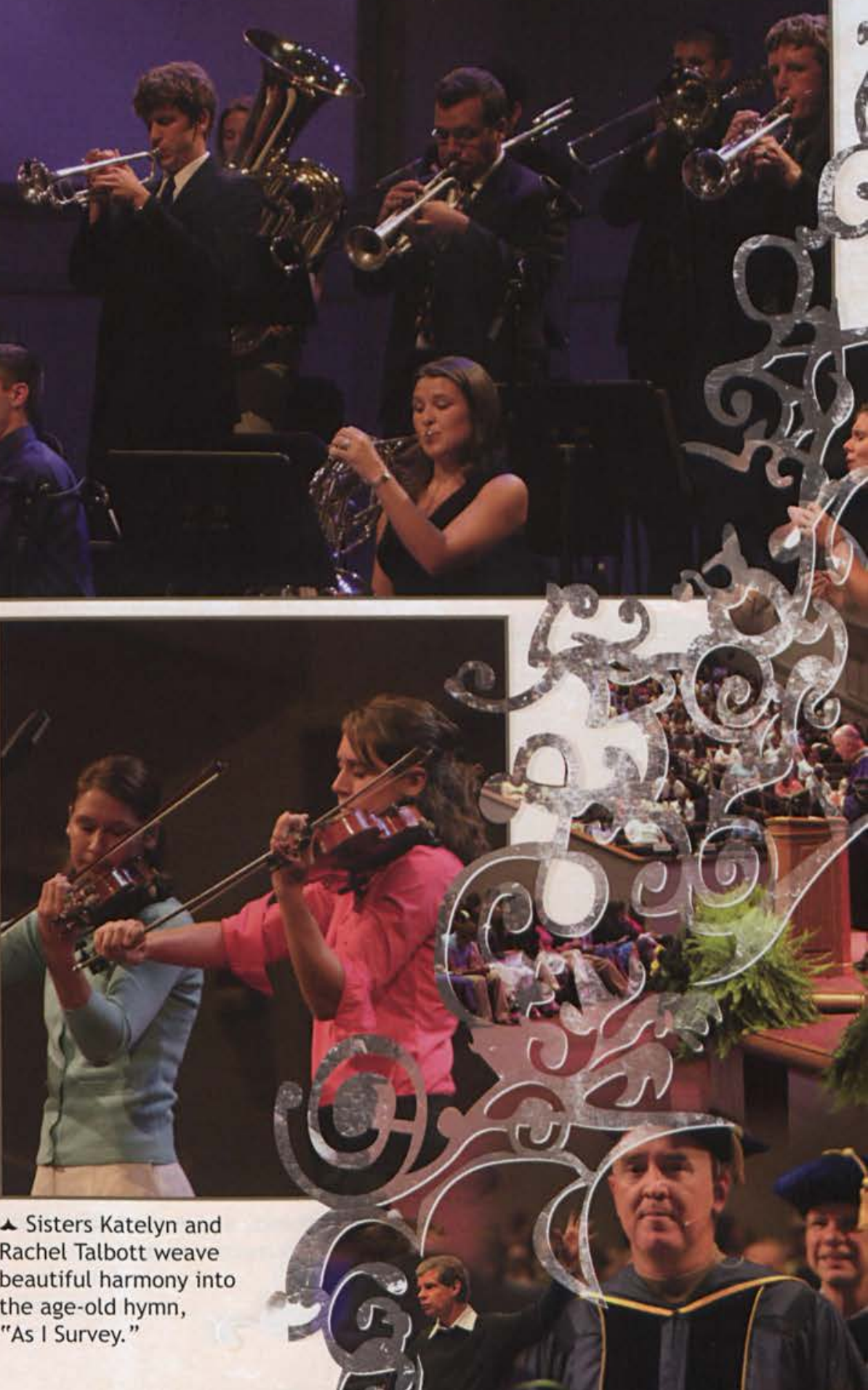



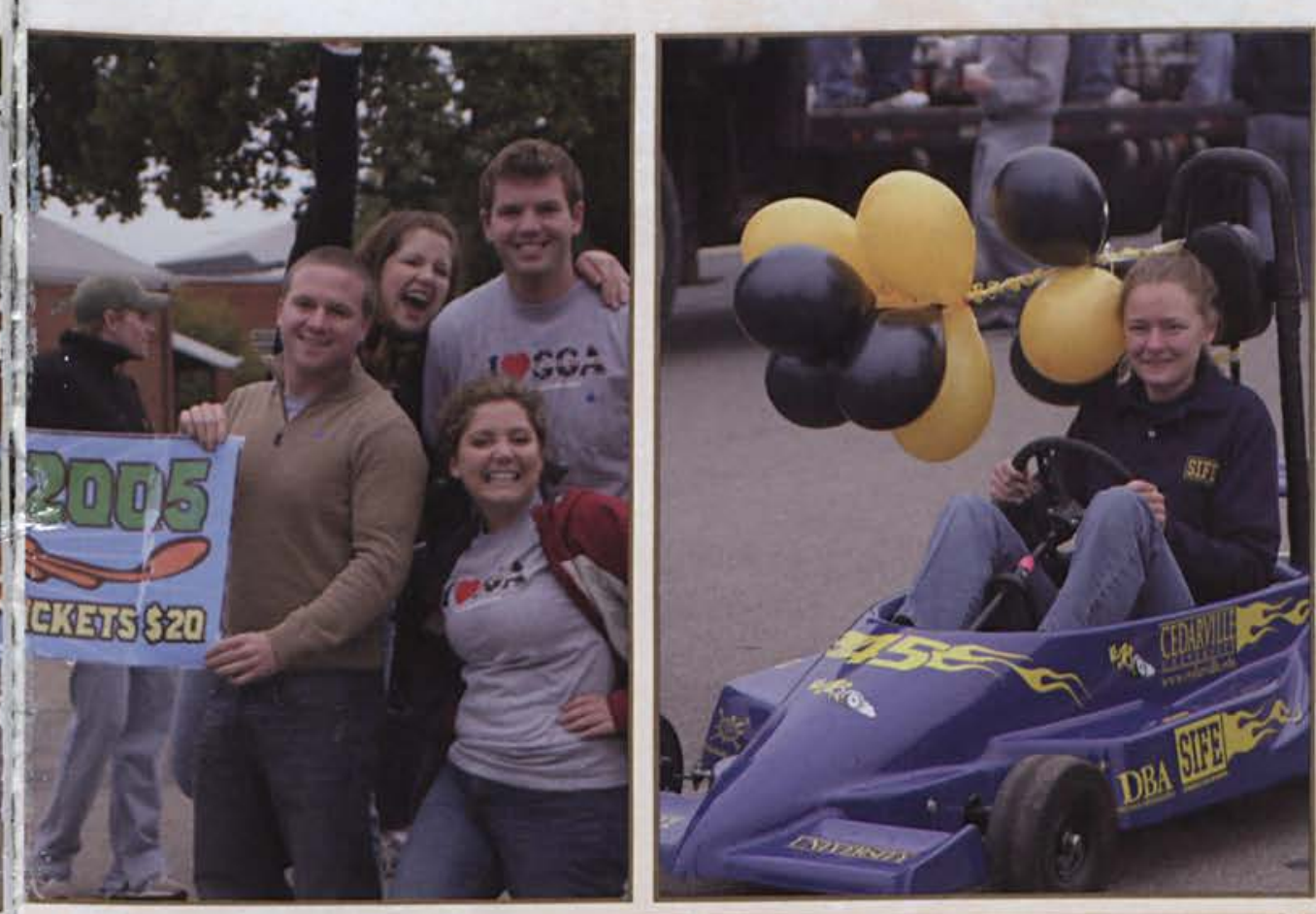

* SGA members Matt Shiraki, Ben Waddell, Holly

Luiken, Lizzie Orrison, and Justin Ely promote the 1950's Homecoming Theme. Lizzie stated, "I was so excited. We had the first performance ever in the parade. Floats are old news; performances are the wave of the future."

$\checkmark$ Resprestenting the SIFE organization, Julie Campbell said, "It's not everyday you get to ride down Main Street in a go-cart!"

A Homecoming Queen Stephanie Hueni and escort Drew Flamm ride in style down the parade route. "My biggest concern was making sure I didnt fall off the back of the convertible as it thrusted forward!" said Stephanie.

- OPE members Andrew Black and Nate Molby pose as members of the Andy Griffith TV show, their theme for this year's float.

$\checkmark$ Delta Pi Sigma girls Christina Cronshaw, Renee Provo, Robin Provo, Amy Elliot, Kate Martin, Pamela Seaymor, Emily Trevino and Mrs. Debra Halsmith show off their 50 's dress with bottled cokes in hand. "Who can resist girls with style and a refreshing beverage?" said student president Kate Martin.
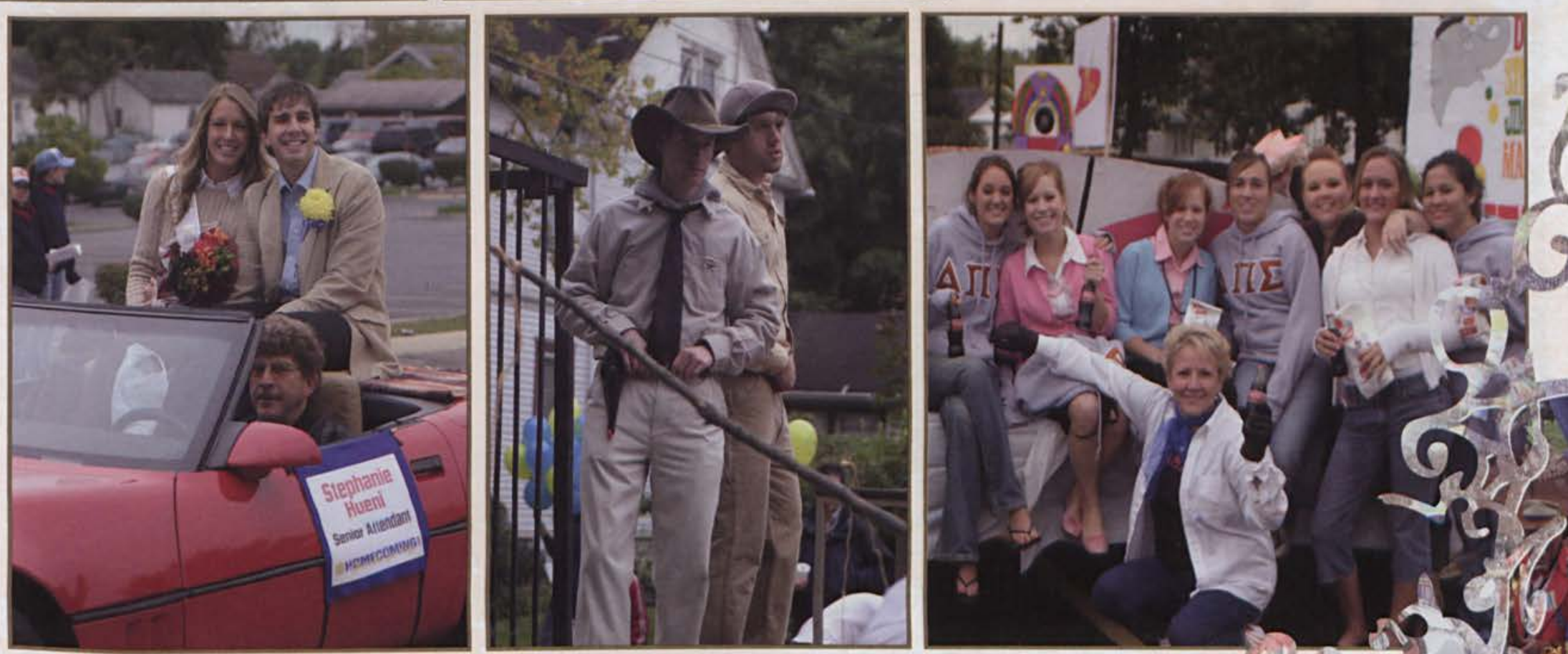

\section{president Kate Martin.}
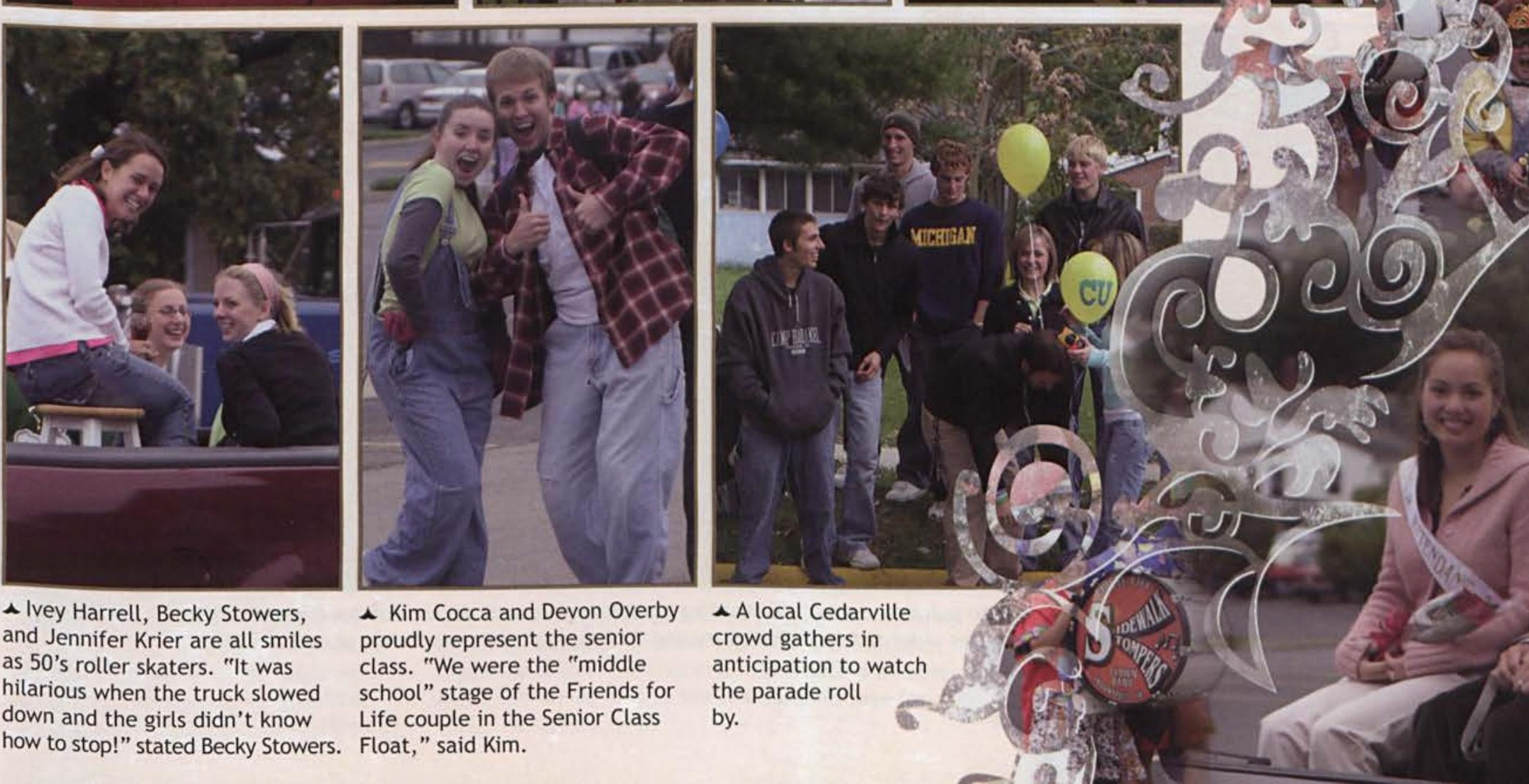

^ Ivey Harrell, Becky Stowers, and Jennifer Krier are all smiles as 50 's roller skaters. "It was hilarious when the truck slowed down and the girls didn't know how to stop!" stated Becky Stowers.
^ Kim Cocca and Devon Overby proudly represent the senior class. "We were the "middle school" stage of the Friends for Life couple in the Senior Class Float," said Kim. 



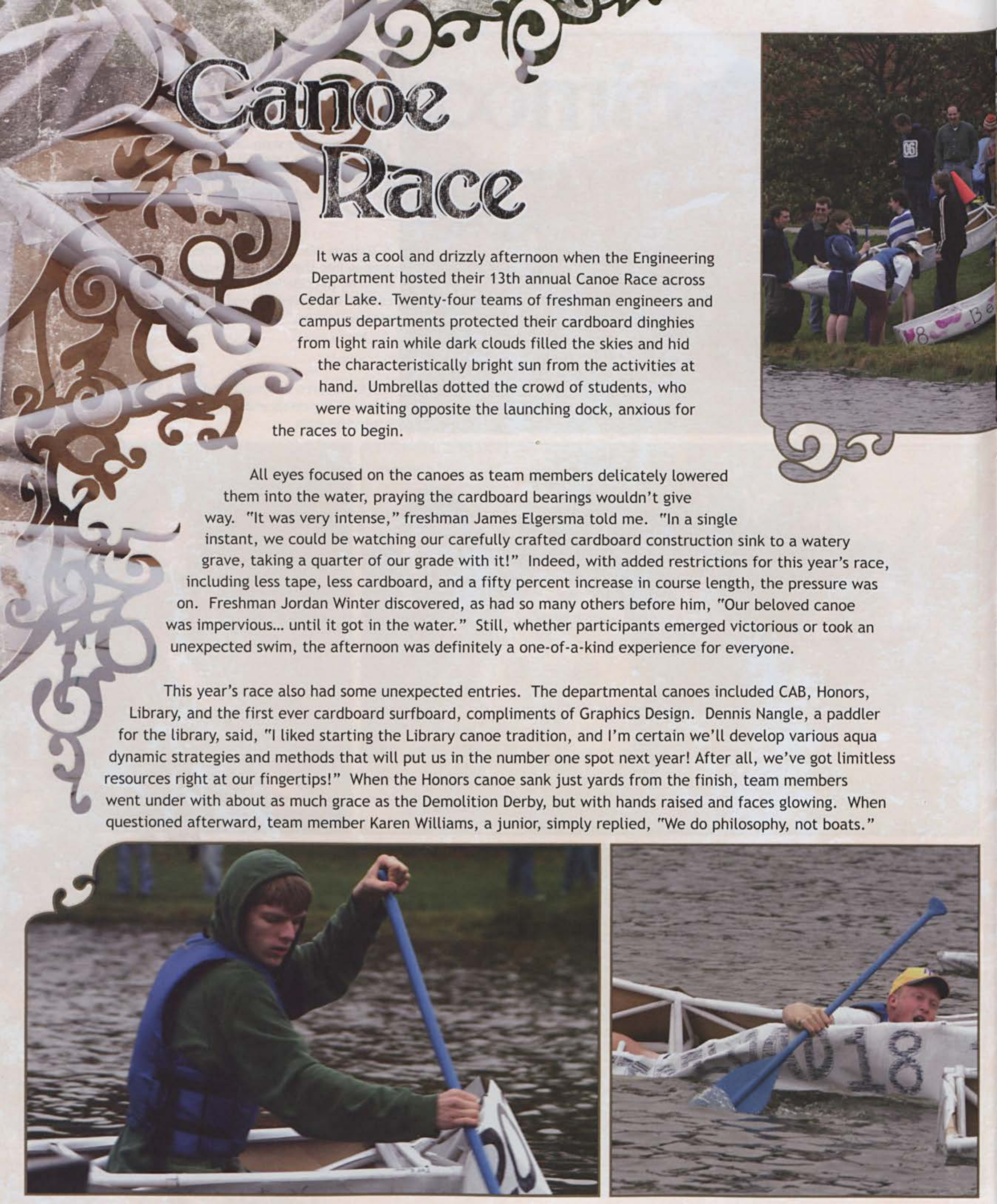

Freshman Miles McGee employs two key strategies to ensure a safe travel across the lake: "We figured out how to use less tape and cardboard for everything, and we paddled across the lake very slowly to make sure we didn't splash water into the boat."

$\Delta$ Clearly a victim of the newly-extended lake route, freshman Taylor Durling takes a spill, similar to several of the shipwrecked freshmen who met their fate on the treacherous waves of Cedar Lake. 


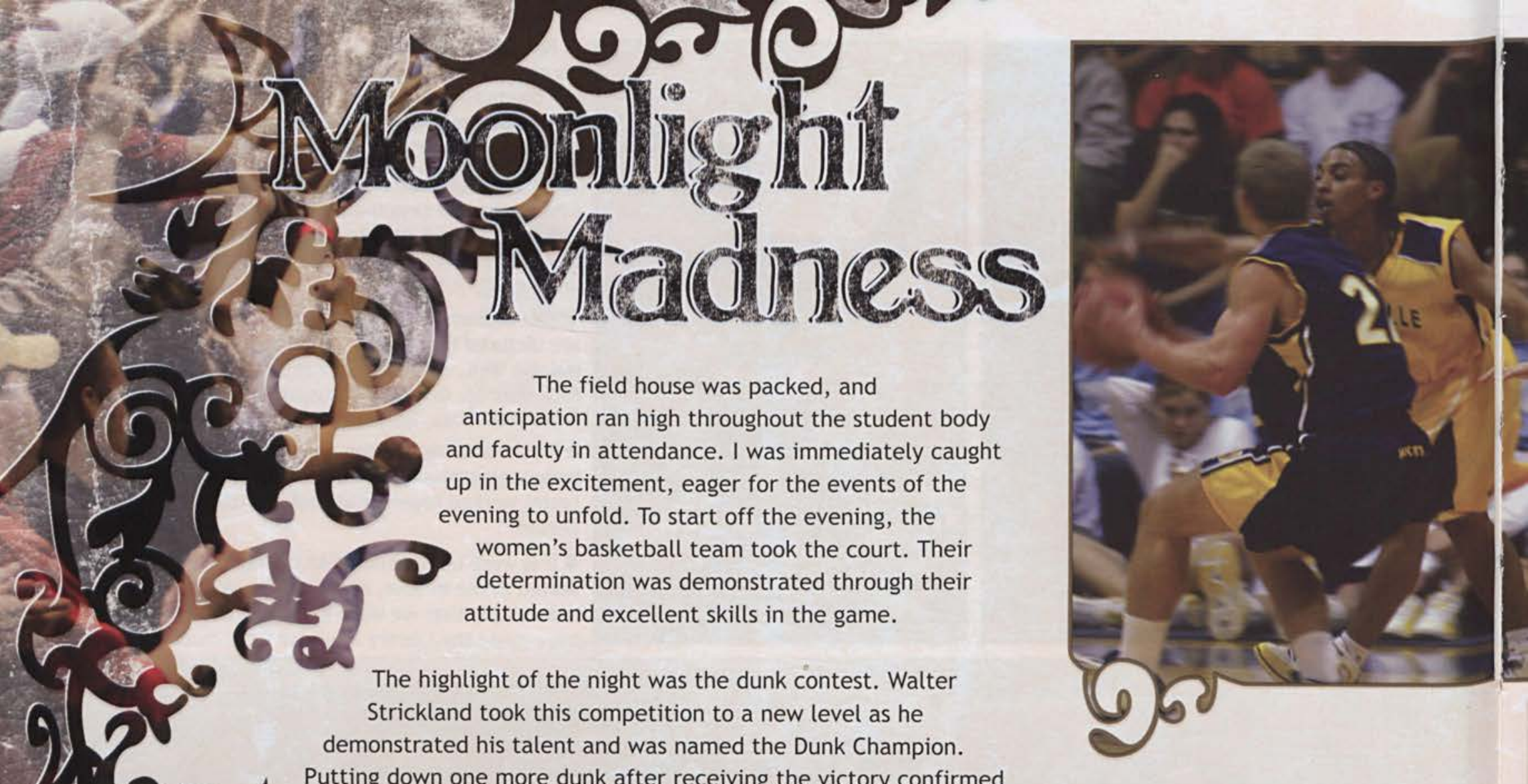

Putting down one more dunk after receiving the victory confirmed

that he was truly the best. The three-point shooting contest between the women's and

men's teams proved to be an exciting event. As the crowd kept score, the men's team easily took over the opposition. In similar fashion, a few faculty members from the Bible Department challenged key administrative faculty to yet another three-point shooting contest. Dr. Scott

Dixon led the Bible Department to victory with ease.

The evening ended with a head-to-head scrimmage of the men's basketball team. The intensity of the game grew quickly as the team showed off their talent and drive for perfection. Halftime gave a few of the male players the chance to goof off with some dunking as well. Freshman Ryan Short brought the fans to their feet as he dunked with ease. "All I could say was 'wow' when Ryan threw down that wicked sweet 360!" said senior Justin Mattern. Freshman Kyle Smith wrapped up his thoughts of the night, saying, "Moonlight Madness was primo. Free drinks, basketballers, hard bleacher seats, overall a good time." This year's Jacket season proves to be promising if both men's and women's basketball teams continue to play with the passion that was displayed on the court tonight.

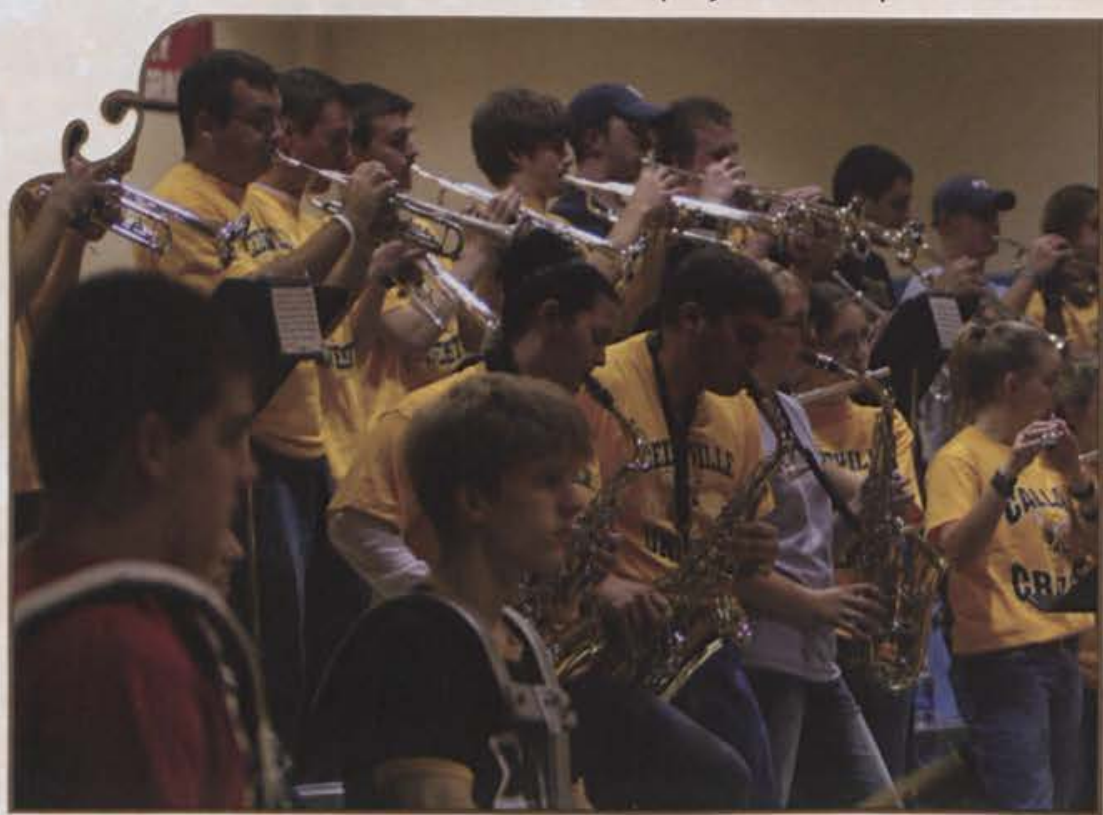

A Michelle Davidson, bass drum player in the pep band, enjoys playing with the group because they make attending the games a lot of fun. "It's good music at a good game!"

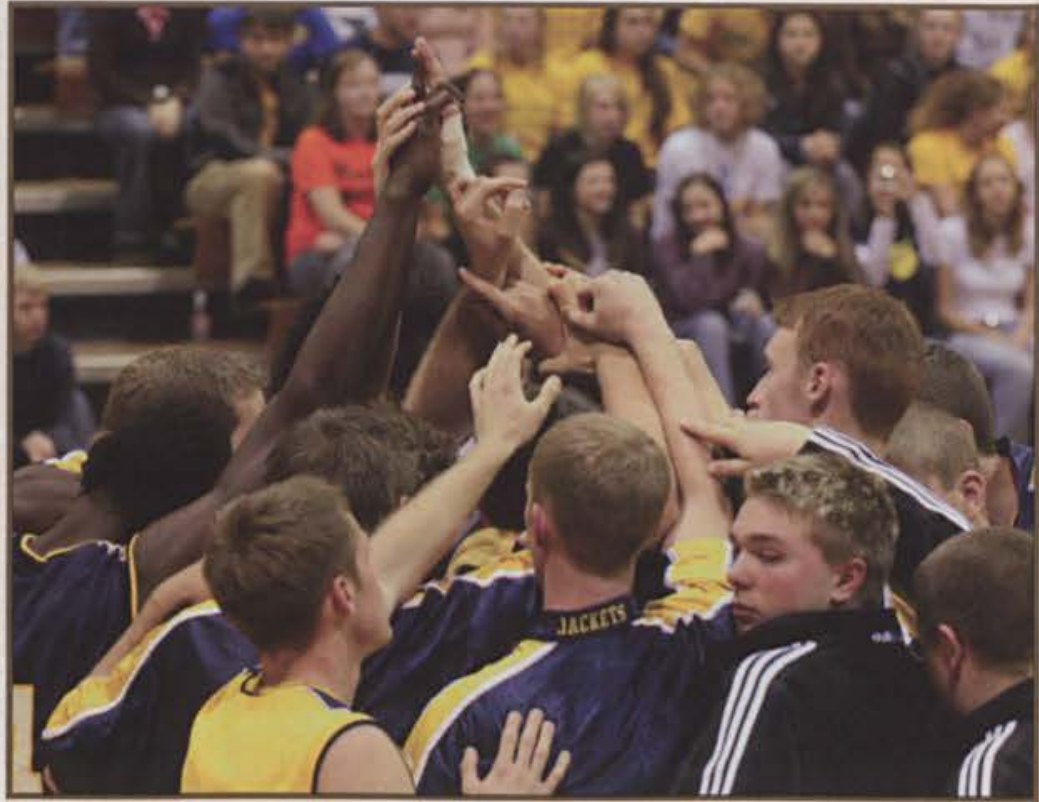

Looking at this picture, it is apparent Who the team plays for. "Major differences coming together in sickness and health for one cause," player Chris Walker states about the team. 


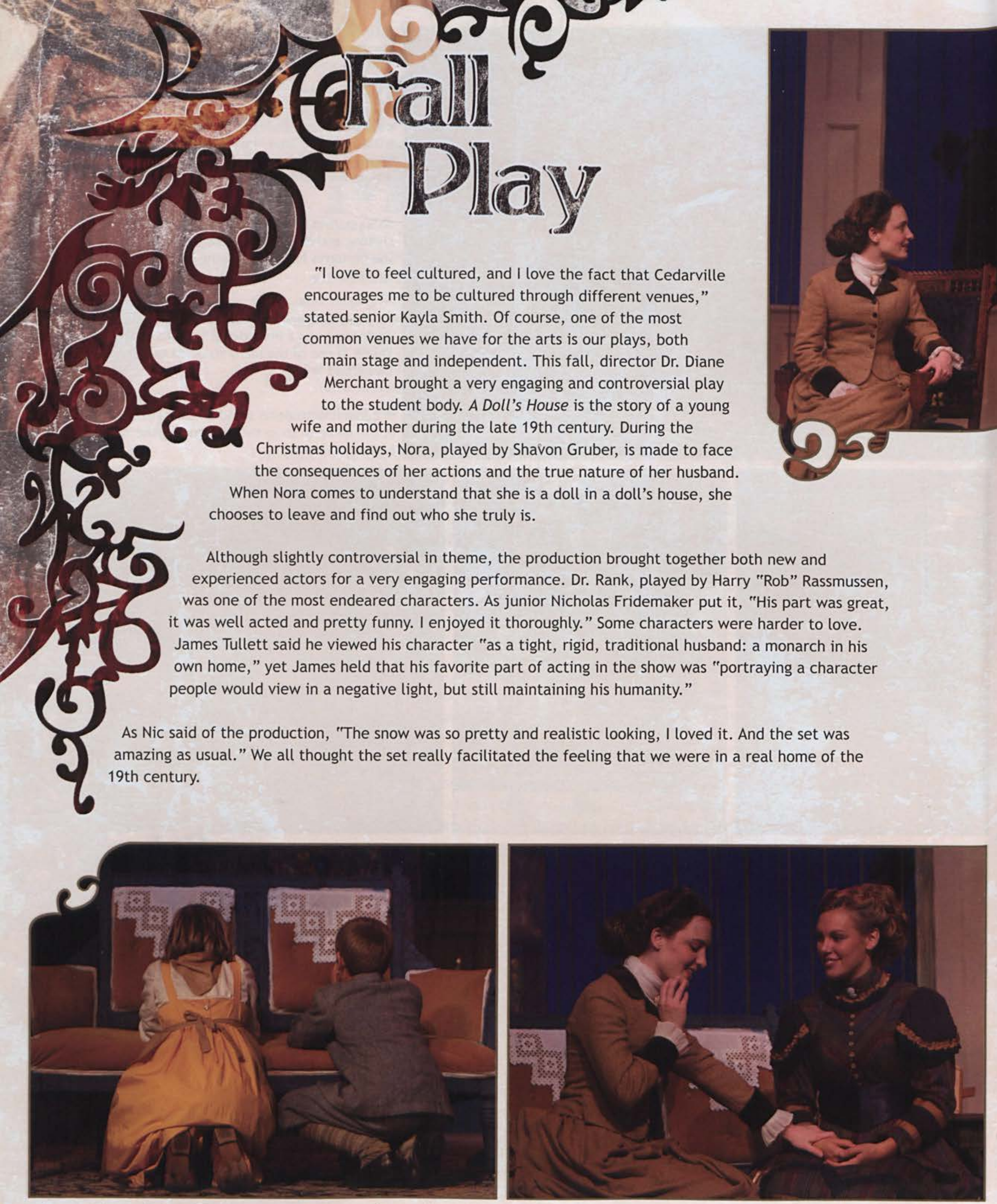

¿ Summer Joy and Adrian Silaghi played the children of Mr. and Mrs. Helmer. Here they are playing "hide and seek" with their playful and childish mother.
Nora's home life is wonderful until her old friend Kristine, played by Victoria Duff, comes into town. Kristine challenges Nora to be honest with her husband and herself. 

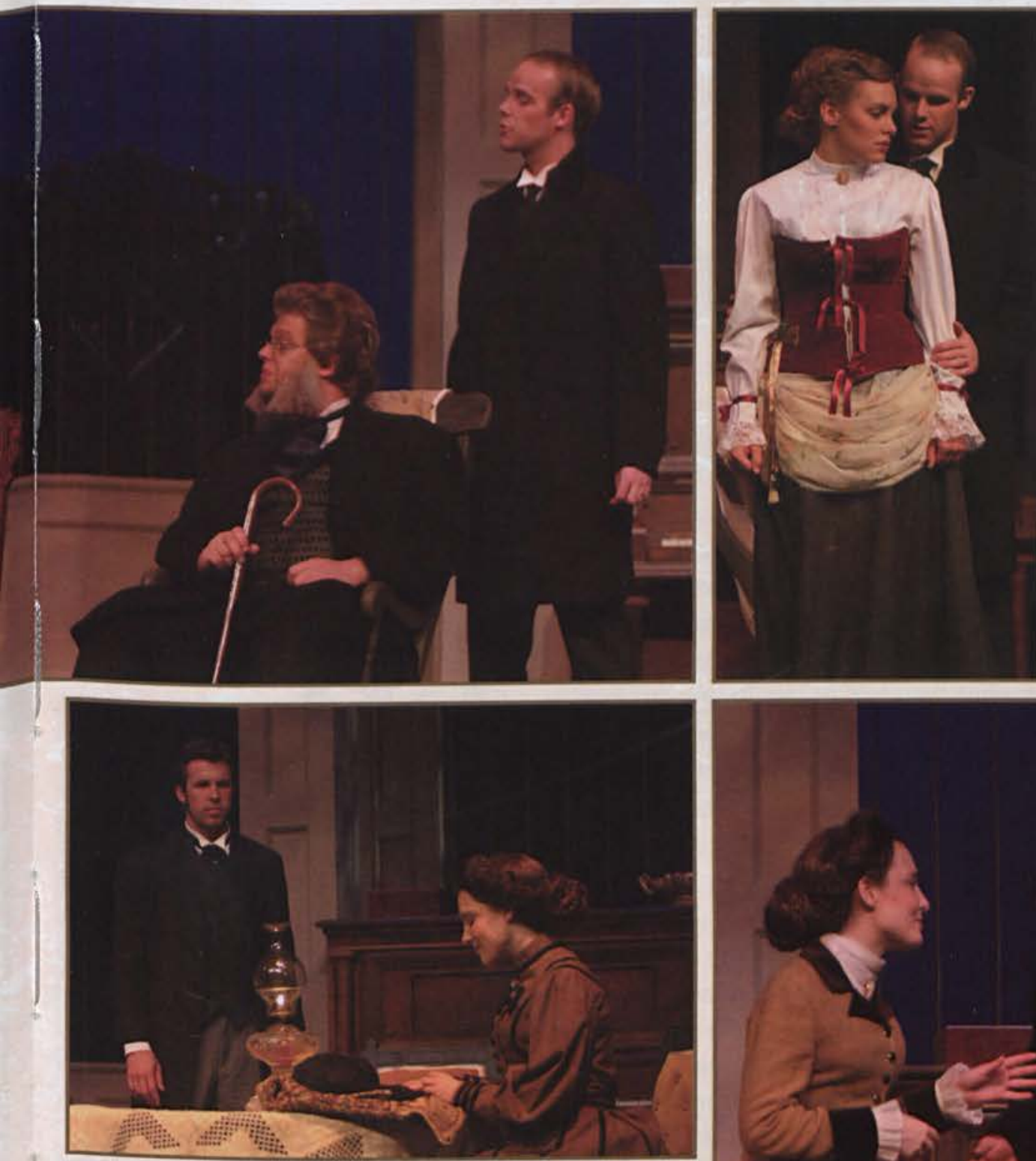

《 The supporting cast of A Doll's House consists of Victoria Duff as Kristine, Rob Rasmussen as Dr. Rank, and Jim Tullett as Torvald Helmer.

$\checkmark$ The nuances of the marriage relationship between Nora and Torvald cause the audience to think about what it takes to be married, a subject some are loath to consider.

Senior Ryan Rotman stated, "I really enjoyed the set on stage. It was very well done. The way they used lights to help with some of the action was excellent, and the snow before the play started really gave it the sense that it was taking place during the winter."

$\checkmark$ "I felt like my character provided the audience with a character with which they could associate and feel empathy for," stated Rob Rassmusen.

A Jeremy Hawkins portrays the antagonist of the story, Krogstad, a severe and cold business man who is only concerned with his business.
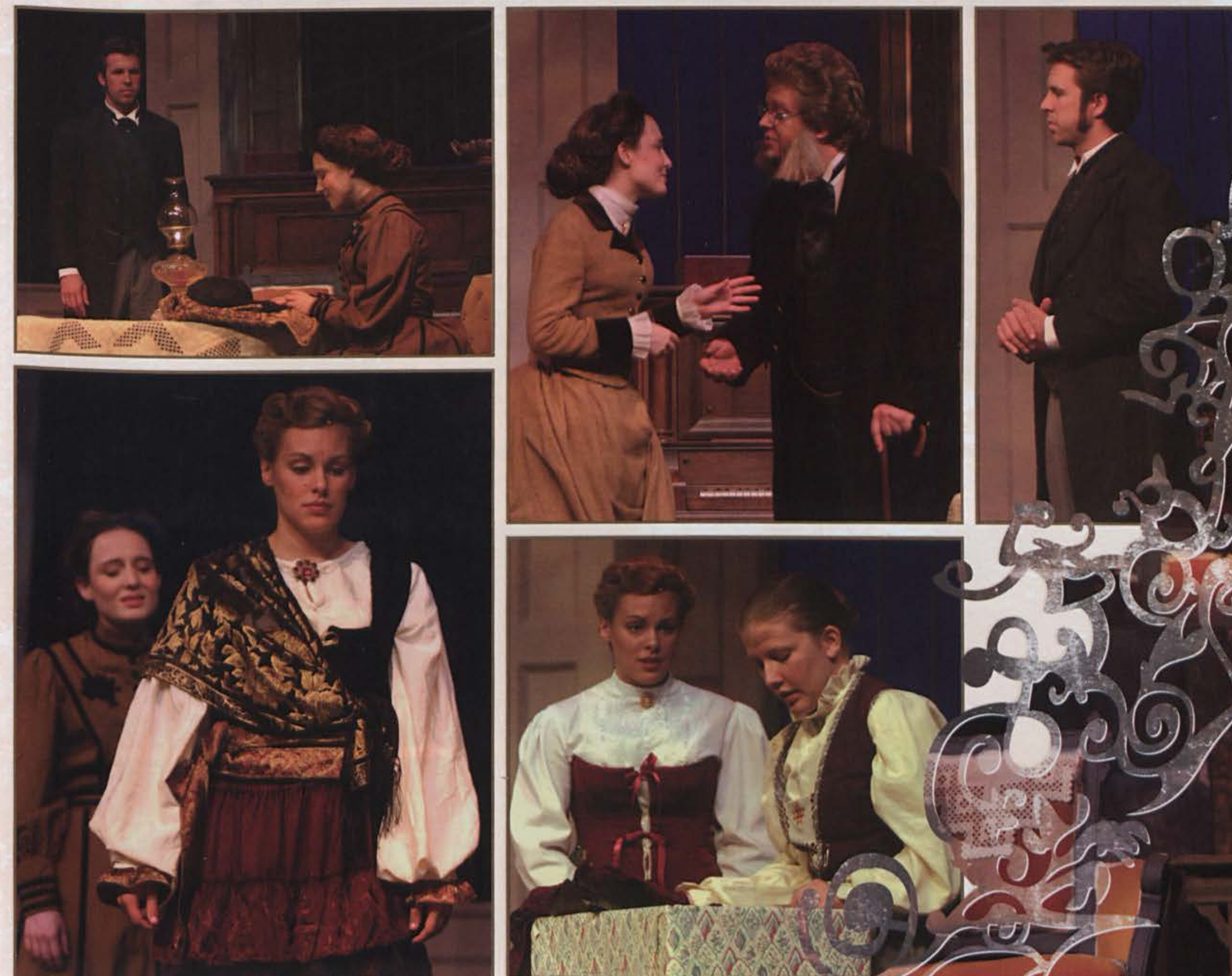

$\alpha$ "I love the play's controversial theme. I think that although the play's message might not align with Cedarville's standards, it raises some issues about divorce and the husband and wife relationship that we as Christians need to examine in our own lives," said Shavon Gruber.

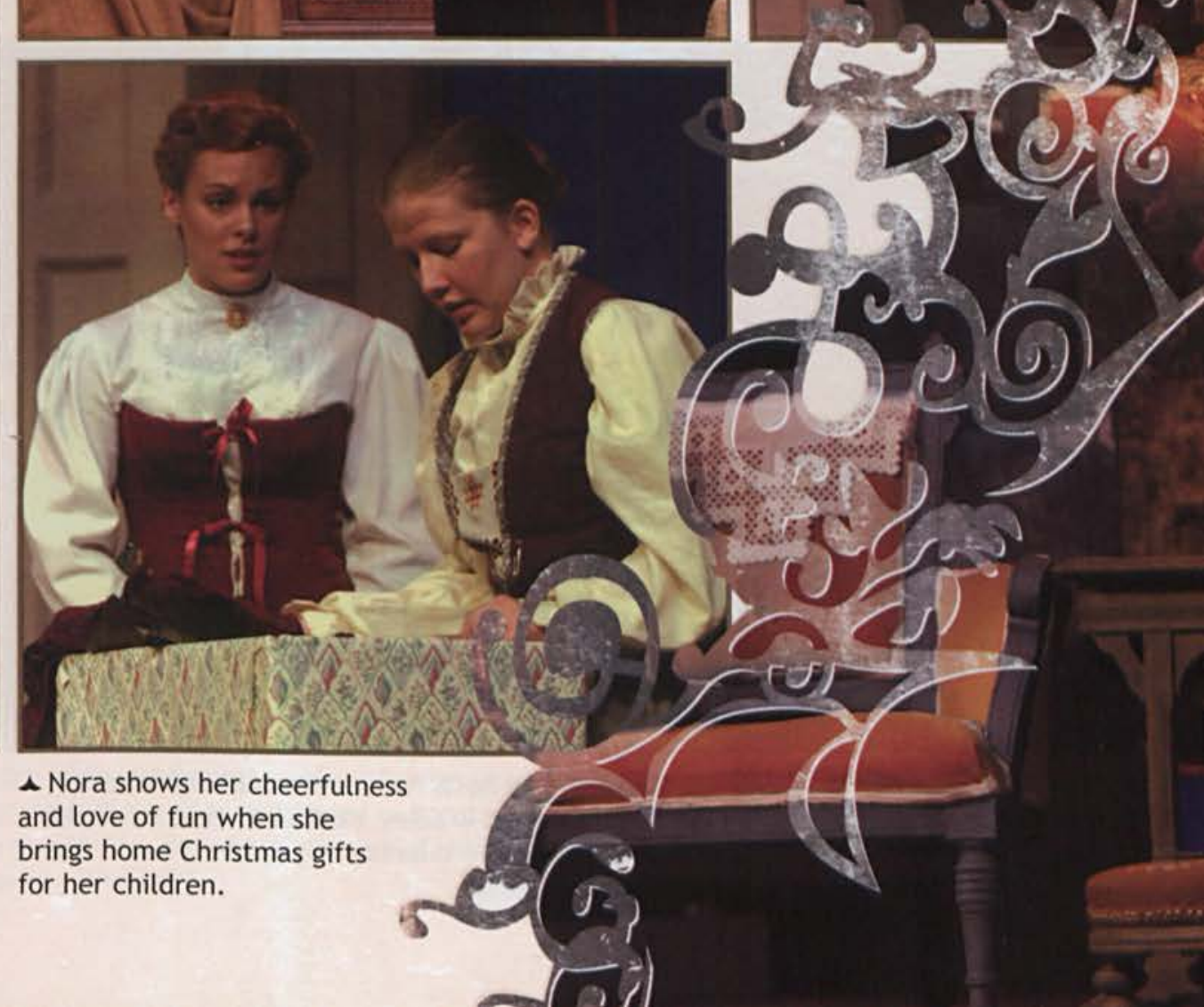



When September is well-worn and the days of summer truly a memory, a crisp edge breaks into the wind, bringing not only the arrival of fall, but the first slump of the school year. Serving as a break from the average and the everyday, the annual Day of Prayer, held September 21, was, as freshman Elizabeth Martin

found, "an excellent time to refocus our thoughts and turn our attention back to prayer."

Light chatter fell away as the Day of Prayer began with a special call to worship by guest singer/songwriter Josh Bales. His unique way of combining traditional hymns and ancient

Christian texts with contemporary music helped set the stage for the prayer and sermon to come. Megan Waters, a sophomore, reminded me that "it is so helpful to remember that we are a part of a bigger story: the historic, Christian narrative."

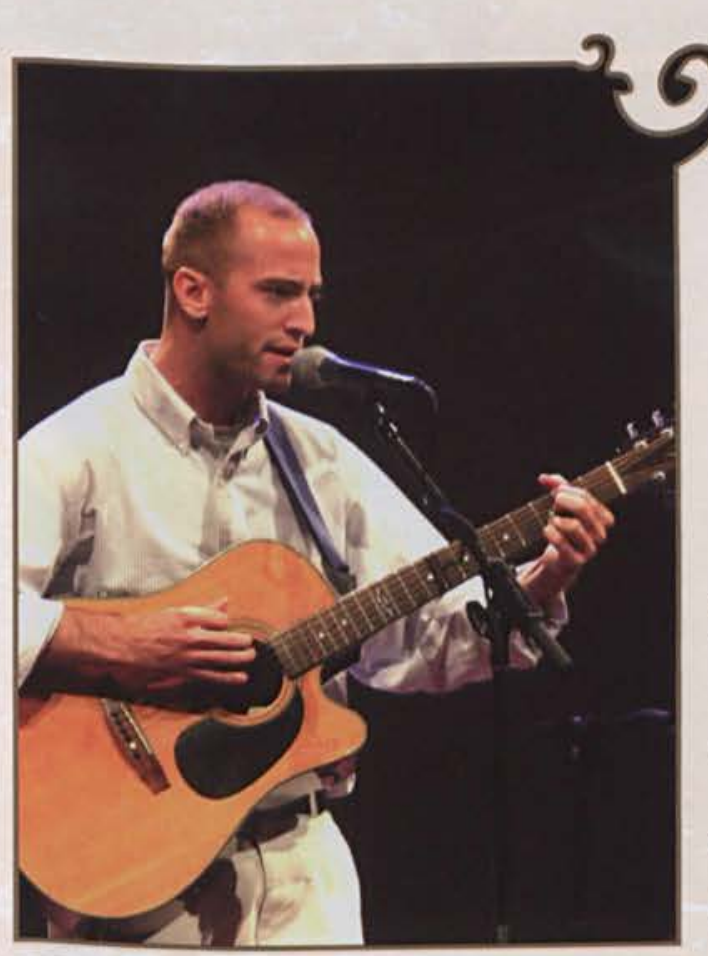

After then praying for the needs of our university

family, country, and world, Dr. Ted Traylor, Pastor of

\section{exise

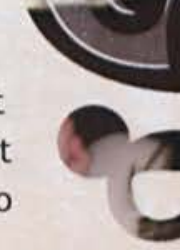

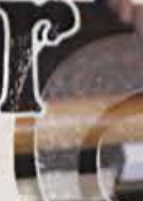

Olive Baptist Church in Pensacola, Florida, encouraged us to learn to pray using the lessons found within the

Lord's Prayer.

The Day of Prayer was anything but ordinary. As sophomore

Debbie Strutton said, "It was a perfect introduction to a day centered around communication with God."

"I found that Josh Bales's emphasis on singing time-worn hymns and reciting communal Christian creeds helped me to see my faith more holistically, as one part of the larger picture of Christianity," stated sophomore Megan Waters.

$\checkmark$ With a refreshing break in tradition of an all-prayer service, Dr. Ted Traylor speaks to the student body about prayer.

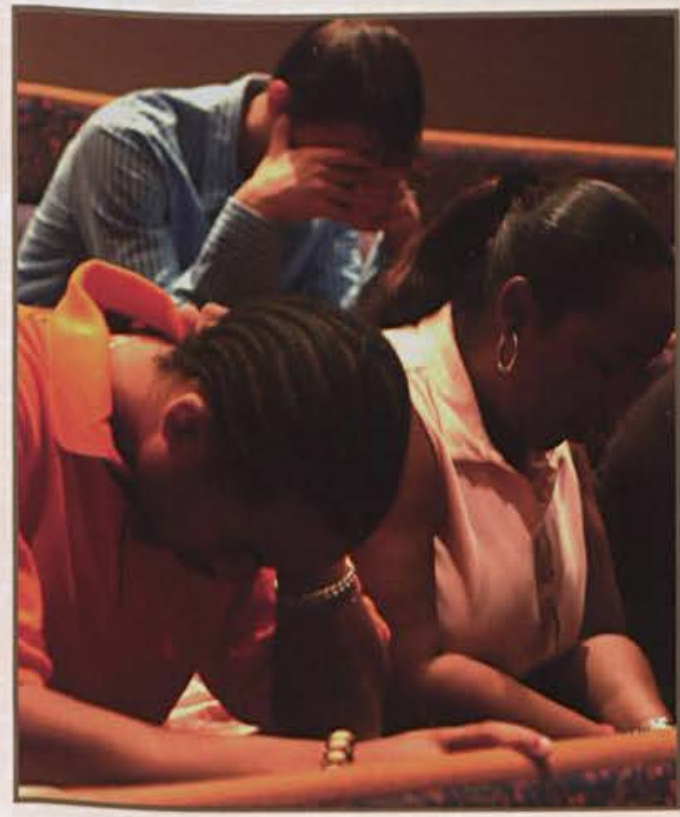

$\downarrow$ Many students take this time to give to God those struggles they are facing. Arielle Dorsey and Mugabe Thomas take the time for some solitary prayer.

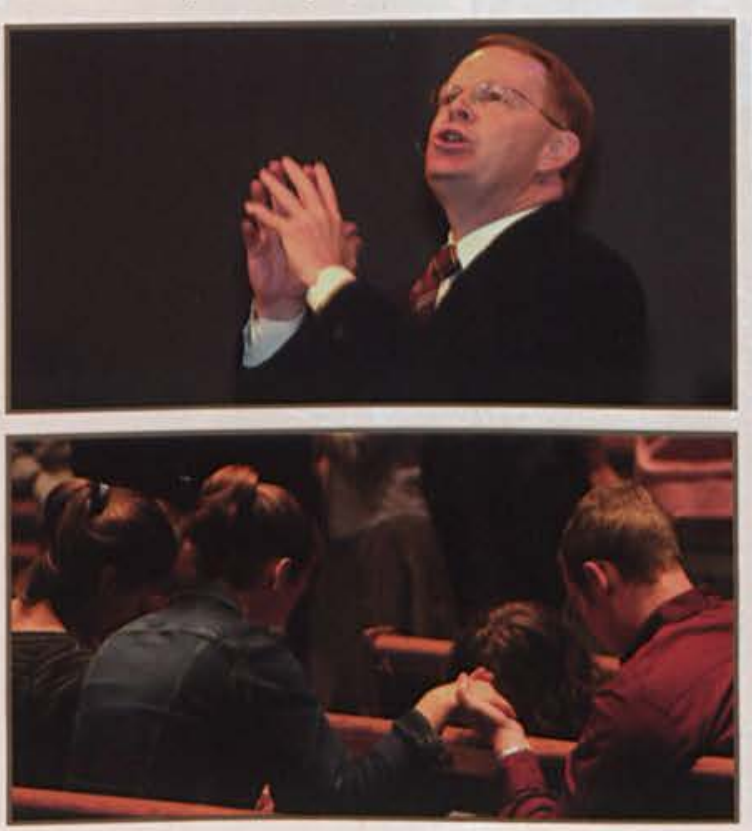

$\downarrow$ Enjoying the time of corporate prayer, freshman Kyle Smith stated, "I am now challenged to focus more on my prayer life, knowing that it will further my relationship with God."

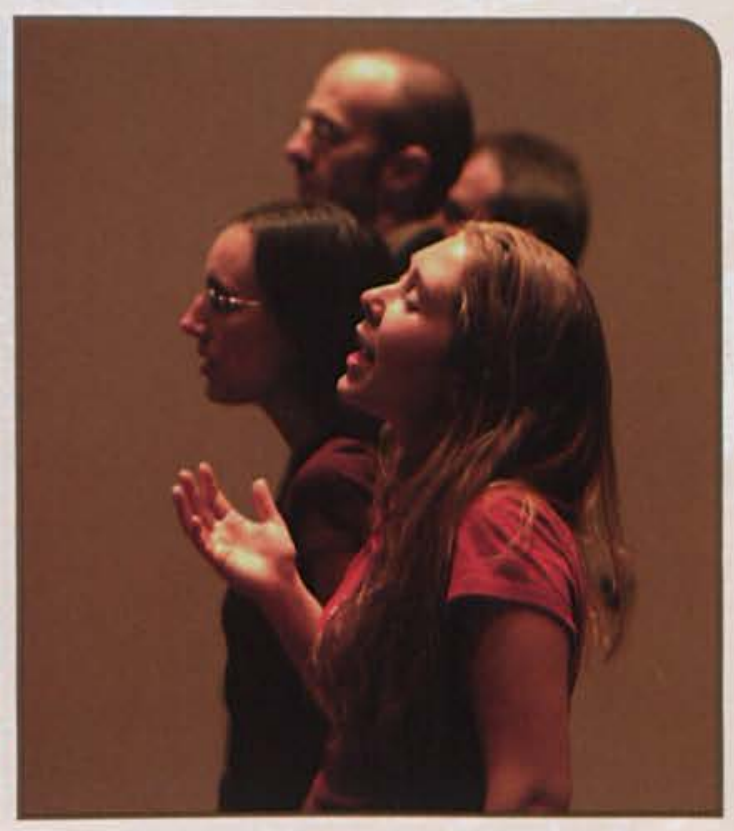

^ Junior Melissa Borton worships her God and praises Him for the privilage it is "to have a day set aside to go before our Heavenly Father as a student body and dedicate our semester to Him for His honor and glory." 
Few events pack the crowds into Cedarville like

Grandparent's Day or Parent's Weekend, and this year the two were combined into one fantastic Family Weekend loaded with activities and events everyone enjoyed.

Ushering in the weekend was the traditional Grandparent's Day chapel, a time for games and laughter, but also tradition and honor. SGA Chaplain, Senior Benjamin Waddel, opened with a theatrical round of "dueling guitars" with his

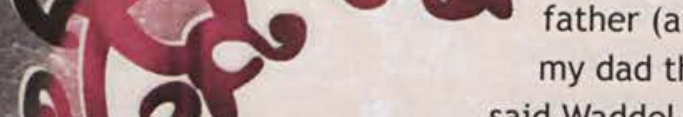
my dad thinks he's better than me, we all know who's better looking," said Waddel, a little tongue-in-cheek. Two pairs of grandparents also competed in a remake of the Newlywed Game for couples married more than 50 years, but they knew how to take it in stride. When asked one trait he appreciated most about his wife, one grandfather simply answered, "She likes me."

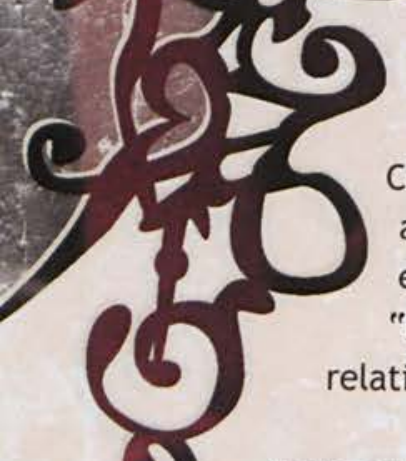

Events continued that afternoon with the traditional choral concert and the Orchestra and Brass Choir concerts. A dazzling mix of spirituals, concertos, and haunting melodies left families moved and in spirited conversation as they planned the rest of their evening. For some, the rest of that evening included a trip to the other side of campus to see Jennifer Knudsen's senior theatre project, "Because I Knew You," a collection of songs and sketches outlining some of the most important relationships we make during our lifetime.

Cedarville's \#2 ranked women's basketball team rounded out the weekend with an amazing performance Saturday against \#3 Indiana Wesleyan at the Cedarville Women's Basketball Classic. Although victory slipped through their fingers in the last moments, the sportsmanship and team spirit the girls displayed will live on in the memories of spectators, as will the remnants of Family Weekend 2005.

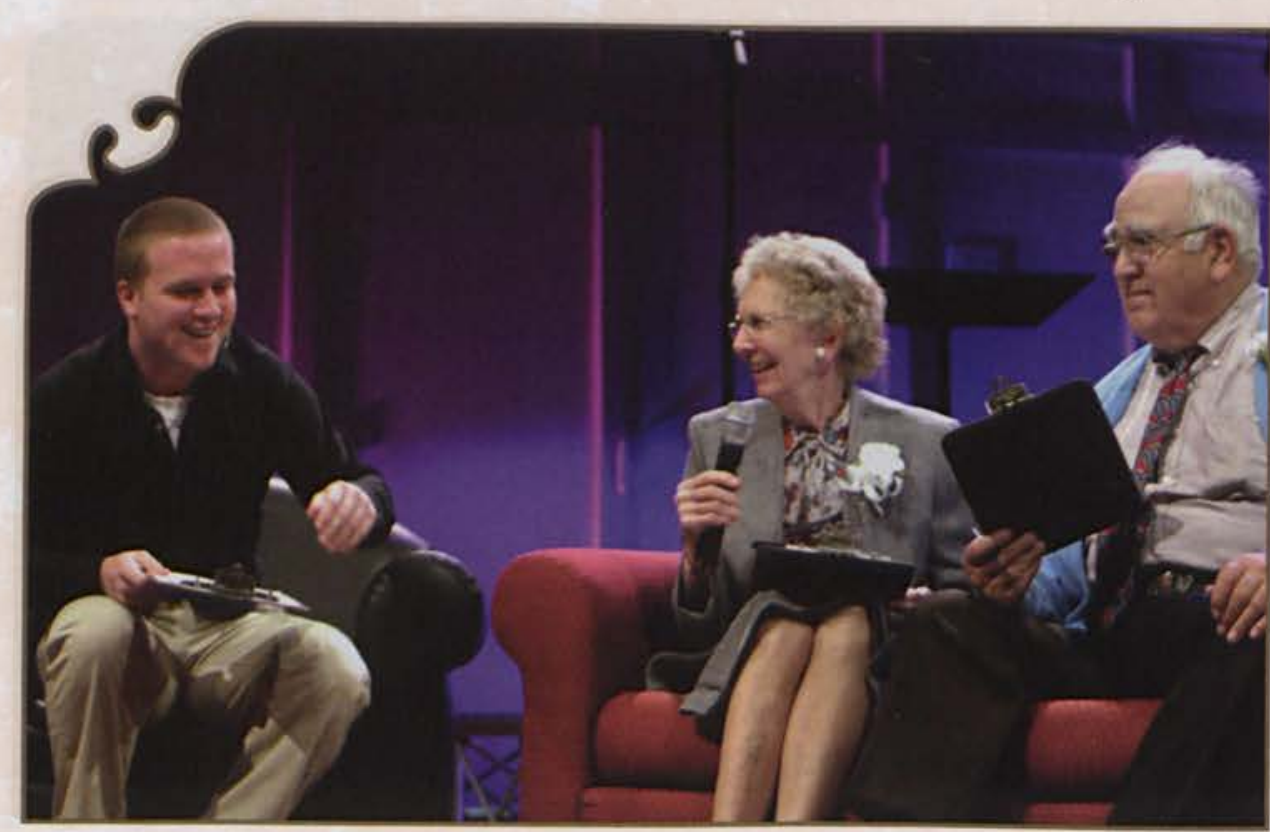

¿ SGA chaplain and host of the weekend's version of "The Newlywed Game," senior Ben Waddell interviews the pair he dubs "the model for the ideal married couple." After fifty years of marriage, this gentleman loves his wife because..."she likes me."

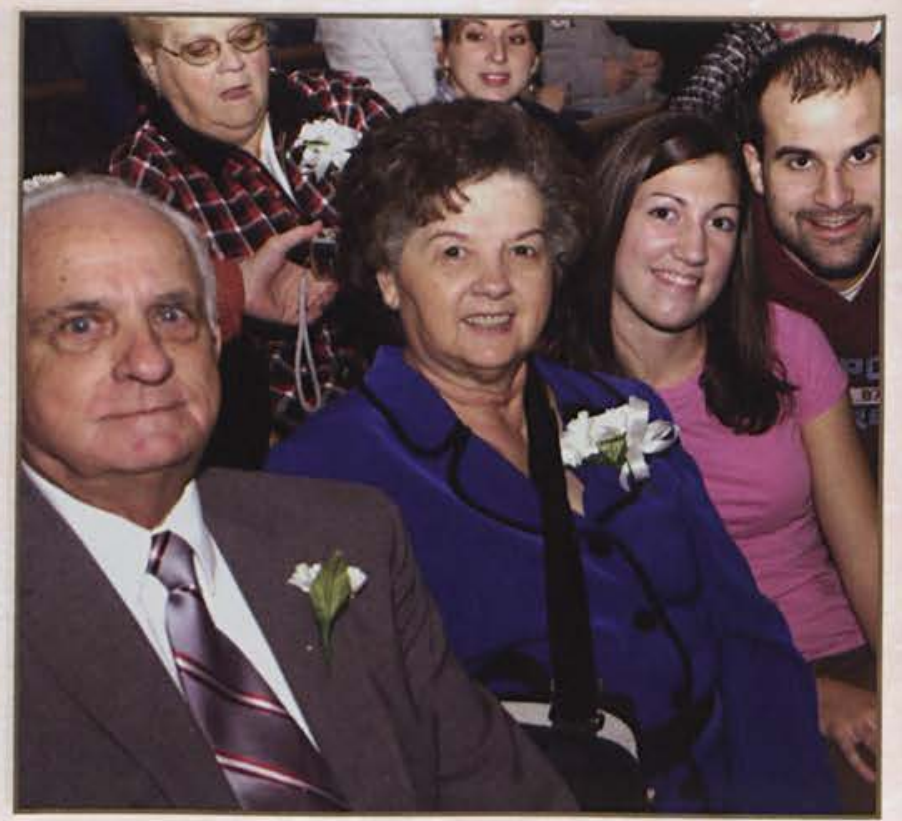

$\downarrow$ Grandparent's Day Chapel holds special meaning for senior Rachel Anderson and grandparents Dave and Carol. "I'm excited they came! It's my senior year, but this is the first time they've had the opportunity to come because I've been out of town for volleyball tournaments every year!" 


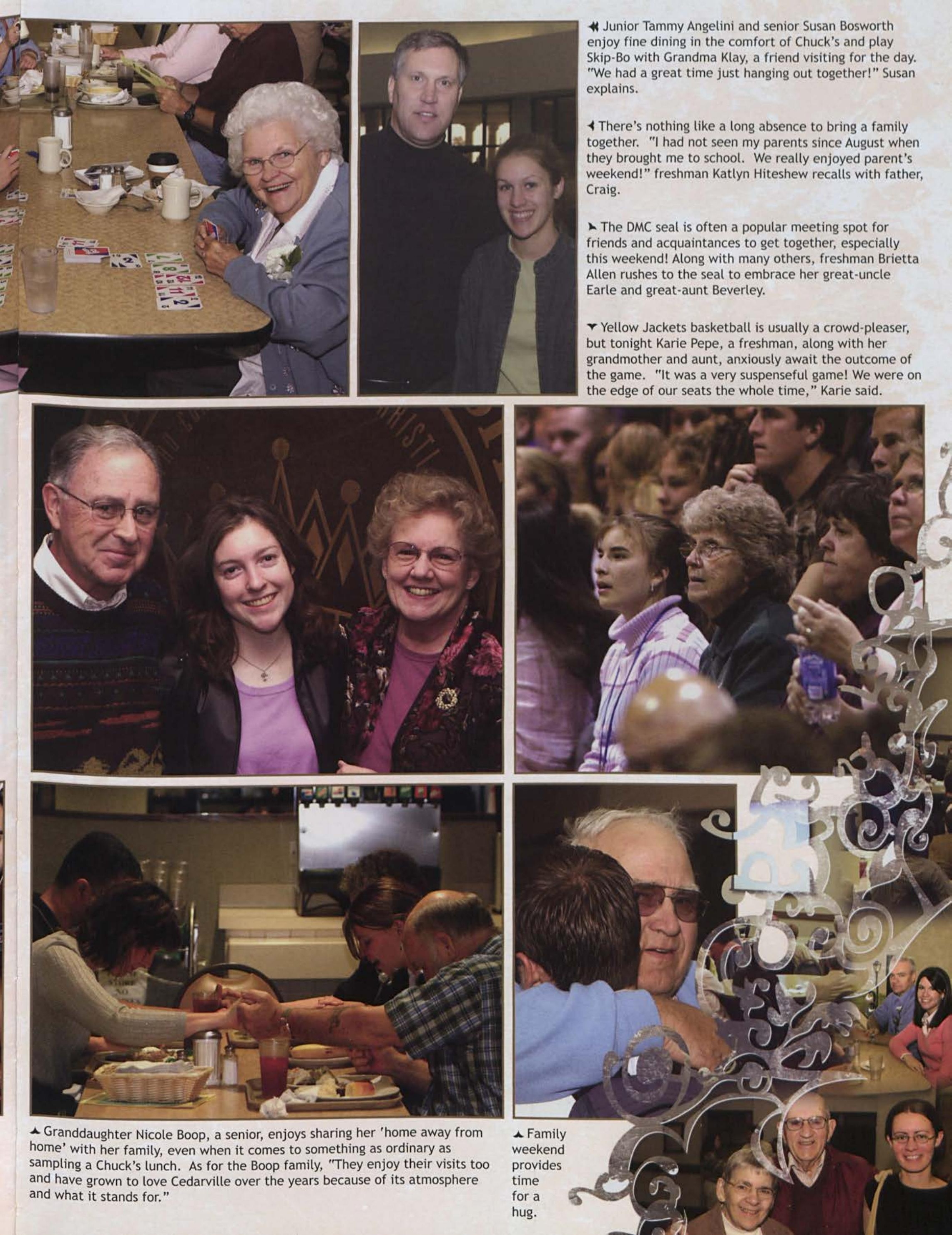




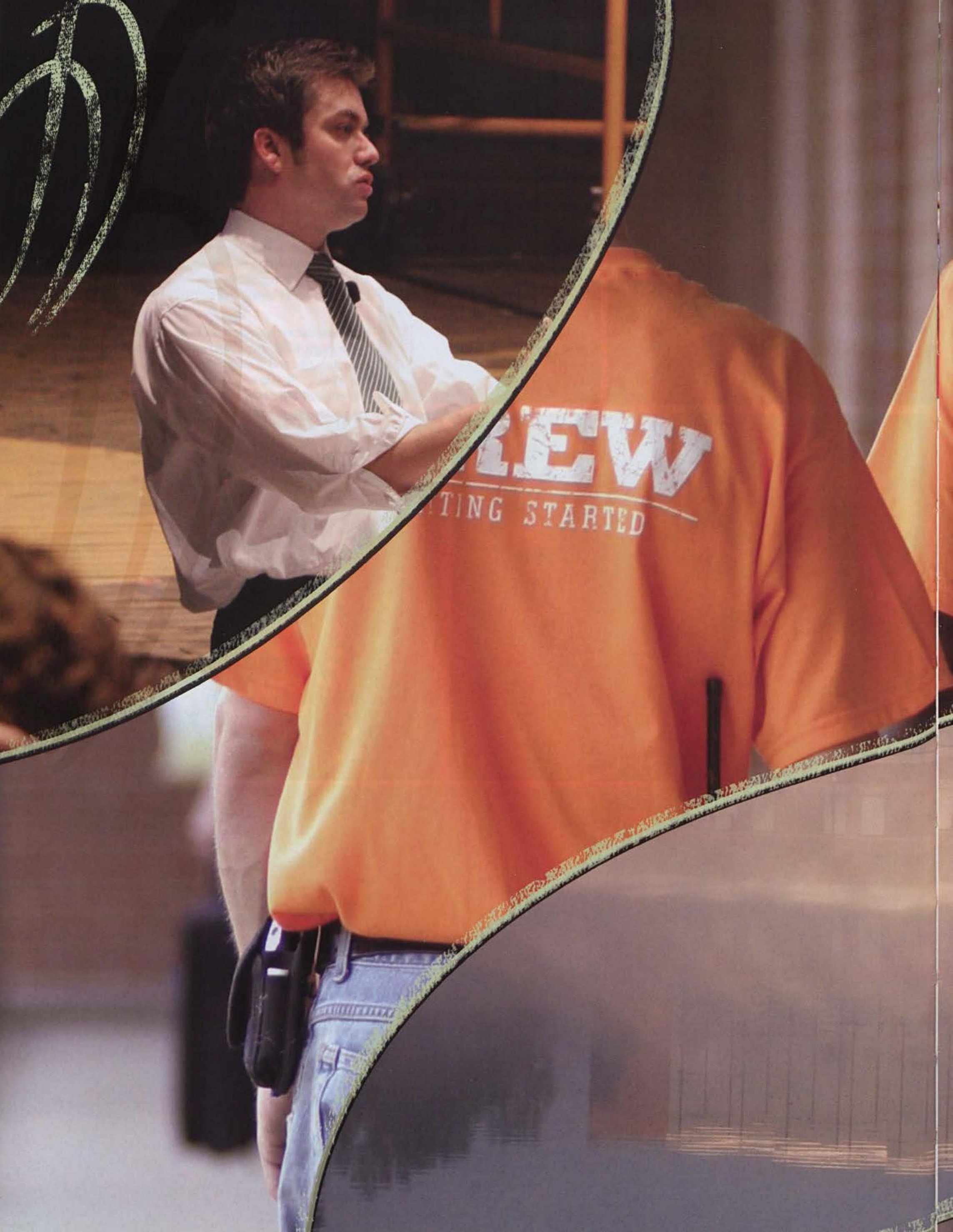




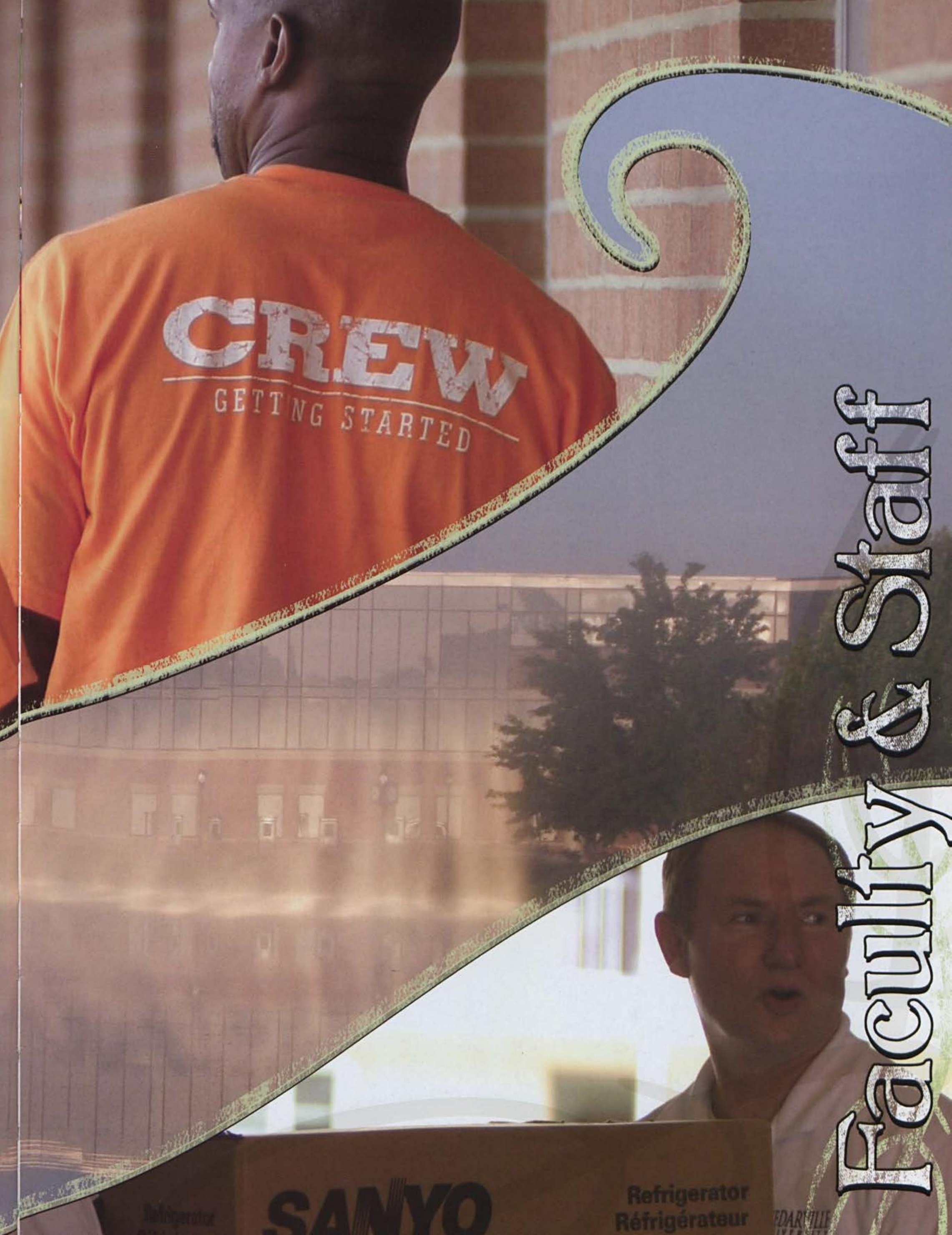




\section{Administration}

Dear Cedarville Family,

2005-2006 - what a year! It will be remembered as a year marked by sorrow and loss; a year filled with sacrifice and giving; and a year colored with achievement and joy.

Chapels, concerts, games, and plays have all added to my memory of this important year. In many ways, this is the passing of an era. The class of 2006 is the last class to have been enrolled under the leadership of another President. The torch has been passed.

From all of us who serve in the administration of Cedarville University, we thank you for your constant encouragement and indomitable spirit. You are the reason we are here, and we never forget our responsibility to serve our Lord by serving you.

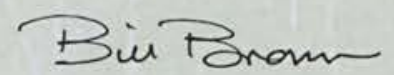

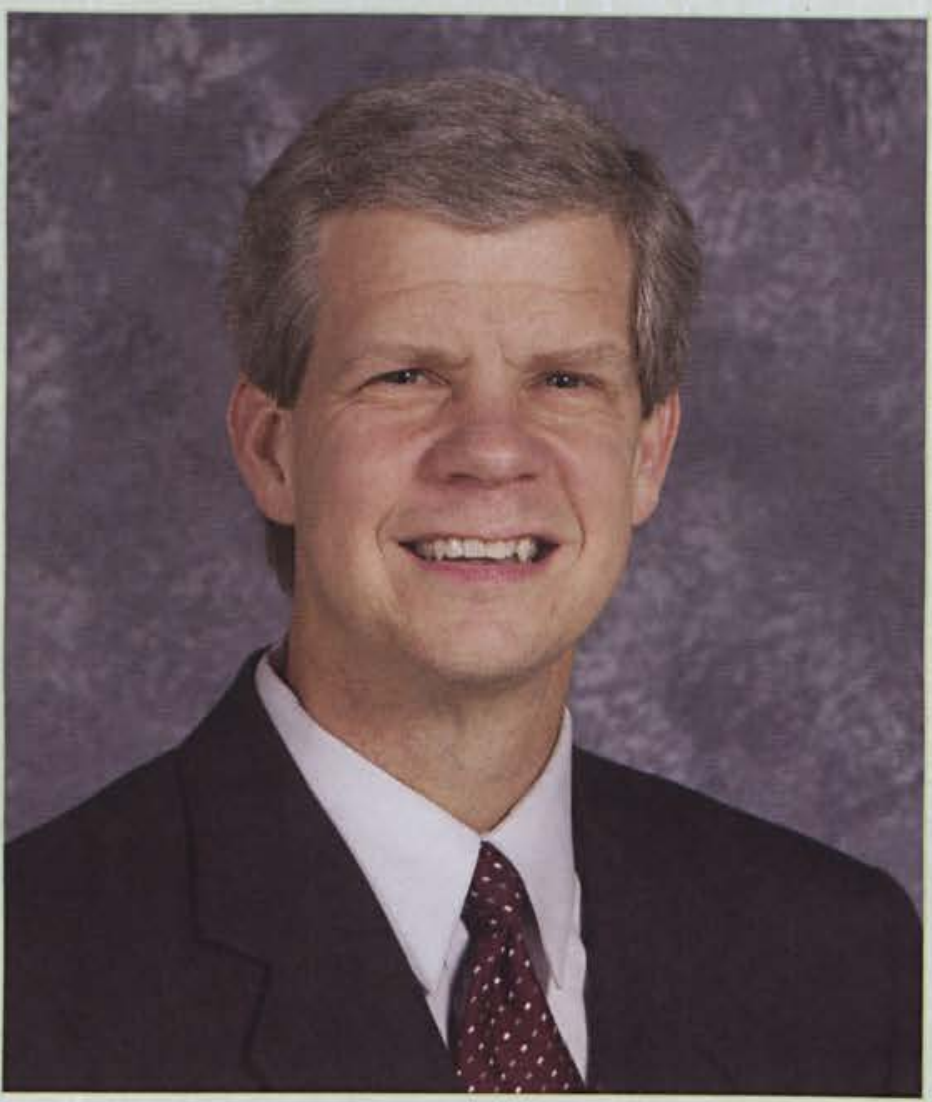

President Dr. Bill Brown

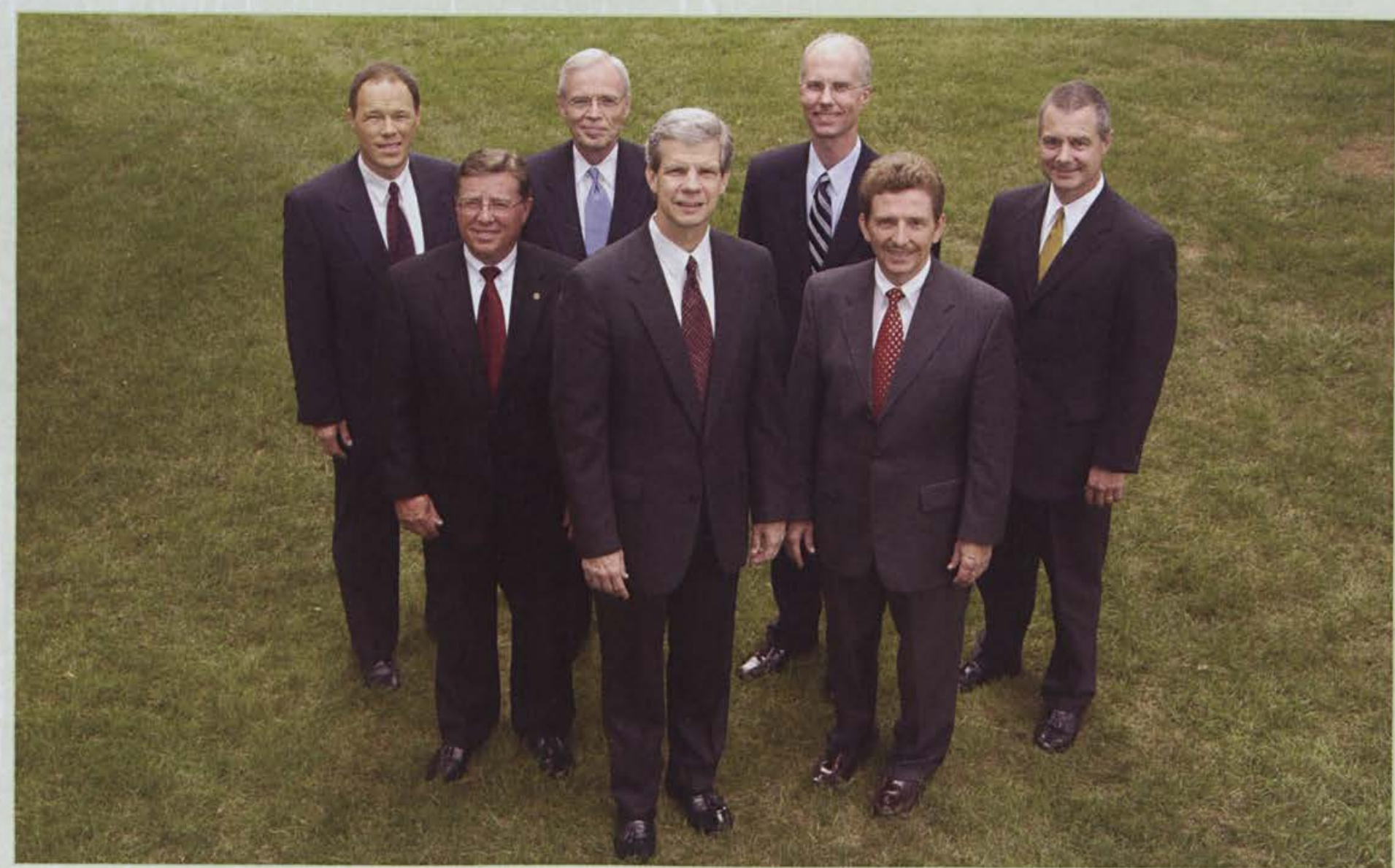

Administrative Council 


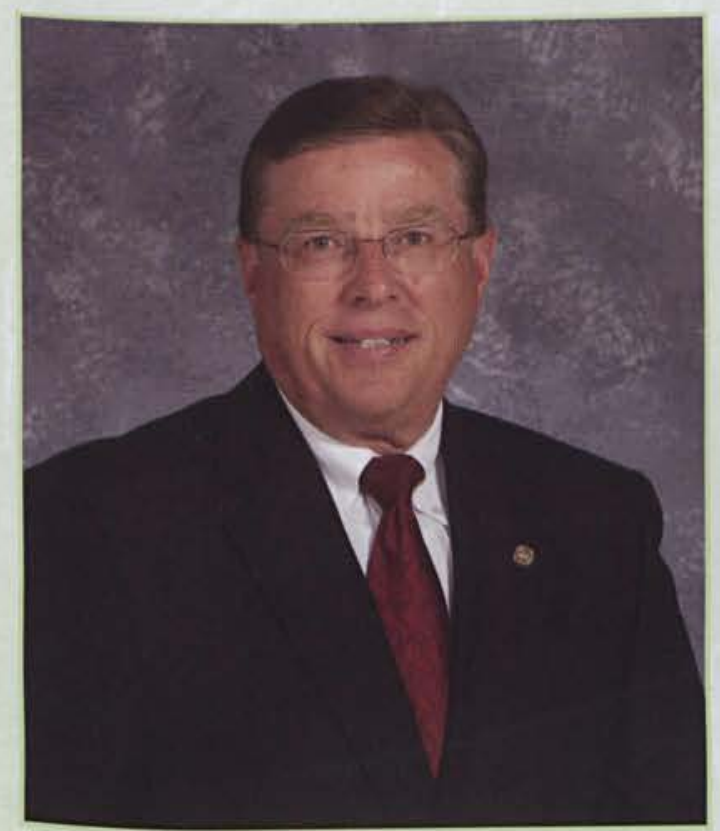

Dr. John Anglea

Vice President for Business

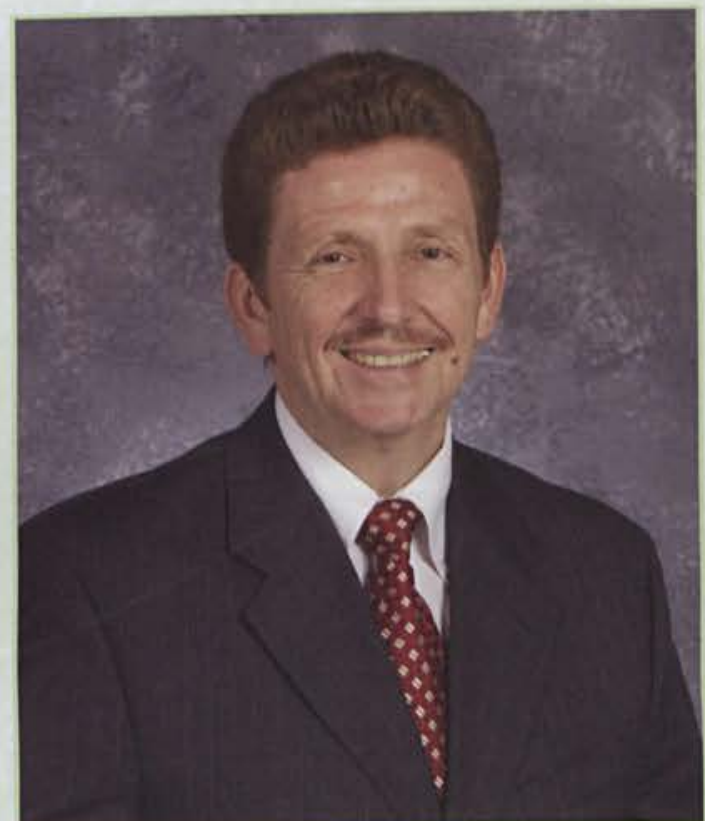

Dr. John Gredy

Vice President for Enrollment Management

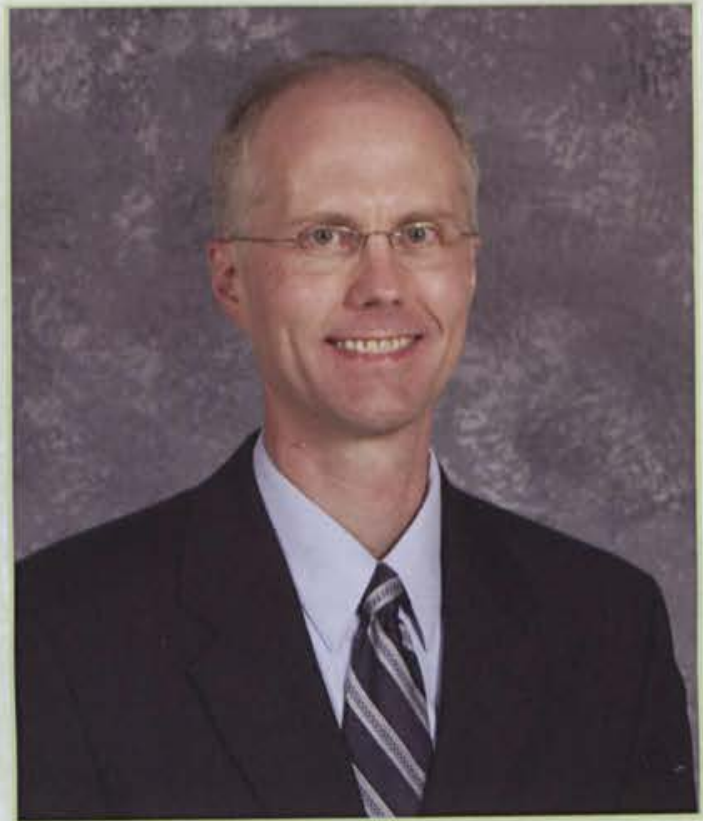

Dr. Bob Milliman

Vice President of Academics

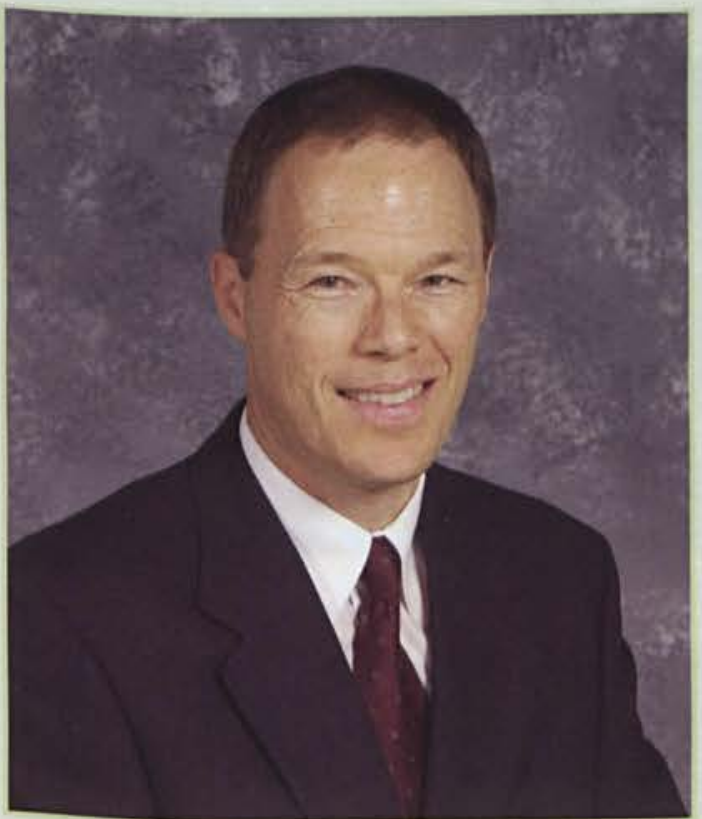

Mr. Dave Ormsbee

Vice President for Advancement

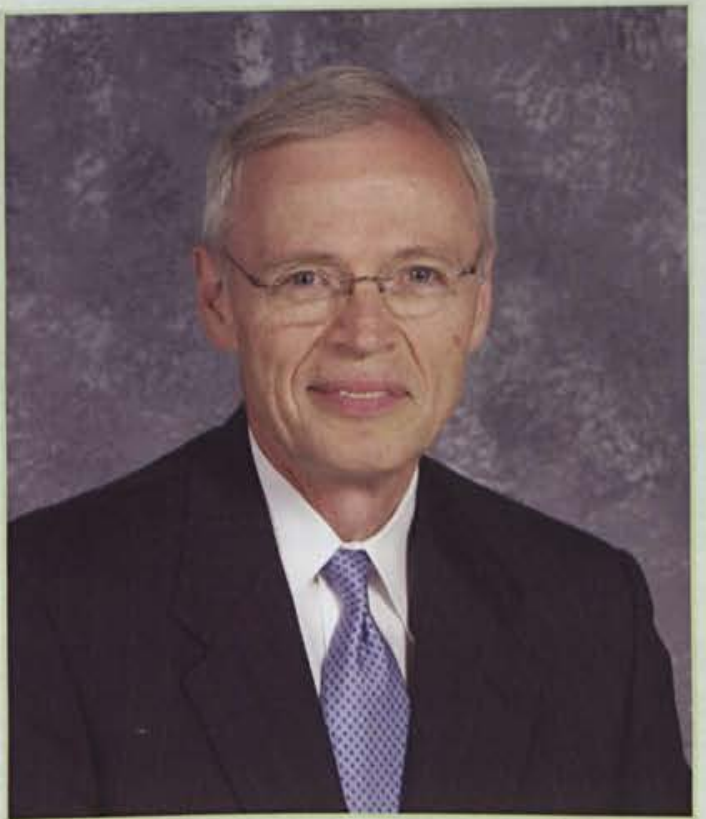

Mr. Bob Rohm

Vice President of Christian Ministries

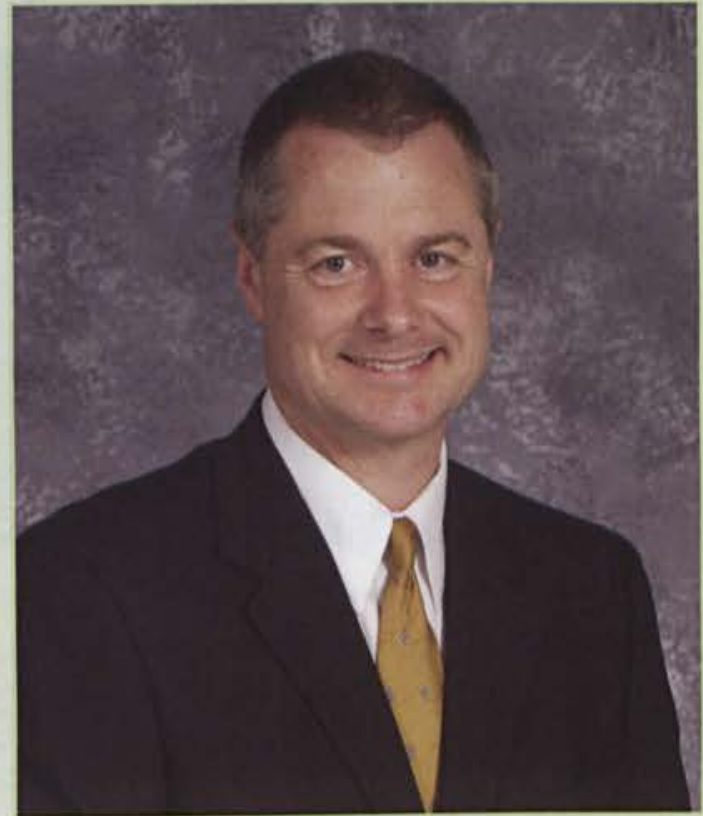

Dr. Carl Ruby

Vice President of Student Services 


\section{Board of Trustees}

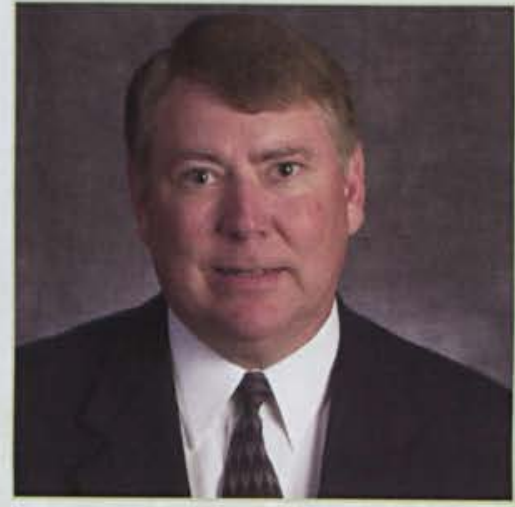

Mr. Ron G. Becker

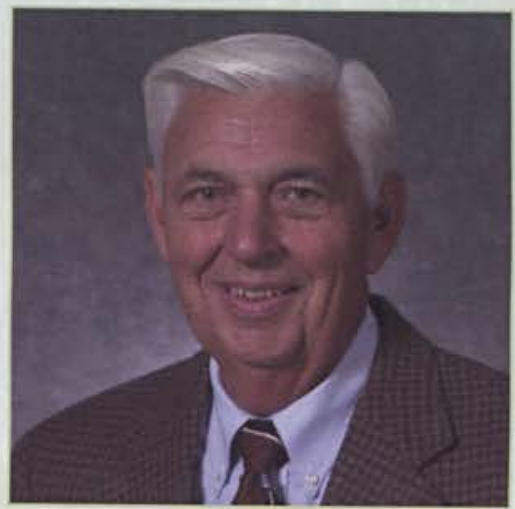

Mr. Gilbert Bruechner

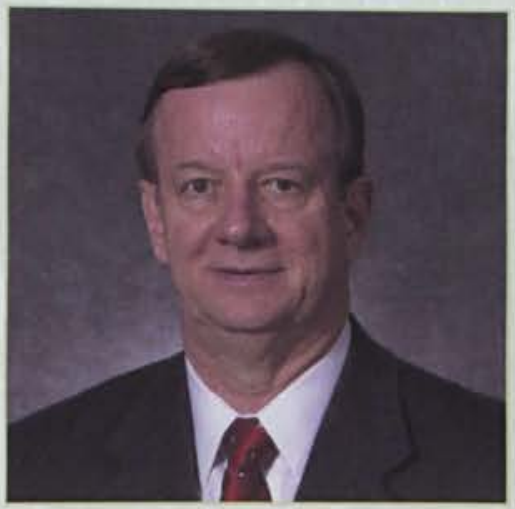

Dr. David Gower

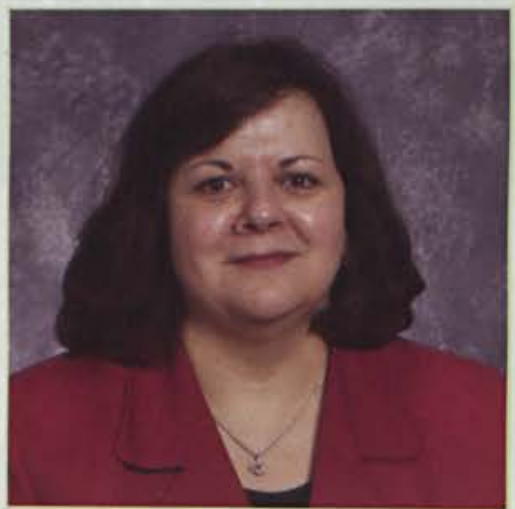

Miss Mary Howard

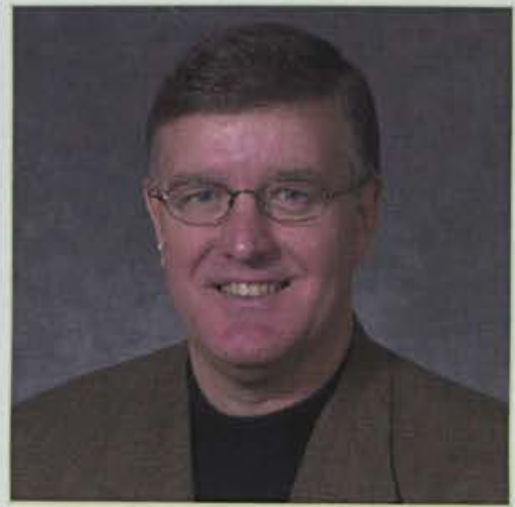

Rev. William Bernhard

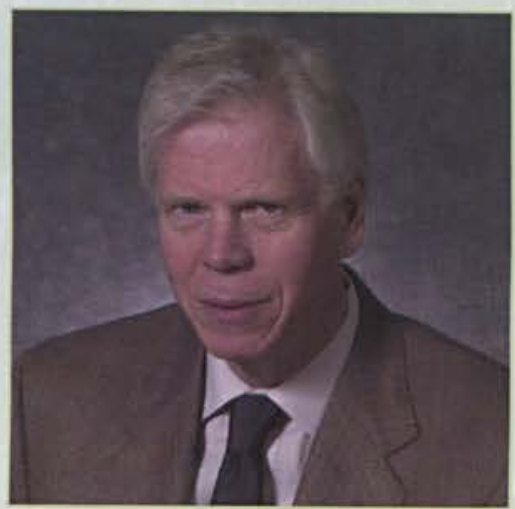

Dr. James H. DeVries

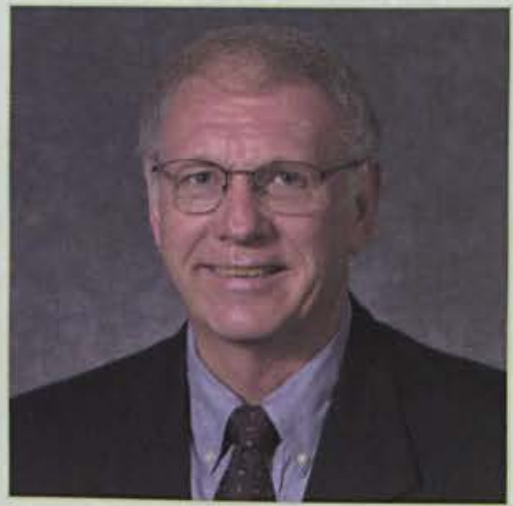

Rev. David Graham

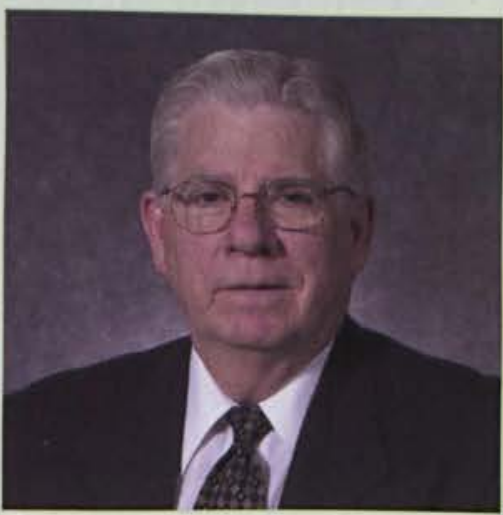

Dr. Jack Jacobs

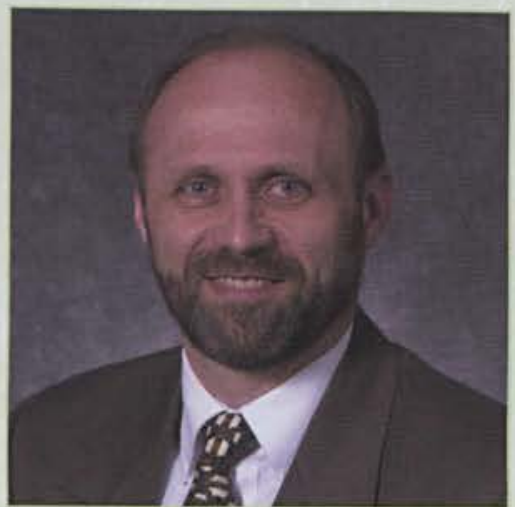

Dr. John Blodgett

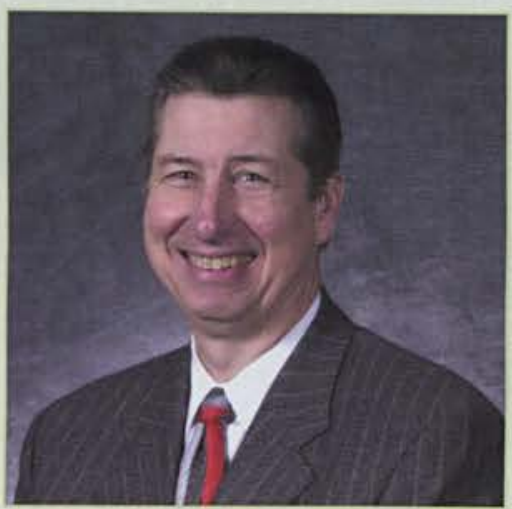

Mr. Daryle Doden

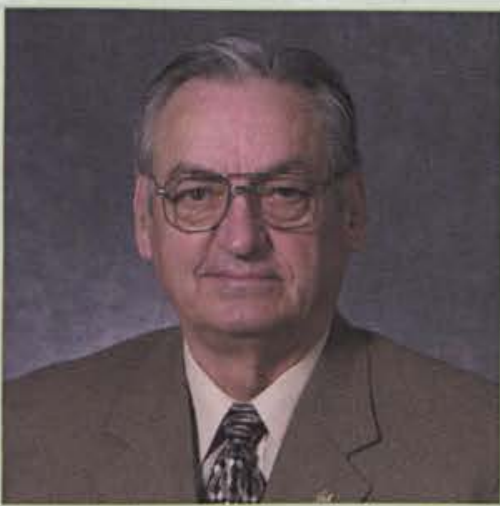

Mr. Roy G. Guenin

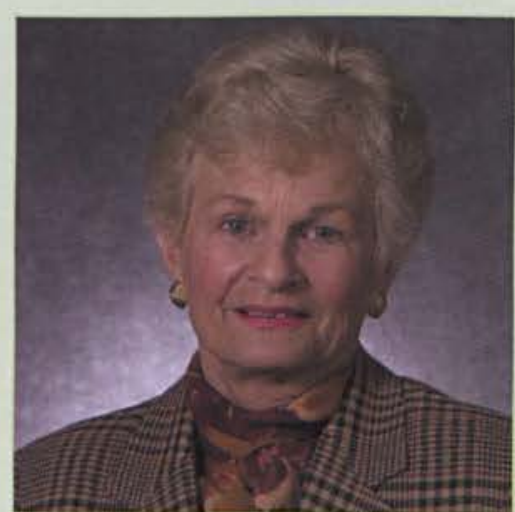

Mrs. Ruth Kempton

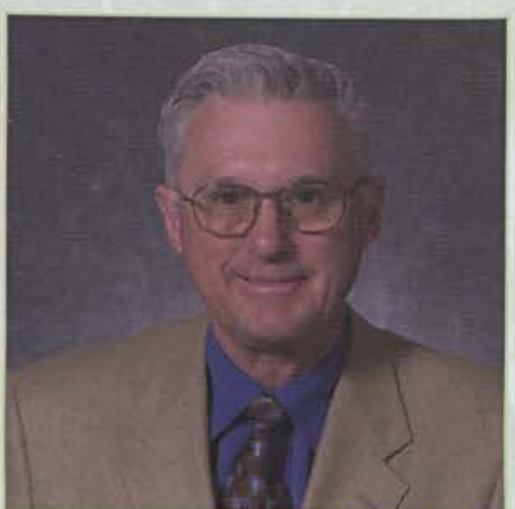

Mr. William Bolthouse

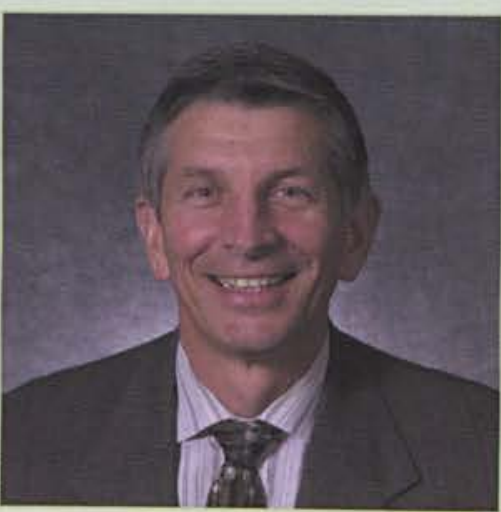

Mr. James D. Engelmann

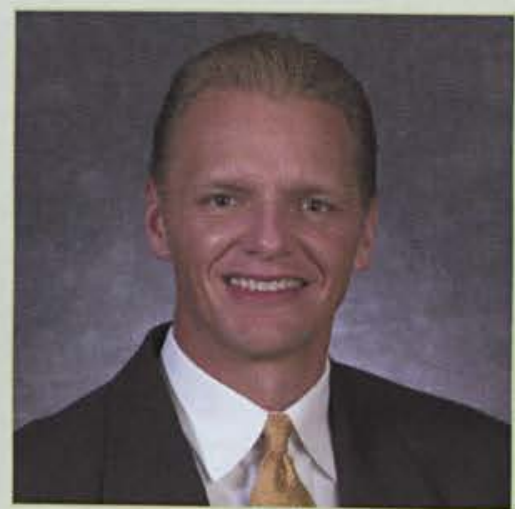

Rev. John Hays

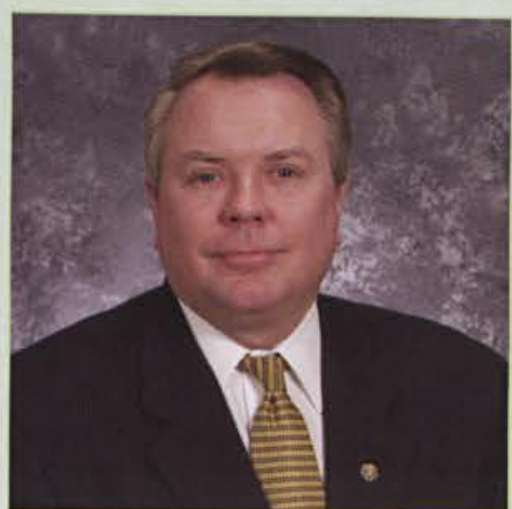

Dr. Jack Kwok 


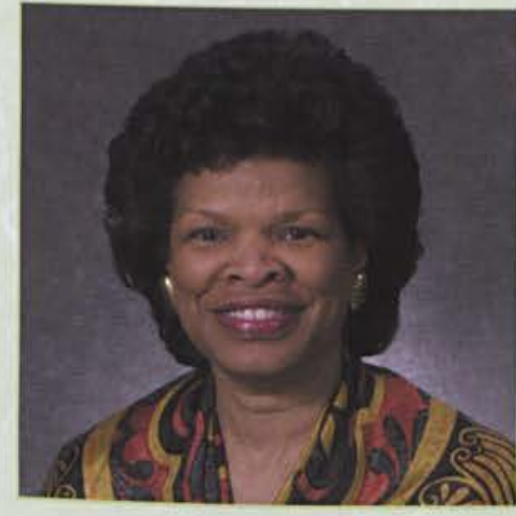

Dr. Deforia Lane

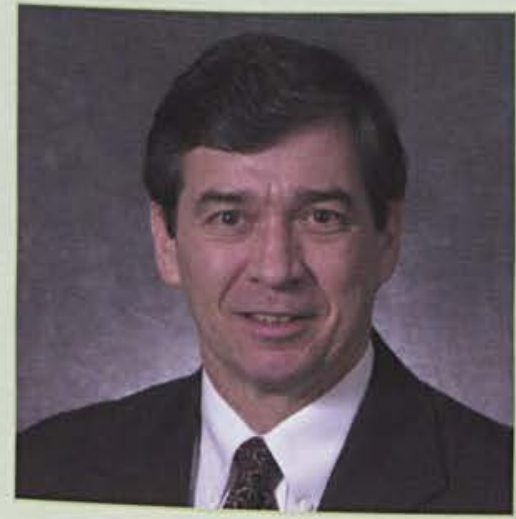

Dr. William Rudd

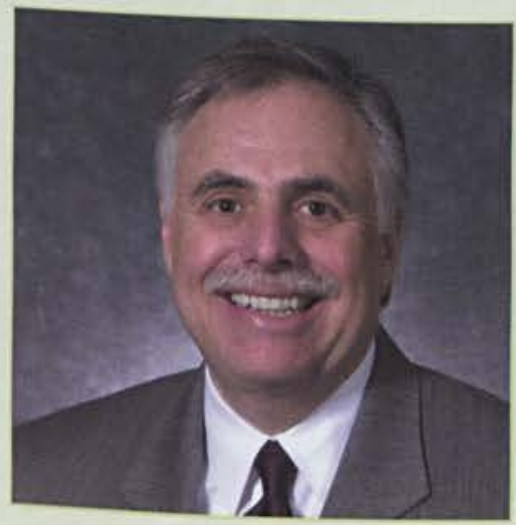

Hon. Robert R. Thomas

\section{Emeriti}

Dr. Eugene F. Apple Mr. Francis Bresson Mr. James Carraher Dr. Jack Cline Mr. John Draxler Rev. Joseph Godwin Dr. E. L. Hawkins Mr. C.E. Miller Rev. Irwin Olsen Rev. Lynn Rogers Mr. Bill Smith Dr. Robert Sumner Dr. Paul Vernier

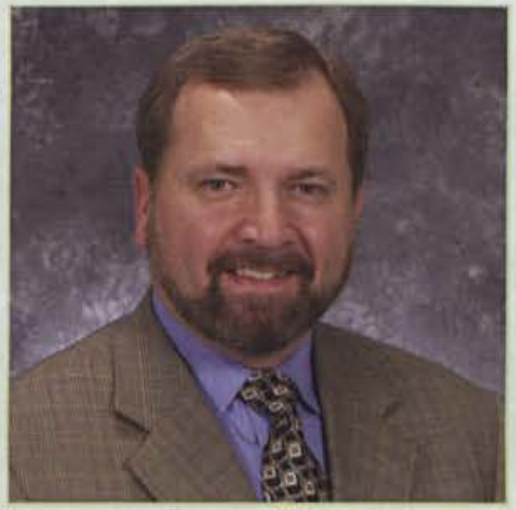

Dr. Michael Loftis

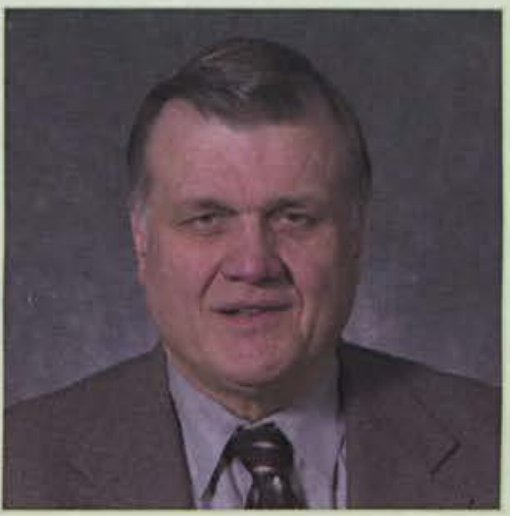

Mr. Lorne Scharnberg

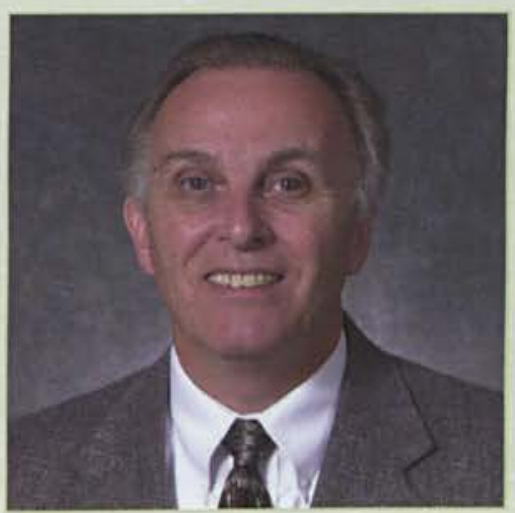

Dr. David Warren

Not Pictured

Mrs. Karyn Brookes

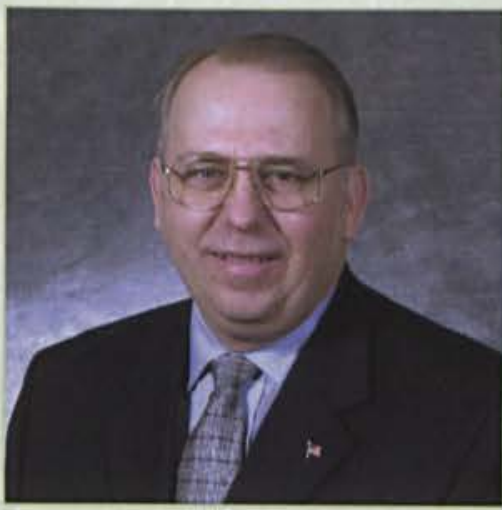

Rev. Randy Patten

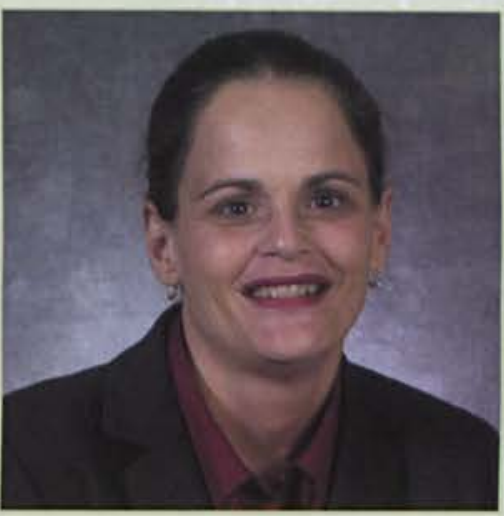

Mrs. Debby Stephens

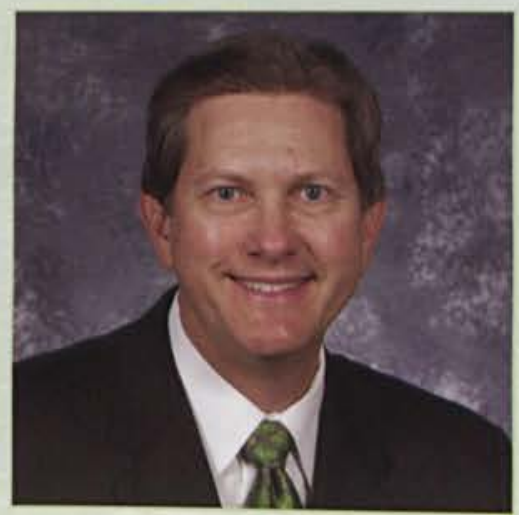

Dr. Hayes Wicker

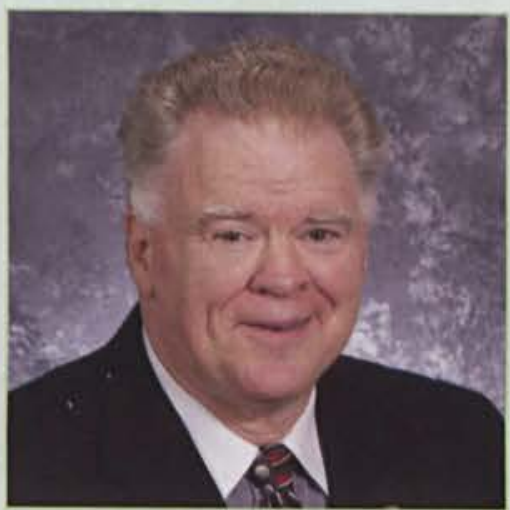

Dr. L. Paige Patterson

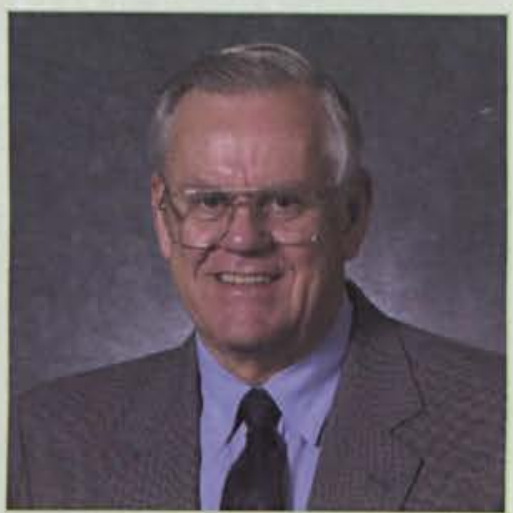

Mr. Albert Stevens

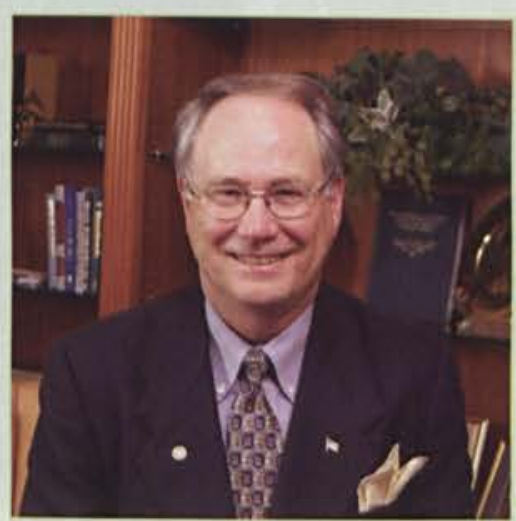

Dr. Paul Dixon, Chancellor 


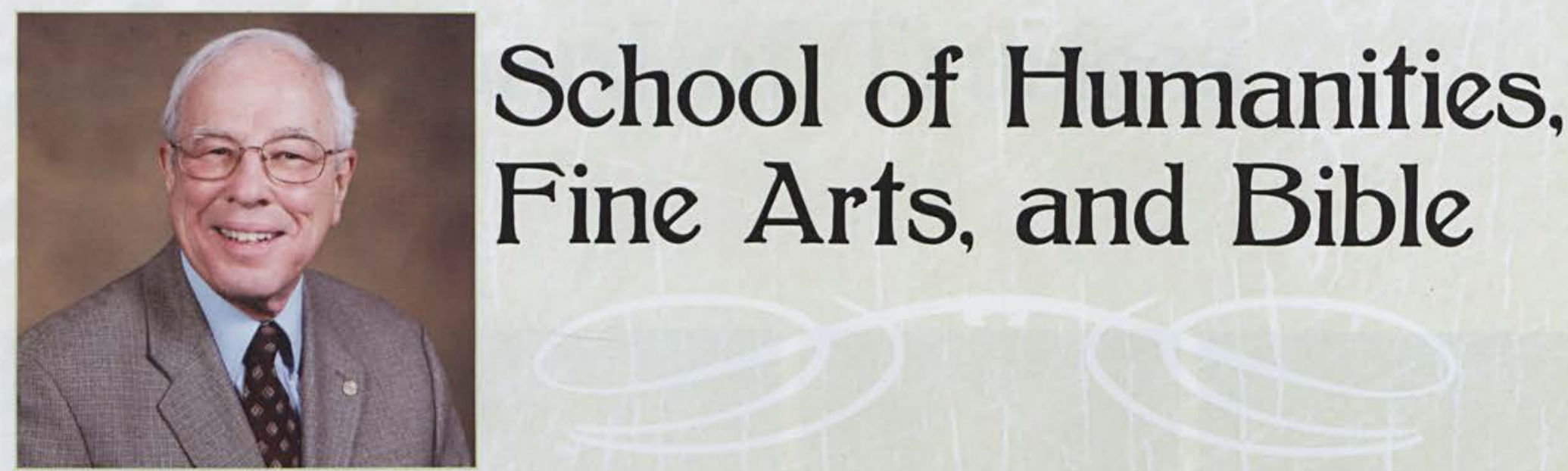

Dr. Jack Riggs Dean

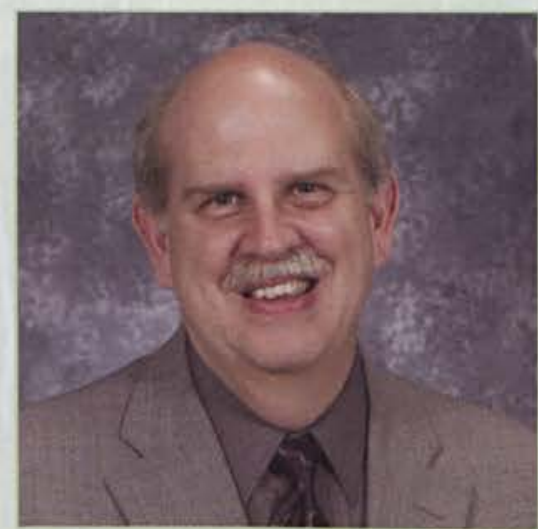

Dr. Charles Elliott Department Chair

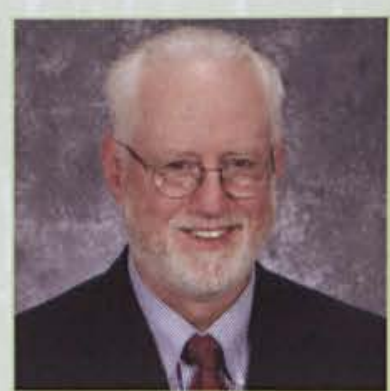

Bob Clements Assistant Professor
The school is largely responsible for a number of artistic endeavors at Cedarville, including plays, films, painting, literature, and more. For HFAB, this year could be summarized with one word: success. The Biblical Education department succeeded in maintaining its focus on student development of biblically based worldviews as well as its pursuit of professionalism. Two professors published books for both scholarly and general audiences. The Language and Literature department also saw many achievements throughout the year. Attendance at the Foreign Film Series skyrocketed 300\% this year, clearly indicating success in student awareness in foreign art and culture. In May, a handful of British Literature II students participated in the first May Term abroad in Dublin, Ireland. The Communication Arts department presented nothing but the highest caliber in performances and presentations. The fall play, A Doll's House, was selected by the Dayton City Paper as the "Critic's Best Pick" for the week of October 12-18. The Forensics and Debate team won recognition in many national competitions. The Music and Art department had two major successes this year. This fall, the department developed a unique and exciting major: Studio Art. This is ideal for students who enjoy multiple disciplines of art.

\section{Department of Communication Arts}

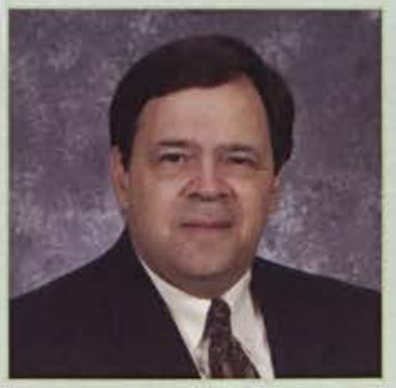

Dr. Wes Baker Distinguished Professor

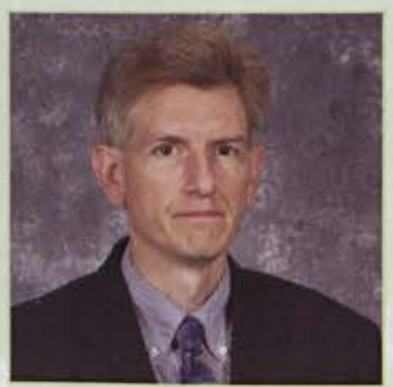

Dr. Clark Greer Associate Professor

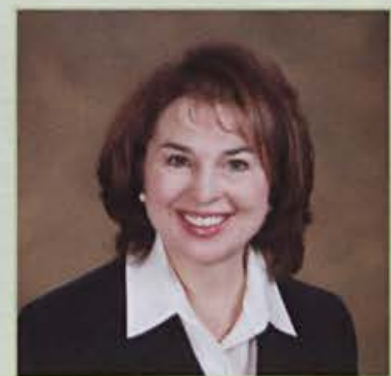

Rebecca Baker Assistant Professor

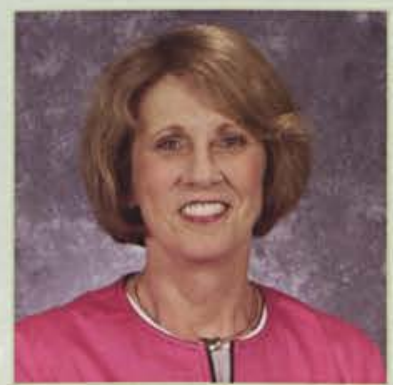

Dr. Deborah Haffey Professor

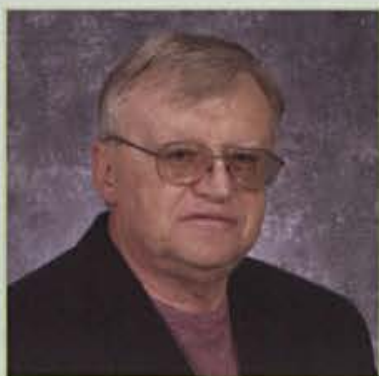

Dave Bastress Electronic Media Tech.

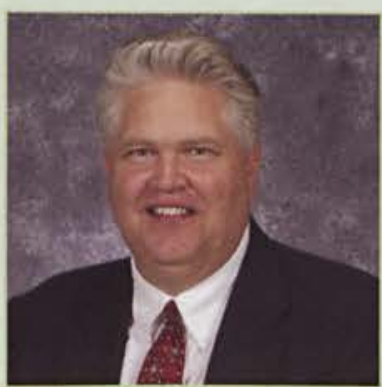

James Kragel Associate Professor 


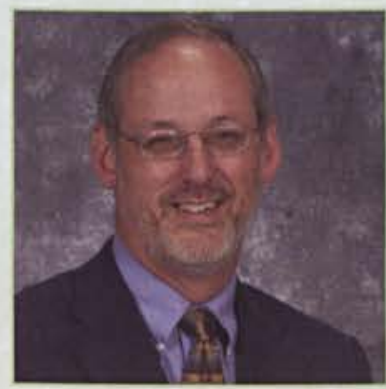

Dr. Mike Lopez Professor

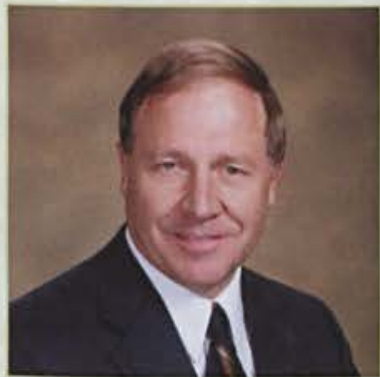

Dr. Jim Phipps Professor

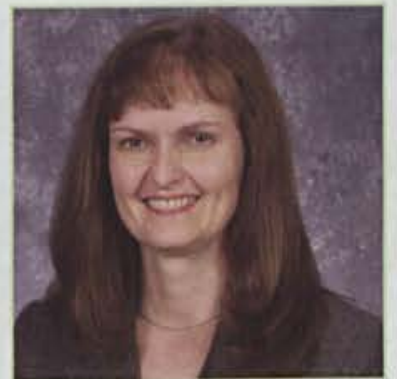

Mischelle McIntosh Assistant Professor

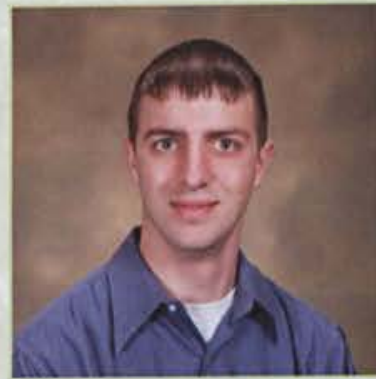

Timothy Phipps Asst. Technical Director

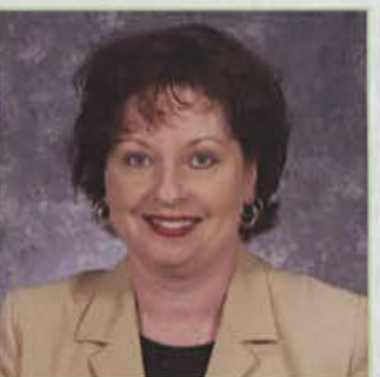

Dr. Diane Merchant Associate Professor

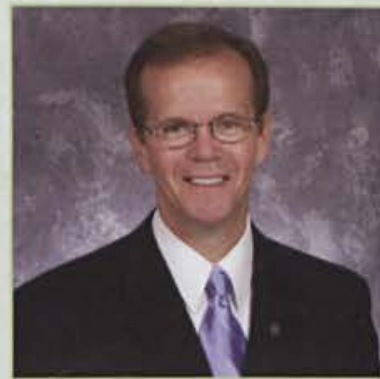

Dr. Dave Robey Professor

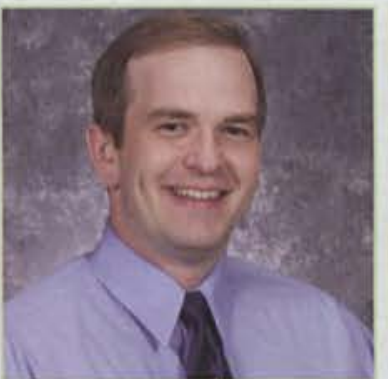

Matt Moore

Assistant Professor

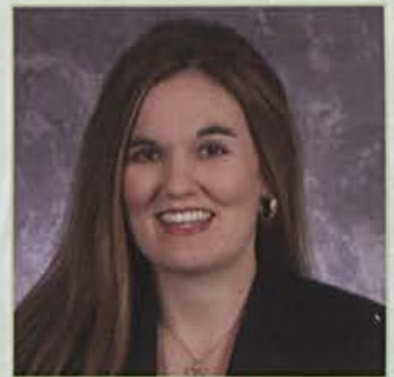

Dr. Rebecca Sietman Assistant Professor

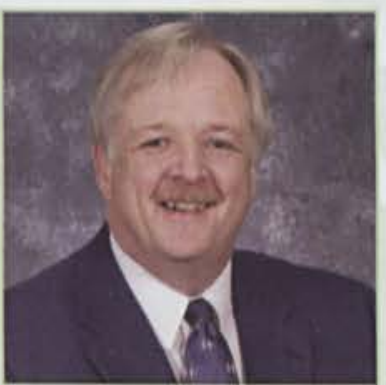

Kurt Moreland Associate Professor

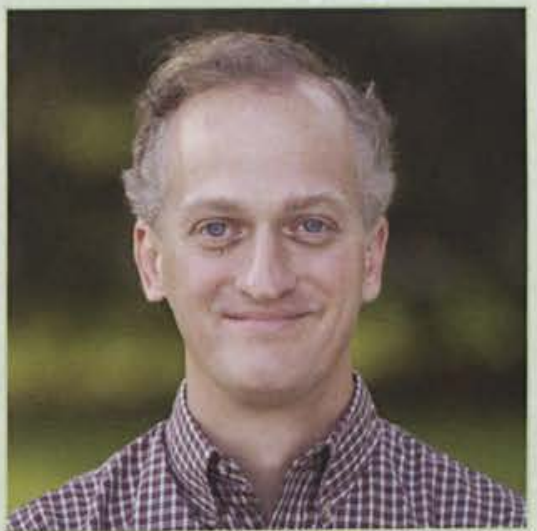

Dr. Thomas Cragoe

Department Chair

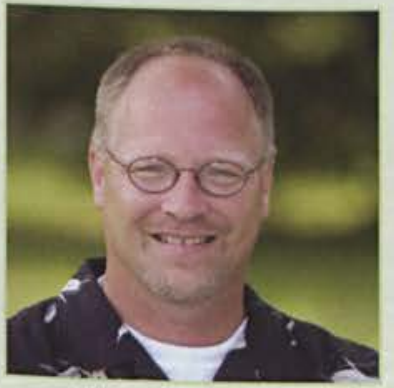

Dr. Scott Dixon Associate Professor

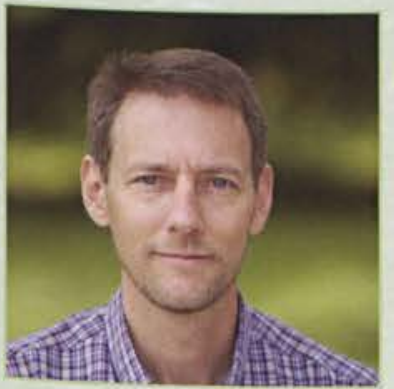

Dr. Don Grigorenko Assistant Professor

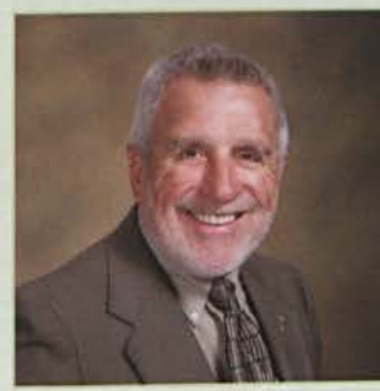

Dr. David Drullinger Professor

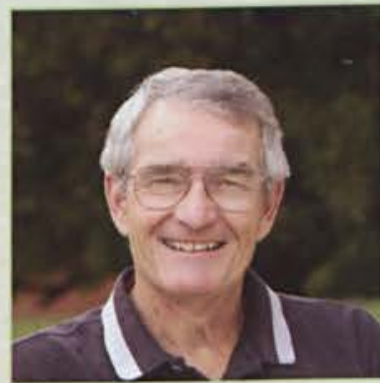

Dr. Bob Gromacki Distinguished Professor

\section{Department of Biblical Education}

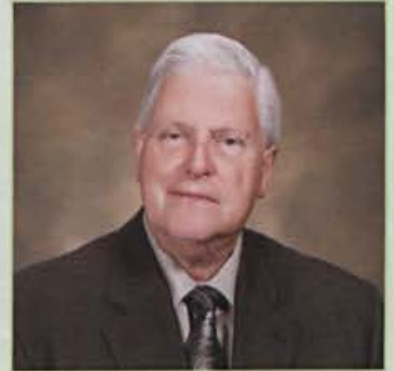

Dr. James Bjornstad Professor

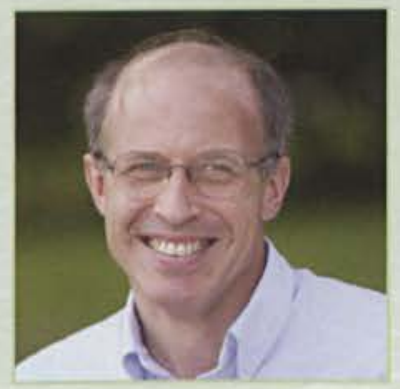

Dr. Dan Estes Distinguished Professor

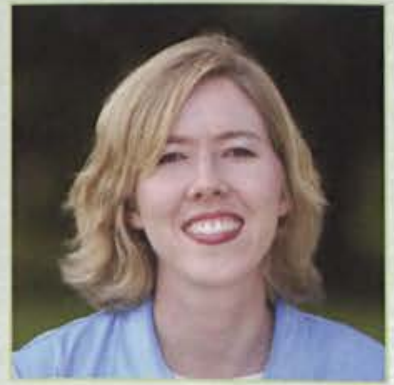

Amy-Hope Guisleman Assistant Professor

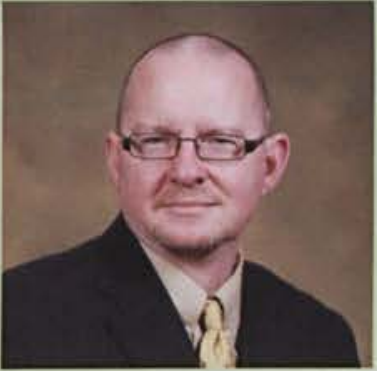

Dr. Jeff Cook Associate Professor

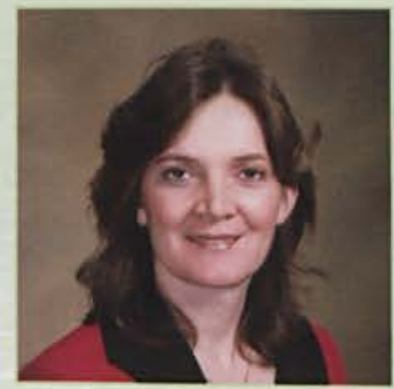

Dr. Joy Fagan Assistant Professor

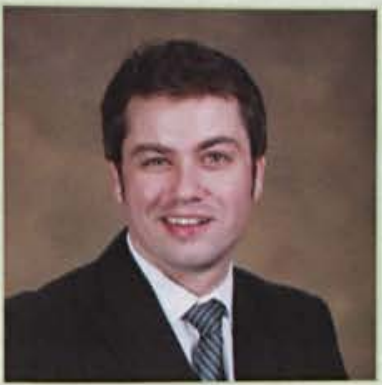

Dr. David Hoffeditz Assistant Professor

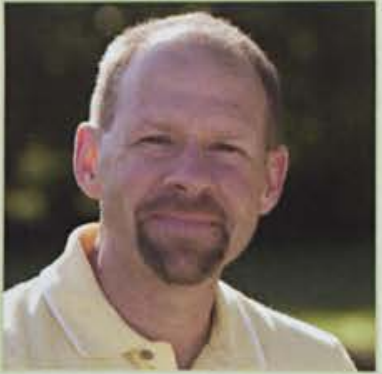

Dr. Gregory Couser Associate Professor

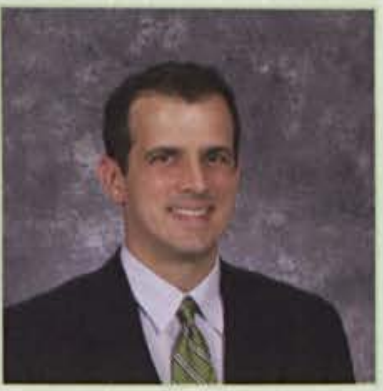

Dr. Timothy Gombis Assistant Professor

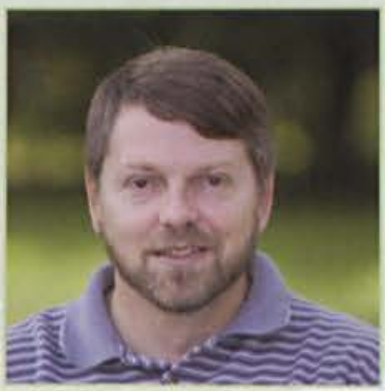

Dr. Tom Hutchinson Associate Professor 


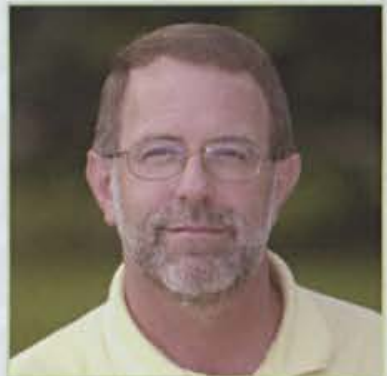

Dr. David Mappes Associate Professor

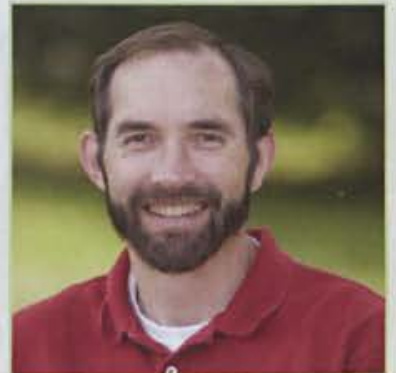

Dr. Chris Miller Professor

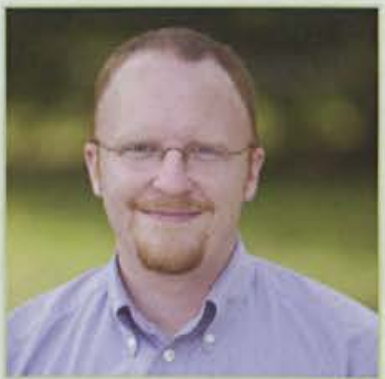

Dr. David Mills

Associate Professor

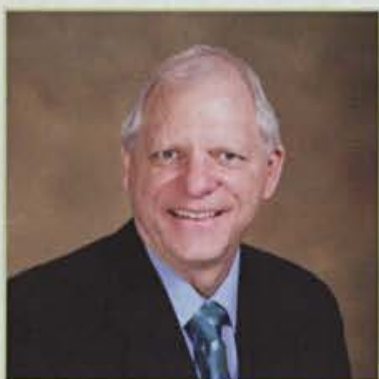

Mike Parrott Assistant Professor

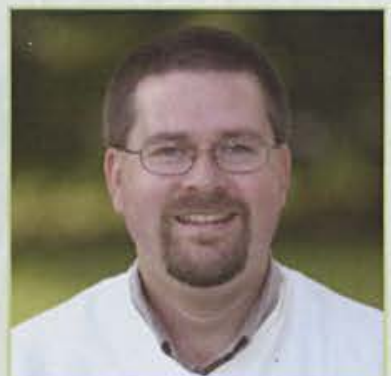

Mike Thigpen

Assistant Professor

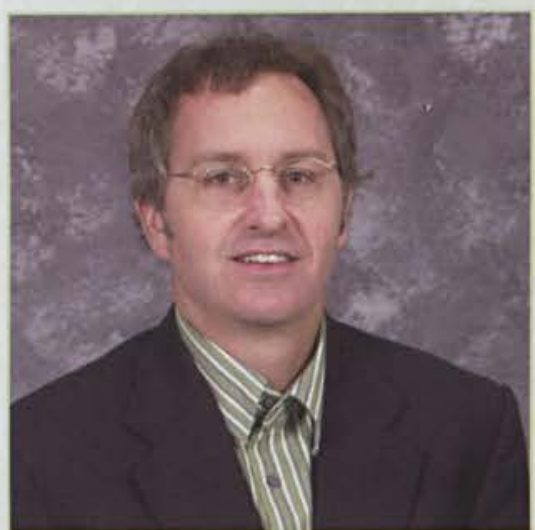

Dr. Kevin Heath Department Chair

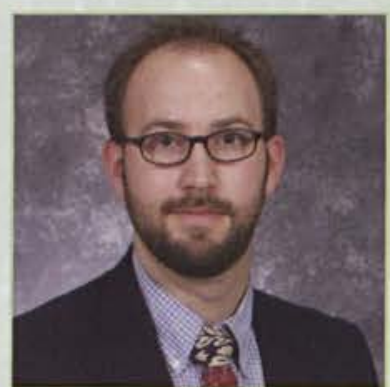

Ryan Futrell Assistant Professor

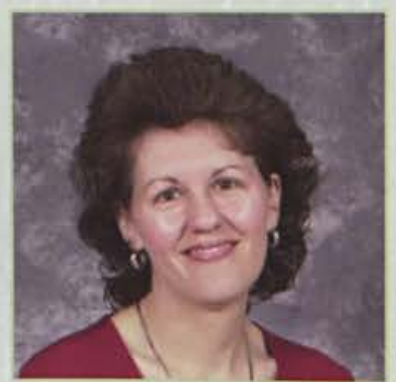

Julie Moore Assistant Professor

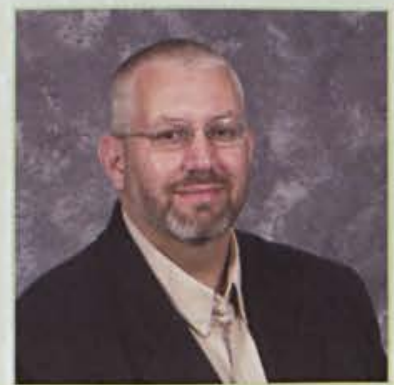

Daniel Sternsher Assistant Professor

\section{Department of Language and Literature}

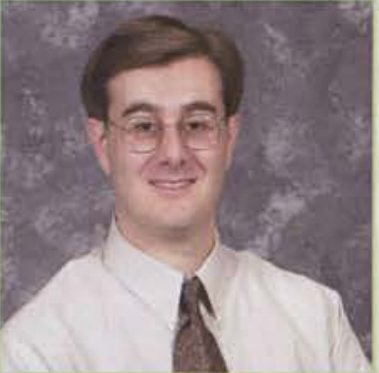

Dr. Scott Calhoun Associate Professor

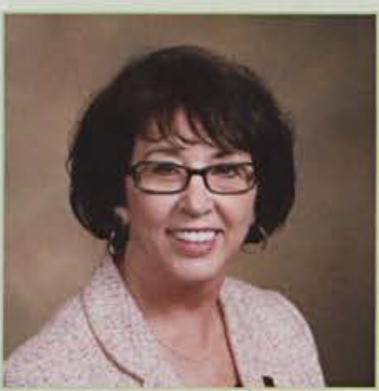

Sandi Harner Professor

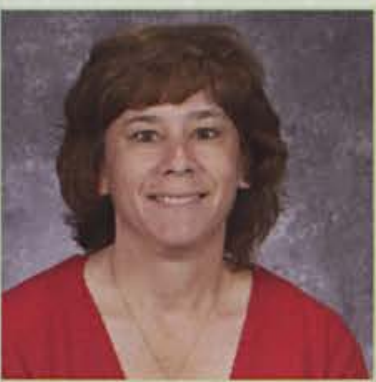

Monique Muncy Administrative Asst.

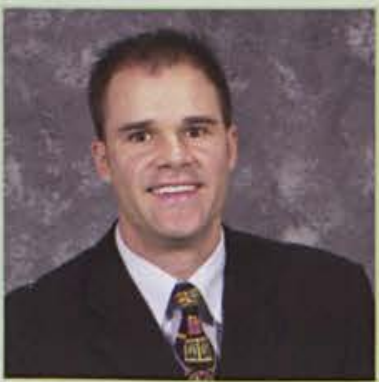

Dr. Andrew Wiseman Assistant Professor

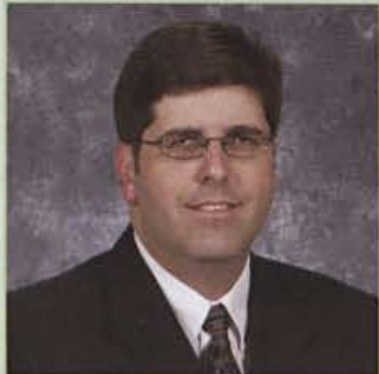

Daniel Clark Assistant Professor

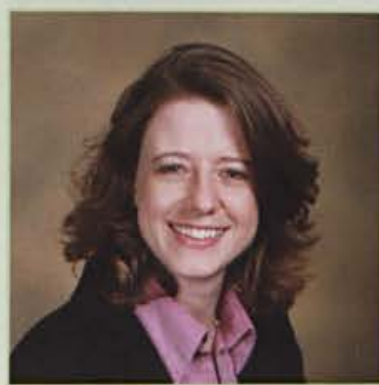

Shannah Hogue Assistant Professor

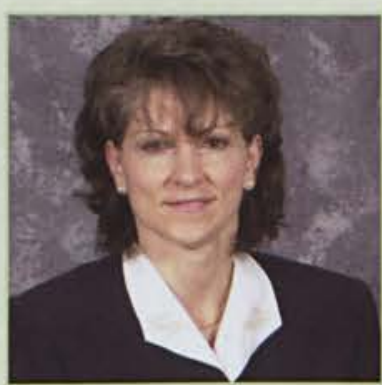

Karen Power Assistant Professor

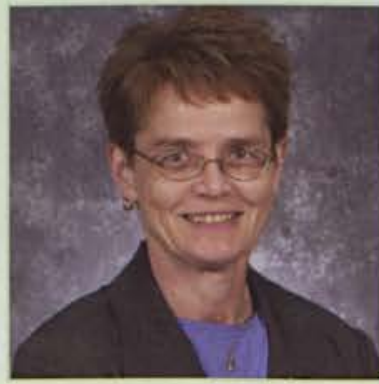

Dr. Peggy Wilfong Associate Professor

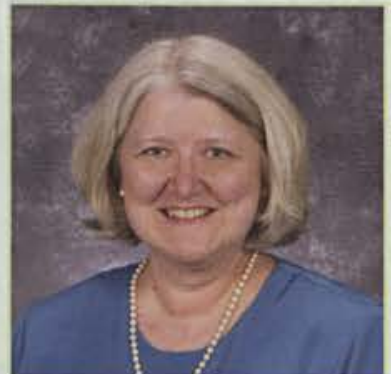

Terry Comers Instructor

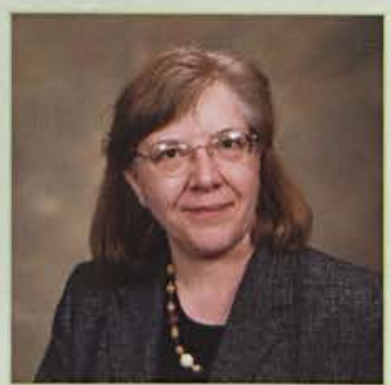

Dr. Barbra Loach Professor

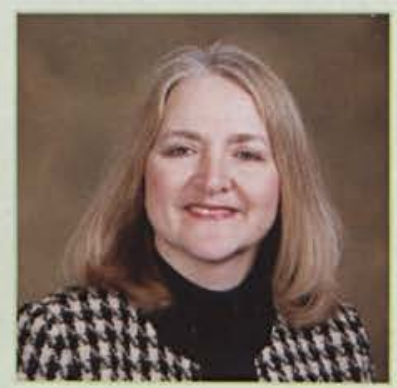

Dr. Annis Shaver Assistant Professor

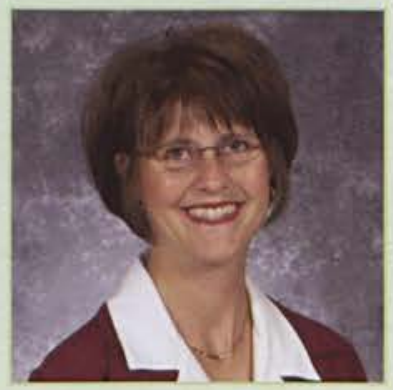

Michelle Wood Assistant Professor 


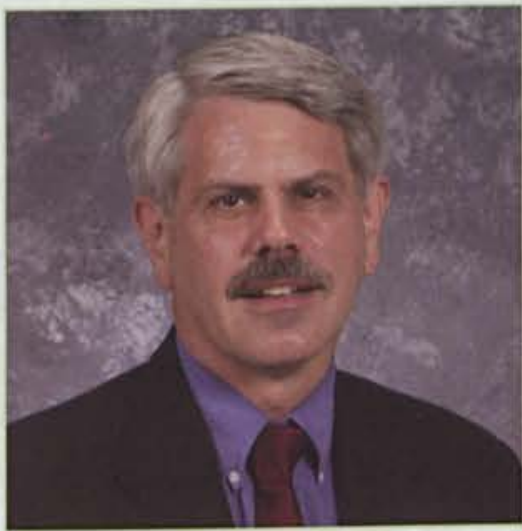

Dr. Steve Winteregg Department Chair

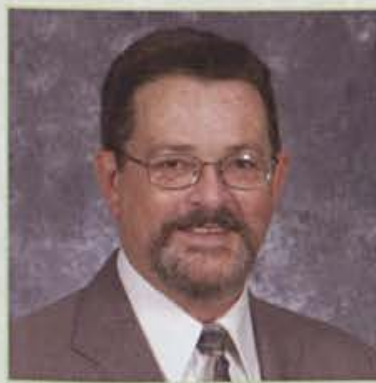

Dr. Chuck Clevenger Professor

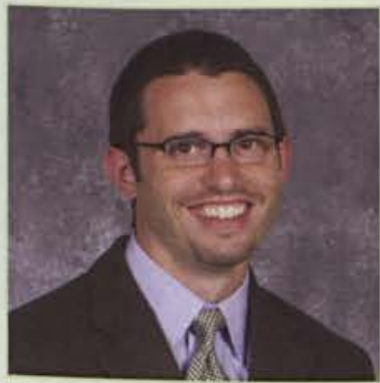

Aaron Gosser Instructor

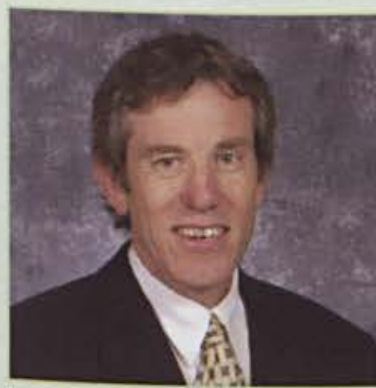

Charles Pagnard Professor

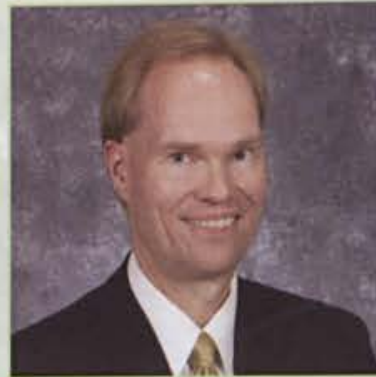

Dr. Bruce Curlette Professor

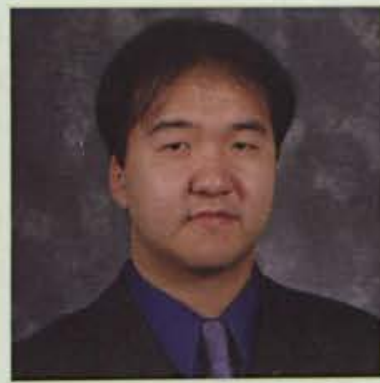

Jun Kim

Associate Professor

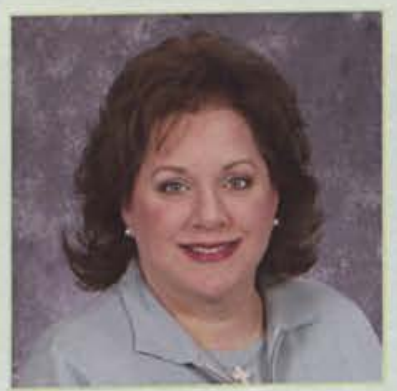

Beth Porter

Assistant Professor

\section{Department of Music and Art}

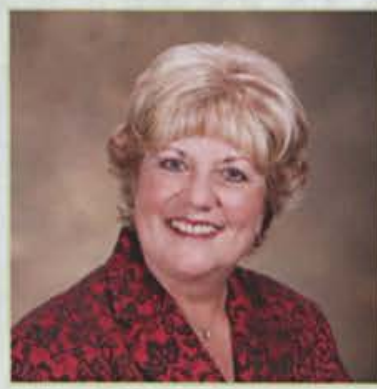

Connie Anderson Assistant Professor

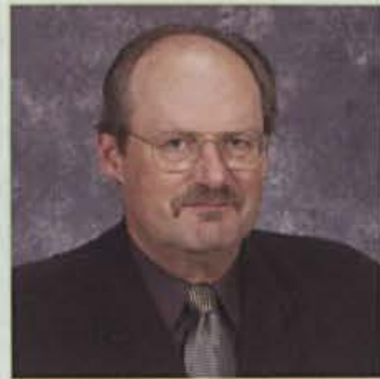

Mike Dicuirci Professor

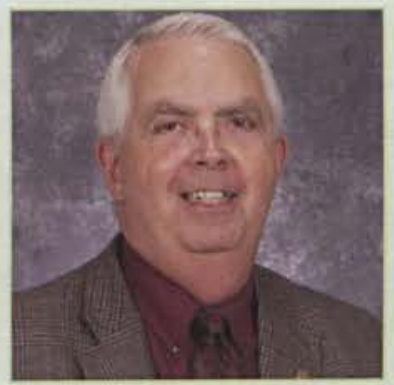

Dr. Dave Matson Professor

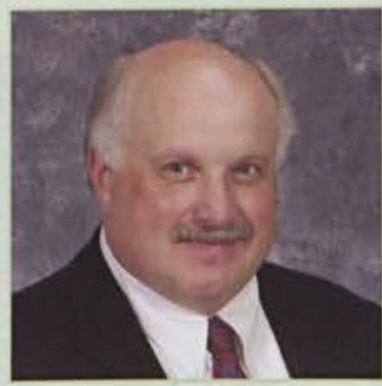

Dr. Mark Spencer Associate Professor

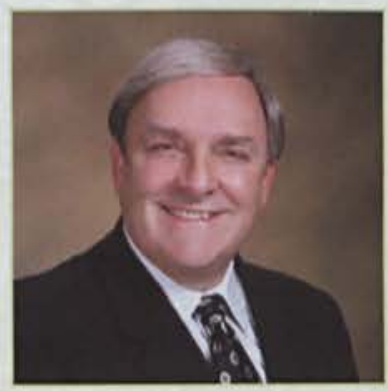

Dr. Lyle Anderson Professor

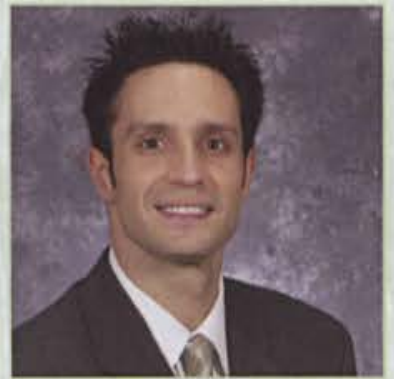

Dr. Taylor Ferranti Assistant Professor

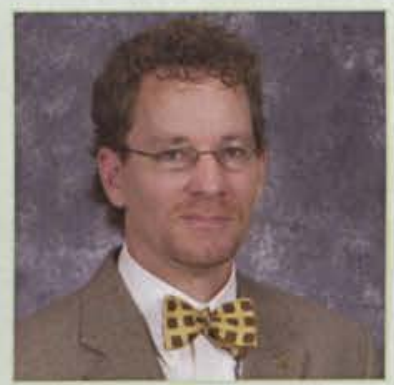

Dr. John Mortensen Associate Professor

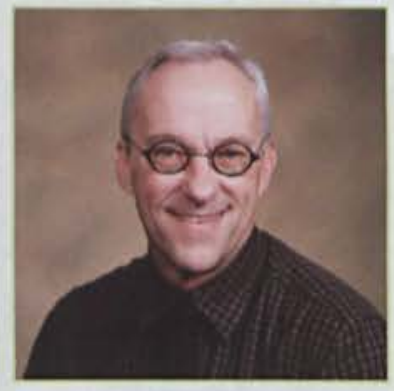

Terry Chamberlain Associate Professor

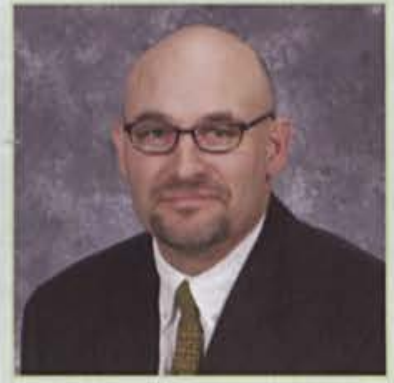

Timothy Frame Instructor

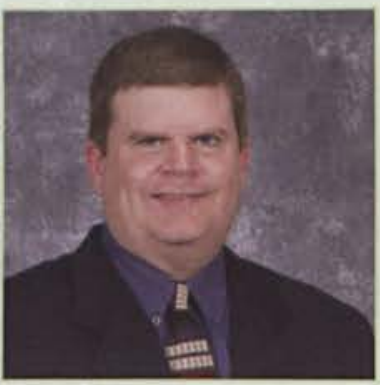

Dr. Roger O'Neel Assistant Professor 


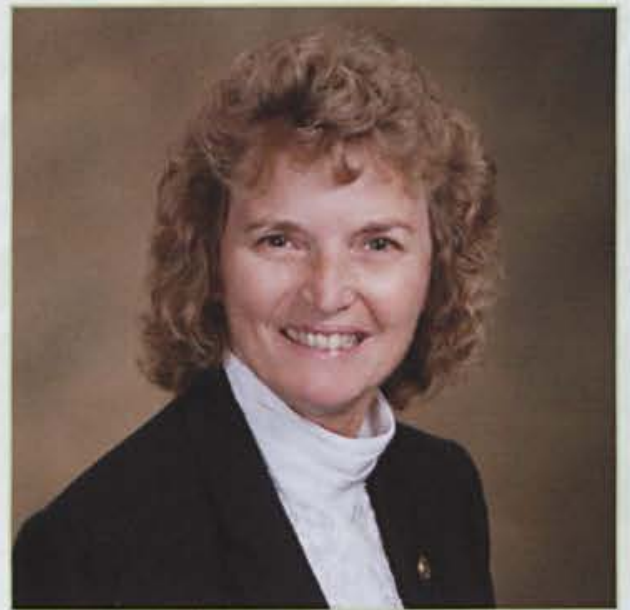

\section{School of Health and Human Performance}

Dr. Pam Johnson Dean
Changes aplenty touched nearly every office and classroom on campus over the past year, including the School of Health and Human Performance, which encompassed the Athletic Training, Athletics, Campus Recreation, and Exercise and Sports Science majors. The Sports Management department hired their first full-time faculty member - Dr. Alan Geist, who taught various classes within the major and oversaw Sports Management practicum and internships. He was also involved in several departmental projects, including an accreditation for the Sports Management major and a student club. Dr. April Crommett, Assistant Professor of Exercise and Sports Science, began the process of starting a new club for health students. Perhaps the most visible change within this department was first observed in the university bookstore as underclassmen found they could no longer get away with renting used versions of the infamous PACL book. A fourth edition of Physical Fitness and the Christian: Exercising Stewardship, by Dean Pamela Johnson and Professor L. Delyte Morris, was approved and quickly implemented into classroom instruction. Finally, this past summer, the Campus Recreation Program made the move from the Student Life Division to the School of Health and Human Performance. Incorporating over 40 activities and opportunities for students to have fun and improve their health, Campus Recreation, along with the entire School of Health and Human Performance, fulfilled their purpose to "develop (in students) an awareness of the Christian's stewardship responsibility with regard to the care and use of the body" in a unique and practical way.

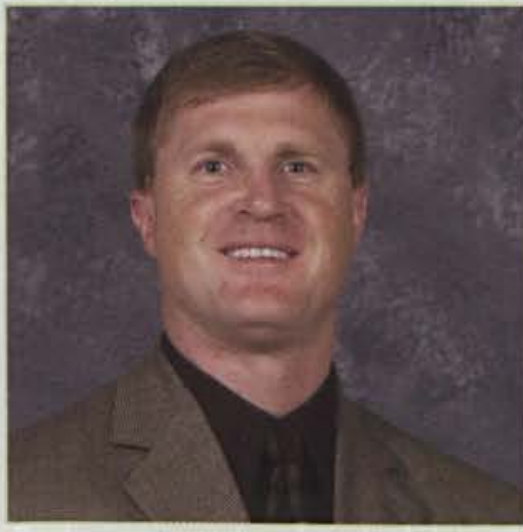

Dr. Evan Hellwig Department Chair

\section{Department of Athletic Training}

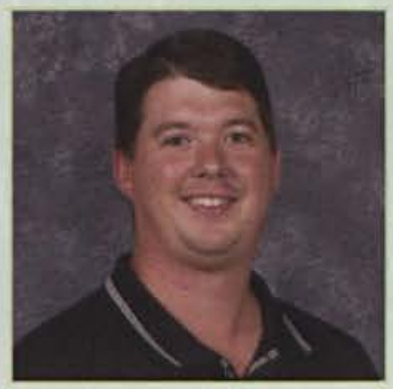

Chris Cross Assistant Professor

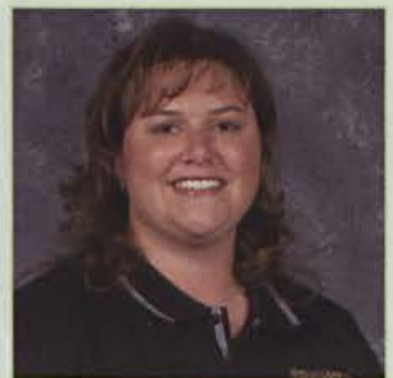

Lisa Martin Athletic Trainer

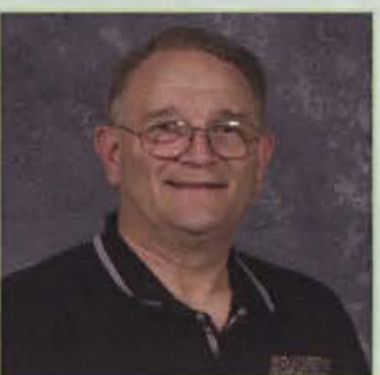

Bob Durchardt Assistant Professor

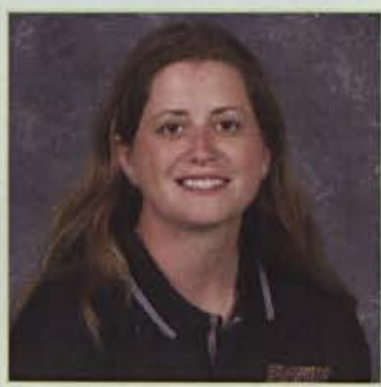

Siobhan Fagan Assoc. Athletic Trainer 


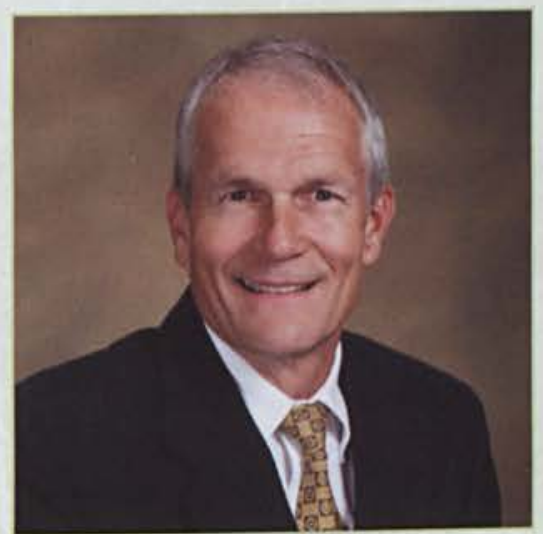

John McGillivray

Department Chair

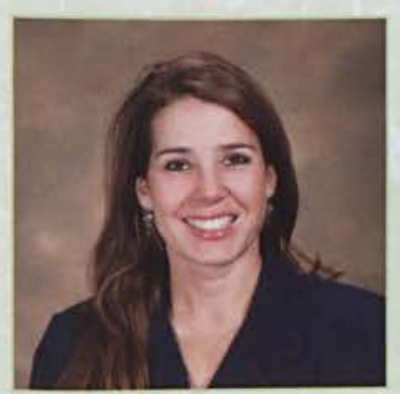

Dr. April Crommett Assistant Professor

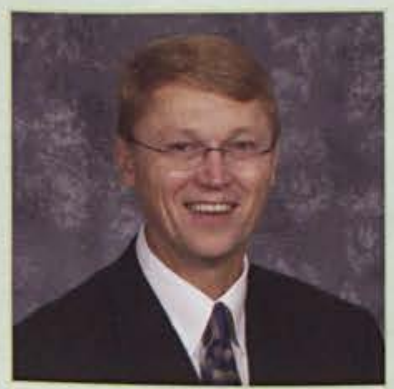

Kirk Martin Assistant Professor

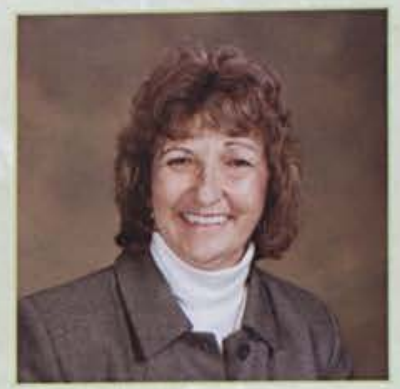

Kathy Freese Associate Professor

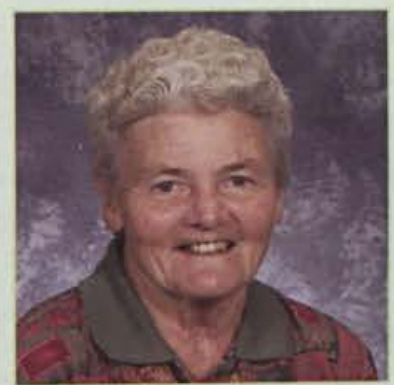

Dr. Dee Morris Adjunct Instructor

Department of Exercise and Sports Science

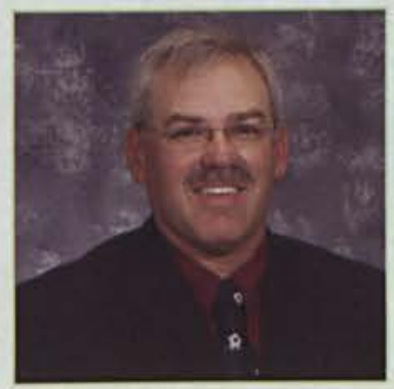

Ben Belleman Assistant Professor

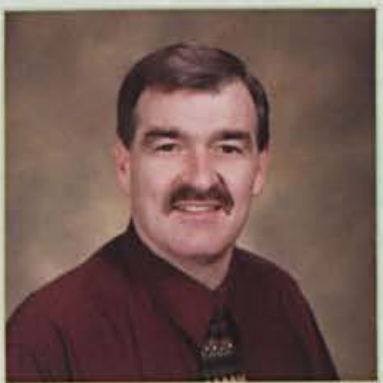

Dr. Alan Geist Assistant Professor

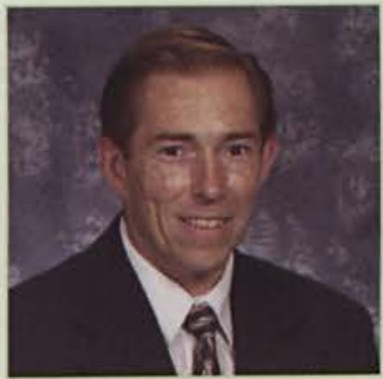

Paul Orchard Assistant Professor

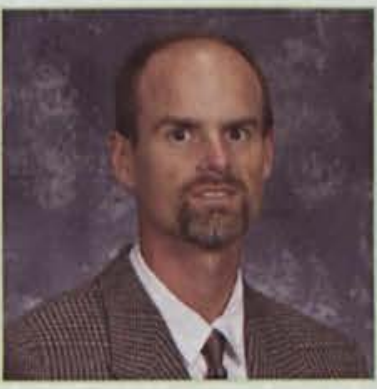

Jeff Bolender Assistant Professor

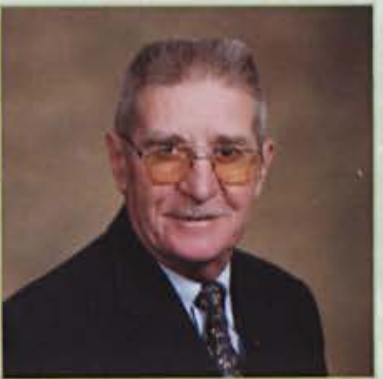

Elvin King Associate Professor

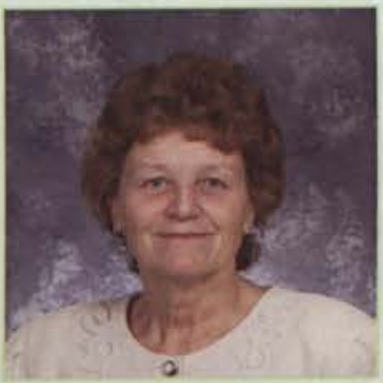

Libby Shearer Instructor

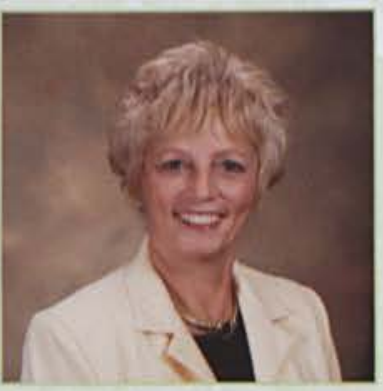

Teresa Clark Assistant Professor

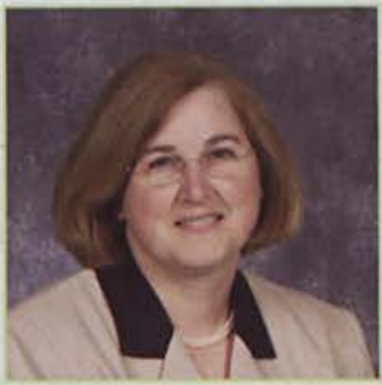

Becky Kuhn Instructor

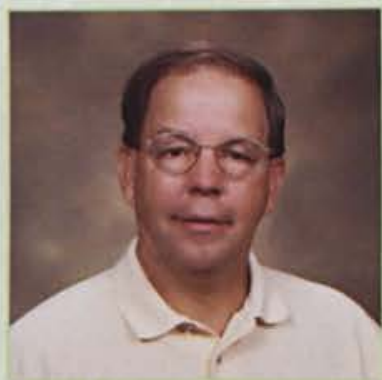

Ray Slagle Assistant Professor

\section{Department of Athletics/Campus Recreation}

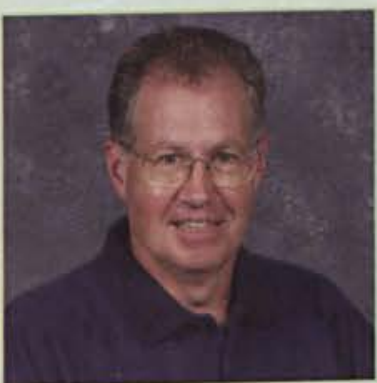

Jim Clark Asst. Sports Info. Dir.

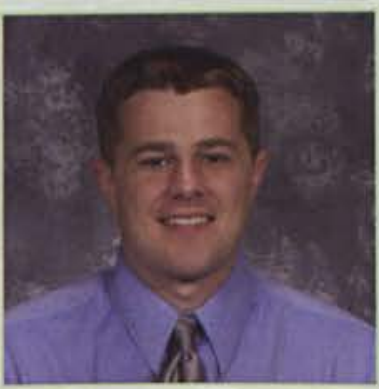

Pat Estepp Assistant Coach

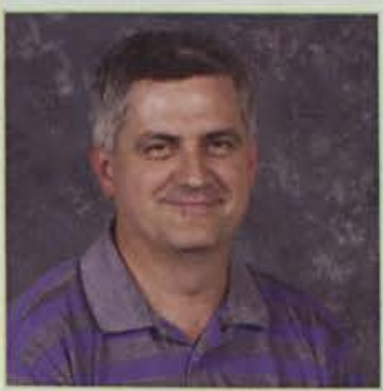

Mark Womack Sports Info. Director

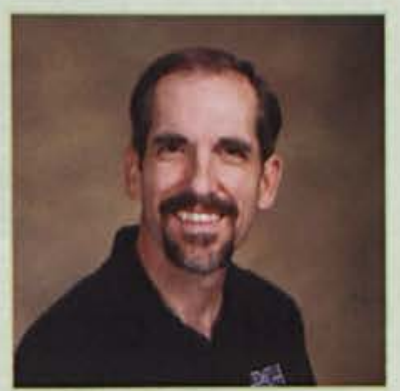

Mark Matthews Director 


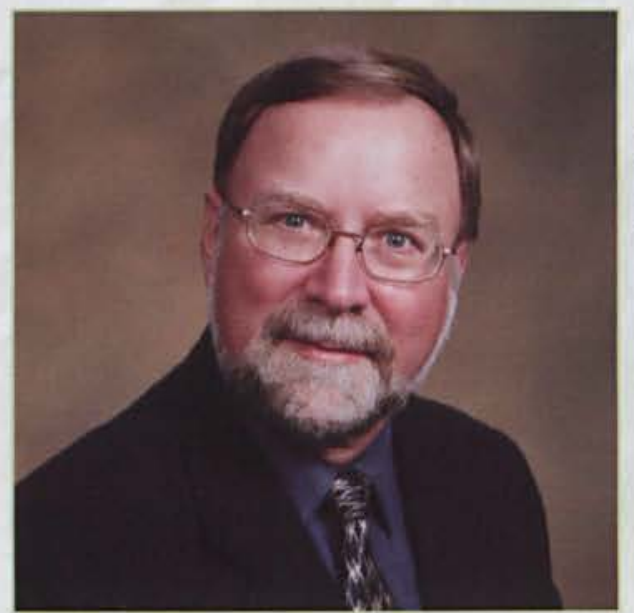

\section{School of Engineering, Nursing, and Science}

Dr. Stanley Baczek

Dean

This year, the School of Engineering, Nursing, and Science sought to fulfill their mission to offer an education in the theoretical and applied sciences that is consistent with biblical truth. On July 1 , the Computer Science major officially crossed over, becoming a part of the Engineering Department. Among other things, this eased class scheduling problems and allowed senior Computer Engineering majors to work with Electrical Engineering and Computer Science majors for their senior capstone design project. The Nursing Department was also alive with change. Four new instructors - Denise Martin, Rachel Parrill, Susan Salladay, and Amy Voris - taught on a variety of nursing matters while upholding the departmental vision statement. A masters' program was also in the works, which would build on the successes, stability, and reputation of the baccalaureate to build a comprehensive nursing program to construct Christ-like leaders for the medical field. Finally, the Math and Science Department hit the ground running. A Forensics Science major was planned to be implemented the following year, and the first Cedarville University graduates with a Bioethics minor graduated in May. Perhaps the most exciting change, however, was Dr. Whitmore's brand new Geology of the National Parks, a course designed to study the geology observable in our national parks during a May term travel study. Whatever the department, no matter the change, the School of Engineering, Nursing, and Science has nothing but excellence behind it and vision ahead.

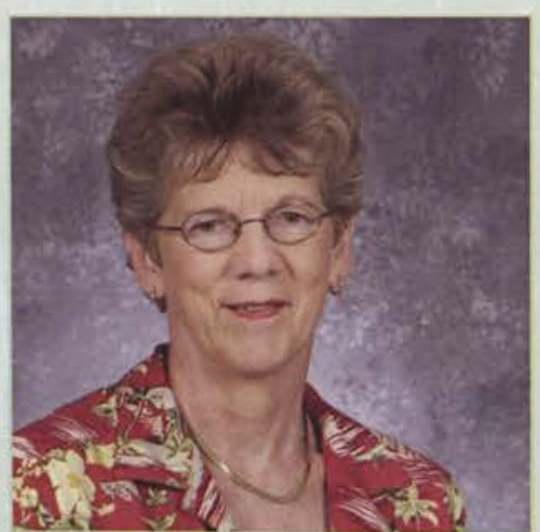

Dr. Irene Alyn Department Chair

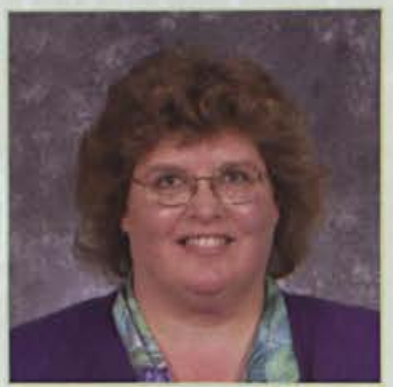

Linda Cave Assistant Professor

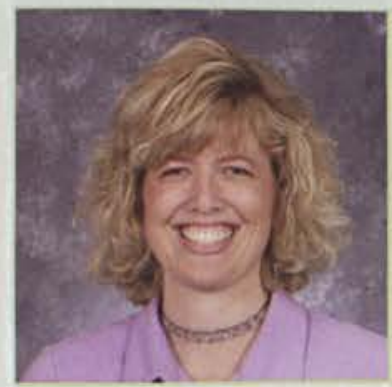

Dr. Charon Christman Associate Professor

\section{Department of Nursing}

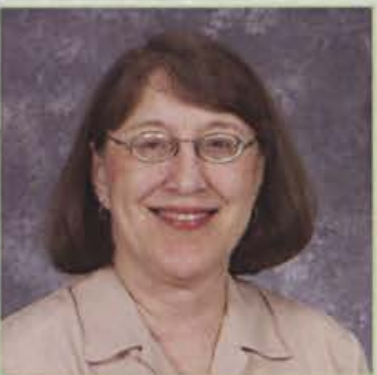

Dr. Lois Baker Professor

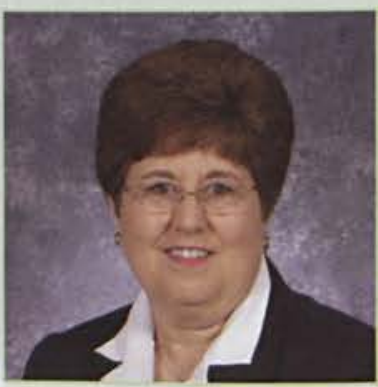

Dr. Janet Conway Senior Professor

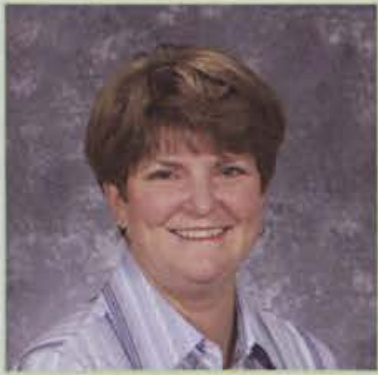

Karen Callan Instructor

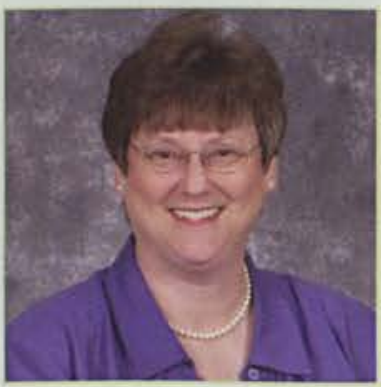

Connie Ford Assistant Professor

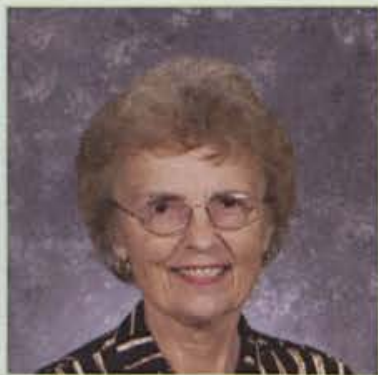

Dr. Carolyn Carlson Distinguished Professor

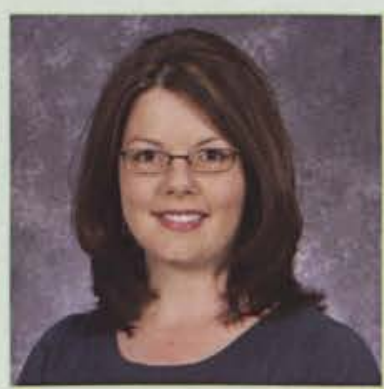

Kim Higginbotham Assistant Professor 


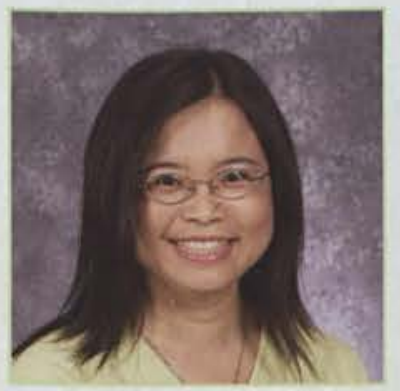

Chu-Yu Huang

Assistant Professor

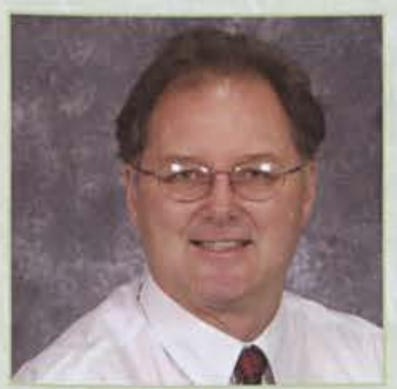

Dr. David Sharp Associate Professor

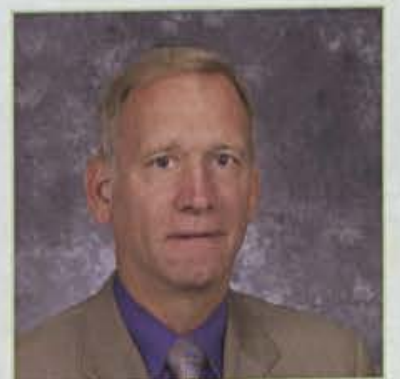

Mark Klimek Associate Professor

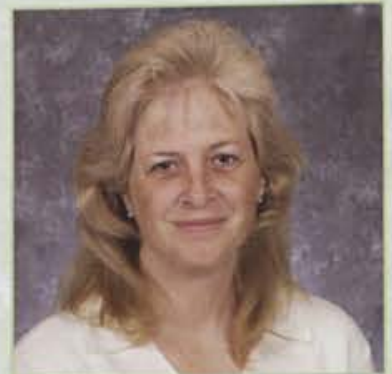

Dr. Kimberly Sharp Associate Professor

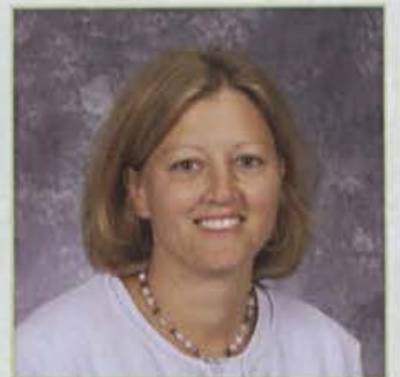

Suzanne Leafever Assistant Professor

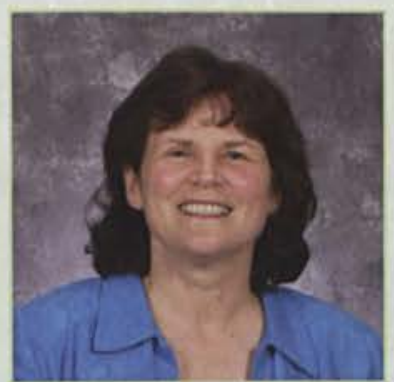

Dr. Sandra Shortt

Adjunct Instructor

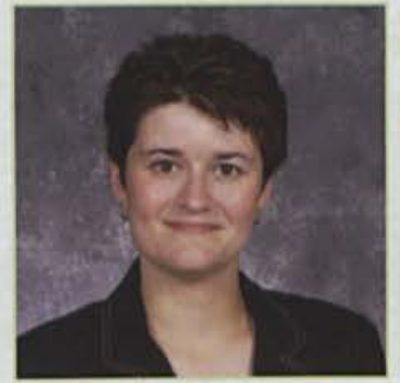

Rachel Parrill Instructor

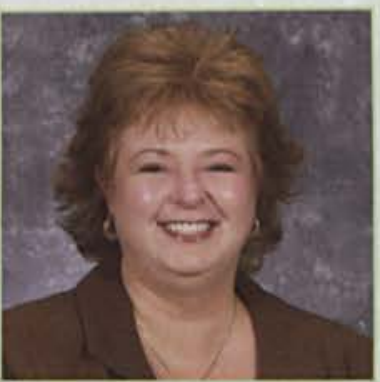

Rhonda Smith Adjunct Instructor

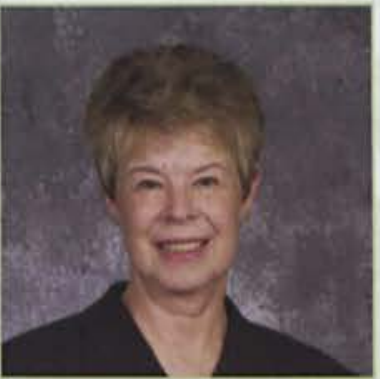

Dr. Susan Salladay Professor

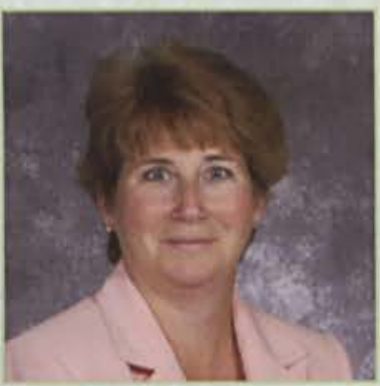

Amy Voris Instructor

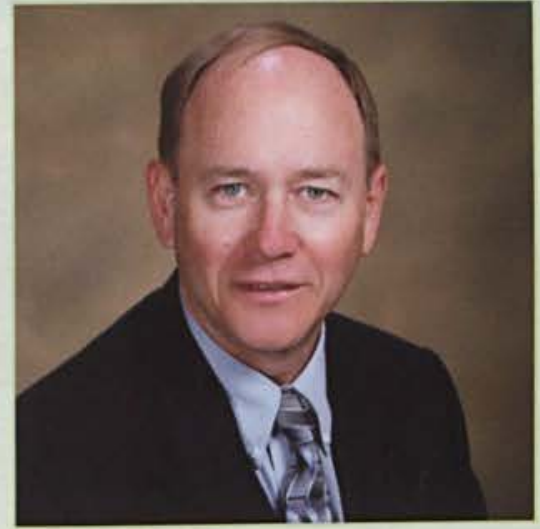

Dr. Lawrence Zavodney Department Chair

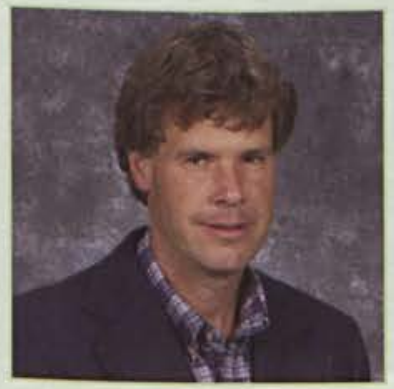

Dr. Timothy Dewhurst Professor

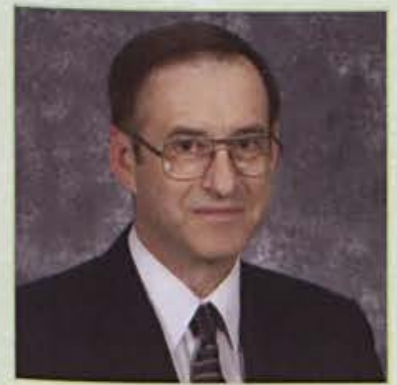

Robert Laramore Professor

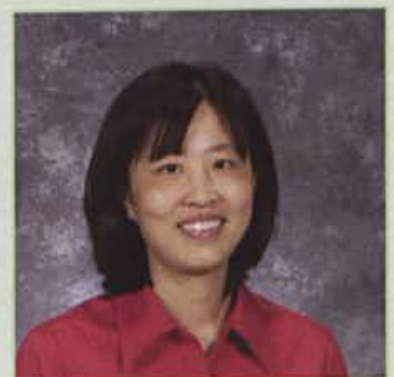

Dr. Vicky Fang Assistant Professor

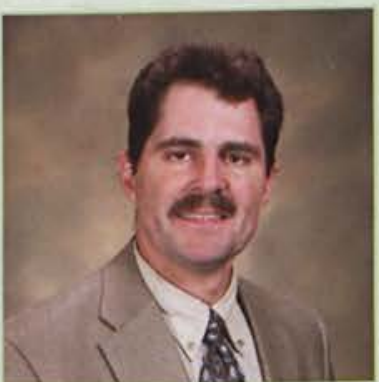

Dr. Timothy Norman Professor

\section{Department of Engineering}

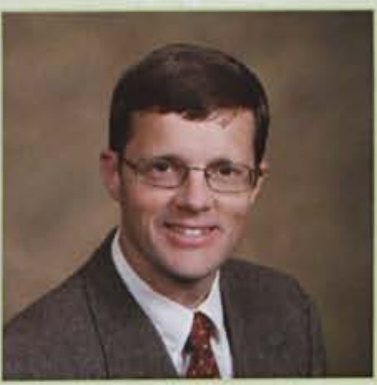

Dr. Gerry Brown Assistant Professor

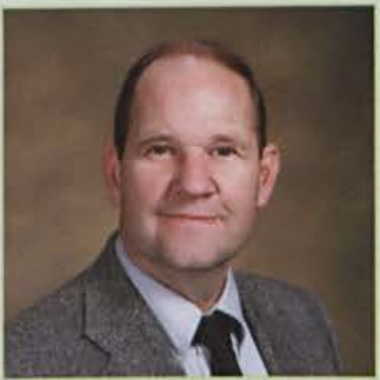

Dr. David Gallagher Associate Professor

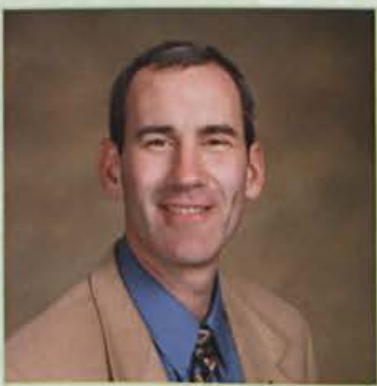

Dr. Sam SanGregory Associate Professor

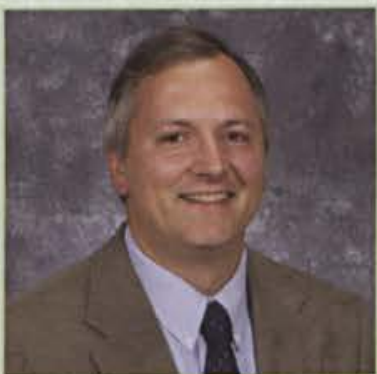

Dr. Pete Burban Associate Professor

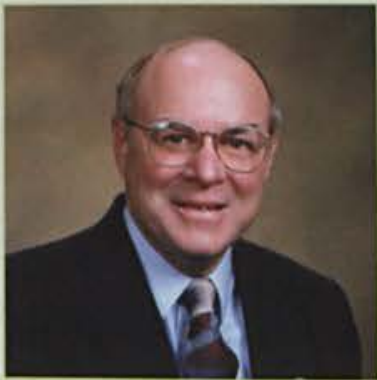

Dr. Harwood Hegna Professor

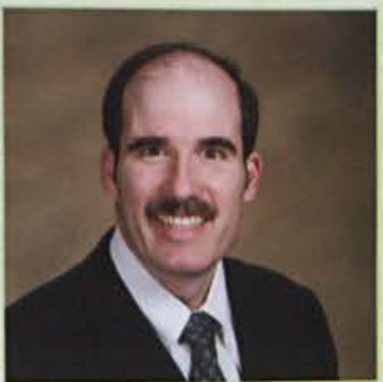

Dr. Keith Shomper Associate Professor

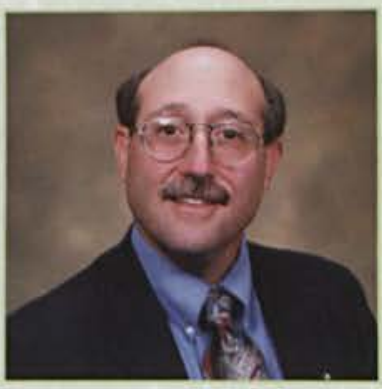

Dr. Robert Chasnov Professor

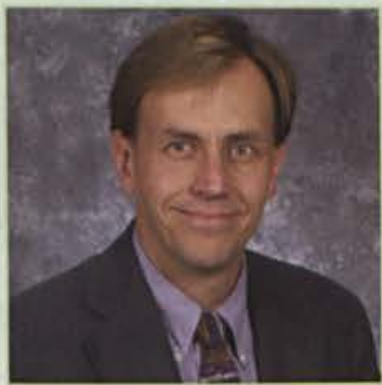

Jay Kinsinger Assistant Professor

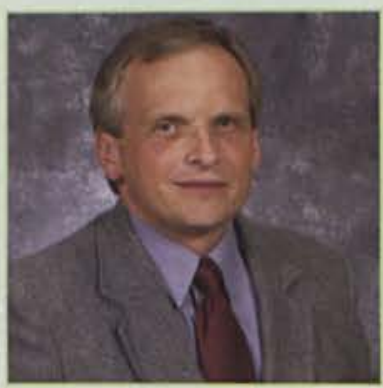

Dr. Jeff Shortt Associate Professor 


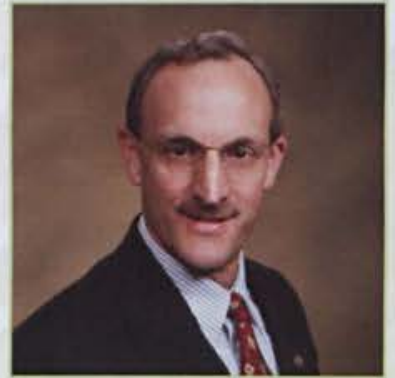

Dr. Thomas Thompson Associate Professor

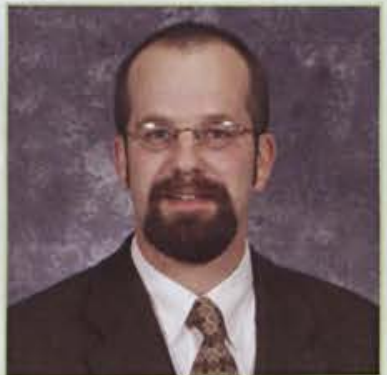

Tim Tuinstra Assistant Professor

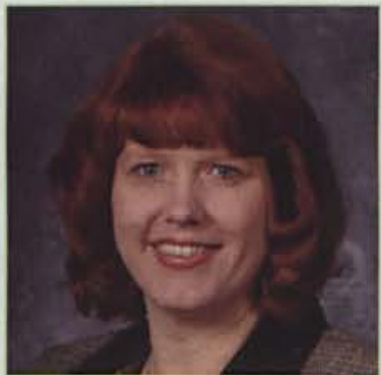

Jean Weyandt Ac. Dept. Secretary

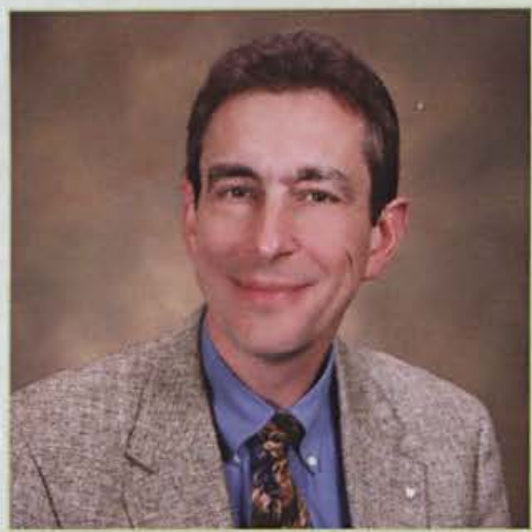

Dr. Dennis Flentge Department Chair

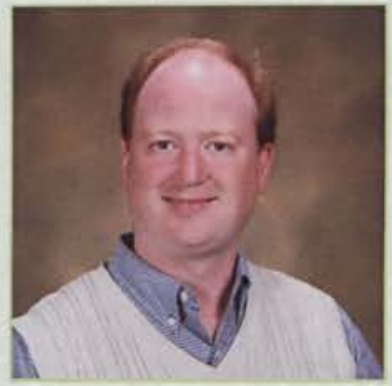

Dr. Darrin Frey

Associate Professor

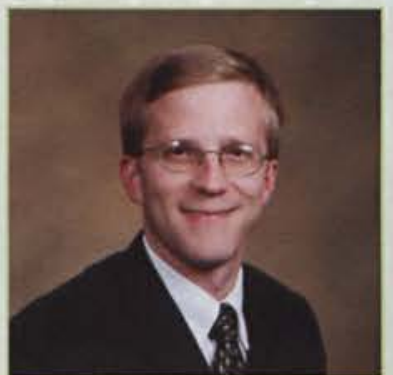

Dr. Mark McClain Associate Professor

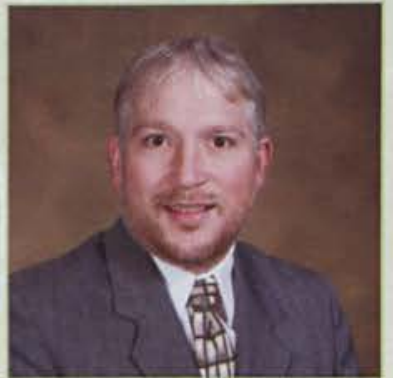

Dr. John Whitmore Associate Professor

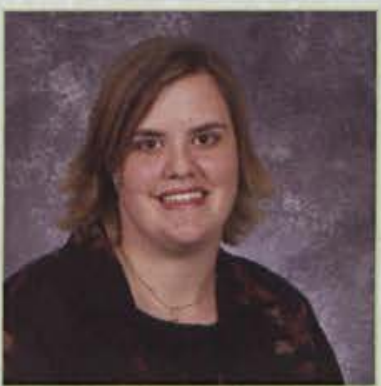

Sarah Gilchrist Instructor

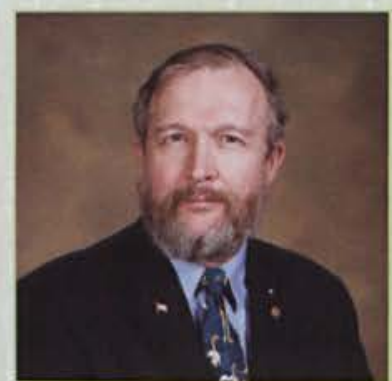

Robert Schumacher Assistant Professor

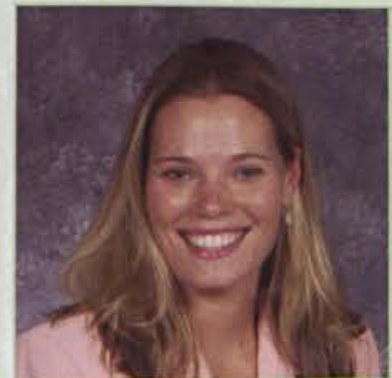

Cindy Wingert Instructor

\section{Department of Science and Math}

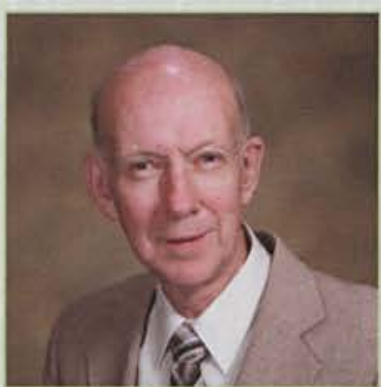

Dr. Donald Baumann Professor

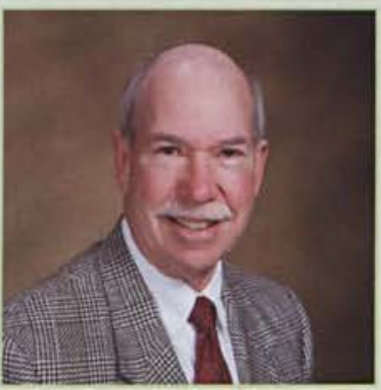

Dr. Larry Helmick Senior Professor

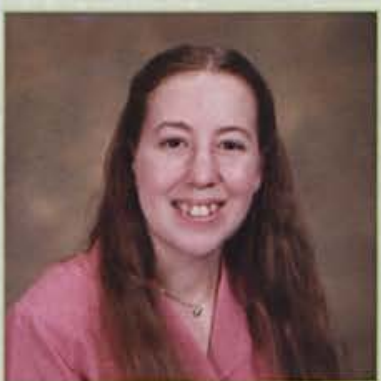

Jennifer Secor Instructor

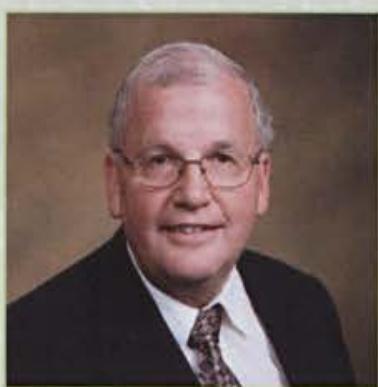

Dr. Edwin Braithwaite Professor

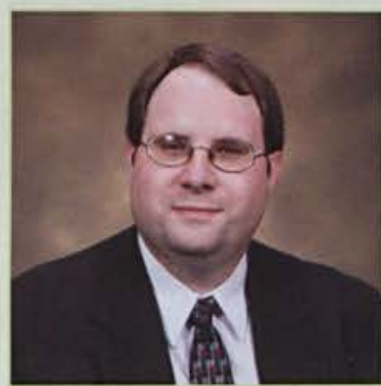

Aaron Hutchinson Assistant Professor

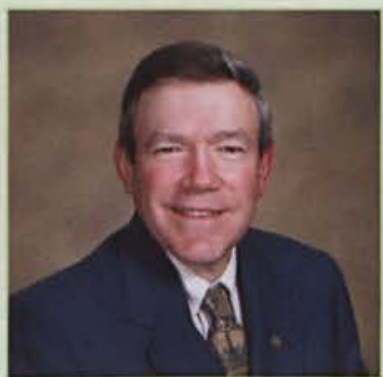

Dr. John Silvius Senior Professor

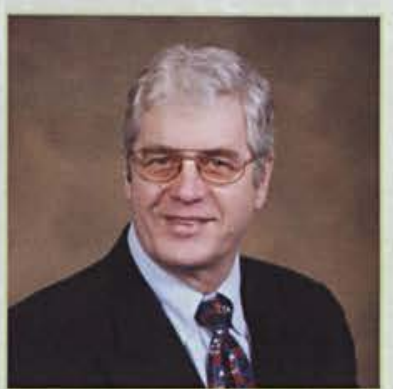

Dr. Lee Eimers Professor

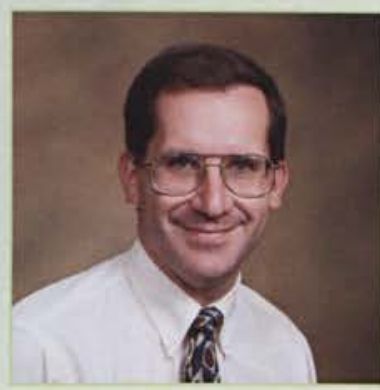

Eric L. Johnson Laboratory Technician

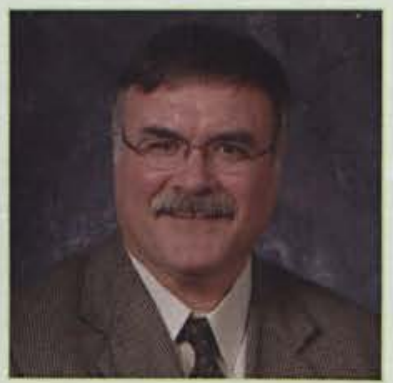

Dr. Dennis Sullivan Professor 

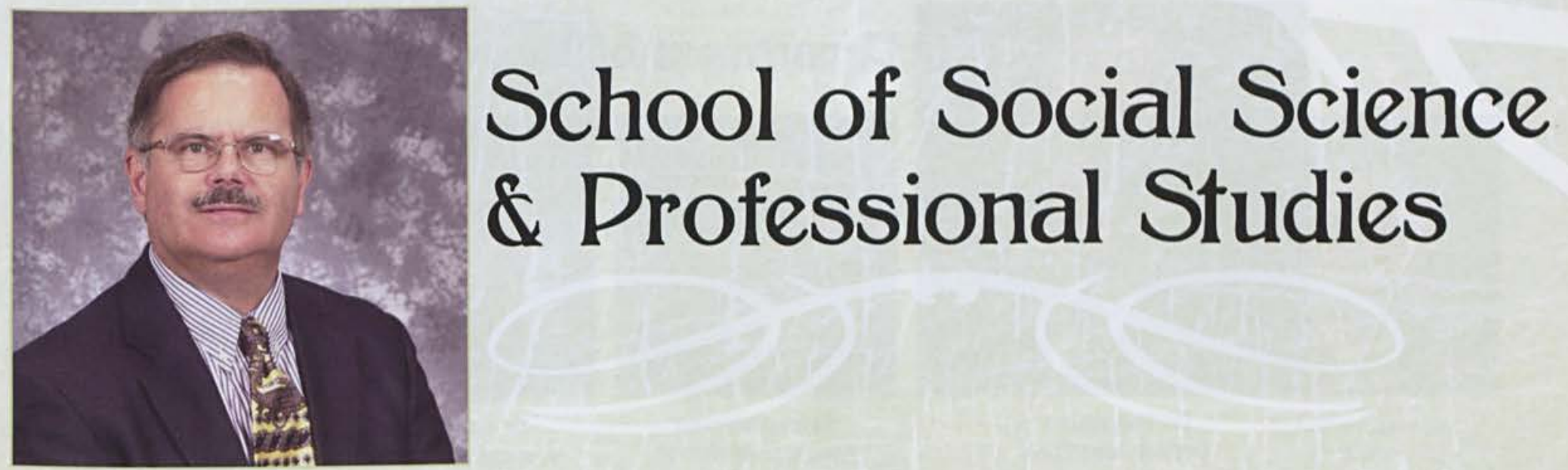

Dr. Kevin Sims Dean
The School of Social Science and Professional Studies strived to create Christian leaders who understand the proper integration of faith and their professions. The Business Department intended to develop business leaders who are discerning, competent, and influencing their organizations, professions, and culture from a biblical paradigm. The department furthered this goal by adding Dr. Richard Martinez as a professor of management, funding $\$ 150,000$ to develop a professional trading room, and creating a student/faculty consulting group to assist local businesses. The Education Department endeavored to prepare quality Christian teachers with a biblical world view, which will be manifested in their ability to teach students effectively around the world. In accordance with their strive for quality, on March 25-26, the department went through a rigorous six- point test and visitation by the National Council for the Accreditation of Teacher Education to become NCATE accredited. The Psychology Department introduced students to the world of research with a strong emphasis on classroom critical thinking that they might understand people and life from a biblical perspective. In September, a number of CU Psychology students participated in the World Conference of the American Association of Christian Counselors. The Social Sciences and History Department's mission was to serve competently in a multicultural society and global community while integrating a Christian worldview. In accordance with this goal, Professor George Huff received a summer grant to research "Integration of Faith and Learning" and presented his results at a social work conference in Chicago in February.

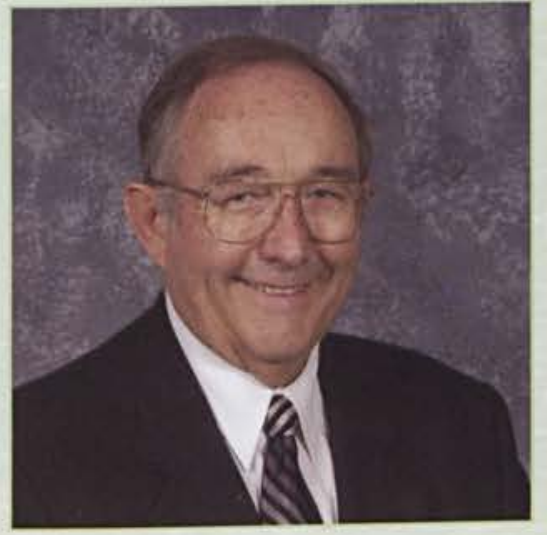

Dr. Murray Murdoch Department Chair

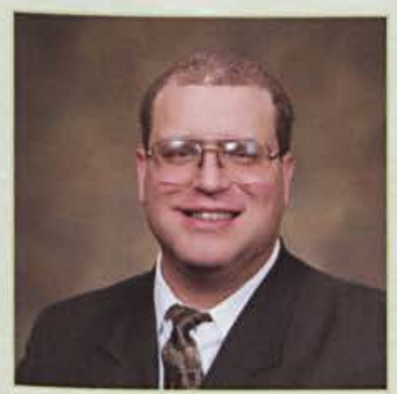

David Meyer Assistant Professor

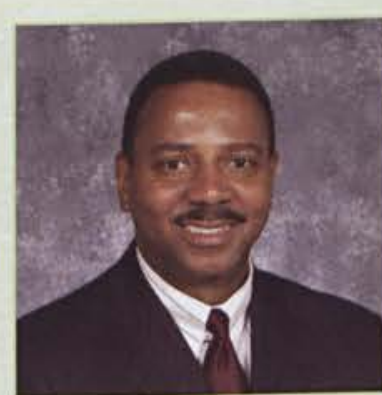

Patrick Oliver Assistant Professor

\section{Department of Social Science and History}

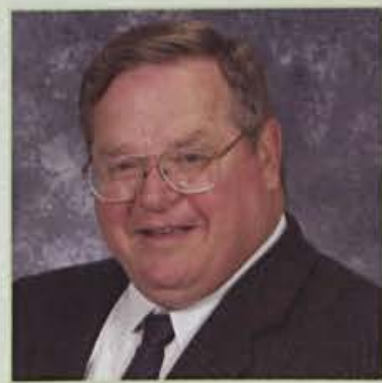

Dr. Nelson Henning Associate Professor

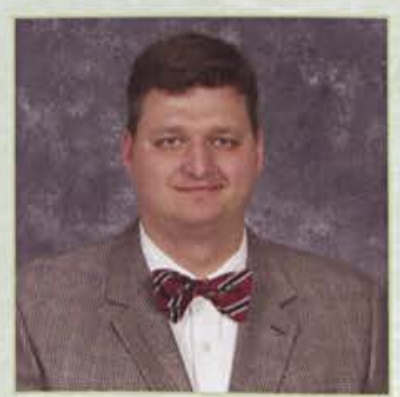

Dr. Mark Smith Assistant Professor

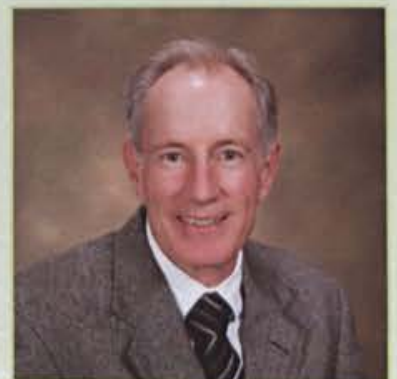

George Huff Assistant Professor

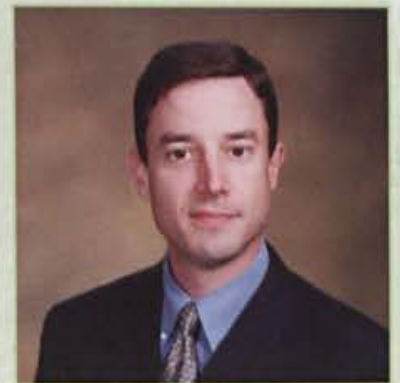

Dr. Tom Mach Associate Professor 


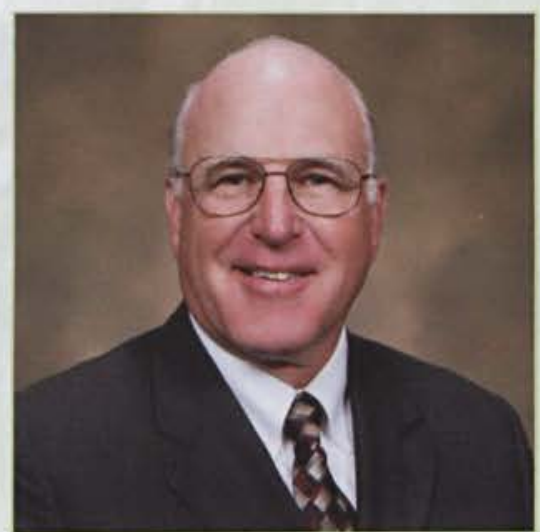

Dr. Ron Walker Department Chair

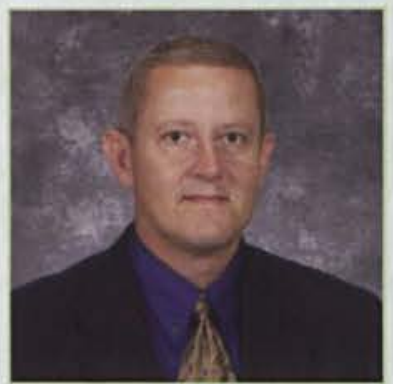

Dr. Alan Dillman Associate Professor

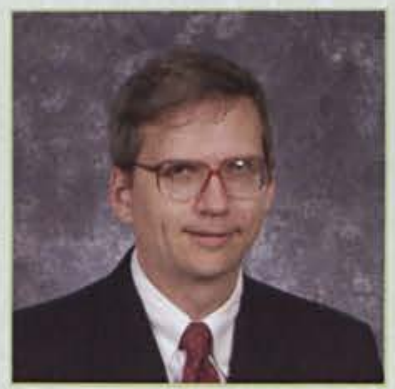

Charles Hartman Associate Professor

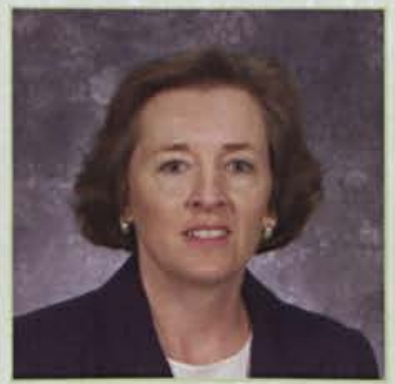

Anne Rich Assistant Professor

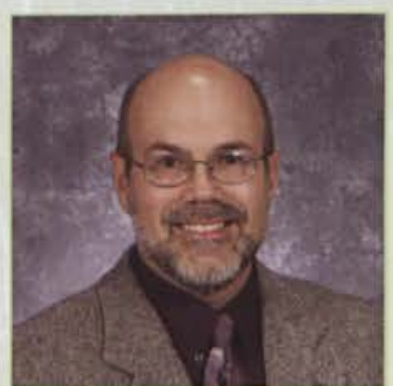

Dr. Bert Wheeler Professor

\section{Department of Business Administration}

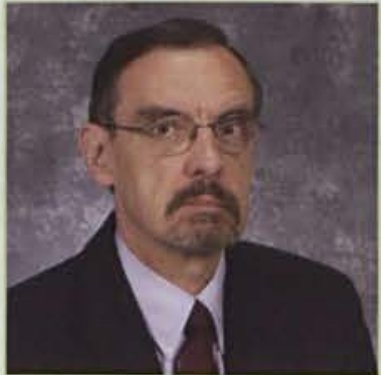

Harry Anderson Assistant Professor

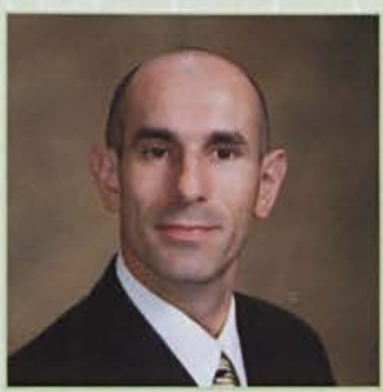

Dr. Franco Gandolfi Associate Professor

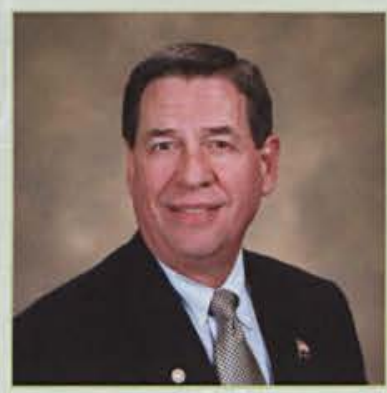

John LeBlanc Associate Professor

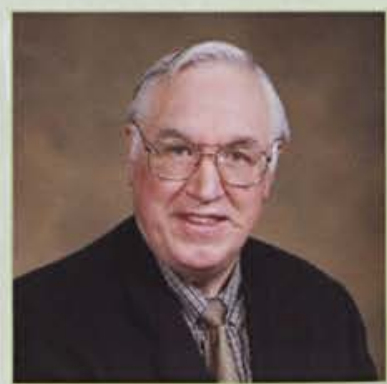

Dr. Galen Smith Professor

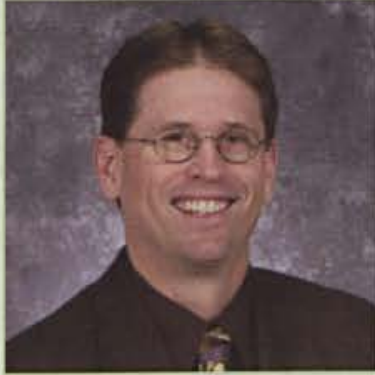

Dr. Jon Austin Associate Professor

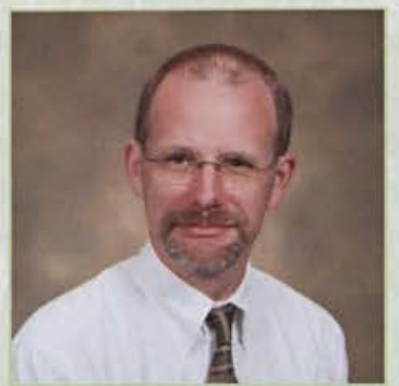

Jeffrey Guernsey Assistant Professor

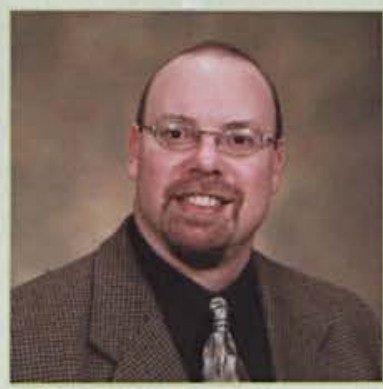

Dr. Richard Martinez Associate Professor

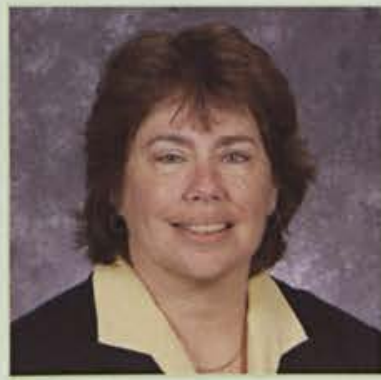

Dr. Sarah Smith Associate Professor

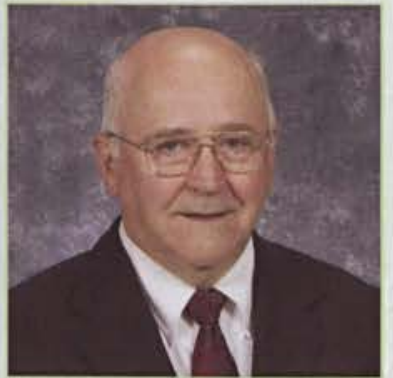

Dr. Dick Baldwin Professor

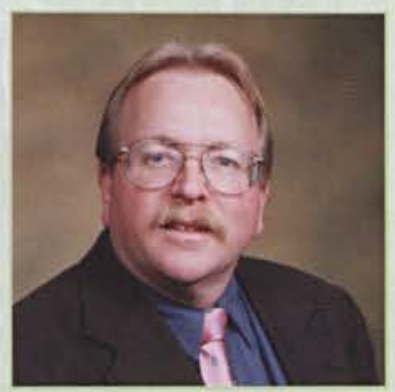

Tim Hansell Assistant Professor

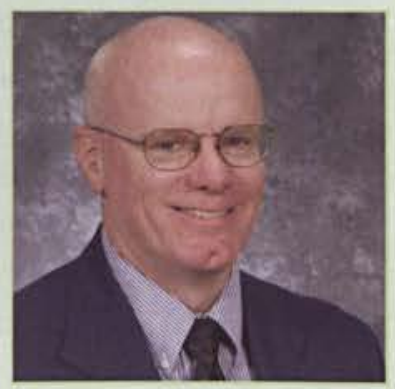

Dr. Bill Ragle Associate Professor

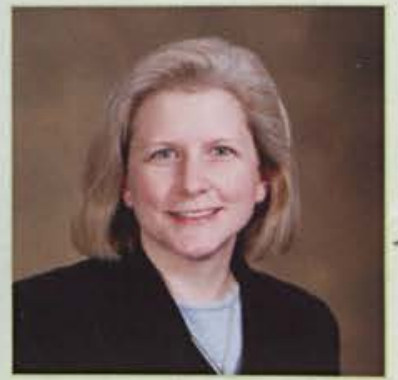

Susan Terkelsen Assistant Professor 


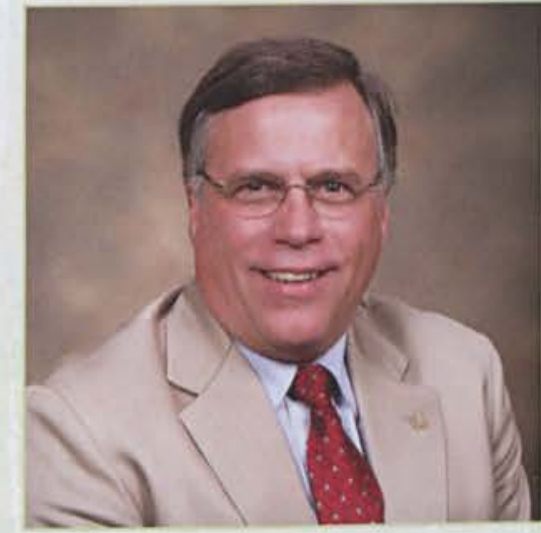

Dr. Steve Gruber Department Chair

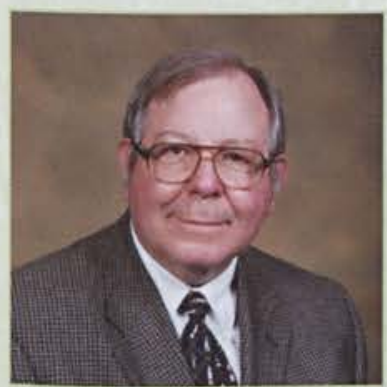

Omer Bonenberger Associate Professor

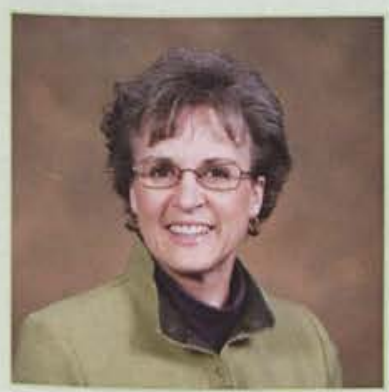

Dr. Cheryl Irish Associate Professor

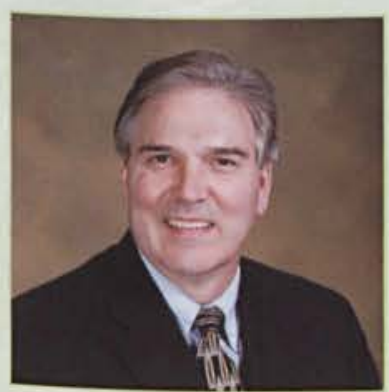

Dr. Bryan Moore Assistant Professor

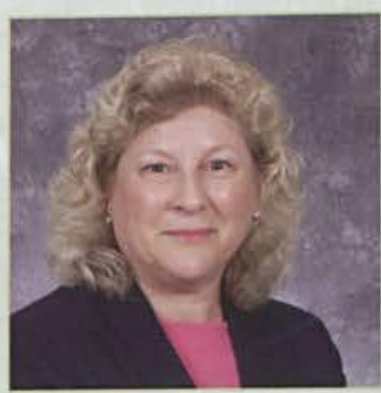

Carol Estes Assistant Professor

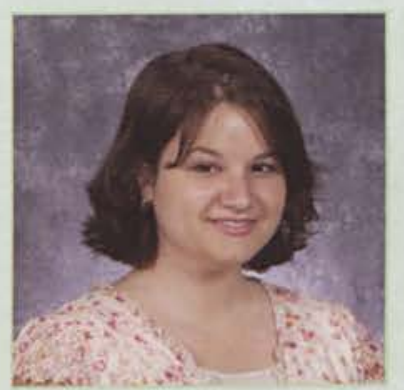

Laura Jolly Lic. \& Testing Cord.

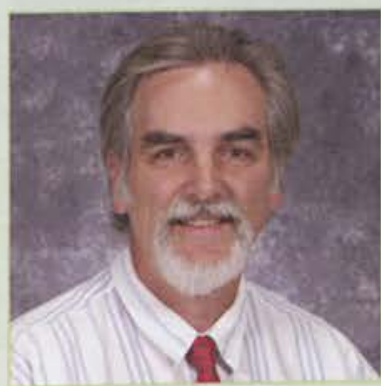

Dr. Tom Sweigard Assistant Professor

\section{Department of Education}

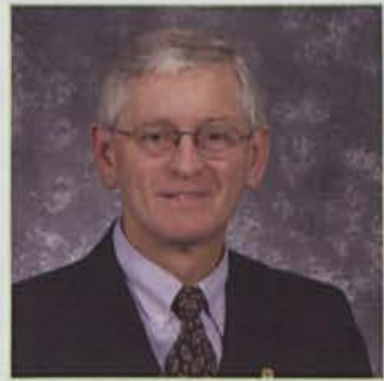

Dr. Merlin Ager Professor

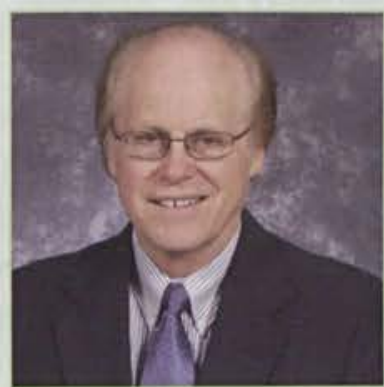

Dr. Dwayne Frank Professor Emeritus

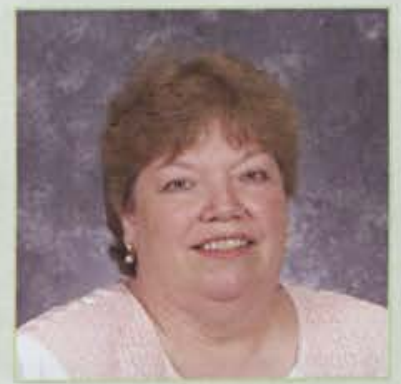

Toi King

Ac. Dept. Secretary

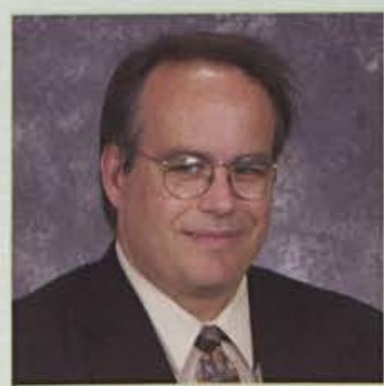

Kevin Winslow

Assistant Professor

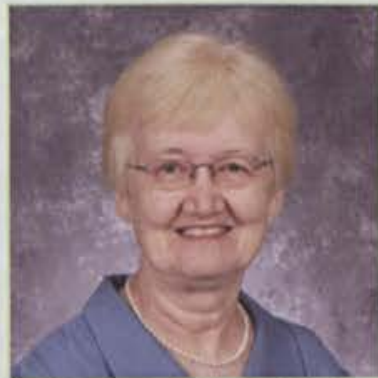

Dr. Sue Baker Professor

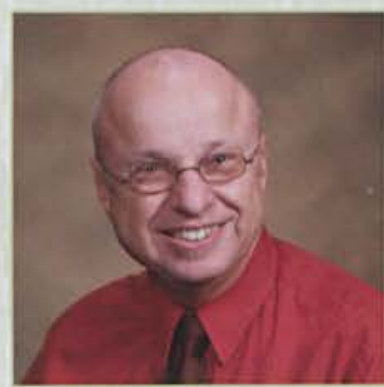

Dr. Tim Heaton Associate Professor

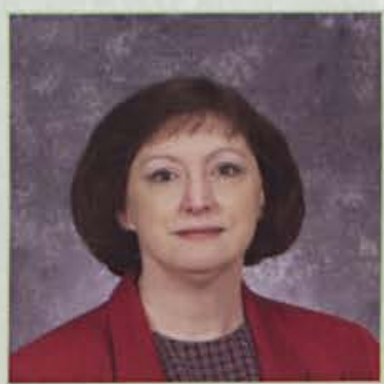

Deb Mallonee Adjunct Instructor

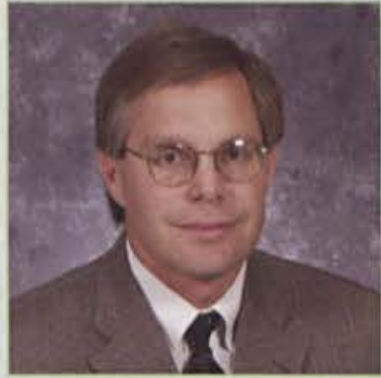

Dr. Eddie Baumann Professor

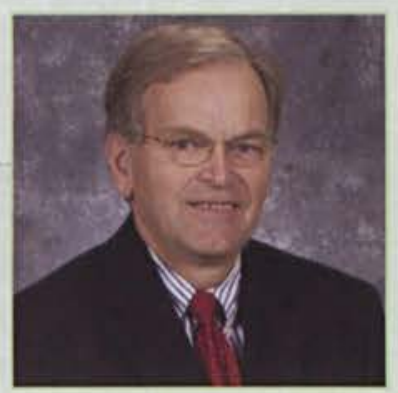

John Hess Director

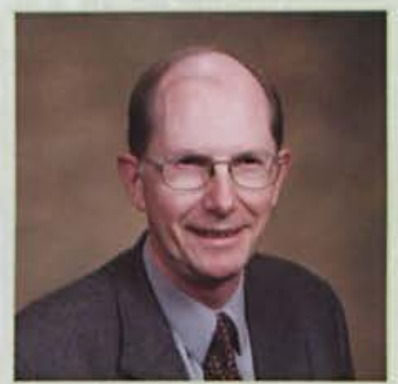

Dr. Don Meissner Assistant Professor

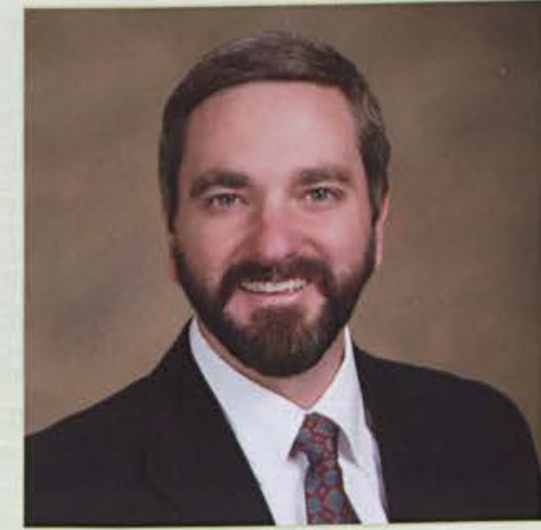

Dr. Michael Firmin Department Chair

\section{Department of Psychology}

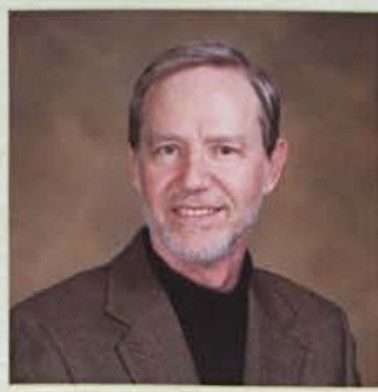

Dr. Milt Becknell Associate Professor

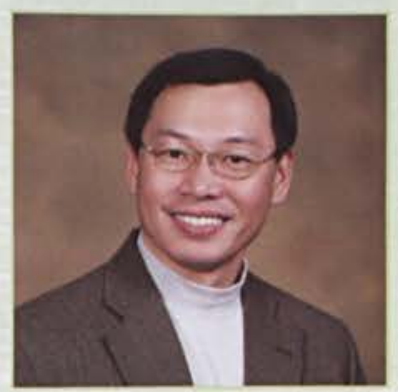

Dr. Luke Tse Assistant Professor 


\section{Academic Division}

Though often hidden from students' eyes, Dr. Robert Milliman, former dean of the School of Humanities, Fine Arts, and Bible, led the Academic Division through a number of changes in his first year as Academic Vice President. The Academic Division covered a vast area of expertise and included Computer Services, Graduate Studies, Institutional Research, the Centennial Library, Summer School, Travel Studies, and the newly revamped Academic Assistance Center - the Cove. A much appreciated project undertaken by Computer Services was the implementation of the outdoor wireless network across campus. Now, students are able to access the Internet from nearly anywhere outdoors. They also replaced an abundance of lab and dorm computers this past summer $-1,200$ of them! Both the Summer
School and Travel Studies departments sought to expand students' cultural consciousness. This was the first semester Cedarville had been a study-abroad institution for another country. During the spring semester, six students from South Korea's Handong Global University experienced life in a quiet college town. The Cove sought to promote authentic scholarship and provided students with a less intimidating place to seek help with schoolwork and further their learning experiences. Within a corner of the Callan Athletic Center, the tutoring program and testing accommodation of students with disabilities skyrocketed. Director Kim Ahlgrim hopes more students will take advantage of the Cove as they begin to "educate faculty, students, and staff about who we are."

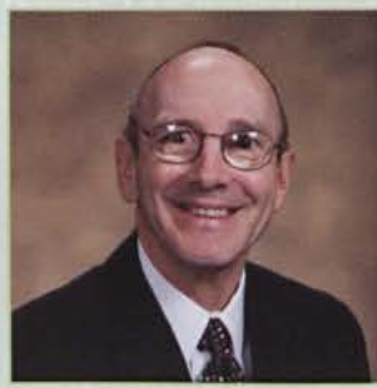

Dr. Duane Wood Executive Director

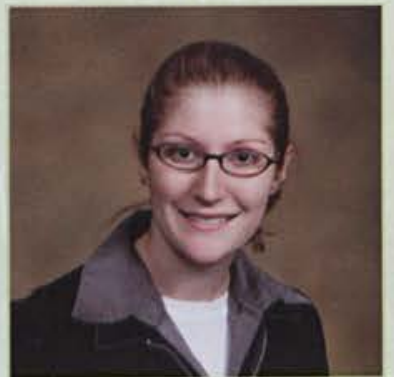

Ashley Rohne Comm. Assistant

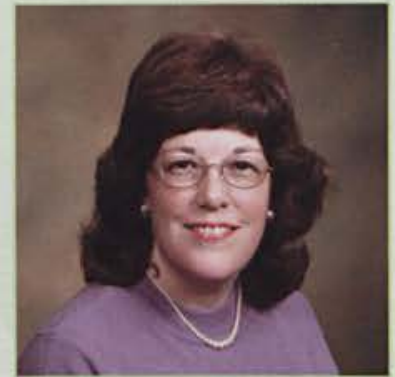

Linda Chrystal Admin. Assistant

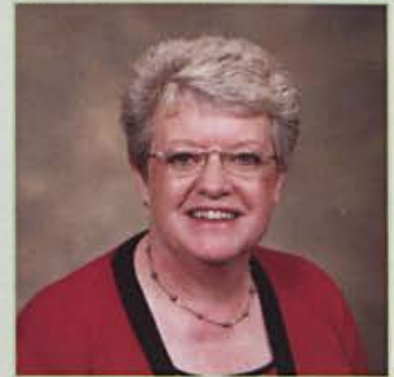

Fran Campbell Registrar

\section{Assistants \& Secretaries}

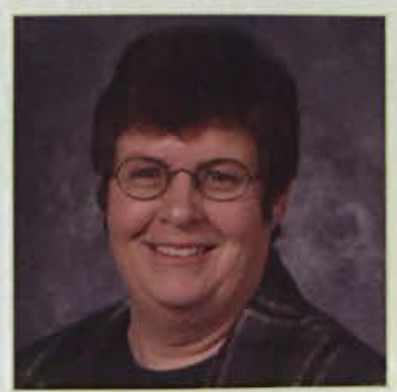

Deb Bissett Admin. Assistant

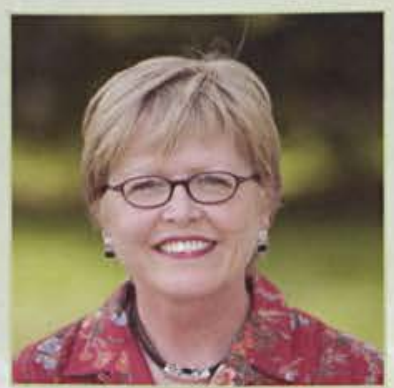

Pam Bromer Admin. Assistant

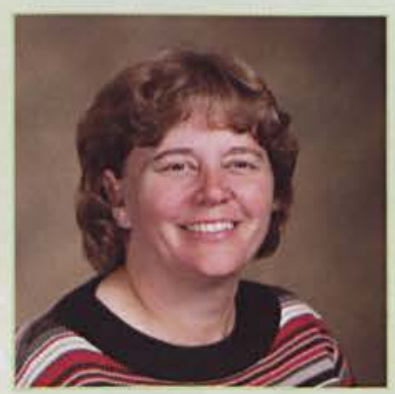

Vicki Edem Departmental Assistant

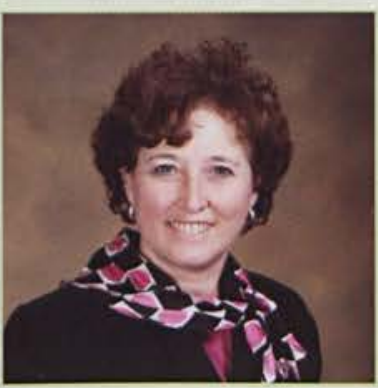

Carol George Admin. Assistant 


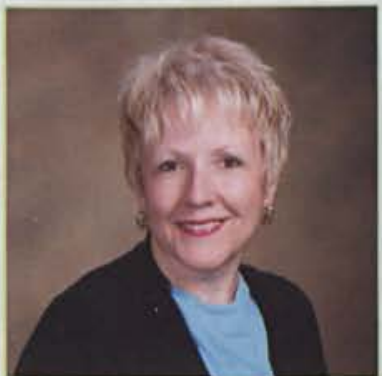

Debra Halsmith Admin. Assistant

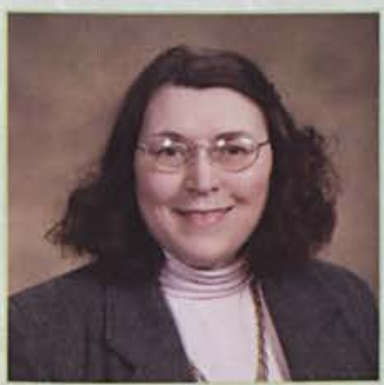

Esther Lanham Admin. Assistant

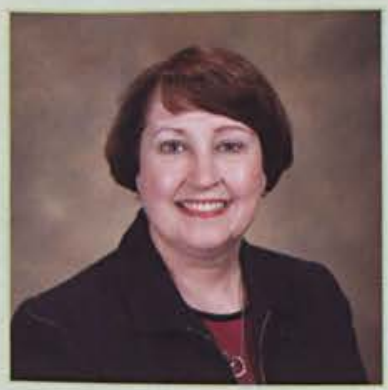

Lynn Rohm Admin. Assistant

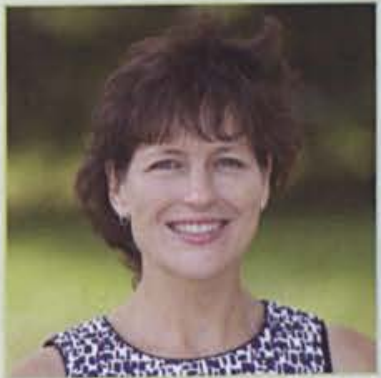

Kelly Hellwig

Secretary

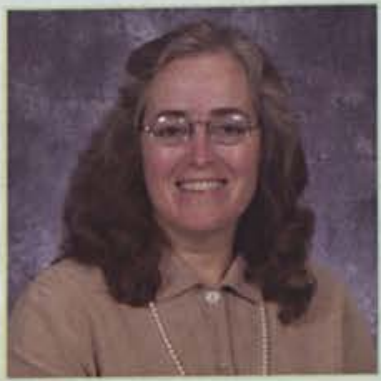

Kay McFarlane Acad. Dept. Secretary

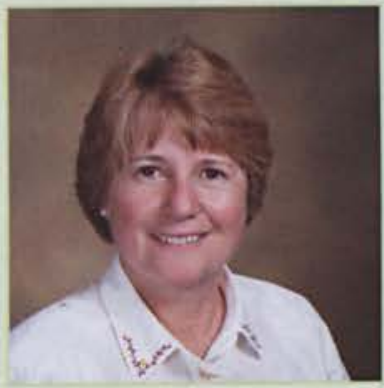

Joy Williams

Admin. Assistant

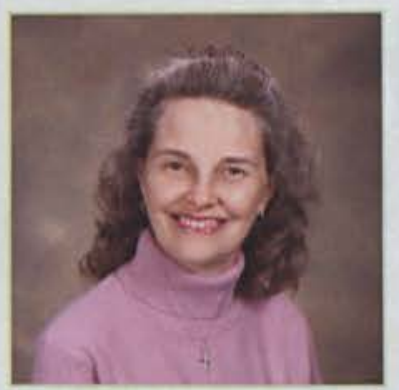

Virginia Huff Department Secretary

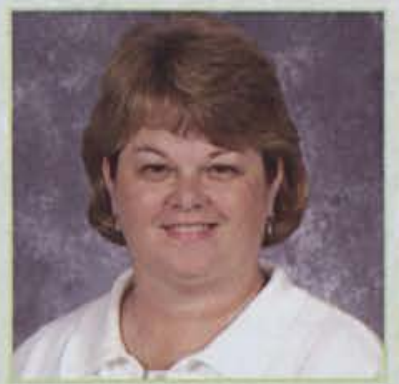

Pam Miller Admin. Assistant

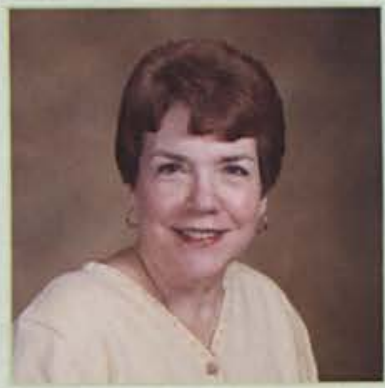

Sherrie Wood Dean's Assistant

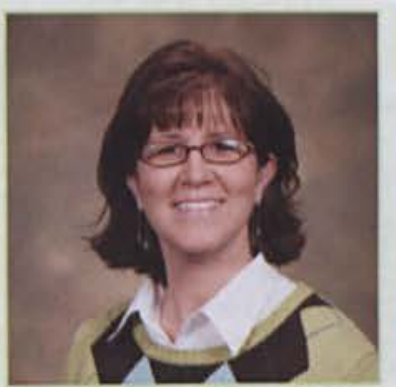

Elaine Keller Admin. Assistant

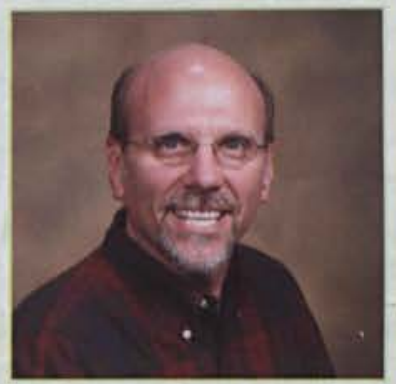

Jon Purple

Assistant to the VP

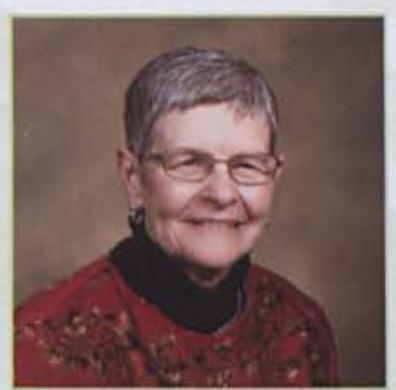

Nancy Knauff Secretary

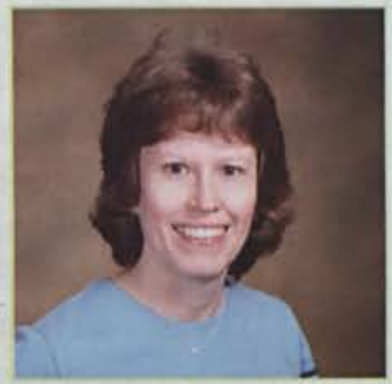

Nancy Ranger Dean's Assistant

\section{Computer Services}

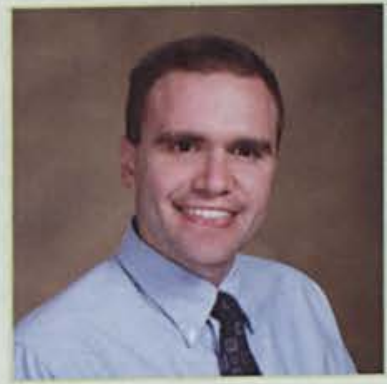

Gabe Custer Programmer

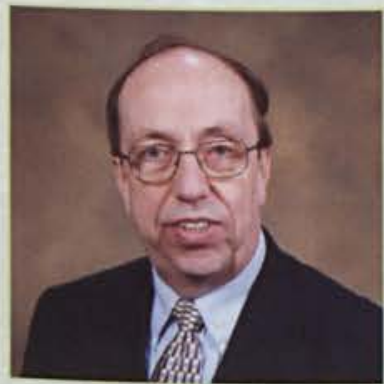

Dr. Dave Rotman Director

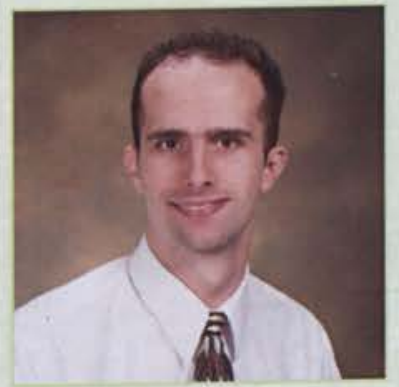

Brad Voumard Systems Analyst

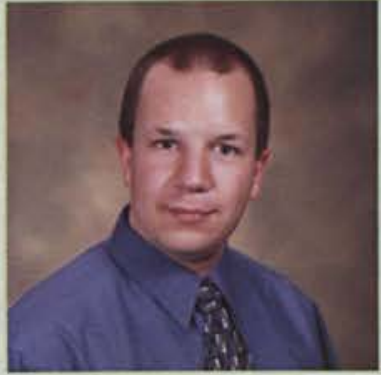

Nathan Hay Network Engineer

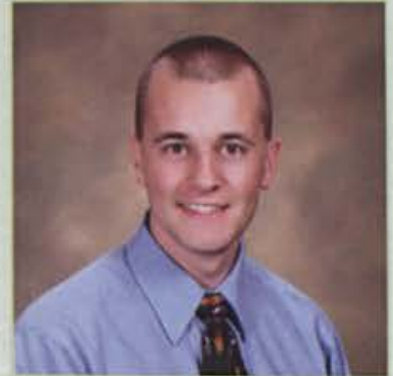

Devon McCarty Programmer

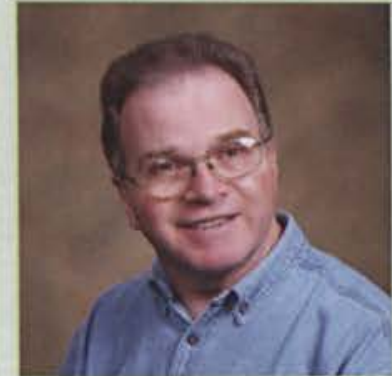

Gary Olin Software Specialist 


\section{Student Life Division}

Cedarville University students were blessed with the multiple Student Life services that were offered to us throughout the campus. The mission statement of Counseling Services was to provide individual counseling, referrals, and support groups consistent with biblical principles. In order to provide more services for students, Rachel Heffield was added to the counseling staff, which now includes four full-time counselors. The Campus Activities Office continued to maintain a large role in the student's lives this year through various activities such as the EXCEL Leadership workshops, Getting Started Weekend, Commencement, and the ever-important service of "survival kits" from parents. Career Services gave students the opportunity to meet with various professionals during ten different Career Link days throughout the school year and continued to assist students in résumé composition, networking, internships, and graduate school test preparations. University Medical Services added two new staff members and instituted a new service in order to serve the student body more fully. This "Nurse Line" feature was available to students, who could talk to an RN, schedule appointments with a physician, or get advice for self-care. Students also had the luxury of having three physicians and a nurse practitioner on campus. Overall, the Student Life Division did an amazing job of helping students to get the very most out of their college experience, and in doing so, fulfilled their goals.

\section{Resident Directors}

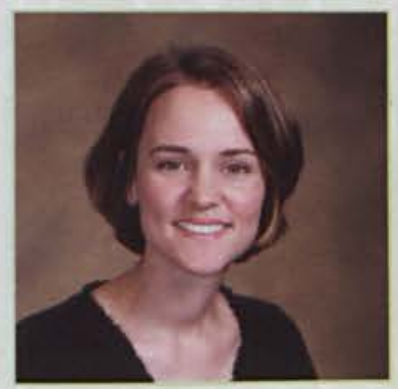

Meredith Irving Resident Director

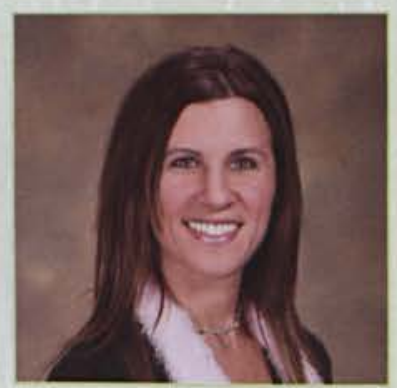

Bonnie Kuvshinikov Resident Director

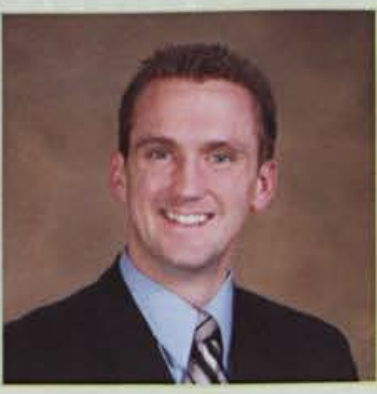

Jason Merkle Assistant Dean

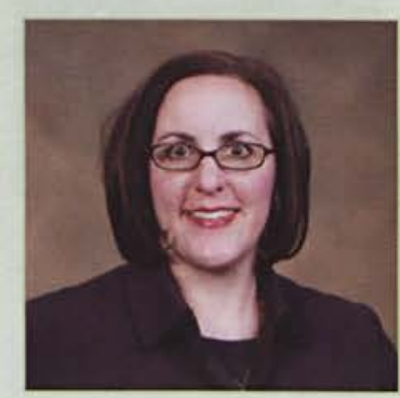

Kirsten Gibbs Dean of Students

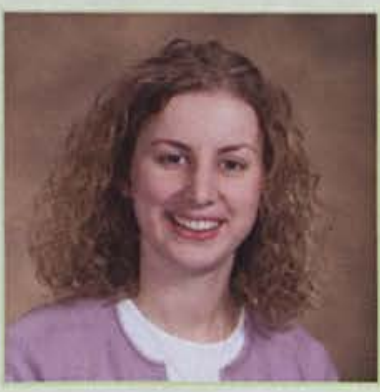

Becky Stowers Resident Director

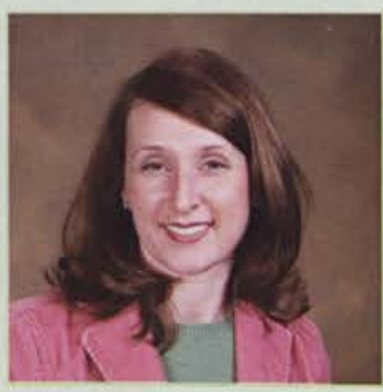

Susan West Resident Director

\section{Career Services}

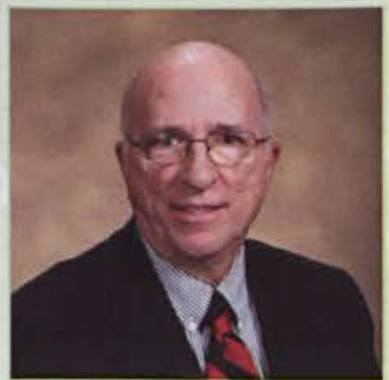

Lew Gibbs

Director

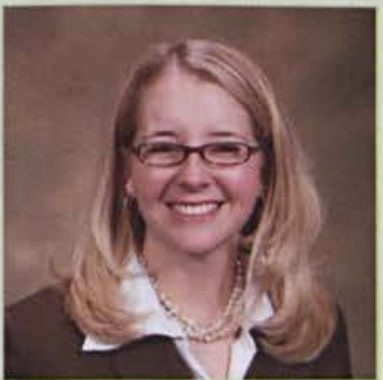

Mandy Hutchinson Admin. Assistant

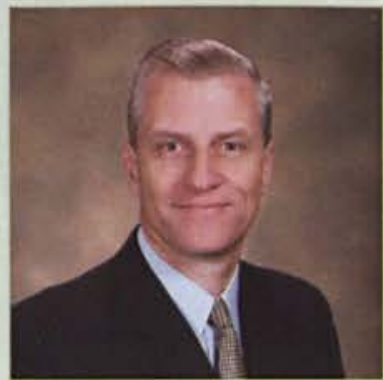

Jeffrey Reep Assistant Director 


\section{Counseling Services}

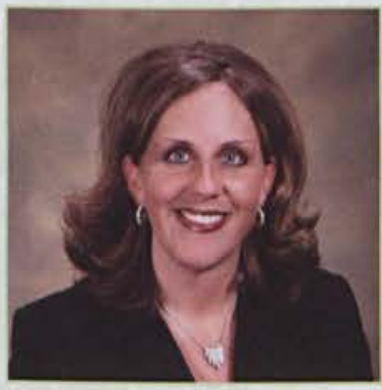

Dr. Lori Anderson Counselor

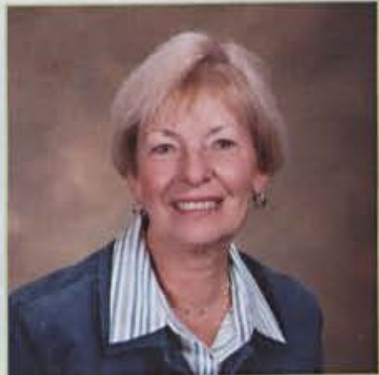

Helen Blumenstock Secretary

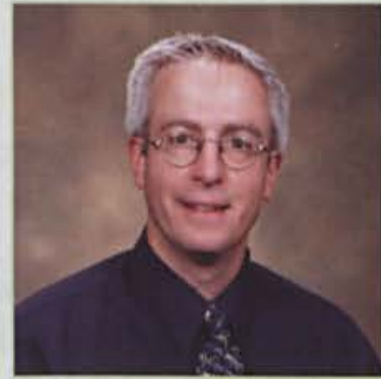

Jeffrey Fulmer Counselor

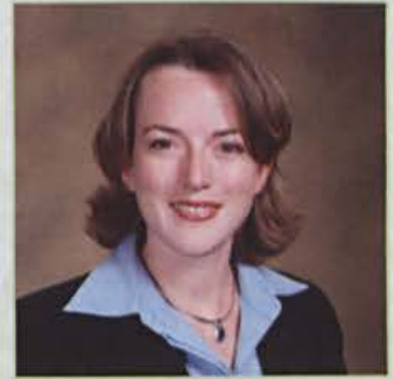

Rachel Heffield Counselor

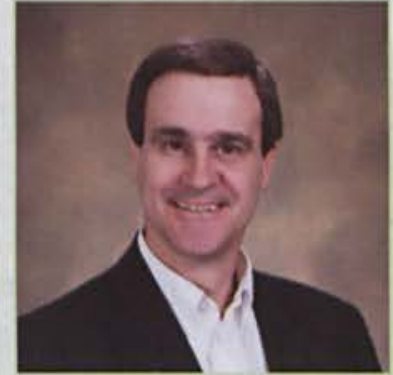

John Potter Director

\section{Campus Activites}

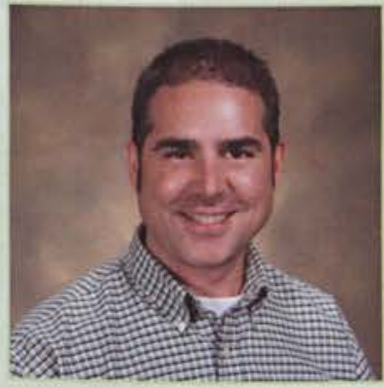

Jeff Beste Assistant Director

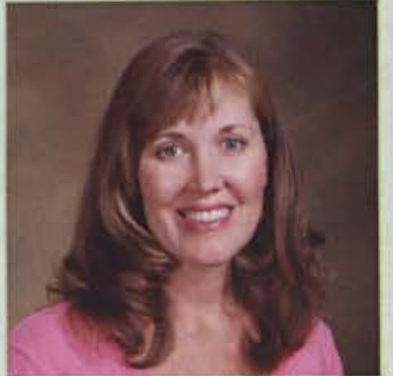

LeAnne Hill Secretary

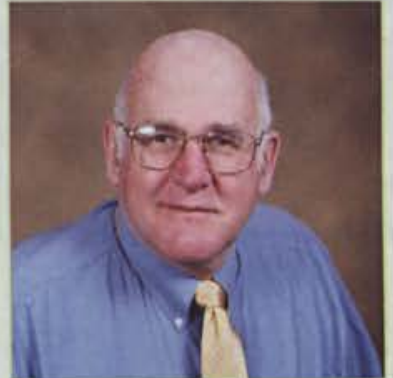

Dick Walker Director

University Medical Services

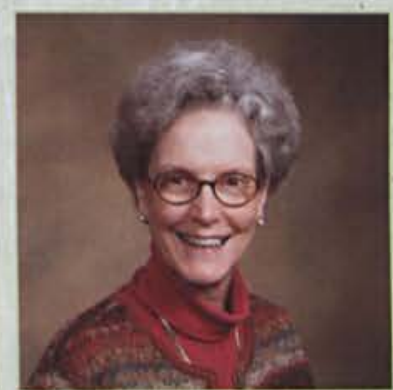

Martha Kaercher Student Ins. Cord.

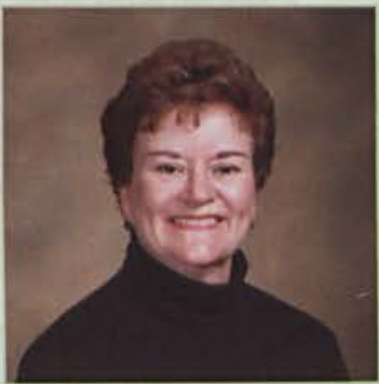

Beverly Robey Nursing Supervisor

\section{Advancement Division}

Though not typically one of the most visible of Cedarville's departments, the Advancement Division had another busy year working behind-thescenes making life at Cedarville University available to more students than ever. This division encompassed the Annual Fund, Alumni Relations, CDR Radio, Gift Planning, and Production Services. The Annual Fund continued its effort to "bridge the gap" between the cost of running Cedarville's many educational programs and the money students actually pay in tuition. It also offered a wide range of endowed scholarships, available to new and returning students of all majors. Alumni Relations, in accordance with its mission, began the long and tedious process of setting up alumni chapters for graduates all over the United States and was in the planning stages of developing their first international chapter in Hong Kong. Volunteers from DAE, the student organization that aids Alumni Relations, clocked thousands of miles traveling all over the United States, representing the student body to alumni. CDR Radio continued their ministry to Cedarville and the surrounding areas through their support of events such as Joni and Friends' Walk ' $n$ Roll and Building Blocks for a Christian Worldview, featuring Joel Belz of World Magazine. CDR also began planning for several expansion stations that will serve four additional Ohio cities - Greenville, Sidney, Bellefontaine and Logan. Though students may not always notice these simple, behind-the-scenes changes brought about through the work of the Advancement Division, its contribution to the Cedarville Family, past and present, is invaluable.

\section{Alumni Relations}

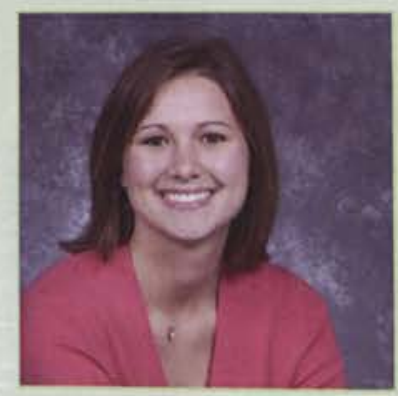

Kristin Borton Administrative Assistant

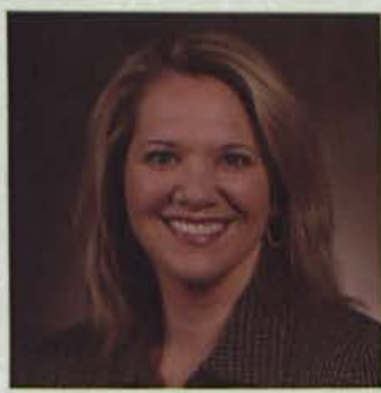

Faith Linn Dir. of Alumni Relations 


\section{Business Division}

There have been many changes in the Business Division this year to accommodate students better. The offices that made up the Business Division were the bookstore, payroll office, Project Development, Building and Grounds, Campus Safety, Cashier's Office, Human Resources, the post office, and Chuck's. The Bookstore had various authors conduct book signings throughout the school year. After speaking in chapel, Gracia Burnham visited with students and faculty as she signed copies of her book, In The Presence of My Enemies. Other featured authors included Cedarville's own Dr. Hoffeditz and Dr. Estes, as well as alumna Sarah Young. The Post Office purchased a new machine in order to make mailings faster and more time-efficient for the students. The post office worked with multiple packages and letters daily and was a blessing to the campus in their hard work and cheerful attitudes. Chuck's had set the standards of college cafeterias for yet another year. Theme nights including Italian, Mexican, Chinese, and American were a hit as always as students listened to music from different countries while enjoying ethnic food. The Buildings and Grounds crew made a good impression on students as they returned to campus. The crew updated many areas around campus such as the addition or repairing of sidewalks for safety reasons as well as to accommodate the rise in attendance. Students had a lot to be thankful for as these businesses continued to advance our technological resources and efficiently meet our needs.

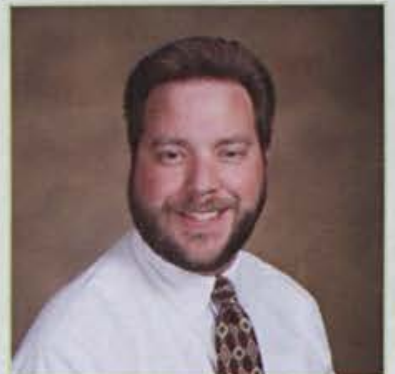

Ben Smith Assistant Controller

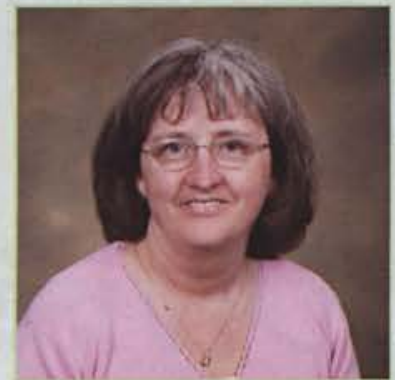

Cynthia Davis Asst. to the Registrar

\section{Post Office}

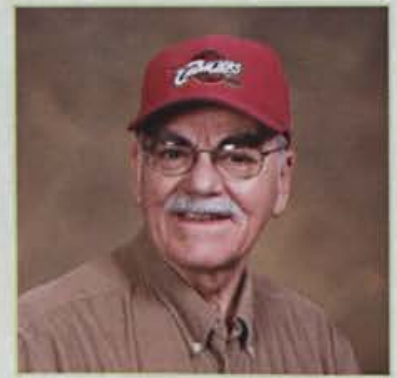

Lee Belleman Delivery

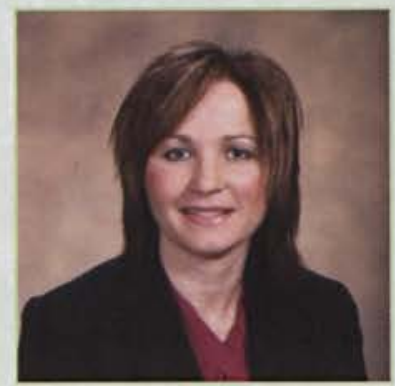

Mary Martindale Postal Clerk

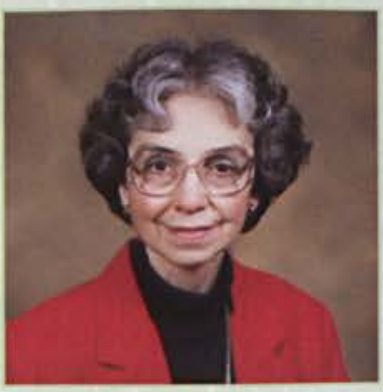

Bonnie McGillivray Senior Postal Clerk

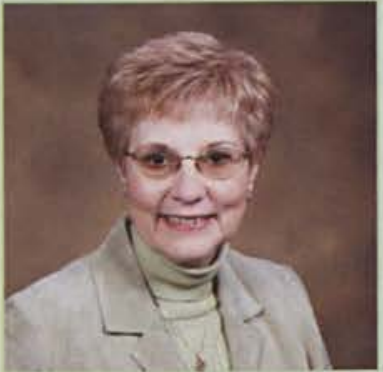

Connie Bradds Supervisor

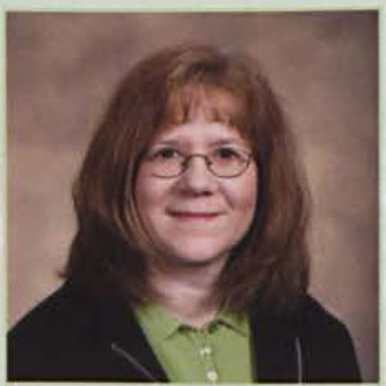

Carrie Minor Postal Clerk

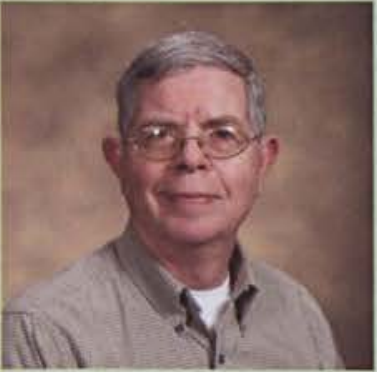

Roger Bradds Temporary Worker

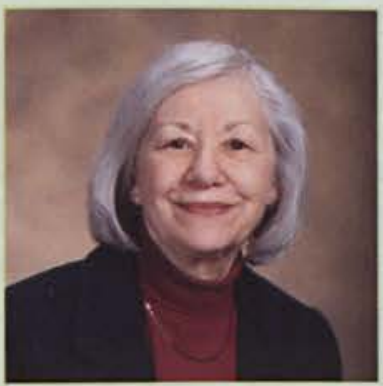

Phyllis Morris Postal Clerk

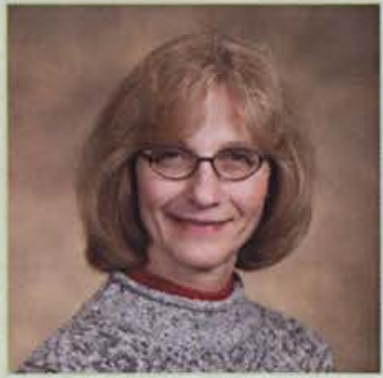

Melody Cato Postal Clerk 


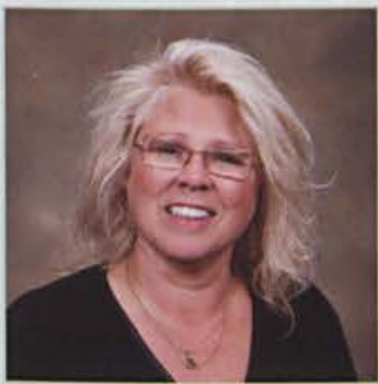

Jan Bosma Associate Director

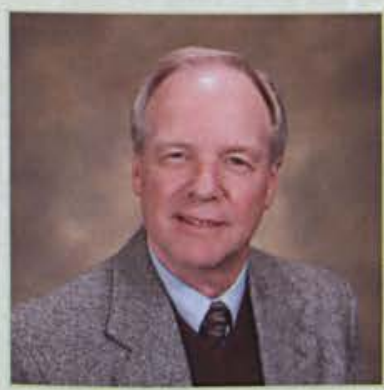

Lynn Brock Professor

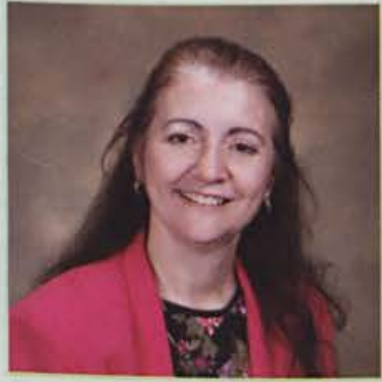

Tonya Fawcett Assistant Director

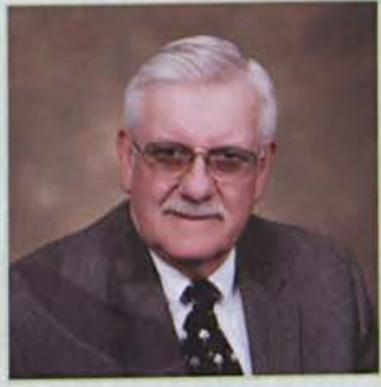

Carl Brandon Assistant Director

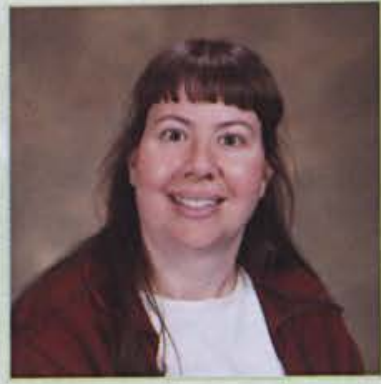

Julie Deardorff Associate Director

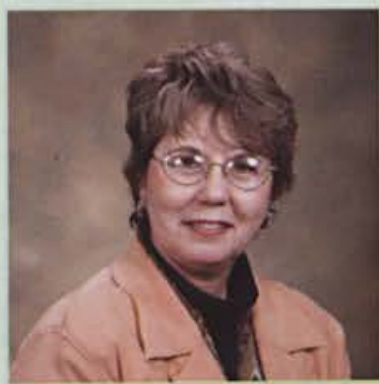

Lynne Funtik Senior Reference Librarian

Between the inauguration of the Centennial Cartwheelers in the Homecoming Parade and the Centennial Schooner in the annual canoe races, the Centennial Library made quite the impression on campus this year. The new retro furniture and plasma TV in the casual reading area drew more students into the library to "study" while they watched the Winter Olympics or March Madness. In addition to these changes, the library also expanded the research database, adding six new databases to the approximately 180 already in existence. However, the library did more than serve the students and faculty here at the university. April 2-8 was National Library Week, and through the book sales and donations taken, more than $\$ 2,500$ was collected for the Benjamin Franklin High School Library in New Orleans, Louisiana. So between the newly extended hours, comfortable furniture, high-tech equipment, and a wide range of resources, the library faithfully served the student body.

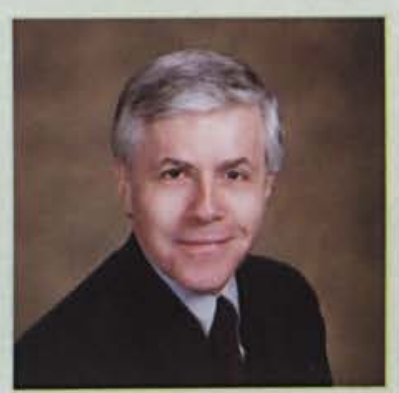

Gregory Martin Assistant Professor

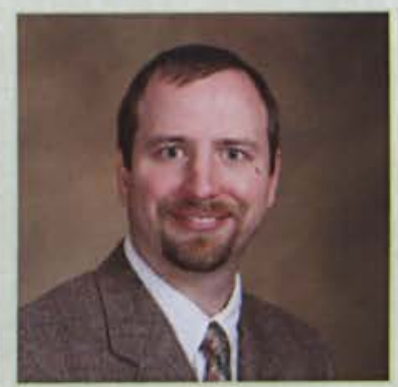

Rory Patterson User Education Librarian

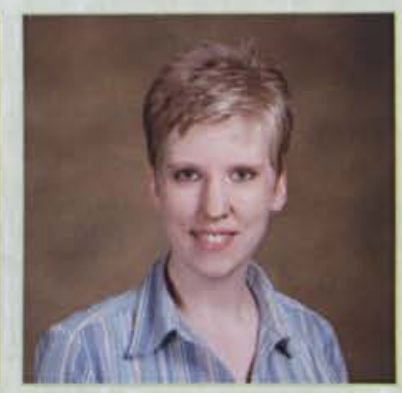

Susan Winn Reference Librarian

\section{Staff}

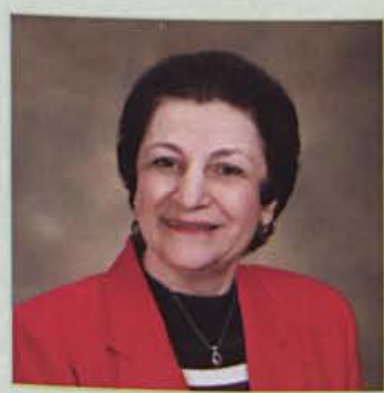

Fran Andrews Office Manager

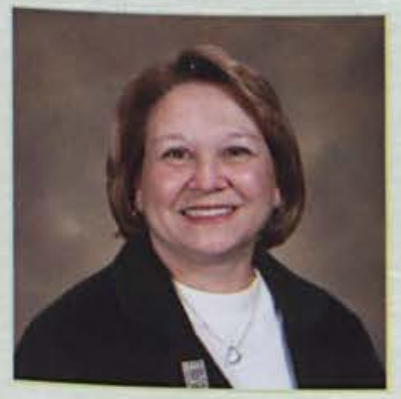

Andi Mounts Assistant Manager

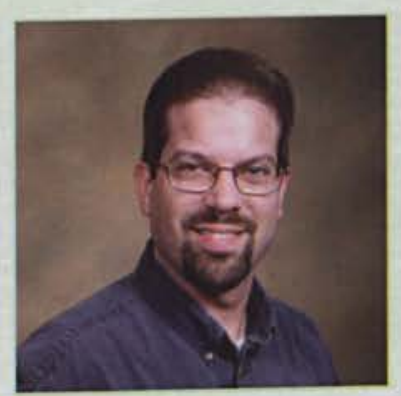

Scott Deetz AV Services Manager

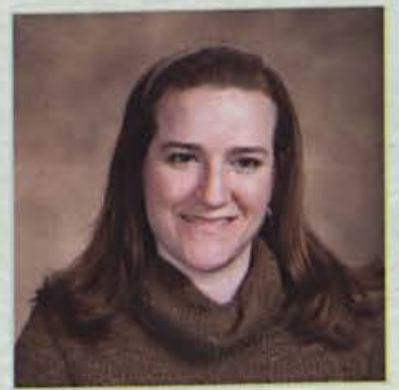

Jennifer Myers Cir. Services Manager

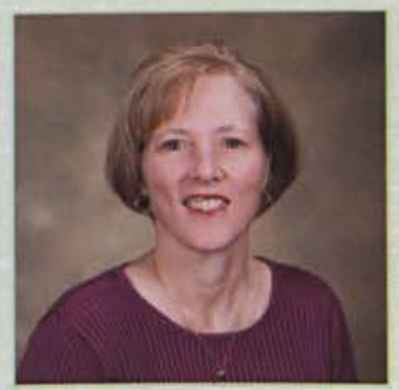

Terry Delong AV Services Office Coor.

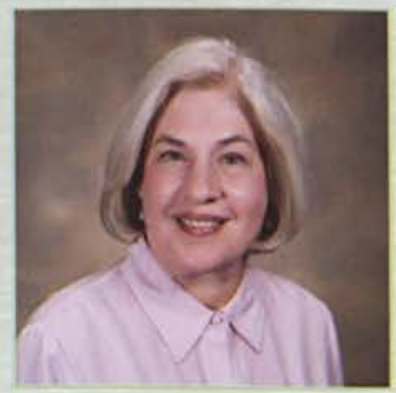

Dianne Seals

Graphic Tech. Assistant

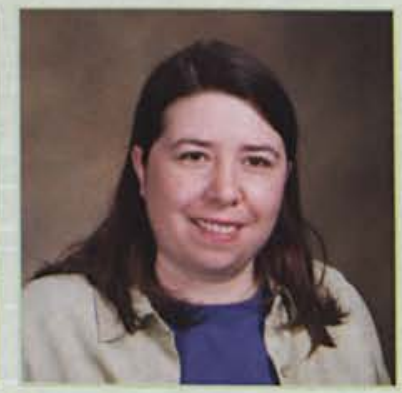

Dara Fraley Production Specialist

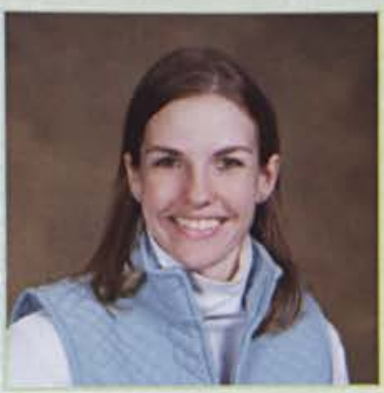

Tricia Walker Technical Coordinator

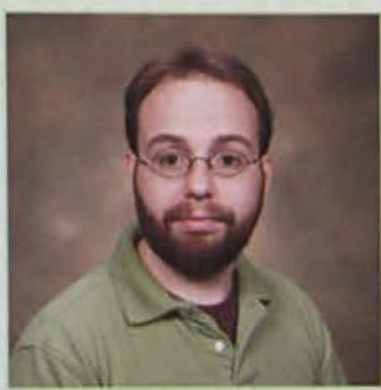

Josh Heinrich AV Services Technician

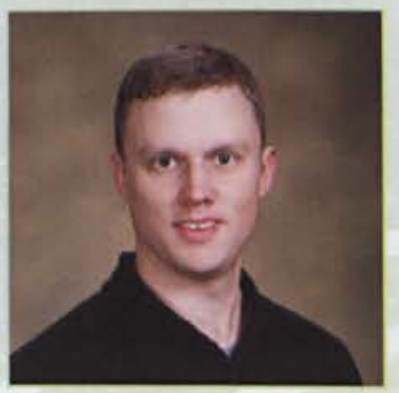

Wesley Warriner AV Services Senior Tech. Faculty \& Staff 53 


\section{Enrollment Division}

For the Enrollment Management Division, the year passed quickly and with more than enough changes and alterations to existing programs to keep everyone busy. Perhaps one of the biggest changes this year to the public recruiting face of Cedarville University was the inception of Cedarville Ambassadors in the Admissions Office. Gone are the days of average tour guides; today, volunteers known as ambassadors show incoming students around campus and travel off-campus to promote Cedarville at college fairs and promotional events. Marketing Services combined to encompass Church and School Relations, External and Public Relations, the Registrar, and Financial Aid. It continued to bring exciting events like the ever popular CedarMania and
Junior Jam, along with a fresh take on the Cedarville image, as seen through promotional materials. Lastly, Financial Aid worked hard to bring about a number of changes, the first of which was the implementation of the Ohio College Opportunity Grant, a new state grant which allows more funding to be allocated for Ohio students. Steve Winey, who made the transition this year to Assistant Director of Financial Aid from his previous post in Academic Records, helped implement this important program. Financial Aid was also instrumental in creating the brand new Church Matching Grant, which allows churches to generously contribute monetary gifts to the education of their Cedarville students, while the university matches church donations up to $\$ 1,000$.

\section{Admissions}

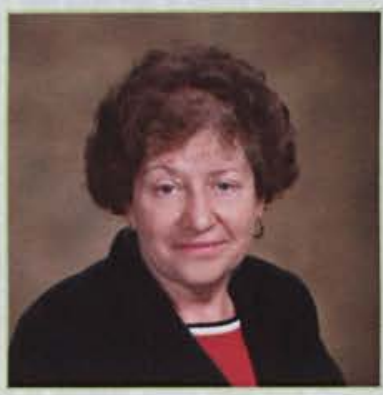

Judy Awabdy Assistant Director

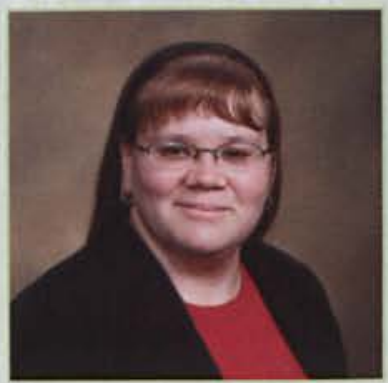

Kristy Lester Sr. Comm. Assistant

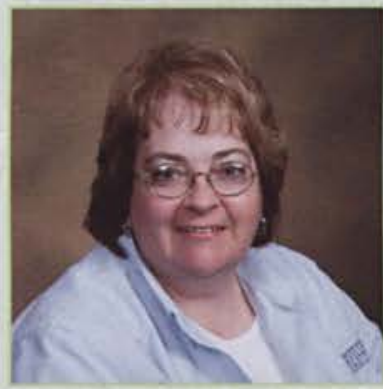

Amy Bielek Sr. Comm. Assistant

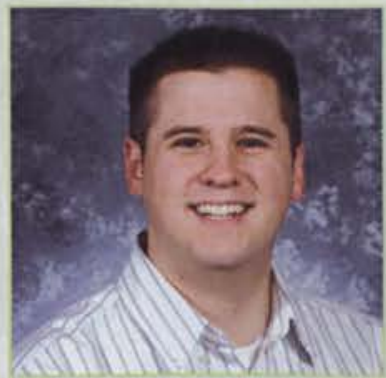

Aaron Mahl Admissions Counselor

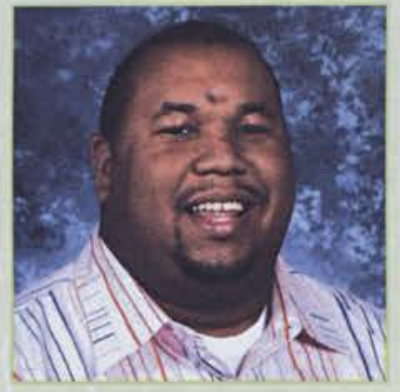

Mike Dorsey Coordinator

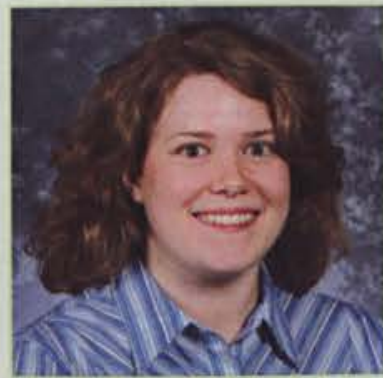

Donna Paulsen Assistant Director

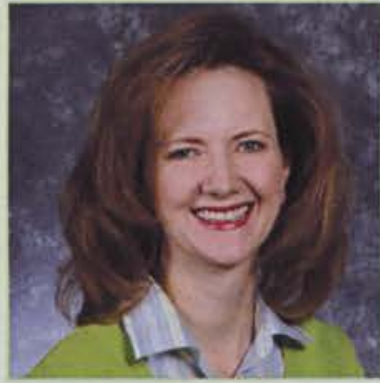

Amy Holderby Associate Director

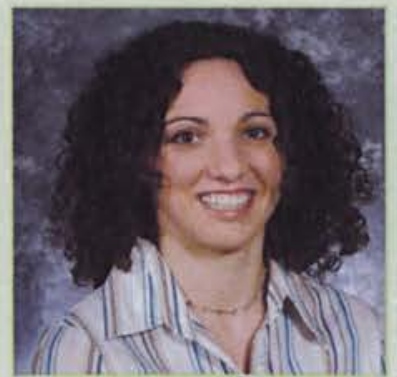

LaVaughn Ricci Admissions Counselor

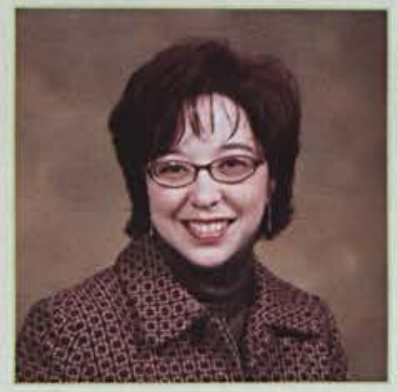

Yuki Johnson Office Manager

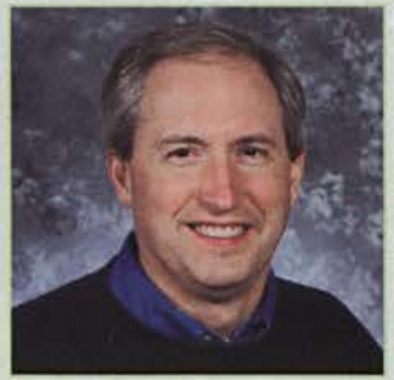

Roscoe Smith Director

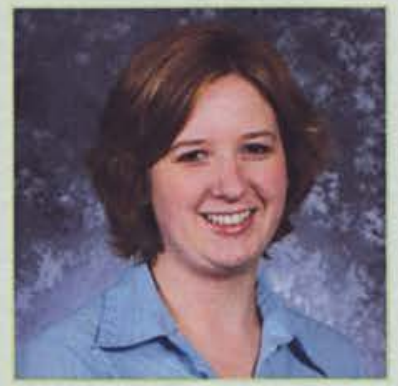

Kenna Stark Admissions Counselor 


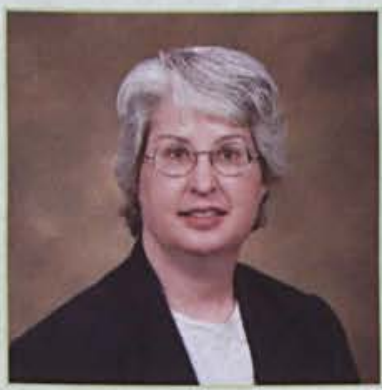

Pam Flippin Student Loan Mng.

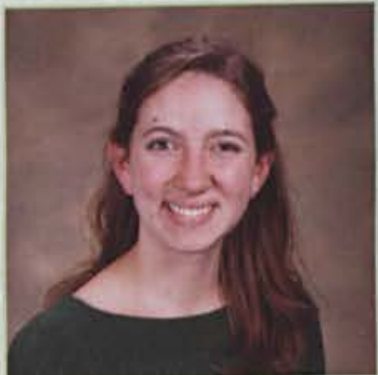

Jaime Hawkins Admin. Assistant

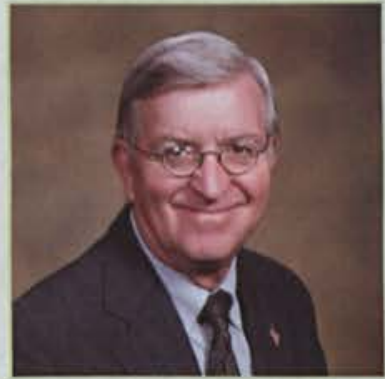

Ken Rotroff Assistant Director

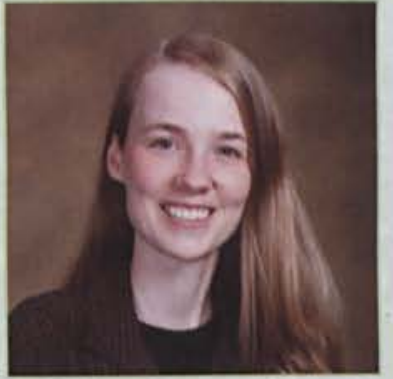

Kara DeMustz Assistant Director

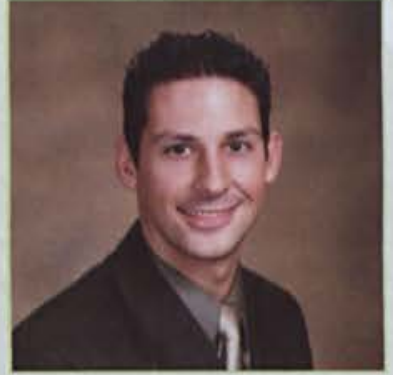

Chad Jackson Prod. Coord. Admin.

\section{Marketing Serivices}

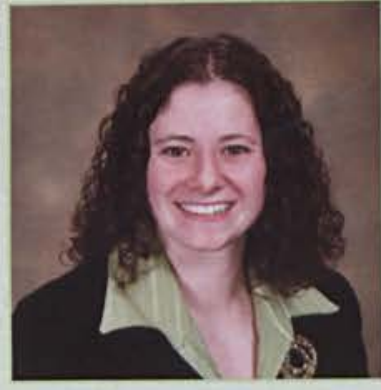

Chrissy Faulkner Event Services Coord.

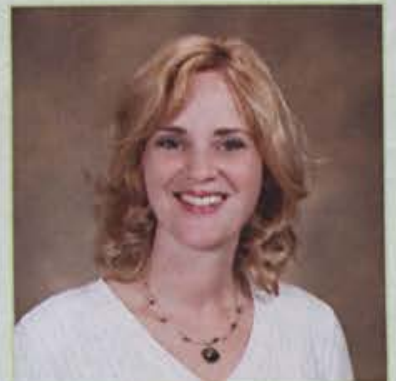

Kristy Hill Church \& School Relations

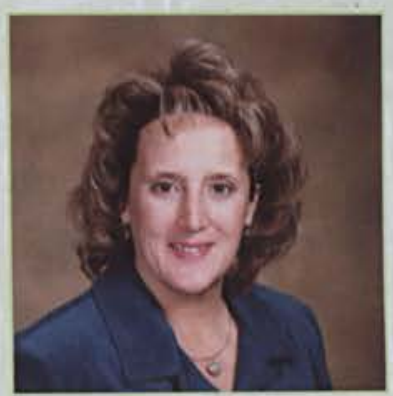

Joan Wilson Church \& School Asst.

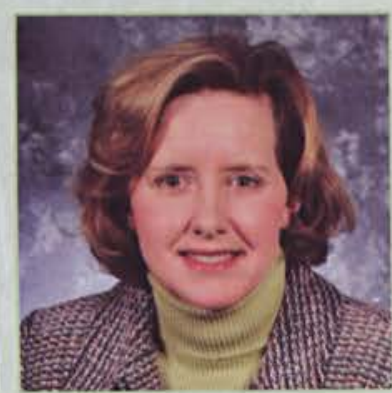

Cessna Winslow Public Relations Writer

\section{Christian Ministries Division}

This year we have seen the Christian Ministries department minister to a record amount of students and non-students. In the wake of Hurricanes Katrina and Rita, Christian Ministries oversaw the collection and use of over $\$ 100,000$ raised by the school. They also coordinated service teams for Fall Break, Thanksgiving, Christmas, and Spring Break to help those people affected by Katrina and Rita. The Christian Ministries department encompassed Community Ministries, Discipleship Ministries, the LifeLine Players and HeartSong touring teams, and Missions Involvement Services. Current ministry teams saw 423 students serving in organizations and 248 serving in local churches, with an additional 162 requests for service on file. In Discipleship Ministries, so many students wanted to be involved that many groups had waiting lists. There were 635 students participating in small groups, 130 in prayer groups, and 84 in the Fit to be Tied premarital counseling program. Senior Stephanie Hueni stated, "My experience with the discipleship ministries has been spiritually challenging and valuable to my walk with the Lord. Discipleship is a wonderful opportunity for encouragement and accountability with my fellow sisters in Christ. Proverbs 27:17 sums up the relationships we've built: 'As iron sharpens iron, so one man sharpens another.'”

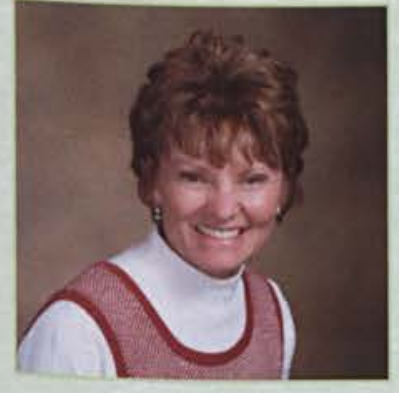

Vicky Hines Admin. Assistant

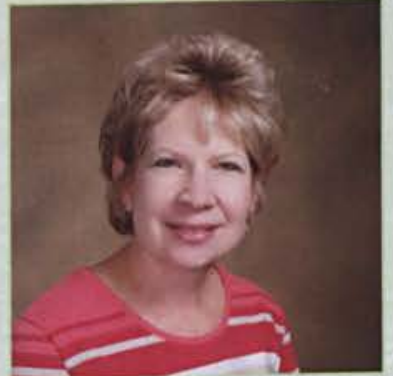

Jacki Pyles Receptionist

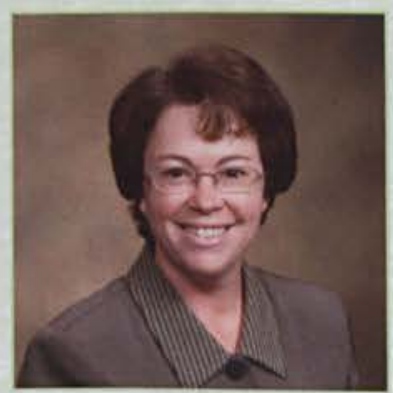

Cheryl Shupe Executive Secretary

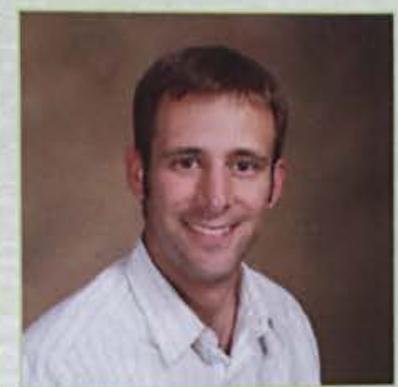

Mark Irving Director

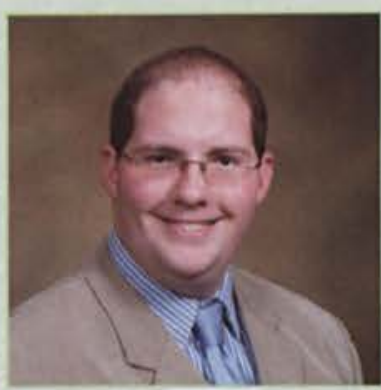

Joel Tomkinson Admin. Assistant 

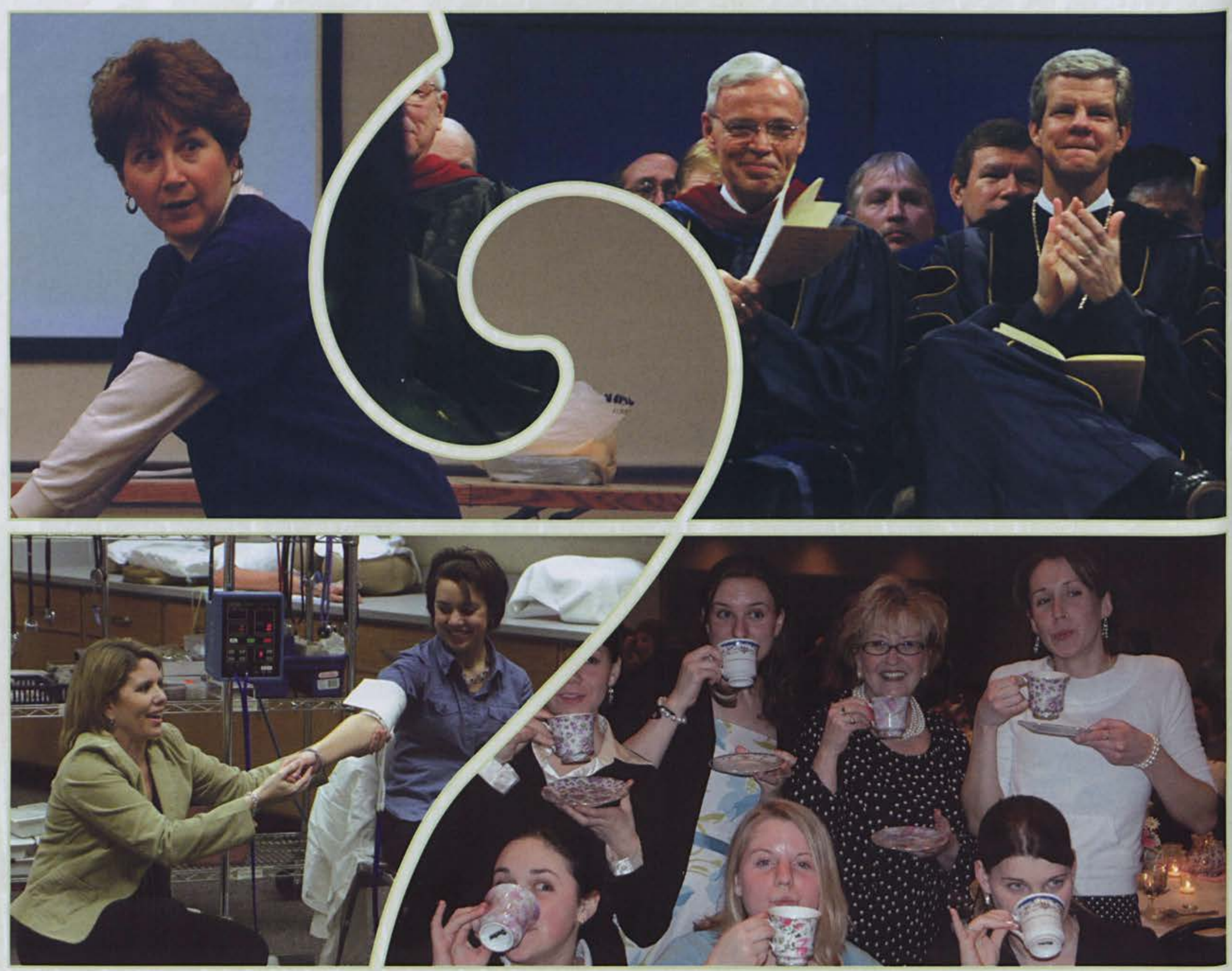

^ Nursing professor Sharon Christman demonstrates a key skill her students will need when they begin their clinicals.

* Rookie nursing professor Mrs. Amy Voris enjoys taking time to make her classes useful and practical to her students in their future careers.

* Attired a bit more formally than usual, Pastor Rohm and University President, Dr. Brown, preside over this year's Convocation excercises.

Y Senior girls enjoy taking tea with Mrs. Ruth Hess, an adjunct education professor, during the Senior Ladies High Tea, hosted in part by Mrs. Hess and the Campus Activities Board.

$\checkmark$ Dr. Baczek, Dean of the School of Engineering, Nursing and Science, is never one to let a fun time with students pass him by -- even a simple game of Uno with some of his students.

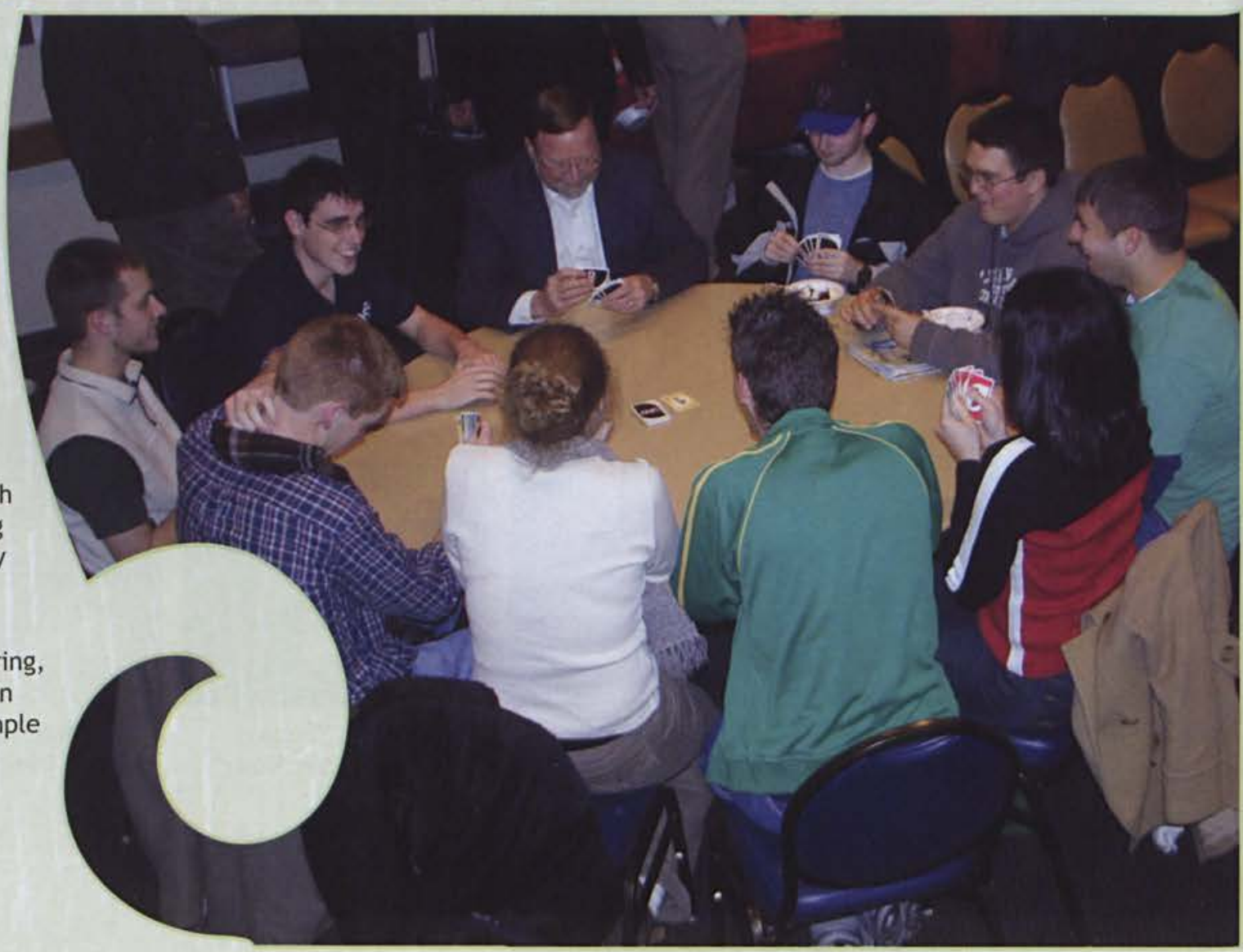




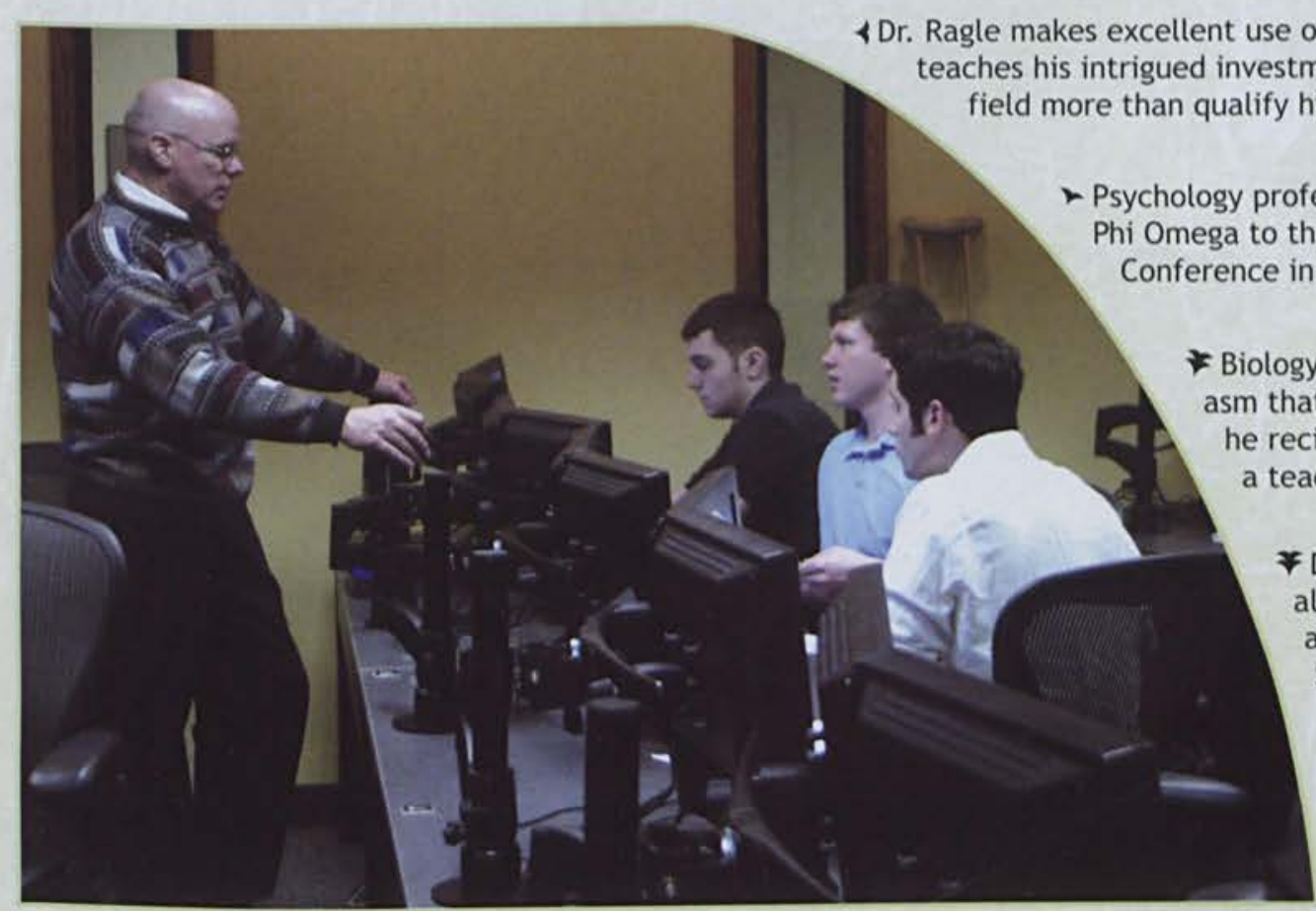

teaches his intrigued investment students. Dr. Ragle's knowledge and expertise in his

Psychology professors Dr. Firmin and Dr. Tse welcome students from Alpha hi Omega to the American Association for Christian Counselors World Conference in Nashville, Tennessee.

* Biology professor Mr. William Jones teaches with a great enthusiasm that spreads to his students. His love of learning and the joy teacher.

₹ Dr. Brown strives to relate to each of his students personally. Though this sounds near impossible, one way he is assured to meet a vast number is through the tradition of Sophomore Breakfasts.

$\checkmark$ University photographer Scott Huck is a welcome sight across campus. His formal shots, studio portraits, and campus candids all attest to his remarkable skill of capturing the perfect moment.
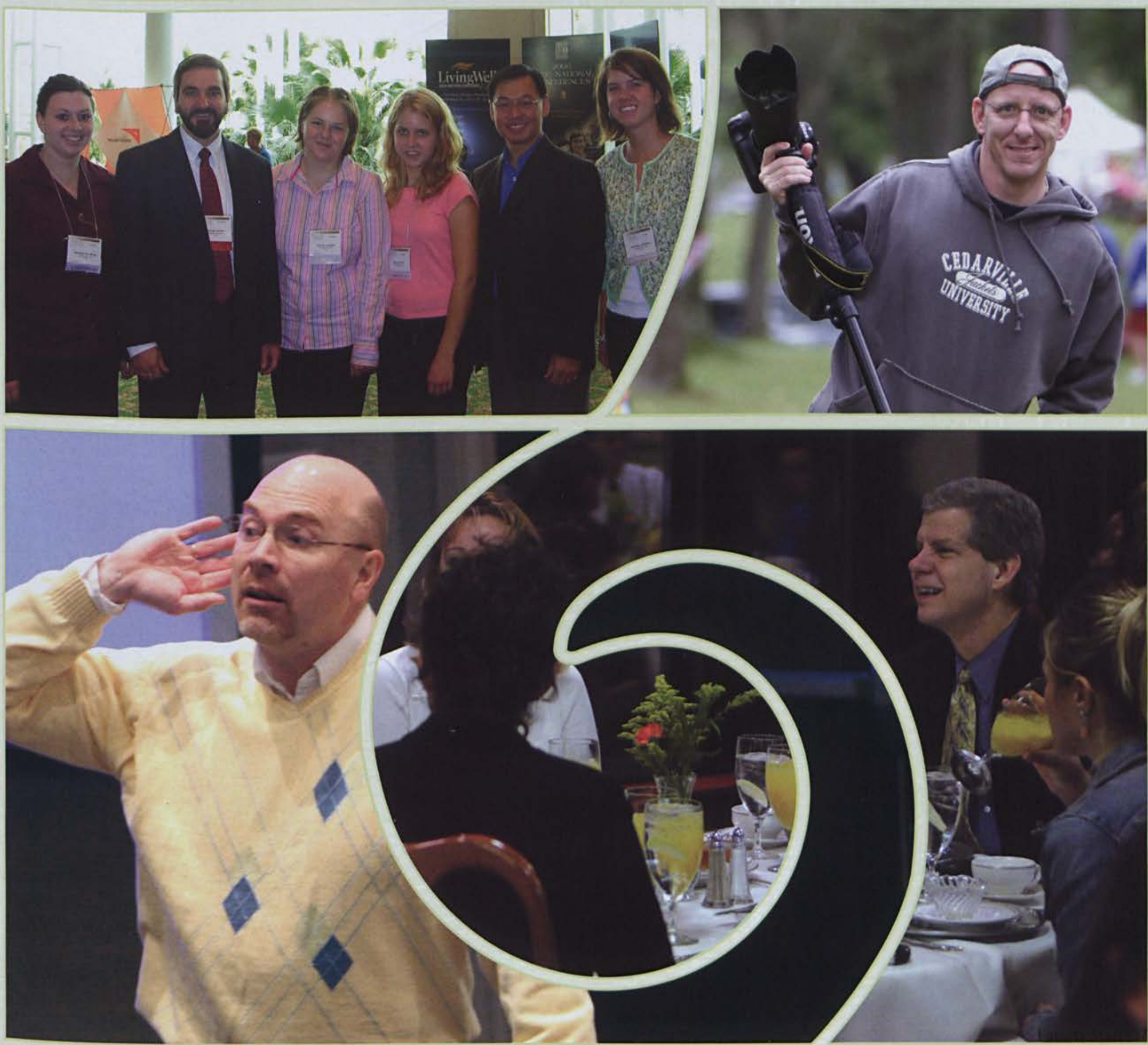


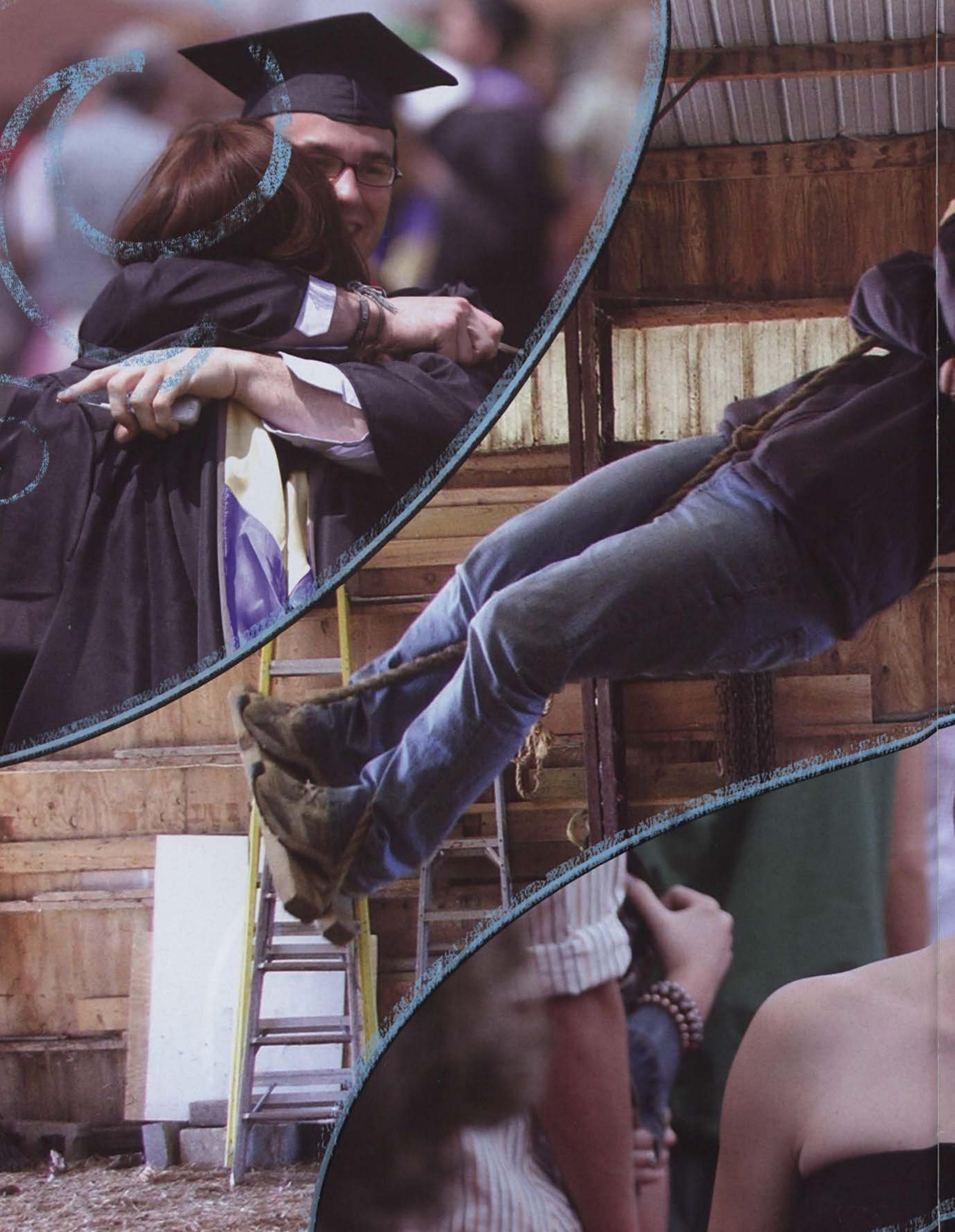




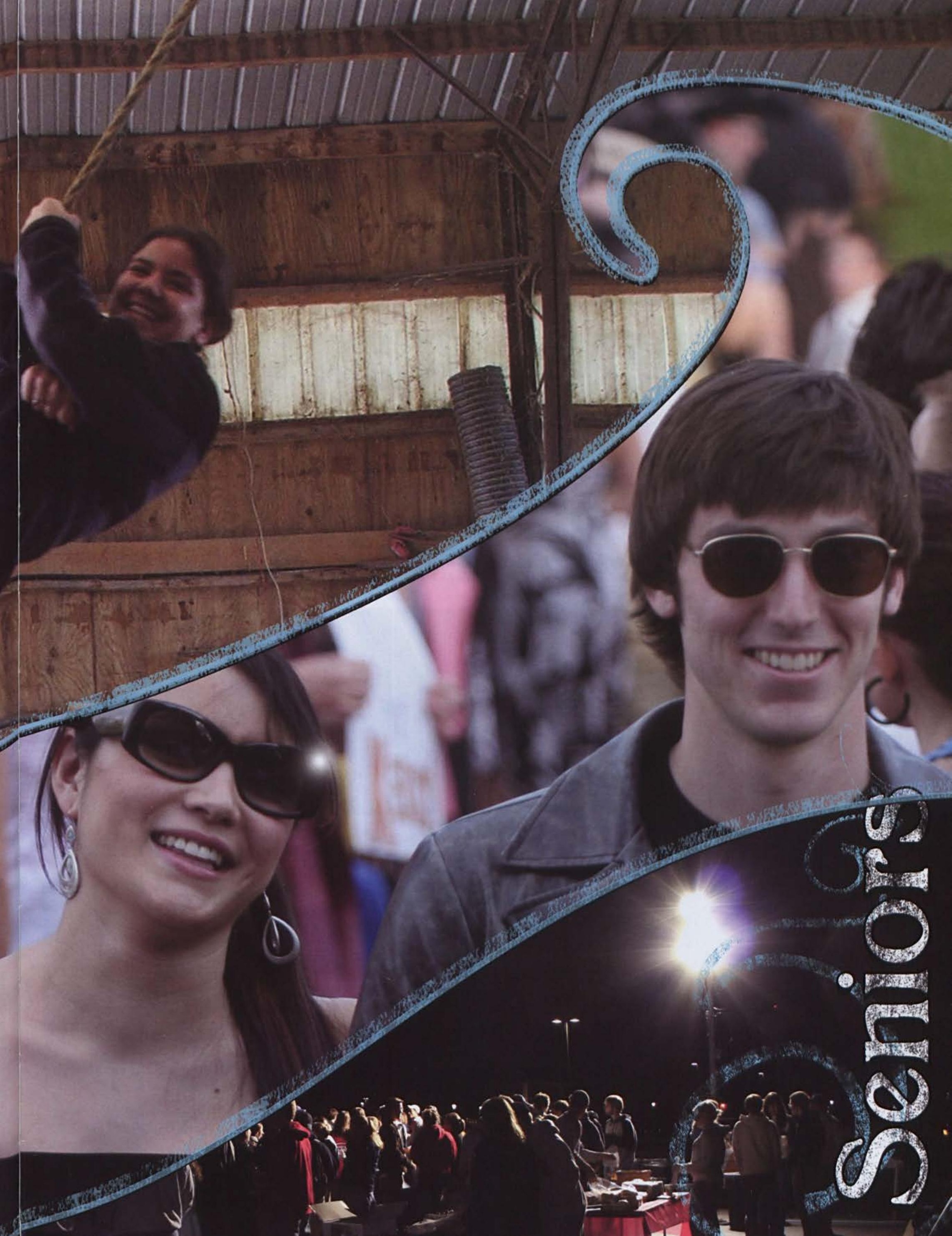




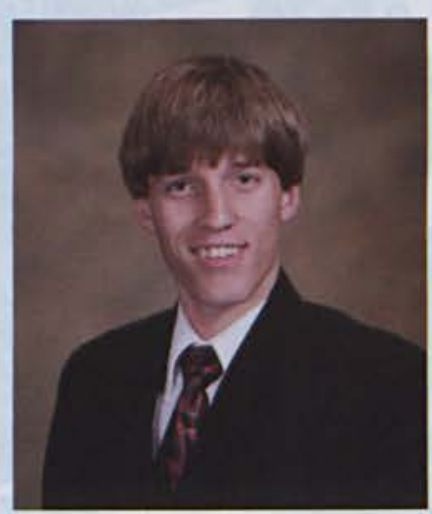

Isaac Morse Abbott Electrical Engineering

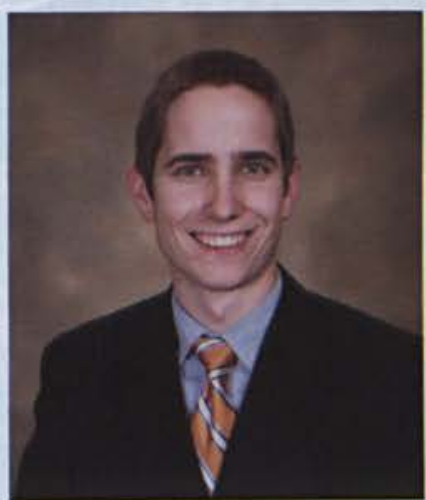

Ryan Kirk Adams Finance \& Accounting

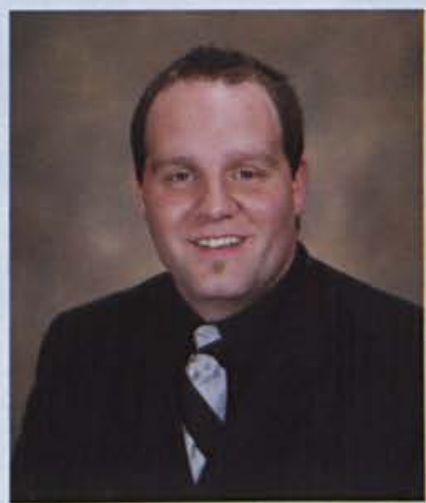

Matthew David Anderkin International Studies \& Social Science

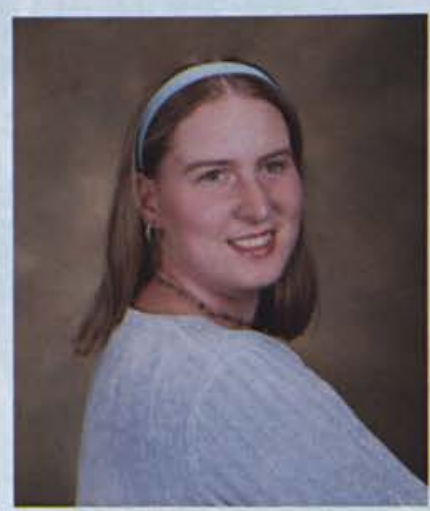

Amanda Joy Anzalone AYA Math Education

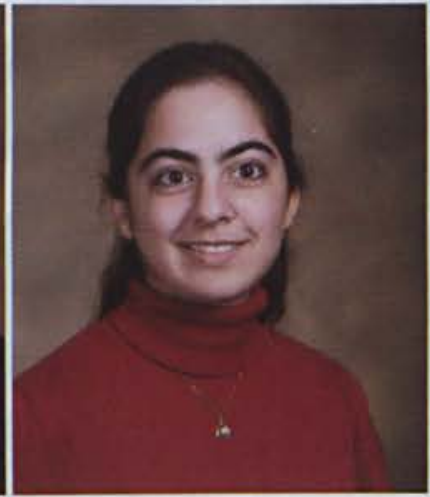

Heidi Jean Abraham Chemistry \& Biology

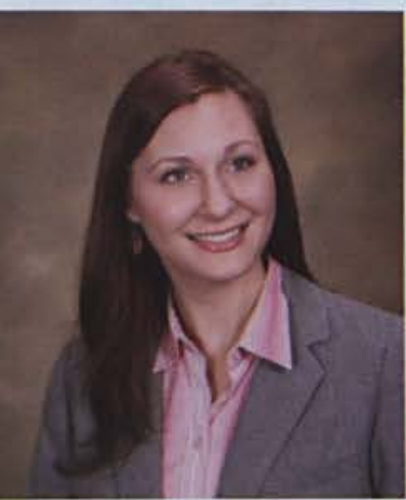

Sarah Jane Alexander Nursing

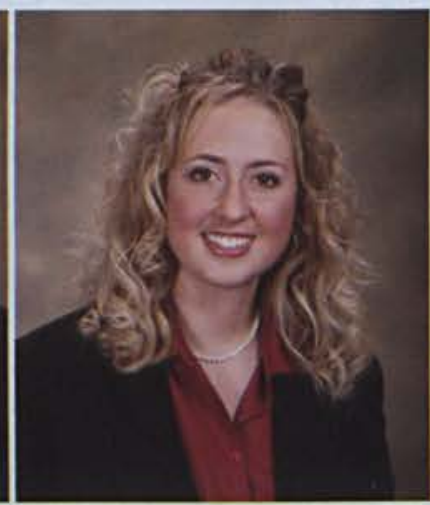

Jillian Rene Anderson Organizational Communications \& Theater

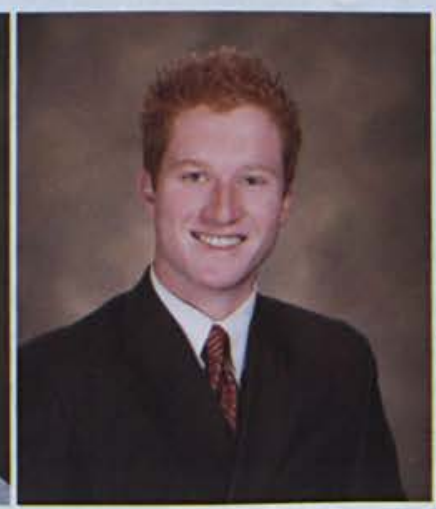

Cameron Joel Arch English

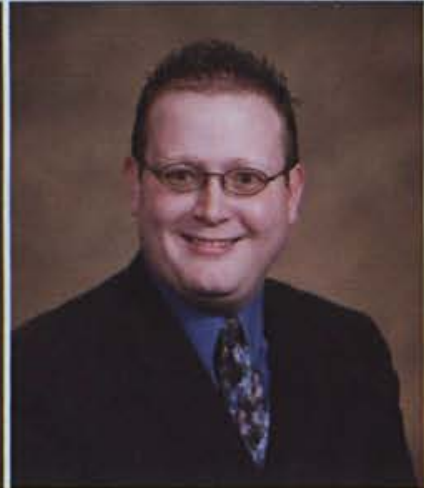

Allen M. Acker Exercise Science

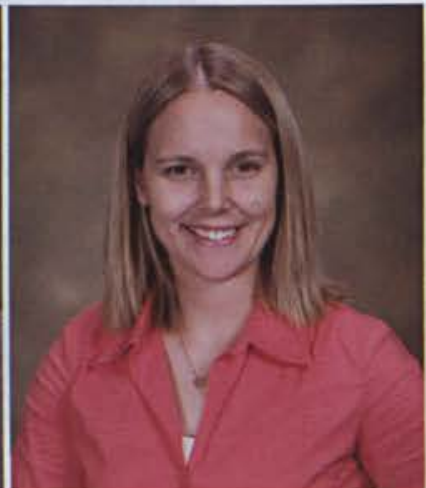

Allison Denise Allen

Psychology

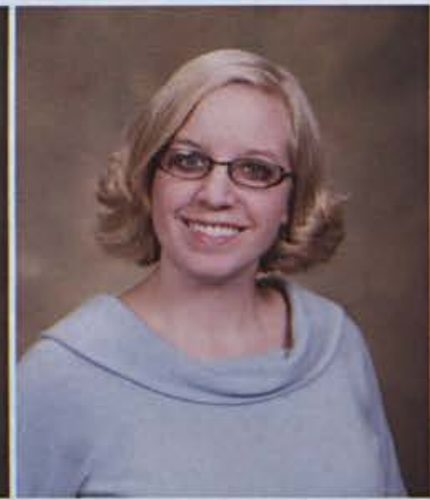

Karen Elizabeth Anderson Early Childhood Education

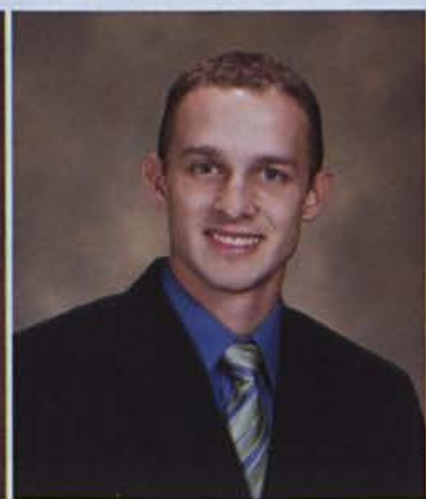

Jason Matthew Ayers Mechanical Engineering

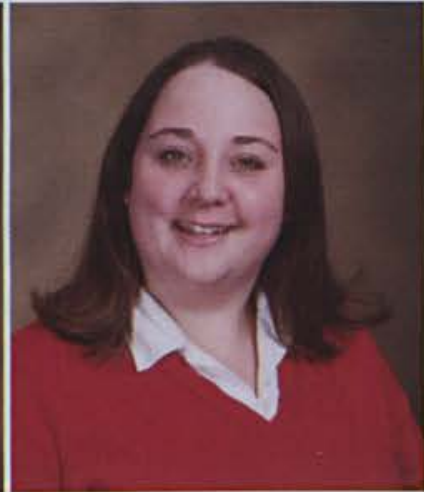

Juliet Marie Ackerman Nursing

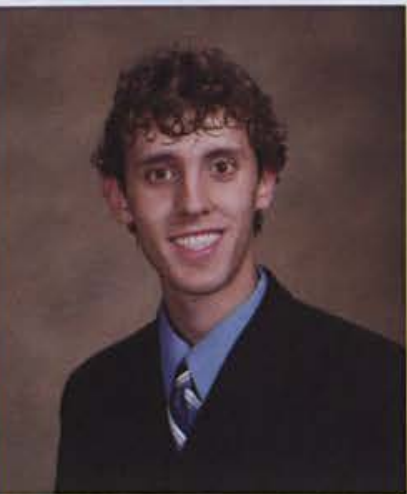

Benjamin Joel Amberg Biology

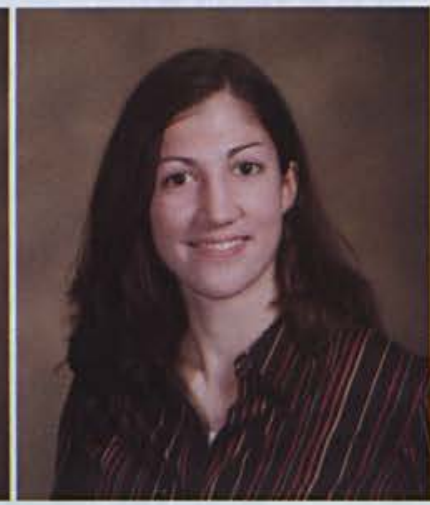

Rachel Leigh Anderson Psychology

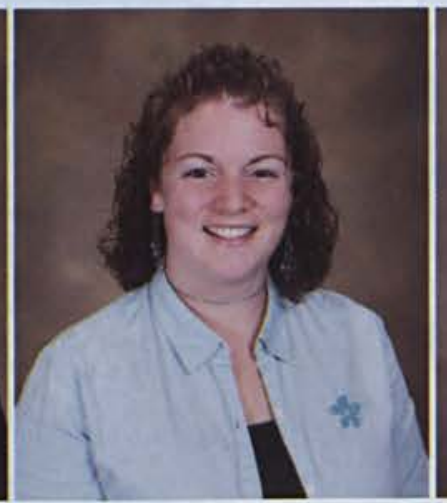

Megan Christa Bailey Psychology

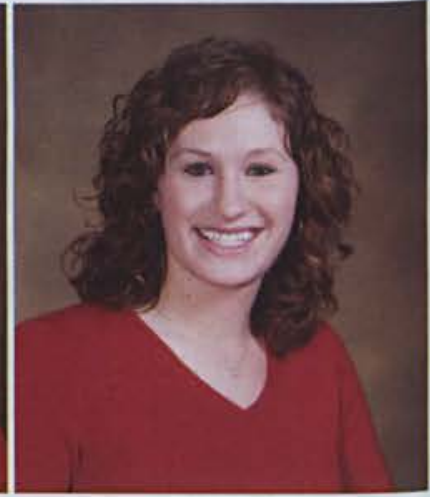

Katherine E. Ackerman

Communications

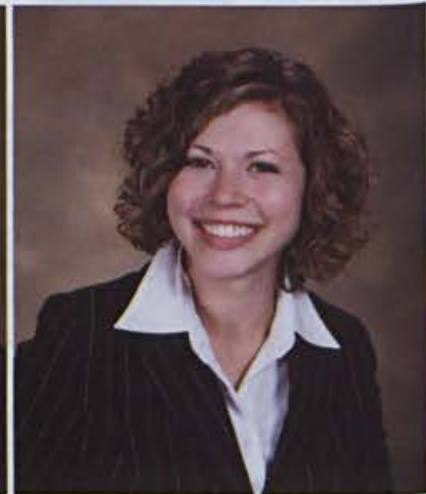

Jenee Christina Amstutz

Communications

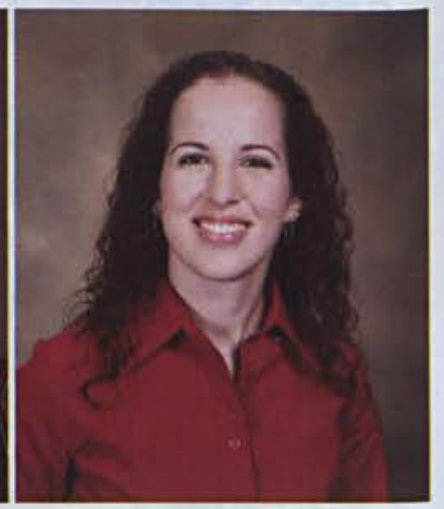

Jamie L. Anfang Social Work

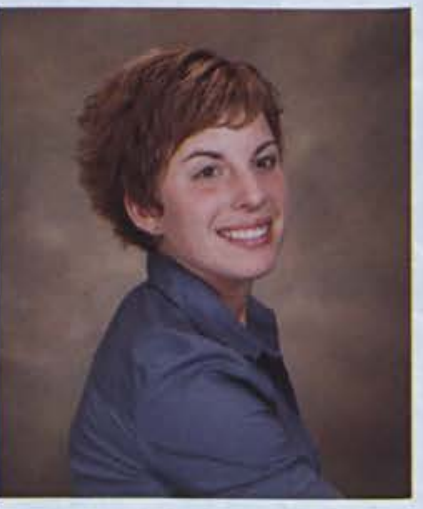

Sarah Katherine Bailey Early Childhood Education 


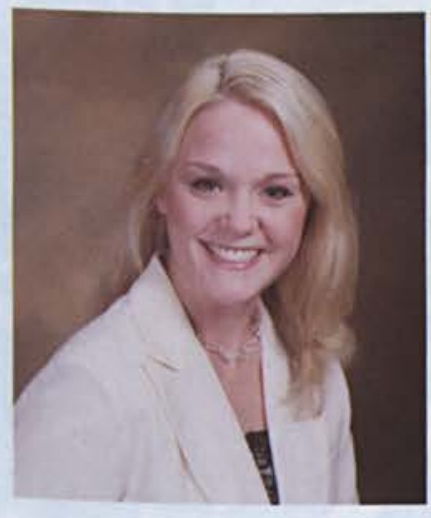

Megan Elizabeth Baker

Comprehensive

Communications

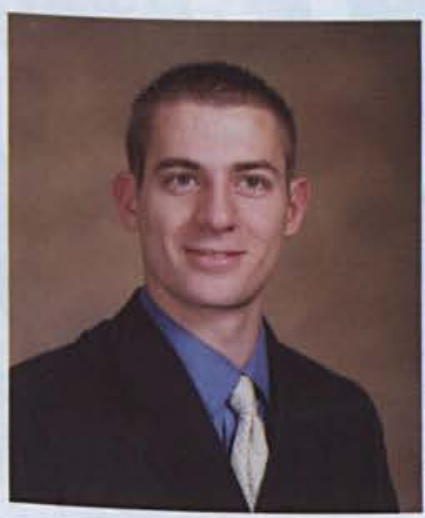

David John Bartlett Mechanical Engineering

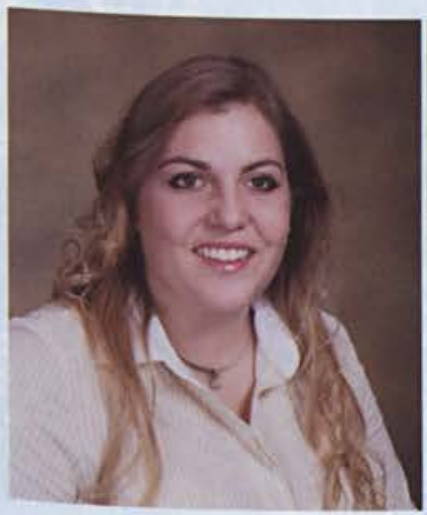

Lisa M. Battaglia

Criminal Justice \& Psychology

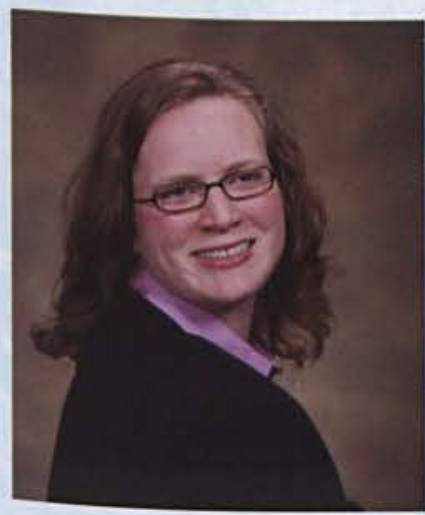

Mary Lee Becker Nursing

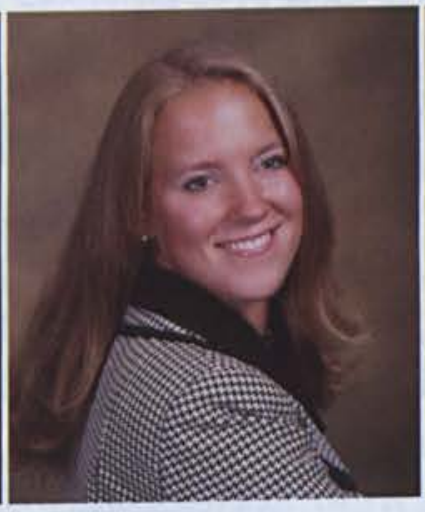

Erin Rae Baranski Nursing

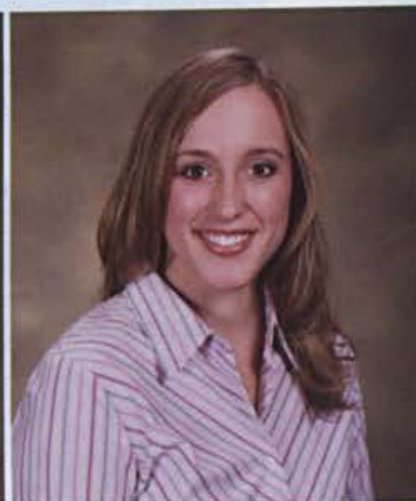

Megan Leigh Bartoletti Middle Childhood Education

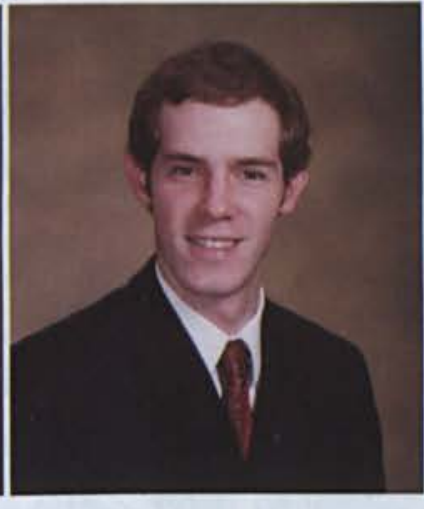

Brandon David Barnes Youth Ministries

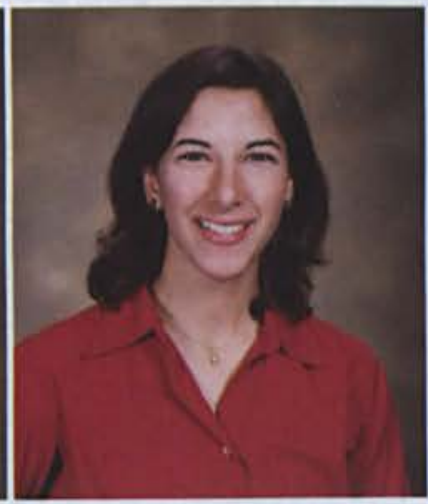

Andrea Lauren Barnett Middle Childhood Education

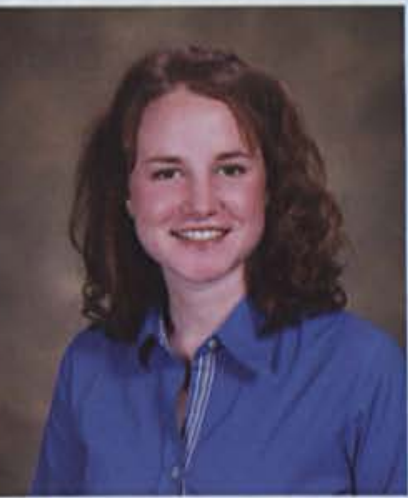

Jessica Lynn Barnette Nursing

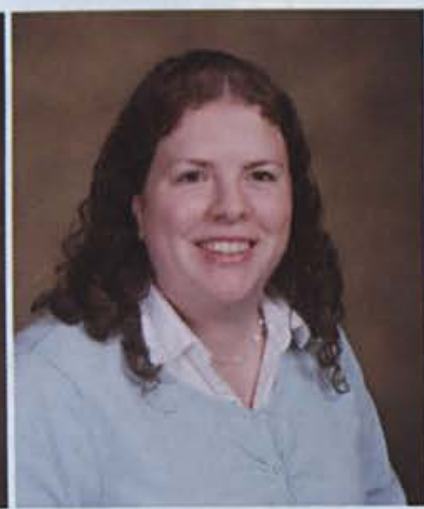

Charity Barton

Electronic Media

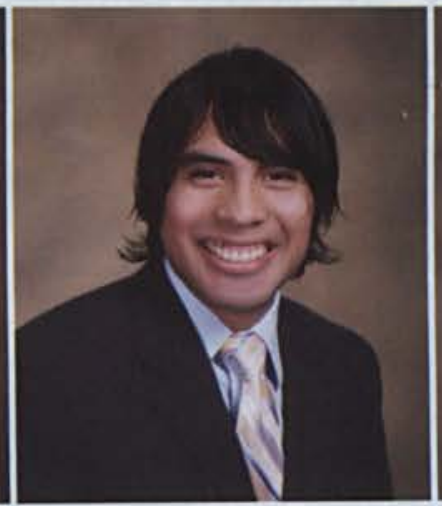

Ivan Ricardo Batista Nursing

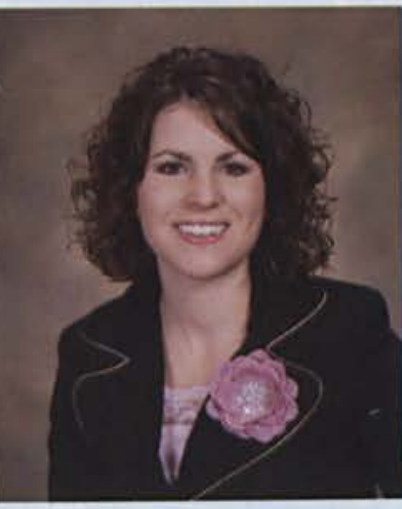

Kendra Brooke Battaglia Applied Psychology

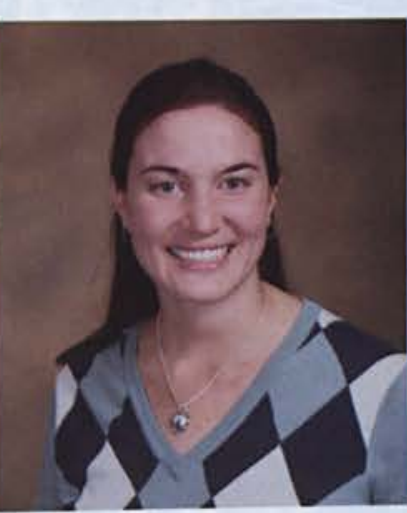

Alison Whitney Bauer Early Childhood Education

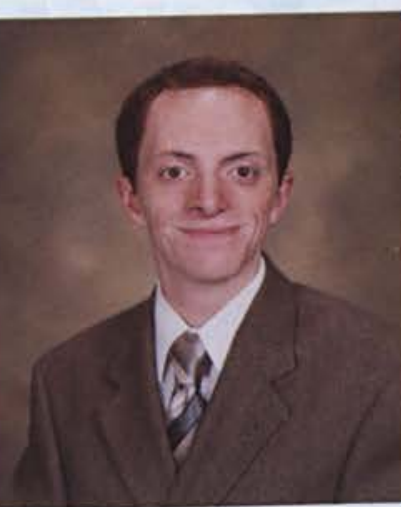

Lewis Herbert Beeler Pre-Seminary Bible

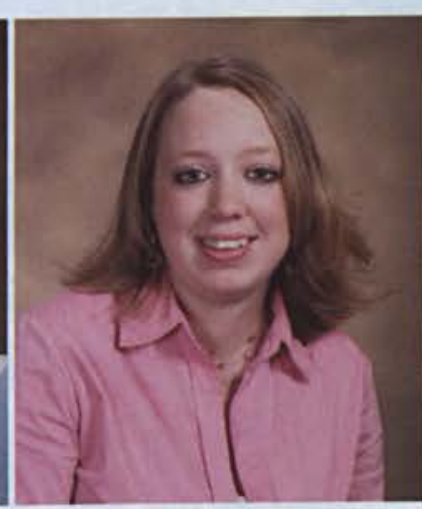

Sarah Anne Bayne Political Science

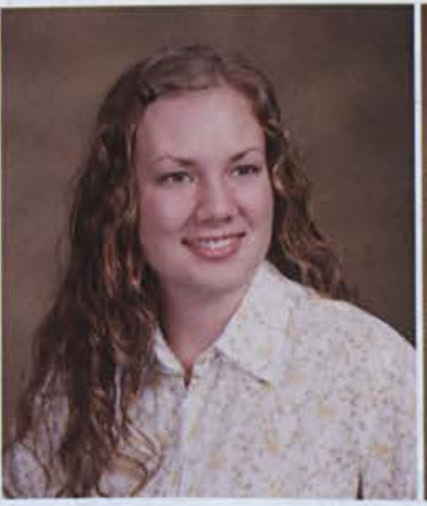

Melanie Dawn Befus Nursing

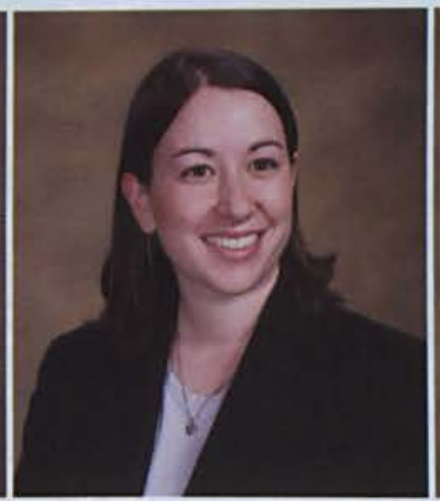

Kassandra Lynne Beachy Organizational Communications

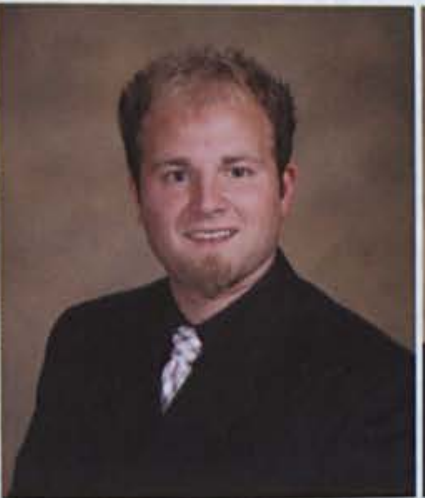

Andrew Seth Belleman Multi-Age Physical Education

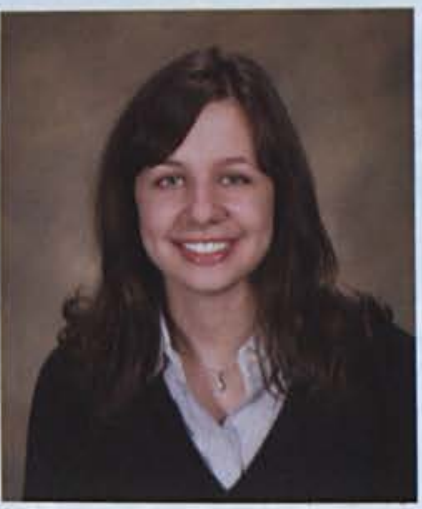

Stacey Ellen Beaty English

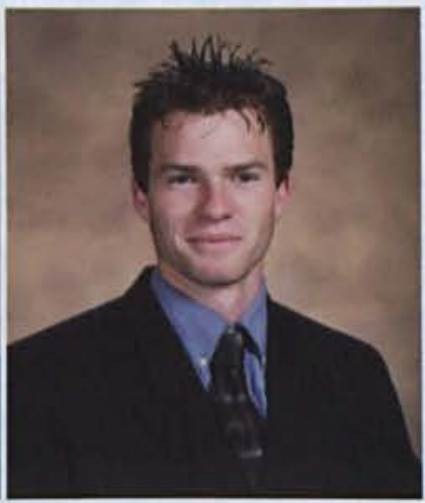

Justin Tyler Benz Physics

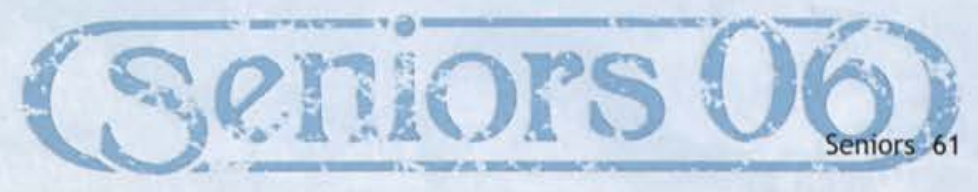




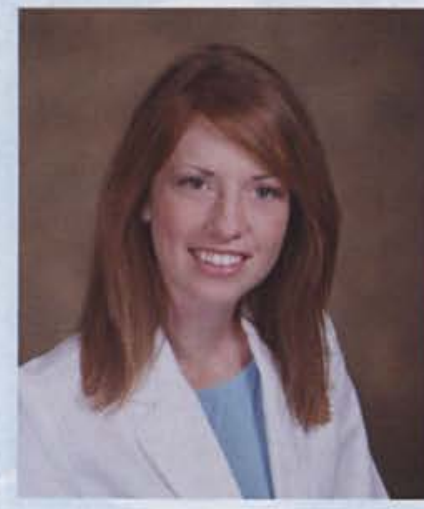

Michelle Colleen Bicking Exercise Science

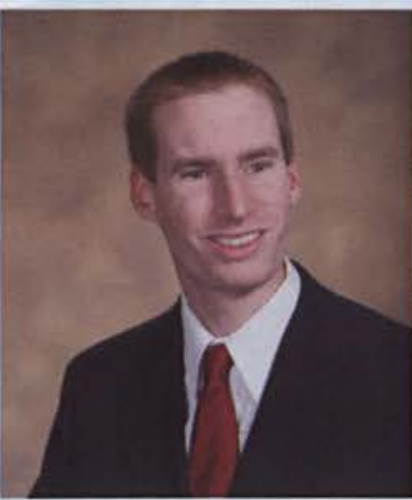

Andrew Phillip Black Biology

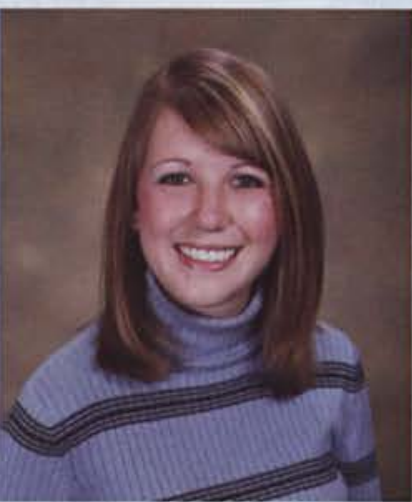

Cara R. Bodenmiller

Global Economics \& International Business

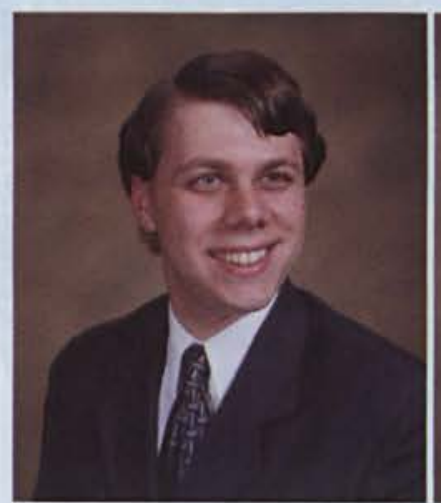

Jonathan David Boston Mechanical Engineering

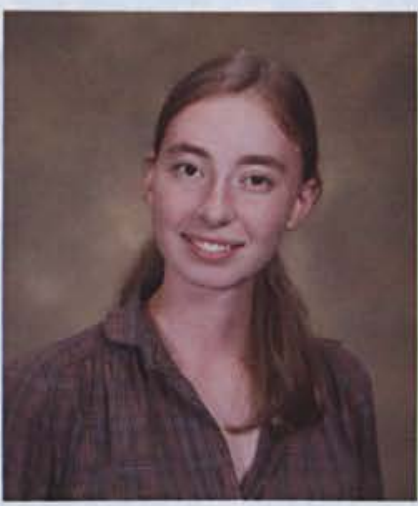

Brandiann April Biggar English

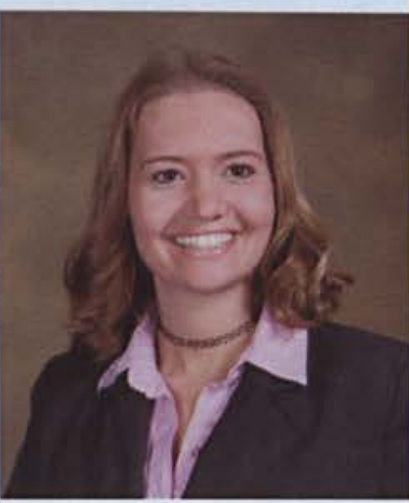

Jessica Lori Black Psychology

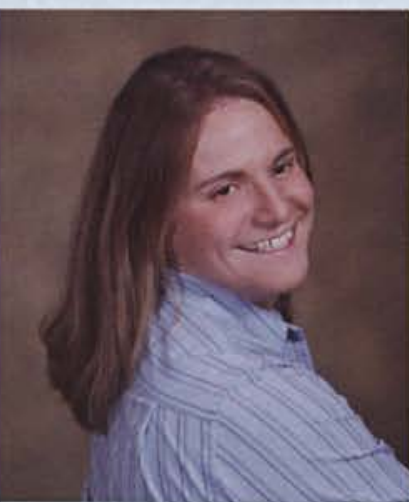

Rebekah Ruth Bole Nursing

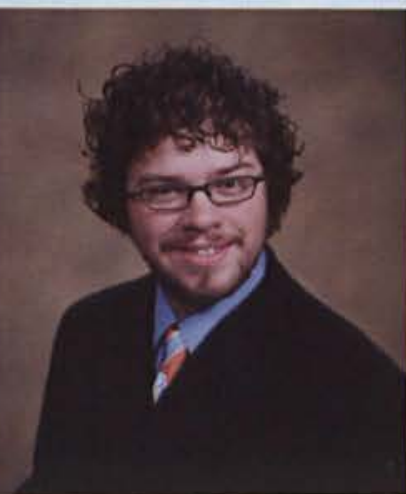

Michael D. Bouchard Music

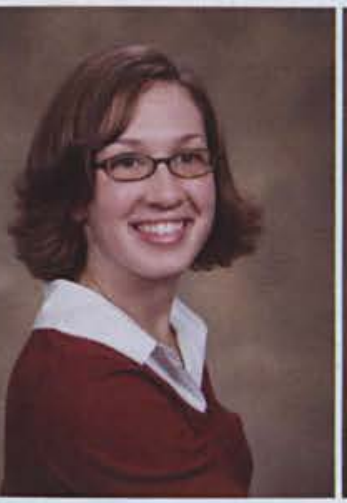

Virginia Erin Bishop Nursing

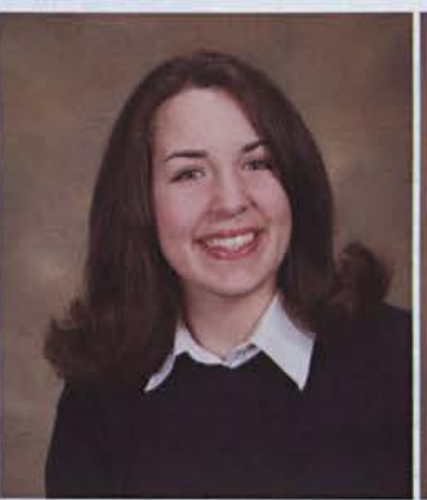

Julie Marie Bledsoe Marketing

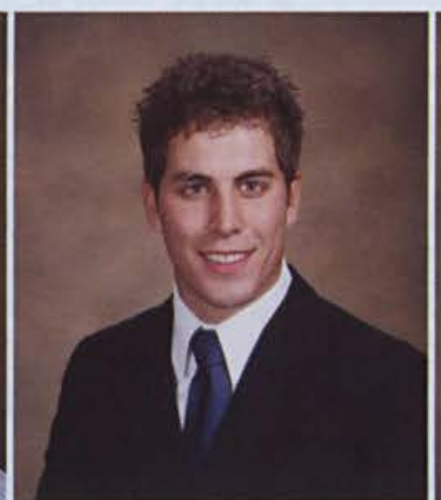

Matthew Jordon Bonin Spanish Education

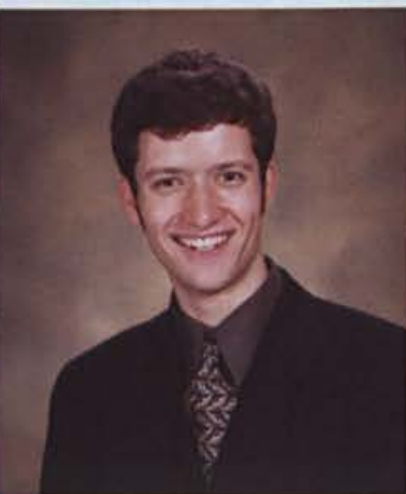

Curtis Brandon Boudreau Mechanical Engineering

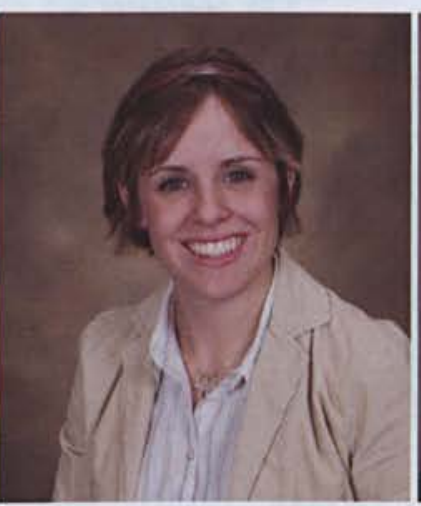

Karis Marie Bissett Nursing

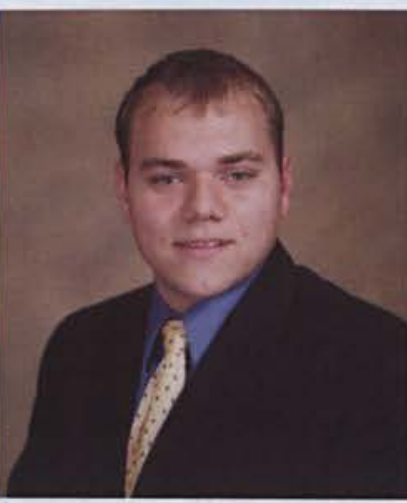

Philip J. Blodgett Youth Ministries

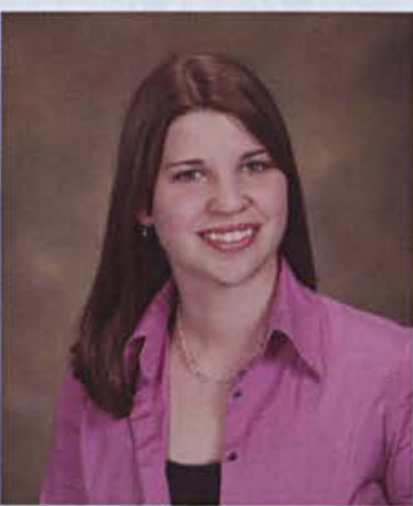

Nicole Marie Boop World Missions

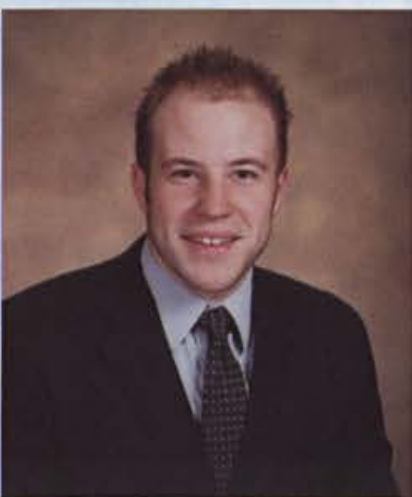

Jeffrey Ryan Bowen Business

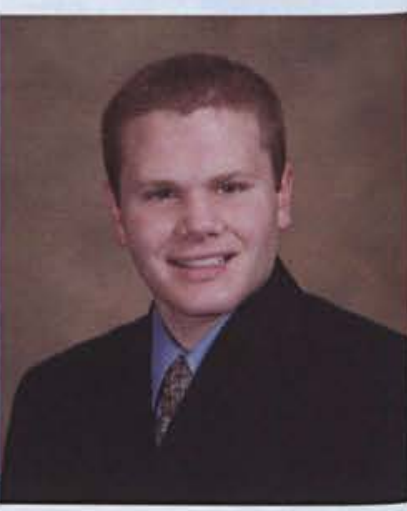

Tyler Wayne Bixel Electrical Engineering

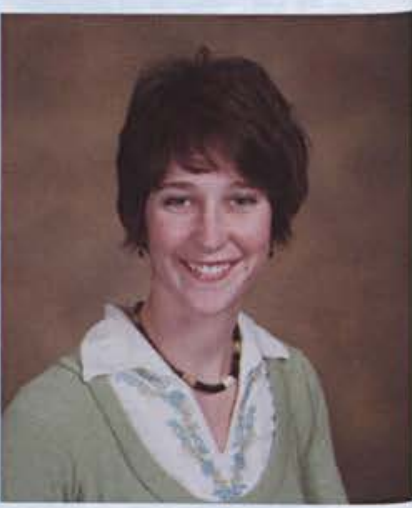

Alison Stephanie Blythe Graphic Design

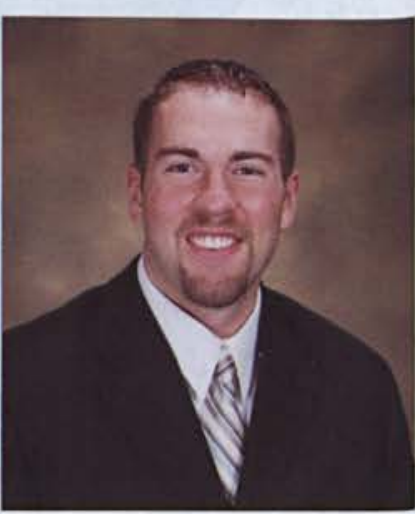

Andrew Peter Borton Mechanical Engineering 


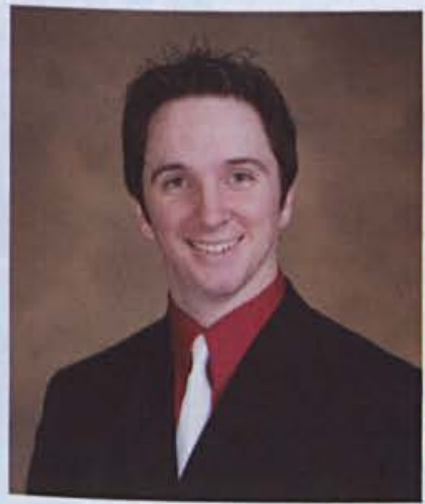

Kiel John Boynton

Physical Activity \& Sport Studies

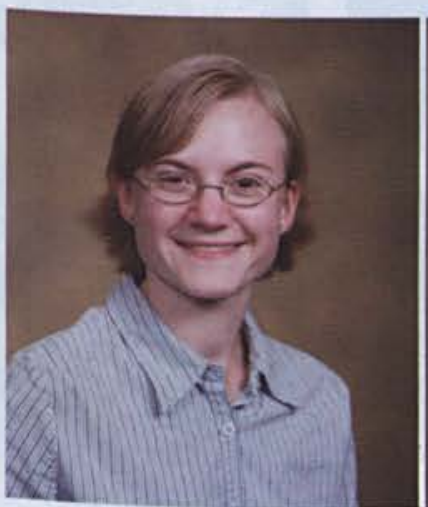

Tiena Britton

Multi-Age Special Education

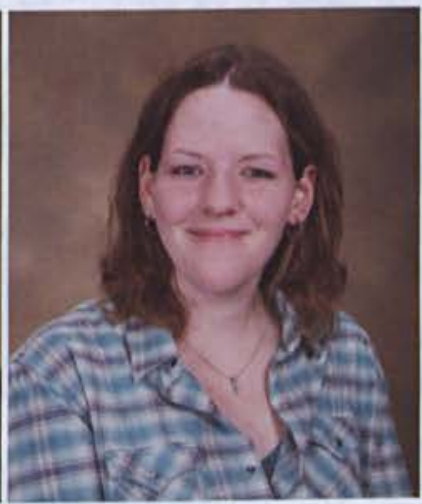

Sarah Amy Boynton Early Childhood Education

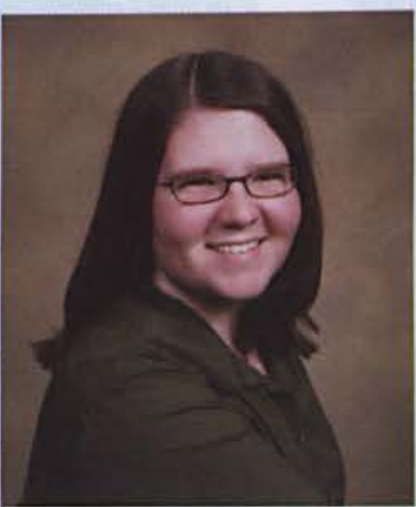

Bethany Lynn Broesder Music Education

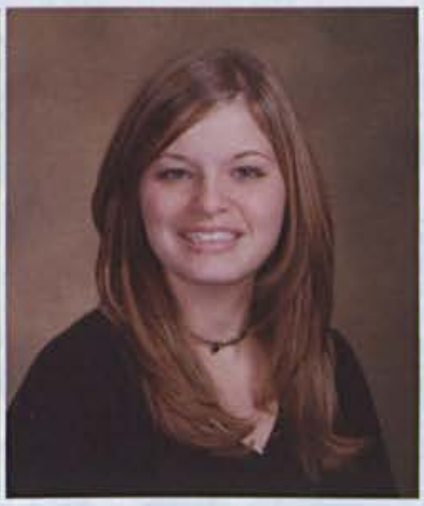

Kathryn Elisabeth Brady Early Childhood Education

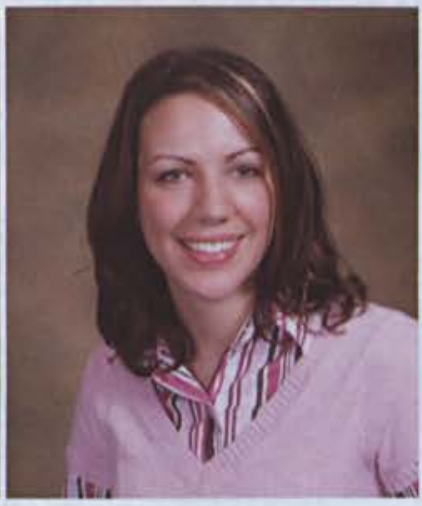

Christen Brady English

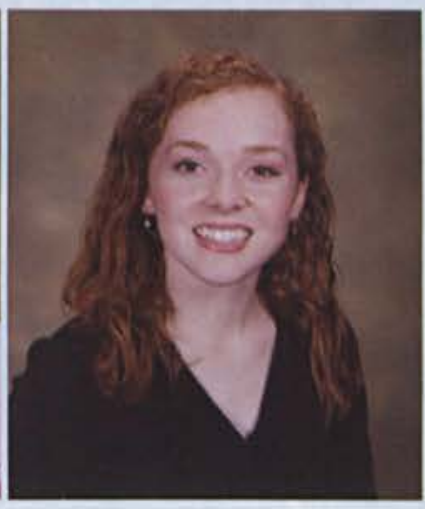

Christina Joy Bremner Marketing

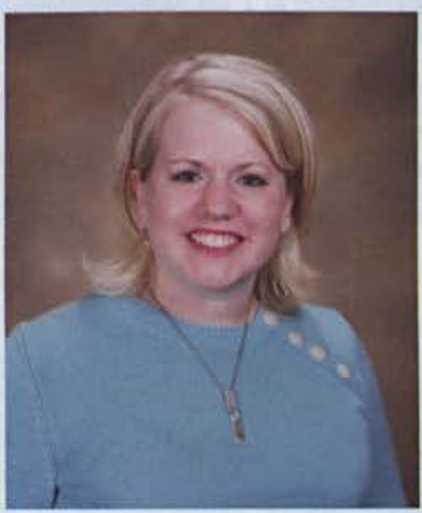

Ashley Nicole Brown Nursing

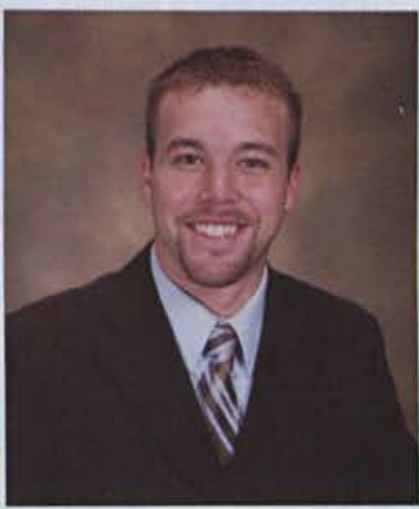

Timothy Lloyd Brownlee Management

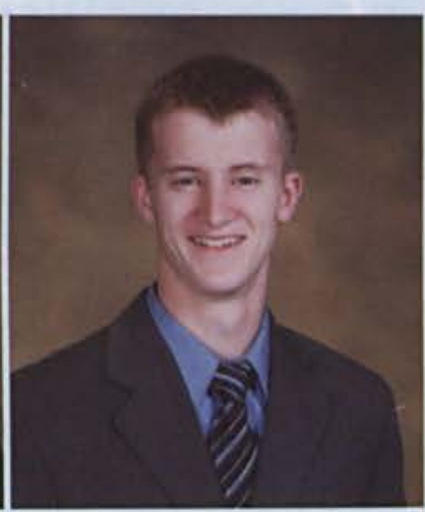

Christopher L. Brubaker Music Performance \& Composition

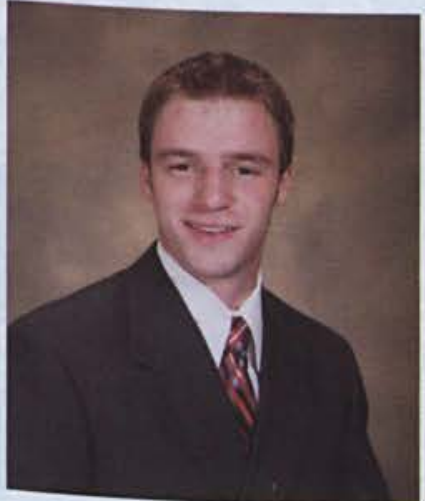

Donald Paul Buczek Public Administration

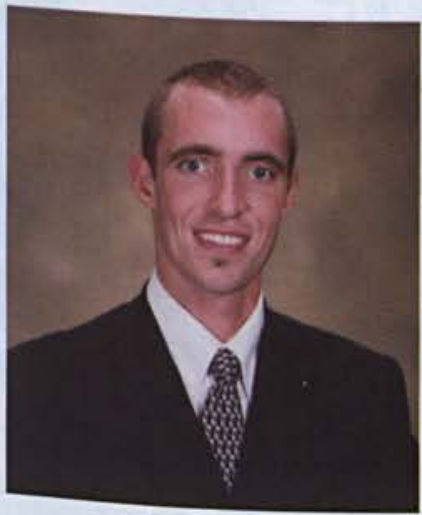

Daniel Edward Campbell Exercise \& Sport Science

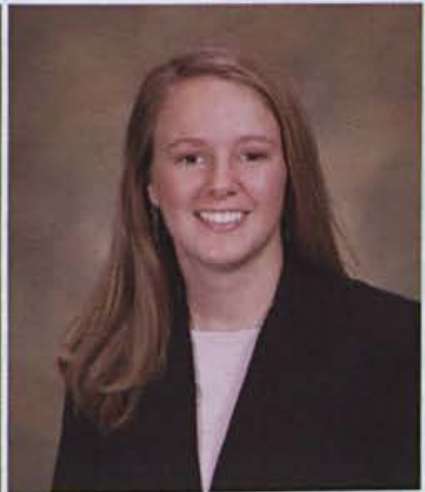

Jennifer Nicole Burdine Middle Childhood Education

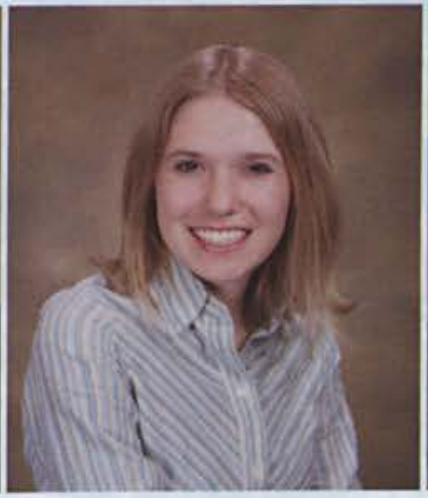

Sara Brianne Bursack Social Work

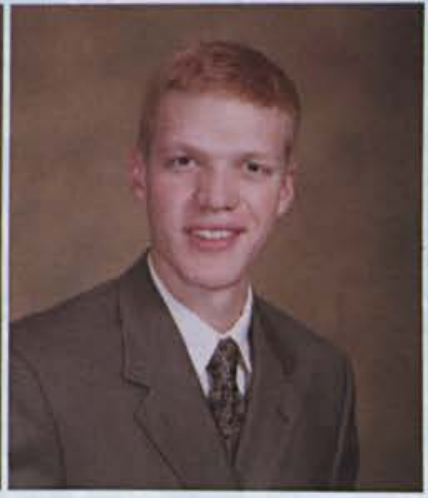

Philip Reuben Buskohl Mechanical Engineering

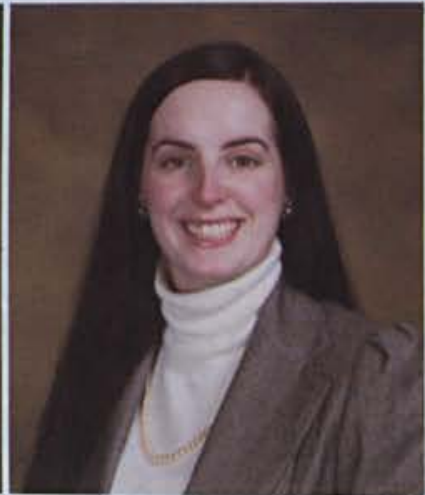

Lauren Jennette Callahan Graphic Design

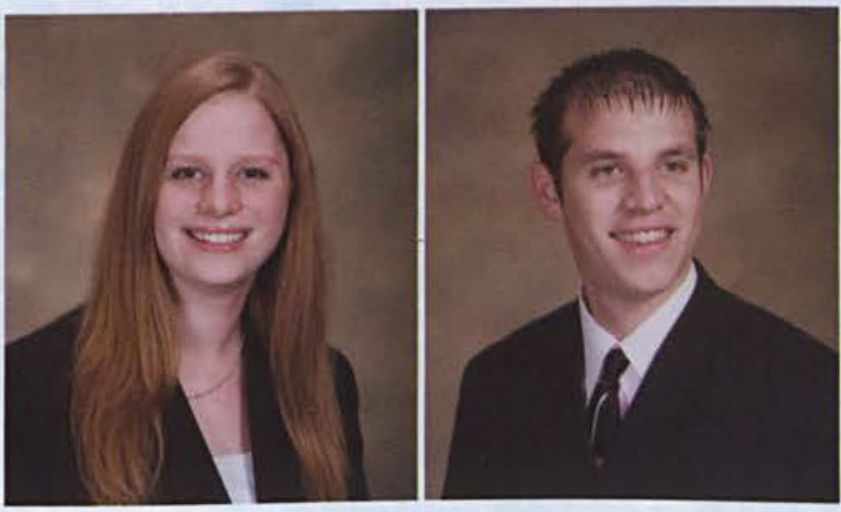

Rachel Diane Capps Psychology \& Criminal Justice

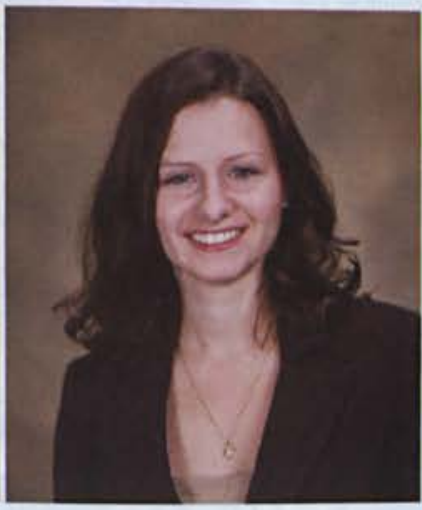

Katherine June Cassity Biology

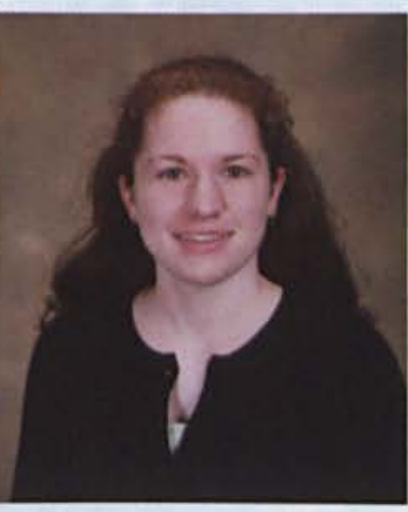

Laura Castle Communication Arts 


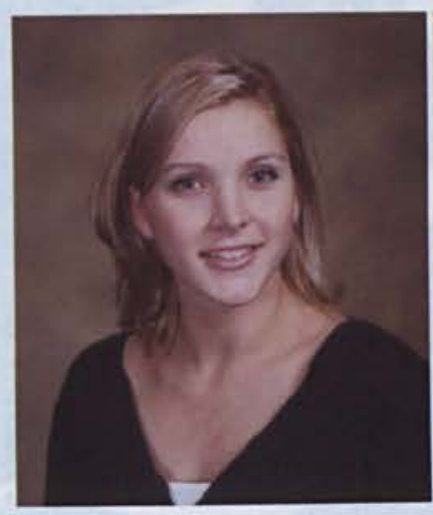

Christena R. Chandler Early Childhood Education

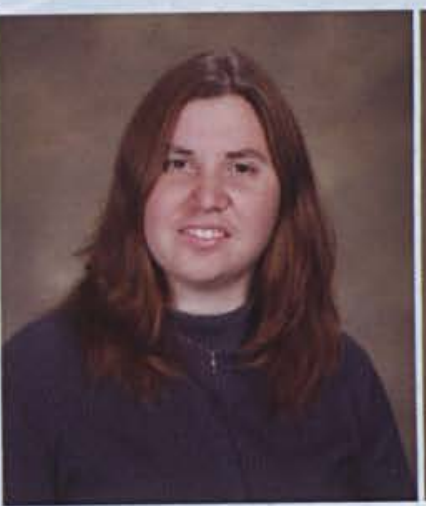

Beth M. Cinson AYA Integrated Math

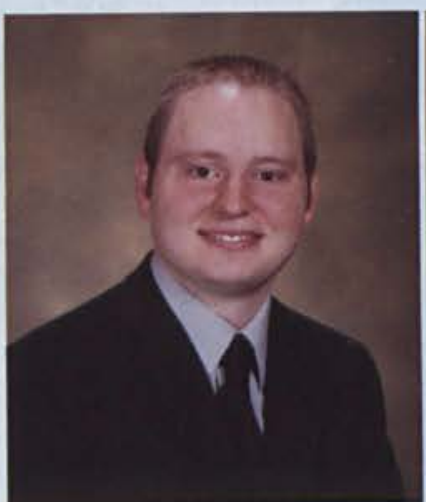

Jeremy Daniel Cobb

Church Music Ministry

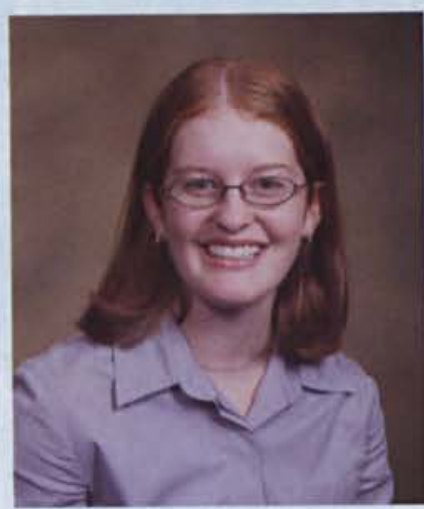

Kathrine Cole

Early Childhood Education

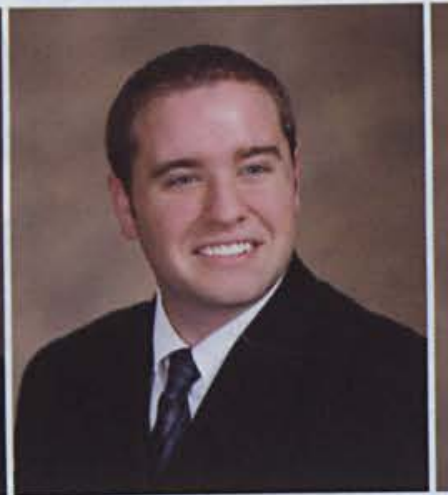

Jeremy William Chandler Finance and Management

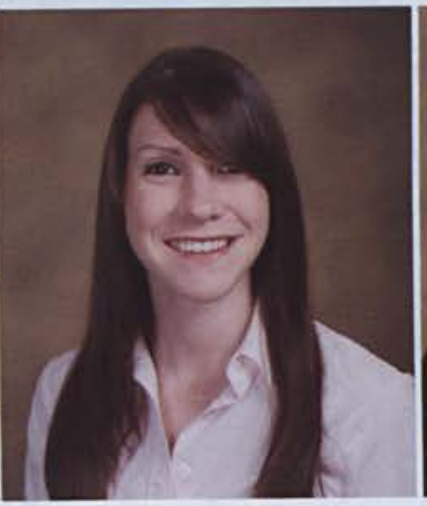

Krysten Joy Clark Organizational

Communications

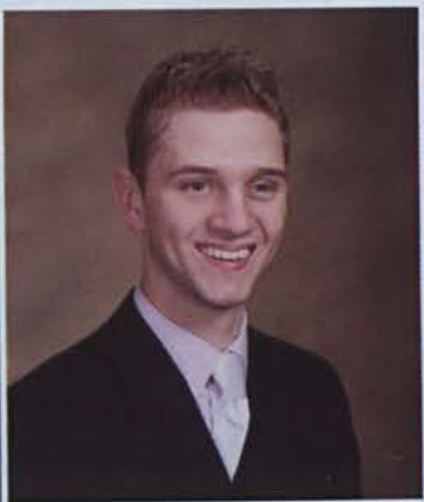

Joshua Mitchell Cobb Theatre

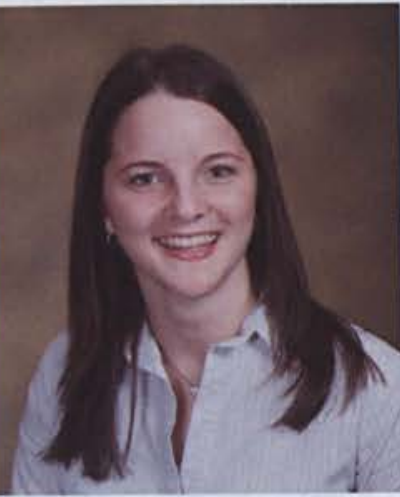

Joy A. Chandler Nursing

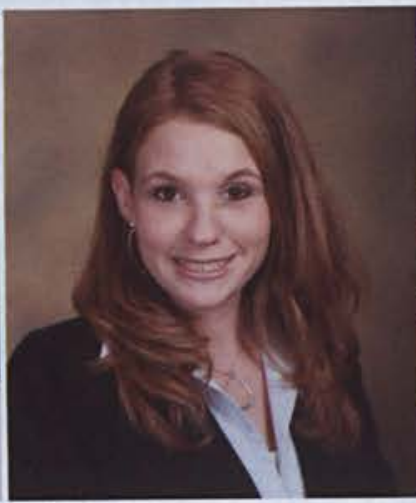

Rachel LeeAnne Clayton Mathamatics

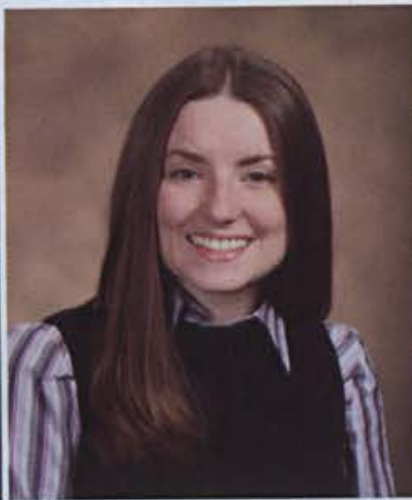

Kimberly Ann Cocca Adoloscent \& Young Adult Language Arts

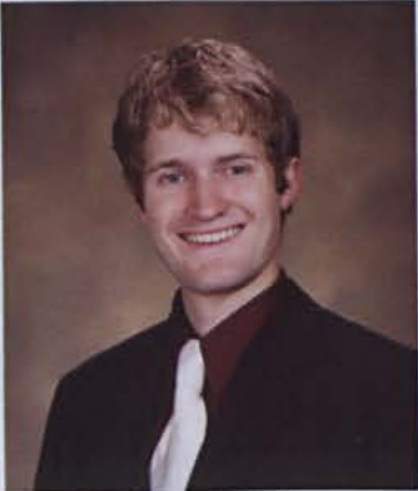

Michael Charles Colletto

Comprehensive Communications

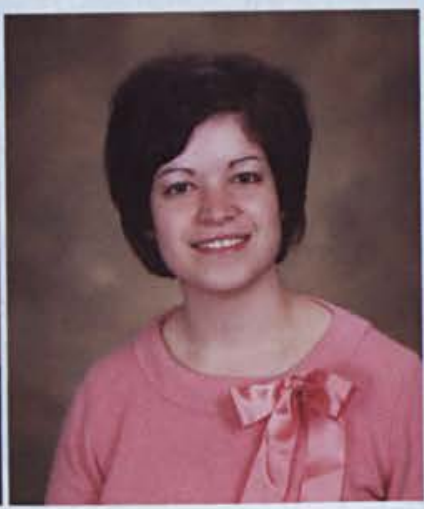

Brianne Leigh Collier Marketing

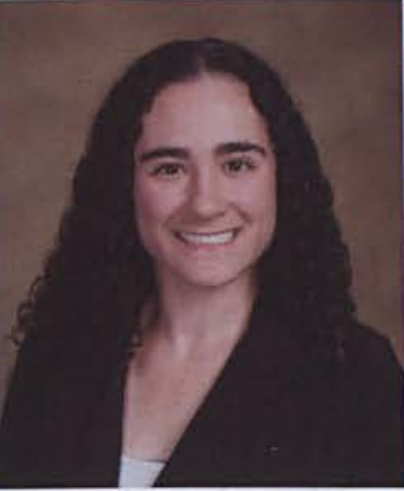

Rebecca Marie Chasnov Music

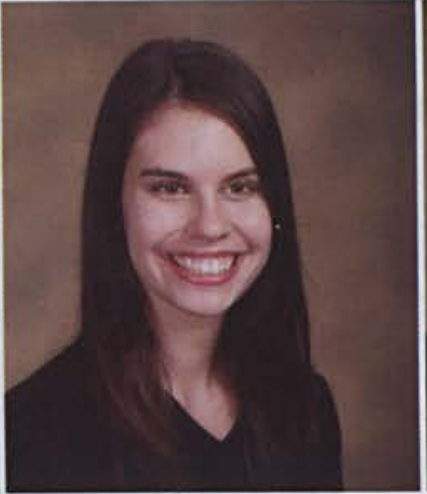

Sarah Elizabeth Clift Management

Joshua T. Clore Biology

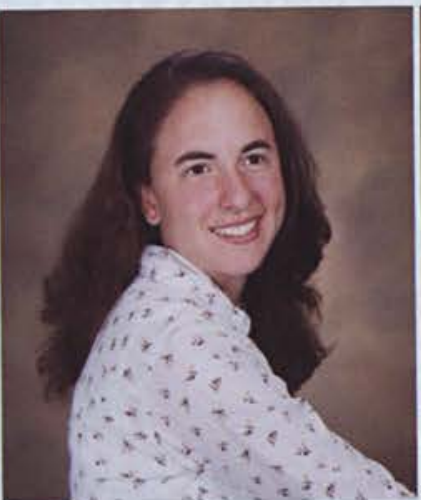

Danielle Elizabeth Colao Early Childhood Education

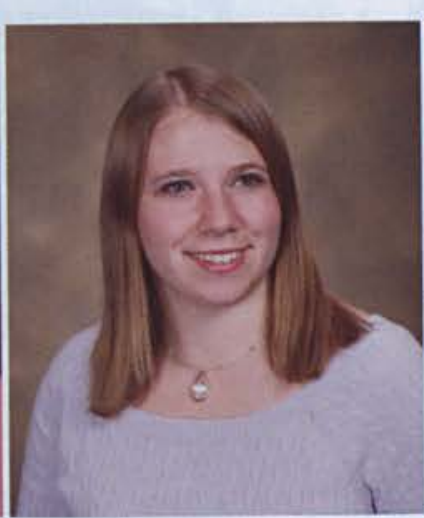

Amanda Jean Collins Church Music Ministry

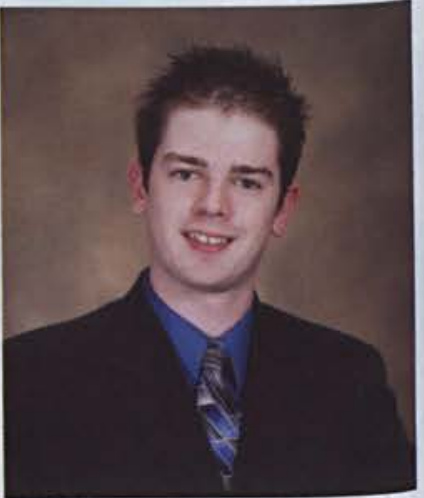

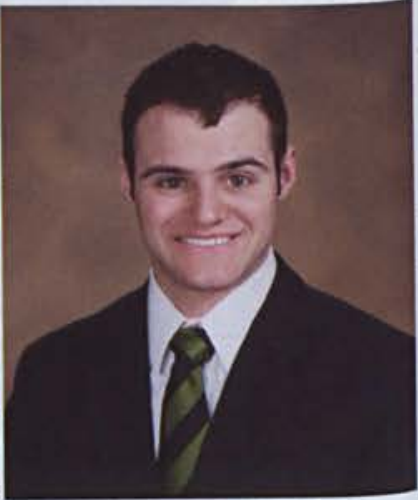

Brandon Michael Colas English \& History

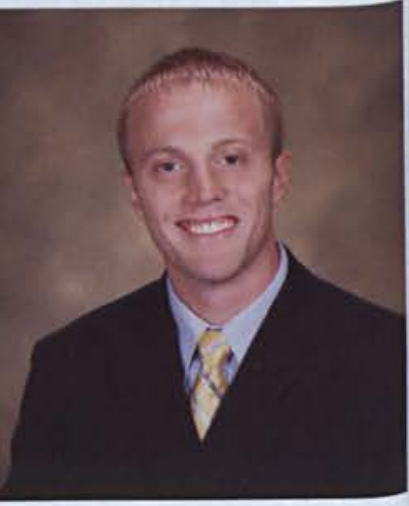

Christopher D. Collins World Missions 


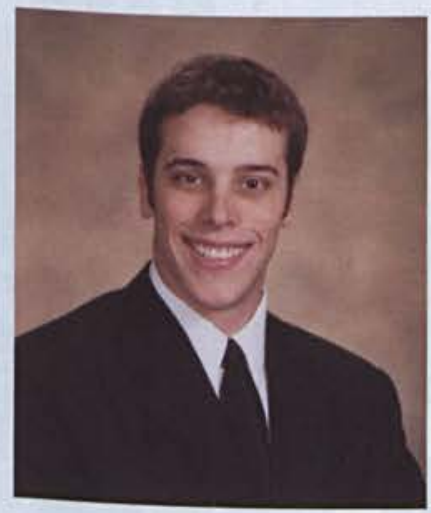

Daniel J. Congrove Electrical Engineering

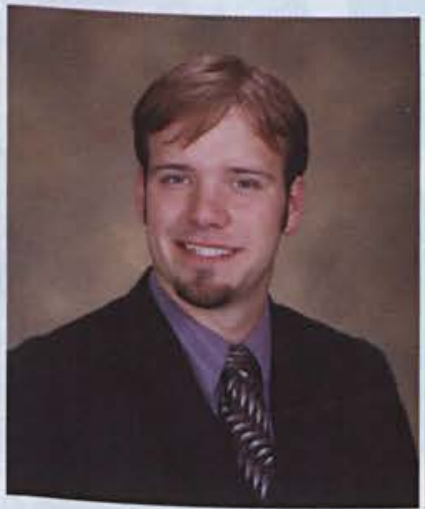

Joshua Charles Craig Management

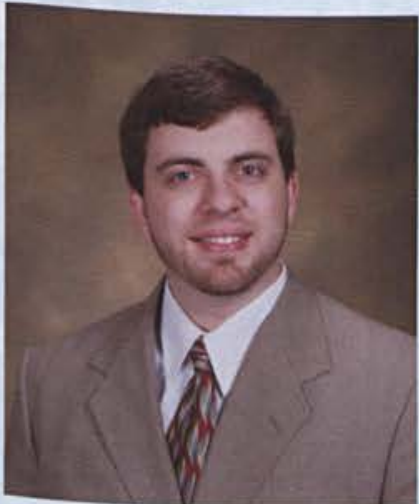

Stephen Allen Danielsen Mechanical Engineering
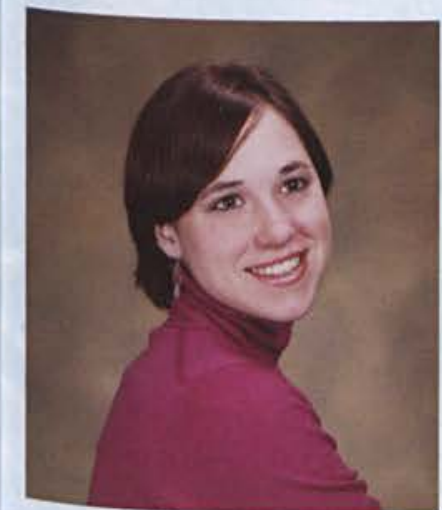

Morgan E. DelGrosso Middle Childhood Education

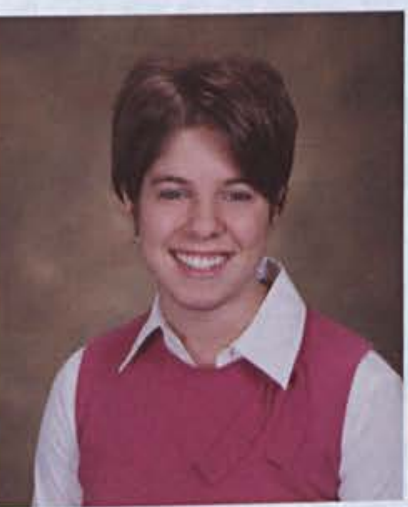

Heather Nicole Coon Nursing

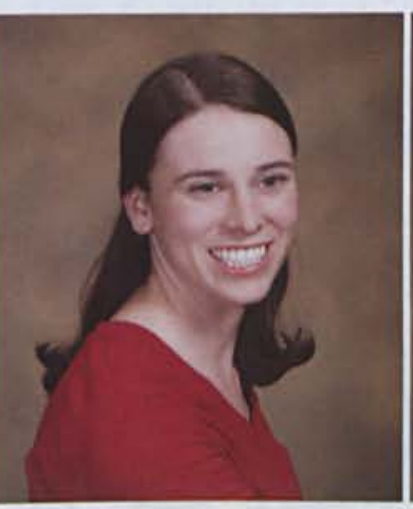

Kristin Renee Corder Integrated Language Arts

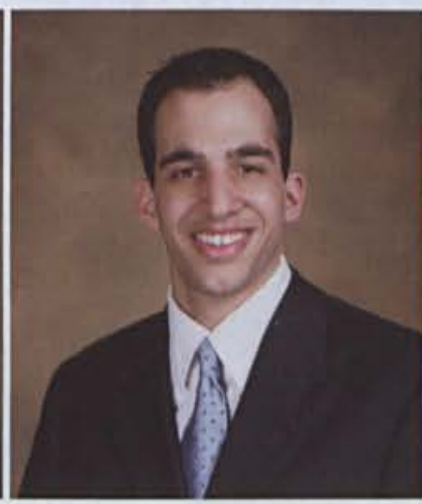

Alexandre S. Costa Marketing \& Mangement

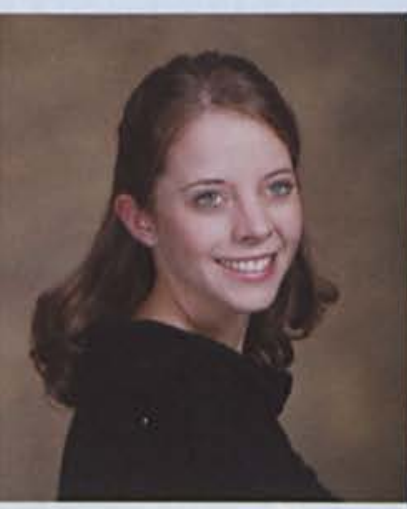

Christina Marie Craig Nursing

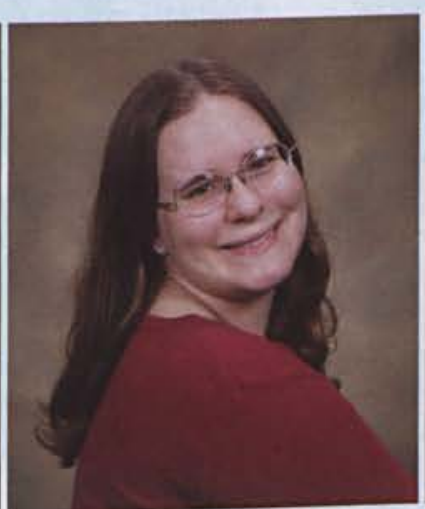

Cassie Wagner Crum Nursing

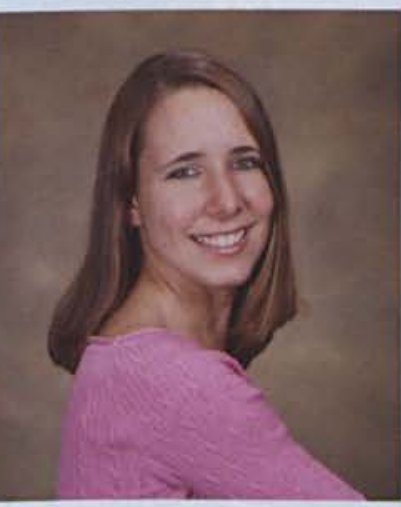

Rebecca Lynn Crunelle Chemistry

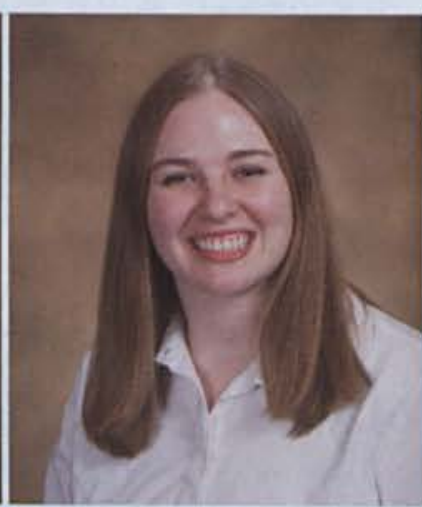

Anna Marie Curby Psychology

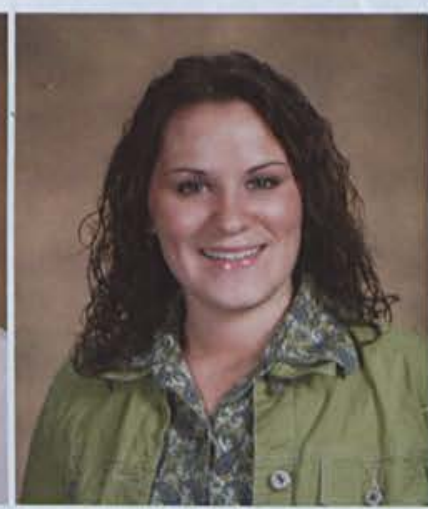

Stephanie Janelle Dager Christian Education \& Youth Ministry

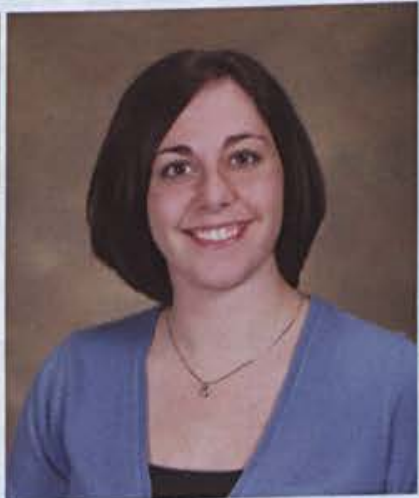

Melissa Joy Davis Nursing

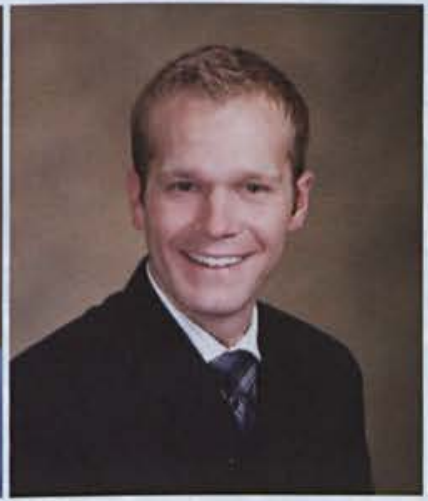

Daron Jeffrey Day Pre-Seminary

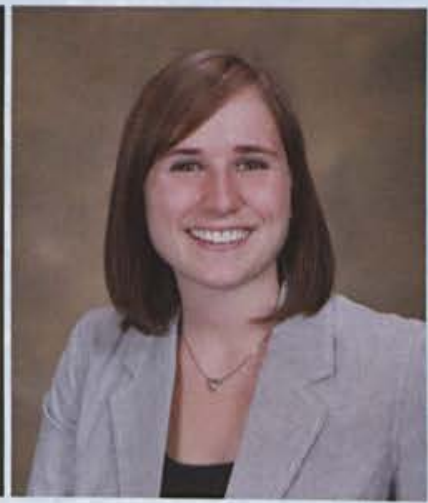

Andrea Marie DeGuglielmo Communications

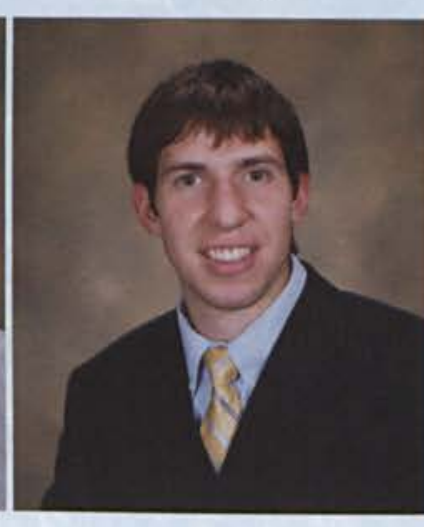

Benjamin Paul DeLarme Middle Childhood Education

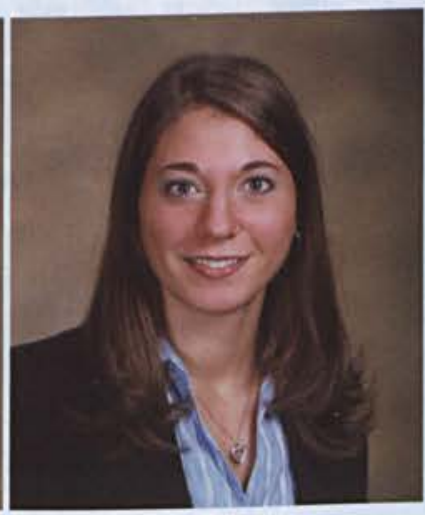

Emily Kae Delimpo Biology

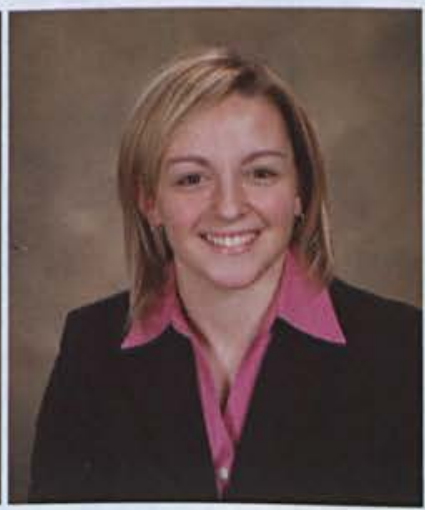

Marie Denio Accounting

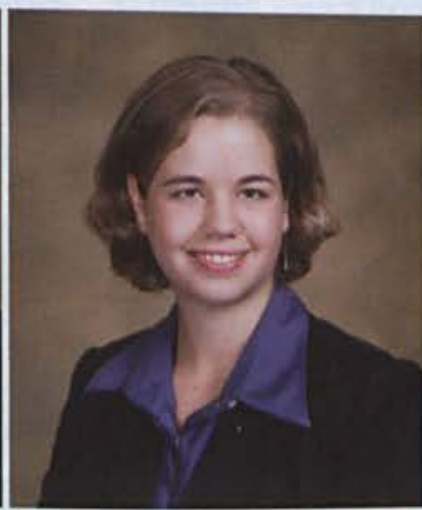

Heather Jean DePree Music Education

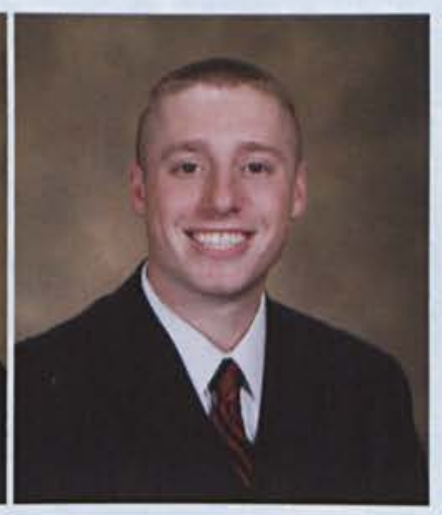

Brian David Diercks Finance 


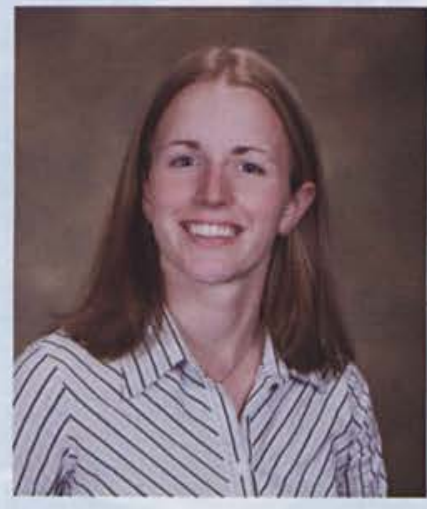

Jacqueline Lee Diercks Biology

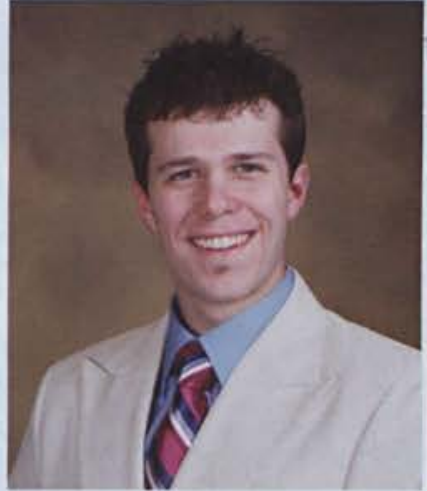

Emanuel Ransom Dolph Biology

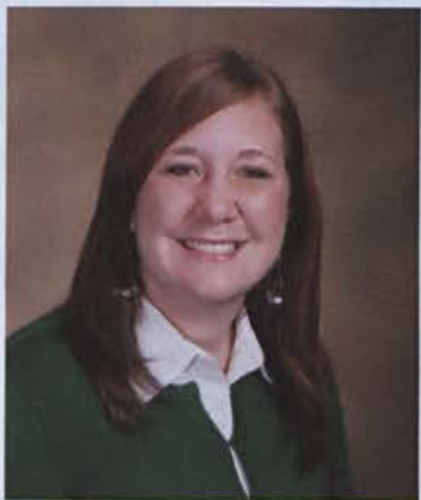

Kimberly Ann Dudick Pre-Law

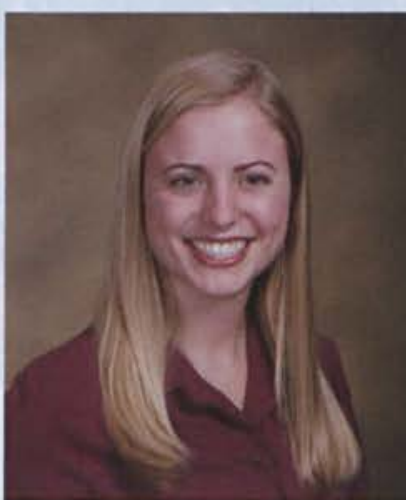

Kathrine Dunn Youth Ministries

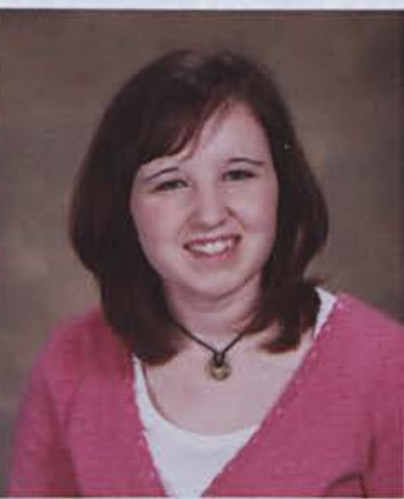

Aimee Kathleen Dilcher History

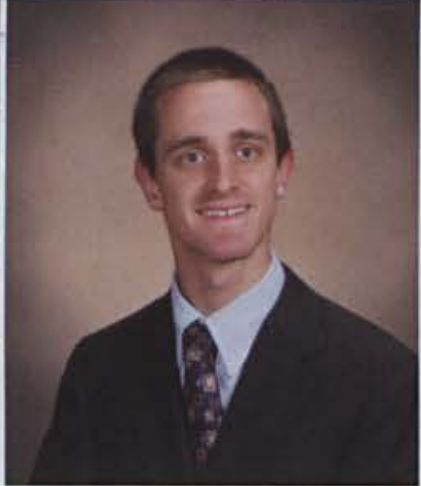

Jeffrey Rowland Doub Biology

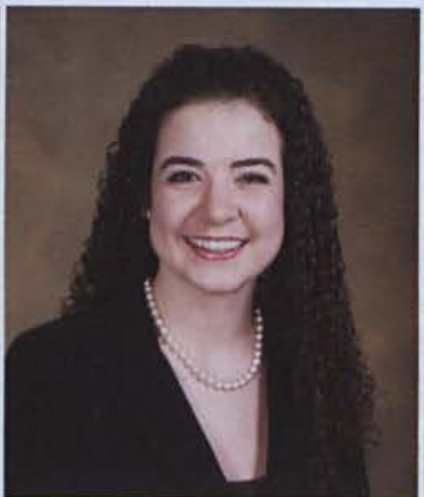

Rachel Duke

Psychology

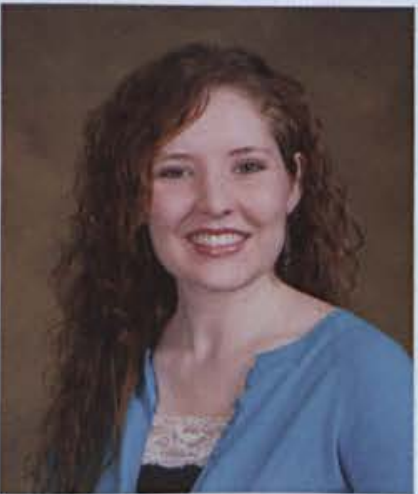

Elizabeth Ann Dye Electronic Media

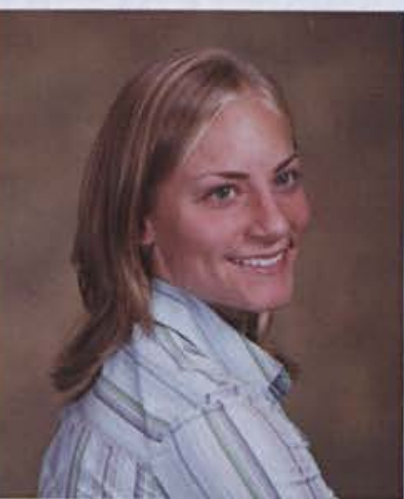

Erin DiMeolo Nursing

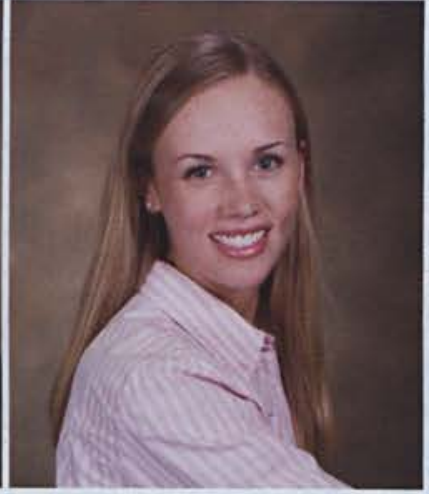

Molly Claire Dovan Exercise Science

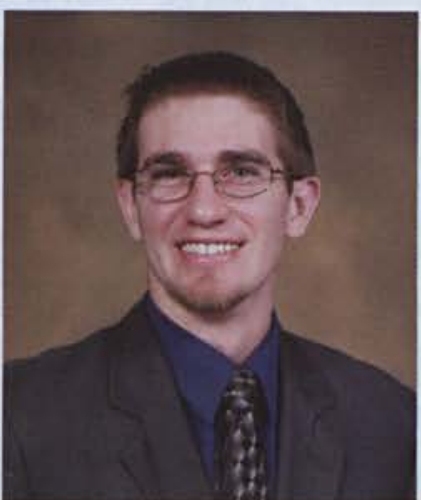

William H. Duncan Political Science

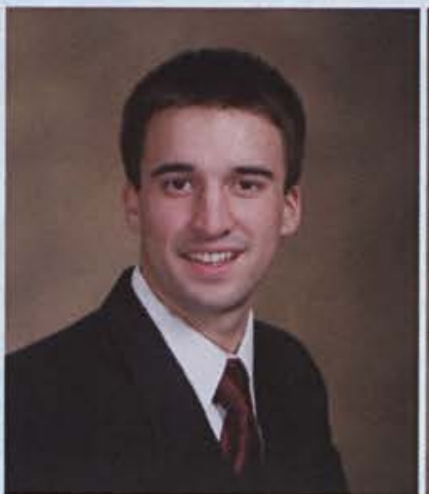

Jonathan Daniel Eaby Mechanical Engineering

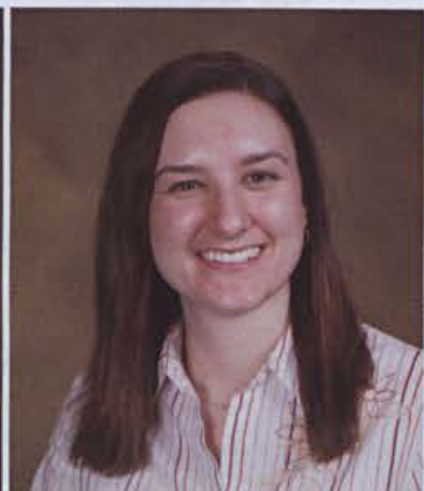

Emily Catherine Doane Integrated Language Arts Education

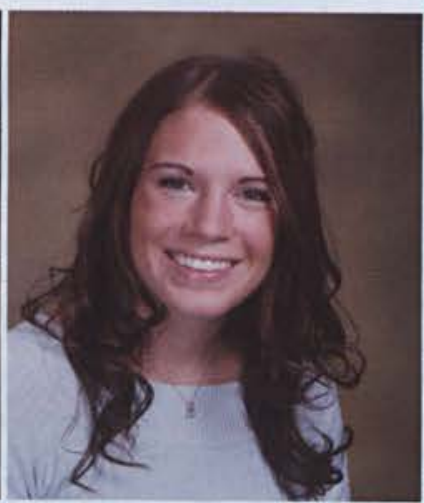

Alyssa Claire Driessnack Nursing

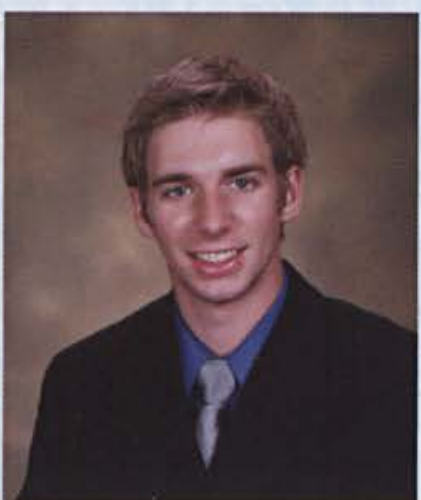

Jonathan Jay Dunkin International Studies

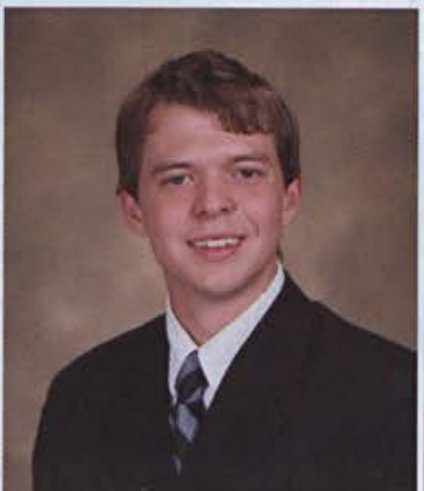

Carey Jay Edgren

Youth Ministries \& Christian Education

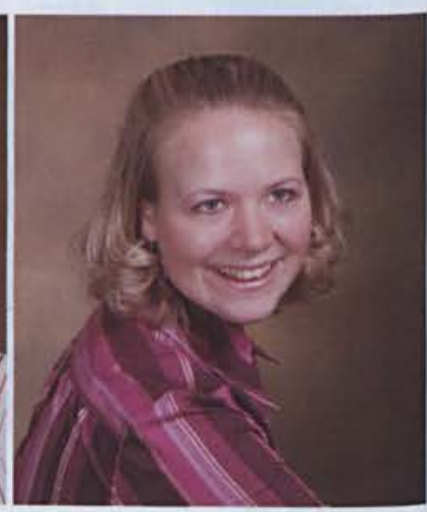

Jessica Rae Dodson Integrated Language Arts Education

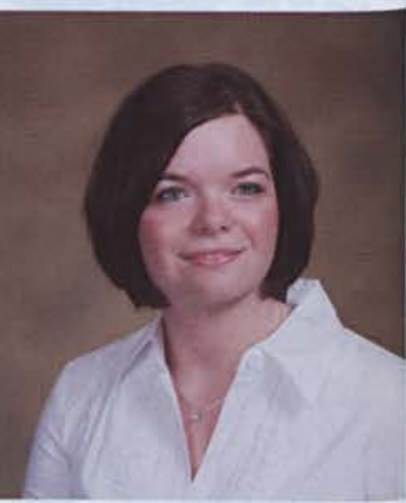

Megan Michelle Driscoll Nursing

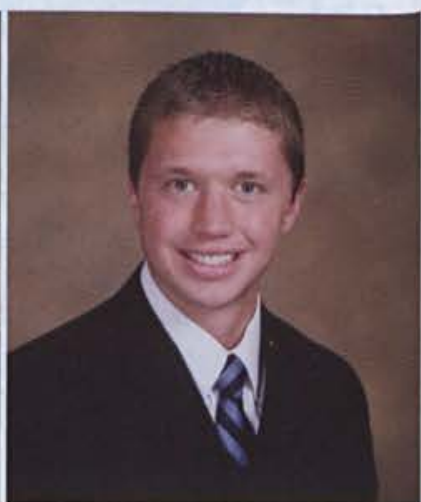

Ryan Matthew Dunlap Accounting

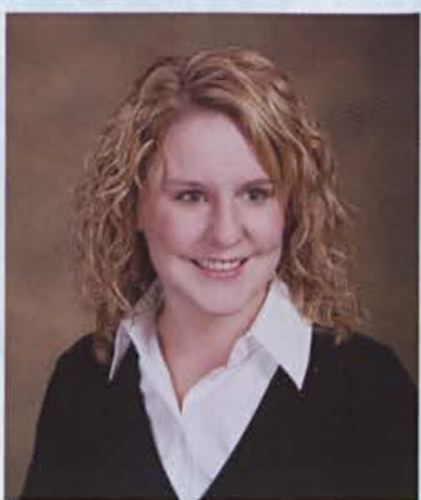

Allison Christine Eklund Early Childhood Education 


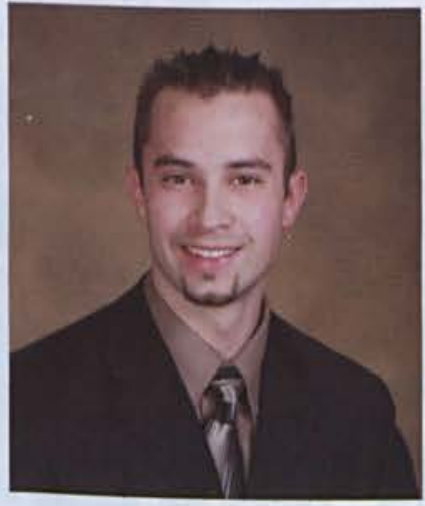

Jonathan Michel Elchison Computer Engineering

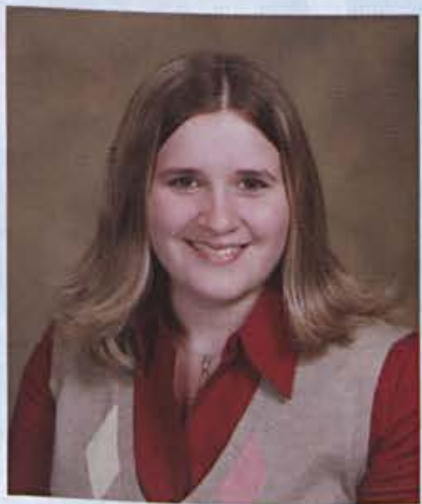

Sara Renee Eplin English

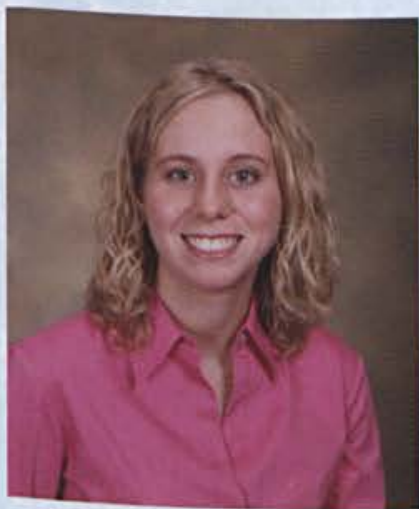

Abigail Leigh Fenton Early Childhood Education

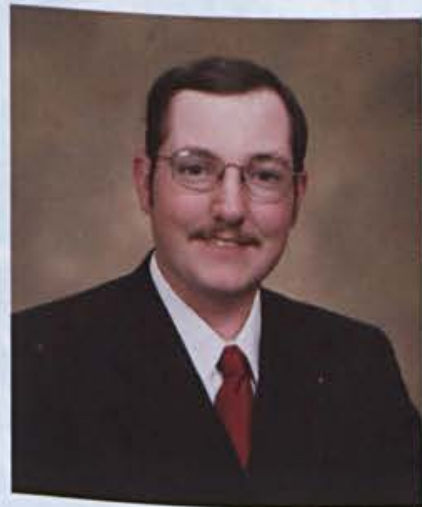

James Todd Filson Psychology

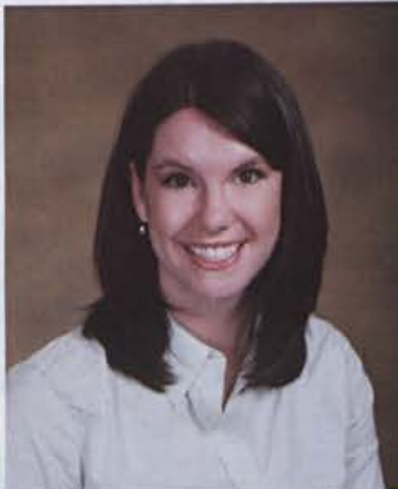

Karen Mae Elliott Nursing

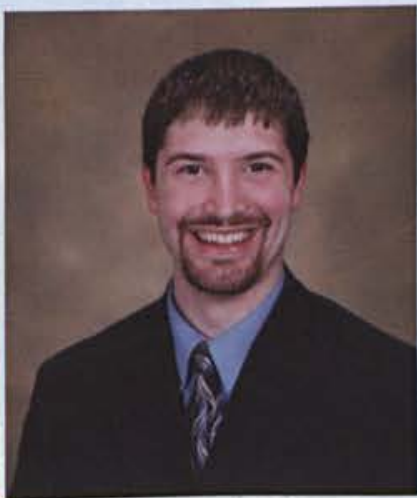

David Arthur Erlandson Church Music \& Music Composition

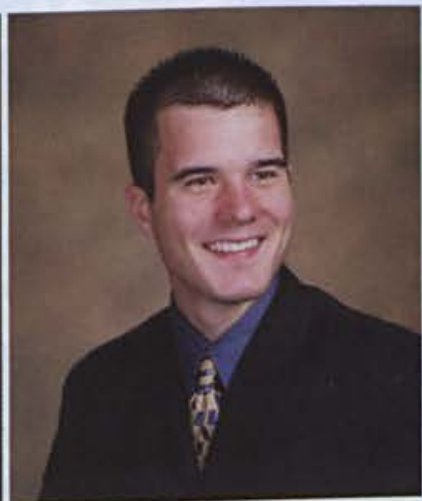

Kyle Lawrence Ferguson Middle Childhood Education Middle Childhood Education

Jeremy Andrew Estes Mechanical Engineering

Martha Jean Ferguson

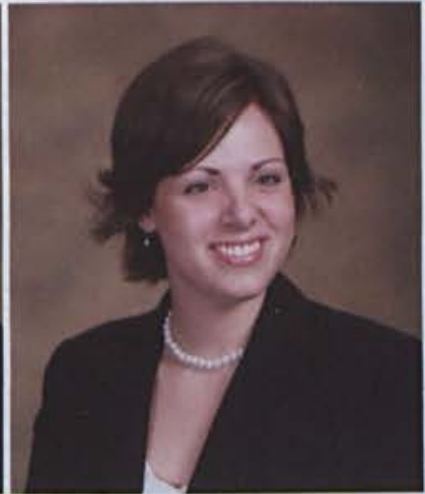

Lisa Marie Engel

Justin Scott Ely Communications

Middle Childhood Education

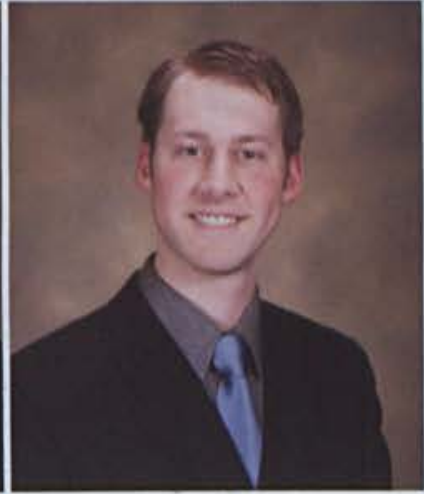

Thomas H. England III International Studies
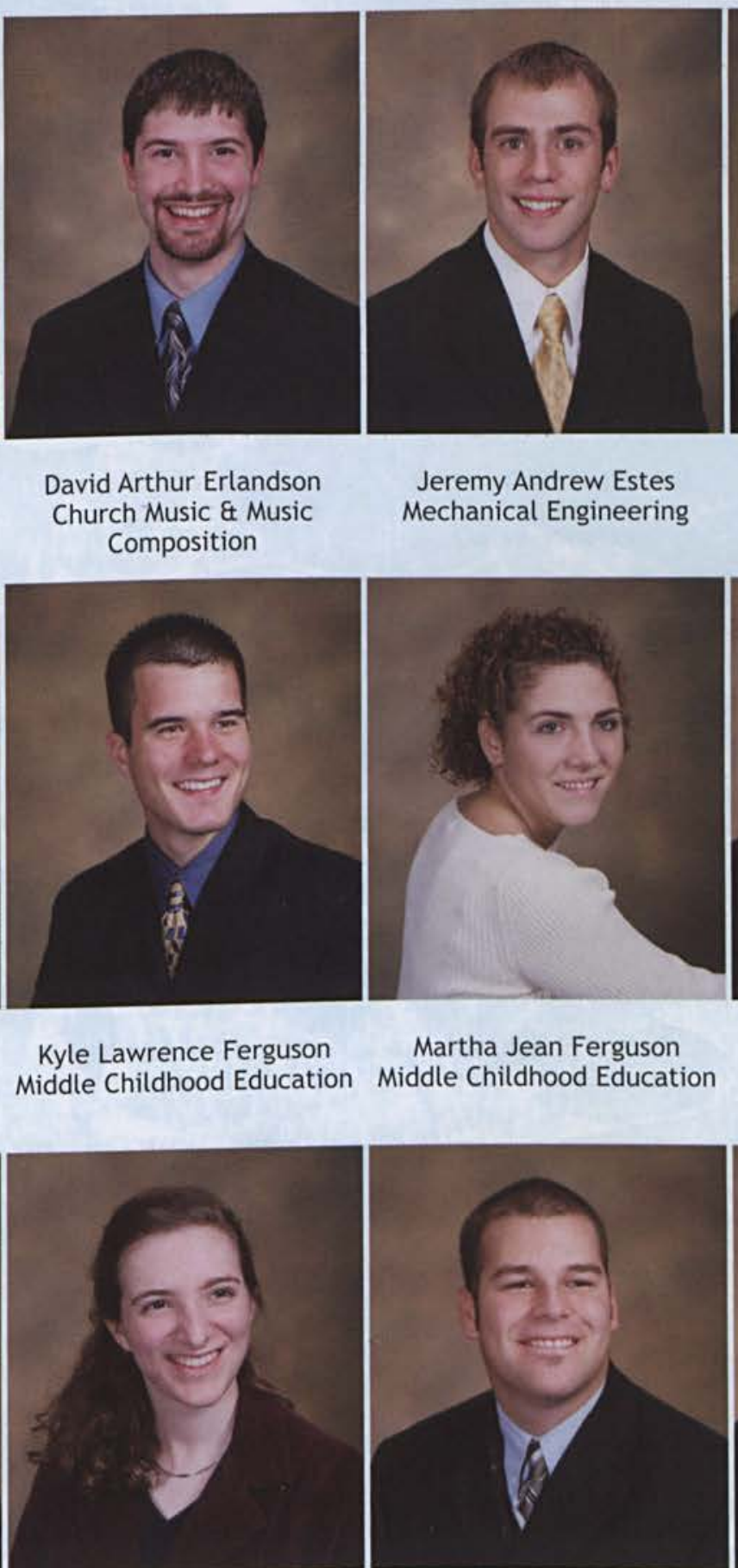

Erin Lorraine Finke Chemistry and Biology

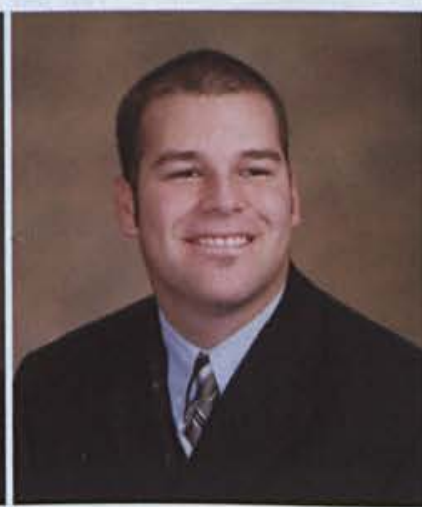

Cody Fisher Youth Ministries

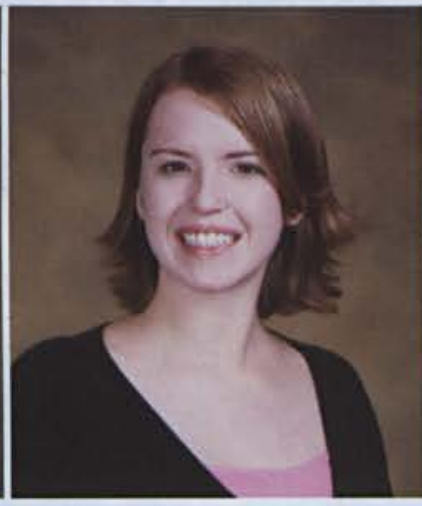

Sarah Marie Fakan Marketing \& International Business

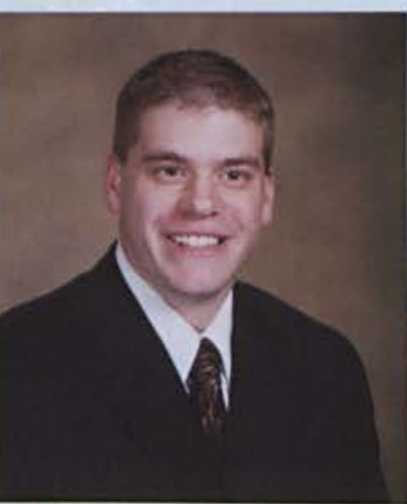

Jonathan Douglas Ferrin Computer Engineering

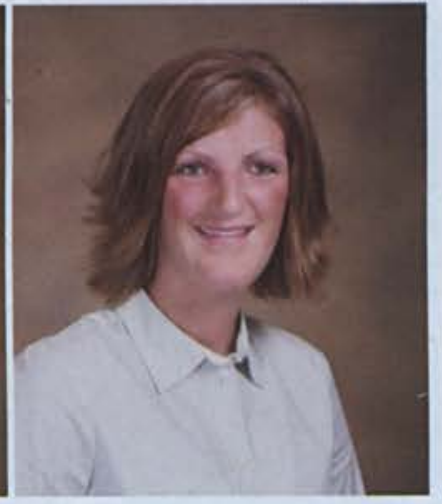

Nicole Ruth Fenstermaker Nursing

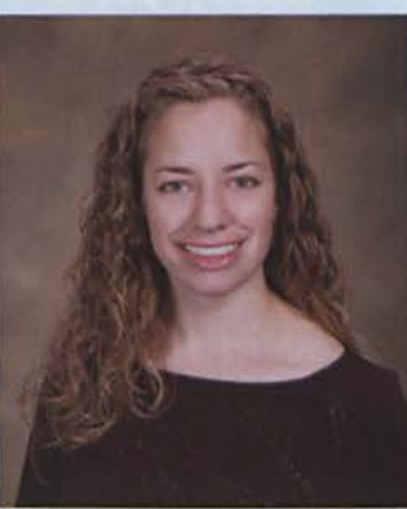

Katherine Louise Fickes Chemistry

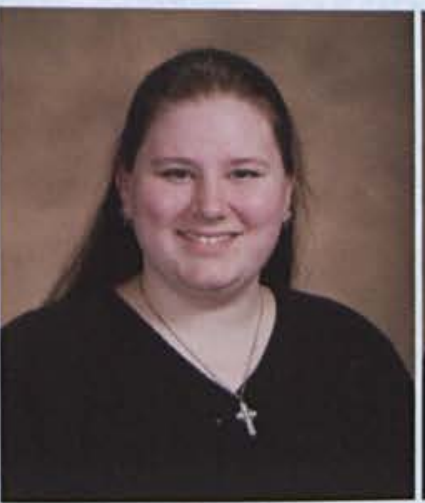

Tammy C. Fitzerald English

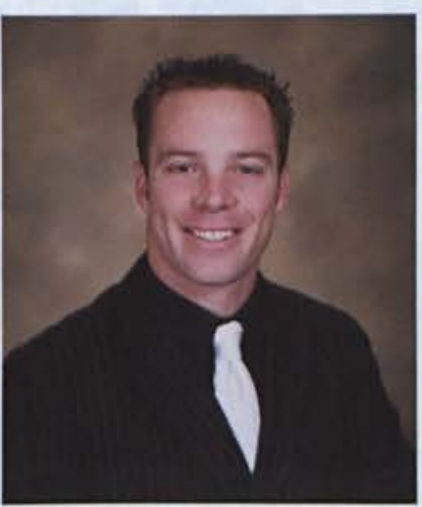

Joshua David Flynn Athletic Training

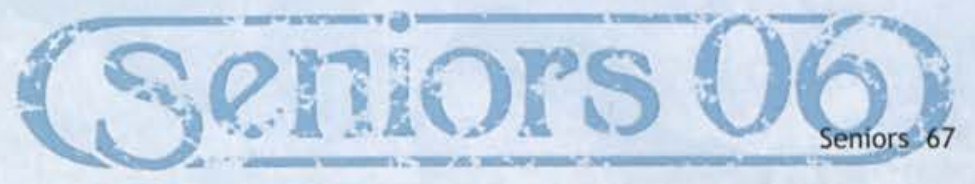




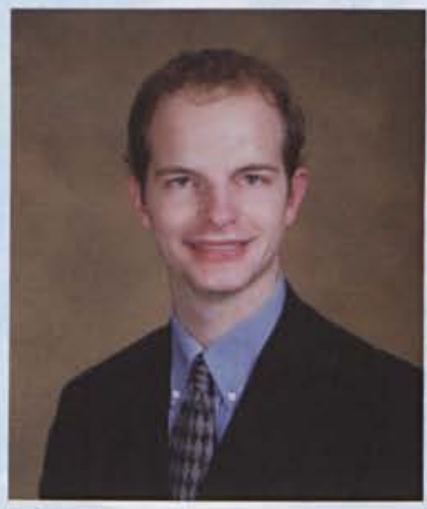

Mark David Forsberg Management

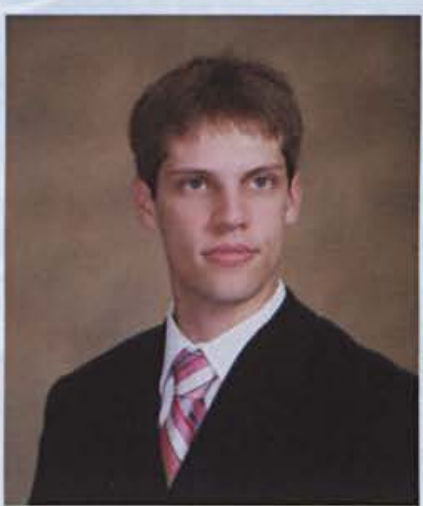

Nathan Douglas Fox Criminal Justice

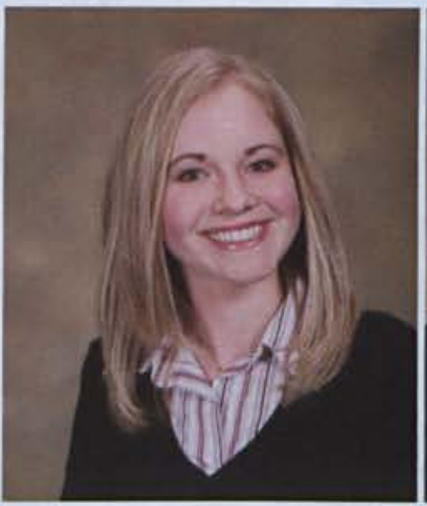

Sarah Renee Friesen

Communications

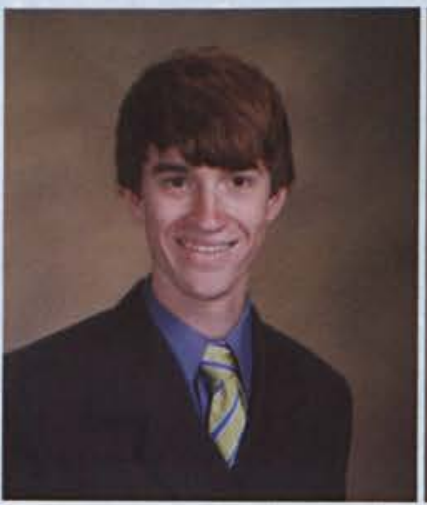

Brandon Pierce Geary Communications

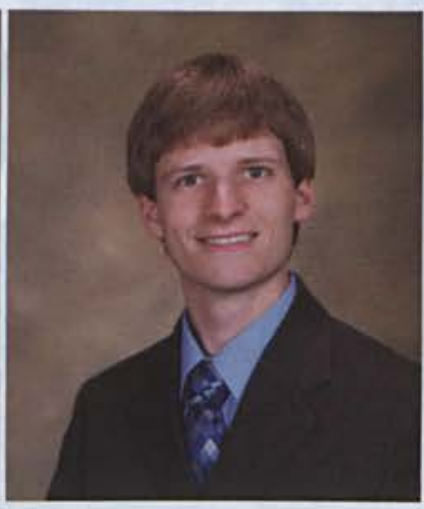

Dustin E. Foster Mechanical Engineering

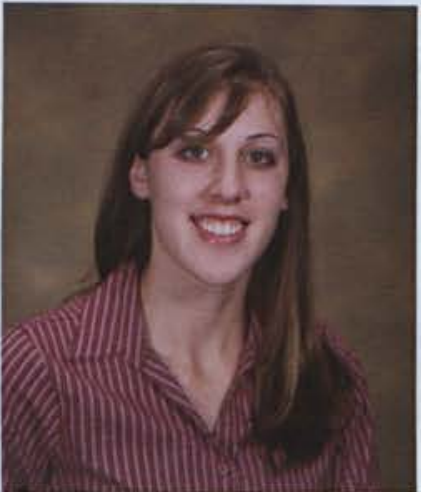

Laurie Irene Fraser Graphic Design

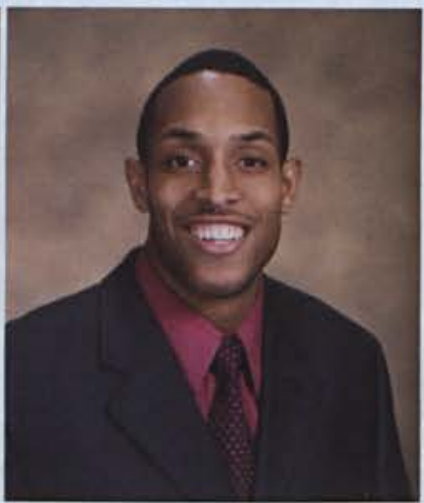

Donyo Michael Frisco

Physical Education

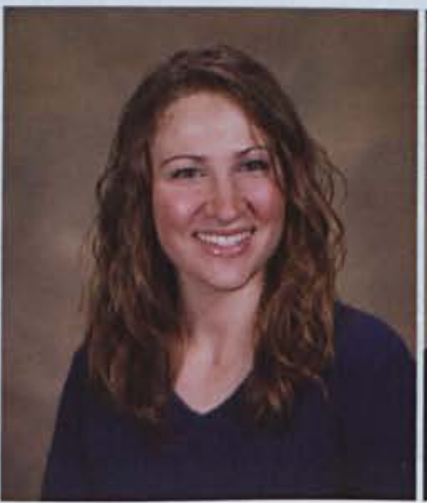

Grace Elisabeth Gebert Global Economics \& International Business

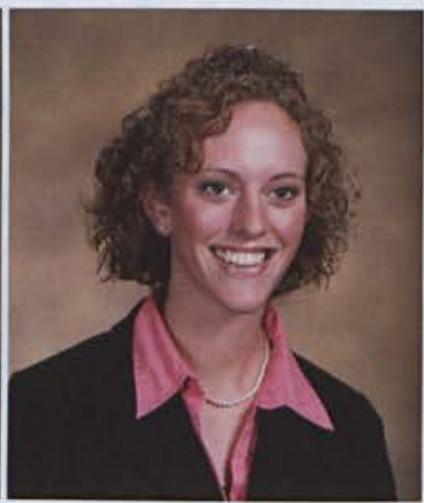

Kathryn Fowler Youth Ministries \& Christian Education

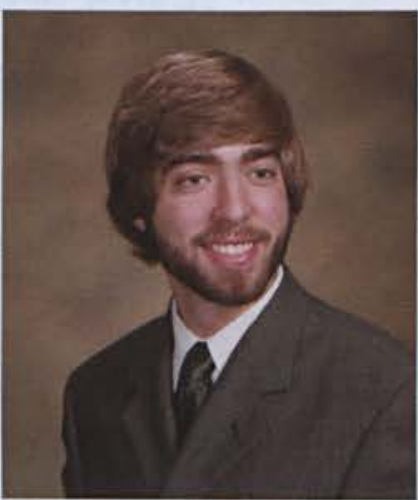

Luke Richard Frederick Biology

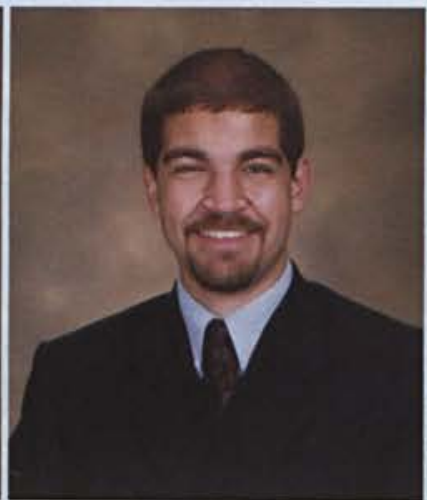

Daniel M. Gallagher Electrical Engineering

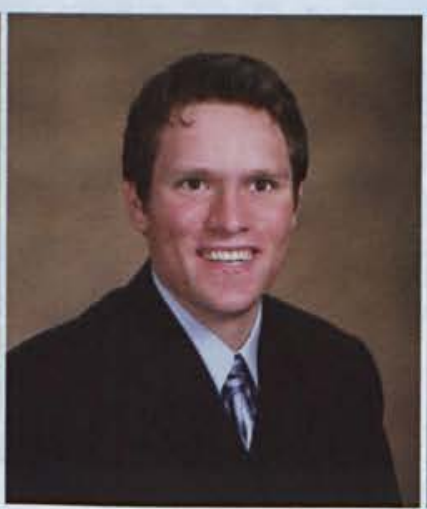

Daniel Paul Gier Management \& Marketing

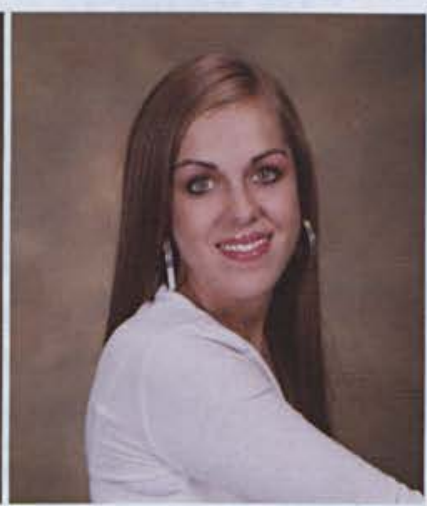

Amanda Rachelle Fox Biology

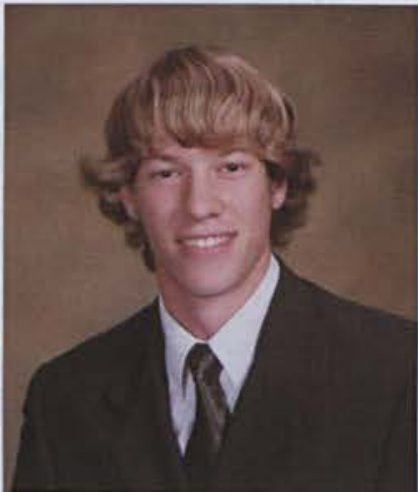

Gabriel Justin Friend Accounting

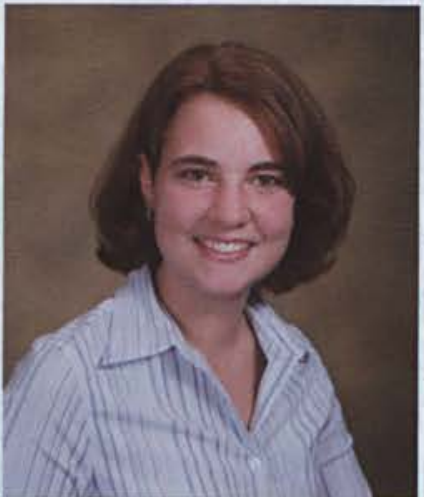

Stacey Ann Galley Early Childhood Education

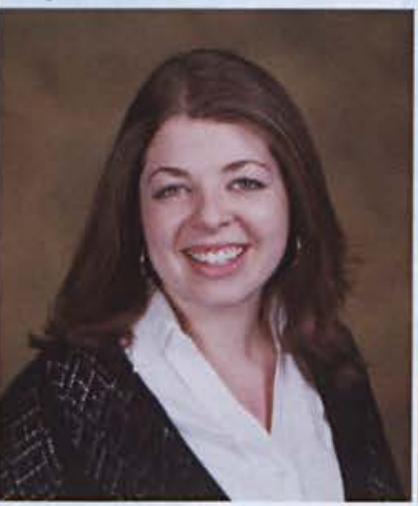

Jessica Noelle Gigliotti Music Education

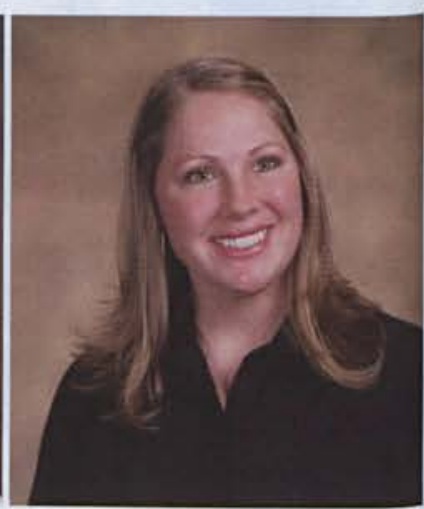

Natalie Mae Fox Nursing

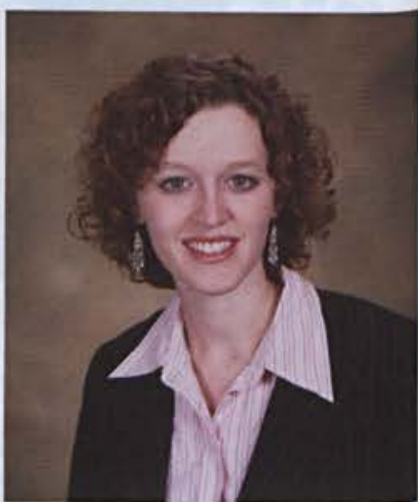

Holly Pauline Friend Integrated Social Studies

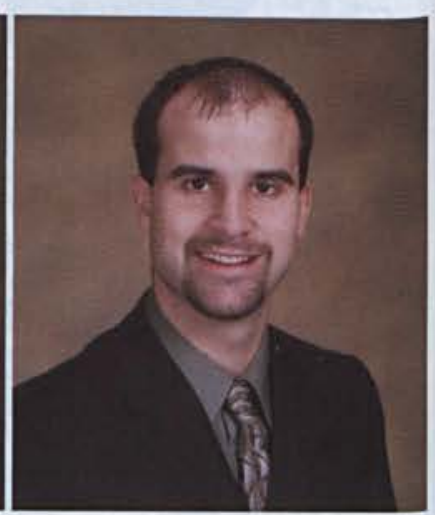

Travis Brandon Gasser Youth Ministries \& Christian Education

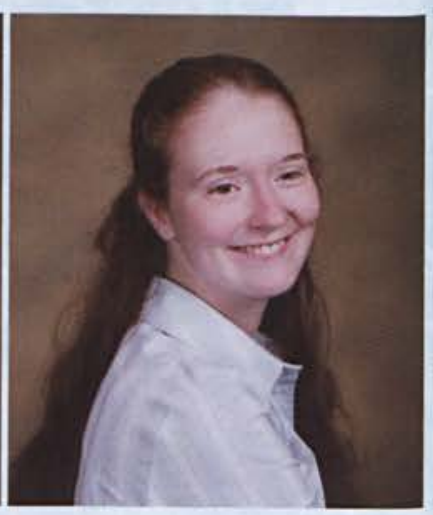

Bonnie Jeanne Gilbert Early Childhood Education 


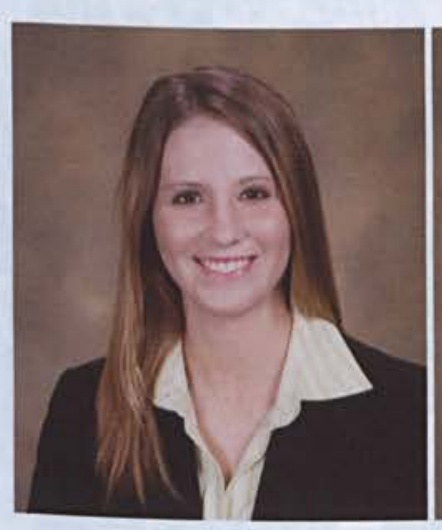

Sarah Ingrid Gilbert Youth Ministry

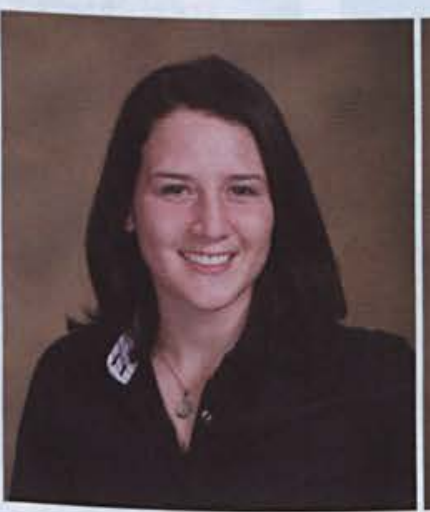

Kathleen Marie Godinez Biology

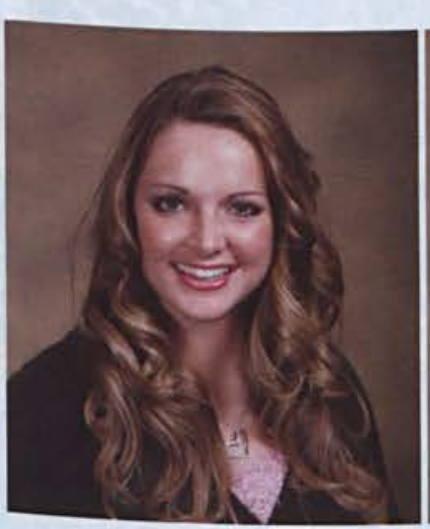

Jacquelyn D. Greetham Graphic Design

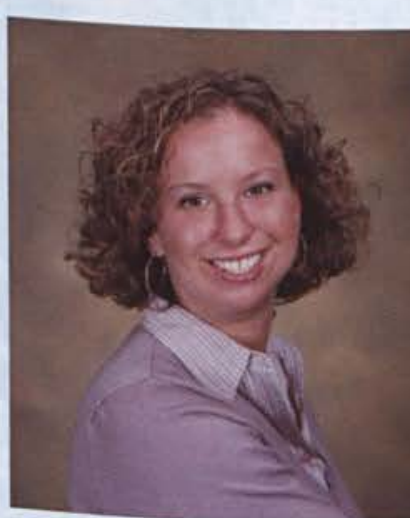

Sarah Beth Grollimund Nursing

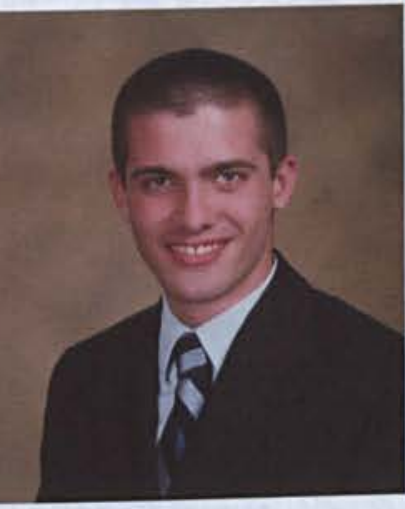

Timothy Gill Finance

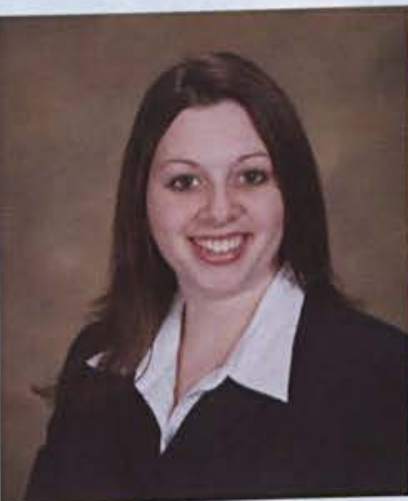

Danielle Elise Goodling Applied Psychology \& Sociology

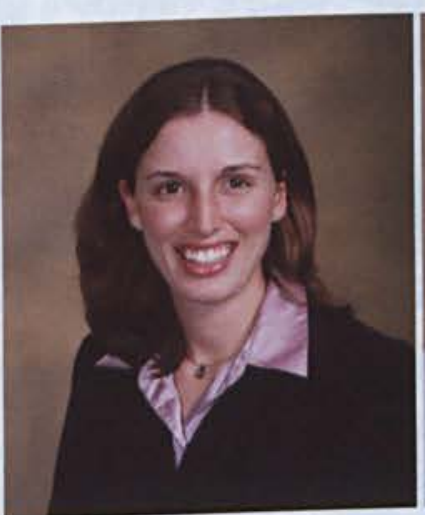

Heather Renee Grier Special Education \& Early Childhood Education

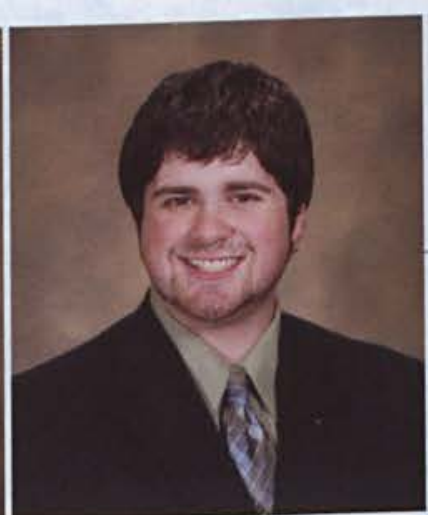

Scott Wesley Gruber Organizational Communications

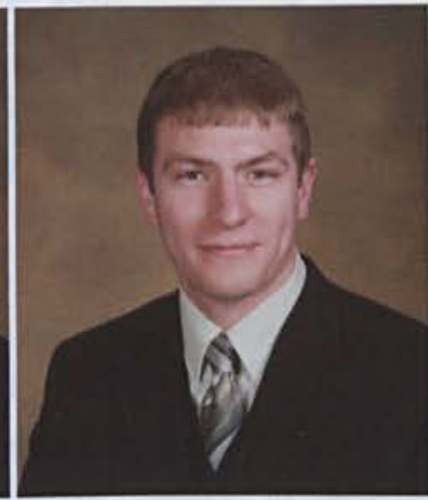

Justin B. Gillett Management

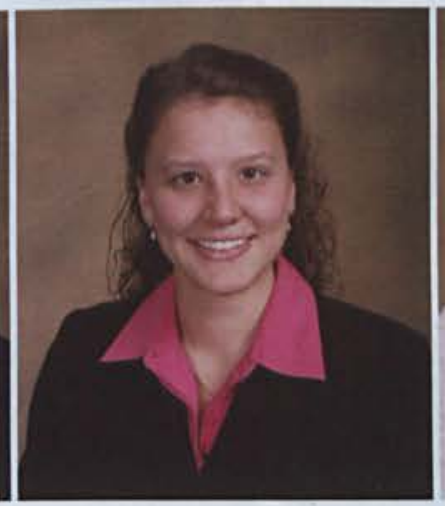

Jennifer Lindsay Grant Organizational

Communications

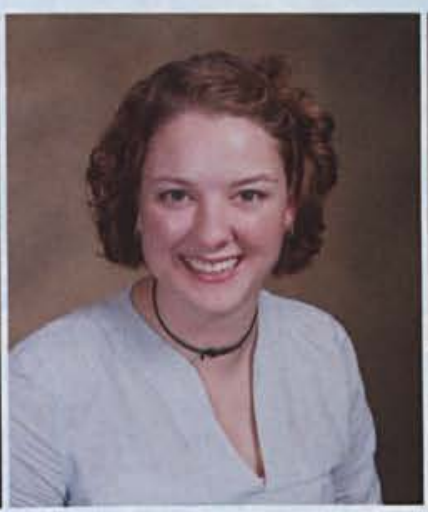

Lyndie Grigorenko English

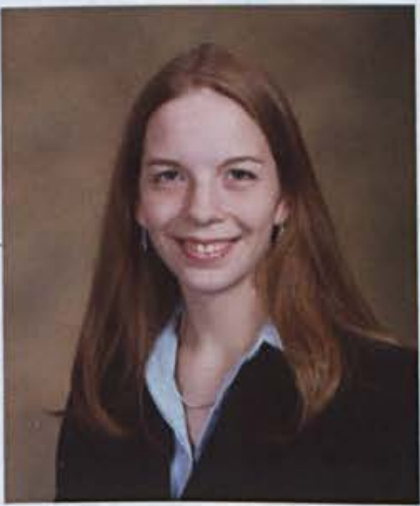

Jessie Lynn Gustafson Applied Psychology

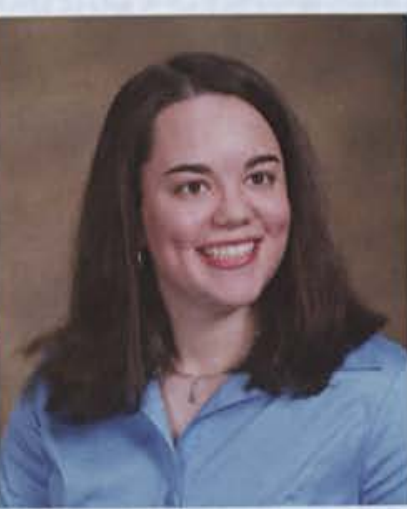

Elizabeth Joy Gingrich Nursing

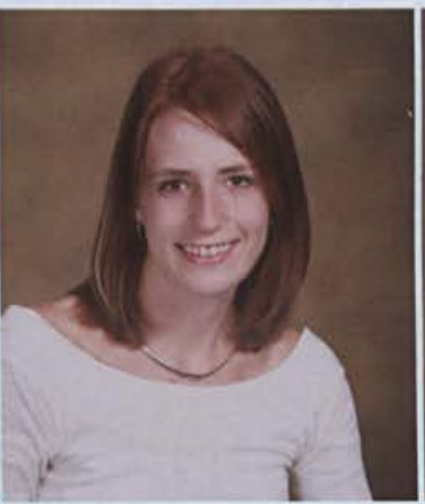

Rachel Lynn Gray Nursing

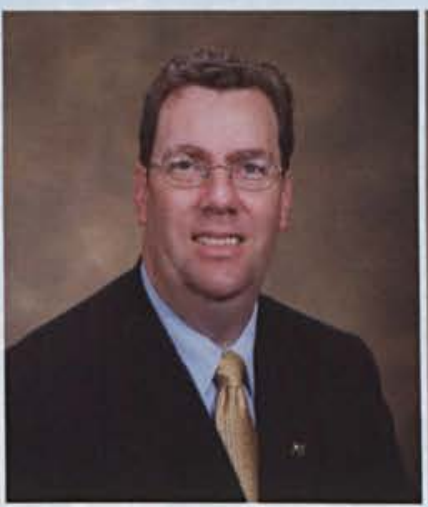

Gerald Scott Grimson Pre-Seminary Bible

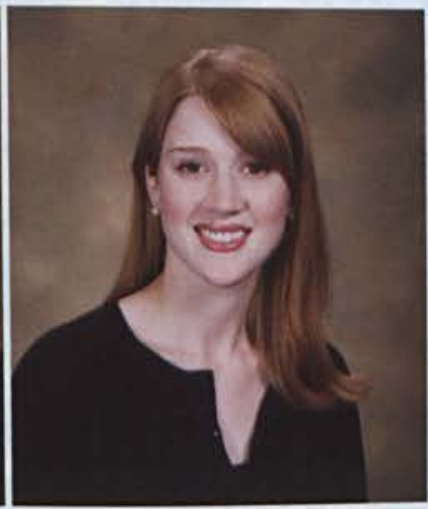

Amanda Rae Haas Music Education

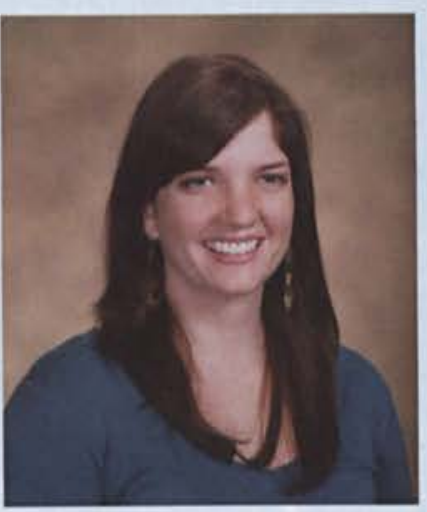

Rebecca Joy Givens Nursing

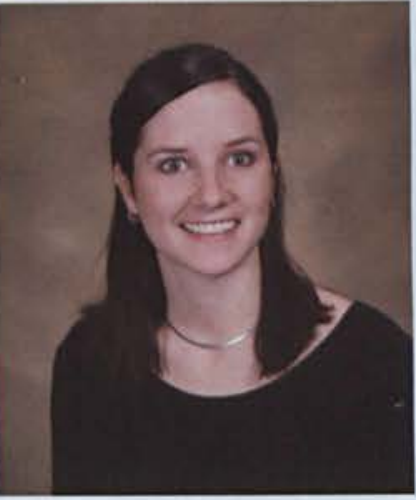

Katherine Holleman Green Nursing

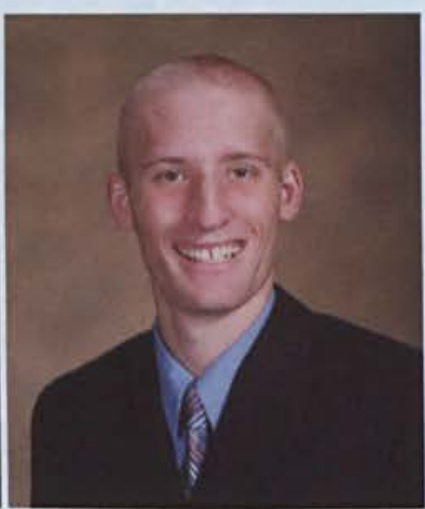

Brian Michael Groff Mechanical Engineering

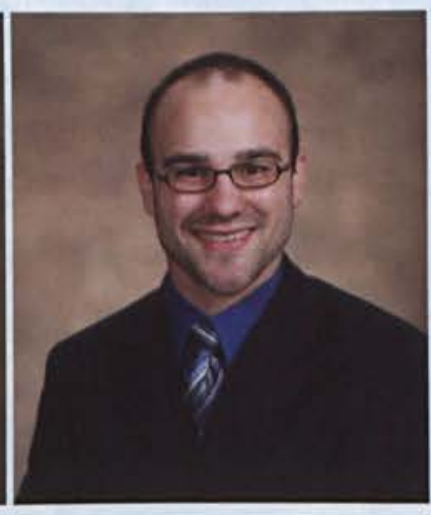

Jonathan Crawford Haber Comprehensive Bible

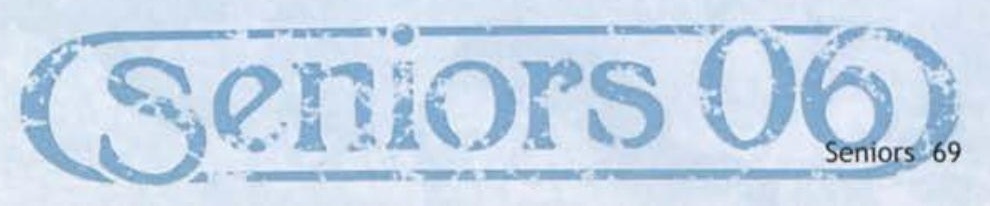




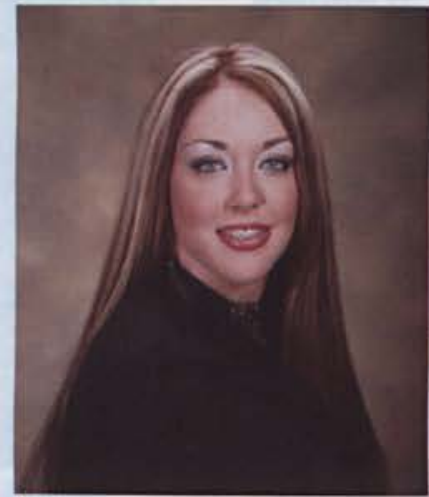

Elizabeth Ruth Hacking Nursing

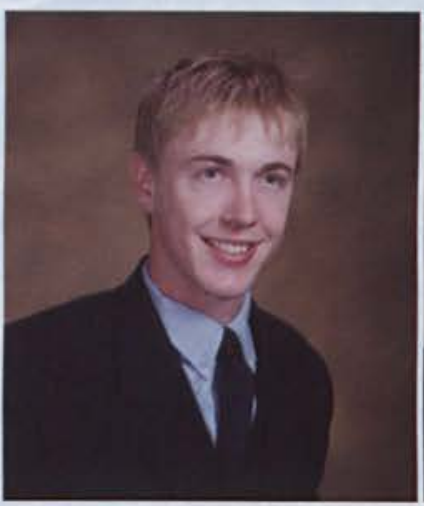

Benjamin Philip Halladay Mechanical Engineering

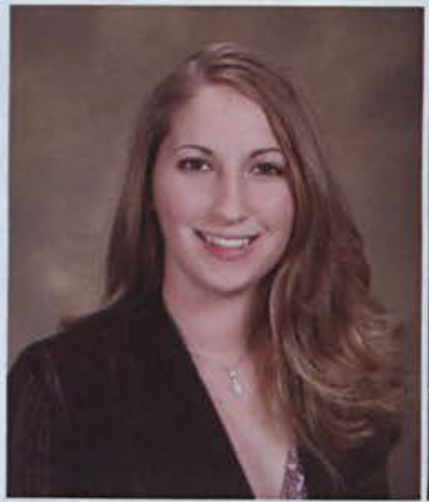

Heather L. Hanson Graphic Design

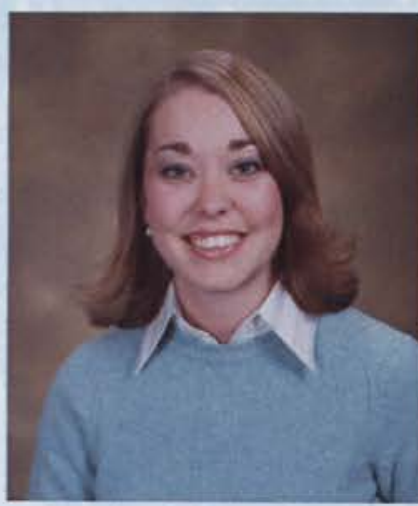

Kristen Elizabeth Heiner Psychology

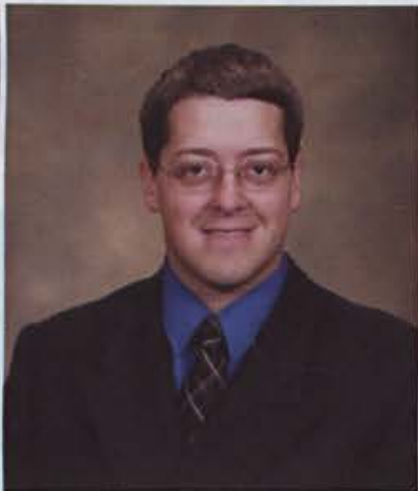

Joseph Daniel Hague Mechanical Engineering

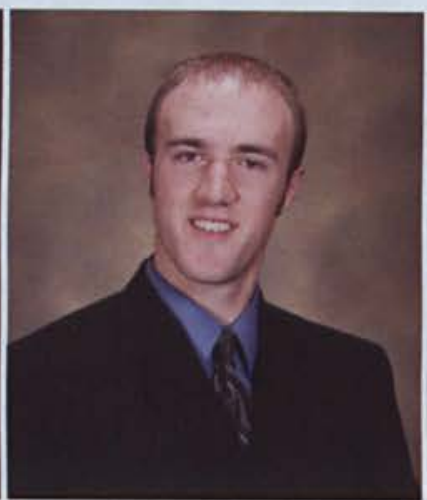

Bradley James Halladay Computer Engineering

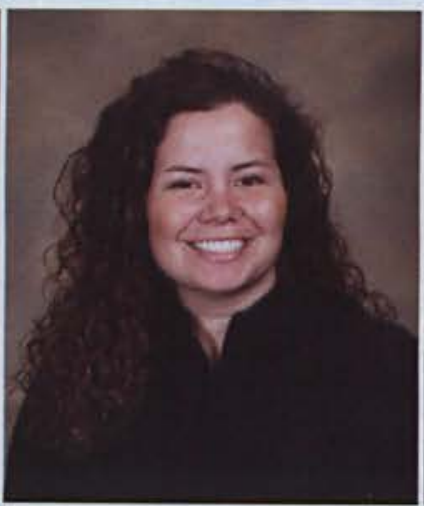

Karie Renea Harpest Middle Childhood Education

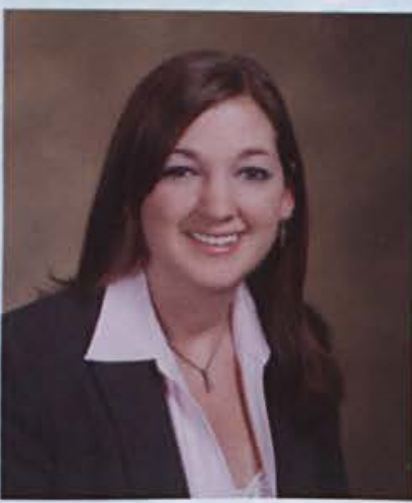

Lindsay Jeanette Hennig Christian Education

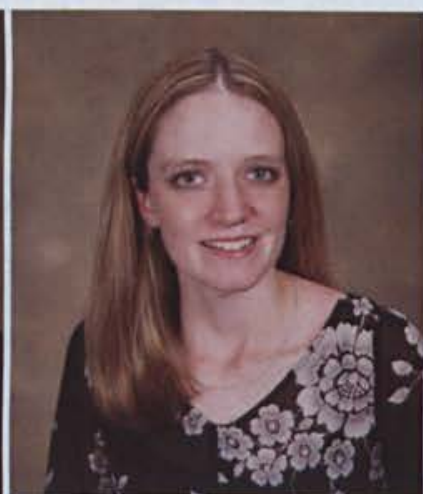

Jessica Nicole Hall Accounting

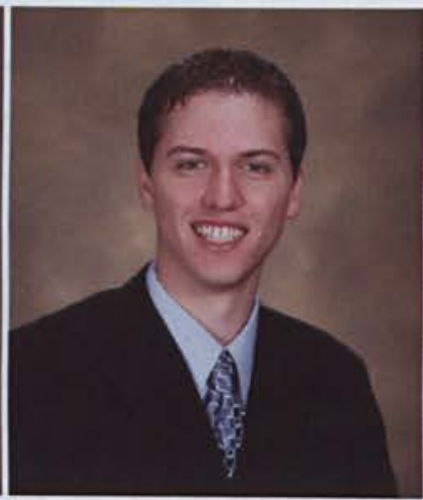

Thomas Jefferson Hancock Marketing \& Accounting

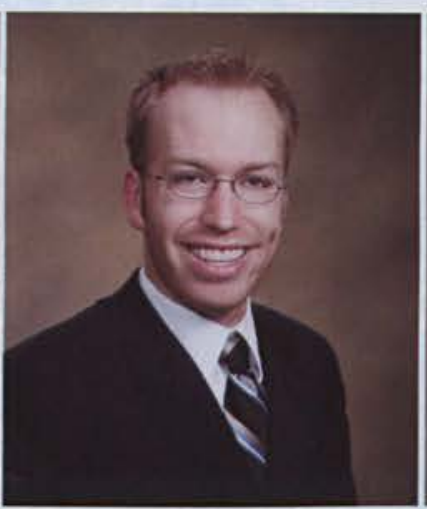

Andrew John Harris Mechanical Engineering

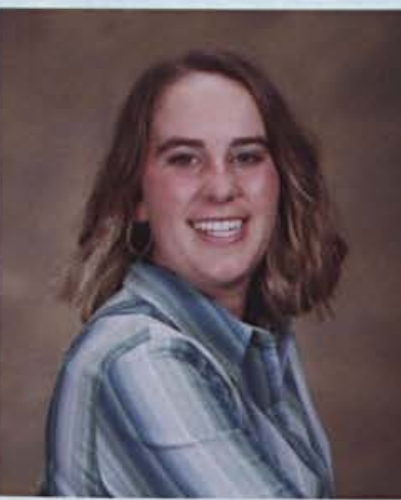

Rebekah Ann Henschel Early Childhood Education

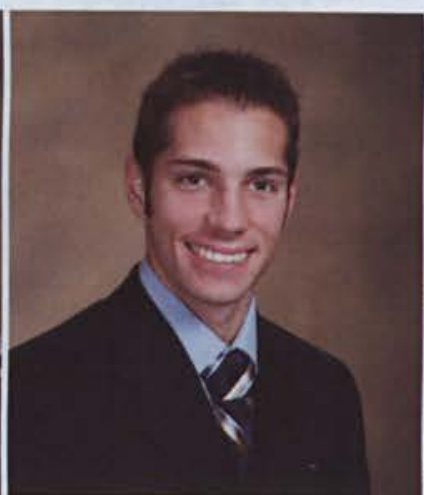

Kevin Scott Hall Comprehensive Bible

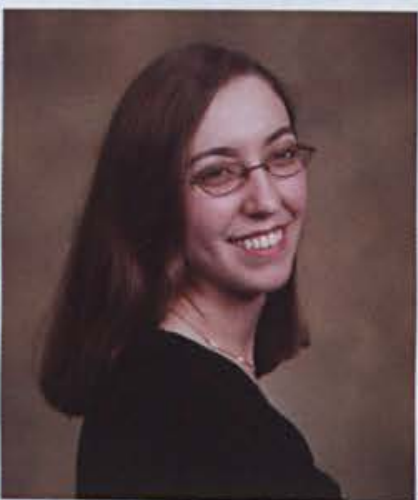

Michaela Diane Hand Music Composition

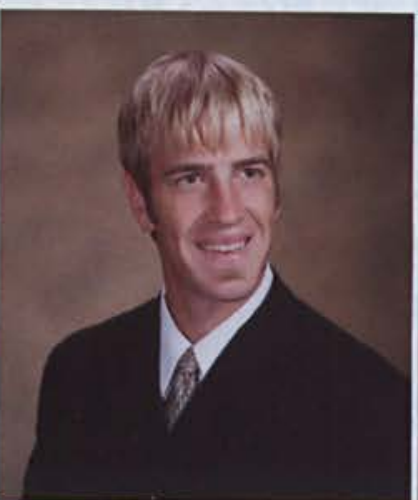

Timothy Benjamin Hartman Mechanical Engineering

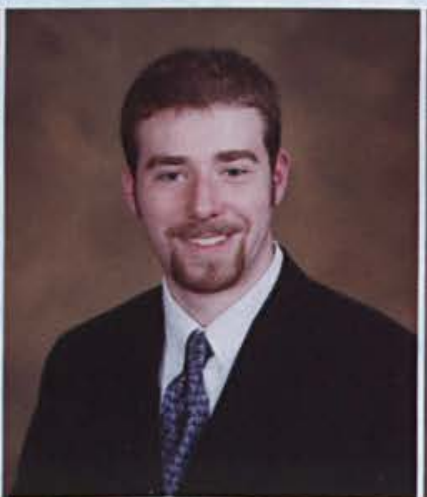

Justin Herby History

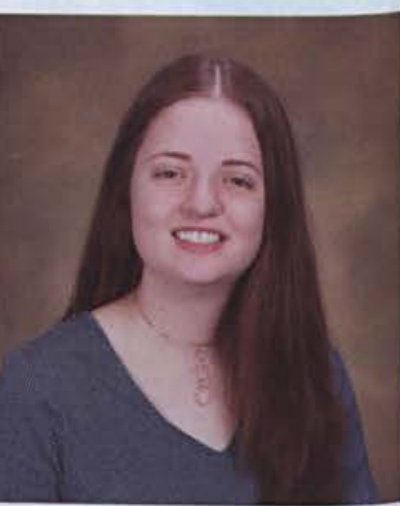

Stacia Elisabeth Hall Early Childhood Education

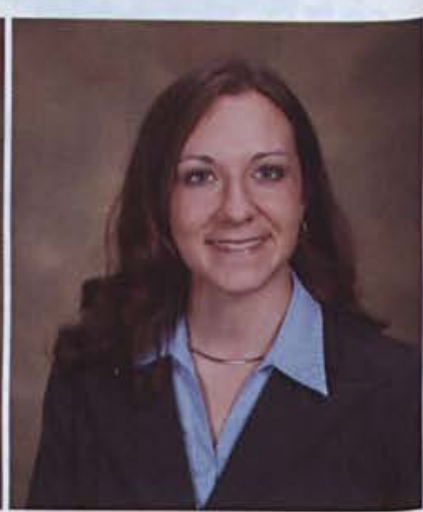

Lauren Elizabeth Hanna Exercise Science

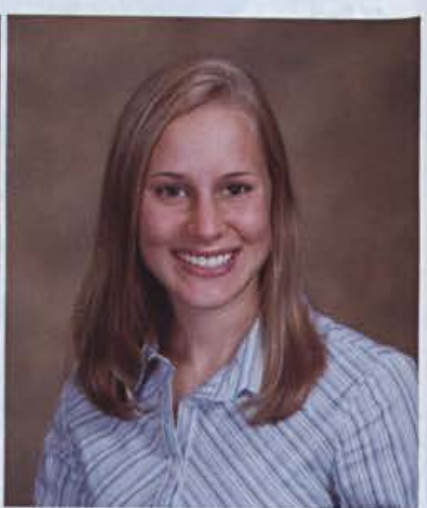

\section{Megan Elizabeth Heagy} Nursing

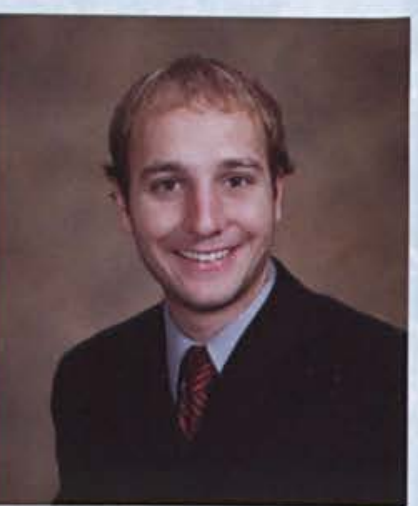

Kyle Gates Hiestand Middle Childhood Education 


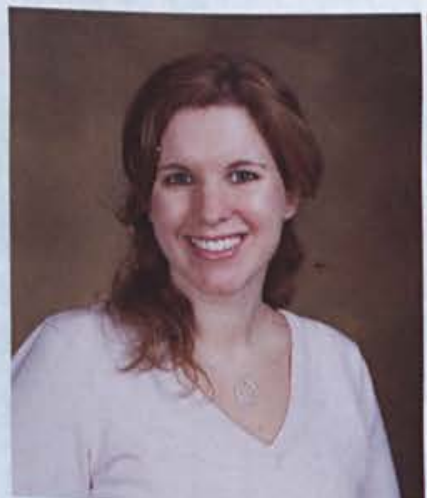

Erin Higley

Music Education

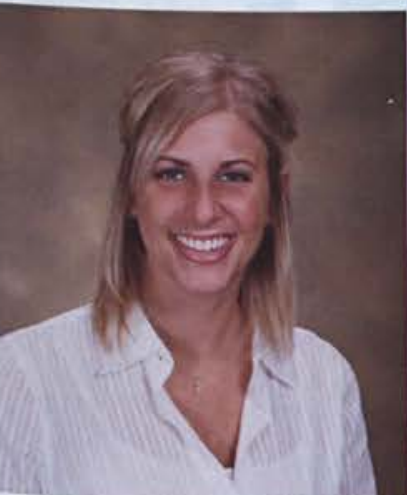

Jamie Catherine Hishmeh Media Communications

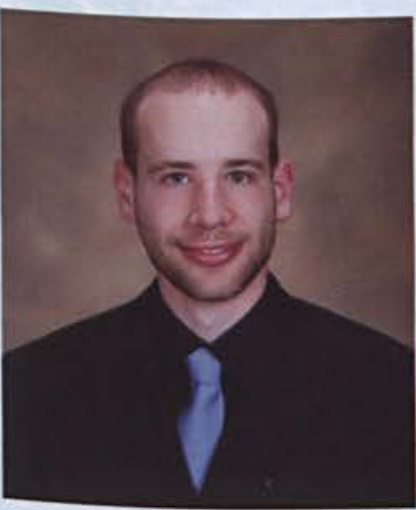

Darren Eugene Holland Mechanical Engineering

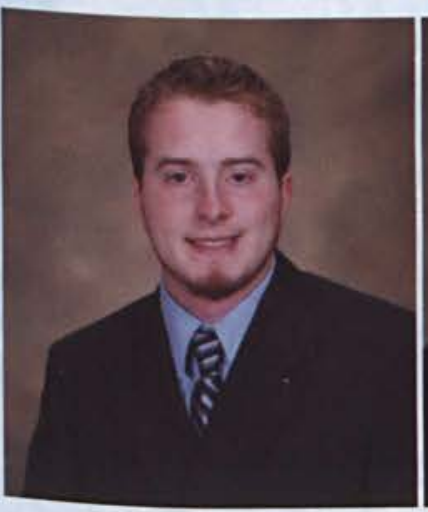

Timothy James Hornbrook Social Work

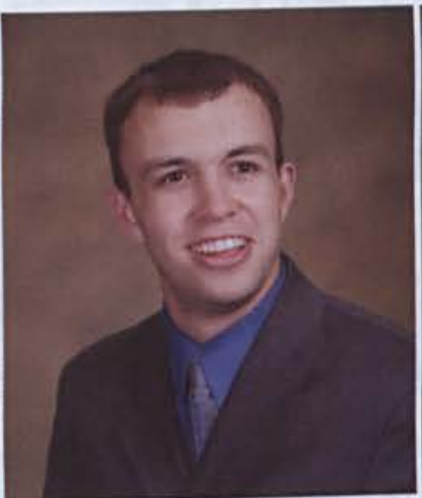

Samuel Lewis Hilbert Electrical Media

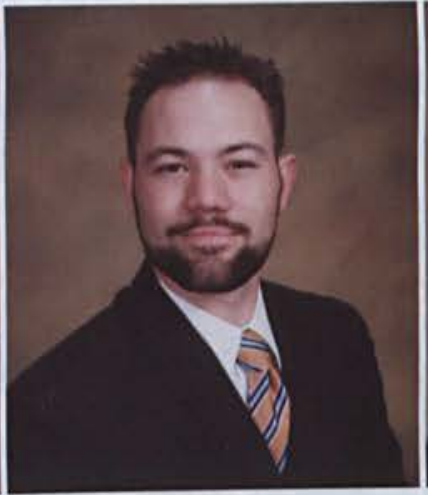

Jonathan Russell Hill Pre-Seminary Bible

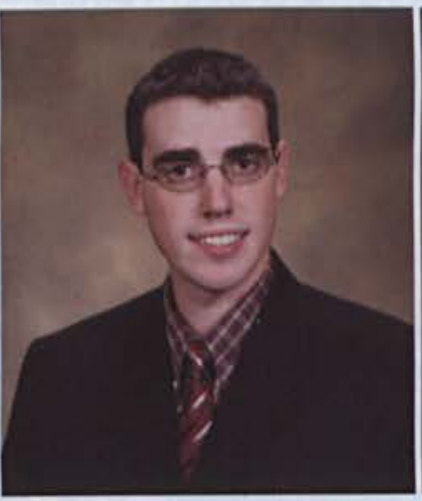

Robert Benjamin Hill Mechanical Engineering

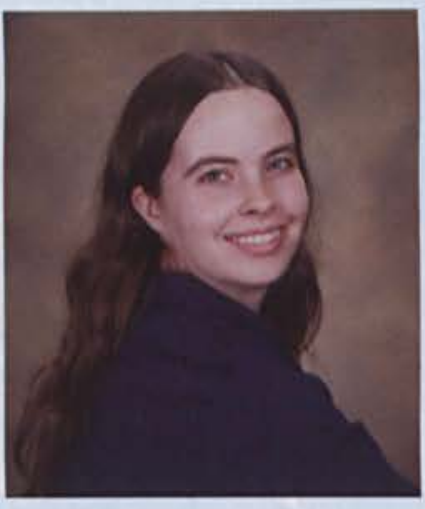

Joanna Christine Hinks Mechanical Engineering

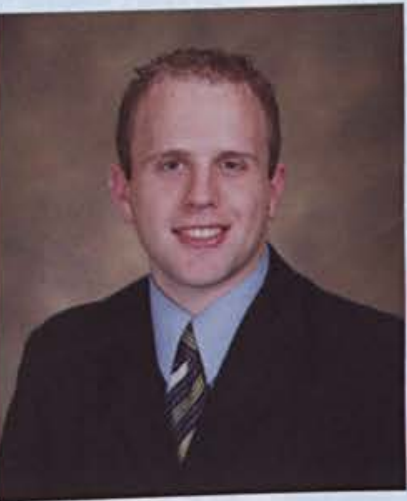

Matthew Dean Hobbs Integrated Social Studies Education

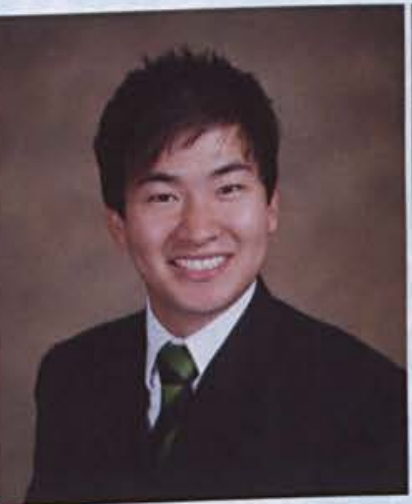

Alexander D. Hollander Biology

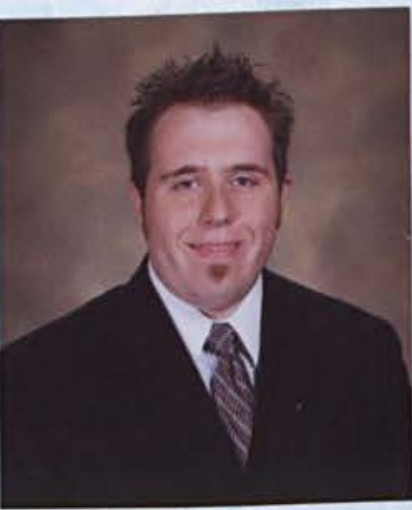

William Horosz Electronic Media

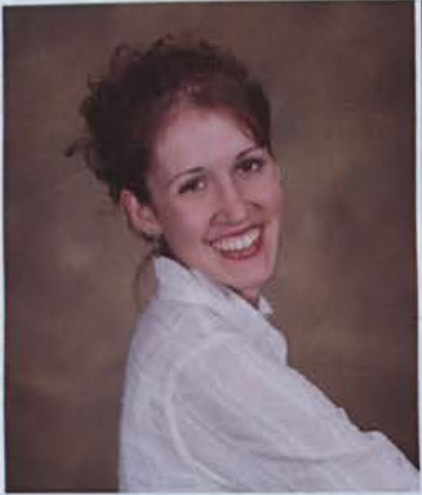

Bethany J. Hochstaetter Nursing

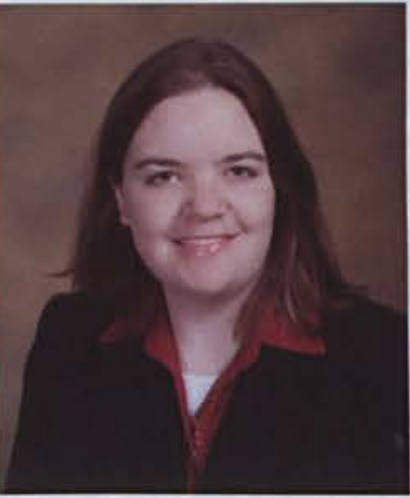

Ashley Marie Hoopes Early Childhood Education

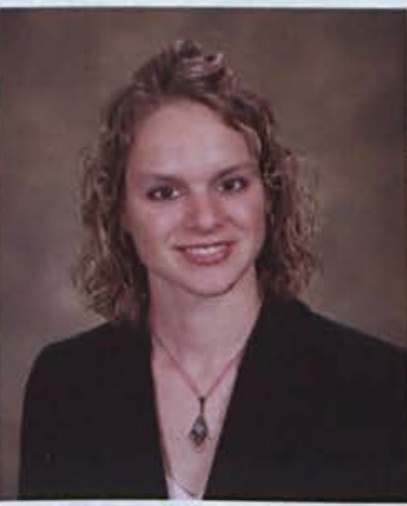

Jacqueline Lee Horst Special Education \& Early Childhood Education

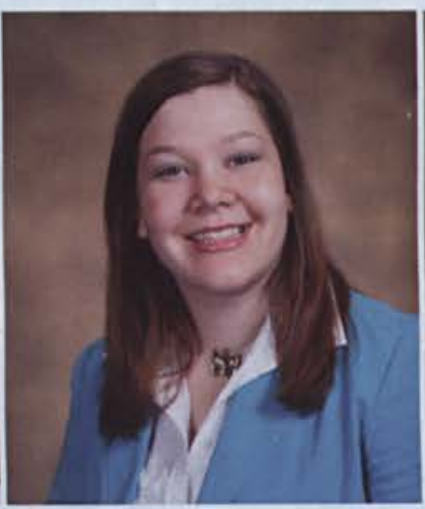

Amelia Hoganson Communication Arts

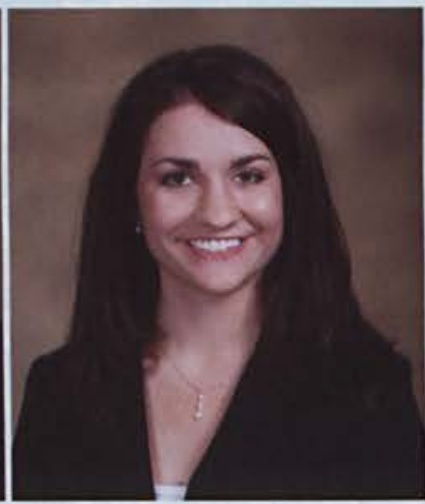

Allison Kay Hope Nursing

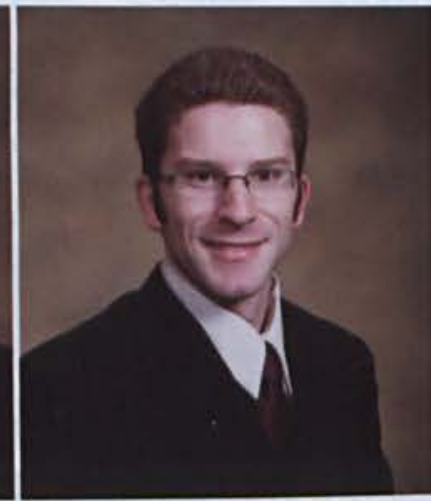

Mark Jacob Howard English

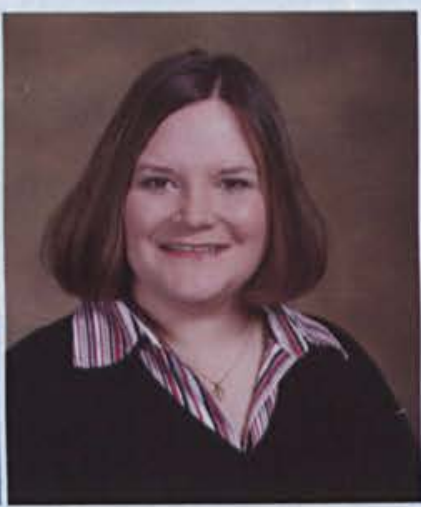

Sarah Jean Holden Early Childhood Education

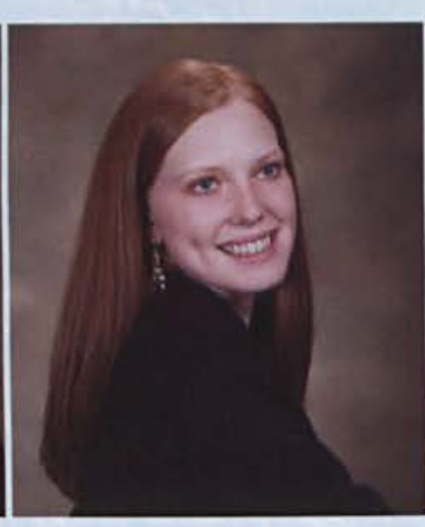

Rebecca Jean Hornbrook Biology

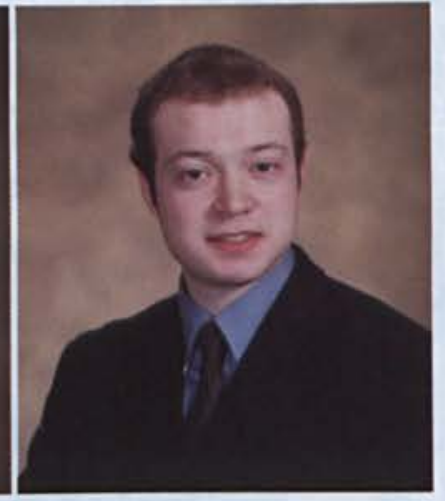

John Stephen Hudson Electronic Media

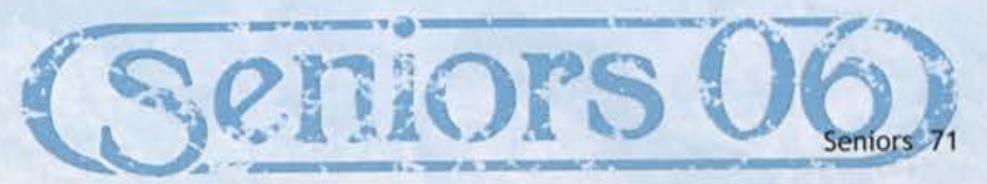




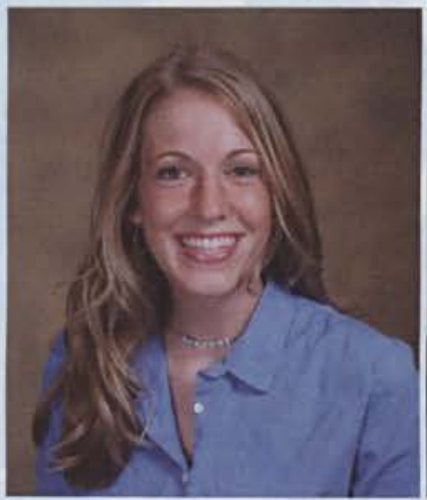

Stephanie Grace Hueni Early Childhood Education

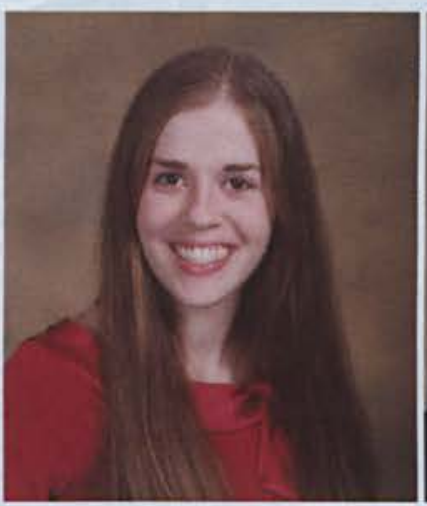

Brigette Ann Hunter Music

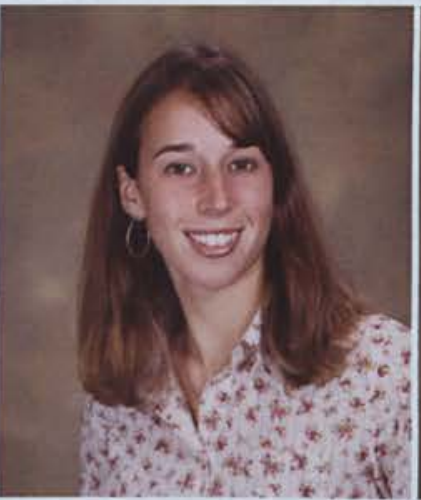

Nicole Elizabeth James Integrated Social Studies Education

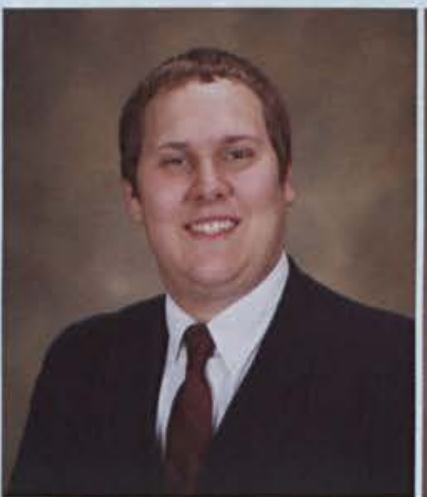

Benjamin Robert Johnson Youth Ministry \& Christian Education

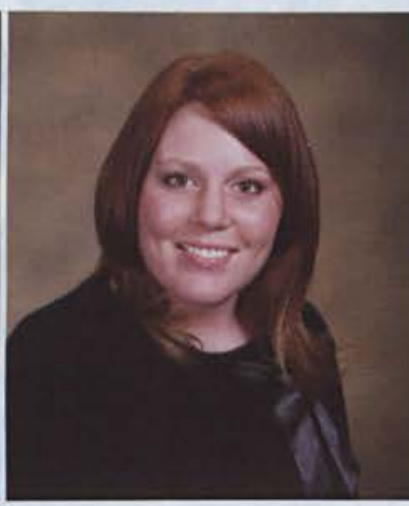

Laura Mae Humberson Psychology

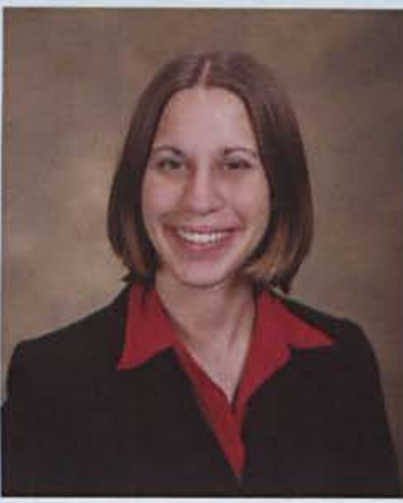

Jennifer Marie Huster Middle Childhood Education

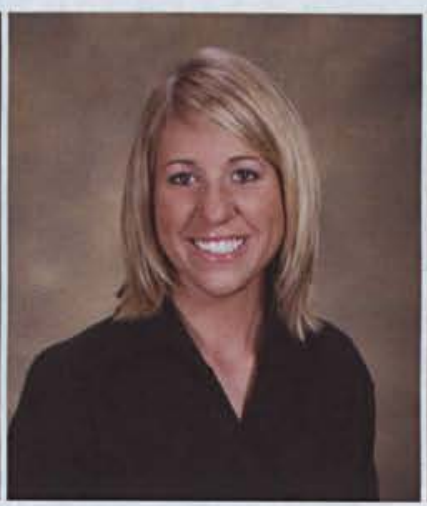

Aftan Leanne Hunt Marketing \& Management

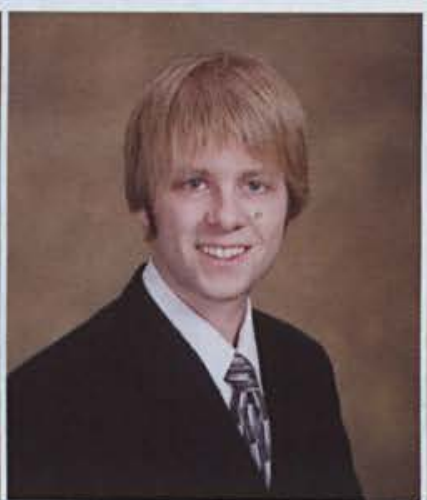

Todd Phillip Huster Electrical Engineering

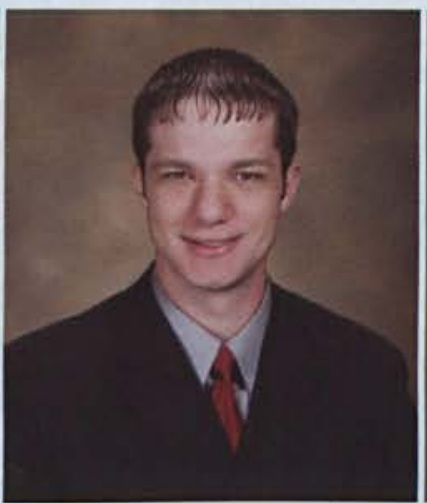

Phil Stephen Jarvis Mechanical Engineering

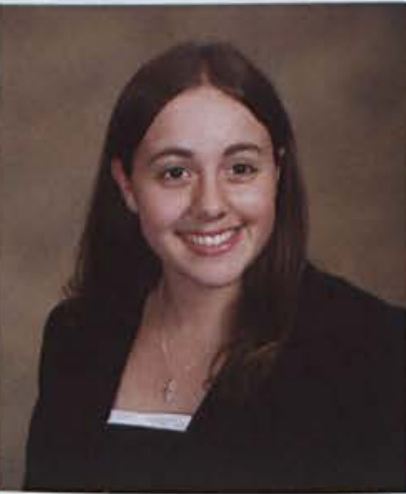

Jennifer Marie Johnson Integrated Mathematics Education

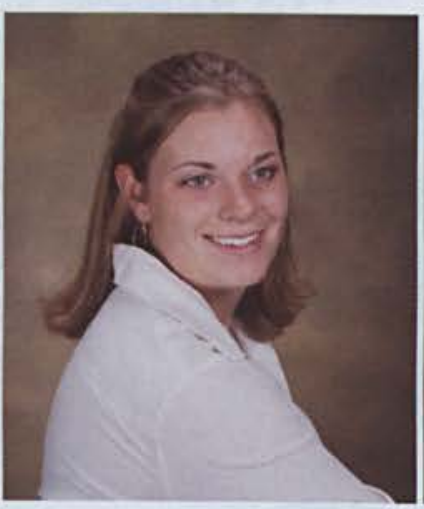

Hillary Jo Hunt Chemistry

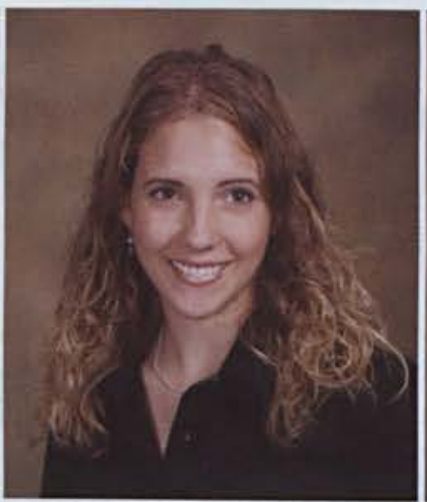

Jocelyn F. Izor Graphic Design

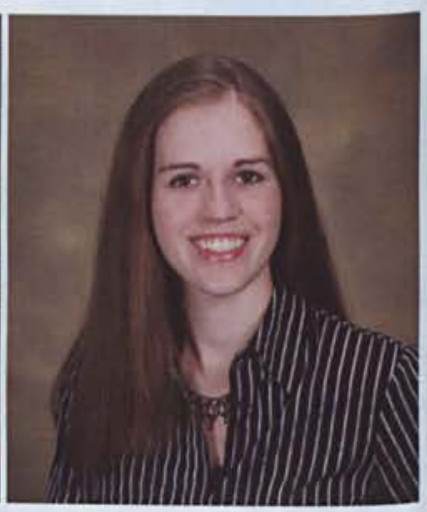

Amanda Marie Hunter Music

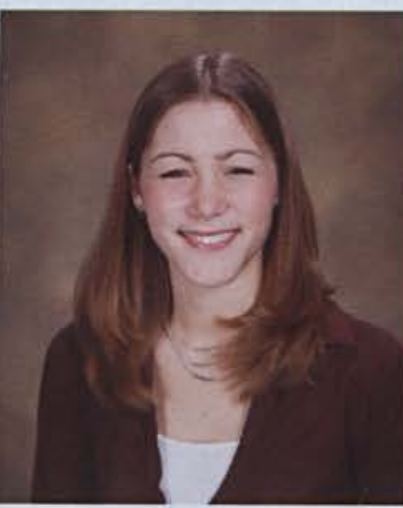

Jessica Jantzen Psychology

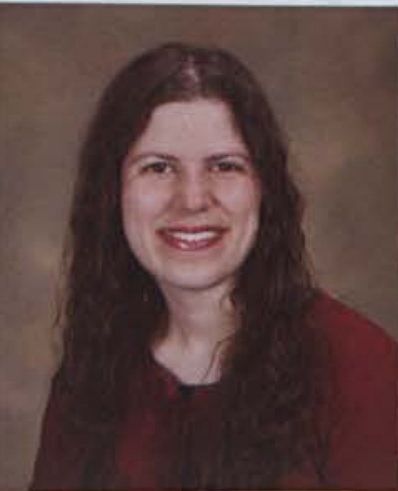

Heather Renee Johnson Music Education

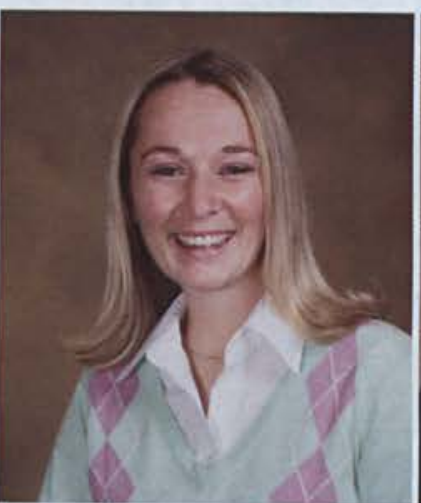

Candace Jelinek

Communications

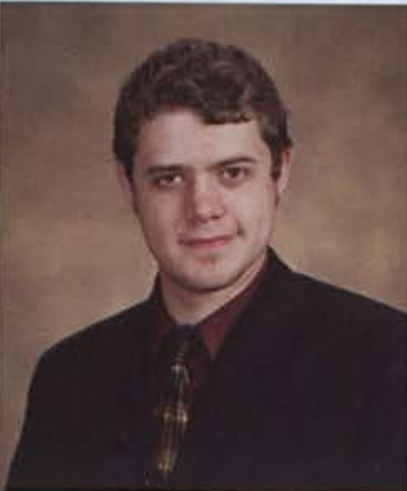

Joseph Dwight Johnson American Studies

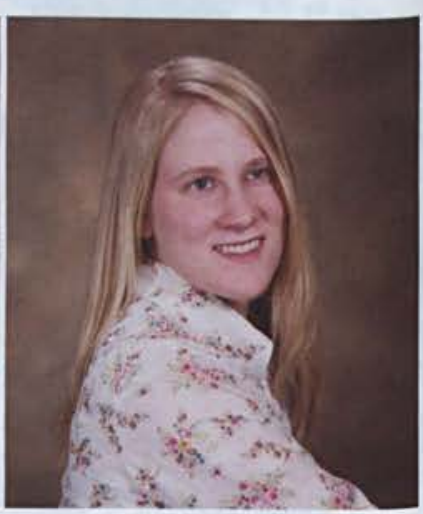

Heather L. Jacobs

Computer Science

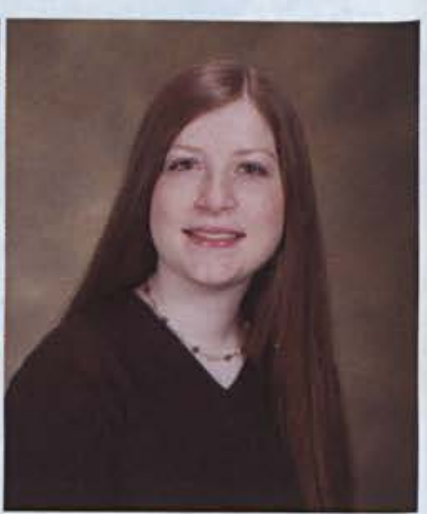

Sarah Michelle Jett Keyboard Pedagogy

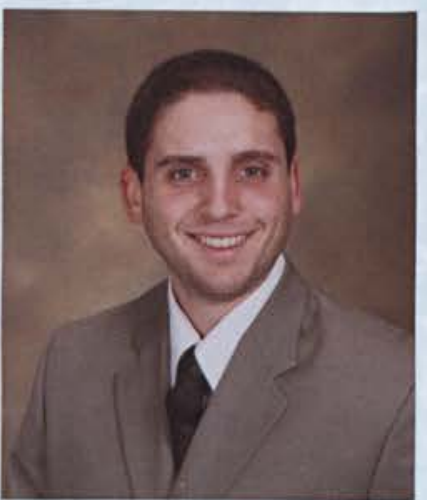

Nathan Scott Johnson AYA Integrated Social Studies 


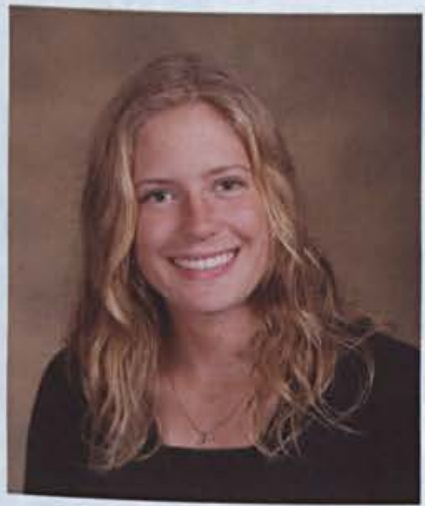

Rachel Elizabeth Johnson Athletic Training

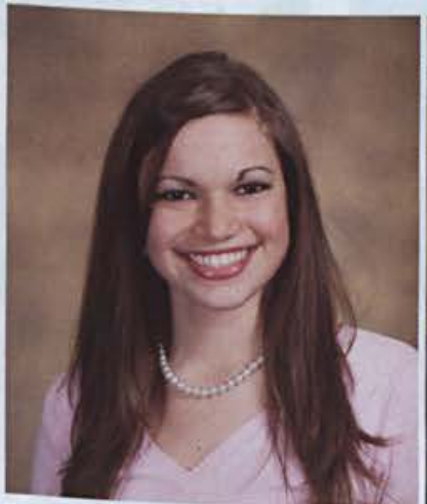

Abigail Sue Kauffman Music

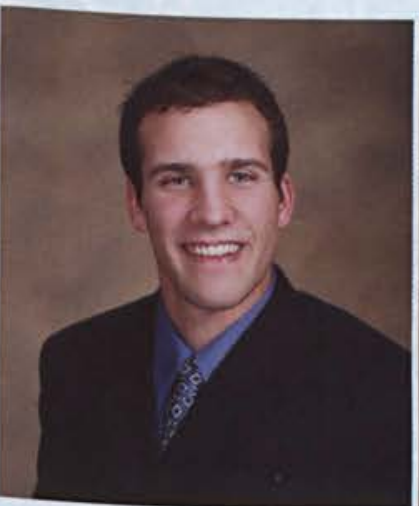

Clair Lamar King Accounting \& Finance

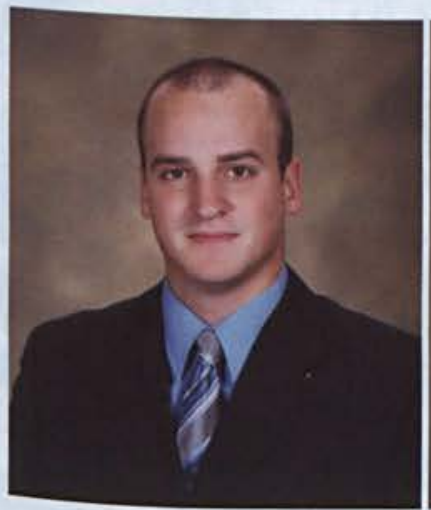

Corey J. Knoedler Management

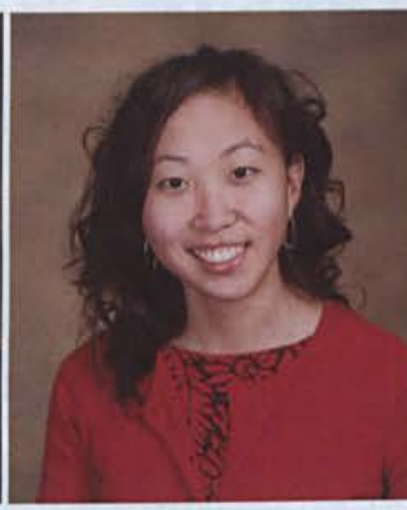

Sara Johnson Athletic Training

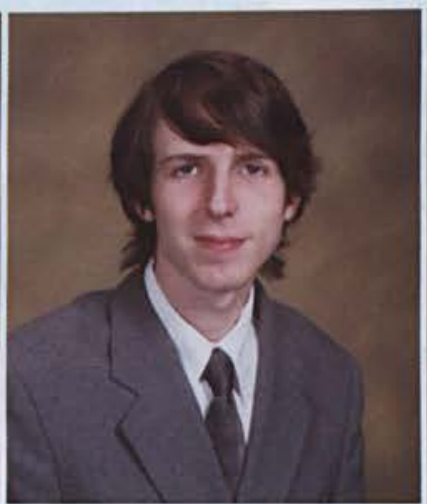

\section{Benjamin Paul Kaufman} Graphic Design

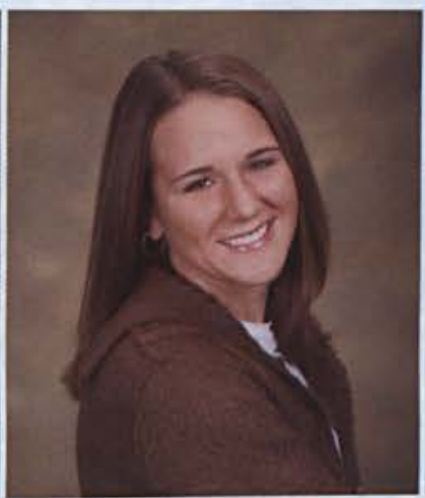

Colleen Beverly Kirby Criminal Justice

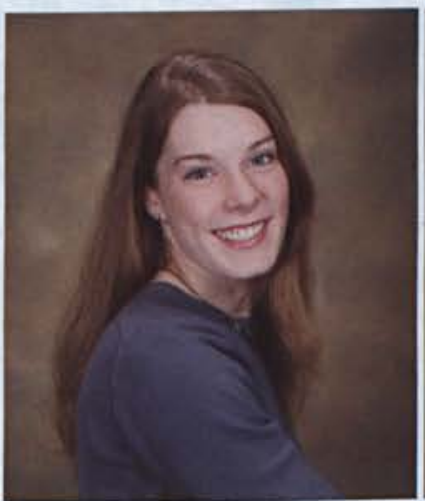

Jennifer Ann Knudsen Theatre

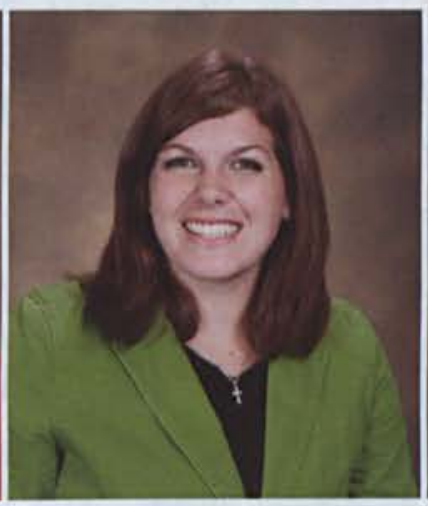

Kelsey Elizabeth Jones Multi-Age Physical Education
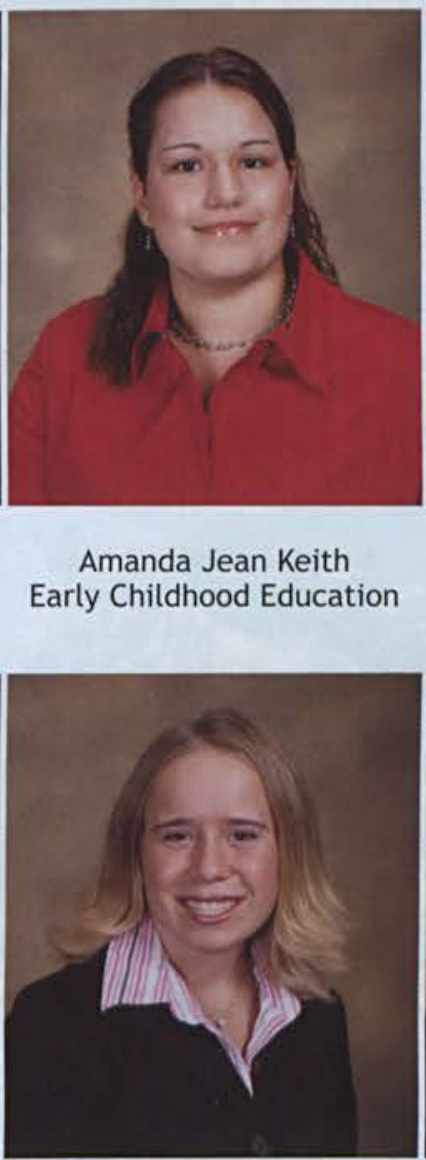

Bethany Ann Kirkendall Exercise Science

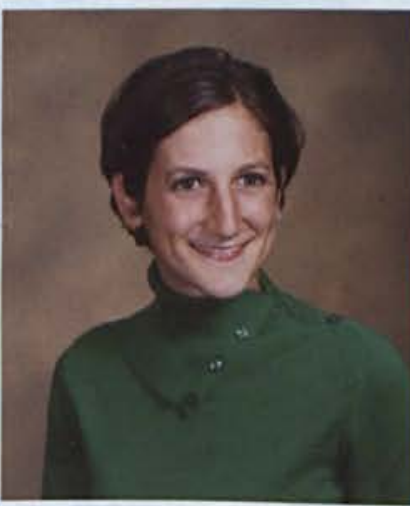

Megan Lynn Kolanko Graphic Design

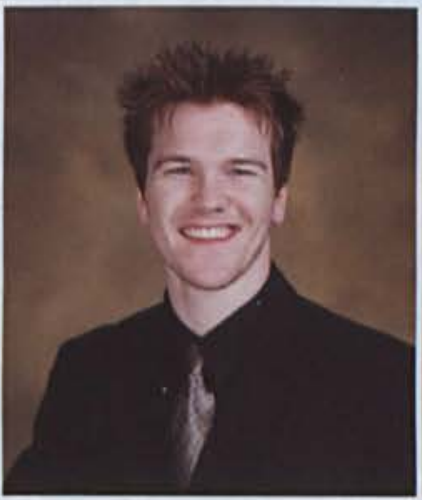

Darin Kaiser Marketing \& Management

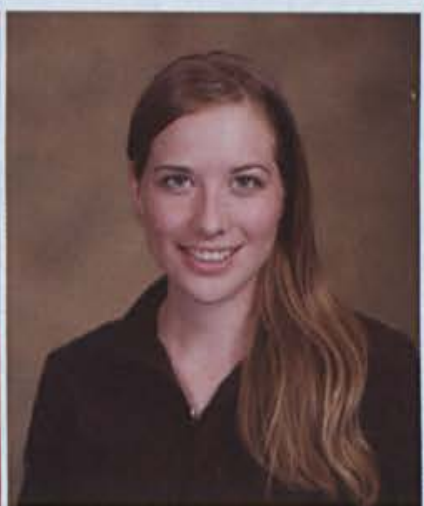

Naomi Kenner Biology

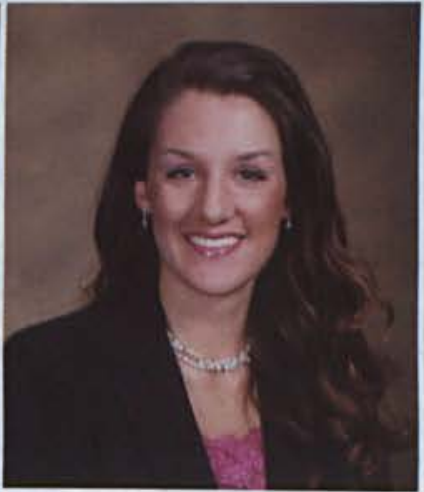

Anna Elizabeth Klein Biology

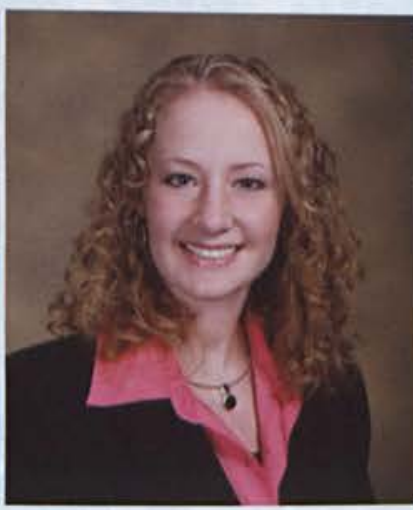

Suzanne Michelle Kooy Communications

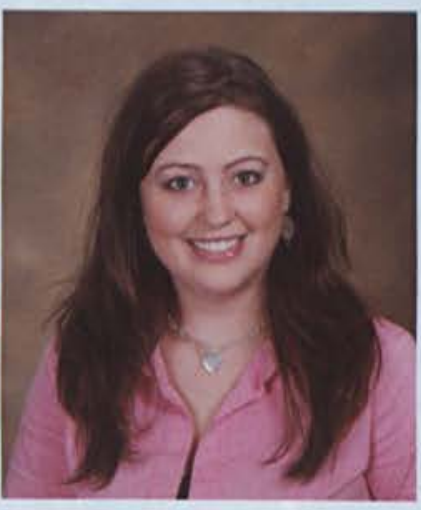

Janelle Marie Kamauf Finance \& Marketing

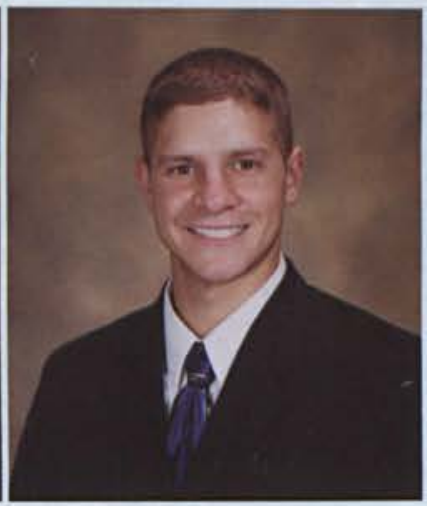

Daniel Edward Keslar Comprehensive Communications

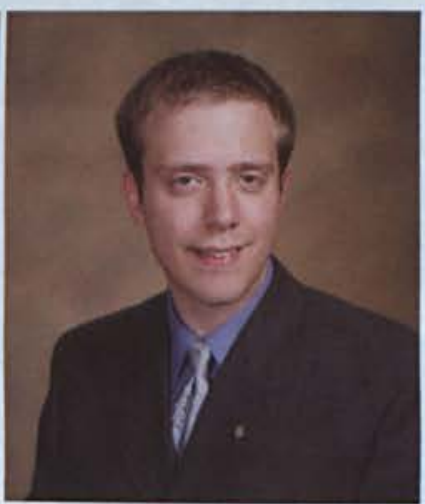

David Paul Knierim Computer Engineering

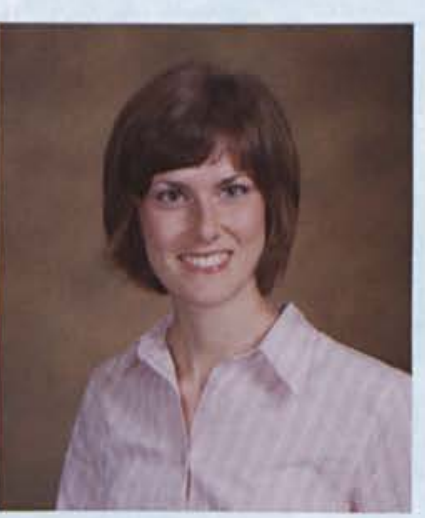

Elizabeth Grace Korver International Studies \& Missiology 


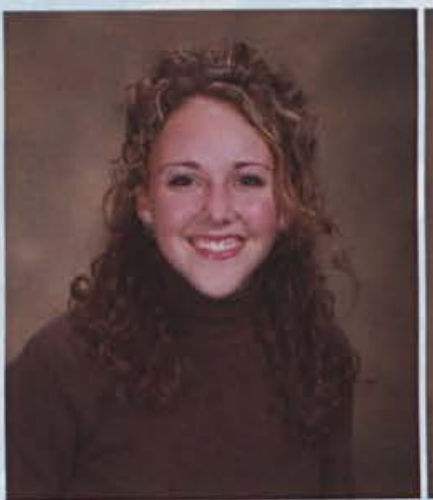

Hannah Lynn Kowatch Nursing

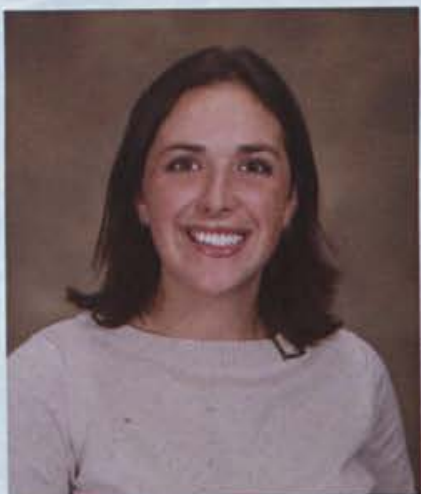

Katie J. Krumeich Nursing

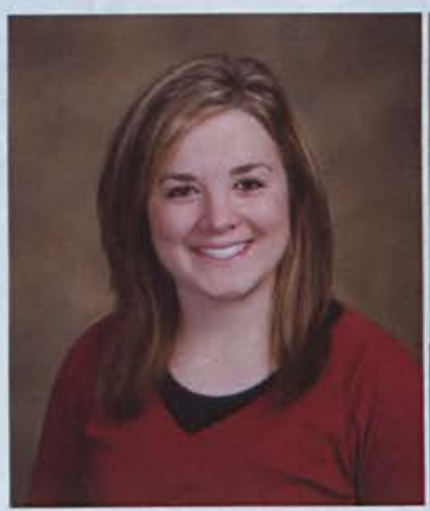

Sara Joy Larsen Nursing

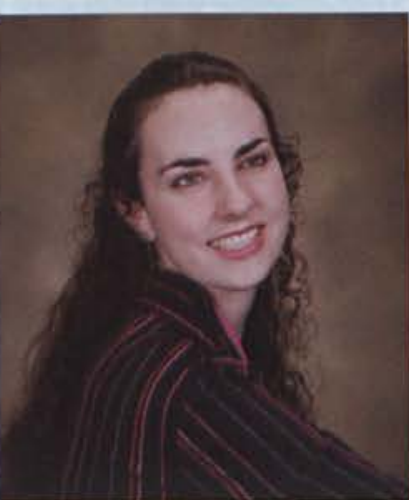

Rebecca Sue Lescure Early Childhood Education

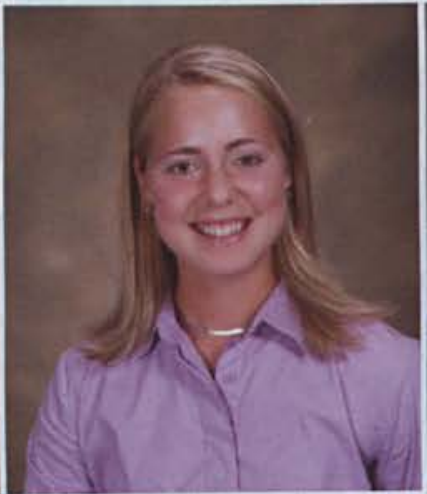

Jodi Kay Kraker

Exercise Science

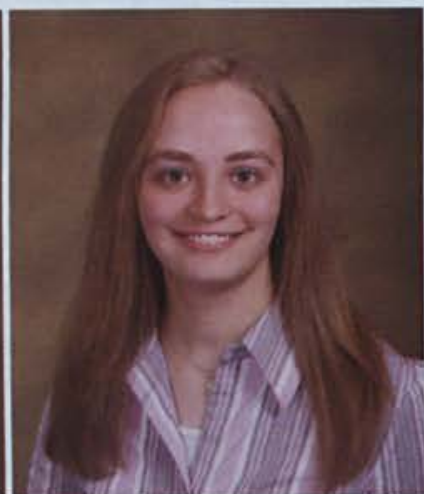

Diana Frances Kurin Keyboard Pedagogy

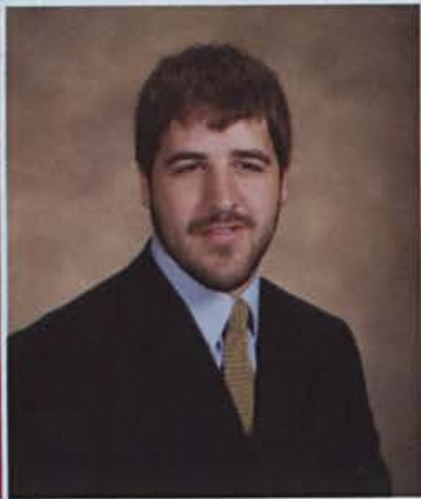

Benjamin Steven Lawrence Mechanical Engineering

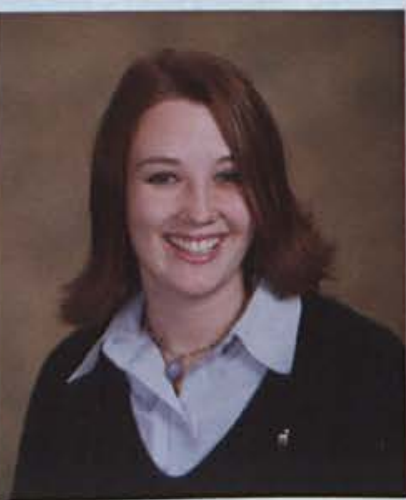

Julianne Nicole Lesh Communications

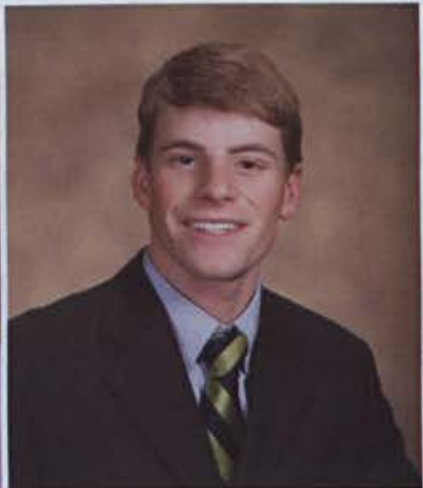

Brent Allen Kruithof Accounting

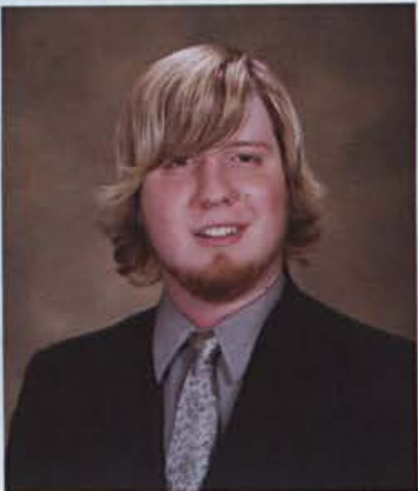

Benjamin Thomas Lake Management \& Marketing

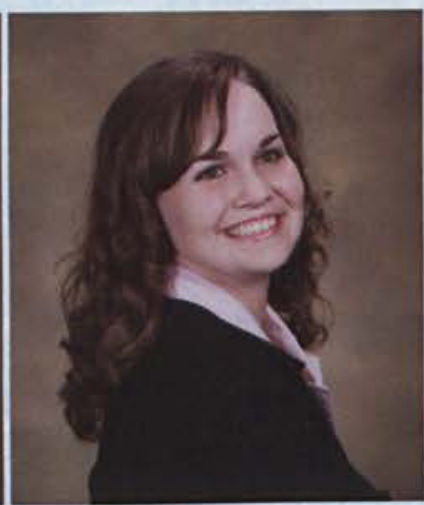

Sarah A. Leary Integrated Mathematics Education

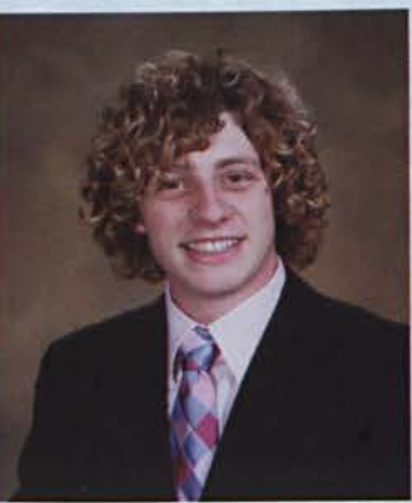

Jonathan Ellsworth Lett Finance \& Marketing

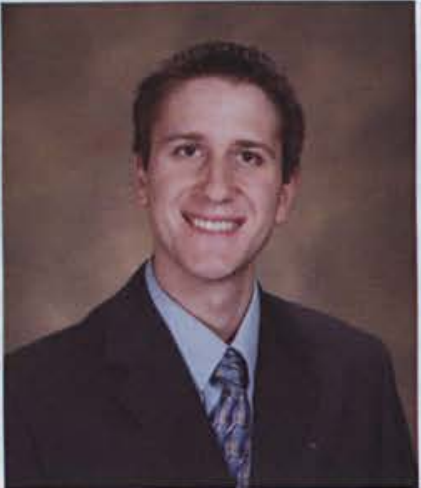

Jonathan Andrew Krull Music Education

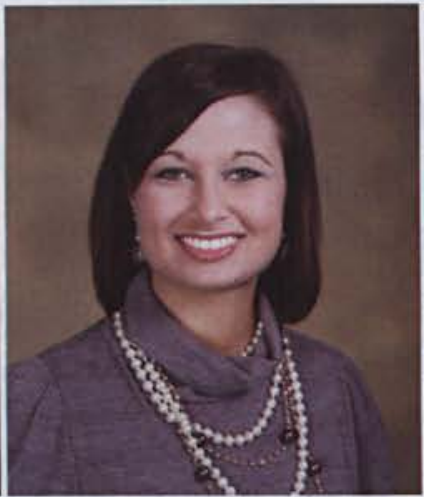

Caroline K. Landenberger

Organizational

Communications

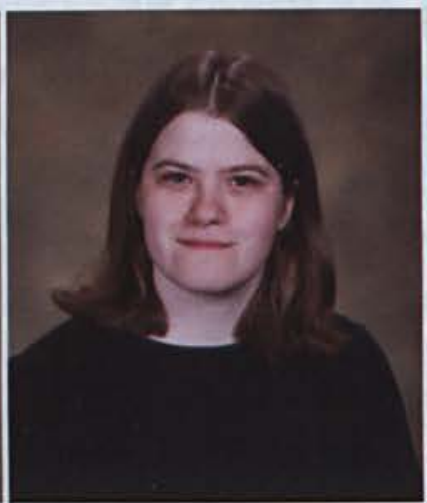

Jennifer Marie Leasure Marketing

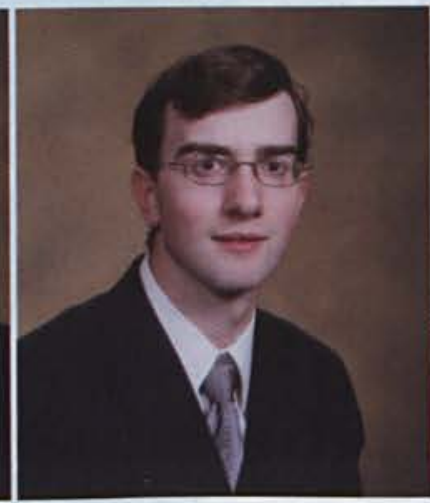

Matthew Morris Lewellyn Computer Science

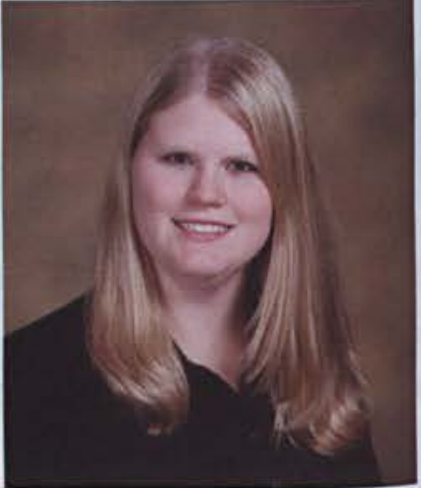

Kristin Ann Krull Music Education \& Instrumental

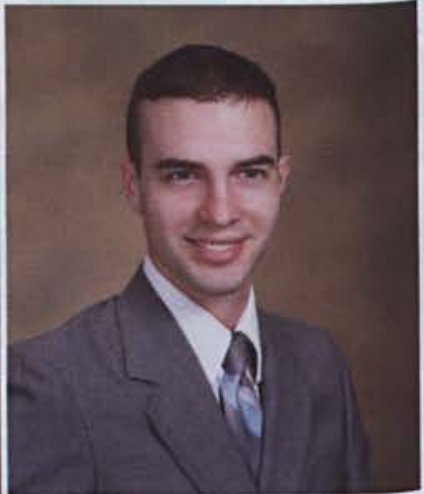

Joel William Lansford Mechanical Engineering

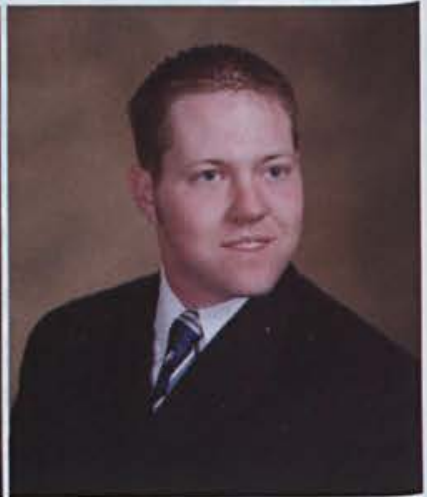

Adam Marc Leffel Mechanical Engineering

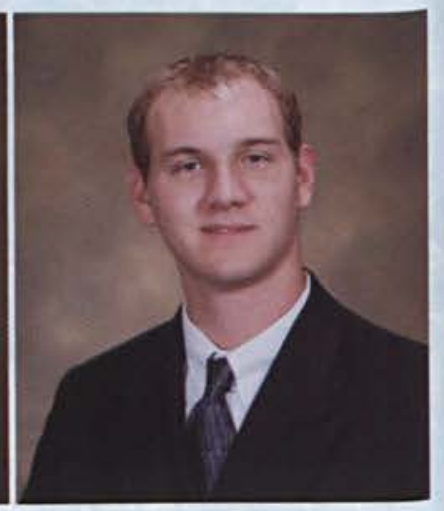

Joseph Nathaniel Leykam Nursing 


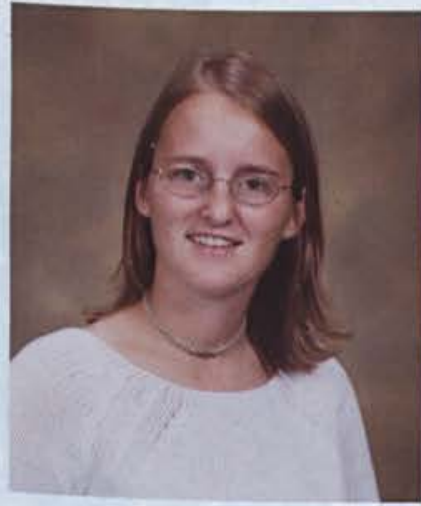

Clarissa Carr Linde Social Work

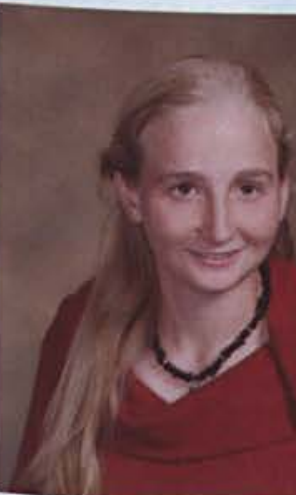

Holly Nichole Livingston International Studies \& Social Science

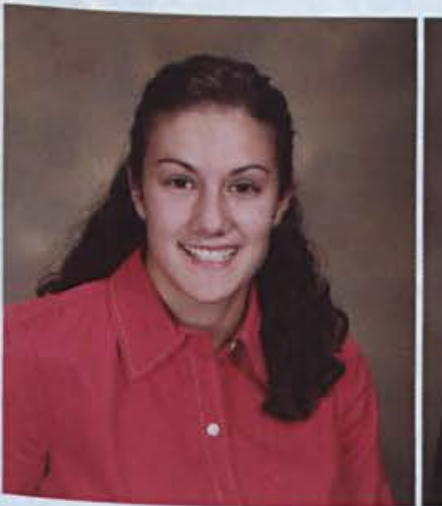

Lauren Elizabeth Mable Early Childhood Education

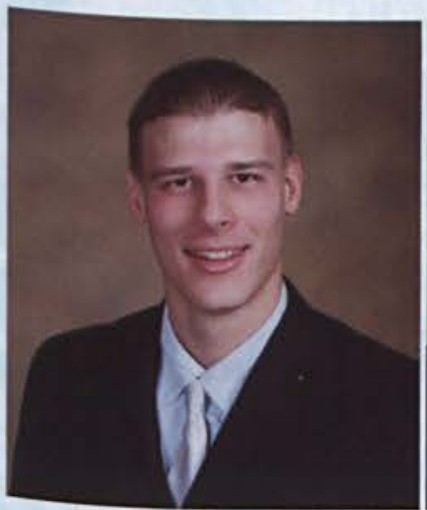

Anthony Michael Mandela Finance \& Management

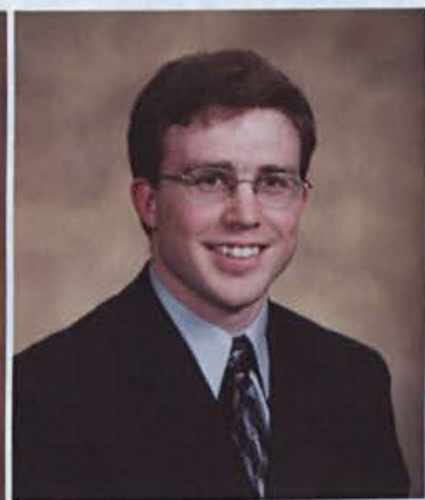

Timothy Ray Linden Computer Engineering

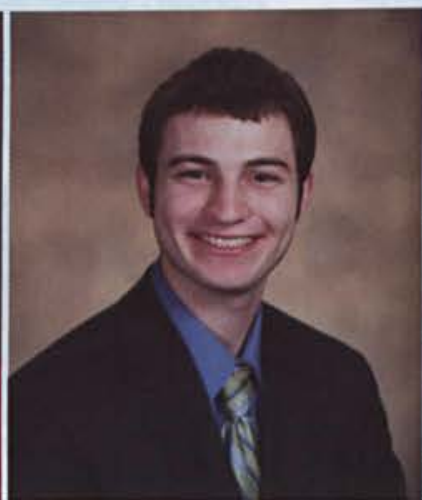

Peter D. Locke Mechanical Engineering

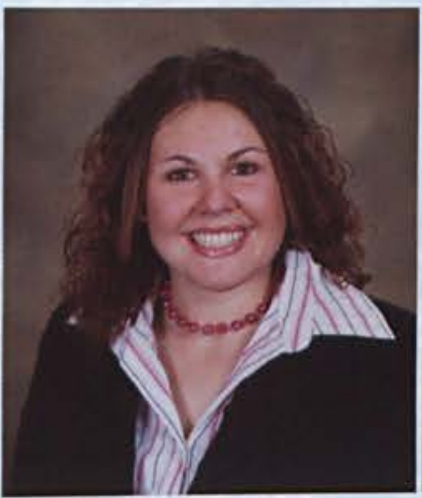

Amber Marie Mack Athletic Training

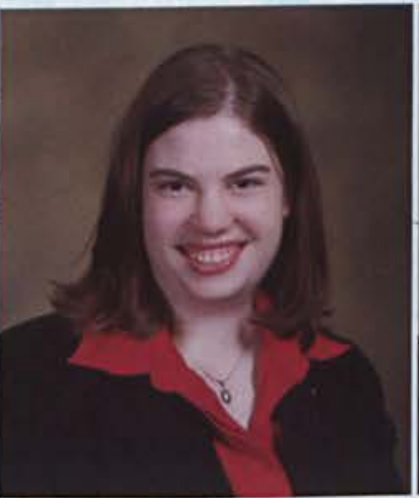

Ellen Johanna Mapes Applied Psychology

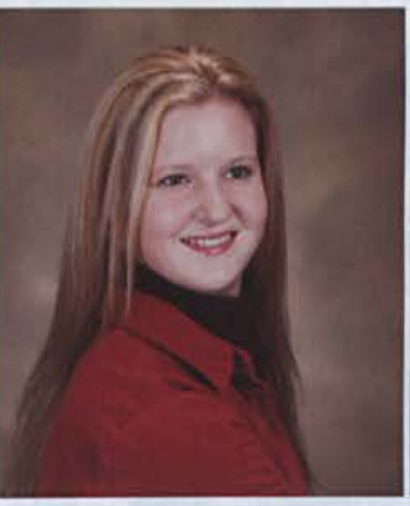

Christine Littell Electronic Media

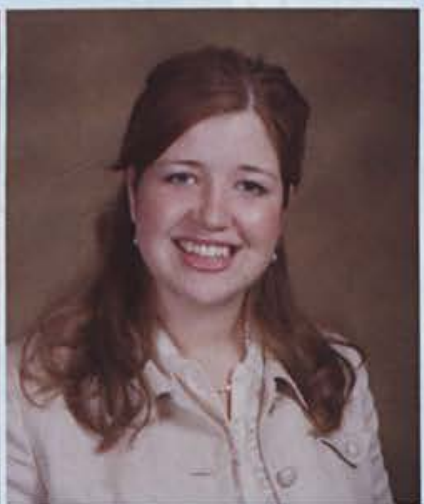

Holly Rhea Luiken Organizational Communications

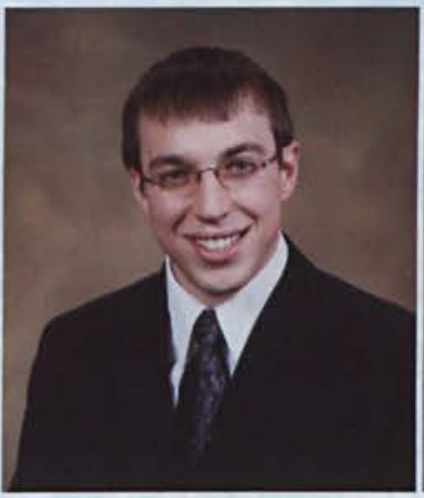

James Andrew Mackiewicz Integrated Mathematics Education

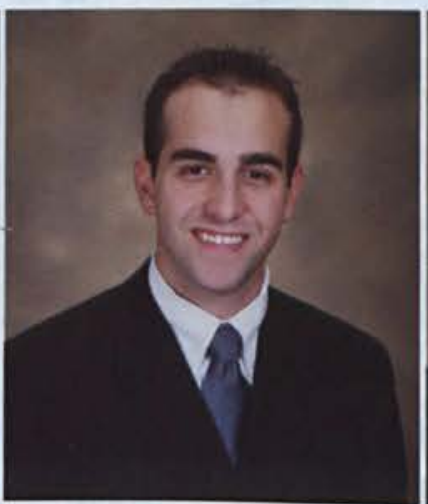

Robert David Marcello Pre-Seminary Bible

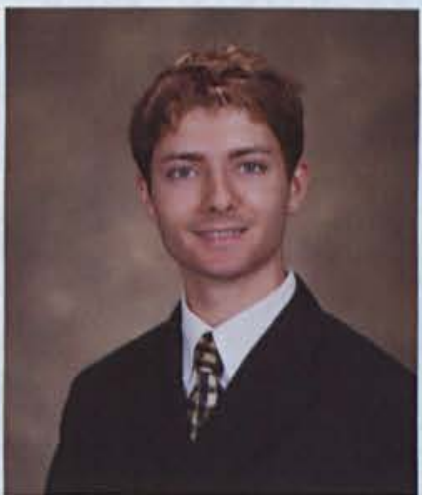

Stephen Brent Livesay Biology

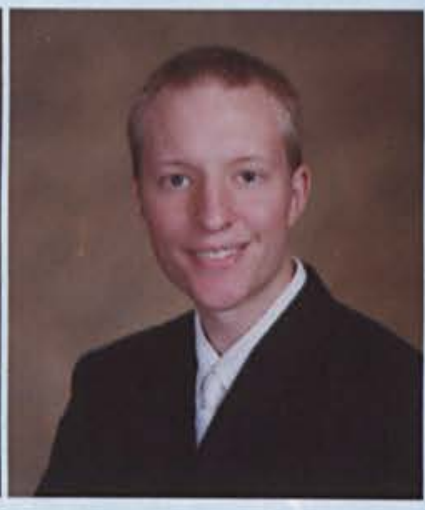

David William Livingston Marketing \& Management

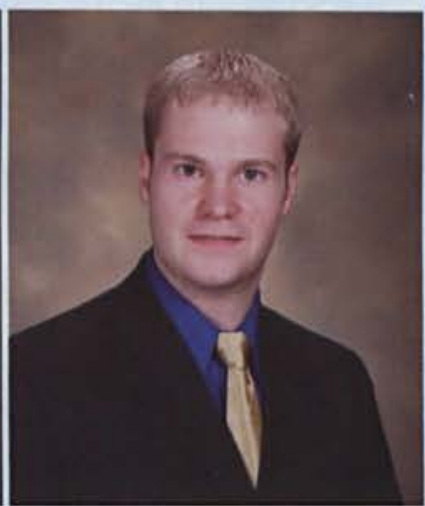

Joshua John Lumpkin Biology

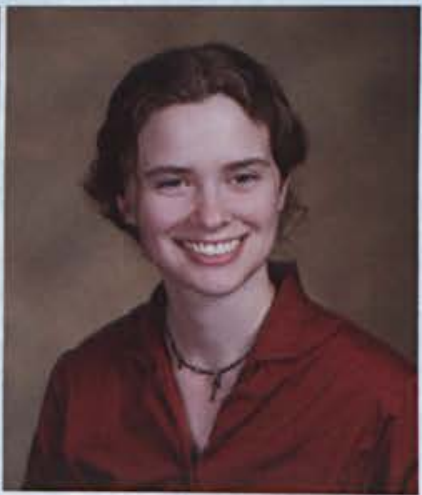

Lisa Marie MacKillop Psychology

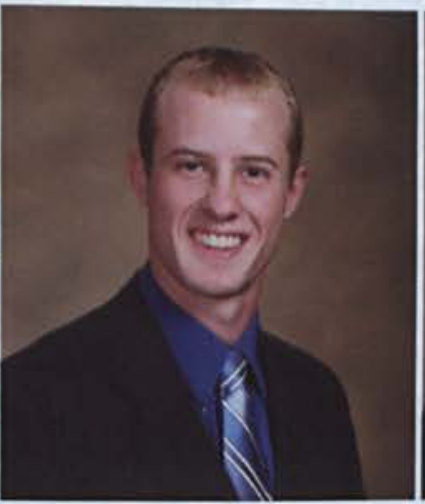

Scott John Marquardt Middle Childhood Education

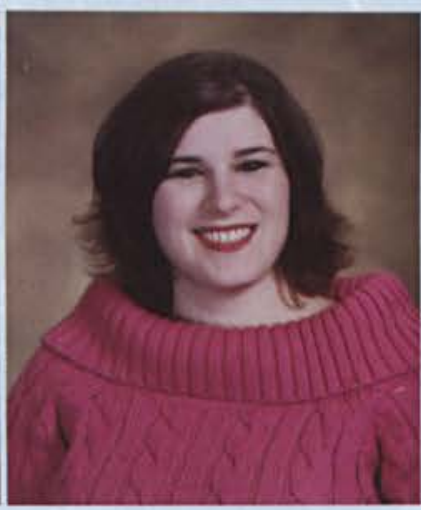

Jennifer Anne Lusk Psychology

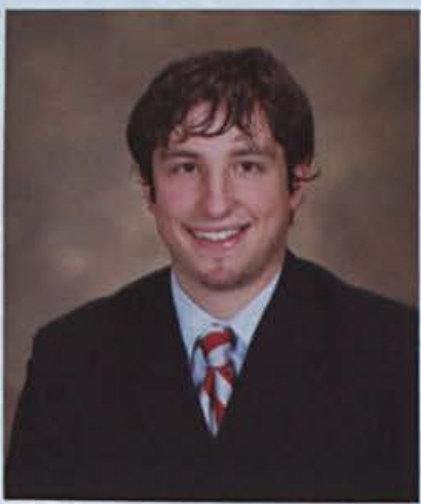

Matthew Malone Communication Arts

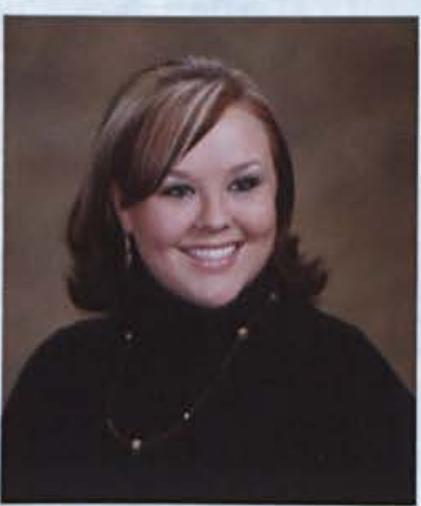

Kate Martin

Youth Ministries \& Christian Education

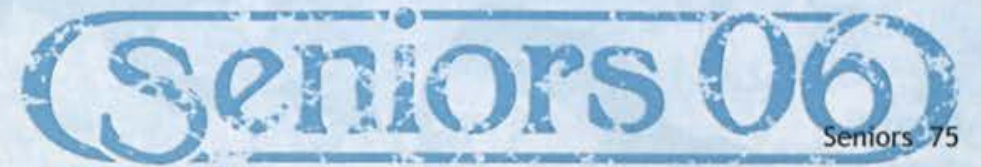




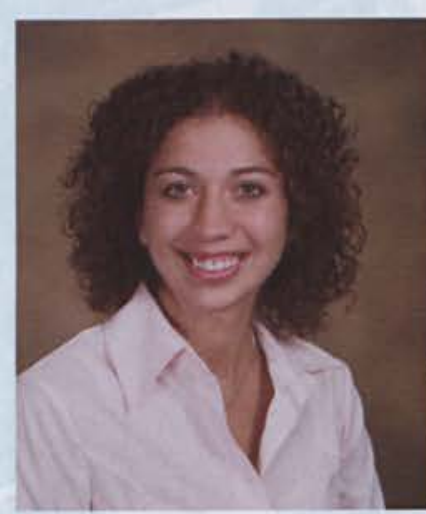

Amy Lauren Masowich

Special \& Elementary Education

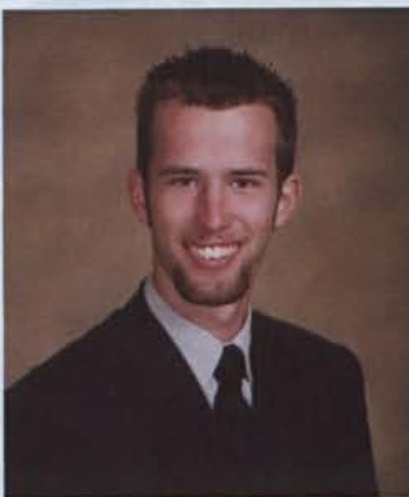

Jason Alan Mattern

Physical Activity \& Sport Studies

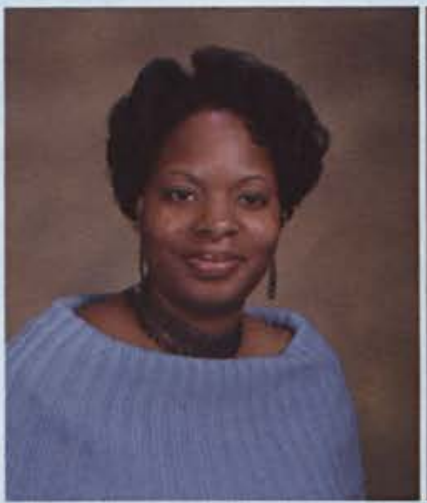

Monique Lynn McCoy Middle Childhood Education

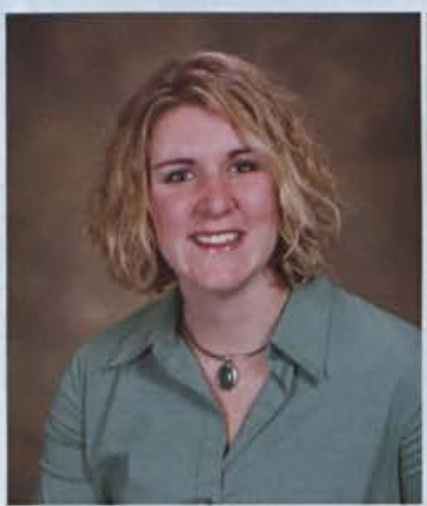

Jaclyn Marie McKeon Psychology

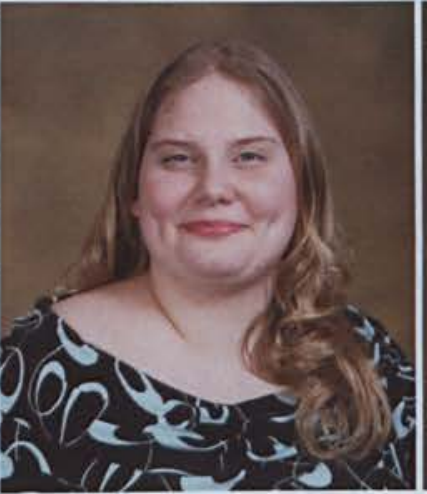

Georgia Marie Massar Marketing

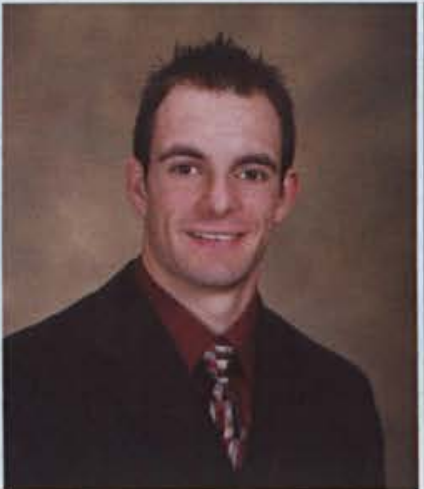

John Darren Mattheis

Management

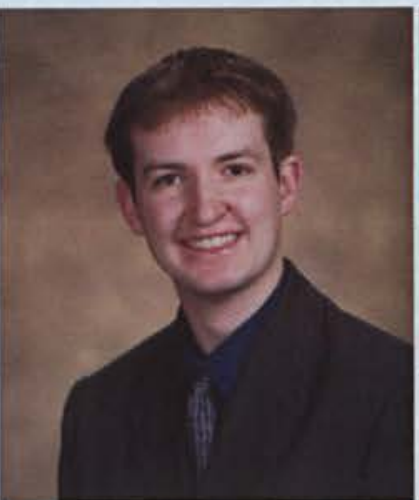

Philip Grant McCune Music

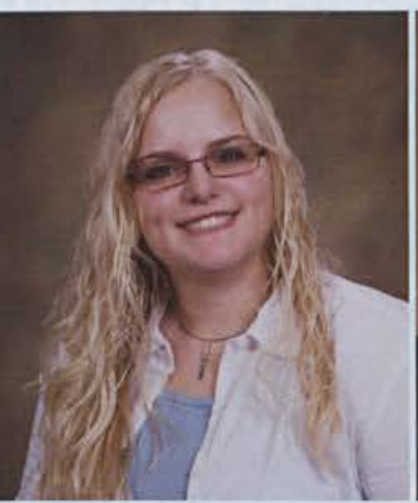

Melody Leanne Massar Mathematics

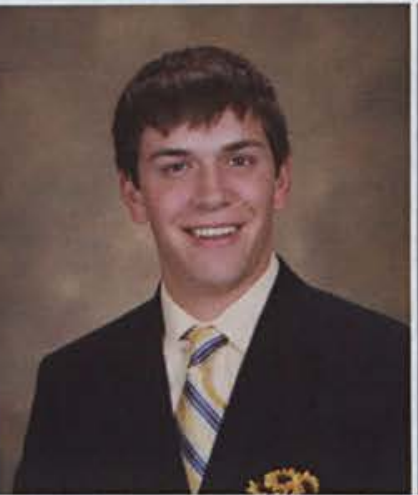

Travis John Mathiesen Accounting

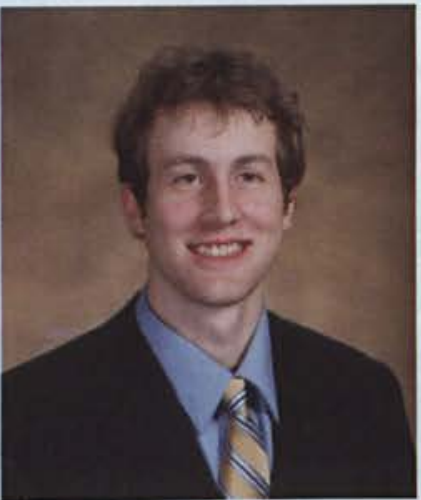

Aaron James MCDivitt Middle Childhood Education

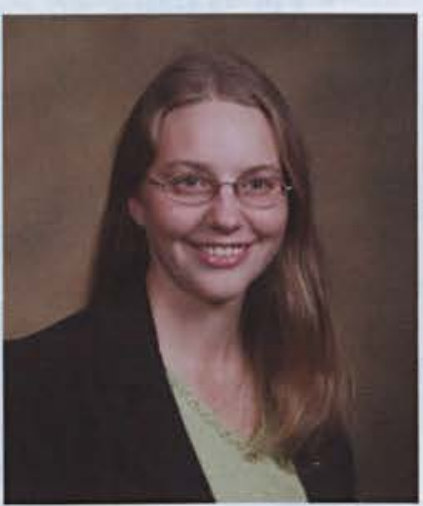

Erica Sue Massie

Elementary Education

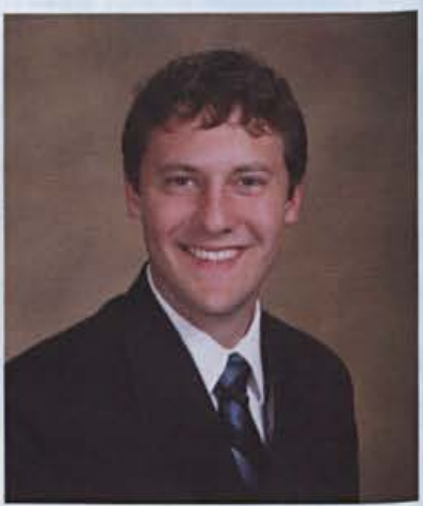

Aaron L. Mast Youth Ministries

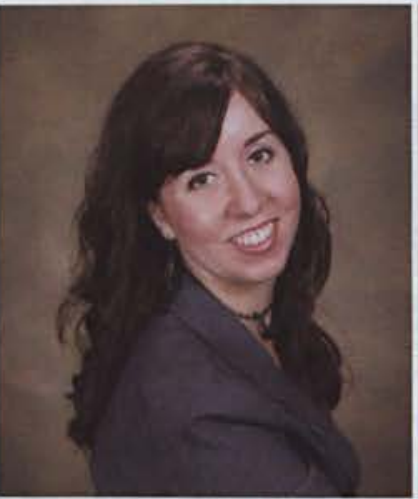

Andrea E. McCathy History \& Political Science

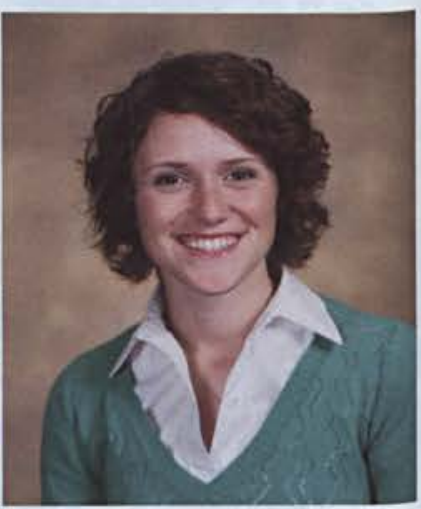

Lauren Eileen McCotter Psychology

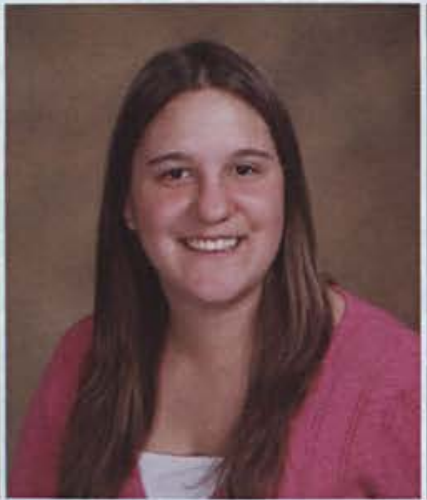

Katie Lynn McGinn Adolescent \& Young Adult Integrated Language Arts

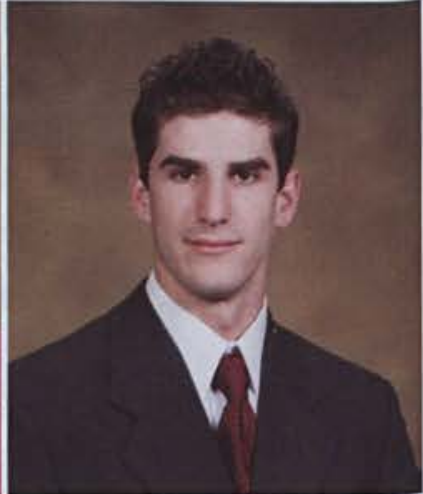

Alan Scott Mclver Mechanical Engineering

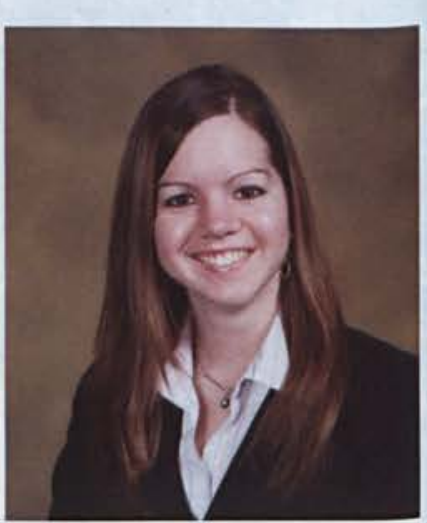
Communication Arts
Cristine LeeAnn McKinney Christian Education \& Youth Ministries
Brittany McNichols Nursing

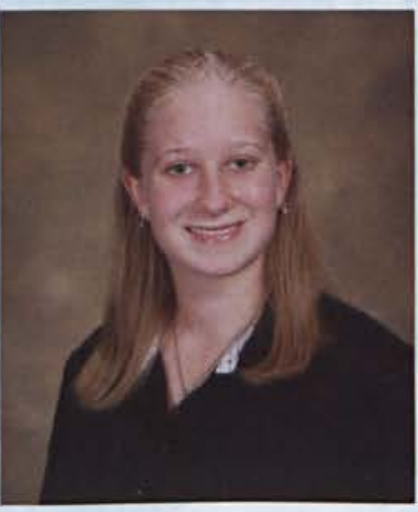

Krista Kay Merrick Psychology 


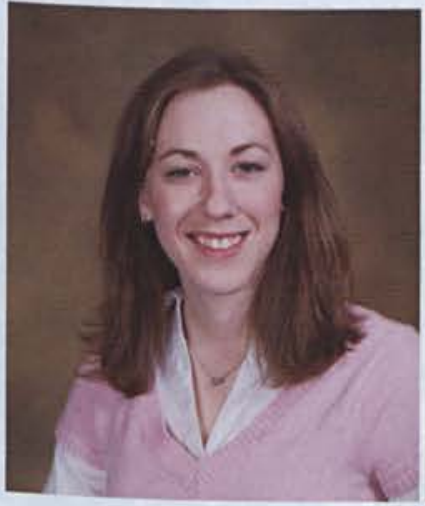

Carin Metsger

Psychology

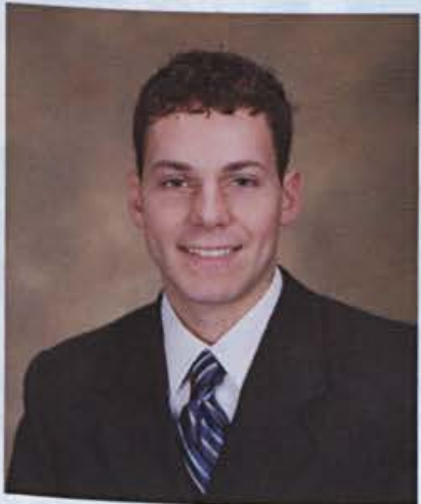

Dane Eliot Miller Biology

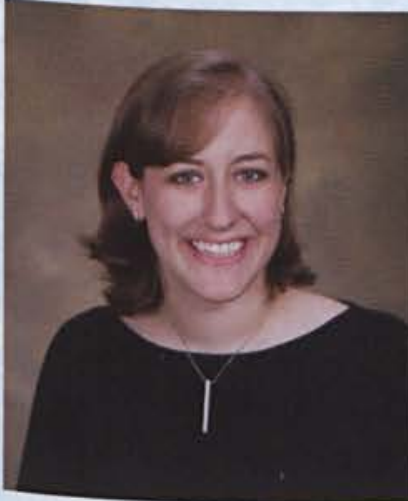

Rachel Sue Miller Pre-Seminary Bible

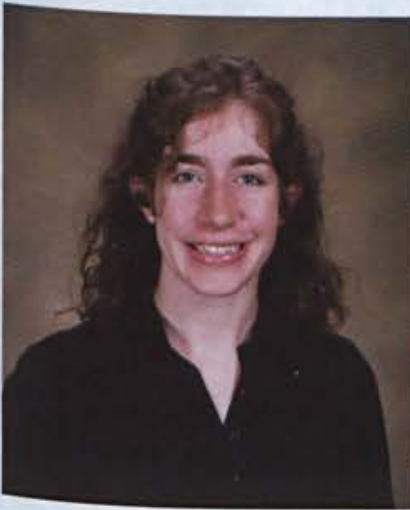

Hannah Christine Morris Nursing

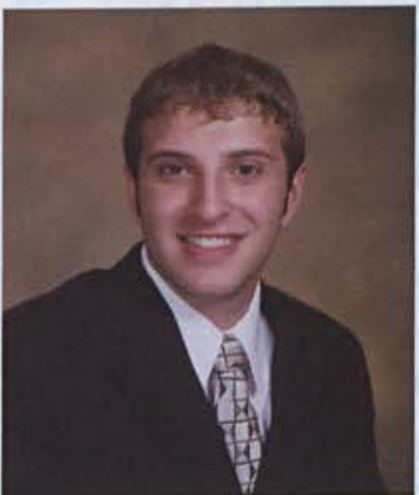

John Jared Michael Mechanical Engineering

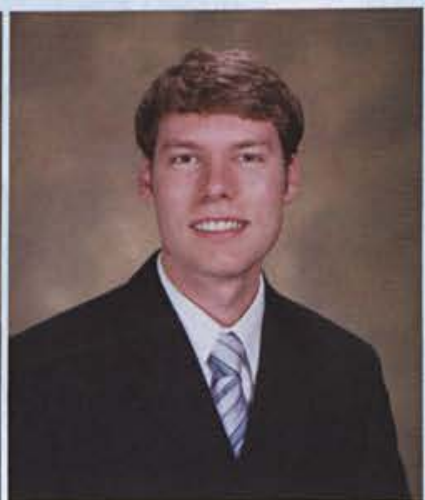

Joseph Daniel Miller Mechanical Engineering

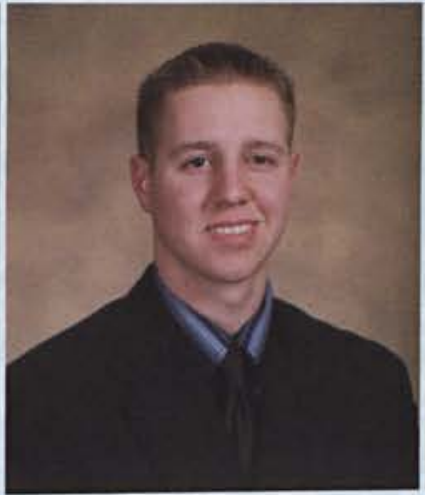

Jonathan Arthur Mingonet Criminal Justice

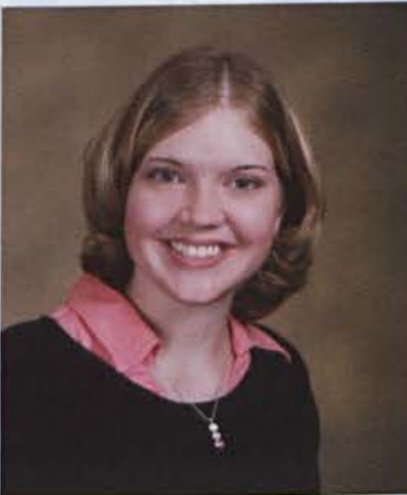

Sara Elizabeth Morrow Applied Psychology

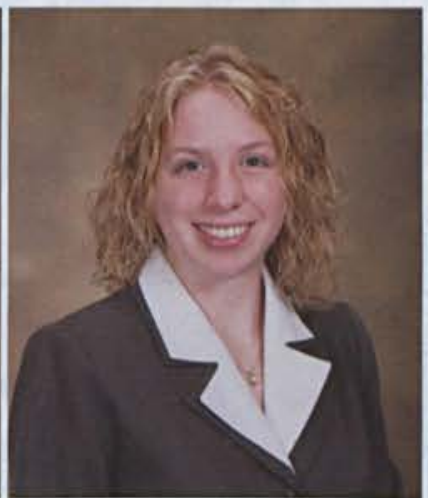

Patricia Anne Mickalko Middle Childhood Education

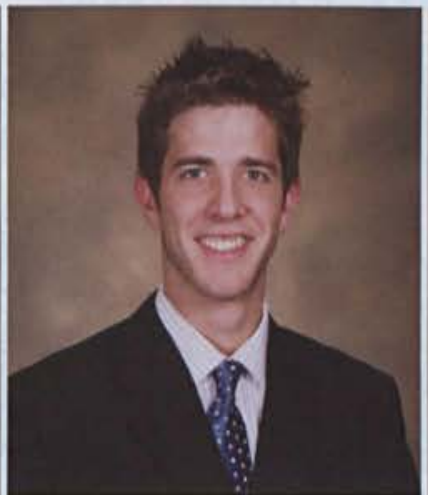

Jared Daniel Michonski Psychology

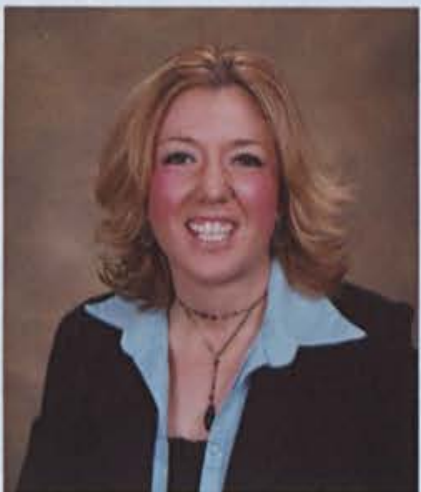

Melody Dawn Midgley Nursing

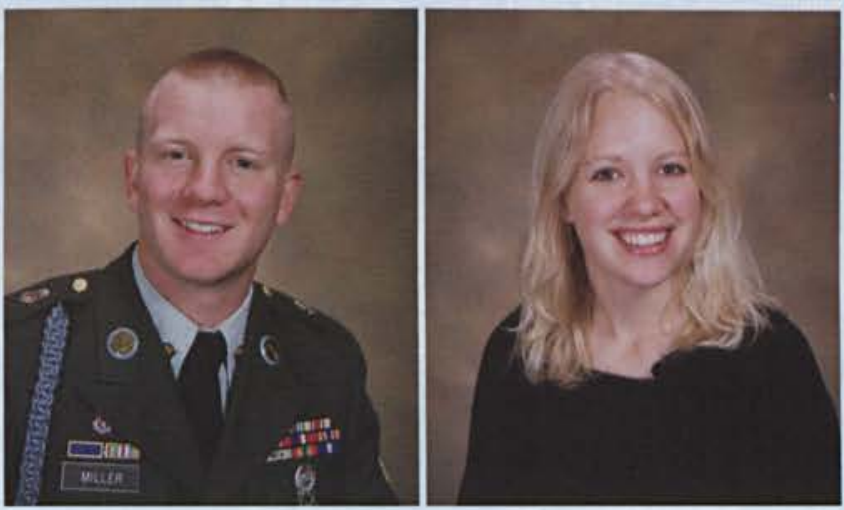

Kate Elizabeth Miller Nursing

\section{Comprehensive Bible}

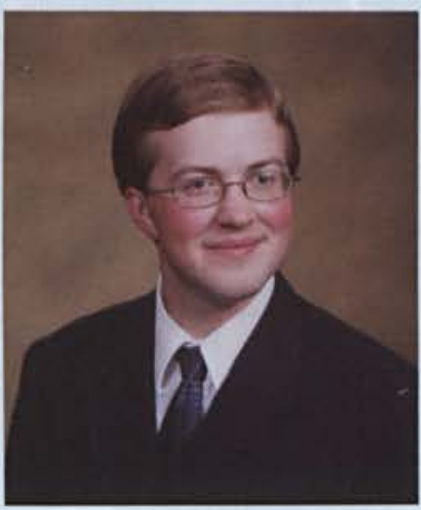

Nathaniel Paul Miller Mechanical Engineering

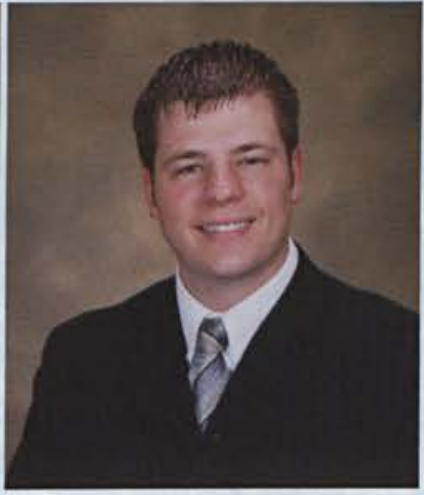

Brenton Arnett Moore Accounting

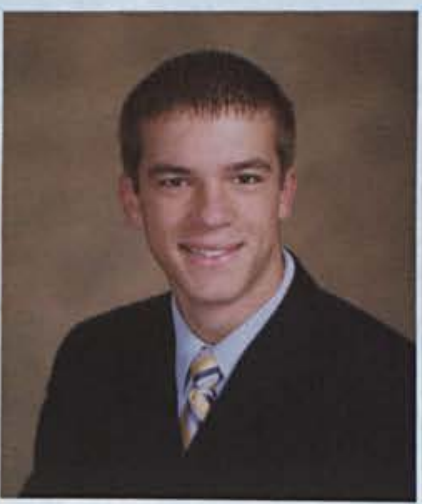

Christopher P. Moran International Business

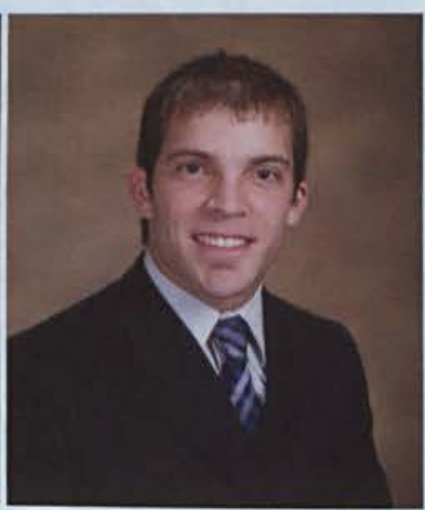

Brody Hunter Morris Psychology

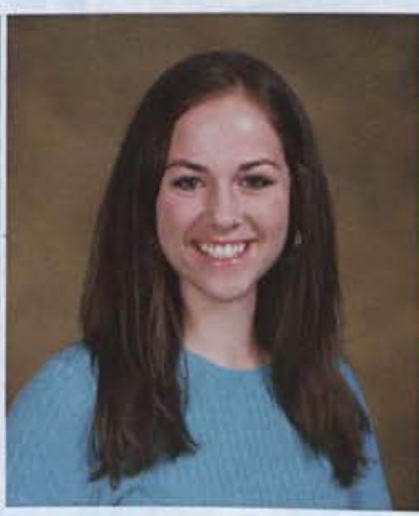

Emily Jean Moser Nursing

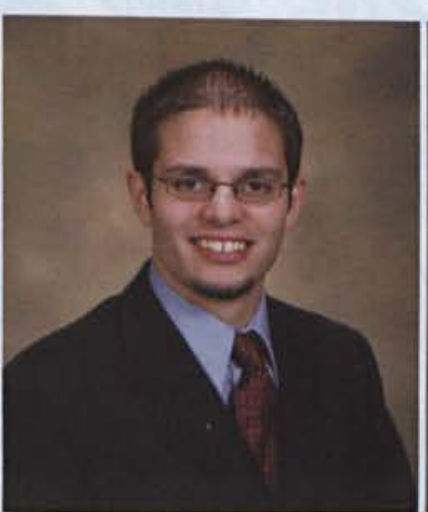

Jeremy Thomas Motta General Psychology

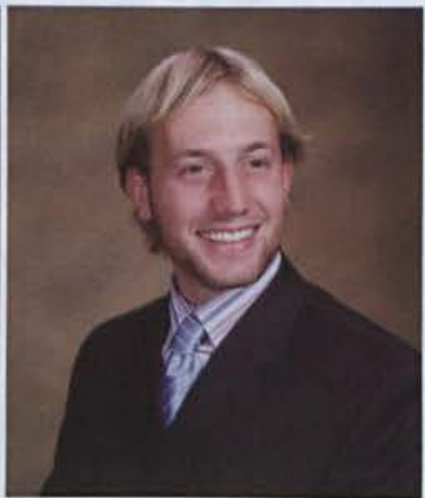

Trevor Thomas Mueller Nursing 


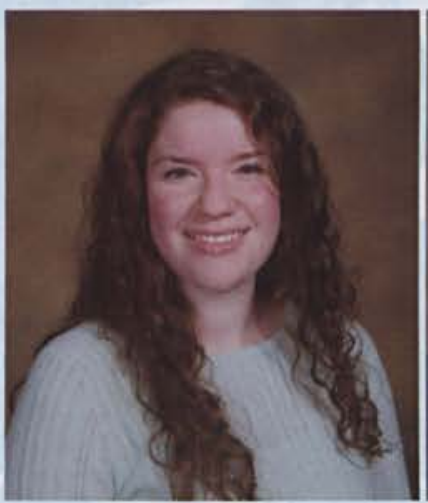

Stephanie Mullenhour Electronic Media

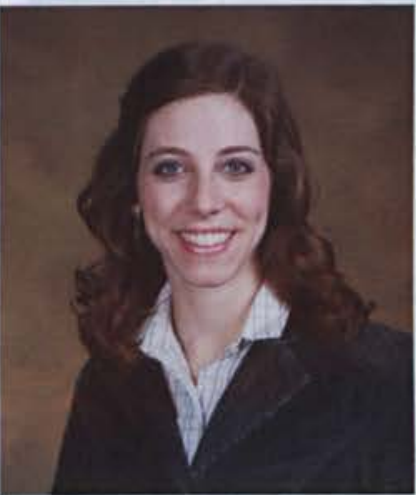

Rebekah Mae Nettekoven Theatre

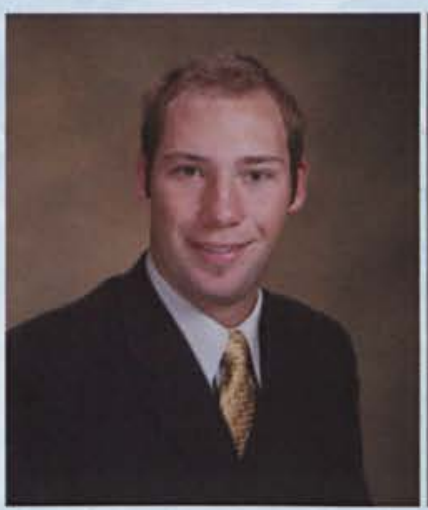

Andrew Joseph Noble Exercise Science

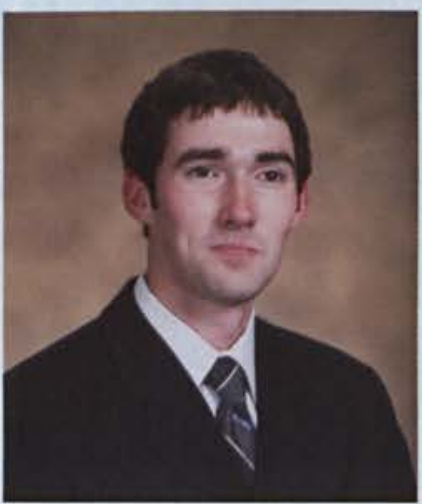

Joshua G. Oliver Mechanical Engineering

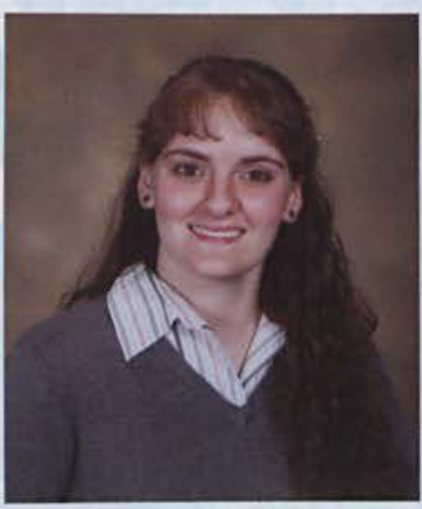

Sarah Elizabeth Mumme Music

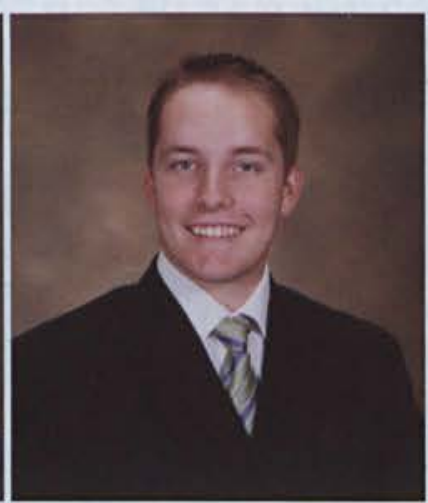

Andrew Reid Myers Mechanical Engineering

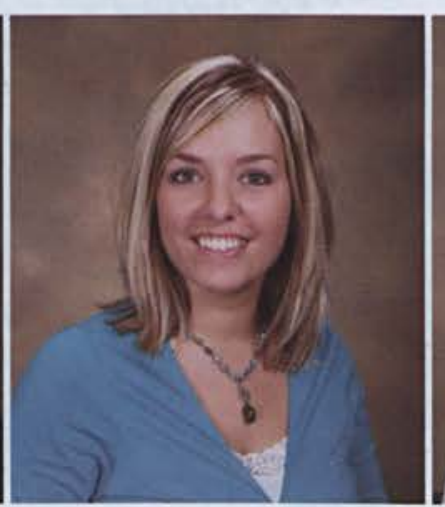

Alissa Neal Management

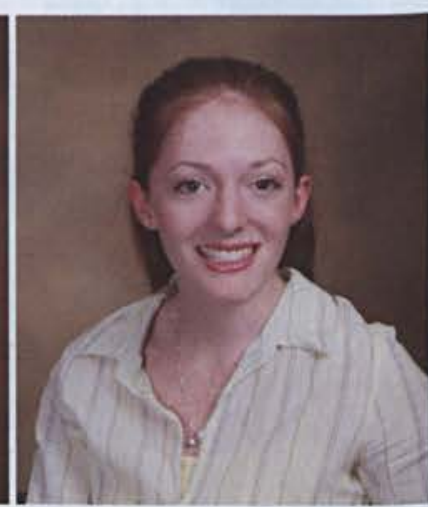

Emily Kathryn Nelson Middle Childhood Education

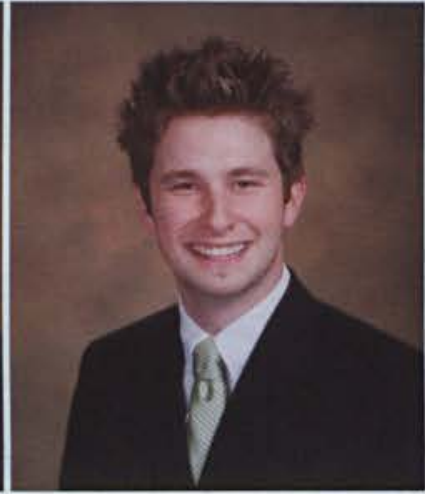

Jason Arthur Ney

English

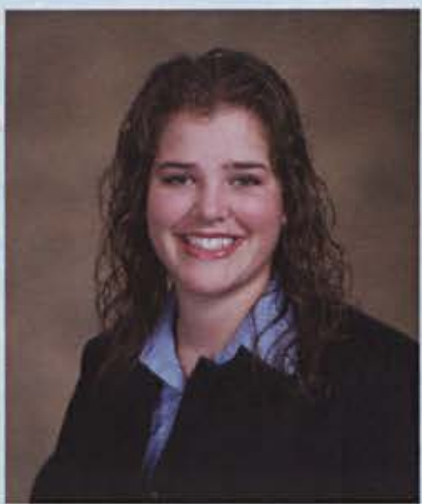

Julianne Michele Null Nursing

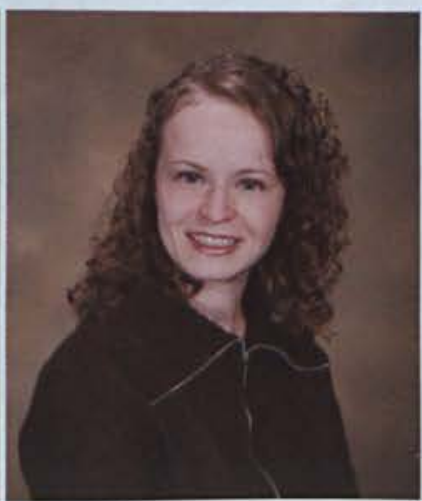

Krista Kathleen Olson Psychology

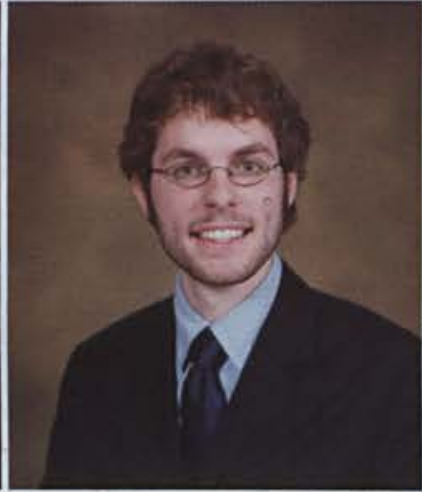

David Martin Nickerson Chemistry

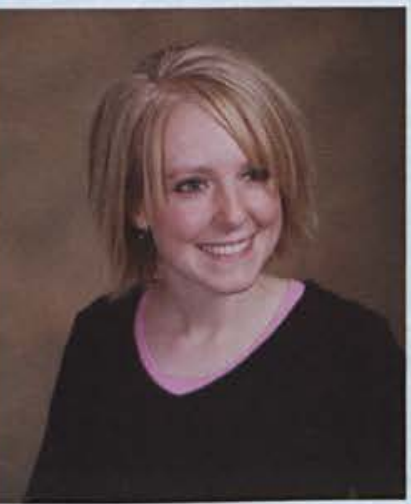

Kelsi Jo Nussbaum Nursing

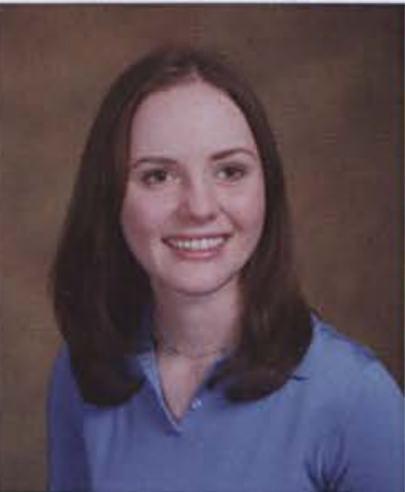

Amber Jean Nielsen Chemistry \& Biology

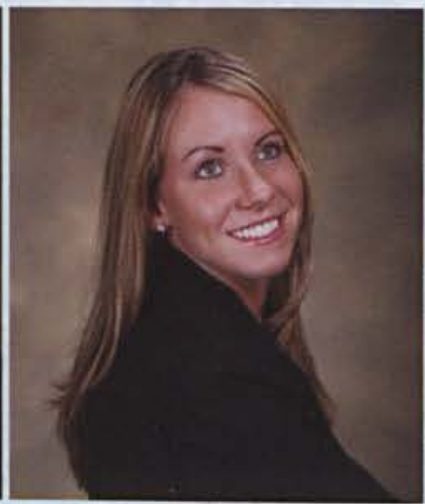

Sara Marie Oddis Management

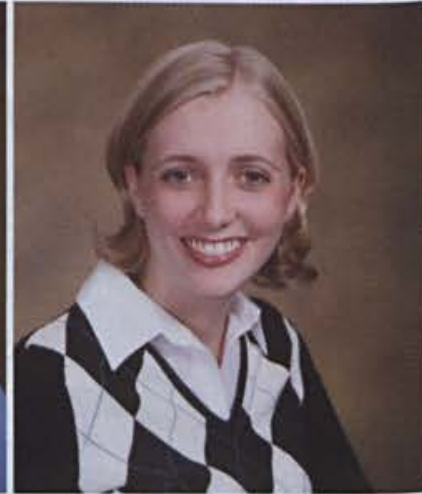

Katie Lauren Nixon Early Childhood Education

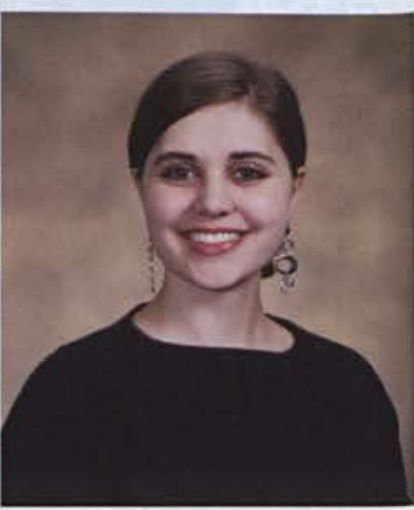

Hannah Lee Officer English

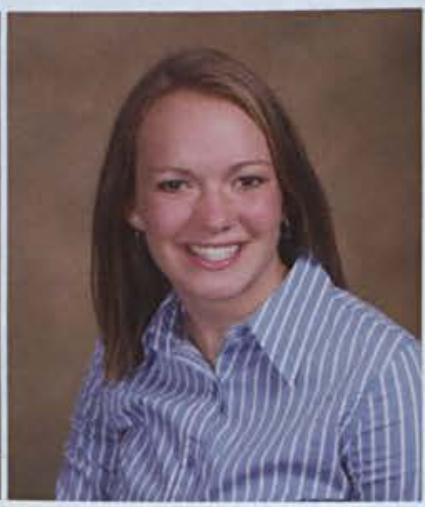

Casey Michelle O'Neal Nursing

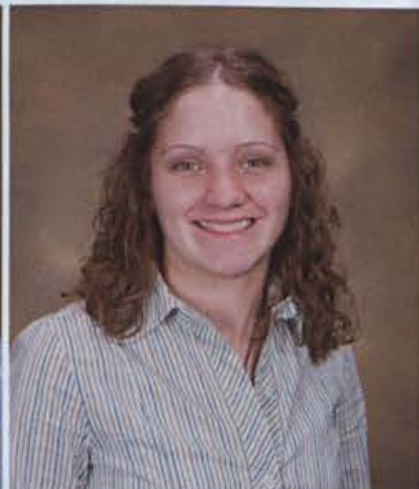

Mary Elizabeth Orr Middle Childhood Education

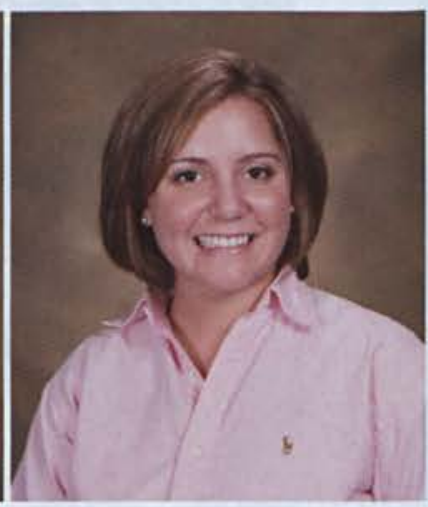

Elizabeth Enders Orrison Special Education \& Early Childhood Education 


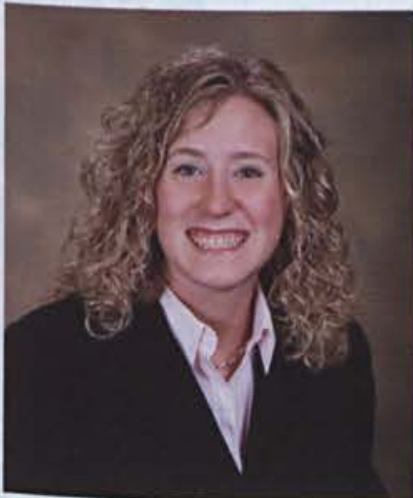

Ashley Marie Ort

Early Childhood Education

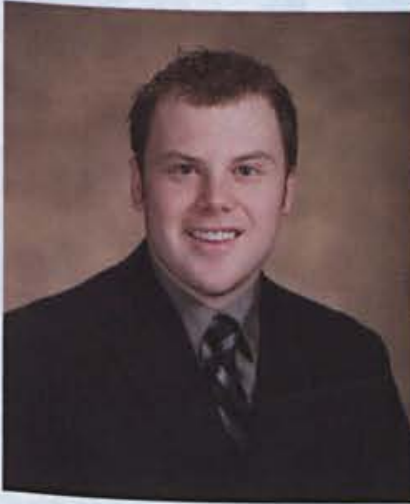

Dave Parmerlee

Middle Childhood Education

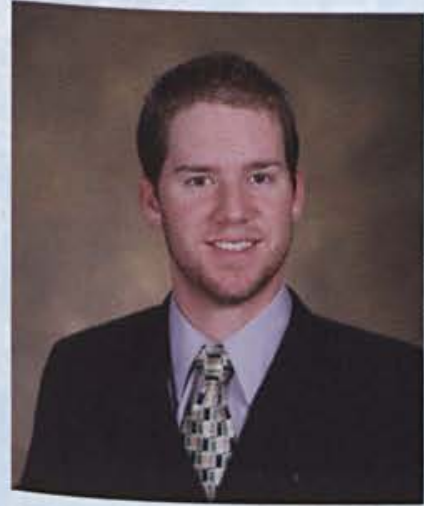

Israel Jacob-Lloyd Peck Psychology

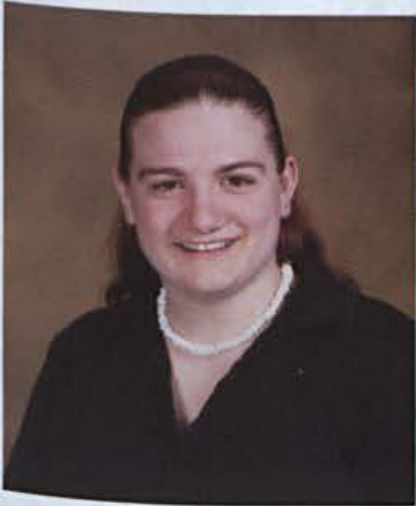

Tabitha Pfoutz

Youth Ministries \& Christian Education

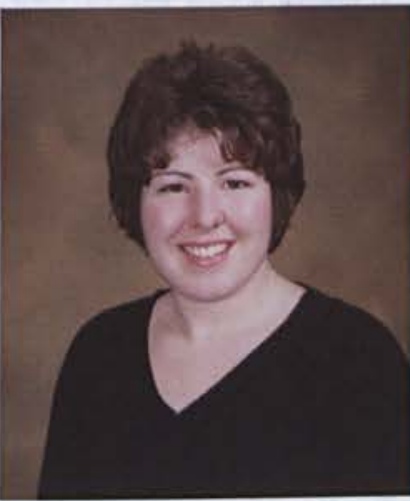

Melissa Esther Osborn History

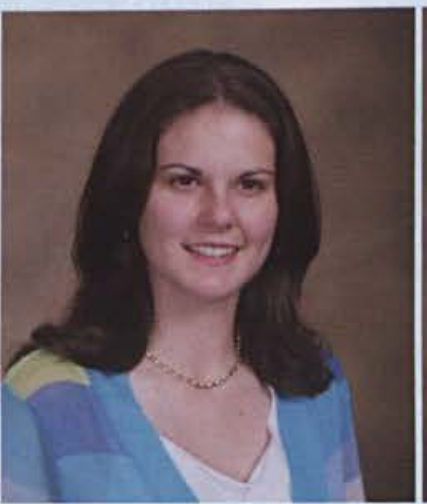

Lisa Ann Partian Computer Science

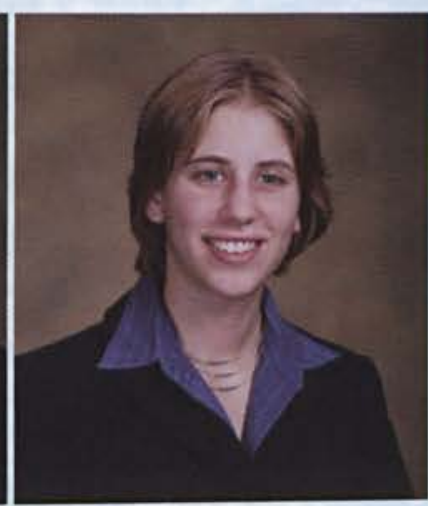

Kristen Marie Pelon Mathematics

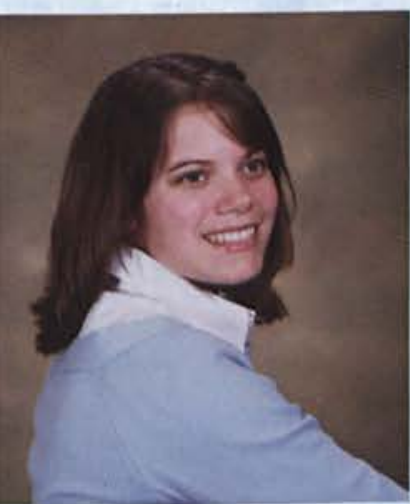

Joanna Pheasant Nursing

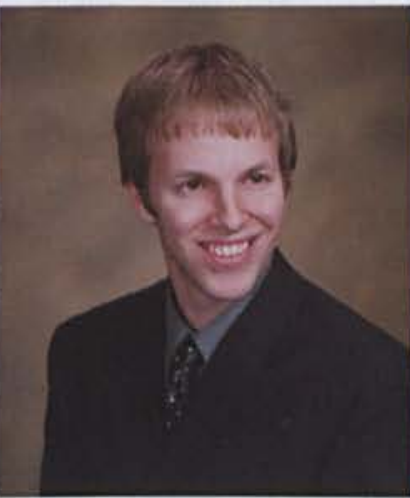

Devon Roy Overby Electronic Media

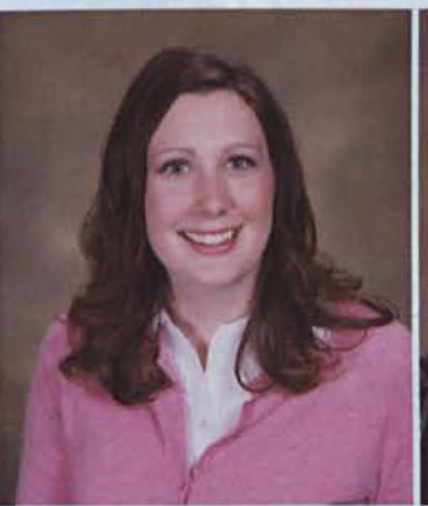

Rebecca Renee Patterson Middle Childhood Education

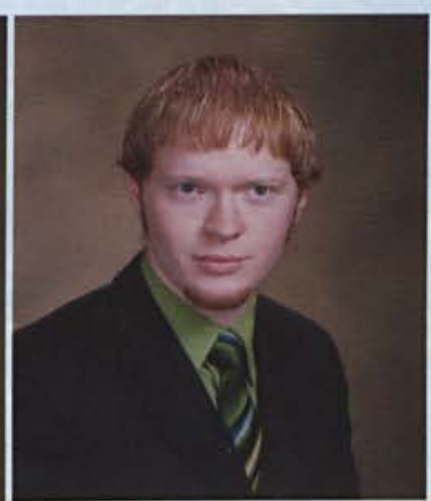

Joshua James Perkins International Studies

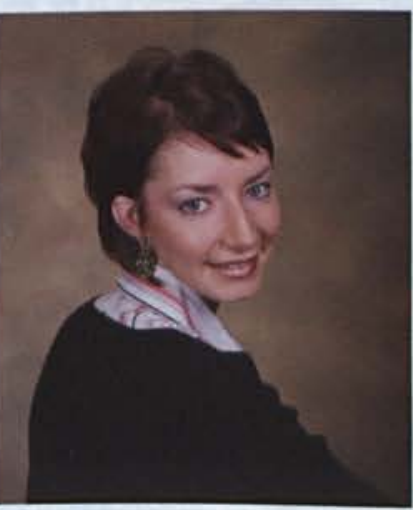

Alissa Joy Phillips Early Childhood Education

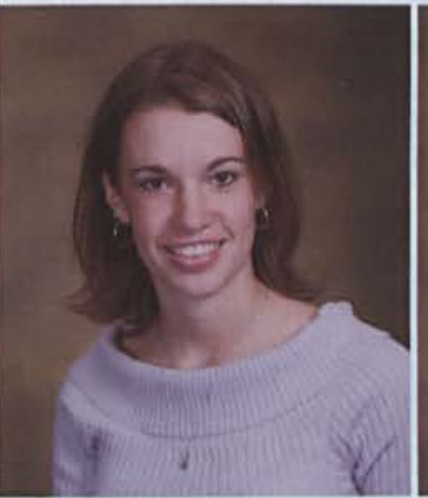

Anna Denae Pabody Early Childhood Education

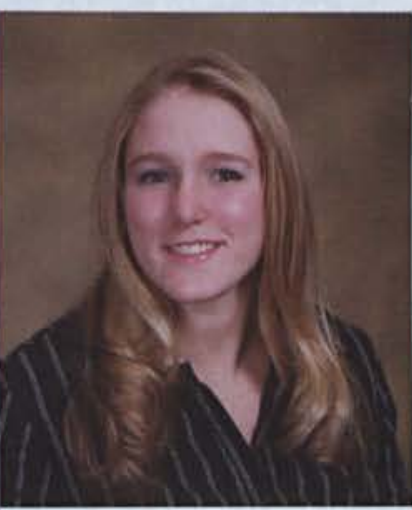

Erica Nicole Paugh Early Childhood Education

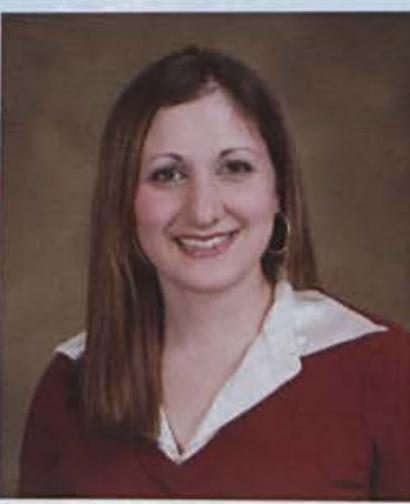

Elizabeth Anne Pesce Music

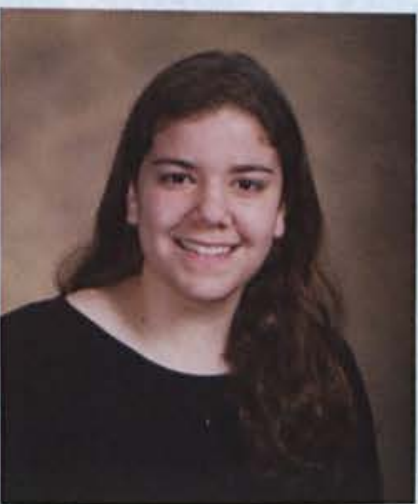

Shannon Picazo Psychology

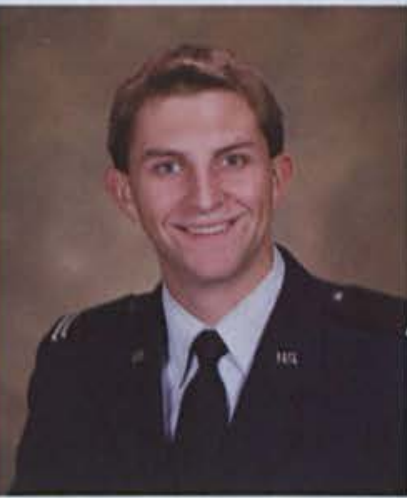

Joshua David Parker Electrical Engineering

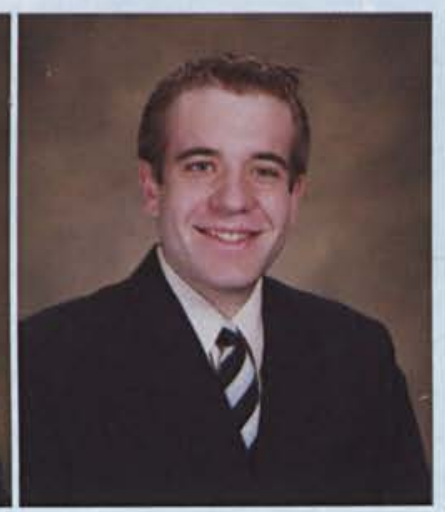

Geoffrey George Peck Political Science

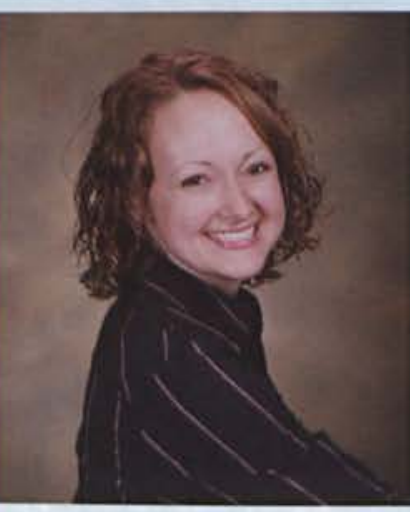

Rachel Amy Petersen Christian Education

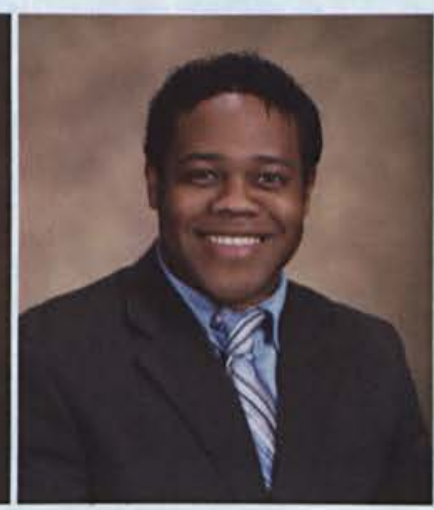

Isaac Pittman Electronic Media

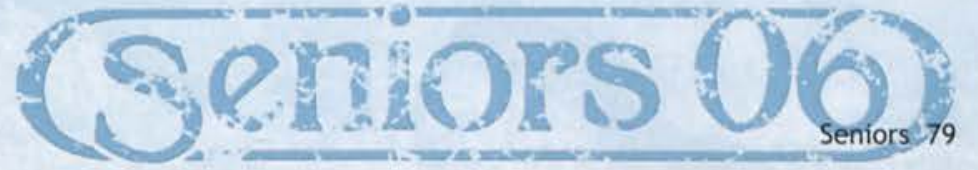




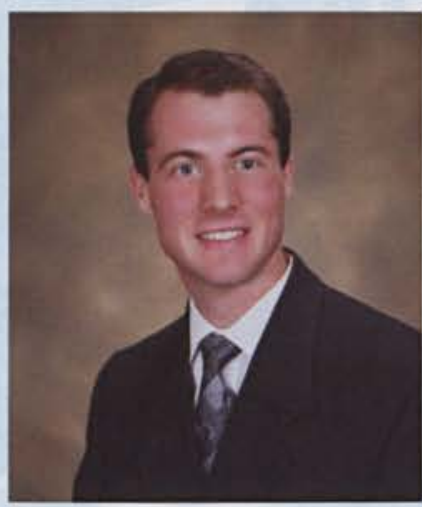

Randall Stephen Plate Electrical Engineering

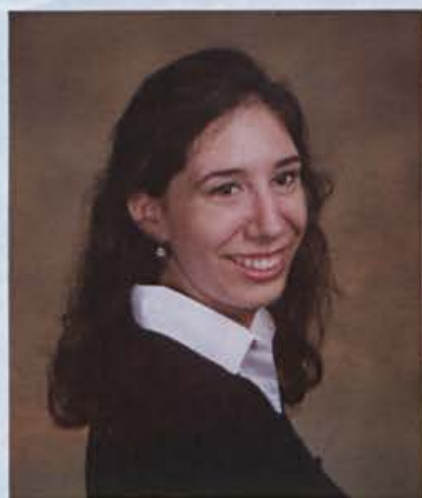

Mary Elizabeth Pritts Mathamatics

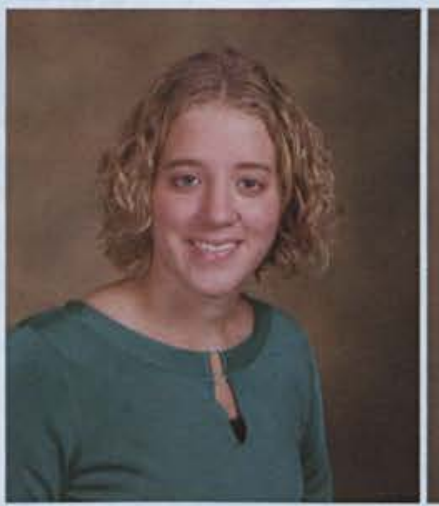

Katie Lynn Rabe Early Childhood Education

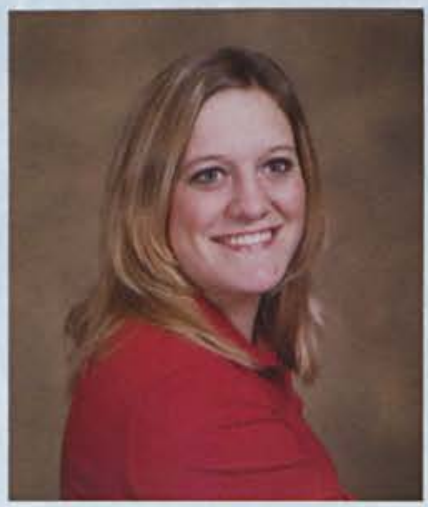

Laura Elizabeth Rapp Sport Management

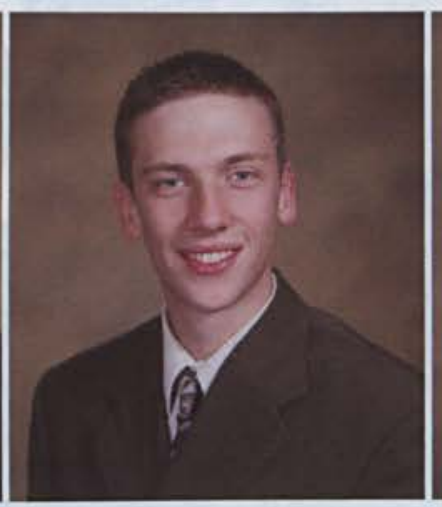

David Michael Podboy Biology

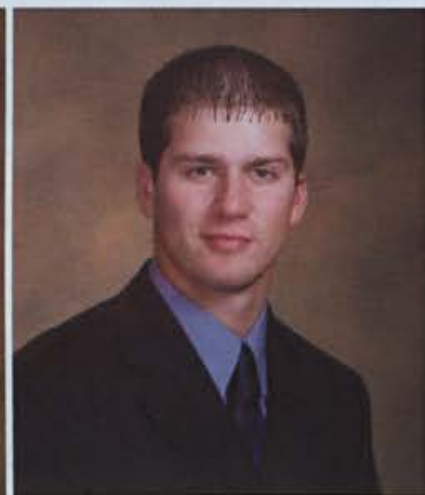

Alexander John Pummell Exercise Science

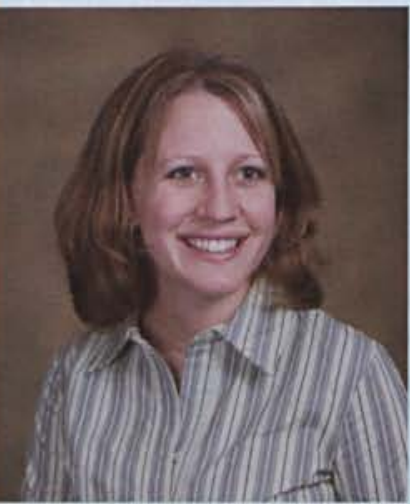

Melissa Anne Raber Integrated Social Studies Education

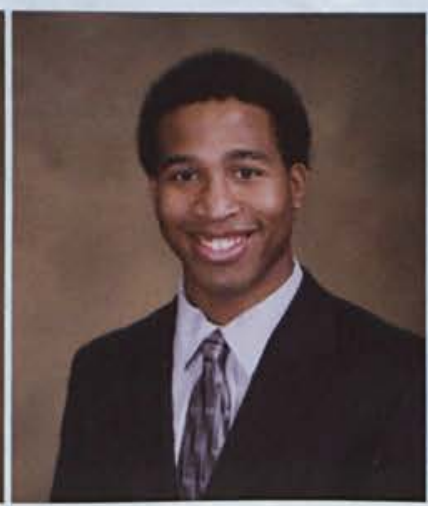

Devon Rawls Psychology

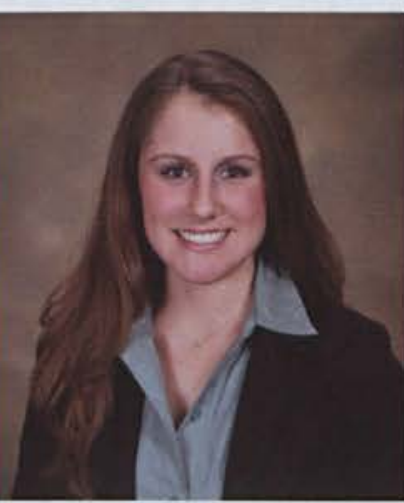

Courtney Joy Potter Music Education

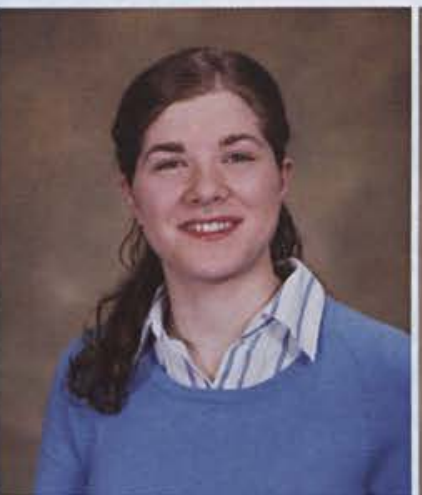

Jennifer Allyn Quidor Nursing

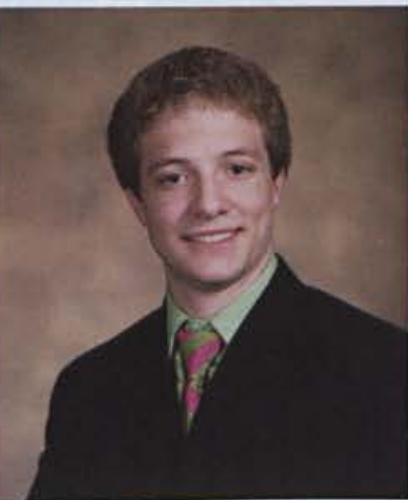

Douglas Andrew Radtke Mechanical Engineering

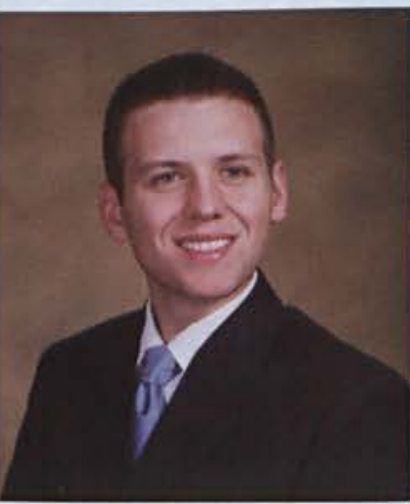

John Mark Raymond Middle Childhood Education

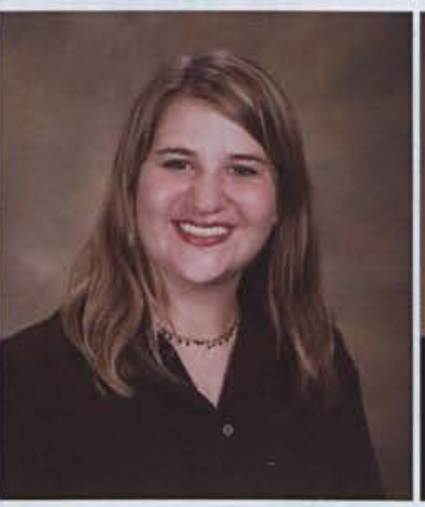

Kathleen Jozena Powers Technical \& Professional Communication

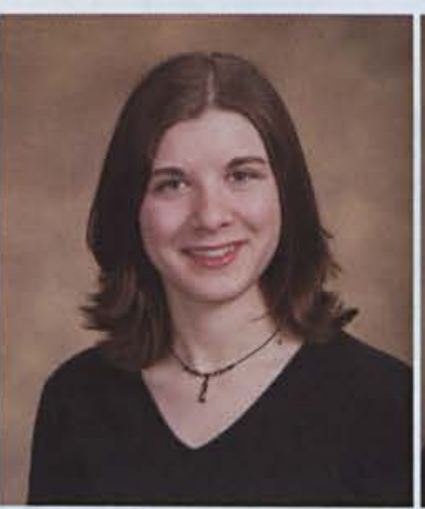

Jessica Anne Quidor Psychology

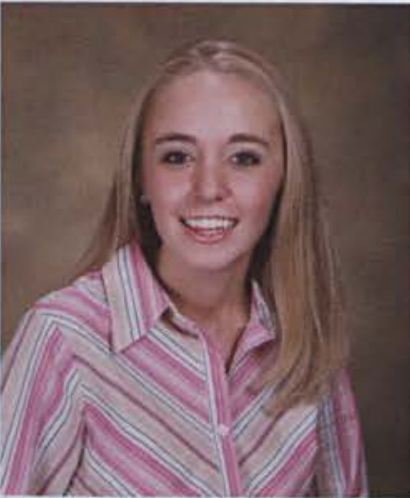

Jamie Lynn Ralston Special Education \& Early Childhood Education

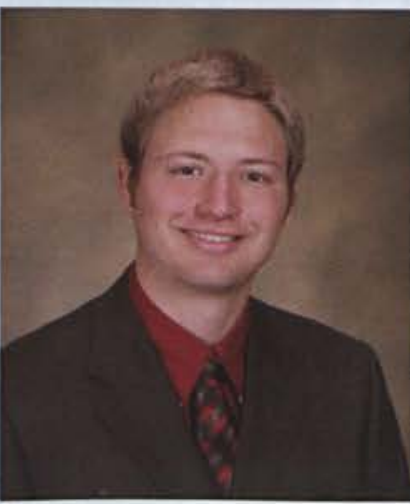

Matthew Philip Redfield Mechanical Engineering

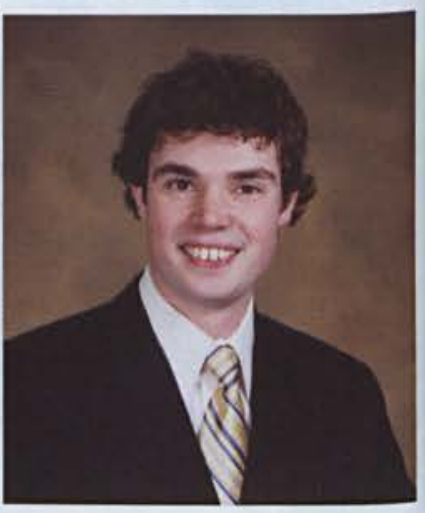

Ty Landon Price Management Information Systems

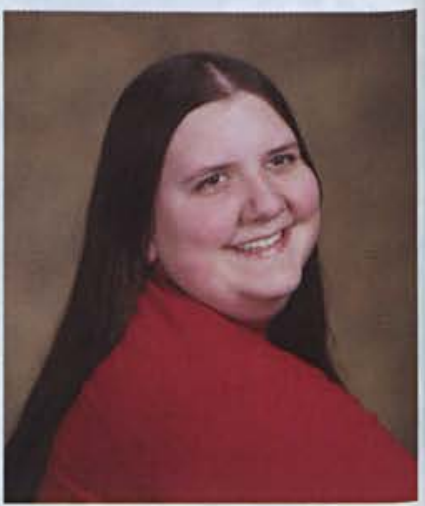

Kathryn Mae Quist Multi-Age \& Early Childhood Education

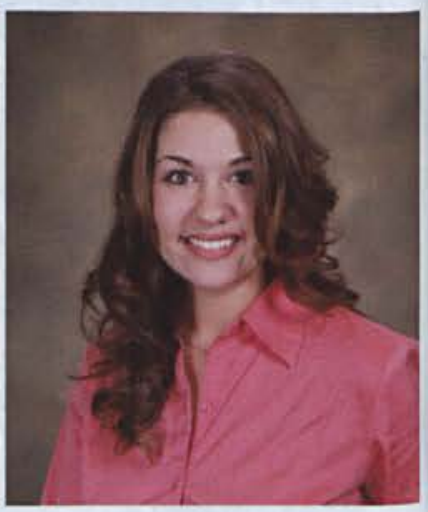

Laura E. Ransom

Christian Education \& Womens Ministry

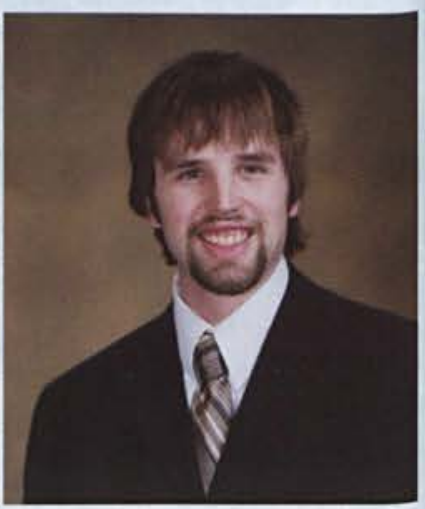

Jameson C. Rehm Electronic Media 


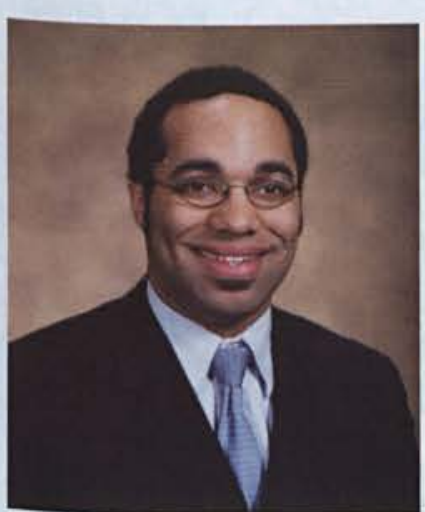

Robert Thomas Reid Music

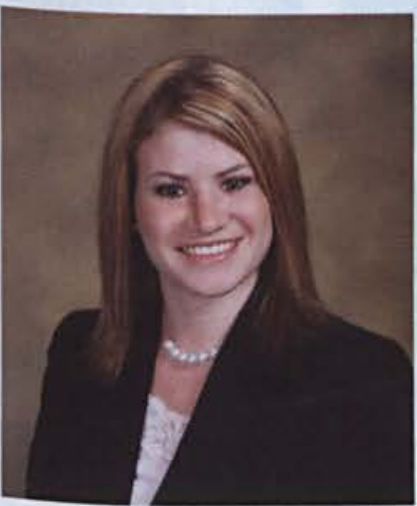

Gretchen Lenore Riedel Theatre

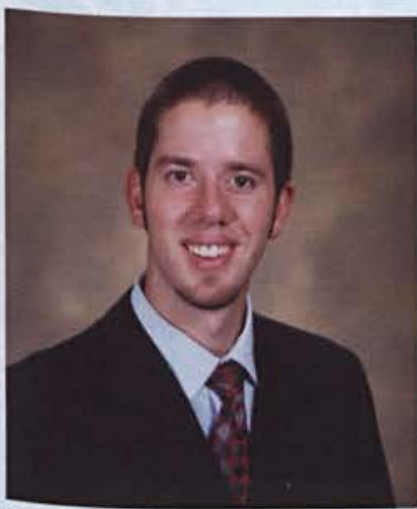

Ryan James Rotman Church Music Ministries

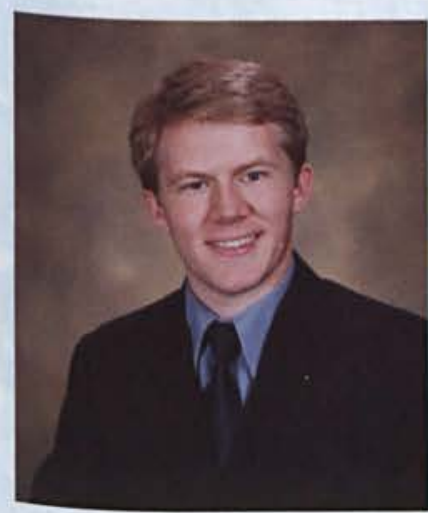

Philip James Schanely Graphic Design

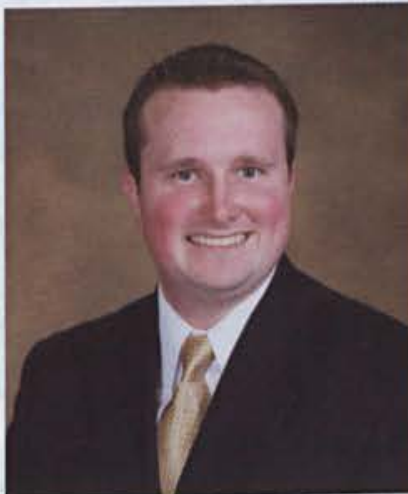

Joshua Daniel Reno Comprehensive

Communications

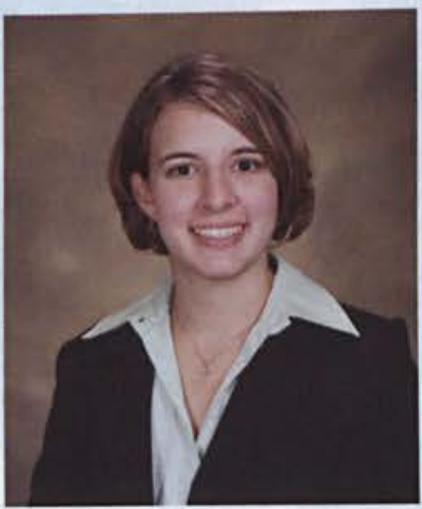

Leah Ruth Robertson Accounting

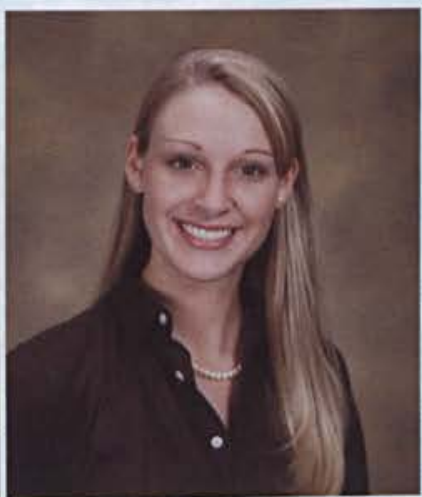

Brittany Rouse Graphic Design

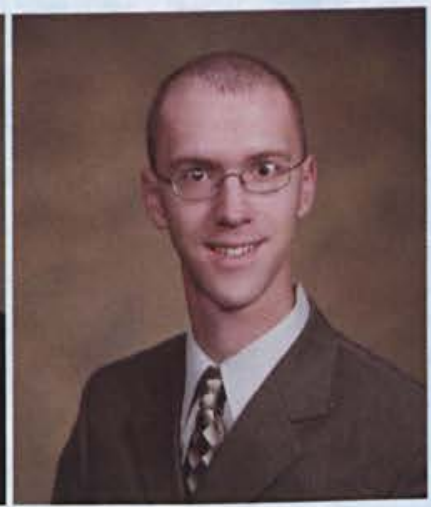

Ryan Andrew Schildroth Pastoral Studies

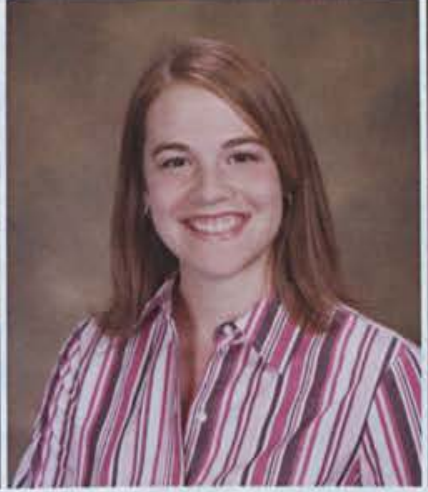

Kathryn Ann Rexford Special Education

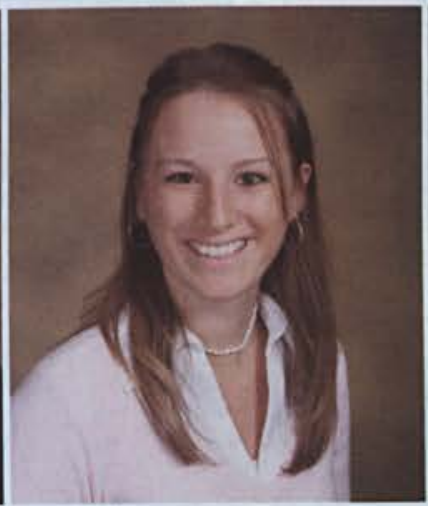

Ginger Renee Roller Communications

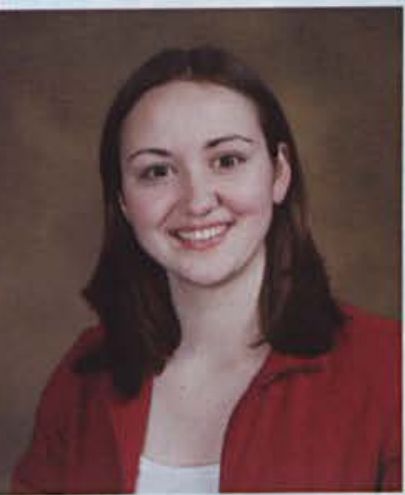

Candace Grace Rowe Middle Childhood Education

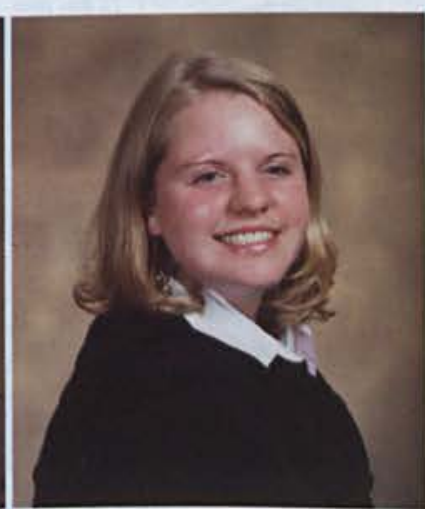

Rebecca Lynne Schillack Multi-Age Special Education

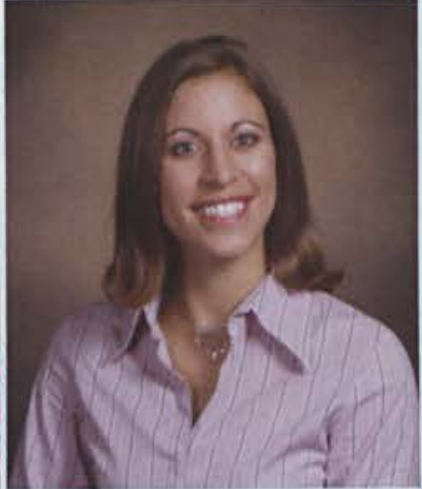

Karissa M. Richardson Multi-Age Spanish Education

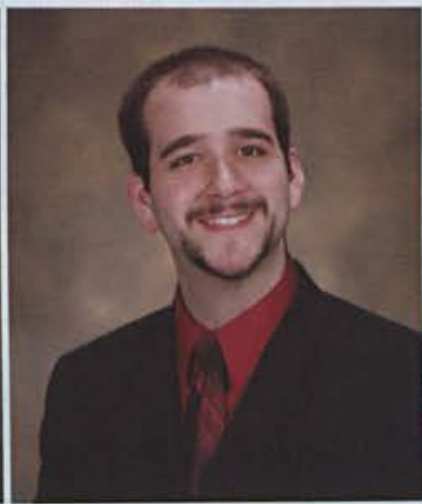

Timothy Daniel Rosie Trombone Performance

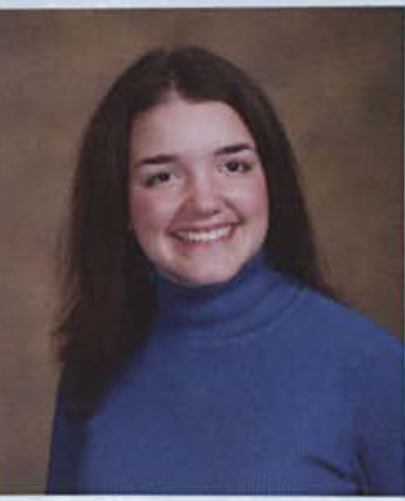

Stephanie Kay Rudd Music Performance

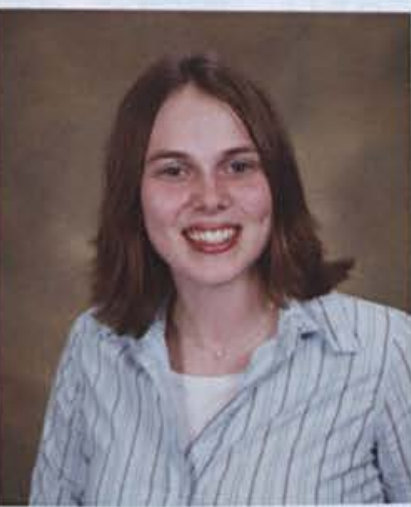

Hannah Elizabeth Schnake Special Education

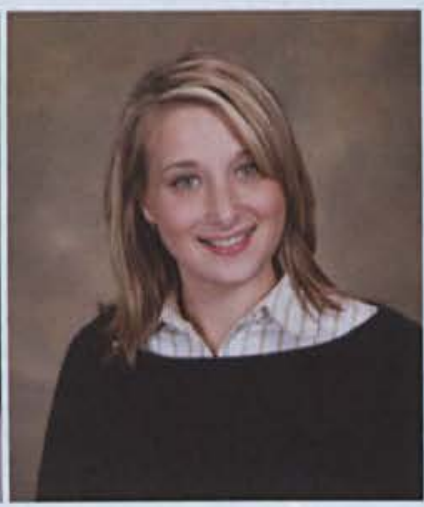

Kathryn T. Rickabaugh Comprehensive Bible

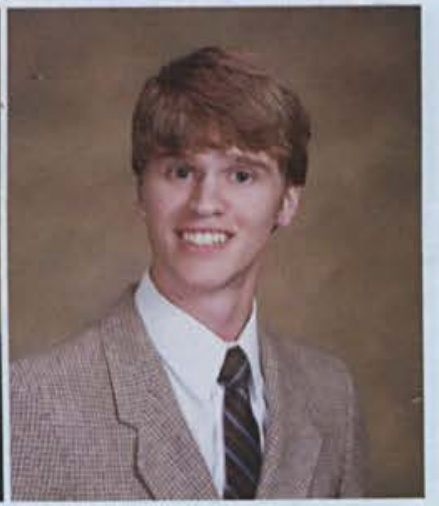

Brian Joshua Rothaar Computer Engineering

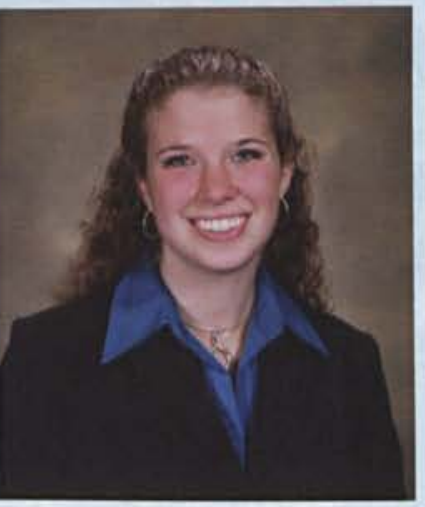

Marybeth Joy Sattler Special Education \& Early Childhood Education

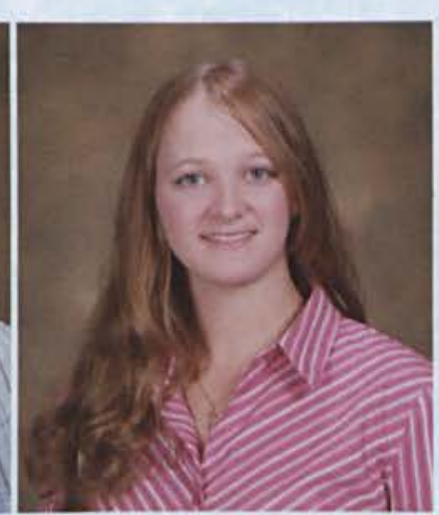

Shannon Alysia Schramm Nursing

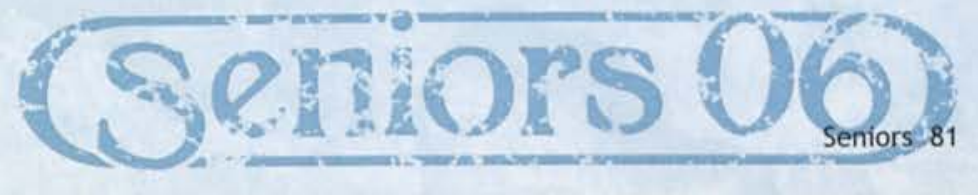




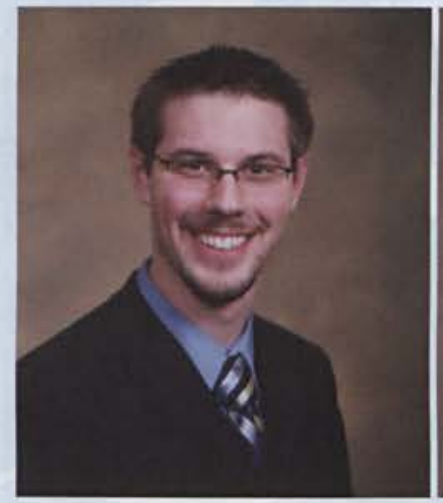

Trevor J. Schuh Church Music Ministries

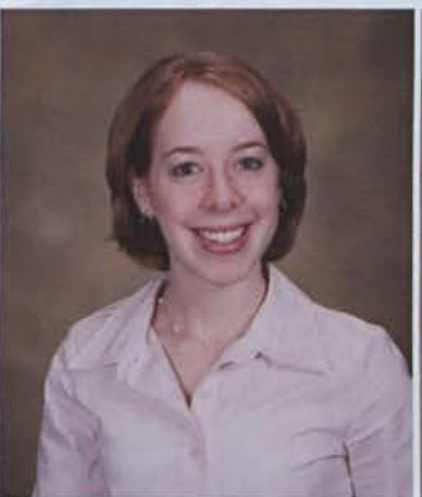

Lindsey Ann Selegue Mathematics

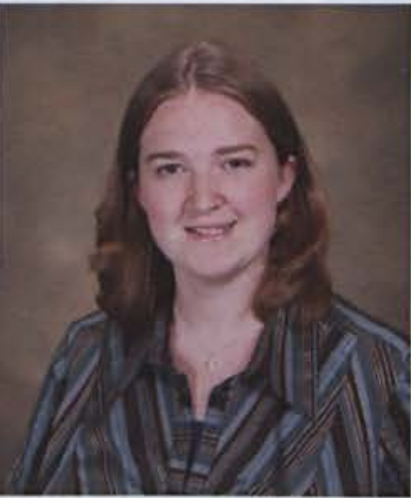

Elizabeth Kay Shimer Middle Childhood Education

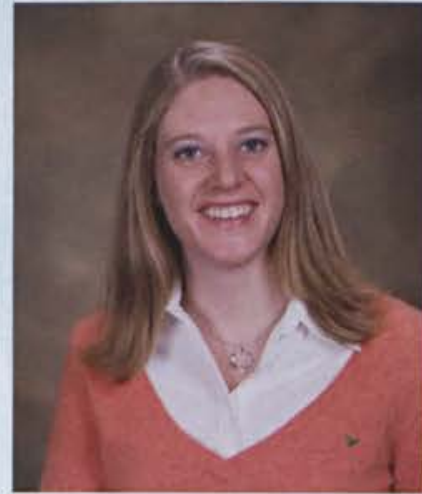

Laura Nicole Shuneson Accounting

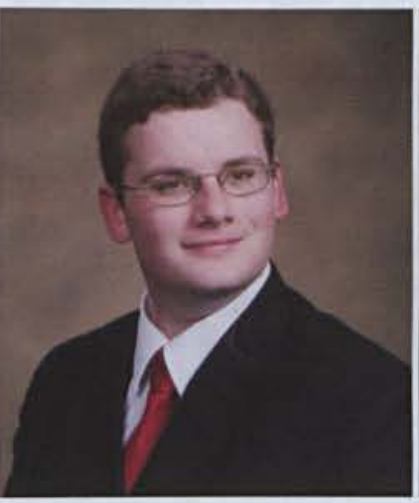

Steven Douglas Schutter Pre-Seminary Bible

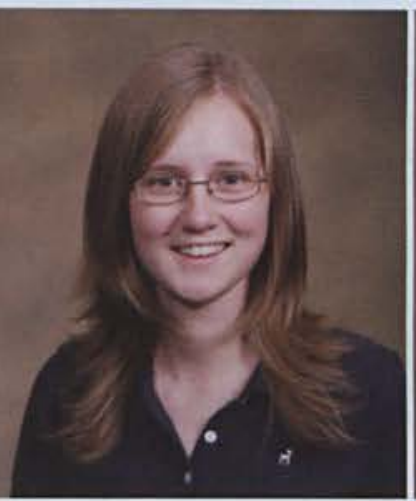

Kimberly Kaye Shank Mathematics

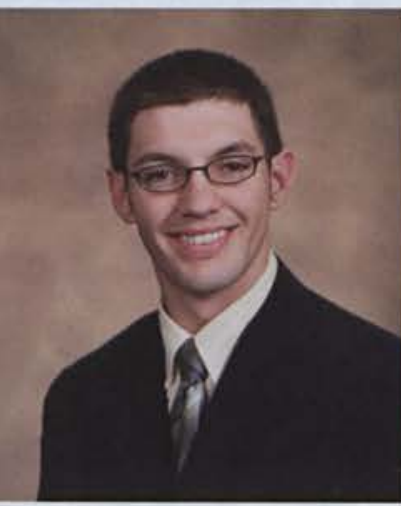

Michael Loran Shipper Middle Childhood Education

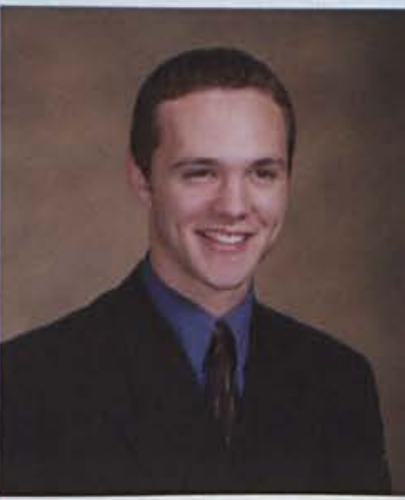

Joel David Sigmon Management

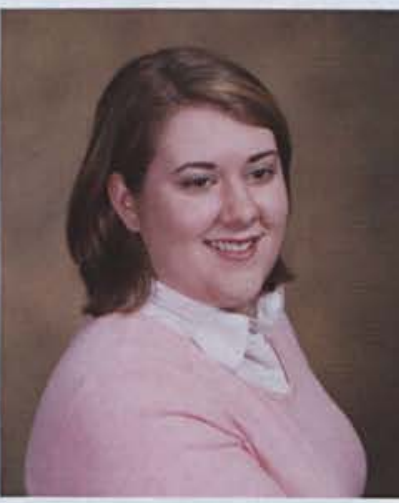

Janna Rae Schwab English

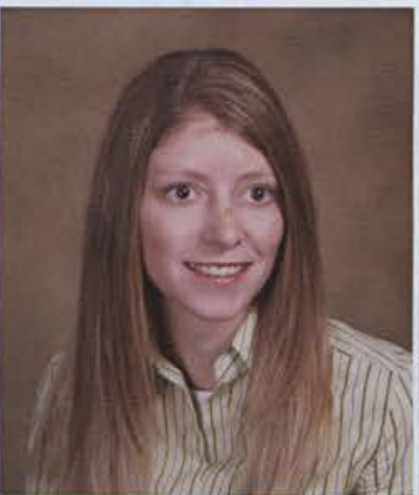

Laura Anne Shank Youth Ministry \& Christian Education

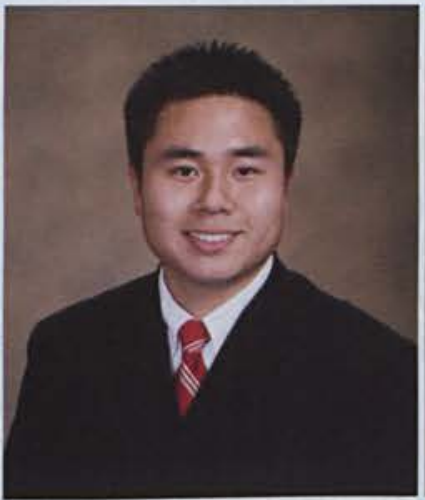

Matthew Kenneth Shiraki Integrated Social Studies Education

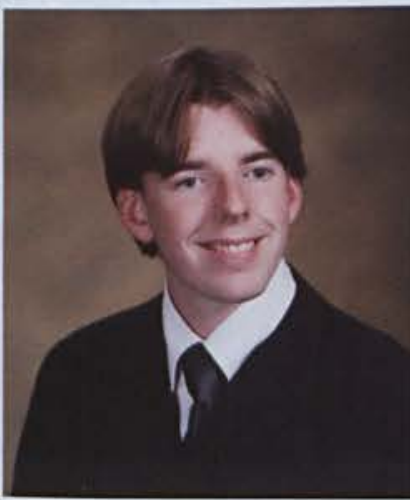

Ryan Anthony Simmons English

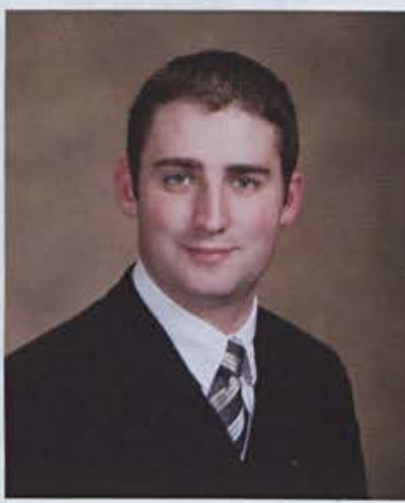

David Michael Scott Mechanical Engineering

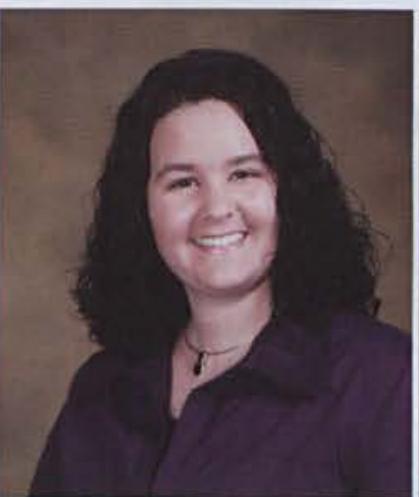

Jennifer Anne Shelamer Nursing

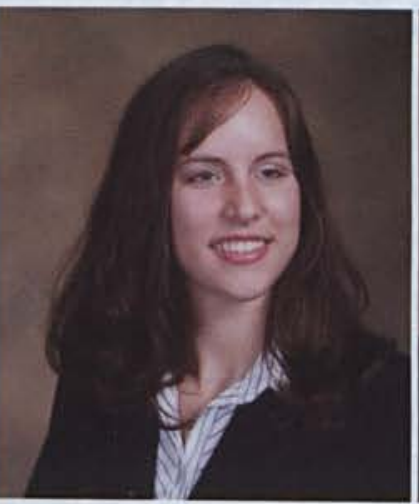

Amanda Lynn Shriber Nursing

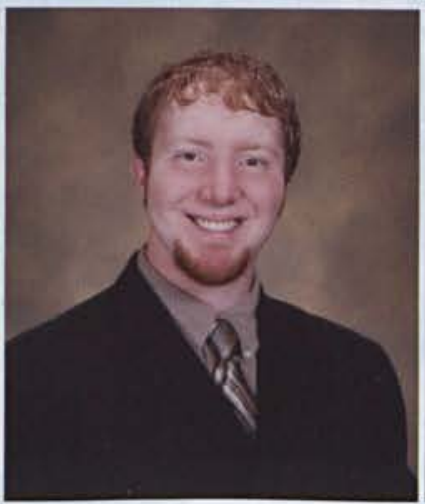

Jeffrey Matthew Simon Electronic Media

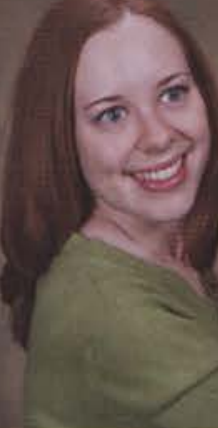

Ashley Dianne Selegue Mathamatics

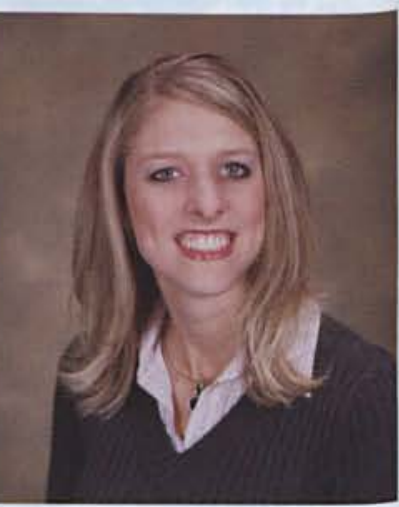

Lindsey Erin Shev Early Childhood Education

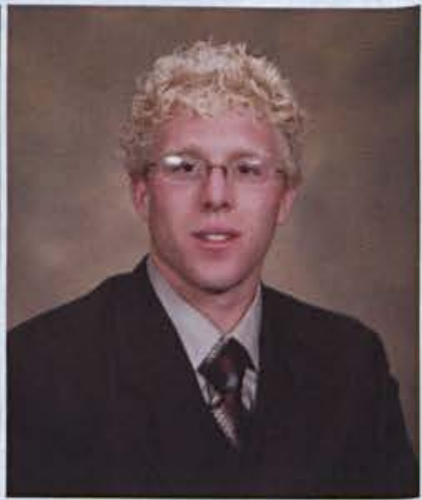

Benjamin Eugene Shroyer Sport Management

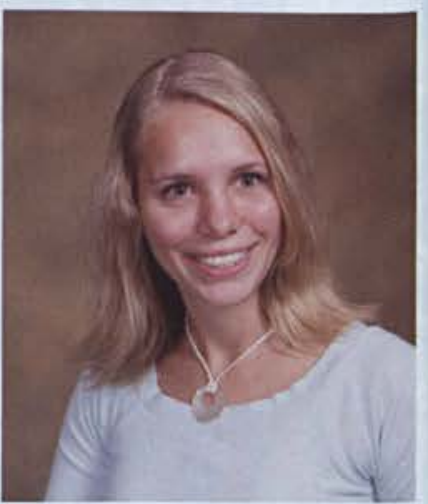

Kara Joy Simons Integrated Science Education 


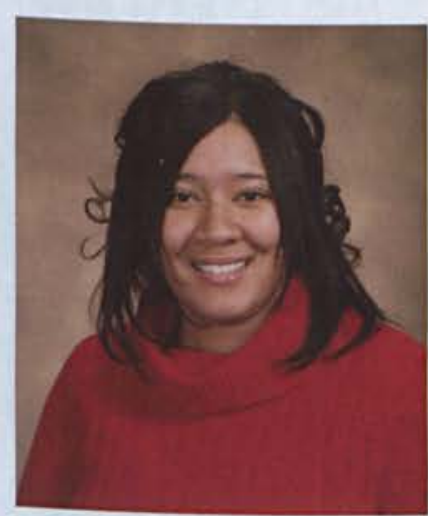

Katherine Simpson

Psychology

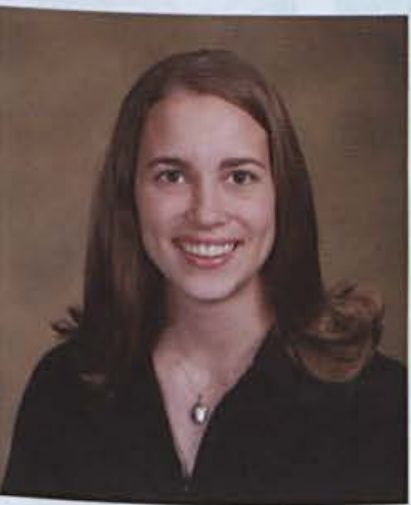

Rachel Lynn Slothower Special Education \& Early Childhood Education

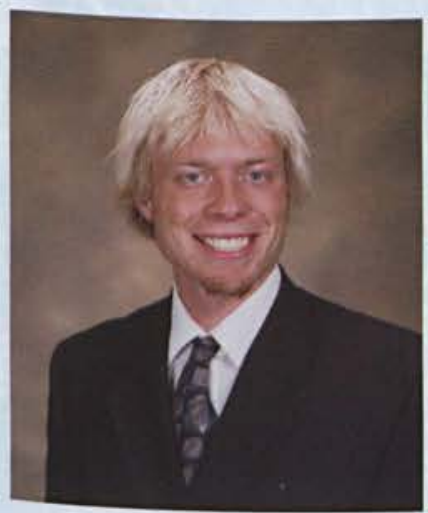

Joel Philip Smith

Exercise Science

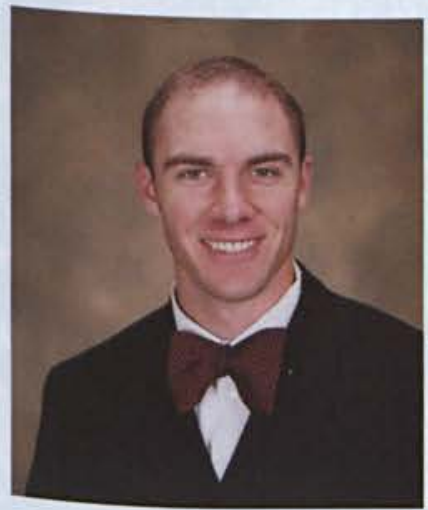

David Gary Snow

Criminal Justice

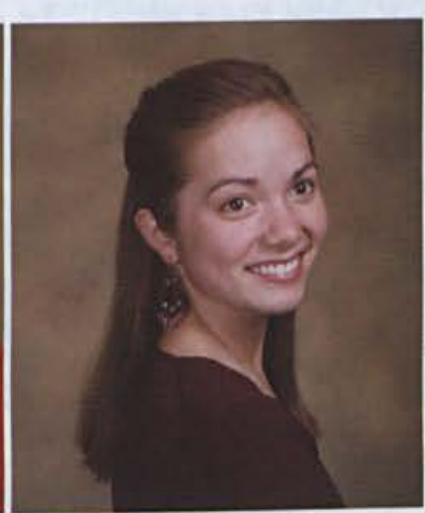

Nikole Marie Simpson Middle Childhood Education

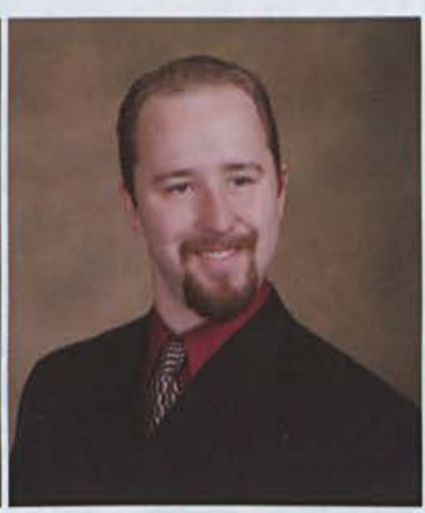

Andrew G. Skaggs History

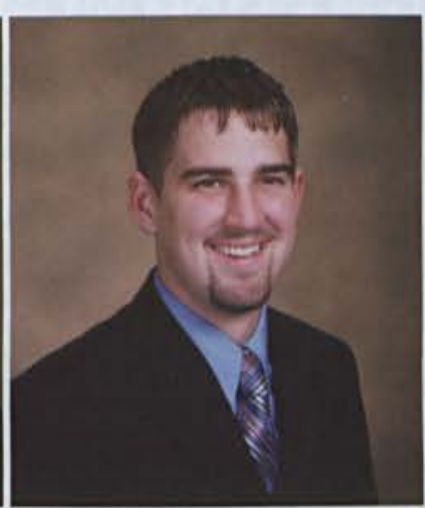

Jason David Skelton Music

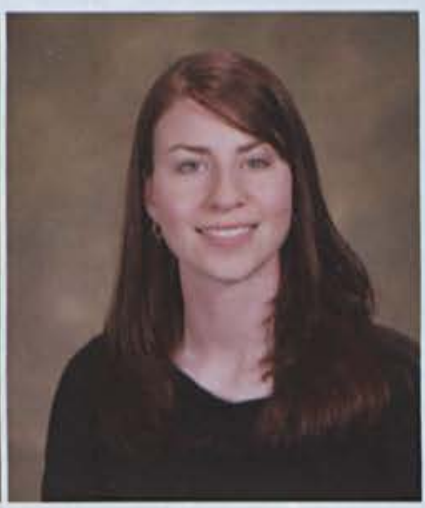

Elizabeth M. Slavish Early Childhood Education

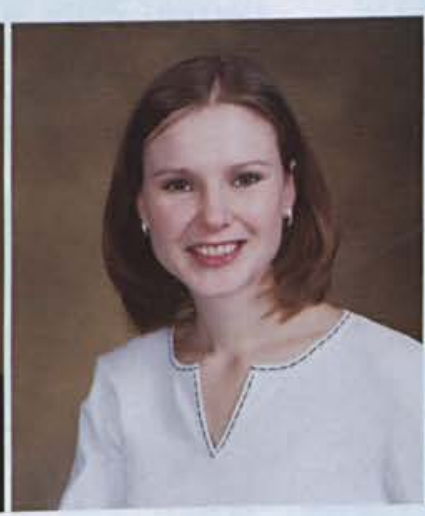

Samantha Marie Slusher Nursing

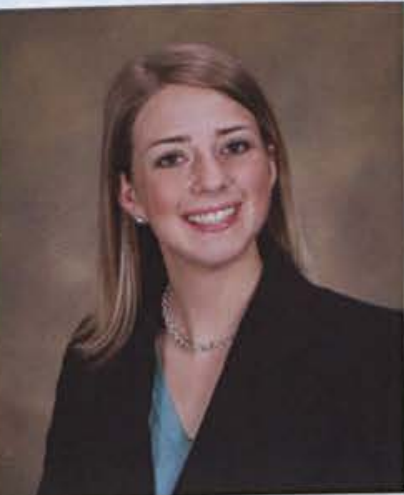

Kayla Marie Smith Marketing

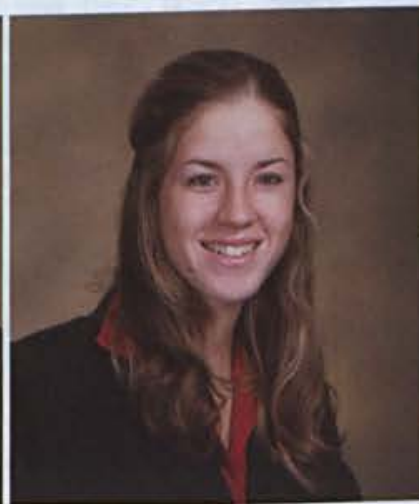

Rachel Elizabeth Solsky Graphic Design

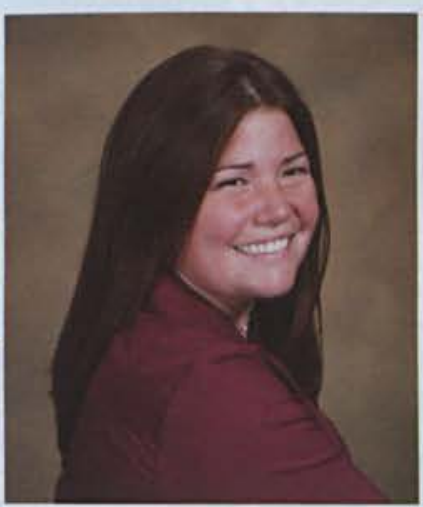

Allison Marie Smith Psychology

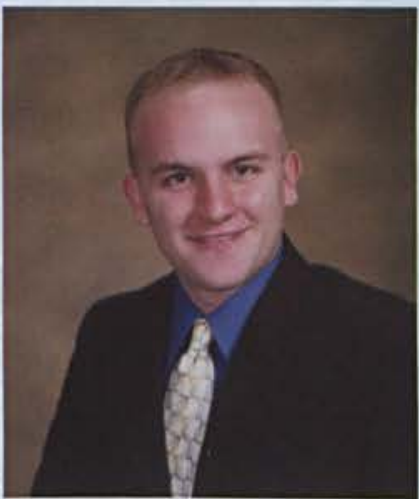

Nathan Daniel Smith Management

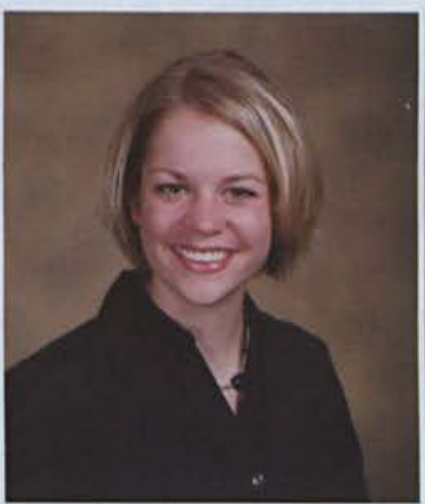

Andrea Laurel Smith Nursing

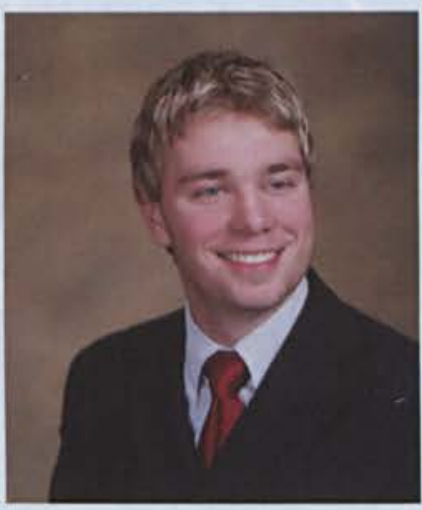

Cody Smith Sport Management

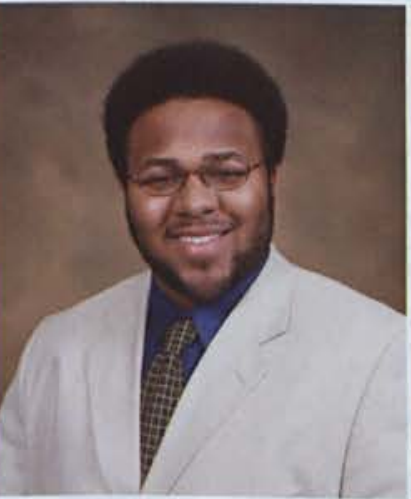

Justin James Spann Management

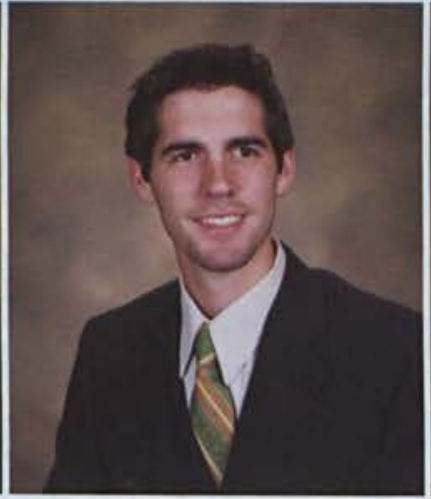

Nathan Paul Smith

Global Economics \& International Business

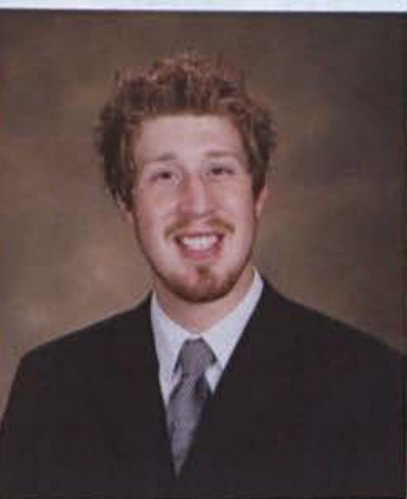

Nathanael Spencer Communication Arts

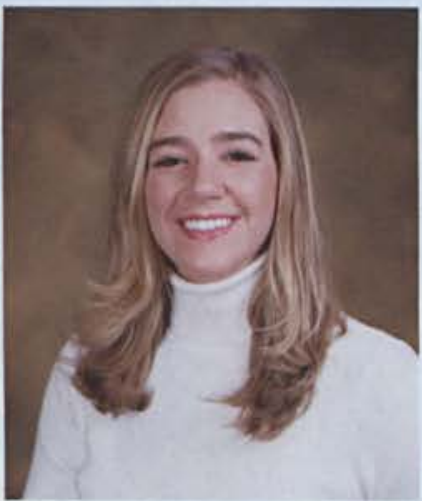

Sarah Adair Smith Multi-Age Special \& Early Childhood Education

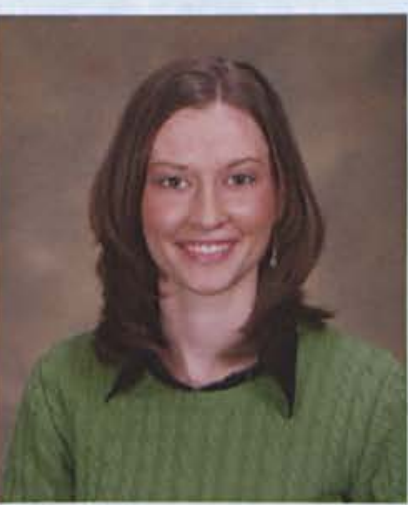

Lacy Louise Sposato Biology

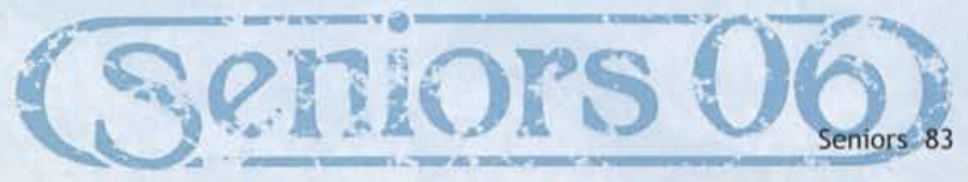




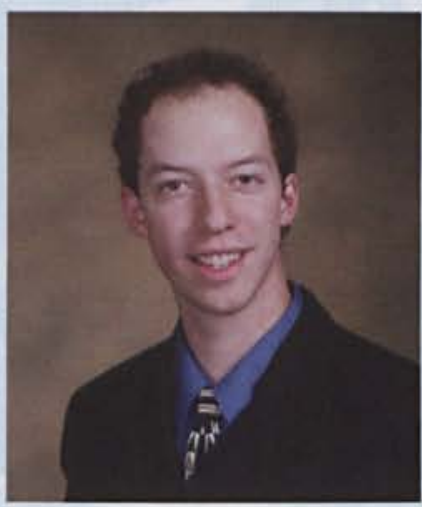

Benjamin Josiah Sprague Computer Engineering

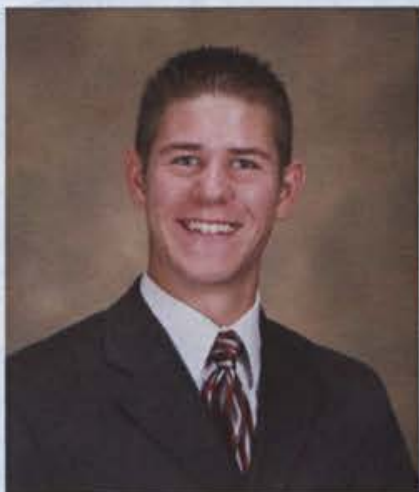

Jason Carl Stephens Biology

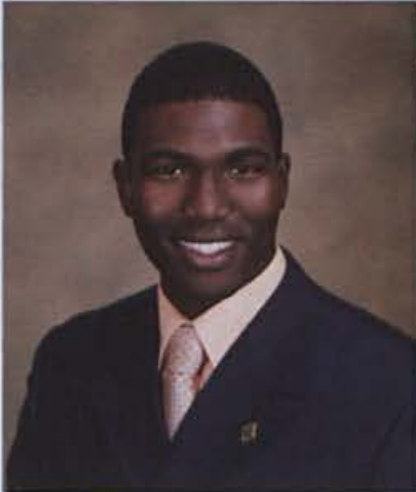

Walter R. Strickland II Comprehensive Bible

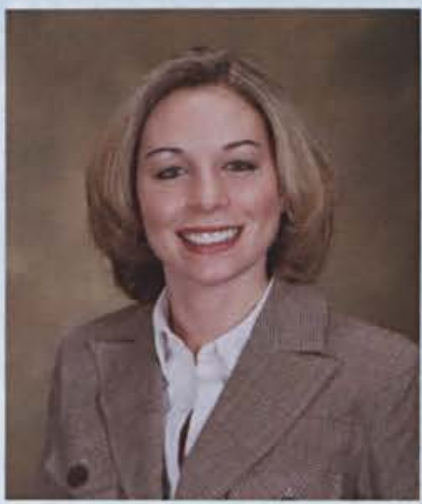

Christina Marie Taylor Accounting

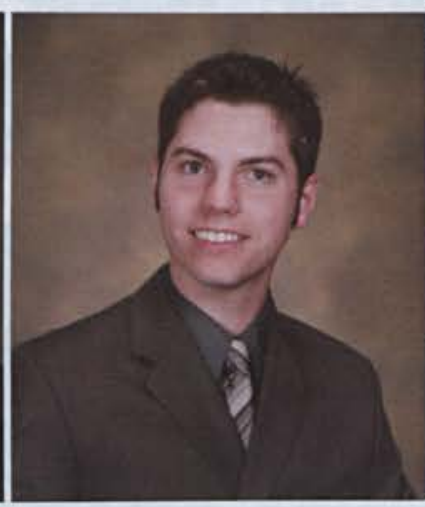

Ryan David Stalker History \& Political Science \& American Studies

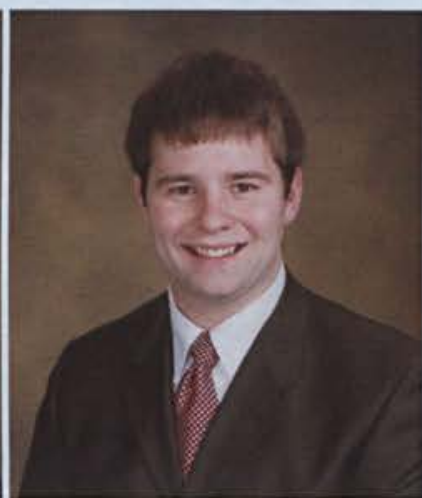

Jeremiah George Stephens Pre-Seminary Bible

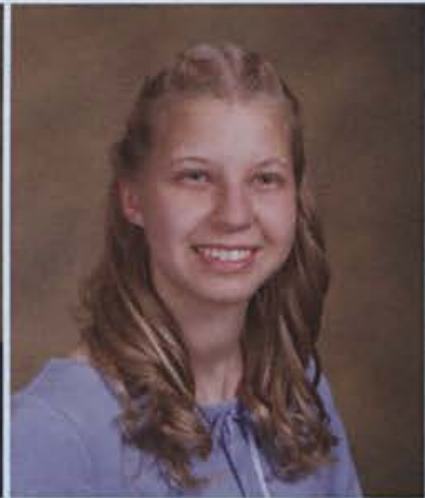

Stephanie R. Struebel Pre-Seminary Bible

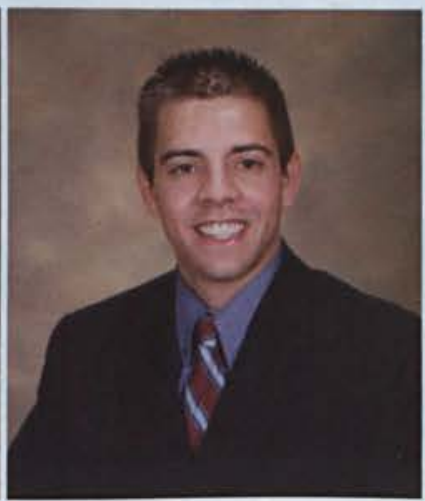

Jonathan Ross Taylor Finance \& Marketing

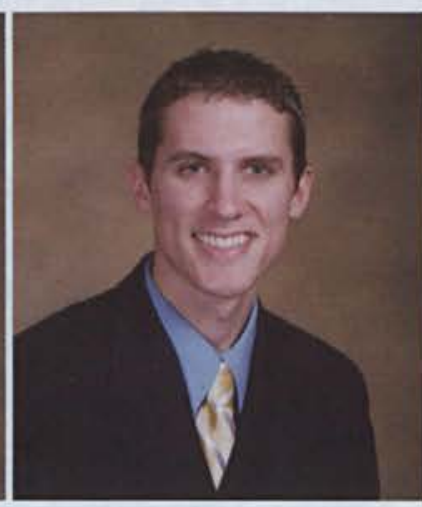

Michael Stuart Standish Youth Ministry

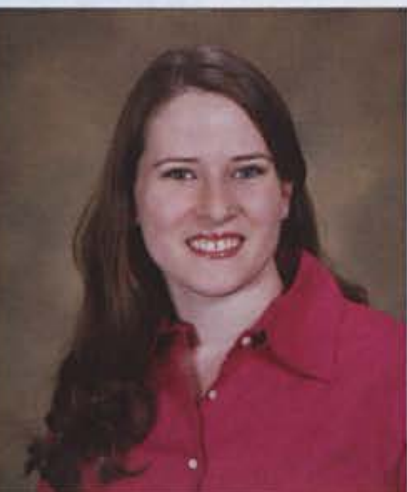

Kristen Marie Stewart Multi-Age Spanish Education

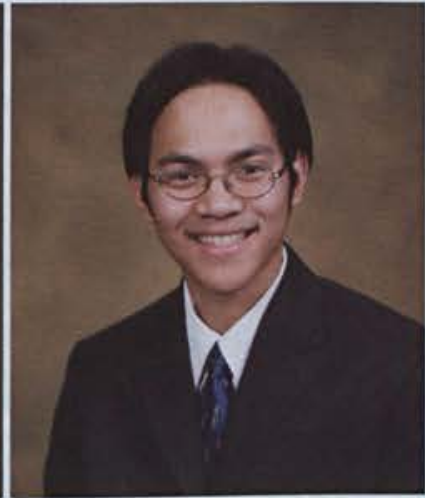

Mark Timothy Sue Church Music Ministries

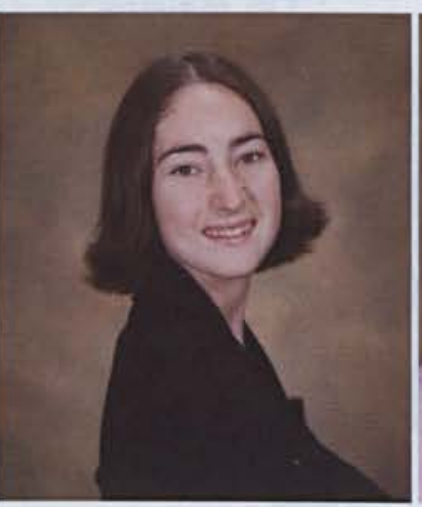

Bethany Anne Staten

Organizational

Communications

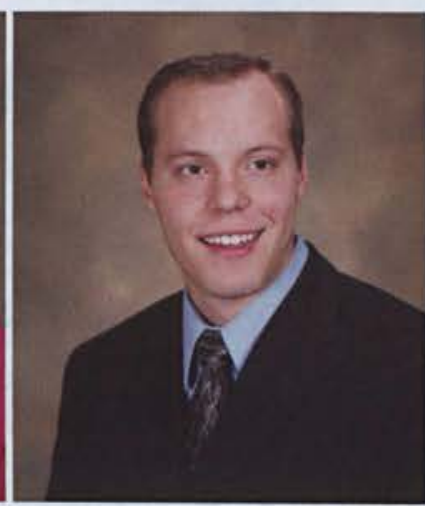

Timothy Aaron Storer Electrical Engineering

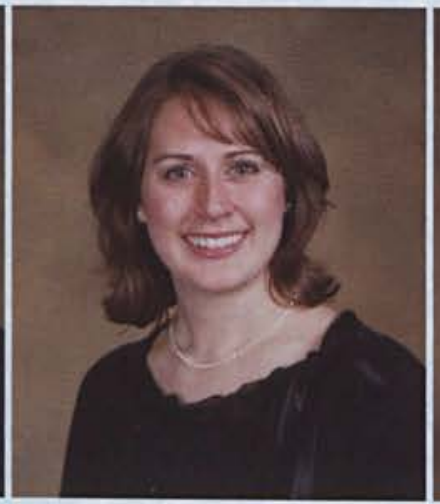

Rachel Maureen Talbott Music Performance

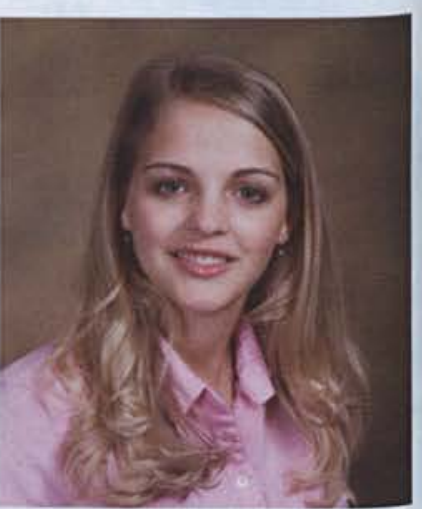

Emily G. Stephens

Special Education \& Early Childhood Education

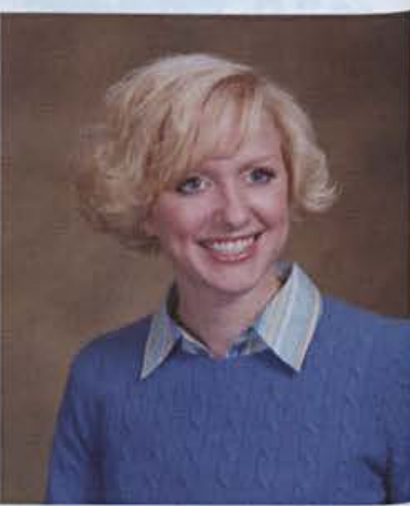

Bethany L. Streitmatter Marketing

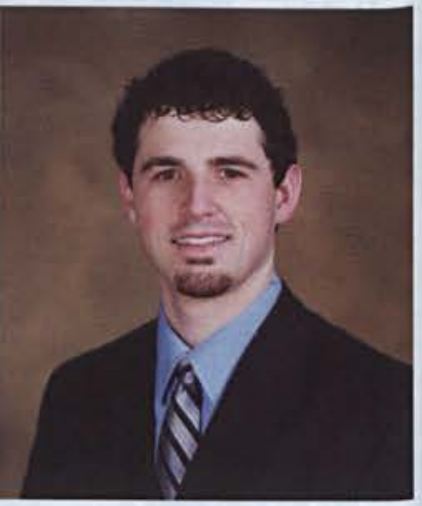

Nate L. Tallman Mechanical Engineering

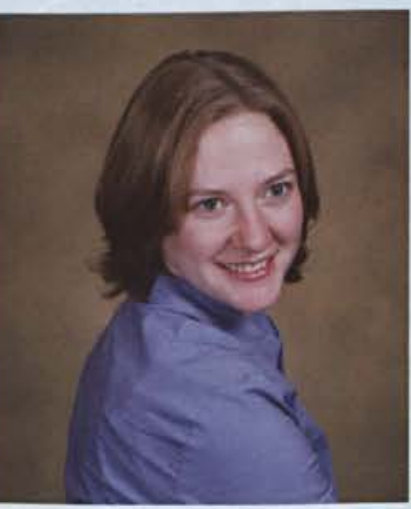

Rachel Ann Tellish Nursing

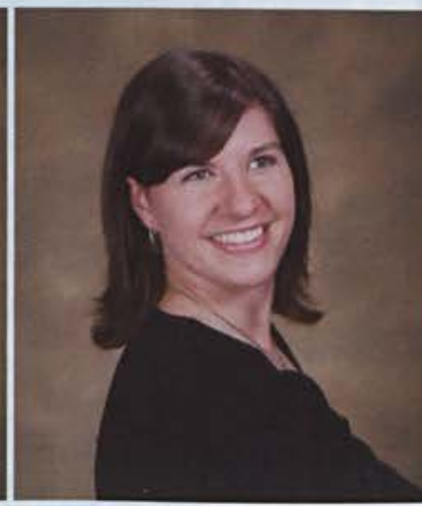

Lana Marie Terpstra Nursing

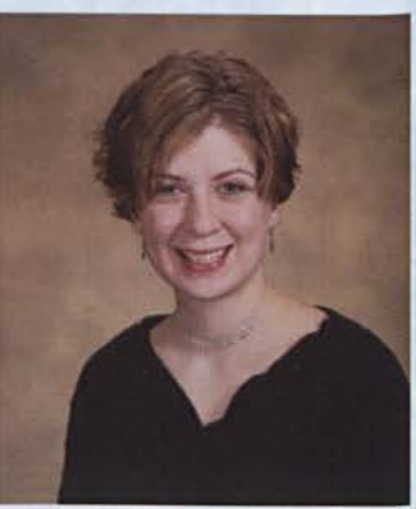

Melody Jolynn Terry Nursing 


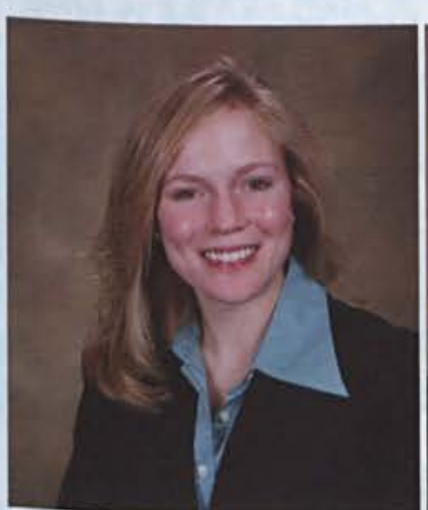

Molly Grey Thayer Management

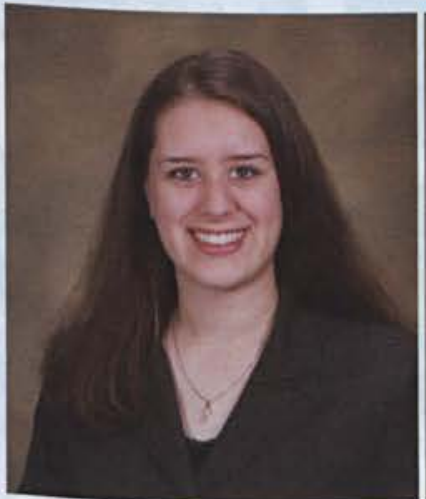

Sara Kathleen Thompson Early Childhood Education

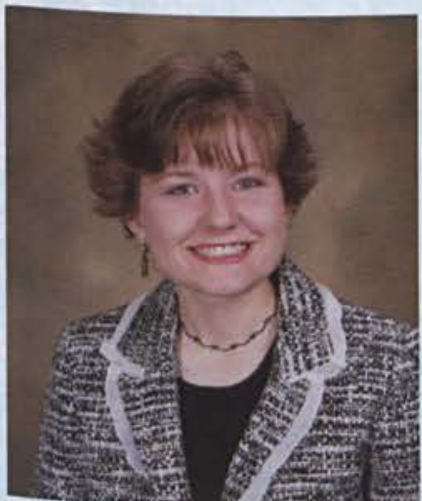

Danielle Nicole Tisdale Early Childhood Education

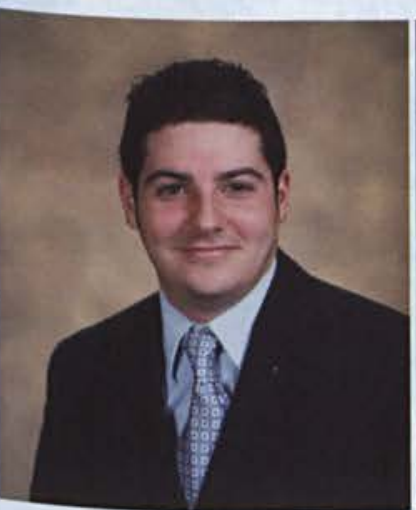

Dustin Michael Tursack Marketing \& Management

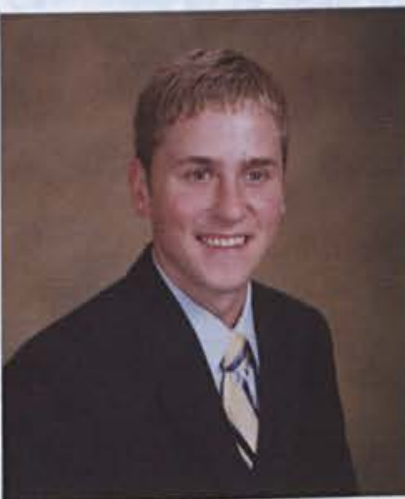

Jonathan Matthew Thomas Marketing \& Management

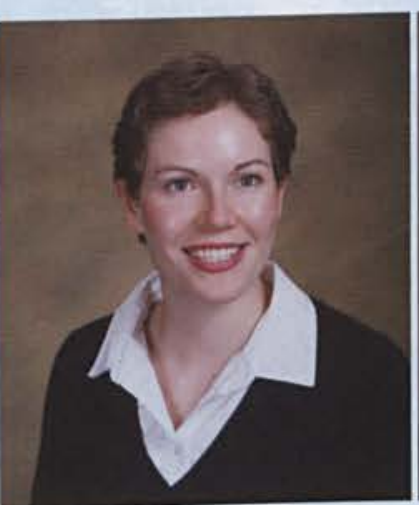

Teah Margaret Thomson Theatre

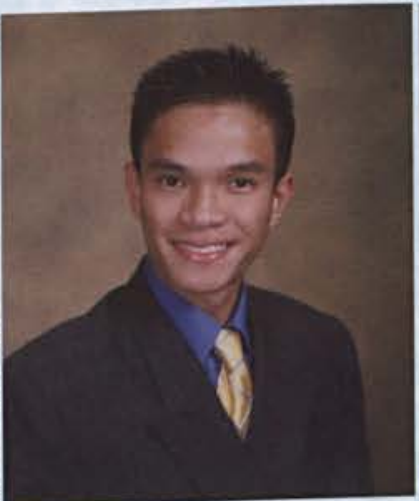

Roleigh Tuazon International Studies

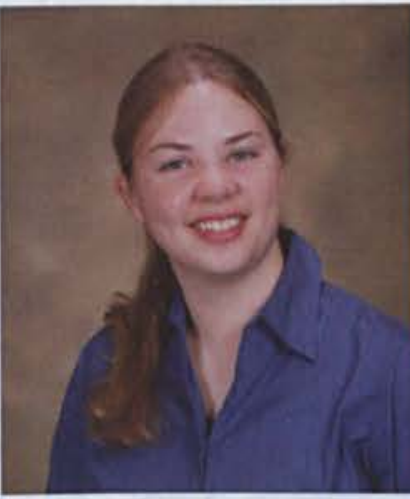

Michele Renee Thomas Electronic Media

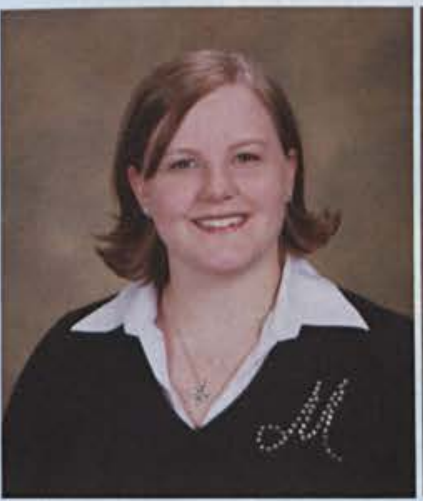

Megan Ashley Thornburg Communications

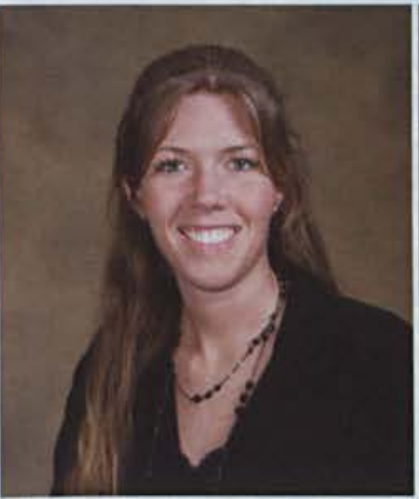

Stephanie Lynn Tucker Graphic Design

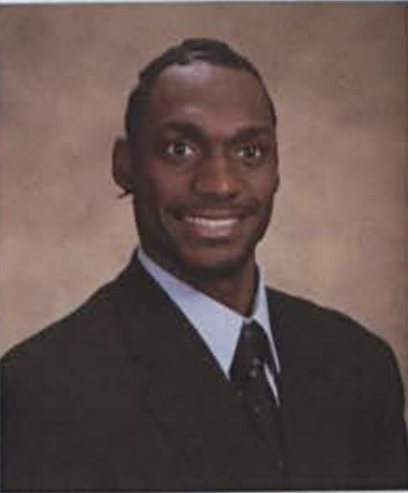

Mugabe Mussia Thomas Comprehensive Communications

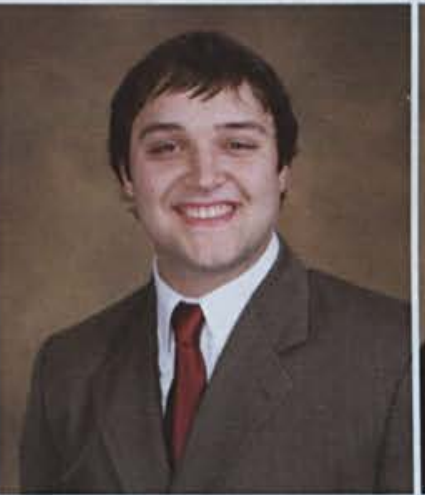

Kipp Tiger

Chemistry

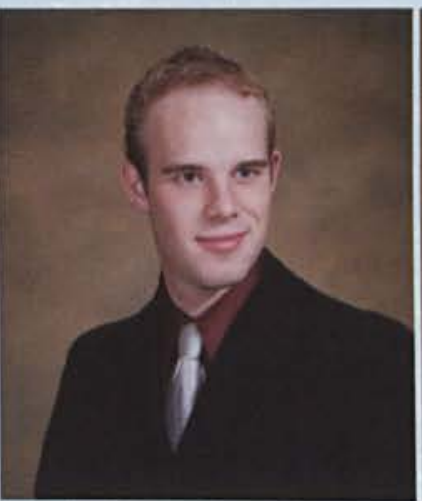

James Michael Tullett Management

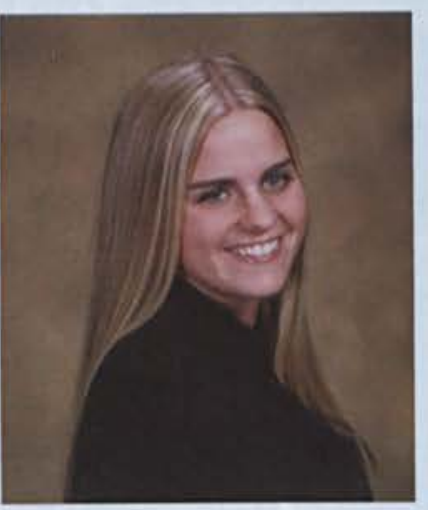

Katherine $\mathrm{E}$. Thompson Nursing

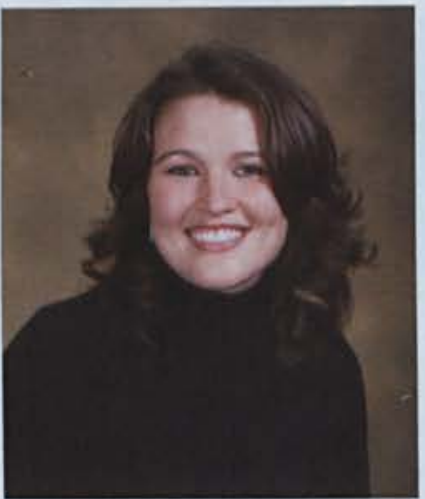

Rachelle Marie Tipton Middle Childhood Education

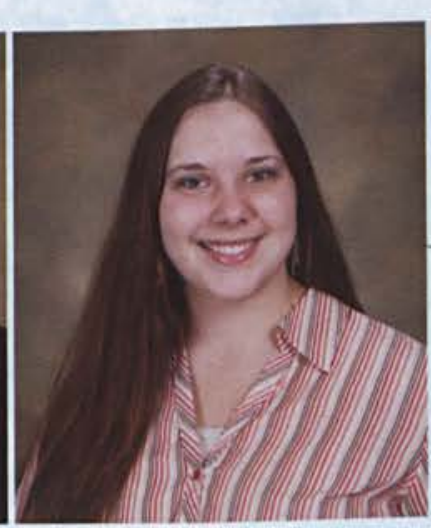

Crystal Lea Tuxhorn Theatre \& Graphic Design

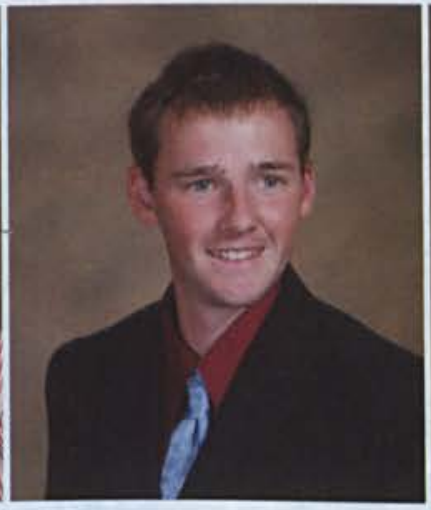

Seth Ivan Unruh Management \& Finance

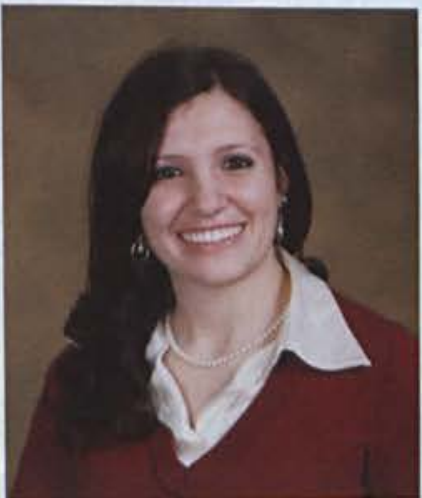

Amanda Valenti Psychology

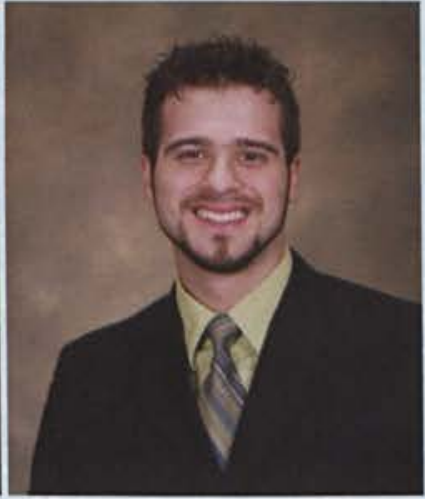

Stephen L. Turkovich Marketing

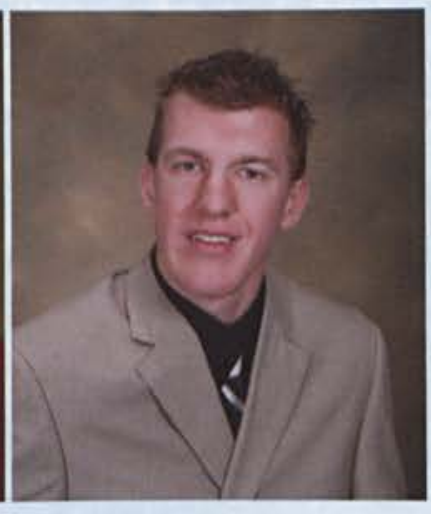

Andrew Caleb Van Curan Accounting

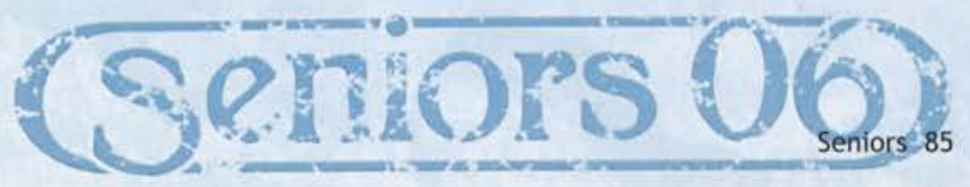




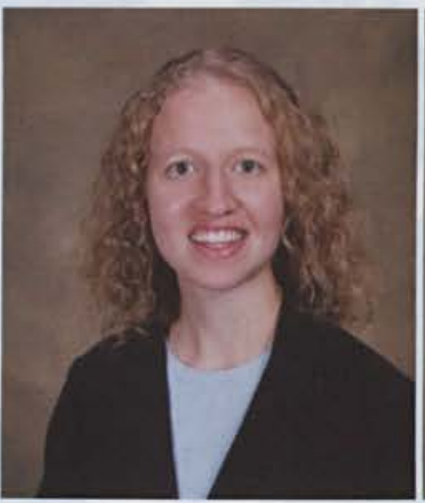

Megan Joy Vance Nursing

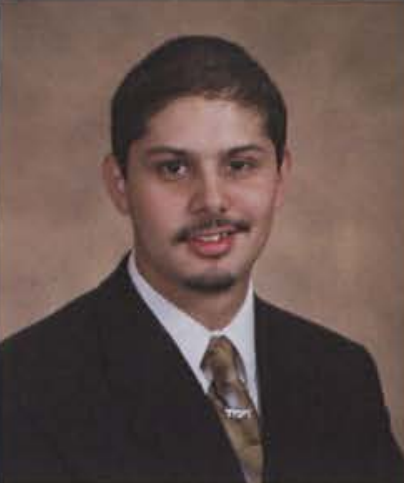

Richard E. Velilla II Computer Science

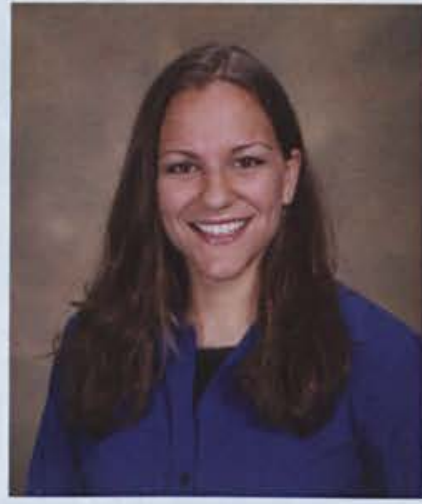

Kimberly Joy Volpe Nursing

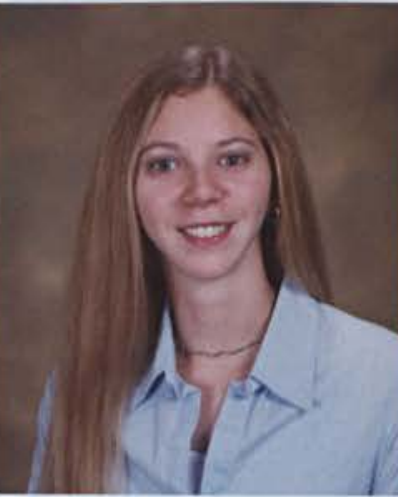

Diane Elizabeth Wagner Early Childhood Education

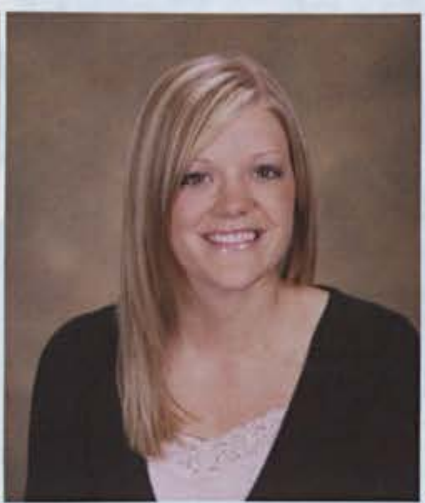

Sarah Joy VanWinkle Social Work

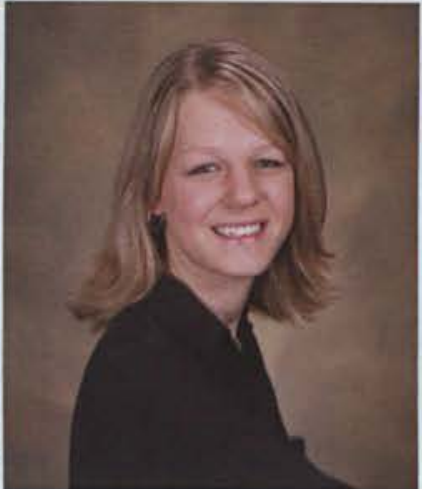

Rachel Erin Verosky Early Childhood Education

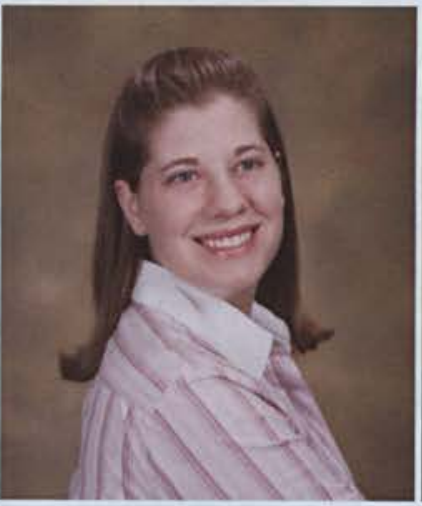

Lindsey A. Von der Mahden Finance

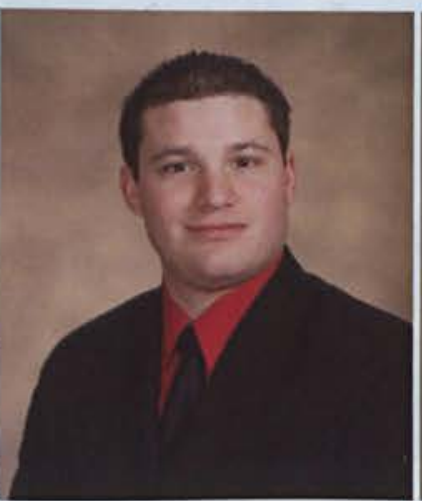

Athan Austin Waldron Mechanical Engineering

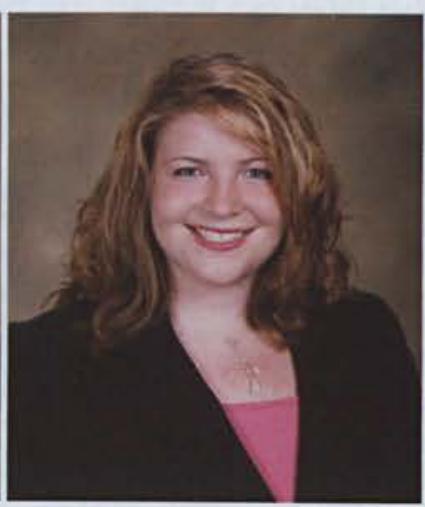

Kate Lauren Varney Psychology

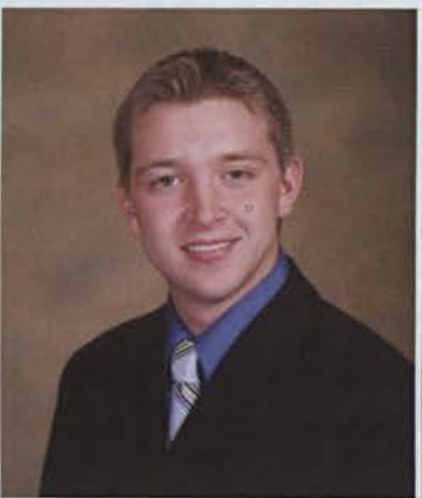

Chad David Viront Youth Ministry

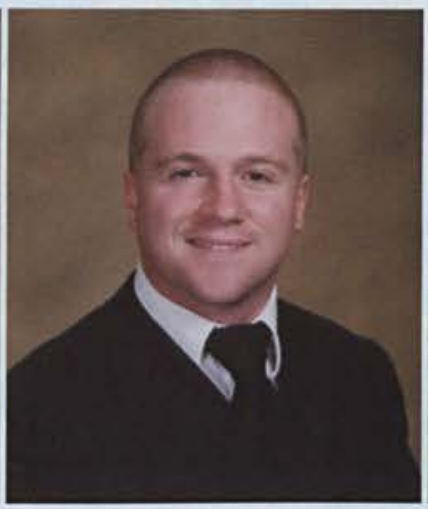

Benjamin Ray Waddell Psychology

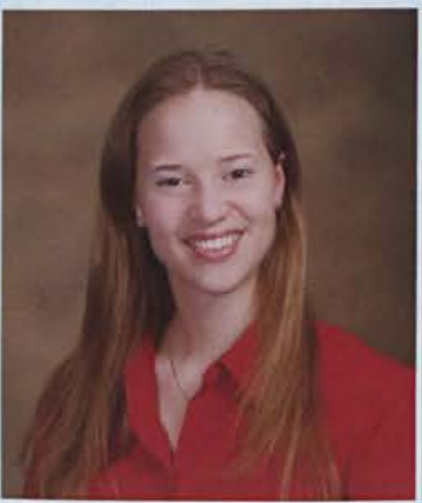

Catherine Kay Walker Early Childhood Education

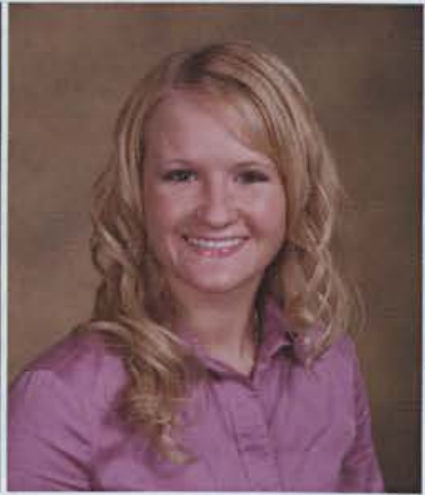

Ashley Dianne Varvel Graphic Design

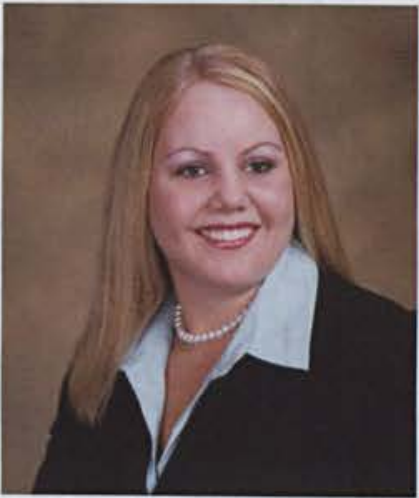

Hannah Edith Vitt Physical Activity \& Sport Studies

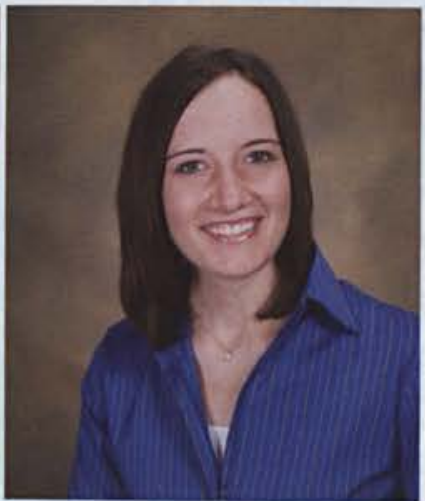

Jessica Joy Waggoner Nursing

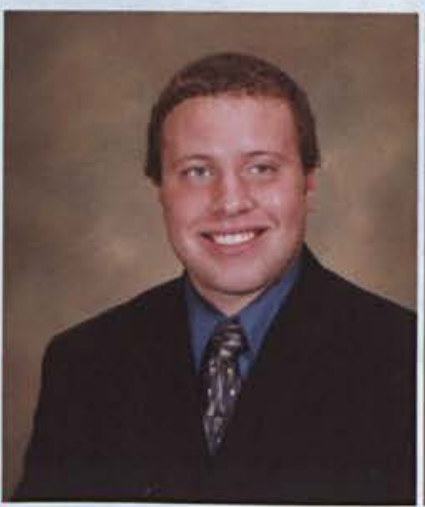

Nicholas George Walker Pastoral Studies

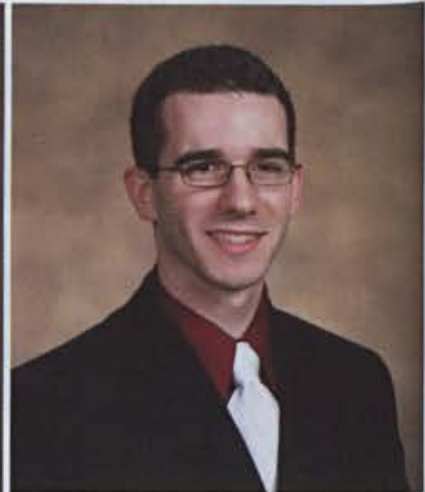

Kevin James Vaughn Piano Performance

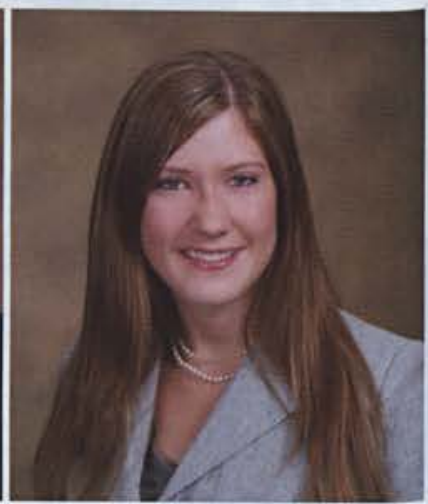

Carol Ann Vollnogle Early Childhood Education

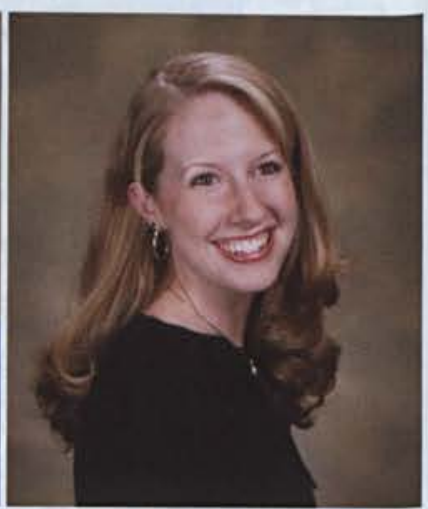

Jessica M. Waggoner Music

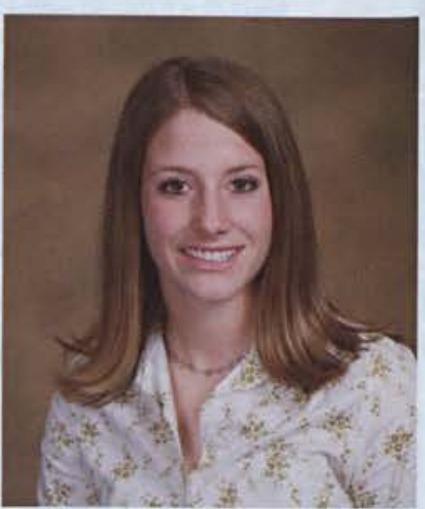

Katharine Nicole Walter Middle Childhood Education 


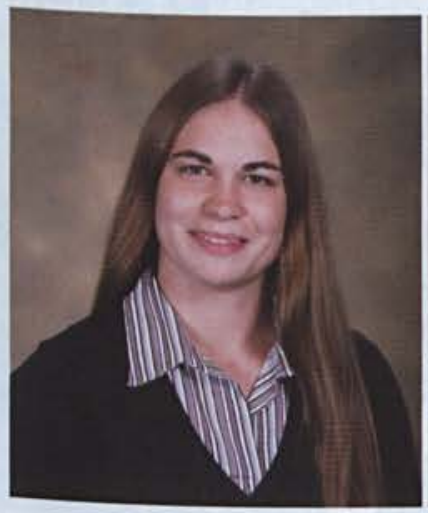

Melissa Marie Walter Social Work

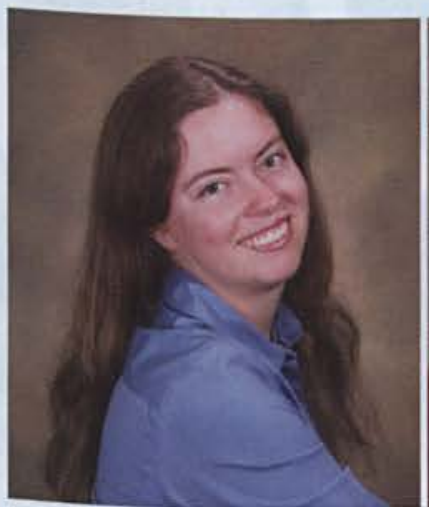

Mallery Anne Waters Individualized Studies

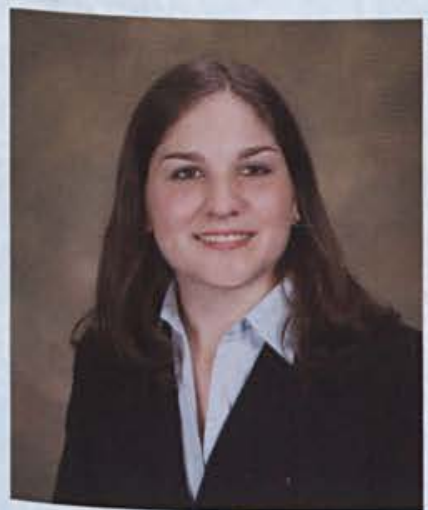

Natalie Eve Weil Mechanical Engineering

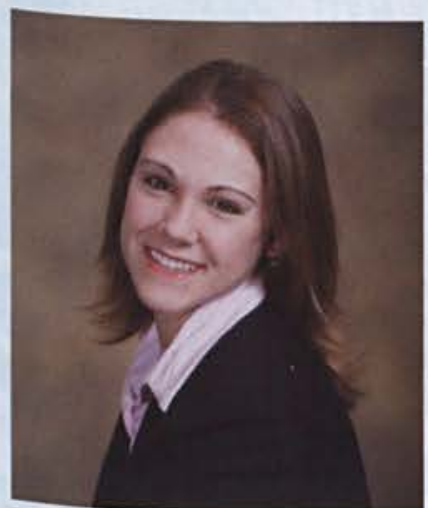

Andrea Hope West Biology \& AYALA

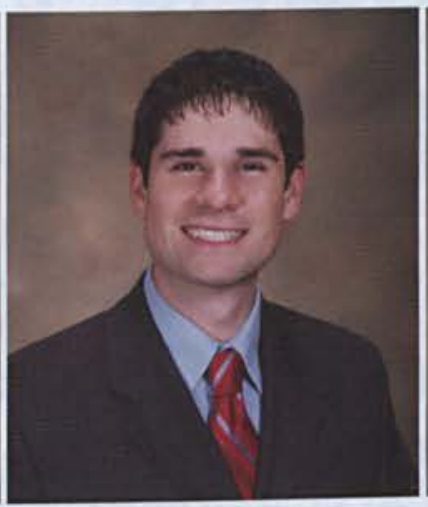

Nathaniel Eric Walter Accounting \& Management

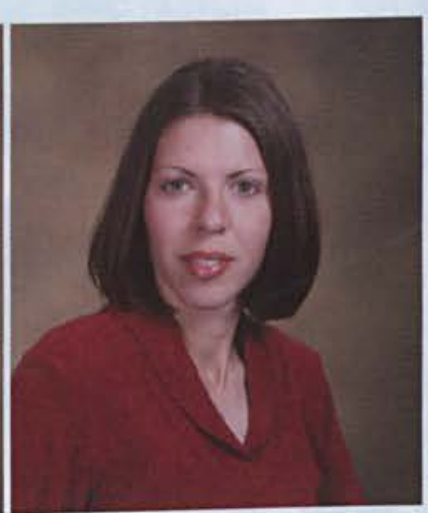

Krista Rene Watson Psychology

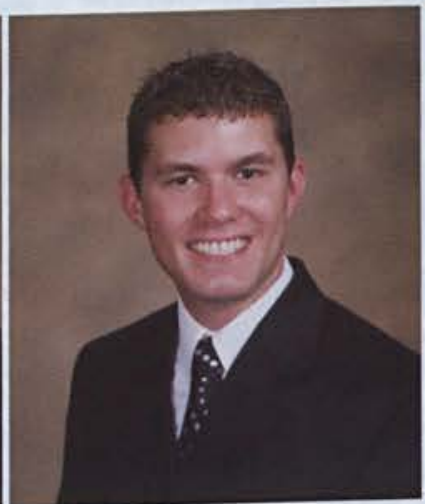

Anthony John Weleski International Studies

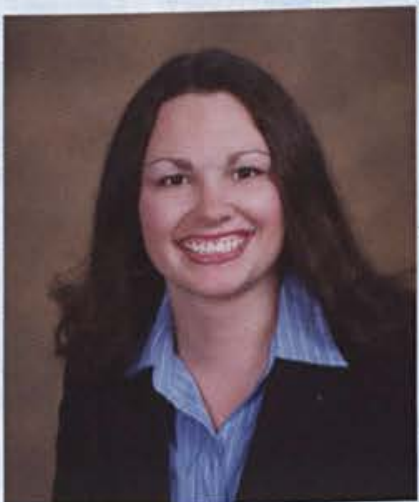

Autumn Elaine West History \& Political Science

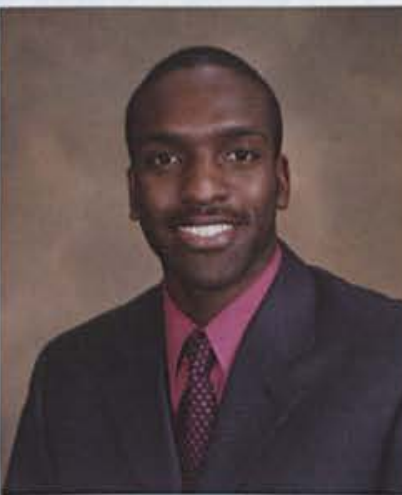

Samuel Mwangi Wanderi Management Information Systems

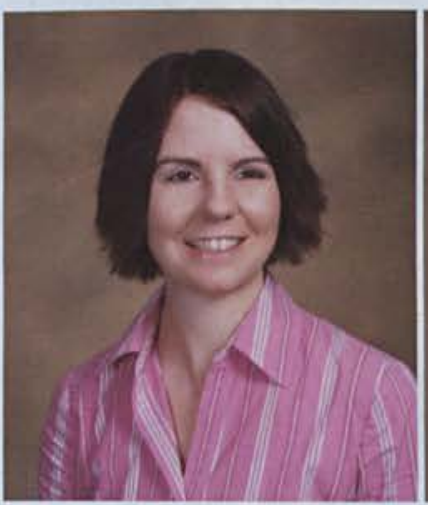

Megan Rose Watson Finance

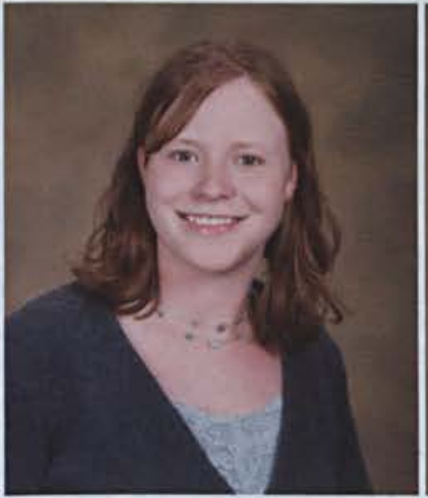

Jillian Leigh Weleski Organizational Communications

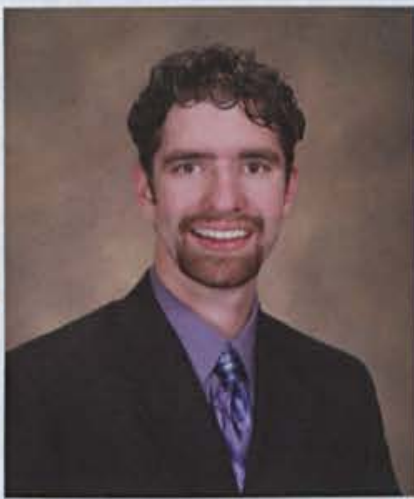

Robert Frederick Ward Accounting

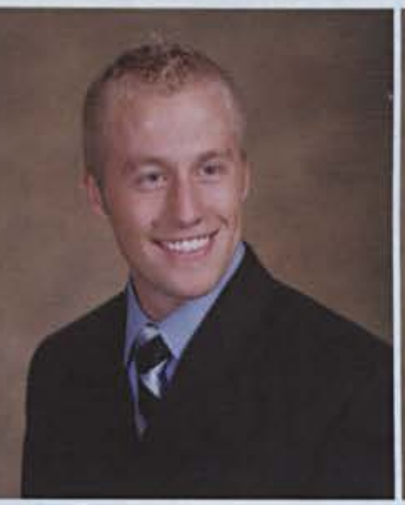

Nathan C. Weeber Finance

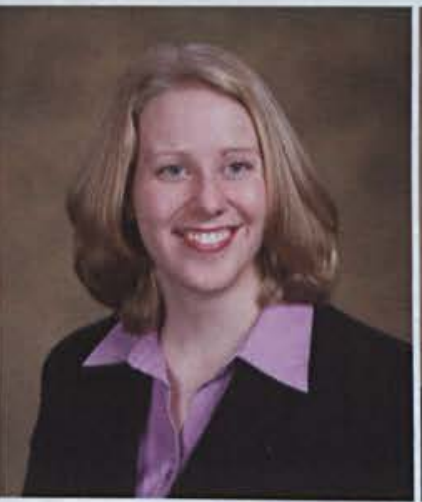

Rebekah Ann Wells Nursing

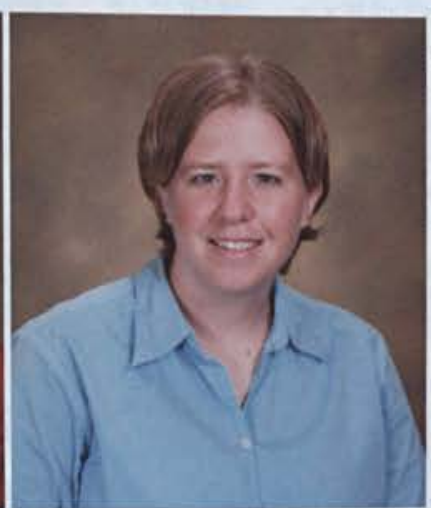

Scott Whitaker

Pastoral Studies

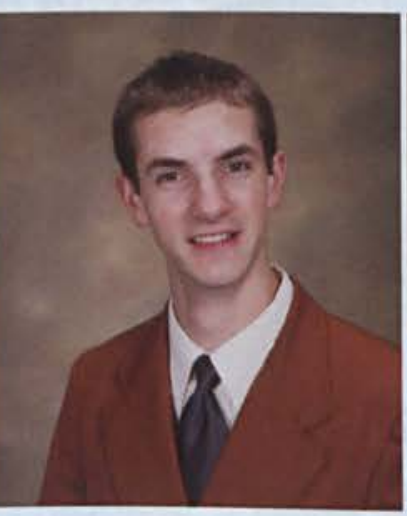

Charis Elizabeth Whitney Biology

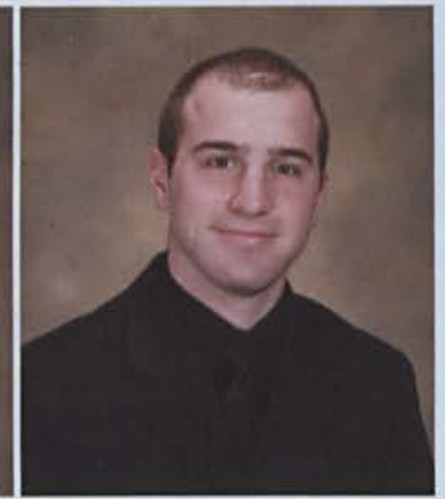

Jonathon Lee Wasson Pre-Seminary

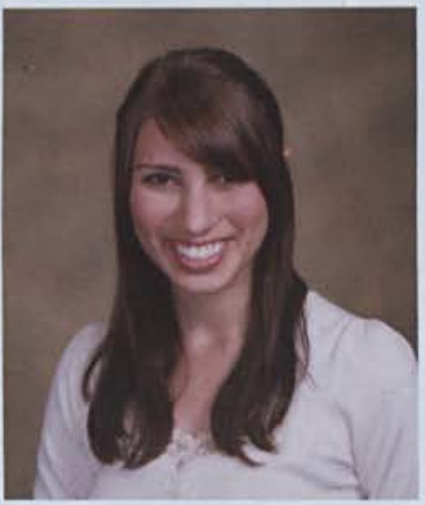
Communication Arts

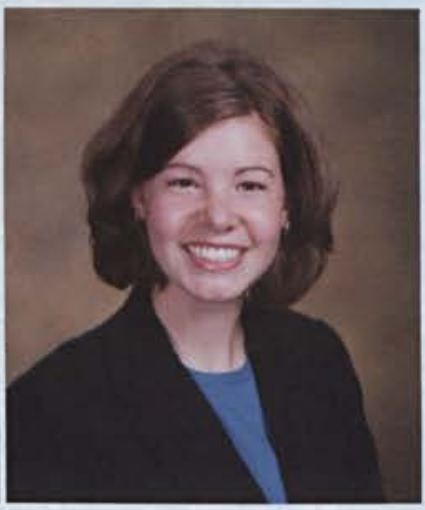

Allison Ruth West Graphic Design

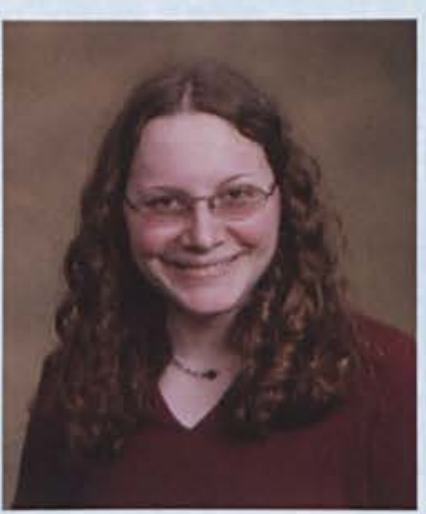

Rebecca Grace Widener Nursing

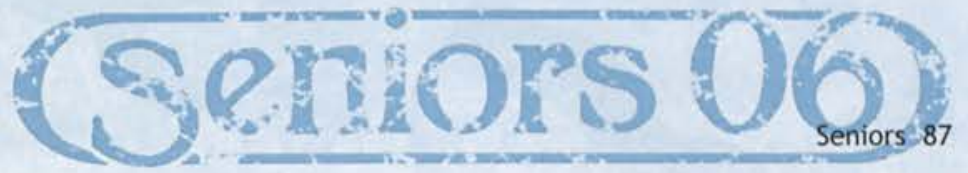




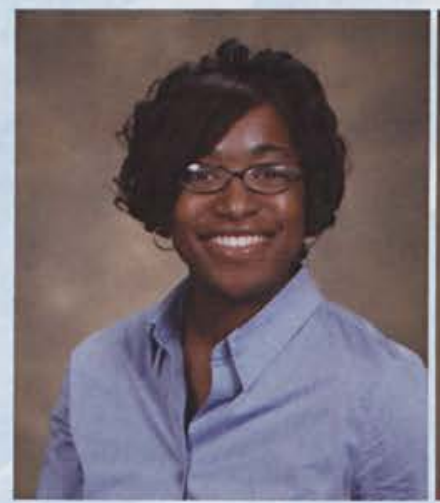

Crystal Jacquel Wiley Sport Management

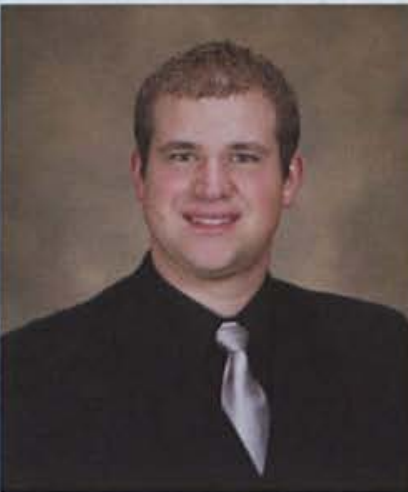

Nathan A. Williams Nursing

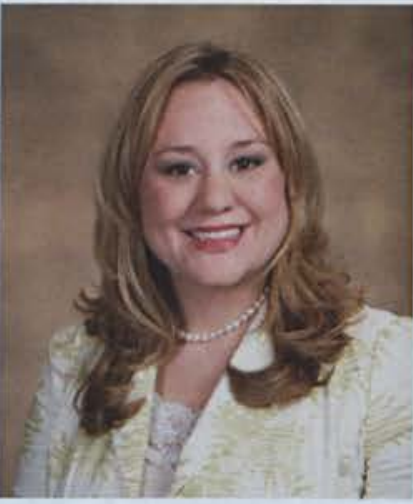

Alissa B. Wingenroth Marketing

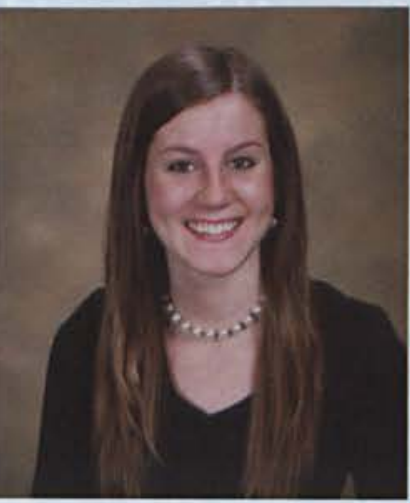

Julie Yinger

Graphic Design

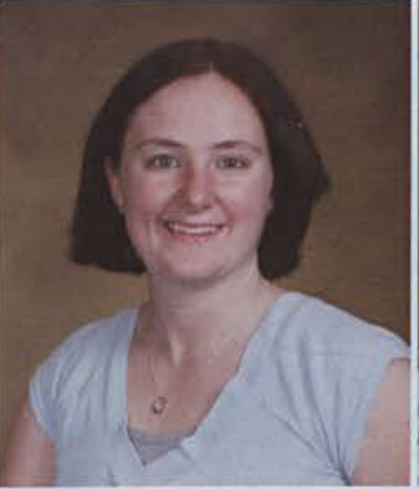

Michelle Renee Wilkey World Missions

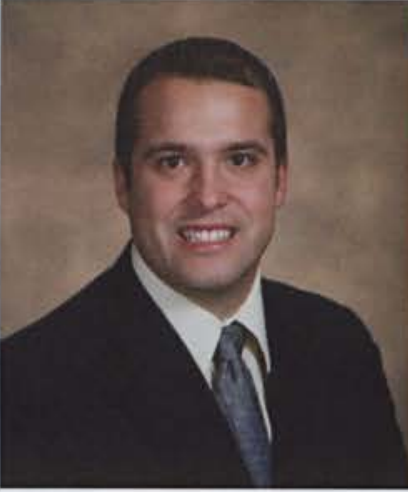

Matthew Lawrence Wilson Athletic Training

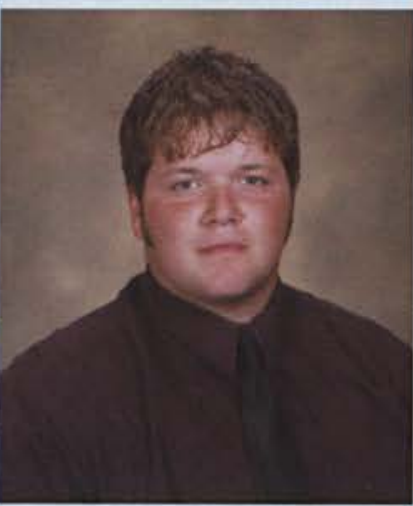

Adam Kent Wise Sport Management

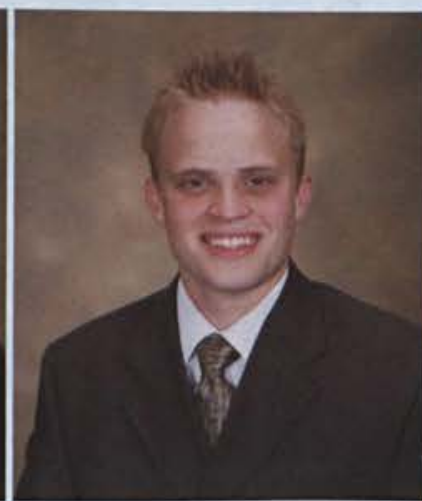

Benjamin Harry Zemmer Electronic Media

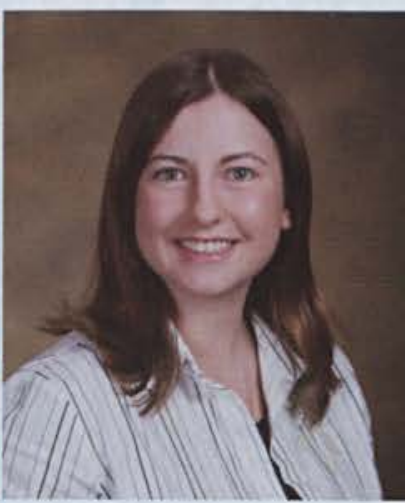

Angela Brooke Wilkinson Chemistry

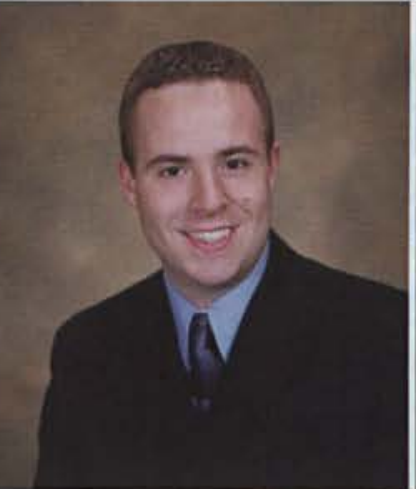

Timothy Steven Wilson Pre-Law

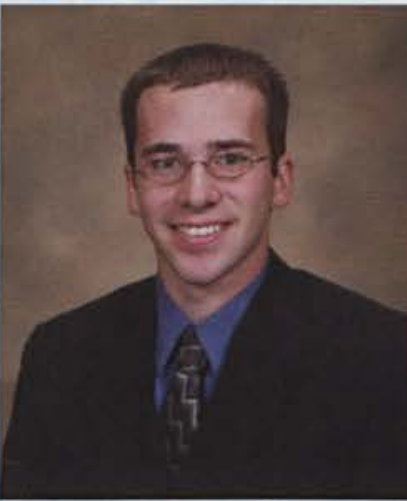

Richard Michael Woordward Mechanical Engineering

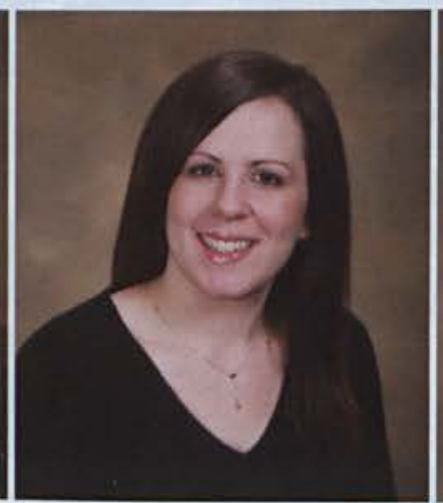

Kristen M. Zeppenfeld Psychology

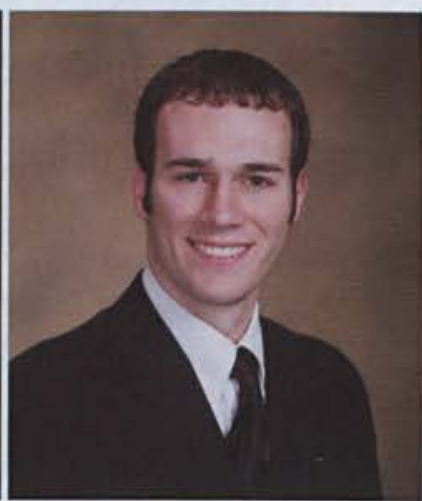

Jeremy Dean Willetts Pre-Seminary Bible

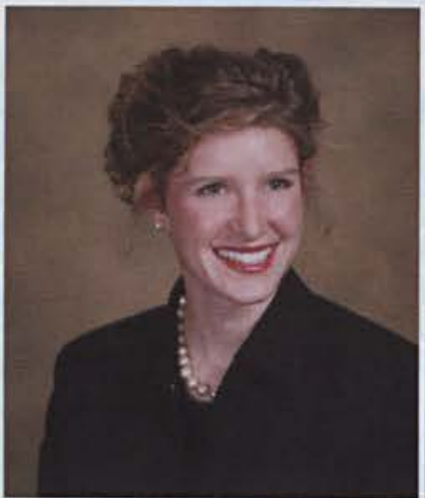

Abigail Jane Winburn Nursing

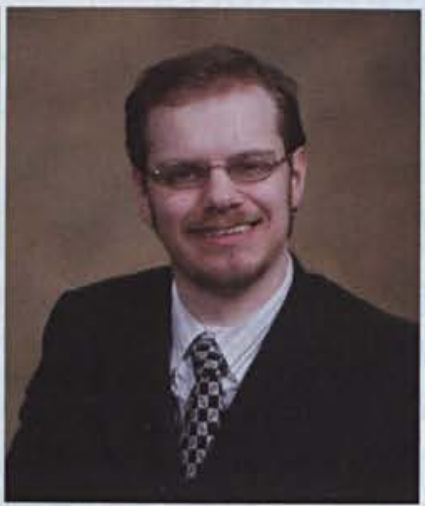

Louis Edward Yaklich III History

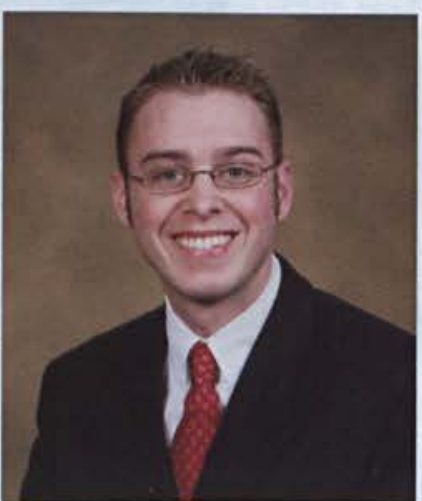

Grant Zerkle Pre-Law

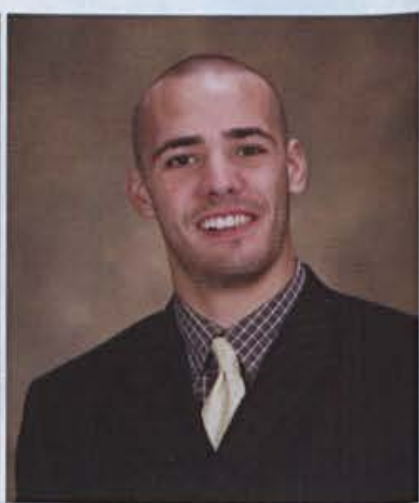

Jordan Williams Missions

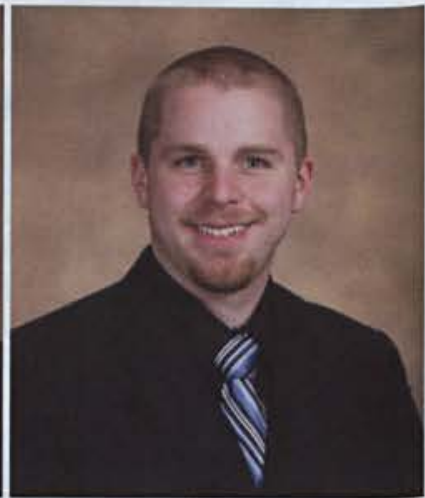

Timothy Chad Winder Church Music Ministries

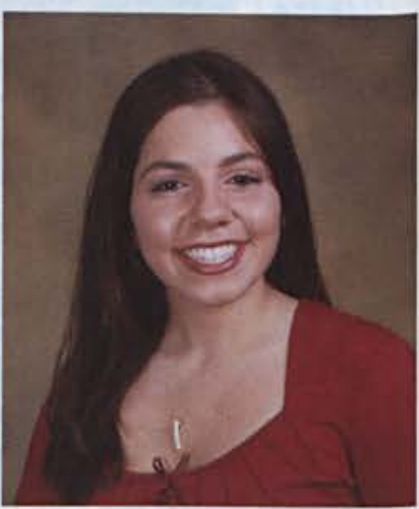

Mirshad Yibrin Nursing

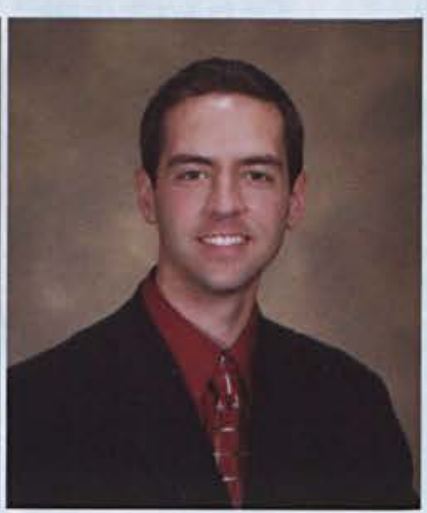

Michael Lee Zerminski Mechanical Engineering 

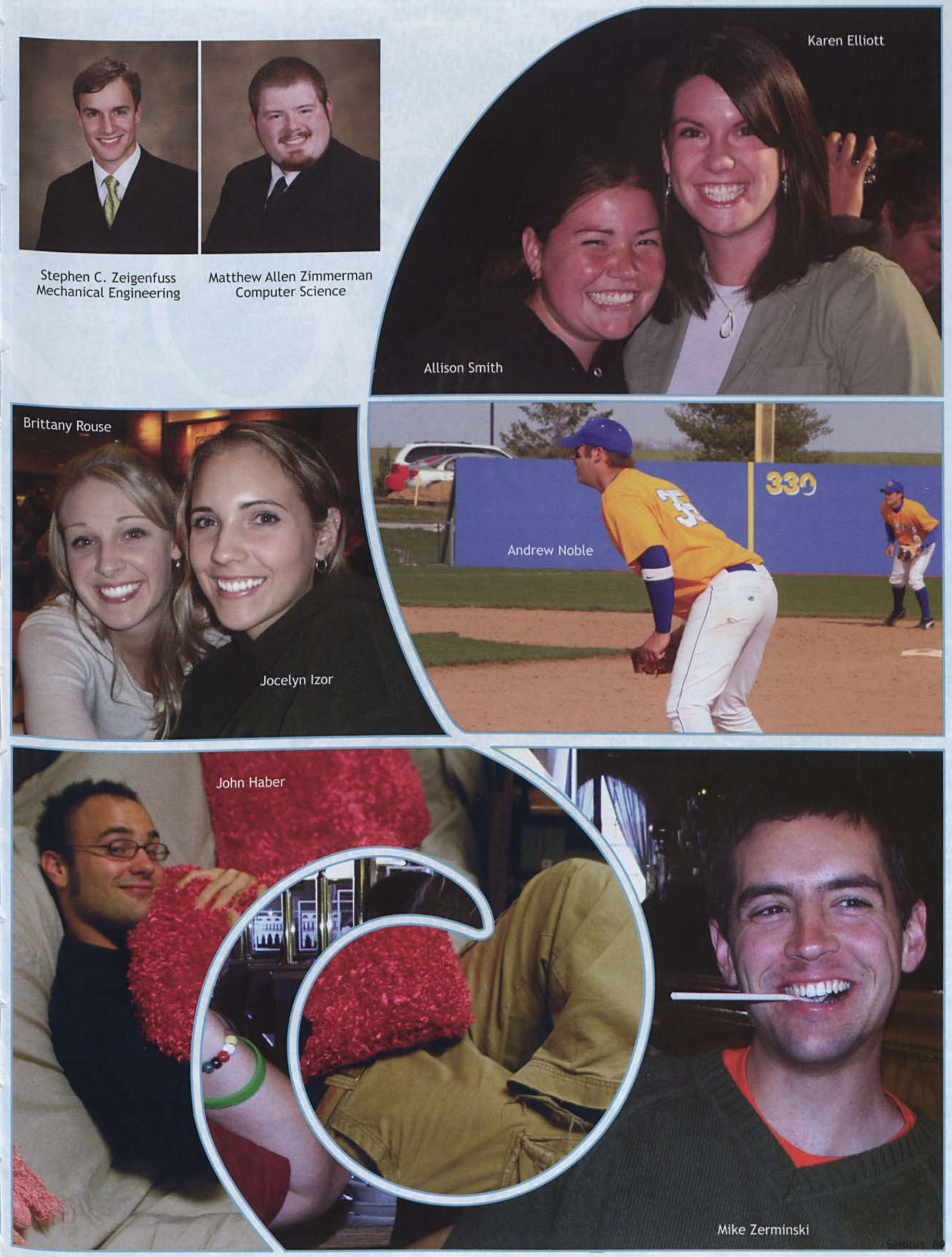

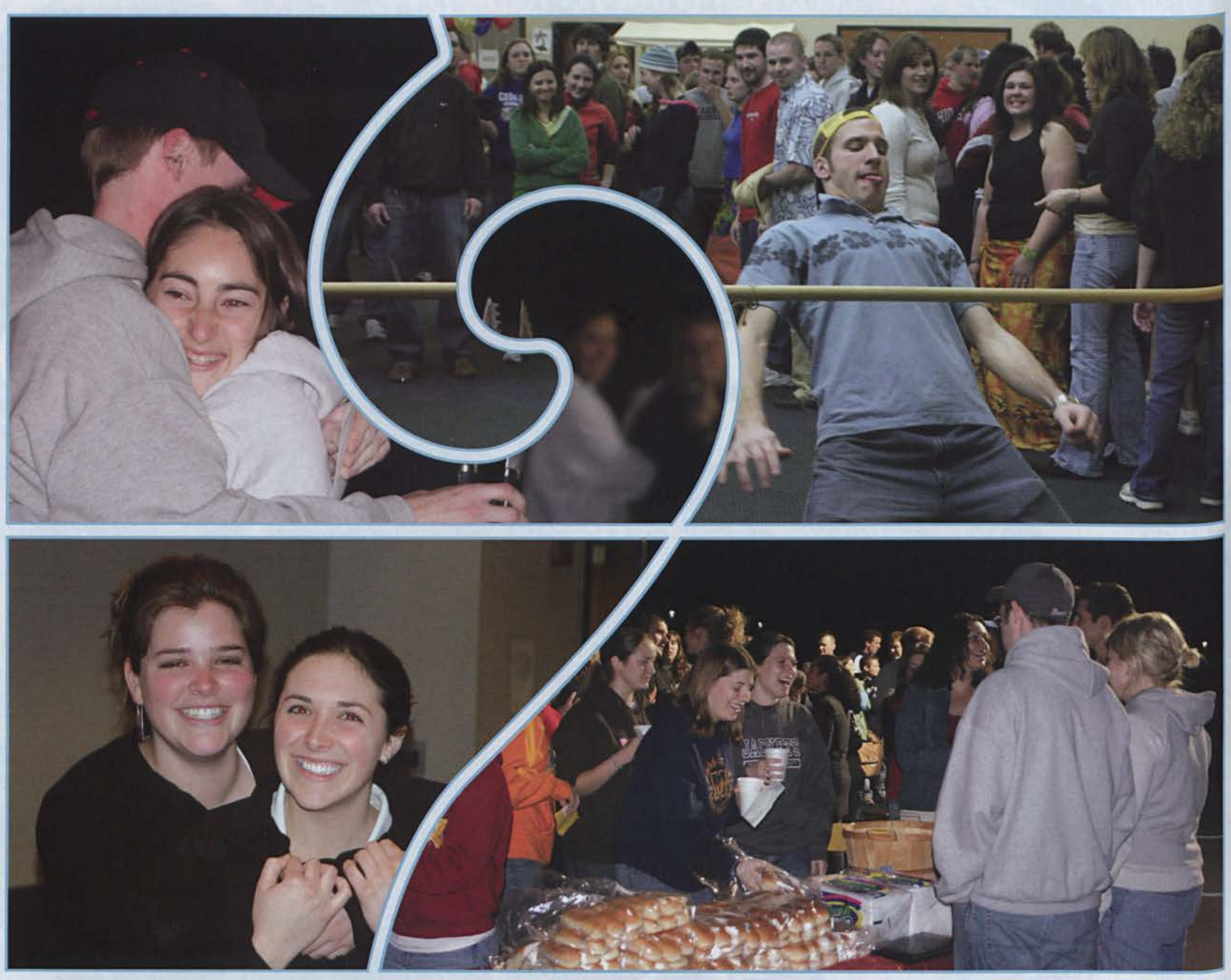

^ Julianne Null and Katie Krumeich relish ther friendship they have developed in their days at the 'Ville.

* The last days on campus for seniors always include those touching moments that just beg for a hug from a friend.

* Limbo! Limbo! The crowd chants as Lamar King bends back under the limbo stick at the senior 100 days party.

Y Seniors enjoy the food and fellowship at the senior party, which was held in the fall on the grounds between the DMC and SSC.

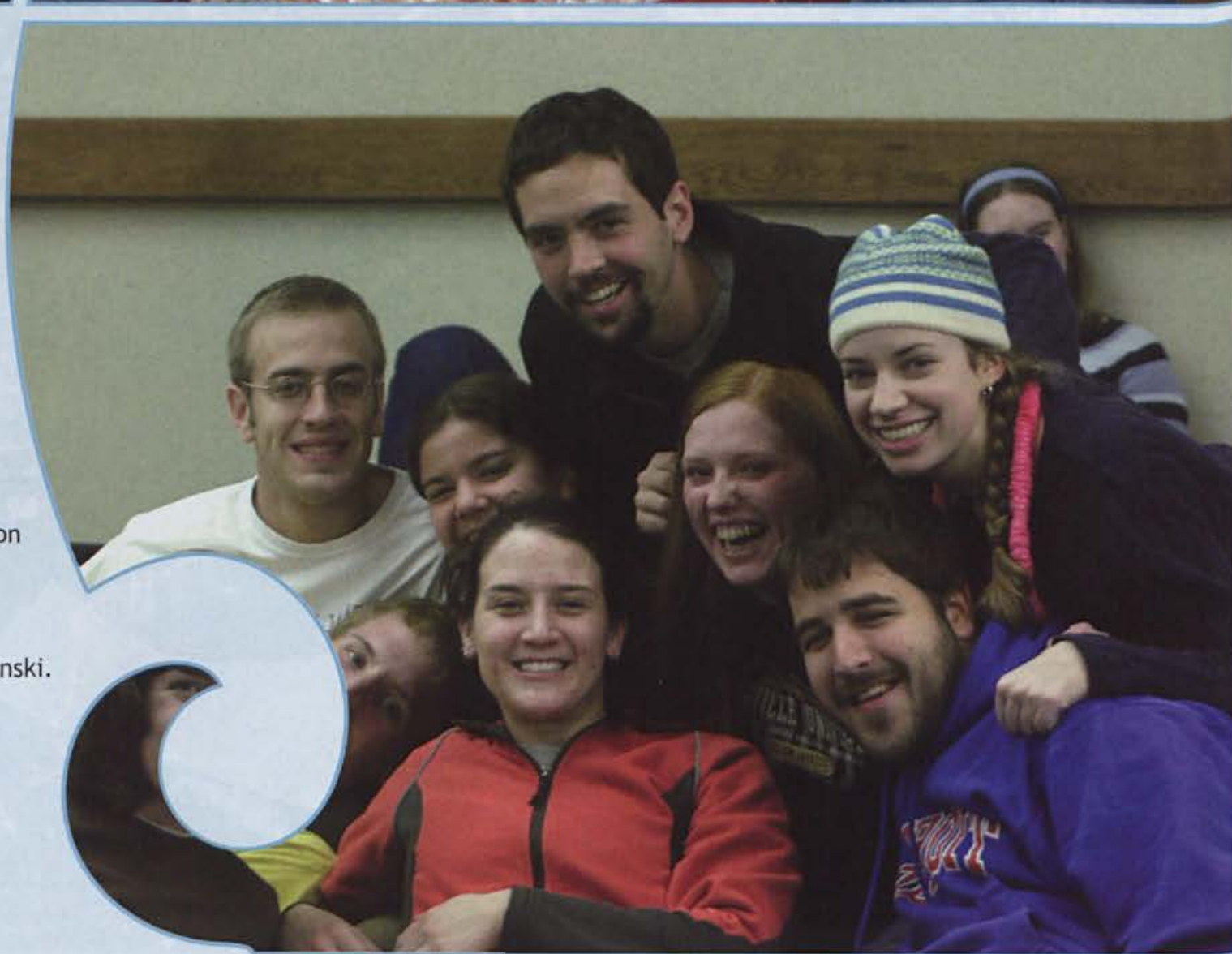

- Tim Hartman, Shannon Picazo, Mike Zerminski. Katie Fickes, Kathy Godinez, Ben Lawrence squeeze together for the group shot. 
Kirsten Ann Gibbs and Tricia Lin Walker, thank

you both for your involvement, support, and help in making our time here such a wonderful experience.
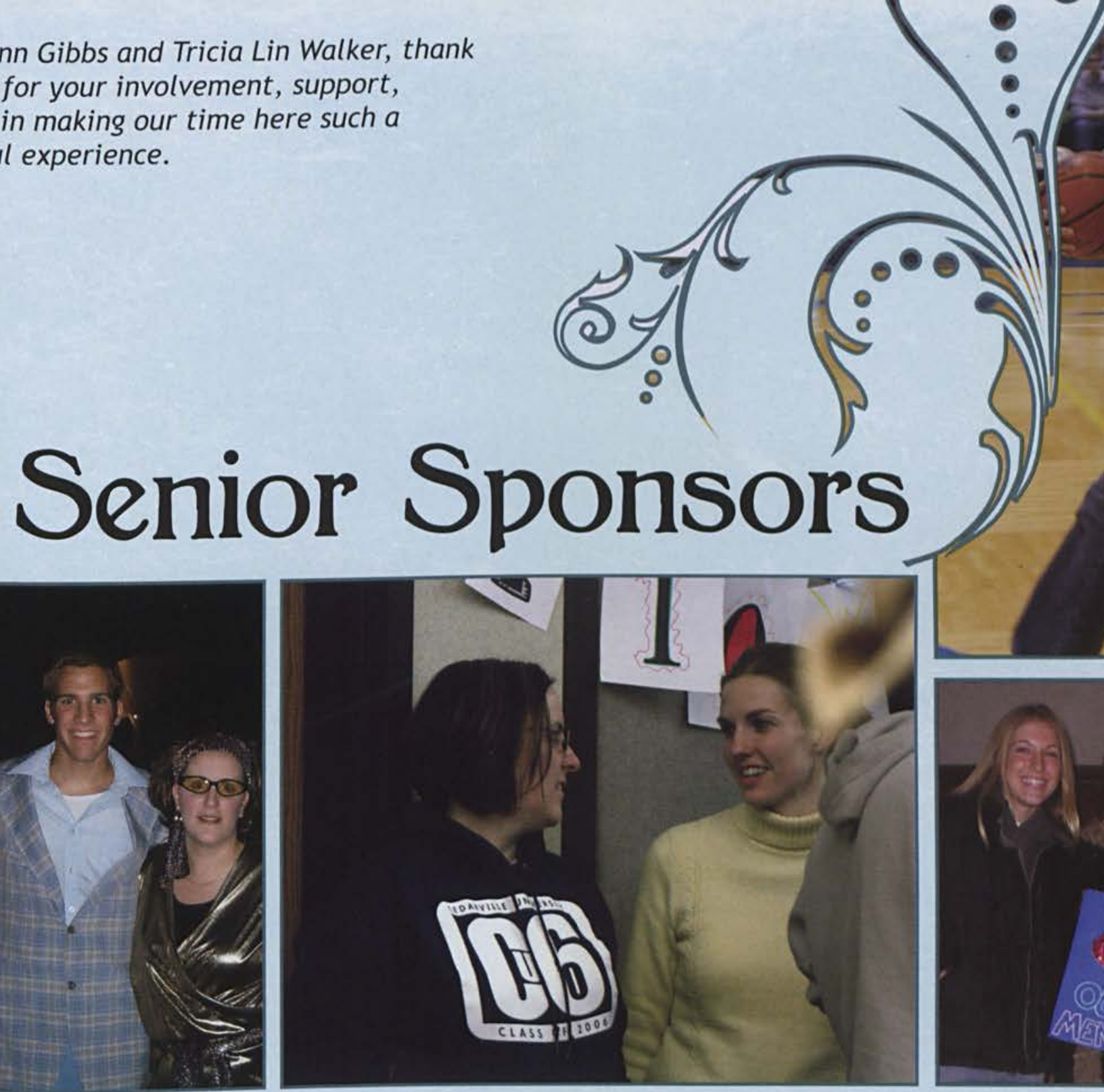


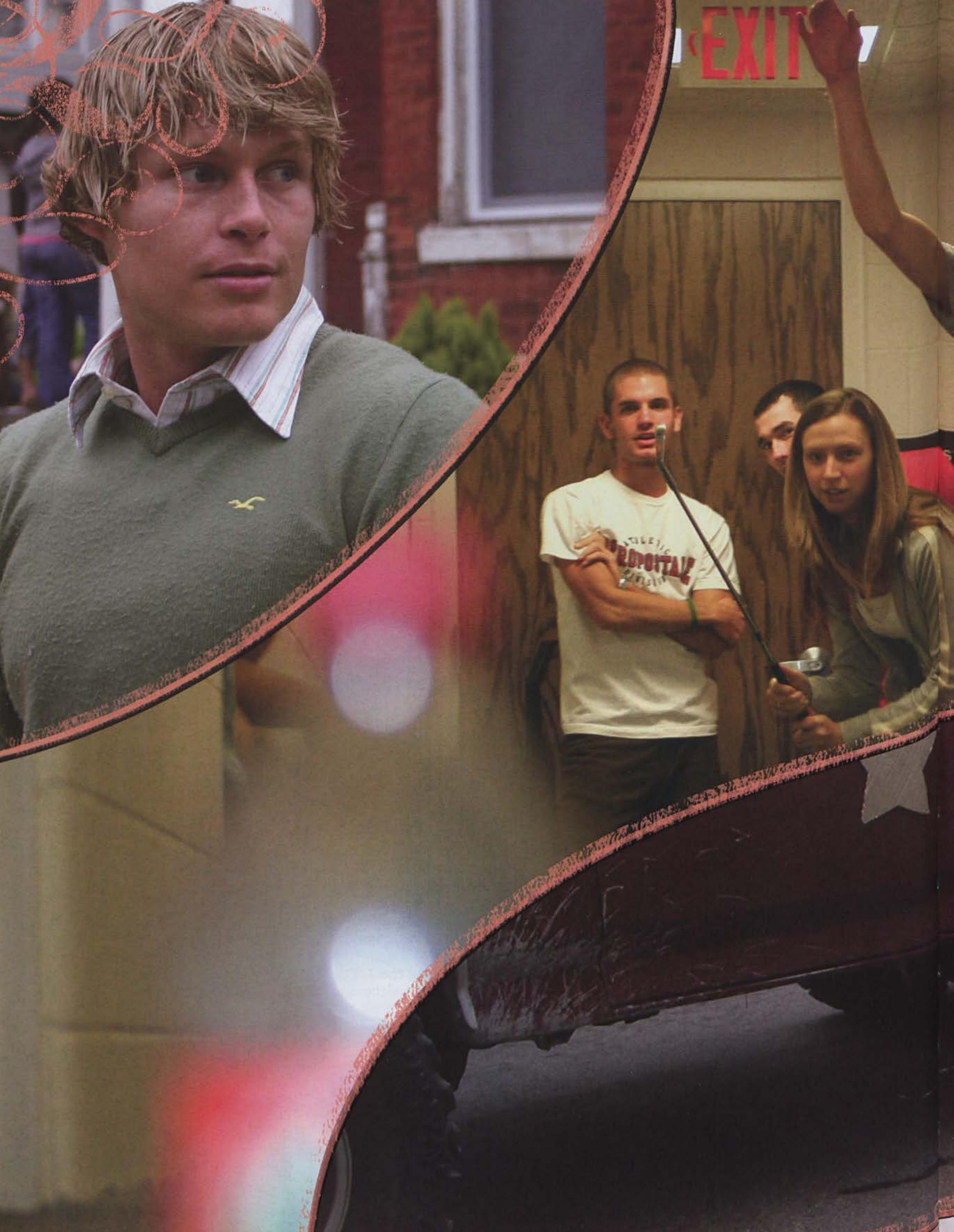




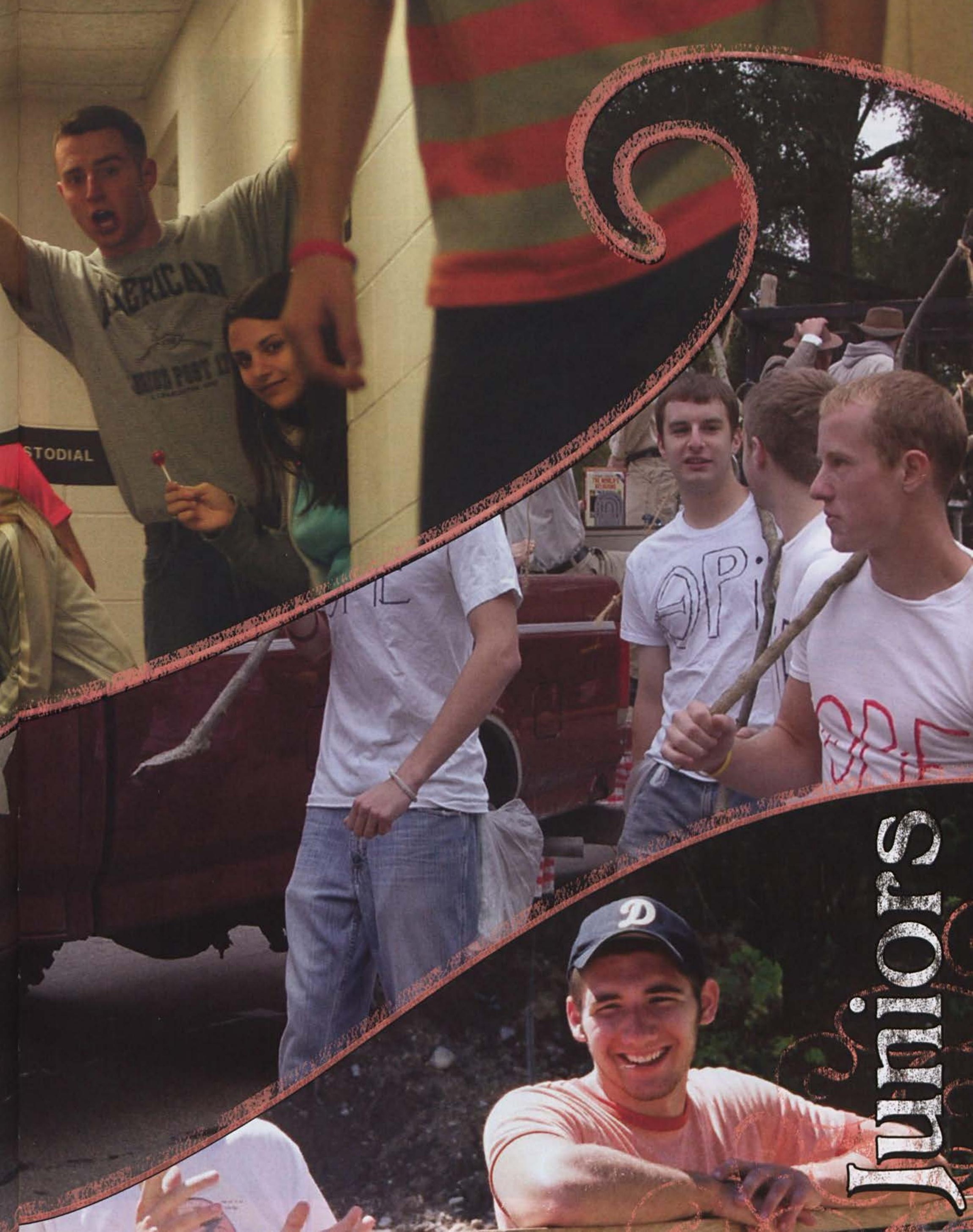




\section{Juniors}

J

Timothy Abel

Amanda Acker

Ellen Ahern

Scott Aker

Brian Alburger

Karen Alenskis

Stacy Alexander

Andrew Althouse

Bryan Amerine

Michael Anfang Jessica Annable

Daniel Archer

Joshua Arndt

Matthew Averbeck

Sarah Baczek

Bryce Bahler

Brian Baker

Crystal Baldwin

Shanna Banks

Erica Bartlett

Daniel Basham

Grace Bates

Aaron Bauer

Ashley Bauer
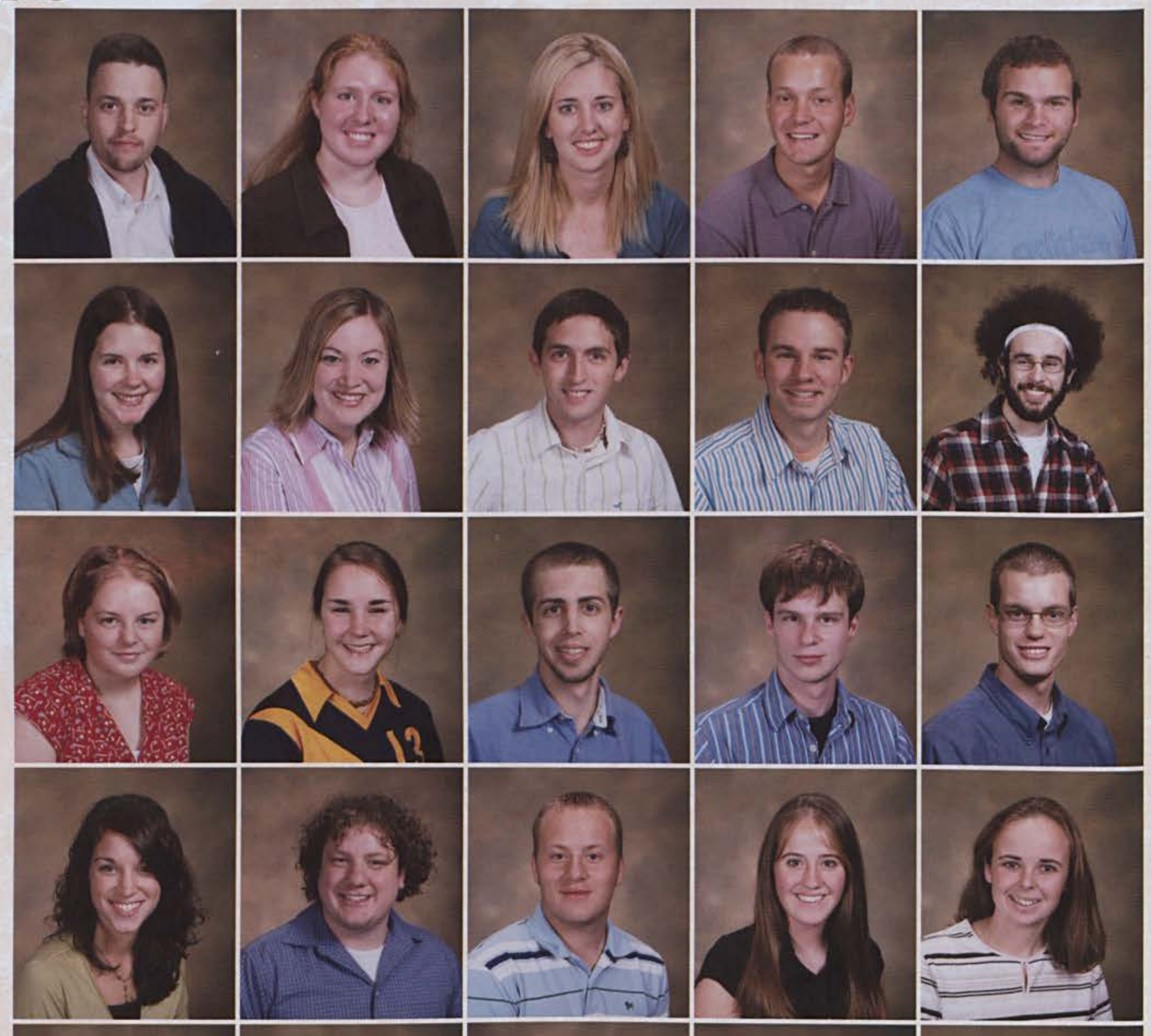

Brendon Bayley

Danielle Beal

Melanie Beatty

Kathryn Behlert Jessica Bellew

Kristi Beougher

Lauren Berry

Debra Birch

Joshua Blackburn

Lisa Blackburn

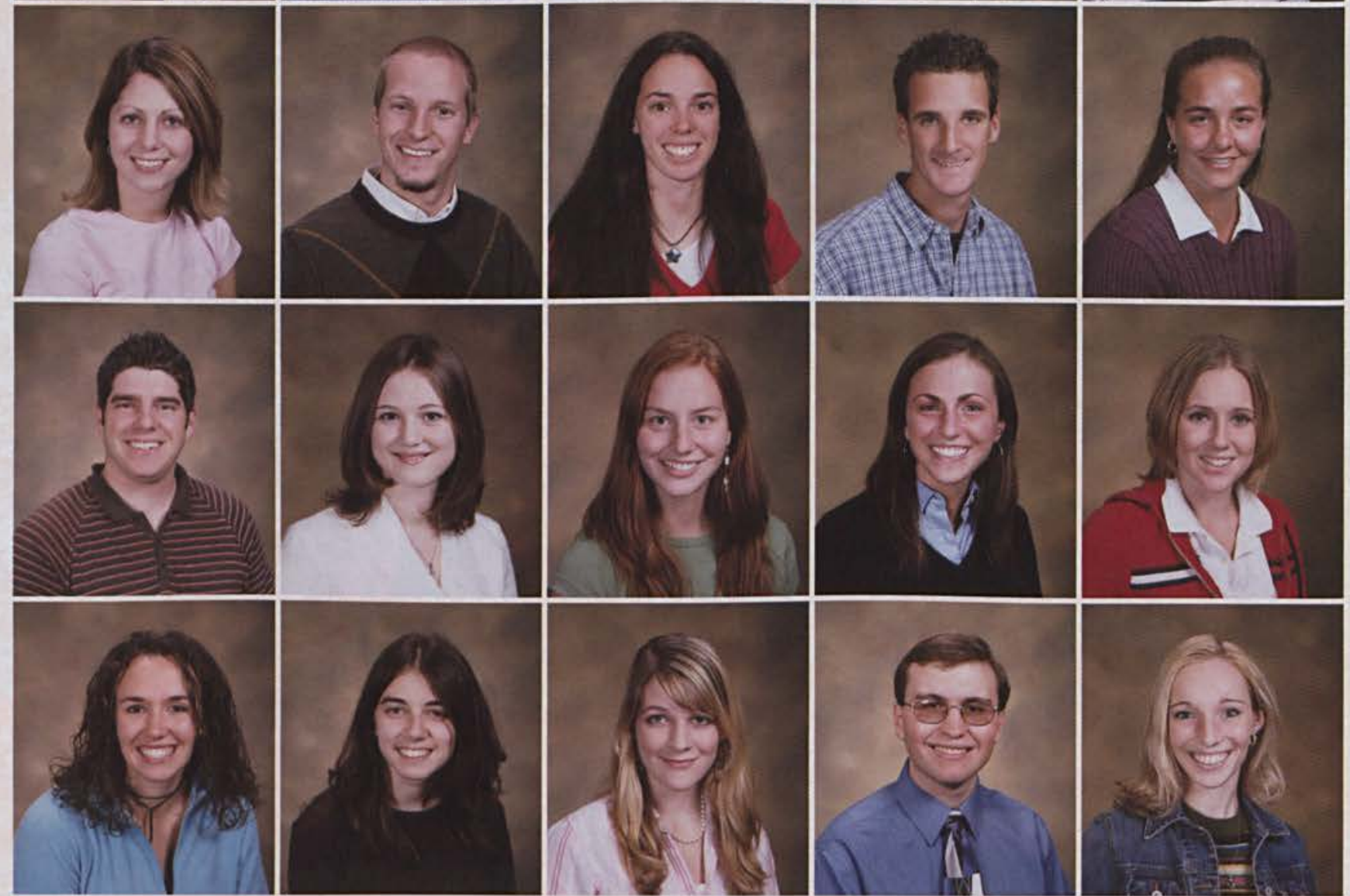




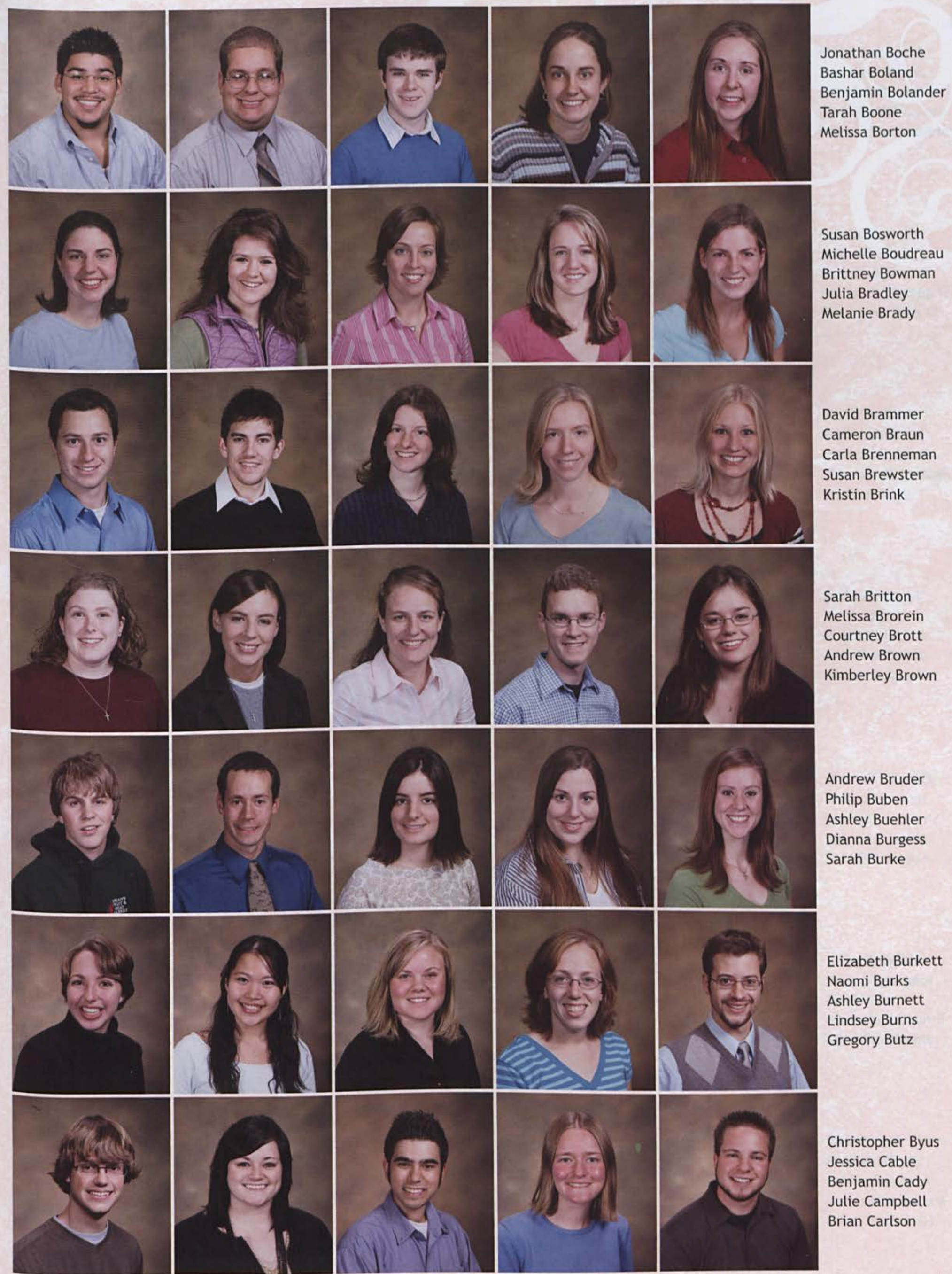




\section{Juniors}

J

Brandon Carrier

Nikki Carroll

Rachel Carter

Tiffany Cary

Stephanie Caywood

Katie Chasnov Evan Chiu

Anne Christensen

Shauna Christensen

Michael Cimba

Matthew Clark

Nathaniel Clark

Andrew Clemans

Steven Clemons Jr.

Jamie Clore
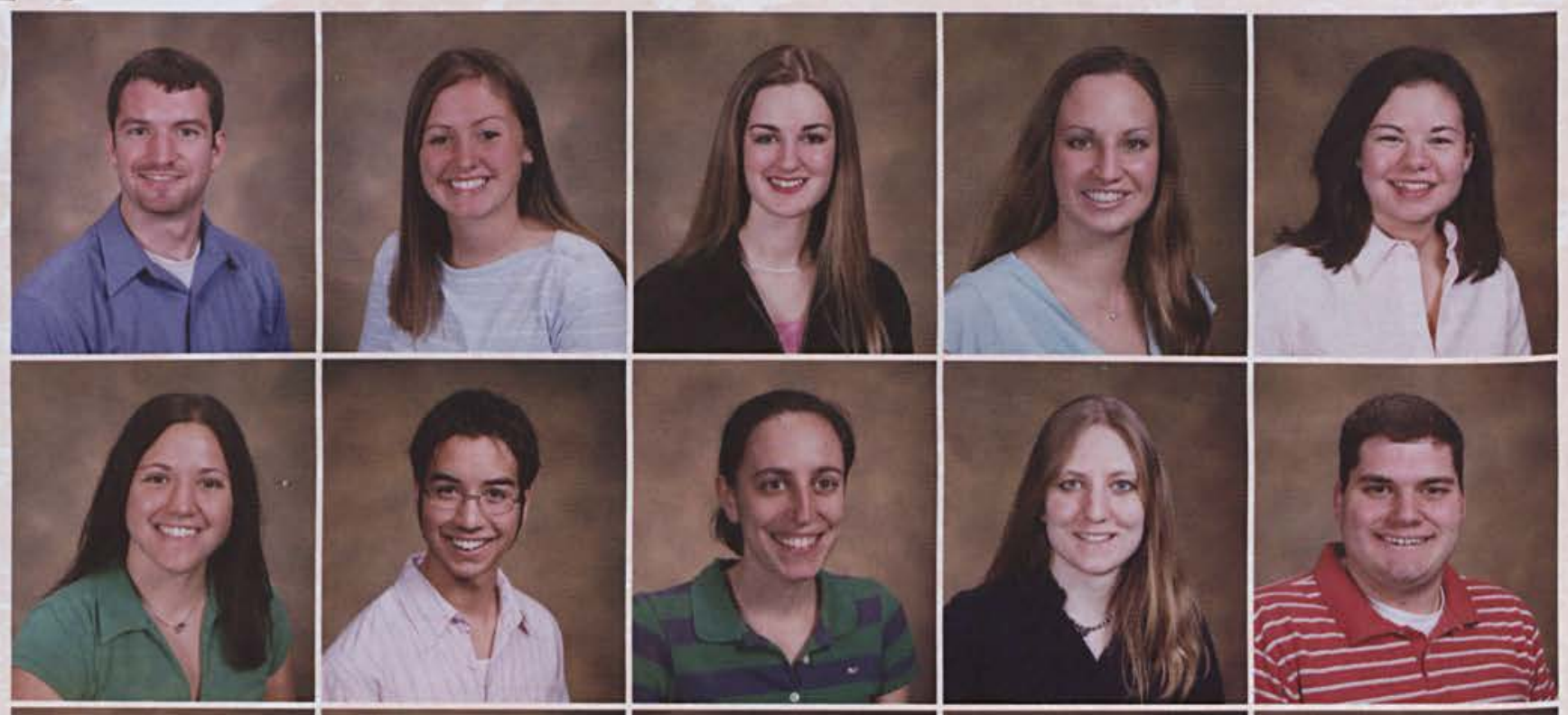

Nicole Coakley John Cody Jr.

Janet Colgain

Kristine Collins

Laura Connolly

Douglas Conway

Jeremy Conyers
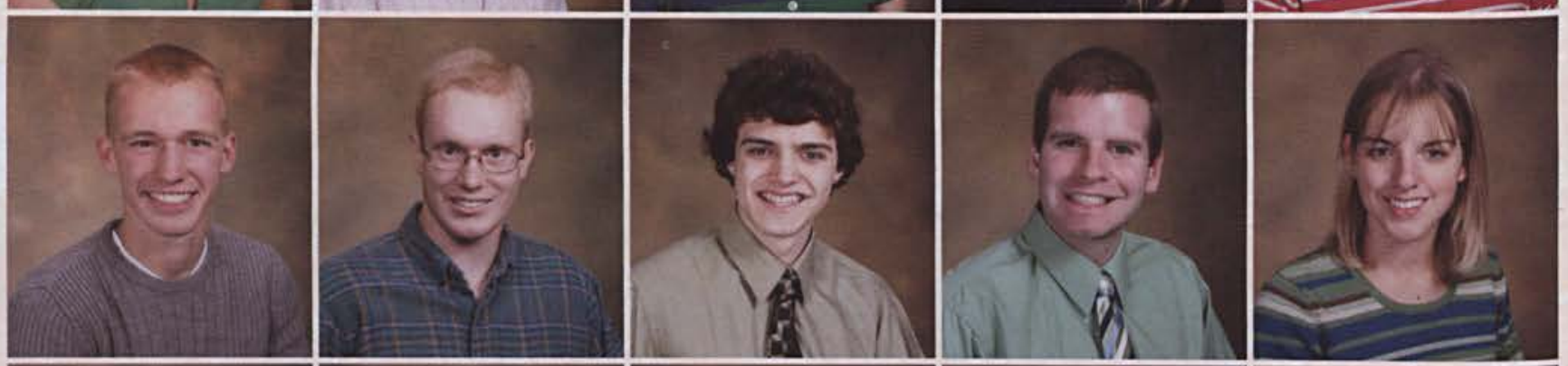

Shannon Cookson

Michael Cooper

Jessica Corbin
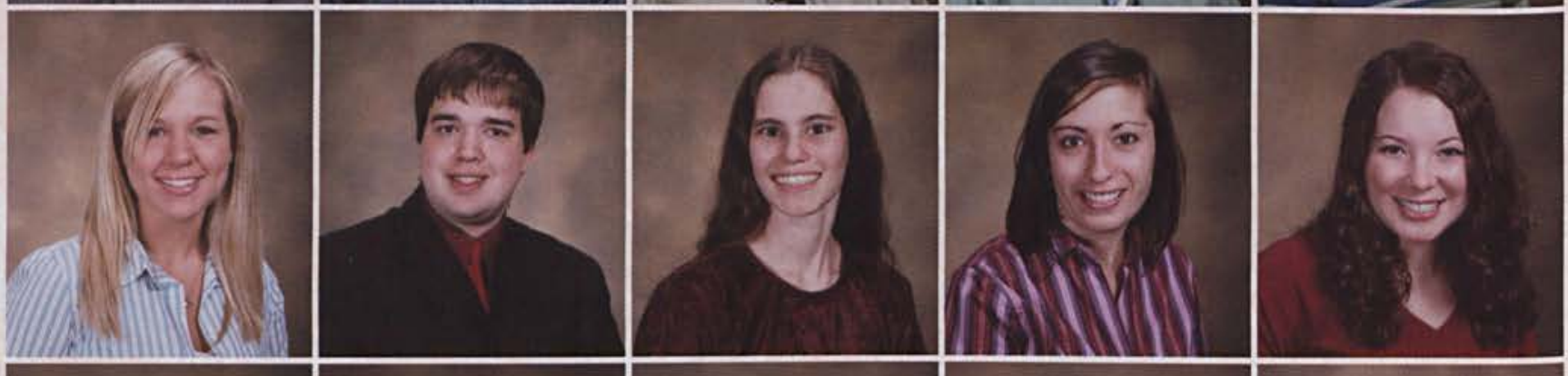

Christi Coulter

Kimberly Cowell

Lori Crain

Erica Cunningham

Jonathan Daiello
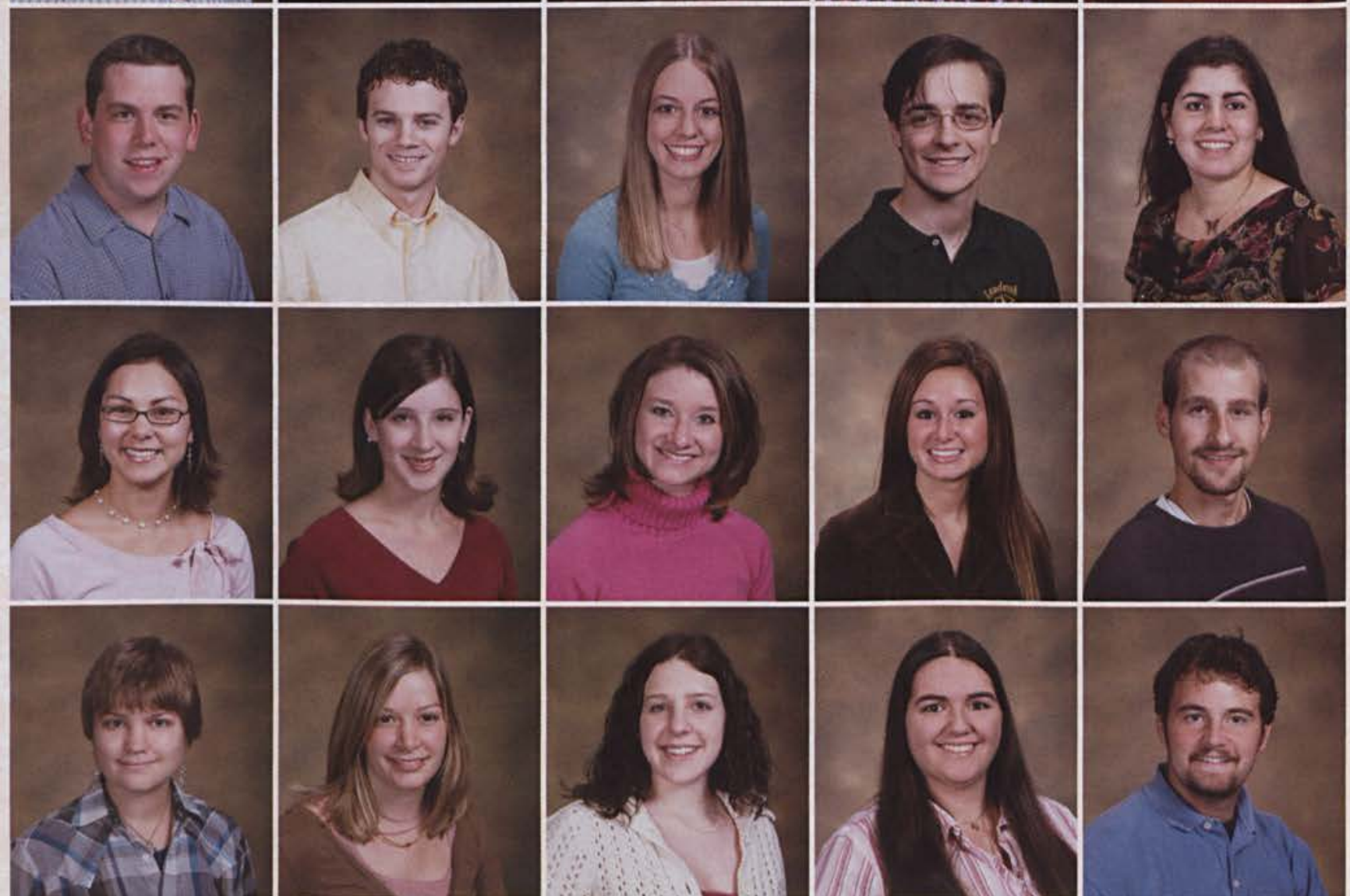

Ruth Danie

Cathy Daniels

Andrea Dankert

Michelle Davidson

Nathanael Davis

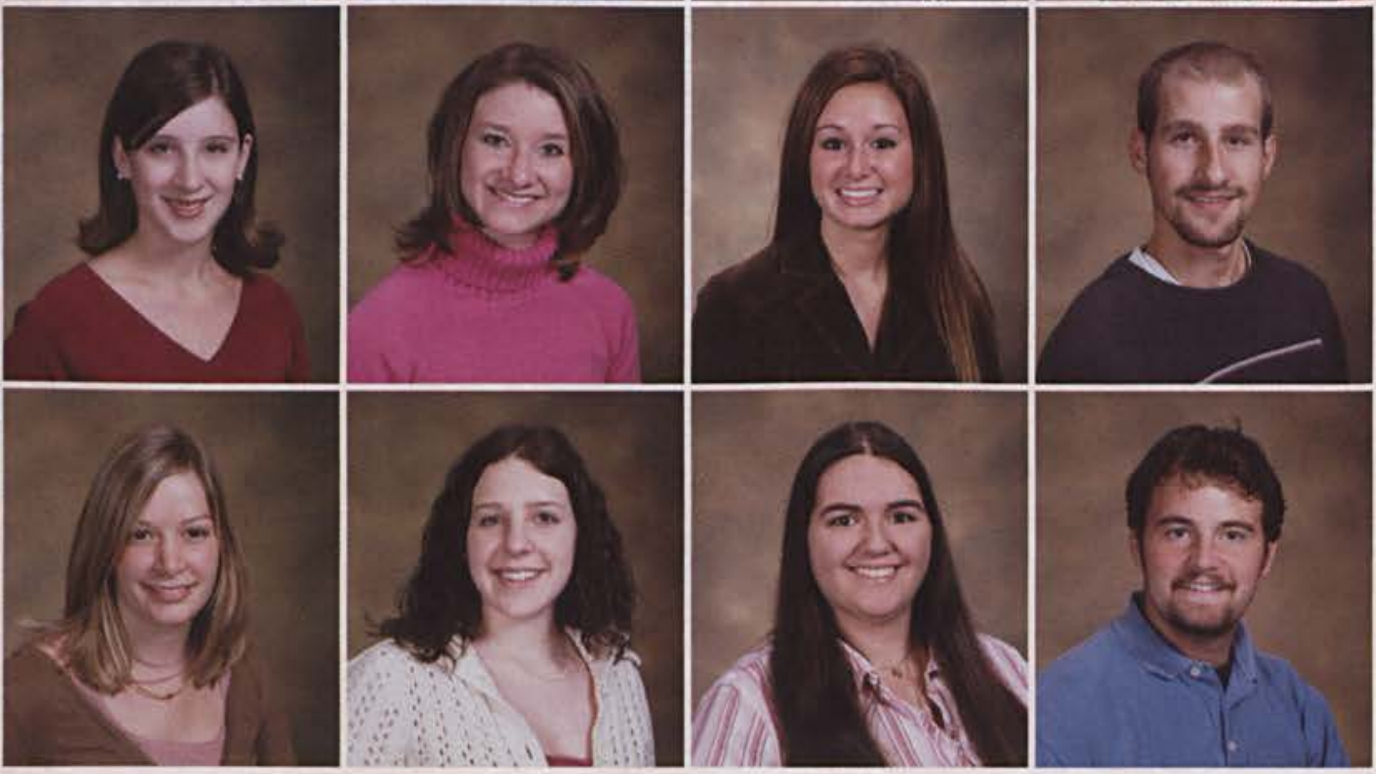




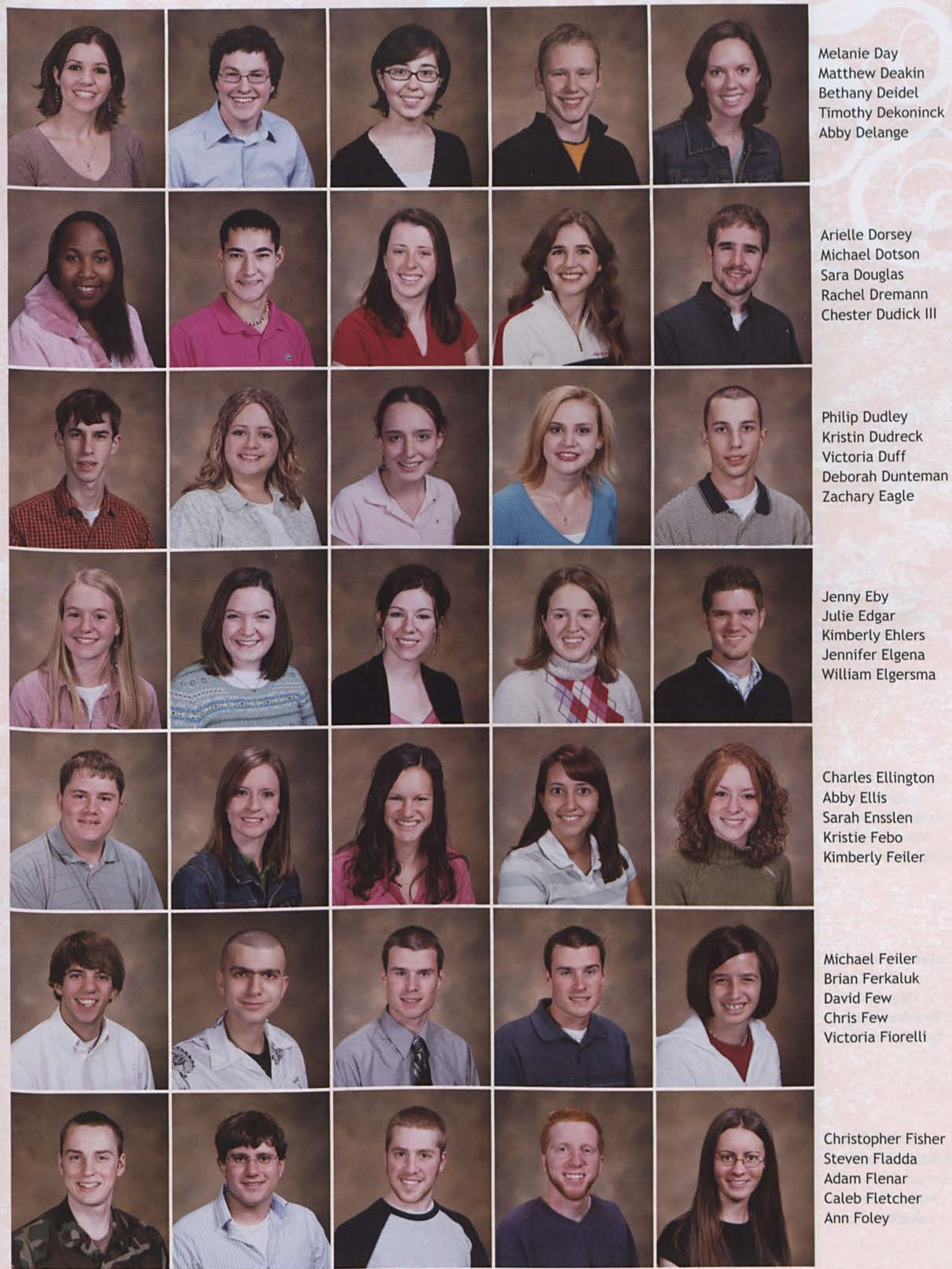




\section{Juniors}

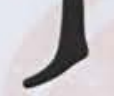
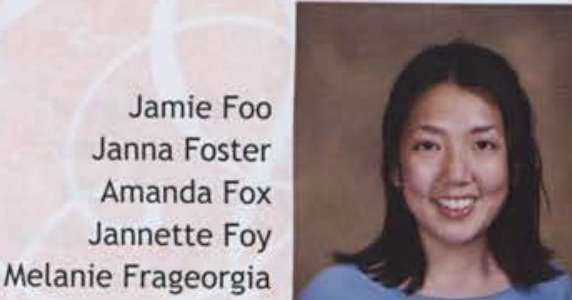

Rachel Freswick Michal Friend

David Fuls

Mark Gainer

Kari Gannon

Christina Genter

Brian Geringer

Kelly German

Michael Gerringer

Sarah Gibson

Zachary Gibson

Daniel Gifford

Shelley Gilbert

Daniel Giles

Kevin Gilsdorf

Cori Ginter

Jessica Glass

Ashley Gochenaur

Monica Godinez

Peter Gohdes

Catherine Gooch

Joel Green

Shawn Green

Jedediah Gregory

Joshua Greve

Joshua Grosso

Christopher Guarnera

Michael Gumprecht

Angela Hagensen

Ruthann Hager

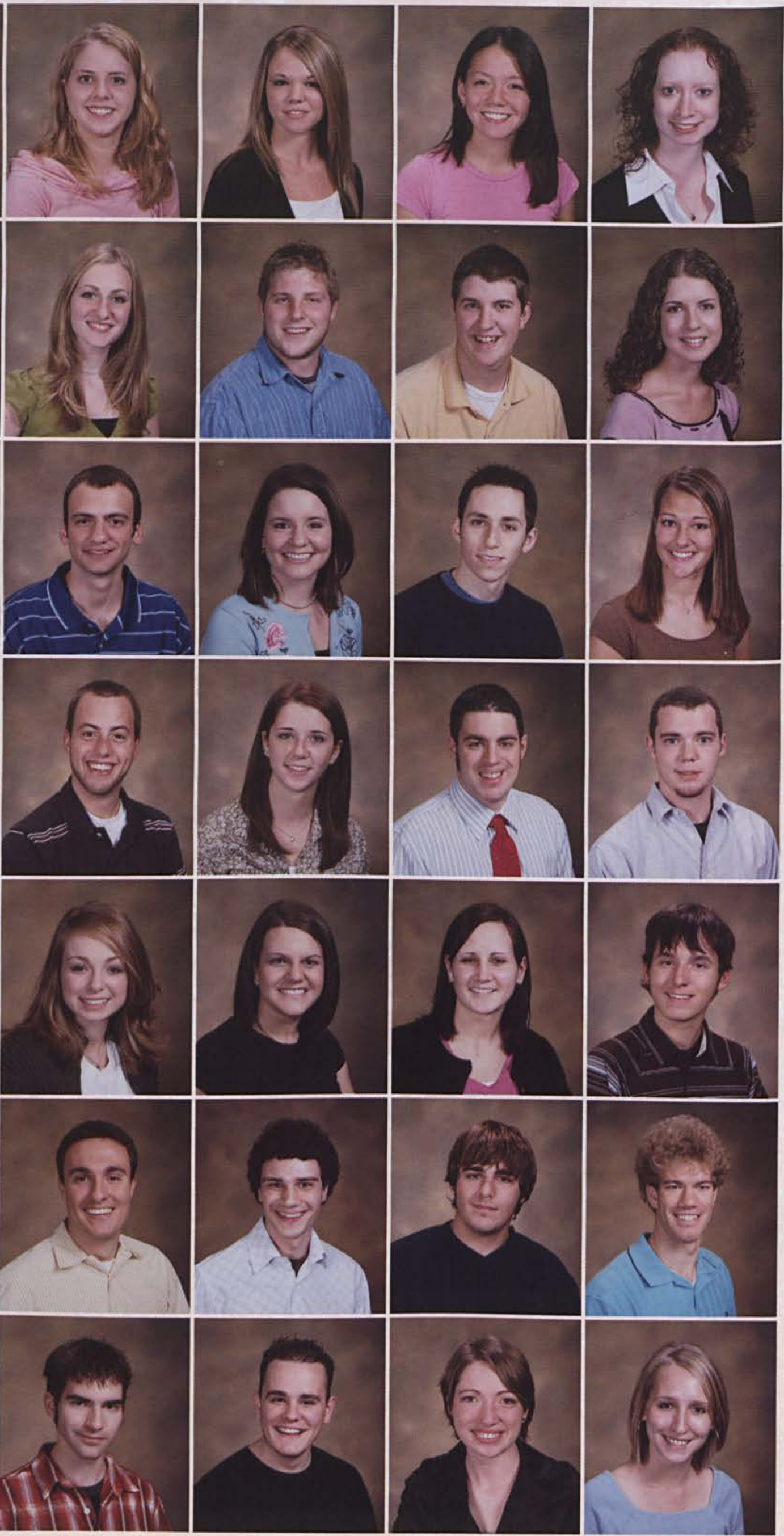




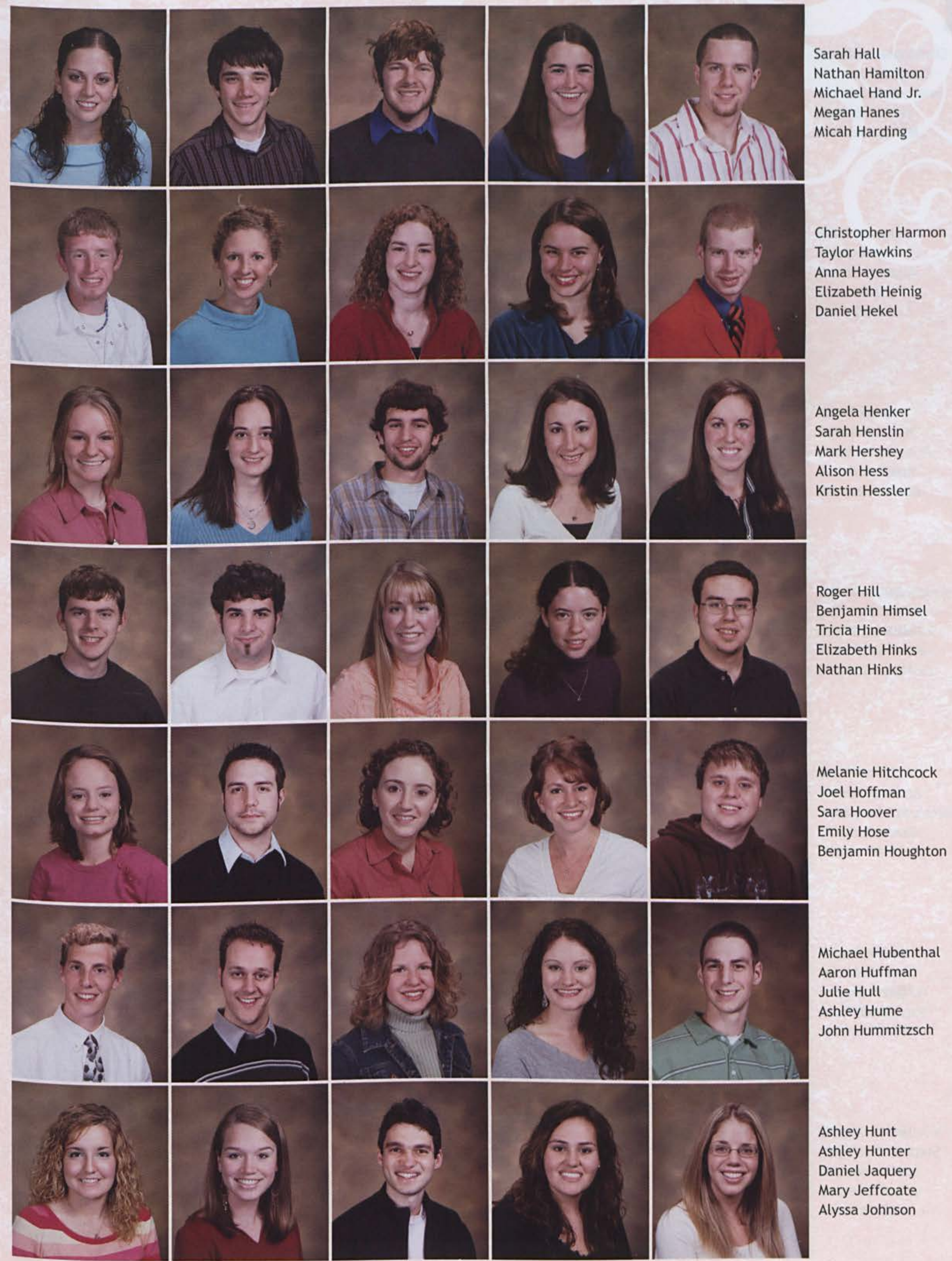




\section{Juniors}

Christopher Johnson Courtney Johnson

Eric Johnson

Holly Johnson Melissa Johnson
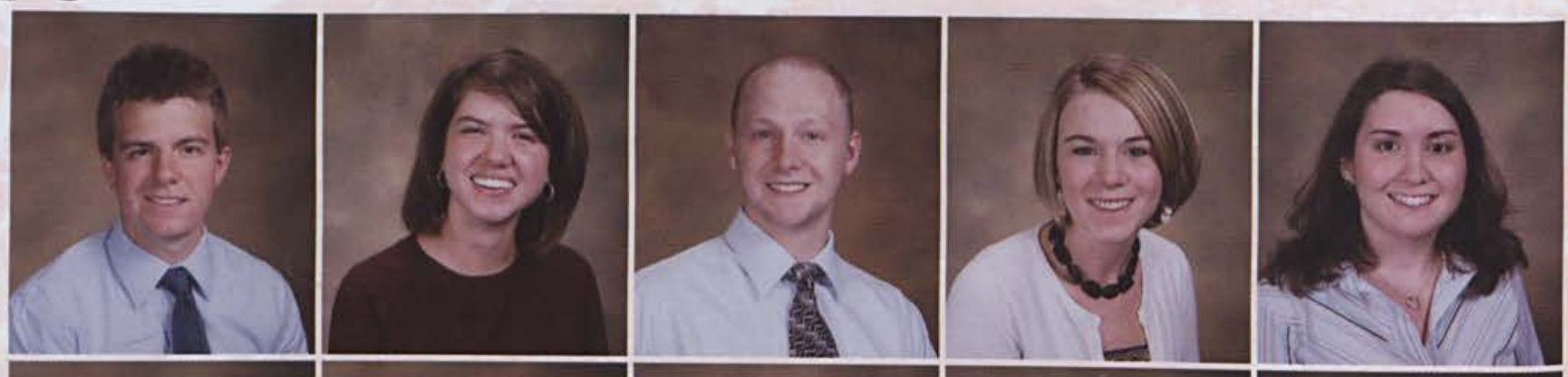

Lauren Jones

Leah Jones

Amanda Joswig

Isaac Judd

Jack Kehl III
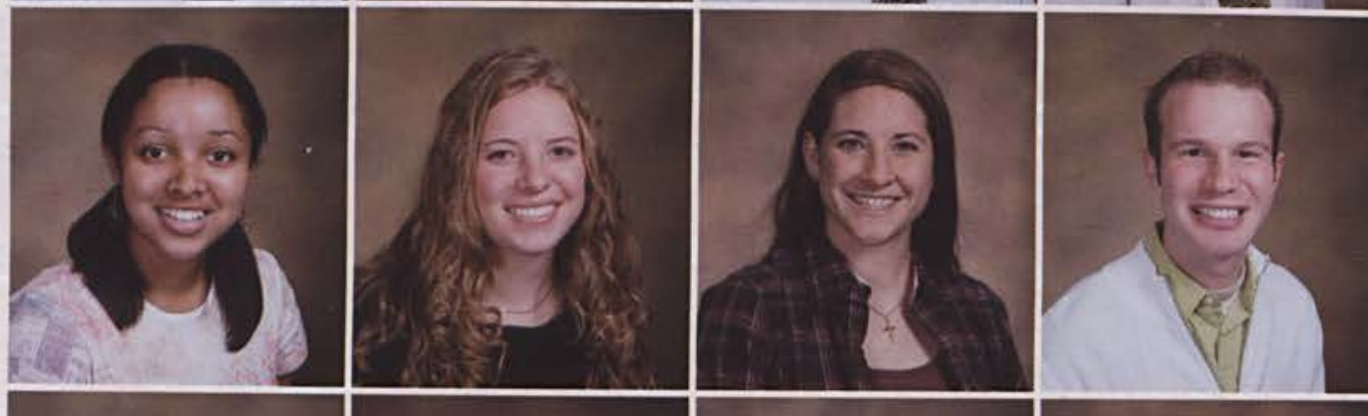

Carol Keller

Justin Keller

Krissy Kelley

Heather Kelton

Rachel Kennelly

ry

Dustin Keslar

Erin King

Diane Kinman

Kelley Kinnard

Amanda Kirby

Susan Knaus

Andrea Kneezel

Jeremy Knickerbocker

Jaime Kobialka

Jennifer Kordic

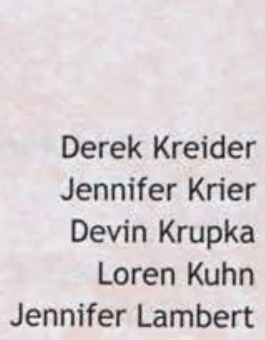

Jennifer Lambert
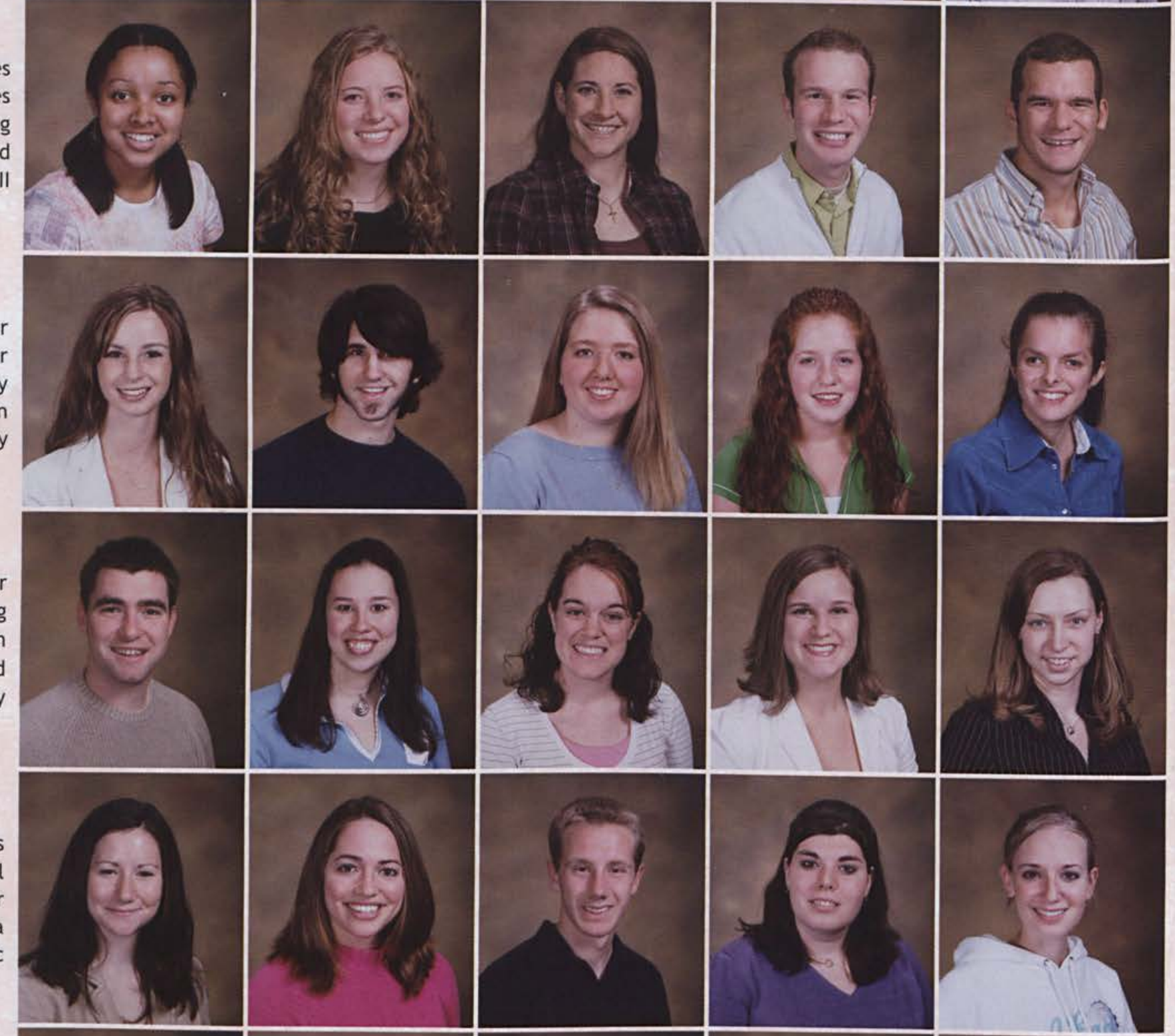

Nathan Landis

Stephanie Langley

Jessica Laporte

Grant Letizia

Jacob Lewis
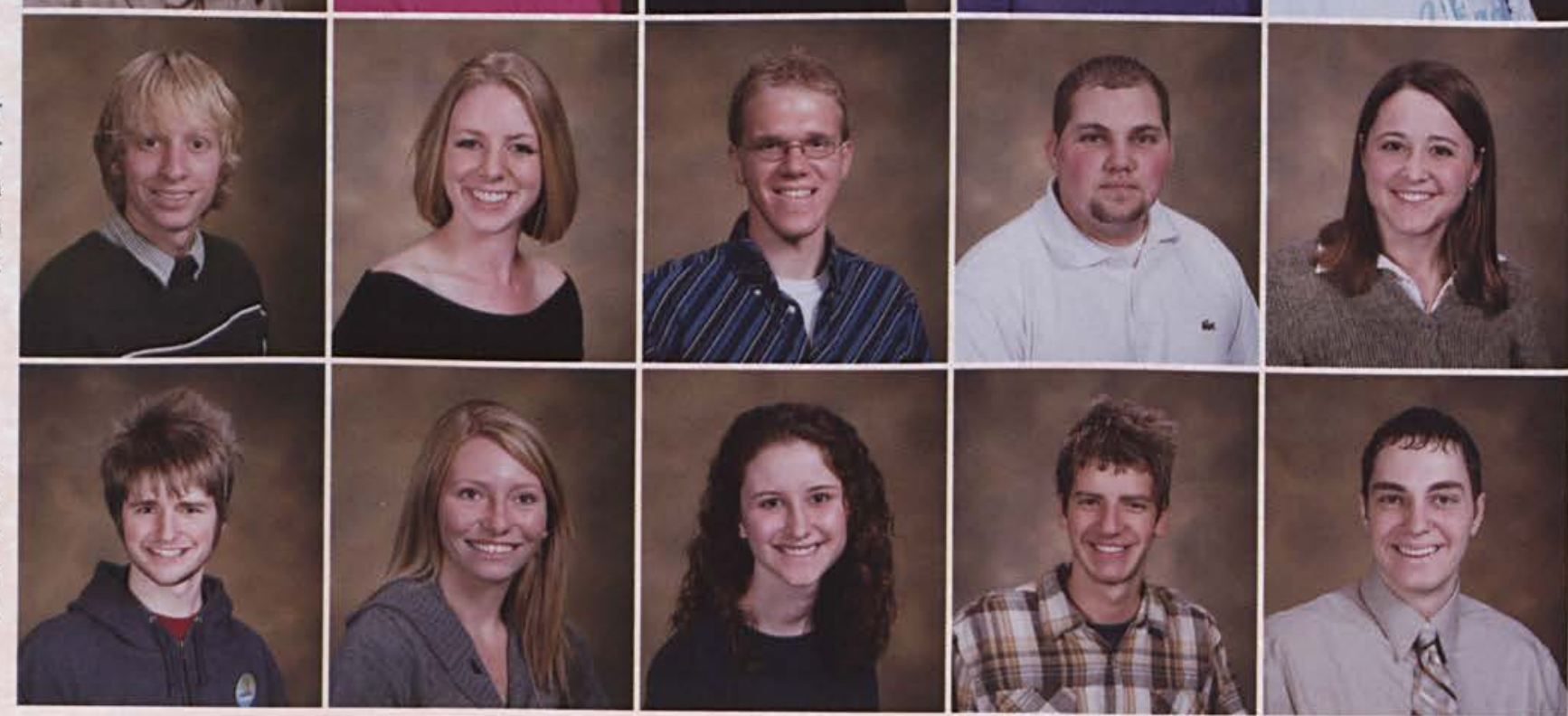


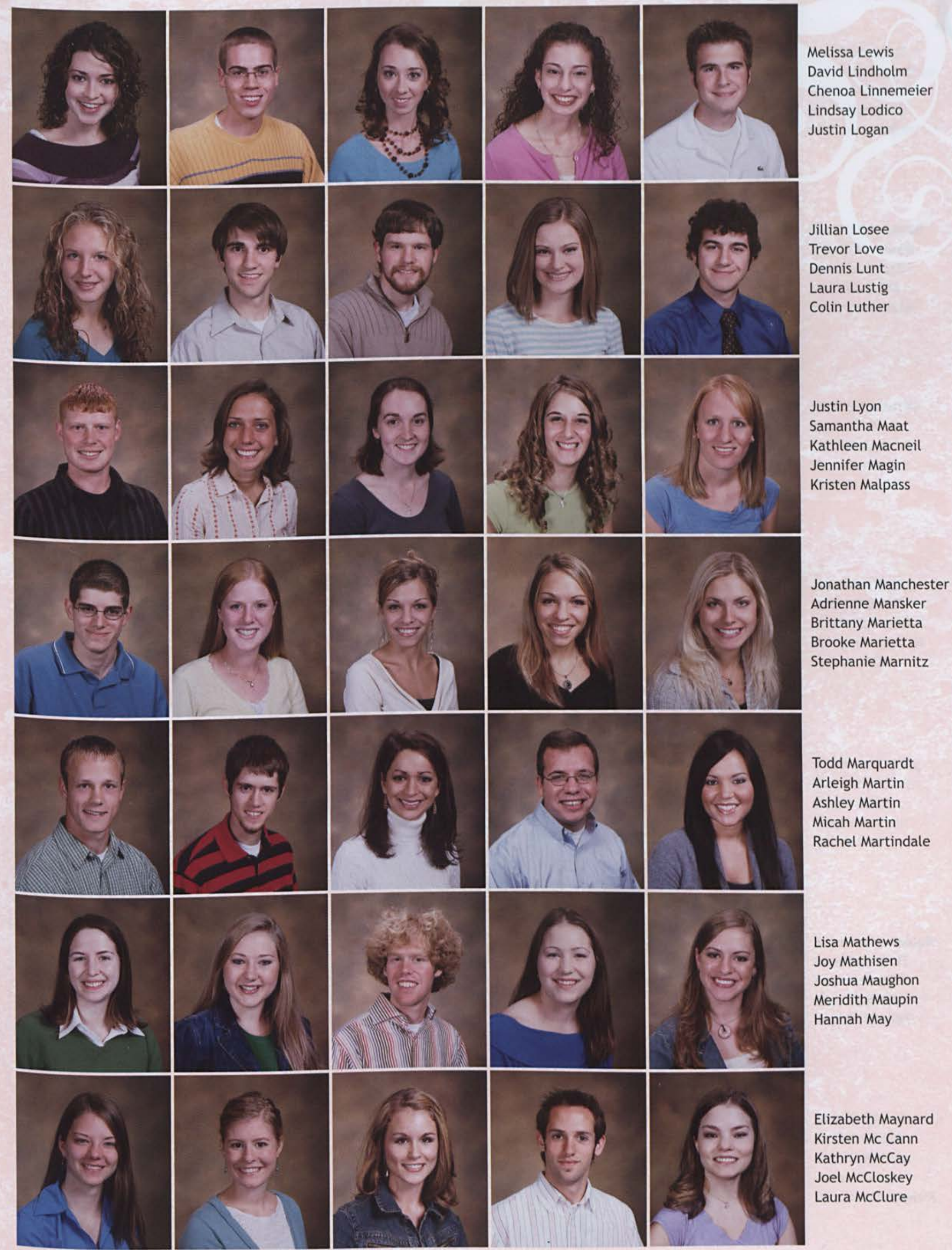




\section{Juniors}

J

Caleb McCollim

Amy McCormack

Laura McCully

Steven McCune

David McCune

Bethany McDaniel

Kaitlin McDaniel

Naomi McDivitt

Abbie McGaha

Lauren McGrew

Tiffanie Meissner

Vashti Mensah

Kara Merrick

Bryan Metcalf

Ashley Mettler

Griffin Meyers

Derek Micucci

Aya Midorikawa

Hannah Mingonet

Eric Mishne

Daniel Moore

Elliot Moore

Jason Myers

Brian Napp
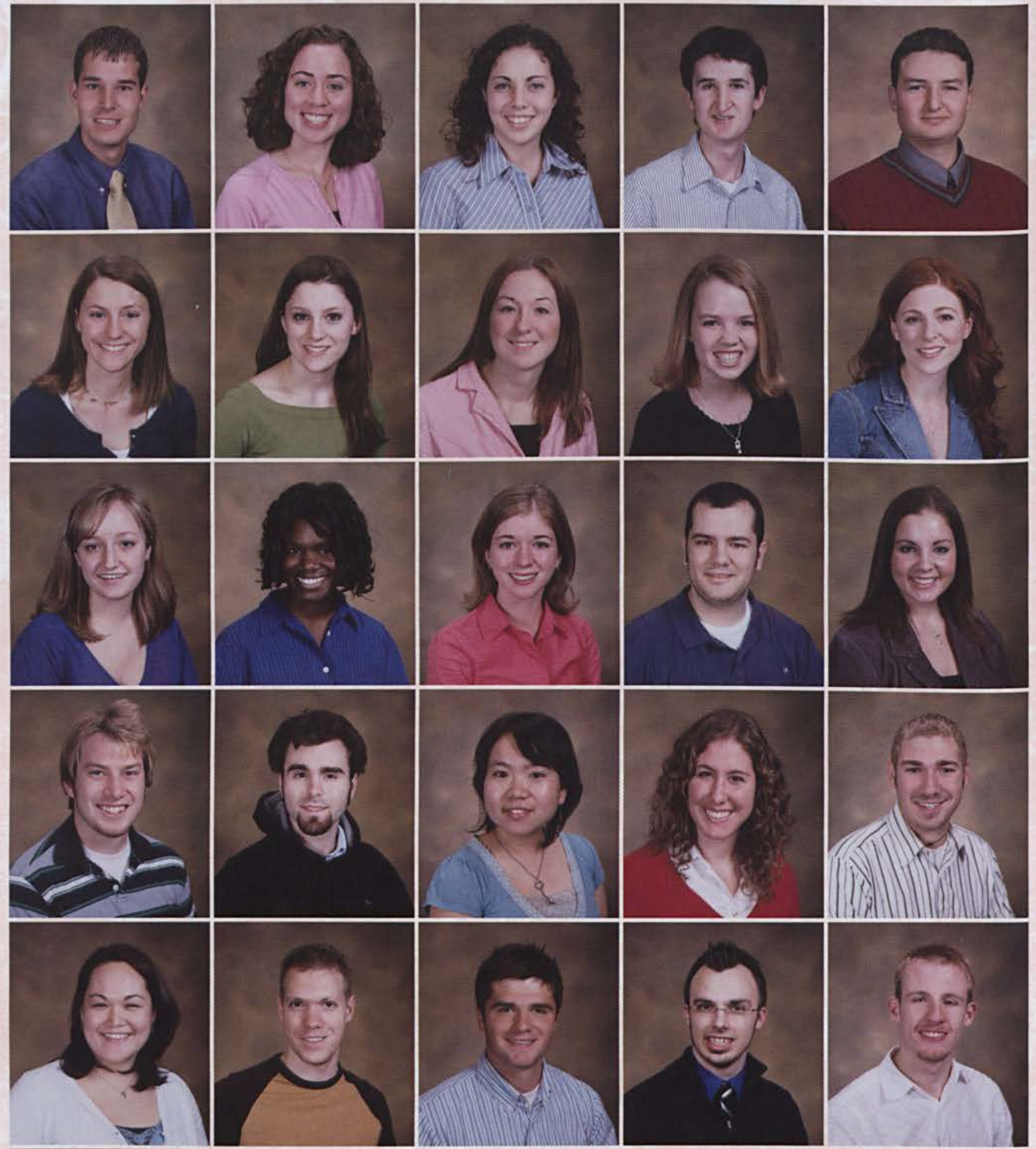

Rebekah Nearhoof

Timothy Neetz

Eric Neumann

Kathryn Nicholl

Tiffany Nickum

Christin Nightingale

Daniel Nikitin

Amberly Noble

Rondi Noden

Ricky Normandeau

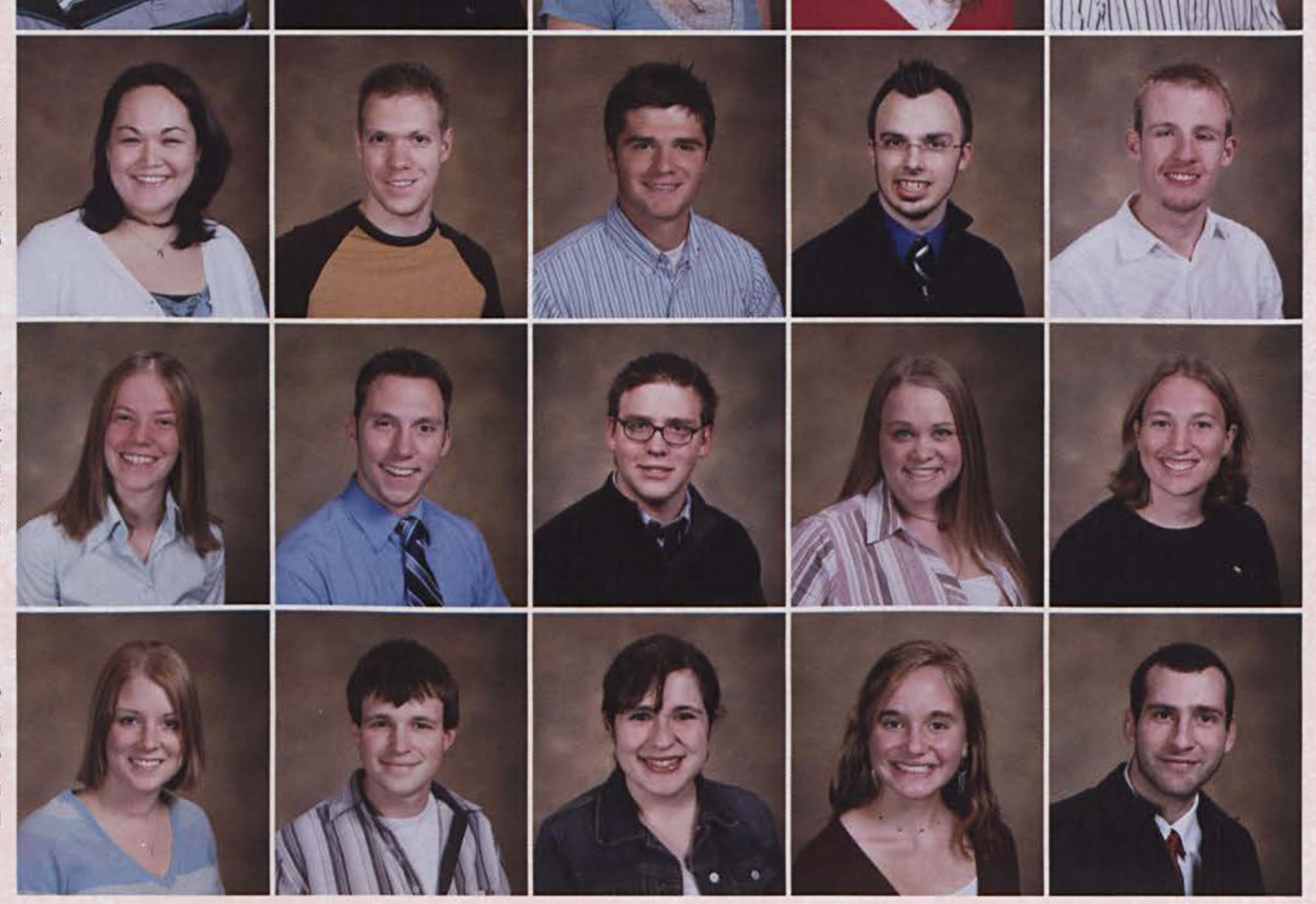




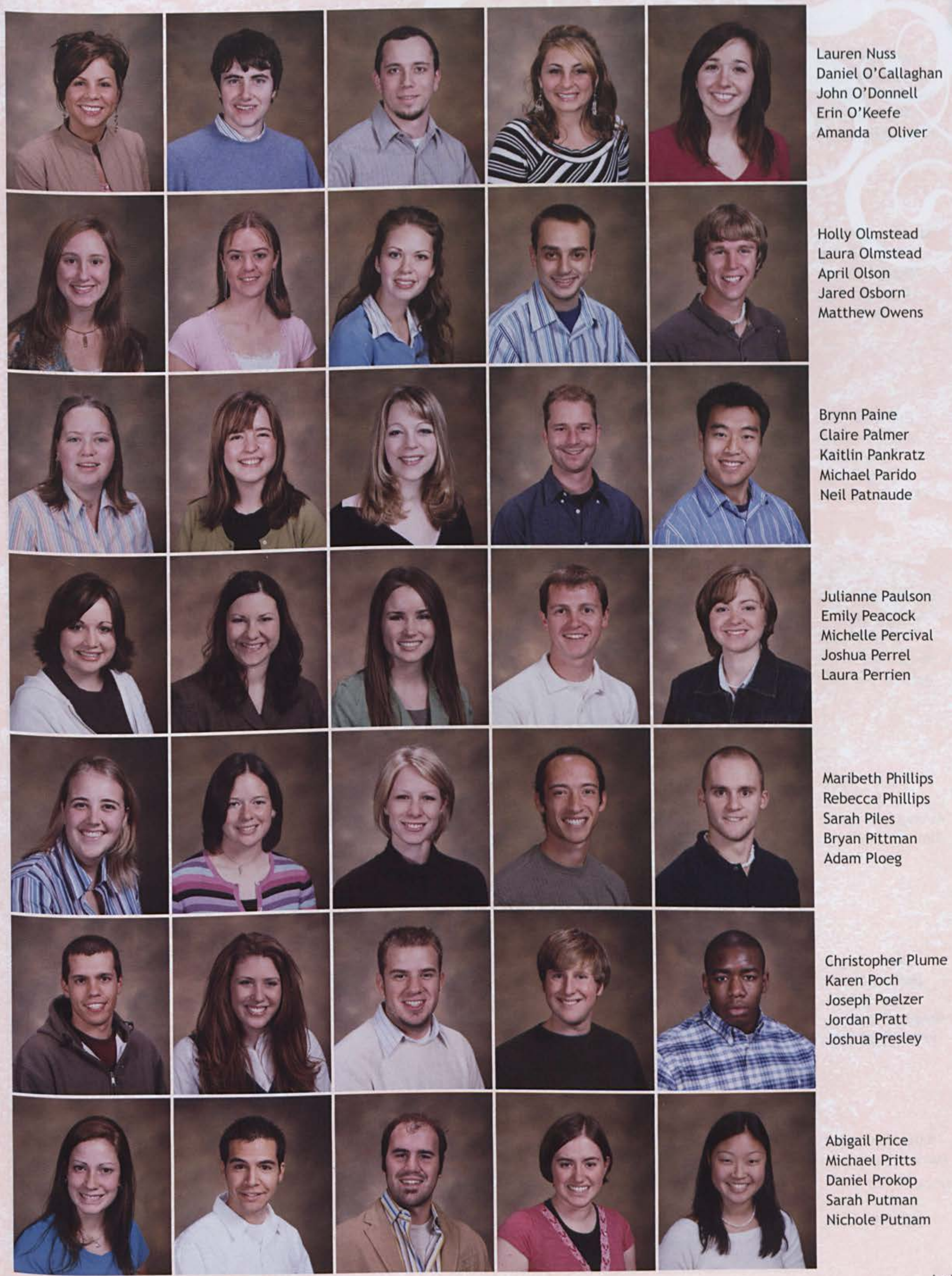




\section{Juniors}

Jonn Rafoss

Michael Rall

Andrew Ralon

Stephanie Ray

Richard Reeder III
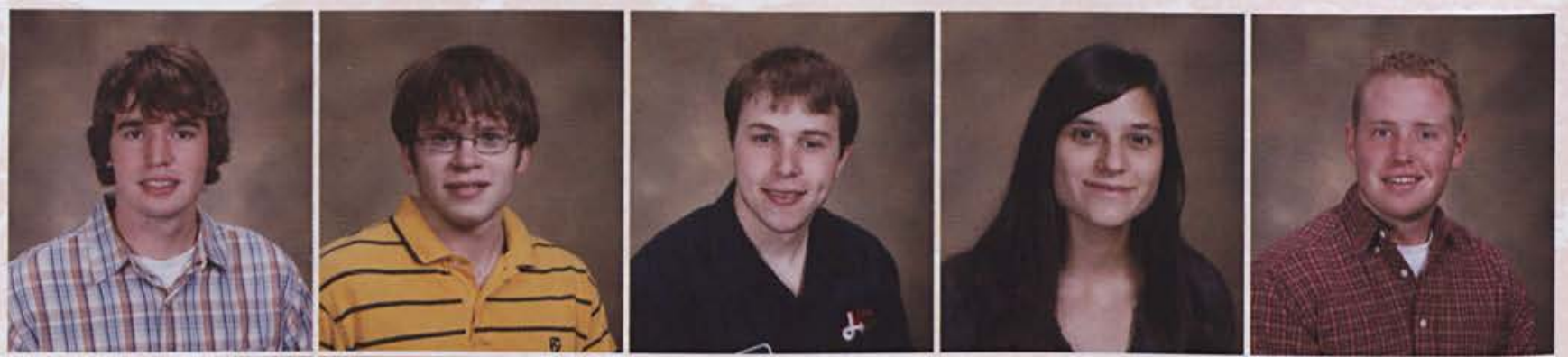

Jameson Reichert Carolyn Reiley Michael Rhoads

Courtney Rice Keith Rice
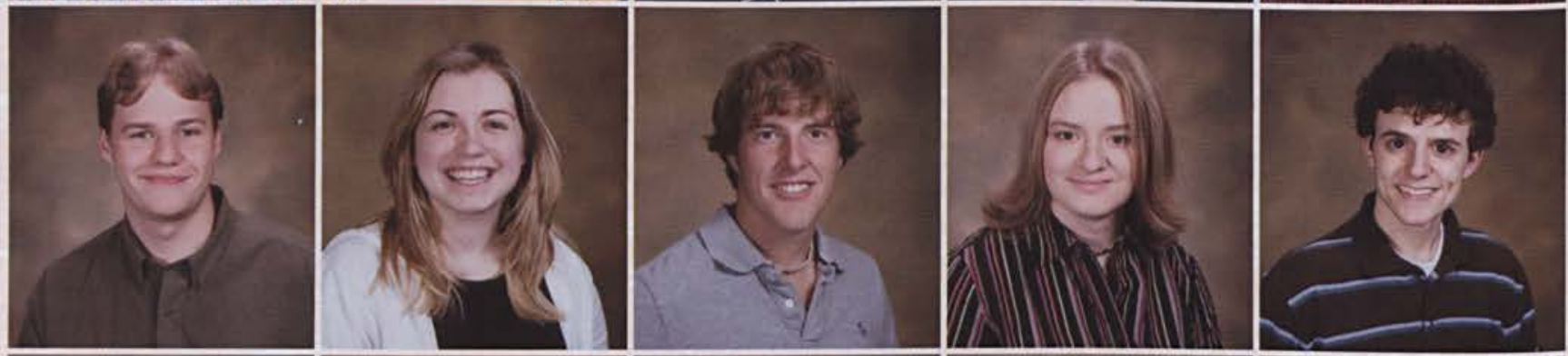

Emmett Robinson Cristine Rozelle

Karen Ruhlman

Mindy Russell

Gary Rutrough

Kelsey Salisbury

Brian Sallee

Aubrey Samuelsen

Timothy Sandlund

Daryl Sando

(1)
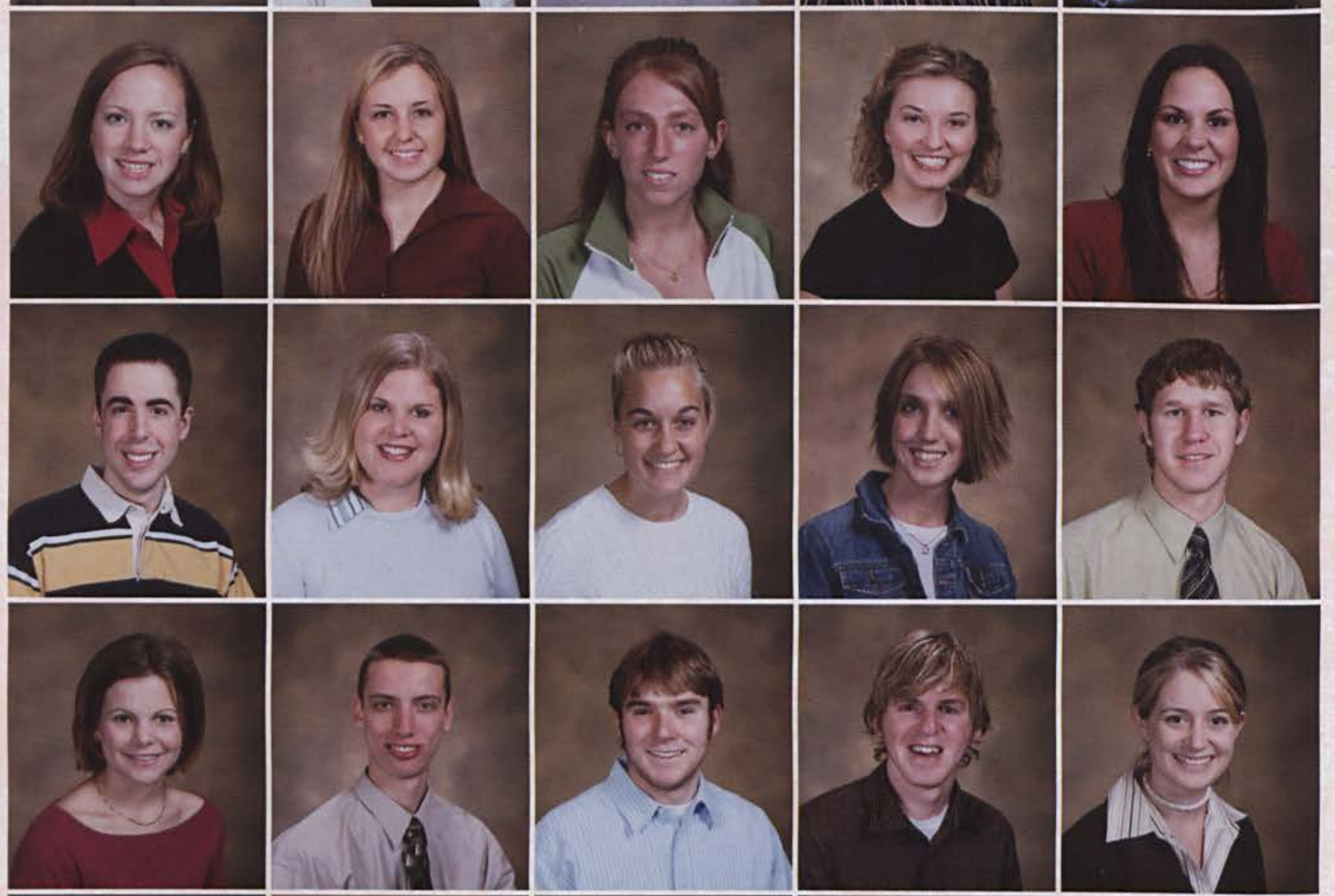

James Sawin

Jessica Scheidt

Josh Schenk

Bradley Schmoyer

Rebecca Schrank
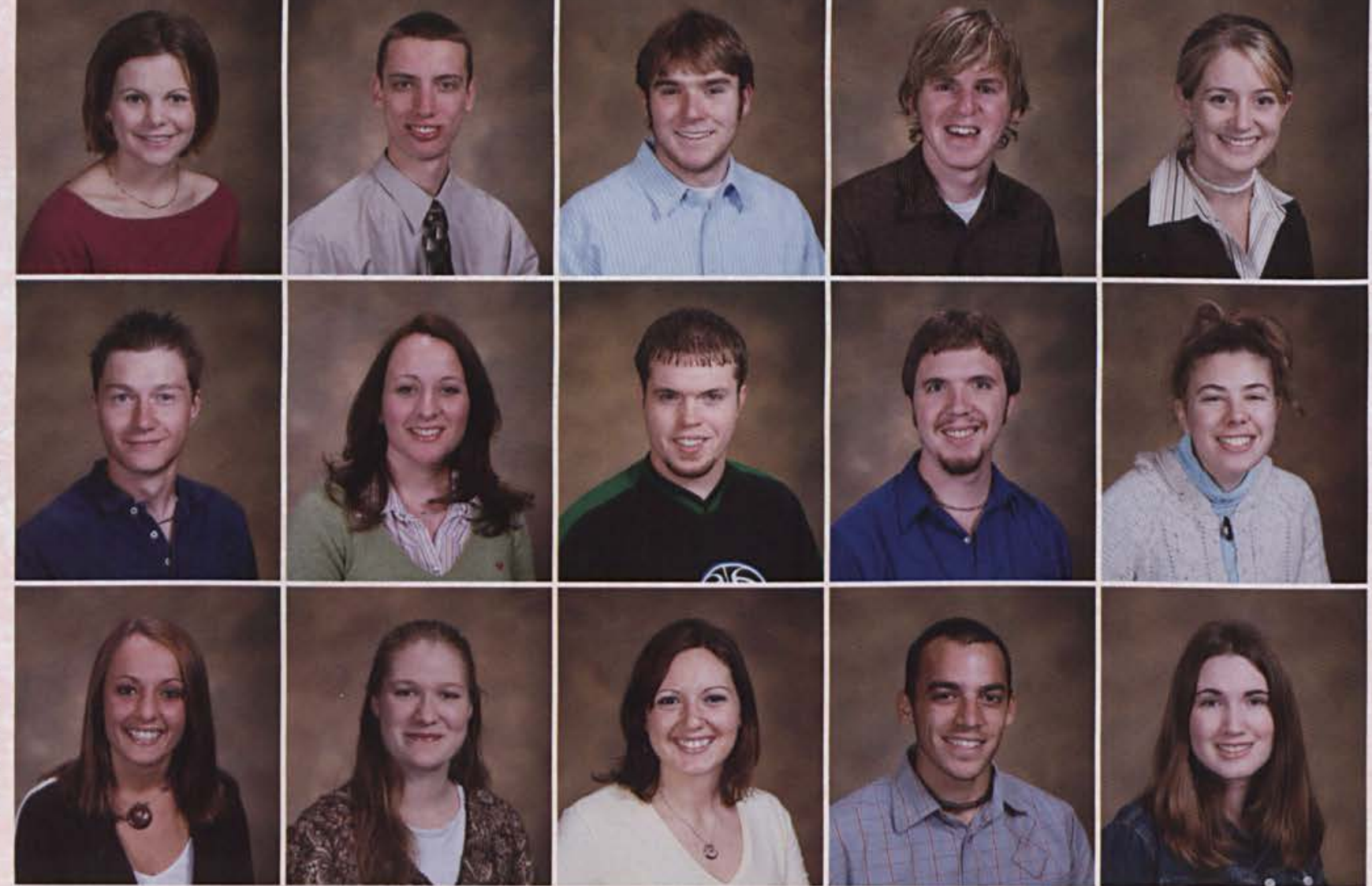

Karah Schroede

Marie Schumacher

Brittny Scott

Jason Scott

Kathleen Scull 


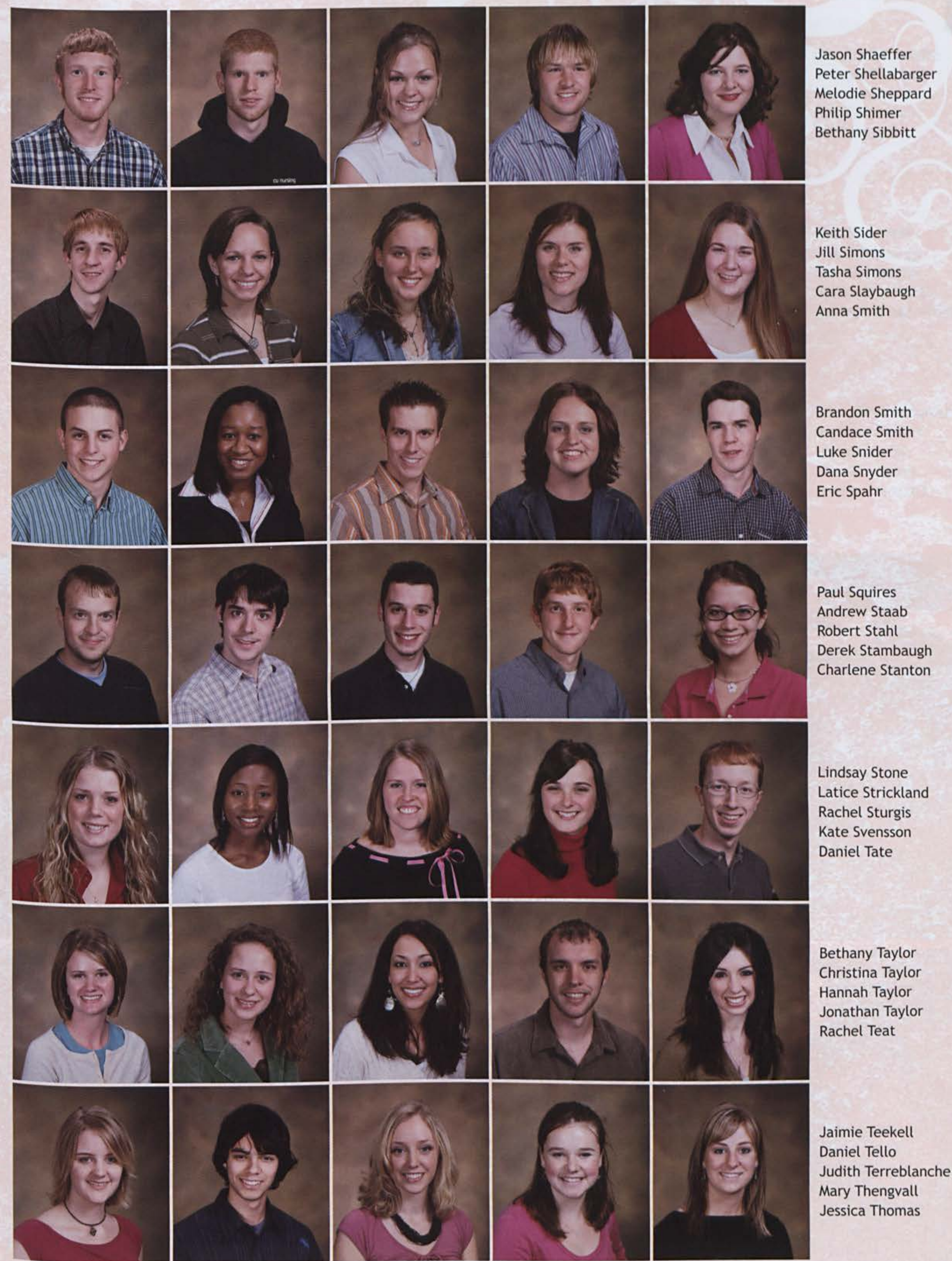




\section{Juniors}

J

Crystal Thompson Martha Thompson

Kristen Tisdale

Stacie Travis

Andrew Tripp

Bethany Turner

Alan Tuttle

Mark Urman

Jennifer Van Dyke

Valerie Van Wingerden

Christopher Vande Lune

William Vandyke

Maicol Venter

Timothy Vernon

Elizabeth Verwys

Craig Vinson

Joel Wallen

Katie Warndahl

Nathaniel Washatka

Matthew Watson

Sarah Welch

Gina Wells

Joshua Wilcox

Katie Willaman

Sara Williams

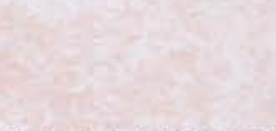

Rebekah Williamson

Kristen Wills

Jordan Winar

Jamie Winchester

Lindsay Wirt
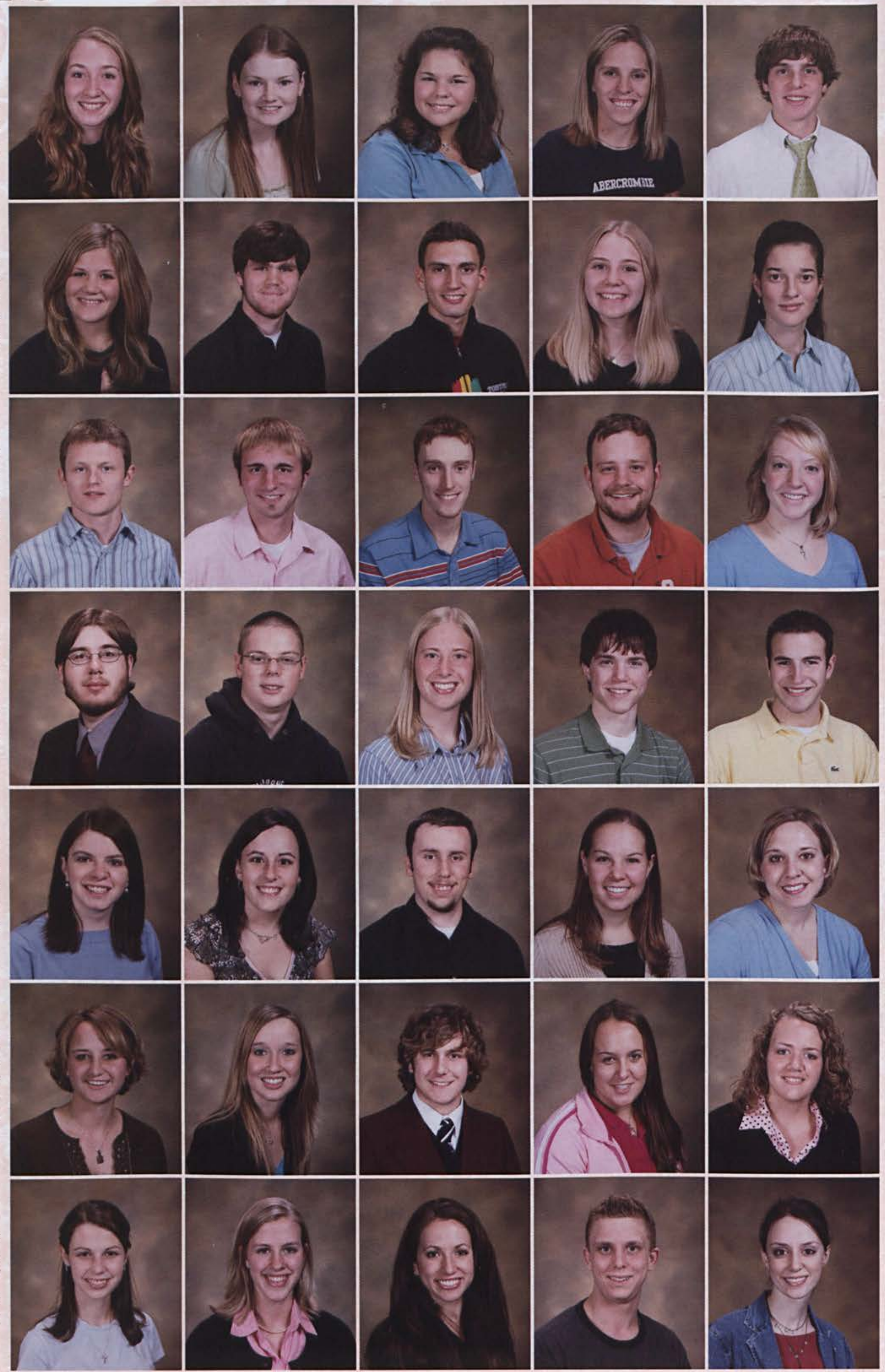

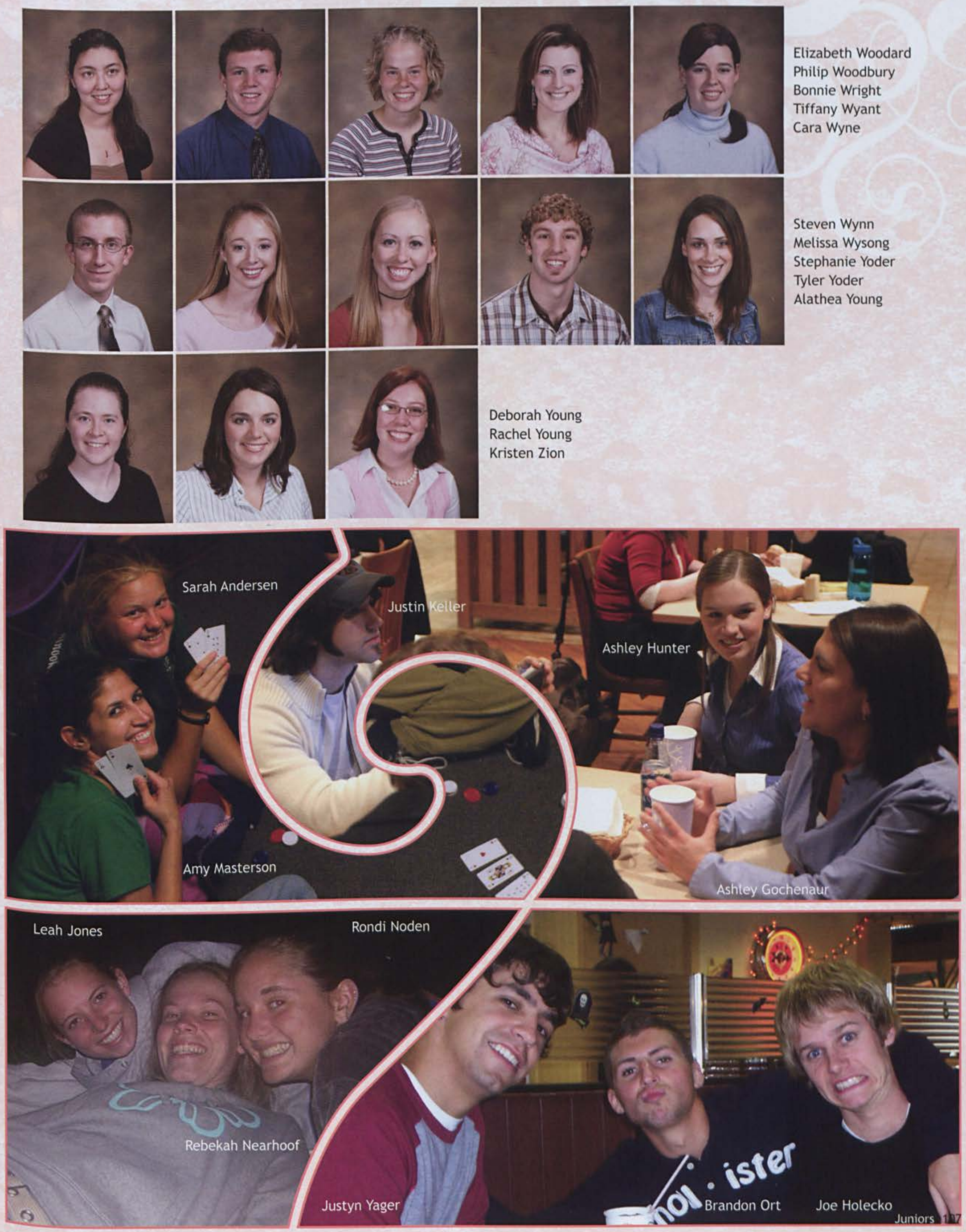


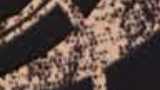

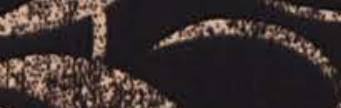

(2)

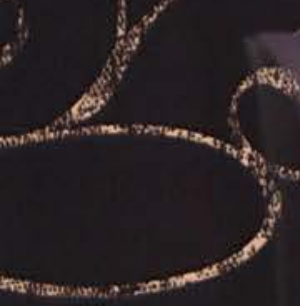

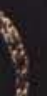

THE COMPLeH infrastructur known as

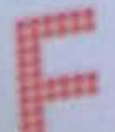
I

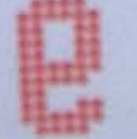

-

ansingesesing

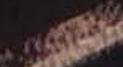

arese
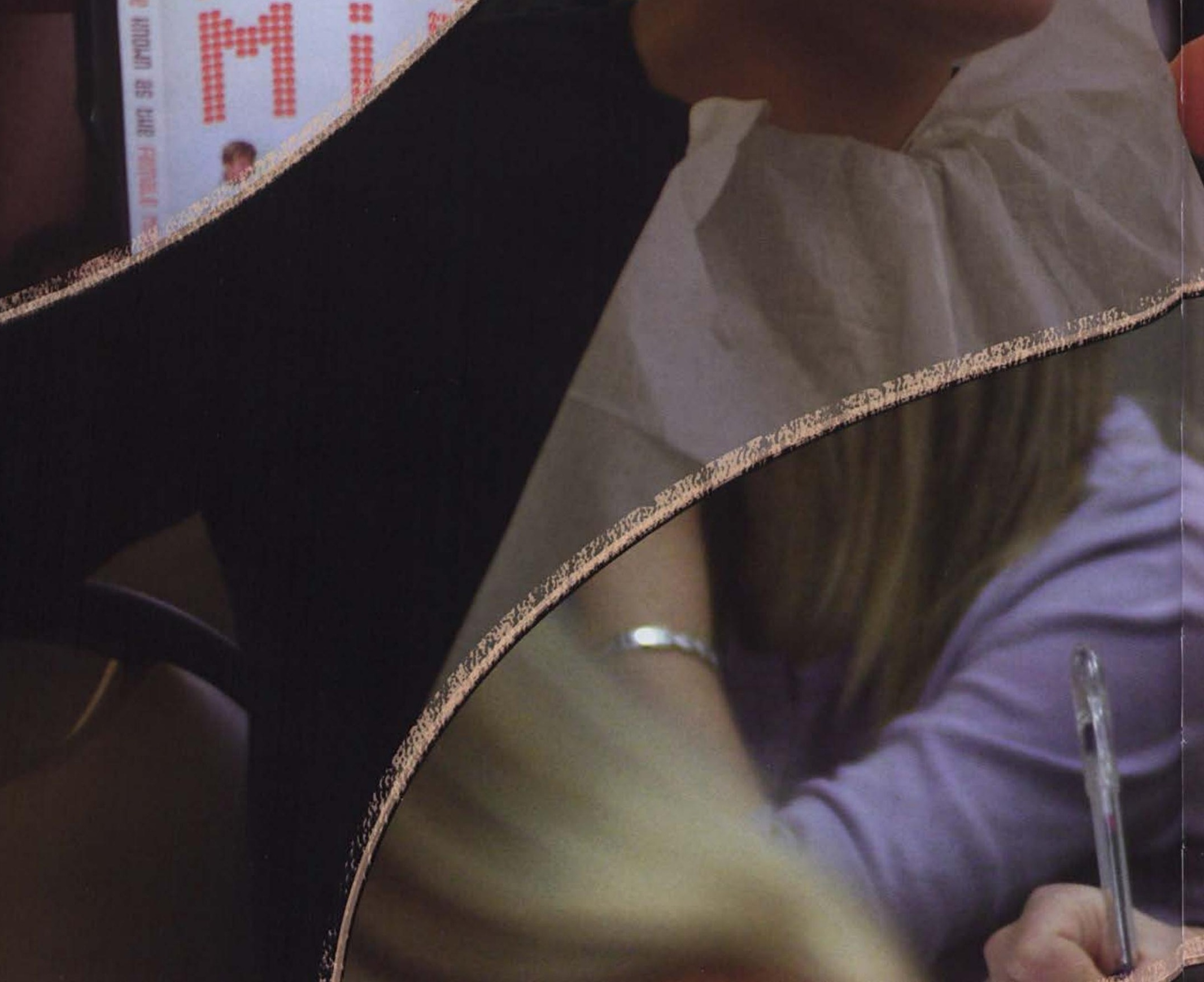

t8

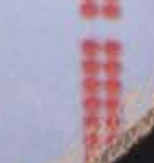




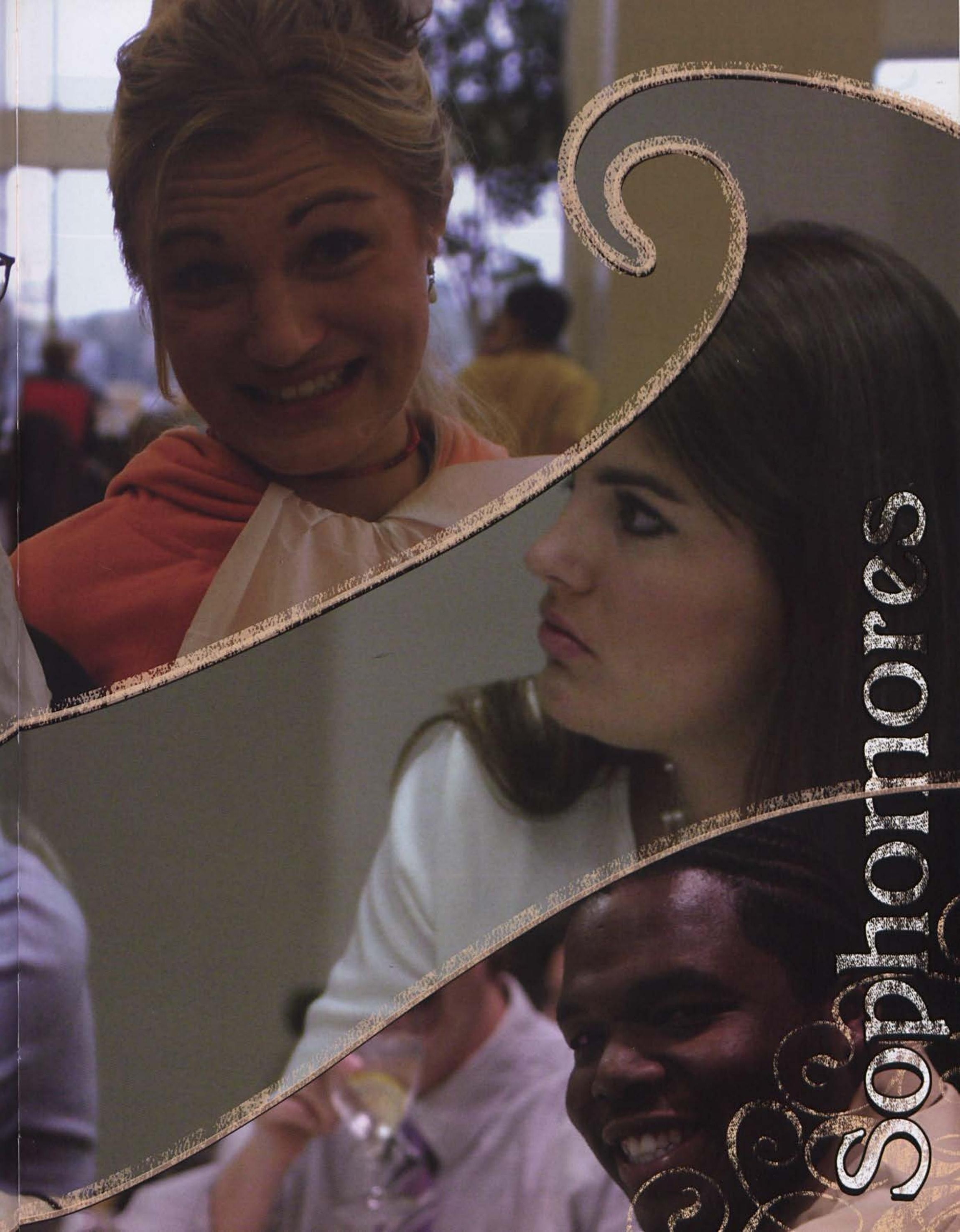




\section{Sophomores}

Matthew Abraham Alyssa Adams

Theodore Adams Jonathan Agler Dannielle Albert

Emily Alexander Jennifer Allen Courtney Anderson Melissa Anderson Amy Ashinghurst

a

Wendy Austin

Amy Babbitt

Becky Bachman

Angela Bacon

Chad Bainbridge

Michelle Baker-Karl Daniel Ballard

Sarah Banas Jonathan Banks Andrew Barber

John Bardakjy Reuben Bassett

Sarah Behn David Bennett Matthew Beres

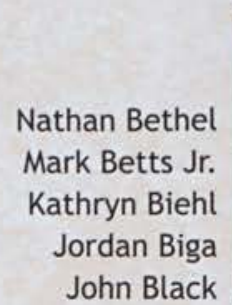
John Black
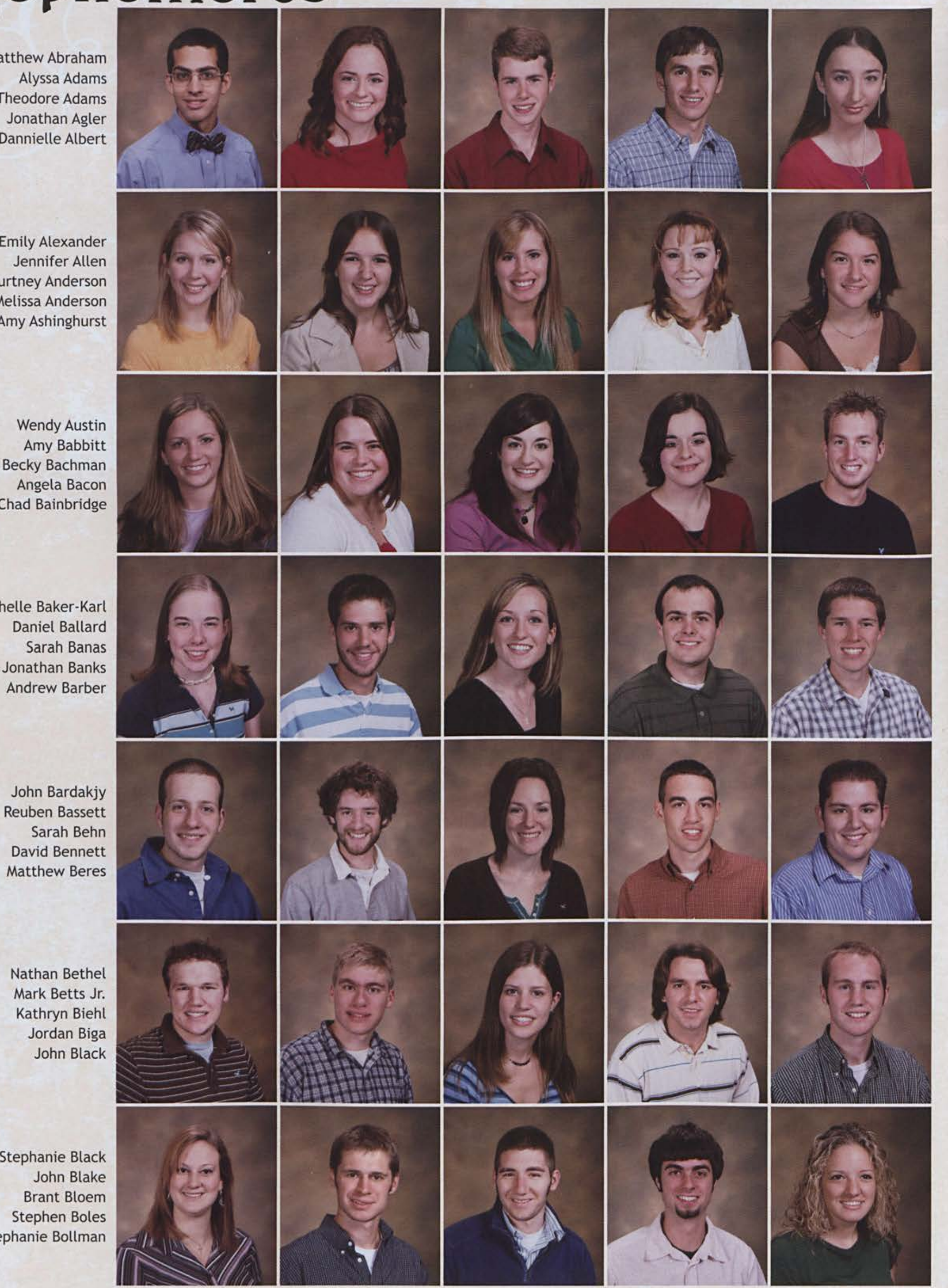


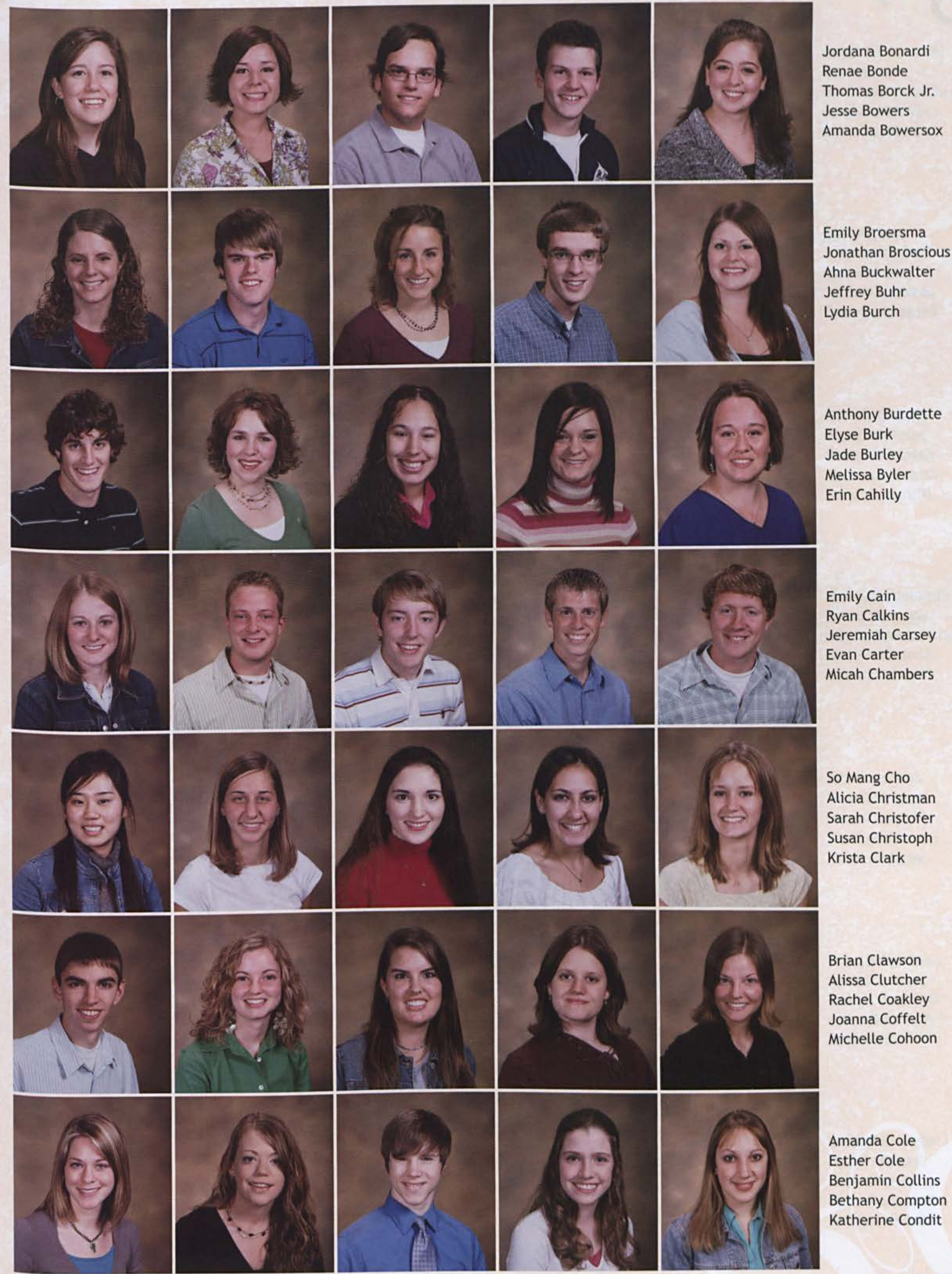




\section{Sophomores}

Elisabeth Congdon Aaron Cook

Matthew Cook

Robert Cook

Gregory Cooley

April Cooper

Daniel Copeland

Shelby Corley

Jessica Coudriet Benjamin Cowell

Lauren Cowell

Gabriel Coyle

Katrina Crandall

Abigail Craycraft

Misheal Crocker

Elizabeth Crowner

Leanne Crunelle

Christopher Cuffman

Anna Cummings

Daniel Cummins

Andrew Curtis

Heather Daniel

Richard Davies

Ellen Davis

Matthew Davis

Michelle Davis

Samuel Delong

Elizabeth Dennis

Merissa Devries

Tobias Dewhurst

Lindsay Di Donato

Jennifer Dixon

Justin Dodson

Brittany Donald

Rebekah Drum

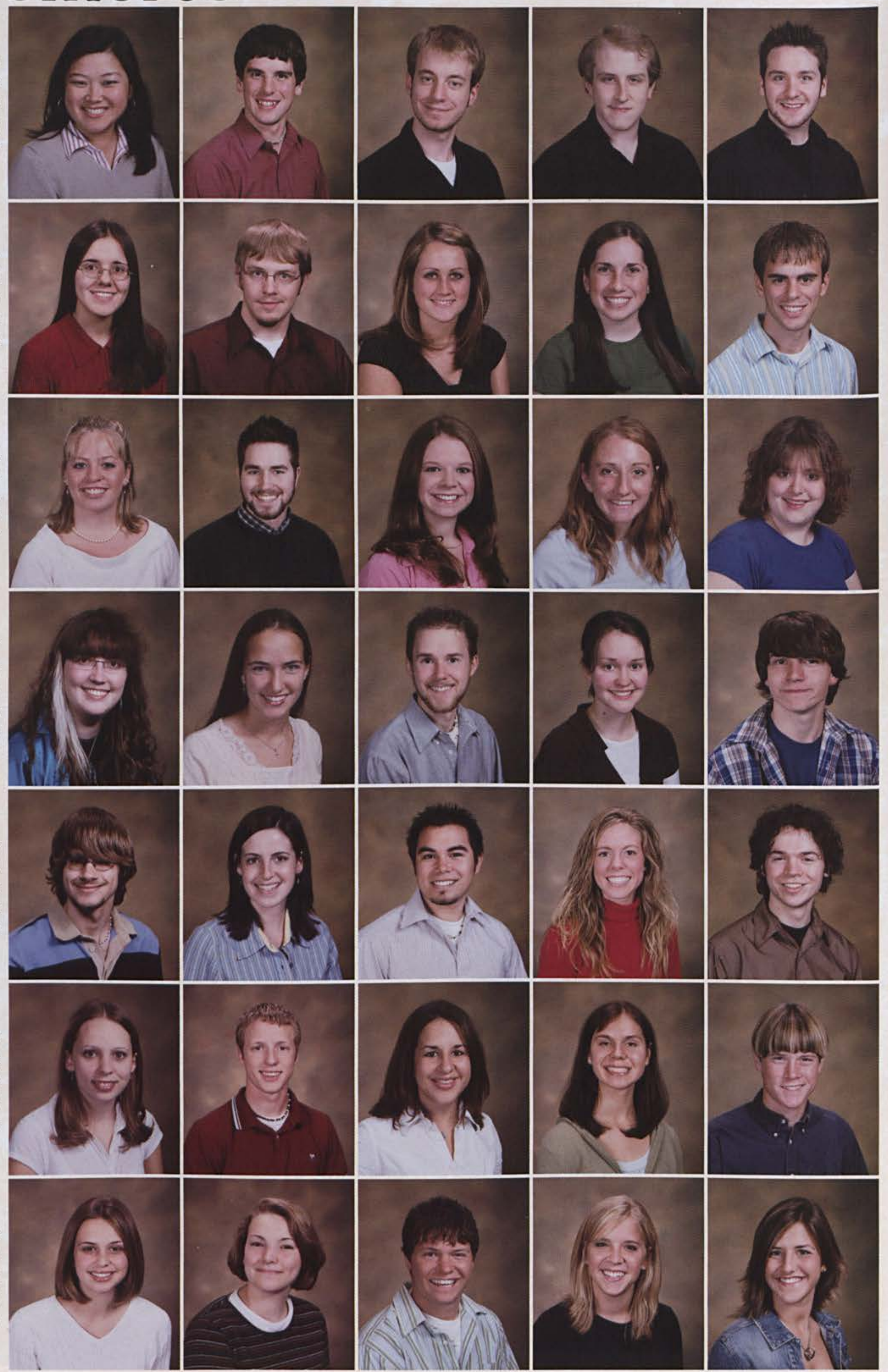




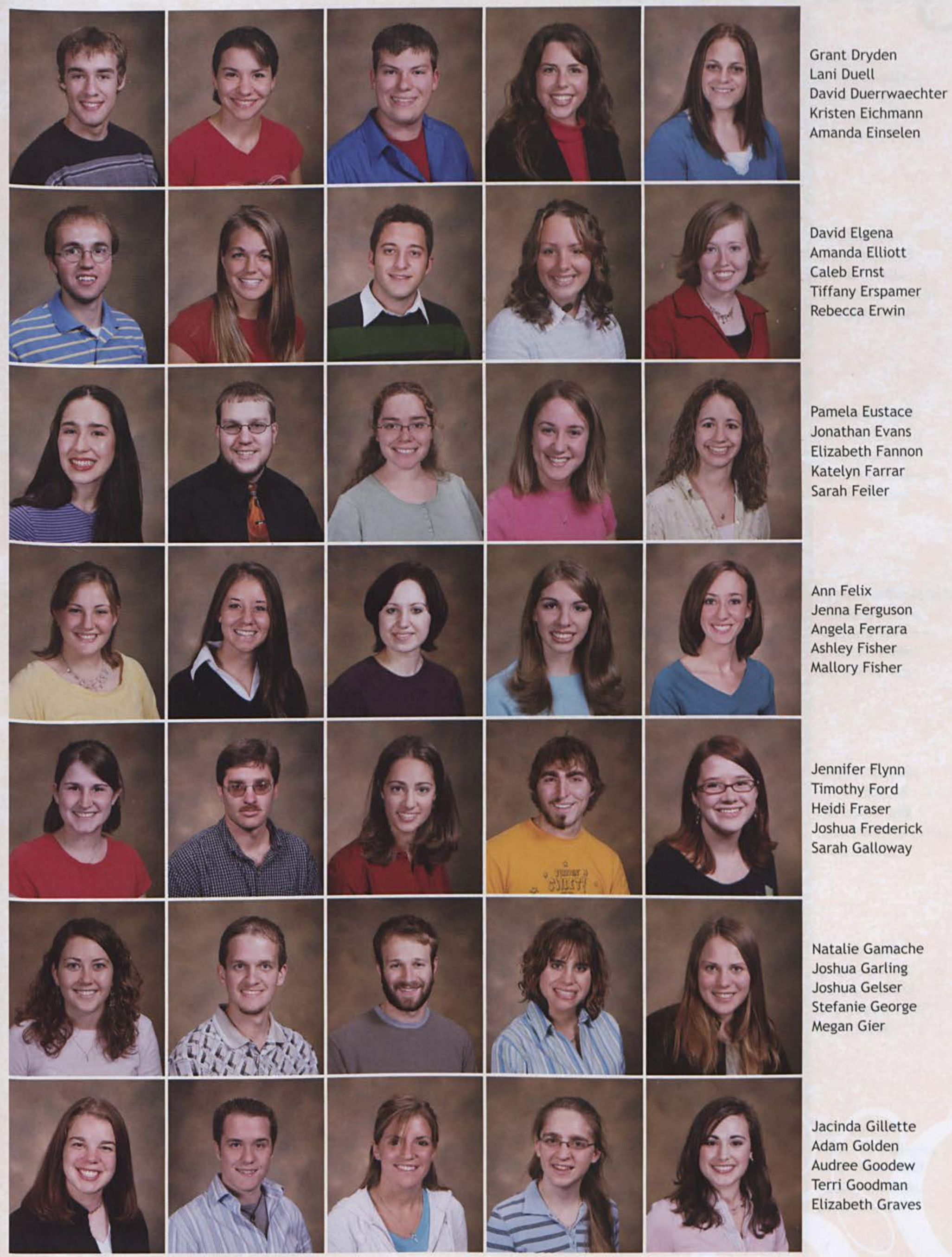




\section{Sophomores

John Gray
Michelle Gray
Kimberly Greer
Jessica Grewe
Aubrey Gries

Jared Griest

Shannon Grosso

David Grudds

Kevin Gulley

Jennifer Guth

Justin Gutierrez

Ryan Haag

Drew Hackney

Amy Haetinger

Paul Hale

Maria Harley

John Harrison II

Andrew Harsh

Heather Hayes

Sara Hayes

Hannah Haynes

Rachel Haynes

Elizabeth Hays

Heather Heagy

Leslie Heiss

Caleb Hensley

Joshua Herbolt

Brittany Herbst

Stephanie Hilerio

Kristi Hines

indsey Hlad

Rachel Hobbs

Chelsie Hoffman

Matthew Hollis

Jada Holloway
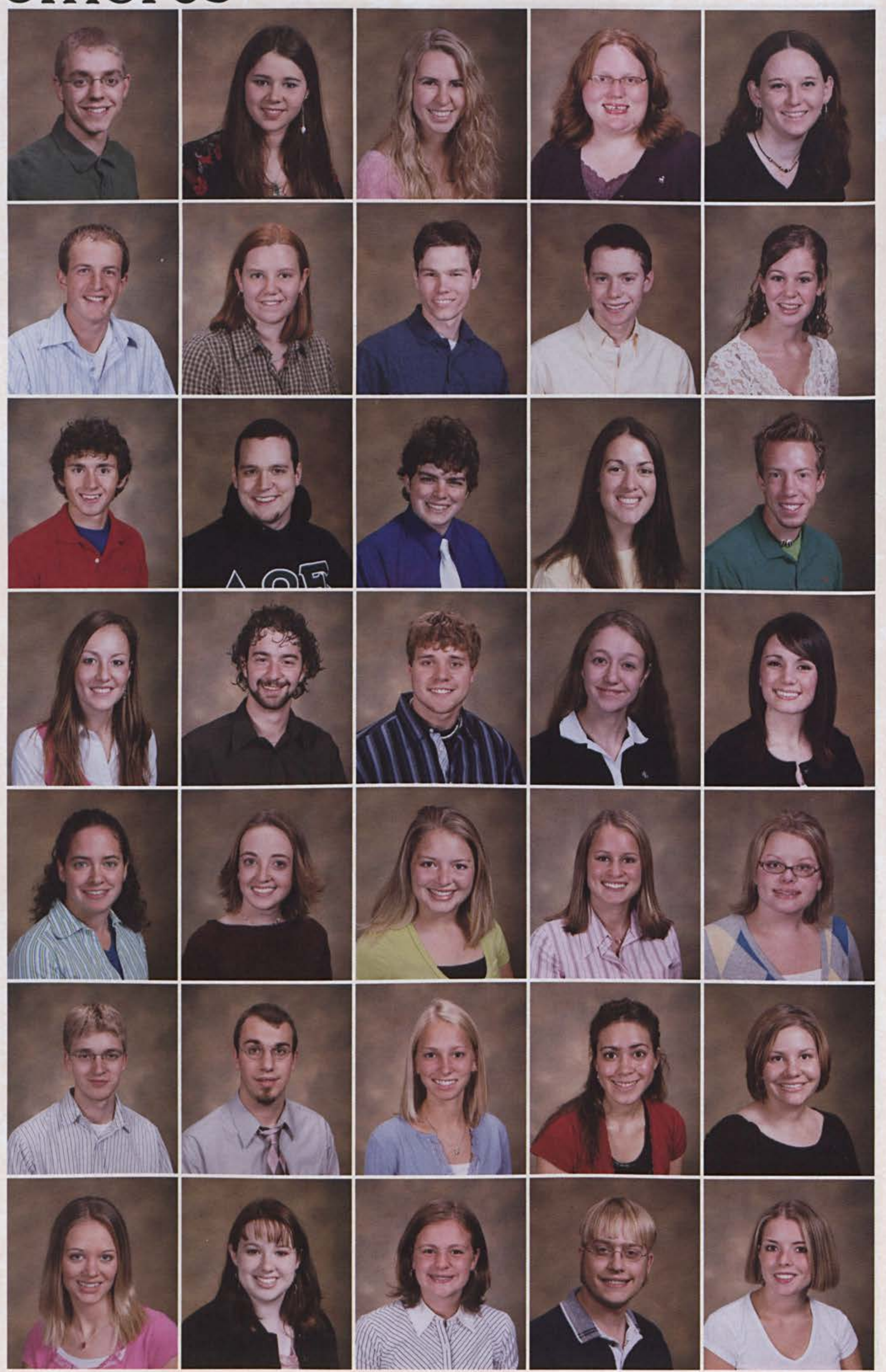


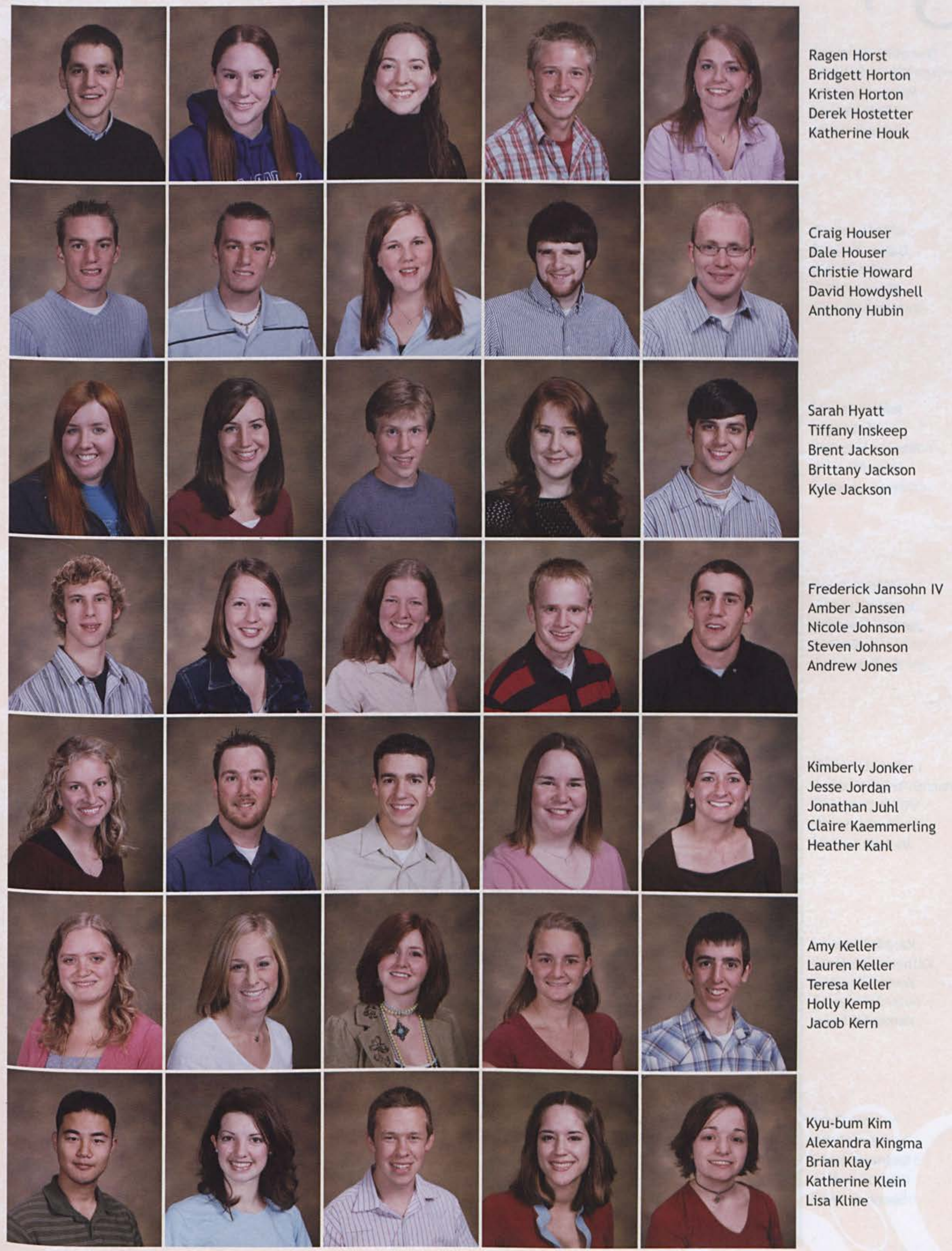




\section{Sophomores}

Daniel Kloosterman Daniel Knudsen

Michael Knudsen

Tiffany Knupp Nicholas Kohne
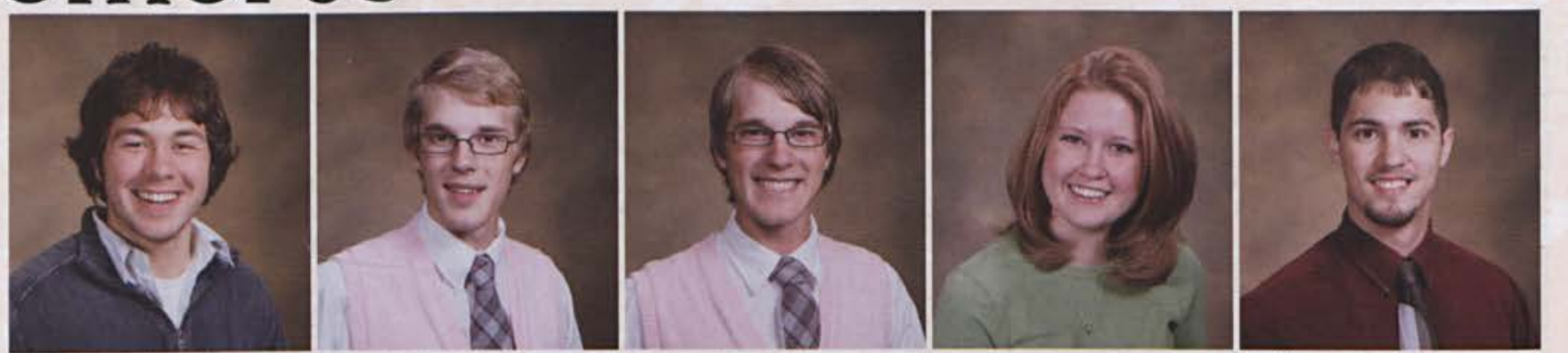

Viki Kooiman Daniel Koranek Justin Kraker Charles Kraus Carol Kreeger

Amanda Kuntz Joel Lagan Rhoda Lambright

Meredith Lang Jesse Lansford

Lisa Largent Jonathan Lawler Jessica Lawrence Laura Lee Andrew Leeds

Lindsay Lehmann Johannah Leightenheimer Philip Lengefeld Kimberly Lincoln Michael Lincoln

Kara Lindemann Katherine Lippman Kevin Livengood John Locke Katherine Loper
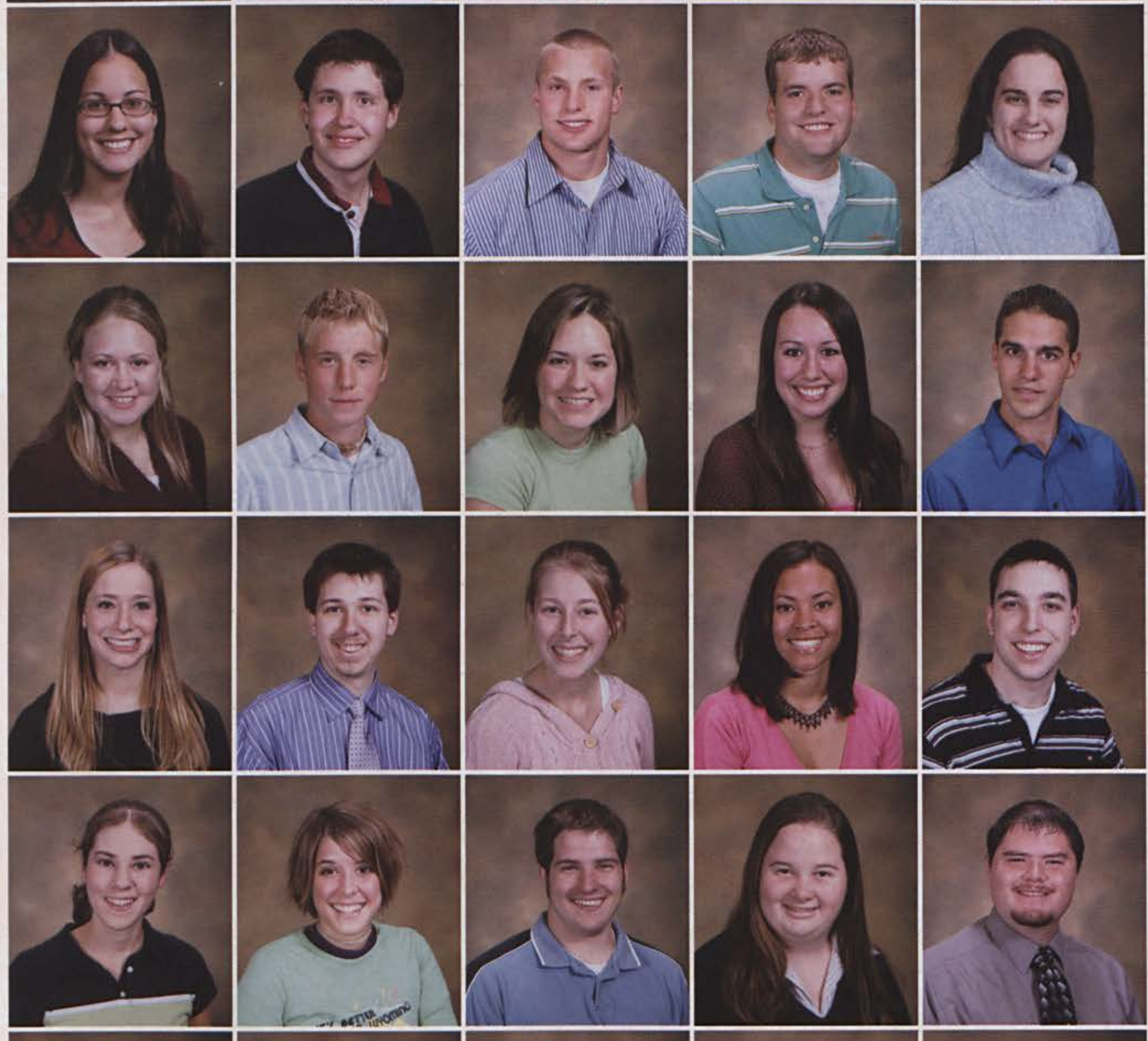

Joel Losch

Aaron Louie

Sharayah Lucas

Clayton Ludlow

Shannon Lynch
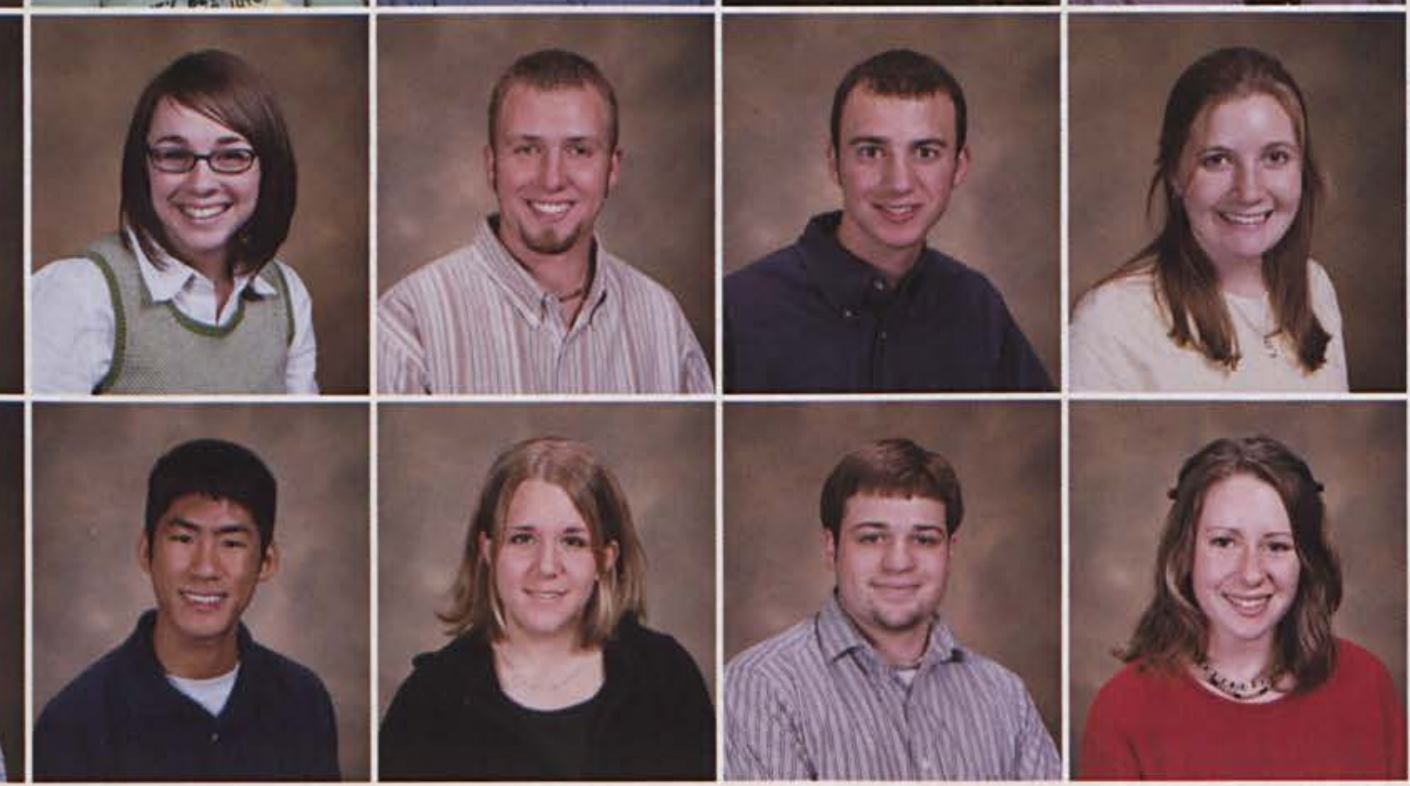


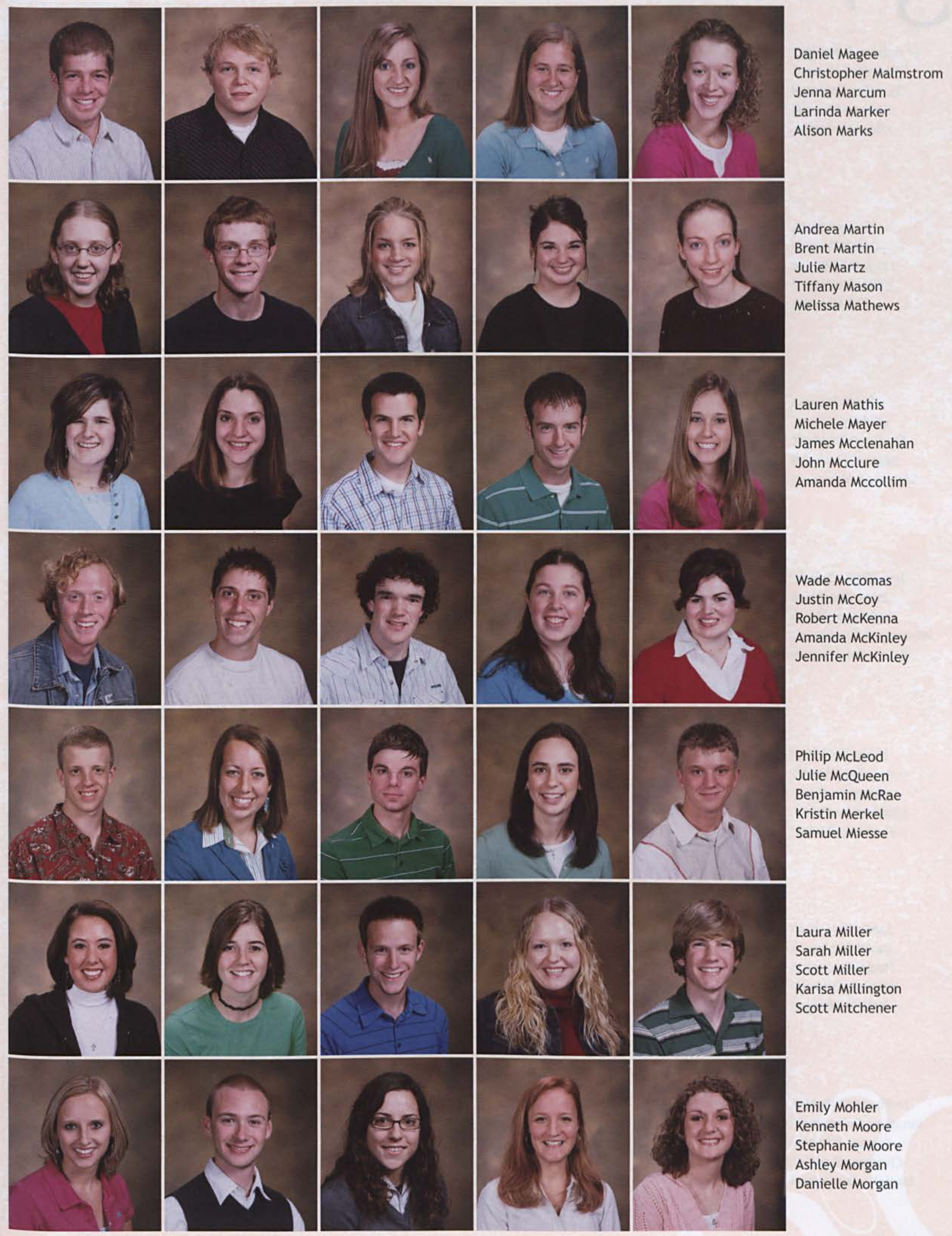




\section{Sophomores

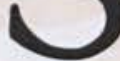

Darrell Morgan Jonathan Morgan Jennifer Moriarty James Morrow Camille Morse

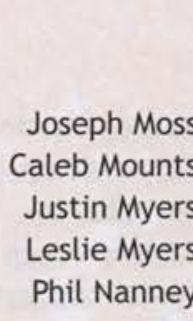

Brian Nelson Kelsey Nelson Benjamin Nesbitt Allison Newfield Sarah Niedermayer

Jonathan Niknam Bryan Norman Joshua Ohms Whitney Oosterhouse Paul Organ

Nicholas Parry Erin Pasma

Rebecca Peak Katherine Pelon Kelly Perrel

Sarah Petersen David Peterson Paula Pheasant Kathleen Picard Kaitlyn Picazo

Timothy Piette Jonathan Polsdorfer Kathleen Pritts

Christina Pupillo Danielle Randolph
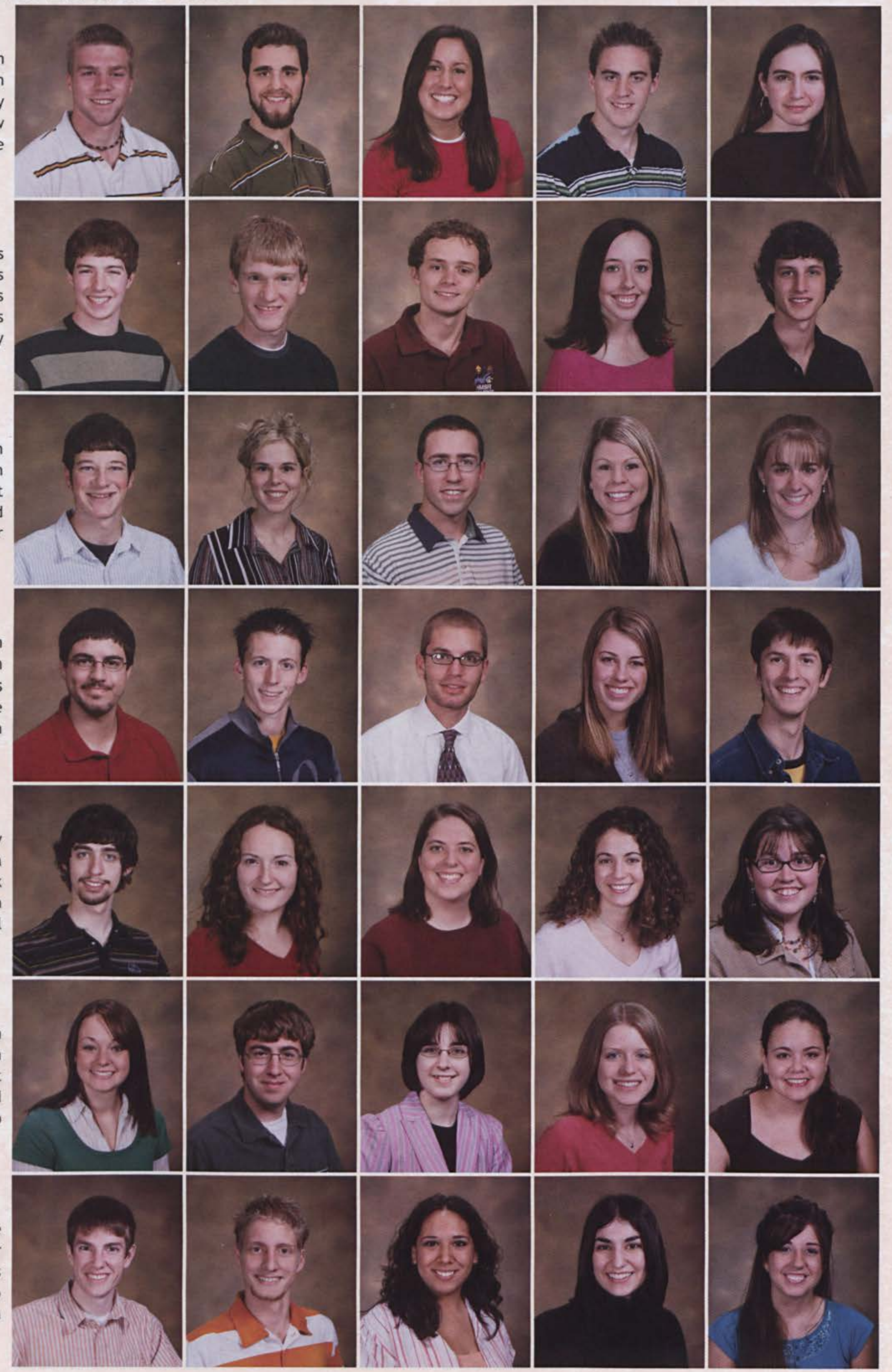


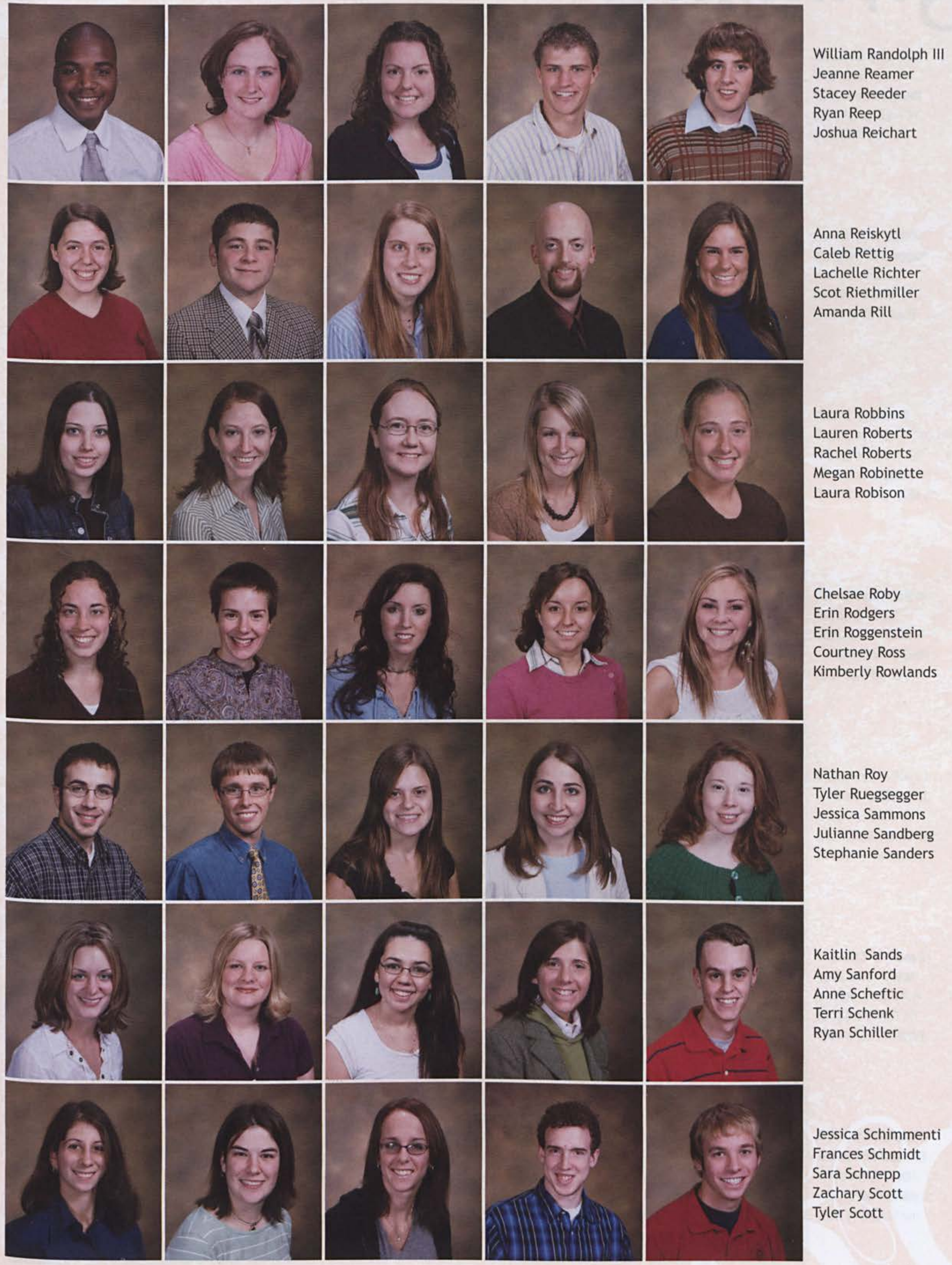




\section{Sophomores}

Bethany Seawell

Tara Self

Pamela Seymour

Lane Sheats

Karlee Shelton

nifer Siegrist

Ashley Simon

Trenton Simon

Paul David Sims
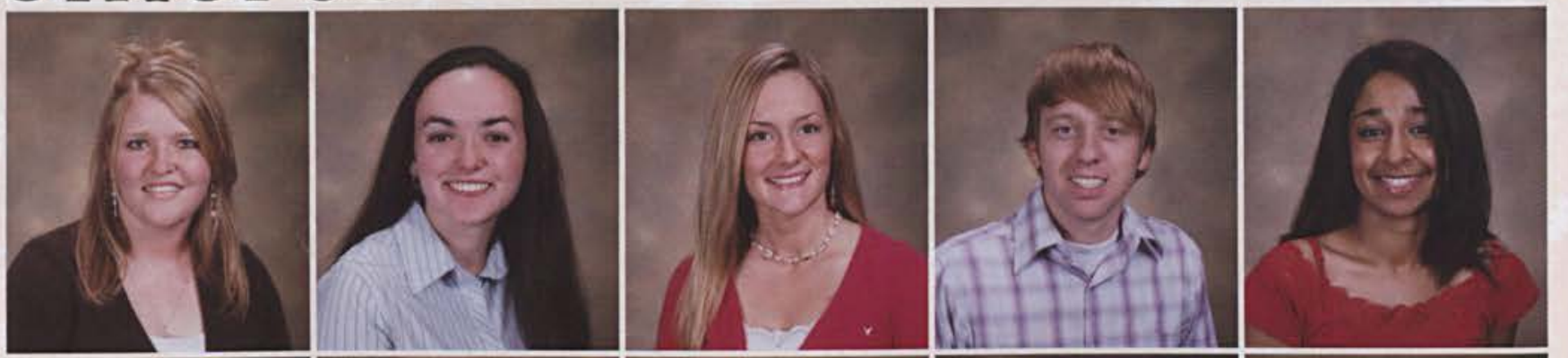

Brittany Smart

Brett Smith

Mark Smith

Matthew Smith

Nathaniel Smith
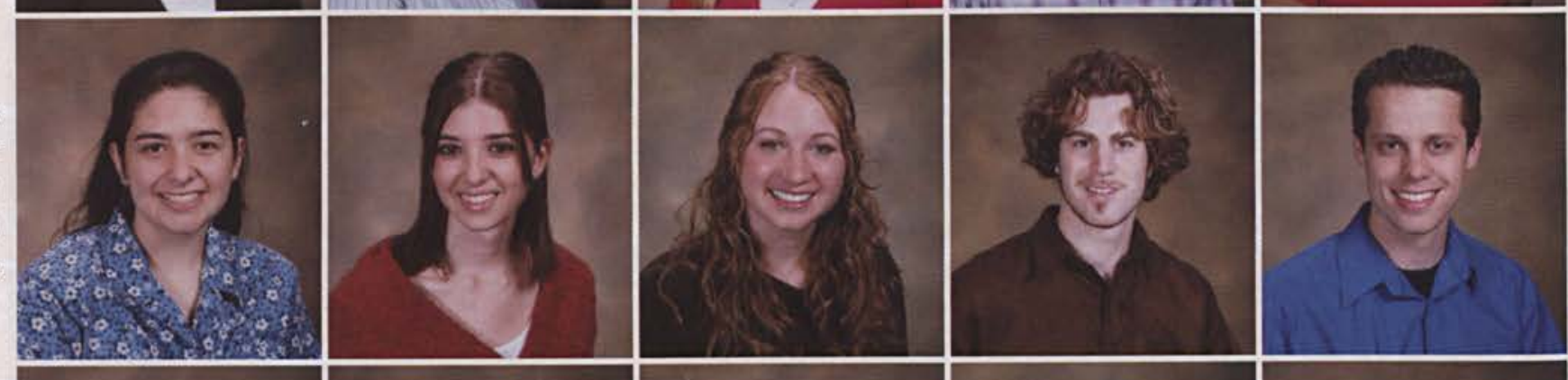

Rebekah Smith

Joel Smoyer

Rachelle Snavely

Nathaniel Sowder

Jacob Spencer
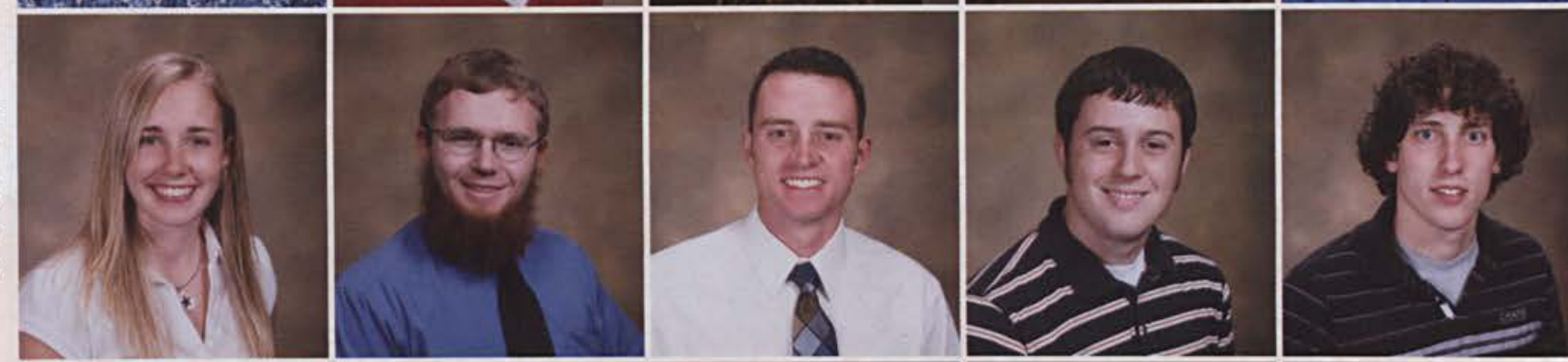

Eva Stark

Johnathan Stefan

Kelly Steingass

Amanda Stephens

Jennifer Stevens
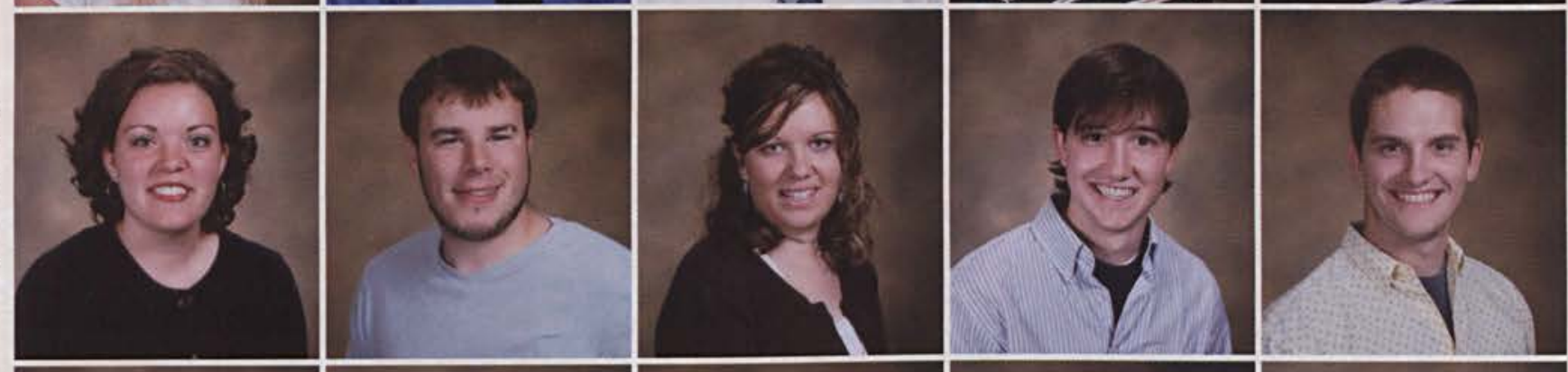

Caroline Stewart

Mary Stockdale

Laura Stokes

Jacob Stout

Michael Strawser
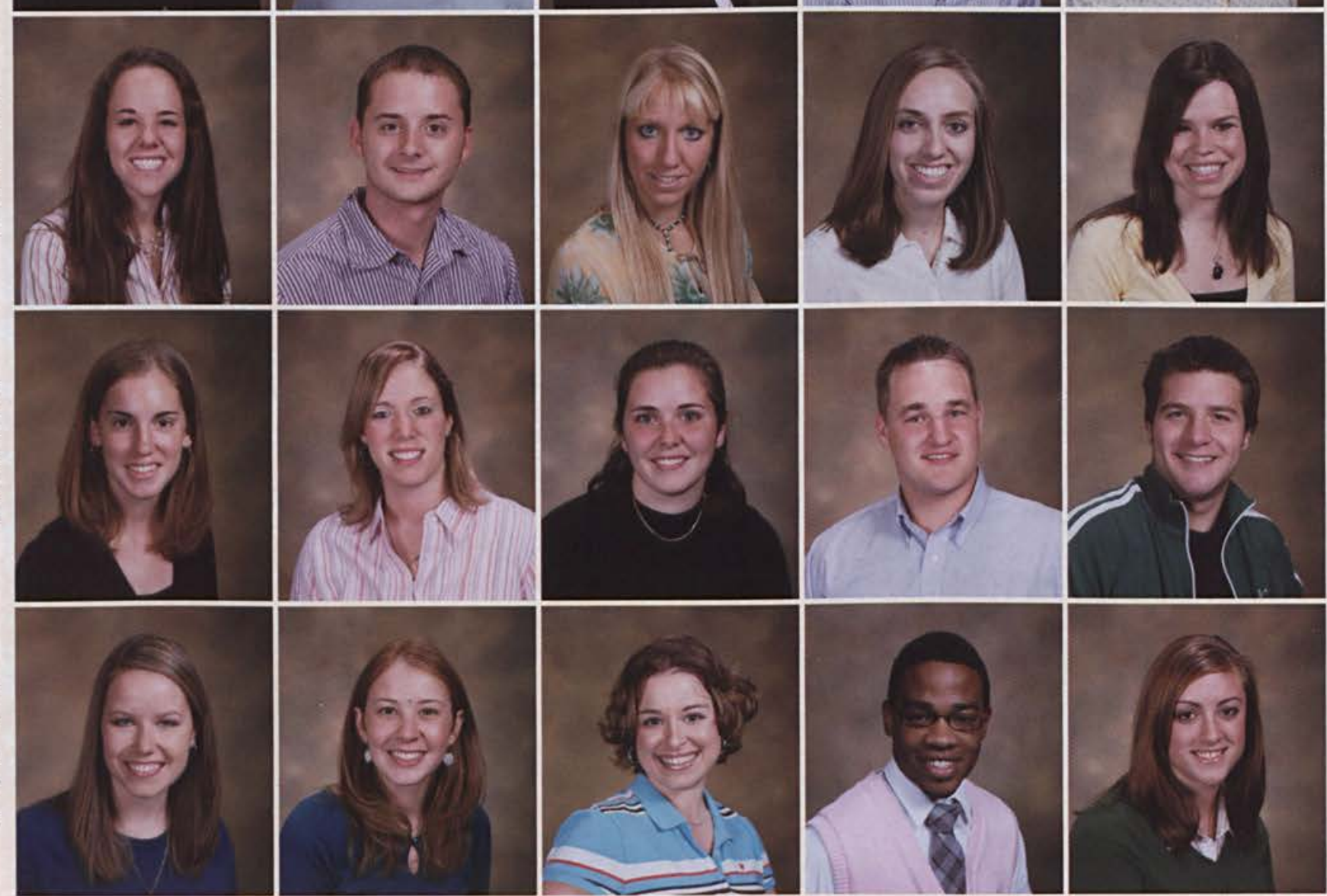

Deborah Strutton Elisabeth Sudlow

Donna Sweede

Stephen Sylvester

Ashley Taft
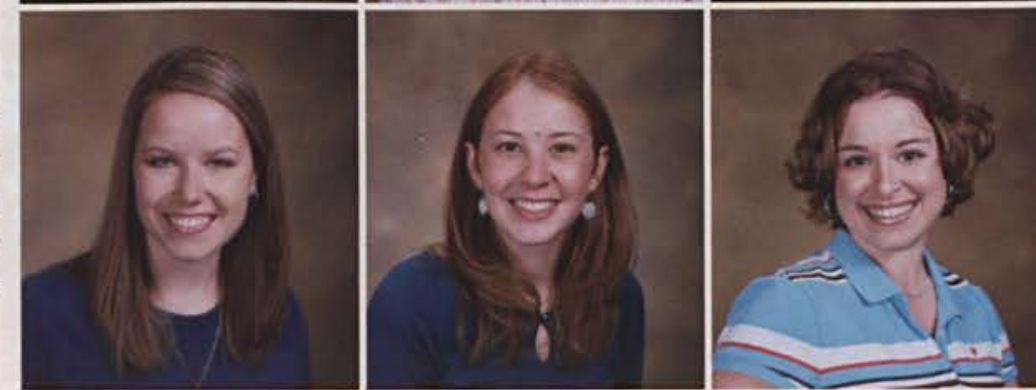


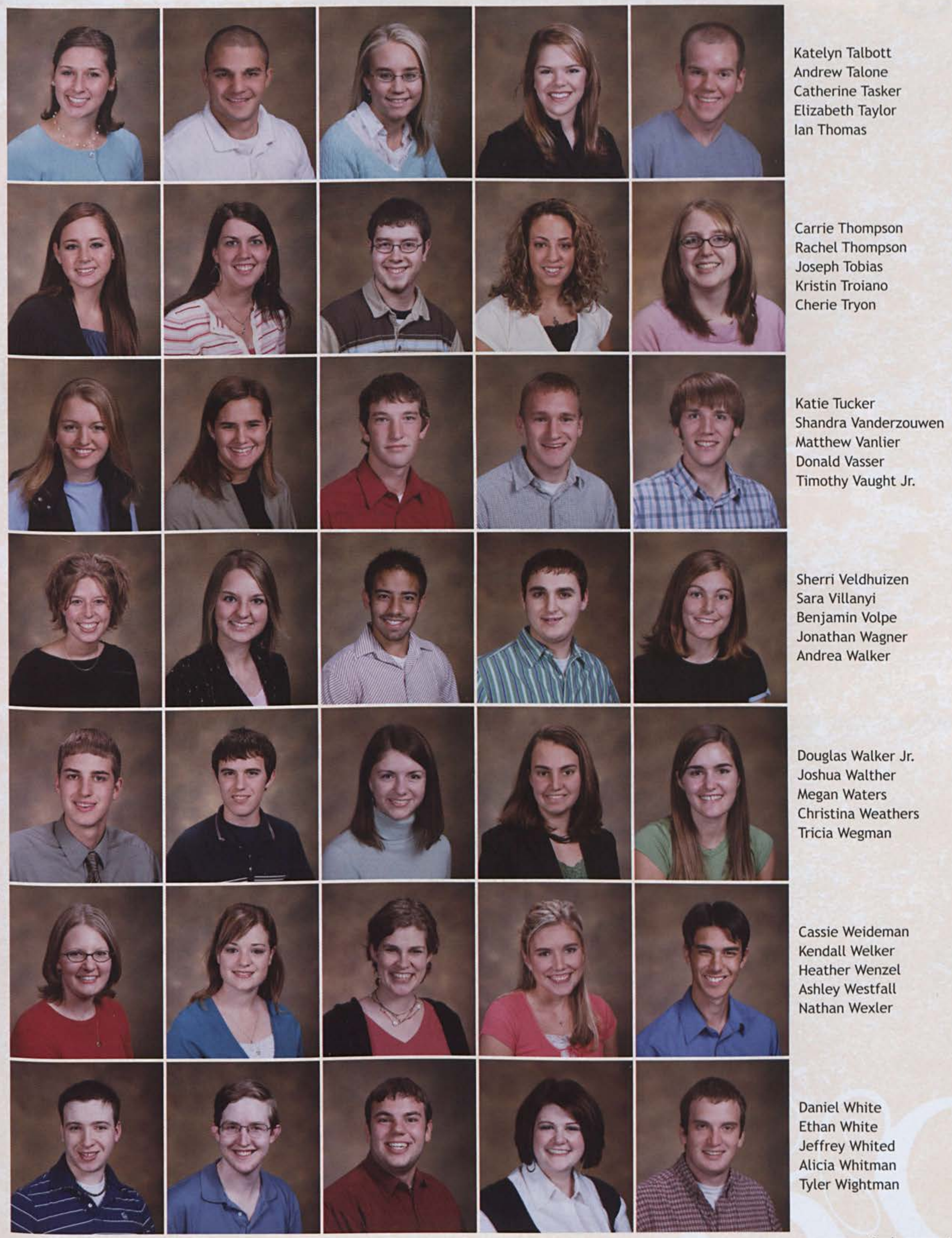



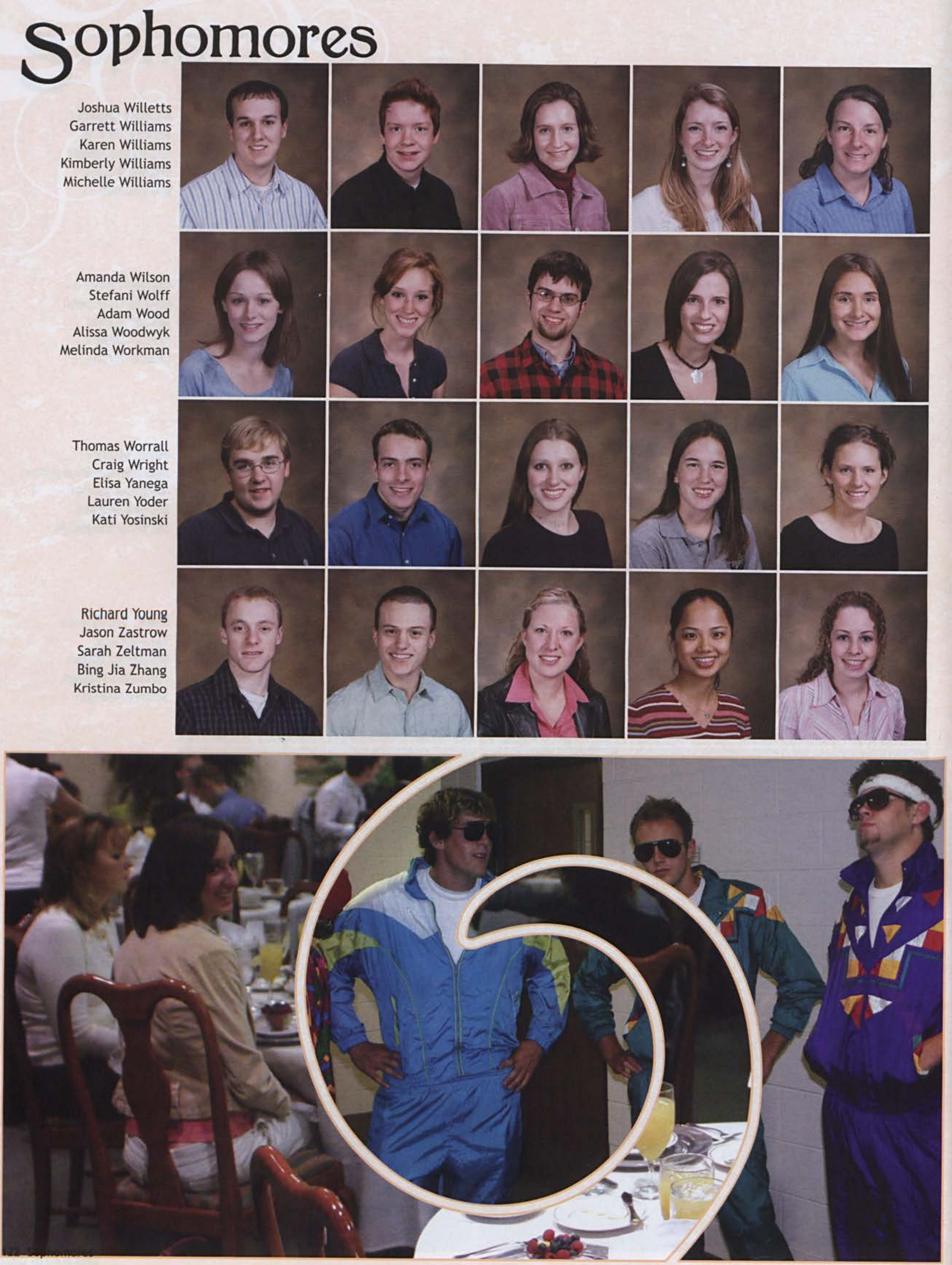


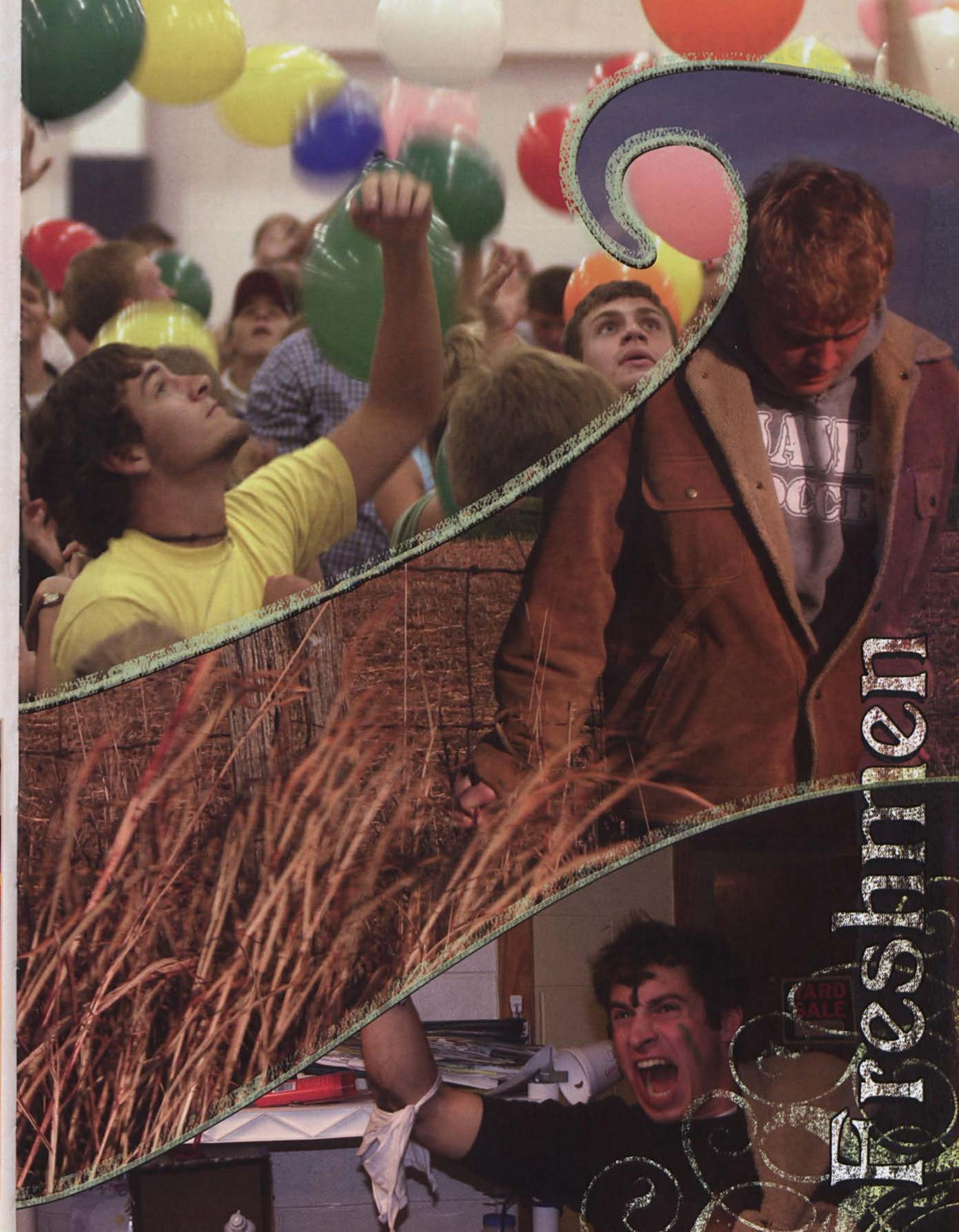




\section{Freshmen}

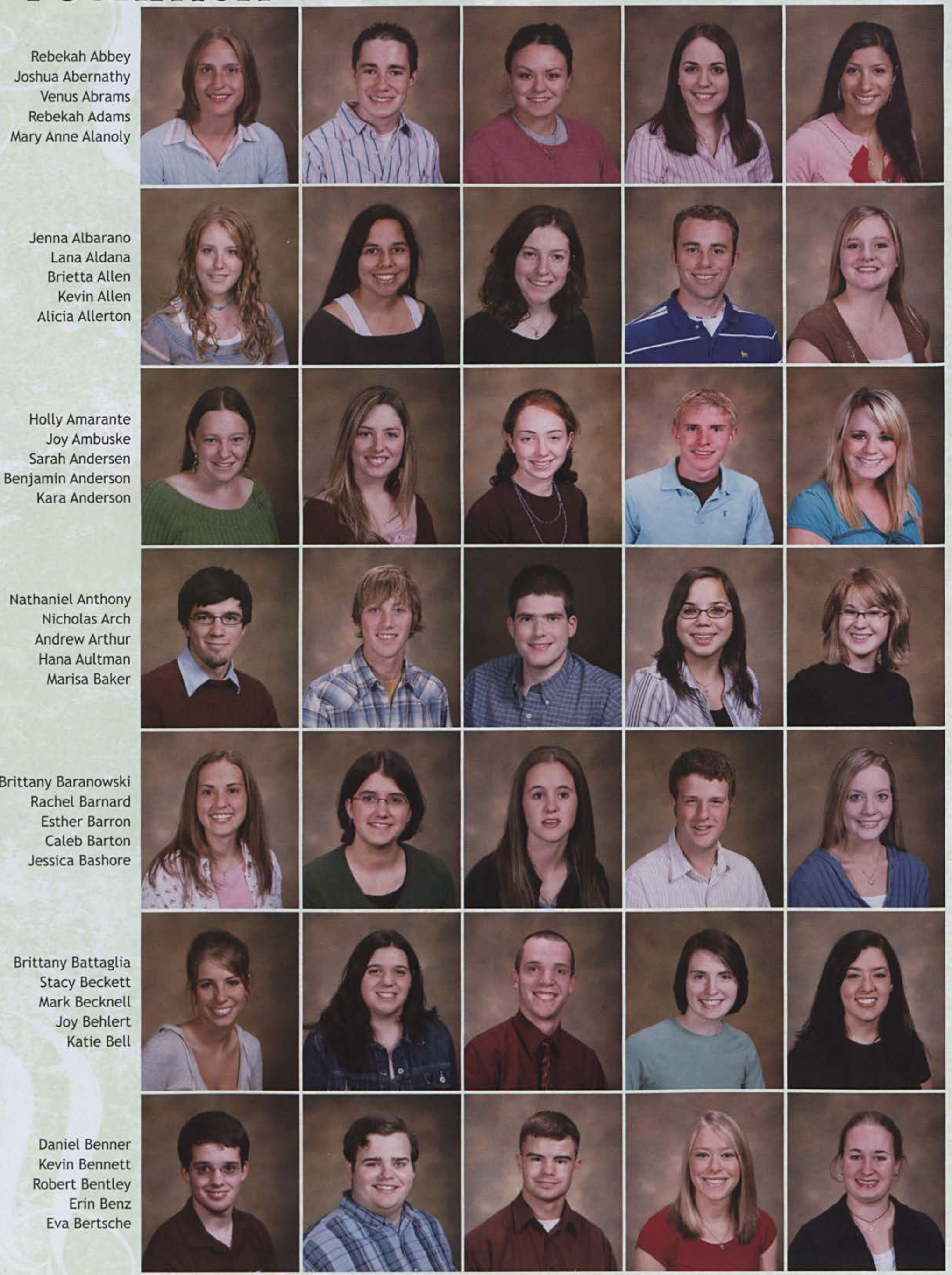




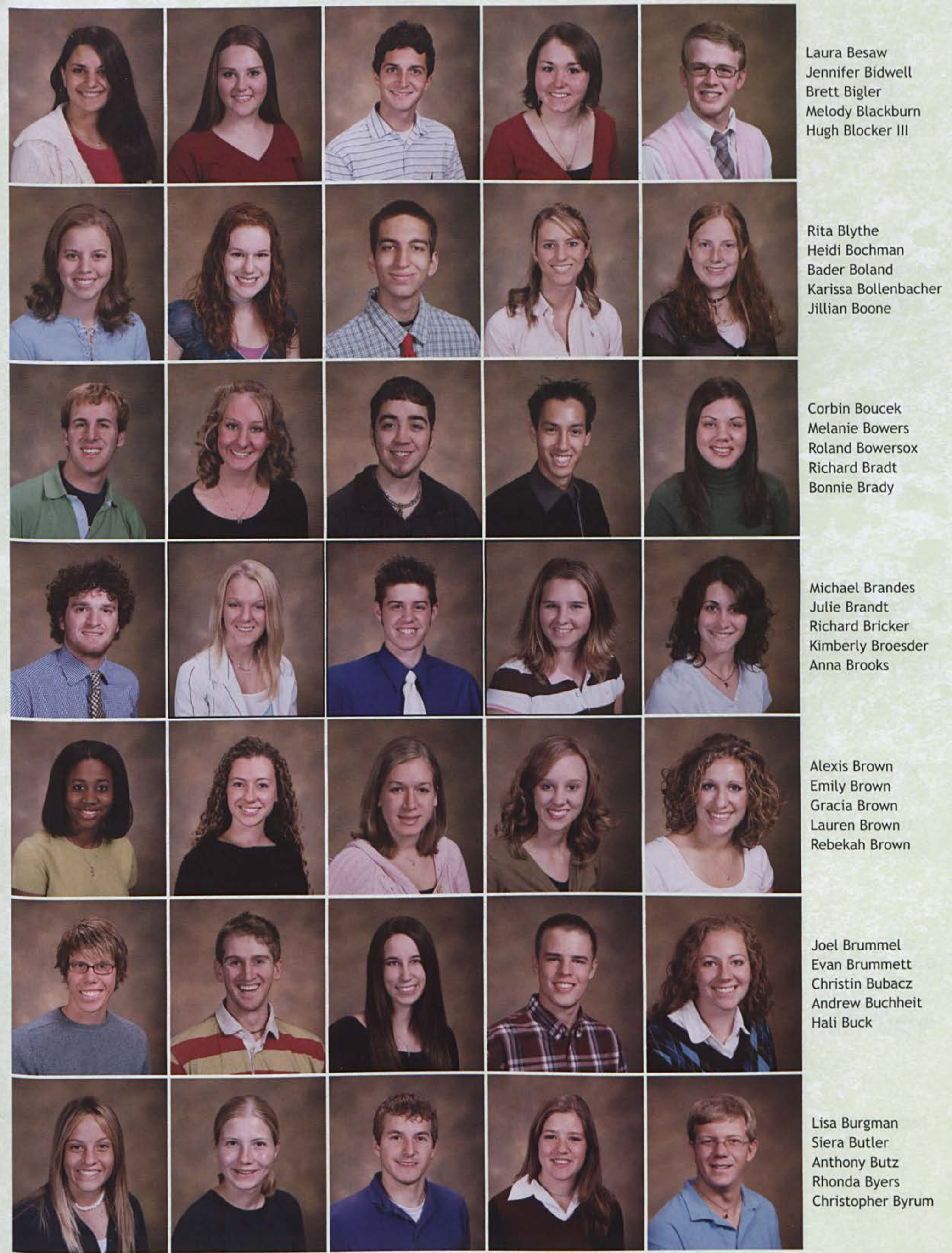




\section{Freshmen}

John Carey

Abigail Carroll

Ashley Carter

Jace Carter
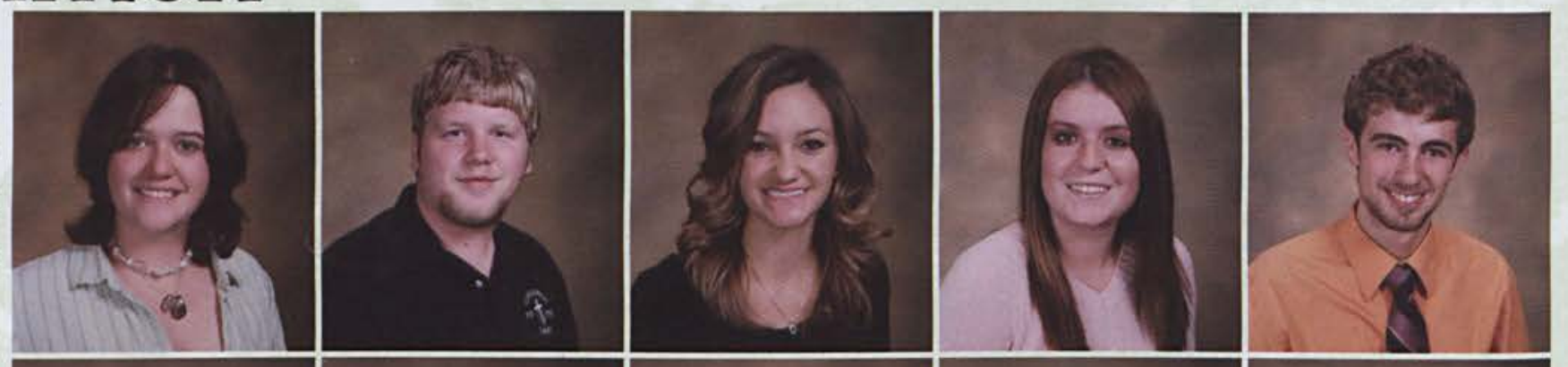

Ian Casper
Celeste Cato

Matthew Chamberlin

Sarah Cheatham

Heidi Christiansen

Lindsey Christman

Abby Clark

Amy Clark

Elizabeth Clark

Melissa Clawson

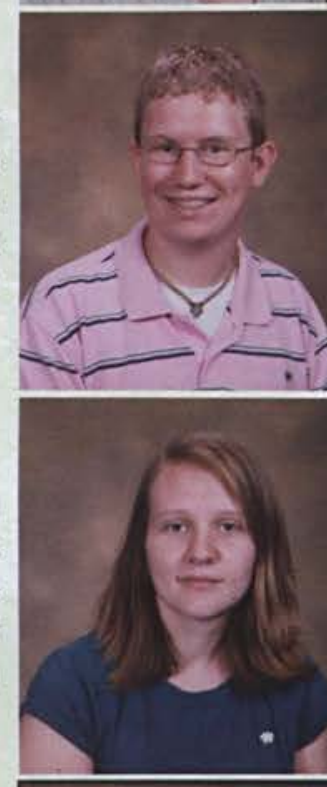

Jordan Clay

Alexandra Clements

Kaydrie Clymer

Stephen Cobucci

Timothy Cochran Jr.

Kari Coffindaffer

Jennifer Coffman

Kraig Cole

Laura Coleman

Sarah Coleman

Elizabeth Conkling

Mary Connolly

Margaret Cook

Andrew Costerisan
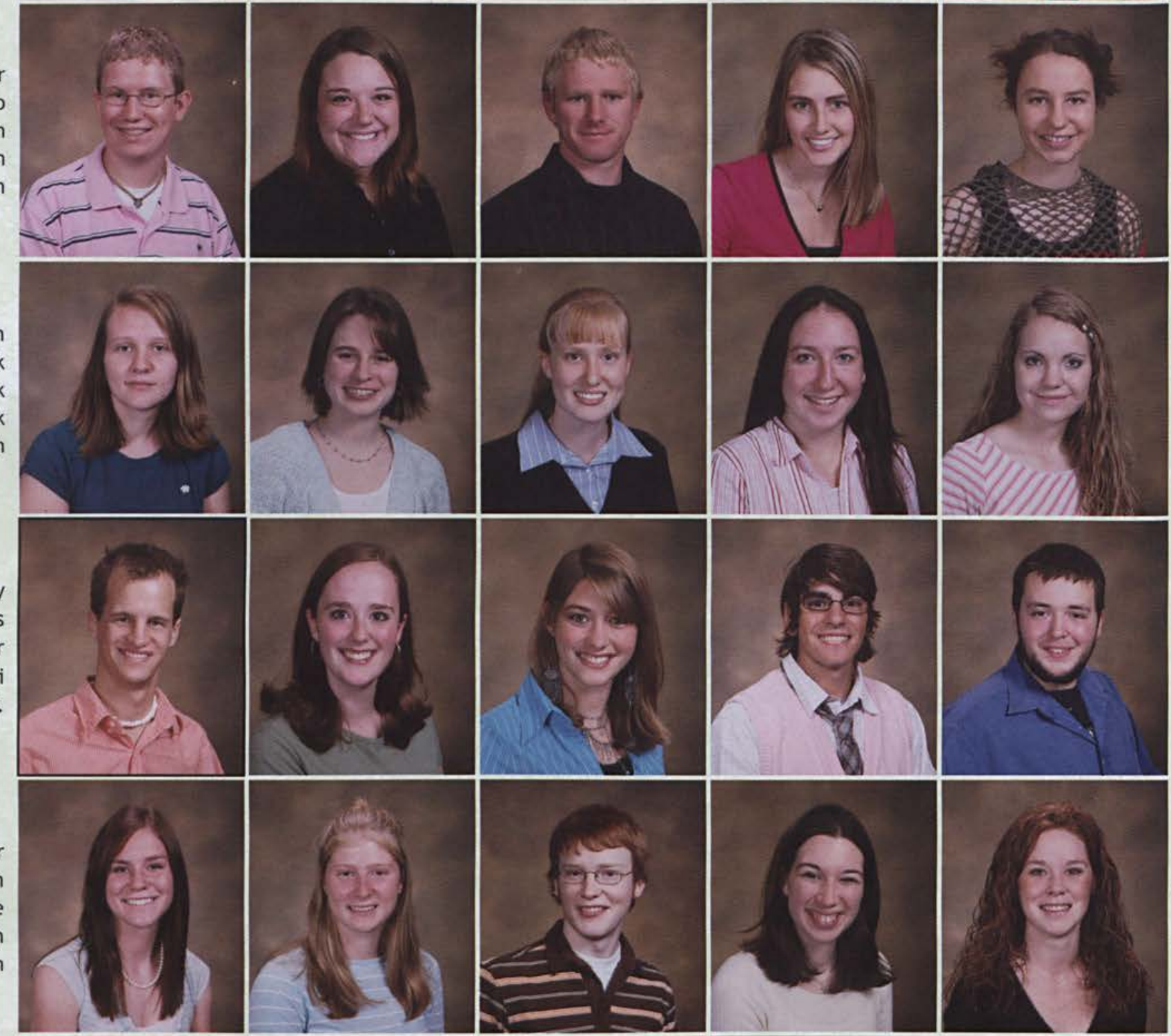

Andrew Costerisan

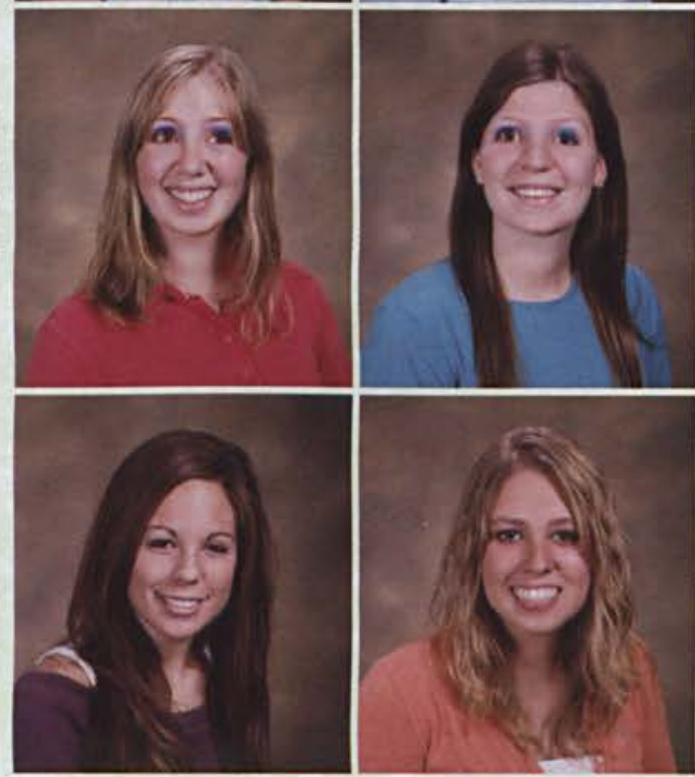

Bethany Cottings

Laura Cover

Amanda Cox

Sara Craig

Scott Crawford 


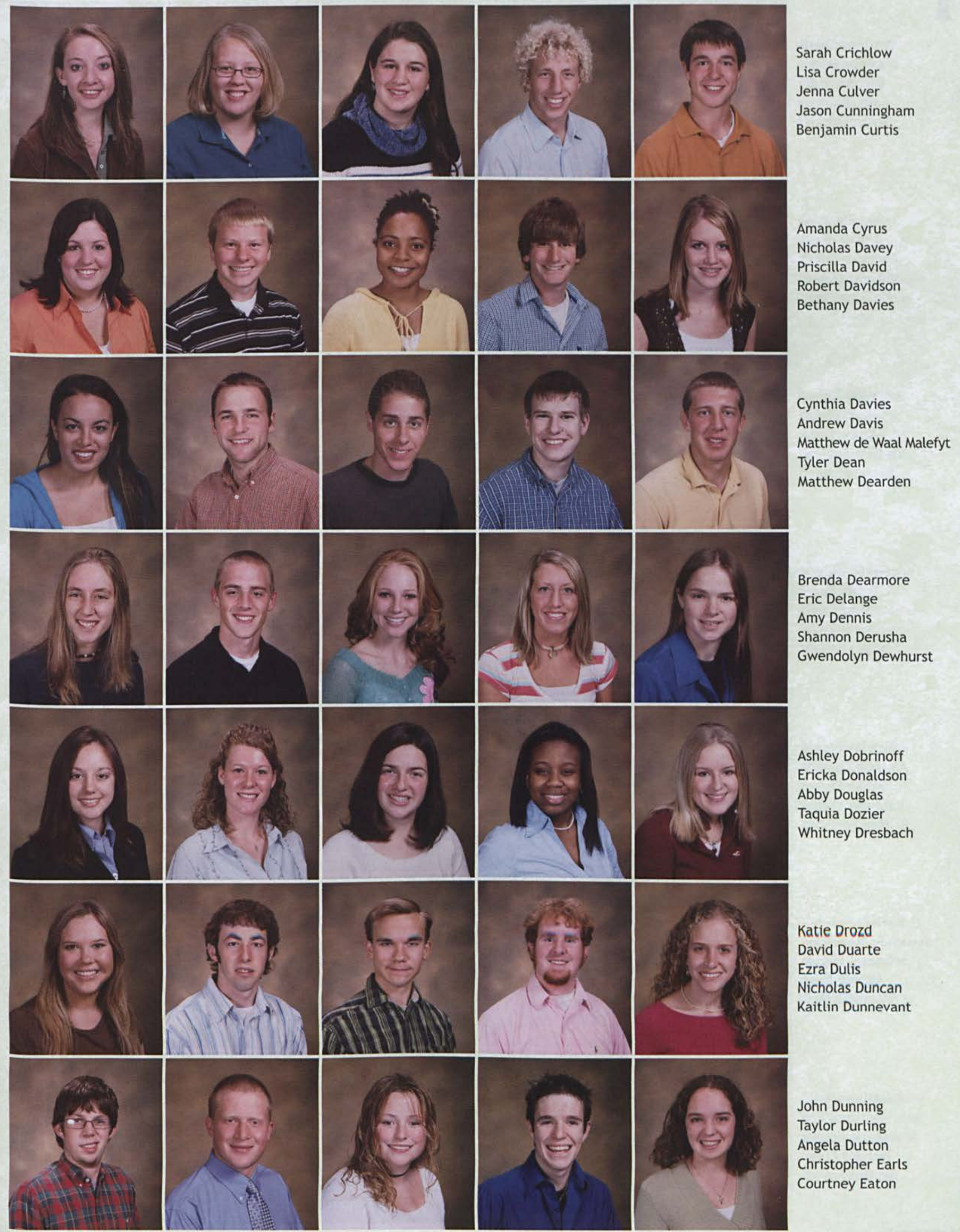




\section{Freshmen}

Matthew Eberle Ashley Eckard Timothy Elder Kaleb Eldridge Sarah Eldridge

James Elgersma Ashley Elliott Camaren Elliott Kimberly Elliott Sara Elliott

Jennalee Elmore

Aaron Engel

Austin Engel Jessica England Valerie England
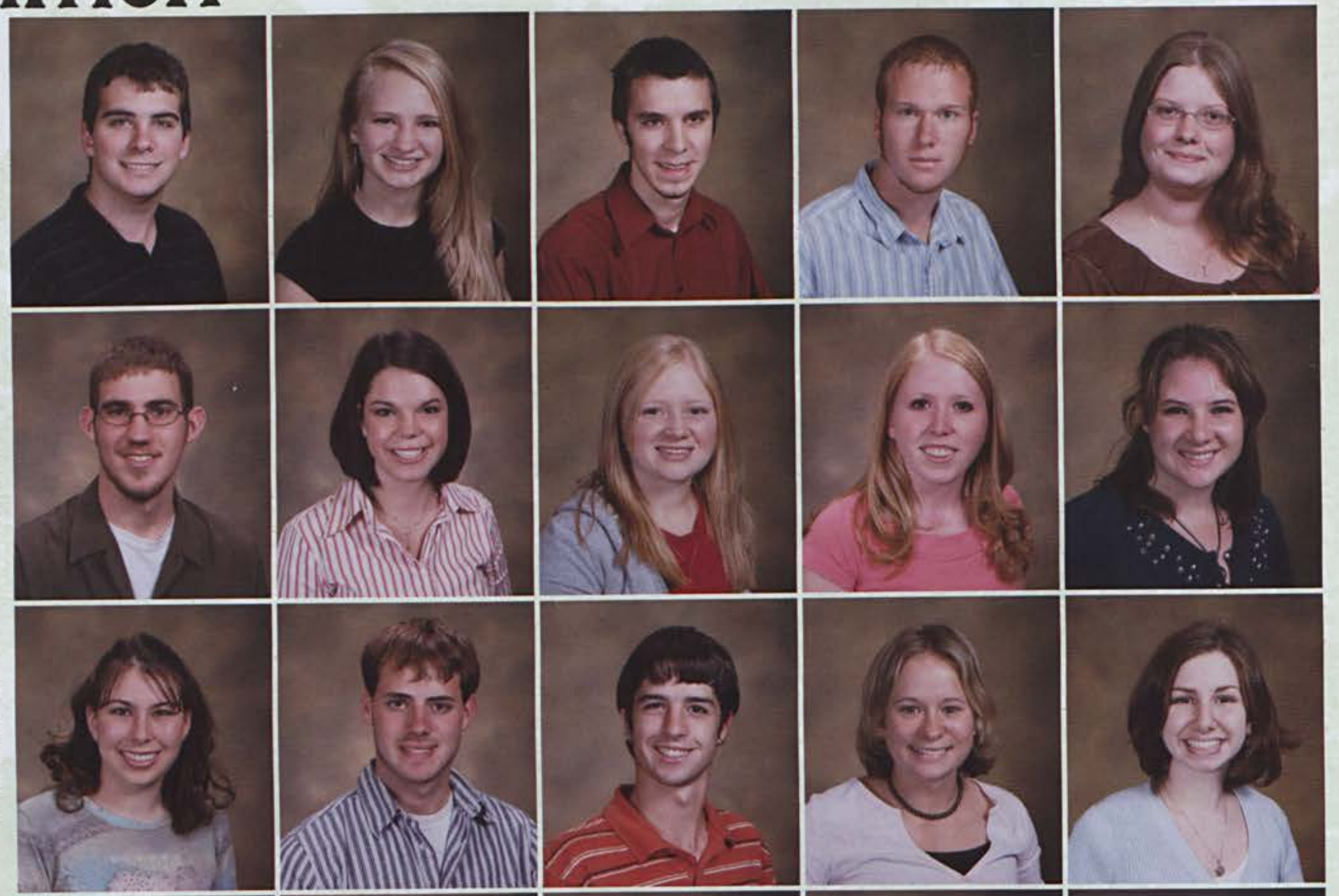

Kristen Ensminger
Adam Evans
Lacy Ewers
Dea Fallin
Megan Fansler

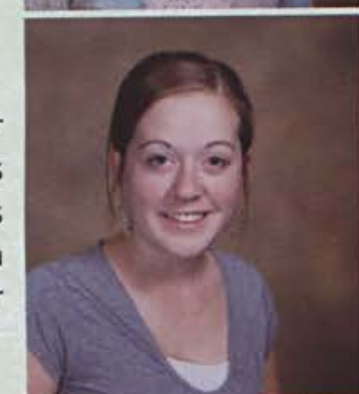

Katherine Farrell

Amanda Fawcett Jillian Feczer

Katherine Feiler Kristen Ferguson Jonathan Fleetwood

Crystal Flippin Bonnie Flora

\section{Elizabeth Flow}

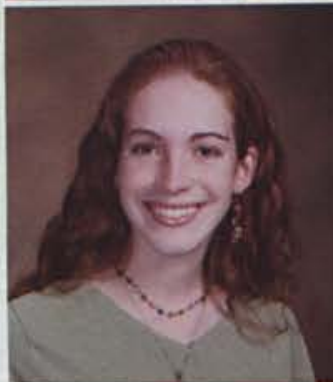

(a)
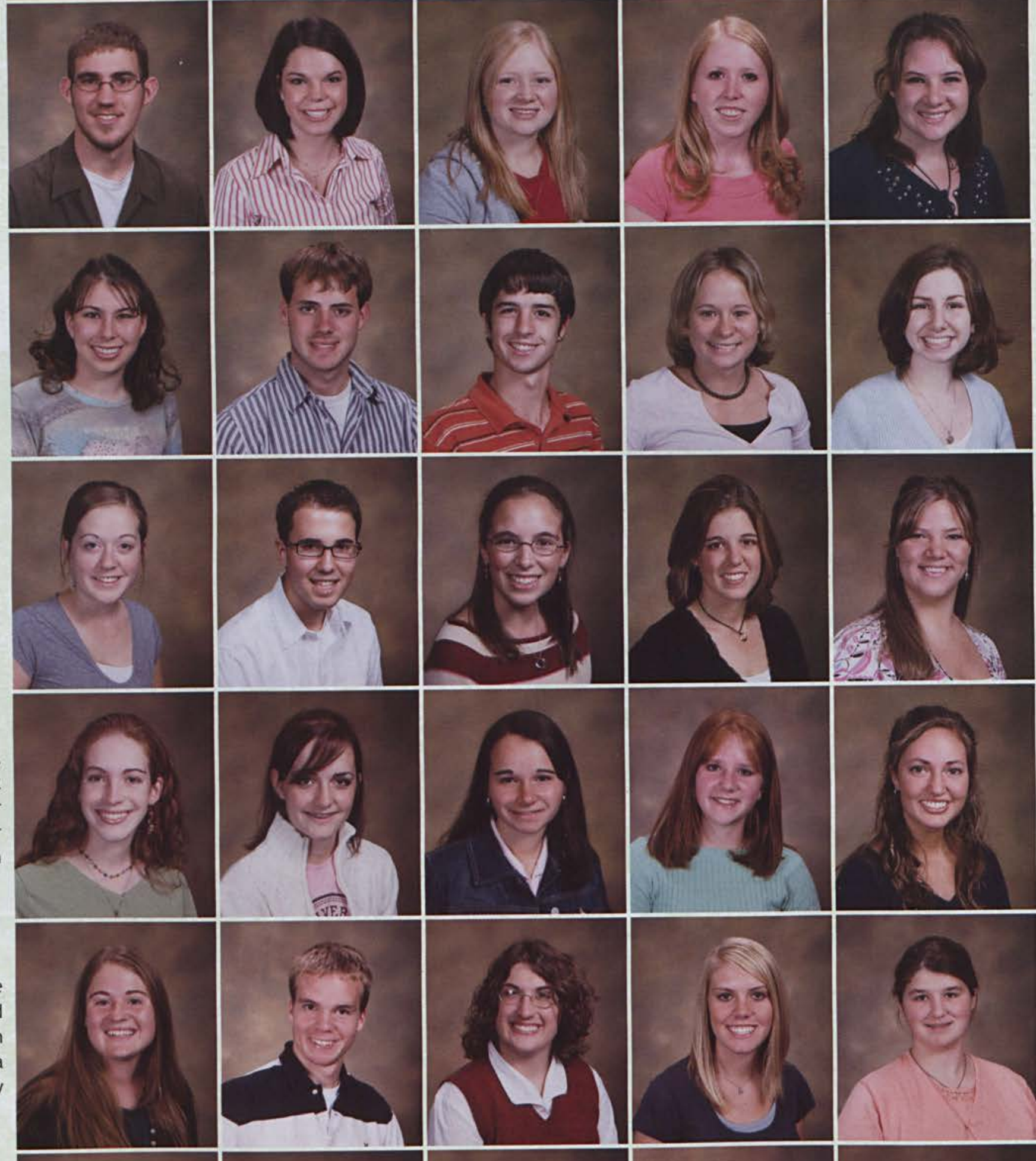


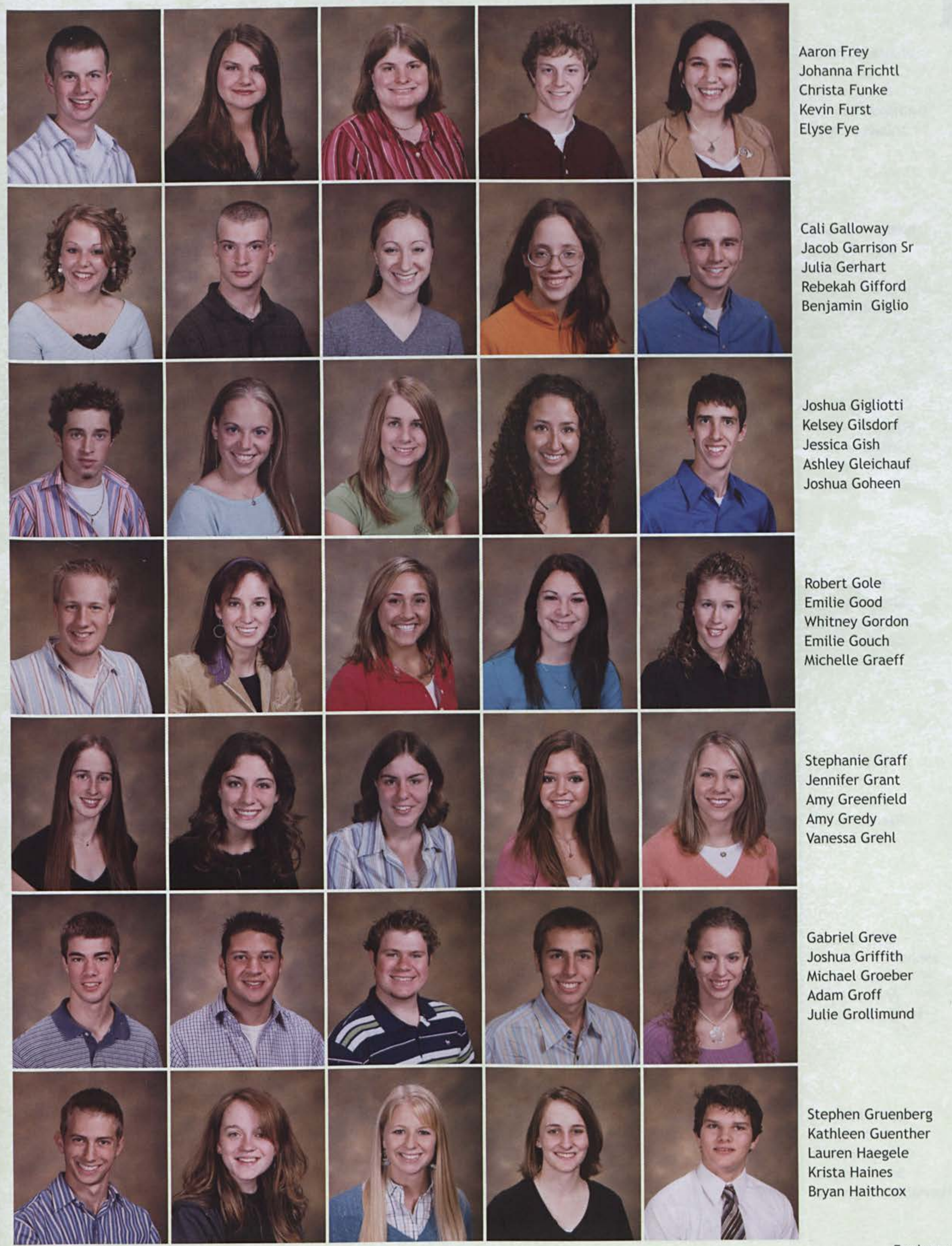




\section{Freshmen}

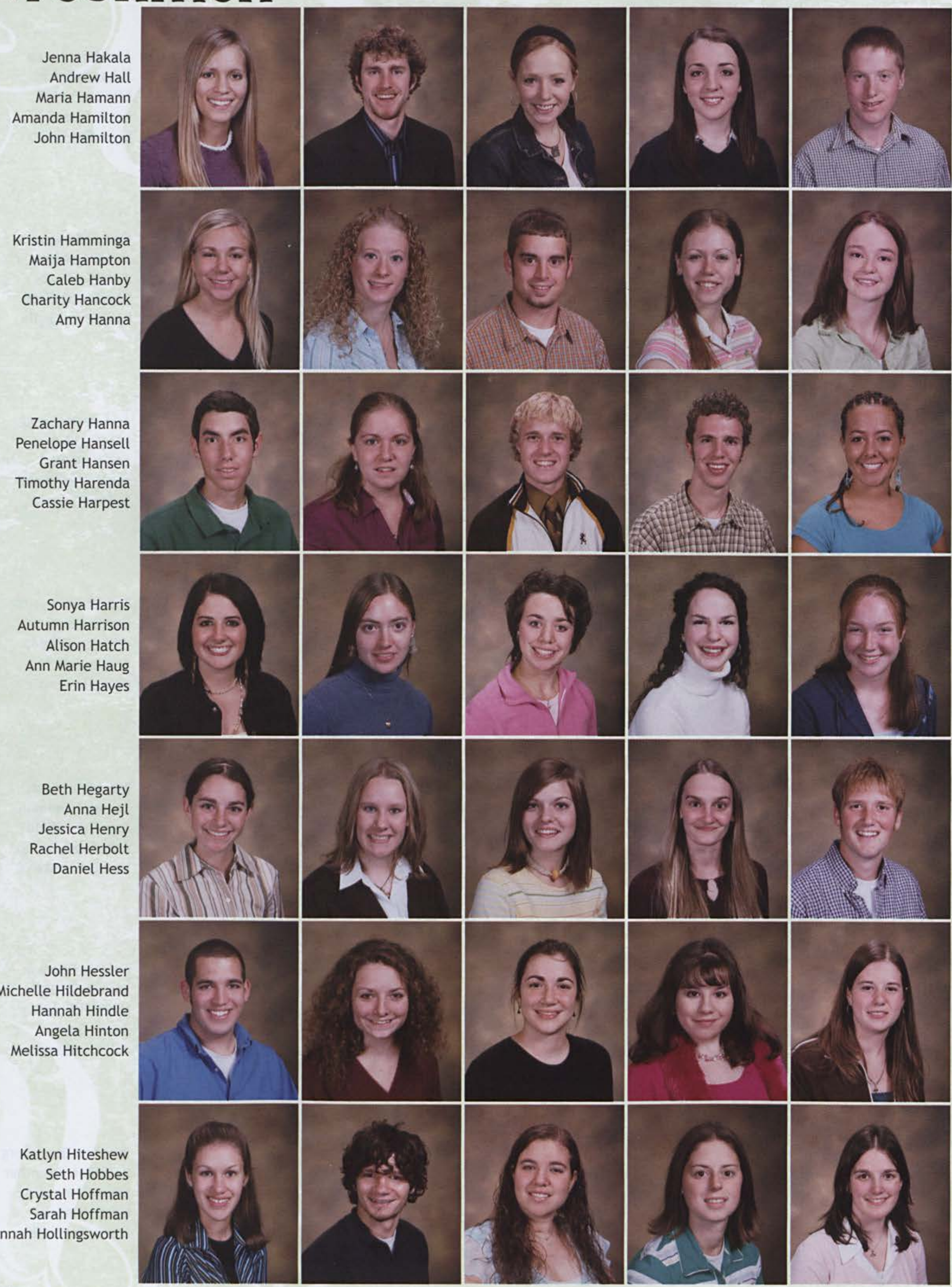




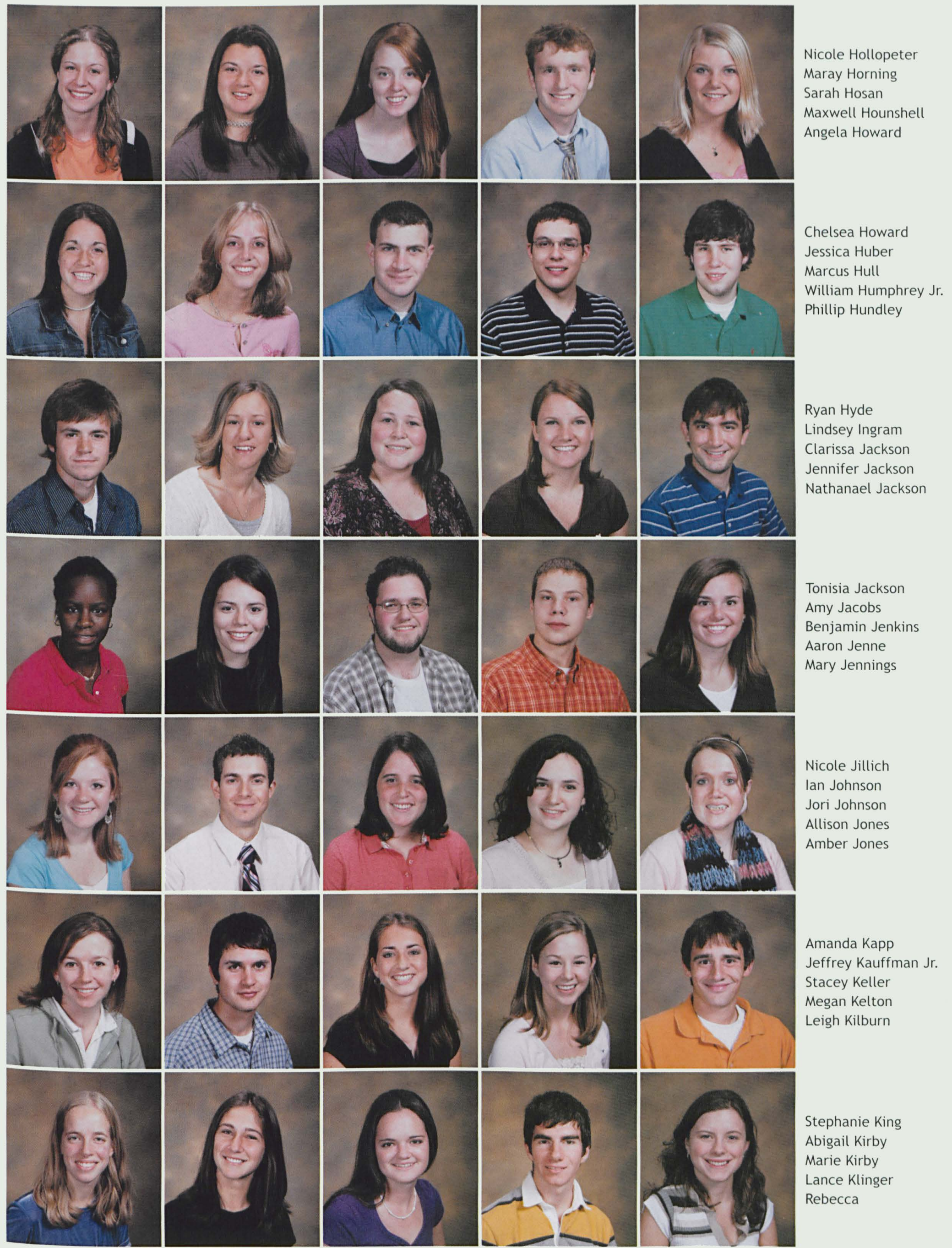




\section{Freshmen}

Christine Knott Kathryn Koch Benjamin Kochanowski Ryan Kochersperger Dezirae Kolmerten

Timothy Kolody Emily Kraft Alicia Krey Michelle Kropf David Kurin

on

Timothy Labrado

Daniel Lagan

Natalie Lagar

Amber Laing

Christina Landry

Nathan Lane Jennifer Larose Sandra Larsen Ashleigh Laswell Joel Lawrence

Rachel Ledgerwood Andrew Lehman Christina Leonard Ashley Lerma

Kelly Liggett

Jesse Lear

Alicia Lease

Andrew Leber

Peter Ledger
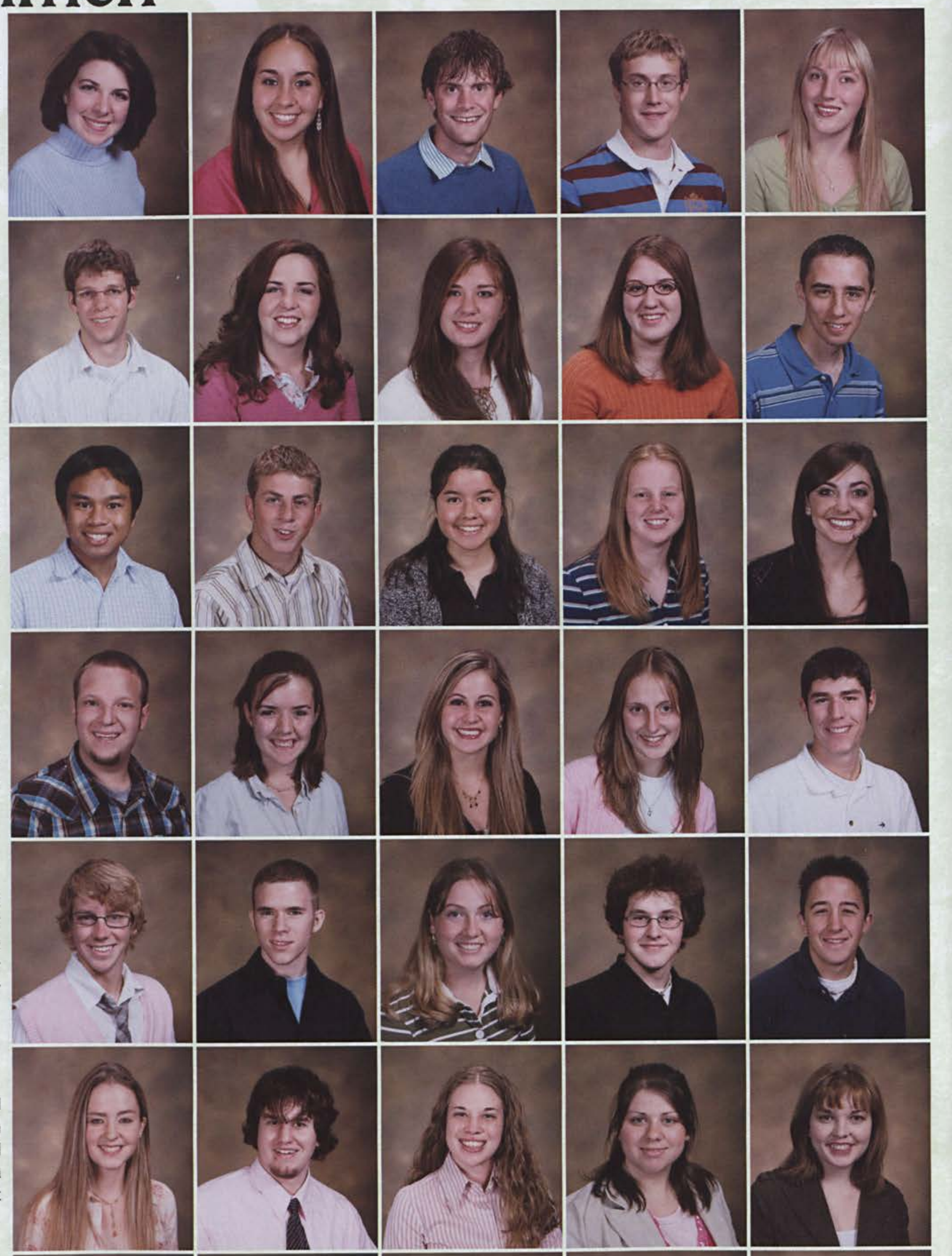

Kyle Linden

Jordan Link

Amanda Littell

Brittany Little

Annette Liu
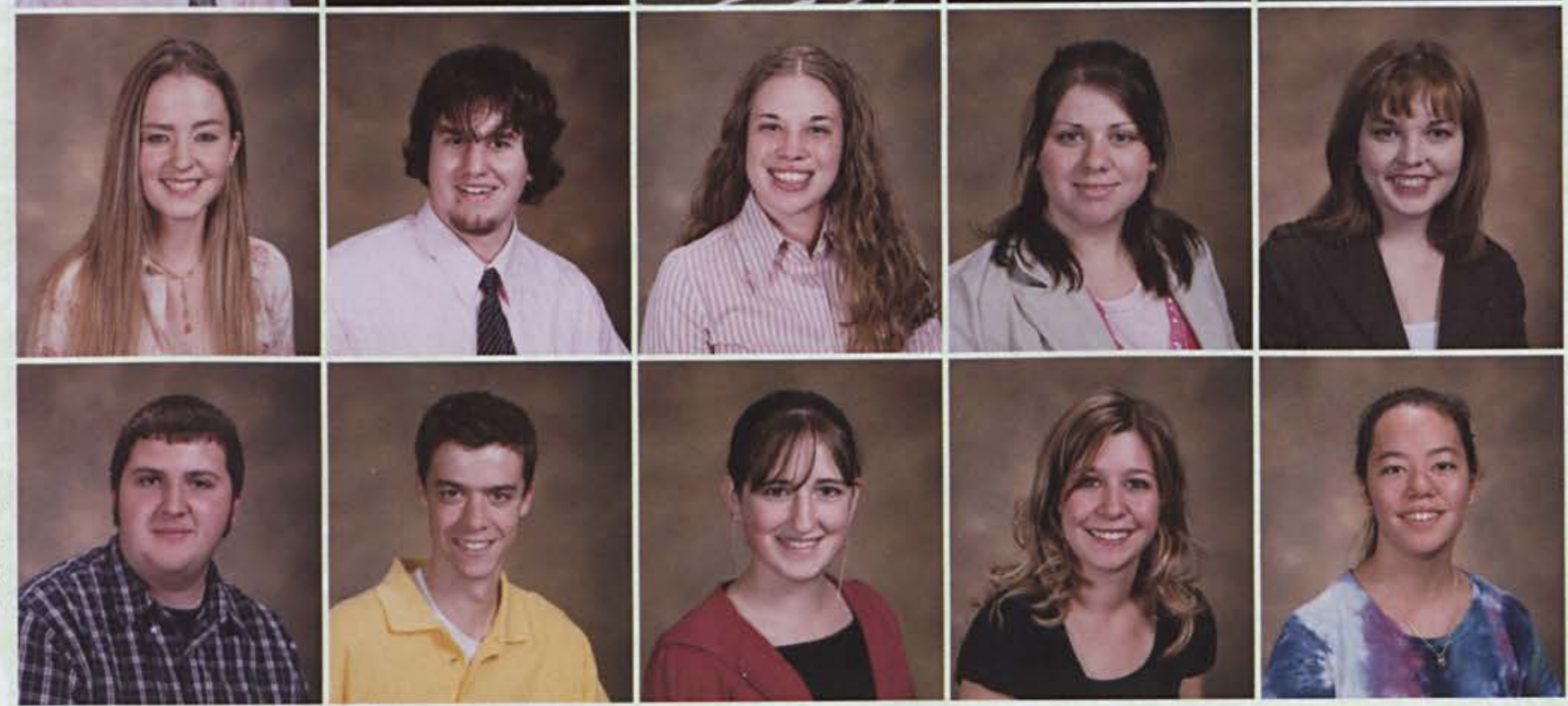


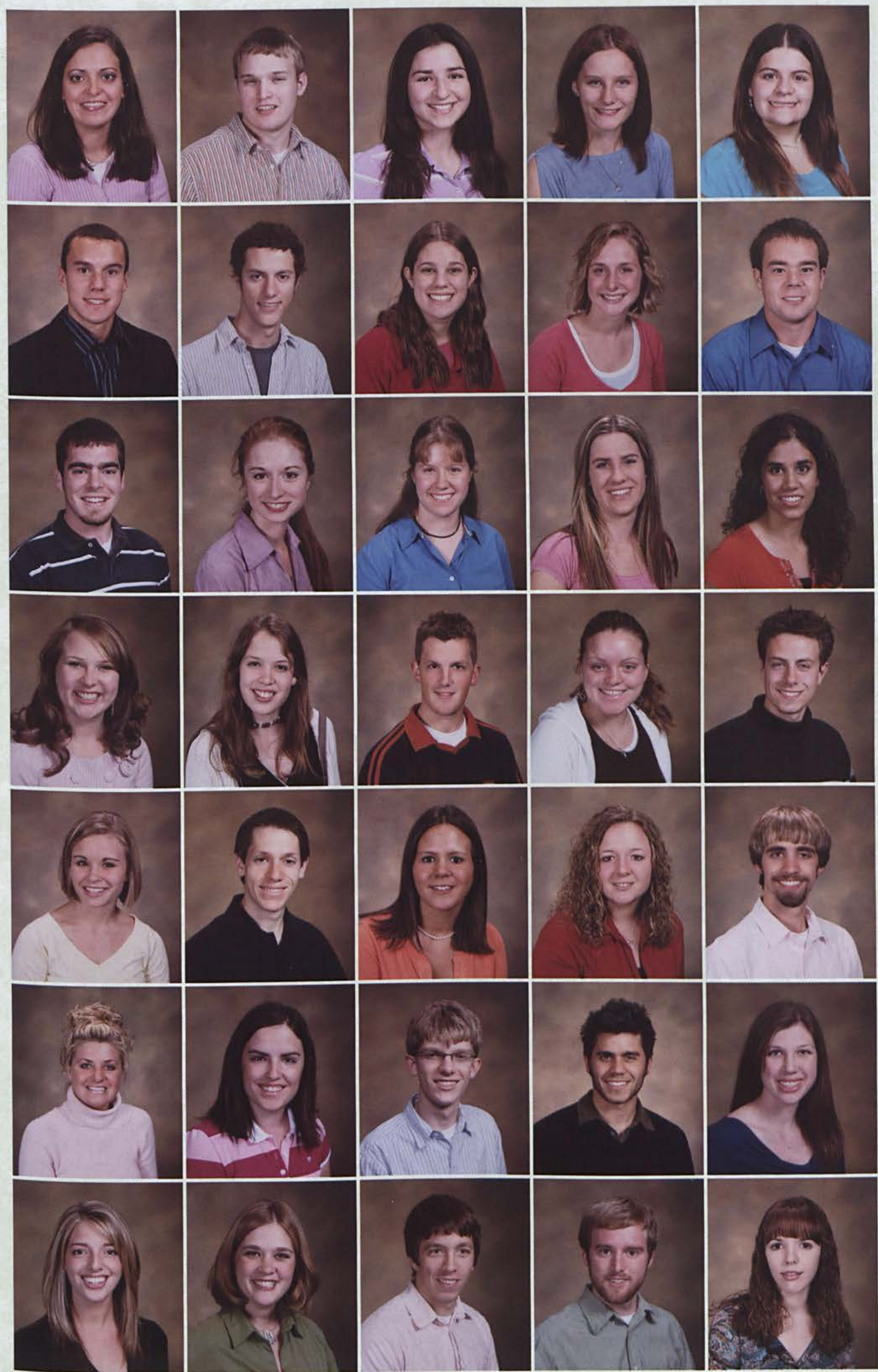

Racquel London

Kyle Lowry

Ashley Lupka

Felicia Lustig

Kathryn Lutz

Josiah Mackenzie

Matthew Mackenzie

Sarah Mahler

Kristen Mansker

Justin Marshburn

Kevin Martelli

Amanda Martin

Elizabeth Martin

Bethany Mast

Sushila Mathai

Sarah Matney

Kendra Mattson

Matthew Maver

Nicole Maxwell

Isaac Mayeux

Carrianna McCay

Nathan McCloskey

Amber McCowen

Casey McCown

Michael McCoy

Merilee McElveen

Heather McGee

Miles McGee

Jonathan MCGill

Kristine McGunnigal

Kate McKee

Brittany McKenna

Nathan McLeese

Kaleb McMaster

Jacquelyn McMillan 


\section{Freshmen}

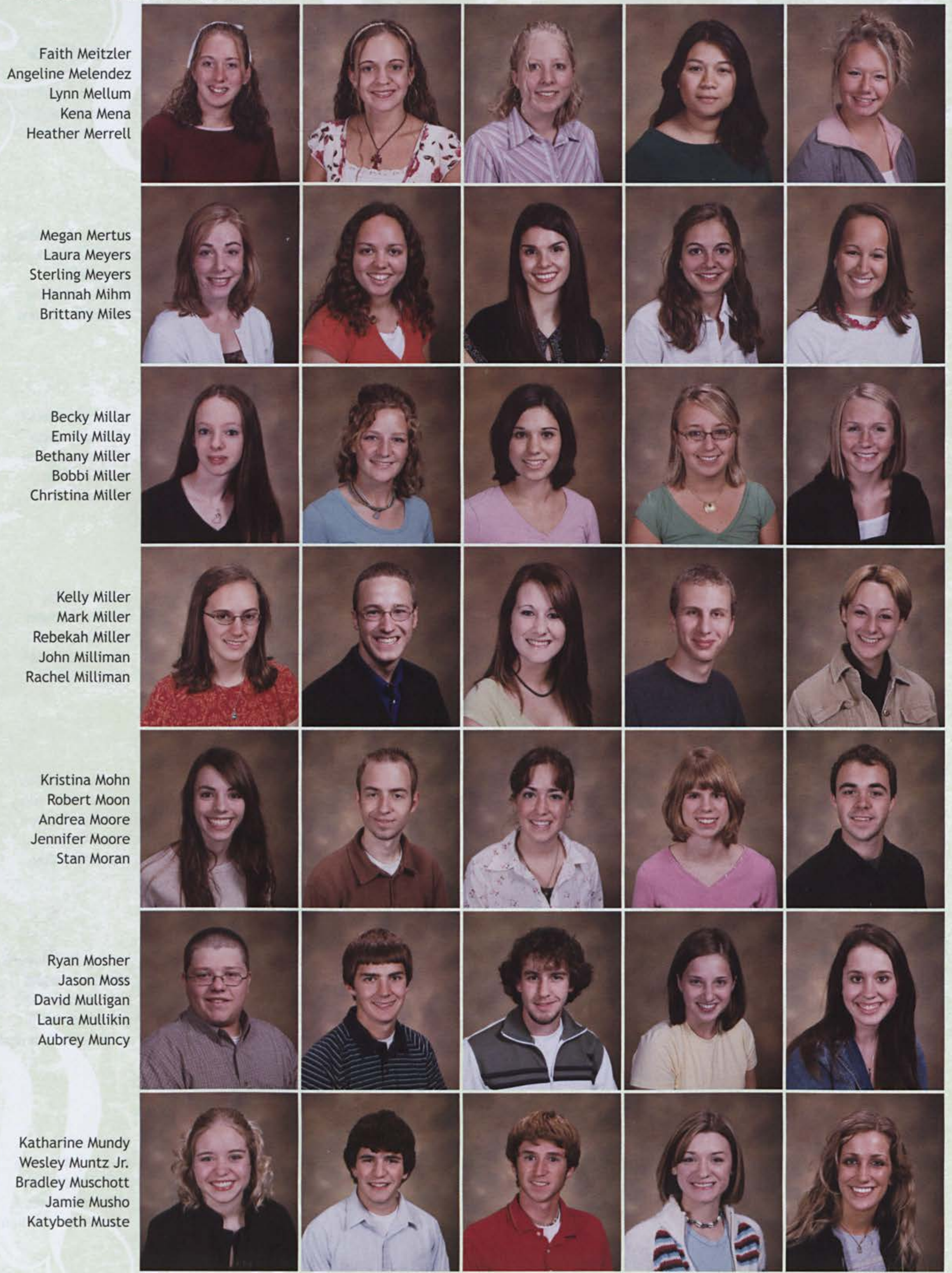




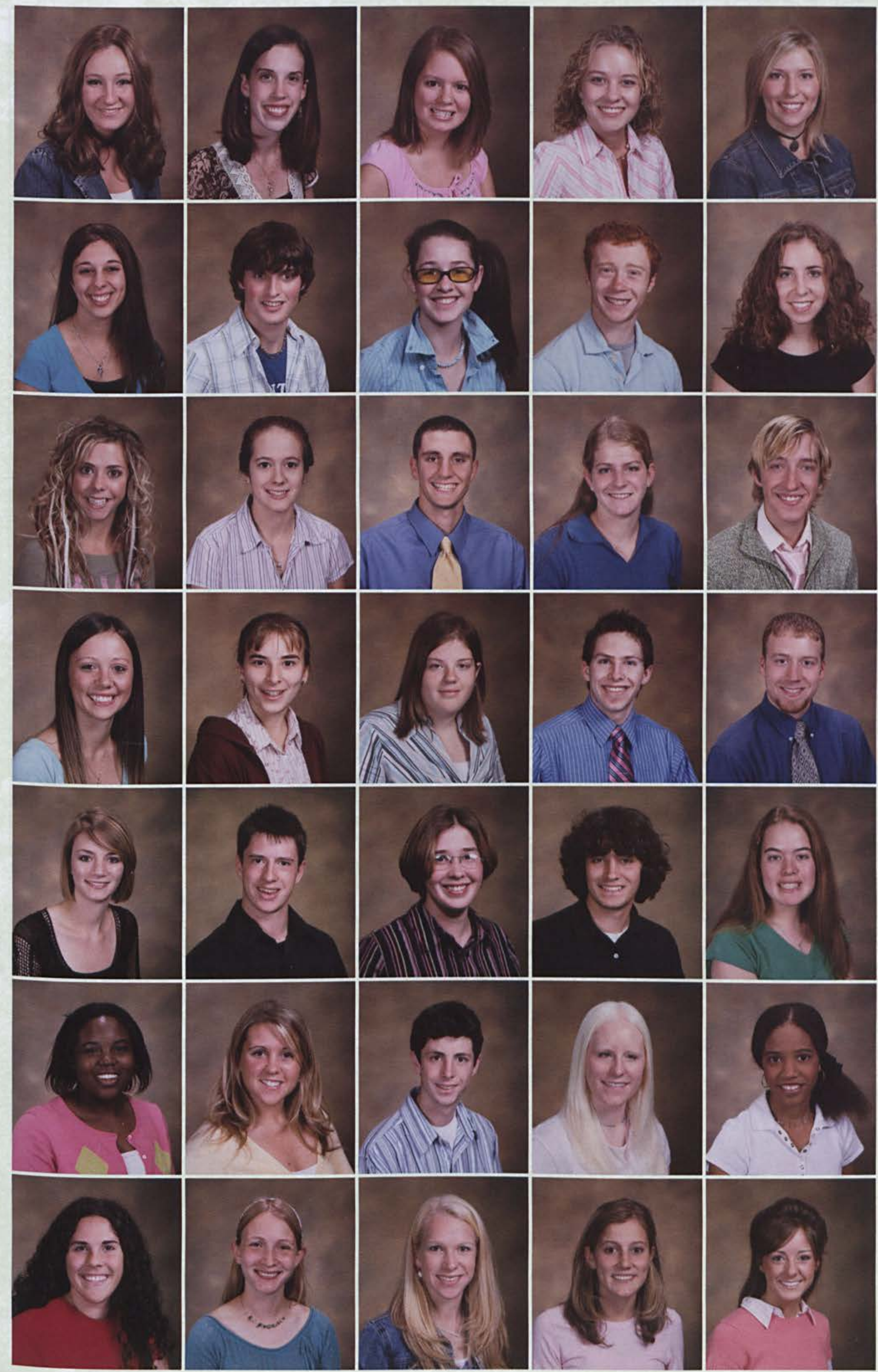

Kristen Neal

Megan Nelson

Micaela Neuhart

Danielle New-Day

Sara Ney

Katie Niemi

Garrett Noble

Nicole Normandeau

Kelly Nowels

Lucy Olney

Toryn Olson

Julie Otis

Tyler Owens

Laura Parker

Daniel Parrott

Cassandra Peletis

Karie Pepe

Rebecca Perry

Joel Peterson

Jared Petry

Andrea Phillips

Mark Phillips

Amanda Pierce

Daniel Pinkham

Laura Polston

Charmaine Porter

Jennifer Powell

Christopher Powers Jr.

Amy Price

Christen Price

Lisa Prins

Pamela Proctor

Elisabeth Pyles

Amy Rambikur

Brittaney Randolph 


\section{Freshmen}

Mark Ratliff

Alicia Raymond

Corey Reeder

Rebekah Reeves

Courtney Reid Krista Reilmann

Timon Reiner

Andrew Renke

Jessica Rex
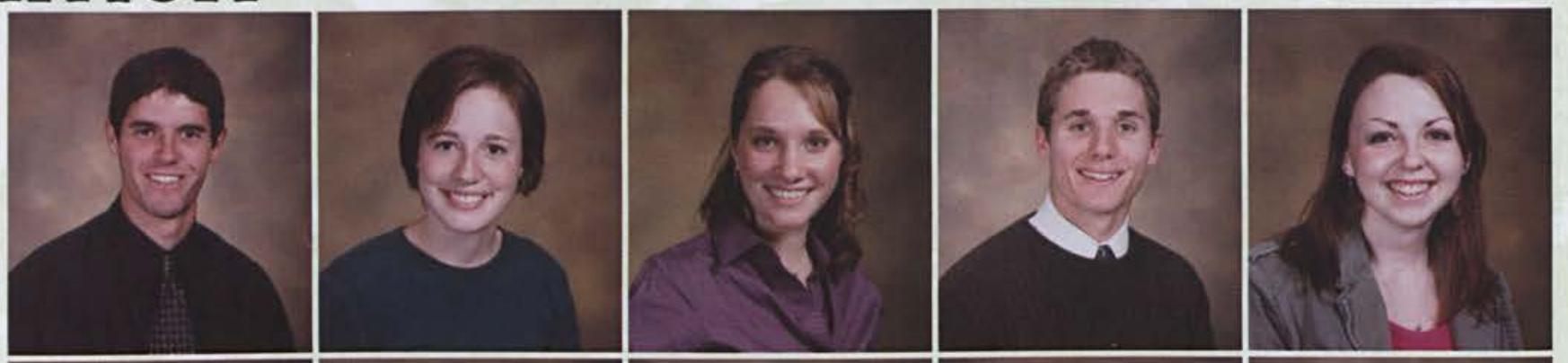

Karen Rhoads

Traci Rhodehamel

Marla Rice

Kasey Richards

Nancy Riddle
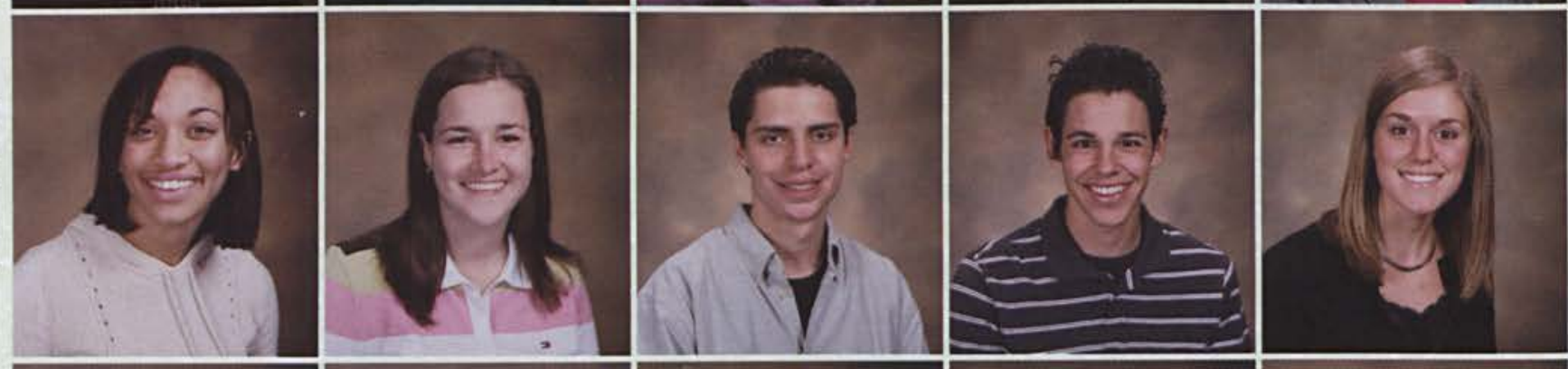

Angela Rill

Janna Roark

Danielle Robertson

Lydia Rodgers

Jacob Rogers

Michelle Romage

Kristina Ronan

Justine Ross

Rachel Ross
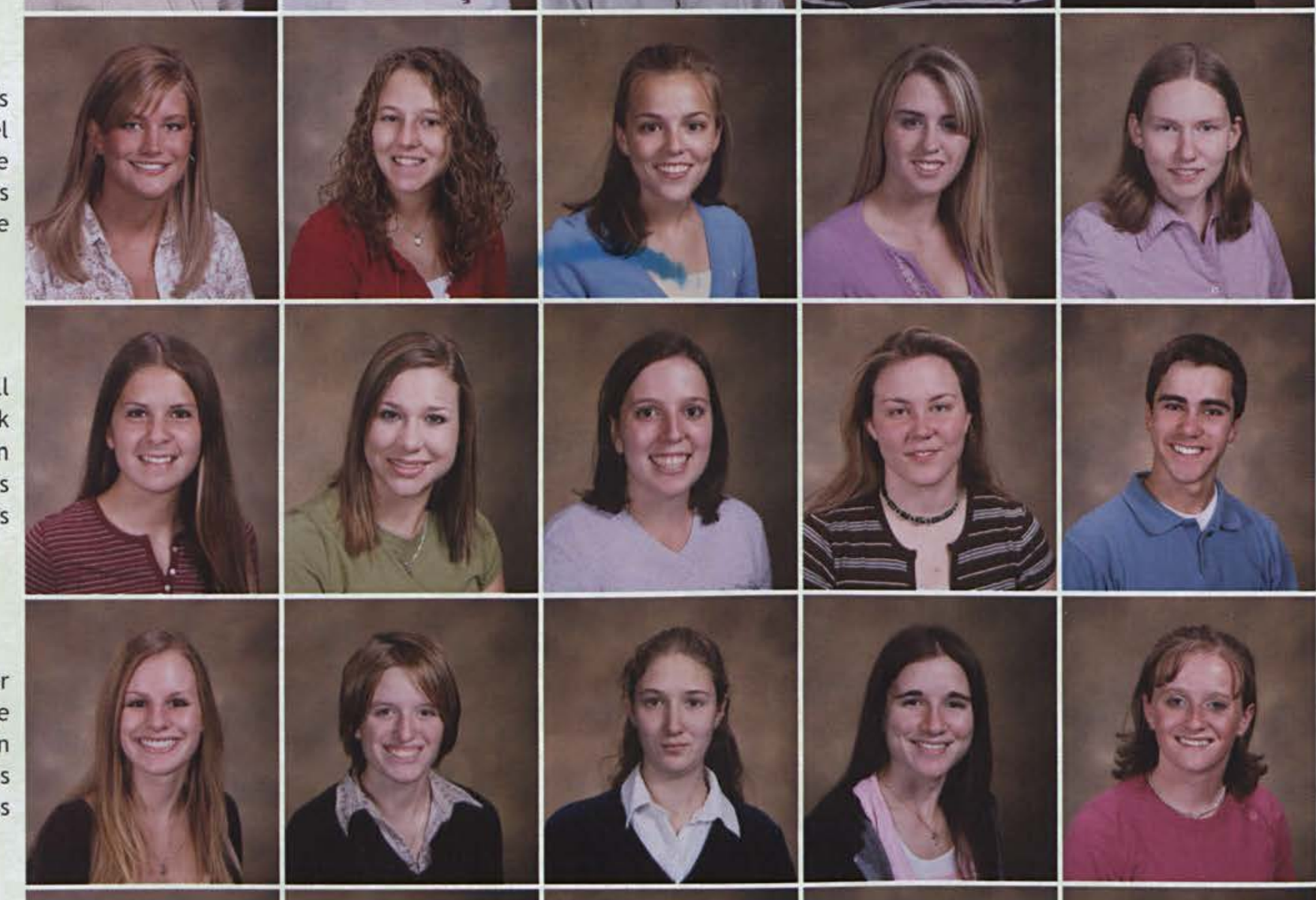

Rachel Rosser

Charissa Rowe

Stacie Rudd

Alisa Rutt

Emily Sammons

Ryan Samuelsen
Angela Sanders
Emily Sandlund
Erin Sangregory

Nicole Santos

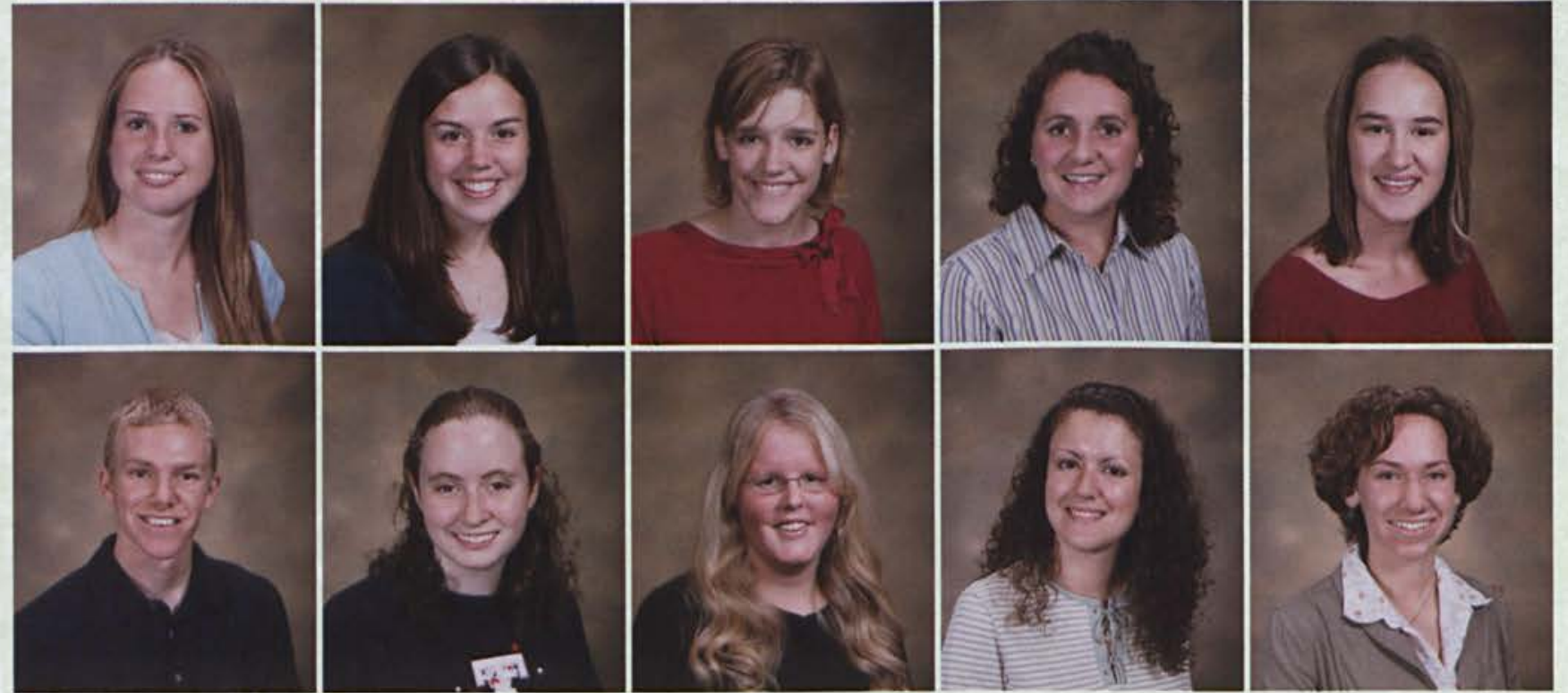




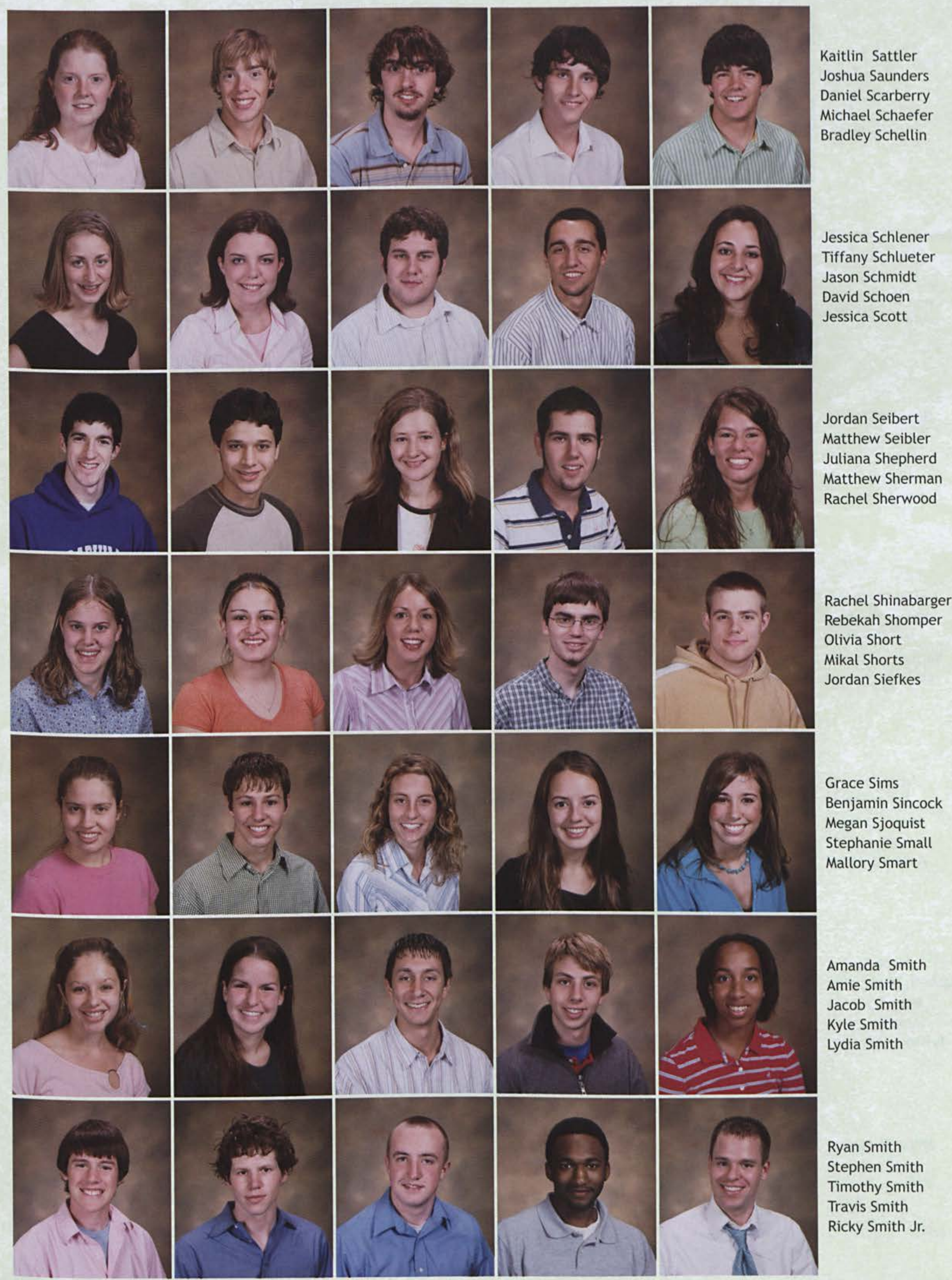




\section{Freshmen}

Kara Sneed

Wade Snyder

Stacey Spain

Kettia St Paul

Jennifer Stackhouse

Stephanie Stahl

Leslie Stamper

Joy Starbuck

Aaron Stauffer
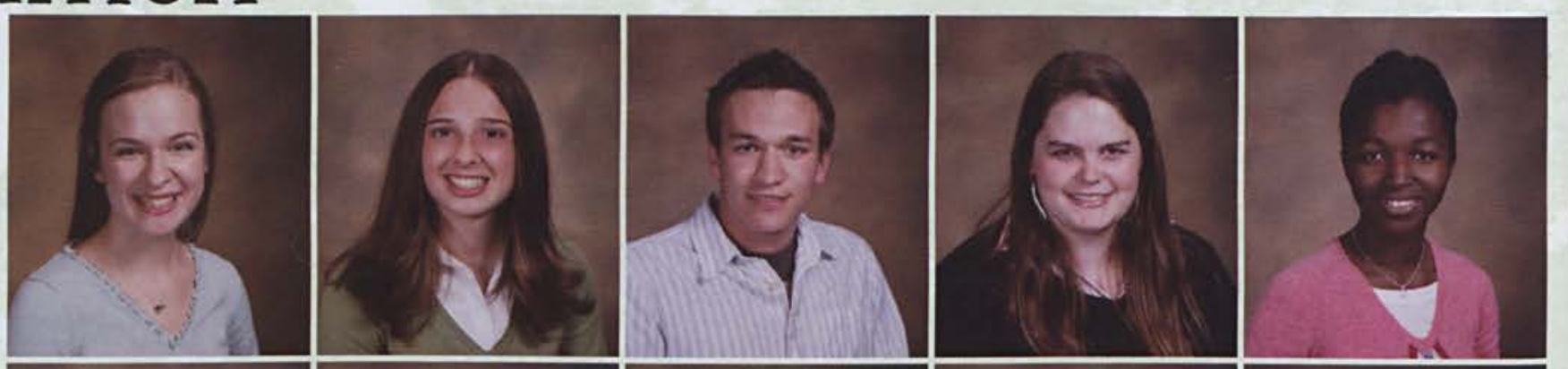

Natasha Stauffer

Jeremy Steckel

Meghan Steele

Michael Steele

Michael Steiner
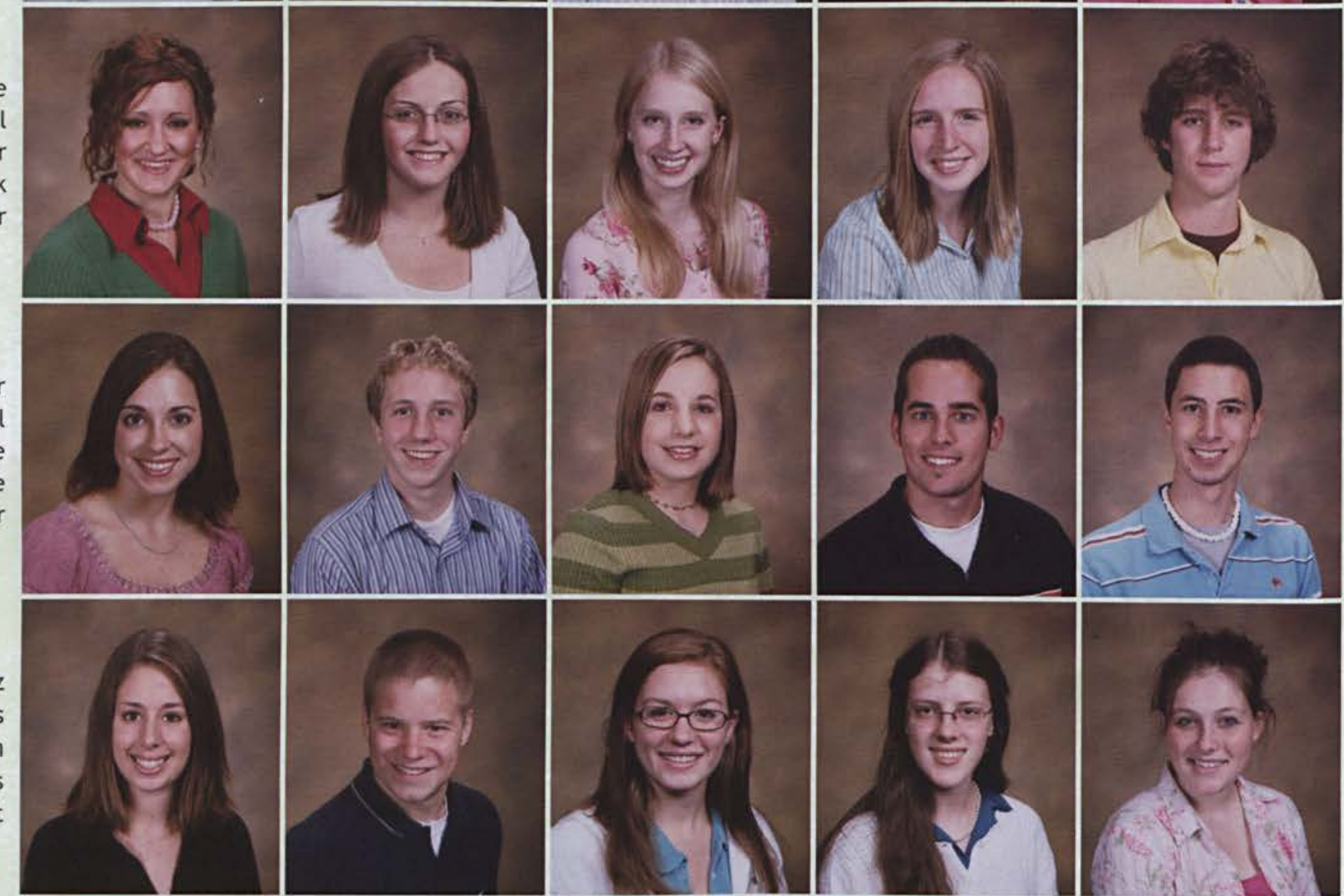

Jessica Steinmetz

Joshua Stephens

Jennifer Stern

Katharina Stevens

Ashleigh Stewart

Amanda Stock

Taylor Storey

Sonja Storhaug

Macy Straton

Anna Strayhorn

Elizabeth Studebaker

Jessica Swayze

Sarah Sweigard

Brittany Sylvester
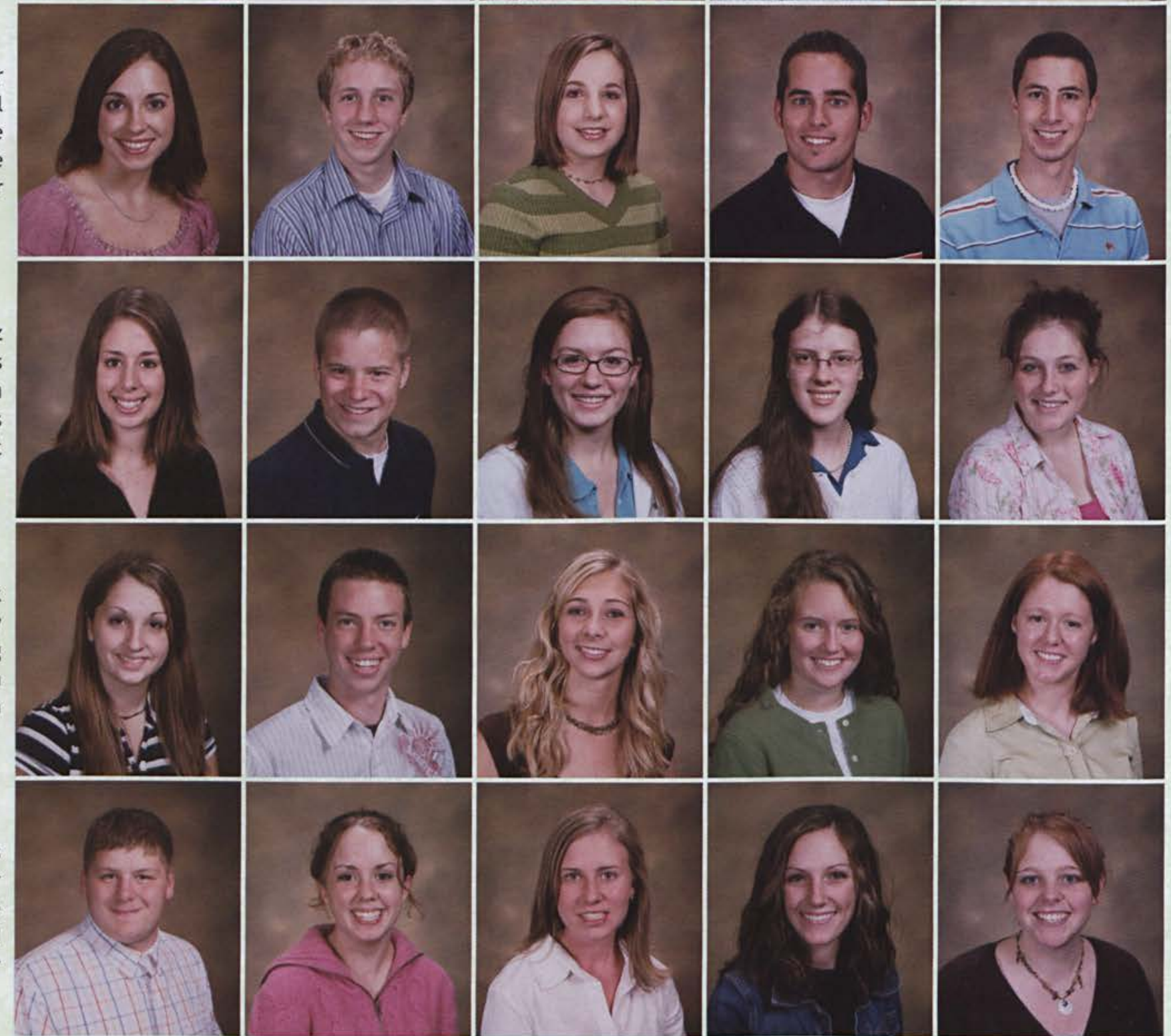

Jenna Tartaglino

Kelly Teague

Kathryn Temple

Emily Tilton

Stephen Tormey
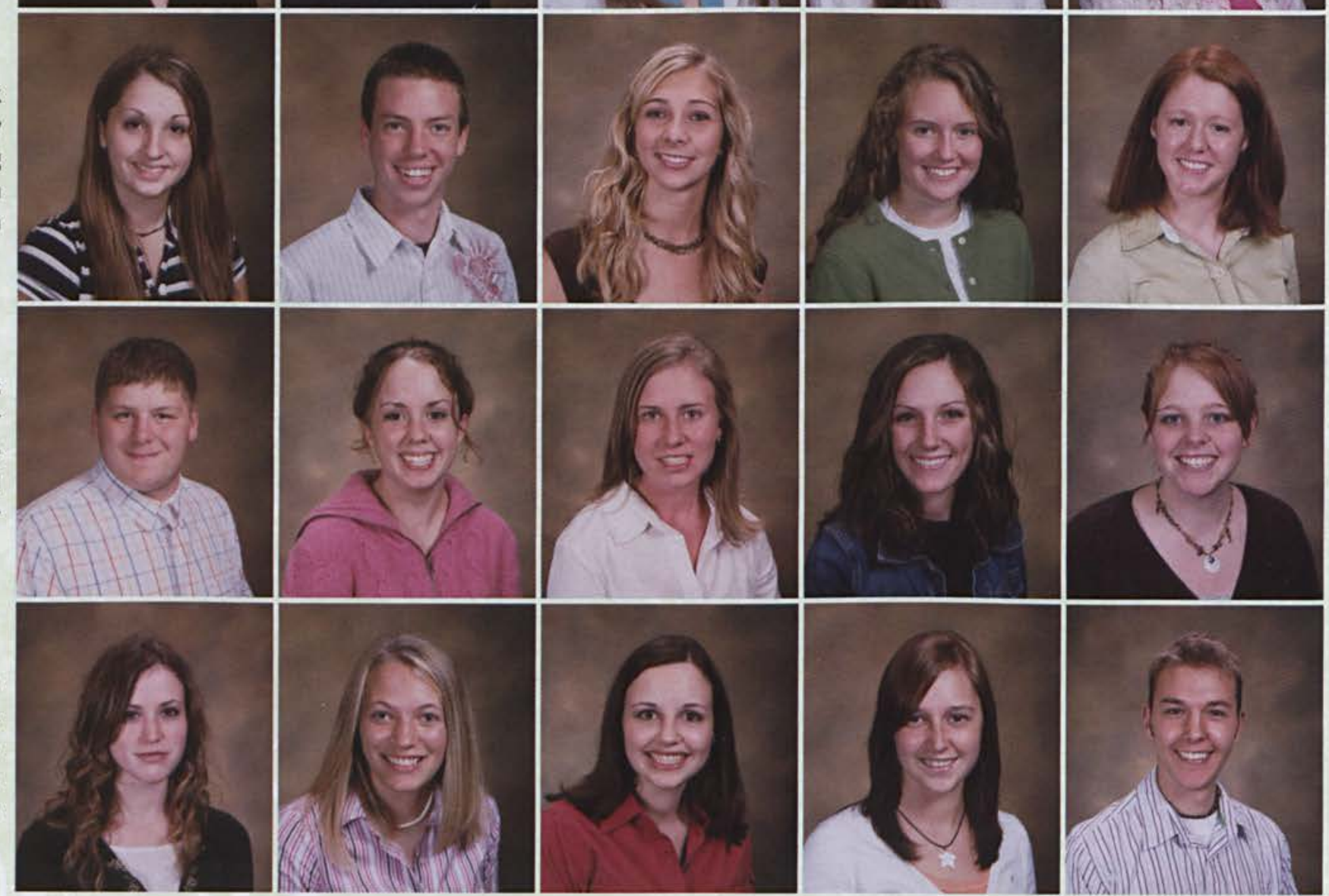


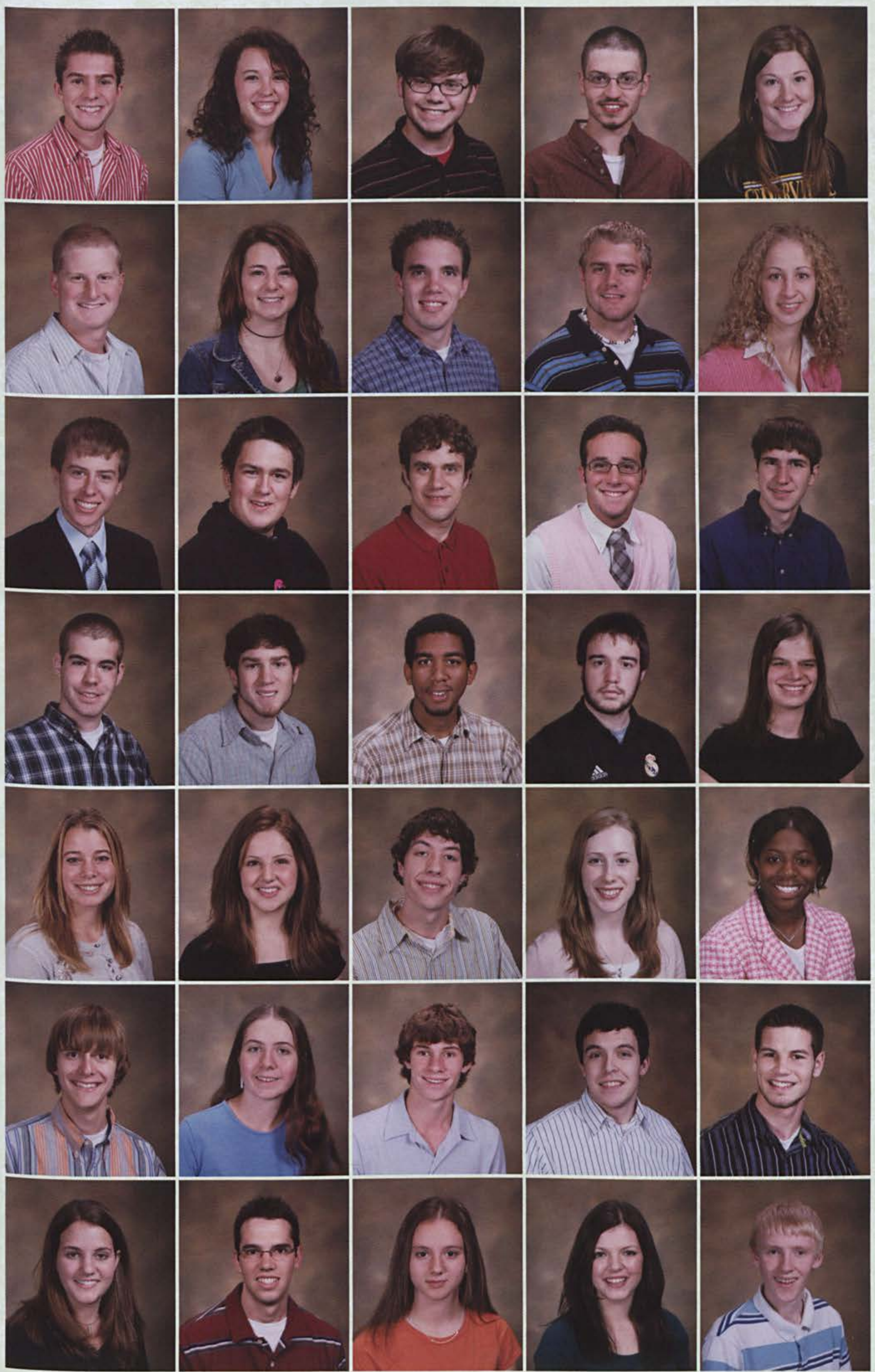

Christopher Travis Mallory Travis

Matthew Trego Brent Trowbridge Aubrey Trulsson

Conor Trulsson Katherine Tuttle John Van Der Wal David Van Schepen Emily Vanmatre

Alexander Vaughan Mordecai Veldt Timothy Vincent Philip Vinson Andrew Vitaliti

Timothy Voltz Sean Wadley Lee Wadlington Jr. Joshua Waggoner Grace Wagner

Laura Walach Elizabeth Walkley Tyler Walter Sharon Waltz Jerica Ward

Stephen Ward Jana Warner Nathan Watkins Jr. Daniel Weiser Justin Wells

Amy Whitacre Jeffrey Whitacre Sara White

Sarah Whitson

Christopher Wiest 


\section{Freshmen}

Abigal Wight John Wildman

Benjamin Wiley Matthew Wilhelm Zachary Wilkinson

Suzanne Wilson Jesse Winchester Karis Winget

Edward Winter Lisa Wirt
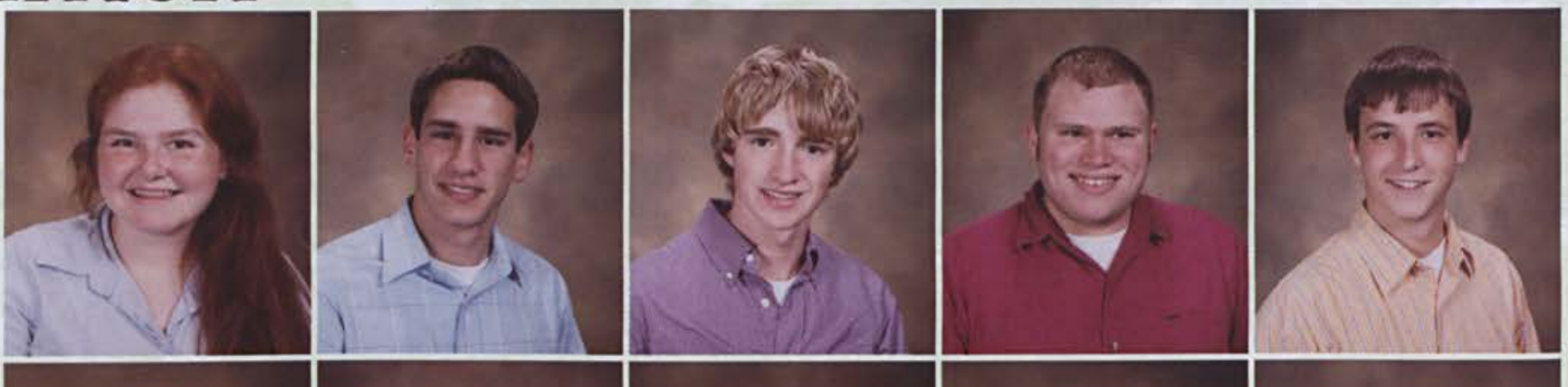

Jonathan Wolverton Nathan Wood Jeremy Woodard Krystal Wooliver Brady Workman

Cassandra Worth Leslie Wuenstel Katie Yahara Micah Yarbrough Jo Lyn Yoder

Rachel Yoder Bethany Young Christopher Young Hilary Young Adam Zagara

Matthew Zellner Michelle Zerminski Bethany Ziemblicki Daniel Zimmerman
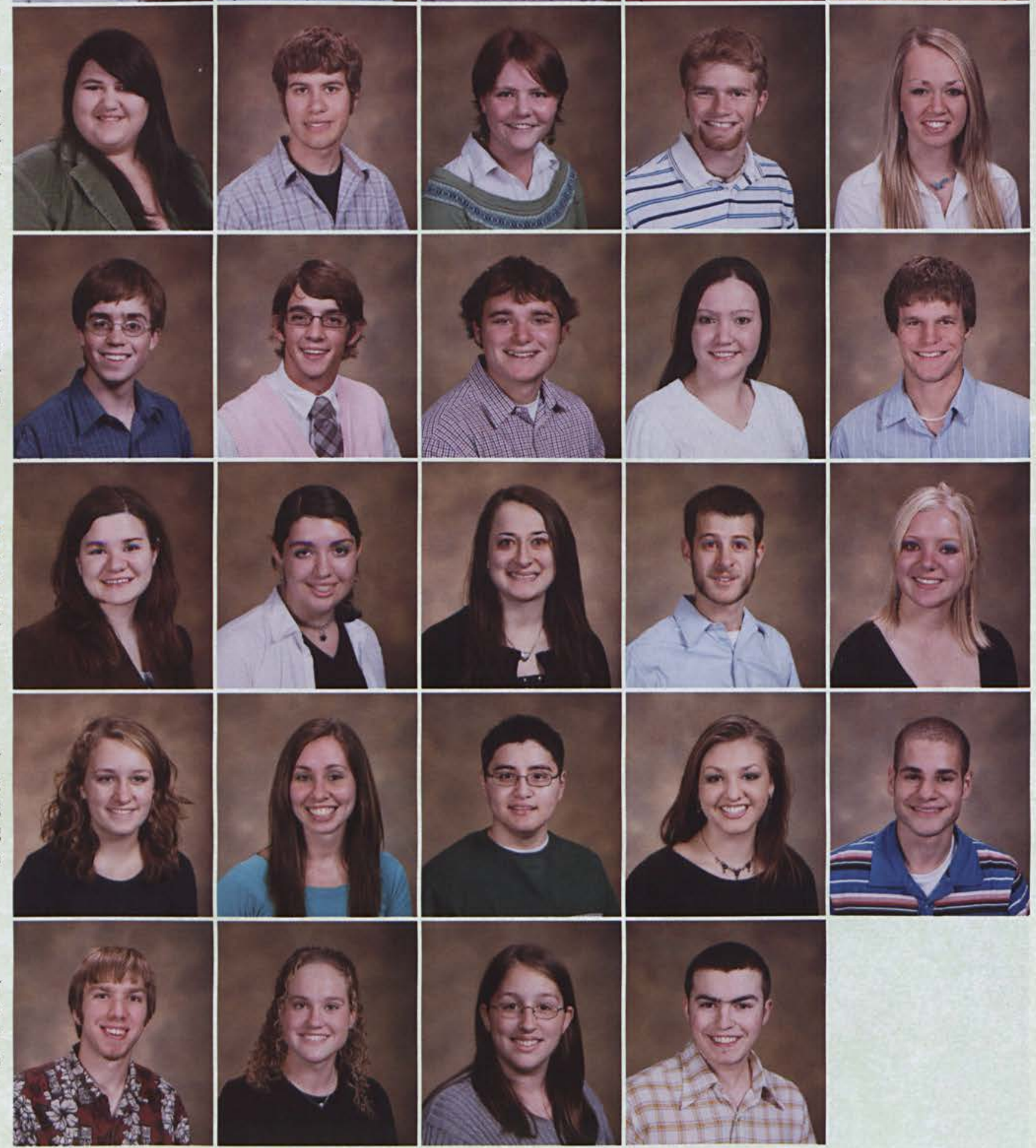

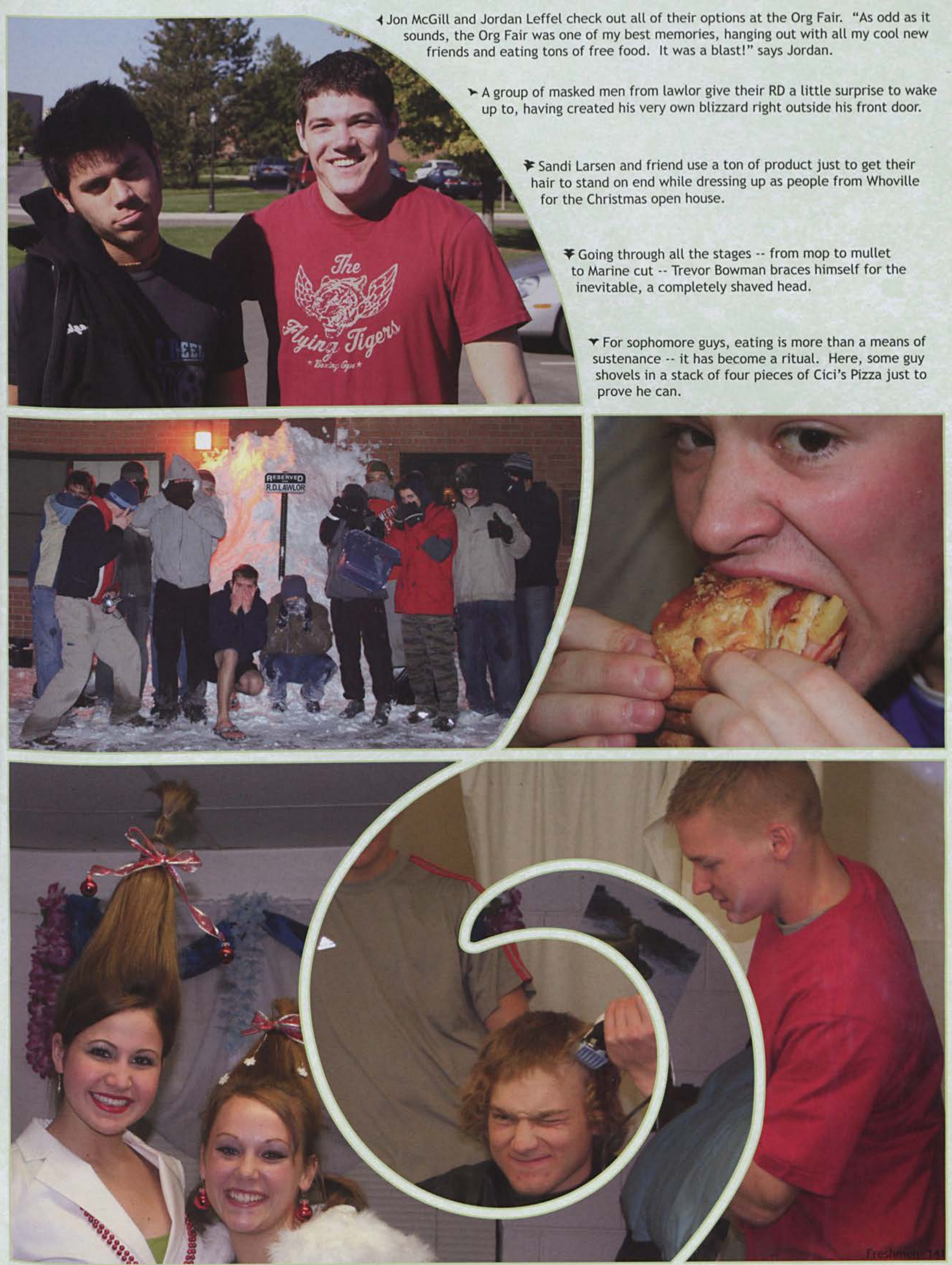


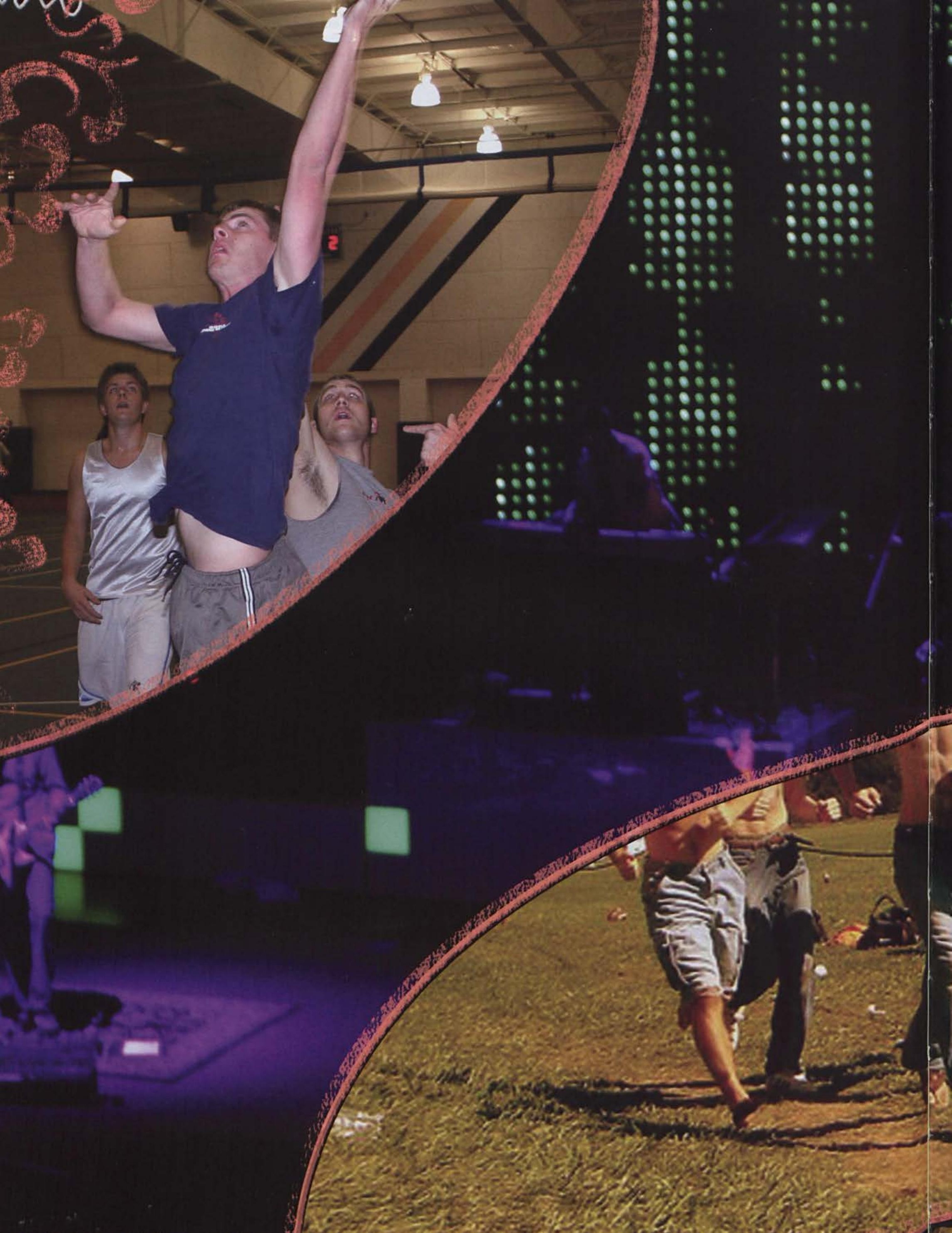




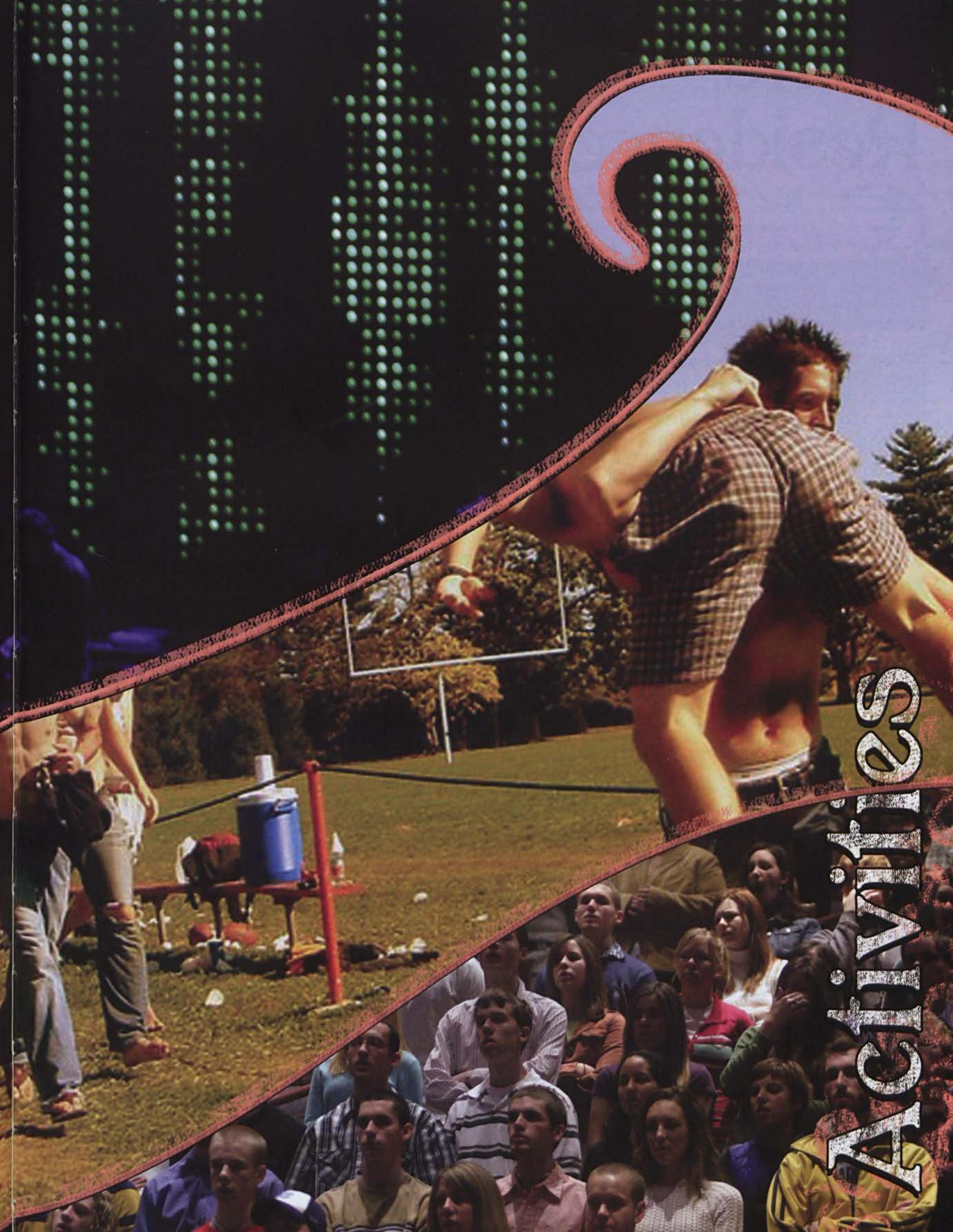



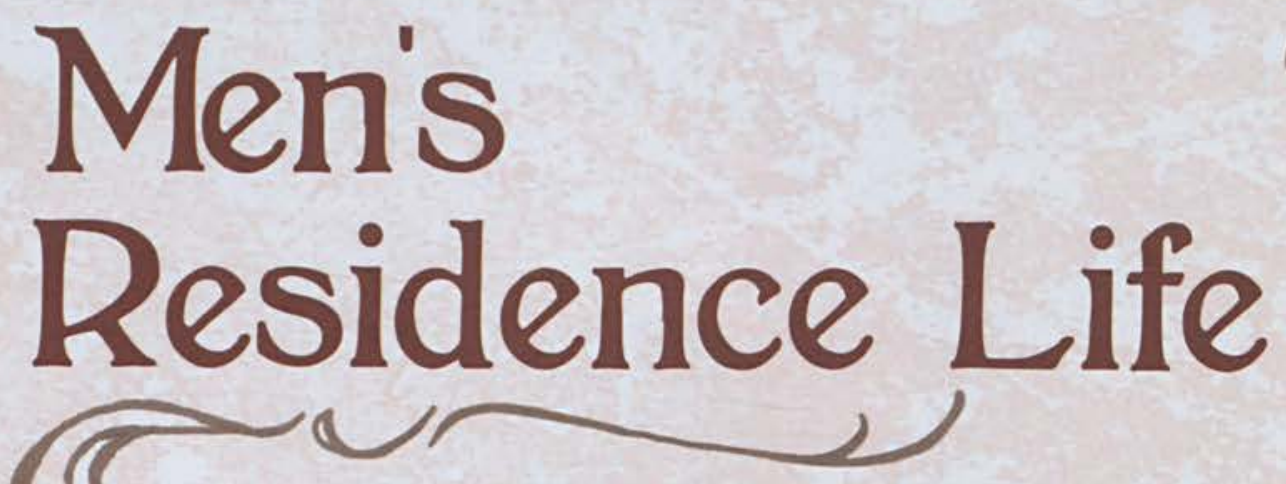

As I walk through campus, I am able to determine where individuals live and to which dorm culture they belong. Each male dorm possesses it owns nuances and quirks that make every dorm experience unique unto itself. By simply learning where a guy lives on campus, it is often easy to know much about him. From Harriman House to the Hill to McChesney, each dorm instilled a sense of grandeur and pride that was often the source of campus traditions and rivalries. Each dorm resident considered his dorm "the best," which made living in them an amazing experience.

Bonds form in the male halls, and this unity was often evidenced in dorm competitions. Freshman Lawlor resident, David DiLernia, commented, "My favorite part about Lawlor is the camaraderie in the units because the people here almost band together to form a brotherhood... and we dislike the Hill." This healthy rivalry with the Hill led to annual football games and various other friendly competitions:

Some dorms, such as Rickard, proved to be much calmer, while others, such as Brock continued annual raucous traditions. Three year Brock resident, Joel Smith, said of his beloved dorm, "Brock sets the precedence for rowdiness among the campus dorms." Other dorms became a mixture of both environments. Junior Tim Neetz said of his hall, "St. Clair is a wonderful place to experience all aspects of residence life while at Cedarville. It's the perfect balance of Brock and Rickard. From the great friends, to the late night spiritual conversations and praying for one another, to the games of Buck Buck, St. Clair is all a guy could ever want, and more!"

While each dorm was different this year, each one became a home that can never be replicated for hundreds of Cedarville University males.

> Junior Wes Yahara takes a leap from a makeshift ramp in a Brock hallway. We always knew we'd need those books someday...

* Luke Snider and Kyle Gunn let their spirit show as they root for the Lady Jackets during a tough scocer match.

$\checkmark$ Brothers and fellow Brock residents, Tim and Matt George, feed the only pets allowed on campus: their very own "little Nemos."

A Matt Massaro, Jacob Smith, Jacob Spencer, Steve Tormey, and Scott Van Dyke unwind with Cedar-appropriate Italian ices during Fall finals.
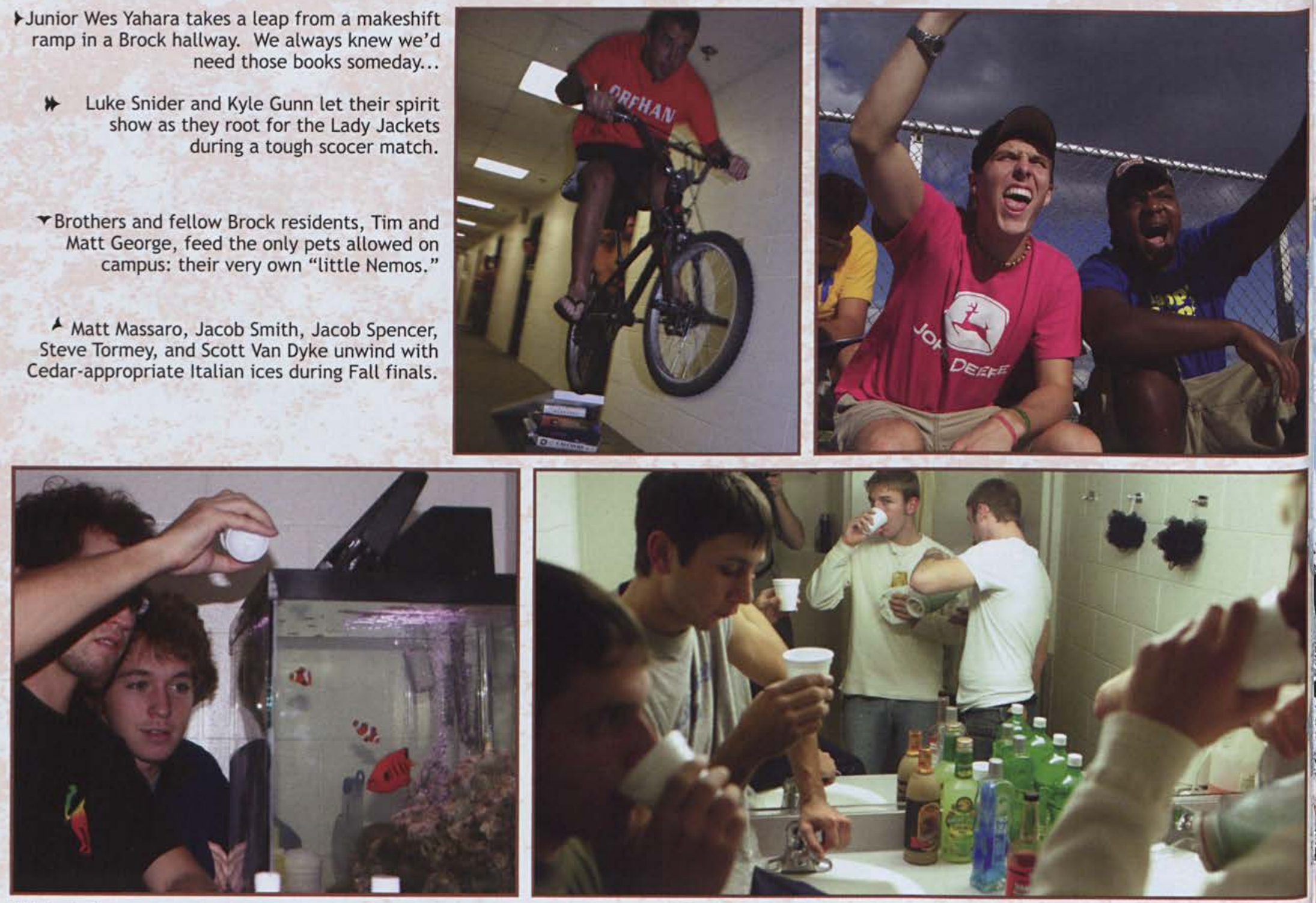


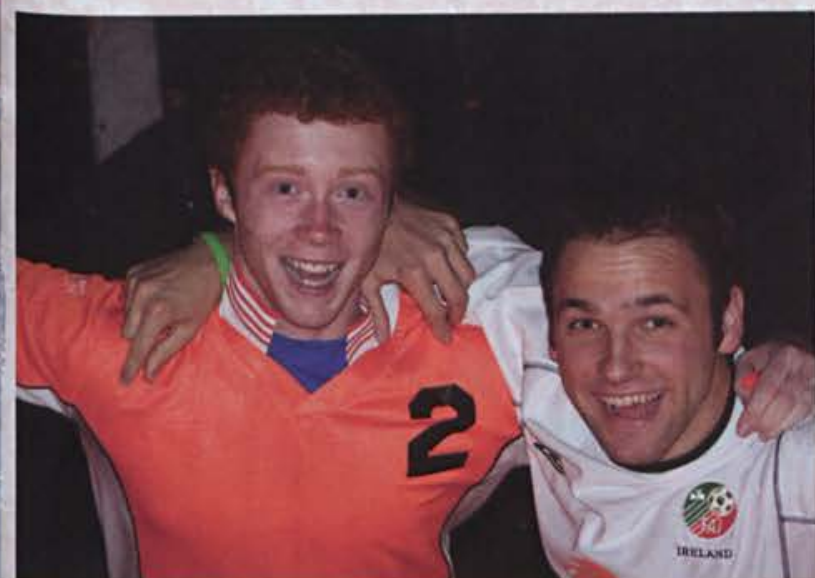

$\checkmark$ Kelly Nowels and Andrew Davis welcome other dorm-dwellers to their unit during Lawlor's Halloween Open Dorm. Andrew Davis commented that "the whole atmosphere [at Cedarville] is focused around growing in your walk with Christ while having an awesome time with friends."

- Lawlor residents Dan Magee, Jon McGill, and Corey Reeder take us on a trip to the wild, wild West during the Christmas Open Dorms.

$\checkmark$ The ever popular game of strategy, and quite a bit of luck, RISK became a phenomenon in certain units this year with games sometimes lasting well into the night and the wee hours of the morning.

\& Seth Buckwalter particpates in a developing trend at Cedarville - the shaved head. The hairstyle took on a popularity hitherto unparalleled this year at Cedarville as this scene grew more and more common.
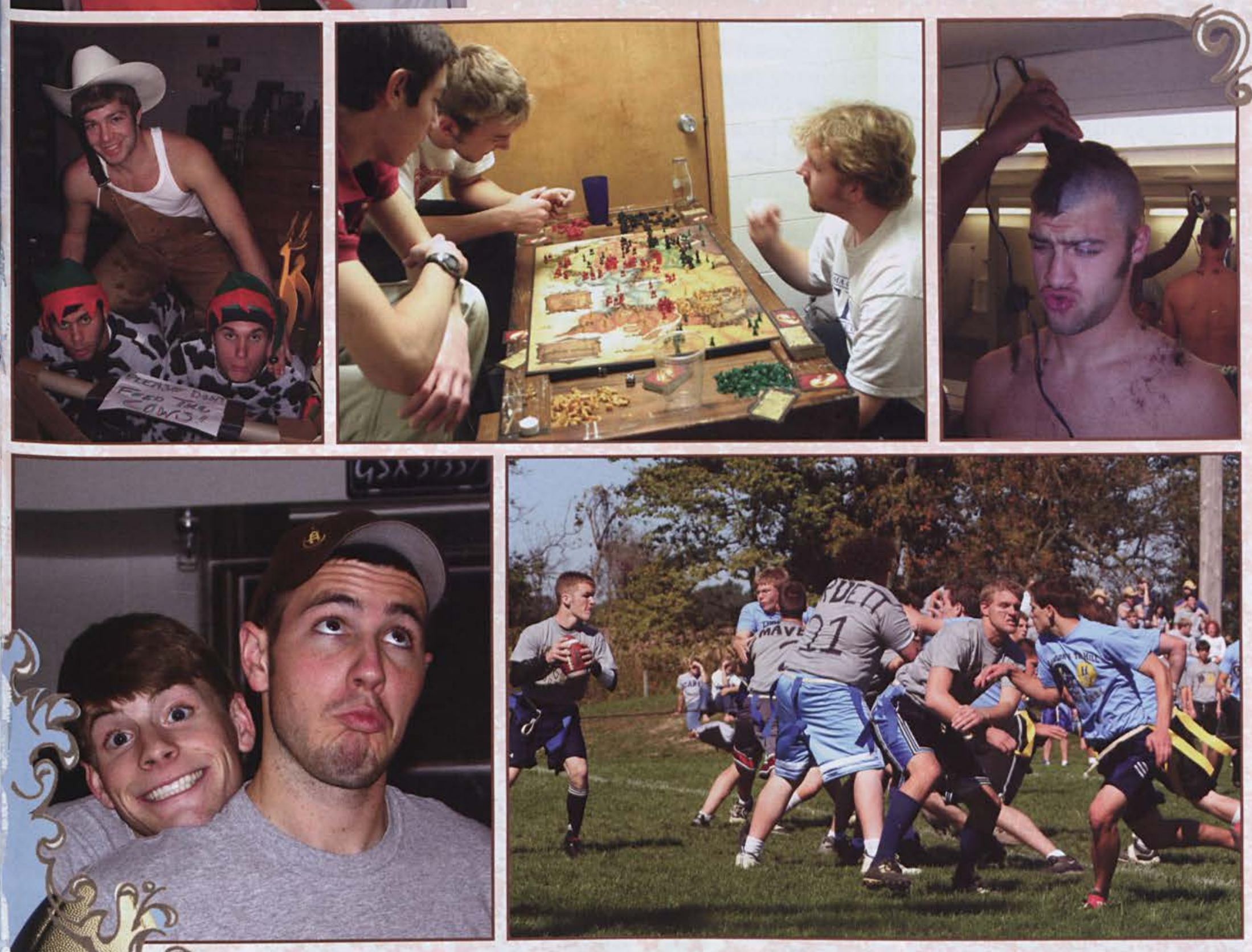

$+1, \pi \times 6$ 90 . (3) 0 , का?

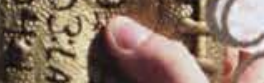
Sह $\sqrt{(2)}$ Kauffman, more commonly referred to as "Bucky" by his friends, enjoy the atmosphere of dorm life at Cedarville. $\downarrow$ Tradition runs deep, rivalries run high, and the fight is intense. Lawlor beat out the Hill for the second straight year at annual Lawlor-Hill football game. 


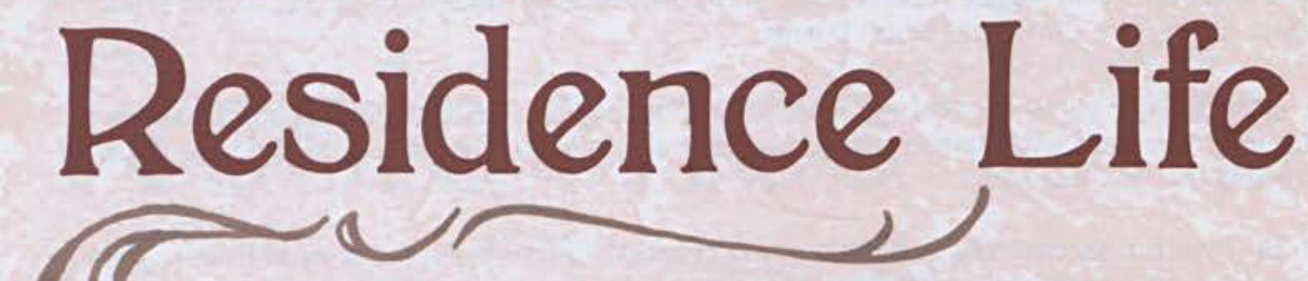

When I transferred to Cedarville this year, I was most excited about dorm life because of

S)

the potential growth and bonding that comes from living with other girls. I have had amazing

opportunities to get closer to the girls in my hall through Bible studies, hall activities, and those crazy

random moments that all the women's resident halls have experienced.

For many girls, Printy is their first dorm-life experience. This year, Printy had their annual "Printy War," which was rumored to have involved syrup, whip cream, and soda. "It was a great night to make memories and meet other girls from different units," freshman Jessica England said.

Many dorms started exercise programs this year. The girls of Willetts and Faith got together to do Pilates and Taebo. "Our exercise nights have been, well, so far...interesting!" laughed Faith resident Emile Brinks as she described the Taebo experiences. While these girls worked to maintain a healthy body, the girls in McKinney could be found at Young's on the weekends, getting their dose of sugar. Junior Hilary Bromely said that she and her friends would "moo for an extra scoop of ice cream."

Johnson had been filled with screams coming from the TV lounge as another member was voted out at tribal council during the latest episode of Survivor. On the other side of campus, the girls in Murphy Upper-Back focused on their destiny as housewives, as displayed by their "Destined to be Housewives" theme for Campus Christmas.

Many guys wonder what happens beyond the walls of Maddox's courtyard. Melissa Clawson shared that, this year "we had a courtyard fiesta with live music, Mexican food, and a piñata. Though our dorm lost the Powderpuff football game, we still know how to have a good time."

$>$ Laura Olmstead, Laura Ramey, and Jenny VanDyke don their costumes and enjoy the food during the Murphy/Rickard Halloween party.

$\checkmark$ Sara Larsen tries to avoid having her picture taken during Faith's open dorms.

\& Victors Casey O'Neal, Tiffany Schlueter, and Elise O'Neal proudly display their trophy after winning the Printy/Maddox Powderpuff game.

A Jen Davis and Alissa Clutcher, residents of Maddox, appear to enjoy the mocha punch just a little too much!
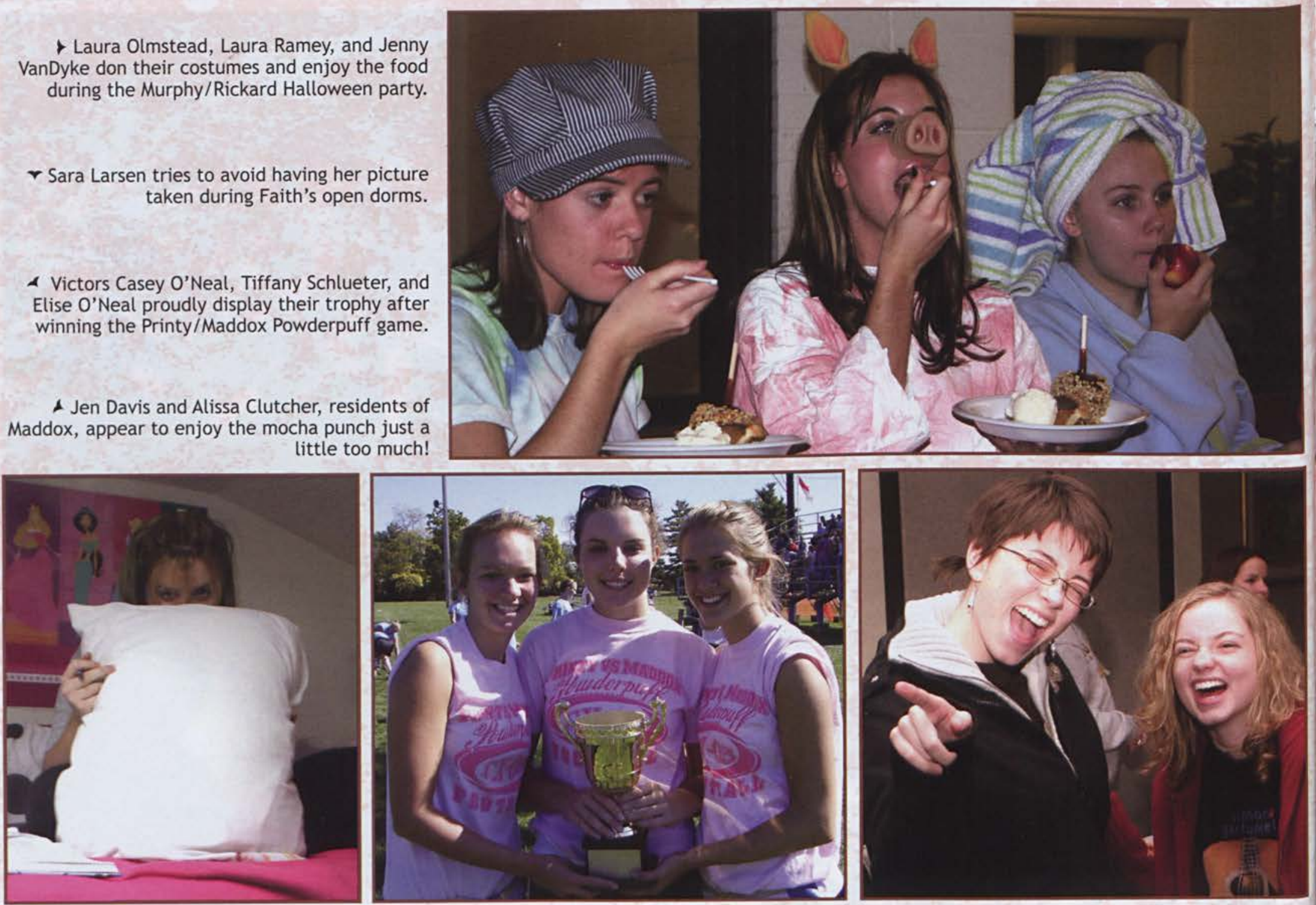


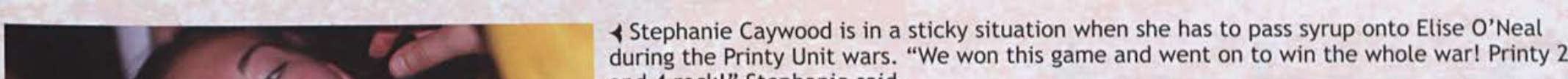
during the Printy Unit wars. "We won this game and went on to win the whole war! Printy 2 and 4 rock!" Stephanie said.

Julianne Null and Bethany Turner express their joy of being roommates in Faith this year.

$\checkmark$ Friends Emily Doot and Lindsay Malone have a great time during the Christmas open dorms. "We visited Lawlor sporting sweet action coffee filters as Jewish yamakas!" exclaimed Lindsay.

4 "Arrrg... Merry Christmas matey... Walk the plank," responds Amber Mack, whose hall won McKinney's Campus Christmas competition.
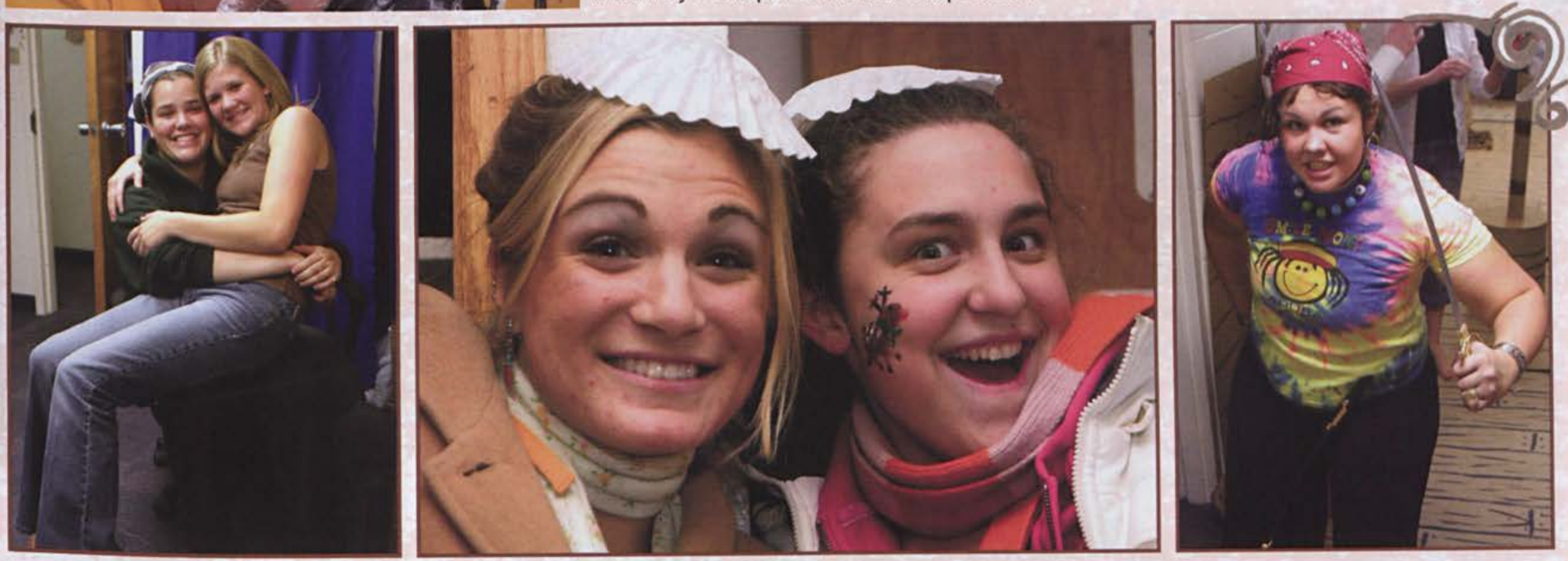

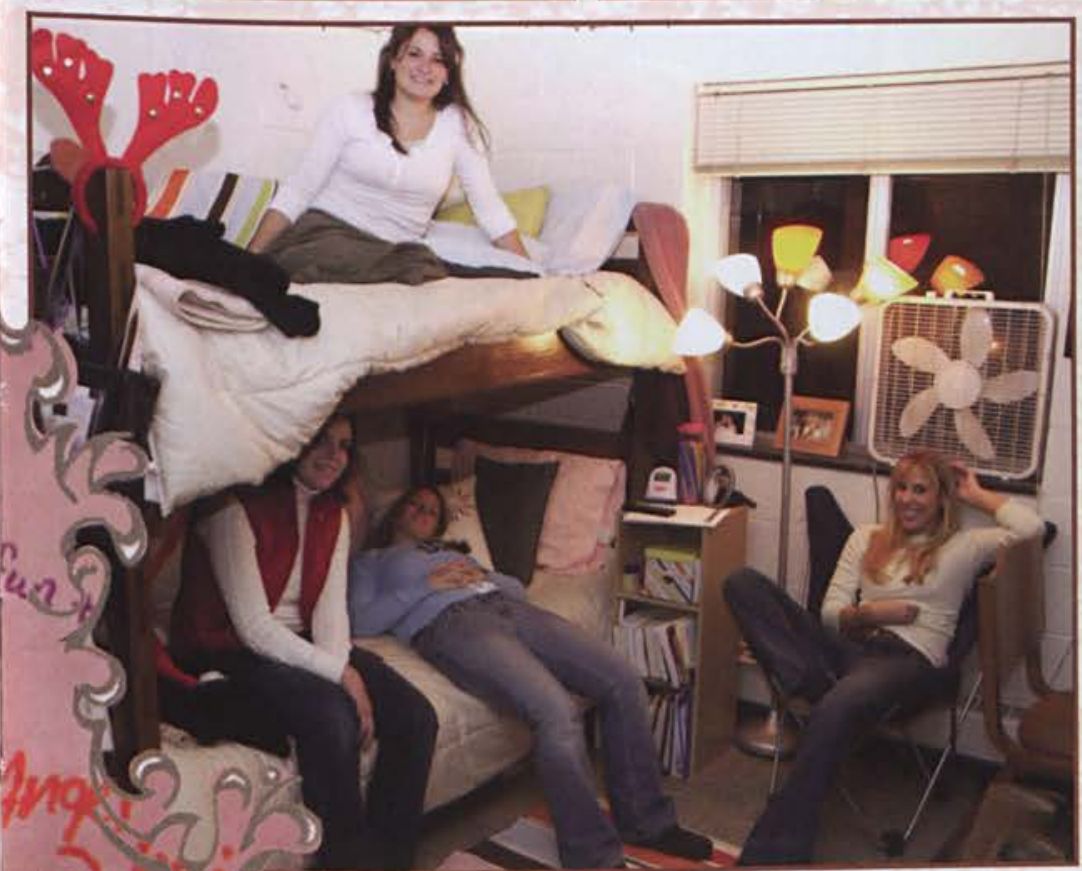

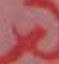

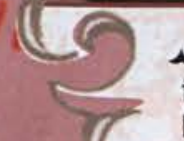

A Debating whether or not they should stay in the dorm or visit the guys' dorms, Brittany

Knoedler, Tiffany Schlueter, Kelsey Pfeifer, and Lynn Verlander decide to stay in the comfort $+C^{\prime \prime 2 ~}$ of their sweet-scented room in Maddox.

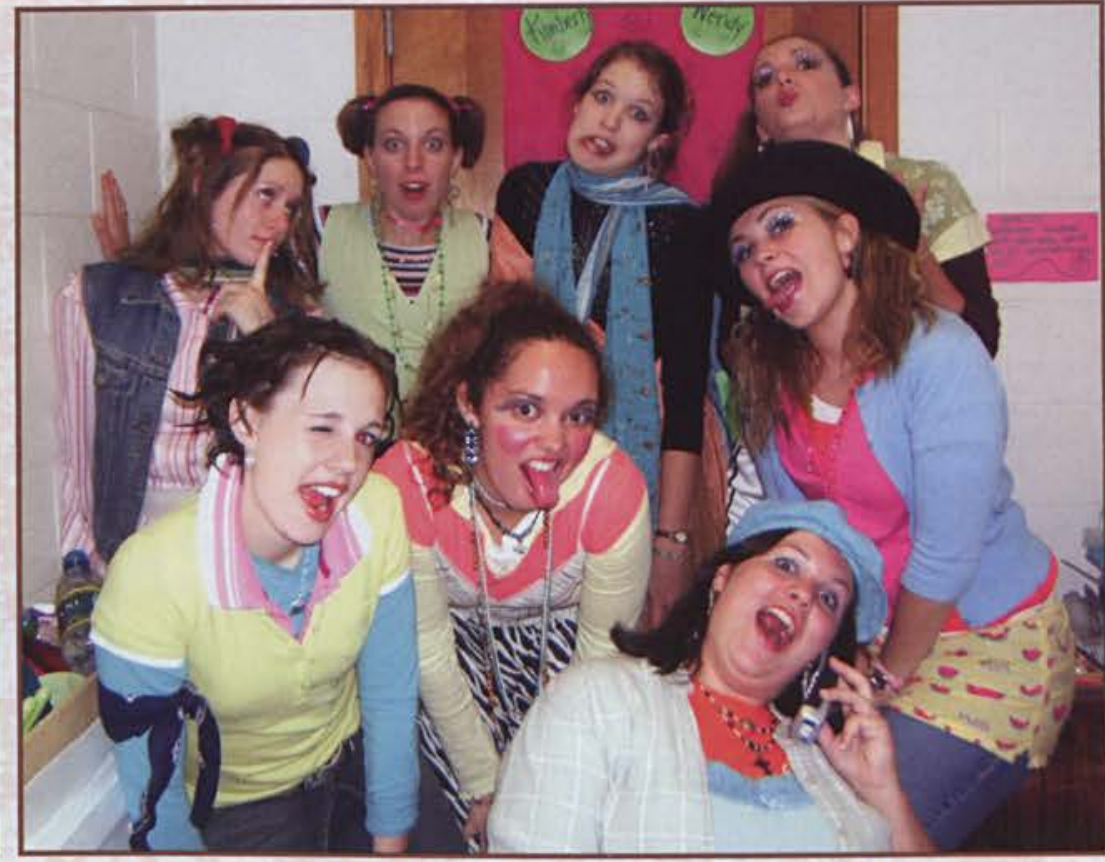

^ Printy units 30 and 32, consisting of Shannon Lynch, Hannah Cozad, Kendra Mattson, Wendy Austin, Kim Rayder, Laura Meyers, Kimberly

Rowlands, and Amanda Cyrus, dress up before visiting their brother hall on the Hill on Halloween.

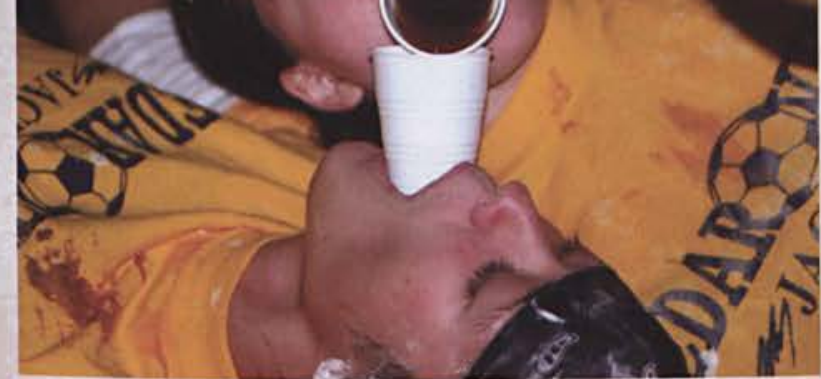

2




\section{Resident}

\section{Assistants}
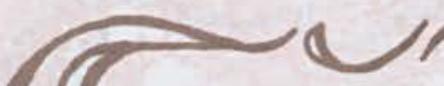

When one thinks of an RA, what comes to mind is perhaps someone older, wiser, an enforcer of the rules, or even that scary person down the hall. However, it's great to discover that RAs are students just like you and me. They have a love for students and a love for investing themselves

into other people around them. Most RAs will share how much they love being an RA and how great an experience it is. Printy RA, senior Megan Bartoletti said, "The coolest part of being an RA is honestly just getting to spend time with the girls. It's awesome getting to know their hearts and seeing God work in their lives." Craig Houser shared his love for "late night conversations about God and life. I also love unit meetings, because they are a blast and provide an opportunity to talk about stuff that is relative to their lives." Even though it is a lot of responsibility, these special students have said it is worth it. It is worth giving of their own free time to get to know someone new or pray with someone who needs support or just to listen when someone needs to talk. I have a great respect for those students who chose to be an RA. It can be a difficult task, but it is also very rewarding.

However, despite all the responsibility, RAs like to have just as much fun as the rest of us. One night, Craig Houser and some of his guys from Lawler "got the idea to fill up the shower with bubbles. For an hour we made bubbles until the shower was completely full. Then around 4 in the morning, we covered ourselves completely in bubbles and randomly walked into different units scaring people. It was topped off by dancing around like idiots in Lawlor's parking lot until the bubbles were all gone."

> Senior RA Jason Briggs helps to move in the freshman boys into Lawlor Hall.

- Justyn Yager \& Joe Holecko don their shades in order to look cool as well as to maintain their air of authority in the dorms.

Printy RAs smile as their wait for their girls to arrive. Back row [left to right]: Kim Cowell, Nikki Carroll, Jacinda Gillette, Katie Pankratz. Front row: Julianne Paulson, Bethany Houts, Alicia Christman.

Apples annyone? Standing [left to right]: Amy Dennis, Nicole Normandeau, Chelsie Hoffman, and Alicia Lease. Kneeling: Sarah Matney and Elise Clowser.
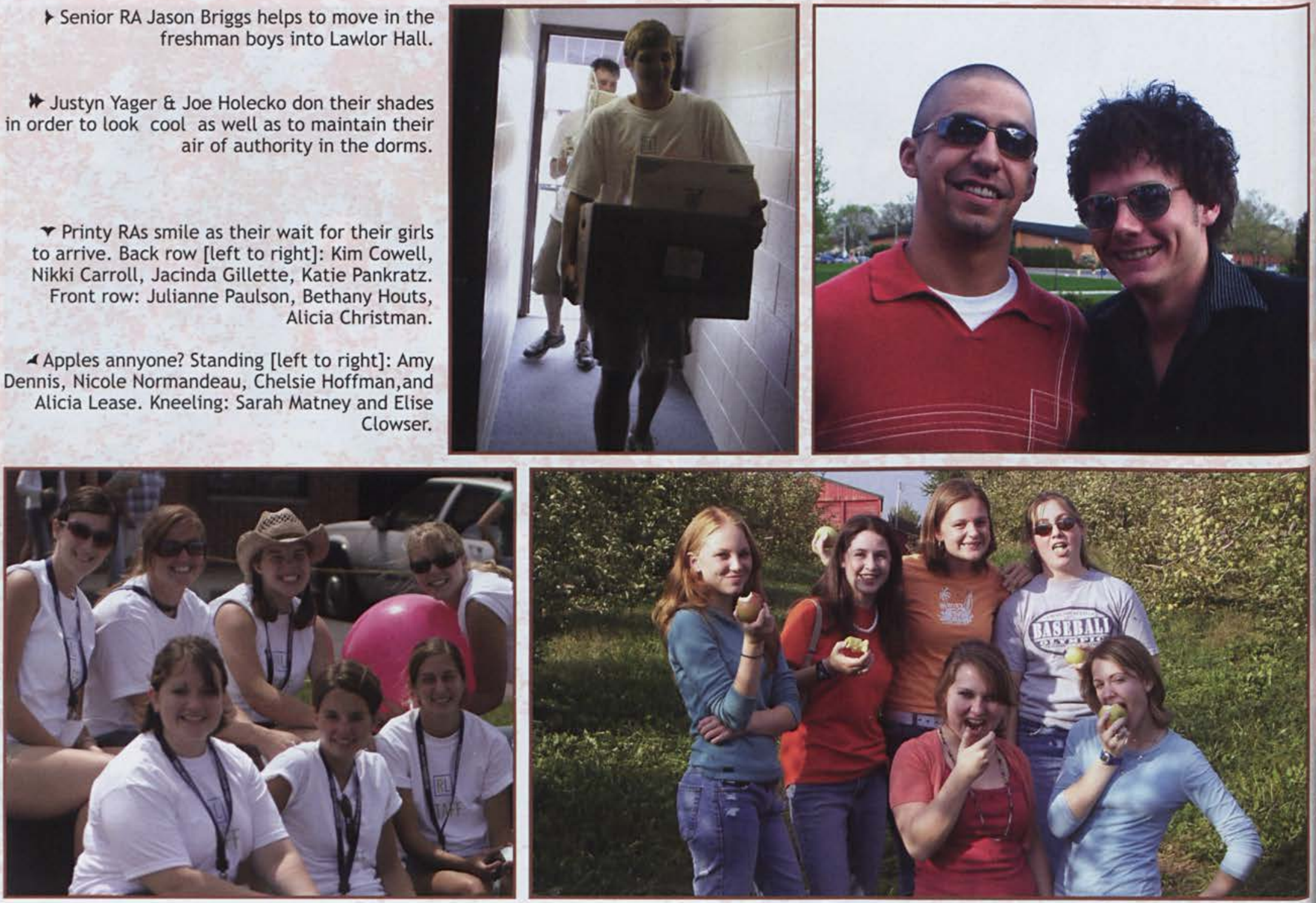


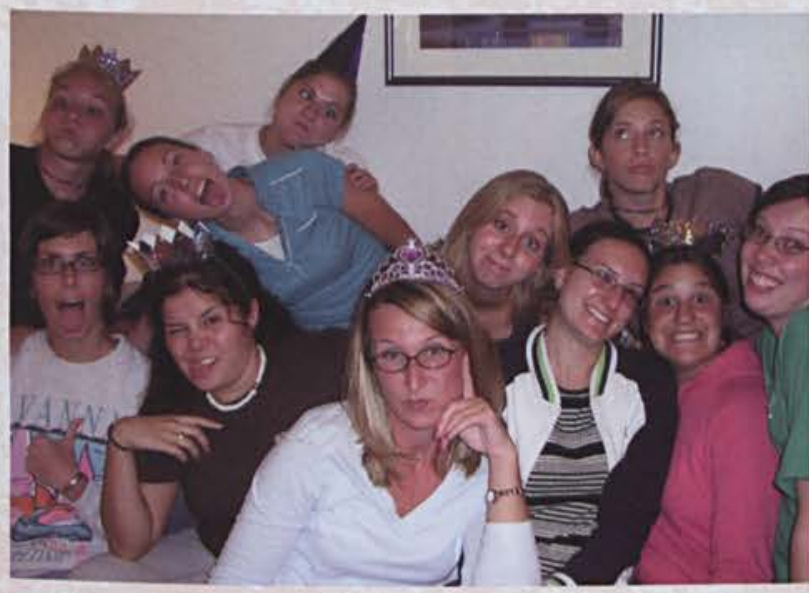

$\checkmark$ McKinney and Johnson RAs enjoy their traditional Thursday Night Randomness. Back row [from left to right]: Cristy Thompson, Danah Sargent, Hannah May, Abby Fenton, Michelle Bicking. Front row: Elizabeth Korver, Karie Harpest, Susan West, Emily Doane, Katie McGinn, Kristen Zion.

- Merry Christmas! Upper North Willetts welcome guests for the Christmas open house by featuring a different Christmas song for each room. Standing [from left to right], Rachel Geier, Katherine Sawdon, Holly Johnson, Samantha Maat. Nealing, Chenoa Linnemeier, Katie Behlert, Stephanie Yoder, Connel TerreBlanche.

$\checkmark$ You wanna mess with us? Murphy Upper Middle and Rickard Upper Middle (MUM \& RUM) have a work day at Scioto Hills. [From left to right] Melanie Hitchock, Siera Butler, Katie Wheeler, Kat Fowler, Jake Lewis, Rob Stahl.

A Saint Clair RAs Jason Ney and John Hummitzch try out Jason's electric scooter over Getting Started Weekend to see what Campus Safety would do.
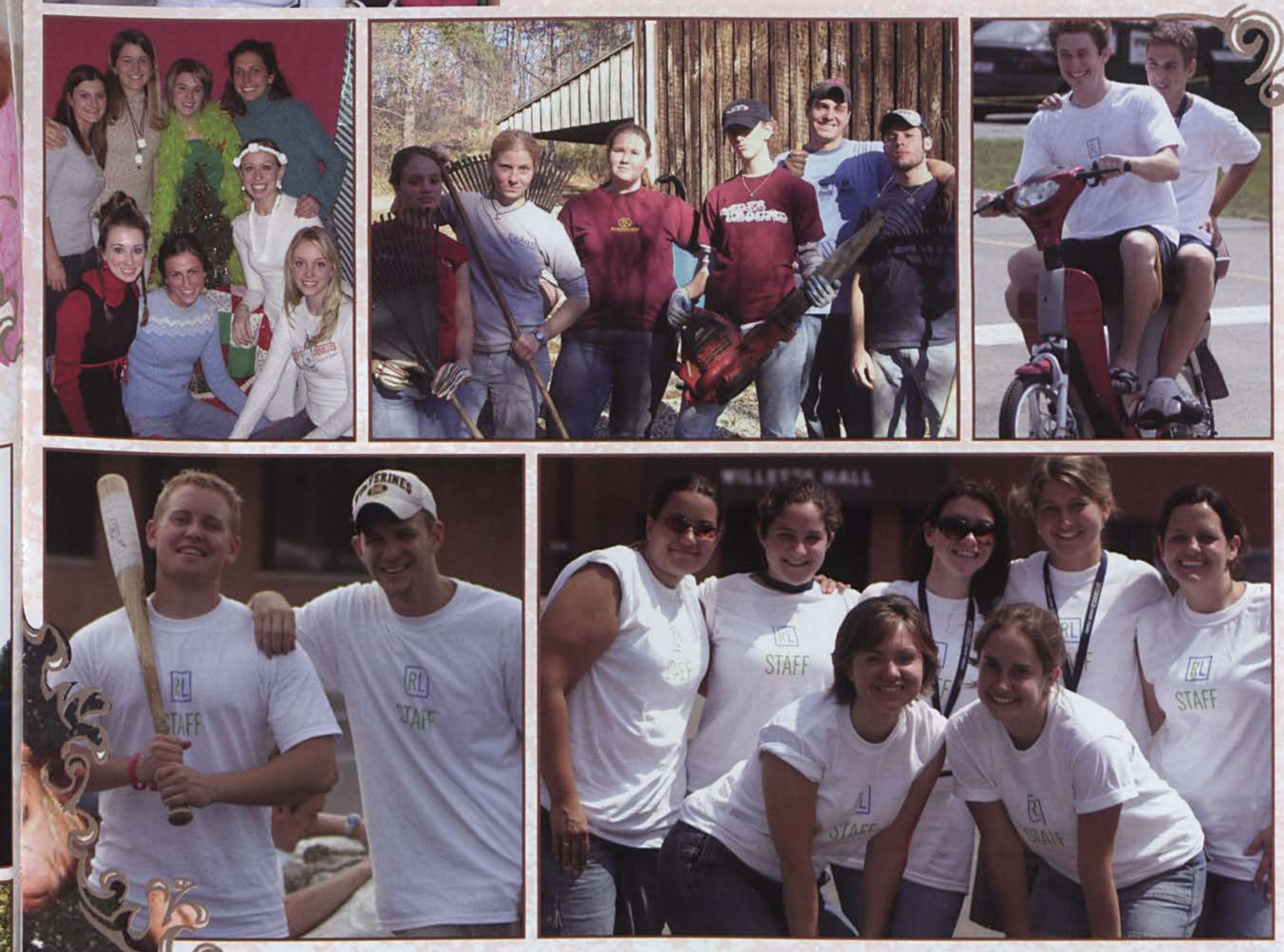

A Watch out new kids, these

Lawlor RAs might just keep you

^ Willetts RAs take a break during Getting Started Weekend. Back row [left to right]: Amanda Keith, Cory Green, Krysten Clark, Katherine Sawdon, Sarah Sargent. Front row: Laura Perrien and Tarah Boone. to bat. RAs Richie Reeder and Scott Miller prepare to meet their boys.

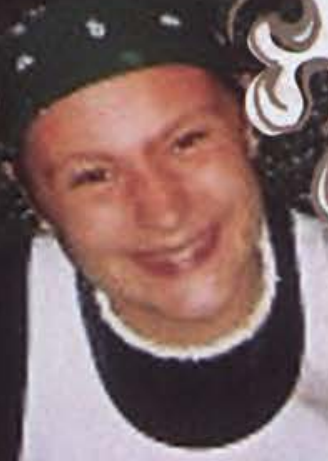
1 


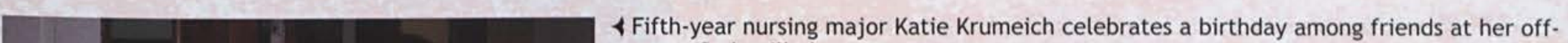
campus Cedarville home.

- Senior nursing majors Erin Dimeolo and Jessica Waggoner relax together at their Cedarville home, lovingly dubbed "the Mansion."

$\checkmark$ Daryl Sando works alongside other Cedarville students at the Dayton Art Institute during Cedarville's Night at the Museum. The Institute was celebrating the arrival of its Egypt exhibit and invited area college students to join in their excitement.

A Tim DeKonick enjoys the aroma of burgers grilling while he attends a cookout at a friend's house.
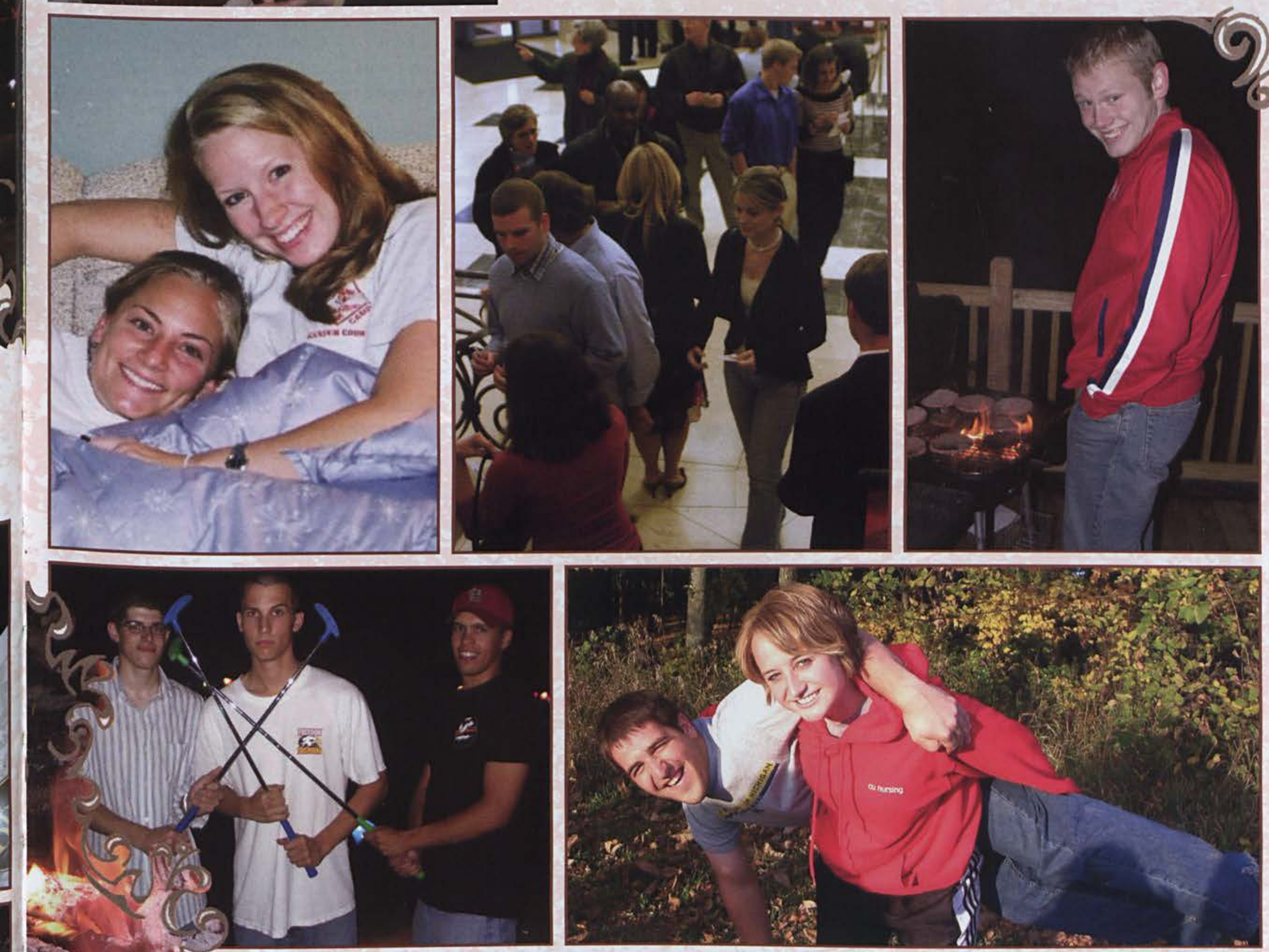

^ Friends Ben Lawrence and Rebecca Williamson hang around one of several parks

^Jon Manchester, Zack Eagle, and Chris Plume clash "swords"after a in the Cedarville area.

night of mini-golf at Young's.

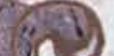

(c)

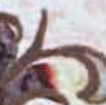

$1 \times$

$\checkmark$ The sharp crackle of wood receding into red flame, the scent of burnt hot dogs and

marshmallows, laughter of friends and family, the crunch of fall leaves- all point to one very popular off-campus activity: the bonfire. 


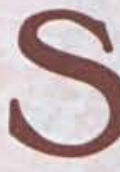

Sports

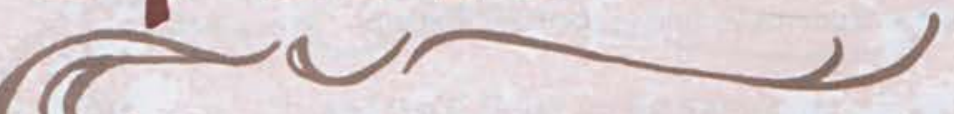

I felt a wave of excitement rush through me as I got ready for the big game. As I carefully applied the intimidating face paint, I began to strategize and replay our team's strategies over and over in my head. And if we lost? Who cares! The most important thing that I carried with me this season was the memory of some awesome games and great times with my friends.

This year's intramural seasons were full of exciting participants and good, clean competition. For many athletes, playing intramurals was a great way to stay involved in their favorite sport without the pressures and time constraints of collegiate play. Sophomore Dan Knudsen, a participant in various intramural sports, said that his favorite aspect of intramurals was "simply the chance to play. . . I love sports, but with my obvious lack of talent for any Cedarville team, intramurals let me play for fun and competition.".

Other participants in intramurals valued the relational component that comes with team sports. Freshman Emilie Gouch explained that joining intramural teams opened up doors to meet people that she would not normally be introduced to. She continued, saying, "I believe it helped me transition to college and form new friendships my freshman year, much faster and better than if I had chosen not to get involved." For senior Nathan Martindale, however, the motivation to be in intramurals was as simple as having "a great way to stay in shape and still play the game I love."

Rachel Teat dons a rather unique uniform before a rousing game of basketball.

- Taylor Storey attacks the ball during an intense intramural soccer game.

ASteve Ziegenfuss, Mike Zerminski, Tim Storer, Brian Groff, Adam Groff, Ben Hill, Becky Hornbrook, Shannon Picazo, Emily Van Vliet, Katie Fickes, Paula Thompson, Kat Godiness congratulate each other after a successful basketball game.

$\checkmark$ David Fuls, Tyler Anderson, and Trenton Simon get ready to charge the opposing team during a flag football game.
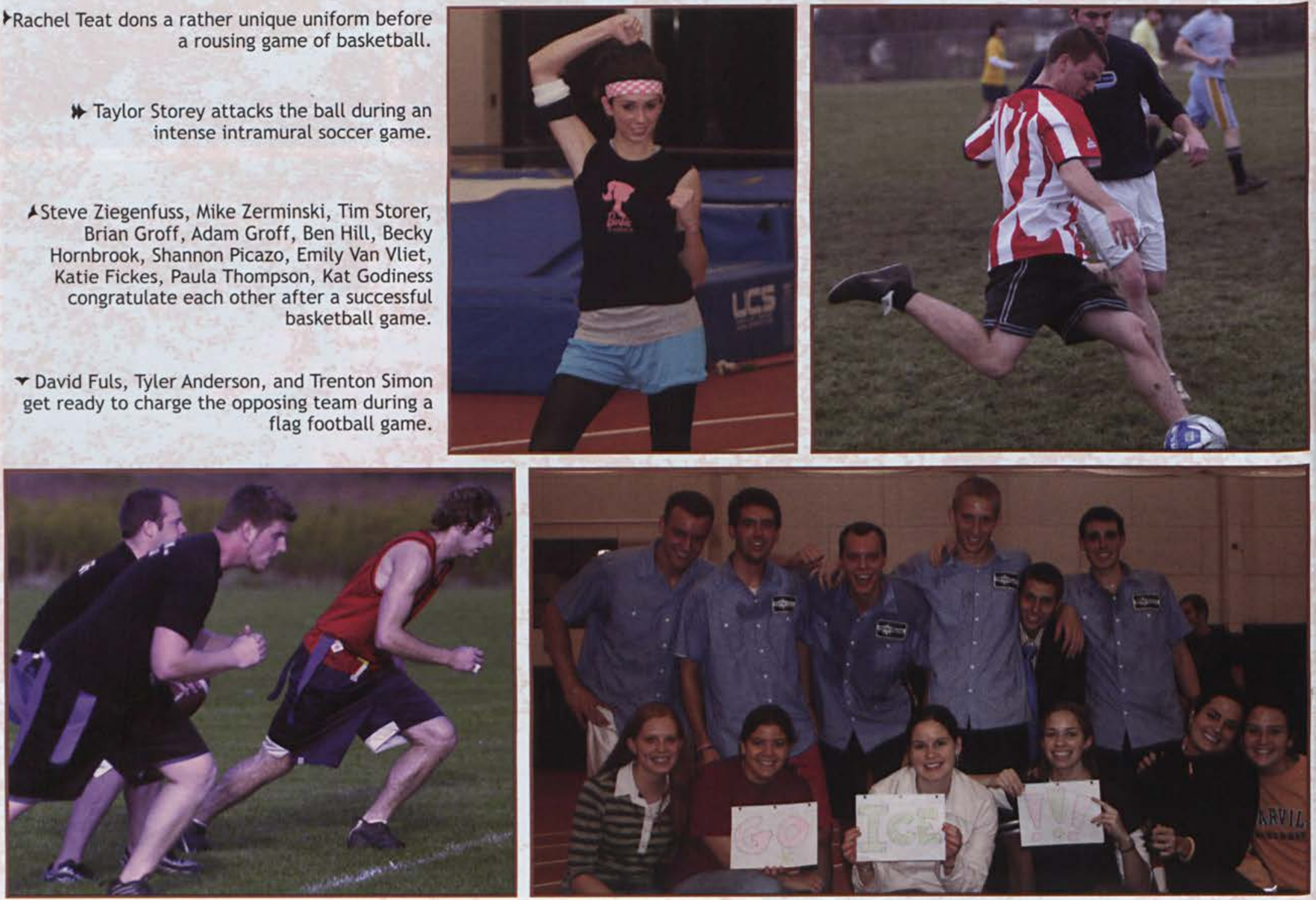



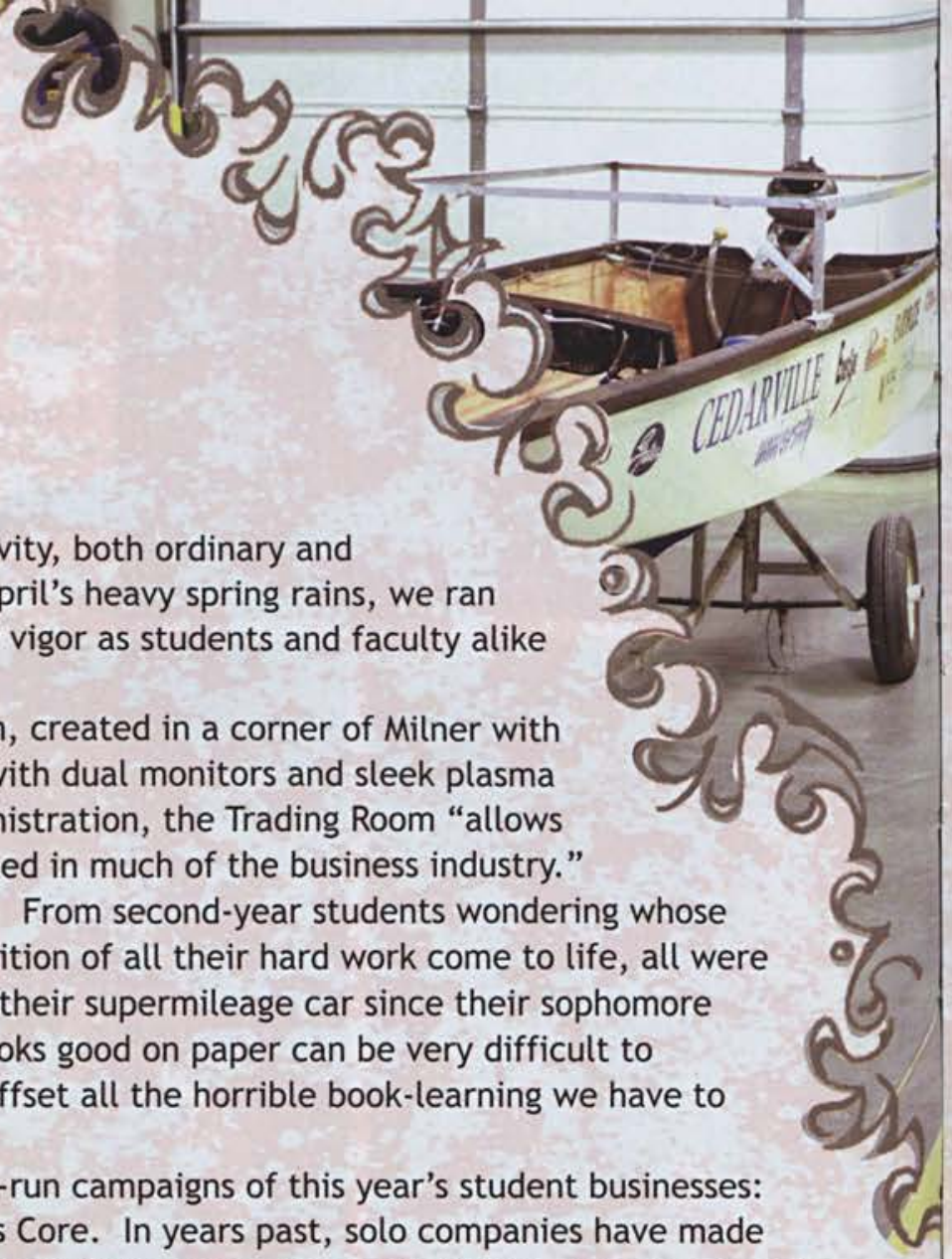
their debut on campus during this experience-building time, but the competition of two companies made this year's campaign all the more exciting. Truly, we will never be able to forget the promotional ads that introduced us to Professor Spencer's alter ego, "Spence Dogg," no matter how much we wished it!

D Dave Scott concentrates on welding together parts he needs to complete his senior design project: a formula car.

- Phil Buskohl screws in a small, but critical piece as he attaches a wheel to his formula car.

4 The members of Mugs Inc. take a break from creating and marketing in order to get a group photo

$\checkmark$ Ben Lawrence, Andrew Harris, Alan Mclver, Doug Radtke, and Peter Locke display their senior design project. Andrew said, "This was an amazing year! I've really learned a lot and had a great time working with this group of guys."
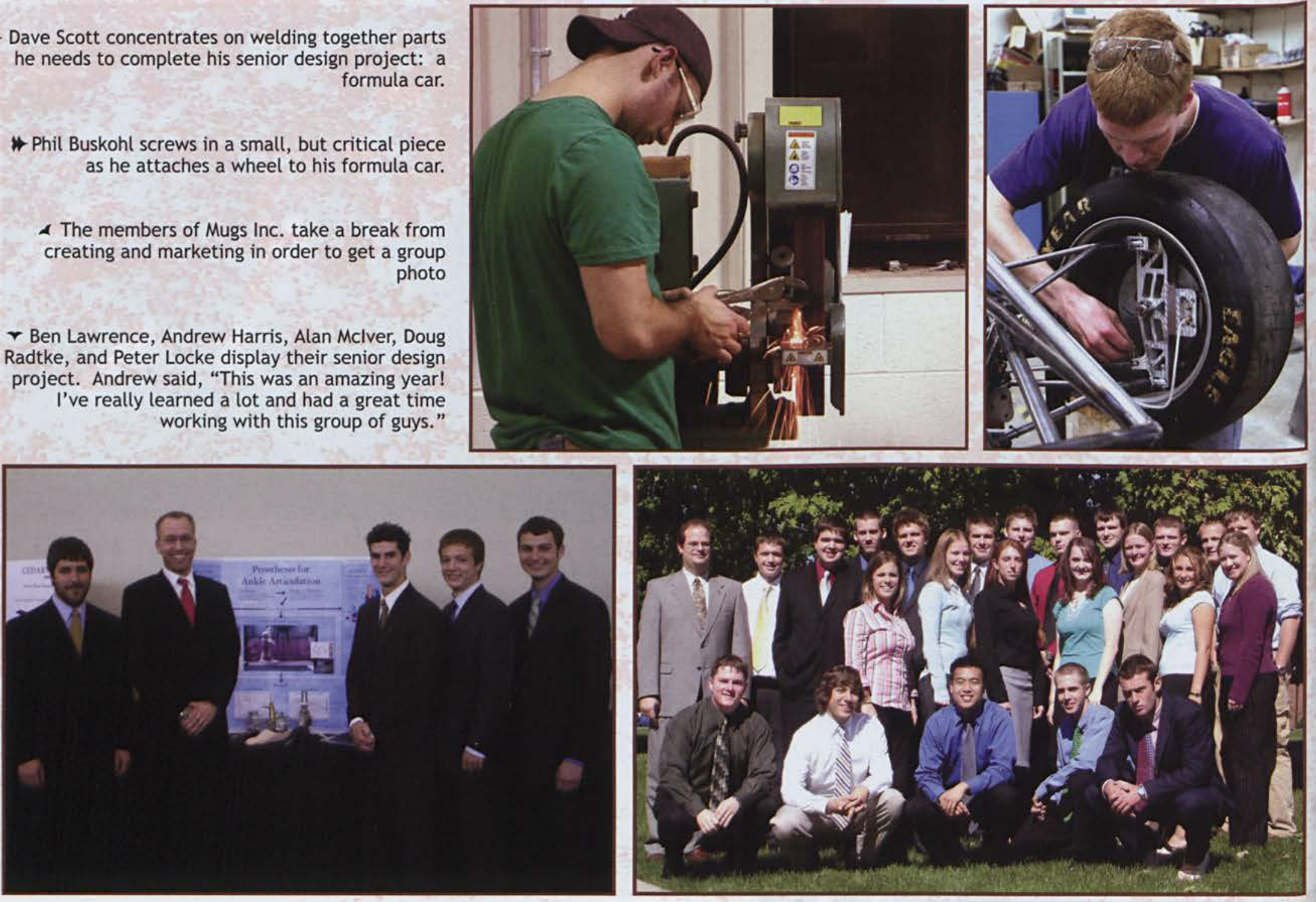


\section{SGA}
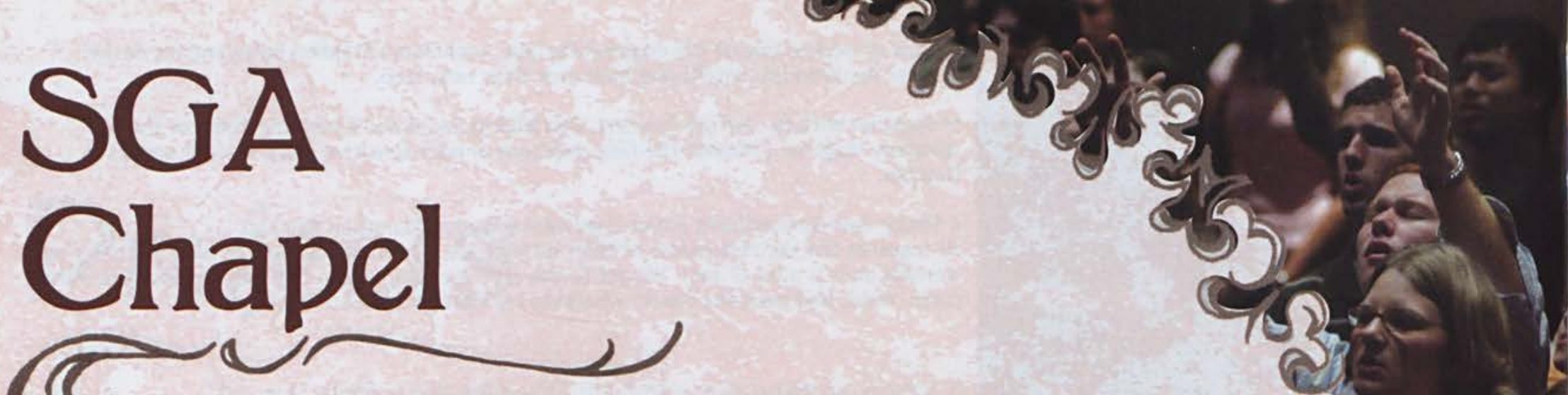

Survey students about what chapel services they enjoy the most, and the number one
ould be Student Government Association chapels. Who does not like to spend an hour of response would be Stude watching a video clip about Pastor Rohm's crib, enjoy the first annual turkey drop, try to get

a date with SGA president Matt Shiraki, and sing funny songs? SGA chapels this year have encouraged me, given me hope, and challenged me daily with the struggles that I go through in my Christian life. SGA worship leader Rob Reid shared why SGA chapels this year have been so successful: "Everyone comes expecting to have fun, and I think that's the main reason why we have so much of it: we're all willing to."

SGA chapels would not have impacted the student body the way it did this year if it were not for SGA chaplain Ben Waddell. Ben's most memorable chapel service was sharing about our hope in heaven. "I feel like God has really had His hand in the student body this year, and that has been reflected in SGA chapels... with the largest turnout ever for opening worship night, the largest offering ever for Katrina relief, and even seeing Pastor Rohm dressed up like Mr. Rogers!" reflected Ben Waddell. Senior Mary Orr reflected that SGA chapels "were a time where students can be real with one another but also enjoy worshipping the Lord together. The videos and 'dates with Matt Shiraki' make SGA chapels memorable."

Overall, SGA chapels were a time to relax, have fun, and relate to the rest of the student body through laughter, tears, and vulnerability. They brought the Cedarville family together in a way that often promoted discussion, changed lives, and created favorite memories.

- Courtney Reid, female vocalist for SGA chapels this year, enjoys coming before the throne of God with her brothers and sisters in Christ.

\# Freshman transfer Nate Cawood has the opportunity to show off his Plinko skills in front of the student body during SGA chapel. What did Nate have to say about it? "I'm good at Plinko!"

\& During the SGA Christmas chapel, Ben Waddell and Rod Bradley laugh at Pastor Rohm as he does his Mr. Rogers impersonation.

$\checkmark$ Mark Hershey and Jason Skelton play guitar during the praise and worship time at SGA chapel.
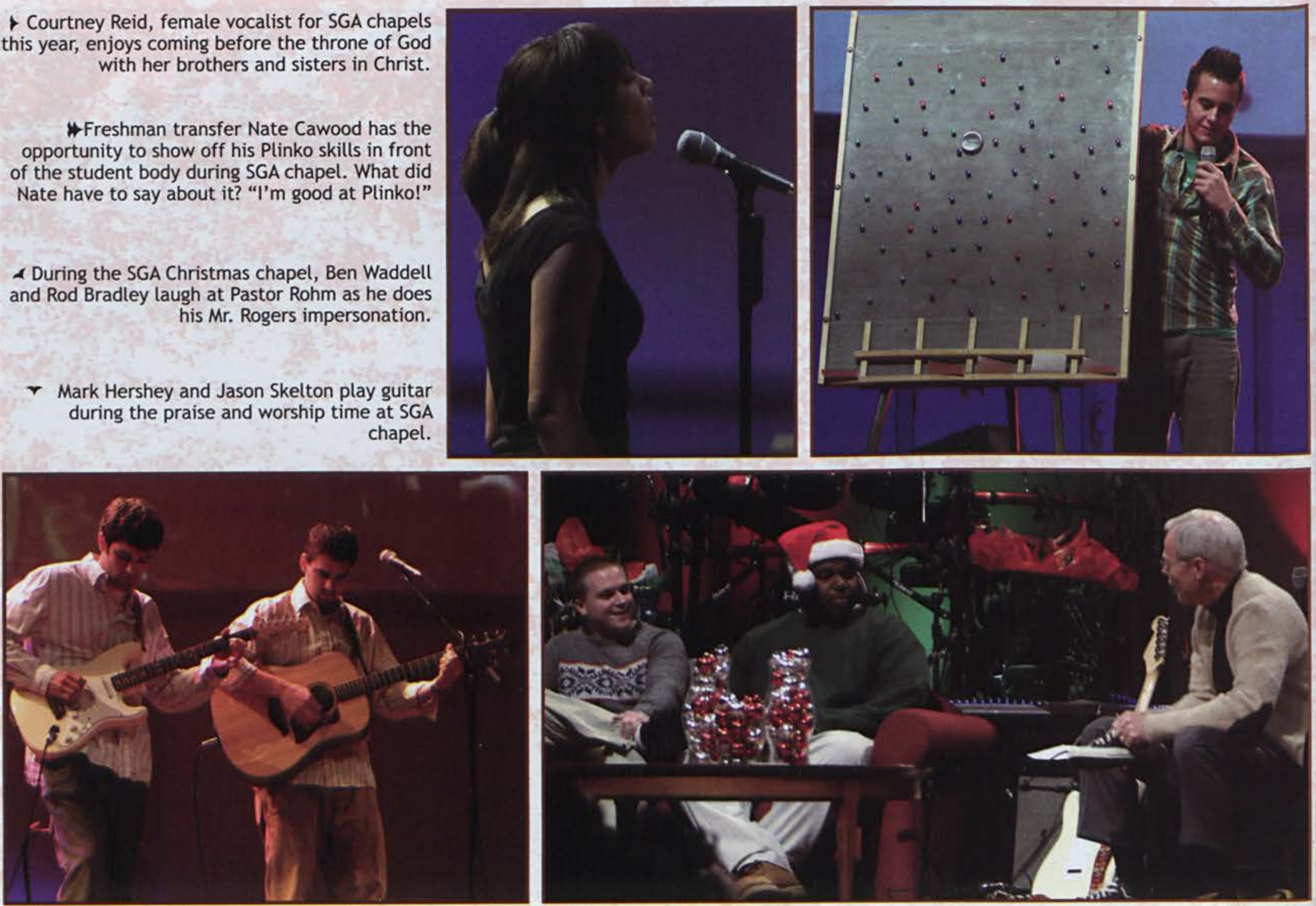

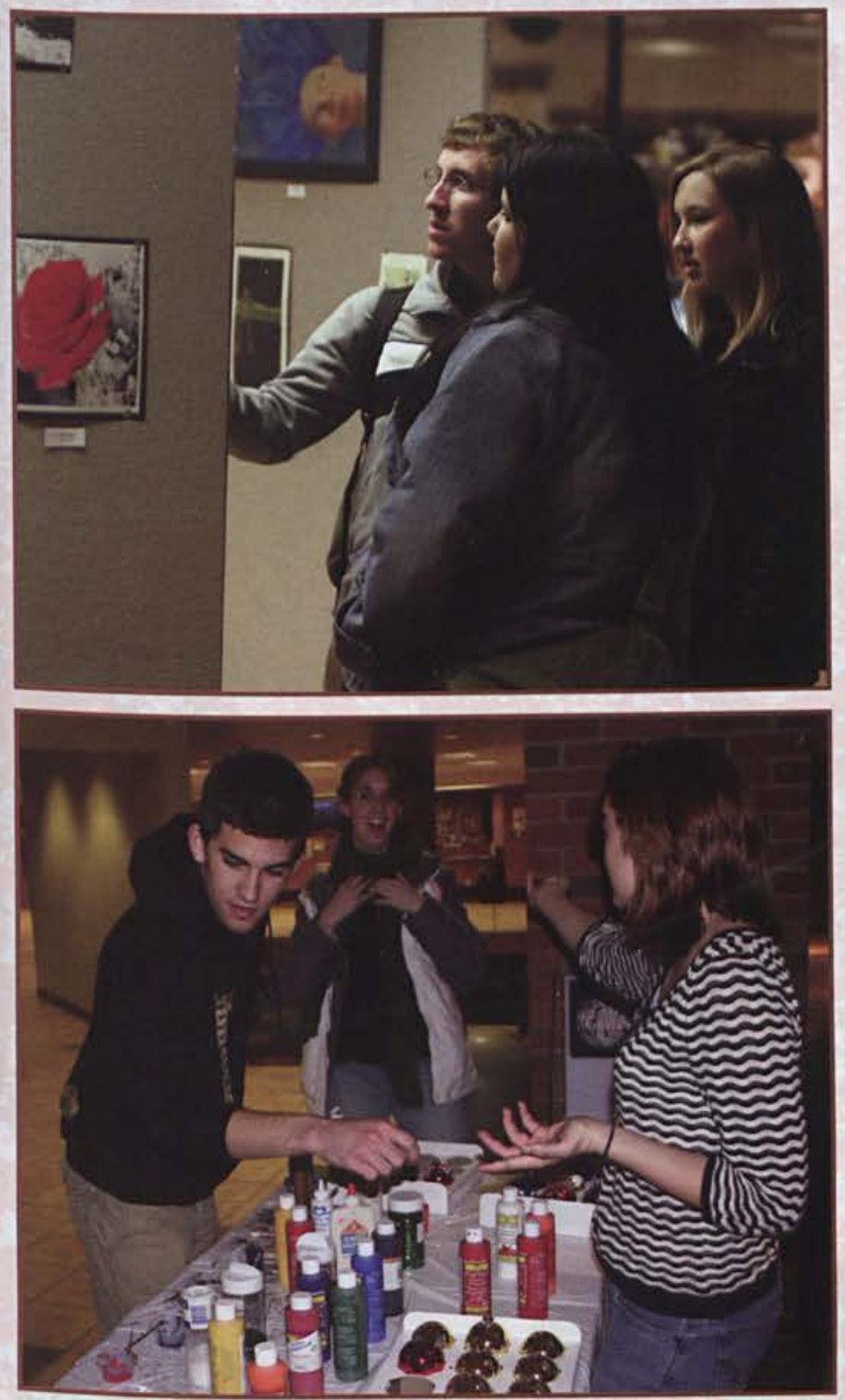

^ Christmas Stress Relief for Cameron Braun, Mindy Russell, and Rachel Petersen give the friends some much needed rest in-between studying for finals.

* Suzanne Wilson, Steve Wynn, and Kristen Wills admire student artwork during SGA art week. "It's nice to know that there are at least a few artists on campus that aren't afraid to produce raw, honest pieces," reflected Suzanne.
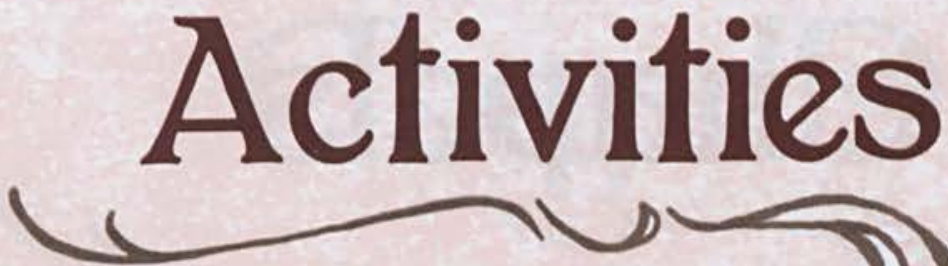

This year's SGA was all about the details, especially when it came to the very untraditional SGA activities which have come to be expected. Activities Board Director, Senior Holly Luiken, said the Board's focus, was directed by the question, "How can we take usual activities and give them a unique personality all their own?"

Their success was evident to all of us from the very beginning - the carnival games at Homecorning and the opening lines of the New Student Talent show, which got a major revamping in order to give it the feel of an old-time game show.

The fall semester's Stress-Relief Night was another hit with its special "Professor Karaoke," hot cocoa, and create-your-own holiday ornaments. As the kick-off to the basketball season, Moonlight Madness proved to be another get-on-your-feet-and-cheer extravaganza which, as a new addition, involved both faculty and students in its traditional halftime shoot-out. The excitement in the air was electrifying; we breathed in the whole scene as our favorite professors battled it out against our friends and hall-mates.

November saw us through with One Week and challenged our hearts to reach out beyond ourselves and minister in our community. Art Week got an upscale twist as SGA organized exhibits into galleries and held an opening night complete with a harpist, hors d'oeuvres, and sparkling grape juice. The week also included oral interpretations in the Hive, live music, and poetry readings, and was coordinated with HALO Films' Student Film Festival. Junior Allison Newfield remembered, "I was blown away by all the talent here at Cedarville and encouraged to see the ways our student body will be able to influence our culture in the future through art and media."

$\checkmark$ During finals week, SGA hosted a "Christmas Stress $\checkmark$ Laura Austin, Kelly Ramsey, and Joy Shaughnessy decorate cookies Relief" with karaoke. Joel Green and Alissa Wingenroth during their dorm's stress relief. sing along to the Backstreet Boys.
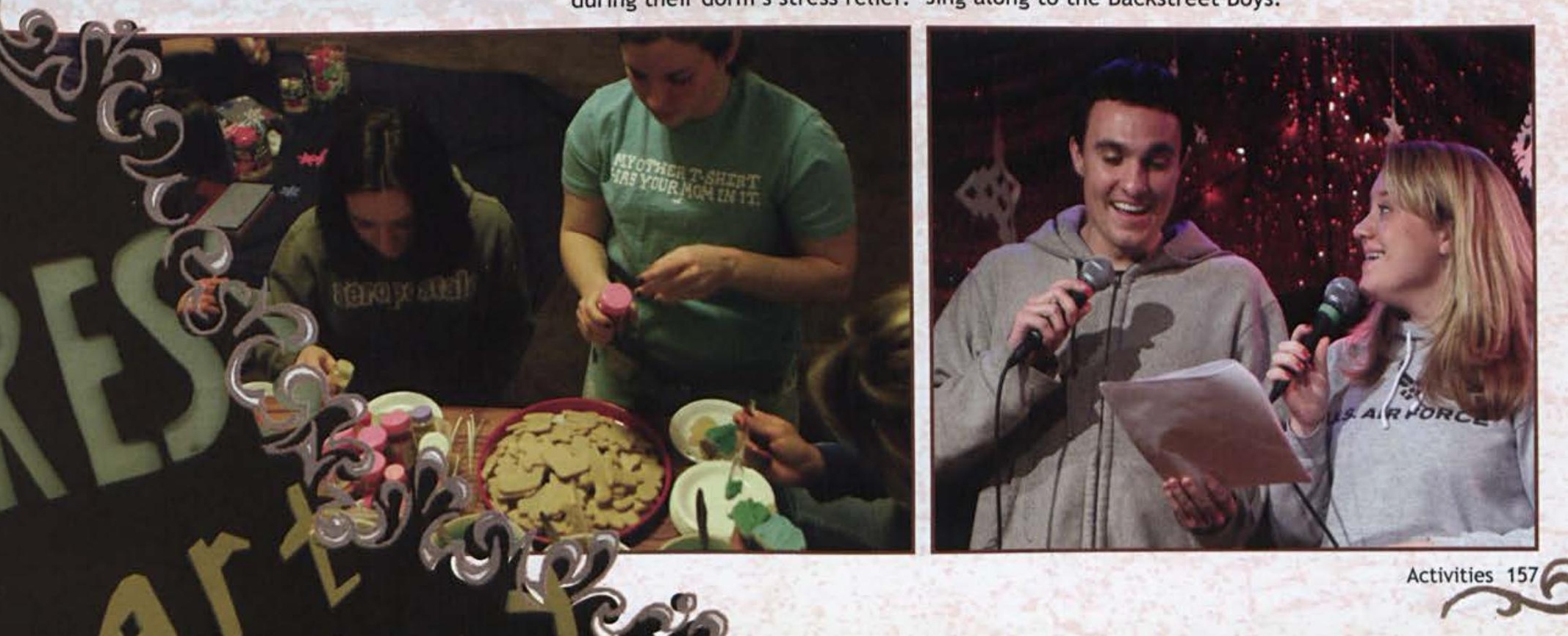

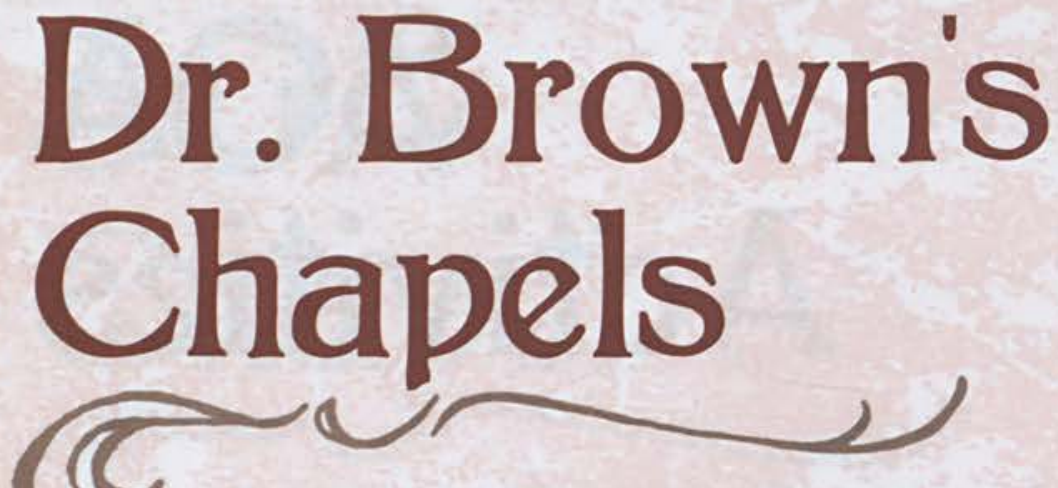

It's not hard to figure out what Cedarville students are doing at 10 a.m. on a Monday

morning. No, not sleeping or cramming for their 11 o'clock exam, but filling vacant chapel pews, ready for their weekly address from University President, Dr. Bill Brown.

The theme for this year's series of messages was "Engaging the World for Christ" and centered on building character and transparency as we live our lives before a watching world. Ethan Sanborn, a sophomore, commented, "Dr. Brown's chapels served to provide common ground for the wide diversity of chapels provided to us students. It is a true blessing to have such a Godly man as our president and to hear insight from him and solid biblical knowledge that is applicable to the student body."

Since Dr. Brown's arrival at Cedarville three years ago, we have been hearing phrases akin to Brown's chapel messages circulated around campus. However, this is the first year we have focused on the subject to such an extent.

Many students appreciated the diversity Dr. Brown included in his chapel messages, making each one a unique blend of Biblical teaching and applications. In one, for example, we concluded with the chorus "Sing Alleluia" in seven different languages - English, Shona, Chinese, French, Russian, Spanish, and Korean. He also gave a series of conversational lectures with the help of Bible Professor, Dr. Chris Miller, which addressed many of the questions Cedarville students ask regarding truth and reality during their college years. "I think that Dr. Brown's chapels with Dr. Miller were really memorable. It was good that they answered and helped us discover answers to a lot of tough questions that are being asked in our society of Christians," senior Ashley Ross said.

$\checkmark$ Dr. Brown plays electric guitar in (or perhaps out) of tune with engineering professor, Dr. Tim Norman.

* Keith Rice thoroughly enjoyed Dr. Brown's chapels.: "His witty sense of humor and keen insights on popular culture make every Monday something to look forward to."

Dr. Chris Miller, professor of Bible, joins Dr. Brown for a two-day stint during which they engage in a conversation aimed at guiding students through the difficult questions of life and of God.

$\checkmark$ Dr. Brown leafs through the day's newspaper before delivering a message concerning how we, as Christian students, can engage our world for Christ.
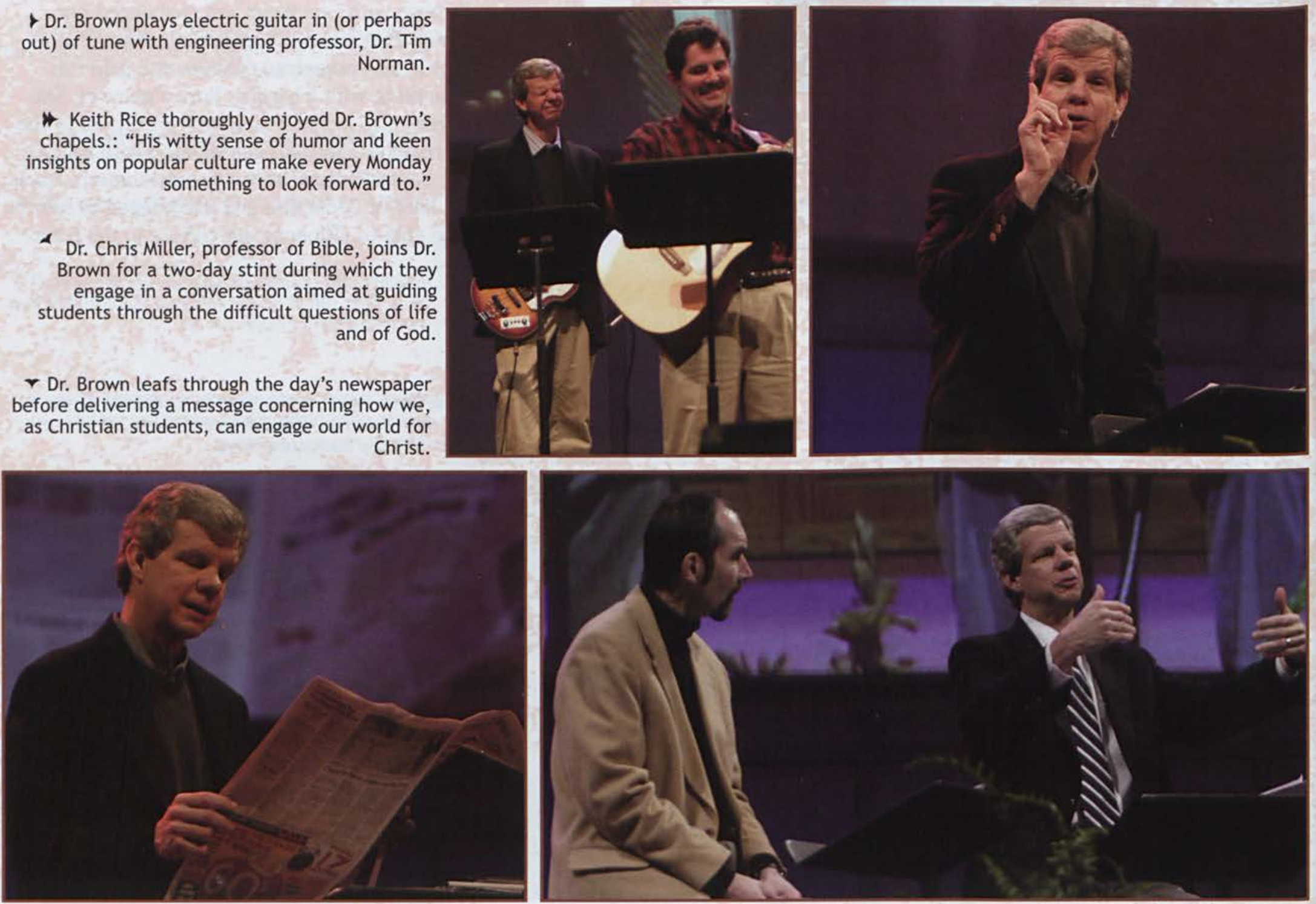

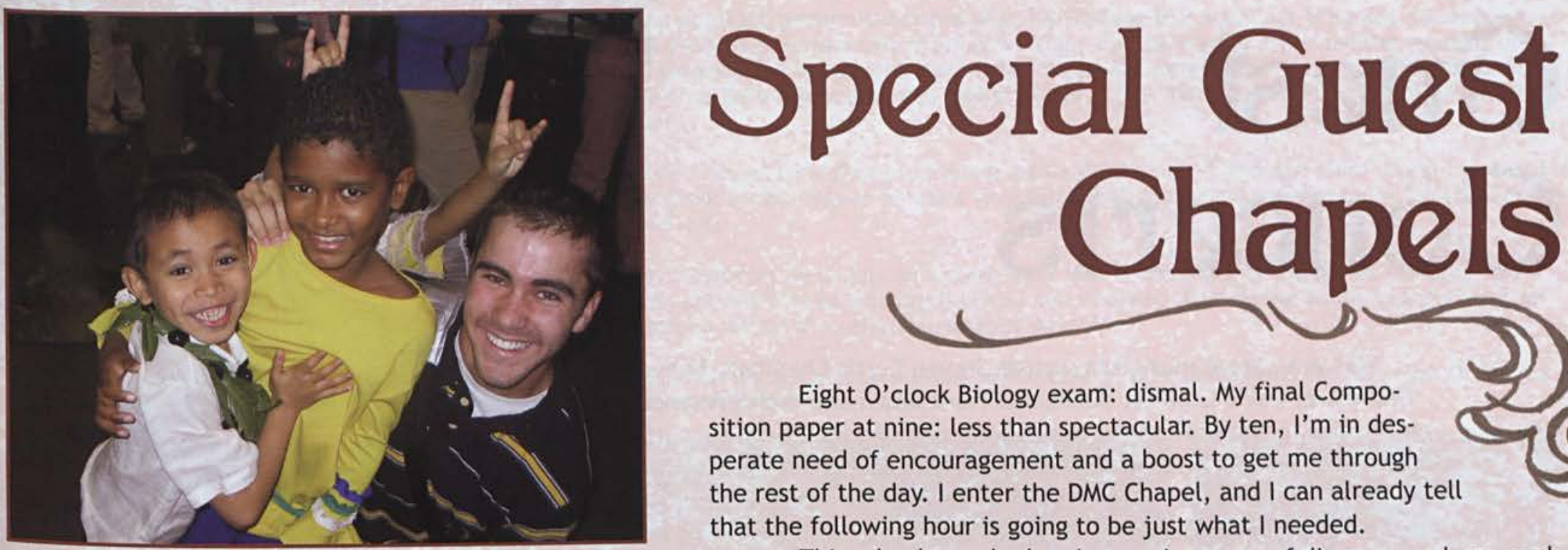

Eight O'clock Biology exam: dismal. My final Composition paper at nine: less than spectacular. By ten, I'm in desperate need of encouragement and a boost to get me through the rest of the day. I enter the DMC Chapel, and I can already tell

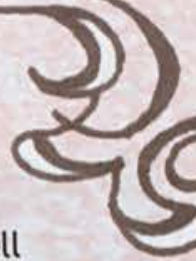
that the following hour is going to be just what I needed.

This school year had an impressive array of diverse speakers, each

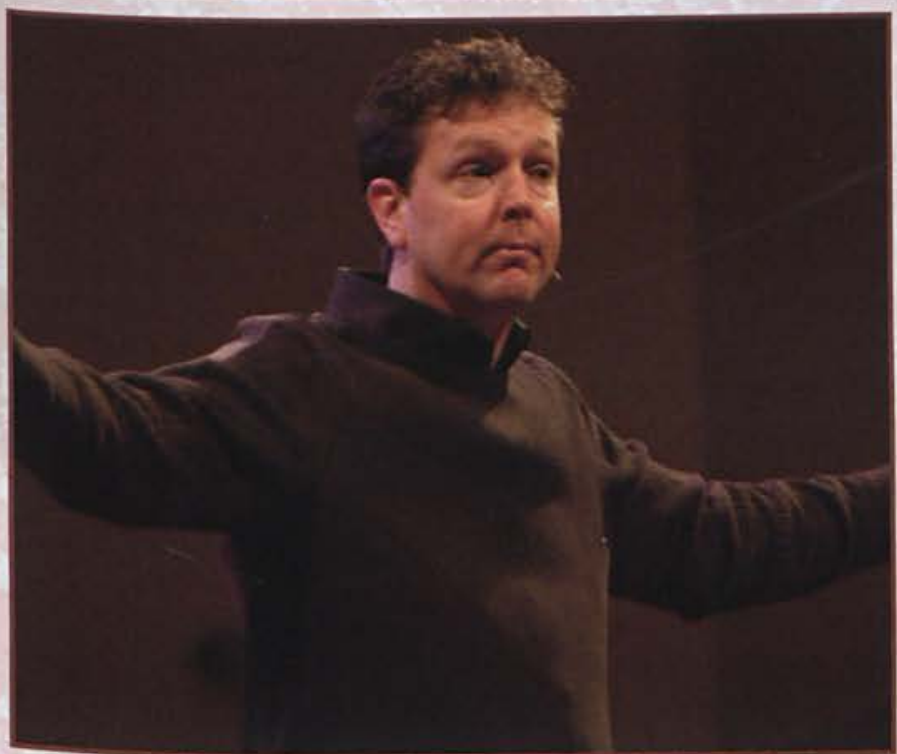

ఎ Dr. Tim Elmore, from "Growing Leaders," a non-profit organization dedicated to developing emerging Christian leaders, encourages the student body.

* Jacob Rogers goofs off with the Children of the World choir. "I loved hearing the joyful singing of the kids as they praised God, proclaiming the truth that children of all kingdoms and tribes were created for God's glory," remembered William Duncan. bringing his or her own unique contribution to the spiritual lives of students. Jon Kitna, a quarterback for the Cincinnati Bengals, was a favorite of many students. Sophomore Jacinda Gillette enjoyed his unique perspective and said that "it was also a good reminder not to give up on my friends who are unbelievers because God uses even the worst of circumstances to bring people to Himself."

Others appreciated certain speakers' fresh approaches to sometimes typical topics. According to sophomore Danny Kloosterman, speaker Chris Williamson did exactly that with the topic of racism and diverse cultures. Danny said, "Instead of becoming uncomfortable by a sense of guilt, I left challenged to change my way of thinking and to not be intimidated by brothers and sisters of different cultures."

Dr. Tim Clinton, a world-renowned Christian psychologist, had a widespread impact on the students, especially those involved in the Psychology field. Joe Poelzer, a junior Psychology major, said Dr. Clinton "was able to shed a new light into the reality of Christian counseling. He got to the heart of the issue, and it really resonated well with me."

Probably one of the more unconventional "speakers" of the year was the Children of the World Choir, who always seem to charm the student body with their energetic singing and dance routines. For senior William Duncan, their enthusiasm for God transcended language: "I couldn't understand all the words the children tried to speak in English, but I knew they were praising God with all their hearts, and that got me excited!"

$\checkmark$ Dr. Tony Evans, with The Urban Alternative, addresses $\checkmark$ Former Cedarville University President, Dr. Dixon, speaks to the the university family during the Southern Baptist university community. Convention's Evangelism Celebration.
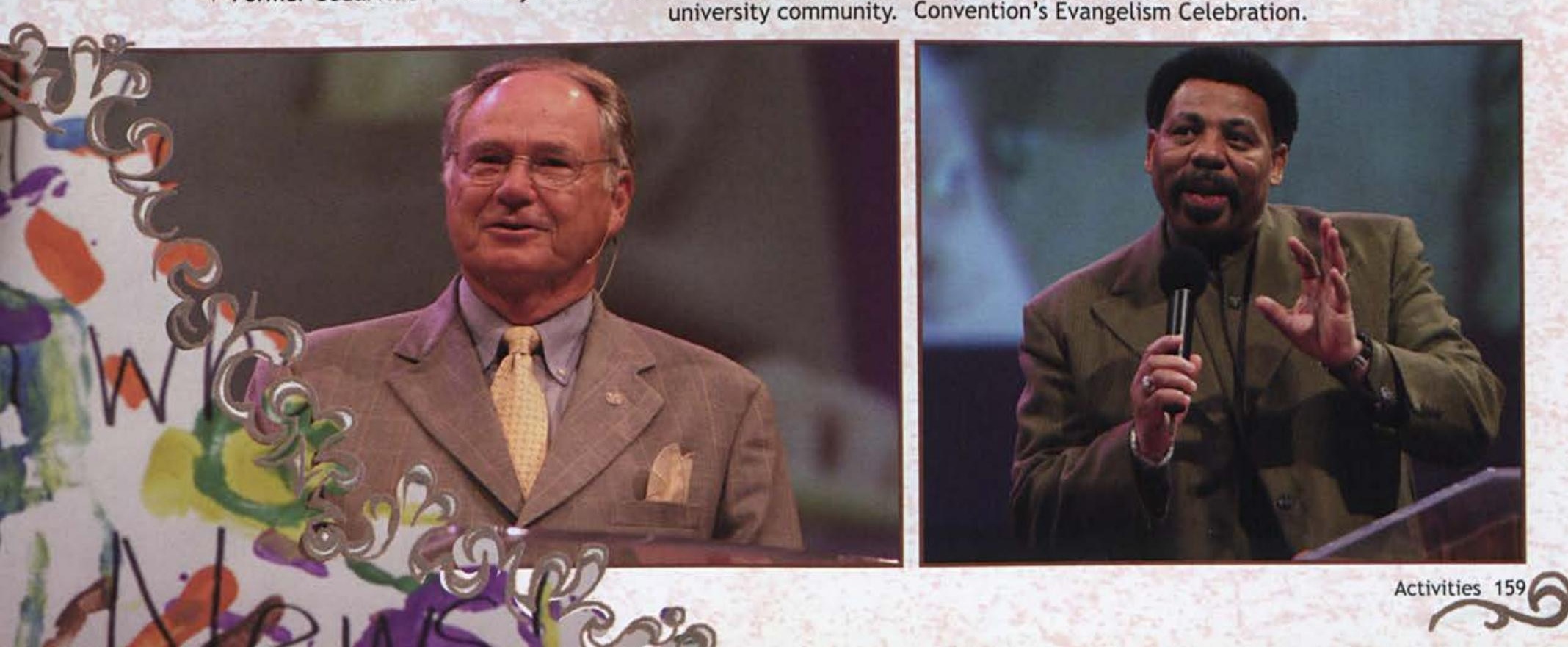


\section{Guest}

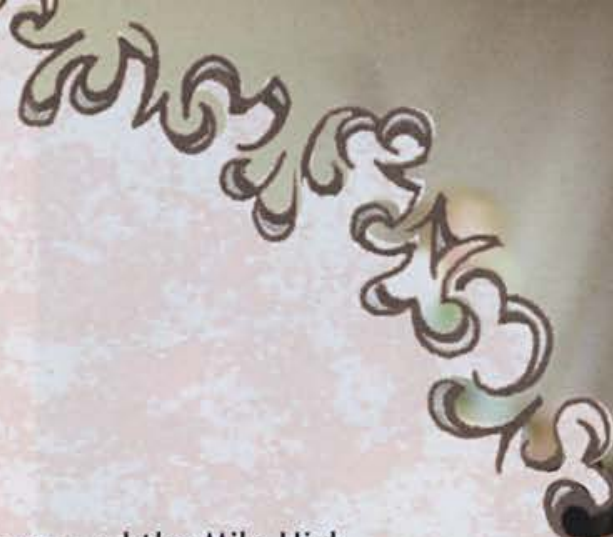

This year, great musicians such as Steven Curtis Chapman, Denver and the Mile High

Orchestra, David Crowder Band, and Third Day made their presence known on campus by

performing memorable shows in the Jeremiah Chapel. These concerts were attended by an amazing

number of Cedarville students, including myself, as well as people from the community, who looked

forward to nights filled with incredible musicians and wonderful times of praise and worship.

Steven Curtis Chapman kicked off this year's string of concerts during Homecoming Weekend in October. Playing many of his famous songs such as "Dive," "I Will Be Here," and "Live Out Loud," kept the student's on their feet for the entire show. During the concert, Chapman expressed the importance of adoption, something near to his heart. When it comes to musicians we tend to think that they are perfect. However, Steven expressed that he too is human, when he forgot the lyrics to one of his songs!

Denver and the Mile High Orchestra performed during the Christmas season. "Every musician in the band demonstrated high musical ability," reflected Elizabeth Fannon. "The guitar player [Keith Cooper] at the beginning of the set was amazing." The band showed clips from classic Christmas shows during their songs. As luck would have it, the first major snowfall of the season happened while they were here. As the band left campus, Cedarville students gave them a final farewell by pelting their tour bus with snowballs!

The last concert of the year sold out with David Crowder Band and Third Day making an appearance. Katie McGinn, an avid Third Day fan, said that "the concert was great! I loved being with over 3000 people and two great bands worshiping our Savior!" David Crowder played many familiar songs as did Third Day. The encore for Third Day provided a time of praise and worship and thought-provoking comments.

\Mac Powell, lead singer of Third Day, workes the crowd with classic hits such as "Consuming Fire" and "Cry Out To Jesus."

* Junior Stephanie Caywood, a Josh Bales fan, said, "I loved the expression of his heart for God at the Day of Prayer. It came through in the songs he sang and the way the chapel was organized."

A For many students, the concert Denver and the Mile High Orchestra put on was a nice study break from exams. Michelle Boudreau said, "Their performance was a therapy session for us before our Pharmacology exam, and we were not let down...it was amazing!!"

$\checkmark$ Mike Hogan, member of David Crowder Band, uses his God-given talents to play worship songs during the Wherever You Are tour that came to Cedarville University.
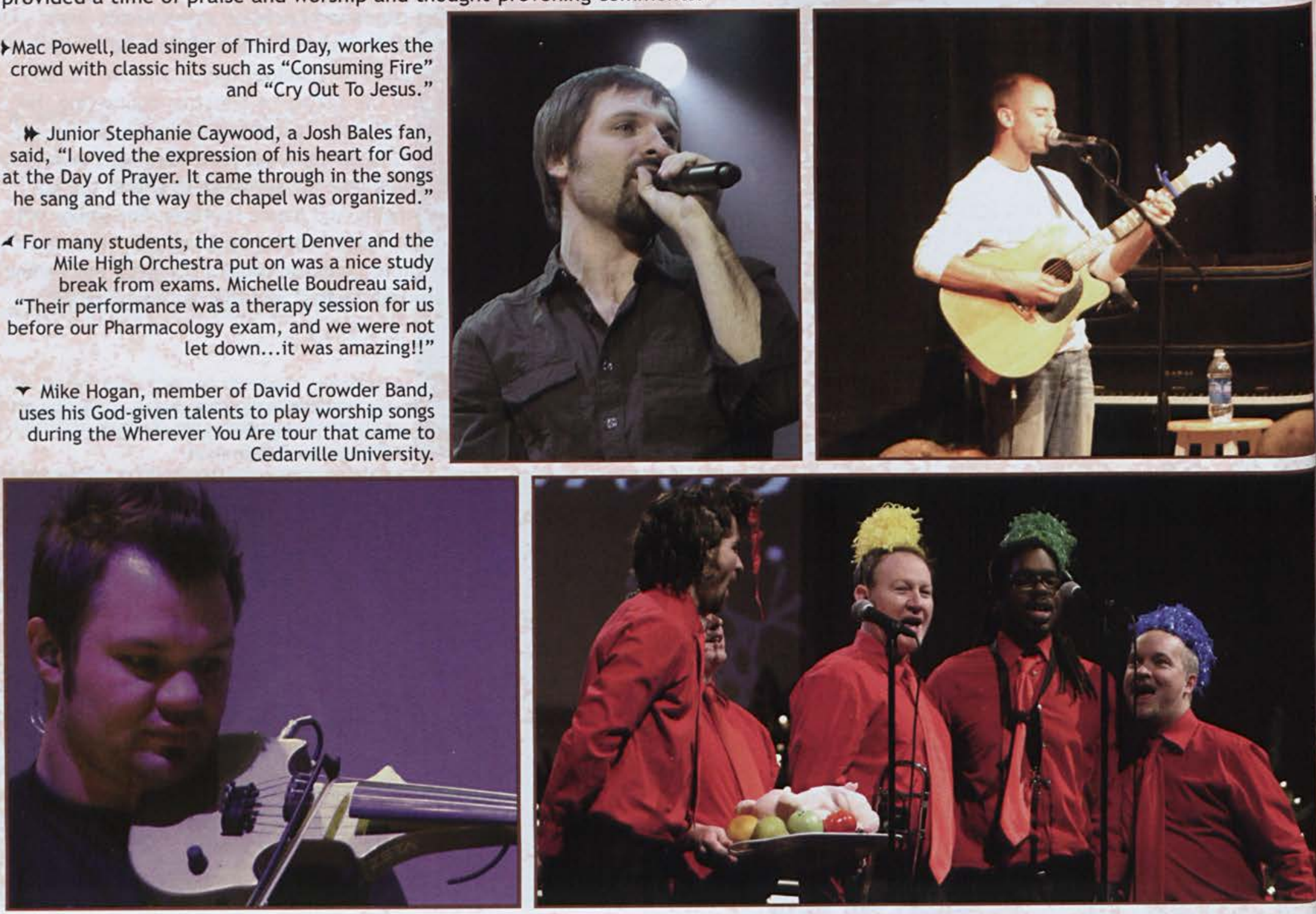


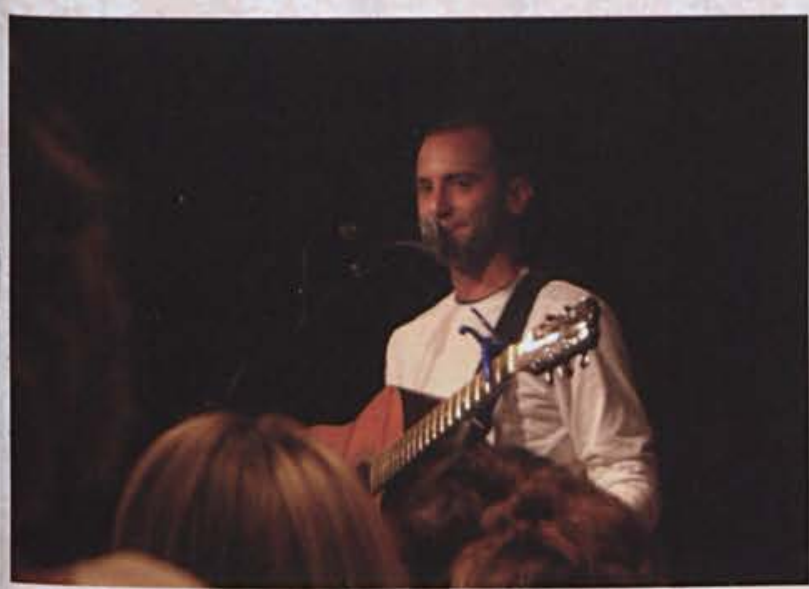

$\checkmark$ "Out of all the performances this year, his was one that stuck out the most. He displays what it means to have a true heart of worship, and the way he explained the meaning of each song made it even more meaningful. I know his song I Need You is a favorite among many of us here because we can relate so well," Julie Martz reflected about the Josh Bales concert.

- David Carr, of Third Day, plays along during the encore. "I thought Third Day put on a great show. Their encore was an amazing time filled with praise and worship songs," junior Jessica Grewe said.

$\checkmark$ David Crowder Band puts on a great show that is conducive to Cedarville students, the public, and the li'l sibs who are there for the weekend. Putting the words to their songs on the screen made for a reflective night of worship.

A Christmas music takes on a whole new meaning when Denver and the Mile High Orchestra perform. Their energy on stage is contagious.
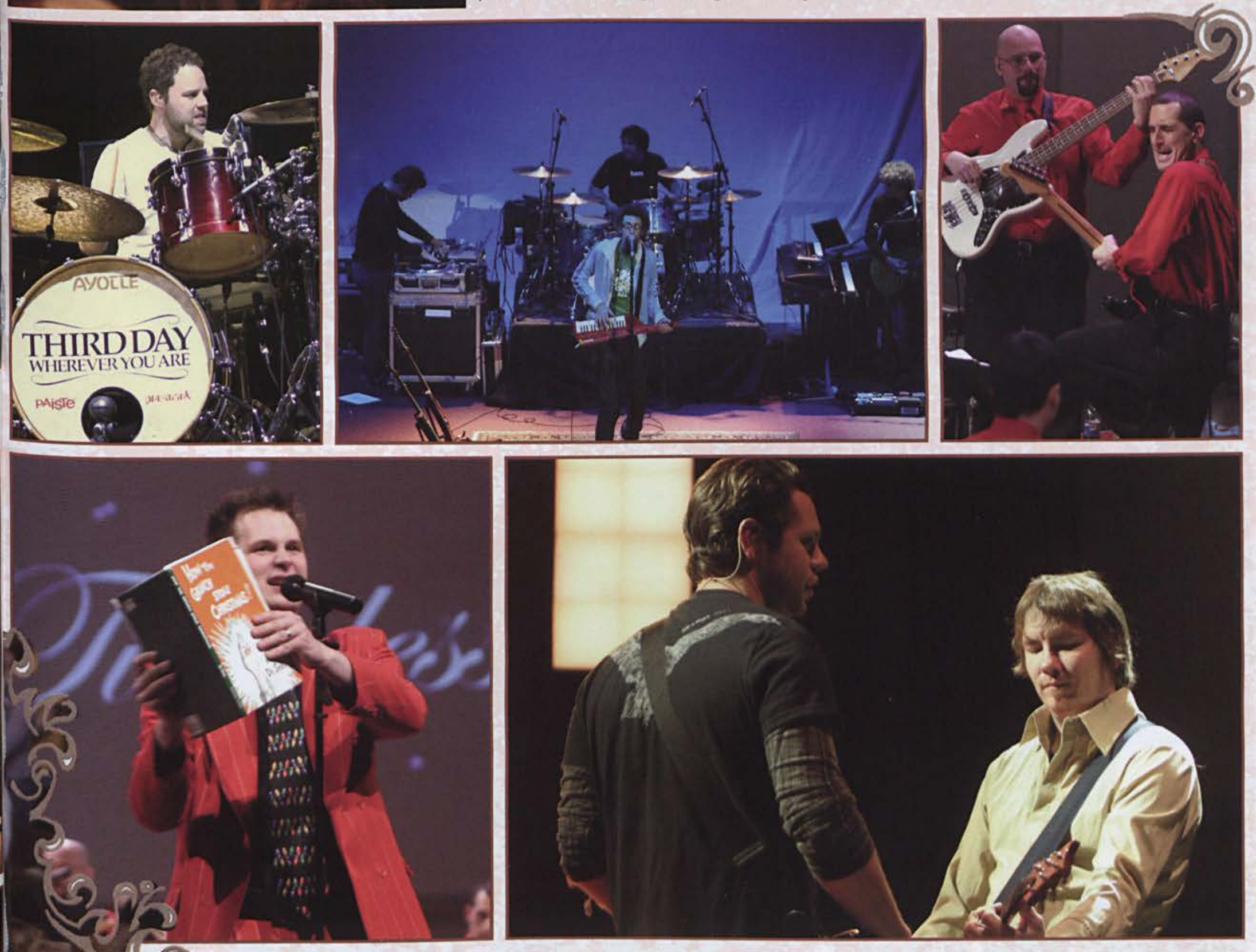

^ Taking many students back to their childhood, Denver Bierman reads Doctor Suess, accompanied

^ Tai Anderson and Mark Lee of Third Day strum notes to familiar Third Day songs that a sold-out audience could not resist singing along to. by clips of classic Christmas movies.

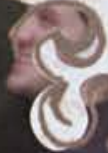
1 


\section{Independent}
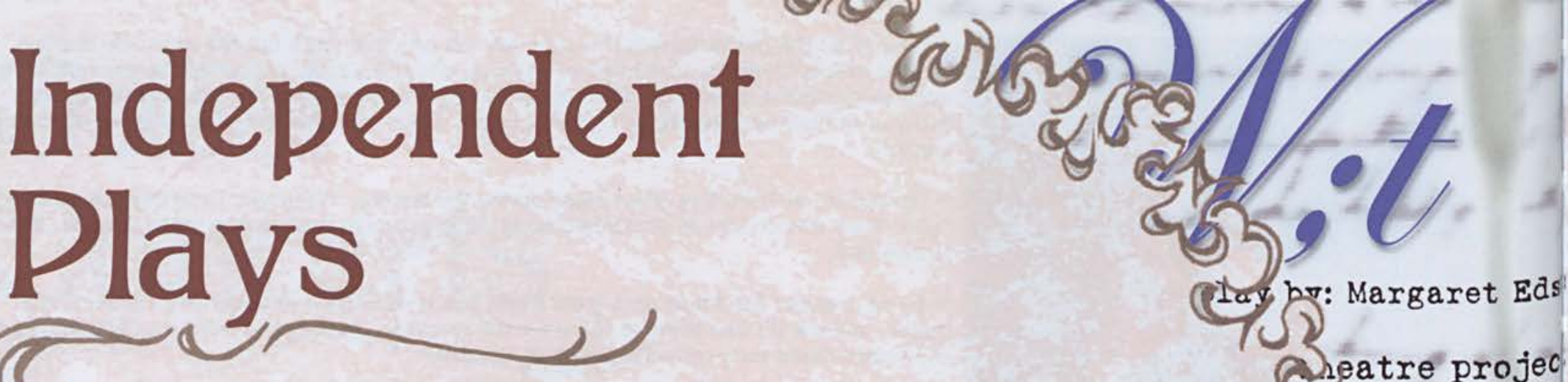

Cedarville University prides itself in its ability to cultivate and send out talented people in every area of vocation. The independent plays and senior theater performances that occur each year exemplify such talent. During the fall semester, we were challenged by Bang, Bang, You're Dead, the true story of a teenage delinquent that shot his parents to death before shooting classmates at

school. This story brought to mind the incredible role parents play in a child's life, but more importantly, the issue of taking responsibility for our actions. We were also introduced to Scriptic, a new production by Alpha Beta Phi, the theater organization on campus. "Scriptic was a stage reading of plays written and performed by students," stated director Jim Tullett.

To start off the spring semester, students performed the play Bridge of Blood. This play looked into the lives and hearts of those who served to bring the Gospel to the Auca Indians of South America and correlated with the theme of the missions conference.

The spring semester also contained several senior theater productions. Aimee Auclair performed the first of these. $W ; t$ is the story of Dr. Vivian Bearing and her thoughts as she battles advanced metastatic ovarian cancer. Aimee said of the production, this play reminds us that "life is shorter than we realize; make the most of it." In Abbie's own words, this unsettling piece "exposes the truth behind the legend of Picasso from the perspective of the eight women (mistresses and wives) in Pablo's life."

Other senior performances included Jillian Anderson's For Such a Time as This, Josh Cobb and Michael Colletto's K2, Teah Thomsons and David Erlandson's The Lily of Persia, and Rebekah Nettekoven's The Bell Jar.

Through the differing performances we saw a variety of talents and look forward to the futures that these performers hold in each of their fields.

- Michael Colletto and Josh Cobb portray what it is like to survive a night on the side of a mountain at 27,000 feet while suffering from injuries, in their senior theater performance, $K 2$.

$\checkmark$ Samantha Stream plays the ghost of a student Josh shot in the play Bang, Bang You're Dead. She, along with the other four students Josh shot, haunt his prison cell.

1 In the initial scene from Bridge of Blood, the five families gather on the eve of the great expedition to the sandbar where they hope to meet the Auca Indians.

- Star of the show, Bang, Bang You're Dead, James Tullett explains this scene: "Josh's grandpa describes to him the details of hunting a buck." In the background, Michael Anfang, the ghost of a student Josh killed, watches intently.
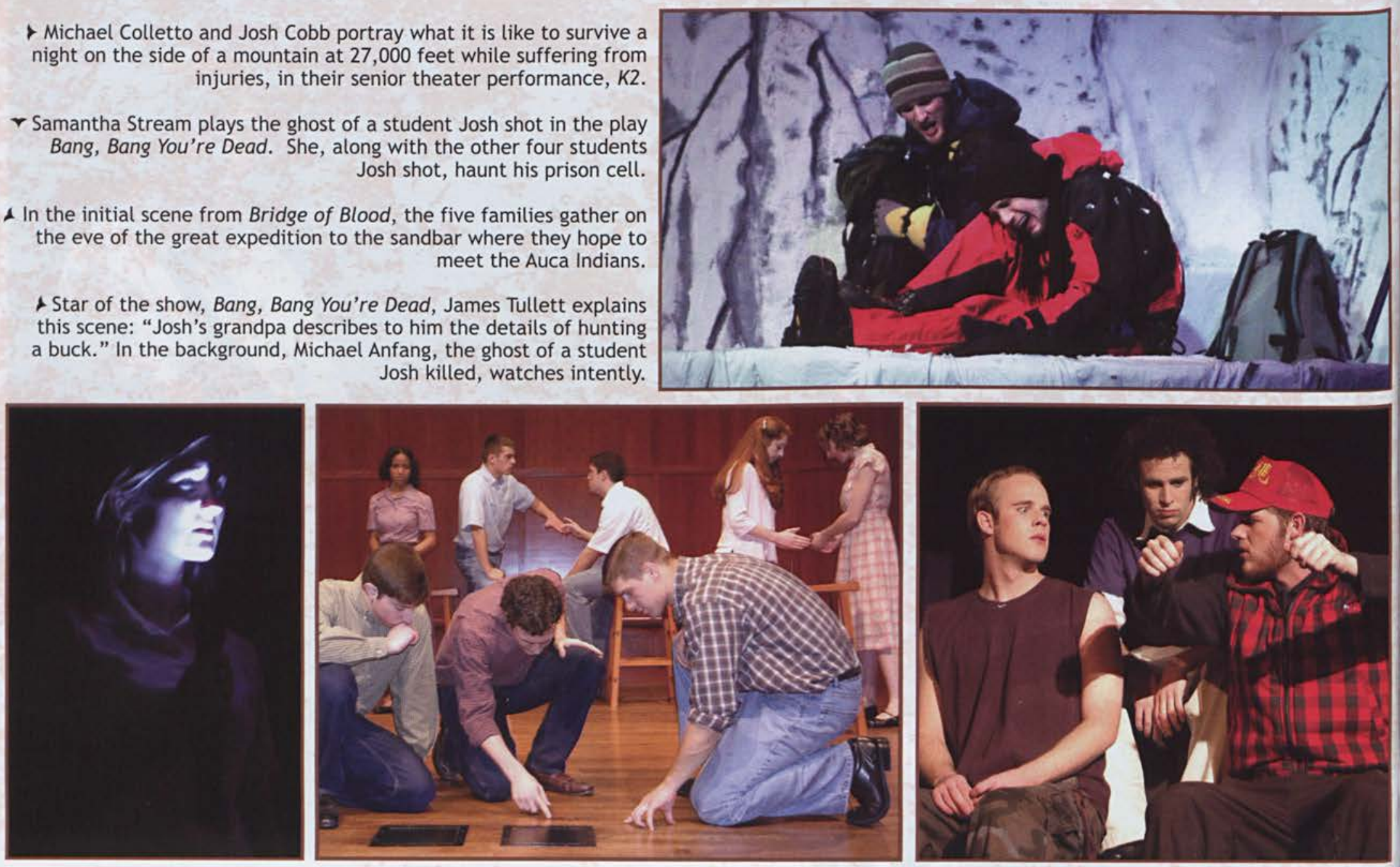


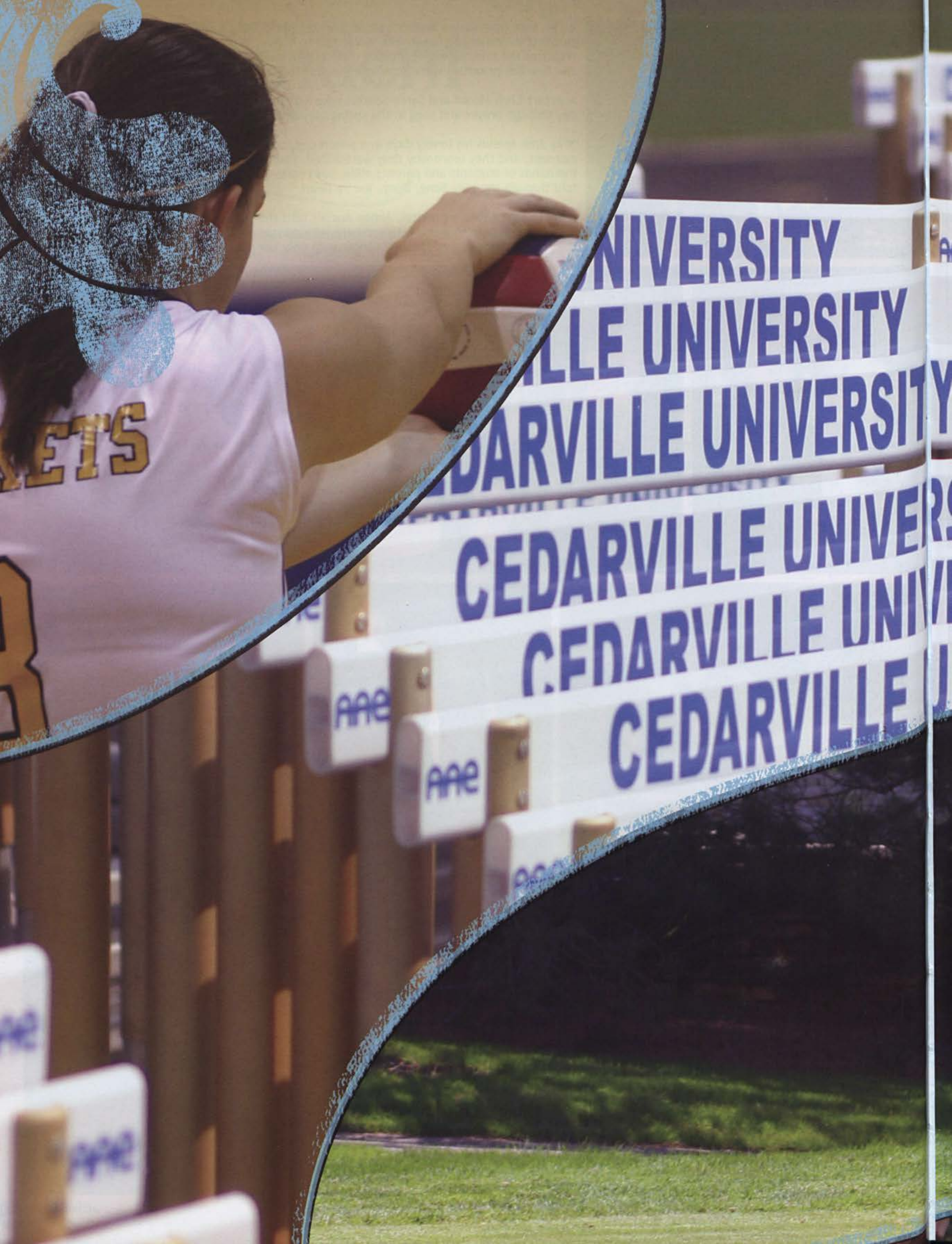




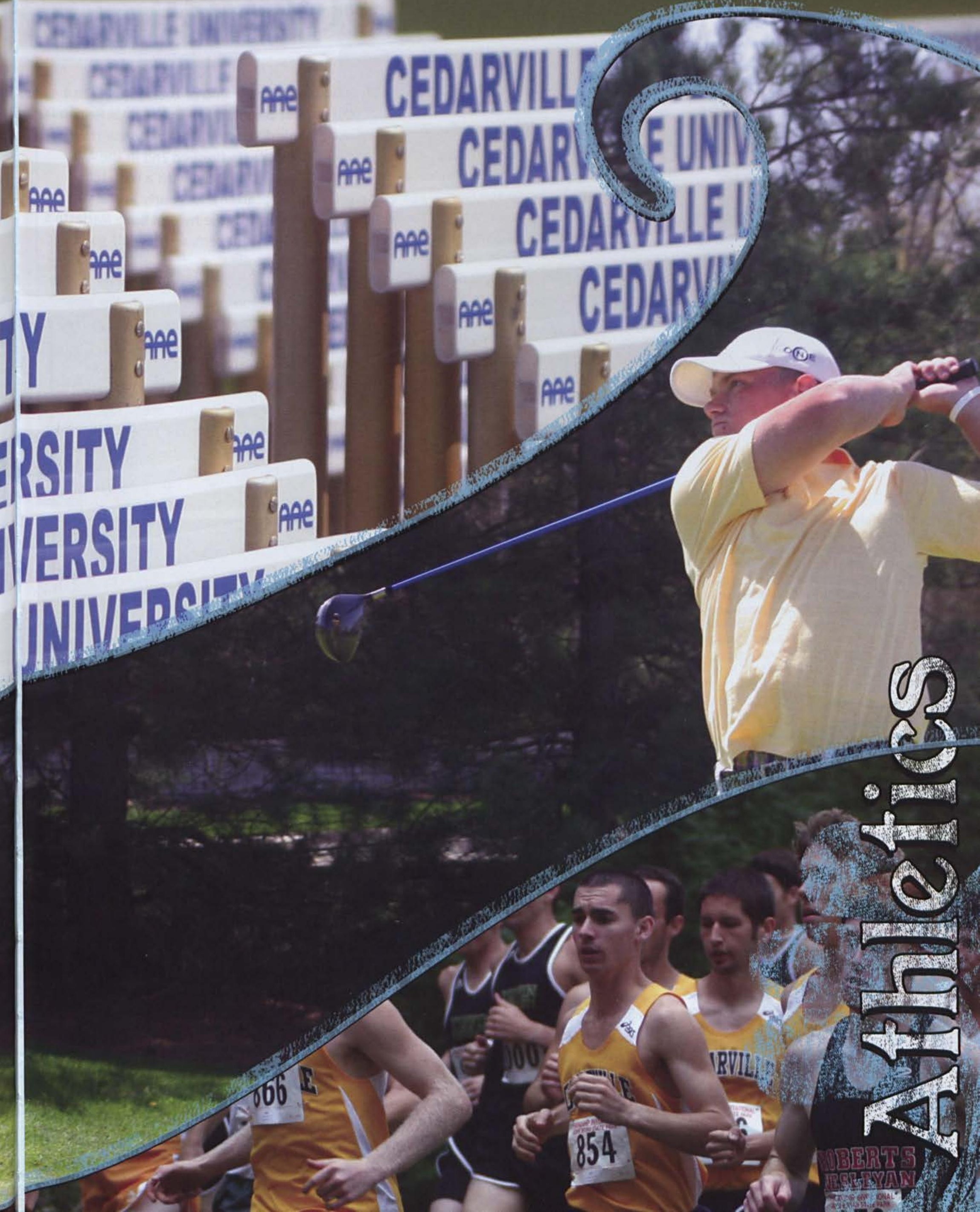




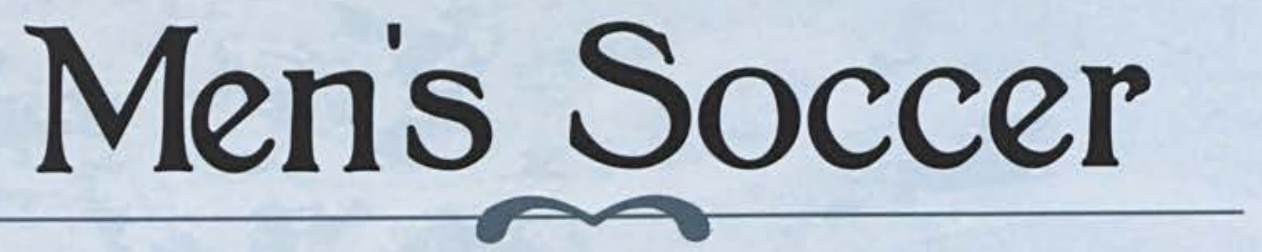

There is nothing I find more exciting

then a Cedarville University Men's

soccer game under the lights on

a chilly autumn night. Grabbing a

blanket and some spare change for hot chocolate, I head to the field.

This year's men's soccer season was quite exhilarating!

The team pulled away at the end of the season with a 15-4-1 overall record, placing them in the NAIA Region IX Final Four. After a hard loss against Rio Grande (ranked 2nd in the NAIA), the Jackets finished 2 nd in the AMC South. The season was highlighted with a rematch against Ohio Dominican; after a loss early in the year, the Jackets forced overtime with the help of Luke Marietta's penalty kick save and Phil Shimer's second half goal.

As the season rolled around, senior goalkeeper Luke Marietta, senior defender Phil Ellis, and senior forward Justin Benz were chosen to the All-AMC South Division First Team. Ellis, Marietta, and Benz were

also selected to the 14-player All-NAIA

Region IX Team. Junior midfielder Jesse Fox and sophomore forward Ken Davis were voted to the Second Team. Coach Belleman was also awarded with the AMC South Division Coach of the Year. As Junior Jon Taylor said, "We grew both as players and as a program. We represented Cedarville well through our wins and our losses." Sophomore Ken Davis netted the golden goal in overtime to put the Jackets in the NAIA Regional tournament. Davis said of the season, "I was both blessed and honored to be a part of such a successful and God-honoring team this year. We have had a level of faith and gratefulness for the opportunities we have to make an impact on others." Here is an event on campus that is well worth attending.

Front row left to right: Jordan Leach, Ryan Hyde, Jon Taylor, Jon Miley, Jesse Fox, Elliot Moore, Ken Davis.

Second row: Phil Shimer, Ryan Lustig, Steve Cobucci, lain Bryant, Josh Gelser, Grant Knight, Ryan Stutzman.

Third row: Assistant Coach Brent Davis, Associate Athletic Trainer Lisa Martin, Andrew Belleman, Scott Crawford, John

Norton, Jeremy Auyer, Luke Marietta, Andrew Elliott, Head Coach Ben Belleman.

Back row: Assistant Coach Kevin Roper, Scott DeLange, Tyler Schumacher, Marcus Mitchell, Justin Benz, Jason Cunningham, David Malpass, Phil Ellis, Assistant Coach Josh Radcliffe.
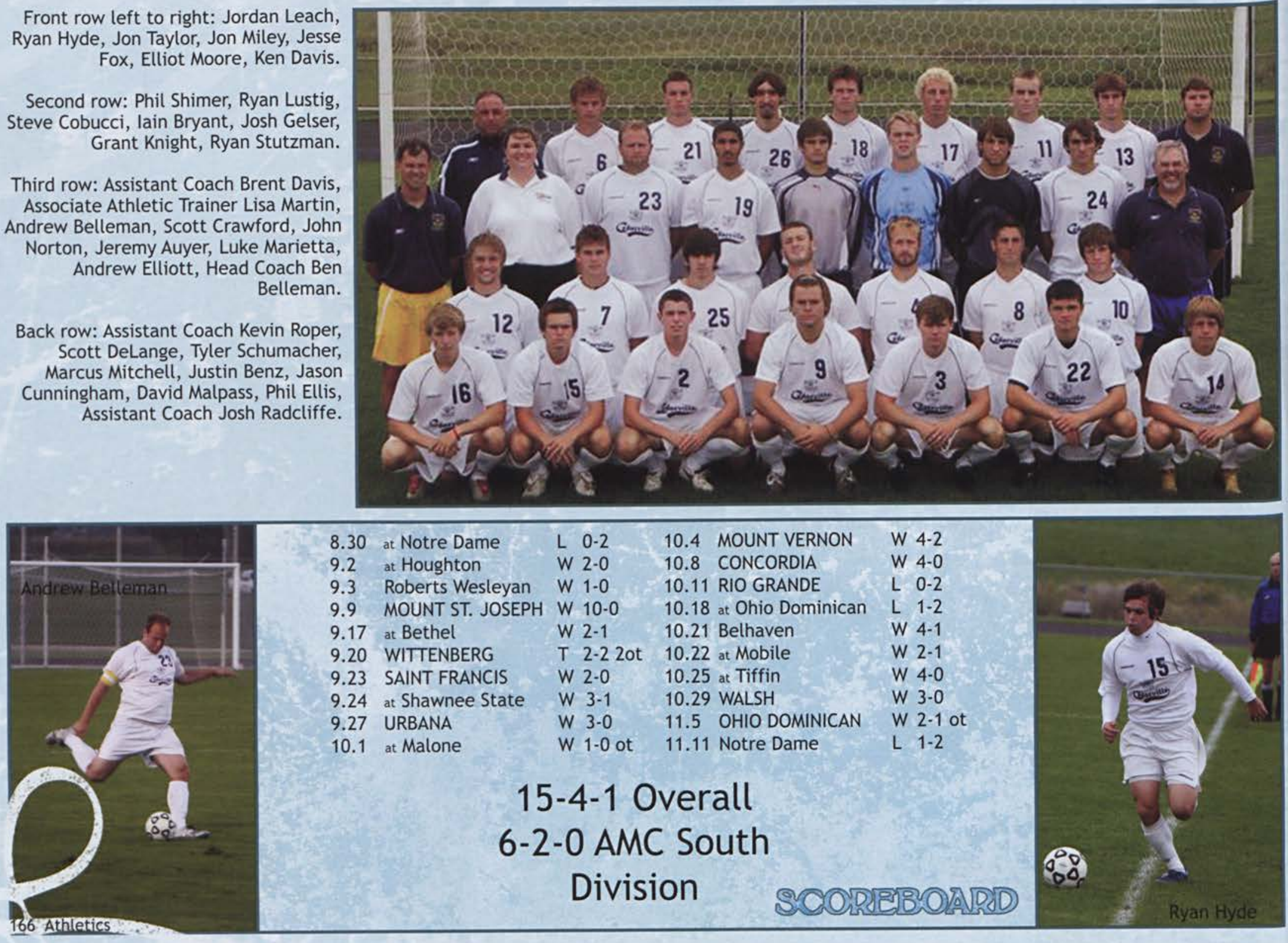

$\begin{array}{llllll}8.30 & \text { at Notre Dame } & \text { L } 0-2 & 10.4 & \text { MOUNT VERNON } & \text { W } 4-2 \\ 9.2 & \text { at Houghton } & \text { W } 2-0 & 10.8 \text { CONCORDIA } & \text { W } 4-0 \\ 9.3 & \text { Roberts Wesleyan } & \text { W } 1-0 & 10.11 \text { RIO GRANDE } & \text { L } 0-2 \\ 9.9 & \text { MOUNT ST. JOSEPH } & \text { W } 10-0 & 10.18 \text { at Ohio Dominican } & \text { L } 1-2 \\ 9.17 & \text { at Bethel } & \text { W } 2-1 & 10.21 \text { Belhaven } & \text { W } 4-1 \\ 9.20 & \text { WITTENBERG } & \text { T } 2-22 \text { ot } & 10.22 \text { at Mobile } & \text { W } 2-1 \\ 9.23 & \text { SAINT FRANCIS } & \text { W } 2-0 & 10.25 \text { at Tiffin } & \text { W } 4-0 \\ 9.24 \text { at Shawnee State } & \text { W } 3-1 & 10.29 \text { WALSH } & \text { W } 3-0 \\ 9.27 \text { URBANA } & \text { W } 3-0 & 11.5 \text { OHIO DOMINICAN } & \text { W } 2-1 \text { ot } \\ 10.1 & \text { at Malone } & \text { W } 1-0 \text { ot } & 11.11 \text { Notre Dame } & \text { L } 1-2\end{array}$

\section{5-4-1 Overall 6-2-0 AMC South} Division

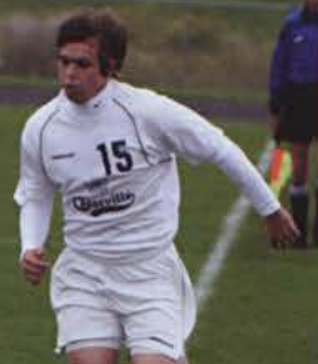


$\checkmark$ Junior Defender Elliot Moore goes up for a header. He later said of the team's goals, "Our team really improved this year on scoring and finishing which allowed us to reach one of our main goals -- to advance to the NAIA tournament."
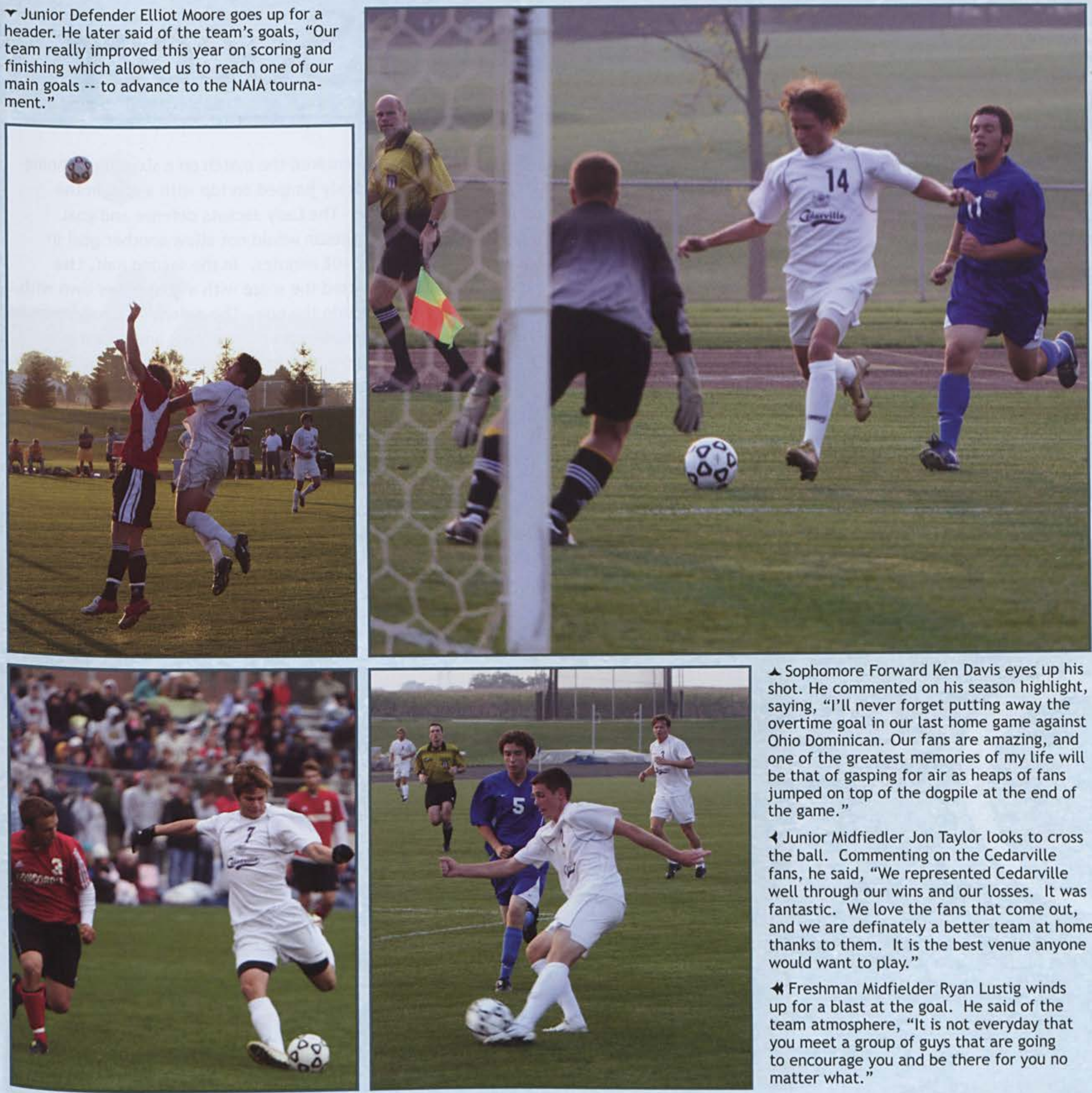

¿ Sophomore Forward Ken Davis eyes up his shot. He commented on his season highlight, saying, "I'll never forget putting away the overtime goal in our last home game against Ohio Dominican. Our fans are amazing, and one of the greatest memories of my life will be that of gasping for air as heaps of fans jumped on top of the dogpile at the end of the game."

$\checkmark$ Junior Midfiedler Jon Taylor looks to cross the ball. Commenting on the Cedarville fans, he said, "We represented Cedarville well through our wins and our losses. It was fantastic. We love the fans that come out, and we are definately a better team at home thanks to them. It is the best venue anyone would want to play."

* Freshman Midfielder Ryan Lustig winds up for a blast at the goal. He said of the team atmosphere, "It is not everyday that you meet a group of guys that are going to encourage you and be there for you no matter what."
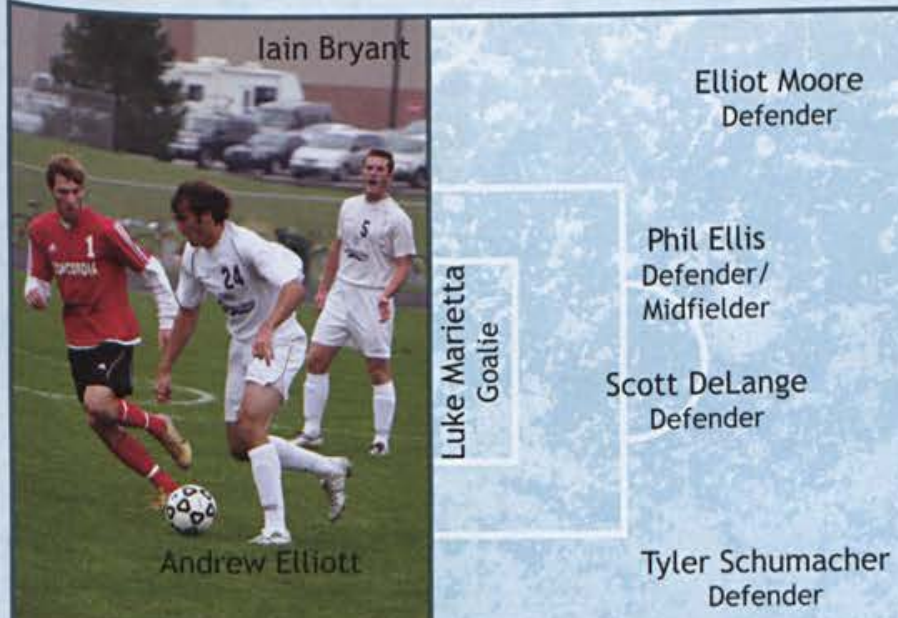

Jon Taylor Midfielder

Andrew Elliott

Midfielder

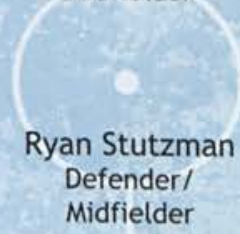

Defender/
Midfielder

Jesse Fox Midfielder

Justin Benz Forward

Defender

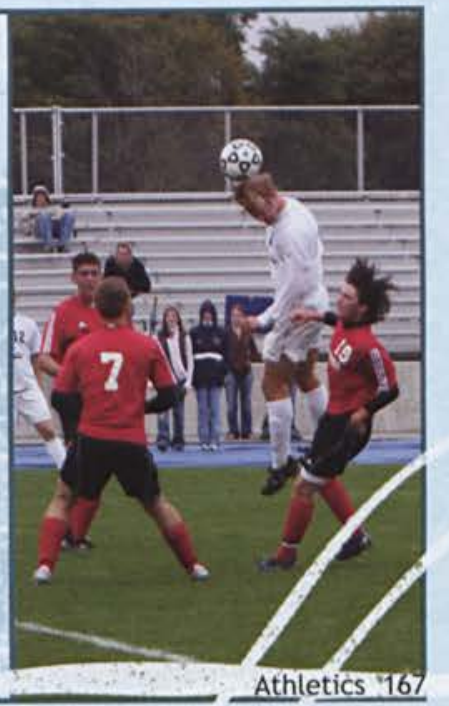




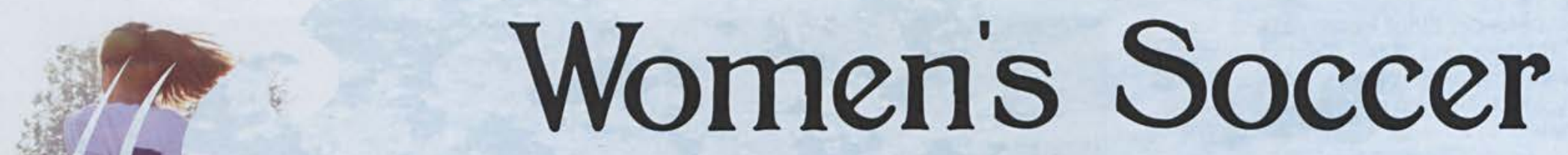

In late October, the Cedarville Mount Vernon entered the match on a six game winning University women's soccer team streak and quickly jumped on top with a goal in the wrapped up their season with an impressive $3-0$ victory over Shawnee State. Leading scorer eighth minute. The Lady Jackets defense and goal keeper Sarah Gibson would not allow another goal in the remaining 102 minutes. In the second half, Lisa

Lisa Blackburn's goal in the first half

proved to be the game winner. Later in the game, senior Nicole Blackburn knotted the score with a goal of her own with a blast from outside the box. The defensive struggle lasted through both sudden death overtimes and ended in a 1-1

James netted the last goal of her college career to finish off the scoring for the Lady Jackets 2005 season.

After the fifth game of the season, the Lady Jackets found themselves with a 4-1 record and riding a four game winning streak. Hopes were high following the previous season's NCCAA National Tournament appearance, but the team struggled through the back stretch of their season. However, the second half of the season was highlighted with a tie against No. 3 ranked (NAIA Region IX) Mount tie. Nicole James said of the match, "It was the best we played all season."

The Lady Jackets ended with a 5-10-1 record, 2-5-1 in the AMC South. After losing numerous players from last season's roster, unity became a key issue throughout the 2005 campaign. Junior captain Abby Price said, "I felt that the unity on the team this year was the best it has been in my three years with the program. I really felt this team did a great job working together, and I am so thankful for the unity and bond that we had in the Vernon Nazarene. players, as well as the coaching staff."

Front row left to right: Lisa Burgman, Katie Mariani, Lisa Blackburn, Jillian Losee, Colleen Derry, Jessica Schmidt, Amanda Cole, Katie Walter, Erin Baranski, Deb Sweede.

Middle row: Assistant Coach Dr. Kevin Roper, Assistant Coach Kelli Zlateff, Amber Lang, Abby Kirby, Becky Kirby, Kristen Malpass, Allison Heavner, Amanda Elliott, Abby Price, Assistant Coach George Weber.

Back row: Lisa Martin, Katie Koch, Kari Coffindaffer, Karen Ruhlman, Sarah Gibson, Ann Felix, Kelly Teague, Kristin Merkel, Nicole James, Jessica Thomas, Head Coach John McGillivray. Not pictured: Krista Watson
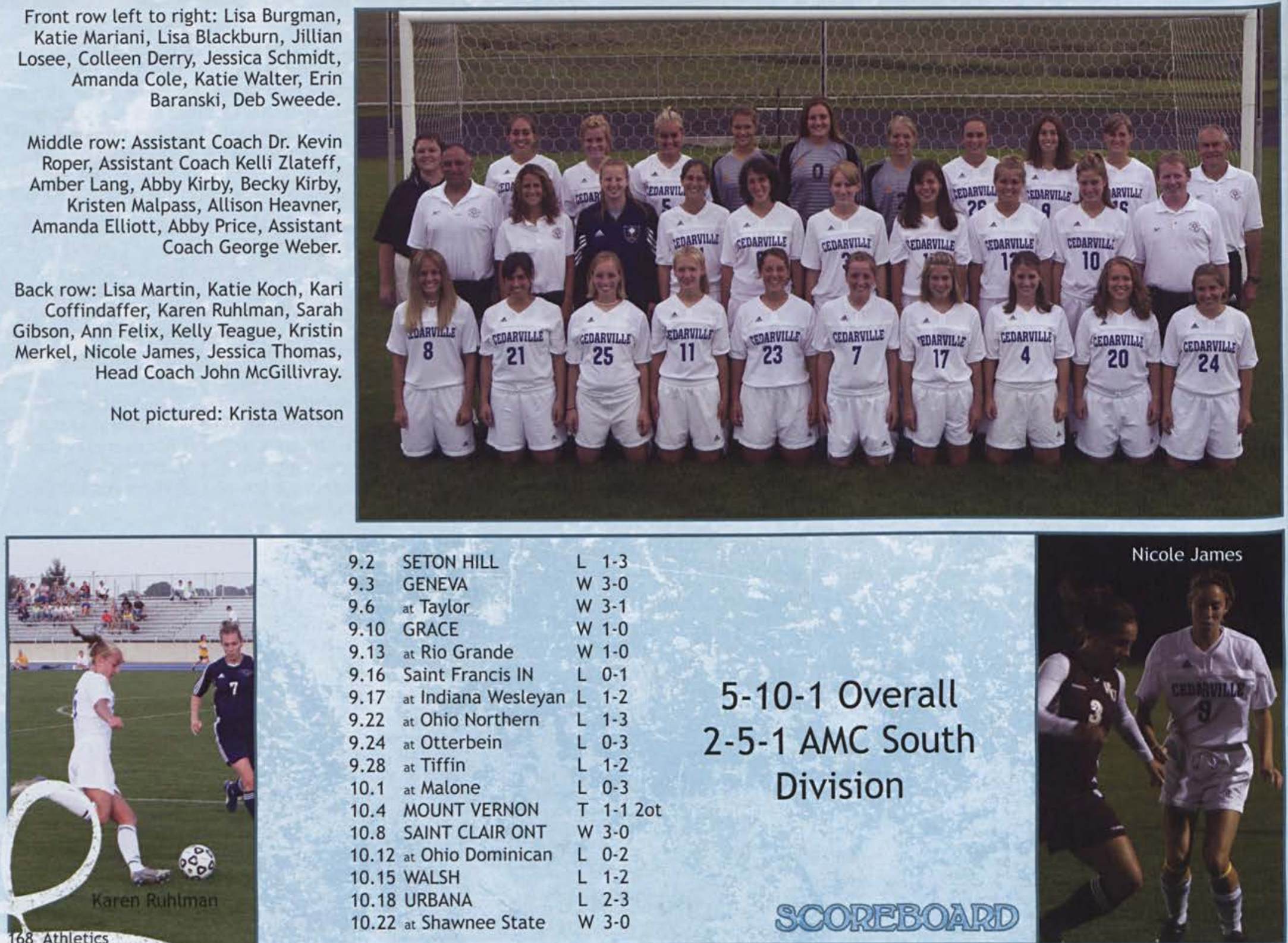

\begin{tabular}{|c|c|c|}
\hline 9.2 & SETON HILL & L 1.3 \\
\hline 9.3 & GENEVA & W 3-0 \\
\hline 9.6 & at Taylor & W $3-1$ \\
\hline 9.10 & GRACE & W 1.0 \\
\hline 9.13 & at Rio Grande & W $1-0$ \\
\hline .16 & Saint Francis IN & L 0.1 \\
\hline 17 & at Indiana Wesleyan & L $1-2$ \\
\hline .22 & at Ohio Northern & L $1-3$ \\
\hline .24 & at Otterbein & L 0.3 \\
\hline 9.28 & at Tiffin & L $1-2$ \\
\hline 10.1 & at Malone & L $0-3$ \\
\hline 10.4 & MOUNT VERNON & T $1-12$ \\
\hline 10.8 & SAINT CLAIR ONT & W $3-0$ \\
\hline 10.12 & at Ohio Dominican & L $\quad 0-2$ \\
\hline 10.15 & WALSH & L $1-2$ \\
\hline 10.18 & URBANA & L $2-3$ \\
\hline .22 & at Shawnee State & W $3-0$ \\
\hline
\end{tabular}

\section{5-10-1 Overall 2-5-1 AMC South Division}


$\checkmark$ Sophomore Jessica Schmidt throws the ball in to teammate Junior Katie Mariani. Senior Katie Walter described the team, saying, "We were unified. Although we struggled to have a winning season, I was so thankful for the unified and good spirit of the team."
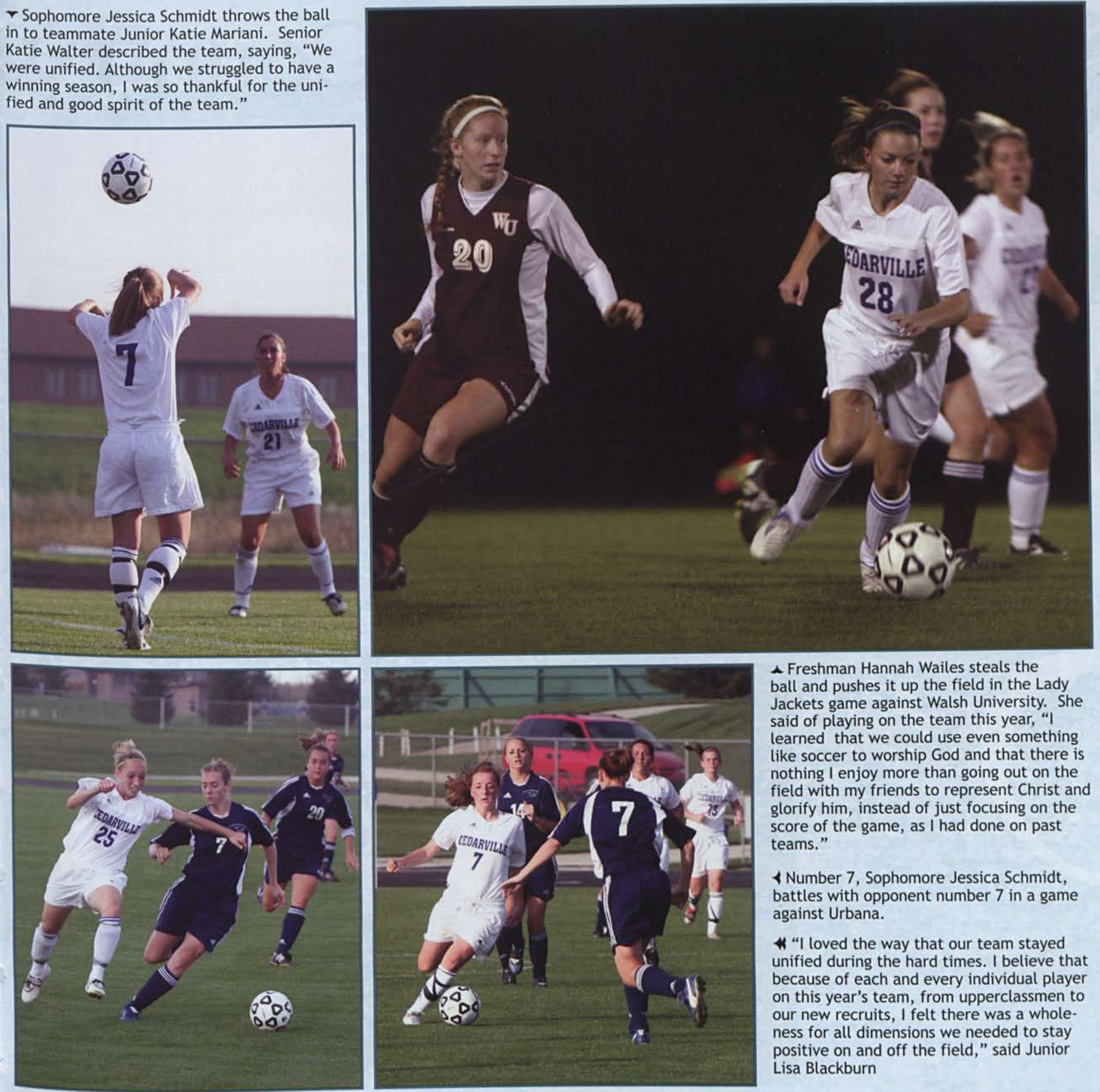

Freshman Hannah Wailes steals the ball and pushes it up the field in the Lady Jackets game against Walsh University. She said of playing on the team this year, "I learned that we could use even something like soccer to worship God and that there is nothing I enjoy more than going out on the field with my friends to represent Christ and glorify him, instead of just focusing on the score of the game, as I had done on past teams."

4 Number 7, Sophomore Jessica Schmidt, battles with opponent number 7 in a game against Urbana.

* "I loved the way that our team stayed unified during the hard times. I believe that because of each and every individual player on this year's team, from upperclassmen to our new recruits, I felt there was a wholeness for all dimensions we needed to stay positive on and off the field," said Junior Lisa Blackburn
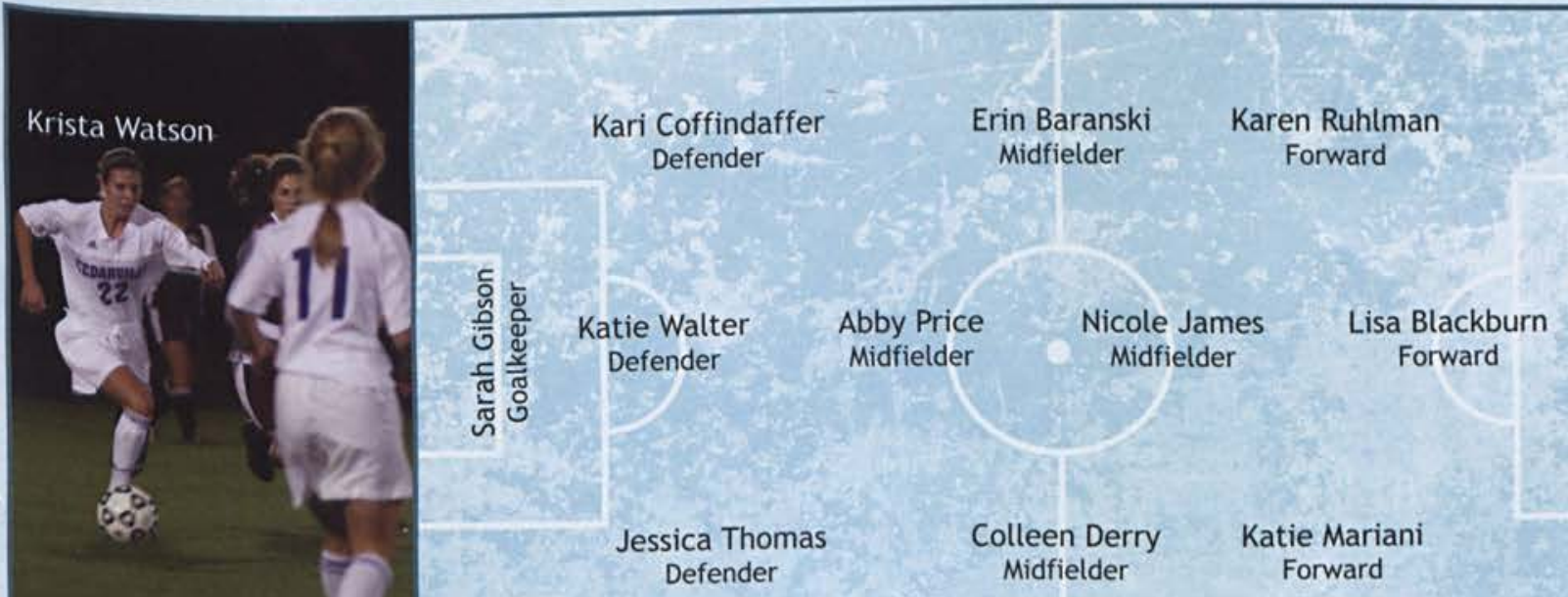

Jillian Losee $\frac{}{5}$

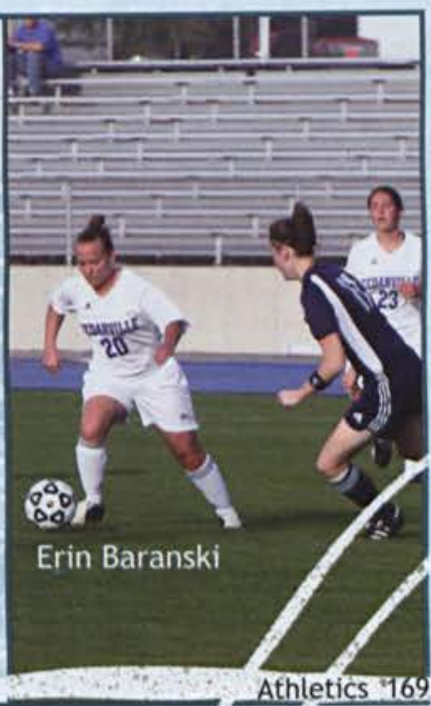


$\checkmark$ Freshmen, Rob Trennepohl and Seth Campbell, compete at John Bryan State Park. Seth, speaking about his footwear, commented, "Our team shoes are pretty sweet."
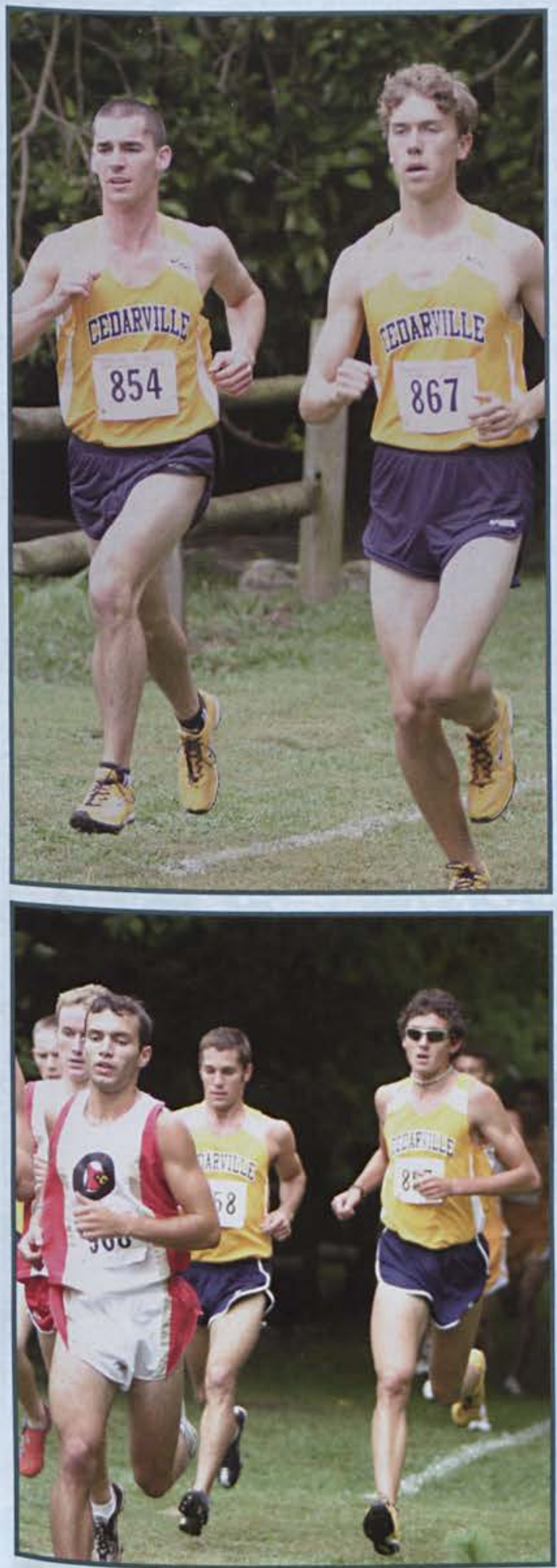
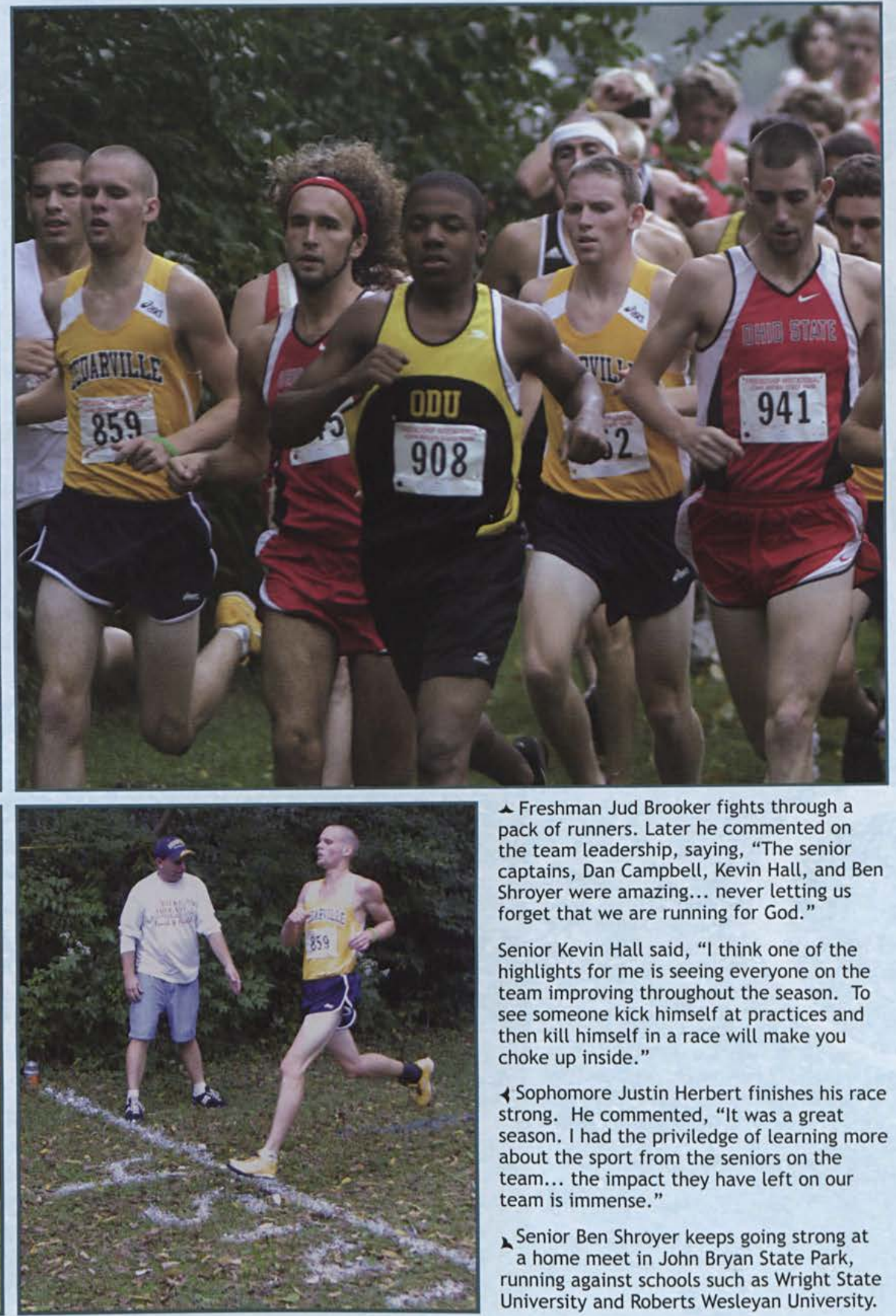

^ Freshman Jud Brooker fights through a pack of runners. Later he commented on the team leadership, saying, "The senior captains, Dan Campbell, Kevin Hall, and Ben Shroyer were amazing... never letting us forget that we are running for God."

Senior Kevin Hall said, "I think one of the highlights for me is seeing everyone on the team improving throughout the season. To see someone kick himself at practices and then kill himself in a race will make you choke up inside."

$\checkmark$ Sophomore Justin Herbert finishes his race strong. He commented, "It was a great season. I had the priviledge of learning more about the sport from the seniors on the team... the impact they have left on our team is immense."

\Senior Ben Shroyer keeps going strong at a home meet in John Bryan State Park, running against schools such as Wright State University and Roberts Wesleyan University.

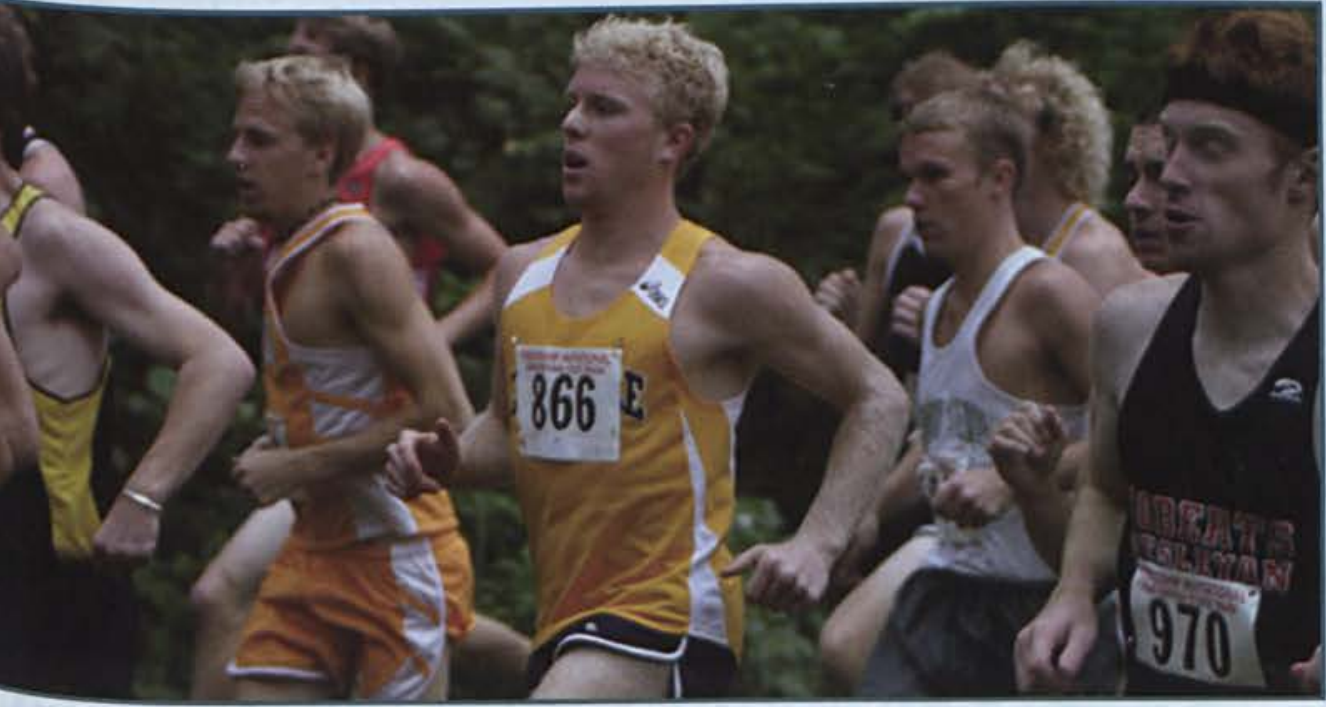

G Gilways love coming into Xc camp a week early before school starts and all the fun activities we do that first week together, besides the intense training.. The infamous "captains" night is always a blast as well; no freshman will forget it... 


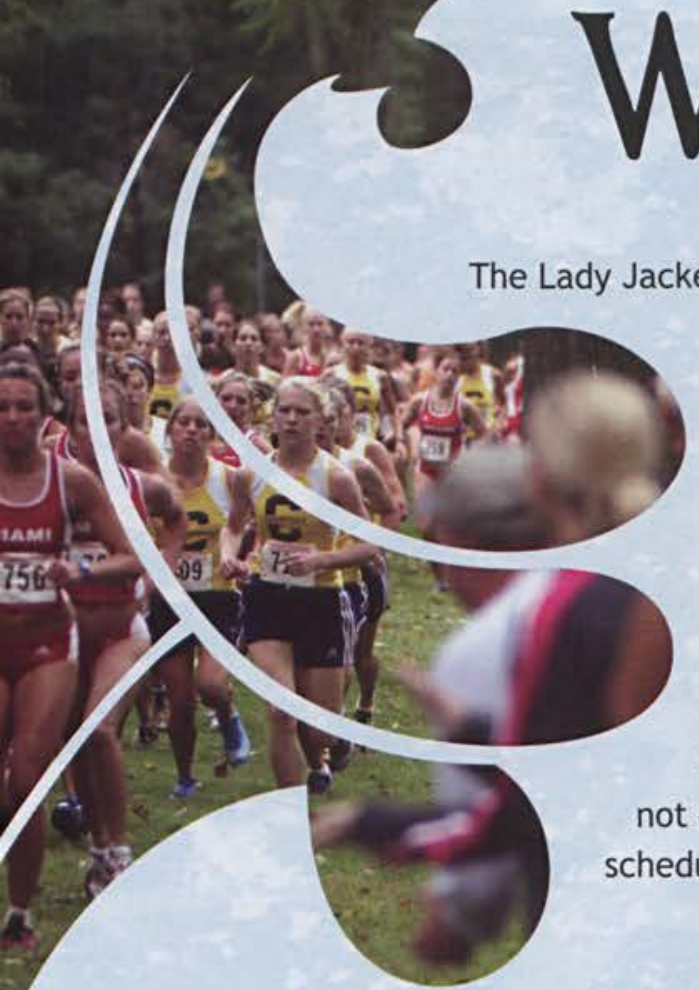

The Lady Jackets both began and finished the season strong. The team took first place in their season opener at the Shawnee State Invitational. The team scored five of the best six times of the meet. They also took back-to-back first place finishes at the Southeast Classic and the AMC Championship. At the Southeast Classic, Samantha Maat finished 3rd overall,

season with a strong group of runners that were willing to push each other to improve. Elisabeth Pyles said of the team, "We not

only had a blast together, but we grew together and challenged

each other." The preseason NAIA rankings listed the team as number 9 in the country, and the Lady Jacket Cross Country team did not disappoint. They tore through their awards as they went.

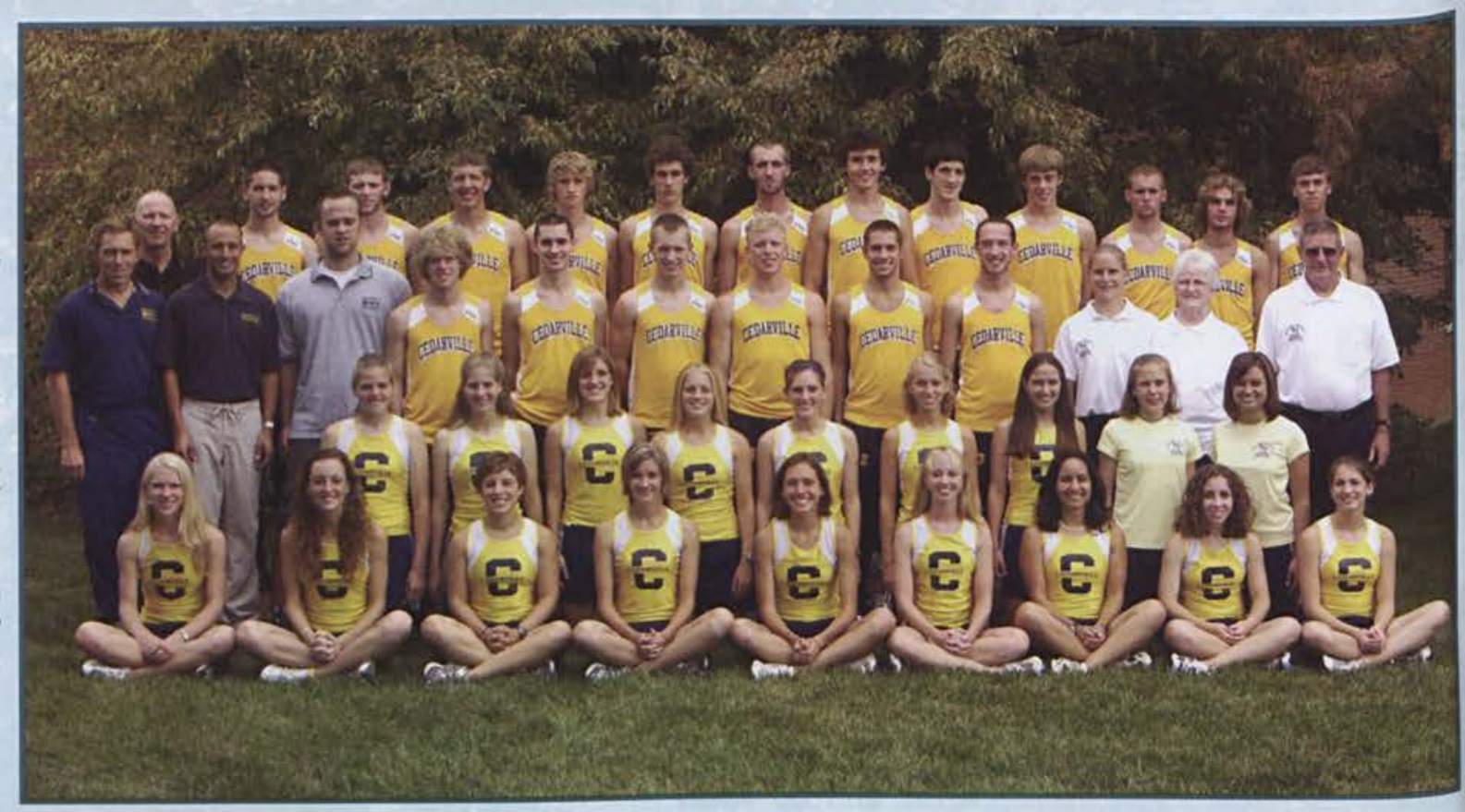

followed closely by Christina Reyes, Elisabeth Pyles, Brittany Simpson, and Audree Goodew. In the AMC championship, the team won first place for the first time in 3 years. Samantha Maat finished with her career best time and said afterward that her favorite moment from the season was, "Winning conference....not just the victory, but seeing how God truly united our team that day by His Spirit."

At the NCCAA Nationals, the Lady Jackets finished second with Elisabeth Pyles earning All-American honors, and Samantha Maat was named All-American as well as receiving the Bullock Award. The NAIA Nationals soon followed with the team ranking 4 th in the nation. True to form, the Lady Jackets took 4th place with Christina Reyes receiving All-American Honors. In addition, Samantha Maat and Sarah Bailey received NAIA AllAmerica Scholar-Athlete Awards. Coach Elvin King was also named to the NAIA Hall of Fame for his work since starting the Cedarville Women's Cross Country team in 1980.
Women's Front row left to right: Elisabeth Pyles, Maria Harley, Sarah Bailey, Keri Hilty, Samantha Maat, Melissa Wysong, Christina Reyes, Lucy Olney, Stacey Keller

Women's Middle row: Brittany Simpson, Jessica Squier, Audree Goodew, Julie Martz, Sarah Sweigard, Brittany Herbst, Leanne Crunelle, Student Assistant Jennifer McKellar, Student Manager Caroline Landenberger.

Women's Back Row: Assistant Coach Kelly Tuinstra, Assistant Coach Joyce King, Head Coach Elvin King.

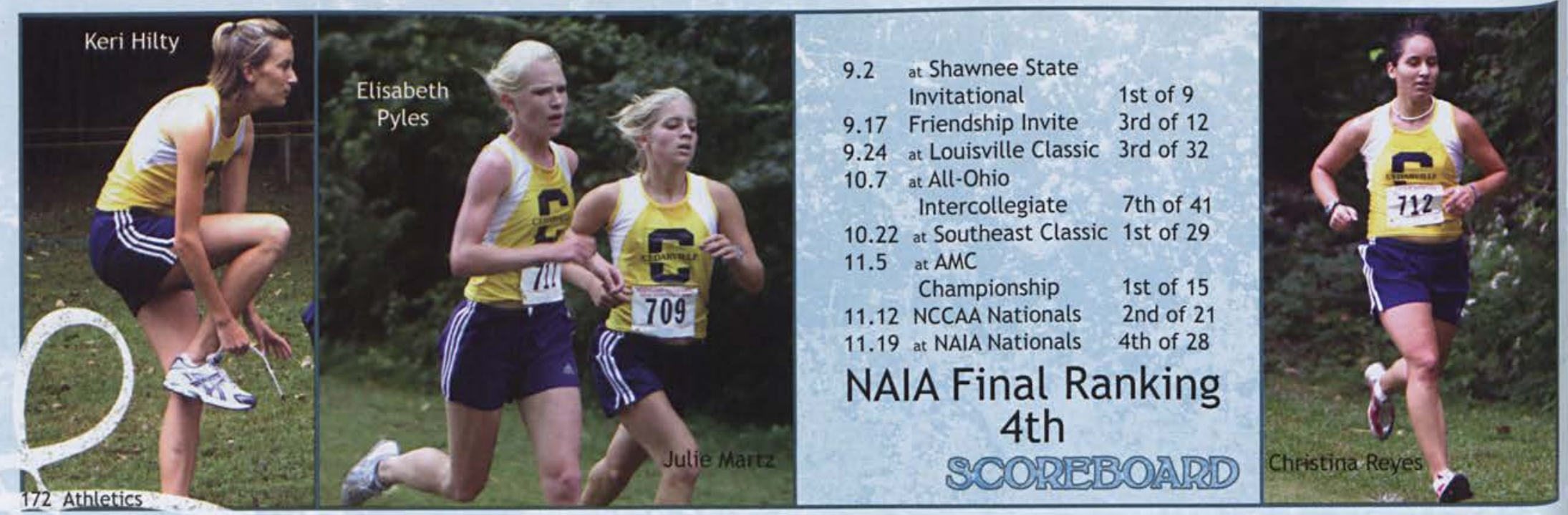


$\checkmark$ Sophomore Maria Harley commented on team goals, saying, "We have a common goal in directing our passion for running toward our greater passion for God, and with how close we became as a team this year, l'd have to say there's no other group of girls that l'd rather run with!"

* Sophomore Brittany Herbst said of the team, "They did a great job of making everyone feel as if they belonged and were neededno matter how long you had been there, no matter how fast you ran."
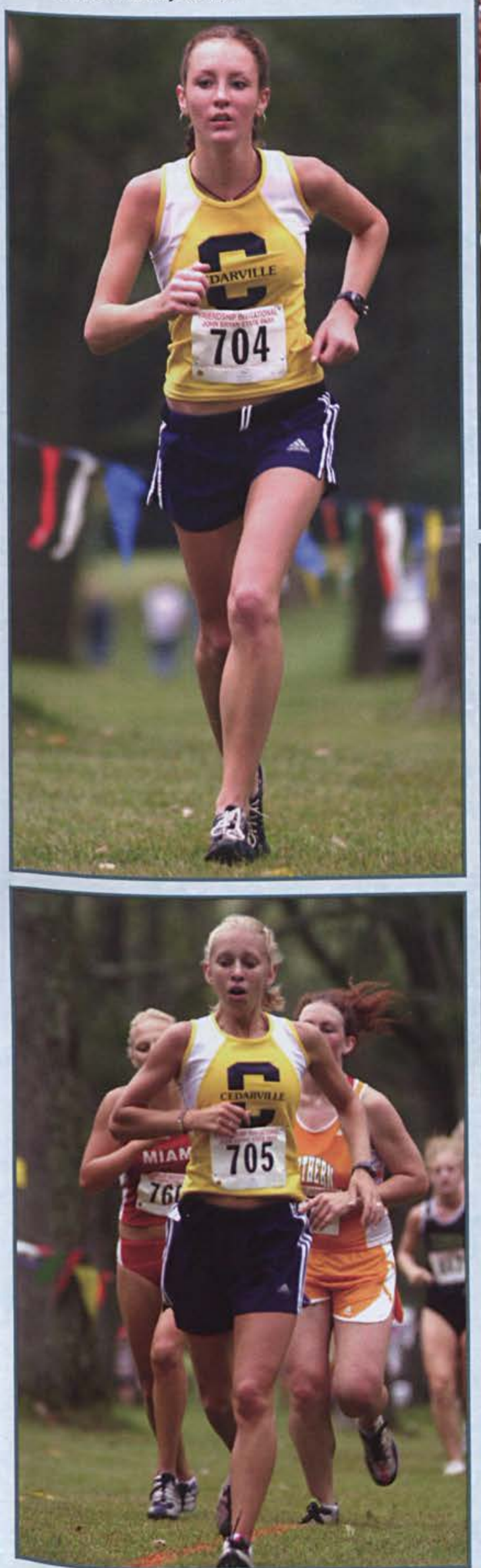
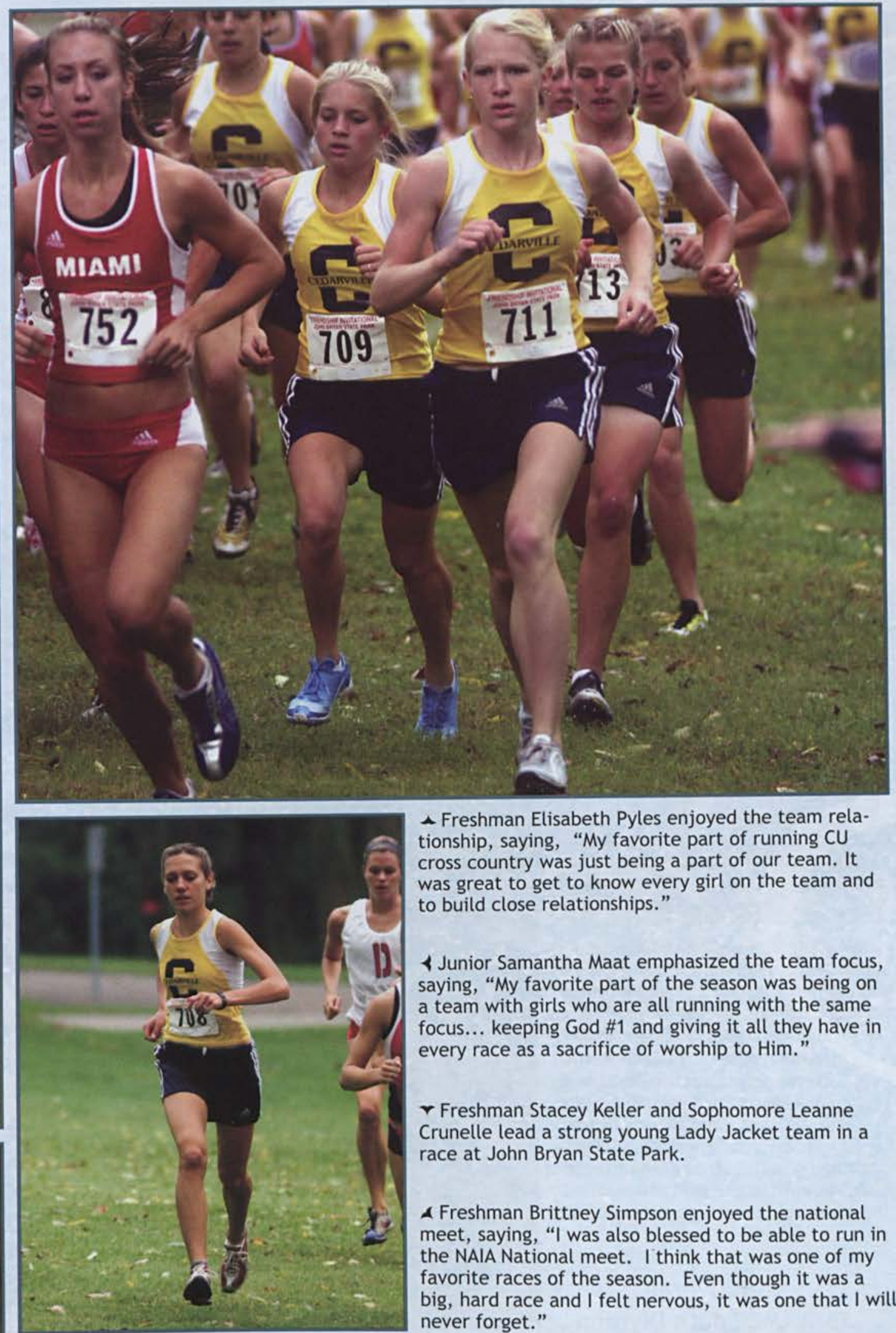

A Freshman Elisabeth Pyles enjoyed the team relationship, saying, "My favorite part of running CU cross country was just being a part of our team. It was great to get to know every girl on the team and to build close relationships."

$\checkmark$ Junior Samantha Maat emphasized the team focus, saying, "My favorite part of the season was being on a team with girls who are all running with the same focus... keeping God \#1 and giving it all they have in every race as a sacrifice of worship to Him."

$\checkmark$ Freshman Stacey Keller and Sophomore Leanne Crunelle lead a strong young Lady Jacket team in a race at John Bryan State Park.

A Freshman Brittney Simpson enjoyed the national meet, saying, "I was also blessed to be able to run in the NAIA National meet. I think that was one of my favorite races of the season. Even though it was a big, hard race and I felt nervous, it was one that I will never forget."
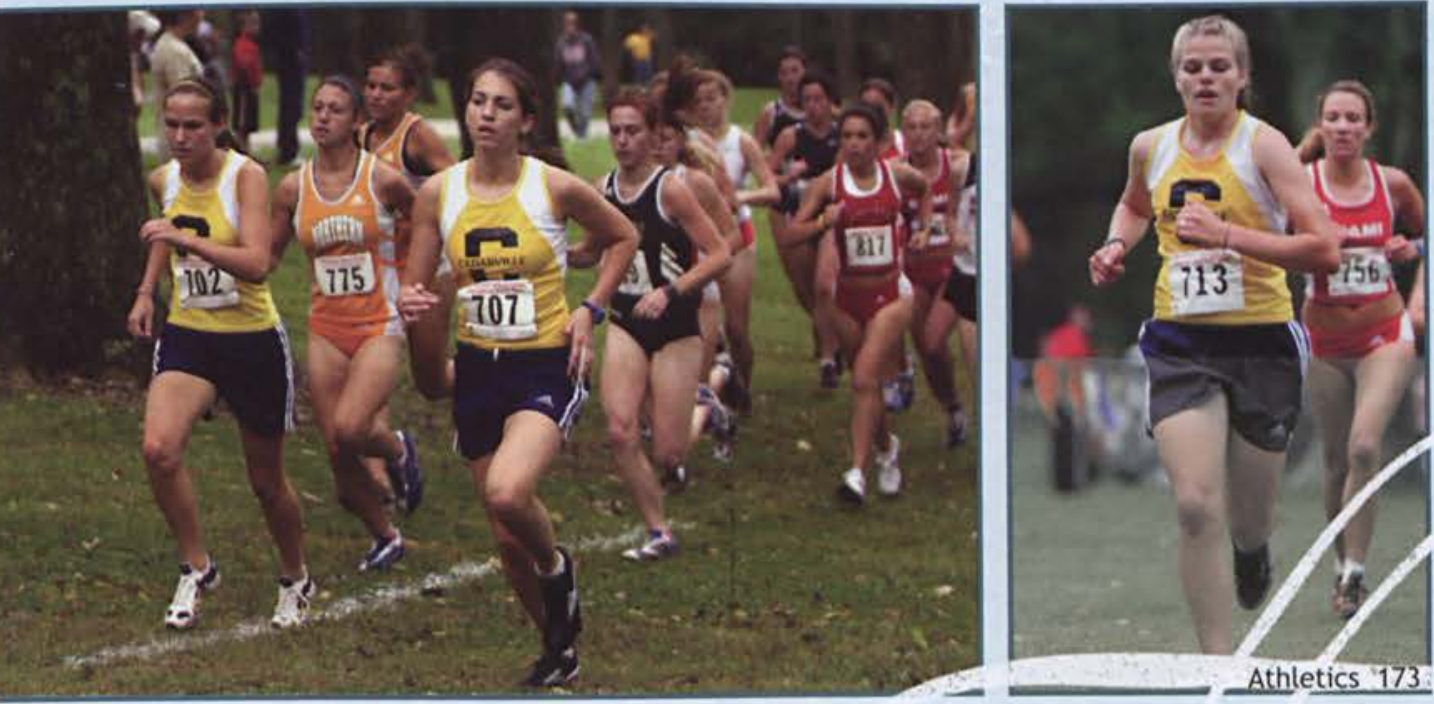


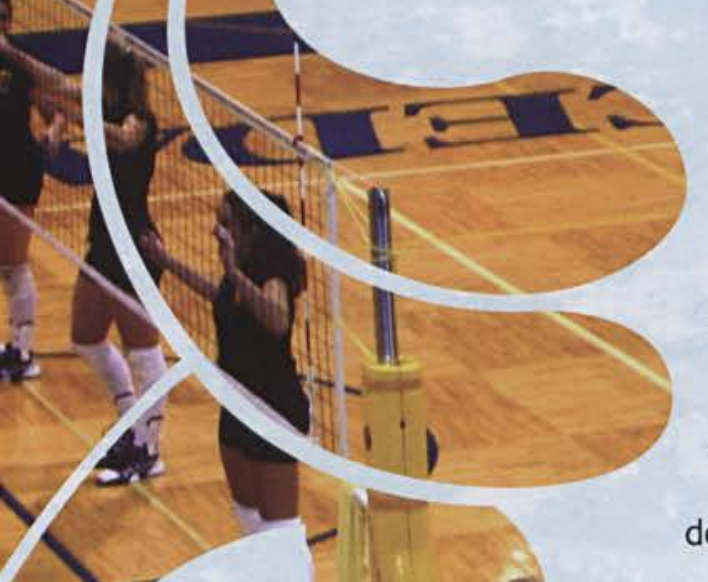

monstrated character both on and off the court.

Wins at the Grace Invitational, Cedarville Invitational, and The Cumberland Classic, as well as regular season matches, placed the Jackets in position for the NCCAA Championship, the AMC Championship, and the NAIA region IX tournament. At the NCCAA tournament, the best Christian colleges and universities from across the country competed in regional and the national tournaments. The Lady Jackets took first place overall, sweeping Bethel in three games led by tournament MVP, Kelsey Jones. In the AMC championship, the Lady Jackets

Dominican Republic and Costa

Rica to winning the first annual Buffalo Funds Five Star Award, made Coach Clark's tenth and final year as head coach a remarkable one. This year's team established unprecedented success throughout the year but, more importantly, finished a second straight season, going undefeated at home and beating Saint Vincent in three games. In the NAIA rankings, the Jackets finished 17 th but found themselves losing to Mount Vernon Nazarene, down two games to none in the Region IX championship match. Led by Julia Bradley, Lauren Mable, and the rest of the Jacket lineup, the team rallied back to take their second straight Region IX championship. This win qualified them for the Division I NAIA National Championship in San Diego, where they competed against some of the best schools in the nation.

At the close of the season, the Lady Jackets had managed two separate 18 match winning streaks and finished with a undefeated $16-0$ conference record, $45-6$ overall. In addition to this performance, the team was awarded the NAIA Buffalo Funds Five star award, given to one NAIA team that demonstrated exceptional character throughout the season. Julia Bradley wrapped up the season, saying, "This year was amazing both physically and spiritually. We played strong until the end, and this team was truly blessed."

Front row left to right: Cari Greetham, Rachel Thompson, Kari Flunker, Lauren Mable, Kelsey Jones, Melissa Parmerlee, Maija Hampton.

Middle row: Student Athletic Trainer Adam Ploeg, Associate Athletic Trainer Bob Duchardt, Student Manager Brittny Scott, Student Athletic Trainer Sarah Johnson, Assistant Coach Melissa Hartman, Assistant Coach Merilee Shank, Head Coach Teresa Clark, Assistant Coach Jim Clark.

Back row: Erica Paugh, Kelly Theiss, Anne Lohrenz, Sarah Zeltman, Julia Bradley, Tiffany Wyant, Libby Short.

\section{5-6 Overall}

16-0 AMC South Division

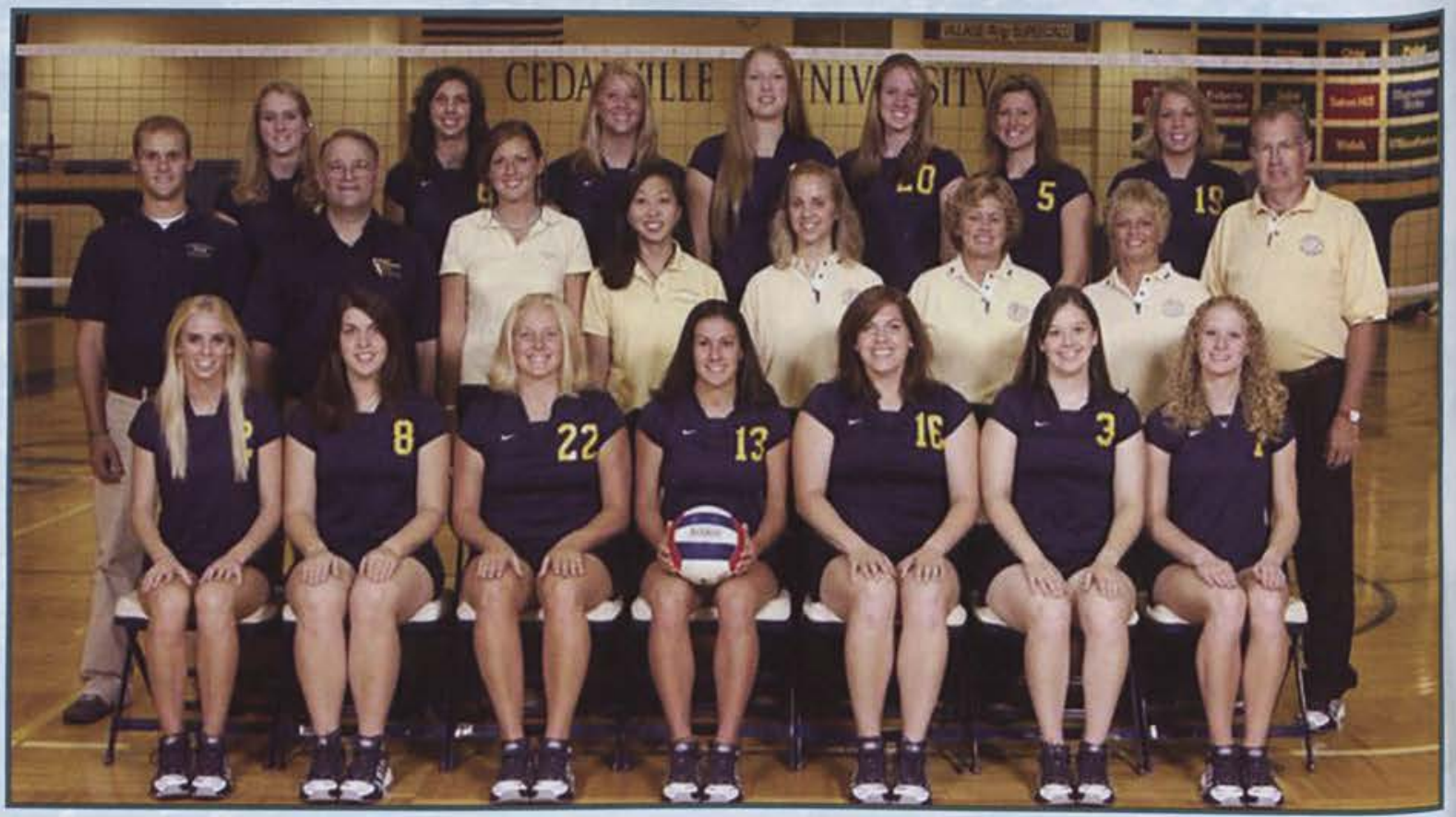

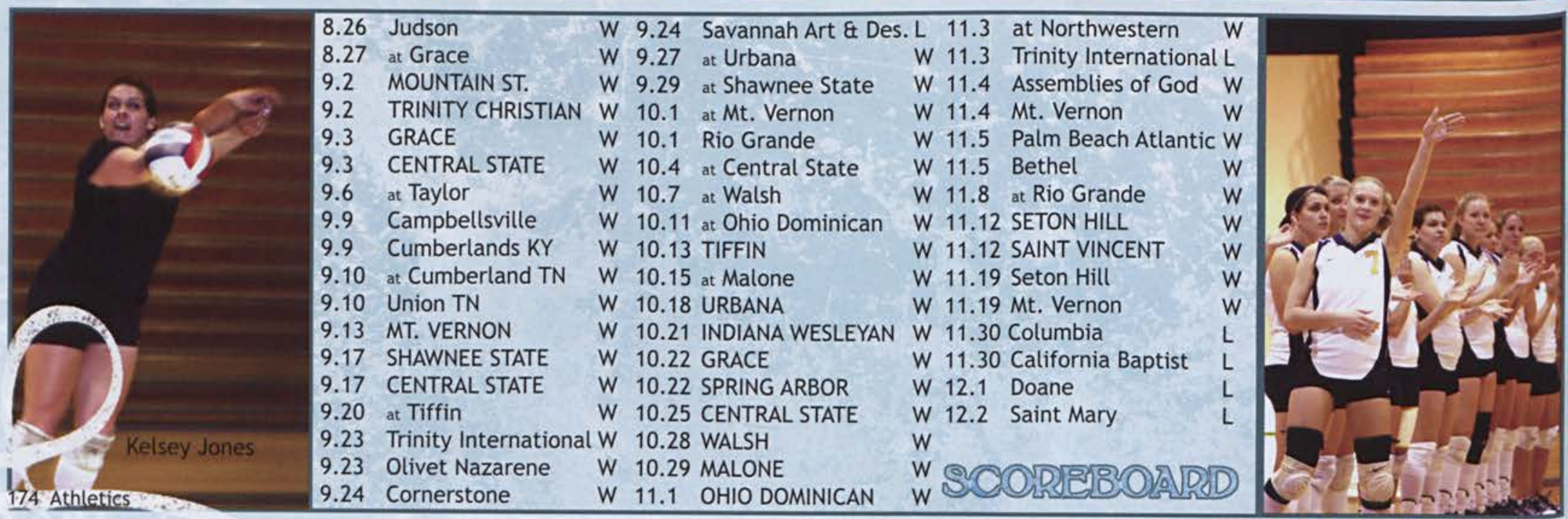


- Junior Middle Hitter Julia Bradley

celebrates with teammates after a point. She commented on the team's focus, saying, "This

is our act of worship and our mission field, and have loved being a part of this team."

- Sophomore Middle Hitter Sarah Zeltman serves up another victory. She emphasized the blessings of this season, saying, "God did so much more than bless us with and incredible season and a National Championship. We got to be used by Him for His glory, and this season was a time for God to show us how awesome He is."

$\checkmark$ Freshman Outside Hitter Maija Hampton sets up her teammate for a spike. She expressed he love for the game, saying, "I would say that this season was the most exciting I have ever experienced. Coach Clark has done a wonder with this program. She will be greatly missed by all of the players."

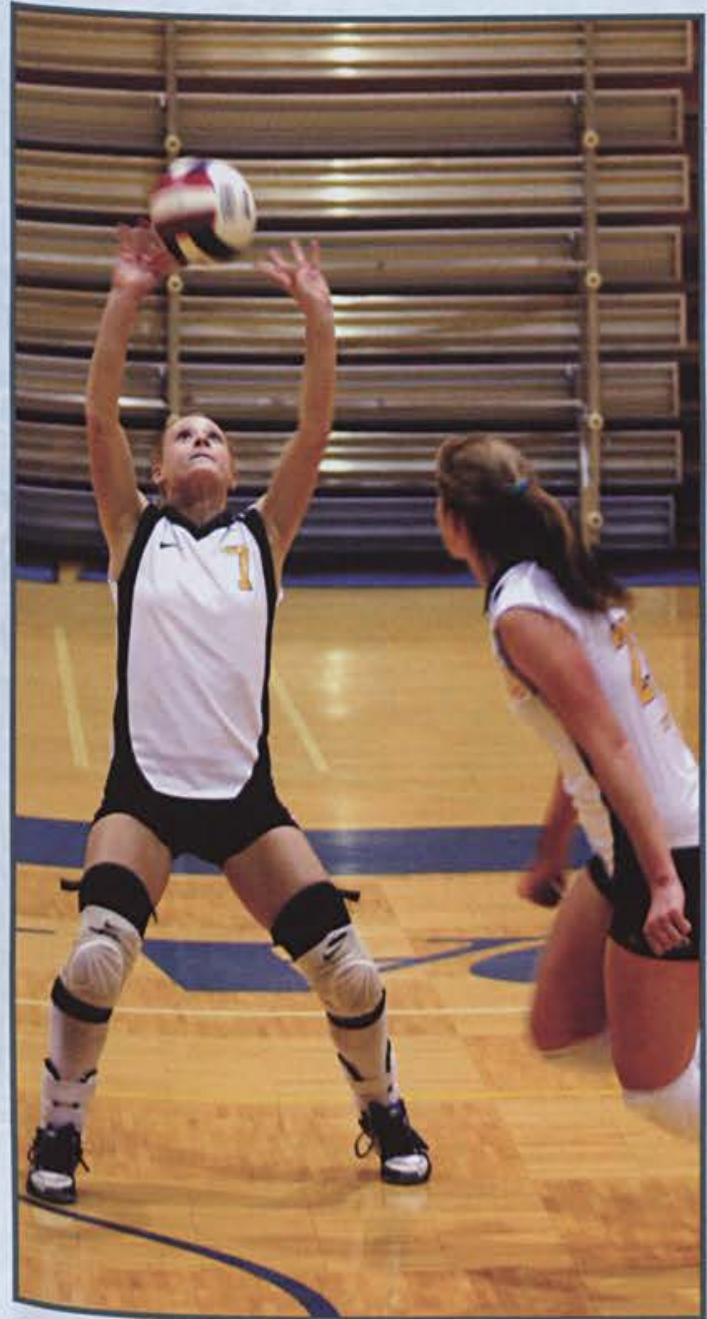

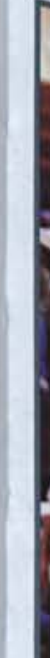
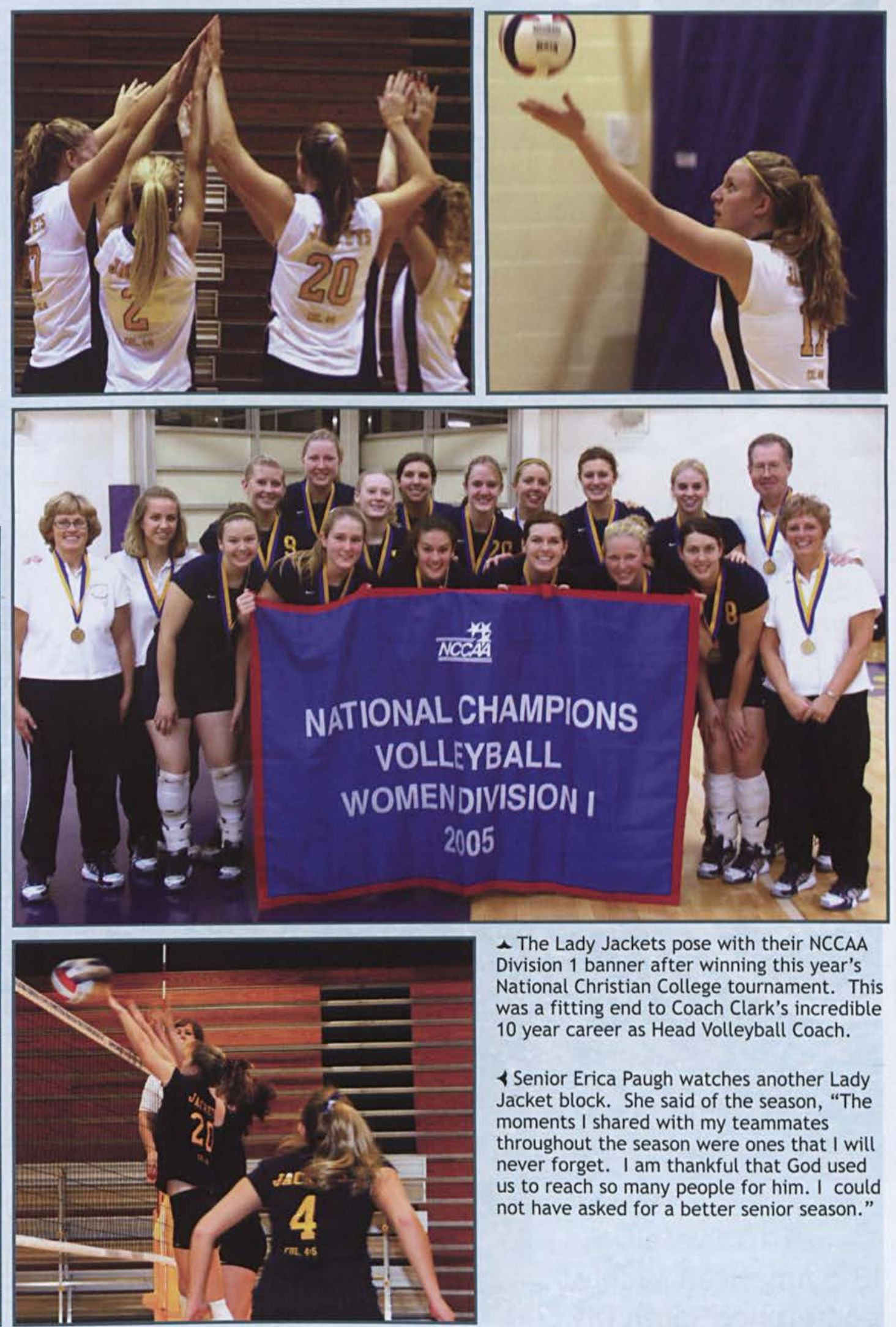

- The Lady Jackets pose with their NCCAA Division 1 banner after winning this year's National Christian College tournament. This was a fitting end to Coach Clark's incredible 10 year career as Head Volleyball Coach.

$\checkmark$ Senior Erica Paugh watches another Lady Jacket block. She said of the season, "The moments I shared with my teammates throughout the season were ones that I will never forget. I am thankful that God used us to reach so many people for him. I could not have asked for a better senior season."

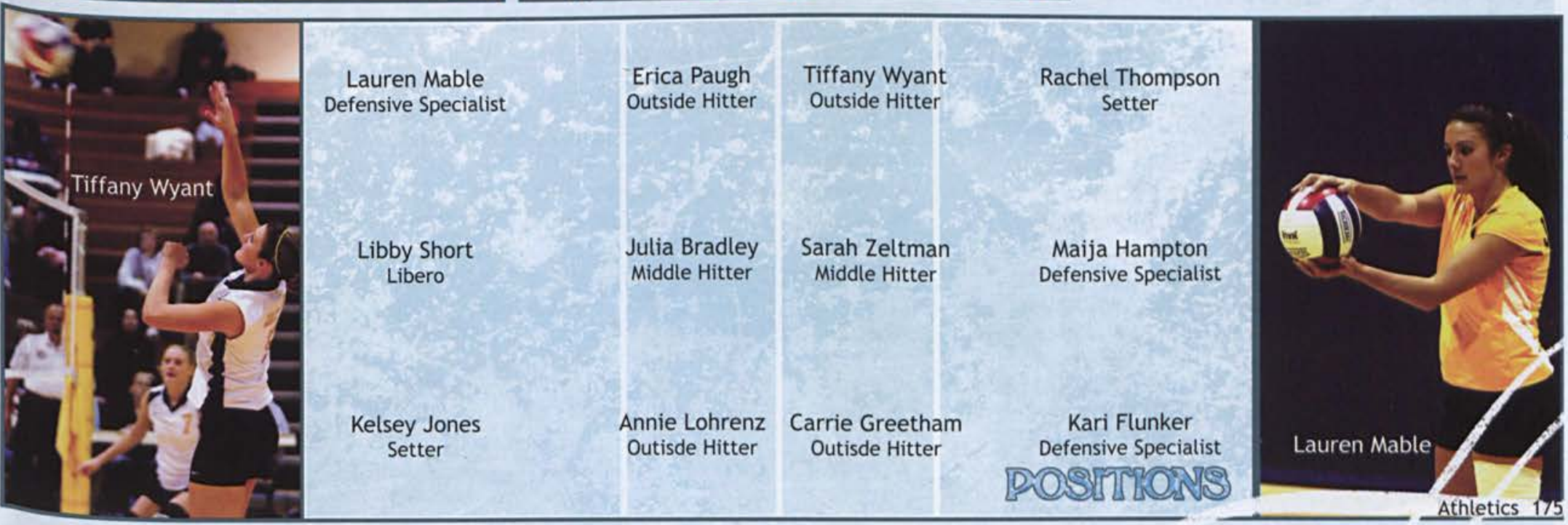




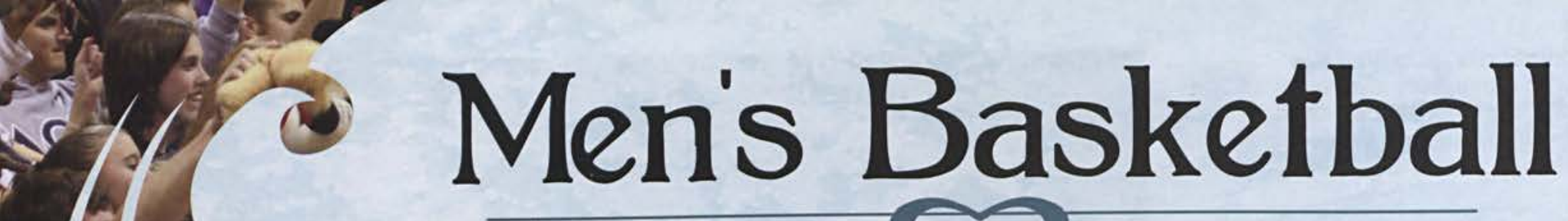

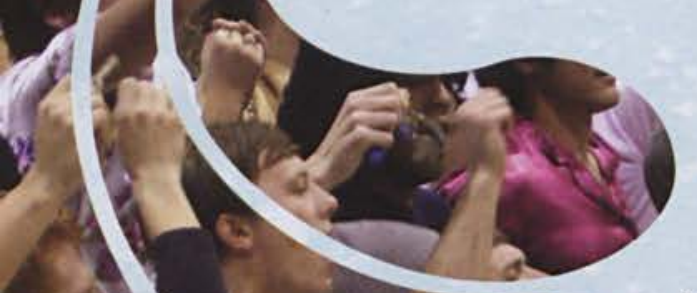

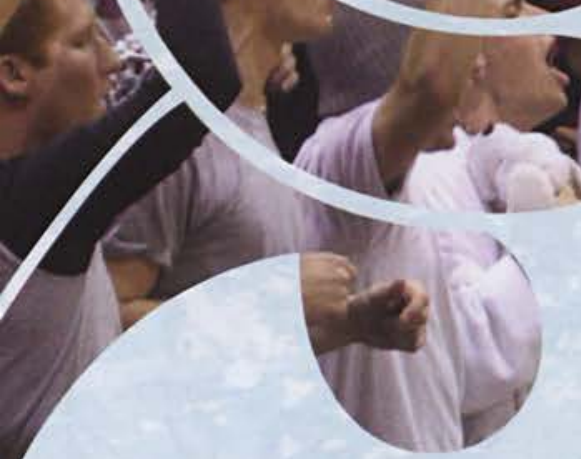

and Walsh is only leading by 3 . Then when we thought all was lost, Ryan Short shot a seemingly impossible three point shot. He made it! The crowd jumped to their feet screaming! The Jackets had managed to tie up a grueling game with only 18 seconds left to go! What a shot, what a shot! History was made here tonight.

Although we might have lost that game, we fought our hearts out. This year's basketball season was definitely rough. But with newcomers like Daniel Rose and Grant Walker, the team was able to post a 20-11 overall season and a 13-5 American Mideast Conference. Senior Mugabe Thomas finished his Cedarville career with 2,043 points and 1,177 rebounds .- only the second player in Cedarville history to have over 2,000 points and 1,000 rebounds. We also said goodbye to team captain Eric Huffer and center Drew Borton, who holds 1,027 career points.

The Jackets made it to the NAIA qualifying tournament but lost to Mount Vernon 56-61. They finished their season at the NCCAA Midwest regional title game, losing $98-92$ to Spring Arbor.

Cedarville basketball games are standing traditions that bring out the true colors of our students. Some go for the sport, some for the social aspect, and most just for a free night of fun and excitement. It is amazing to see how the school comes together to support our players. One night was most memorable when a player from the opposing team was injured, and the Jacket boys gathered on the court and prayed for him in the middle of the game. There was no better way to show the true spirit of $\mathrm{CU}$.

Front row left-to-right: Ray James, Josh Greve, Ryan Short, Eric Leininger, Eric Huffer.

Middle row: Student Assistant Ryan Mosher, Student Assistant Loren Kuhn, Assistant Coach Andy Allgrim, Head Coach Ray Slagle, Assistant Coach Pat Estepp, Student Athletic Trainer Pat Clinton.

Back row: Student Assistant J.J. Krumlauf, Guy Rathmell, Drew Borton, Daniel Rose, Maicol Venter, Mugabe Thomas, Head Athletic Trainer Chris Cross.

\section{0-10 Overall}

\section{3-5 American Mideast Conference South Div.}
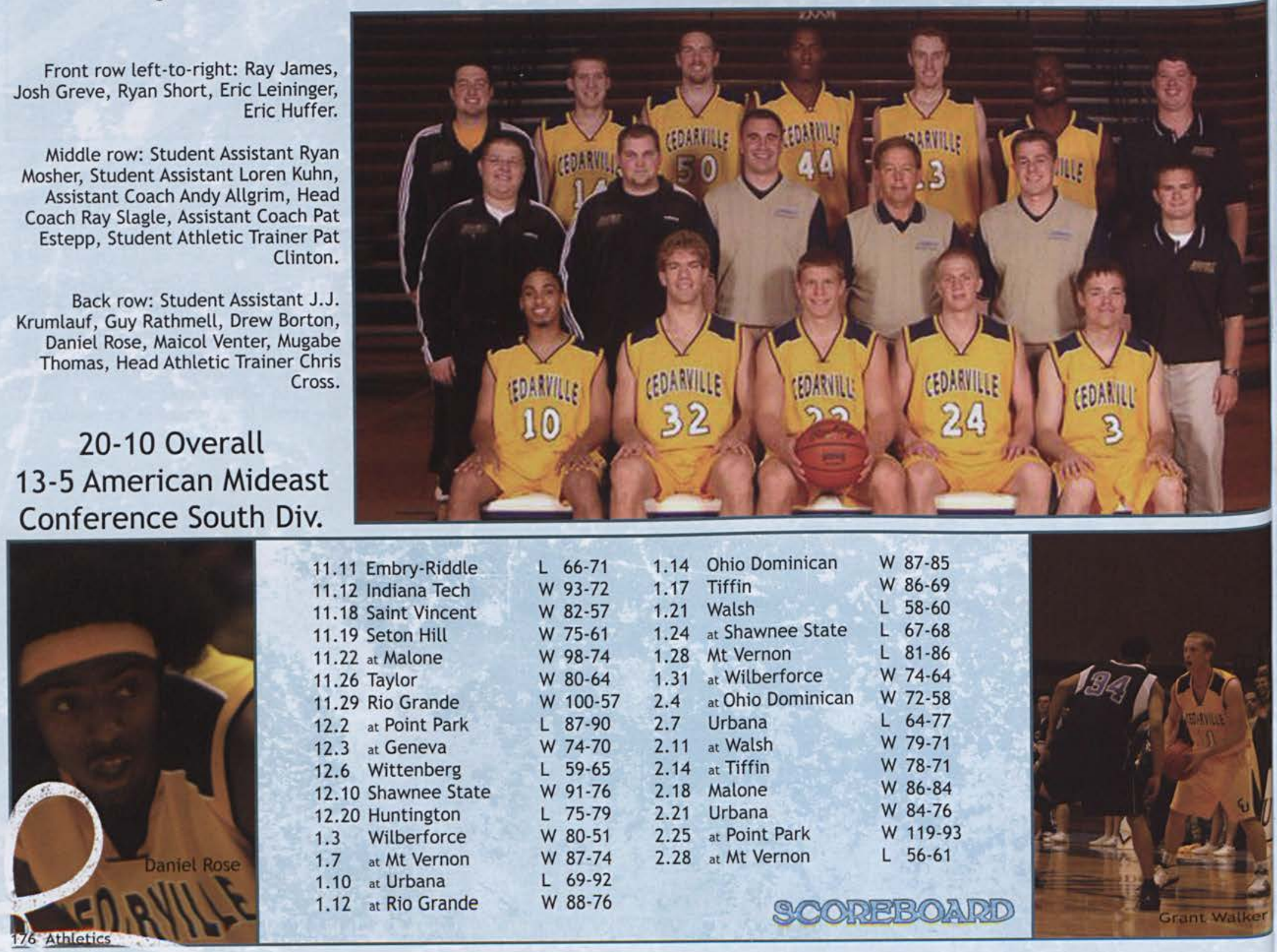

11.11 Embry-Riddle 11.12 Indiana Tech 11.18 Saint Vincent 11.19 Seton Hill 11.22 at Malone 11.26 Taylor 11.29 Rio Grande

12.2 at Point Park

12.3 at Geneva

12.6 Wittenberg

12.10 Shawnee State

12.20 Huntington

1.3 Wilberforce

1.7 at Mt Vernon

1.10 at Urbana

1.12 at Rio Grande
L 66-71

W $93-72$

W 82.57

W 75-61

W $98-74$

W $80-64$

W $100-57$

L $87-90$

W 74-70

L $59-65$

W $91-76$

L $75-79$

W $80-51$

W $87-74$

L $69-92$

W $88-76$
1.14 Ohio Dominican

1.17 Tiffin

1.21 Walsh

1.24 at Shawnee State

1.28 Mt Vernon

1.31 at Wilberforce

2.4 at Ohio Dominican

2.7 Urbana

2.11 at Walsh

2.14 at Tiffin

2.18 Malone

2.21 Urbana

2.25 at Point Park

2.28 at Mt Vernon
W $87-85$

W $86-69$

L $58-60$

L $\quad 67-68$

L 81.86

W 74-64

W $72-58$

L $64-77$

W 79-71

W 78-71

W $86-84$

W 84-76

W $119-93$

L 56-61 in de

\section{the}

*

\section{for}

\section{lor}

$$
\begin{aligned}
& \text { on } \\
& \text { the } \\
& \text { sti }
\end{aligned}
$$$$
\begin{aligned}
& \text { Sh } \\
& \text { of } \\
& \text { do } \\
& \text { ou } \\
& \text { me }
\end{aligned}
$$ 
$>$ Guard Eric Leininger moves to make a pass in the tournament game against EmbryRiddle. Even though the game ended in defeat, Cedarville put up a hard battle that showed the commited nature of the team that carried through the whole season.

- Drew Borton posts up against the center low. Center Borton says of the season, "I was probably the closest I have been to the guys on the team in my five years here. Even though we didn't make it to Branson, it was still a memorable year."

Forward Guy Rathmell and guard Ryan Short drive the ball to the basket. Ryan said of the season, "[It] was filled with ups and downs, but through it all, we maintained our testimony, made some unforgettable memories,e court." for Embry-Riddle, Looking for a pass down
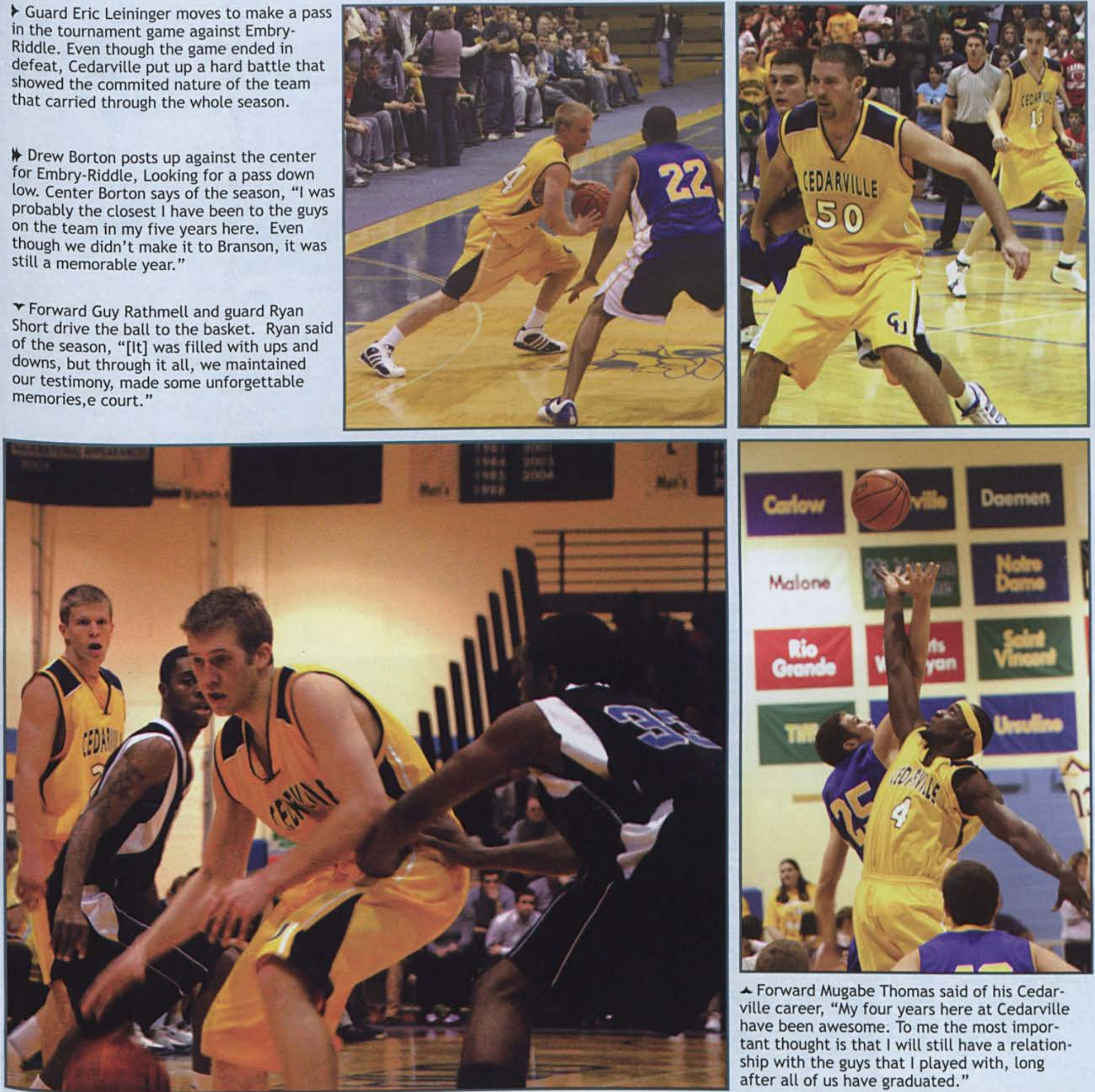

A Forward Mugabe Thomas said of his Cedarville career, "My four years here at Cedarville have been awesome. To me the most important thought is that I will still have a relationship with the guys that I played with, long after all of us have graduated."

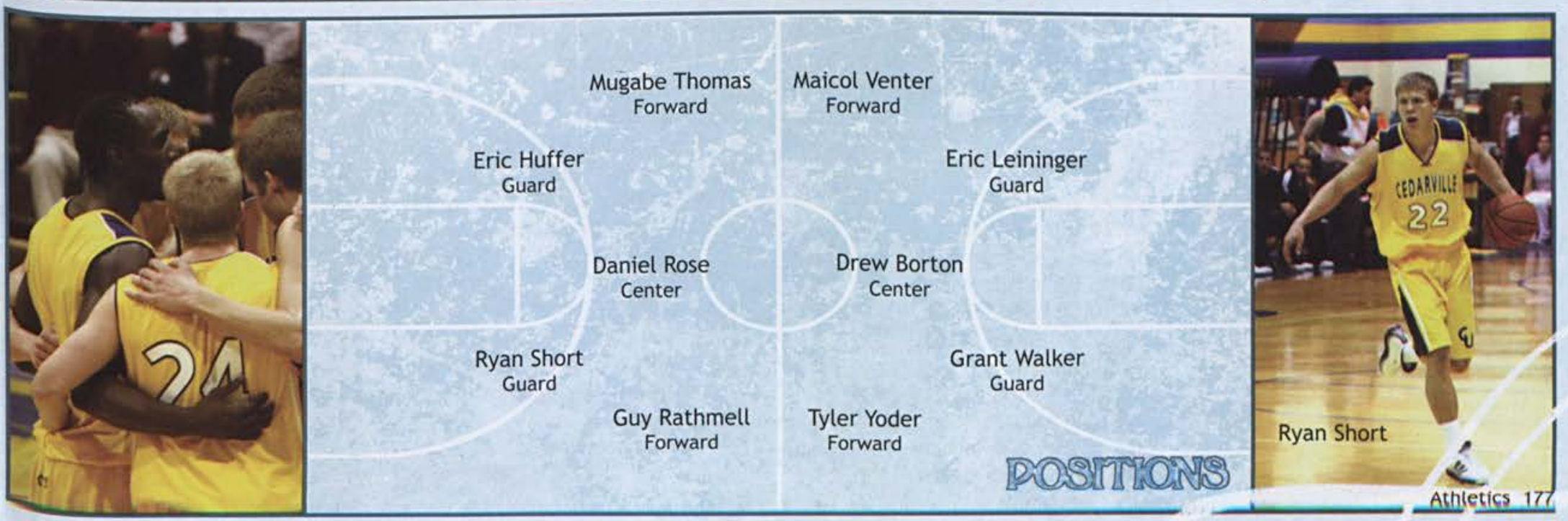


$\checkmark$ Junior Brittany Smart scores an easy layup in a game against Tiffin. She said of the team's perserverance, "This year was a year of ups and downs, concerning sickness and injuries, but our team definitely held the rope and pulled through together. We had a great year, and it was a learning experience for us, but with God's strength, our character stayed strong in the midst of adversity."
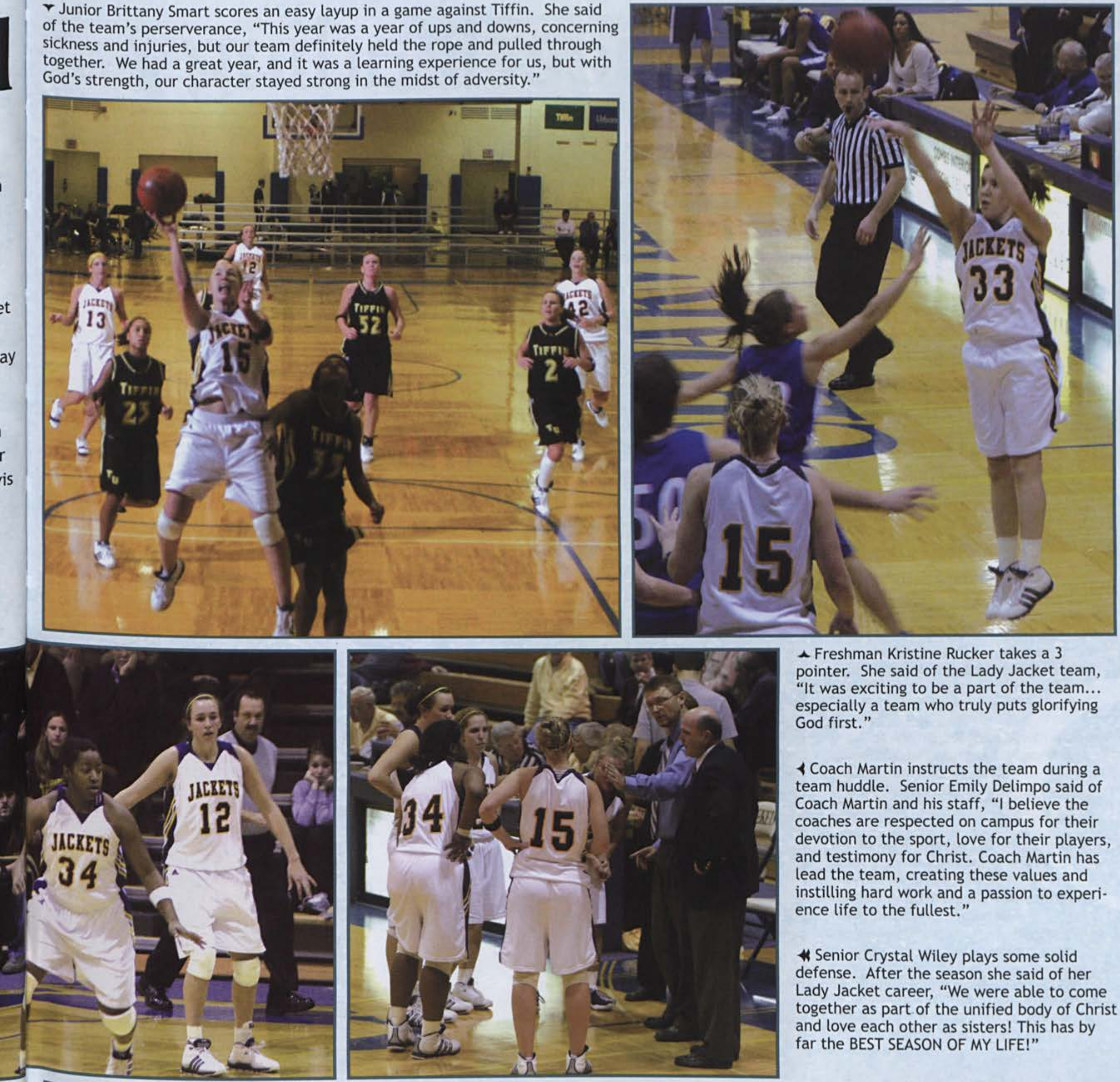

^ Freshman Kristine Rucker takes a 3 pointer. She said of the Lady Jacket team, "It was exciting to be a part of the team... especially a team who truly puts glorifying God first."

$\checkmark$ Coach Martin instructs the team during a team huddle. Senior Emily Delimpo said of Coach Martin and his staff, "I believe the coaches are respected on campus for their devotion to the sport, love for their players, and testimony for Christ. Coach Martin has lead the team, creating these values and instilling hard work and a passion to experience life to the fullest."

* Senior Crystal Wiley plays some solid defense. After the season she said of her Lady Jacket career, "We were able to come together as part of the unified body of Christ and love each other as sisters! This has by far the BEST SEASON OF MY LIFE!"

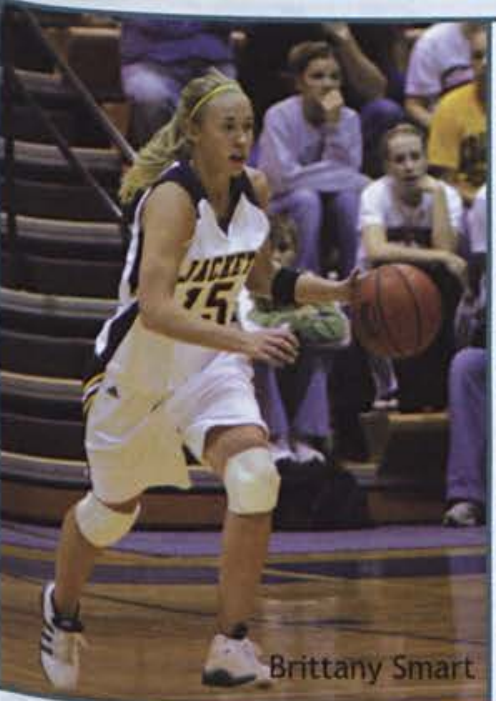

Kristine Rucker Guard Guard

Emily Delimpo Post

Kara Walton Point Guard

Baley Nosal Guard

Kristi Beougher Post

Brittany Smart Guard
Mary Stockdale Post

Crystal Wiley Post/Guard

POSTIRNS

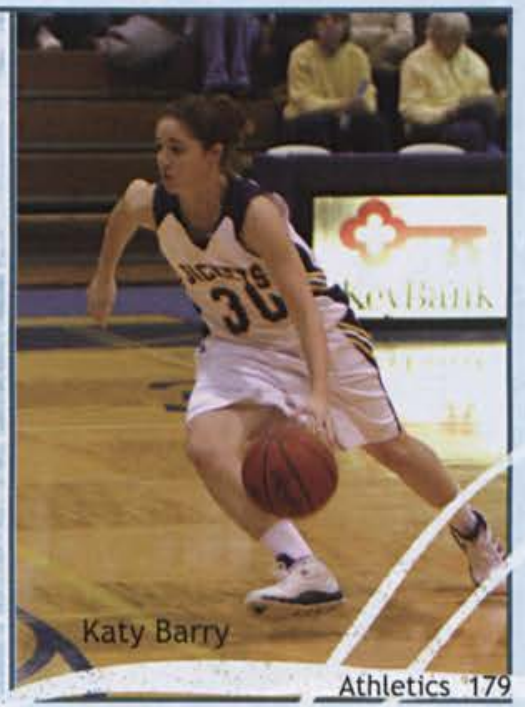


$r$ Whether flipping and flying through the air or just standing on someone's shoulders, junior Laura Teague places all of her trust in her teammates who support her during their stunts.

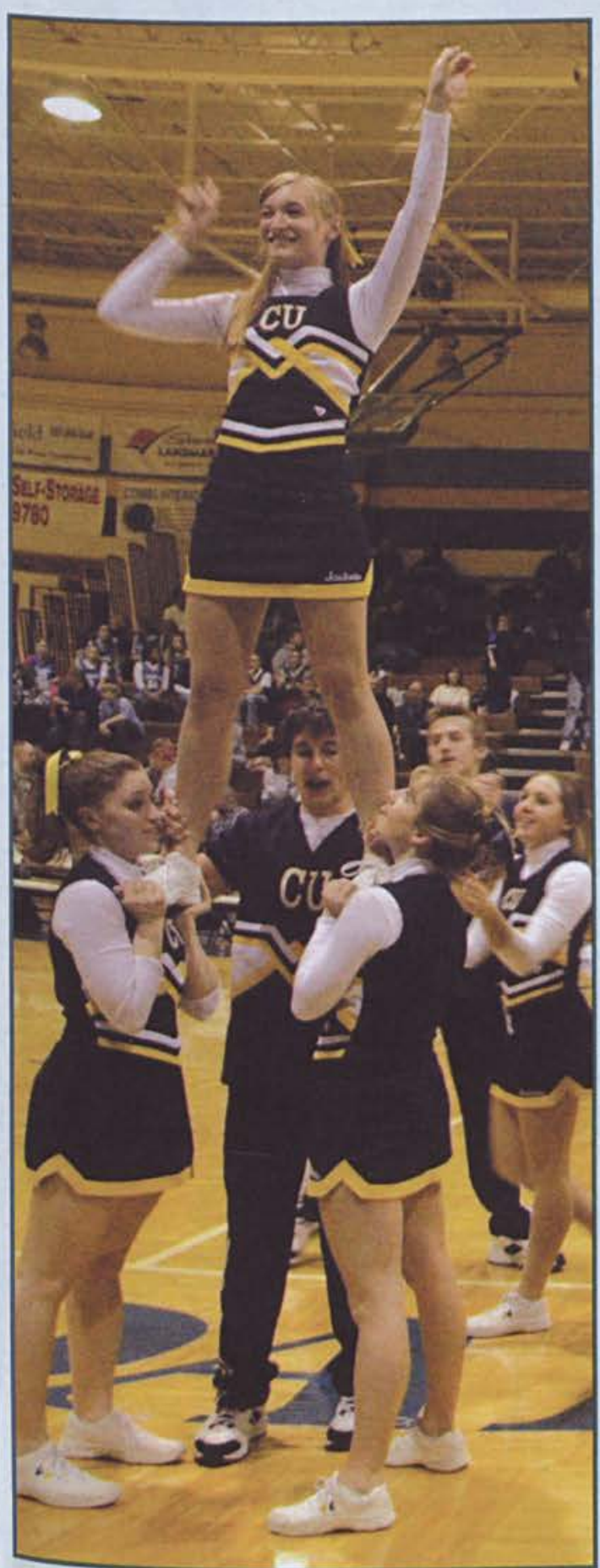

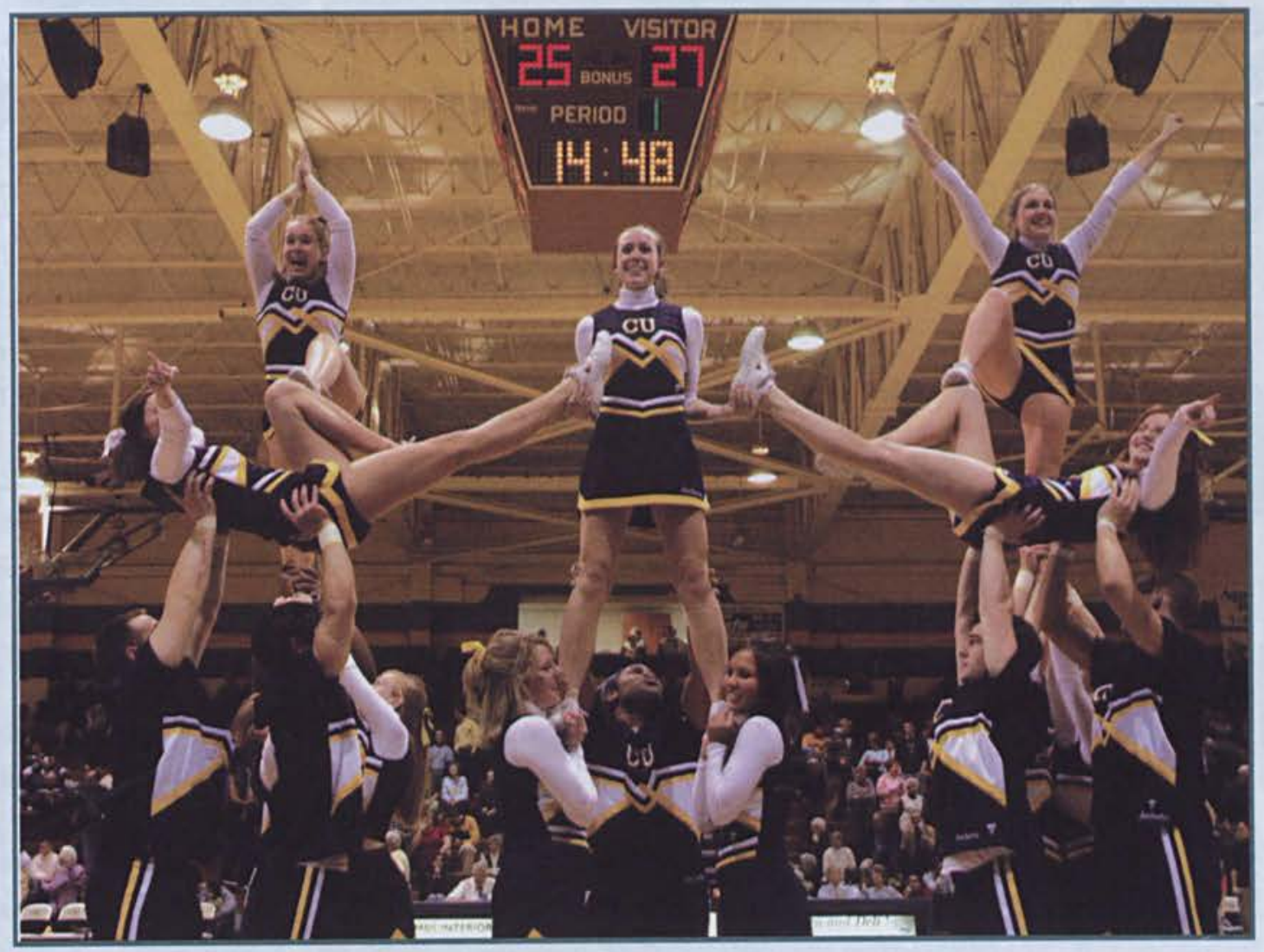

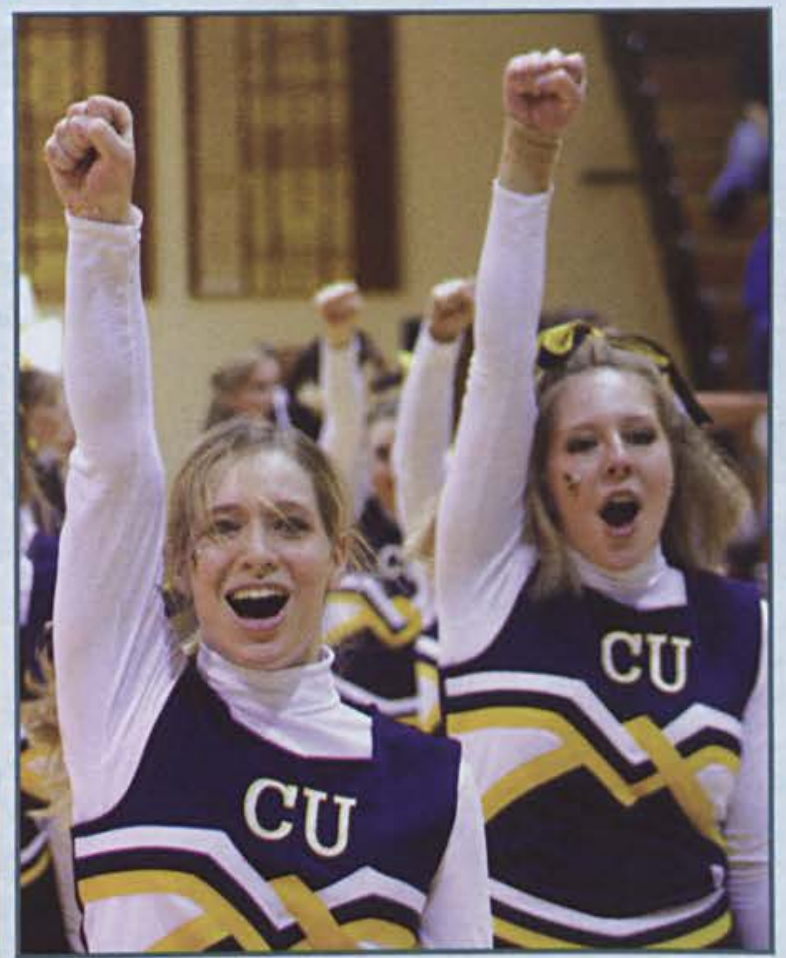

^ Senior Isaac Pittman [center spotter] has been on the team for two years. He says, "I enjoy being on the team because it feels more like a family. Each person has a responsibility and plays a unique role, and most of all, there is love for each team member. The encouragement I get from everyone is incredible."

$\checkmark$ Sophomore Ashley Coale and junior Jessica Forsythe cheer on the Yellow Jacket men's basketball team. These cheerleaders really know how to get the fans on their feet!

$\checkmark$ Cheerleaders Issac Pittman, Heather Kelton, Lisa Largent, Clay Ludlow, Nate Leykam, and Regan Rice stand in front of the students to cheer on the Yellow Jackets.

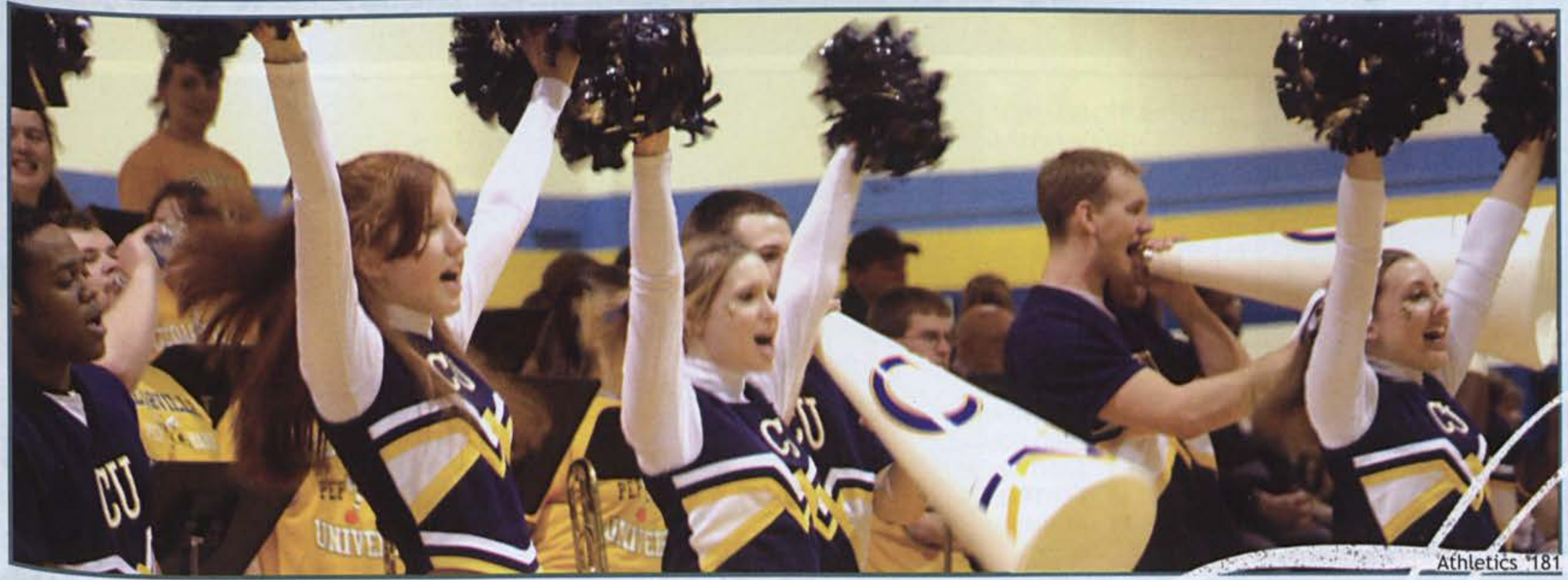




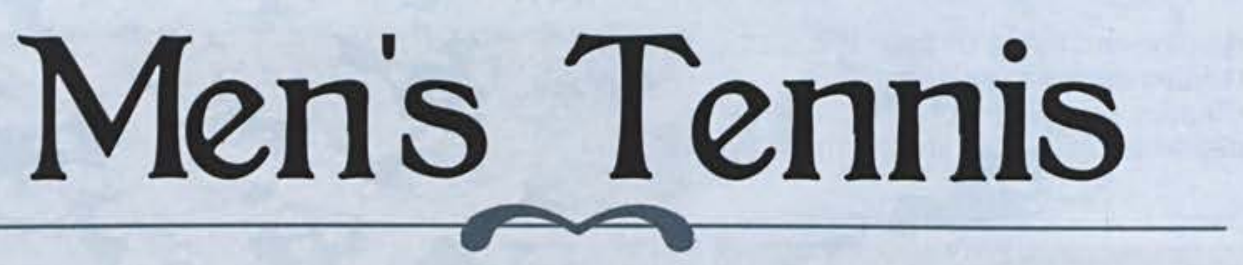

After dropping the first three, the Yellow Jacket Men's Tennis team won all but one match through the beginning of May. The team finished the NAIA Region IX Tournament on a 16-match winning streak with a $20-4$ overall record.

The team enjoyed a spring break full of tennis action with numerous schools down south. Emanuel
Dolph said of the trip, "Spring break was E especially enjoyable getting to know the

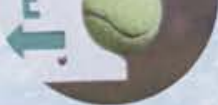
guys while traveling down south. The guys had a commitment to doing well and looking to show God to all of our opponents. It was really great to talk to other teams and hear them say that they like playing us because we were honest and enjoy playing."
Led by Senior Tim Hartman and Junior Jake Bezeck, the Yellow Jackets clinched second place in the AMC with an 8-1 win over Tiffin. The team only dropped five games in the contest and swept the doubles matches. Earlier in the year, the team lost what turned out to be the deciding meet against AMC leader Walsh. The Yellow Jackets dropped a 5-4 decision at defending AMC champion Walsh in the first match of the year.

Fortunately, the Yellow Jackets met up with Walsh coming into the NAIA Tournament seeded number 2 . This time the results were drastically different with the Yellow Jackets winning 5-0. After facing Walsh in the last 5 title matches, the Jackets finally scored the victory, which sent them to the NAIA National Tournament for the first time in 13 years. Tim Hartman, Jake Bezeck, Jacob Rogers, Dan Ballard, Mike Rhoads, and Kevin Furst all posted wins, ending the season on a high note.

Front row left to right: Jacob Rogers, Derek Hostetter, Tim Hartman, Emanuel Dolph, Jake Bezeck.

Back row: Assistant Coach Jamie Hand, Kevin Furst, Mike Rhoads, Dan Ballard, Jared Michonski, Head Coach Alan Edlund.

\section{2-7 Overall}

\section{7-1 American Mideast} Conference South Div.
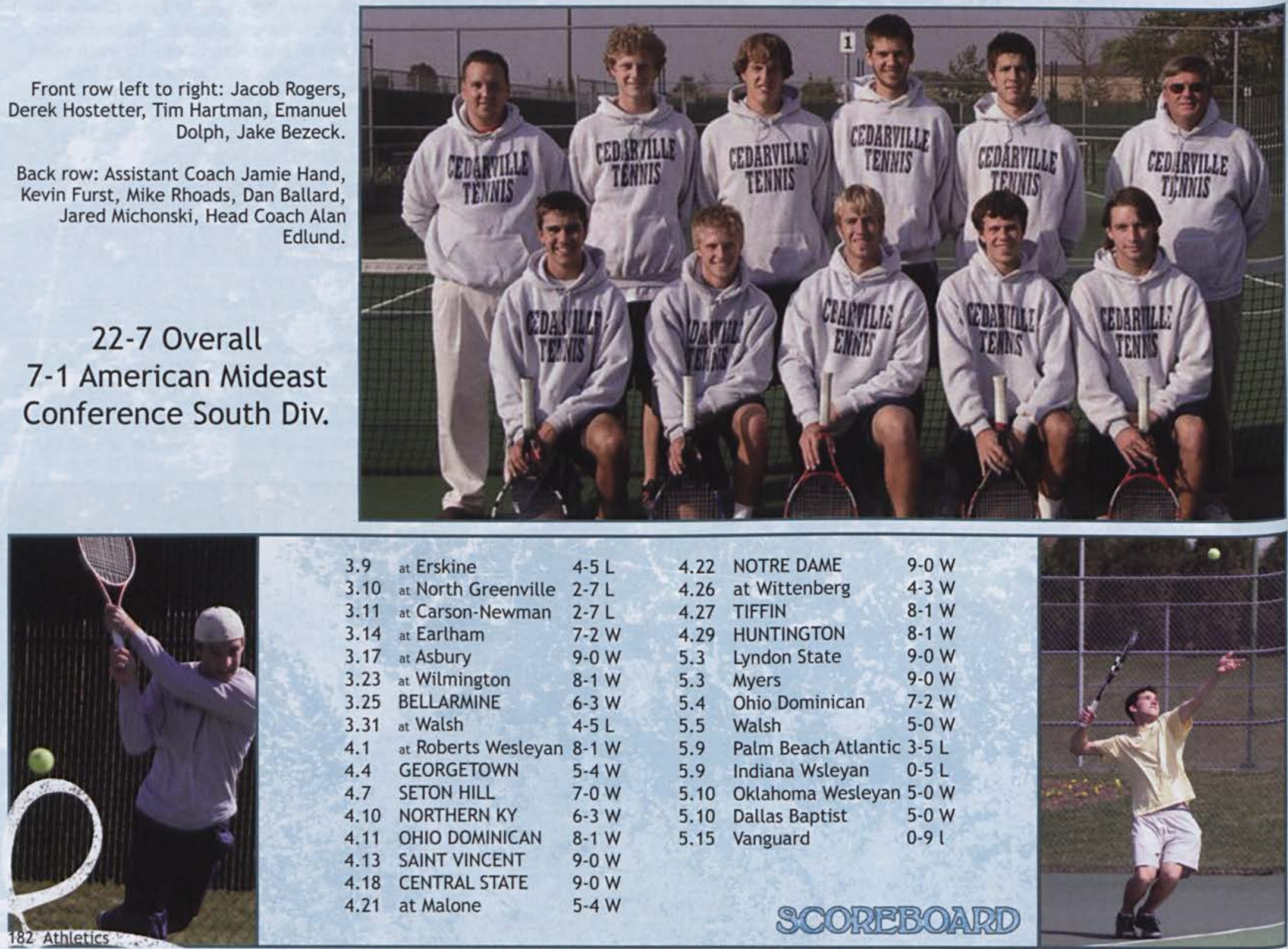

3.9 at Erskine

4-5 L

3.10 at North Greenville $2-7 \mathrm{~L}$

3.11 at Carson-Newman 2-7 L

3.14 at Eartham

$7-2 \mathrm{~W}$

3.17 at Asbury

3.23 at Wilmington

3.25 BELLARMINE

3.31 at Walsh

$9.0 \mathrm{~W}$

4.1 at Roberts

4.4 GEORGETOWN

4.7 SETON HILL

4.10 NORTHERN KY

4.11 OHIO DOMINICAN

4.13 SAINT VINCENT

4.18 CENTRAL STATE

4.21 at Malone

$6-3 \mathrm{~W}$

4-5 L

-1 W

$5-4 \mathrm{~W}$

$7-0 \mathrm{~W}$

$6-3 \mathrm{~W}$

8-1 W

9-0 W

9-0 W

$5.4 \mathrm{~W}$
4.22 NOTRE DAME

4.26 at Wittenberg

4.27 TIFFIN

4.29 HUNTINGTON

5.3 Lyndon State

5.3 Myers

5.4 Ohio Dominican

5.5 Walsh

5.9

5.9

Palm Beach At

5.10 Oklahoma Wesl

5.10 Dallas Baptist

5.15 Vanguard 
$\checkmark$ "This year's team made history. We are the first team to take Coach Edlund to the national tournament. I consider it an honor to have played with this team," states Mike Rhoads.
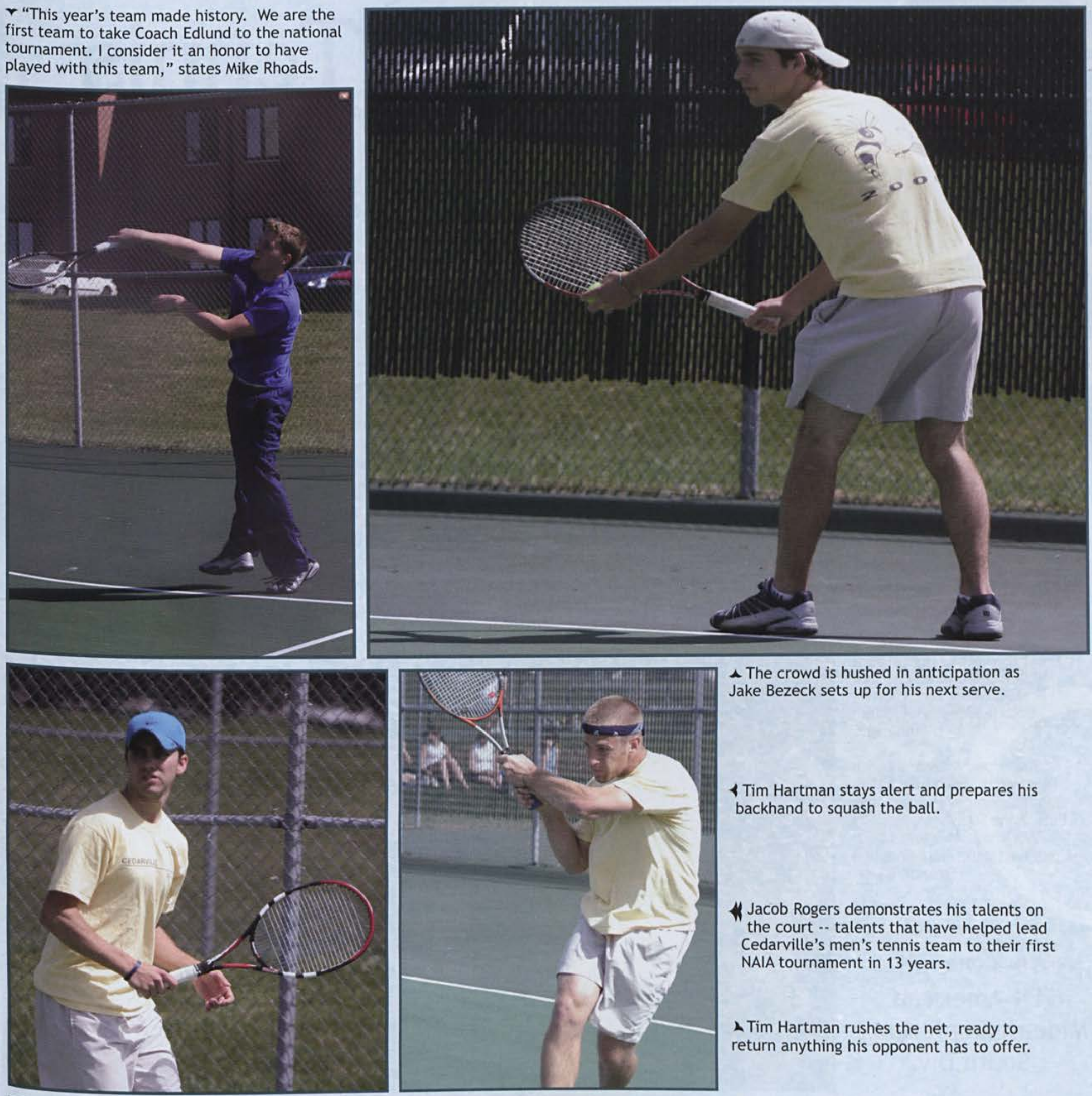

The crowd is hushed in anticipation as Jake Bezeck sets up for his next serve.

$\checkmark$ Tim Hartman stays alert and prepares his backhand to squash the ball.

ฟ Jacob Rogers demonstrates his talents on the court -- talents that have helped lead Cedarville's men's tennis team to their first NAIA tournament in 13 years.

- Tim Hartman rushes the net, ready to return anything his opponent has to offer.

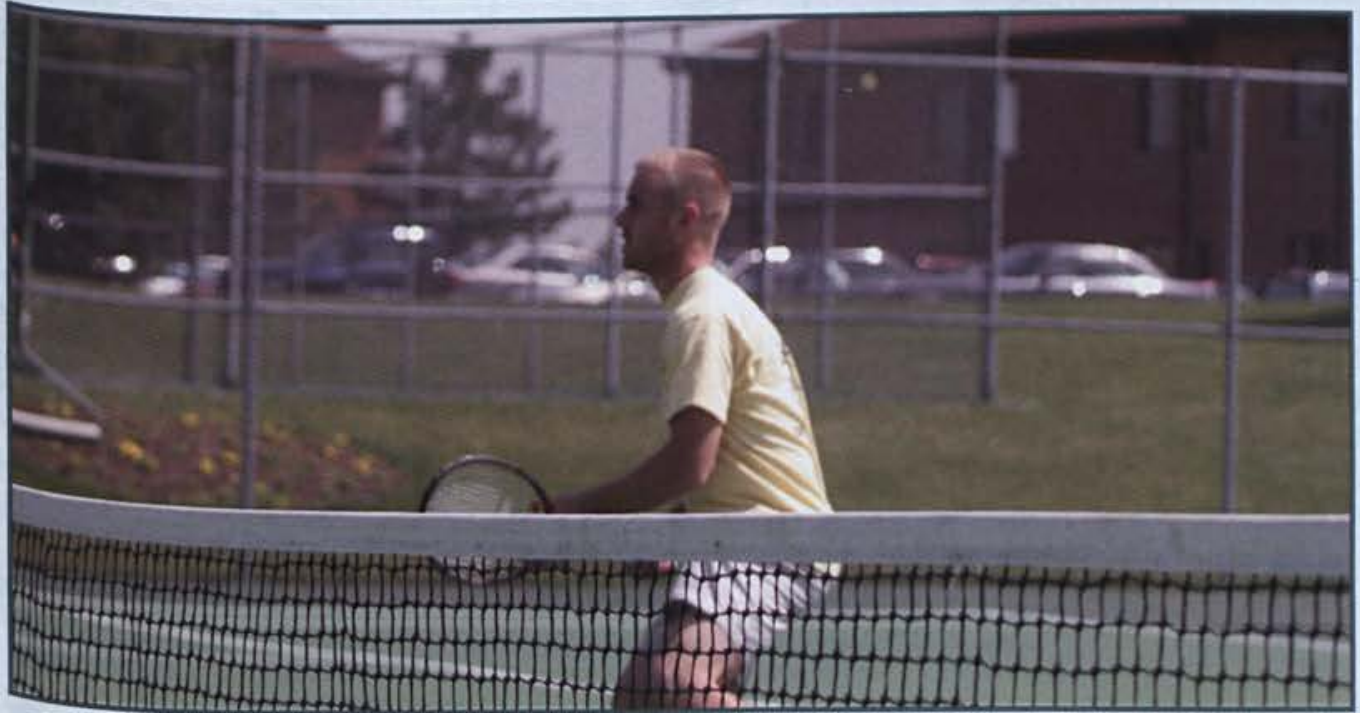

Coach Edlund is a great coach and a spiritual leader who is always encouraging and motivating us to be stronger men of Christ. Tennis is great to play, but it's amazing being on the Cedarville Men's Tennis Team. 
The entire team lines up at the beginning of the match. Their smiles show their love of the sport and each other.
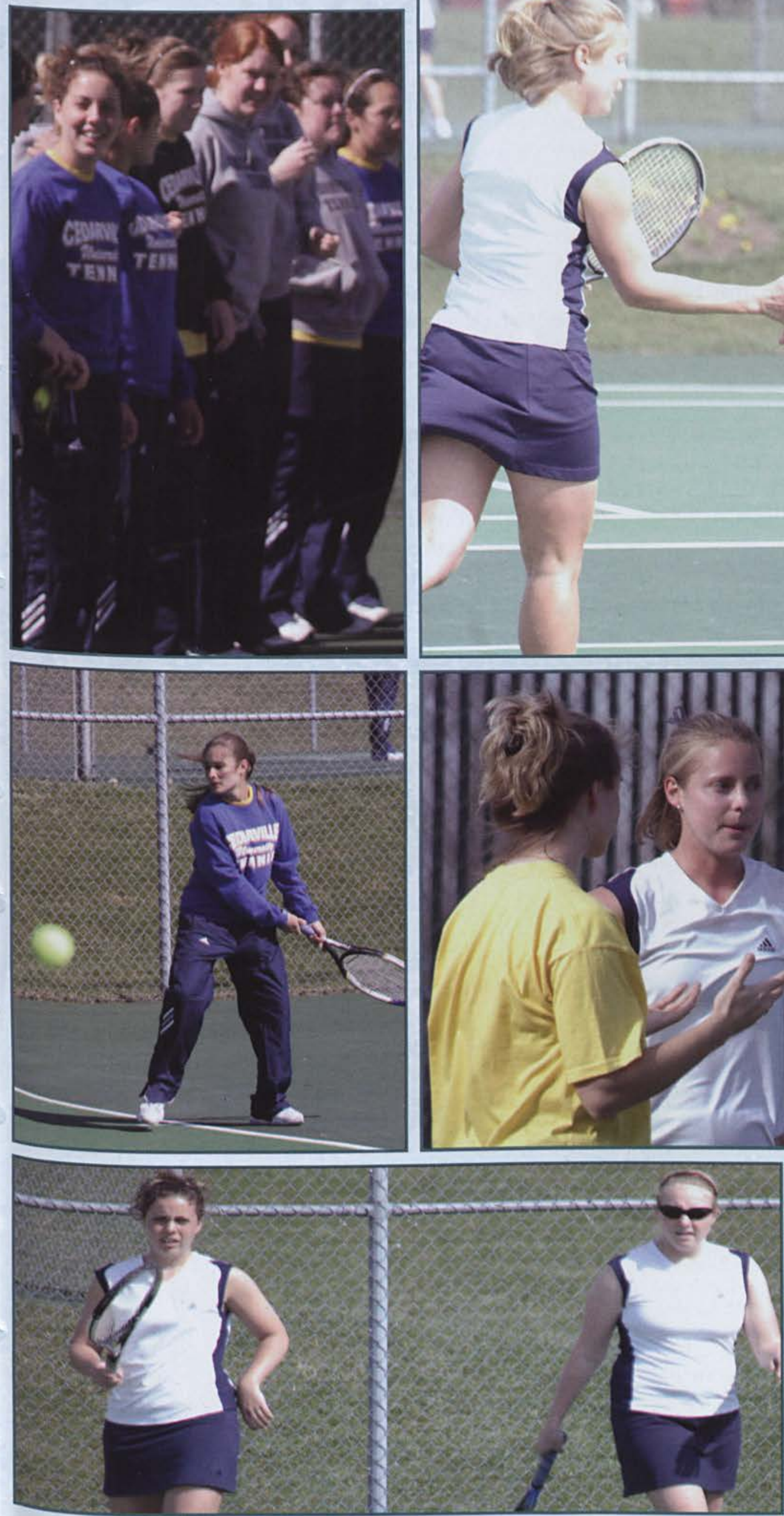
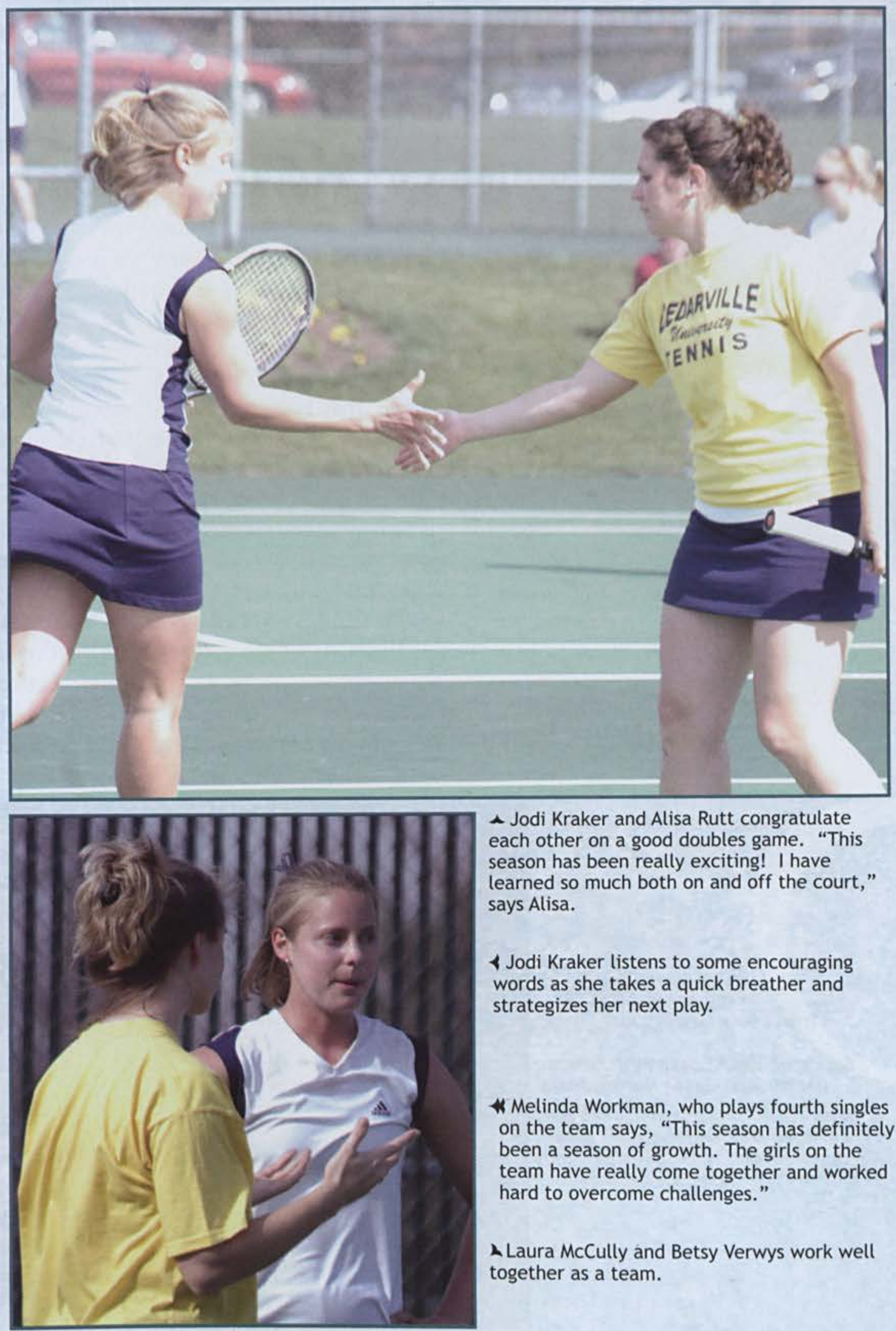

^ Jodi Kraker and Alisa Rutt congratulate each other on a good doubles game. "This season has been really exciting! I have learned so much both on and off the court," says Alisa.

$\checkmark$ Jodi Kraker listens to some encouraging words as she takes a quick breather and strategizes her next play.

* Melinda Workman, who plays fourth singles on the team says, "This season has definitely been a season of growth. The girls on the team have really come together and worked hard to overcome challenges."

L Laura McCully and Betsy Verwys work well together as a team.

\section{This year we worked at starting} our matches with great intensity and, for the most part, have done a great job. Not only was the tennis season memorable, but also the relationships built on the team. We really came together this year. 


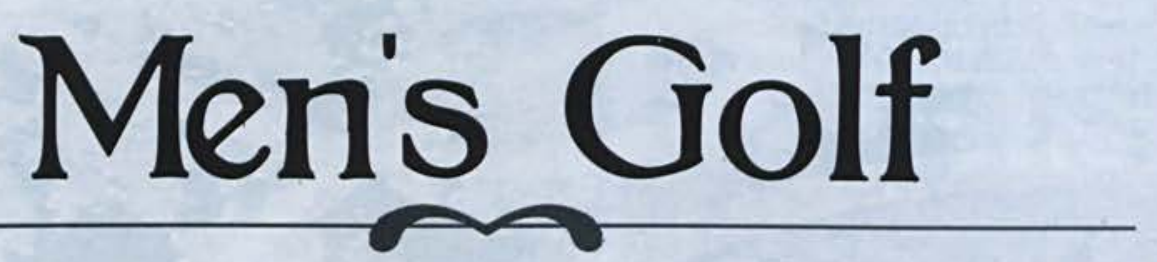

The Yellow Jacket golf team utilized

the entire academic year for their golf season, starting back in early

September and ending in late April. This year's young team was led by juniors Scott Aker, Travis Roach, and Adam Schlappi, and as a group, they broke the school record twice for 36 holes. In the fall semester, the team posted an impressive 1 st and 2 nd finishes in backto-back tournaments at the Wittenburg Invitational and the Ohio Dominican Classic. At the Wittenburg Invitational, the Jackets won by 33 shots and had three players on the All-Tournament team.

At the conclusion of the year, Scott Aker said of the season, "We achieved many of the goals that we set at the beginning of the

season, such as winning a tournament in each of the seasons. We really have a great group of guys." The Yellow Jacket team started the spring off well with a 1st place win at the Urbana invitational. Adam Schlappi led the way with a 76, despite poor conditions. Adam said, "The team this year has been a joy to be a part of. This has been the greatest team that I have ever been a part of. We all get along so well. We will do anything to help out each other also. Throughout the year, we have worked really hard on preparing our games for the best of our ability."

Sophomore Brent Martin had his best set of two rounds with a 157 at the Mt. Vernon Nazarene Invitational, as the team tied for 3rd place. He said of the year, "This past golf season has been incredible. It was filled with highlights from both on and off the course. Off the course is the most important. Our team has really had a great time getting to know each other."

Front row left to right: Brett Bigler, Scott Aker, Trevor Bowman, Brendan Ojala.

Back row: Head Coach Ryan Bowen, Daniel Servi, Brent Martin, Adam Schlappi, Taylor Durling.

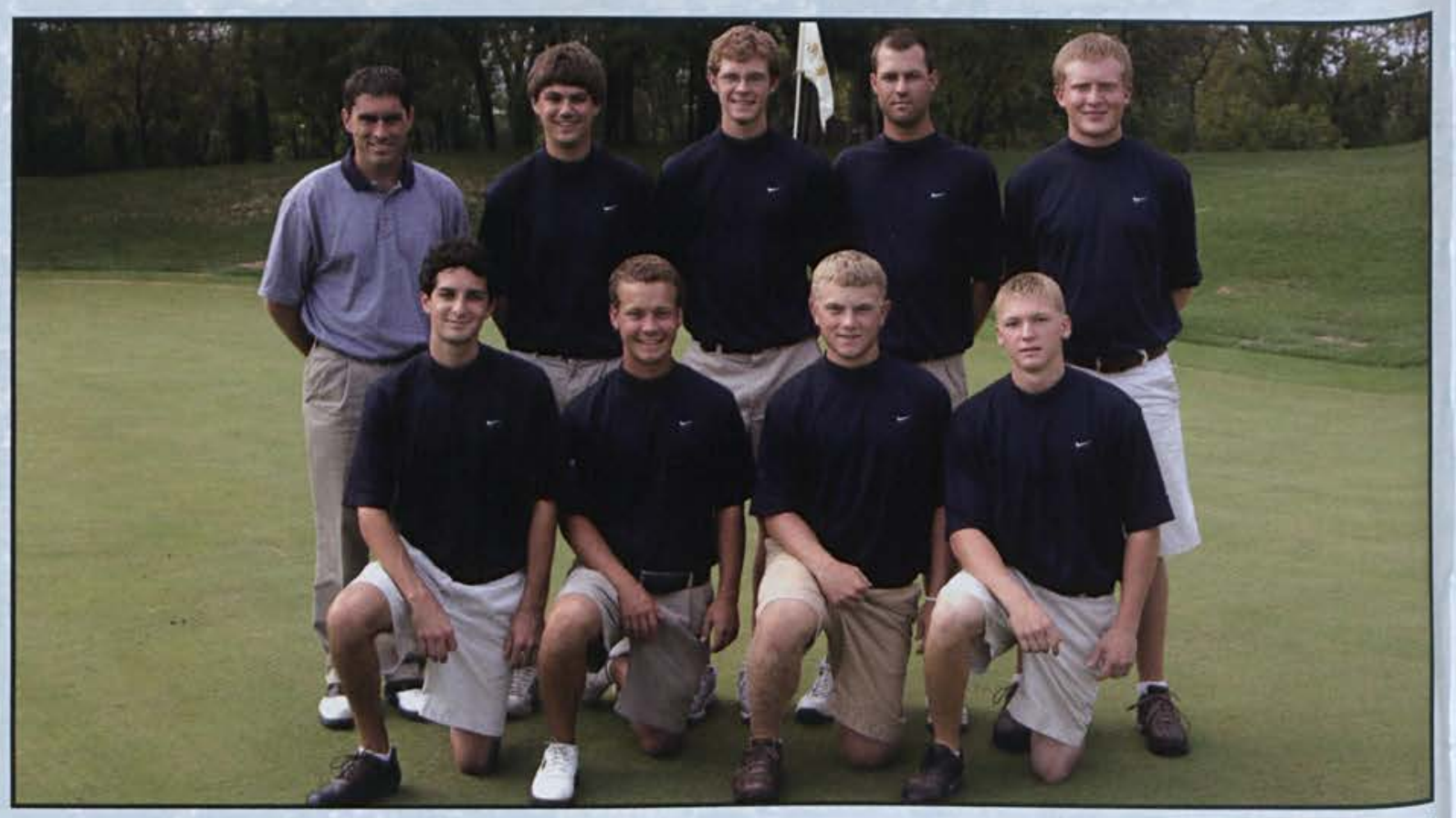

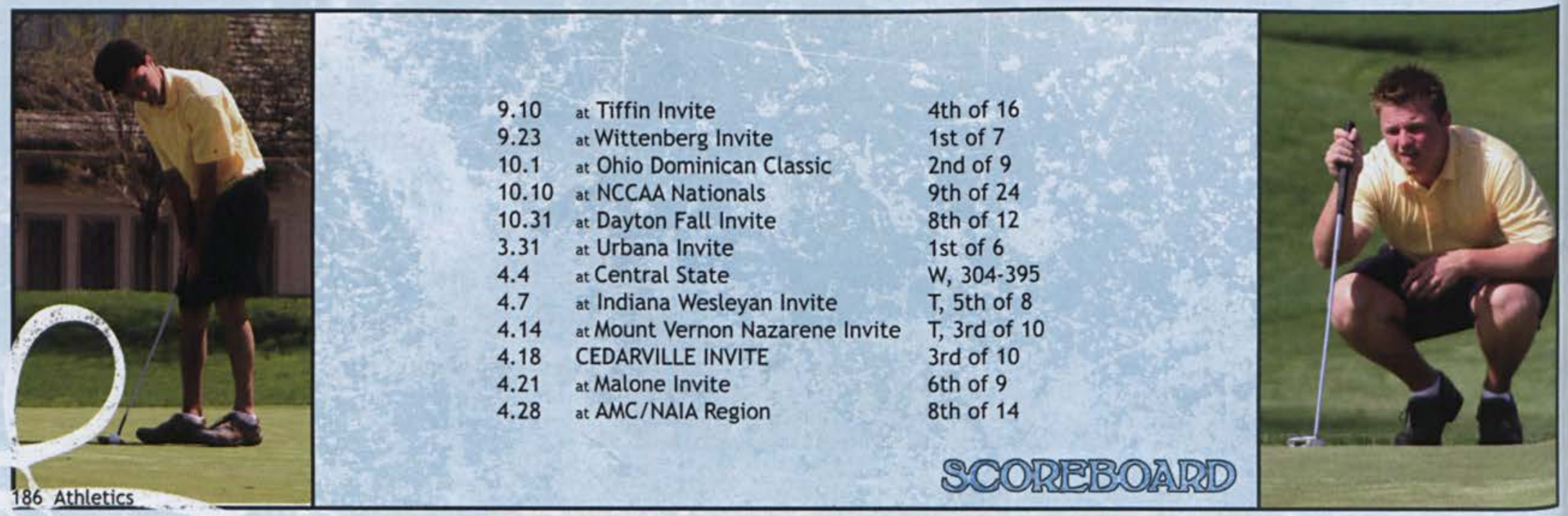


$\checkmark$ Taylor Durling watches as his ball sailes through the air after an impressive swing.
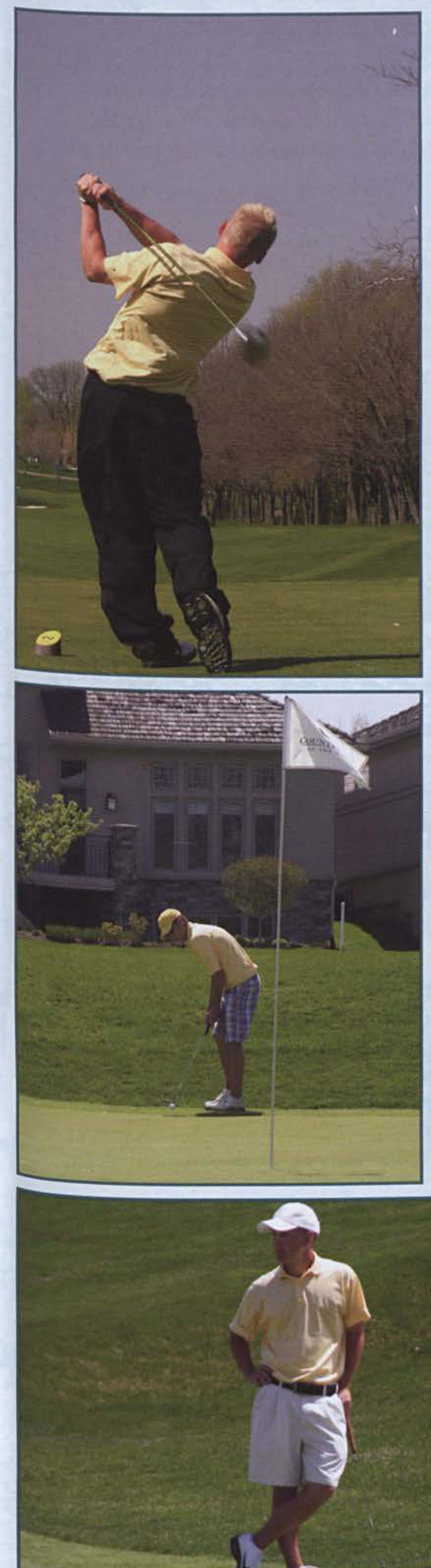

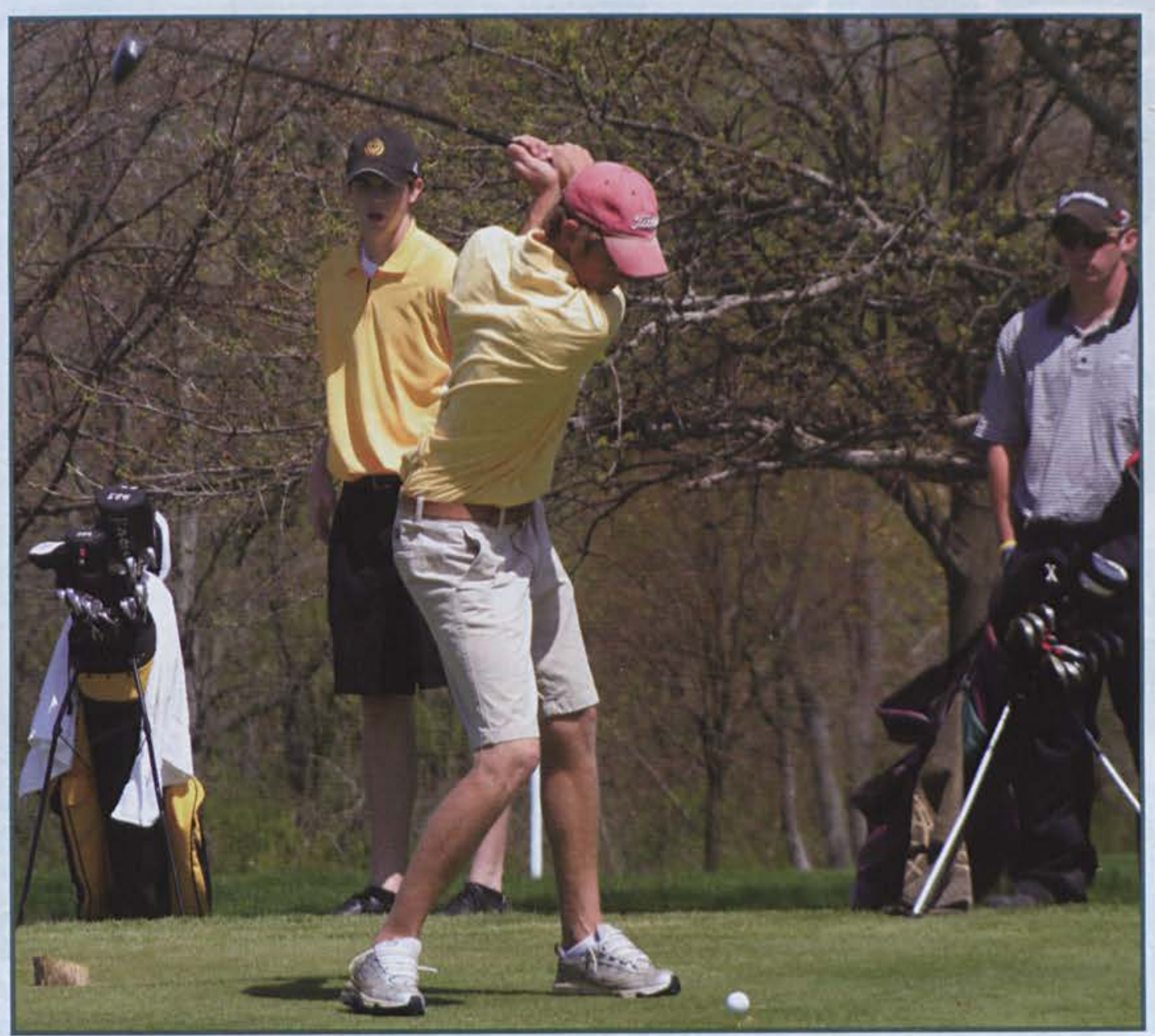

Brent Martin takes a swing as other players watch on.

$\downarrow$ Trevor Bowman said, "This season was a good start. I think that with all the talent that we have and with the youth that we have on this team, we should really be able to compete in the future. It feels good to get a year under my belt."

* "It was so fun being a part of this team this year. It has been my favorite team in all of the teams that I have played for in any sport!" says Adam Schlappi.

4. Trevor Bowman takes a break to asses his situation on the course.

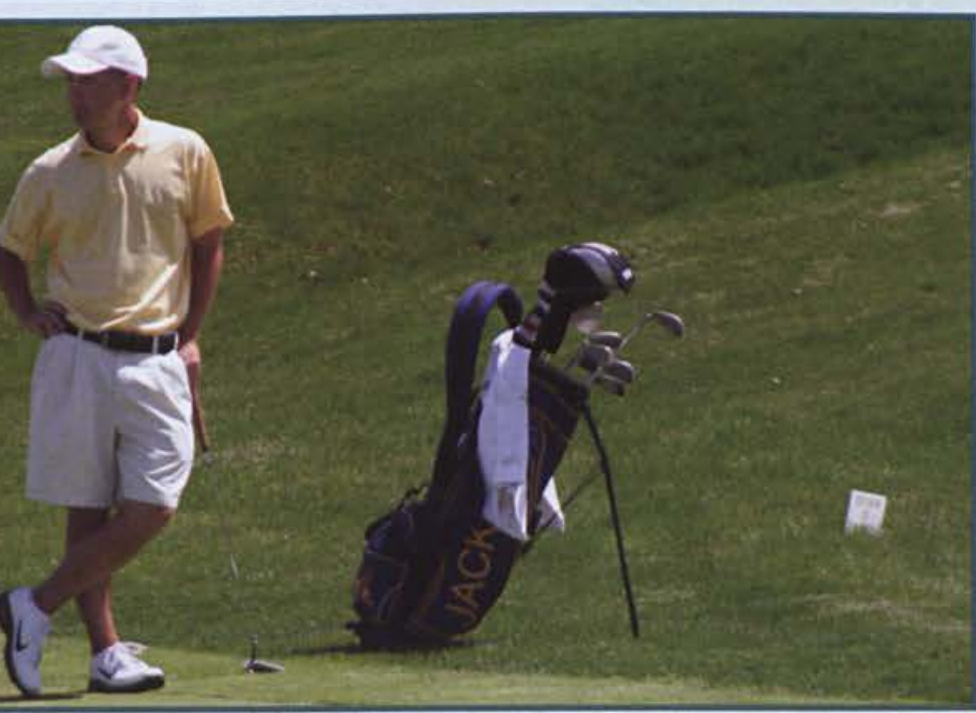

We won two tournaments, which is the first time Cedarville has won a tournament in a while. I think that this year was a big step in the right direction for us. This season allowed us to look to next year with confidence and compete with the league's ber? 
- Ben Shroyer, Justin Gutierrez, Bryan Pittman, and Dan Campbell run in the Cedarville home meet of the outdoor season. They're still wearing shorts despite a sub-freezing wind chill and snow flurries.

$\checkmark$ Brad Muschott concentrates on his set up for the $4 \times 200$ relay during the home meet for the indoor season.
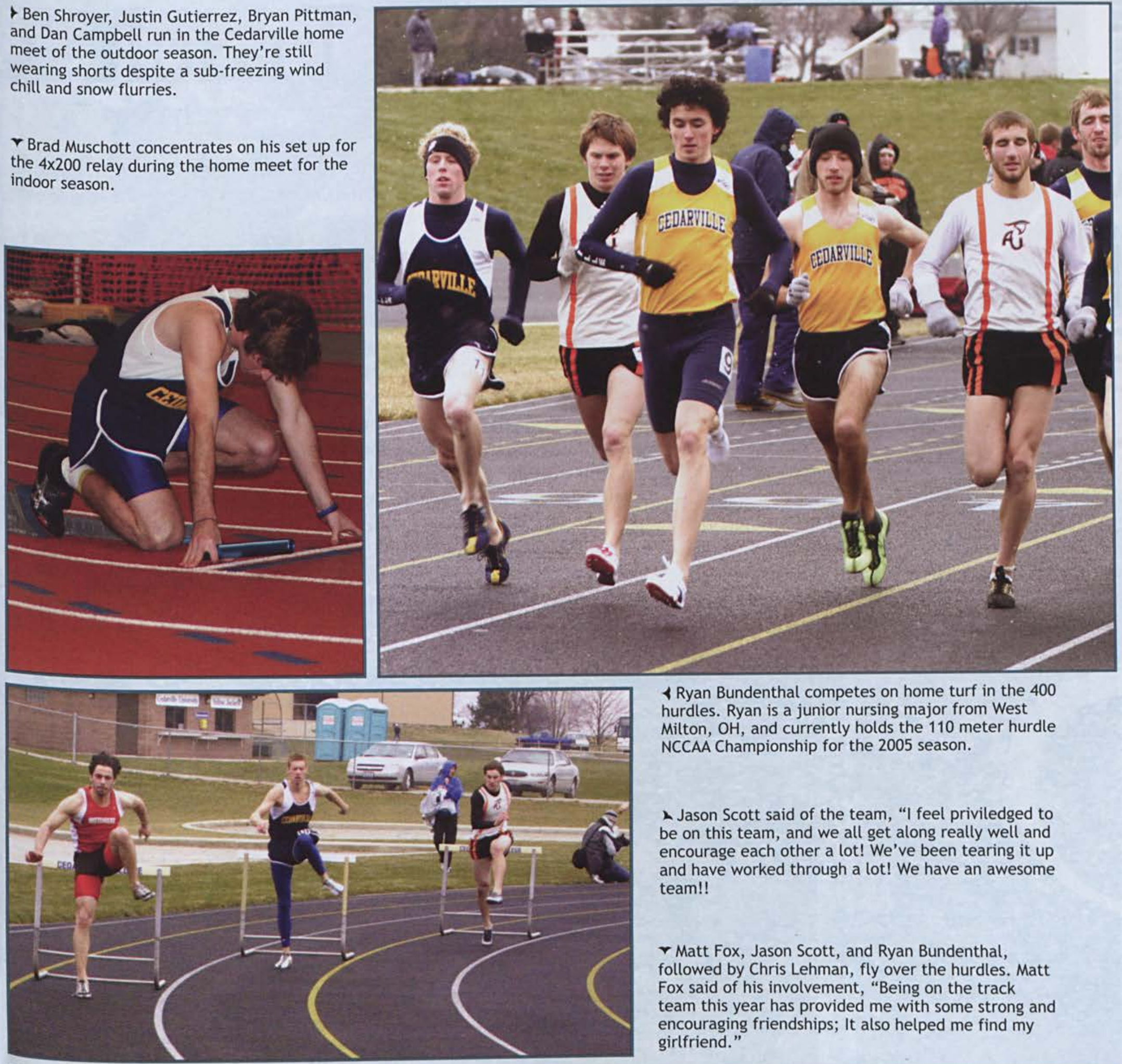

$\checkmark$ Ryan Bundenthal competes on home turf in the 400 hurdles. Ryan is a junior nursing major from West Milton, $\mathrm{OH}$, and currently holds the 110 meter hurdle NCCAA Championship for the 2005 season.

- Jason Scott said of the team, "I feel priviledged to be on this team, and we all get along really well and encourage each other a lot! We've been tearing it up and have worked through a lot! We have an awesome team!!

$\checkmark$ Matt Fox, Jason Scott, and Ryan Bundenthal, followed by Chris Lehman, fly over the hurdles. Matt Fox said of his involvement, "Being on the track team this year has provided me with some strong and encouraging friendships; It also helped me find my girlfriend."
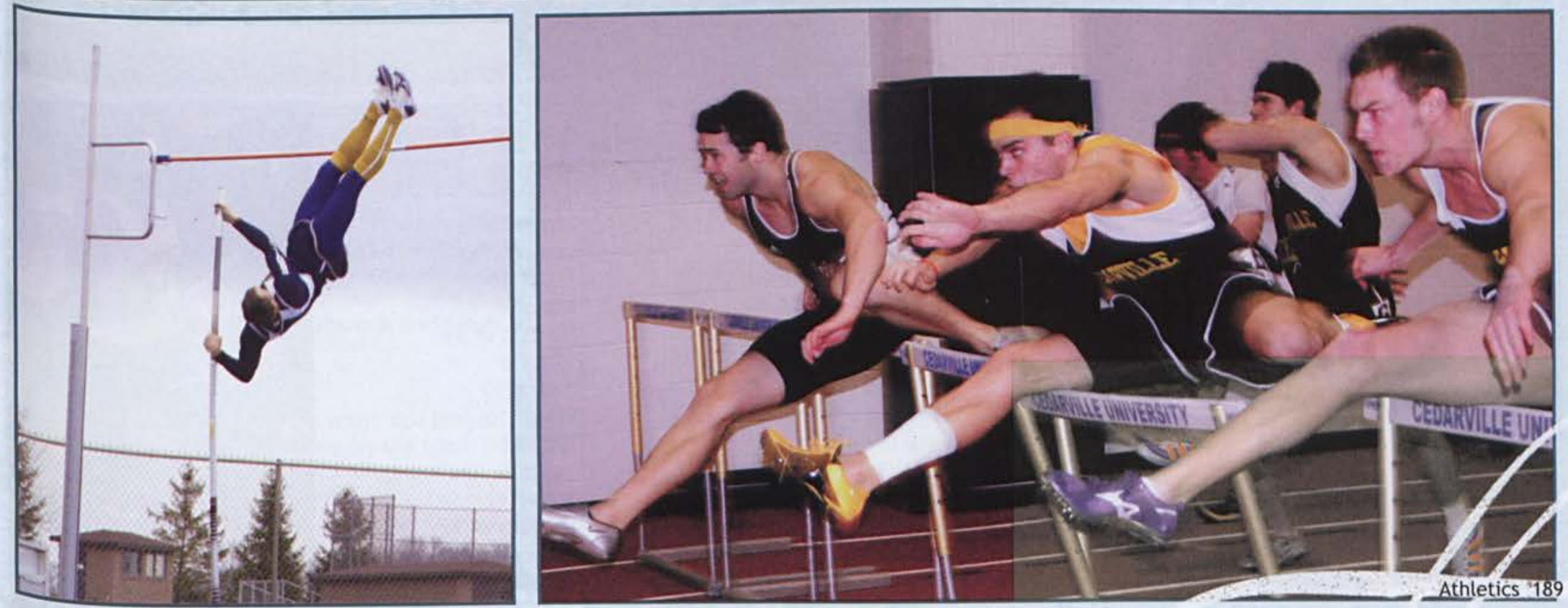

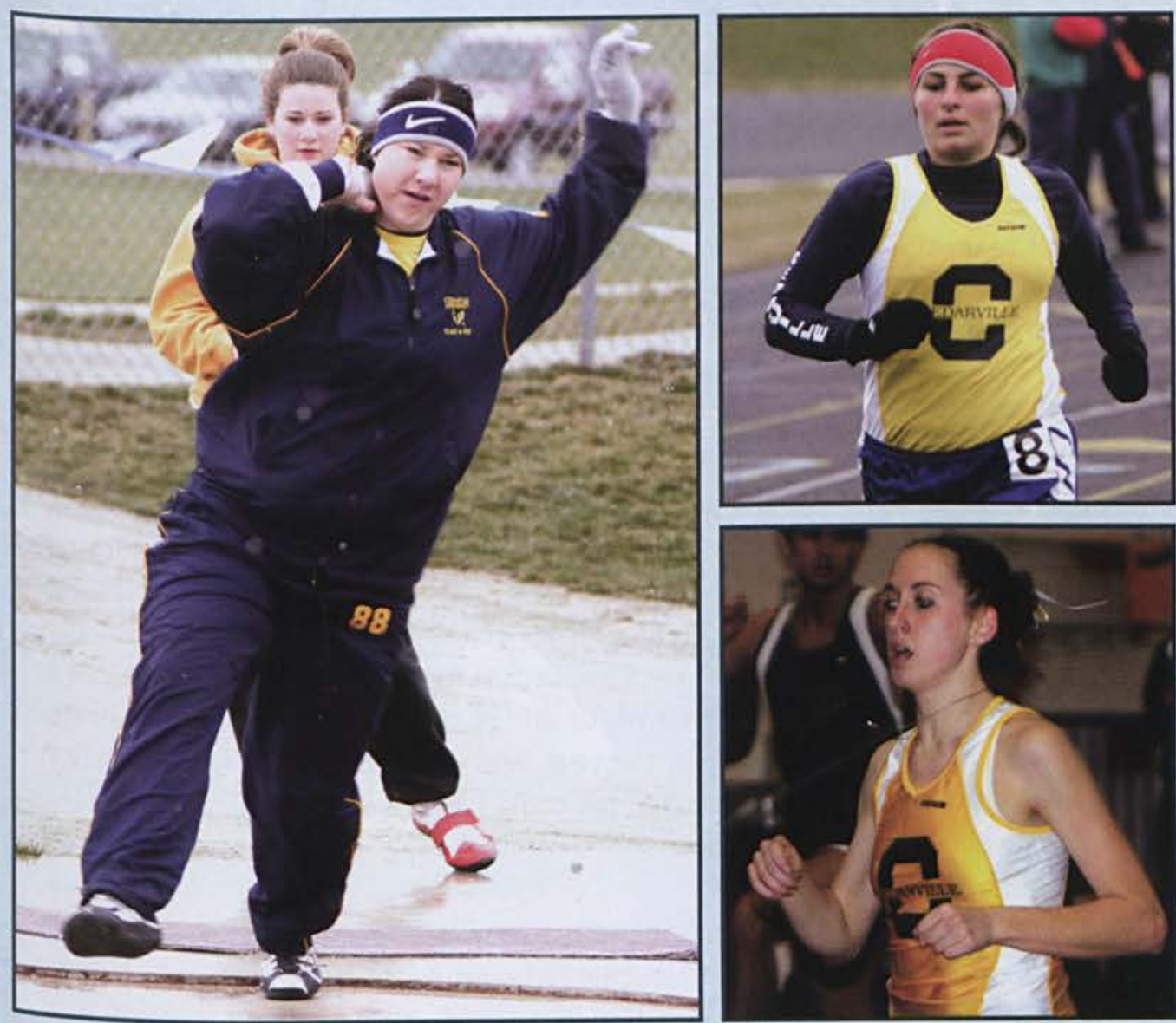

* Emily Peacock throws shot put at Cedarville's home outdoor meet. Emily is a junior psychology and criminal justice major. Competing for the last 3 years, Emily holds several conference titles including this season's AMC Hammer Throw champion.

$\checkmark$ Erin O'Keefe focuses on the finish line as she runs her race hard during one of the teams home meets.

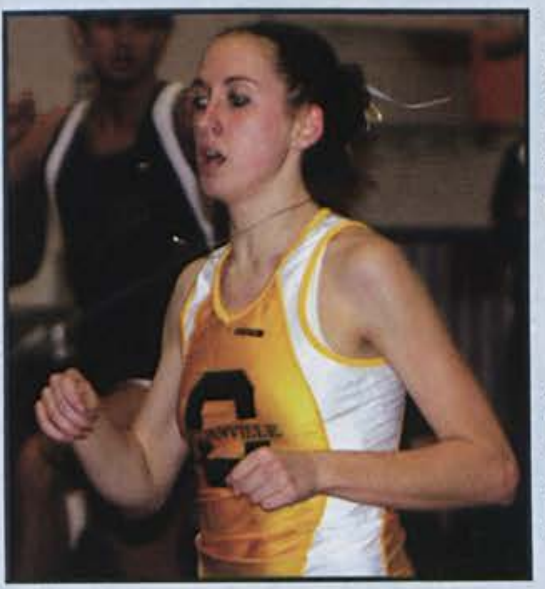

4 Faith Meitzler said of this season, "For me it has been a learning season, I got an injury after the first meet of outdoor season, and I only ran in two meets becasue my injury put me out for the rest of the season and the whole team came around and were encouraging me and praying for me.

4 Marla Rice said of the team, "Competing on a college track team has been an amazing experience, not only through the team unity and level of competition, but through the Christ-focused approach we take all the time."
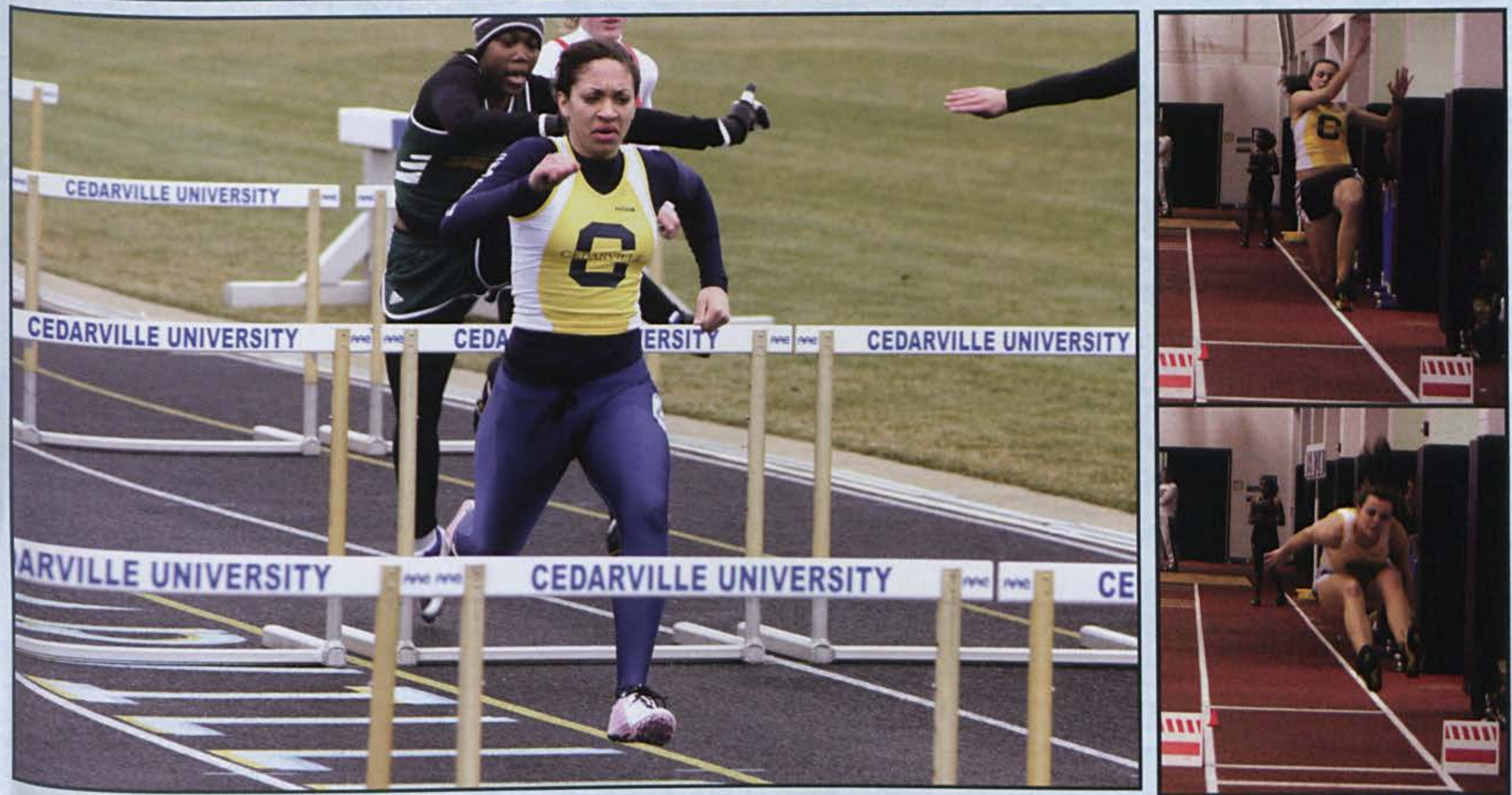

Courtney Reid said of the team, "It is a family We laugh together, cry together, fuss at each other, even fight with each other, but we love one another , and I know that no matter what, someone is going to be there screaming on the sidelines and ready to hug me when I get done."

Marla Rice and Bonnie Wright warm up. Bonnie Said of the team, "Through being on the track team I have learned so much about working hard and persevering through hard circumstances. I love how every year our team is different because of the new dynamic that each group of freshmen brings."
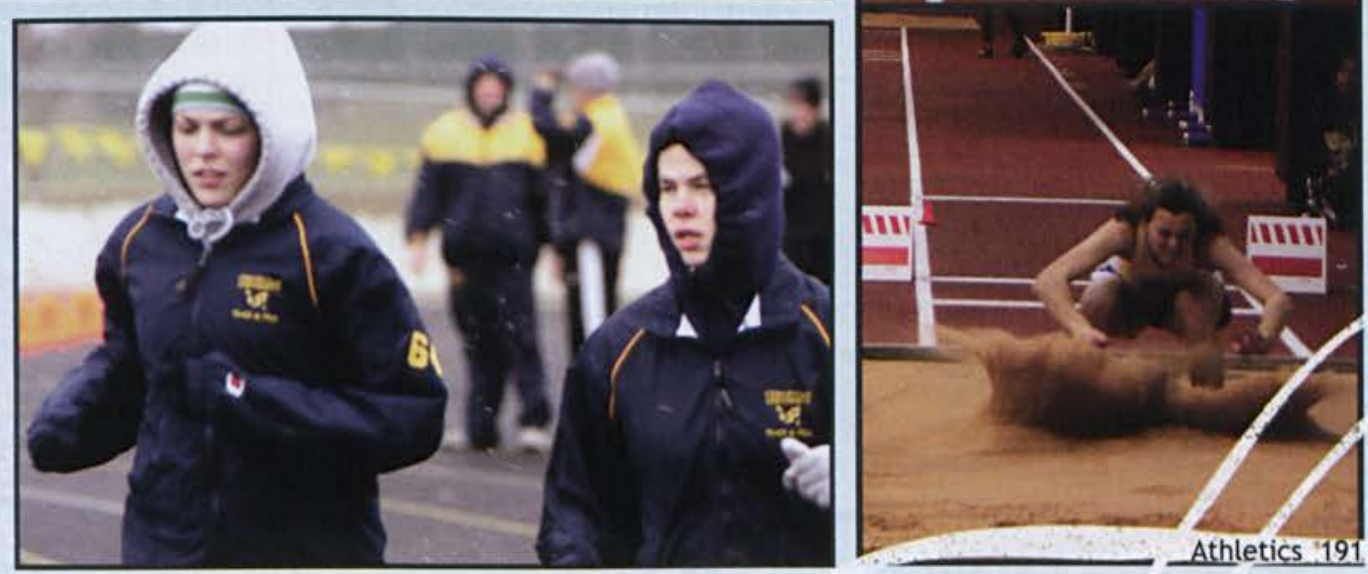


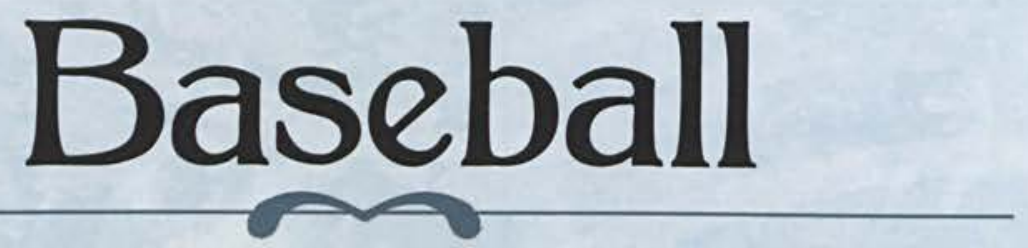

The Yellow Jacket Baseball team started team. More than 500 fans turned out to see Senior Kiel their season well before the grass was green and the flowers were

sprouting. Everyday is a good day for baseball, so their season

began in late February with an extra inning loss against Cumber-

land. Cedarville managed an impressive 17 hits in the defeat. Matt Houchin, Alex Pummell, and Bonyton's 9 inning complete game gem but were disappointed with a 4-1 loss to Wilmington. Richie Reeder had the Yellow Jackets' lone RBI, with a single driving in Paul Wilson.

At the end of April, Paul Wilson was leading in batting with Derek Woloshyn, Matt Totten, and Richie Reeder not far behind. Seniors Matt Bonin and Kiel Boynton headed up the pitching staff while Derek Koogler, Kiel Boynton, and Matt Houchin each finished with 3 wins pitching.

game.

The first half of the Yellow Jacket season was filled with promise, going through 20 games with nearly a .500 record. The team played well throughout the second half of the year, but wins stayed just out of reach.

Highlighting the season was a game hosted by the Yellow Jackets at Fifth Third Field, the home of the Dayton Dragons minor league Pitcher Dan White said of the season, "We competed hard throughout the year, and if we could have continued our early success, we could have done some big things in the post season."

The team ended the year on the bats of Andrew Noble and Matt Houchin, who produced the winning run in a 3-2 victory over Shawnee State. Noble opened the inning with his 13 th double of the season, and Houchin batted him in for the game winner.

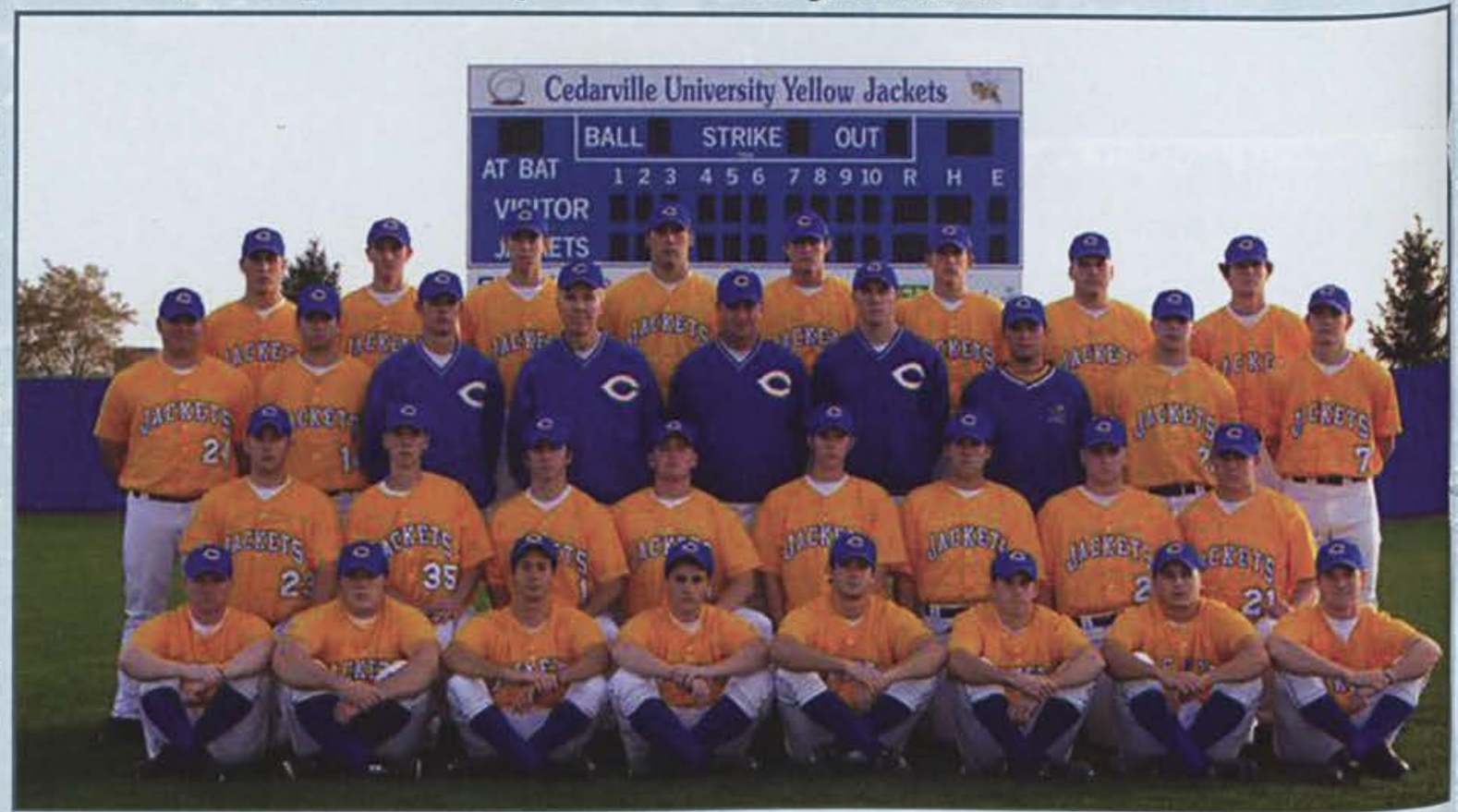

Front row: Matthew Chamberlin, Derek Koogler, Phil Buben, Dave DiLernia, Ian Johnson, Tim Hubler, Paul Wilson, Bryan Lutes. Bonin, Tyler Owens, Zach Hanna, Andrew Noble, Matt Houchin, Jared Griest, Pete Kraus, Brady Workman.

Third row: Phil Westenbarger, Alex Pummell, Assistant Coach Travis Allen, Assistant Coach John Meyer, Head Coach Greg Hughes, Assistant Coach David Snow, Student Assistant Anthony Weleski, Dan White, Derek Woloshyn

Second row: Micah Wilson, Jordan Shumaker, Kiel Boynton, Richie Reeder, Matt Owens, Kevin Brown, Josh Chamberlin, Jordan Siefkes.

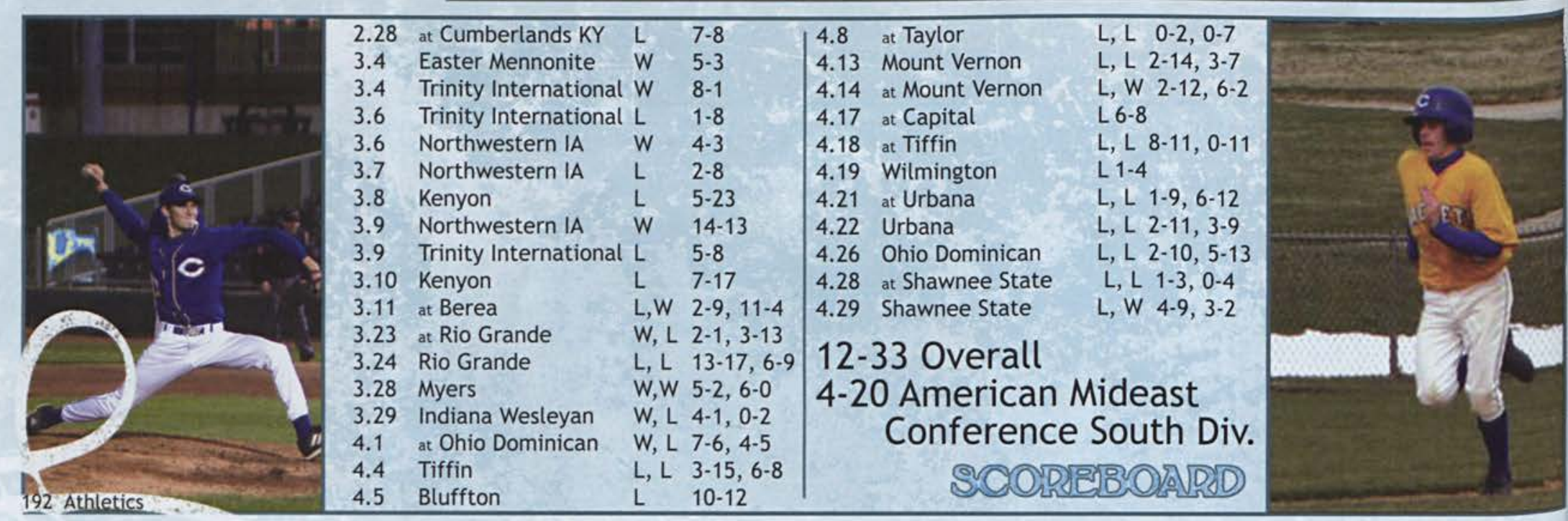




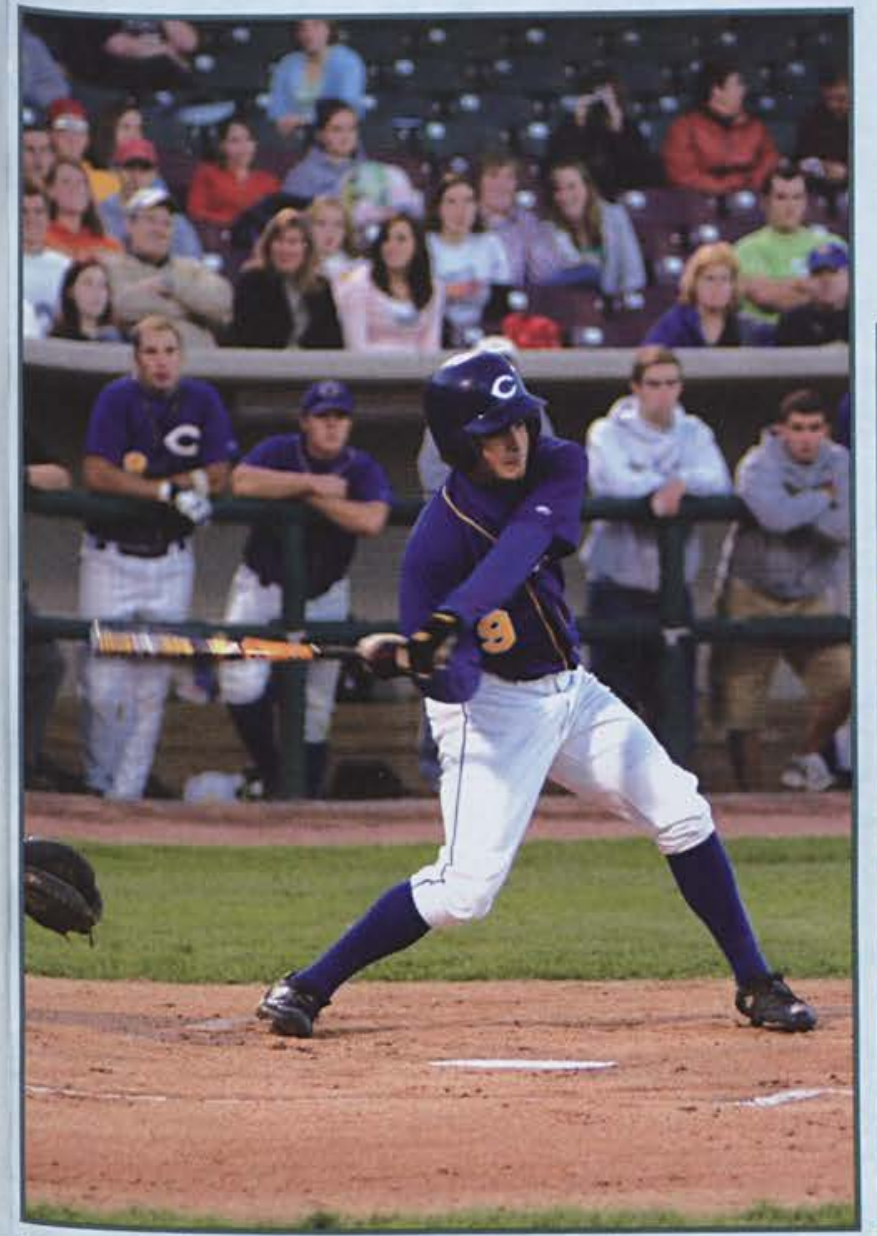

1 Paul Wilson swings at a pitch as the

Cedarville University baseball teams plays

their first game at Fifth-Third field in

Dayton.

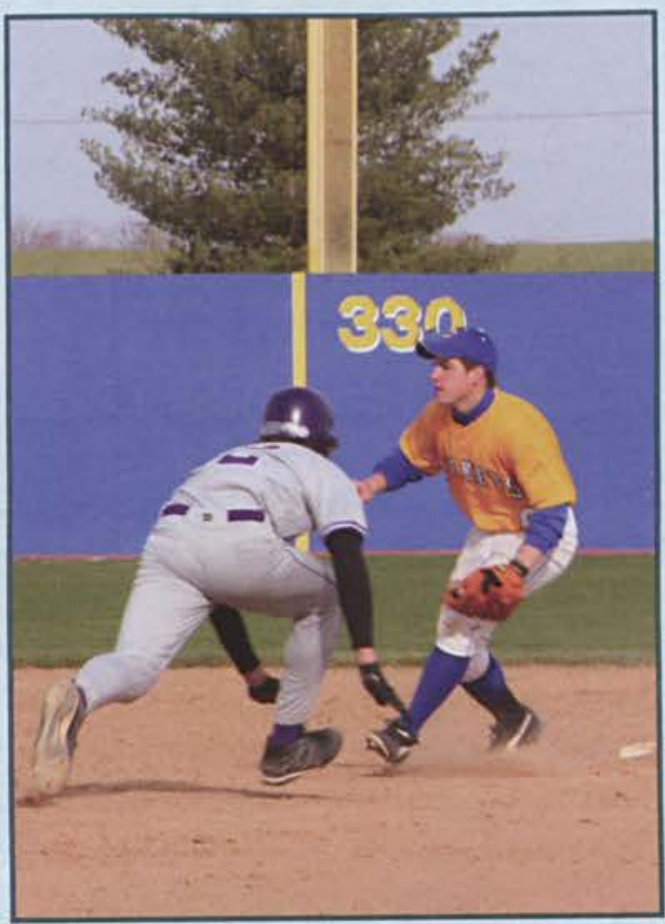

1 A member of the baseball team tags his apponent out as he tries to take another base.

A Jordan Shumaker, freshmen, gets ready to hit one out of the park during a home game.

- A member of the baseball team slides into third during their first game at Fifth-Third field in Dayton.

$\checkmark$ Phil Buben, junior, surveys the teams standing as he heads back out to his possition on the field.
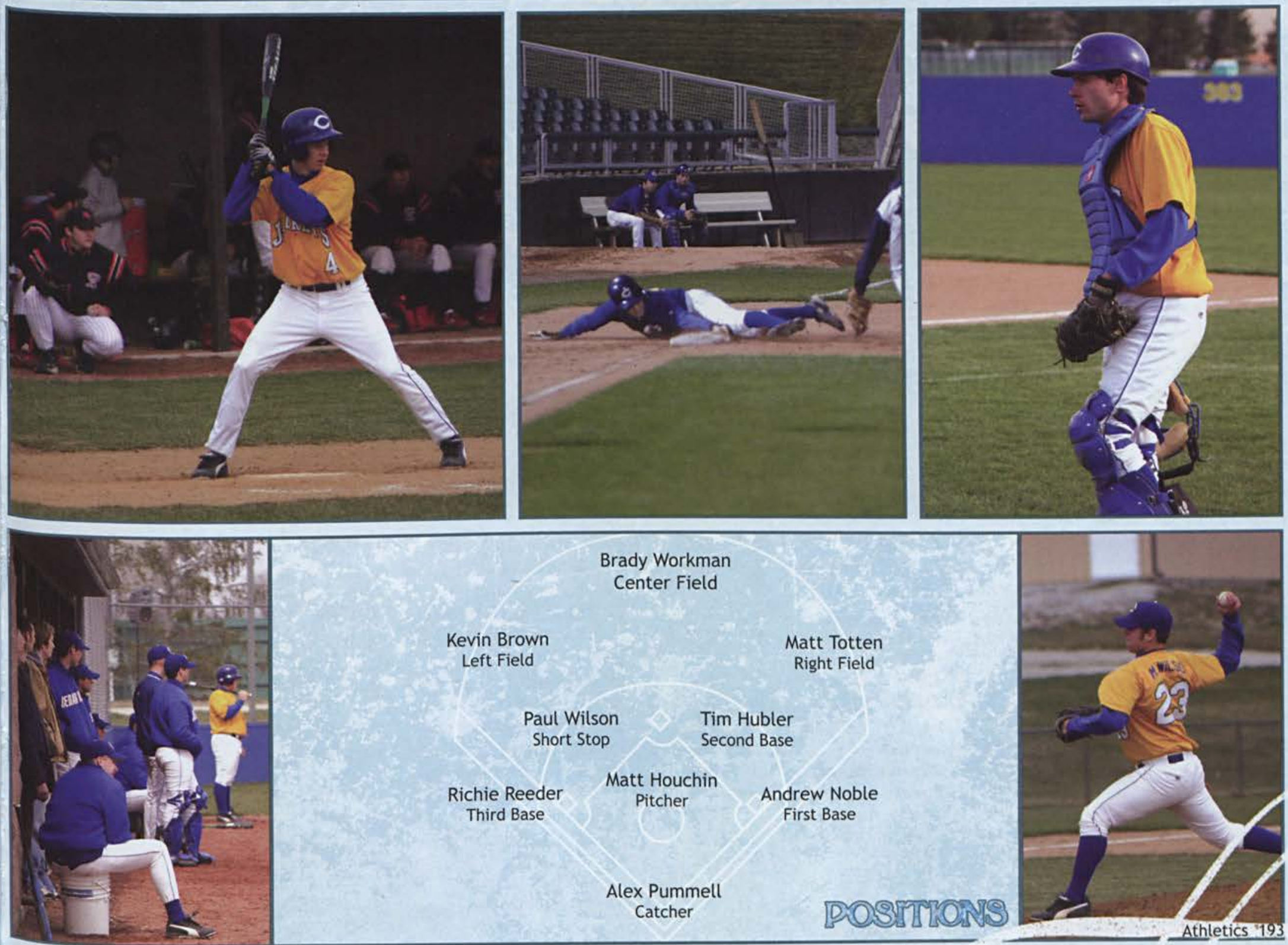

Brady Workman

Center Field

Kevin Brown

Left Field

Paul Wilson Short Stop

Richie Reede

Third Base
Matt Houchin

Pitcher

Andrew Noble

First Base
Matt Totten

Right Field

Tim Hubler

Second Base

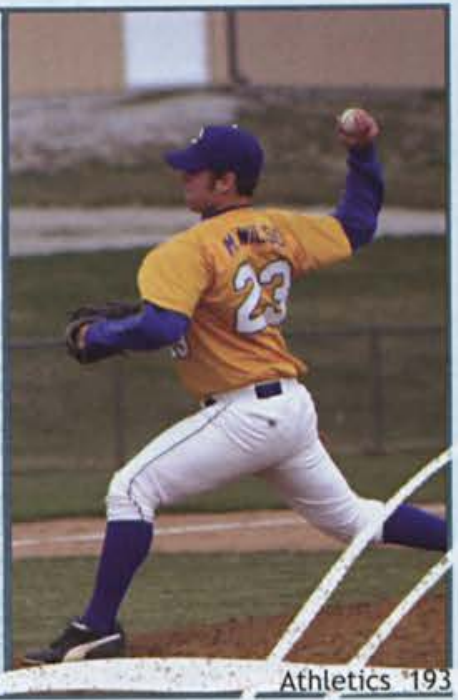




\section{Softball}
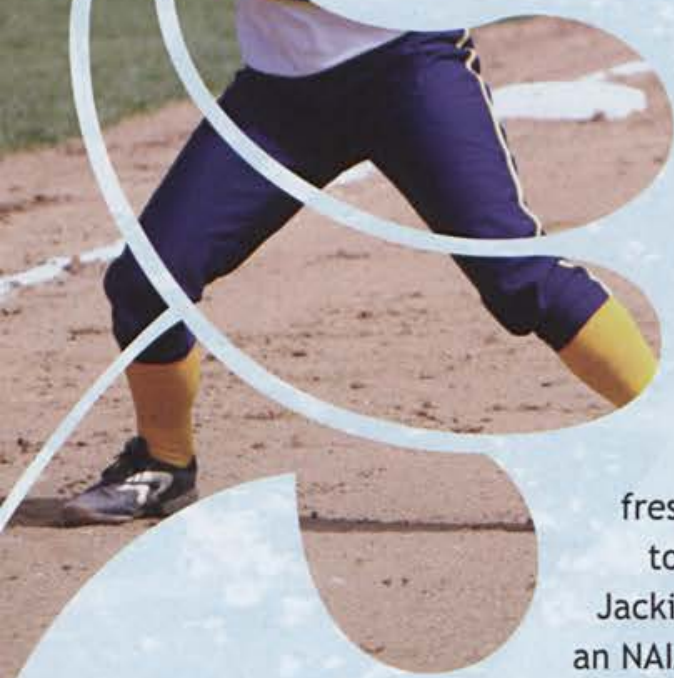

A little known yet integral part

of the Lady Jackets sports here at Cedarville University is the women's softball team. This talented team of 13 women is coached by Sue Carpenter, who returned for the sixth year as head coach.

The team welcomed five incoming freshmen to the group and said goodbye to three seniors. Outfielder and senior Jackie Greetham entered the season with an NAIA All-American Scholar-Athlete and a .344 batting average, making her the second leading hitter on the team. During the NCCAA Midwest regionals on April 22, Jackie Greetham, Erin Dimeolo, and Andrea Walker gained spots on the all-tournament team. Erin was also named to the All-NCCAA Midwest Region squad as were Erica Schroeder, Aubree
The two other senior players were Allie Smith and Erin Dimeolo. Allie was an All-American Mideast Conference South Division Second Team choice as a pitcher with a 9-5 record and a 1.71 earned run average as a junior last year. Erin finished as a third year catcher who posted a .306 batting average last spring.

Erin Dimeolo said of the team, "The season has been a ton of fun. We had new girls added to the team, and each of them has really stepped up and has had a tremendous season. Being on the team has meant that softball is pretty much my life along with school work, but it is a good part of my life. Not only do we get to go out and play a sport that we love, but we get to share the love of Jesus while were at it by giving cards with our testimonies on them with a gift of candy to the opposing team."

Munson, and Allie Smith.

Front row: Emily Millay, Charissa Rowe, Jackie Greetham, Erin DiMeolo,

Hillary Skrabacz, Amanda Einselen.

Middle row: Assistant Coach Dave Stewart, Team Chaplain Kathy Freese, Student Manager Tiffany Mason, Allie Smith, Aubree Munson, Head Coach Sue Carpenter, Head Assistant Coach Jay Skrabacz.

Back row: Erica Schroeder, Kim Bowman, Chelsea Howard, Sarah Hoffman, Kandis Armstrong, Andrea Walker.

\section{2-24 Overall 6-8 American Mideast Conference South Div.}
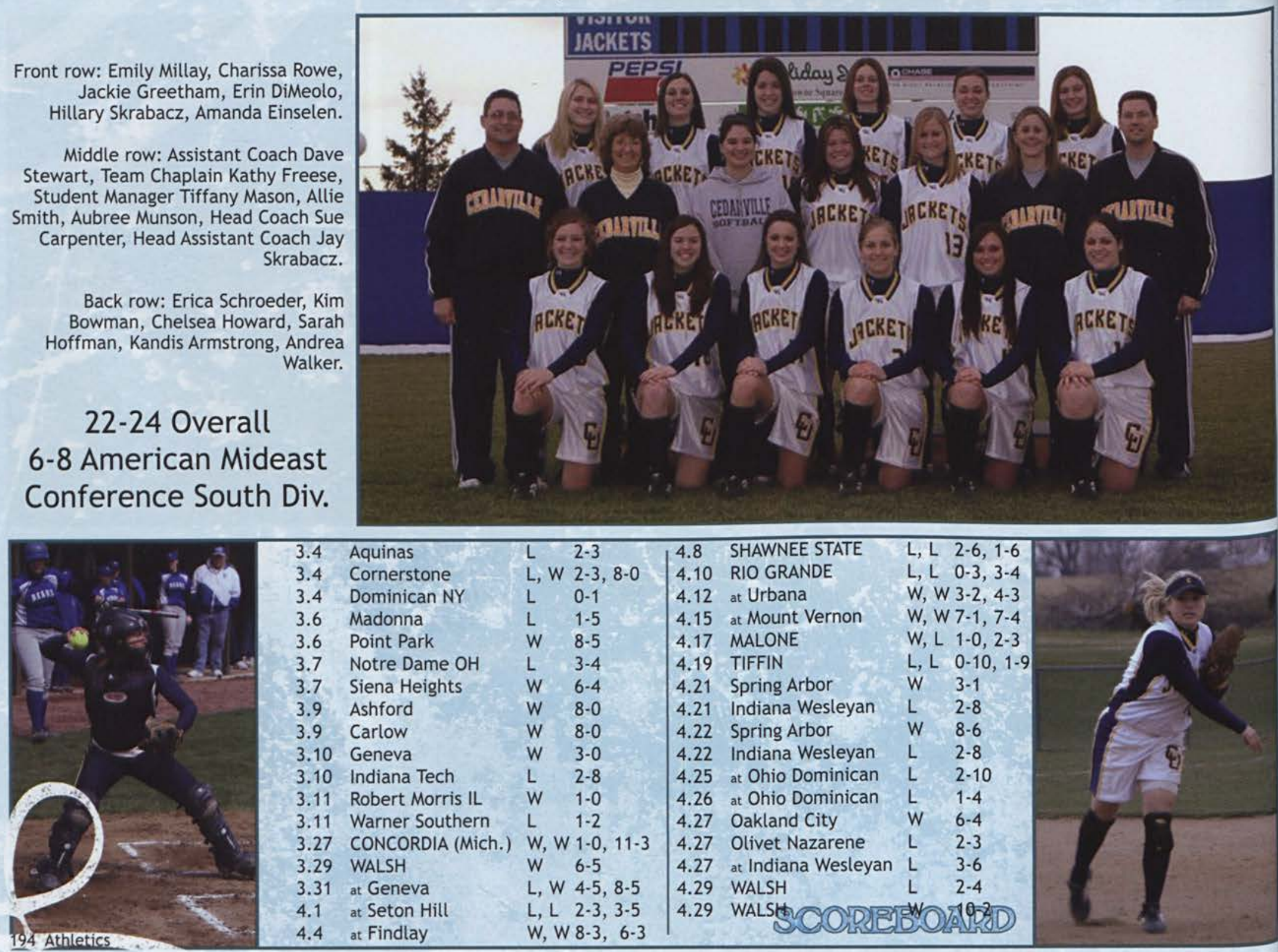

$\begin{array}{llll}3.4 & \text { Aquinas } & \text { L } & 2-3 \\ 3.4 & \text { Cornerstone } & \text { L, W } & 2-3,8-0 \\ 3.4 & \text { Dominican NY } & \text { L } & 0-1 \\ 3.6 & \text { Madonna } & \text { L } & 1-5 \\ 3.6 & \text { Point Park } & \text { W } & 8-5 \\ 3.7 & \text { Notre Dame OH } & \text { L } & 3-4 \\ 3.7 & \text { Siena Heights } & \text { W } & 6-4 \\ 3.9 & \text { Ashford } & \text { W } & 8-0 \\ 3.9 & \text { Carlow } & \text { W } & 8-0 \\ 3.10 & \text { Geneva } & \text { W } & 3-0 \\ 3.10 & \text { Indiana Tech } & \text { L } & 2-8 \\ 3.11 & \text { Robert Morris IL } & \text { W } & 1-0 \\ 3.11 & \text { Warner Southern } & \text { L } & 1-2 \\ 3.27 & \text { CONCORDIA (Mich.) } & \text { W, W } 1-0,11-3 \\ 3.29 & \text { WALSH } & \text { W } & 6-5 \\ 3.31 & \text { at Geneva } & \text { L, W } 4-5,8-5 \\ 4.1 & \text { at Seton Hill } & \text { L, L } 2-3,3-5 \\ 4.4 & \text { at Findlay } & \text { W, W } 8-3,6-3\end{array}$

\begin{tabular}{|c|c|c|c|}
\hline & &,$L$ & 2-6, \\
\hline 4.10 & RIO GRANDE & $\mathrm{L}, \mathrm{L}$ & $0-3,3$ \\
\hline .12 & at Urbana & W, W & $3-2,4$ \\
\hline 15 & at Mount Vernon & W, W & $7-1,7$ \\
\hline 7 & MALONE & W, L & $1-0,2$ \\
\hline 19 & TIFFIN & L, L & $0-10$ \\
\hline 21 & Spring Arbor & W & $3-1$ \\
\hline 21 & Indiana Wesleyan & L & $2-8$ \\
\hline .22 & Spring Arbor & W & $8-6$ \\
\hline 6 & Indiana Wesleyan & L & $2-8$ \\
\hline .25 & at Ohio Dominican & L & $2-10$ \\
\hline 4.26 & at Ohio Dominican & L & $1-4$ \\
\hline 4.27 & Oakland City & W & $6-4$ \\
\hline 4.27 & Olivet Nazarene & L & $2-3$ \\
\hline 4.27 & at Indiana Wesleyan & L & $3-6$ \\
\hline & WALSH & $\bar{L}$ & $2-4$ \\
\hline & & & $10 ?$ \\
\hline
\end{tabular}

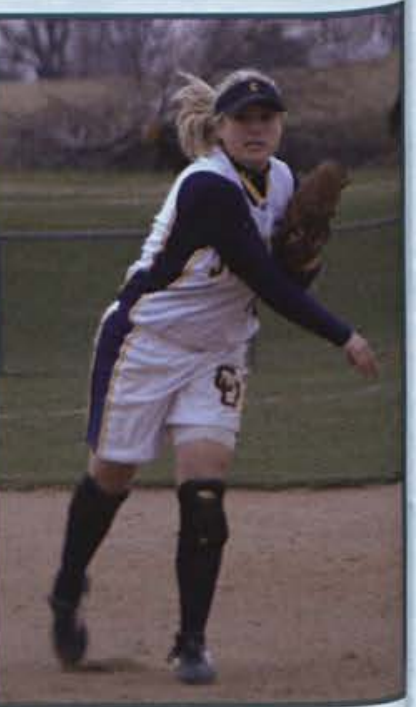



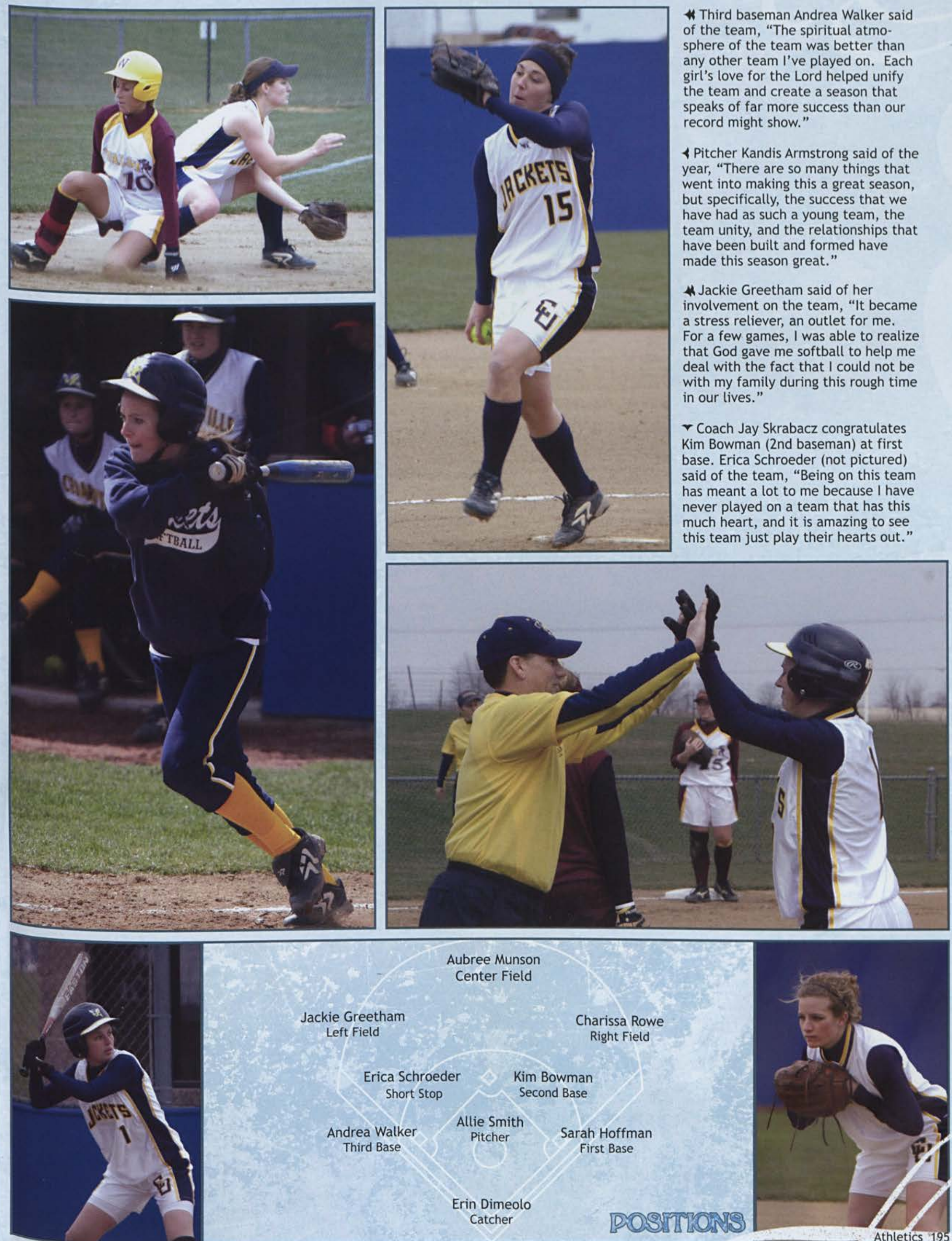

Jackie Greetham

Left Field

Erica Schroeder Short Stop

Andrea Walker

Third Base
Aubree Munson

Center Field

$$
\text { Al }
$$

Allie Smith Pitcher

Kim Bowman

Second Base

Charissa Rowe

Right Field

Pitcher Kandis Armstrong said of the year, "There are so many things that went into making this a great season, but specifically, the success that we have had as such a young team, the team unity, and the relationships that have been built and formed have made this season great."

4 Jackie Greetham said of her involvement on the team, "It became a stress reliever, an outlet for me. For a few games, I was able to realize that God gave me softball to help me deal with the fact that I could not be with my family during this rough time in our lives."

$\checkmark$ Coach Jay Skrabacz congratulates Kim Bowman (2nd baseman) at first base. Erica Schroeder (not pictured) said of the team, "Being on this team has meant a lot to me because I have never played on a team that has this much heart, and it is amazing to see this team just play their hearts out." 
$\checkmark$ Katie Nicholl is just one of the thousands of students who contributed their own money to send toward Hurricane Katrina Relief efforts during SGA's special offering.

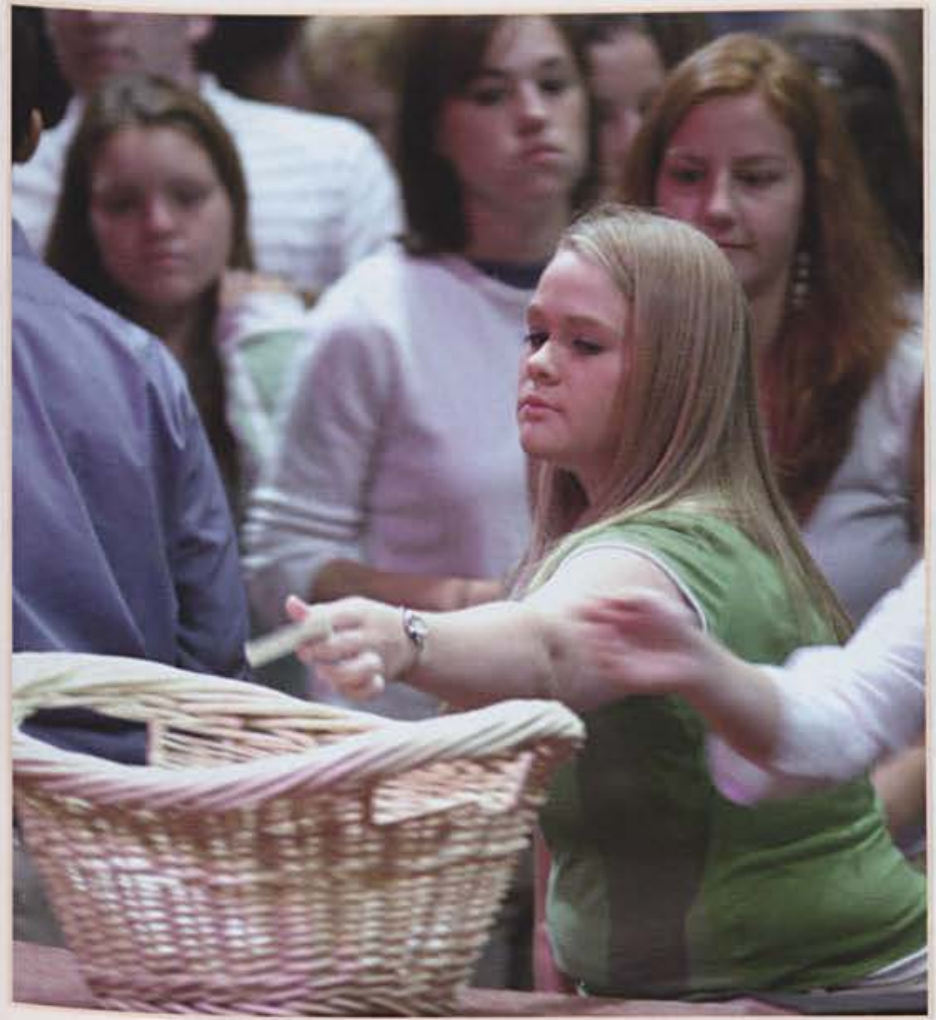

F A student observes the progress in the next room by peering through the large hole he has torn in the center of an unsafe wall.

$\checkmark$ Bethany Turner serves up some bacon for breakfast during one day's clean-up efforts.
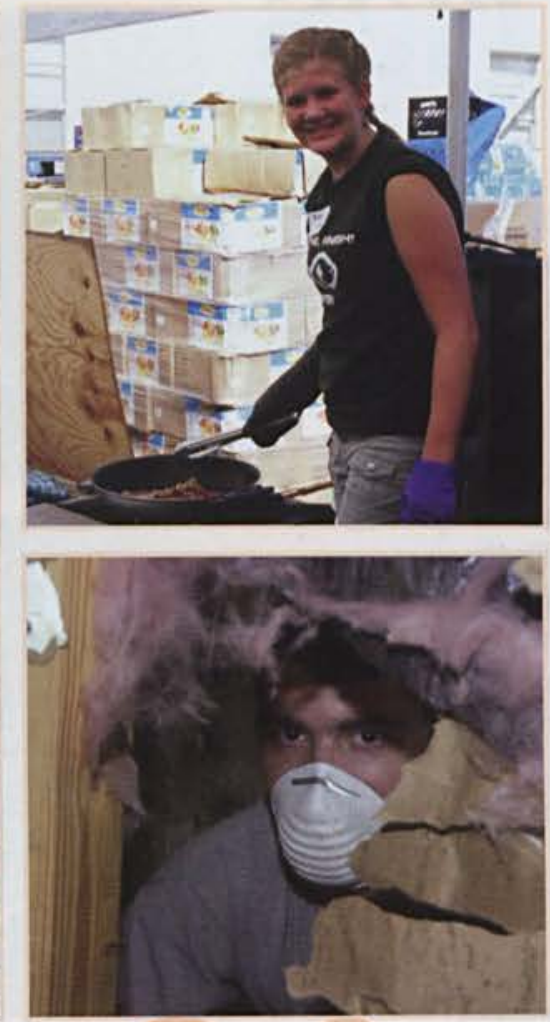

$\checkmark$ Cornel TerreBlanche helps tear down the rotting wall in someone's home. The crumbling tiles are the next to go.

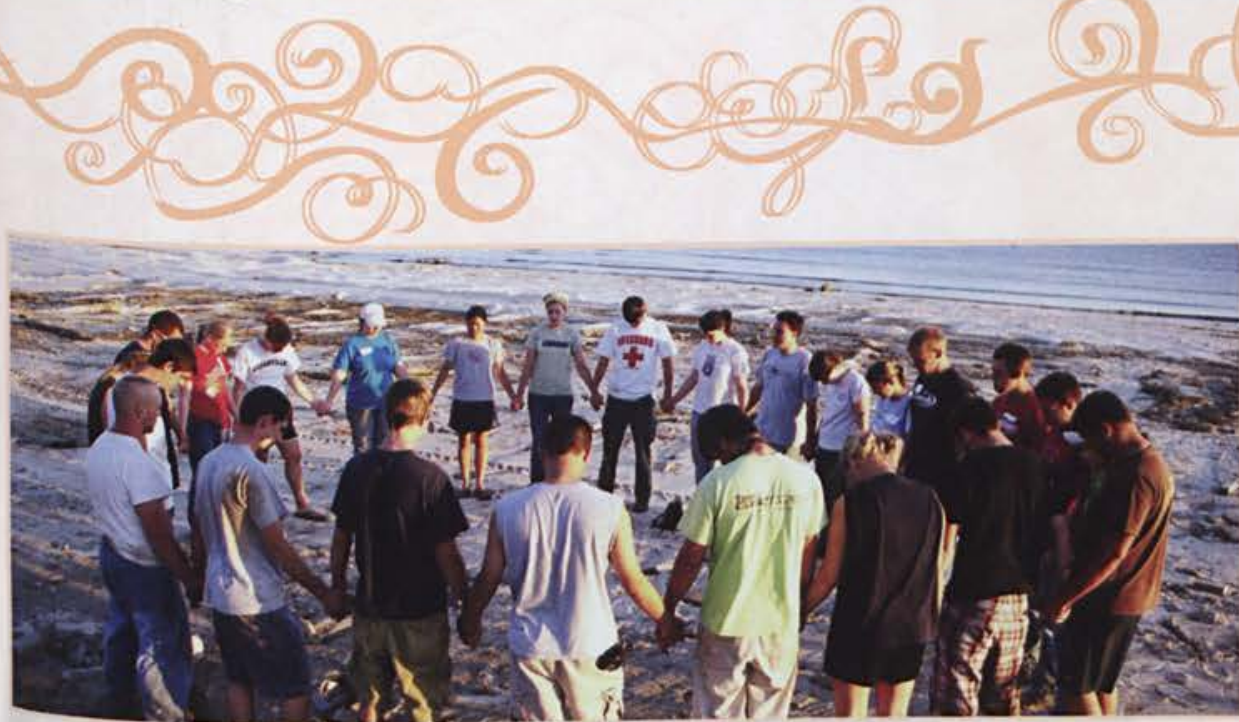

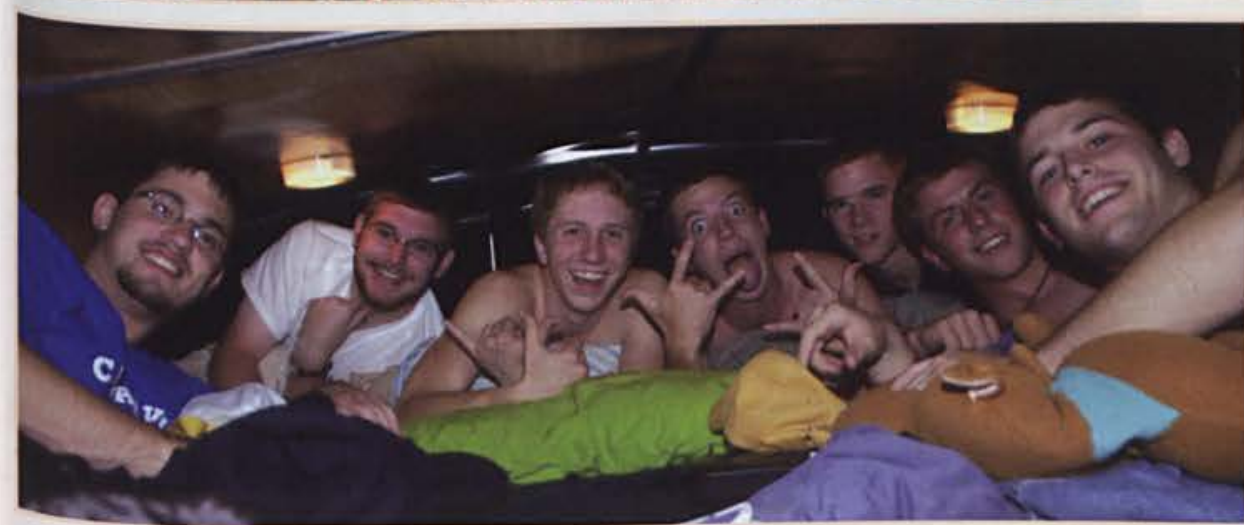

Before arriving to help with Katrina relief, Joel Lagan and friends chill together during the long bus ride south.

A trip to the beach at Spring Break isn't all that unusual, but for the 100 students who headed to New Orleans to aid clean-up efforts, a prayer vigil on the beach was the perfect conclusion to the week's activities.

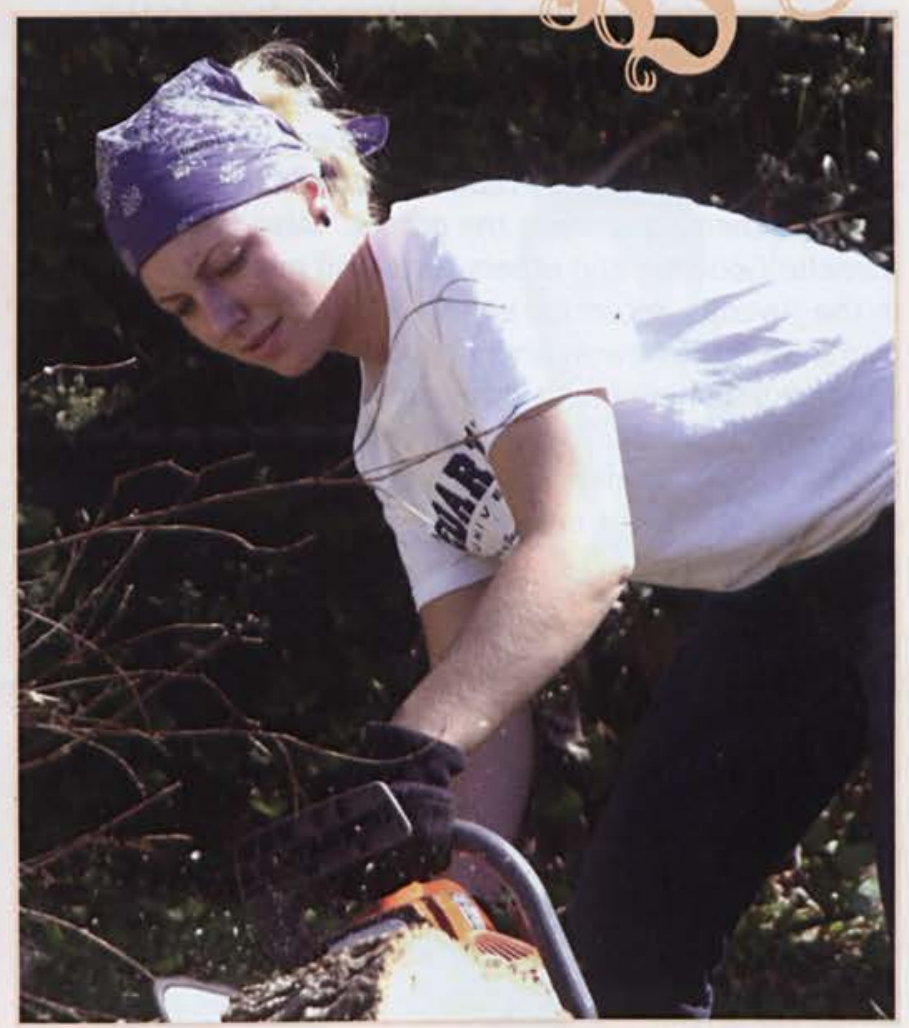

$\Lambda$ Despite the heat, Kristen Cato saws off a piece of tree trunk that had, until late, been lodged on someone's property. 


\section{Community}

F Sandra Lescure and Sue Clarkston are members of the Nehemiah University Chapel team. "We usually call ourselves the 'Sunday Team' to the Dayton Gospel Mission."

$\checkmark$ Nic Fridenmaker, Hannah Pilgrim, and Lauren Berry work with the Truth and Training group at Awana at Calvary Baptist Church in Springfield. These Cedarville students help kids memorize verses and teach them basic lessons Ministries

Cedarville University offers many ministries to students in order for us to engage our world for Christ. Whether working with children in AWANA programs, visiting patients in the hospital and residents at nursing homes, or furthering the education of those in churches around us, Cedarville students use their God-given abilities to reach out to those around them.

One of the biggest community ministries on campus is AWANA. Students work in churches around the area such as Calvary Baptist and County Line Baptist Church. God has been moving through Cedarville students this year by impacting children for the Lord. Nathaniel Miller's AWANA group at Crossroad Baptist Church in New Carlisle started a children's choir. Many children have come to a saving knowledge of Christ through the ministry of Cedarville students in AWANA.

Every Sunday morning Cedarville students can be found in churches helping children with their Scripture memorization, building relationships, teaching English to Chinese residents of the Dayton area, or providing music for church services.

However, God is using Cedarville students outside of the church as well. "We have developed some great personal relationships with the residents," said junior Sarah Ensslen, a ministry volunteer to the residents of Liberty Nursing Center in Xenia. Timothy Rosie, who visited a nursing home weekly, shared the love he had for his fellow team. "You can see the love of Christ in them as they reach out to the new people, befriend them, and share the gospel." Also in Xenia, Danielle Goodling and others tutor and mentor those in the juvenile detention center. Michelle Boudreau also shared the new ministry opportunities that opened up in her church this year in Dayton: "Twice a month the youth can come on Saturday nights and listen to Christian music, watch skits, hear testimonies, and provide an open mike where the kids can show their talents."

It is apparent to all that God is working both in and through Cedarville students, touching the lives of children, teens, and adults all across the Miami valley.

is
from the Bible.
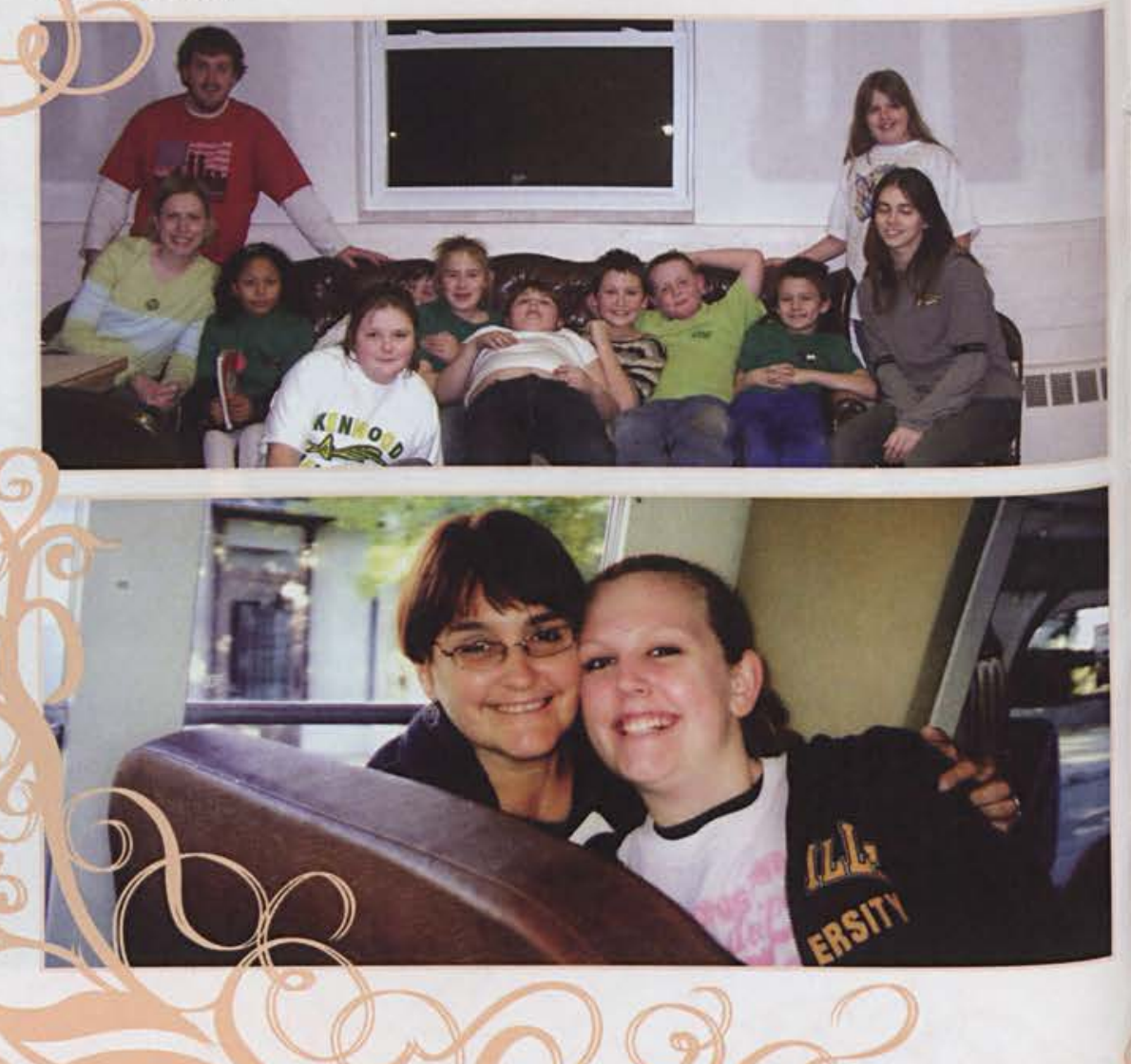
F Kari Gannon shares the gospel in downtown Dayton at the Dayton Gospel Mission.

$\checkmark$ Tarah Boone and Jessica Gallas work with Revolution Blowout. "We had a retreat for the girls. They stayed in the dorm, and then we took them to Revolution Blowout!" reflected Tarah.

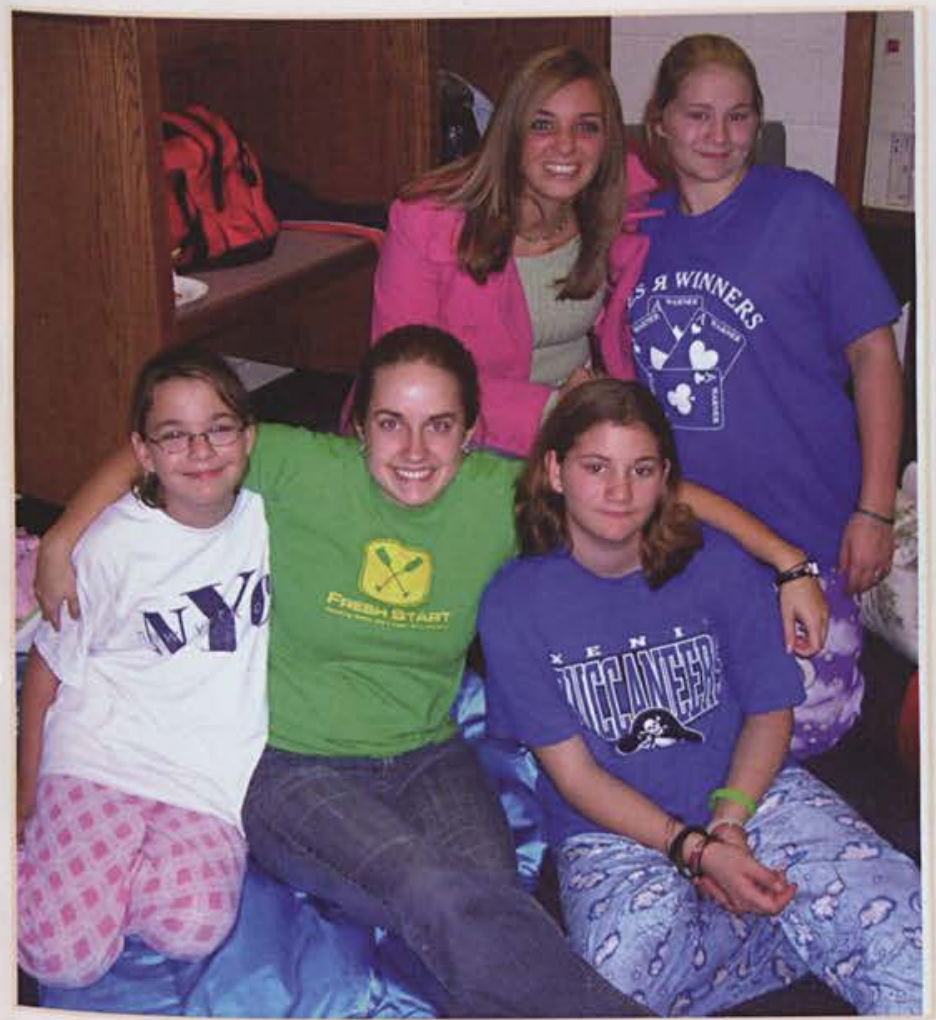

$\checkmark$ Melanie Brady embraces some of her Cubbies at Grace Baptist Church in Cedarville.
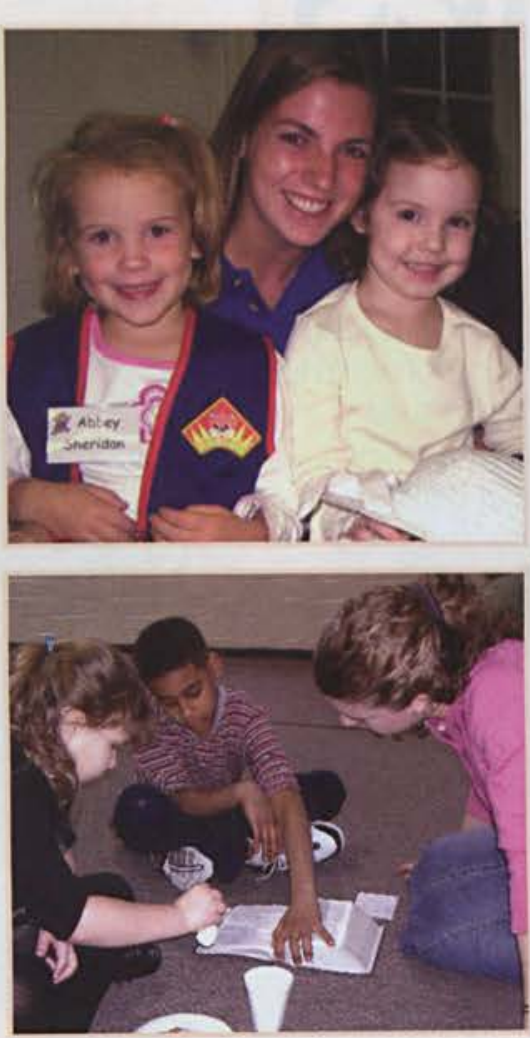

$\checkmark$ Bev Clark and Tarah Boone have a great time ministering to the young girls through the Revolution Blowout ministry.

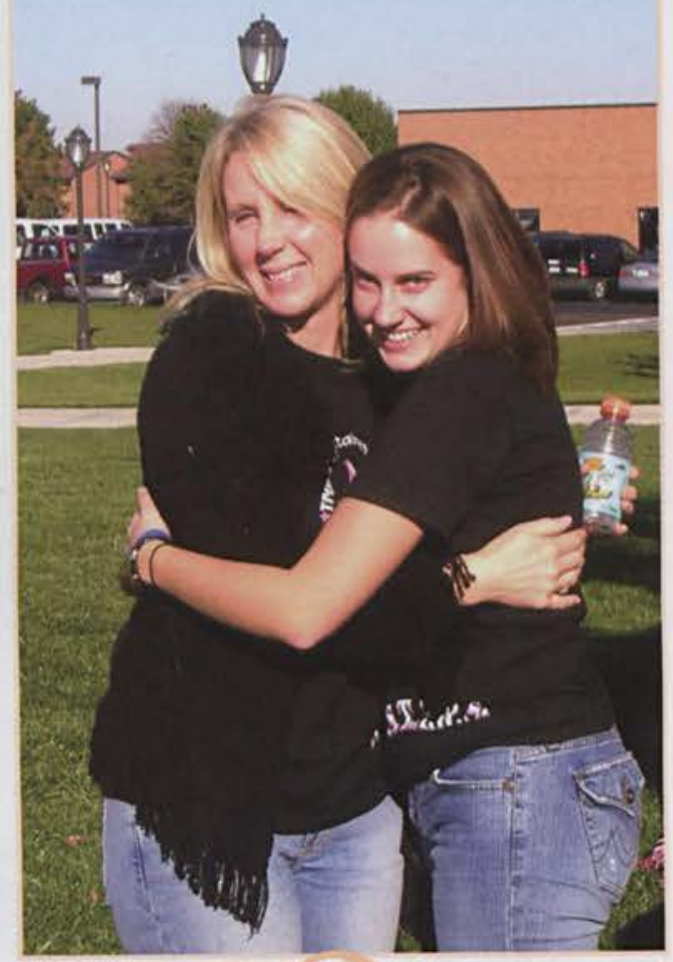

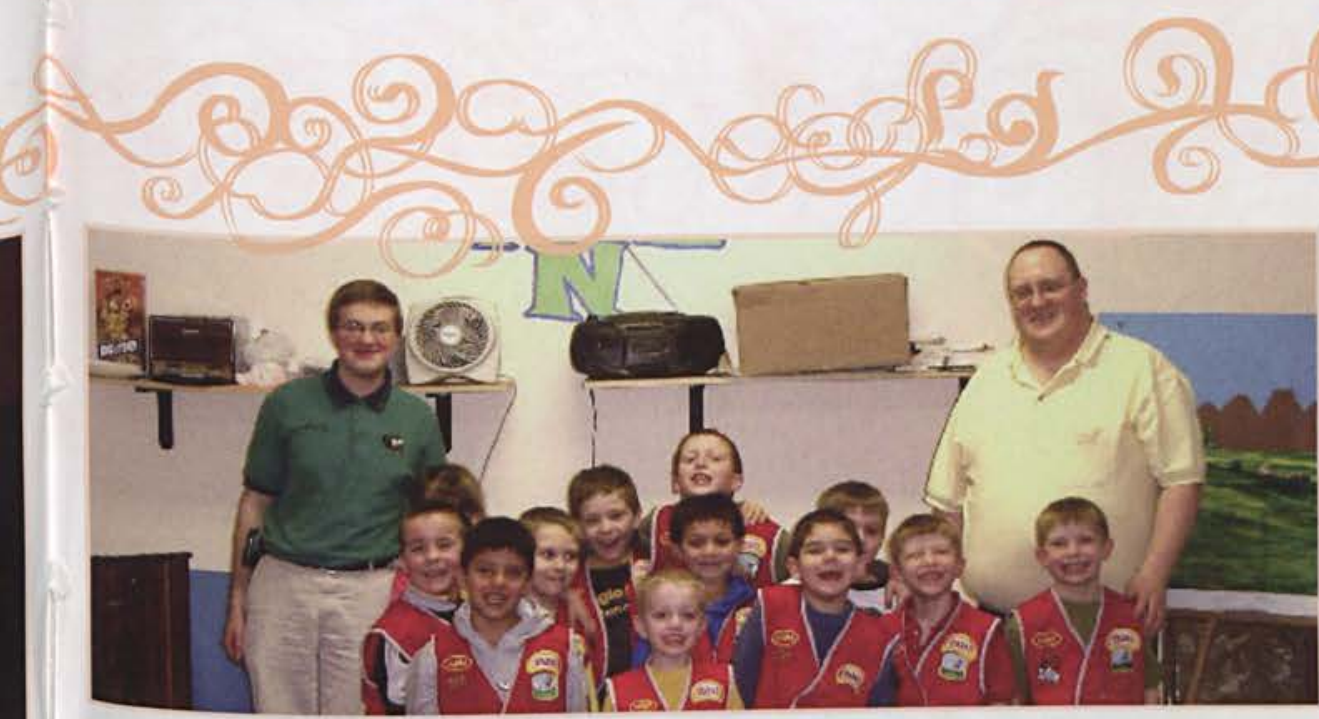

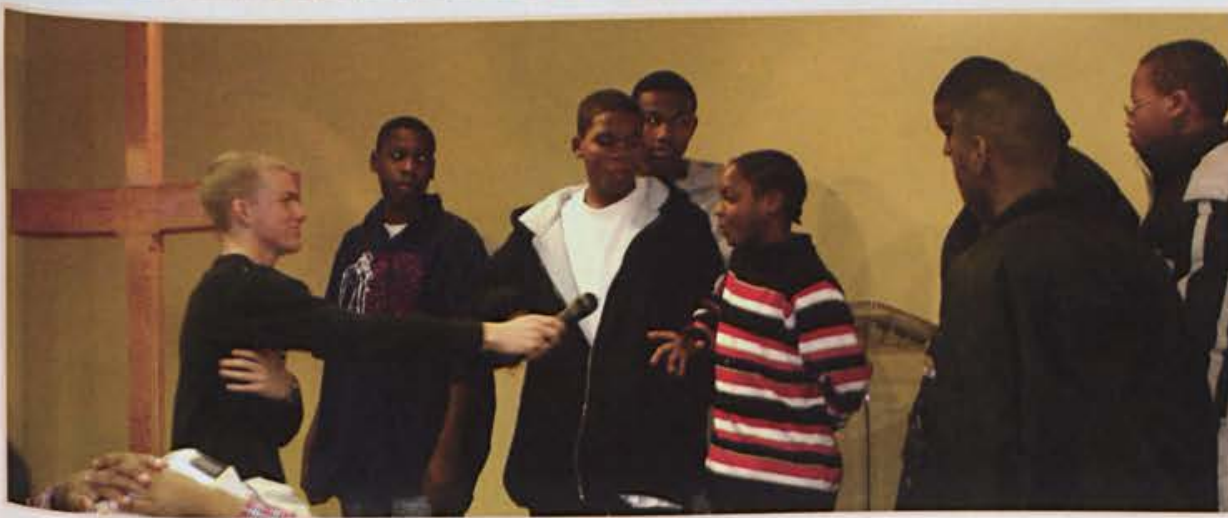

Ryan Samuelsen, pictured with his Sunday school class, report what they learned that morning to their church body, All Nations Bible Fellowship.

Nathaniel Miller, AWANA group leader, works with the Sparks on Wednesday nights at Crossroad Baptist Church in New Carlisle.

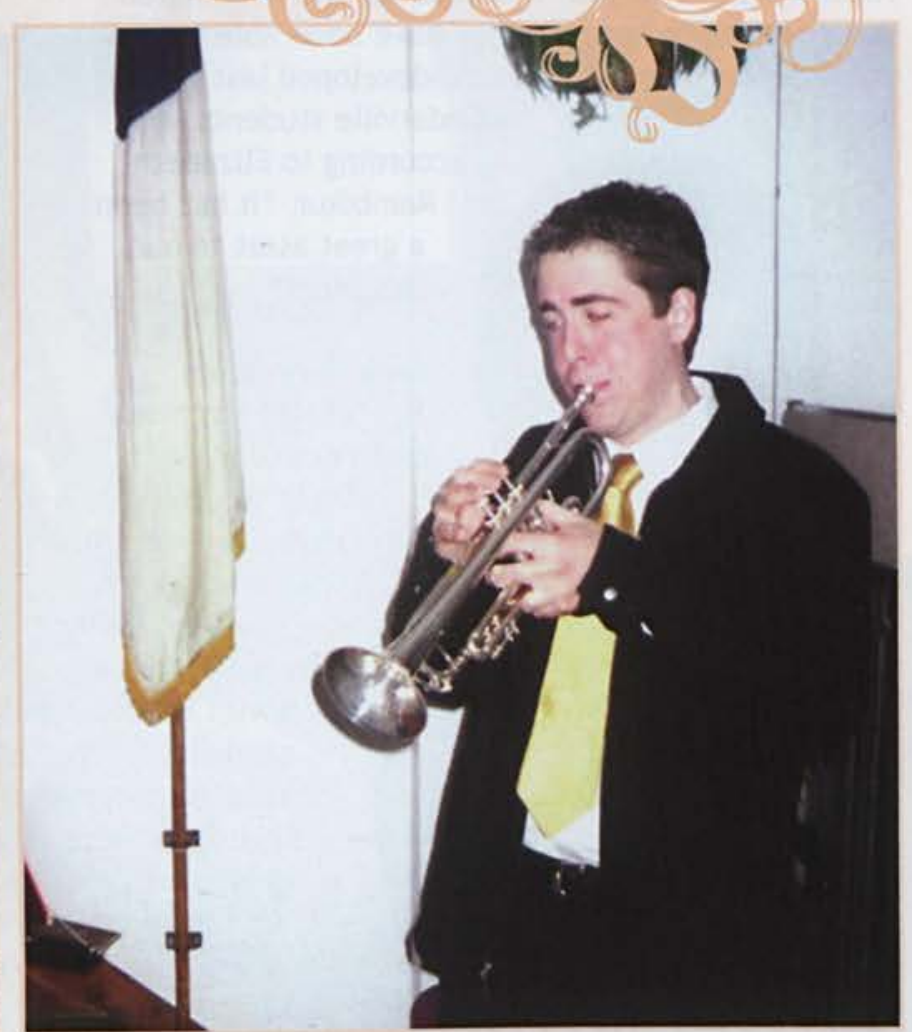

^ Senior trumpet player Emmett Robinson ministers to his church family by playing during the church services. "I enjoy playing special music for the Sunday morning service at the Dayton Gospel Mission," says Emmett. 


\section{Cross-Cultural}

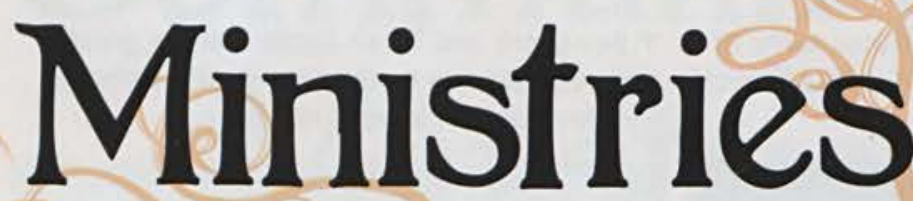

$\checkmark$ Melody Blackburn, freshman member of the Wilmington Hispanic Outreach, shares a children's story. Ministry leader, Sophomore Elizabeth Rambikur, said, "In the past, our average student was a middle range male, but this year we have had friends, spouses, young adults, children, and adolescents."

Cedarville has a foothold in a broad range of ministry areas - churches, soup kitchens, detention centers, even hospitals and nursing homes -- but of all these, cross-cultural ministries are probably the most unique. These ministries include teaching English to immigrants of varying English fluency as well as traveling to various parts of the world on MIS trips as a means to build bridges across cultures and share the gospel.

Jessica Annable, a senior and ministry leader of the Dayton Chinese Church TESL Ministry, said, "We're teaching English to many members of the church, community, and even visitors from China! We're also building great relationships with them."

"They are very open to talk with anyone and are willing to share their life story with you," said senior Joshua Blackburn, co-leader of the Wilmington Hispanic Outreach as he related his enthusiasm about the ministry and the impact it has had on both Cedarville students and tutoring students. "Each week we work with students individually, trying to teach them the next level of English grammar and vocabulary." This year is unique for the Wilmington Outreach teams because they have been able to implement a clear curriculum, developed last year

by Cedarville students, and according to Elizabeth Rambikur, "It has been a great asset to our teaching!"

\section{A ministry called}

Zion's Light has also been active in the community. This ministry worked with Jewish immigrants who came primarily from the former Soviet Union. The students tutored and taught conversational English to these Jews while attempting to build relationships that would eventually open up opportunities to share about the Messiah. As its leader Nate Smith said, "The job of these individuals is to display Jesus in word, deed, and

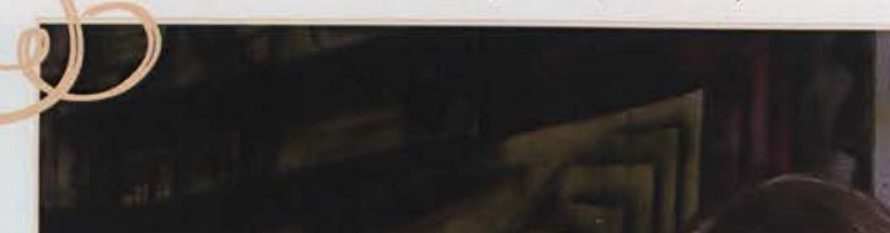

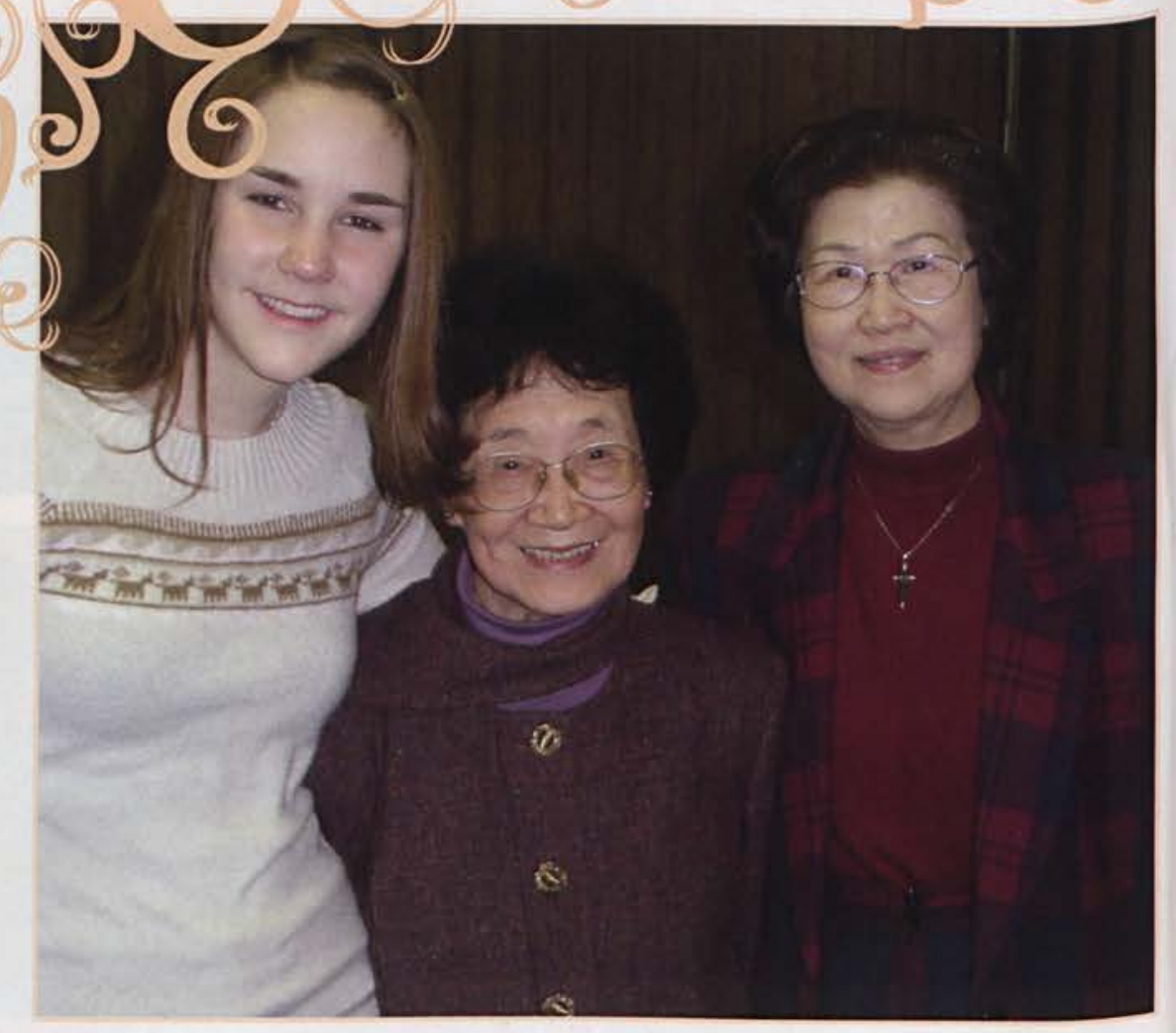

¿ Senior Jessica Annable, leader and veteran member of the Dayton Chinese Church TESL ministry, reflects on a good year: "God has really blessed us. We can always use more members, but those who attend really give us their all." 
$\checkmark$ Senior Steph Hueni works with local children during her missions trip.

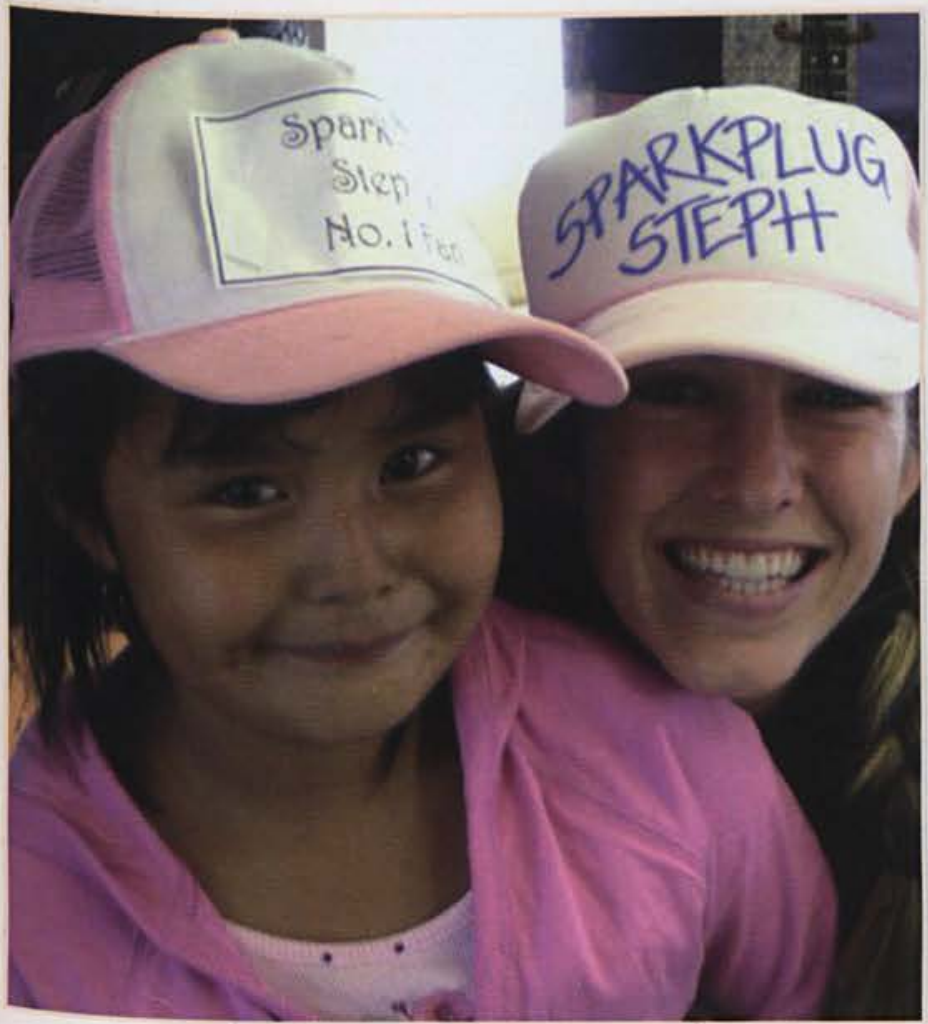

$\checkmark$ A nursing student helps administer medication during her missions trip to Togo Africa.

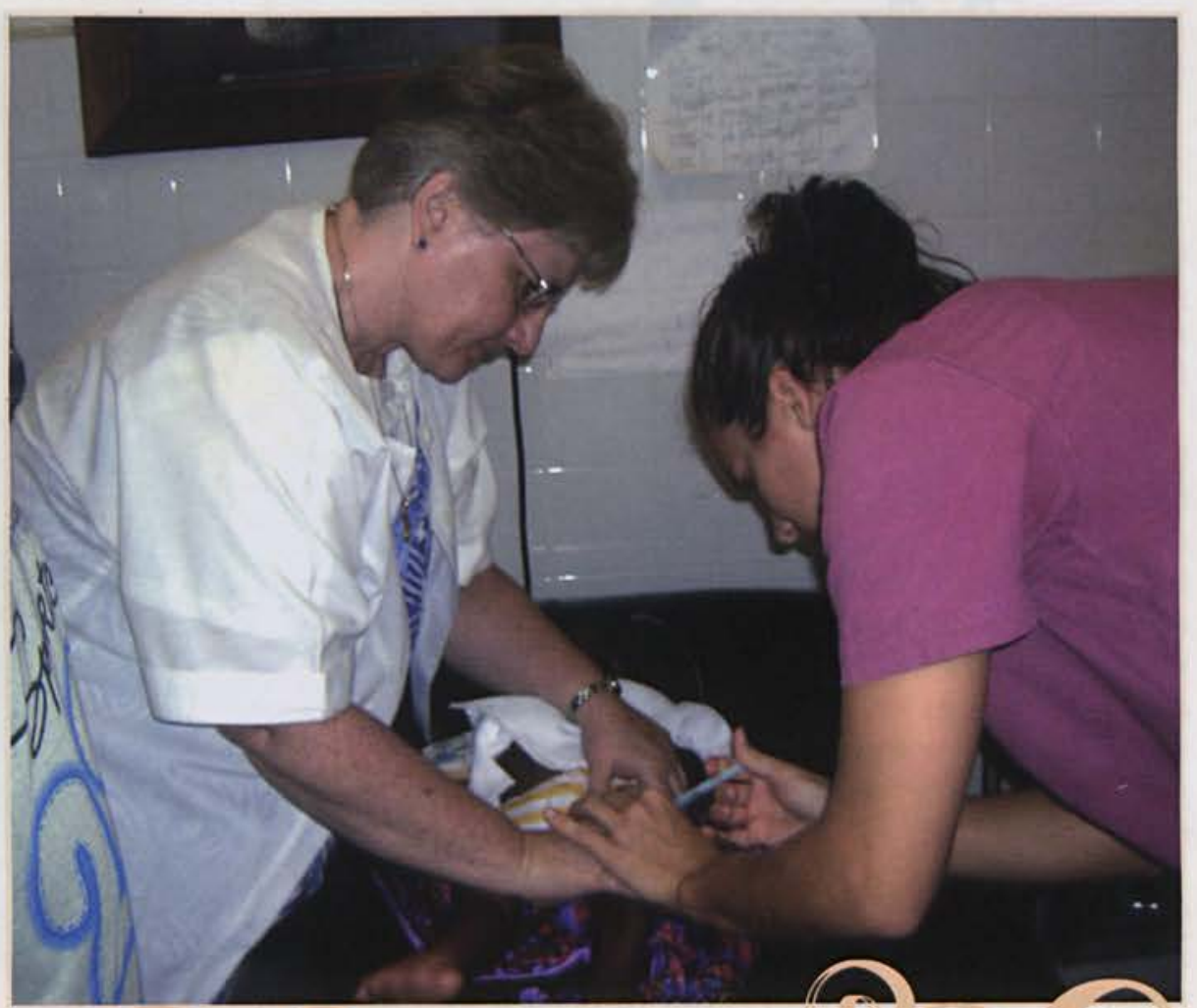

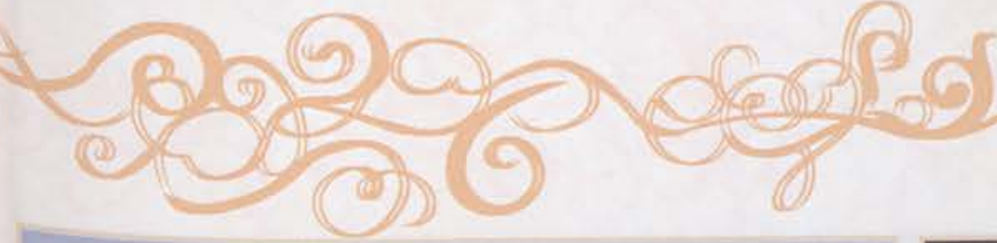
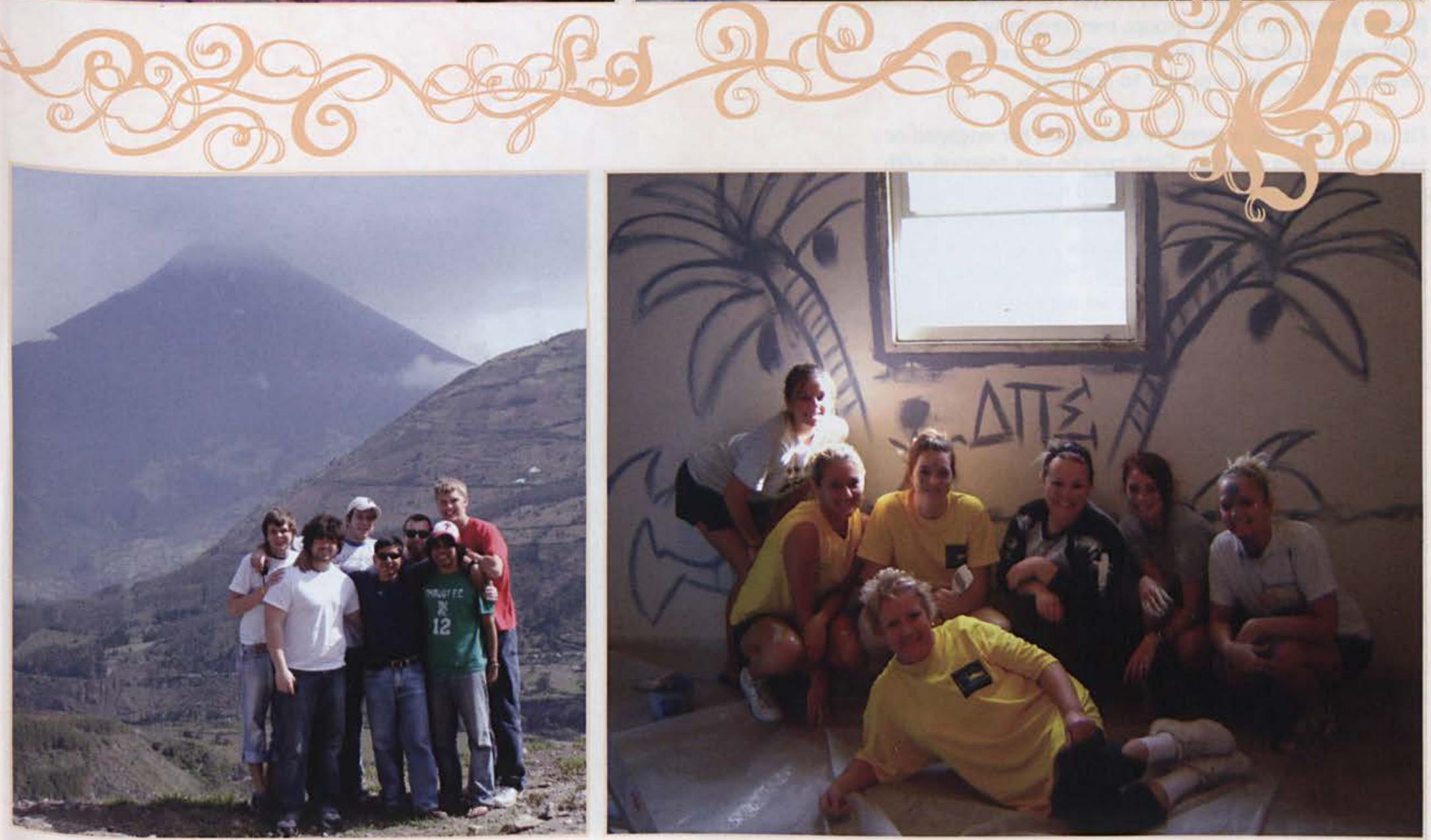

Guys from SPL pose for a picture during their missions to $\Delta$ The girls from Delta Pi Sigma get creative with their painting job during their missions trip to Eleuthera. 
$\checkmark$ Rebecca Patterson leads Whitney Oosterhouse, Amber Janssen, Mallory Fisher, and the other girls of her discipleship group. Mallory Fisher speaks for the entire group as she exclaims, "Of course we read the chapter!"

The purpose of Discipleship Ministries was to "foster spiritual growth and life change on campus through the means of small groups and mentoring relationships." We could see this done through discipleship small groups, prayer groups, and the Fit to be Tied program.

In September, students were given the option of becoming part of a small group, and this year, more than 80 students chose to be leaders of small group Bible studies. With more than a dozen studies offered, the topics ranged from sex to the Fruit of the Spirit. Senior Clarissa Linde said, "I love being able to encourage and love on the girls in my Bible study, even in simple ways like sending them a card through the post office or providing food at Bible studies."

One of the most powerful ways we could support and encourage each other was through prayer, which was what Elijah's Fire, Prayer Force, and the Chapel Prayer Force did. These groups met regularly to uplift each other, their fellow students, and the campus as a whole in prayer to our God.

Fit to be Tied was a mentoring program for engaged or seriously dating couples. Each couple was teamed with a faculty or staff couple who would meet with them throughout the year to discuss issues and questions raised in the group seminars. Junior Natalie Plunket and her fiancée Chad Winder said, "Fit to be Tied has been a great experience as we anticipate our wedding. We have become closer with one another and with God through the seminars and in meeting with our mentors."

Through each of these programs, Discipleship Ministries attempted to plug each student into a small group or accountability situation where they could individually grow in their walk with Christ as well as have an impact on the students around them. In the years that these ministries have been on campus, they have left a tremendous impression on the student body and will continue to do so for years to come.

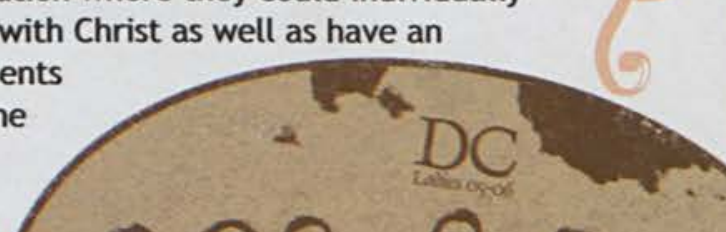


Dan Gier, Evan Chiu, Phil Buben, Chad Imgrund, Dale Houser, Tyler Vawser, and Josh Schenk gather for their discipleship group meeting.

$\checkmark$ Stephanie Hueni and Katelyn Talbott take a break to enjoy the sun on the Discipleship Council's trip to Arizona.
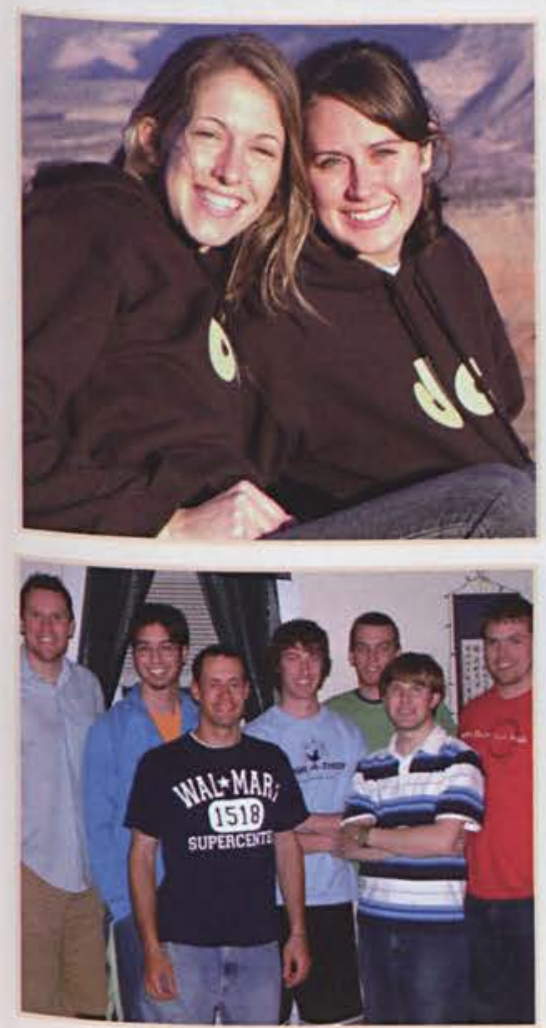

$\checkmark$ Lauren Hanna, Kim Greer, Janelle Moffitt, Emily Mohler, Ivey Harrell, Kara Sneed, Lauren McGrew, Angela Rill, and Alathea Young take a break from their Bible study and run to Beans ' $n$ Cream for coffee. Junior Lauren McGrew feels that "the Bible study was great, and it was amazing to see how we all grew over the course of the year, but it was also amazing to see how a group of girls from different dorms, classes, and majors who might have never crossed paths developed such deep and lasting friendships."
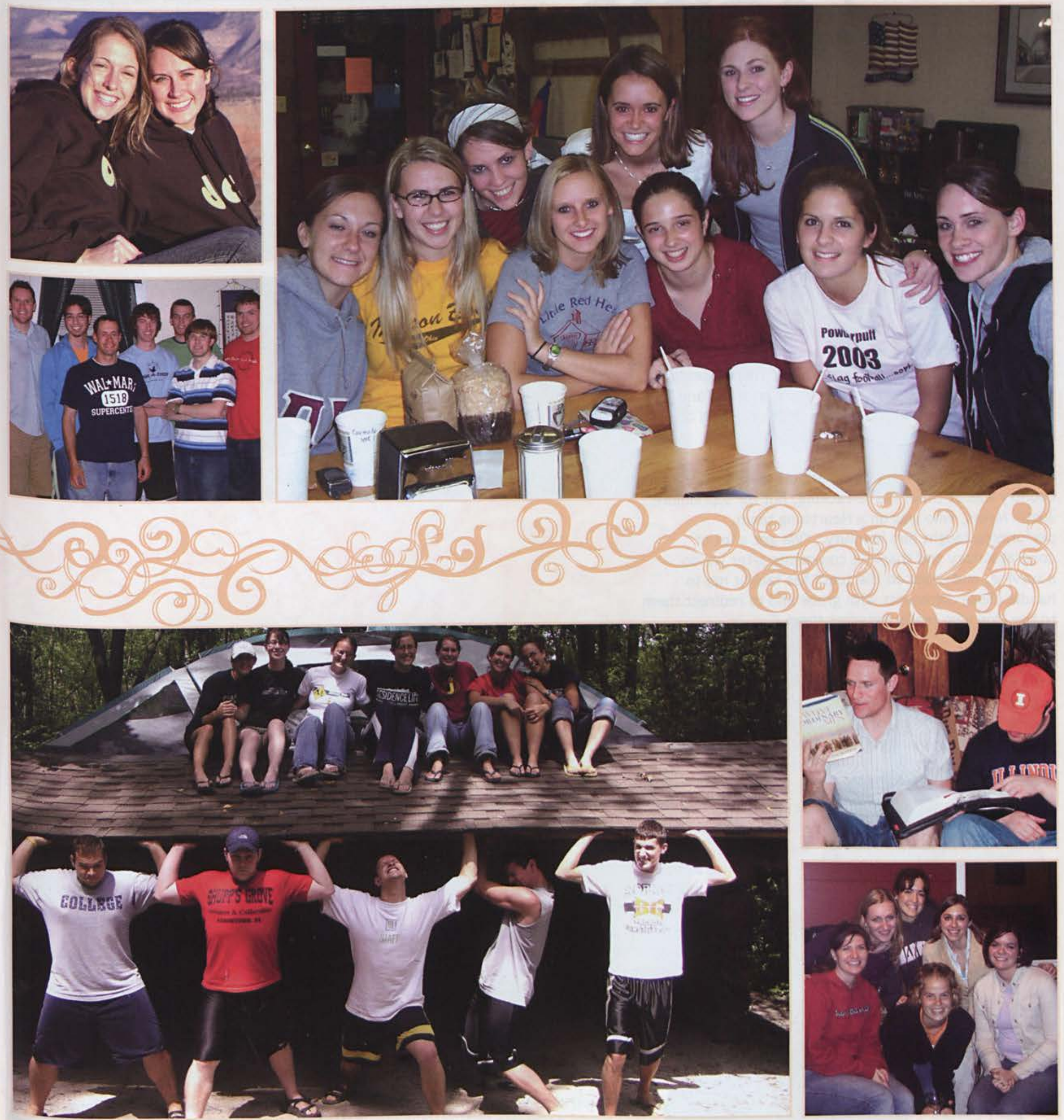

Phil Blodgett, Adam Leffel, Dan Keslar, Dan Gier, and Mike Standish show off those hard-earned PACL

muscles by raising the roof of the Discipleship Council's shelter during their fall camping trip at John Bryan.

Meanwhile, Stephanie Hueni, Elizabeth Slavish, Clarissa LInde, Rachel Miller, Rachel Talbott, Katie Krumeich, and Danielle Colao laugh off their attempts.

^ Michal Friend, Andrea Moore, Bonnie Wright, and Ashley Elliott get together for discipleship group.

$\star$ Dan Gier leads his discipleship group in their study of "Twelve Ordinary Men." 


\section{Touring}

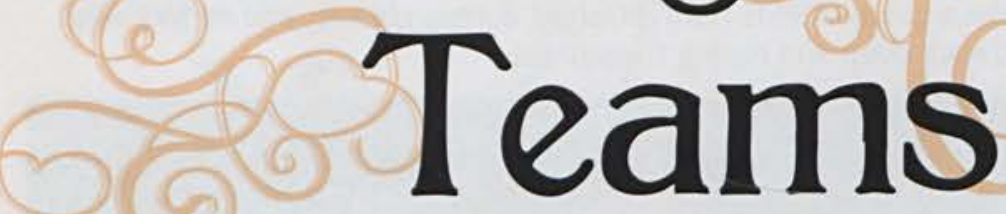

Cedarville's touring teams this year consisted of three Heartsong teams, the Lifeline Players, and two Master's Puppets teams. Touring in the summer and on weekends throughout the school year, the groups traveled to different areas of the country and focused on recruiting students by showing the love of Christ through their talents.

For the drama team, Lifeline Players, several new responsibilities expanded and deepened the team's effect on tour and on campus. According to senior and student leader Josh Cobb, Lifeline worked in Junior Jam, completed the Worldview project, performed in chapel, and even wrote a new program. Senior Abbie McGaha, another team member, expressed what several other touring team members felt, saying, "Ministry is $10 \%$ on stage and $90 \%$ fellowshipping with believers in their homes and churches."

Being on stage for numerous churches across the country was at times nerve-racking, but for sophomore Justin Myers, who was on a Heartsong team, performing taught him humility. "We get a lot of congratulatory and thankful comments after we do a service, and being on the team has taught me to handle those comments with grace and to redirect them to the One who really deserves them."

While Lifeline and Heartsong were able to target a wide range of church members, the Master's Puppets carried with them the responsibility and privilege of ministering to a very special and often overlooked people group. Sophomore team leader James

McClenahan said

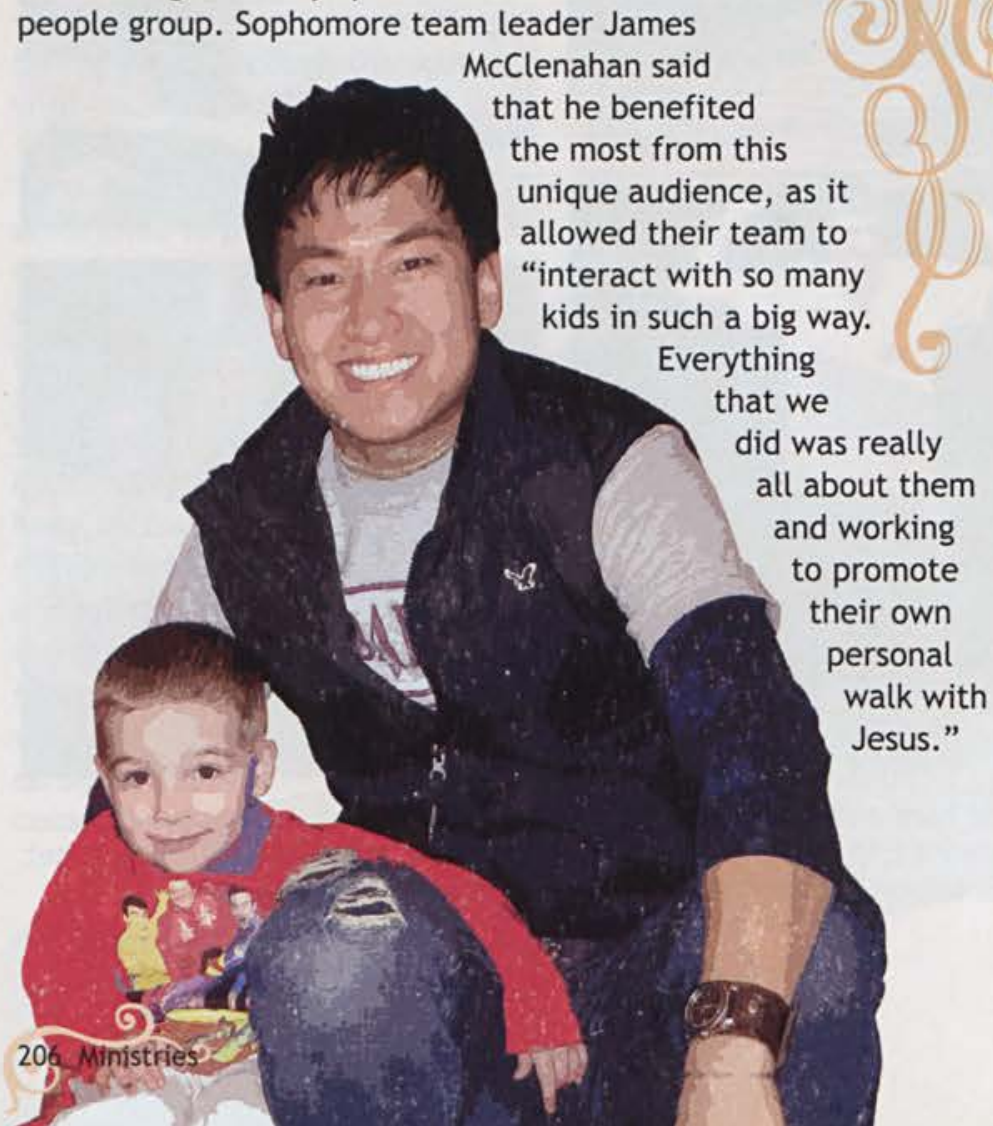

Fauren McCotter, Megan Mertus, and Crystal Baldwin have opportunities to minister through Master's Puppets. As Crystal Baldwin related, "I enjoy having fun with the kids but also teaching them important lessons from the Bible."

$\checkmark$ Heartsong loads into a van before taking off for another performance. Senior Jessica Joy Waggoner shared that once, "someone on our team got Scabies when it was going around, and we had to cancel one of our weekend tours!"
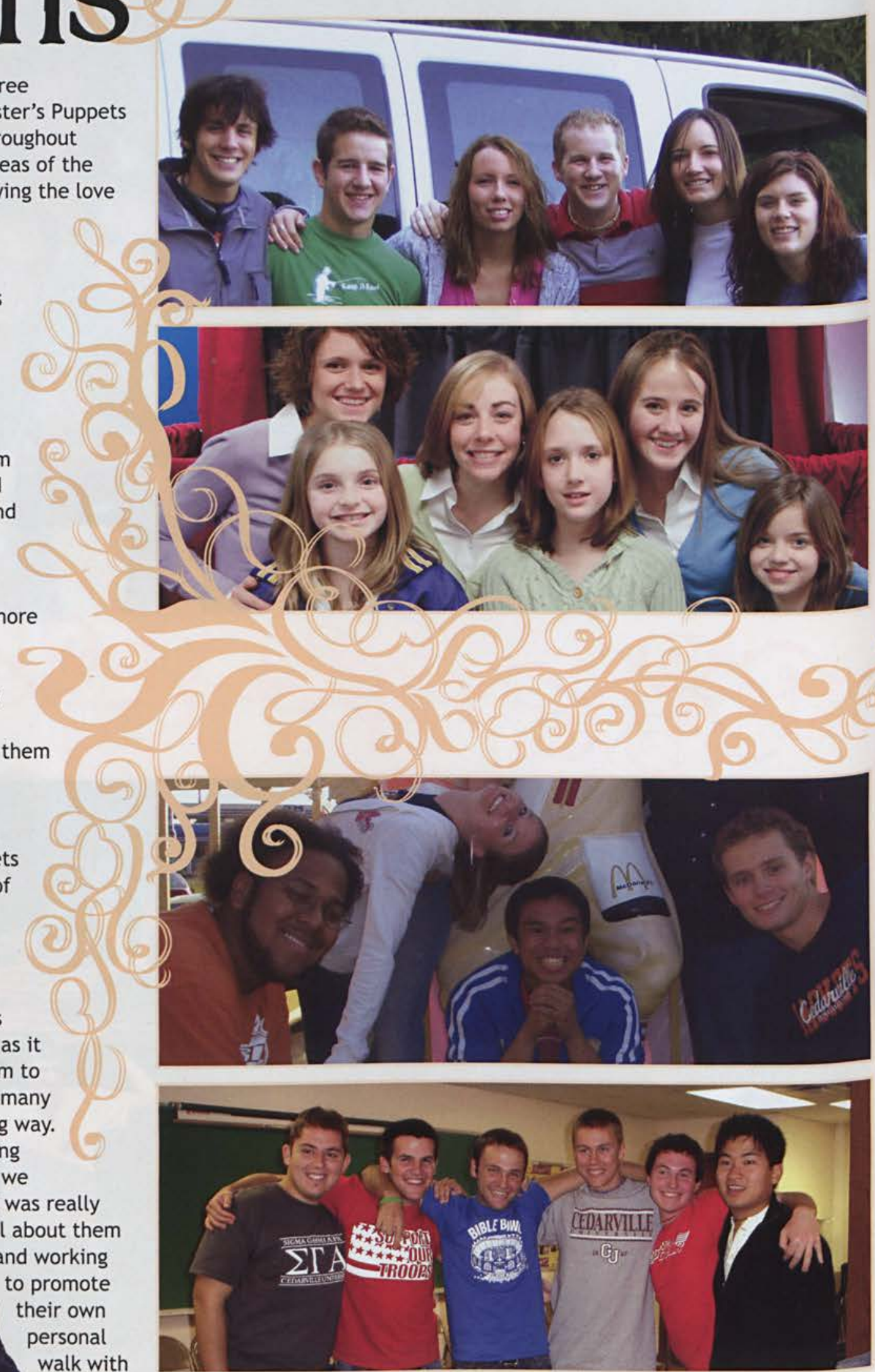

$\Delta$ Both Master's Puppets teams grew very close over the course of the year. According to Alex Hollander, "We were strangers when we met, but by the time the tour was over, we were a family that could make each other laugh by a simple look or a single word."

On their way to another performance, Justin Myers says "travelling is a tremendous opportunity to develop close relationships and this is probably what I love the most about Heartsong." 
Heartsong 1: Brandon Thomas, Seth Gibson, Jess Waggoner, Matt Anderkin, Dana Snyder, Cara Slaybaugh, Gabe Coyle, Becky Roten, Lukas Seelye (student leader)

Master's Puppets 2: Top row - James McClenahan (student leader), Joel Losch, Crystal Nate Weeber, Keith Sider, Jason Skelton, Emily Baldwin. Bottom row - Megan Mertus, Lauren McCotter, Alex Hollander.
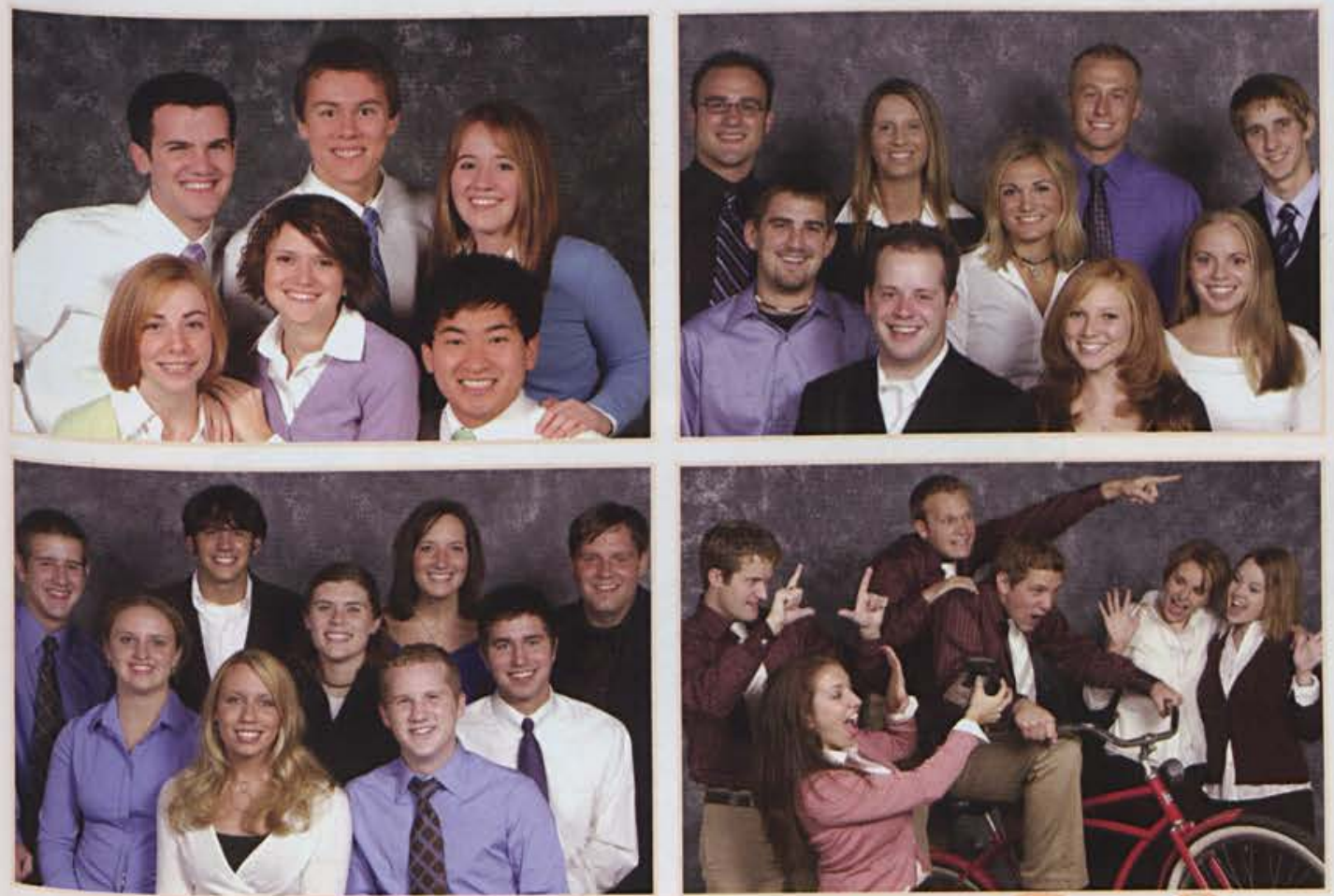

* Heartsong 2: Garrett Williams, Justin Spann (student leader), Sarah York, Tony Bostic, Michael Halsmith, Gretchen Riedel, Justin Myers, Lori Crain, Tim Labrado

$\checkmark$ Master's Puppets 1: Top row - Terri Schenk, Matt Beres (student leader), Matt Deakin Bottom row - Sarah Banas, Amanda McCollim, Andrew Davis.
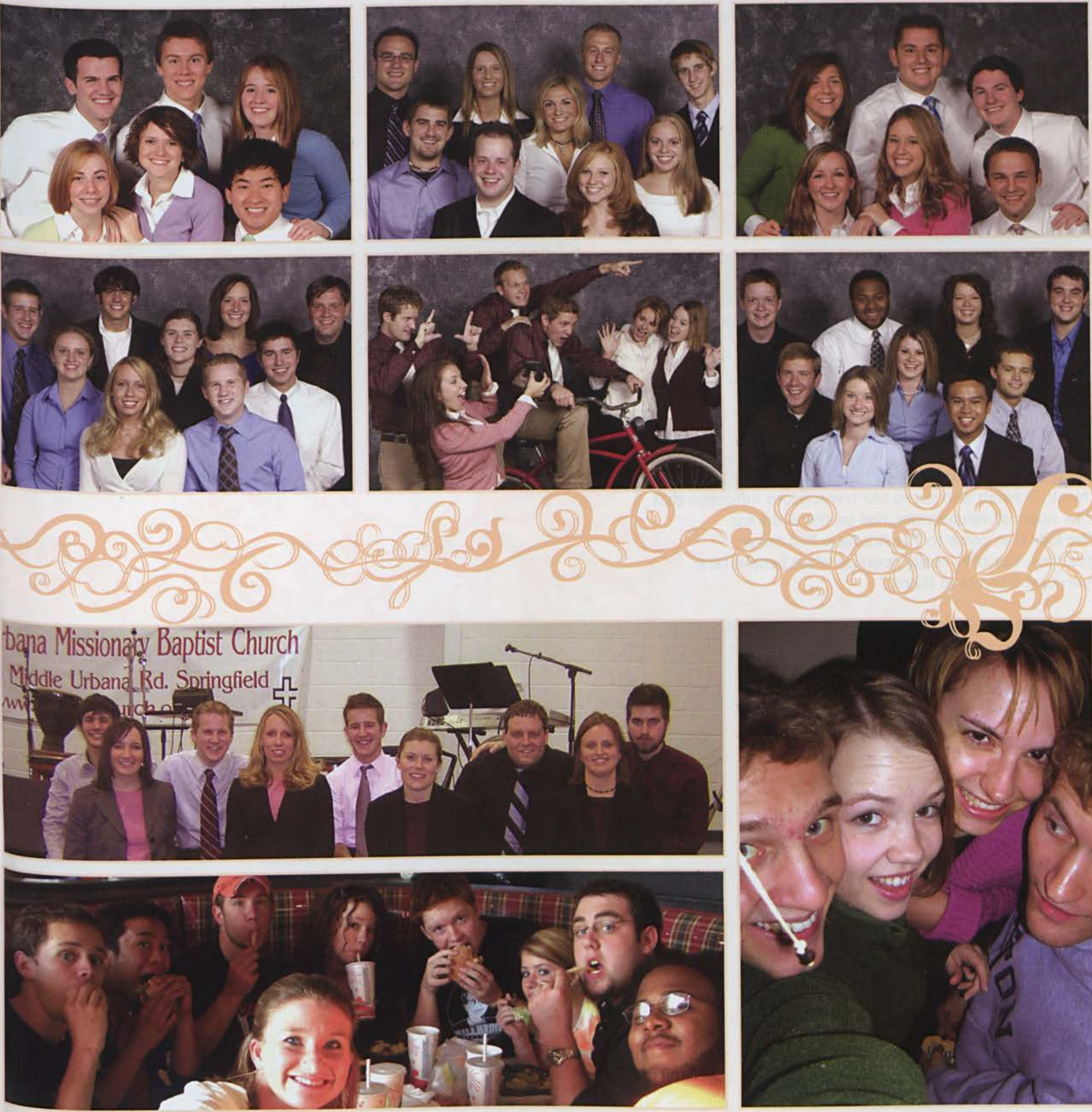

Heartsong chills together at McDonald's on their way to another performance. Senior Gretchen Riedel loves these simple moments. "I enjoy the fellowship and family-like atmosphere of the teams. I feel it's a safe place to be vulnerable and share the deep struggles of our lives" with the other members of my team.

* Heartsong relaxes after another performance, but Dana Snyder echoes sympathies of the entire team: "Everytime I sing I feel out of my comfort zone because I know that I am nothing. I am just an ordinary person with no extraordinary ability, but God has decided to use me for some reason."

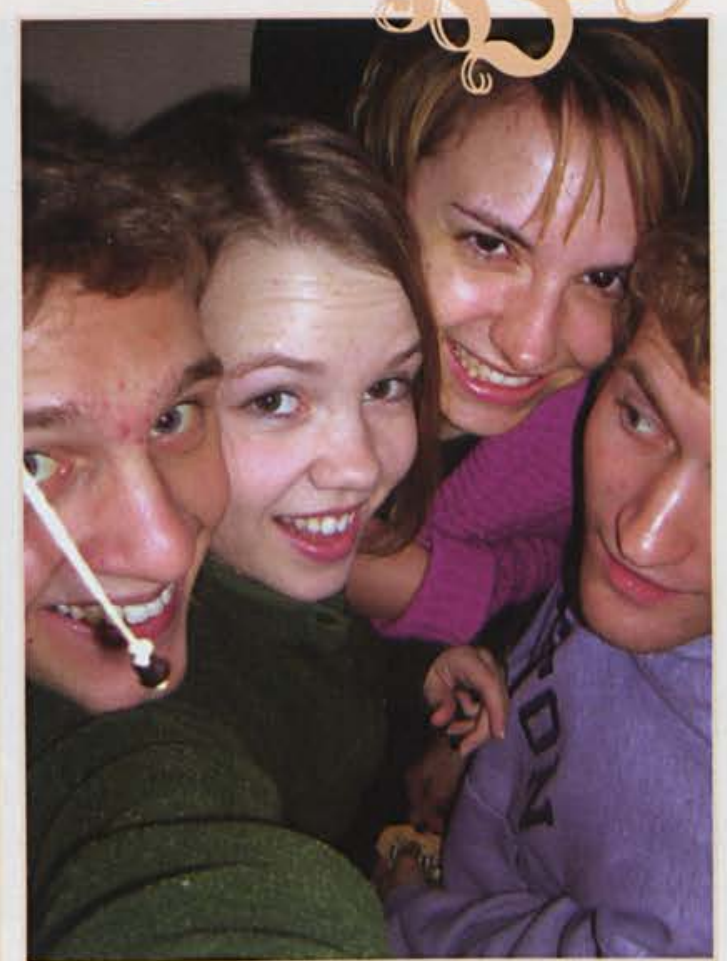

$\Lambda$ Joshua Cobb enjoys time with fellow Lifeline Players. In his own words, "It's a really comforting thing to be surrounded by people who you know love you and care for you." 
$\checkmark$ Senior Janna Schwab reflects on her time working with children at the Ark. "There is a great need for inner city ministry and Cedarville students can make a difference by giving a few hours a week. It was great to see the kids every week and our team had a great time hanging out with all the kids!"

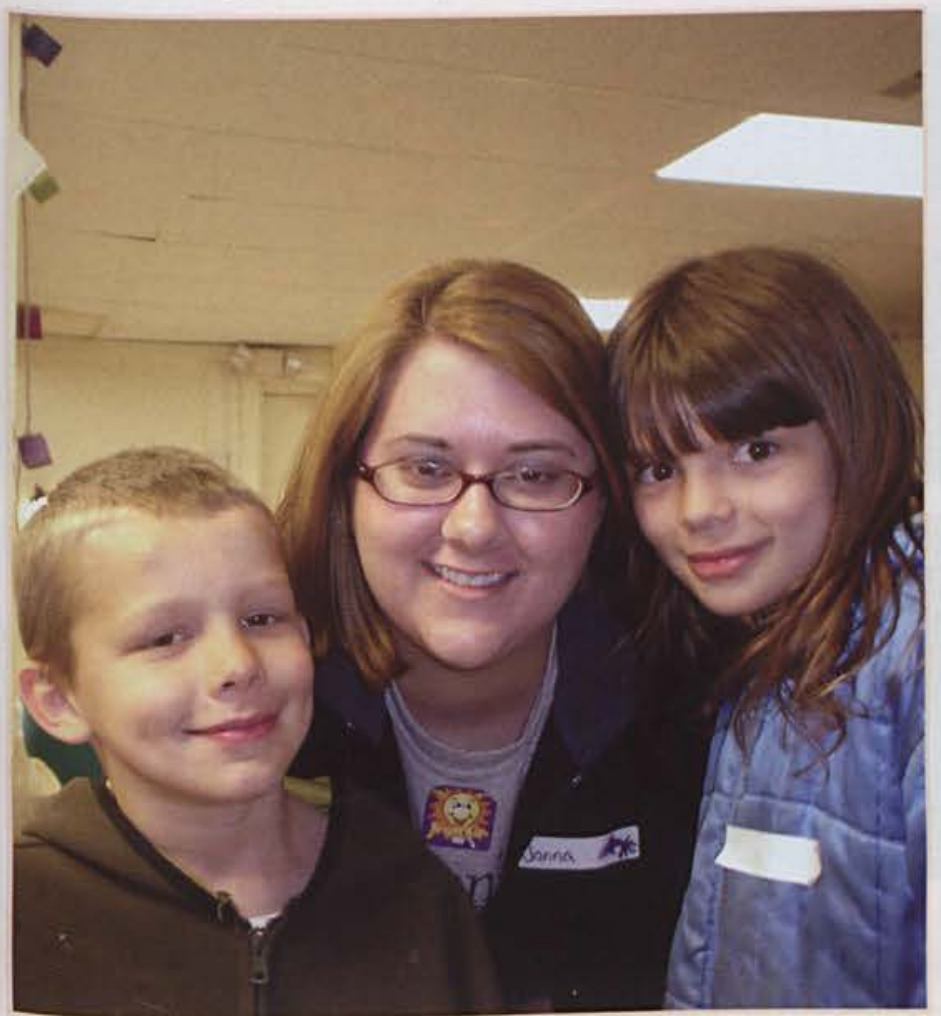

* A young boy waves as he races around the gym during game time at the Sonshine Club, almost as if to say, "take luck!"

$\checkmark$ An exchange student from South Korea's Handong Global University and Janet Colgain minister together.
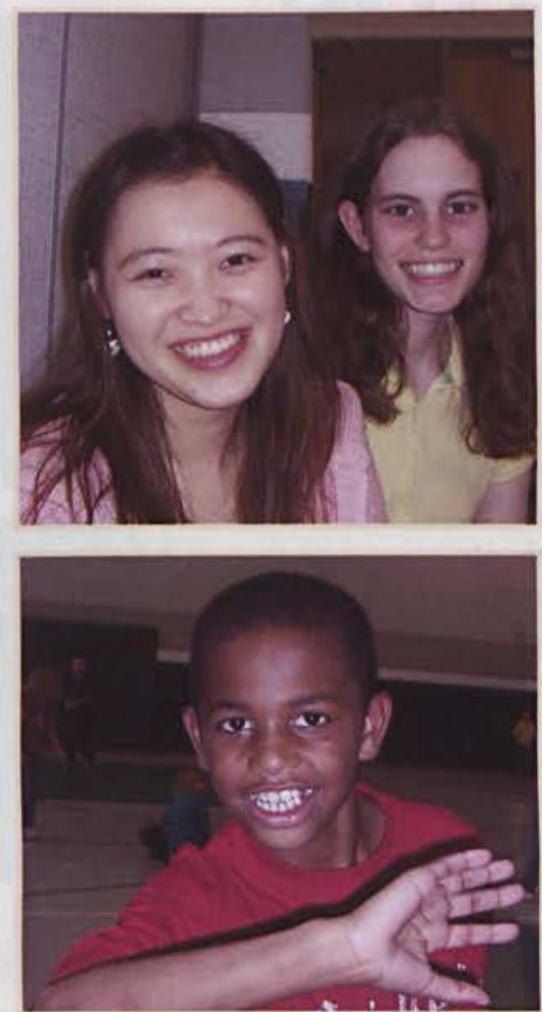

$\checkmark$ Julie Campbell plays her clarinet during a special Christmas party at Hillside Retirement Home where students also presented a short skit based on the Christmas story in Luke. "Sometimes I forget how much a brief visit can mean to the residents, but they are always so glad to see us," Julie says.
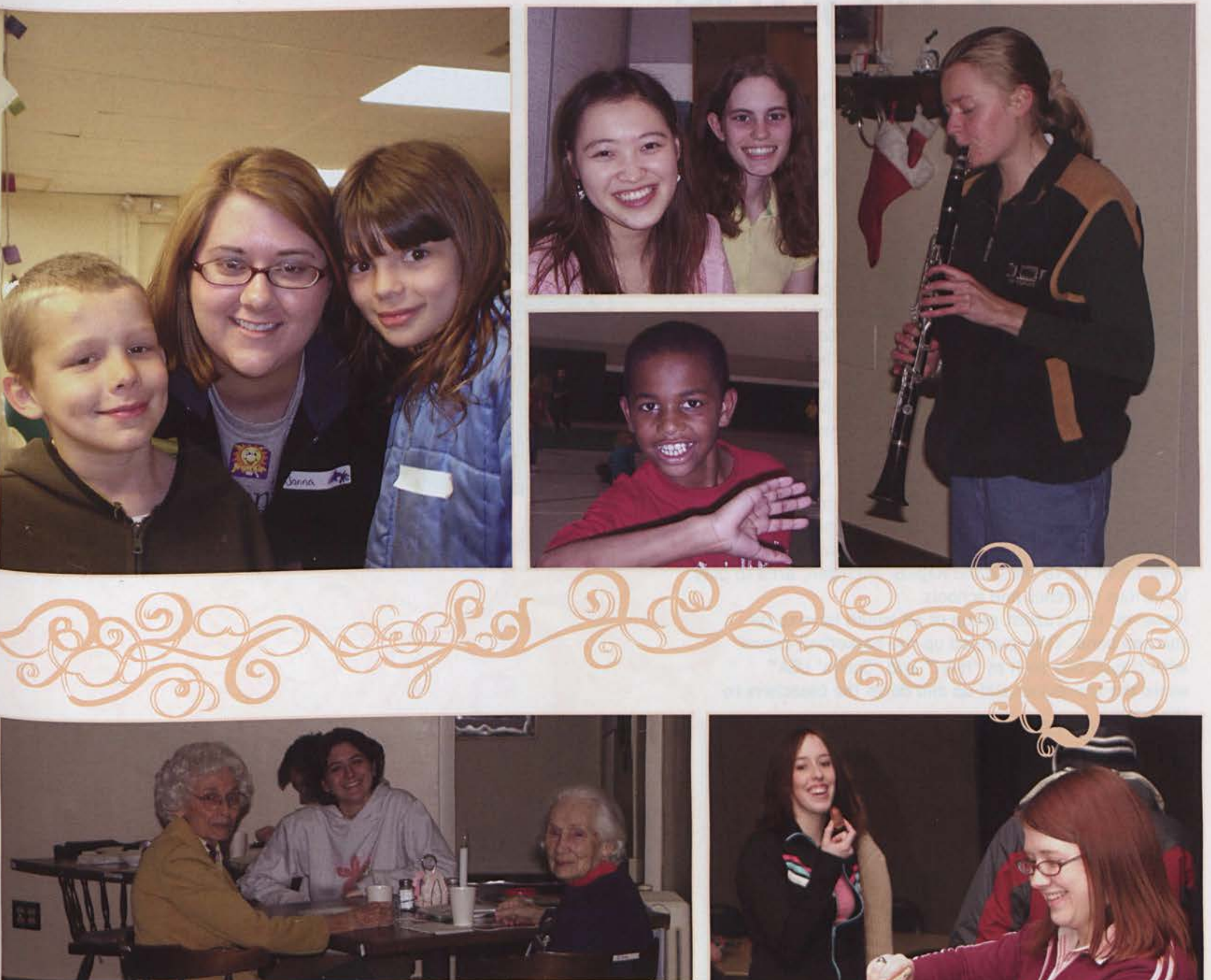
Under the direction of newly-hired conductor Mr. Paul Kim, the string section of the orchestra flourishes. Mr. Charles Pagnard also conducts the orchestra at times, allowing Mr. Kim to ease into his new position.

$\checkmark$ The second clarinet section of the symphonic band concentrates on their music during their fall semester concert.

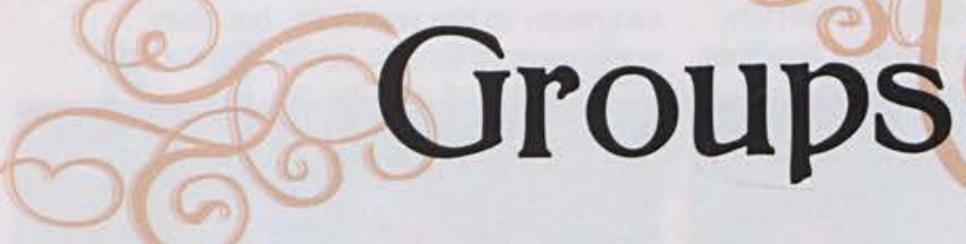

This has been yet another incredible year for the instrumental groups for Cedarville University. Instrumental groups ranging from the symphonic band and orchestra to the flute choir and string quartet performed on campus, in churches, and at the state and national levels.

The Jazz Band caused many listeners both on and off campus to tap their feet to the sounds of the Big Band era of the 30's and 40's as well as contemporary jazz pieces. Along with the Jazz Band, the Symphonic Band performed on campus, having been featured in chapel on such days as Veterans' Day. They also performed at churches and schools in the area, bringing God the glory as they ministered at places such as CedarCliff High School and Faircreek Church.

Playing high quality compositions, the Orchestra and Brass Choir performed at many concerts around the area and on campus. The Orchestra went on the road for the first time in its history this year, taking their music up to the Grand Rapids, Michigan, area to play in various churches and schools.

However, the craziest group of all would have to be the pep band. They pumped up the basketball crowd with classic hits such as "Hey!" and "Surfin' USA" while the trombones slid up and down the bleachers to the tunes.

Cedarville University has a habit of producing great musicians, and this year was no different. Their performances reflected their hard work, and their standard of excellence was clearly seen. The conductors of all of these groups put a lot of time

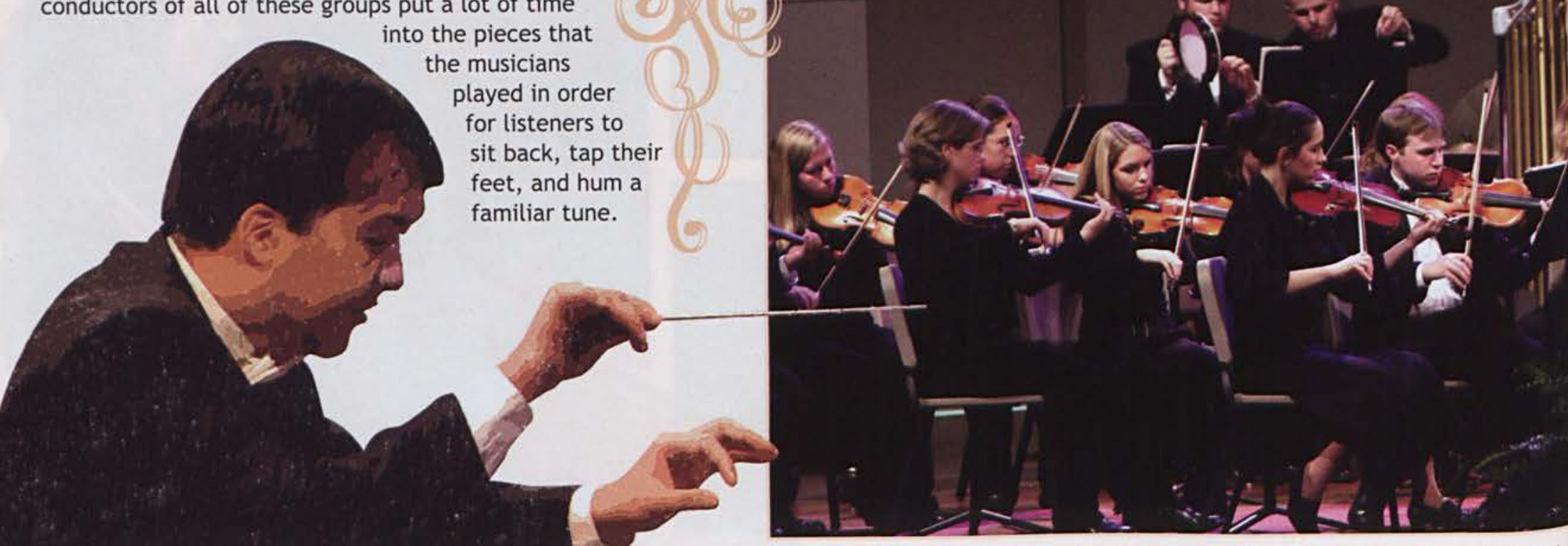

$\Lambda$ Led by section leaders Pamela Eustance and Heather Johnson, the violins of the orchestra concentrate on their music. 
$\checkmark$ Sophomore Kate Talbott is a frequent face on the chapel stage and is a fixture in the string quintet, which played for the Homecoming Prism Concert in October.

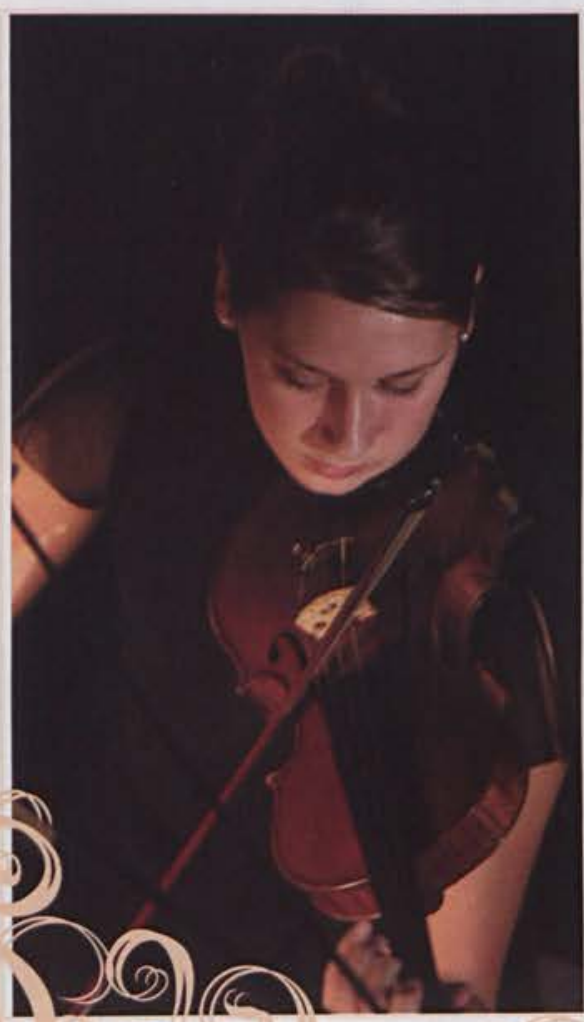

$\checkmark$ Heather Hennessey, Randy Plate, and Dr. Curlette play in a small ensemble accompanied by senior Kevin Vaughn.
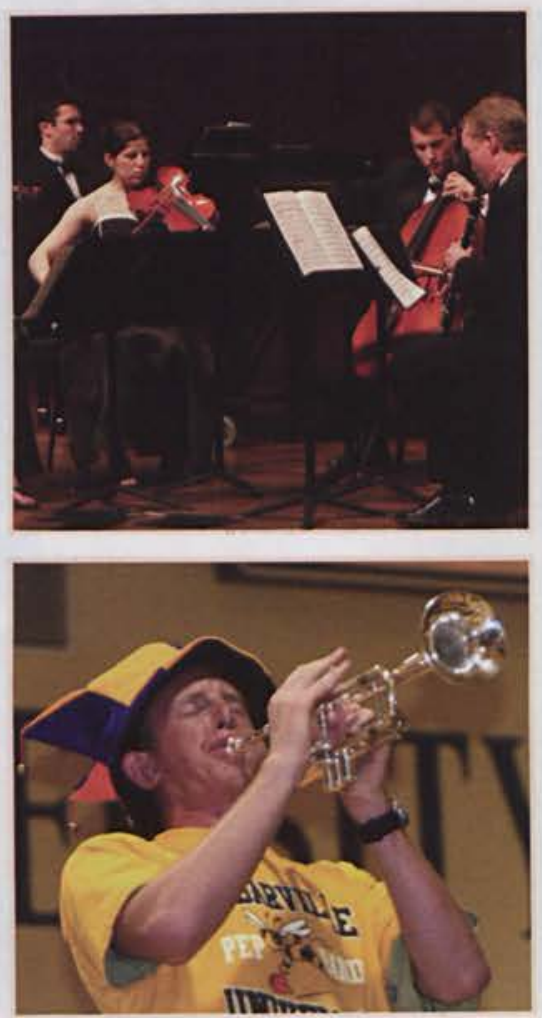

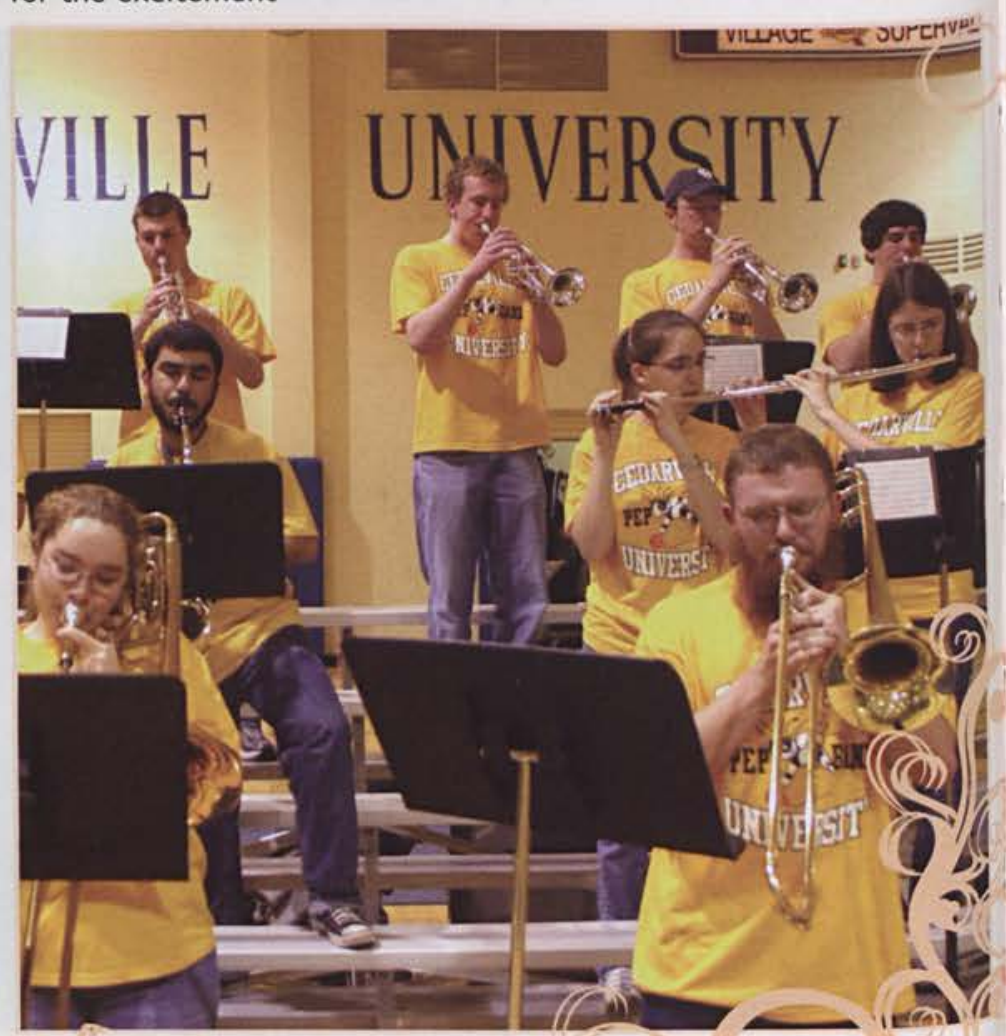

$\checkmark$ The pep band provides lively music during breaks at the Varsity Jacket basketball games and pumps up the crowd, setting the mood for the excitement
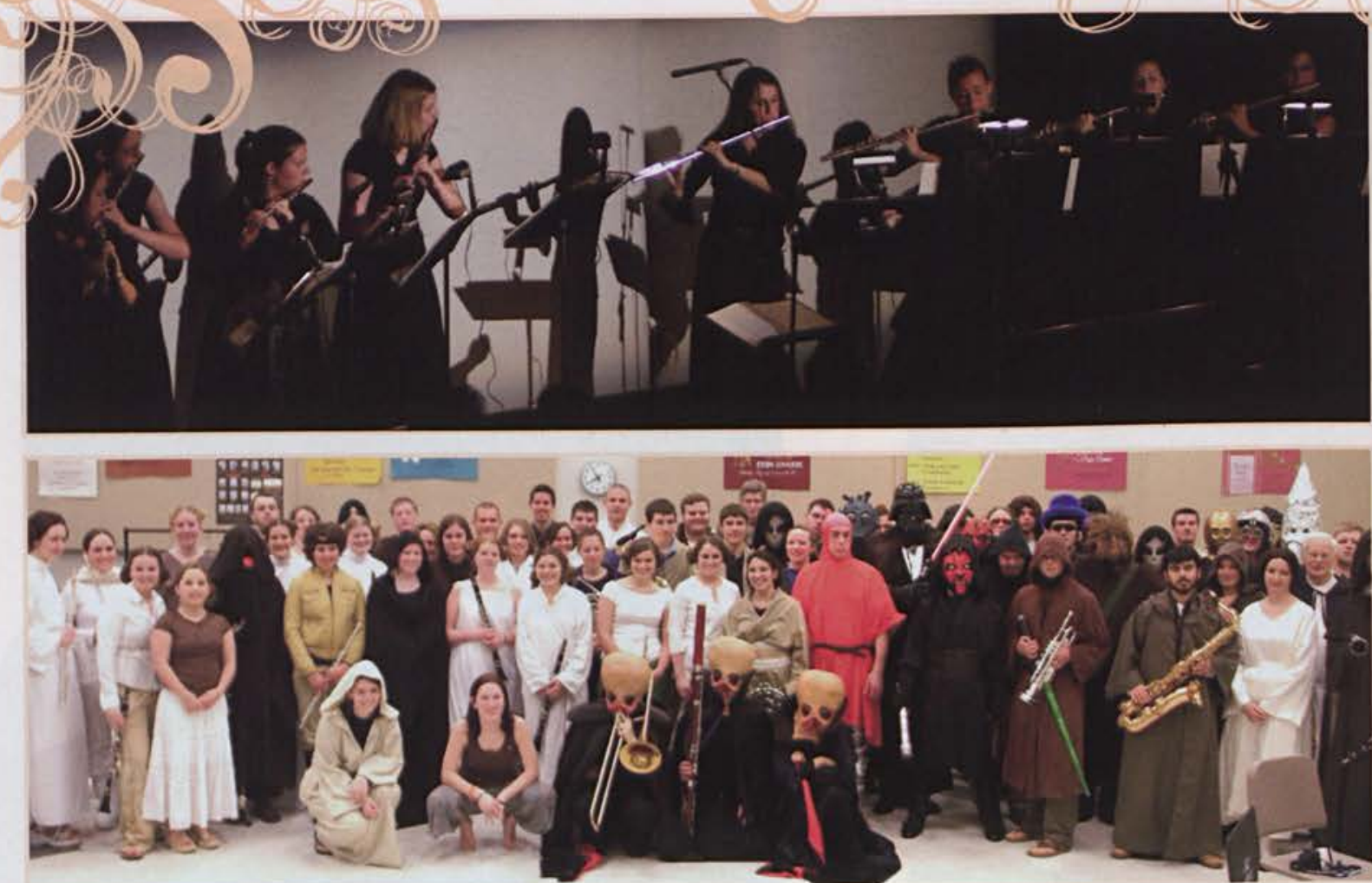

A The symphonic band prepares for the annual Pops concert. Scheduled to play selections from John Williams' "Star Wars Trilogy," the band composes a skit and performs in character.

$\star$ The flute choir, a fixture before many of the main concerts, takes part in the Homecoming Prism Concert.

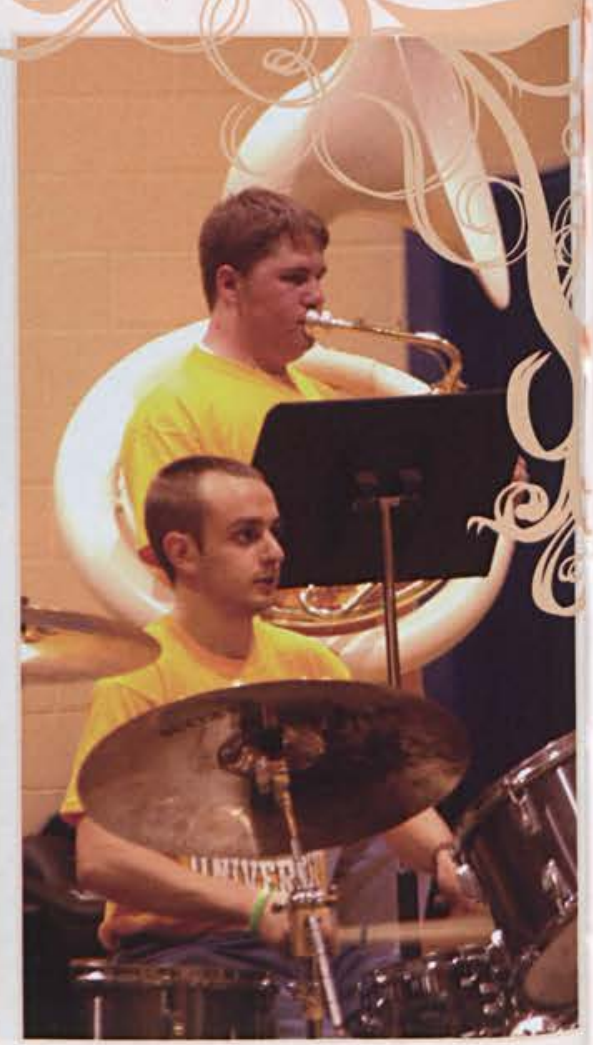

$\Lambda$ Jared Osborn (percussion) and Matt Ellington (tuba) contribute to the big sound of the pep band. 


\section{Choral}

pes.

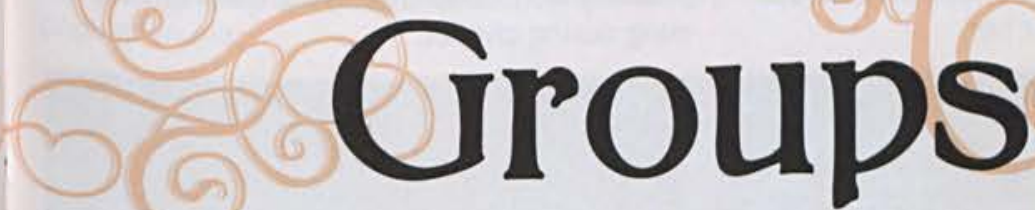

F Concert Chorale, Men's Glee Club, Women's Choir, Jubilate, and Orchestra join together to present Mozart's "Solemn Vespers" and Poulenc's "Gloria" during the annual Choral Masterworks Concert.

$\checkmark$ "One Voice" takes the stage in chapel and ministers through song under the direction of admissions counselor Michael Dorsey.

The chapel was filled with the reverberations of harmonies, melodies, and crescendos as a stage crowded with men and women in tuxedos and evening gowns belted out sacred hymns, southern spirituals, and Latin arias. My skin was covered with goose bumps as the group reached their powerful finale. God definitely blessed these students with the gift of song.

Cedarville's choral groups, consisting of the Men's Glee Club, Women's Choir, Jubilate, and the Concert Chorale, traveled across the country and expanded their musical repertoire during the school year. In addition to the several other countries that they visited, the Men's Glee Club added Scotland to their itinerary this year, as they toured the country for a week in April.

Sophomore Brian Sallee, a member of the Men's Glee Club, remembered the interesting new direction their choir took. He said, "We did a song this year in African dialect. It never really meant much to me, until at one church a lady came up to me and said she understood everything in the song! She was from that region of Africa, and she said it meant so much to her and her family."

Choral groups this year didn't just affect their audience, however. Nila Privedenyuk, a sophomore member of Jubilate, mentioned, “It's funny how you can stand next to someone for two hours, twice a week and not really know who they are. That all changes when we go on tours. During our travels there's a lot of time to just talk to people about life and see what makes them tick."

One thing that the choral groups were constantly reminded of was for Whom they sang as well as their true reason for performing. Freshman Jesse Lansford, a member of the Men's Glee Club, said, "The main point for the choral music we do is to give glory to God, so even if we are only singing to three people, We will still do the concert because we are giving glory to God through our voices." e

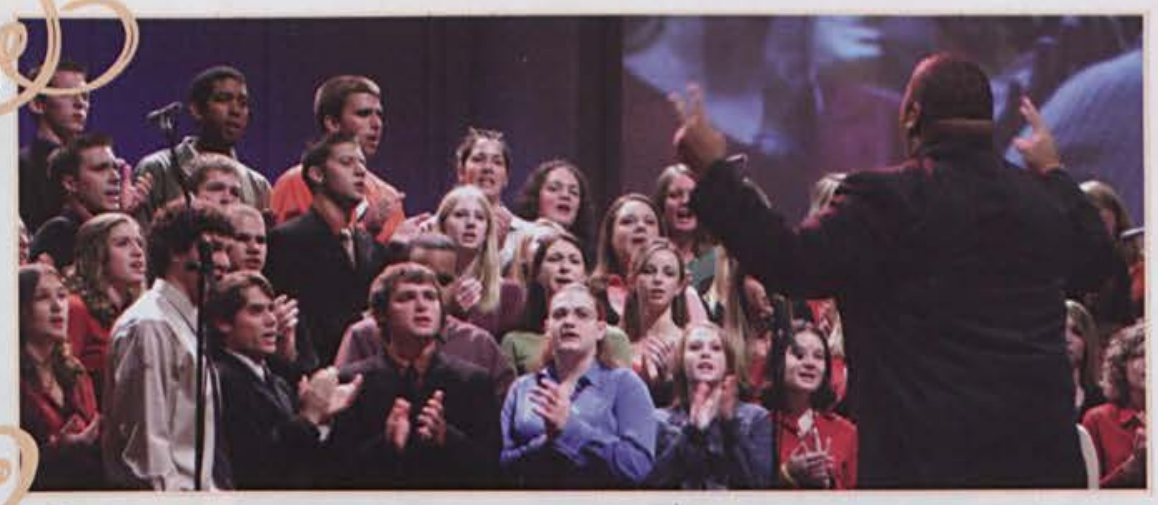
(4)

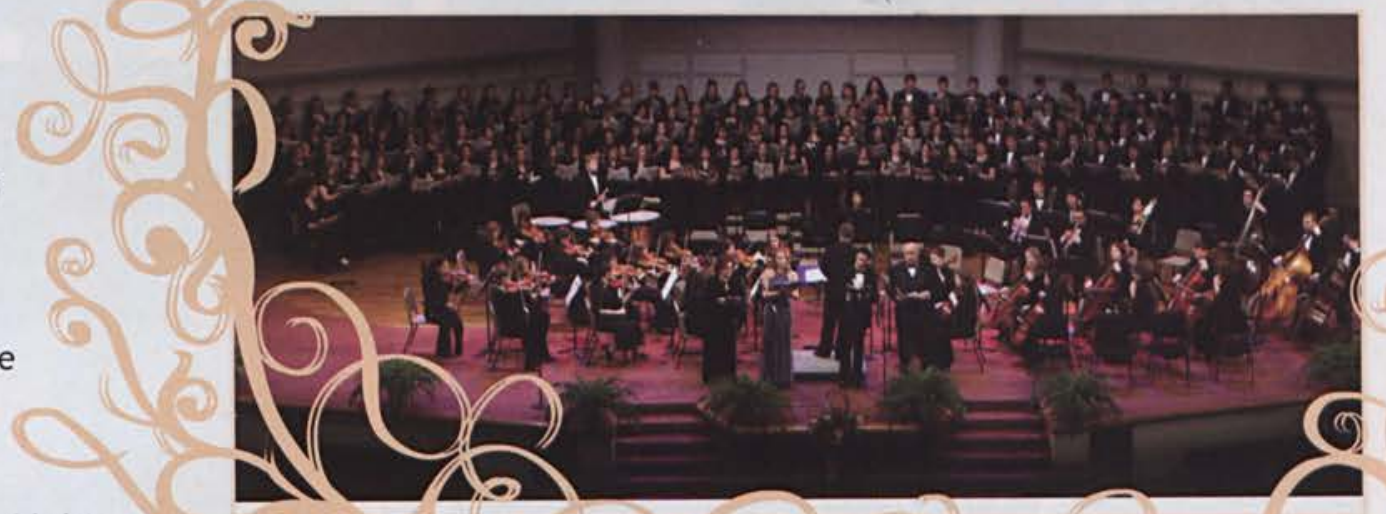


$\checkmark$ Heidi Fraser, Kim Williams, and Joy Mathisen prepare to sing their solos during one of Jubilate's songs at the annual Christmas Concert.
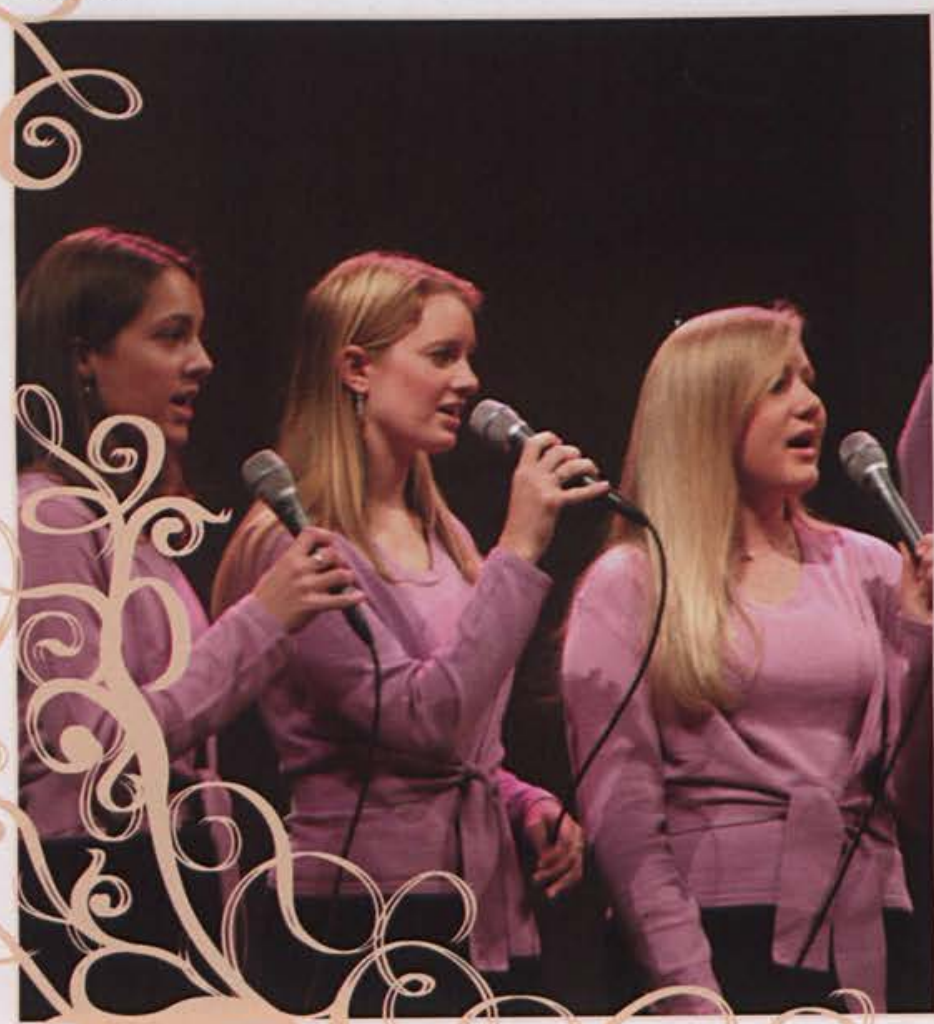
contributes to the ministry of One Voice during a chapel service.

$\checkmark$ Senior Amanda Collins

accompanies Jubilate as Mark Sue turns pages for her. $\checkmark$ One Voice raises their hands in worship, praising God while displaying their talents in song during chapel.
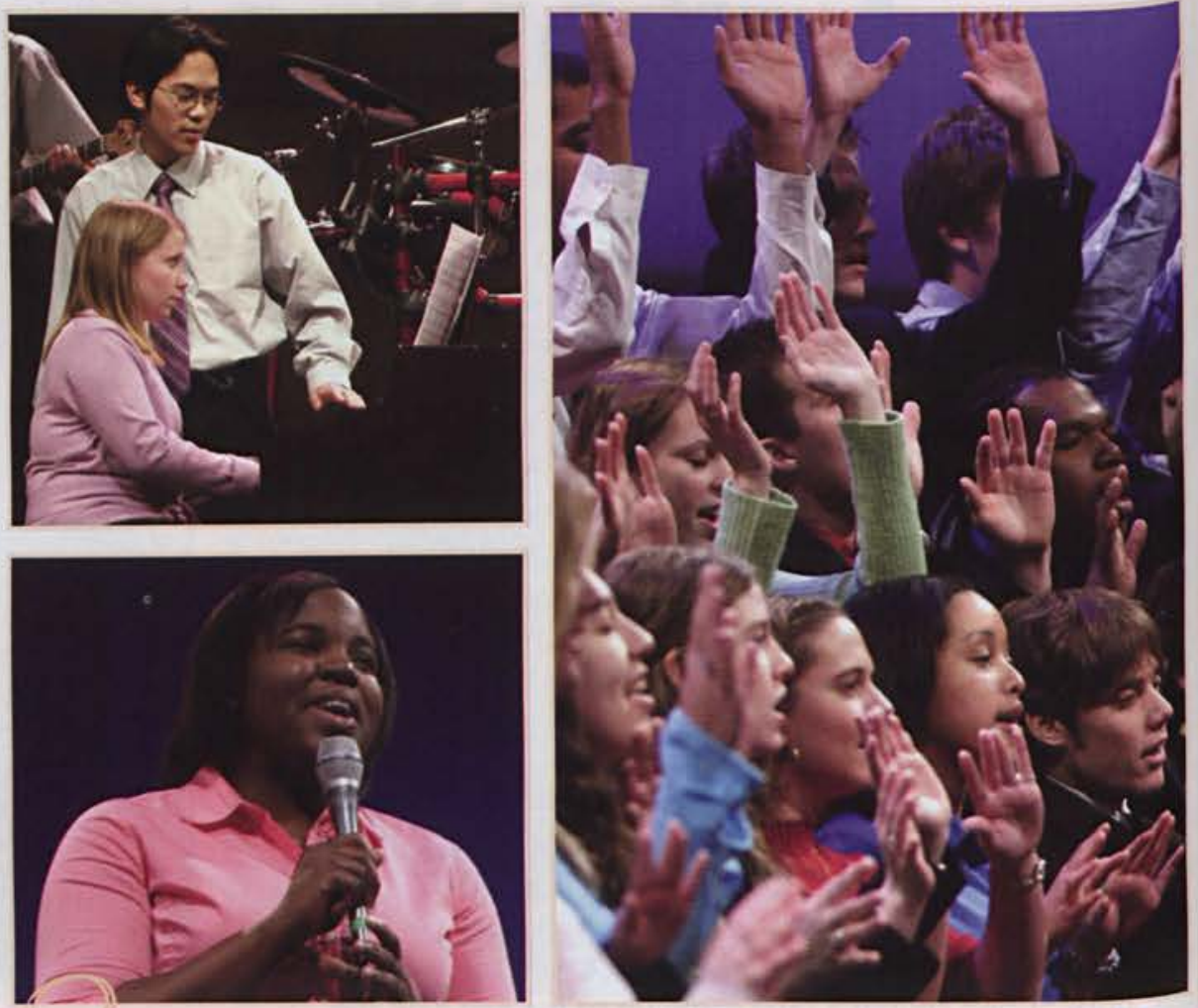
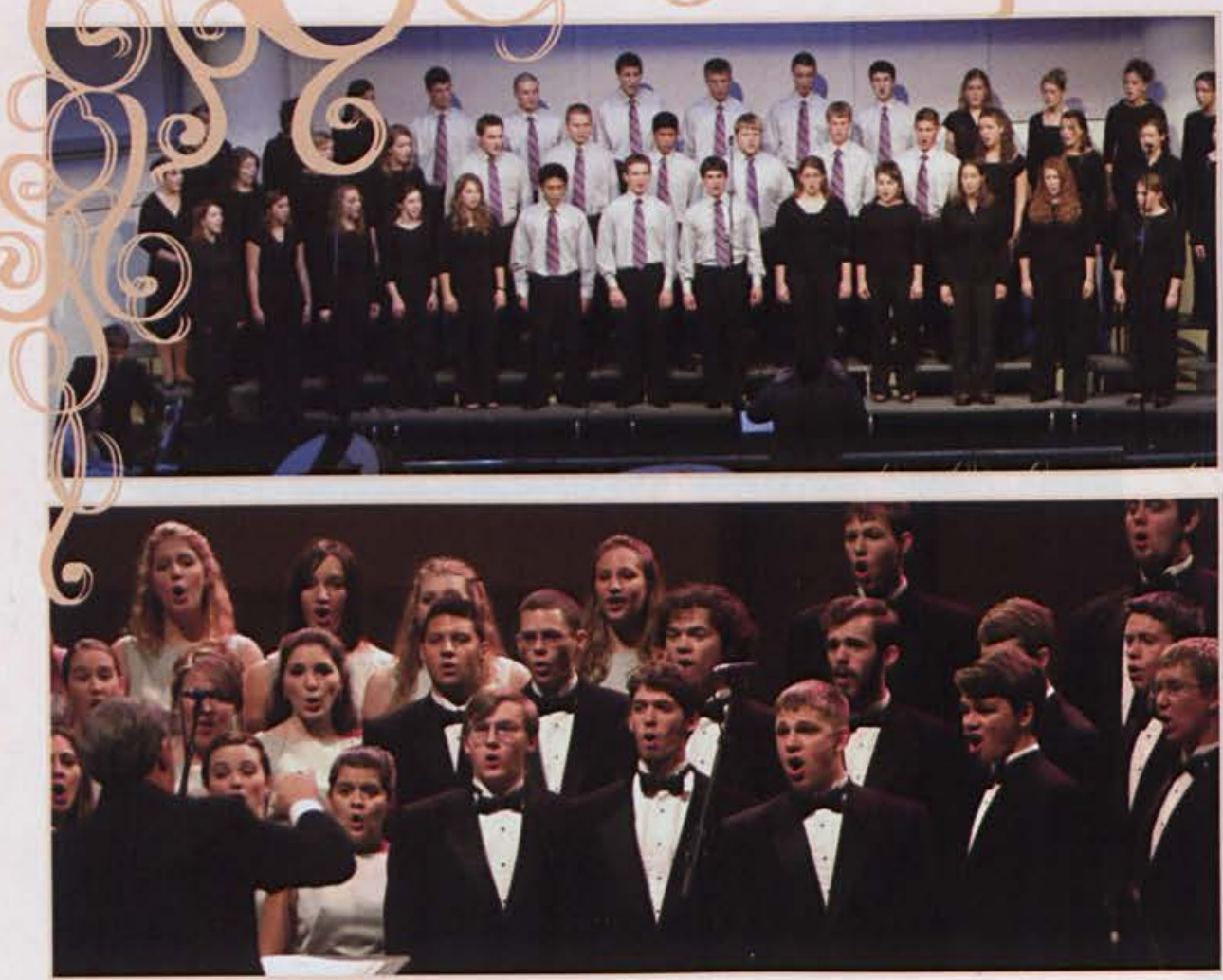

$\Lambda$ Concert Chorale, under the direction of Dr. Lyle Anderson, work hard to please their director and strive to bring glory to God in all they do.

* Jubilate, a ministry team of approximately 40 vocalists and instrumentalists, ministers in many churches over the weekends. They also take part in the many concerts on campus, including the Homecoming Prism Concert.

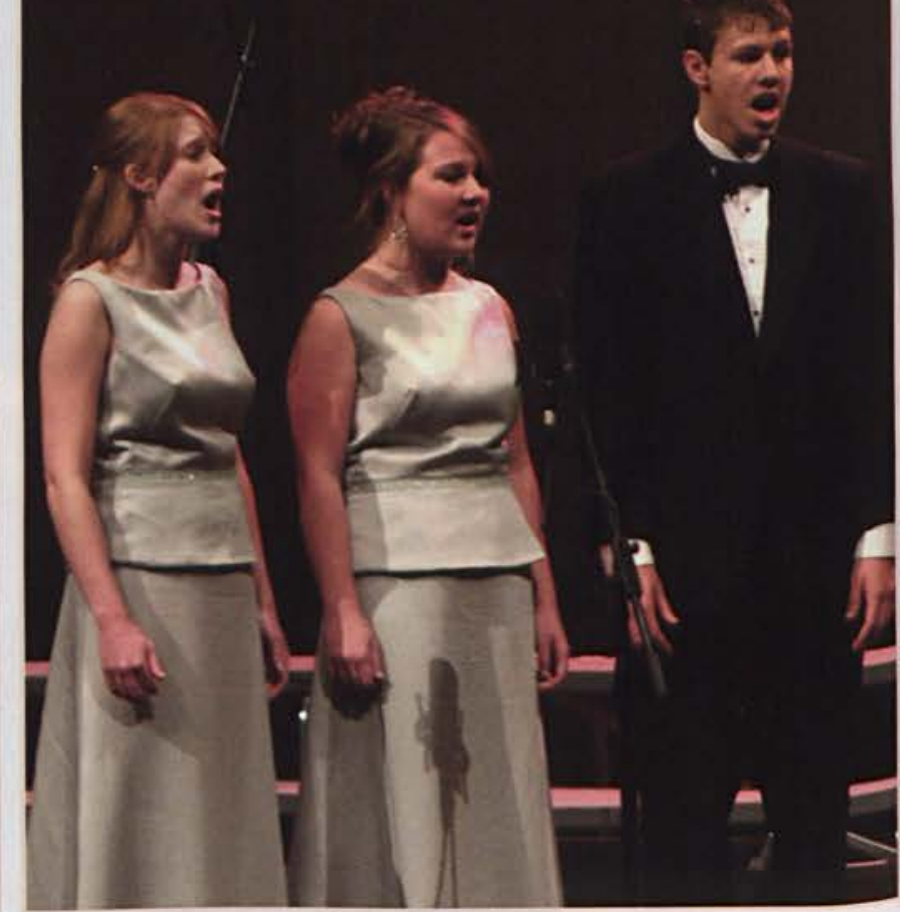

^ Amanda Haas, Sarah Matney, and Ben Sincock sing as a part of the Arts Ensemble, a select group composed of Concert Chorale members. 
The men of the Glee Club sing one of their more classical pieces during a campus concert. The 30 -member group performs concerts both on and off campus, singing spirituals, hymns, contemporary Christian, classical, and popular music.

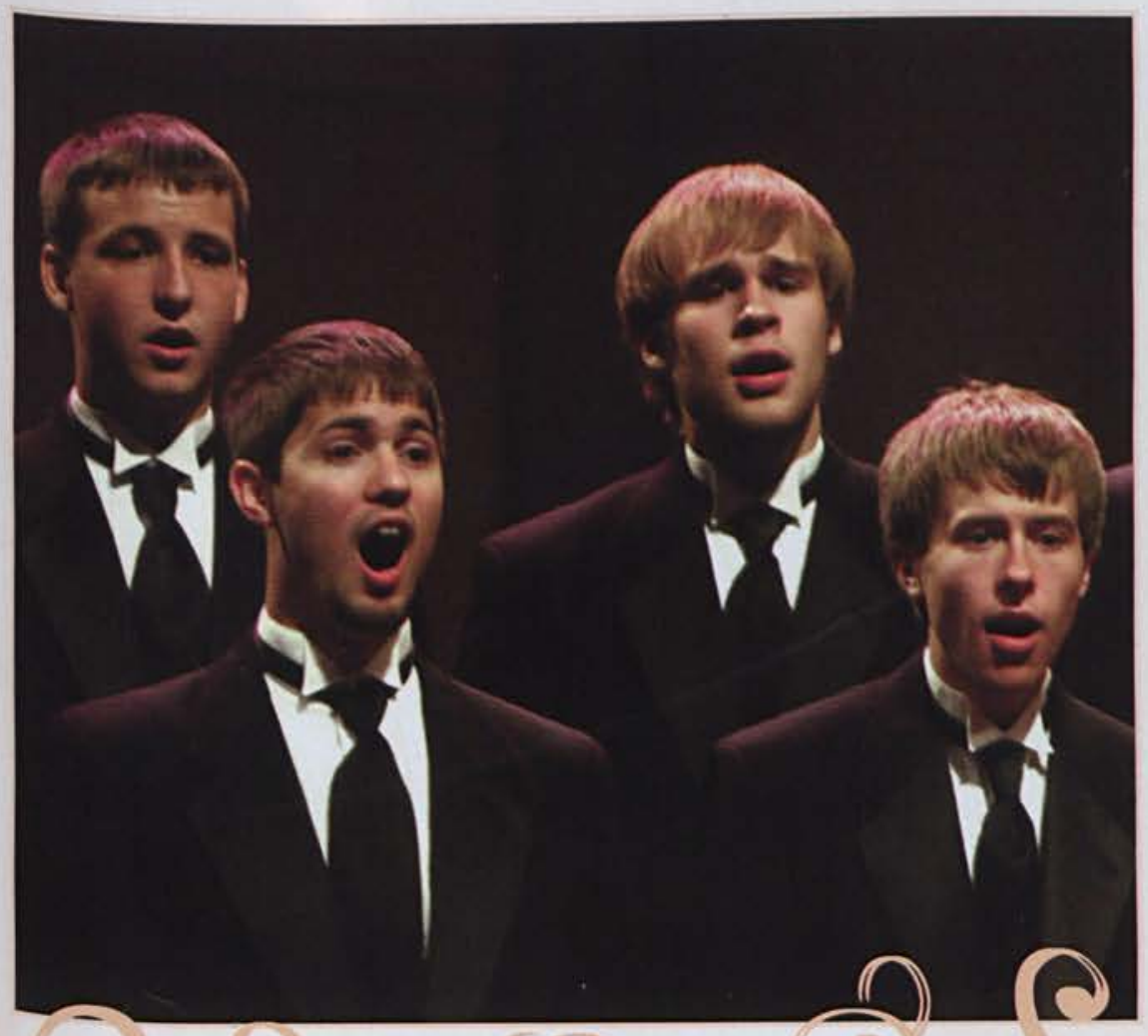

₹ The "'Ville Singers," composed of Matthew Abraham, Nicholas Kohne, Adam Golden, and Ryan Lowry, perform at a recital and are accompanied by Isaac Judd

$\checkmark$ Members of the Men's Glee Club wait for the cue from Dr. Anderson before joining the rest of the choir in singing praises to God.
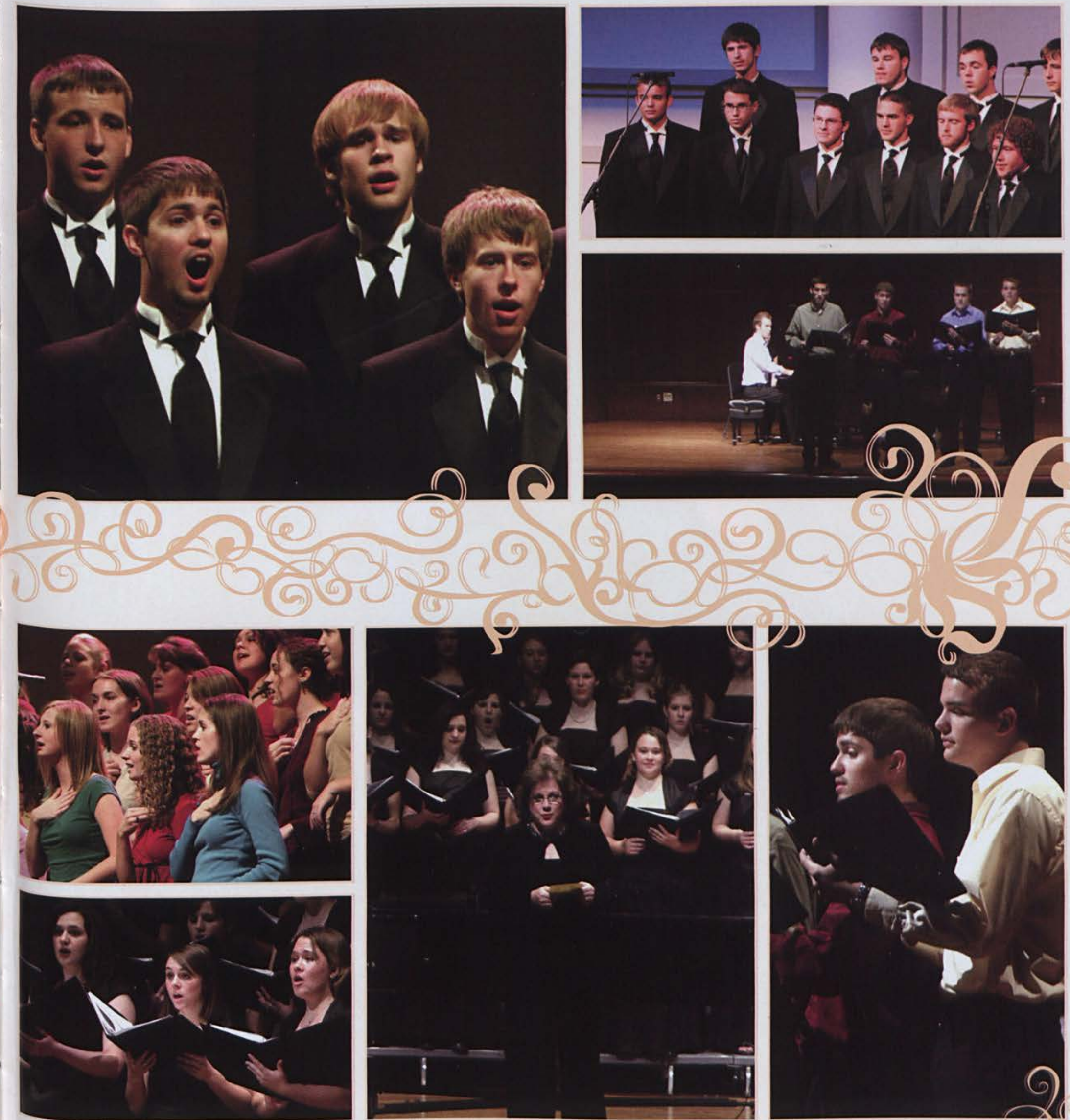

Dressed in their finery, Women's Choir

Prepares to give a concert under the direction of the talented Mrs. Beth Porter.

Watching the director is one of the most

important parts of singing in a group such as the Women's Choir, as sophomore Katie Lippman demonstrates.

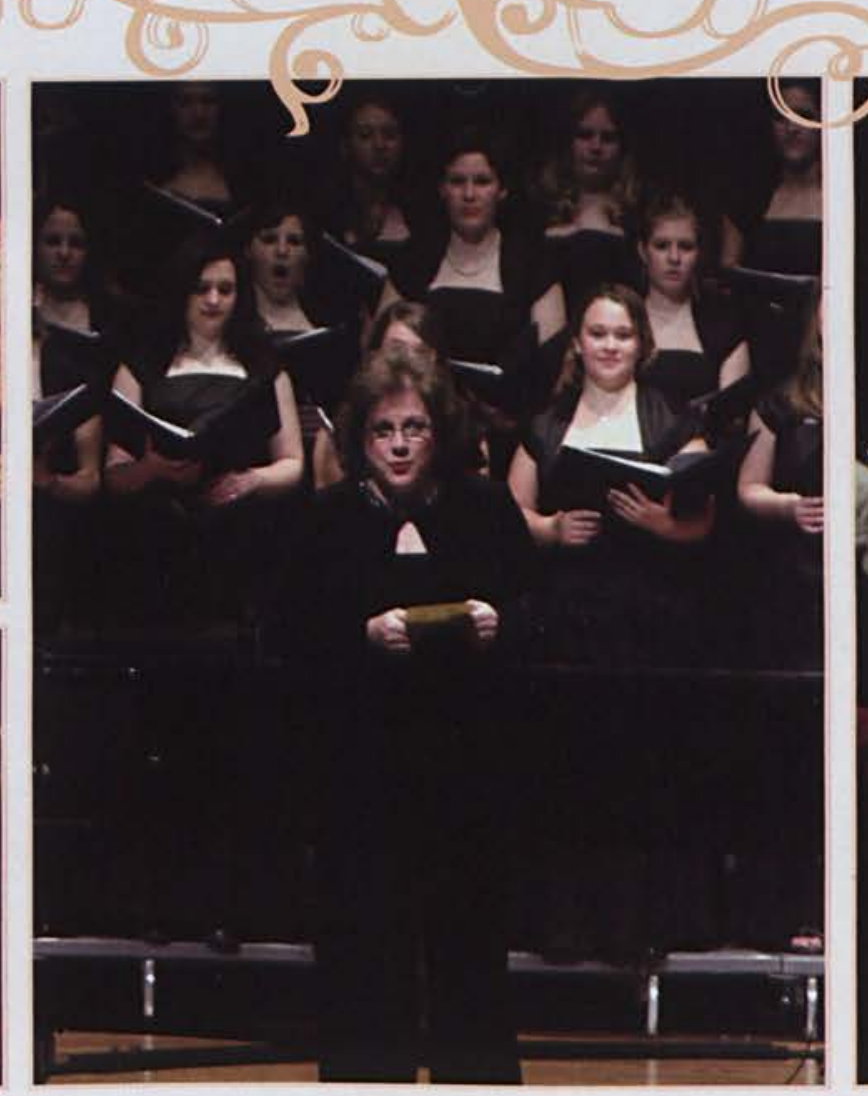

$\Lambda$ Dressed in their finery, Women's Choir prepares to give a concert under the direction of the talented Mrs. Beth Porter.

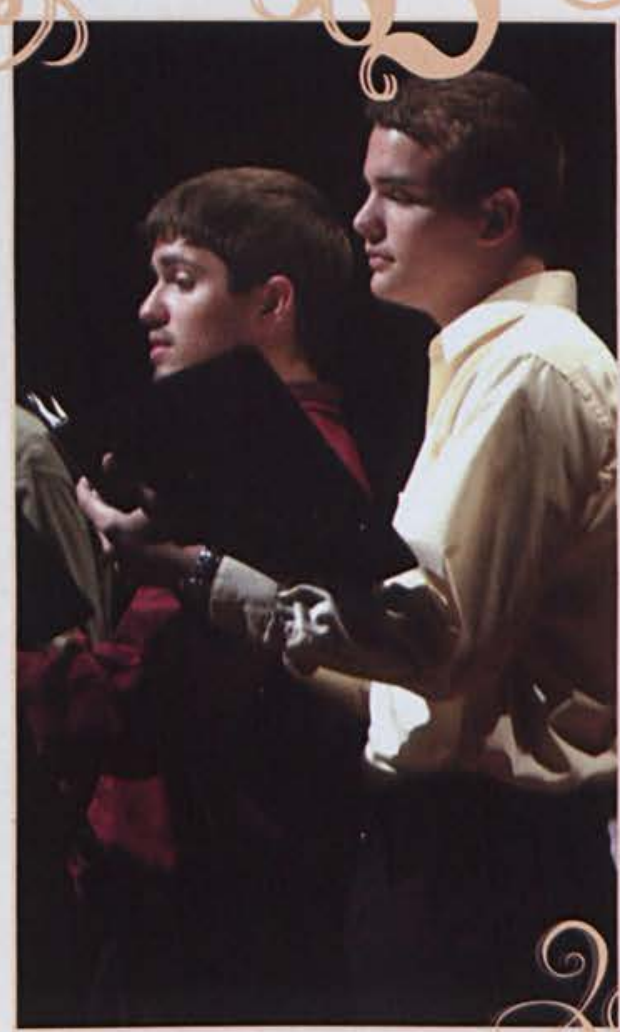

¿ Sophomore Nicholas Kohne and junior Ryan Lowry raise their voices during a performance of the "'Ville Singers," a group composed of Concert Chorale members. 


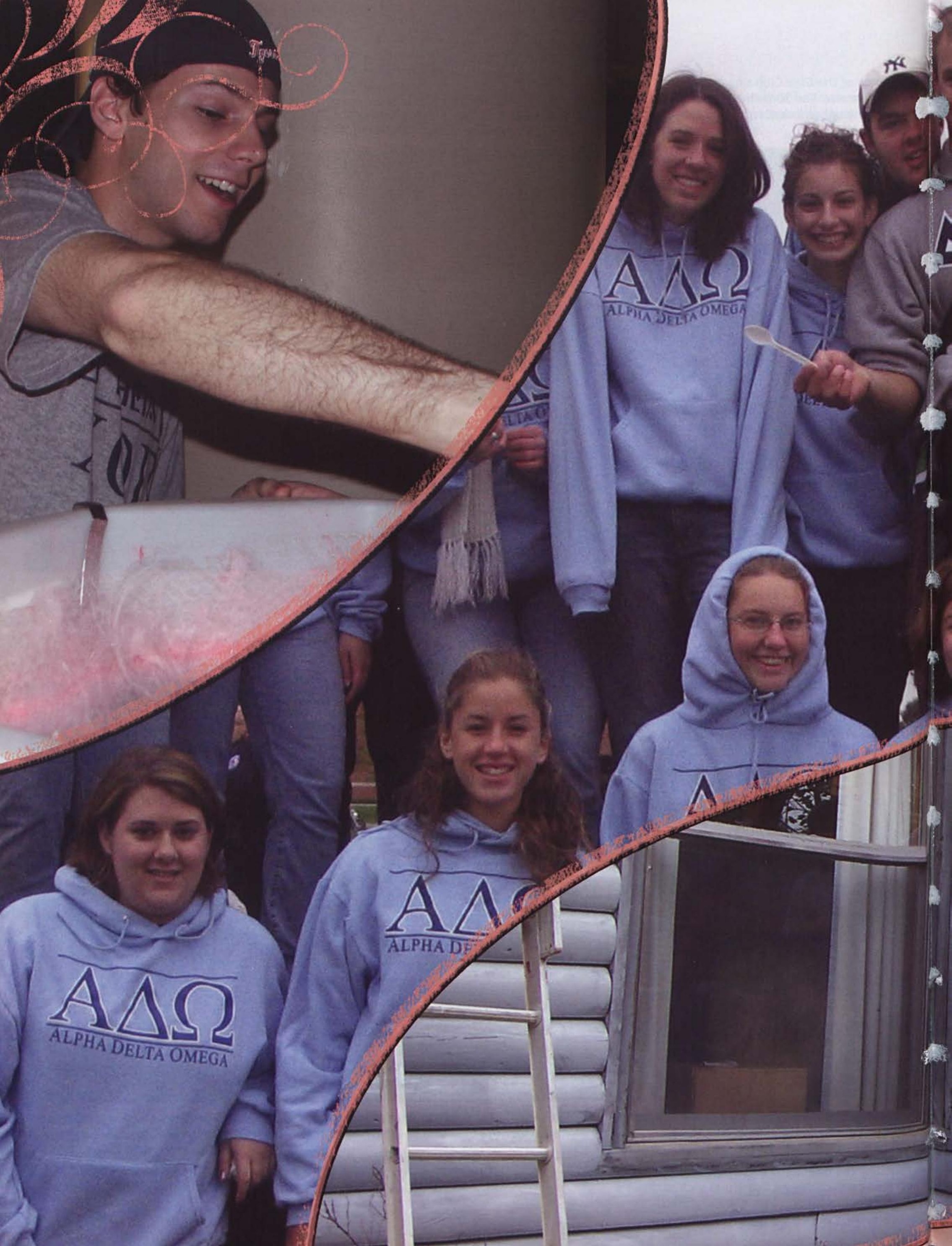




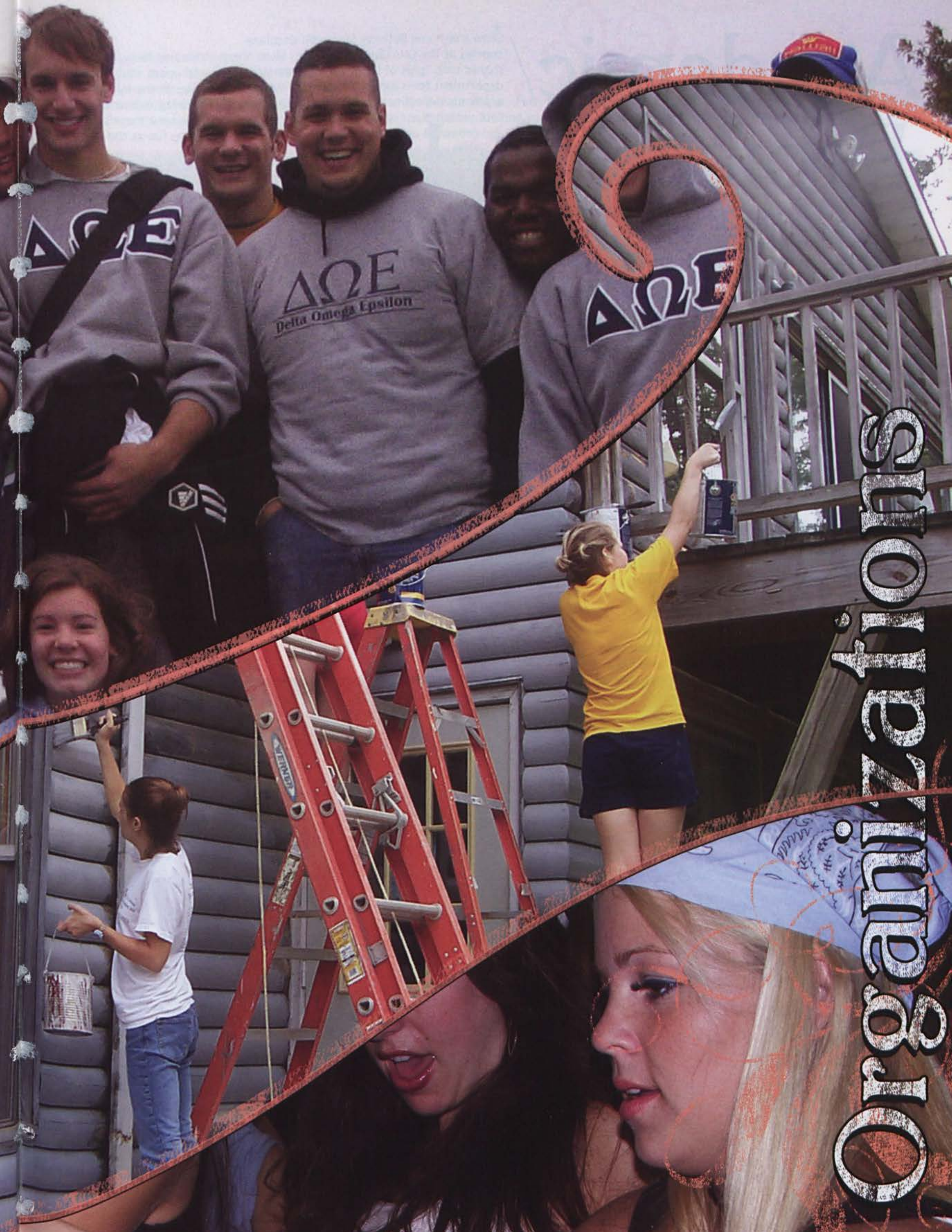



A quick stop by Omega Mu's table at the Org Fair gives an interested student a chance to win a gift certificate and free copy of the U.S. Constitution.
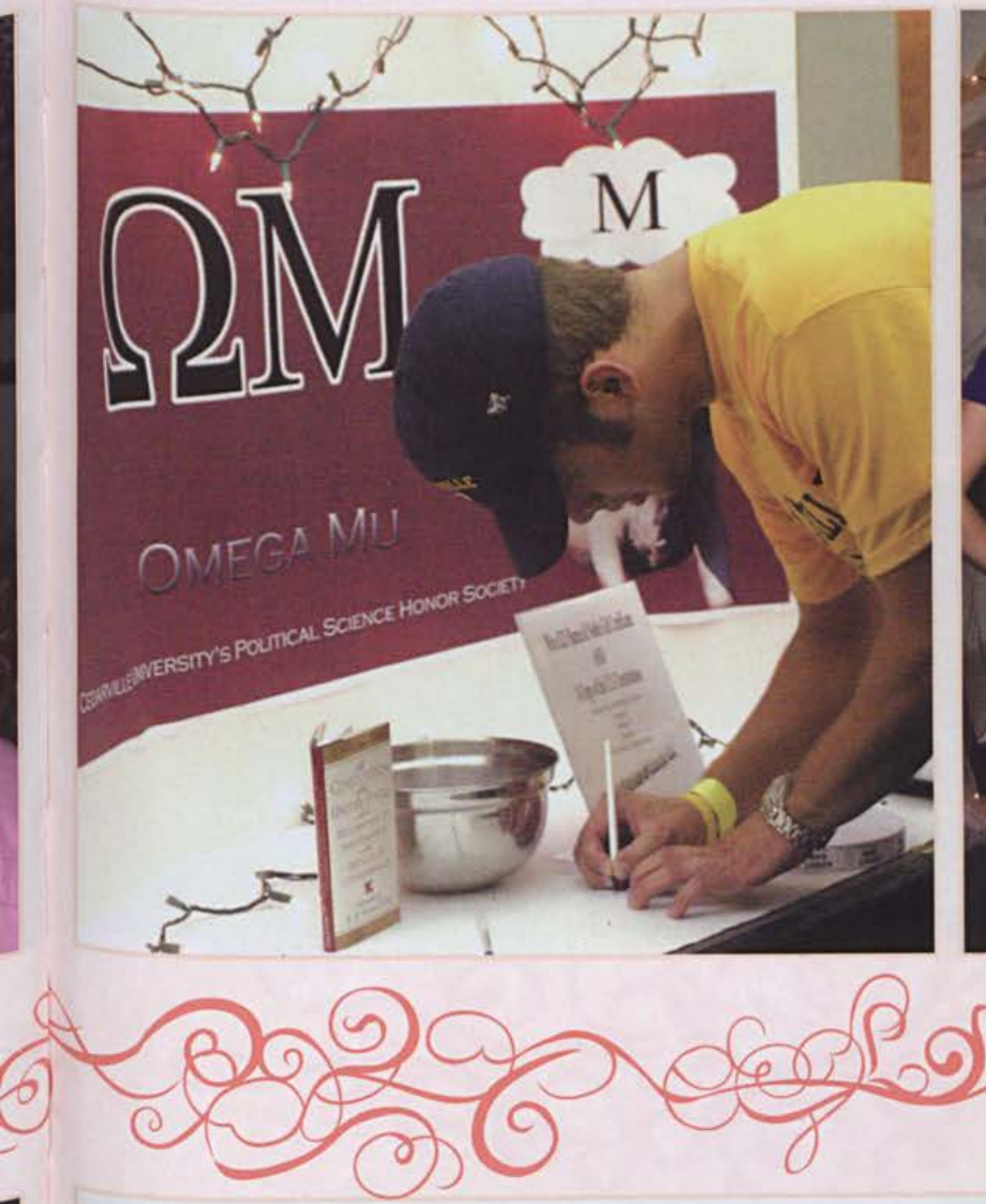

$\checkmark$ Alpha Beta Phi, the theater honors organization, livened up campus and got everyone in a theatrical spirit for their first-ever Halloween Masquerade. Kelsey Nelson, Jim Tullett, Tori Duff, and Colin Luther dress up as the gang from ScoobyDoo. “Jenkies! It was like, far out!” said Daphne (Kelsey Nelson).
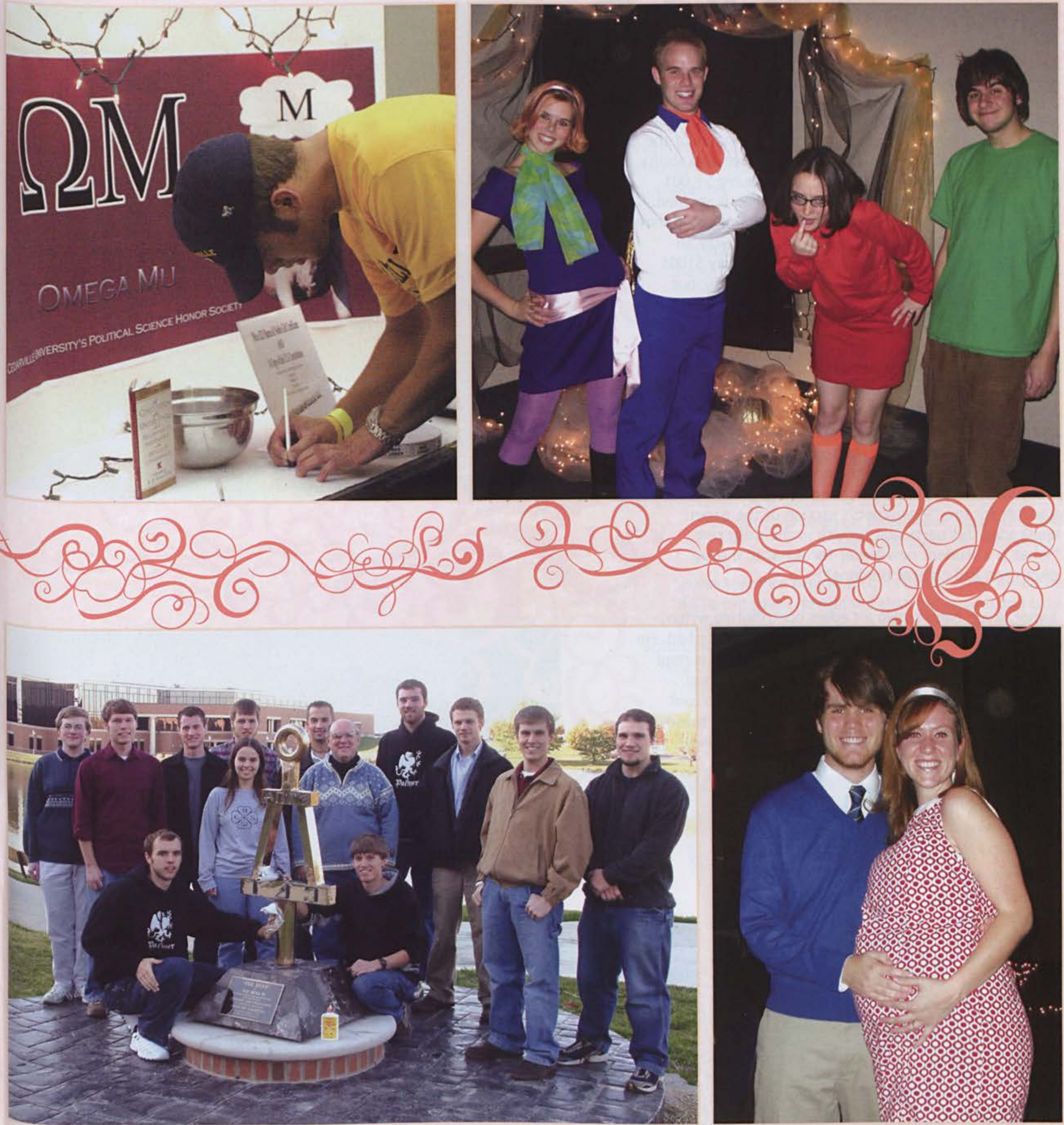

Nathaniel Miller, Joseph Miller, Randall Plate, Joshua Lansford, Jonathan Elchison, Ryan Prins, Jeremy Conyers, Luke Snider, Mark Caterinacci, Joanna Hinks, Dr. Hardy Hegna (Advisor), Jonathan Taylor, and Isaac Abbott round out the members of Tau Beta Phi, the Engineering Honors Organization, and get their hands dirty polishing their beloved "Bent!"

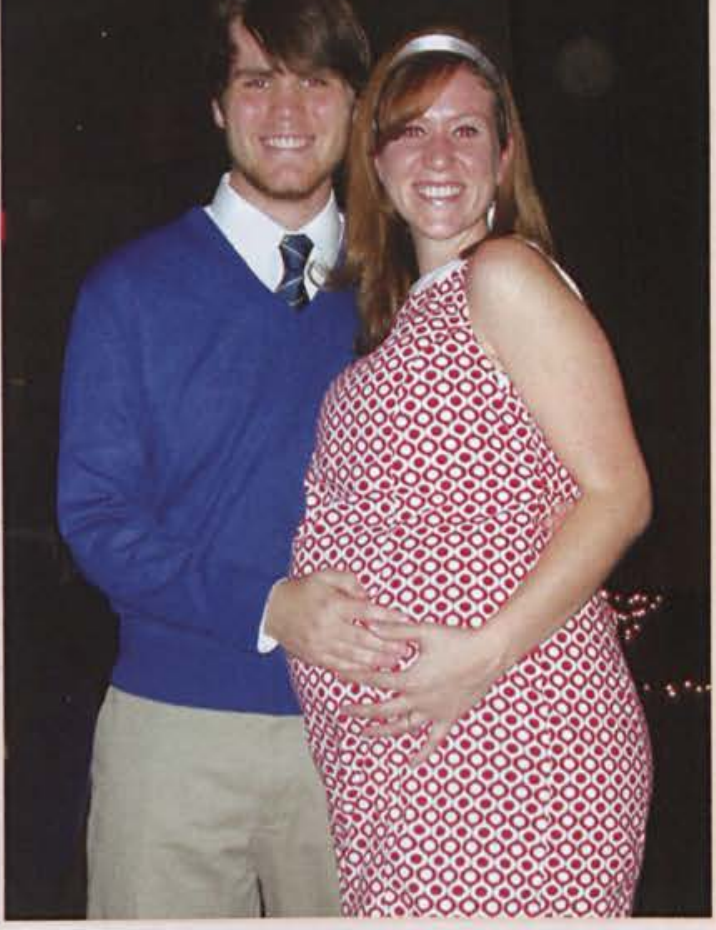

^ Senior Alan Tuttle and junior Sarah Burke impersonate an expectant couple at the ABP Halloween Masquerade. 


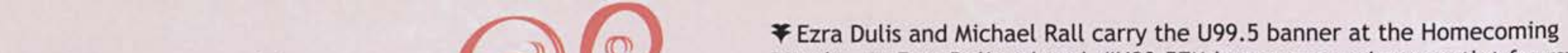

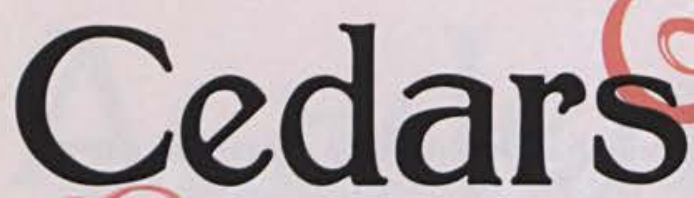
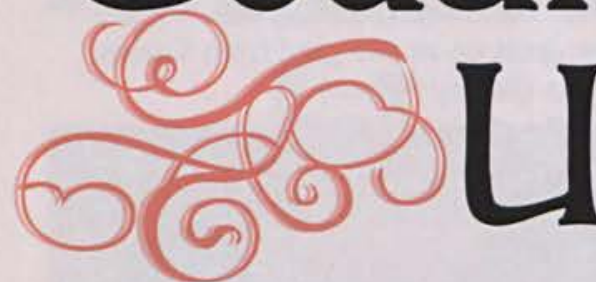

U99.

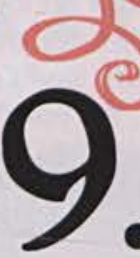

This year, U99.5 FM held a thrilling and entertaining promotion. Enlisting the help of over nine local sponsors, the radio station was able to raise $\$ 1,351$ in prizes for the winners of the grueling competition that tested contestants' stamina and ability to survive frostbite and poor circulation. The big $\$ 1,001$ promotion was a lot more complicated than it seemed. According to sophomore and co-host of the morning show, Jon Broscious, "We said we wanted to give away an XBox 360, and we did. We wanted to give away $\$ 1001$ dollars, and we did. These things were not easy, but we pulled them off through a lot of hard work and coordination."

Similar to U99.5's attention-getting promotions, this year's Cedars gained an impressive amount of readers (and reader reactions) through its thought-provoking and intelligent articles. One issue in particular created so much discourse among the student body, that an entire spread of letters to the editor was printed in a subsequent issue. Sophomore Brittany Jackson, a writer for the "Viewpoints" section of the paper, applauded this particular growth in the magazine. "What is a student newspaper for if not to inspire discussion and critical thinking?"

Junior Bryce Bahler, also a Cedars contributing writer, noted the widespread effect that the newspaper had. He remembered, "People often forget that Cedars is read by more than just students. Faculty, staff, alumni, parents, and community people all take time to look at Cedars." With the tremendous progress that these two student-run media publications have achieved, it's safe to say that Cedars and U99.5 will be grabbing the attention of audiences for years to come.
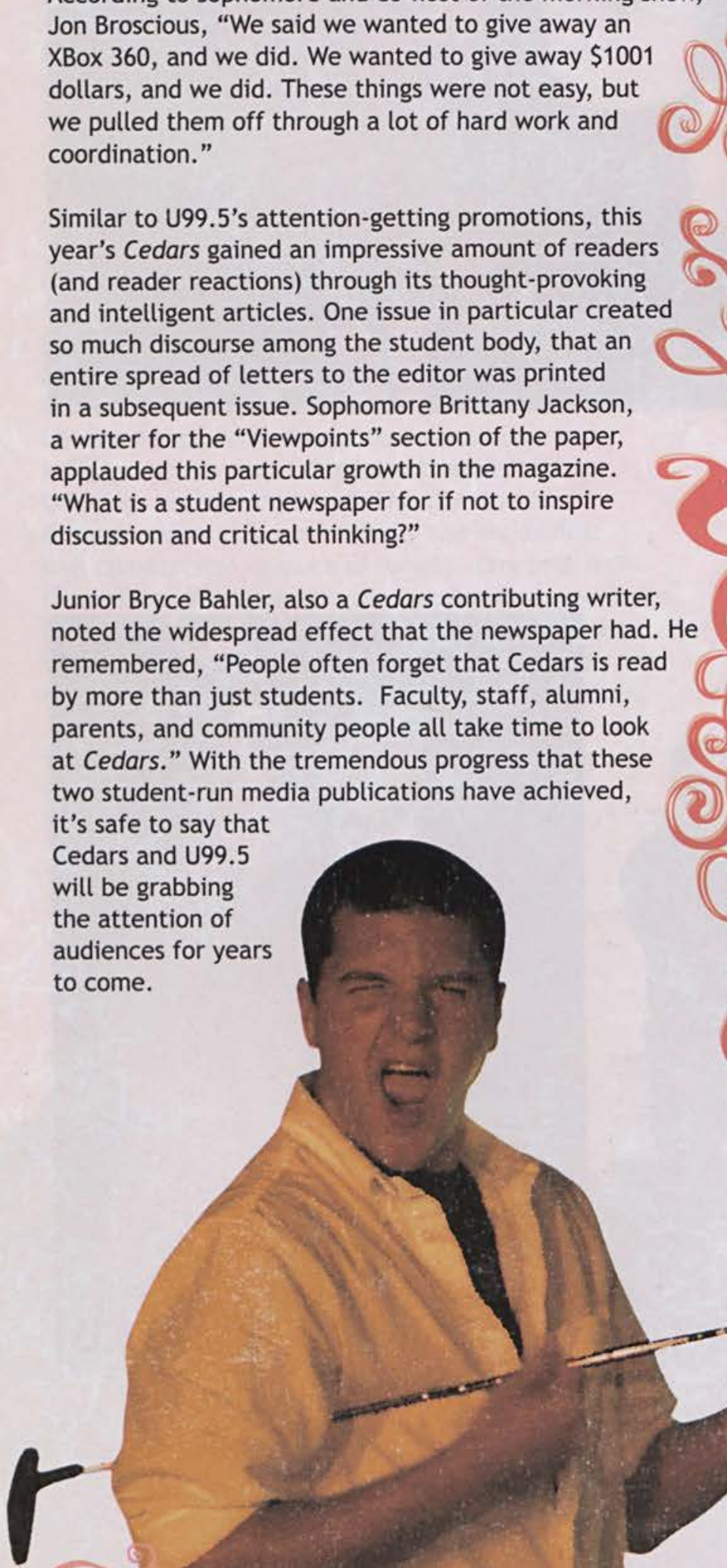

\section{Q} Parade. As Ezra Dulis related, "U99.5FM became my primary outlet for my previously unquenched desire to carry around large banners in freezing cold weather."

$\checkmark$ Sophomore Dan Parrot DJs for U99.5. As Michelle Evington shared, "There are so many different tastes, and we want to please everyone but know it's impossible. We've worked hard to get good feedback that helps us find a balance that reflects what the majority of campus wants to hear."
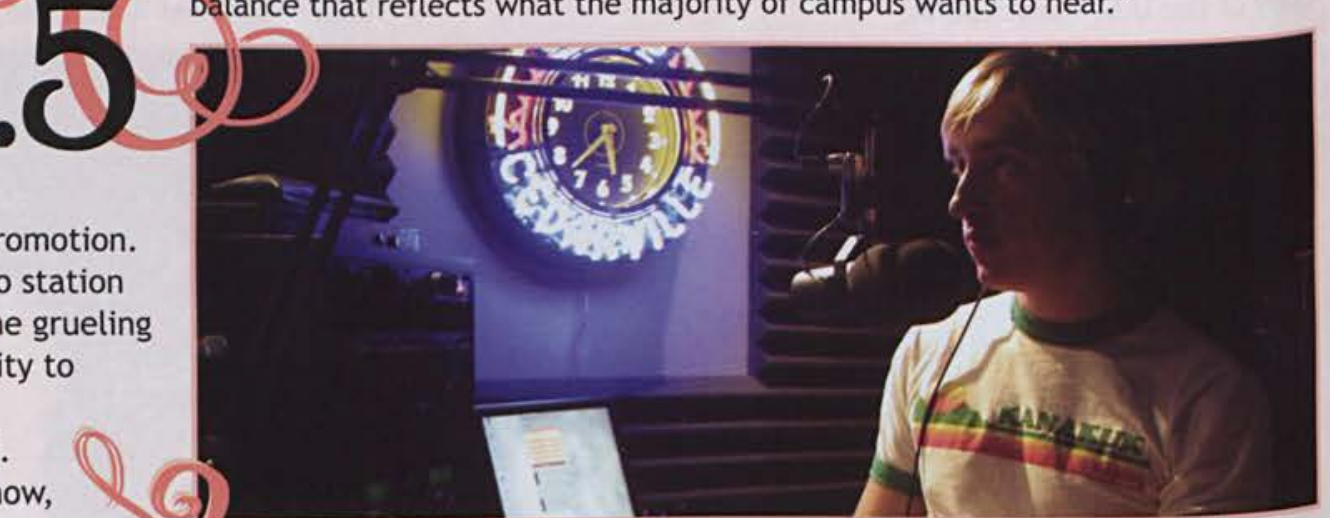
Joshua Miller, Ben Nesbitt, Joel Lansford, and Jake Garrison prepare for Saber Team practice. Sophomore Julia Basford related, "With hard work and the encouragement of my peers, I could actually do things I never thought I could."

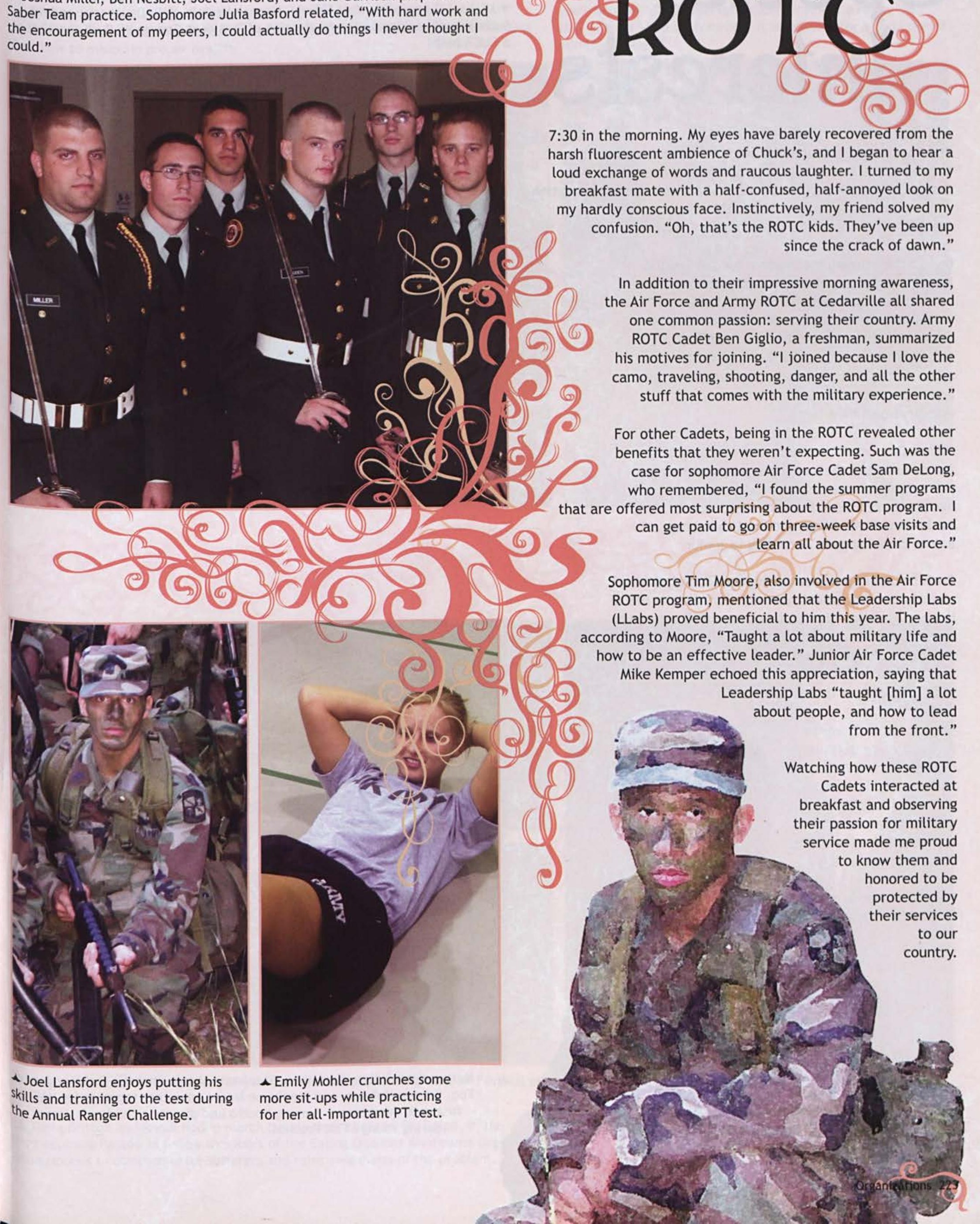




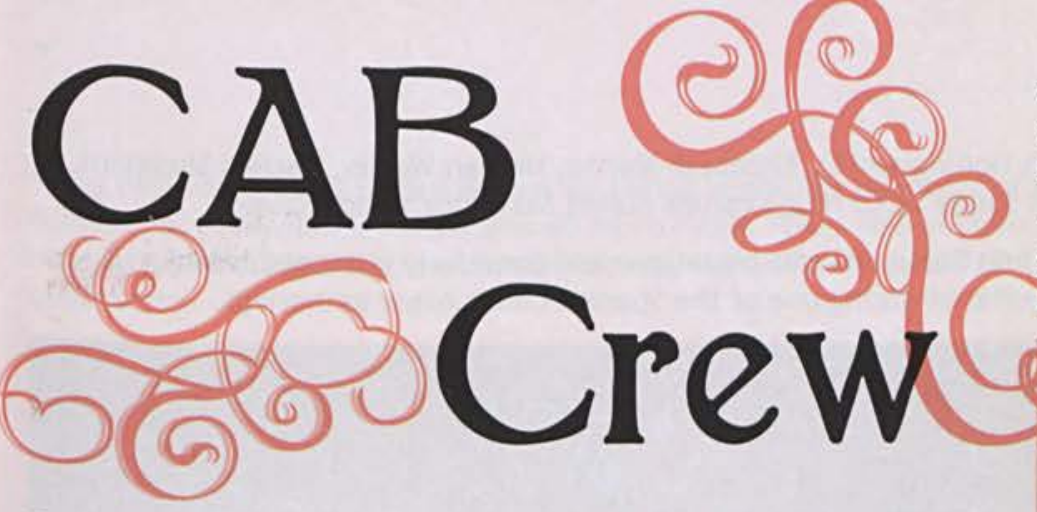

₹ Phil Kirby, Nicole Boop, Becky Schillack, Dan Basham, Dick Walker (aka Chief), Phil McCune, Bethany Hochstaetter, Katie Krumeich, Emily Doane, AJ Clemans, and Tim Warren relax at a CAB retreat.

$\checkmark$ Katherine Sawdon, Michelle Bicking, Phil McCune, Rebecca Schillack, AJ Clemans, Nicole Boop, Dan Basham, Katie Krumeich, Tim Warren, Jannette Foy, Bethany Hochstaetter, and Emily Doane dine together in the PDR.

Campus Activities Board (CAB) and Committed Representative Enthusiastic Willing (CREW) have been behind some of the greatest activities of campus this year. As students volunteered to help out with various conferences, many forgot that previous work needed to be done in order for the conferences to happen. Very often, CAB and CREW were the main groups heading up all of these conferences and putting in a lot of hours behind the scenes.

For the second time CAB hosted the Senior Tea, which 120 women attended. Although this was a relatively new activity, CAB had been behind some of the classics such as Getting Started activities (who doesn't remember the "500 Miles" song and dance?), the Org Fair, High School Leadership Conference, Leadership Institute, Parents Weekend, and Lil' Sibs Weekend. Emily Doane, senior member of CAB, reflects that her past years of working with $C A B$ had been amazing. "Dick Walter, affectionately known as Chief, has modeled for me what it means to be a servant and how to always have my eyes open for different opportunities to serve others."

An exciting new addition to the CREW organization this year was Worldview Weekend. The first Worldview Weekend included world-famous speakers such as Kirk Cameron. Along with this new addition, CREW was also in charge of traditional festivals like Junior Jam, the Homecoming Parade, Revolution @ Blowout, Fall Pastor's Summit, Leadership Conference, Youth Workers Summit, CedarMania, Evangelism Celebration, and ACSI Music and Art Festival. Junior Brynn Paine was a member of CREW and enjoyed Junior Jam because of her passion for children's ministries. "It's rewarding to see how your hard work results in children having fun and learning about God."
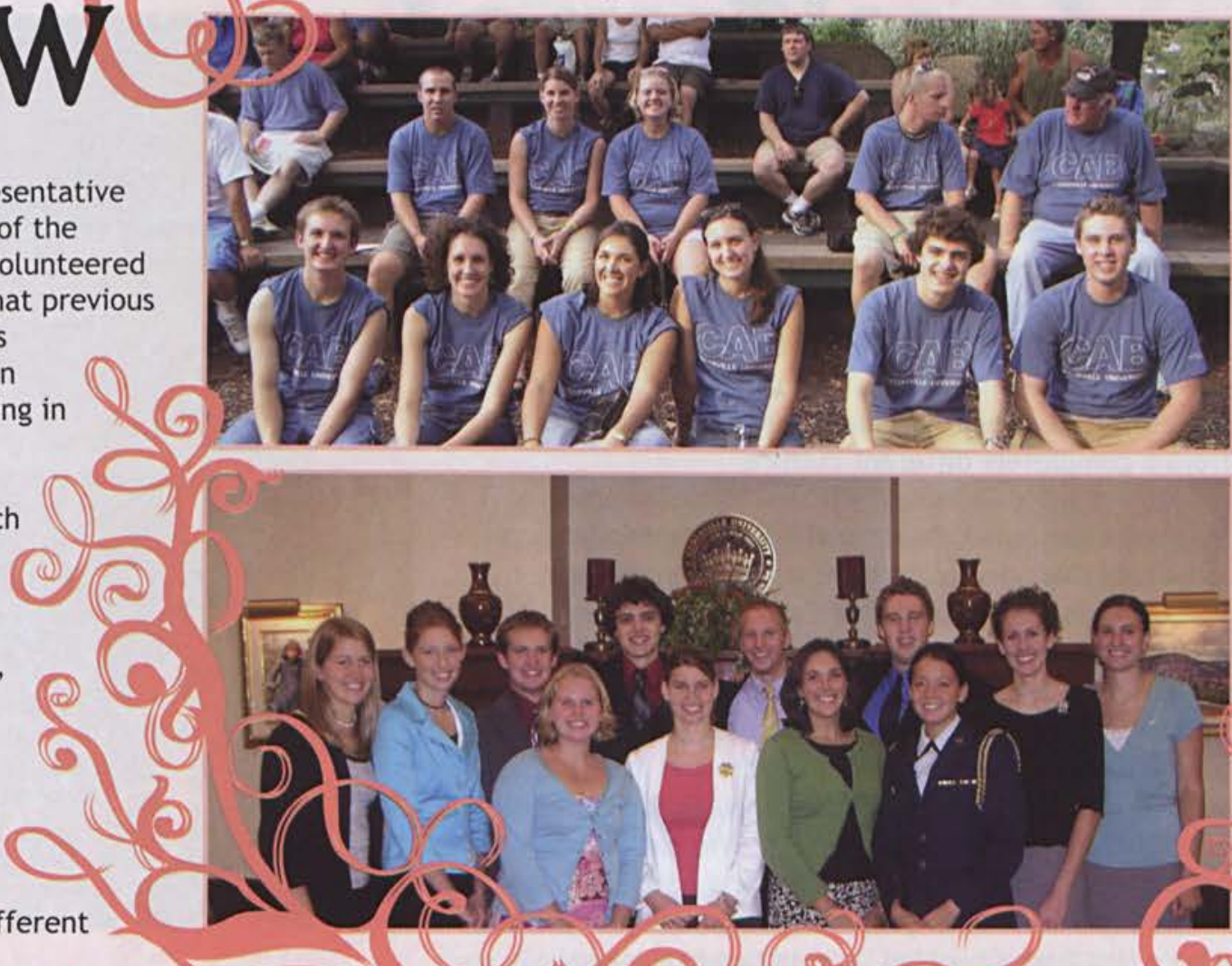

\section{H}

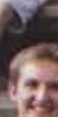
(e)
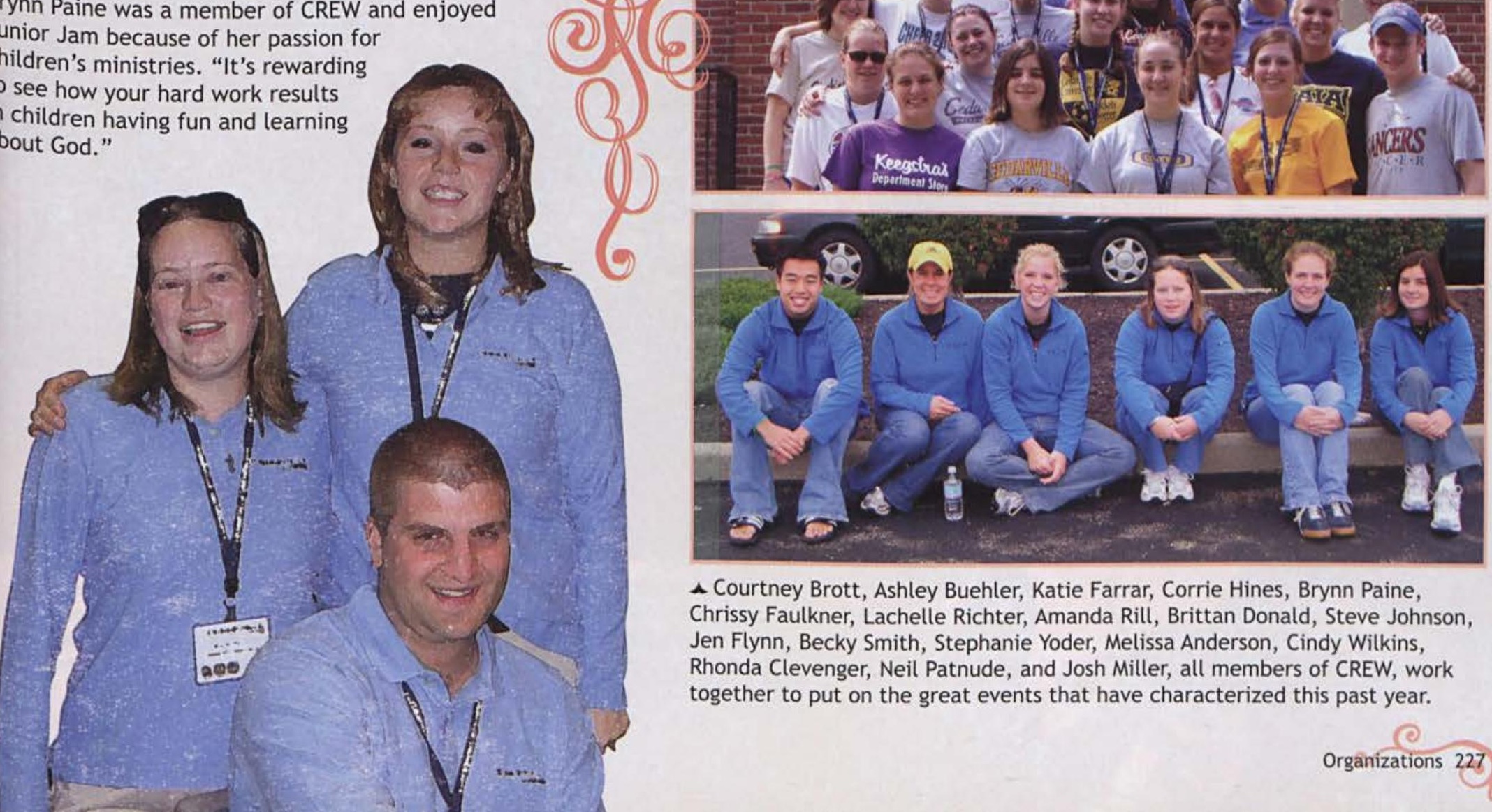

^ Courtney Brott, Ashley Buehler, Katie Farrar, Corrie Hines, Brynn Paine, Chrissy Faulkner, Lachelle Richter, Amanda Rill, Brittan Donald, Steve Johnson, Jen Flynn, Becky Smith, Stephanie Yoder, Melissa Anderson, Cindy Wilkins, Rhonda Clevenger, Neil Patnude, and Josh Miller, all members of CREW, work together to put on the great events that have characterized this past year. 
$\checkmark$ Nicholas Fridenmaker, Ryan Rotman, Emily Stephens, Steve Clemons, Daryl Sando, Kevin Vaughn, Heather Grier, Peter Shellabarger, Sarah Clift, Kelly Myers, and Vashti Mensah, members of DAE, the org that assists Alumni Relations, work hard all day so they can relax at the edge of Lake Michigan.
₹ Murphy R.D., Bonnie Kuvshinikov, and Sara Eplin take a back seat as they are chauffeured back to campus after Homecoming.

$\checkmark$ Kate Martin shares with a boy from Eleuthra while on her trip with DPS.
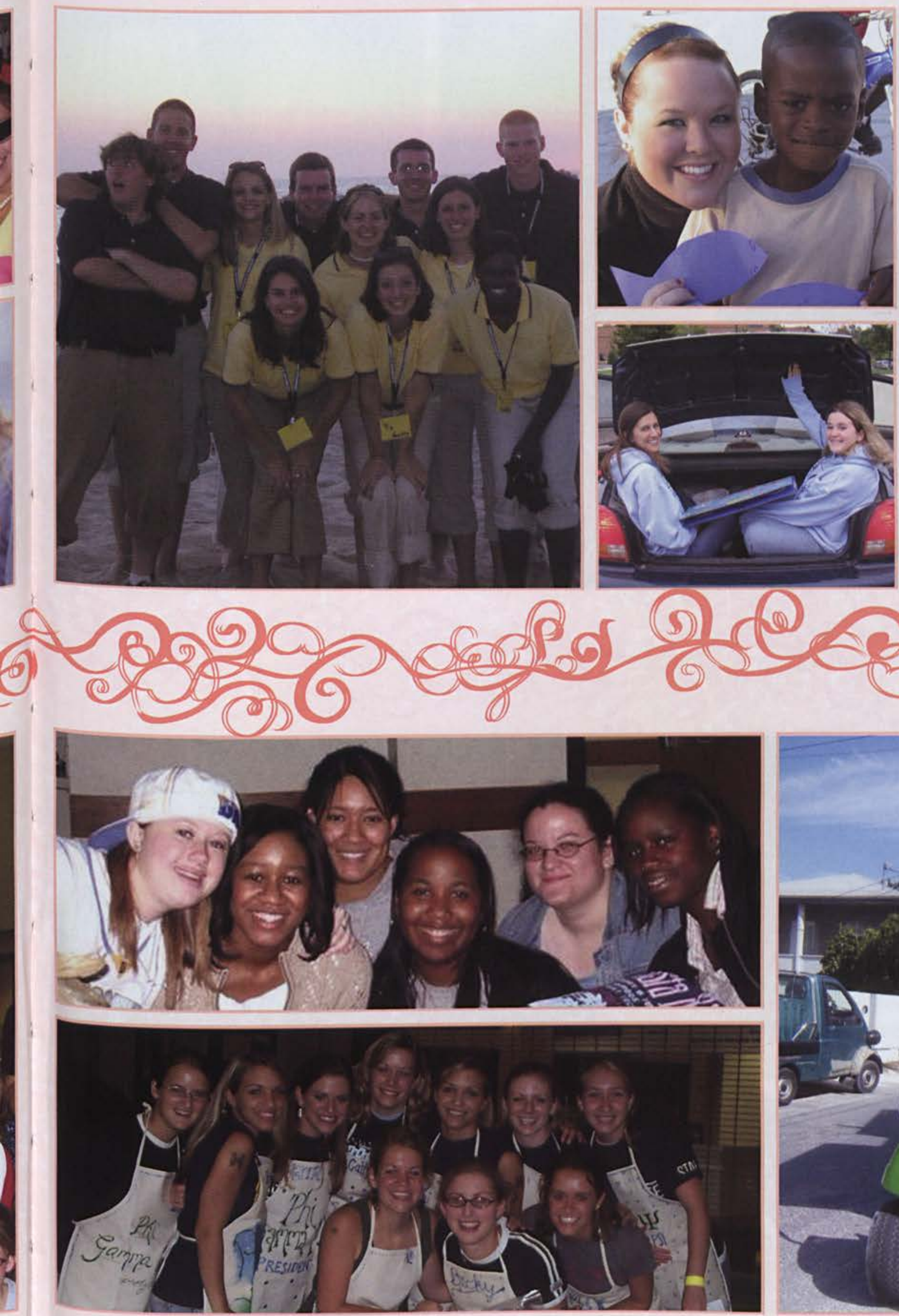

Members of PGP, Clarissa Linde, Brooke Marietta, Hannah May, Cathy Daniels,

Brittany Marietta, Jen Krier, Cristy Thompson, Andrea Smith, Becky Stowers, and Ivey Harrell prepare to make soft pretzels for the Org Fair.

* Kriss Marr, Candace Smith, Kathy Simpson, Arielle Dorsey, Bekah Taylor, and Tonisia Jackson gather at a party sponsored by the P.E.A.C.E. Project. $\checkmark$ Monica Godinez paints "the Rock" on the Gamma Chi/Alpha Chi Homecoming float. Participating in a service org is important to Monica because "it is a great thing to meet any little need someone might have."

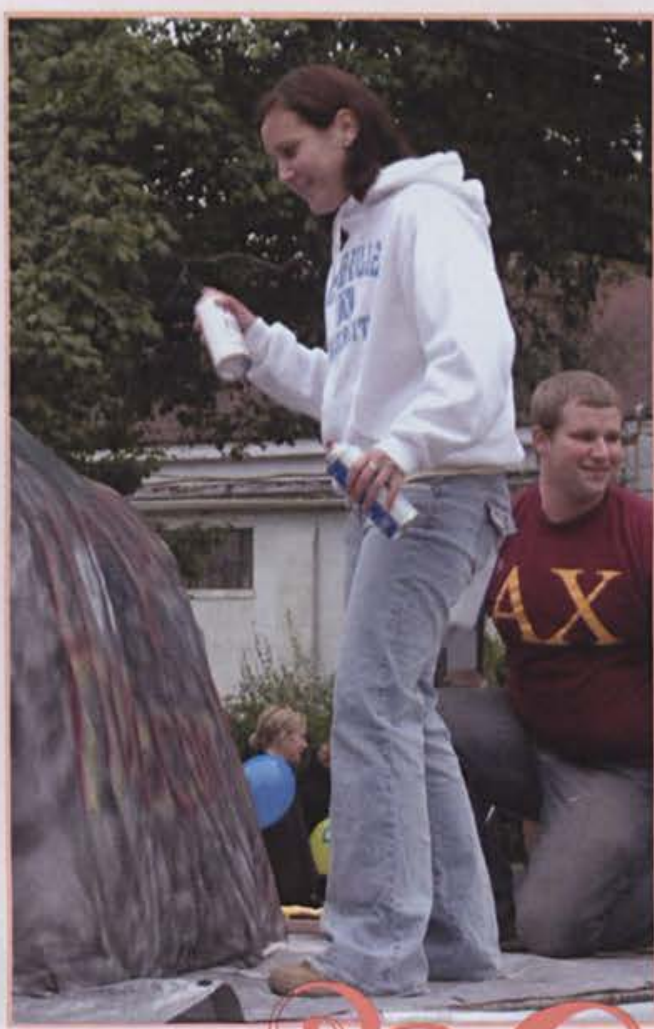

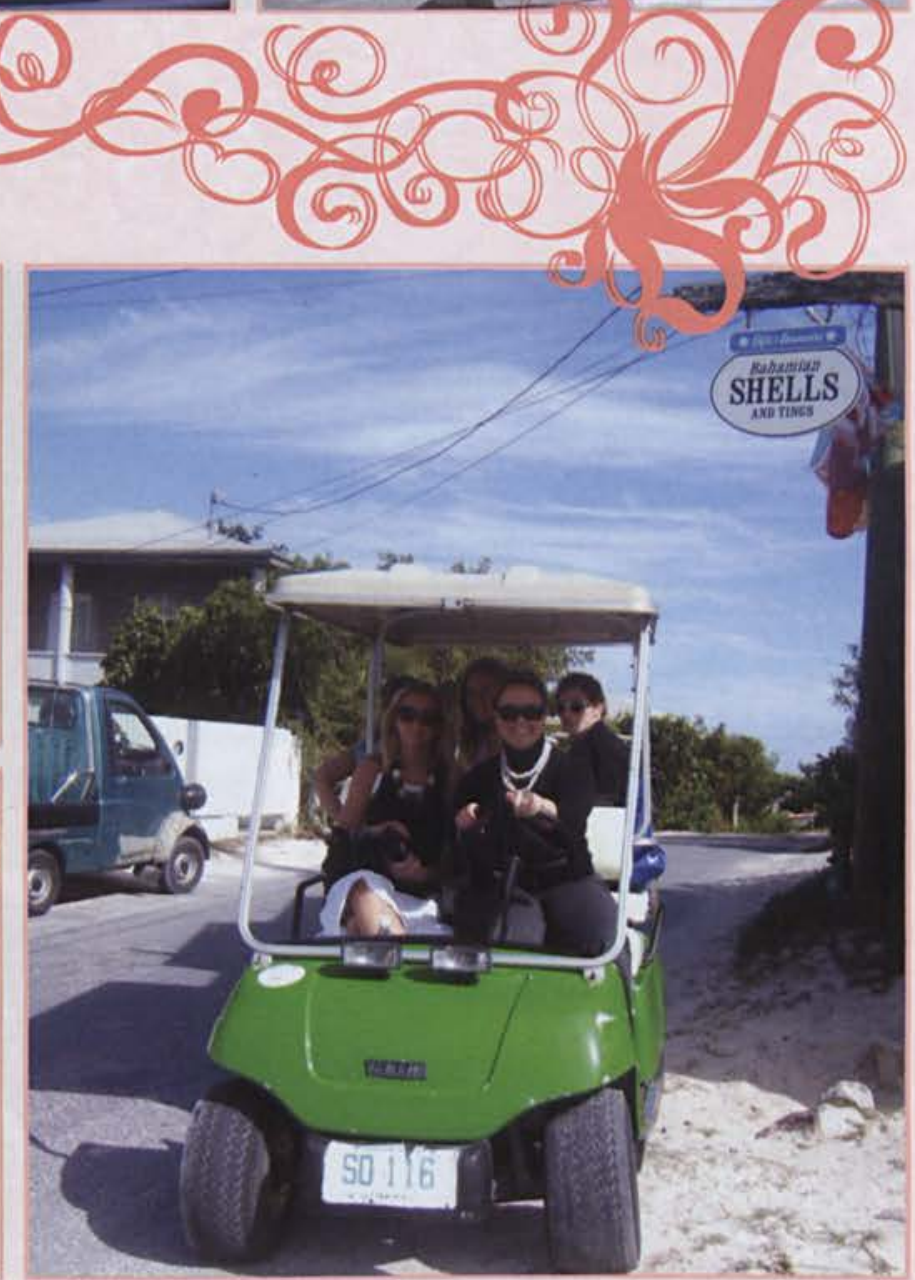

$\Lambda$ Kate Martin and Kelsey Kirby cruise the sand and gravel streets of Eleuthra with the other girls from Delta Pi Sigma. 
$\checkmark$ At the Org Fair, sophomore Justin Gutierrez draws attention to Theta Rho Epsilon, a men's organization that seeks to develop character and friendships through social and service opportunities.
Mr. Spencer is no match for Sara Eplin, Carrie Gumm, and Christen Brady, Bonnie Kushinikov, and Janna Schwab of Alpha Delta Omega!

$\checkmark$ Induction ceremonies for PGP

reach the strange and bizarre - but always fun.

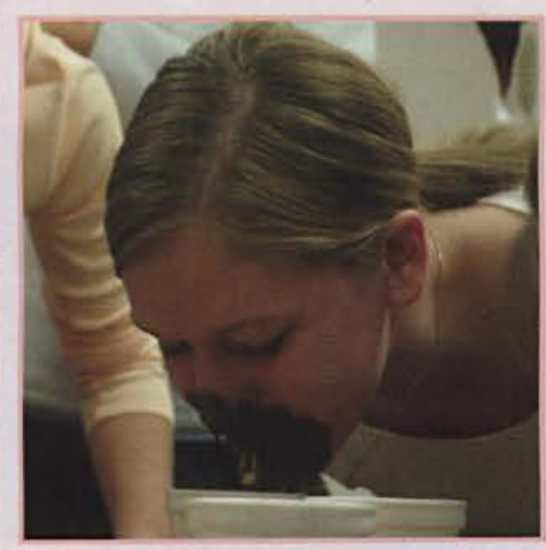

$\checkmark$ Pamela Seymour joins the girls from Delta Phi Sigma in welcoming the newbies during initation rounds with silly string and a handful off DPS roses.
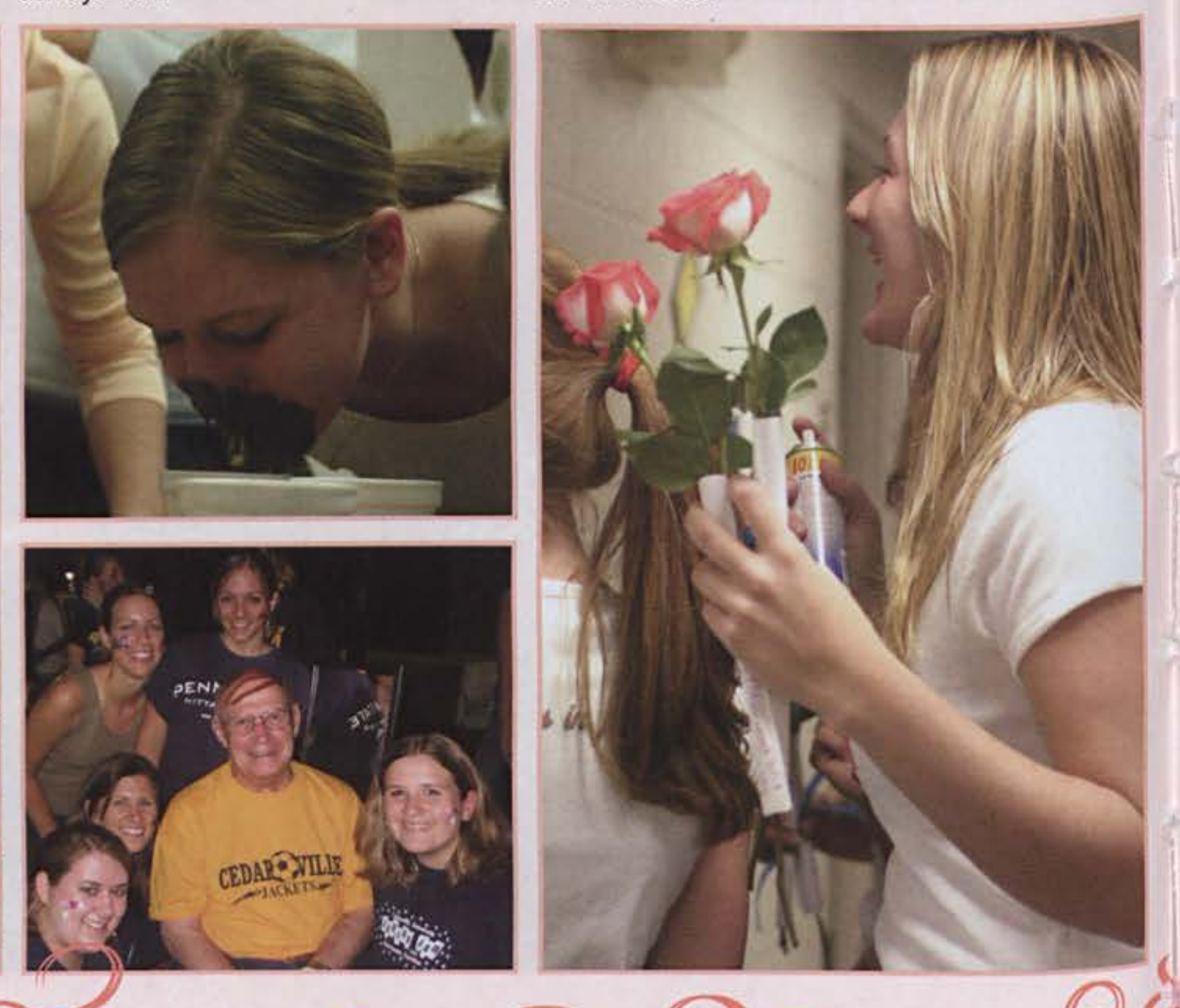
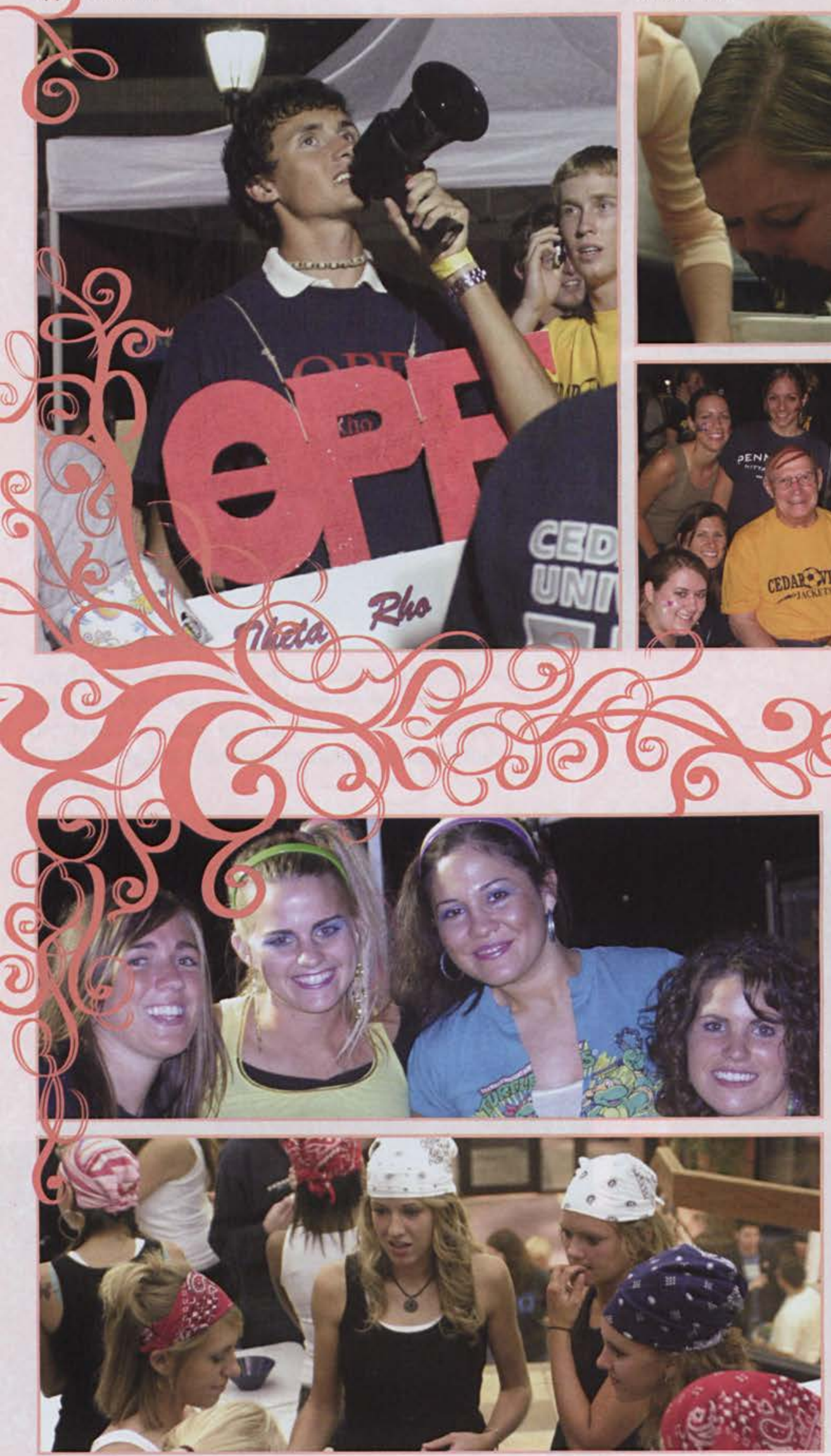

Aftan Hunt, Stephanie Hueni, Casey O'Neal, and Hannah Kowatch apply tattoos (rub-on, of course) to students at the Org Fair. Of her PEB girls, Stephanie Hueni said, "They have sharpened, challenged, and blessed my life in more ways than I can say."

¿ Kate Martin, Lauren Nuss, and Chelsea Bowman sneak some popcorn and old-fashioned Coca-Cola during the Homecoming Parade.

^ DPS girls Amy Elliott, Kate Thompson, Emily Trevino, and Kendra Battaglia chill in their 80 s gear at the annual Org Fair. 
₹ Jessica Forsythe, Robin Provo, and Lauren Nuss support Delta Pi Sigma at the Org Fair. "There was lots of good food, silly games, free things and a lot of fun!" said junior Lauren Nuss.

$\checkmark$ Kate Martin, Amanda Stroop, Debra Halsmith (group leader), Kelsey Kirby, Pamela Seymour, Julie Moran, and Kayla Smith, work at the Eleuthera Bible Training Center with Delta Pi Sigma. All would agree with Kayla Smith's words when she said, "Serving with girls that have a passion for Christ while enjoying God's beauty of the islands was an experience that I will never forget."

$\checkmark$ Jonathan Thomas, Tyler Anderson, and Kevin Gulley are enthusiastic Delta Omega Epsilon. "I love DOE! We are guys who focus on three things: Discipline, Obedience and Excellence. We have a special bond in Christ," said senior Tyler Anderson.
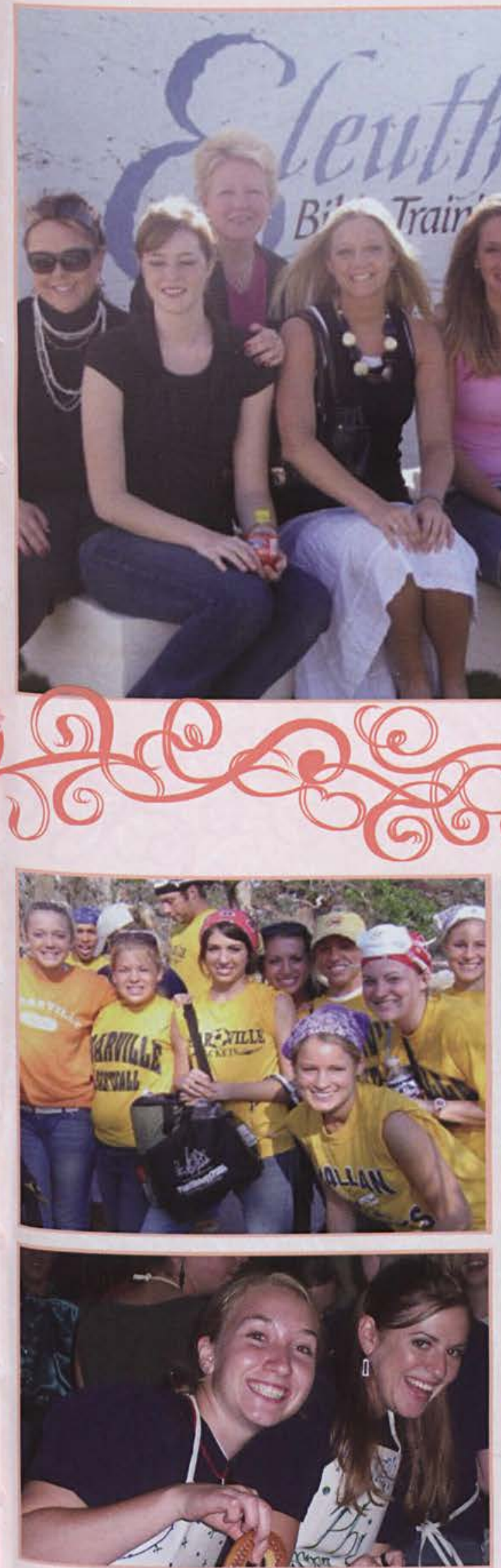

C Cristy Thompson and Hannah May from Phi

Gamma Psi serve soft pretzels to students at the Org Fair.

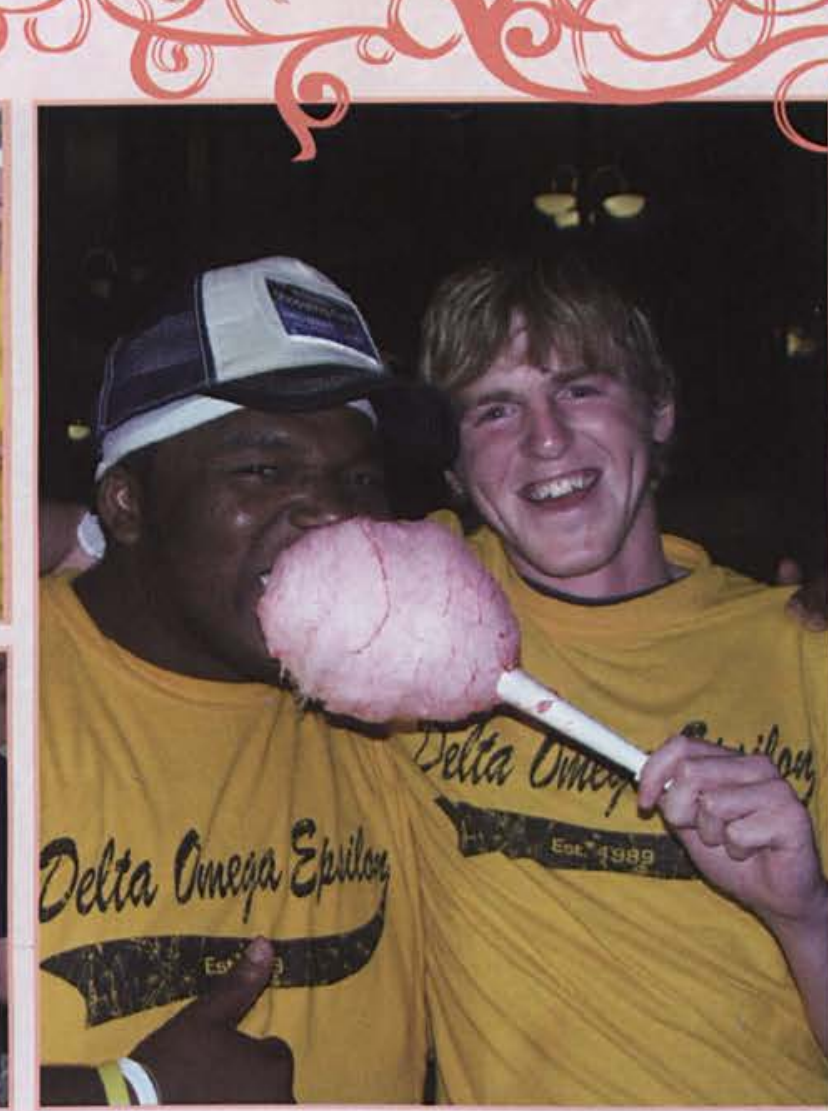

$\Lambda$ Rod Bradley and Tim Sandlund goof off with Delta

Omega Epsilon. Jokingly, Tim Sandlund said, "DOE boys are sweet!"

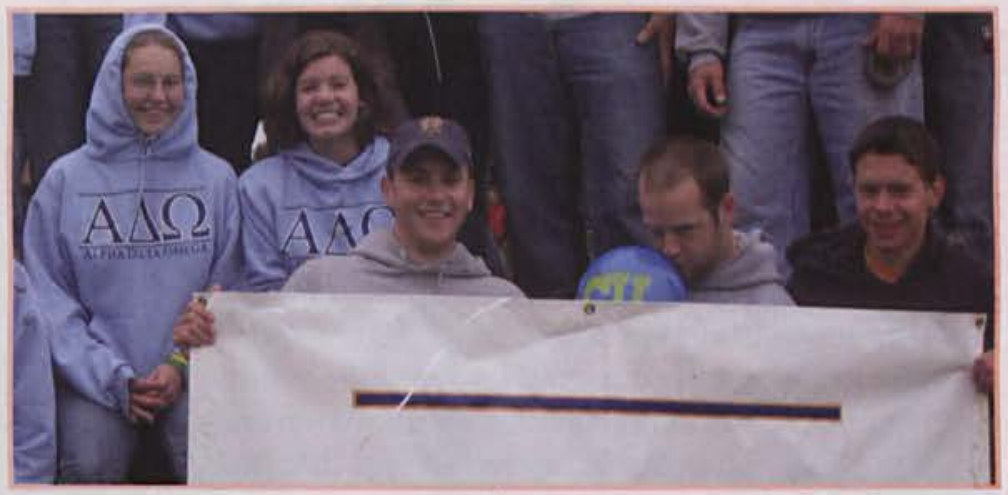

* Kelsey Kirby, Jennifer Boulet, Rachel Teat,

Rebecca Wolff, and Stacy Alexander take a quick

break from work with the rest of PEB. 

$\checkmark$ A member of the MERCY Team guides "Freddie," a loveable boy who attended the MERCY Team's Family Christmas Party, through the events and activities of the evening. $\checkmark$ A rather excitable Israel Peck and other APO members gather at Dr. Dolph's Cedarville home for a bonfire during the Fall semester. Even brief moments like this, hanging out with friends, can make all the difference in the middle of a long and busy semester.
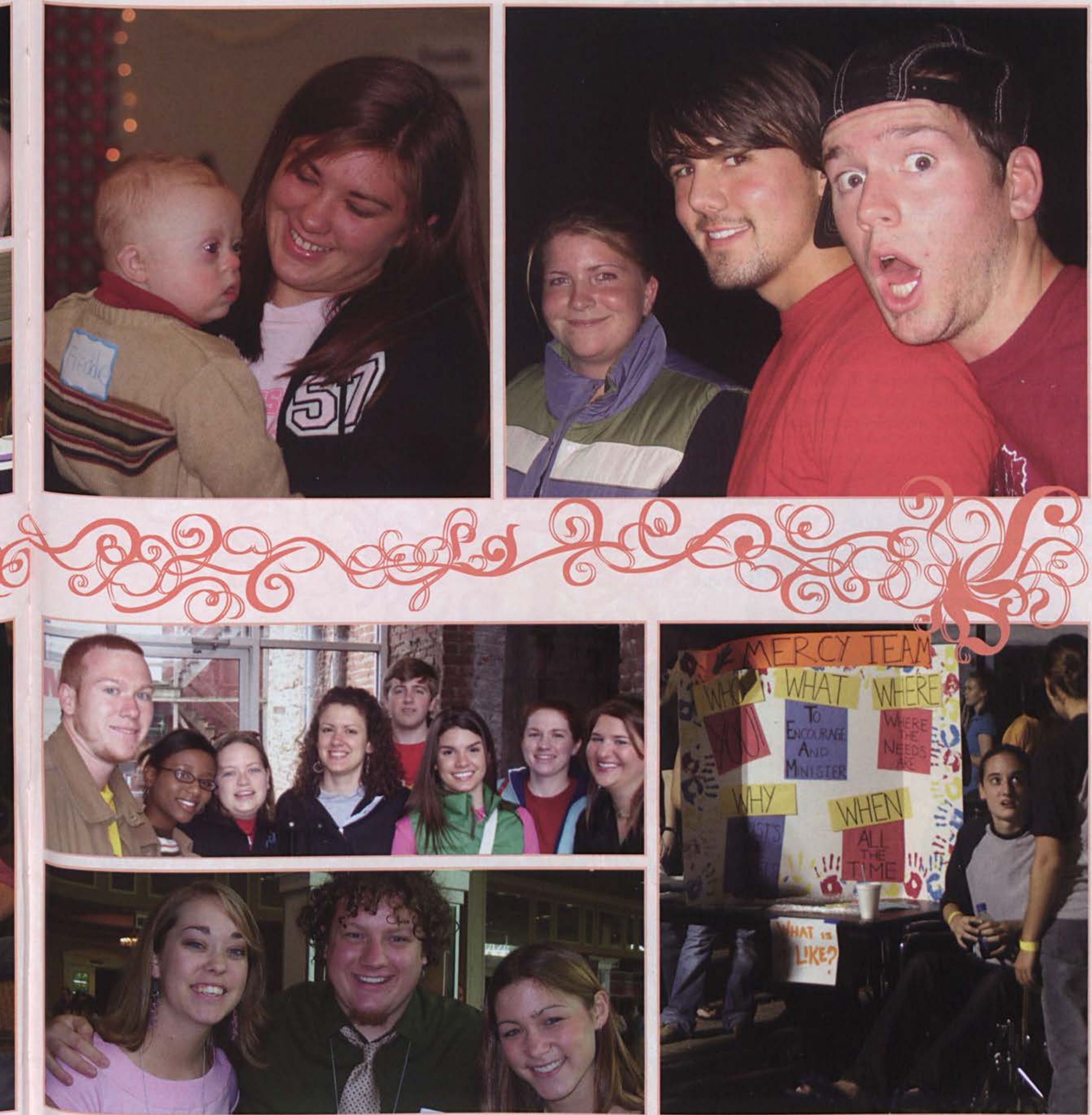

Kristen Heiner, Bryce Bahler, and Jessica Jantzen, from APO, the Psychology Organization, attend the American Association for Christian Counselors World Conference in Nashville, Tennessee.

Members of STC, Kaleb Eldridge, Latice Strickland, Brynn Paine, Erin SanGregory,

Andrew Powell, Sterling Meyers, Laura Castle and Kat Powers tour The Mattress Factory

in Pittsburgh, Pennsylvania -- a modern art museum with "cool and weird exhibits." 
John Hawkins, a tutor in the writing center, said that he "loved having the opportunity to learn what other students on campus were working on through their writings. Being able to encourage students in their writing was a great joy for me."

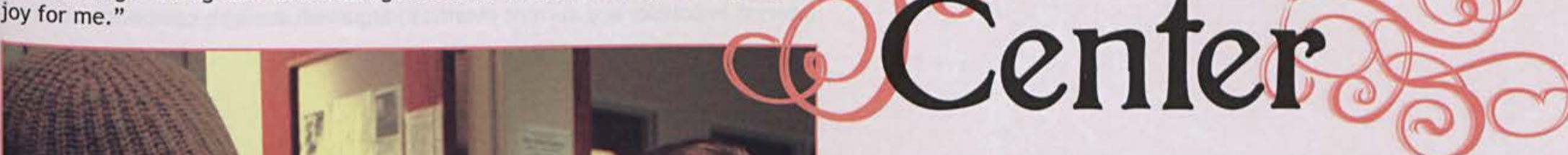

I slipped into a leather lounge chair as I waited for my consultation. Immediately I was relaxed by the soothing atmosphere and friendly faces of the tutors. Without the help

of the Writing Center, I knew that I wouldn't be able to pass Composition, let alone develop such a deep passion for the art of writing. By the time my session was over, I felt a wave of relief and empowerment to communicate my thoughts into the written word.

During the school year, the Writing Center illustrated impressive improvements in a number of areas.

They implemented a "Read to Write" program, encouraging students to read one selected book per month, such as The Chronicles of Narnia by C.S. Lewis and $W ; t$ by Margaret Edson. While the "Read to Write" helped several students in their literacy skills, the Writing Center's greatest improvement this year was their online sign-up program. Faculty director Julie Moore explained the success of this new system, which was the key to their spring semester consultations: "Every spring semester, we've always dropped in our numbers, usually by $200-300$. This is

the first year that we actually have tutored MORE students in the spring than we did in the fall. That's especially noteworthy since far more comp classes are offered in the fall than in the spring."

The Writing Center wasn't just a place for students seeking the help of the tutors. Those who worked at the Writing Center themselves also sought the help of their fellow student workers. Sophomore and Writing Center tutor Nathan Washatka attested to this. He said, "The

Writing Center has a wonderfully

laid-back atmosphere. No

one at the Writing Center is out to make you feel dumb or insufficient as a writer; we're here to improve ourselves and (hopefully!) everyone else who stops by."

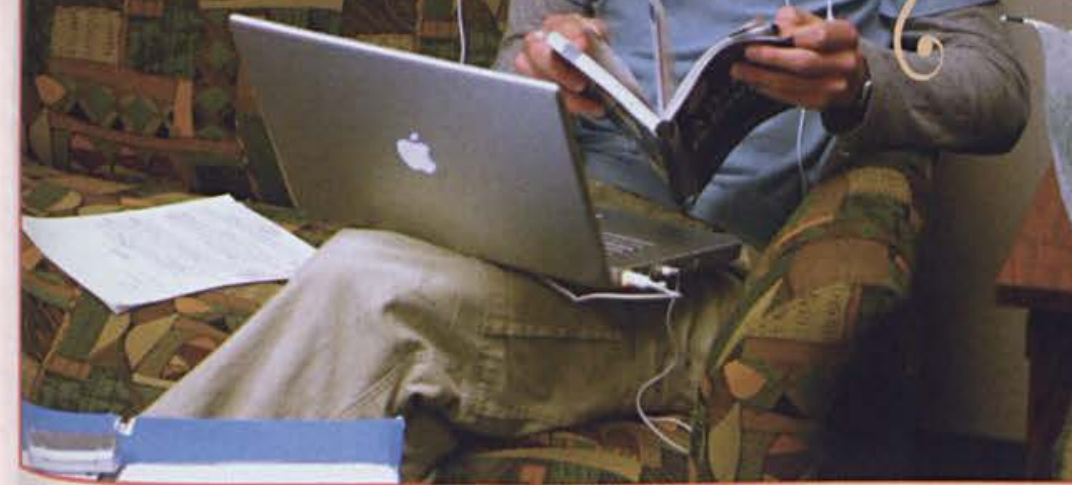

Hanging out in the Writing Center is always a good time for freshman

Nathan Sheppard. "I like the quiet atmosphere, the free chocolate, the

comfortable seats, and the help that is readily available." 


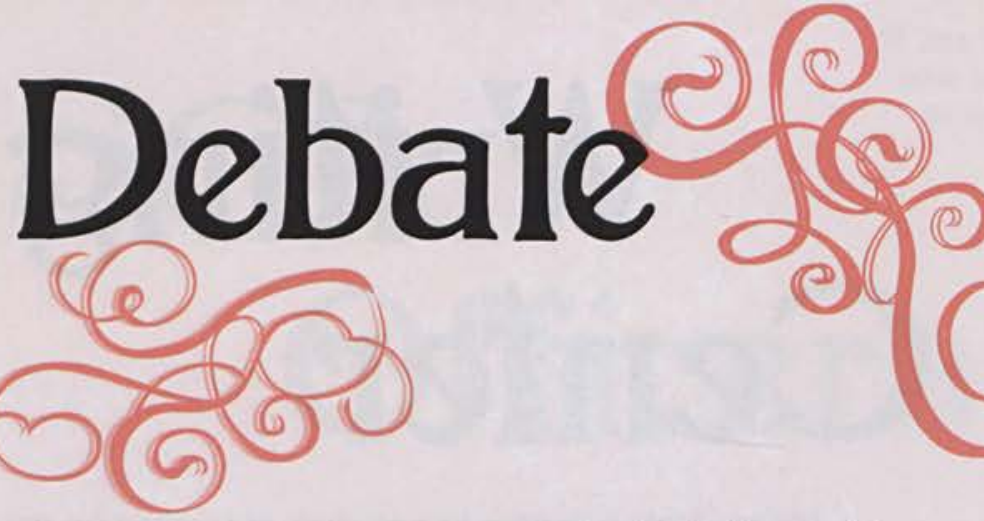

"Preparing students for life." One might think this an odd statement to be applied to team debate, but as coach Dr. Rebecca Sietman explained, "Debate gives students the opportunity to not only improve their critical thinking and speaking skills, but also allows them to engage the current issues facing us from different points of view. This helps to prepare students for life as they will have to deal with these issues each and every day."

Cedarville University's parliamentary debate team was one in the making. Not only was it making a name for itself, but it was making students to be critical thinkers who will have the knowledge and ability to address the world head-on.

This year was Dr. Sietman's third year leading the team, which participated in a total of six tournaments. They placed 2 nd at Purdue and 1 st at Western Kentucky University, Berea College, Hillsdale College, and the University of Notre Dame. At the National Parliamentary Debate Association, the largest national contest in the country, Cedarville was ranked number 14 , a large improvement from the number 40 spot they occupied last year.

Sophomore Mark Miller expressed his ideas about the debate team: "Debate is an awesome opportunity to use the gifts God has given me for His glory. I love the ability to use my mind to think critically about issues relevant to today, and debate gives me that opportunity. Debate is a great opportunity to show nonbelievers that being Christian doesn't mean you 'check your brain at the door.' I think it's great that the majority of our competitors are liberal non-Christians and that we can not only hold our own, but argue issues from a Christian point of view and challenge opposing ideas."

Brandi Biggar's four years in debate has helped her "cultivate a lasting interest in politics and current events." $\checkmark$ Nathan Washatka systematically expands upon the points of his logical argument during a competition.

\section{.}

\section{flai}


$\checkmark$ Shawn Green and Heather Wenzel perform a duo with their usual gusto and flair for the dramatic. It is passionate performances like these that give our Forensics team such high marks in competition.

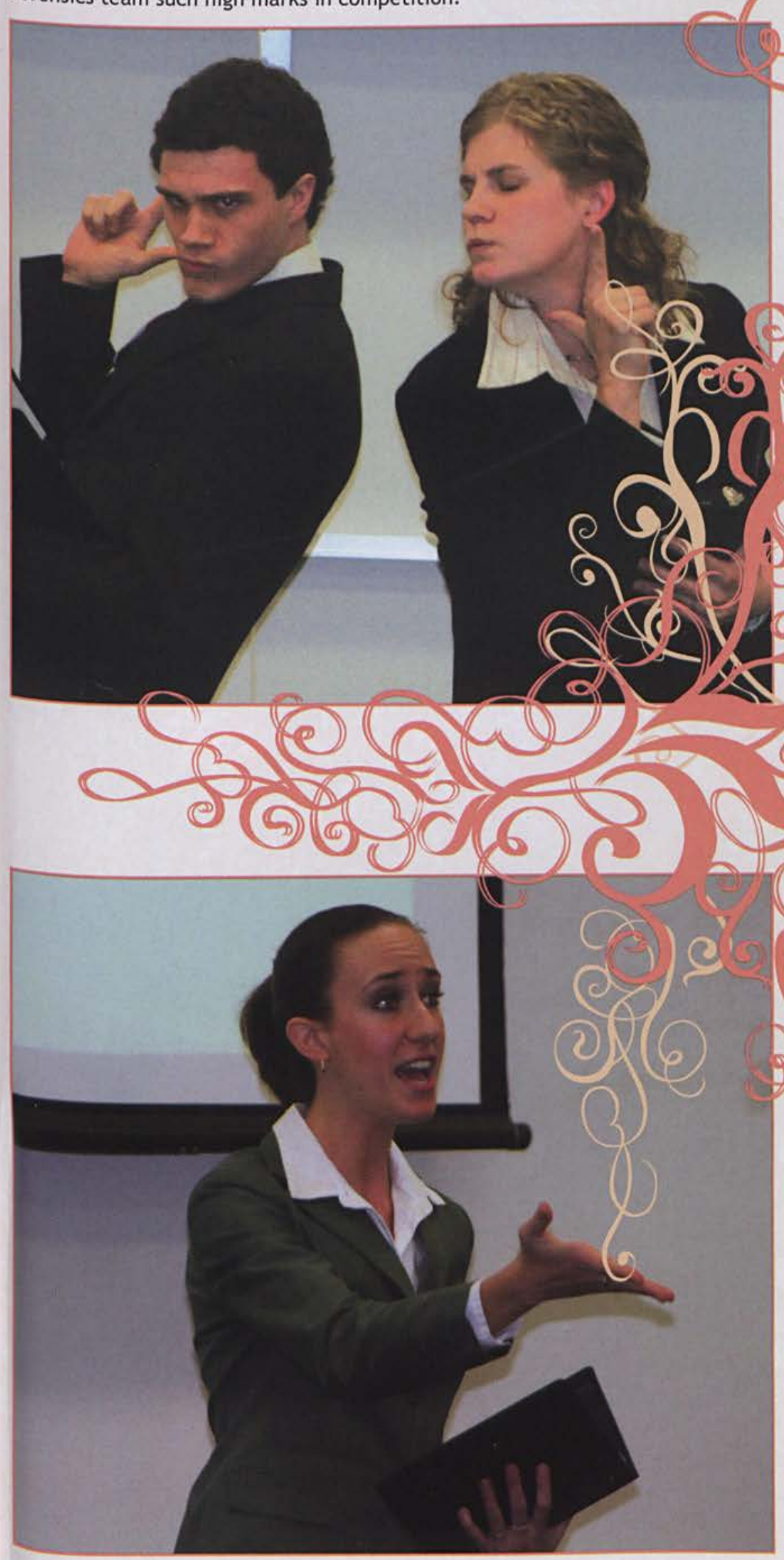

2

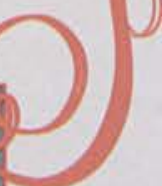

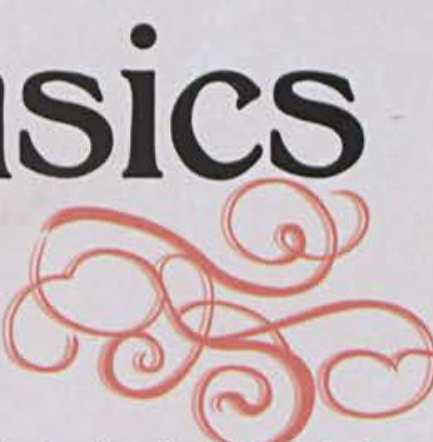

"Forensics. You mean they study dead bodies?" This is the thought that comes to many minds when you use the word forensics, but for a select few across the nation, it means a highly competitive speech organization. Matthew Moore, the forensics team coach, said the purpose of the team at Cedarville University was to "take a student's skill to the next level as a researcher, writer, speaker, and performer.

We also purpose to be a testimony for Jesus Christ as we perform with excellence." Students competed in twelve different events broken into three categories: public address events, limited preparation events, and oral interpretation. Although one might not think it, these speech competitions were very grueling and required many hours of research and preparation.

Matthew Moore explained, "It is one of our team goals to have at least one spiritual conversation with a competitor per tournament. So we are very intentional about building relationships with students from other universities and dialoguing about what we believe and why." To help reach this goal, the Cedarville forensics team invited students from Kent State University meet in Columbus for a time of peer coaching, debating, and fun. Through a "scrimmage" debate

on Saturday, the team had many opportunities to engage the Kent State students in spiritual discussions. Team member Ben Bolander said, "It was an amazing way to strengthen our relationships with the Kent team. There is a great mutual respect between our teams that allows us the chance to talk openly about our faith."

Along with their multiple opportunities to share the gospel, the forensics team experienced many academic successes this year, including taking first place at

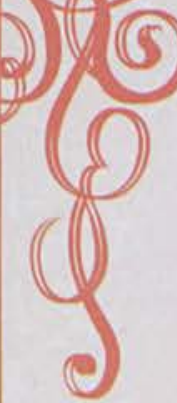
the 23rd Annual Tower Invitational hosted by Eastern Michigan University as well as taking the 2nd place sweepstakes award at the Holiday Frolic tournament at OSU. In doing so, they fulfilled their goals to share their testimony through their competence and skills at both local and national tournaments.

Mallory Fisher fervently portrays a desperate young woman who comes

to realize only she can save herself; not drugs, and not the doctor she is Pleading with in a piece entitled "Scoring." 


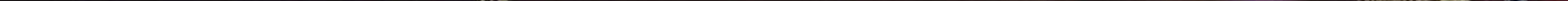




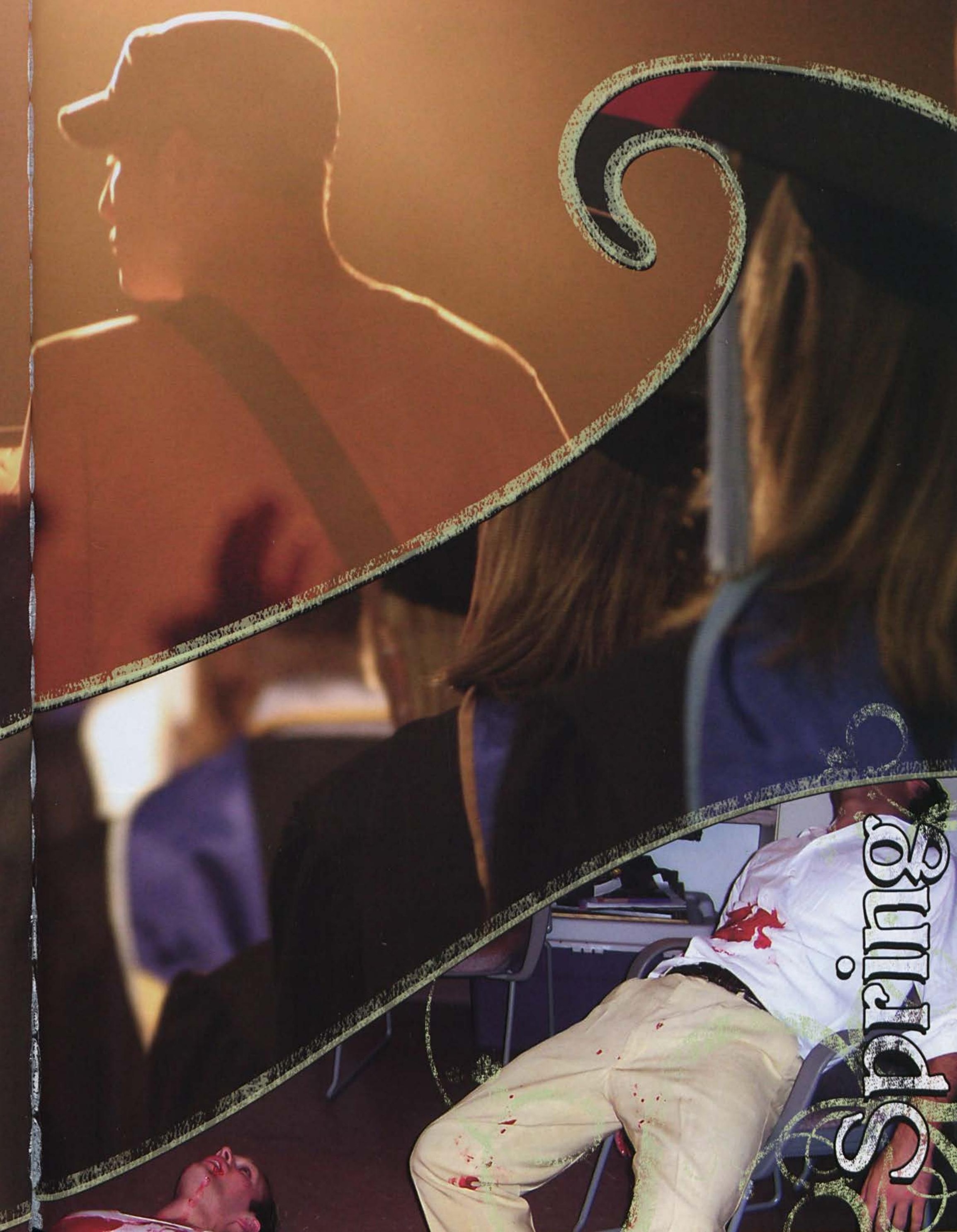





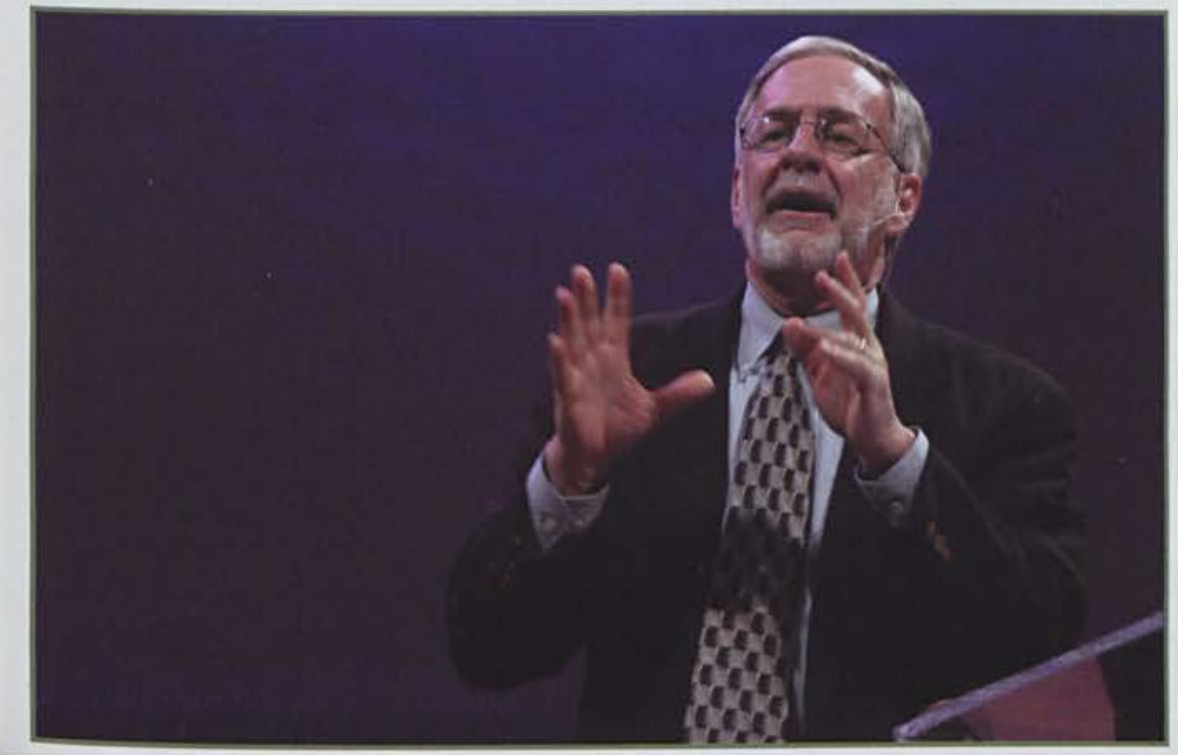

$\checkmark$ Keynote speaker for the Missions Conference was Dr. George Murray. Junior Kristin Hessler said of the conference, "The speaker communicated the importance of giving of ourselves as a means of worship and practicing total surrender. I was challenged to take a road different from the convenient Christian walk."

L Pastor Rohm passes out commitment cards to students. Freshman Jason Moss said of the conference, "The Missions Conference this year taught me that if I want to be a man of God like Jim Elliot, I have to patiently nurture a relationship with Jesus just like I would with any other individual."

$\checkmark$ Each day of the Missions Conference the students are given the opportunity to interact with missionaries from all over the world. "It's great to see the interest of the student body in missions," commented senior Kayla Smith.

* Every year students from the different music groups lend their talents to leading the student body in worship. Pictured here, from left to right, are Elyse Fye, Josh Erlandson, and Becky Chasnov.
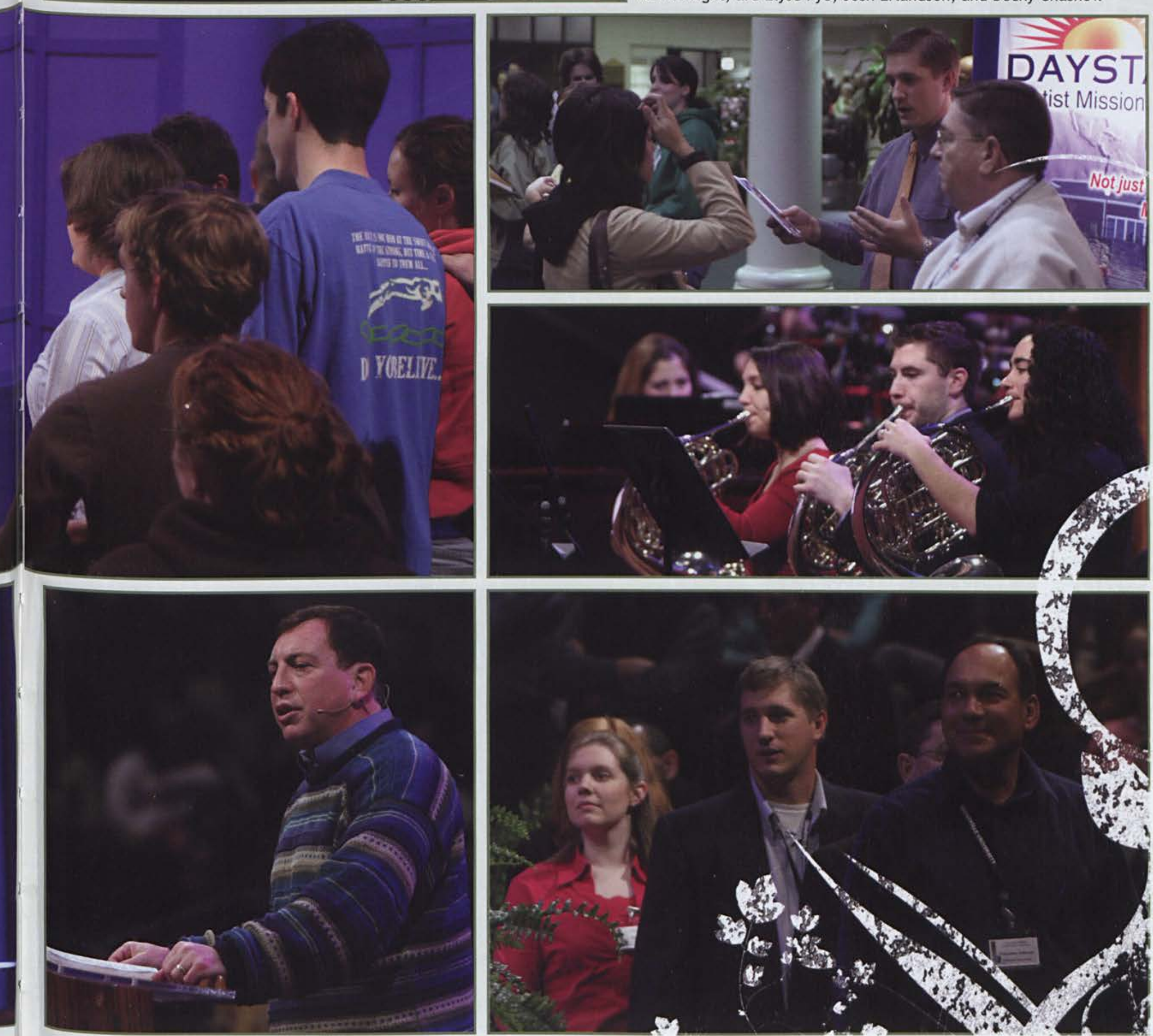

Dr. Mark Thompson, Medical Missionary to the Amazon, shares his experiences.
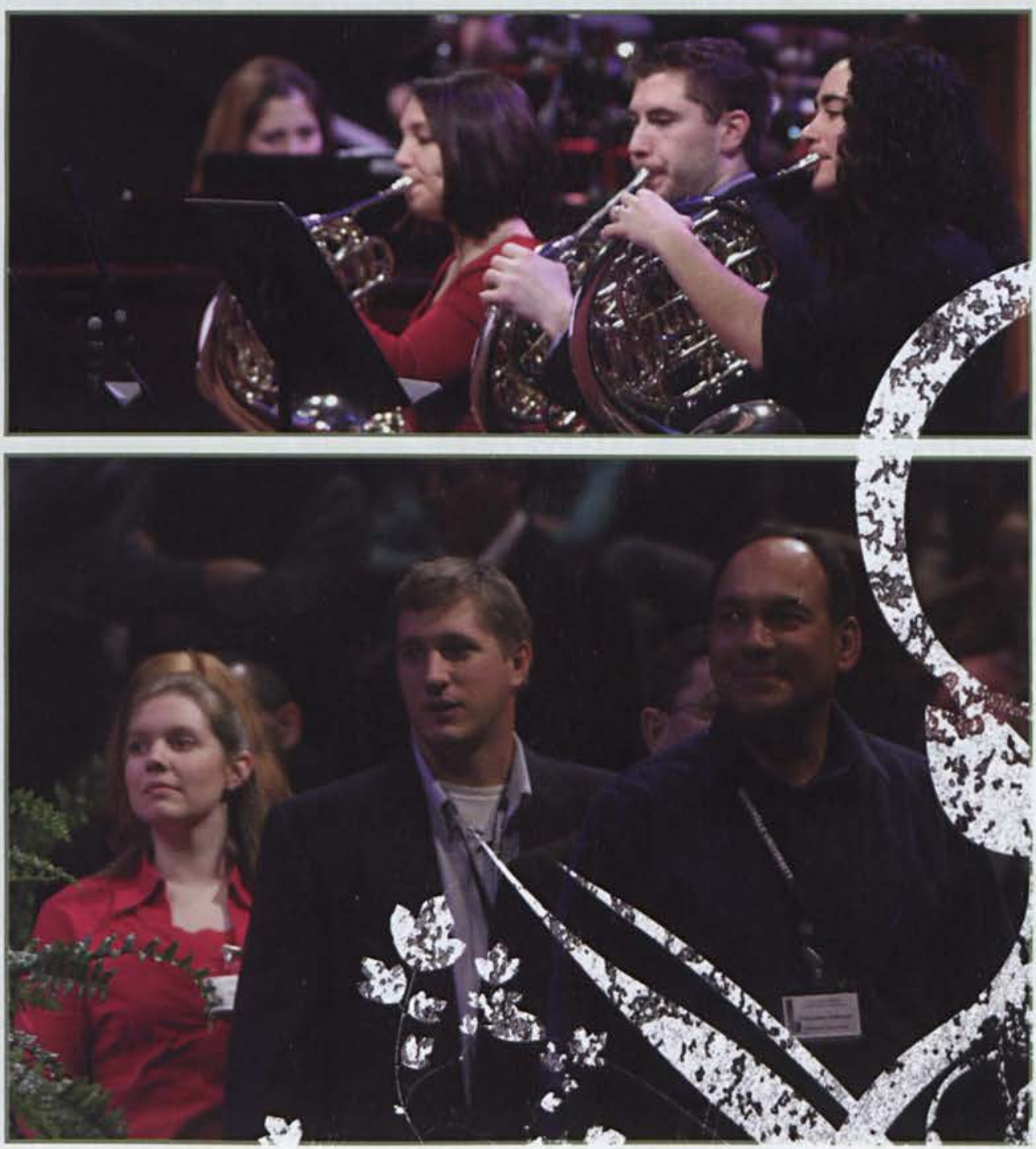

Missionaries and their agencies who joined us for this year's conference wait for their introductions. 


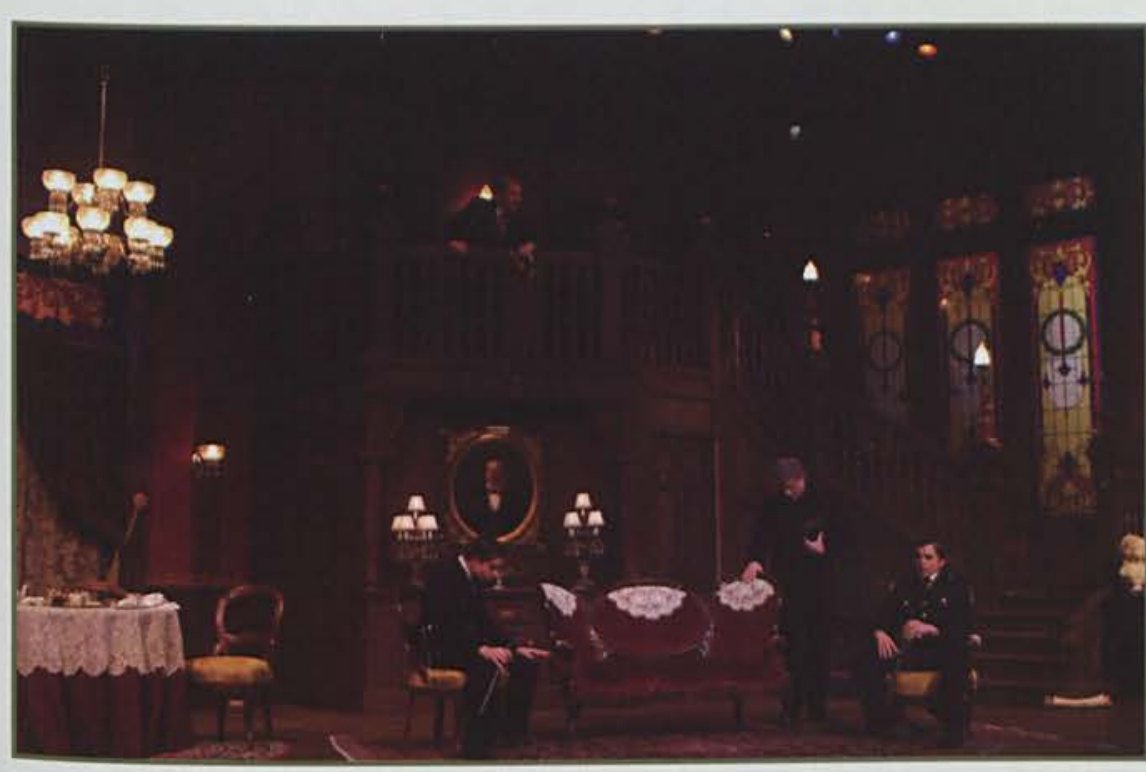

$\checkmark$ As shown here, the "Arsenic and Old Lace" set incorporates the home style of the 1940's including everything from the velvet loveseat to the stained glass windows.

Mortimer, played by Grant Hansen, faces the dilemma of what to say to his fiancé, played by Victoria Duff, after he finds out his aunts are killing people.

F Dr. Einstein, played by the masterful Colin Luther, cowers in fear as Jonathan (not pictured) demands that Dr. Einstein once again change Jonathan's face through a transplant of a dead person's face.

$\checkmark$ Andrew Tripp and Jonathan Wolverton play the oblivious cops. Andrew Tripp said about his character, "I liked the fact that I could carry around a nightstick and hit people with it."
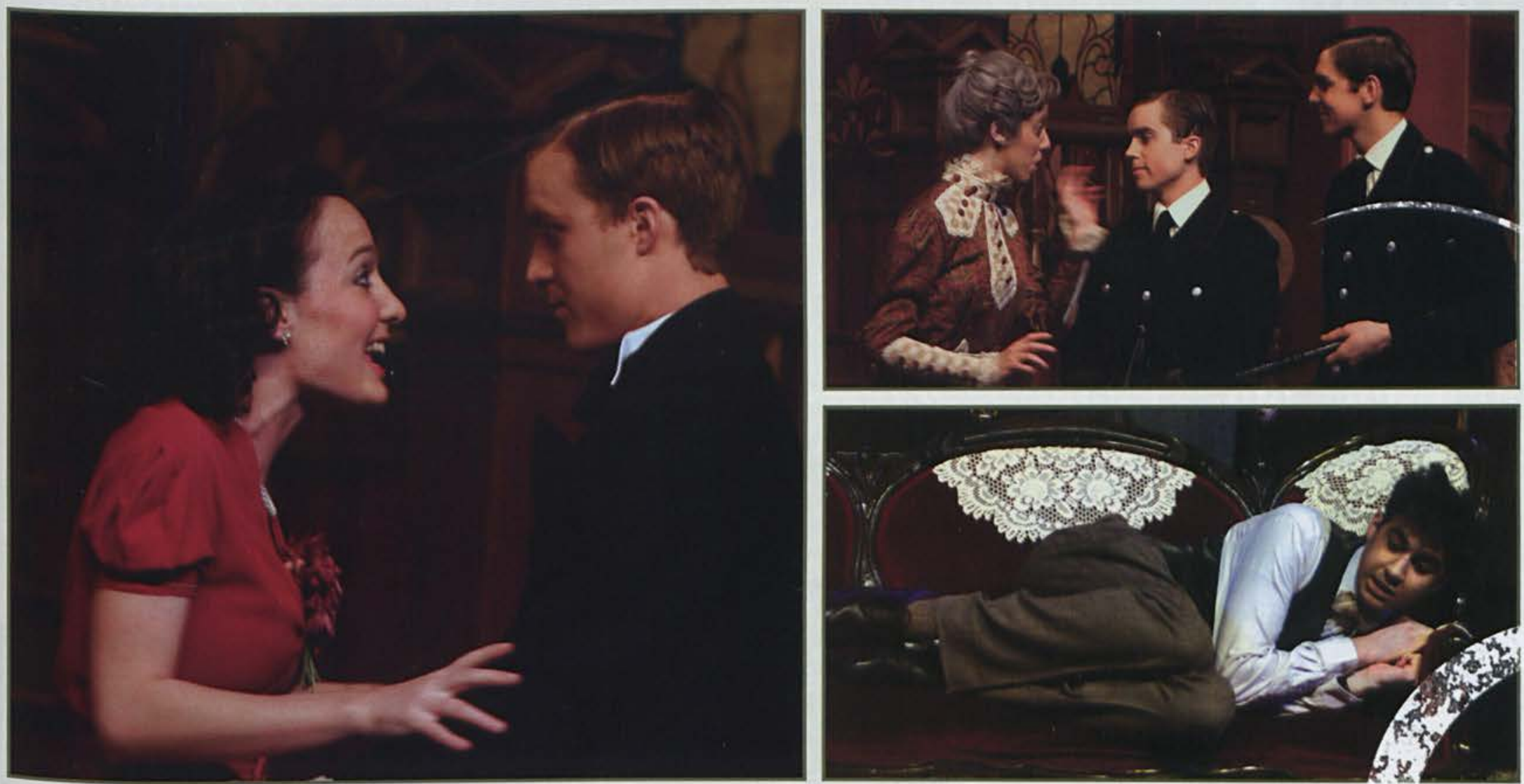

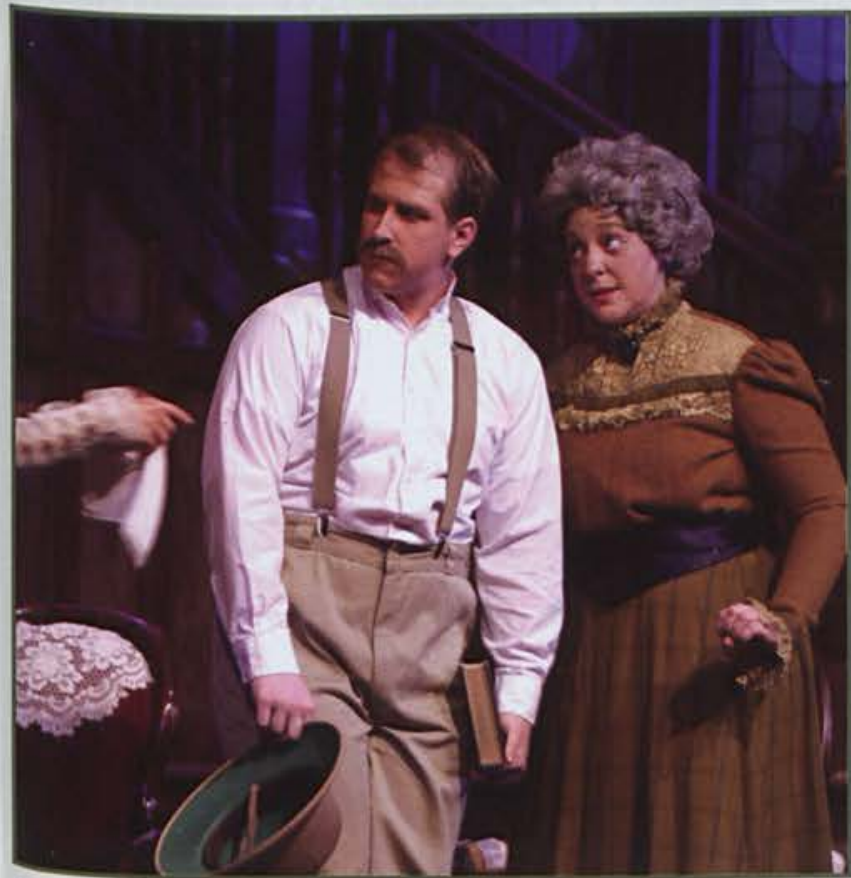

Aunt Abby and Aunt Martha (not pictured) attempt to reason with Teddy, played by Robert Cook, persuading him to "dig the Panama canal."

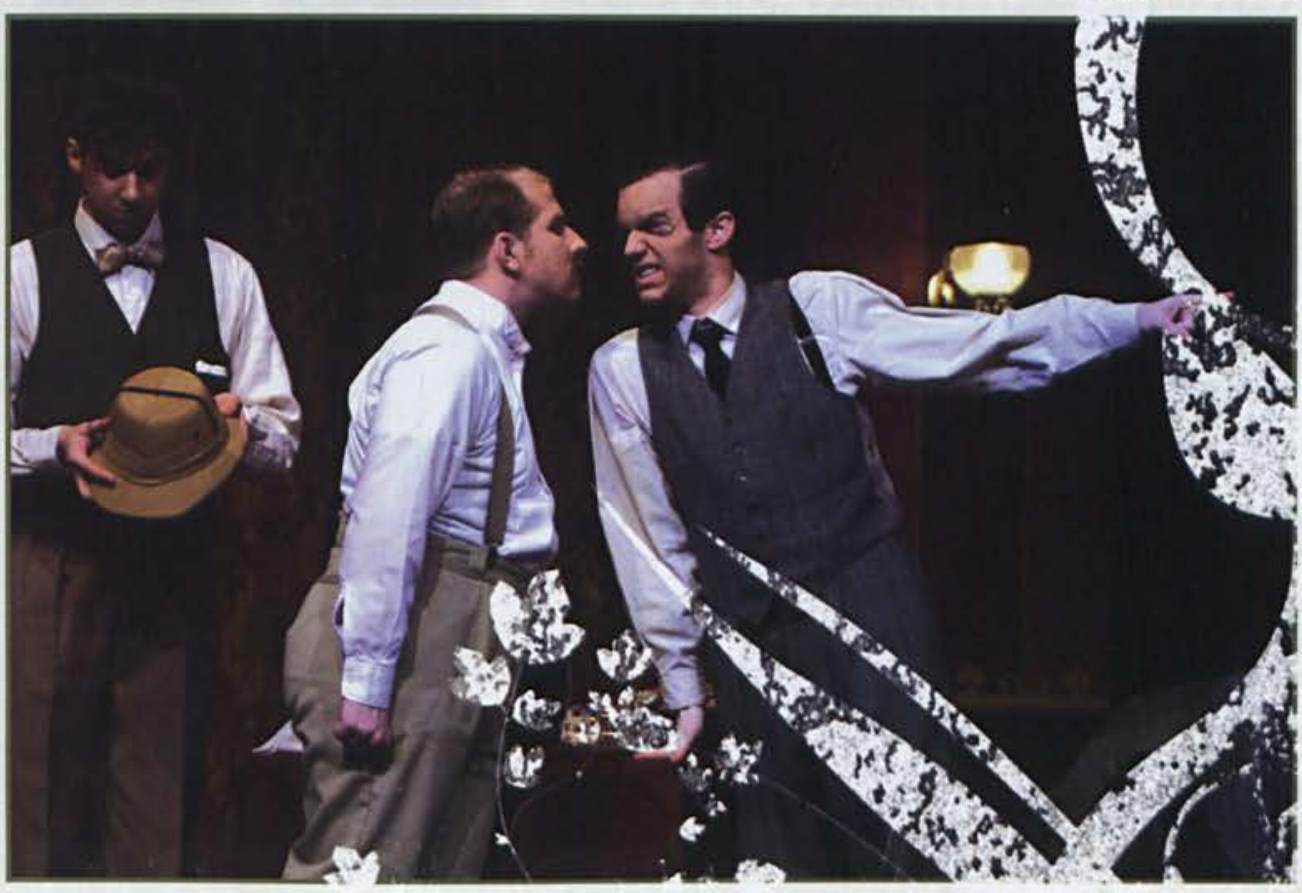

J Jonathan has just ordered Teddy (who thinks he's President Roosevelt) to go to bed. Teddy: Excuse me... who are you?

Jonathan: I'm Woodrow Wilson! Go to bed! 

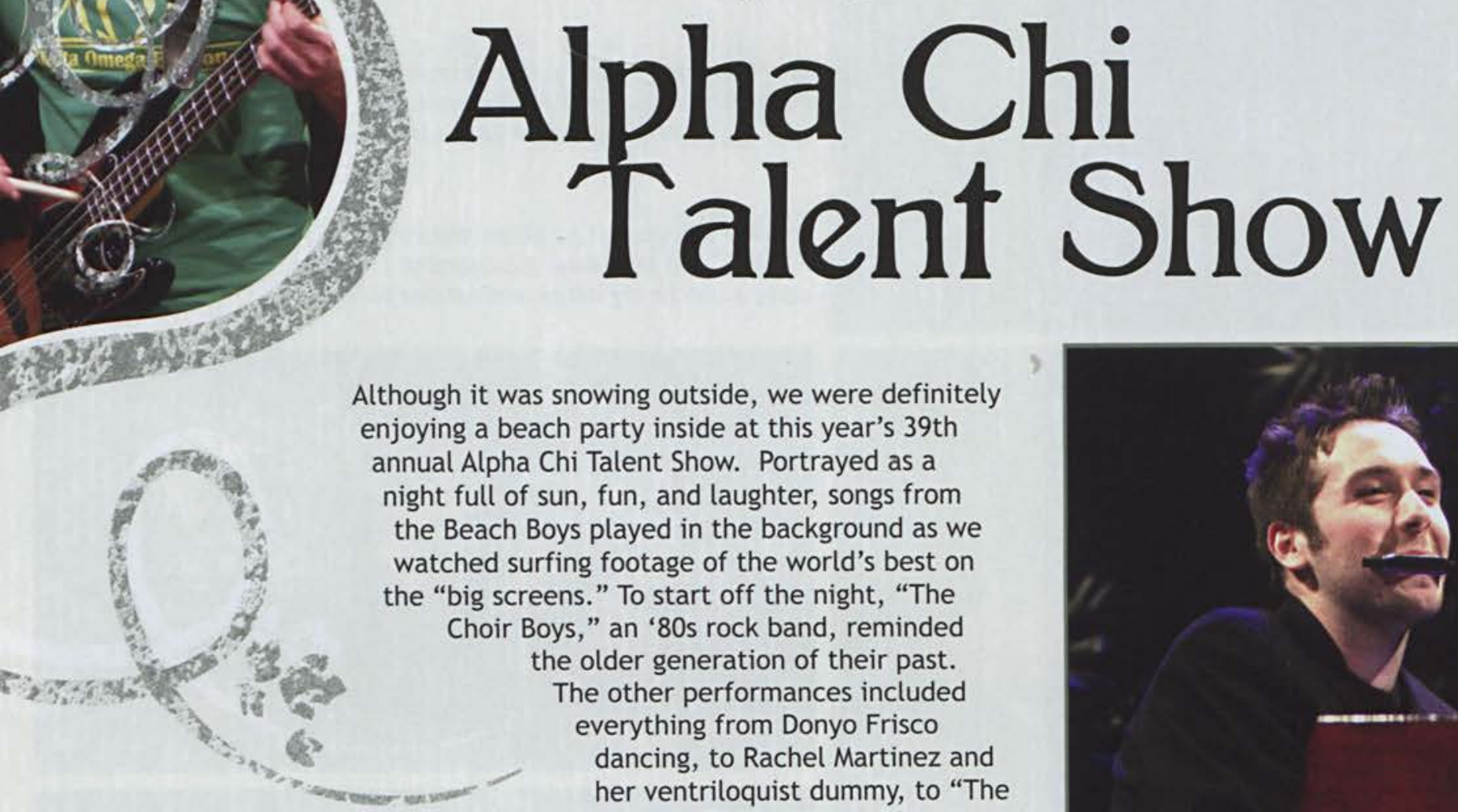

Although it was snowing outside, we were definitely enjoying a beach party inside at this year's 39th annual Alpha Chi Talent Show. Portrayed as a night full of sun, fun, and laughter, songs from the Beach Boys played in the background as we watched surfing footage of the world's best on the "big screens." To start off the night, "The Choir Boys," an " 80 s rock band, reminded the older generation of their past. The other performances included everything from Donyo Frisco dancing, to Rachel Martinez and her ventriloquist dummy, to "The Explosive Trio" with a little twist on

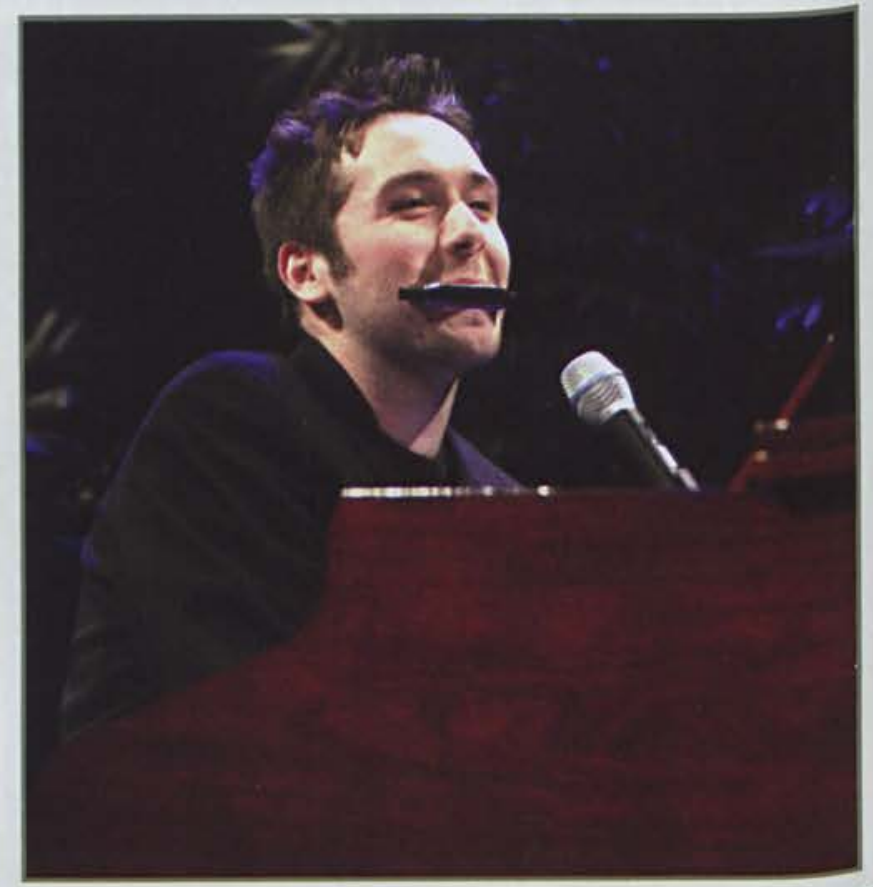
original act as well as 2nd place was "The Untouchables," composed of Corey Knoedler, Ben Waddell, and Walter Strickland. Chris Harmon said of them, "The way they threw the drumsticks around and were still able to jam out, not to mention they were actually able to play the guitars with drumsticks, made them, in my mind, the best act there." Josh Gesler took 3rd place with his slight of hand card tricks, but the ruler of the show and the 1st place winner, was Greg Cooley with "The Piano Man," Cedarville style.

Alpha Chi member and director of the show Nathan Williams said, "During the show, all the performers watched and encouraged each other. A feeling of cooperation and congratulations from the various acts instead of competition made [everyone] enjoyable to work with." The participants might have been competing for cash prizes, but you would have never known. There was a general good-naturedness exhibited in all the performers, and it definitely made for a fun show.

After the night of entertaining acts and a little beach fun, we all were able to face the cold with a smile on our faces.

Winner of the event, Greg Cooley, shows off his abilities of harmonica playing while playing the piano.

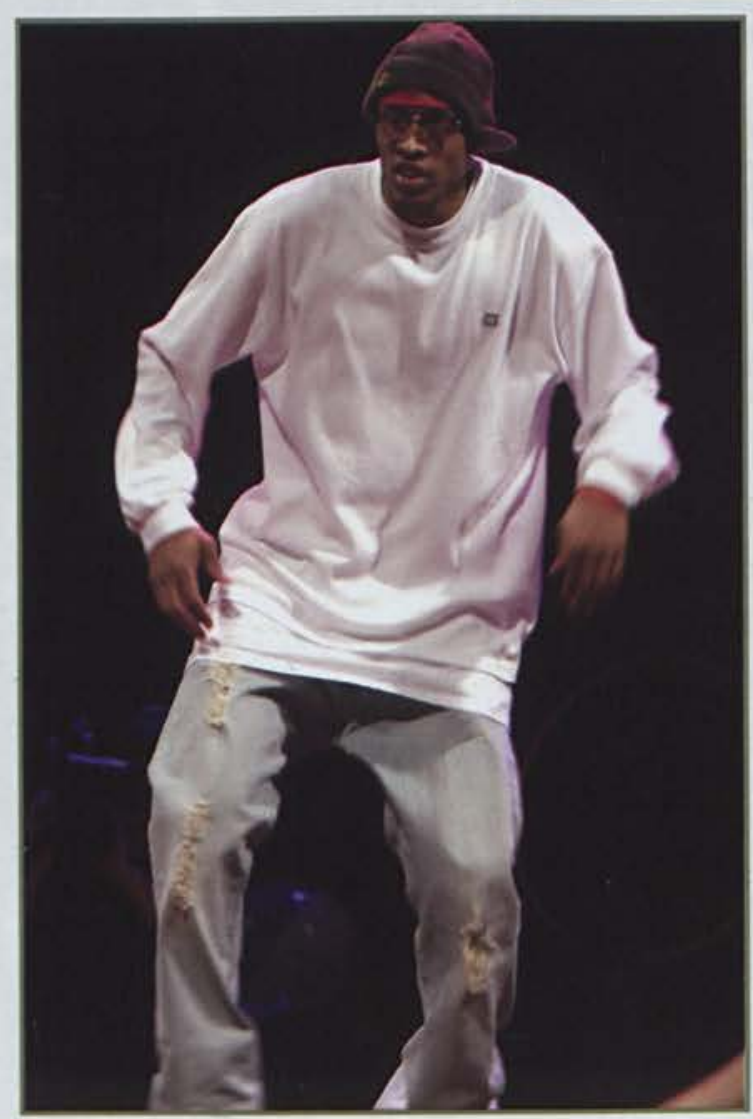

- Donyo Frisco exhibits his dancing abilities. In a school of "no dancing," Donyo has definitely been hailed the "CU Dance King."

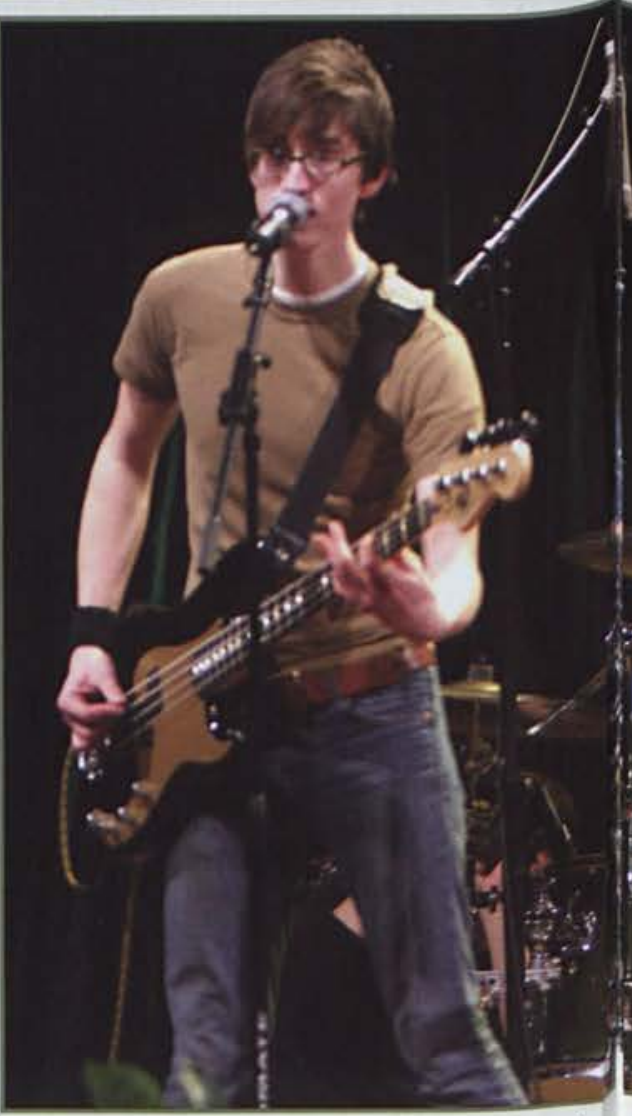

^ The established campus band, Retail 


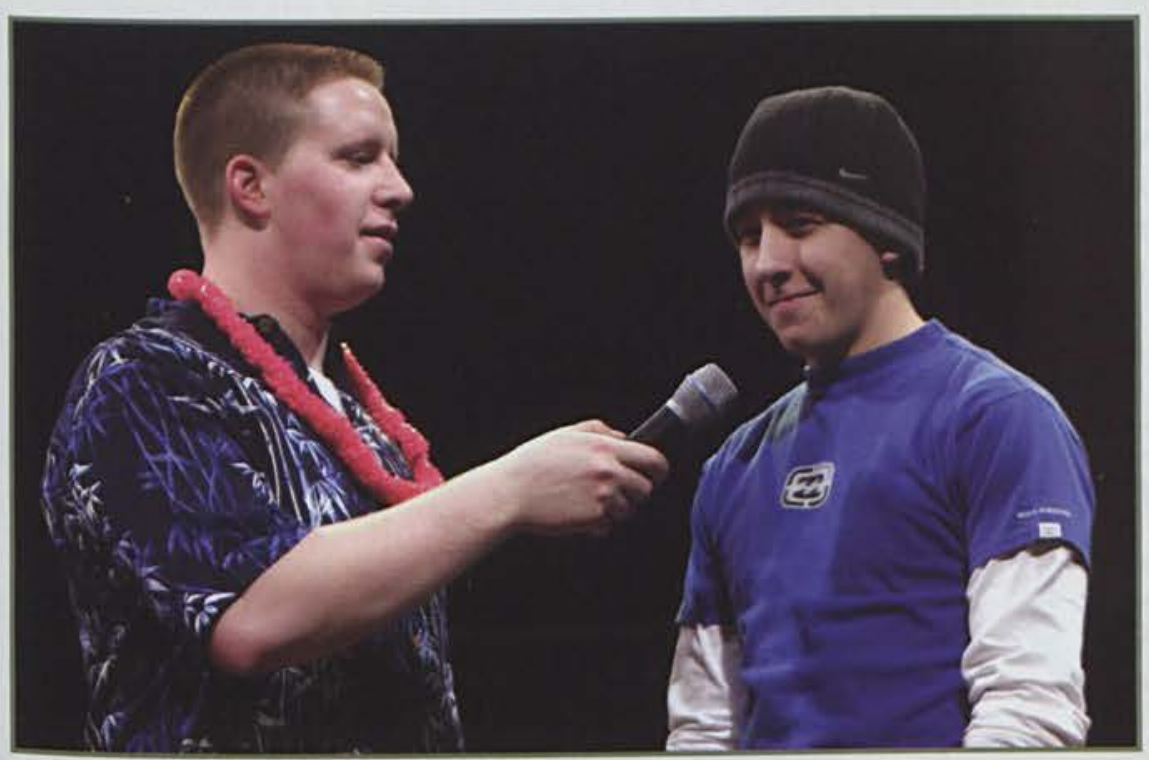

1 Co-host Josh Townsend talks to a crowd member. The night featured not only student talent, but many prizes for attendees as well.

High school student, Alex Brown, shows off his mad break-dancing skills, definitely an incredible addition to the show.

* Co-host Rebecca Wolff said of her involvement in the show, "It was fun to be a part of such a great show, the different acts were all so talented and I loved being able to interact with the lively audience.... it was a good time."

₹ Chris Harmon said of Dr. Brown's involvement, "He did a great job presenting the Gospel in a way that everyone could understand and allowed for those there that might nor know Christ to have a chance to hear about Him."

$\checkmark$ The night featured many talented vocalists, including Liz Pesce and Jenna Albarano, pictured below, who sang "For Good" from the Broadway musical, Wicked.
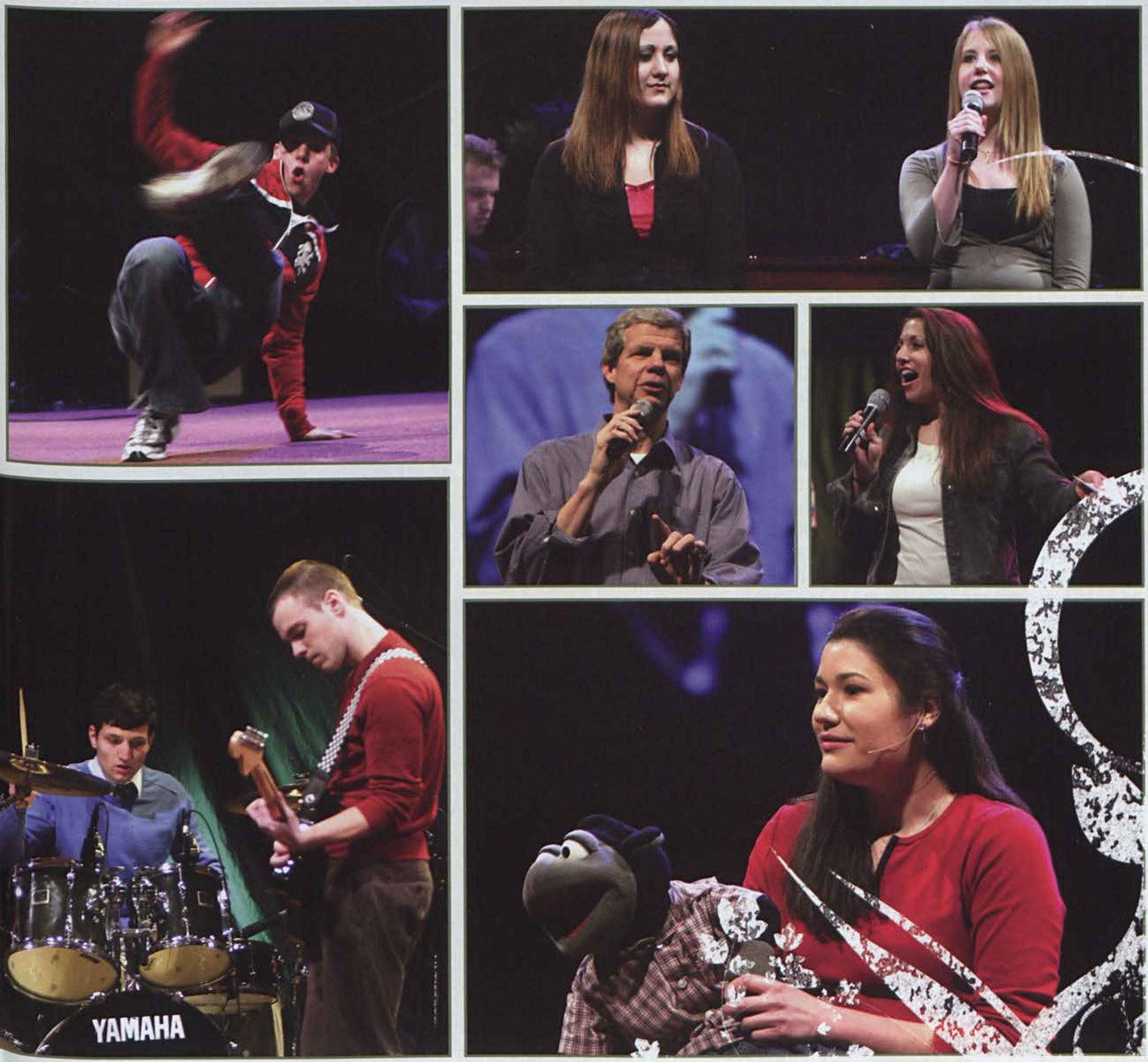

Age, plays to the joy and excitement of the crowd.

^ Rachel Martinez wowed the crowd with her ventriloquist skills. "My ventriloquist figure, J.R., and I did our "Just Trust Me" routine for the audience." 


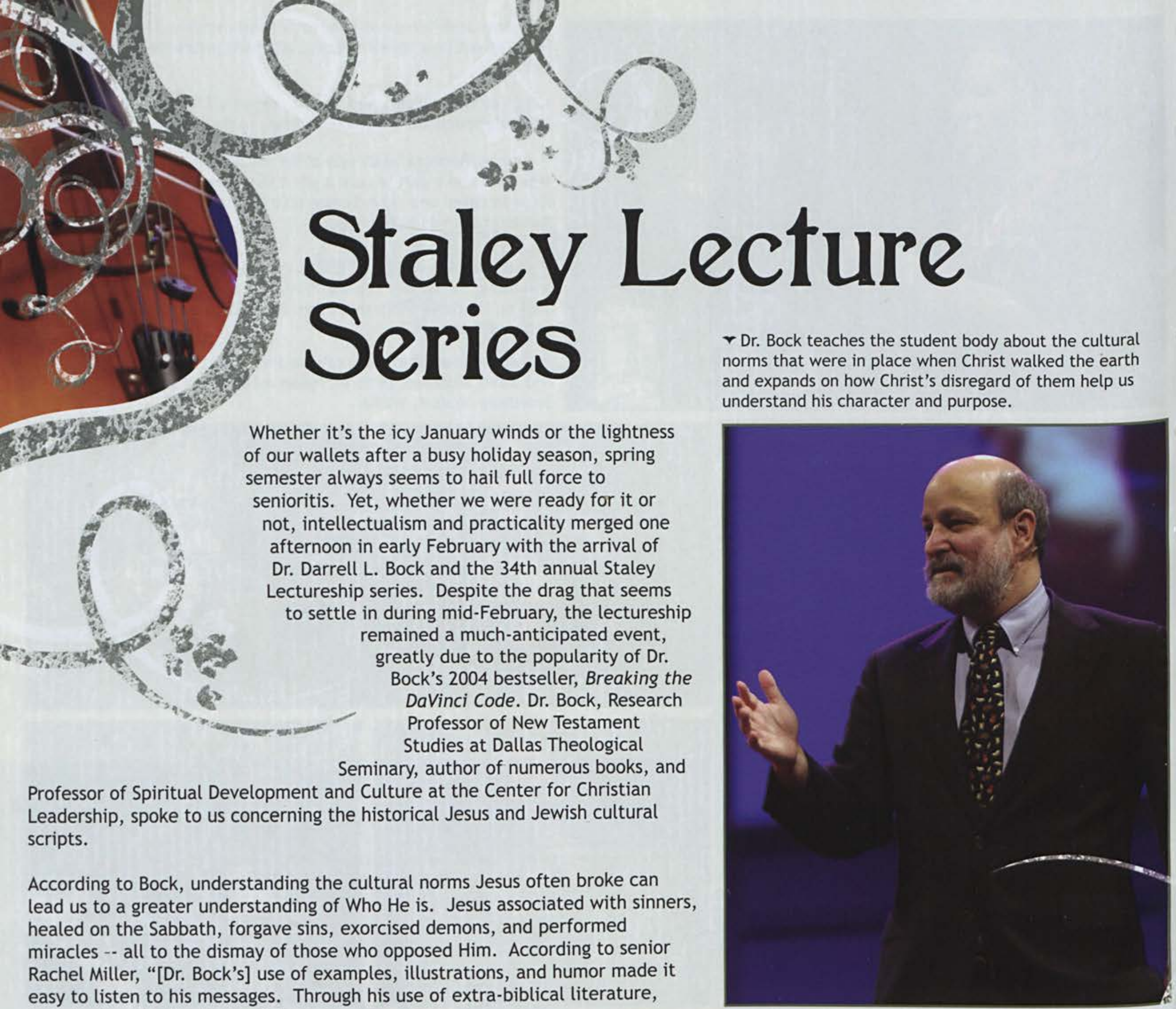
he introduced not only new ideas regarding Jesus, but also new ideas regarding how to best understand and research the Bible in its context."

\section{Dr. Bock's useful messages provided} the stimulation many of us needed to carry through a few more weeks. Senior pre-seminary student Stephanie Struebel spoke for many when she said, "I left impressed with the reminder of Jesus' ministry and that $\mathrm{He}$ is Who $\mathrm{He}$ claimed to be: God, with power over everything. The lectures challenged me to live in the reality that I have a relationship with the God of the universe, the One who has all power."

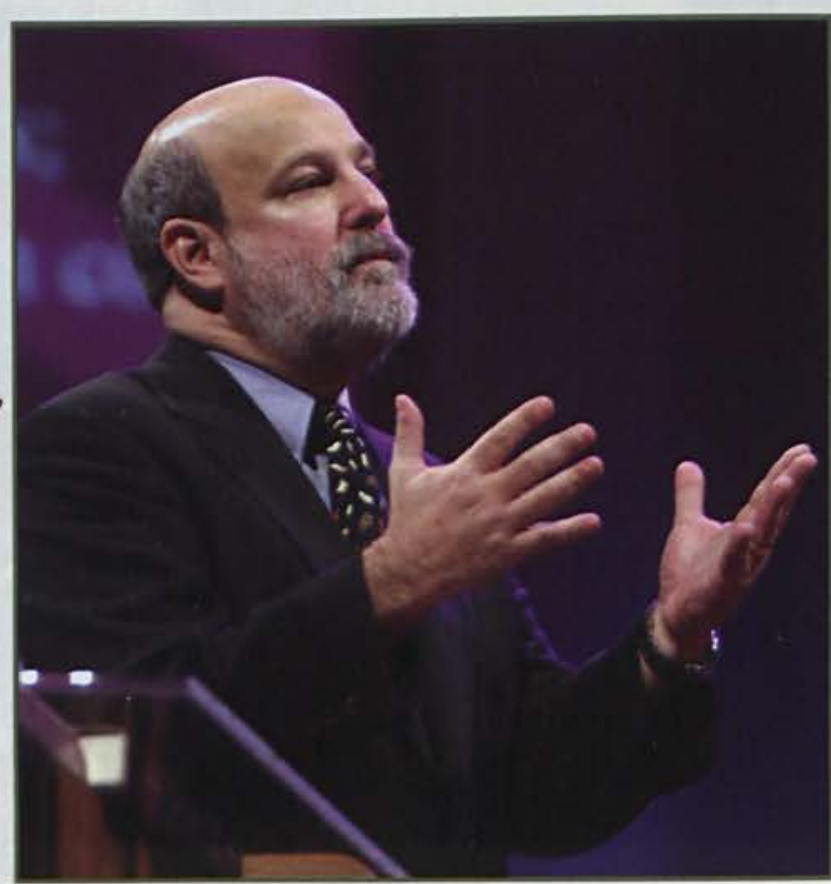

ఓ Junior Chris Guarnera said, "The passion he demonstrated in investigating and exploring the truth of the historical Jesus was both encouraging and challenging."

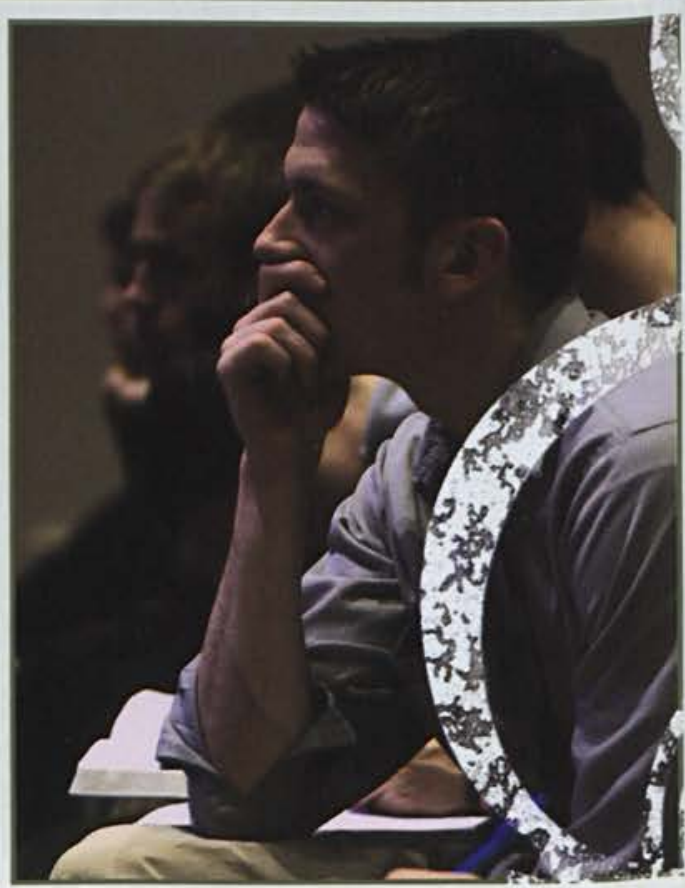

A A student meditates on the well-timed words of Dr. Bock. As Angel Voris said, "He provided just the lift I needed at this point in the year." 
$\checkmark$ Dr. Milliman, Vice President of Academics, congratulates Dr. Moreno-Riaño as is recieves the prestigious title of Cedarville University's 2006 Faculty Scholar.
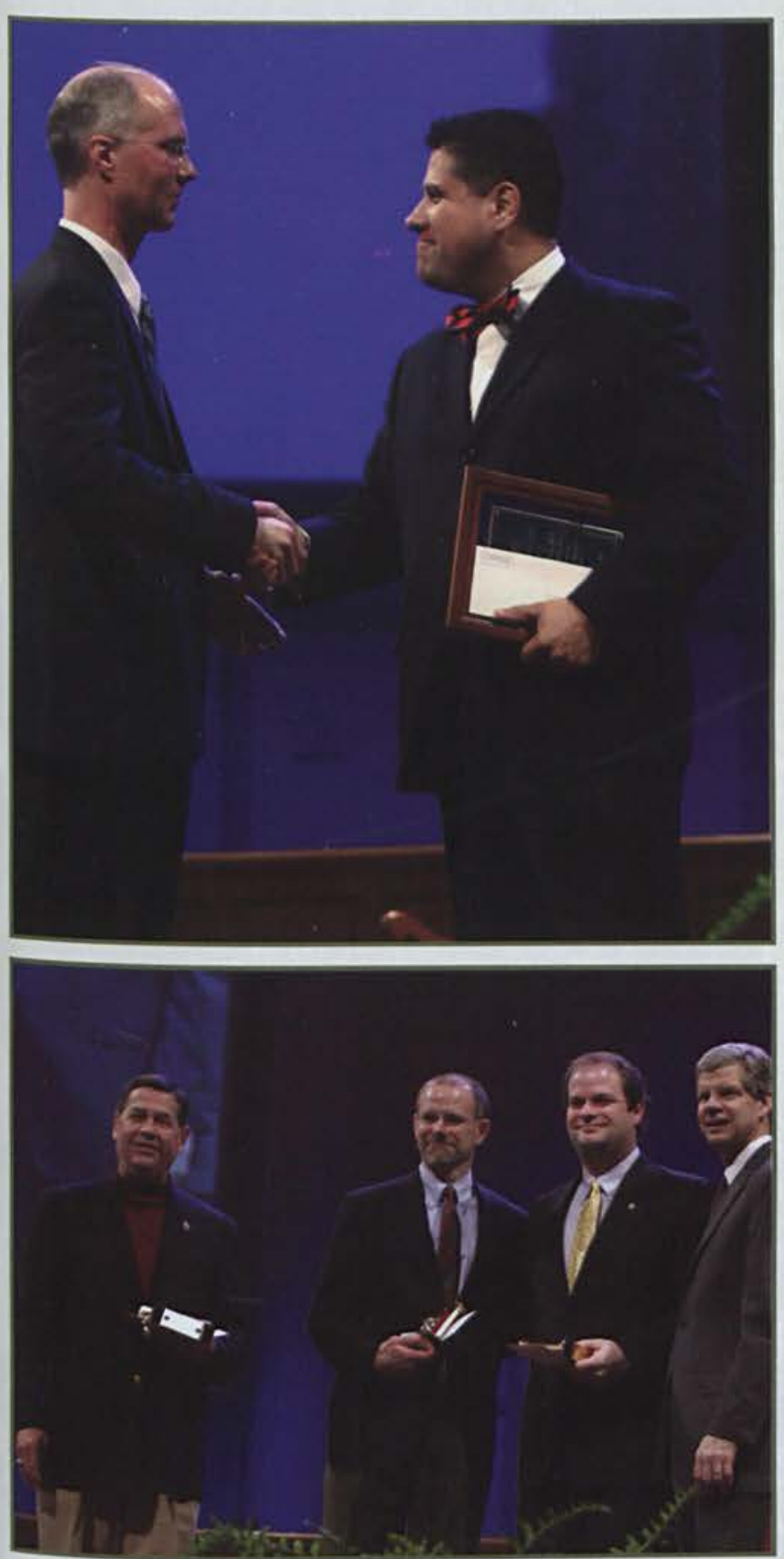

I'll admit it; I wasn't exactly thrilled to be at faculty chapel this year. I figured it would just be a mundane listing of teachers' promotions and achievements, which wouldn't really catch my attention. I couldn't believe how wrong I was. The faculty that we have here are amazing! Not only did they devote their time into teaching their fields of study, but there was so much that they did for the community. Faculty chapel definitely made me realize the caliber of the staff that we have here.

This year's faculty chapel left an impressive mark on students and their opinions of professors. Many

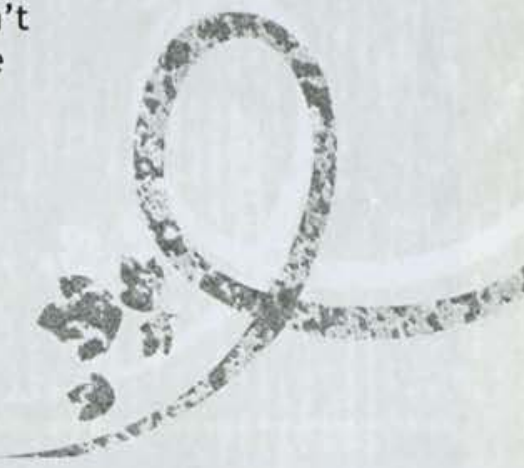
students had no idea that their

favorite professors had achieved so much outside of the classroom.

Sophomore Julianne Sandberg encapsulated the feelings of several students when she said, "Even though I know the faculty members are great, I am reminded at how they have excelled not only at Cedarville University but also in their broader fields of study. I always leave faculty chapel impressed and proud of the professors that choose to serve at this school."

While most students could appreciate the achievements of the entire faculty that were awarded, some faculty favorites were especially appreciated by the students that they've taught. Sophomore English major Garrett Lowe said, "I was especially proud when Dr. Wilfong received her promotion. The passion she has for what she teaches, combined with her extensive knowledge of literature, makes her more than deserving of the award."

Judging by some of the faculty's eloquent acceptance speeches, it seemed like they were fully aware of and prepared for the award. However, such was not the case for Communication Arts professor Rebecca Baker, who explained, "I was completely taken off guard. My response, upon reflection, was to be humbled by the choice and personally challenged to serve more."

Dr. John Leblanc, Dr. Jeff Guernsey, and Dr. Jeffrey Fawcett are awarded the Faculty Innovator of the Year Awards for their groundbreaking work with the Integrated Business Core.

$\checkmark$ Dr. Brown and the rest of the Cedarville family bid farewell to Dr. Abbas and his thirty years of service at Cedarville as he takes the stage for the last time before retiring. 


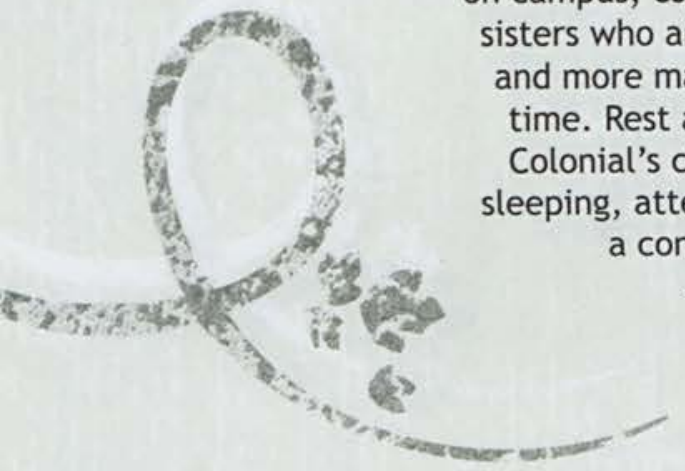

Li' Sibs Weekend is always a fun time to have family on campus, especially those younger brothers and sisters who are desperate to see what their older and more mature college siblings do with their time. Rest assured, my li'l sib enjoyed eating Colonial's cheesy breadsticks, watching movies, a concert. Sarah Zeltman's little brother, Jacob, had a great time as well while keeping busy around campus. As Sarah said, they "went to the Talent Show, ice-skated, played some basketball together, climbed the rockwall, and watched the girls' and guys' basketball games."

Li'l sibs got to experience the best part of the college life. With a Winter Olympics theme this year many youngsters were seen donning red, white, and blue $\mathrm{t}$-shirts. There were many activities going on around campus during the weekend as a source of entertainment. Soldout activities such as late night bowling, ice skating, and the David Crowder Band and Third Day concert provided relaxation and time with family.

Siblings also had the opportunity to attend the girl's and guy's basketball games where red, white, and blue popsicles were passed out. "I really enjoyed having my sister come for the weekend. She had a blast, thinking that all I did on the weekends was sleep, eat in Chuck's, and go to basketball games," said Jessica Grewe.

Chuck's always seems to be a hit with younger kids because of the "all you can eat" aspect and of course the dessert island and ice cream bar. Yet another successful Li'l Sibs Weekend proves that bigger and better ones are yet to come.

* John Hawkins and his little brother enjoy the special SGA Chapel geared toward the little sibs.

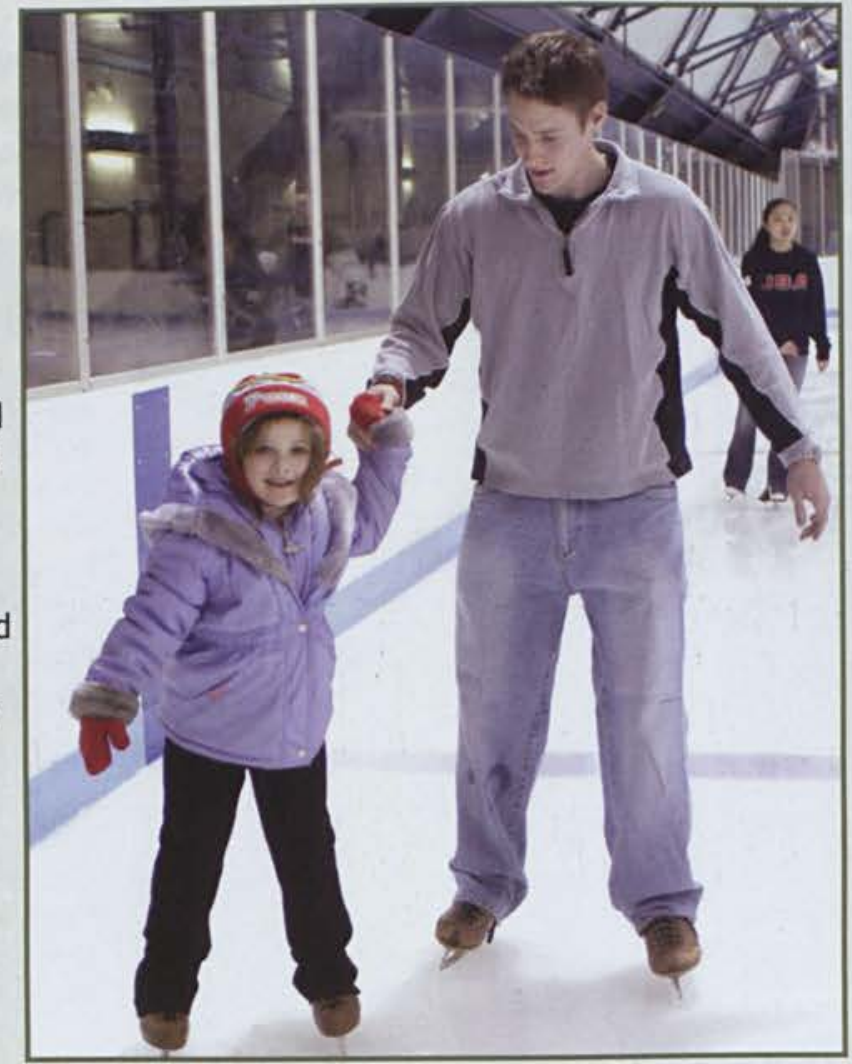

^ A big brother helps his little sister skate around the rink.

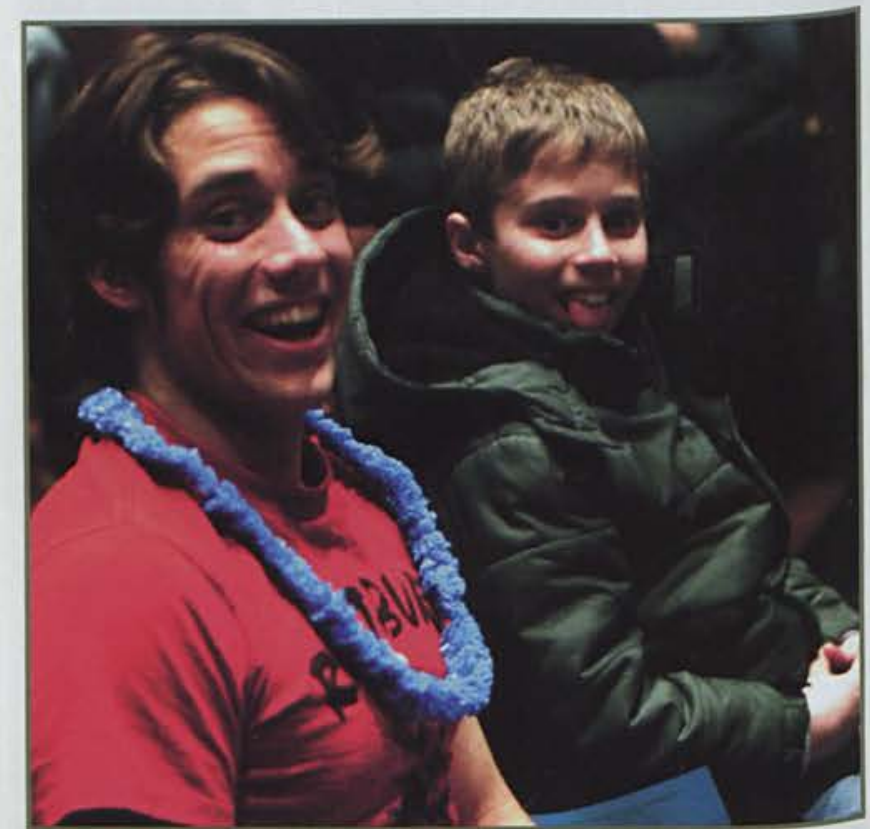




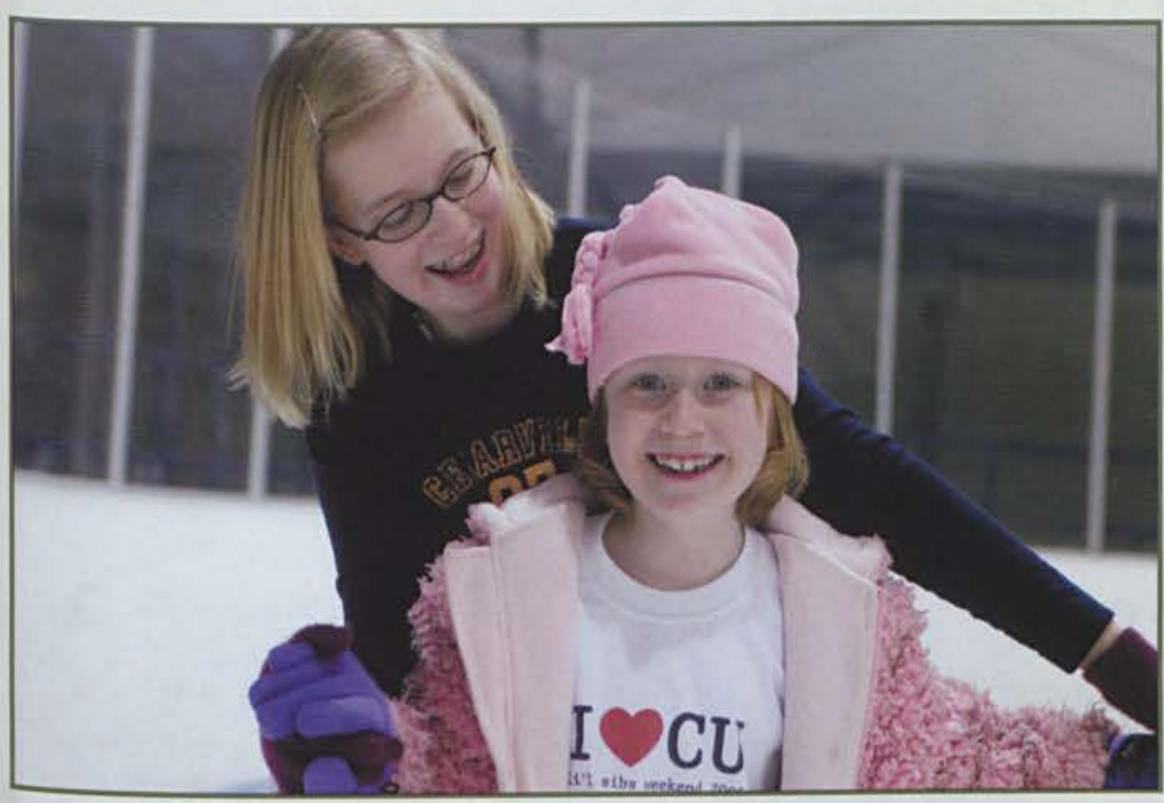

An older sister supports her sibling as they skate around the rink.

4 Emily VanDette and her little sister enjoy the opportunity to spend some time together and improve their ice-skating skills.

F This foursome is bundled up against the cold inside the ice rink, but enjoying their time together despite the chill.

$\checkmark$ The climbing wall was opened up to the little sibs on Saturday, much to the joy of this little brother, who scaled the wall in record time.
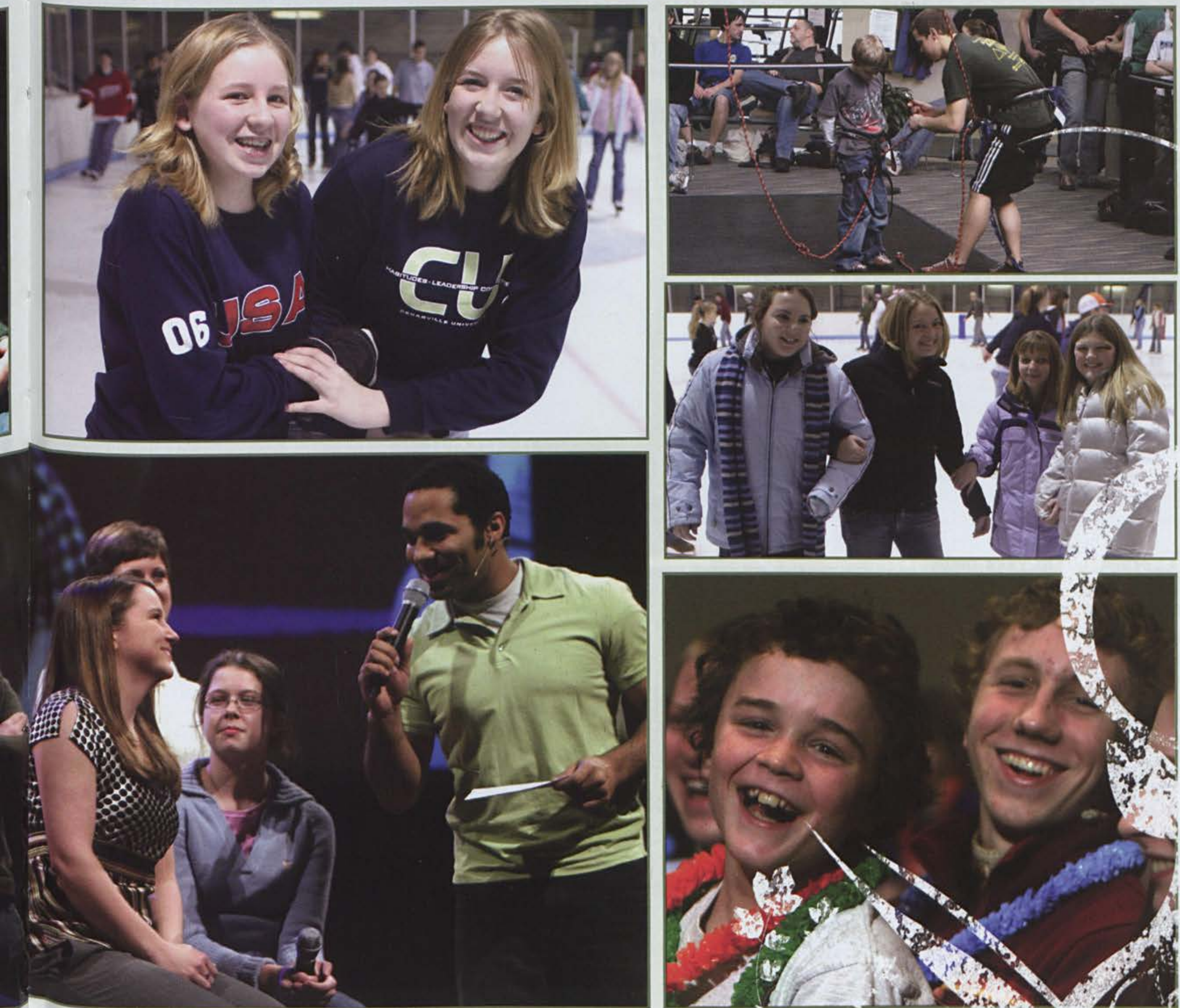

Dairs of sisters up on stage to see just how much they know about each other, younger sister comes up to answer the same questions for her brother.

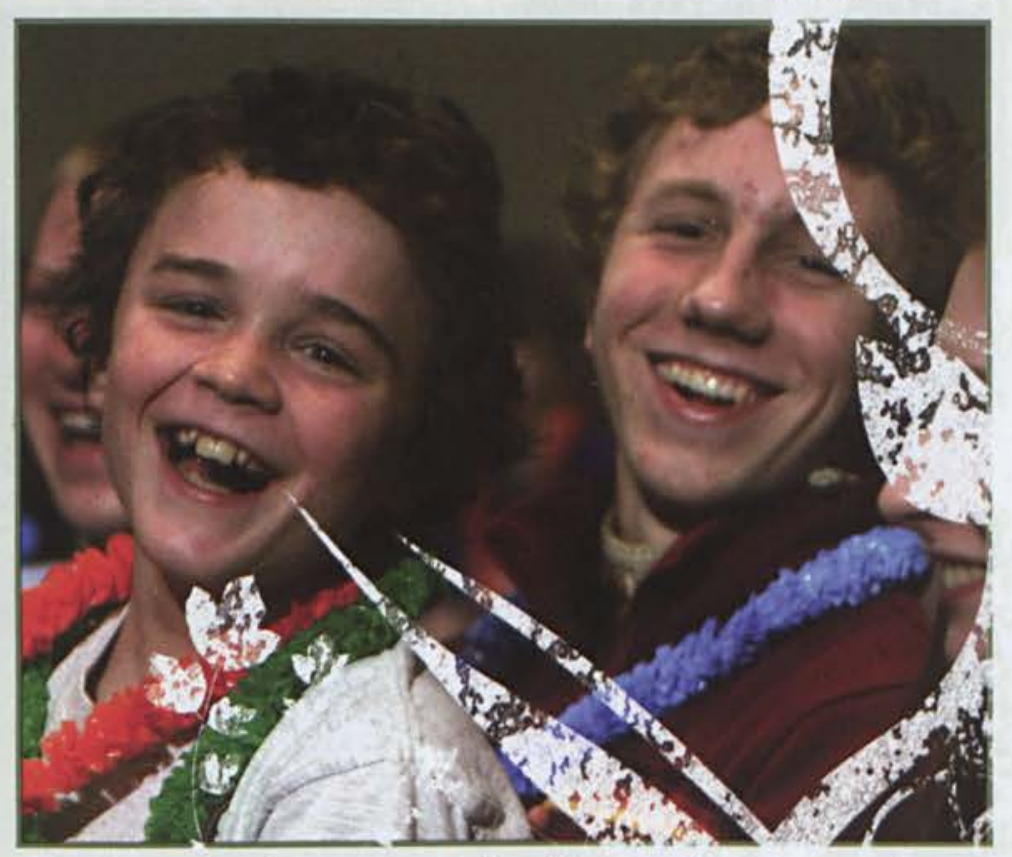

$\Lambda$ The fact that these two are related is more than apparent in their enthusiastic smiles. 

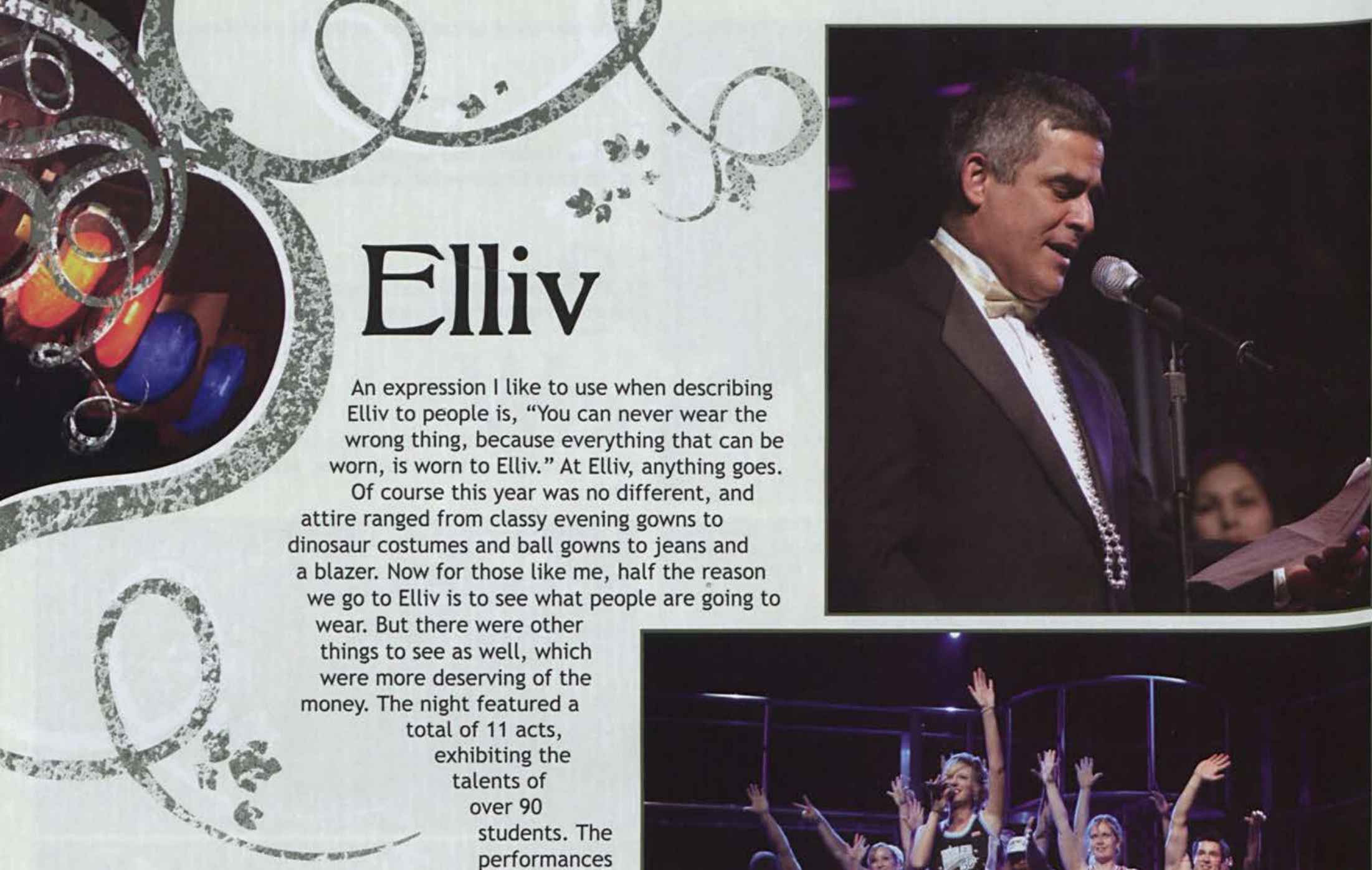
covered songs

from musicians such

as Kelly Clarkson, Gavin Degraw, Dixie Chicks, Daniel Bedingfield, and the Backstreet Boys.

To the joyous surprise of the seniors, former SGA members and Elliv hosts Dave Wenzel and Seth Martin preached to the student body about student loans with the help of a well known R\&B song. The hosts for the evening, Terri Schenk and Daron Day, also added to the entertainment with their dance moves and friendly banter. The purpose of the night was not only to

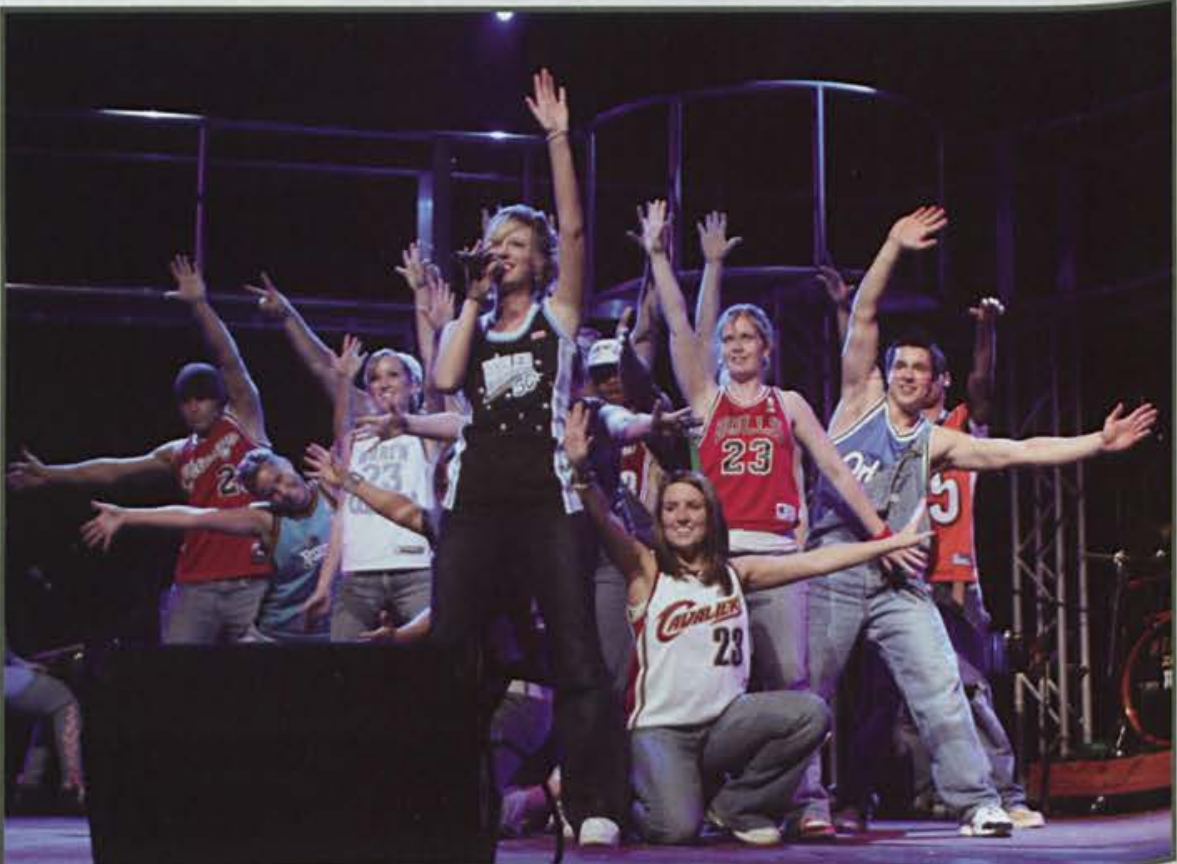
entertain, but to honor those who excelled in their respective fields. Awards were given in the categories of Best Campus Artist, Athlete, Author, Entertainment, Mr. and Ms. Cedarville, Musician, Organization, Play Performance, and Video. A song was dedicated in memory of those whom the Cedarville family had lost this year, and a check was presented to Benjamin Franklin High School in New Orleans for a continuation of the campus efforts for Hurricane Katrina relief. In response to the show, sophomore Abby Colgrove stated, "Elliv is a great "yearender" that kind of ties the whole Cedarville experience together for the student body. It's an awesome tradition to look forward to."

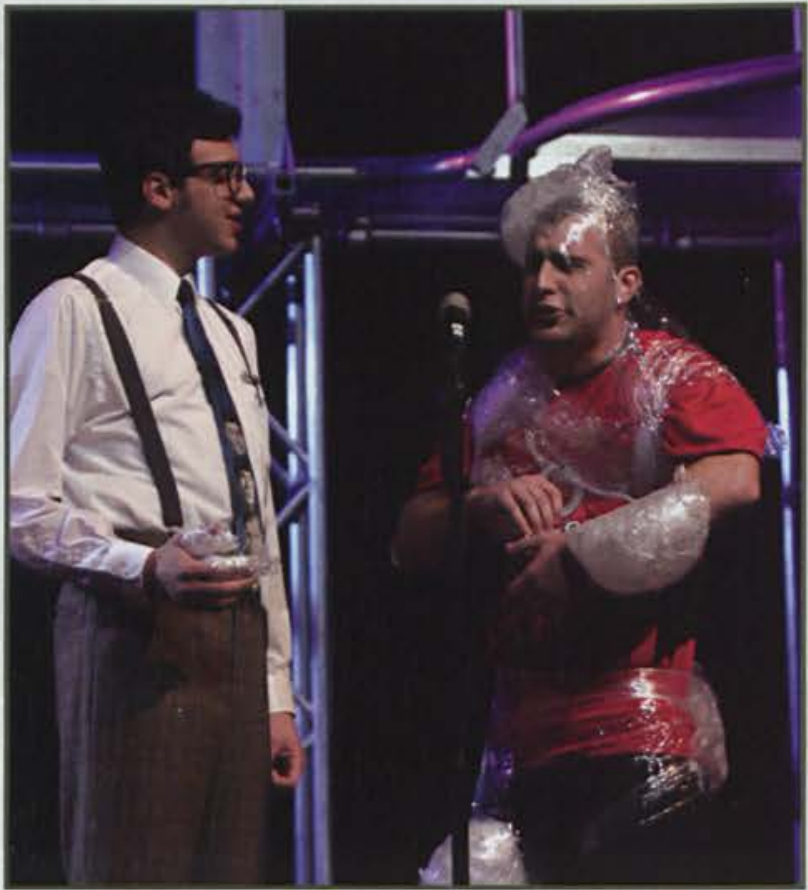

^ Completely covered in ice, Jon Wasson helps Colin Luther present the awards for Best Male \& Female Athletes.

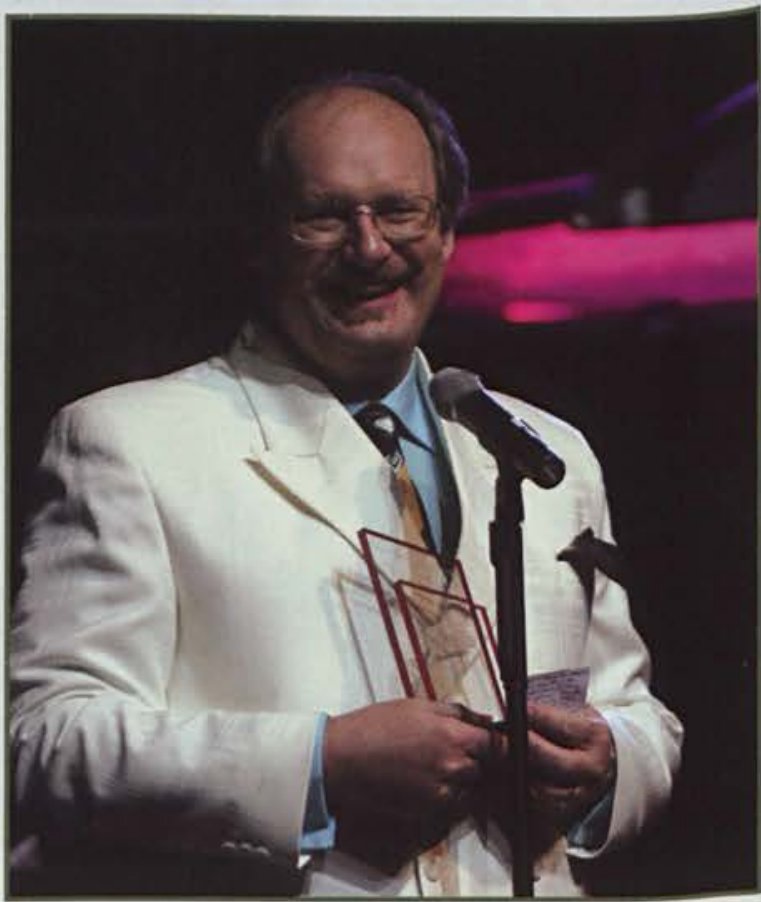

^ Mr. DiCuirci, symphonic band director, jazz band director, and professor of music education, received the much coveted faculty "Man of the Year" award.

exhibiting the

talents of

students. The performances

\author{
(1)
} a 


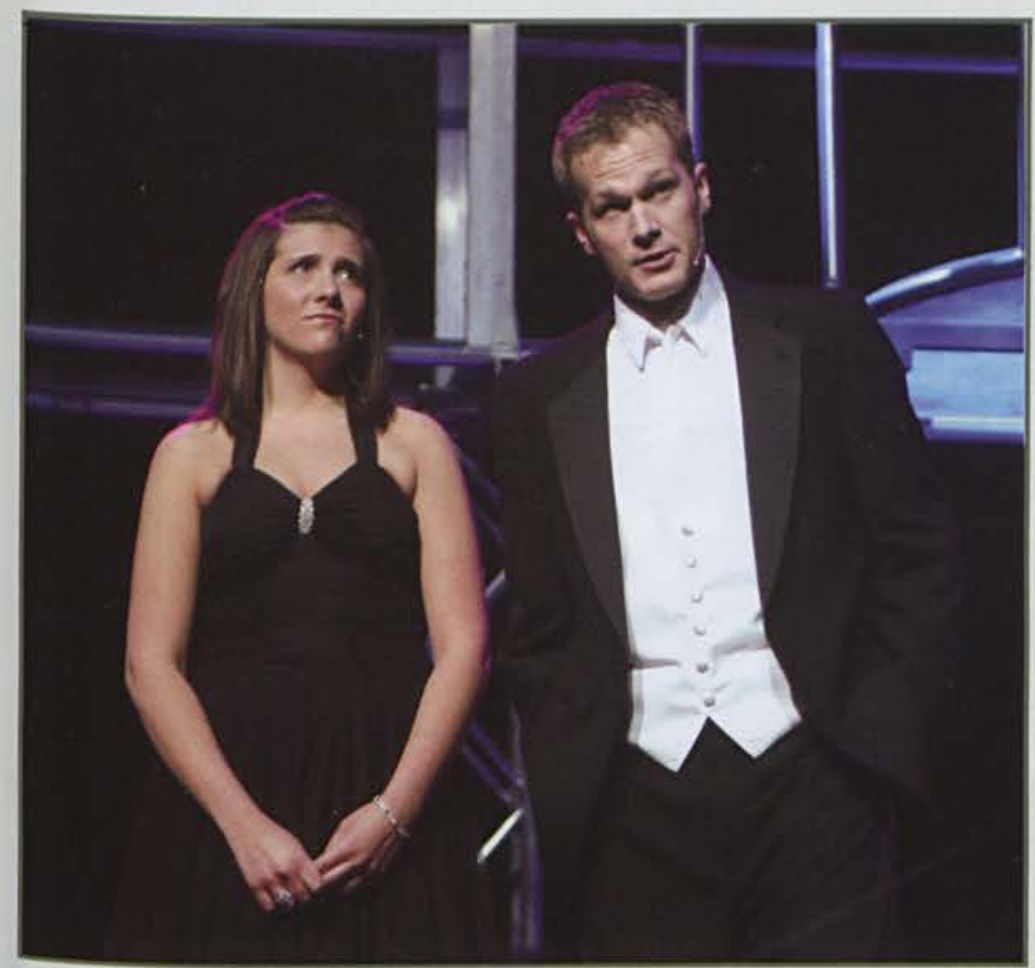

$\checkmark$ Daron Day and sidekick Terri Schenk banter between acts as they hosted this years Elliv events.

* In continuation of the support Cedarville has given to those impacted by Hurricanes Katrina and Rita, a special guest accepts a check given to the Ben Franklin High School in New Orleans.

Abi Cook wows the crowd with the opening act of ELLIV.

A Andrew "Cos" Costerisan plays his mandolin, contributing to the music of Gavin DeGraw's “I Don't Want to Be," the finale for ELLIV 2006.

$\checkmark$ The girls of "Footloose," led by Laura Teague, are fancy-free with their cowboy hats and enthusiastic smiles.
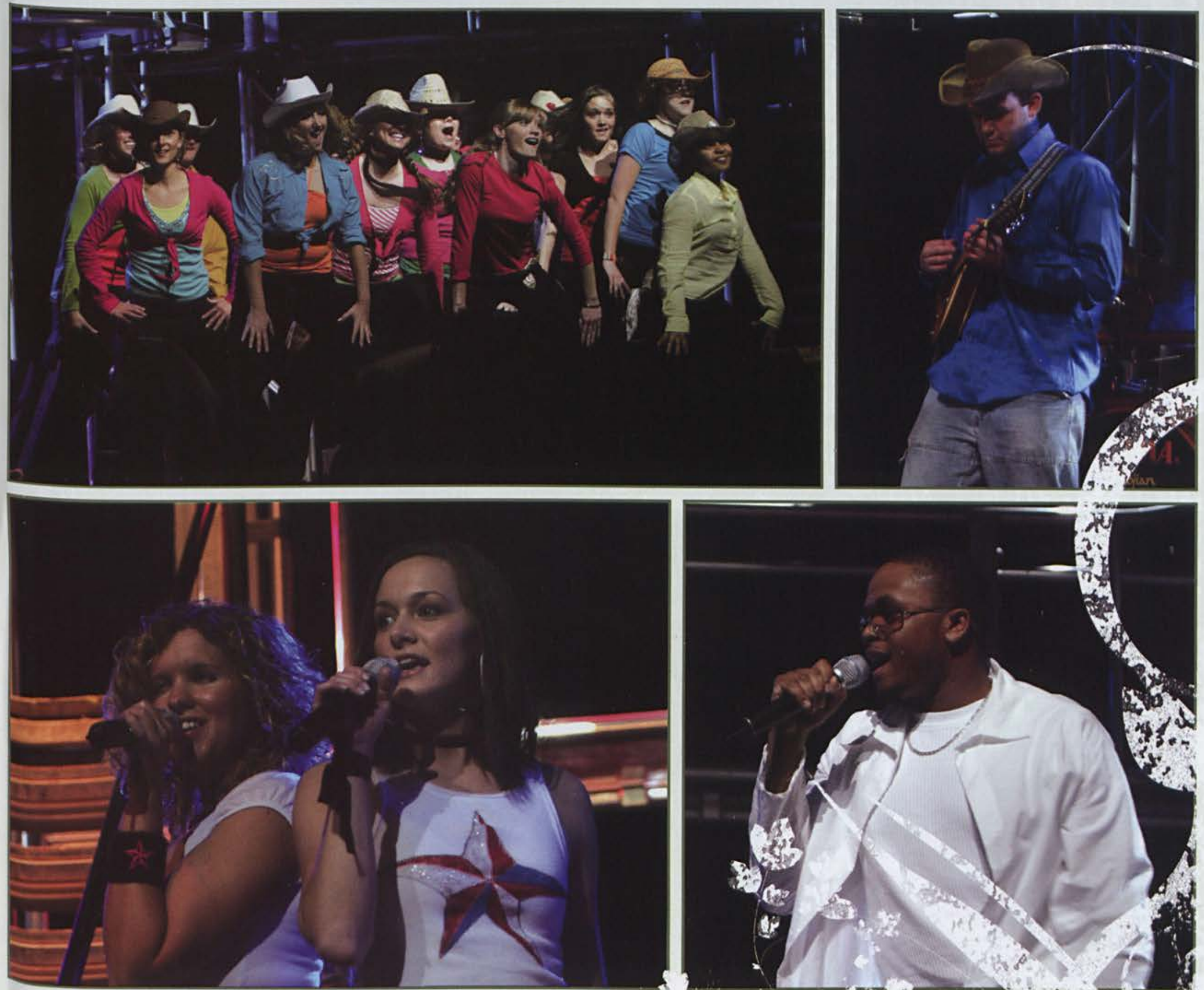

Erin Higley and friend crank out Dixie Chicks's "Ready to Run” with the help of

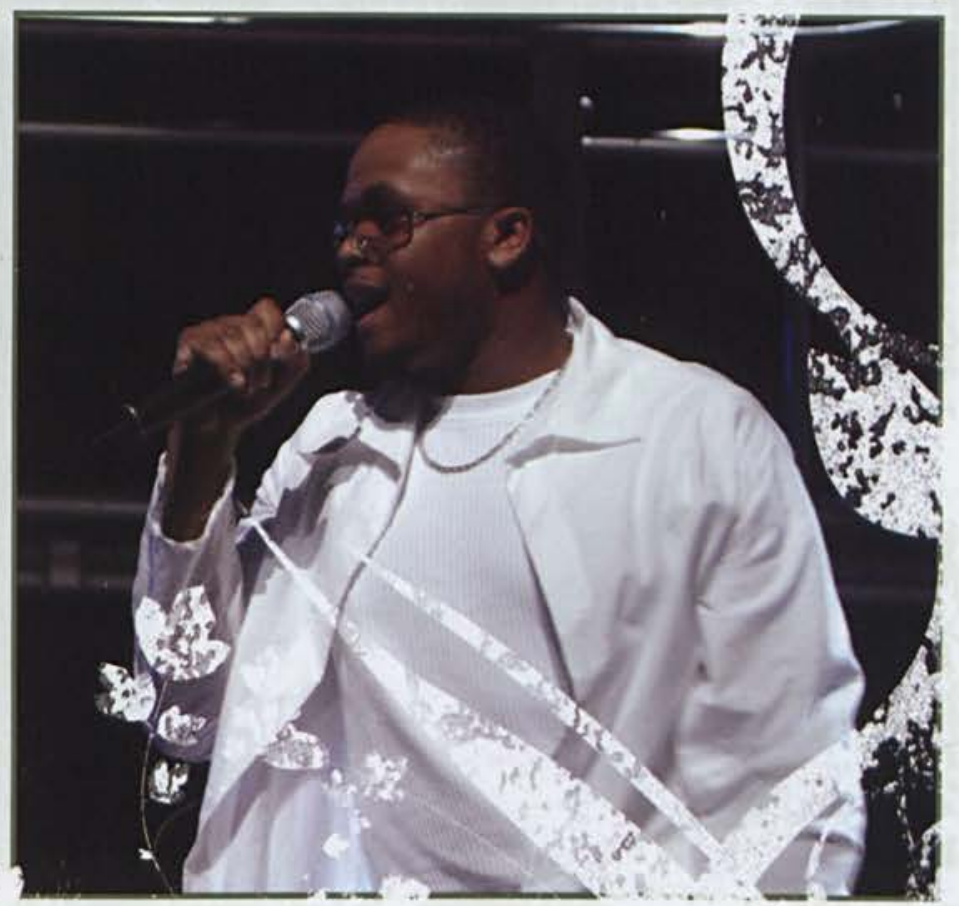

iz Pesce (not pictured).

^ Taking us back to our junior-high days, Mike Smith transforms into a Backstreet Boy, much to the joy of the girls in the audience. 



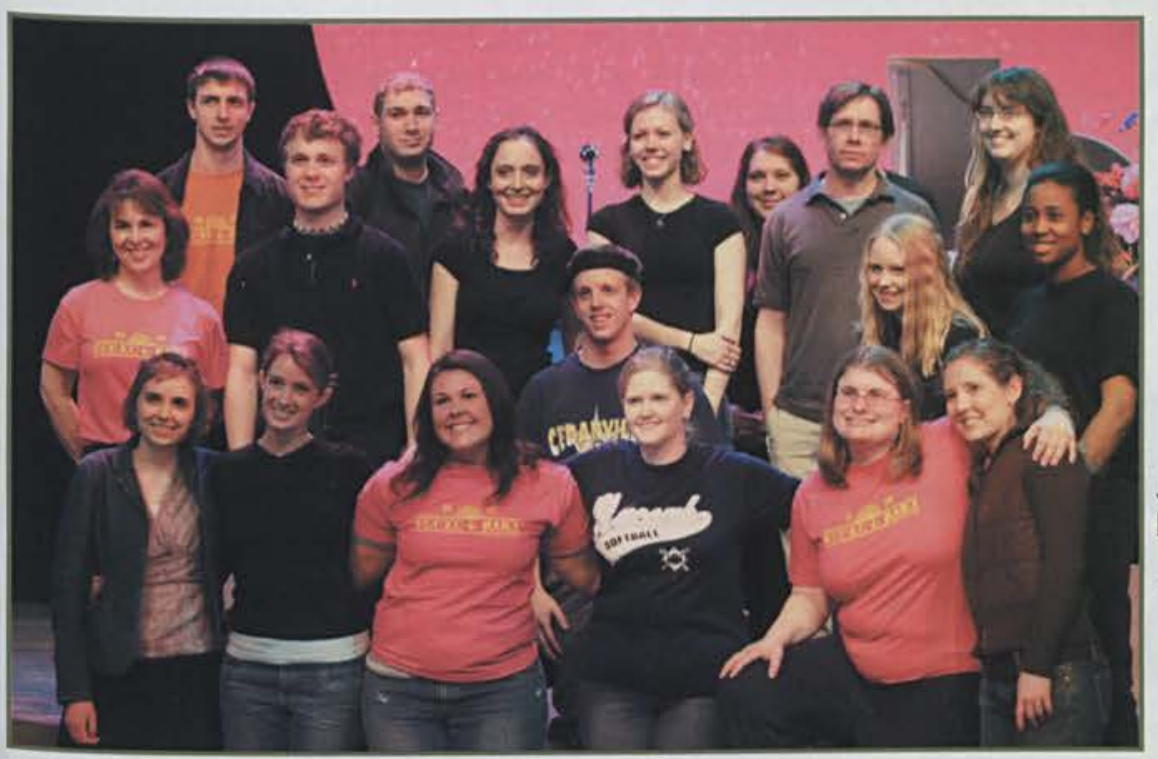

$\checkmark$ No play is complete without its backstage crew. The set designers, lighting and stage crews, sound operator, and costume and make-up/ hair crews gather for their own set of bows.

- In the opening scenes of Romeo and Juliet, servants Abraham and Balthasar, played by Alex Hollander and Matthew Abraham respectively, pick a fight with their counterparts to the Montagues. Before the brawl, the pair greet and observe the sights and sounds of a normal morning in 1930.

In the most haunting scene of the entire play, a heartbroken Juliet chooses to kill herself rather than live without her beloved Romeo.

$\checkmark$ Following the Capulet party, Mercutio (Daron Day) and Benvolio (Josh Cobb) stumble through the street towards home, but not before rising much laughter from the audience.
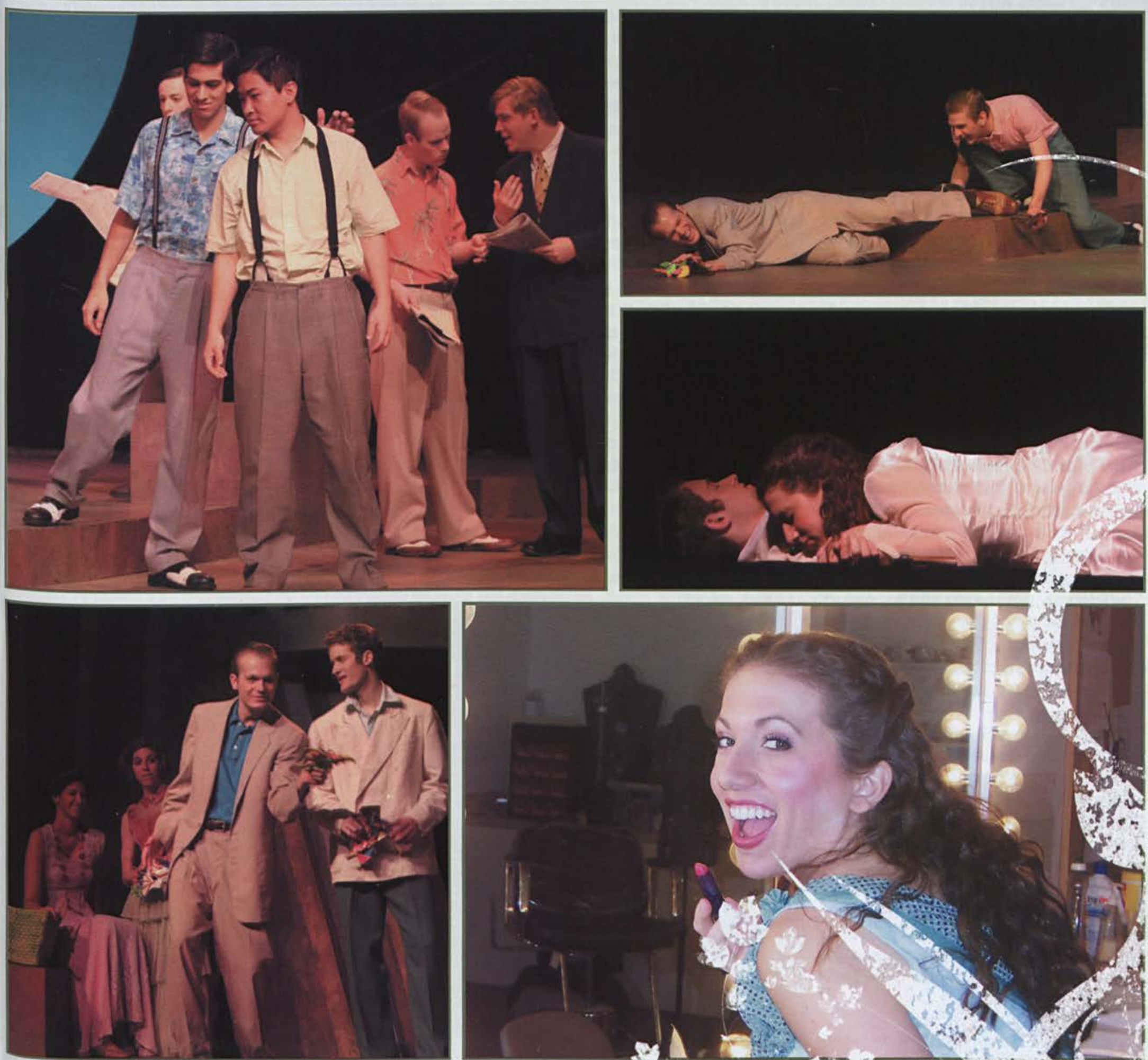

An engaging Mercutio (Daron Day) encourages his good

friend Romeo, played by Mike Colletto, to drown his unre-

^ Junior Rebecca Wolff gets caught off guard while carefully applying her stage make-up.

quited love in the music and laughter of a party. 

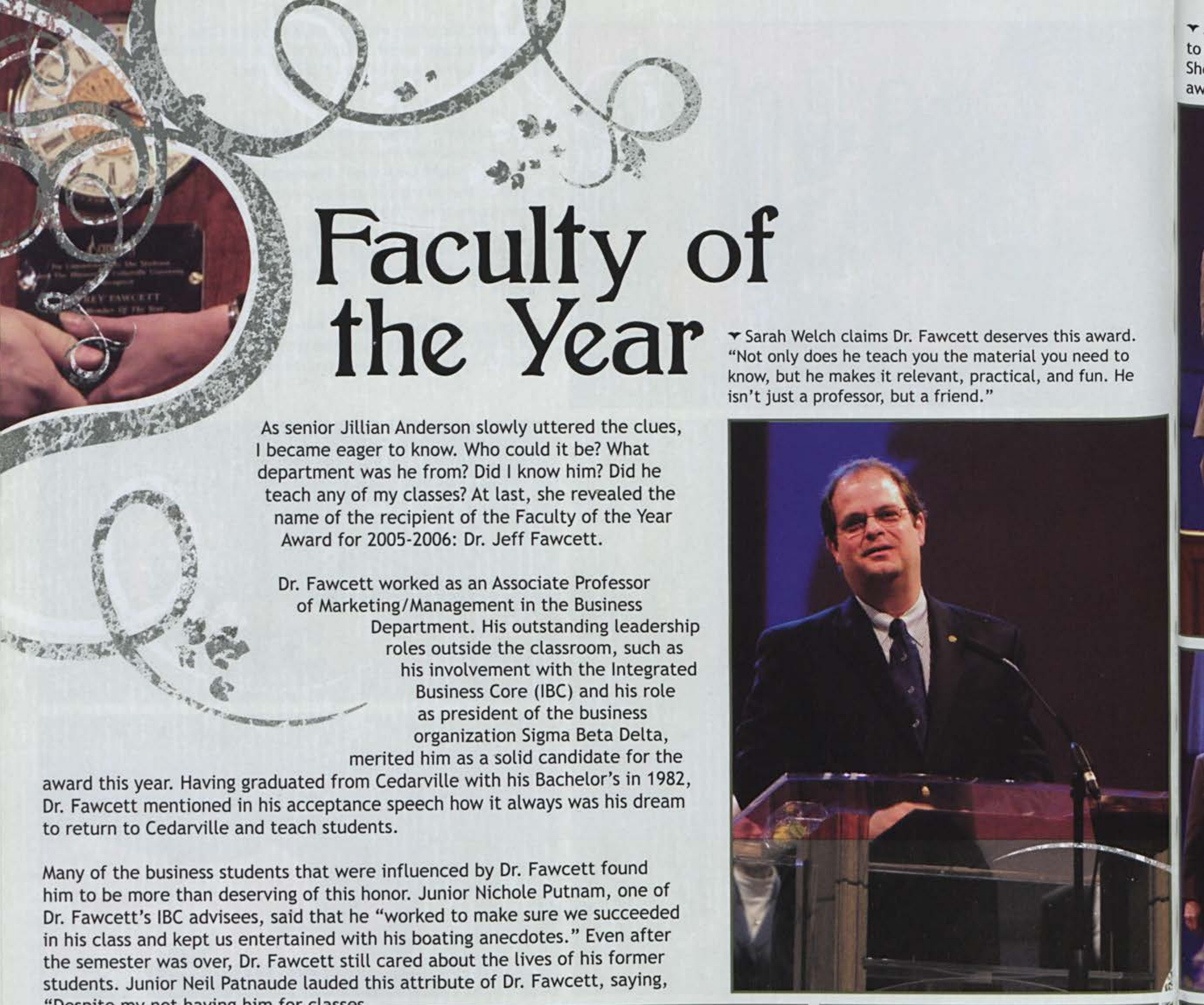
right now and not being a marketing major, he will still take time out of his day to answer questions that I have and meet with me for just about whatever."

The foundation of Dr. Fawcett's commendable qualities was best encapsulated by junior Joel McCloskey when he said, "There's no doubt he cares about his students, and I gained a lot of respect for him through my classes and the IBC." A dedicated spirit, a heart for students, and a genuine love for his field made Dr. Jeff Fawcett an honorable and deserving winner for this year's Faculty of the Year Award.

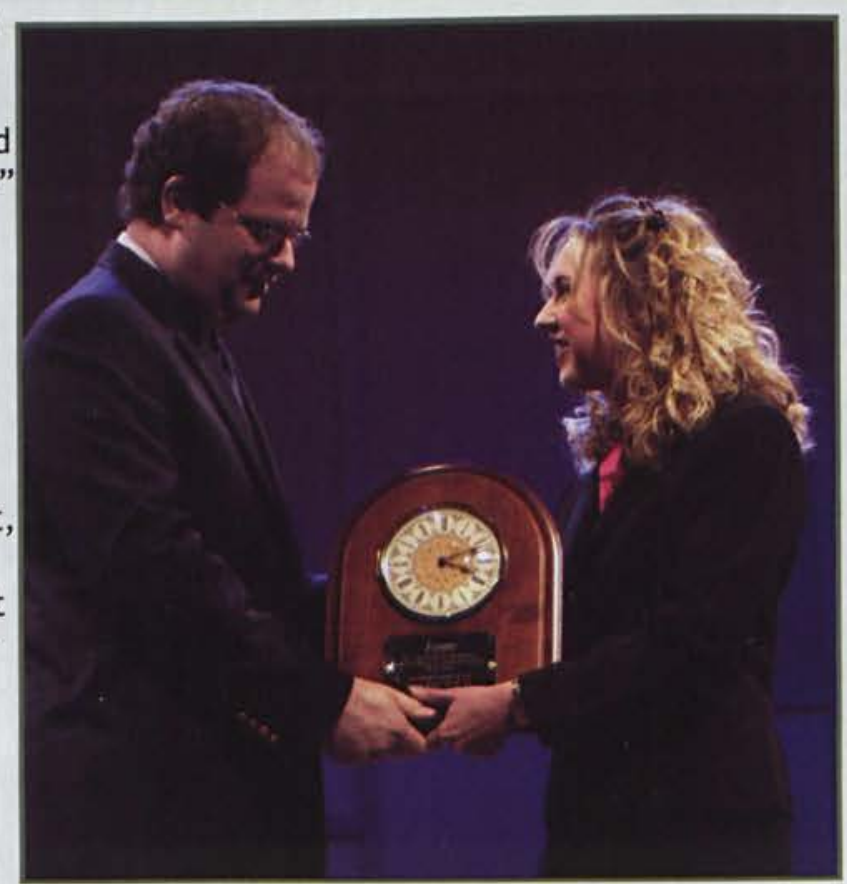

^ Jillian Anderson presents Dr. Fawcett's award. Lindsay LoDico called him "a very down-to-earth professor who continually displays a passion for teaching and students."

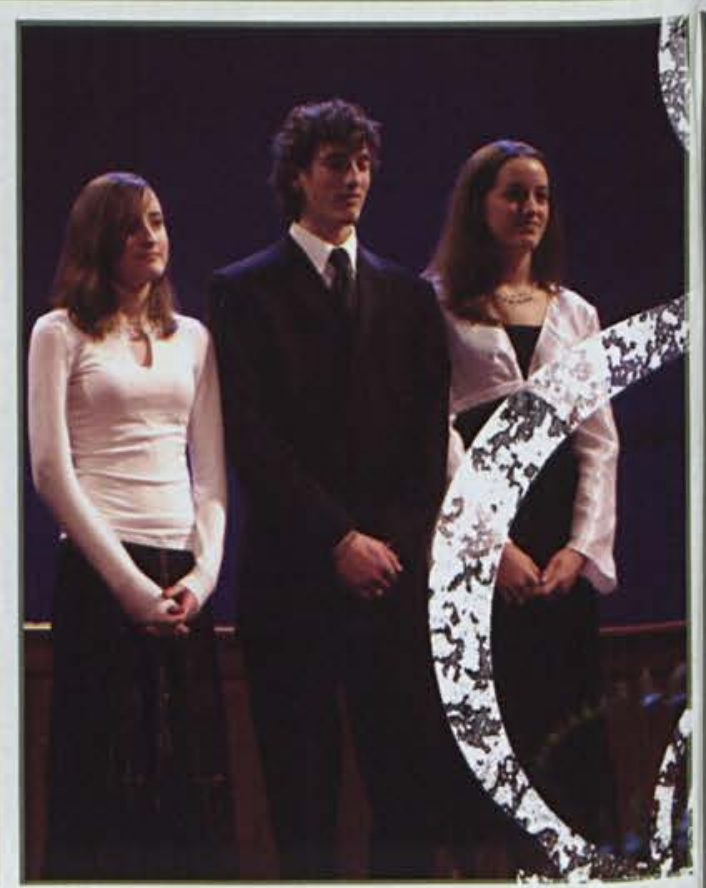

- Three of Dr. Fawcett's four teenage children are present with their mother, Tonya, for the presentation of their father's award. 
$\checkmark$ Jillian Anderson presents the Staff of the Year Award to director of Alumni Relaitions, Faith Linn. Peter Shellabarger, a member of DAE, says, "You always walk away from her feeling like she genuinely loves you."
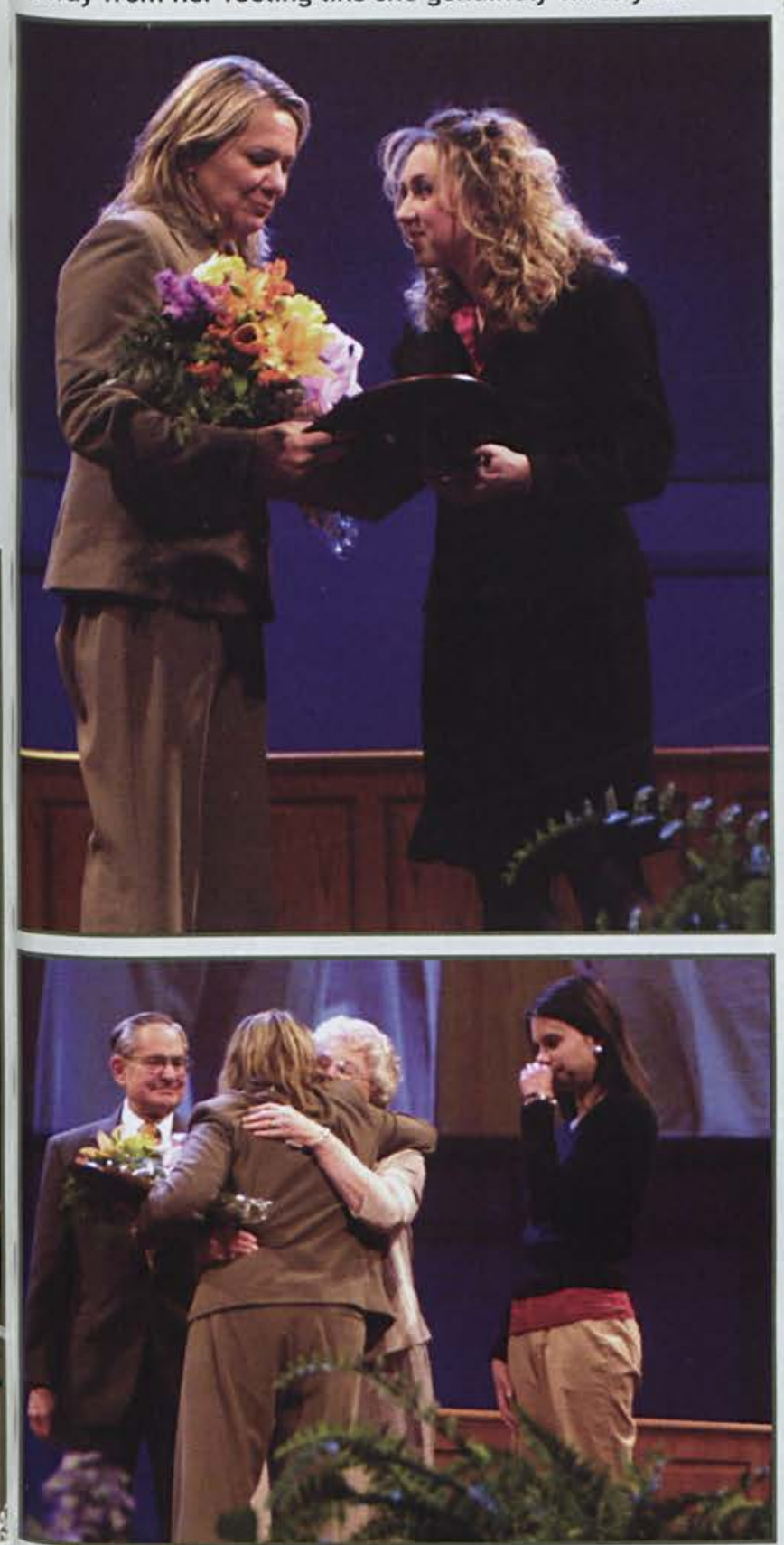

For professors or staff members, nothing is more prestigious than receiving an award from the very students they have dedicated their life to. The Staff of the Year Award was given to a staff member who had shown dedication to both the university and its students. This year's recipient was someone who had committed her life to this school for the past sixteen years. Faith Linn had served as Resident Director of Faith Hall, guest coordinator, and adjunct professor. She currently held the position of Director of Alumni Relations. In this position she kept track of and connected with over 16,000 alumni. Faith also advised and ran the student alumni organization, Delta Alpha Epsilon.

Faith said after receiving her award, "This is the biggest honor I think one can get at Cedarville. I feel humbled because I know there are so many deserving staff members." When asked about Alumni Relations, Faith stated, "We are here to serve students both now and when they leave. I know lots of offices that serve students while they're here, but we keep doing it. We want to help you stay connected with your friends and with Cedarville!"

Students knew Faith as an energetic, fun loving, and committed individual. She brought life to any room she entered. Her devotion to the service of others was overwhelming, yet she never ran out of energy; she would go until the job was done. Junior Peter Shellabarger, member of DAE, said of Faith, "She is one of the most giving people I have ever met. You always walk away from her feeling like she genuinely loves you."

Ms. Faith Linn's parents are on hand for the presentation of their daughter's award, as well as senior Sarah Clift, who works very closely with Faith through DAE.

$\checkmark$ Steven Clemons, DAE member, said, "What I like most about Faith is that she has so much energy. Even when I know she's tired or sick, she still puts forth so much energy to keep us motivated and always gets the job done." 



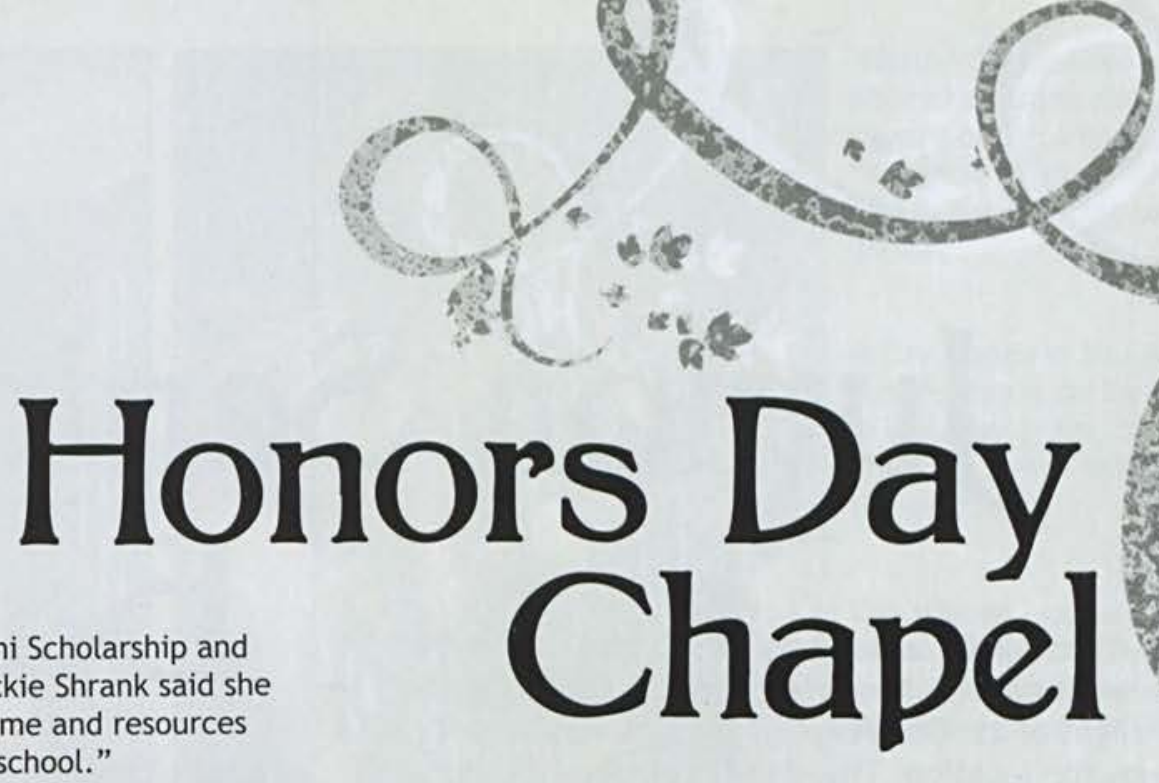

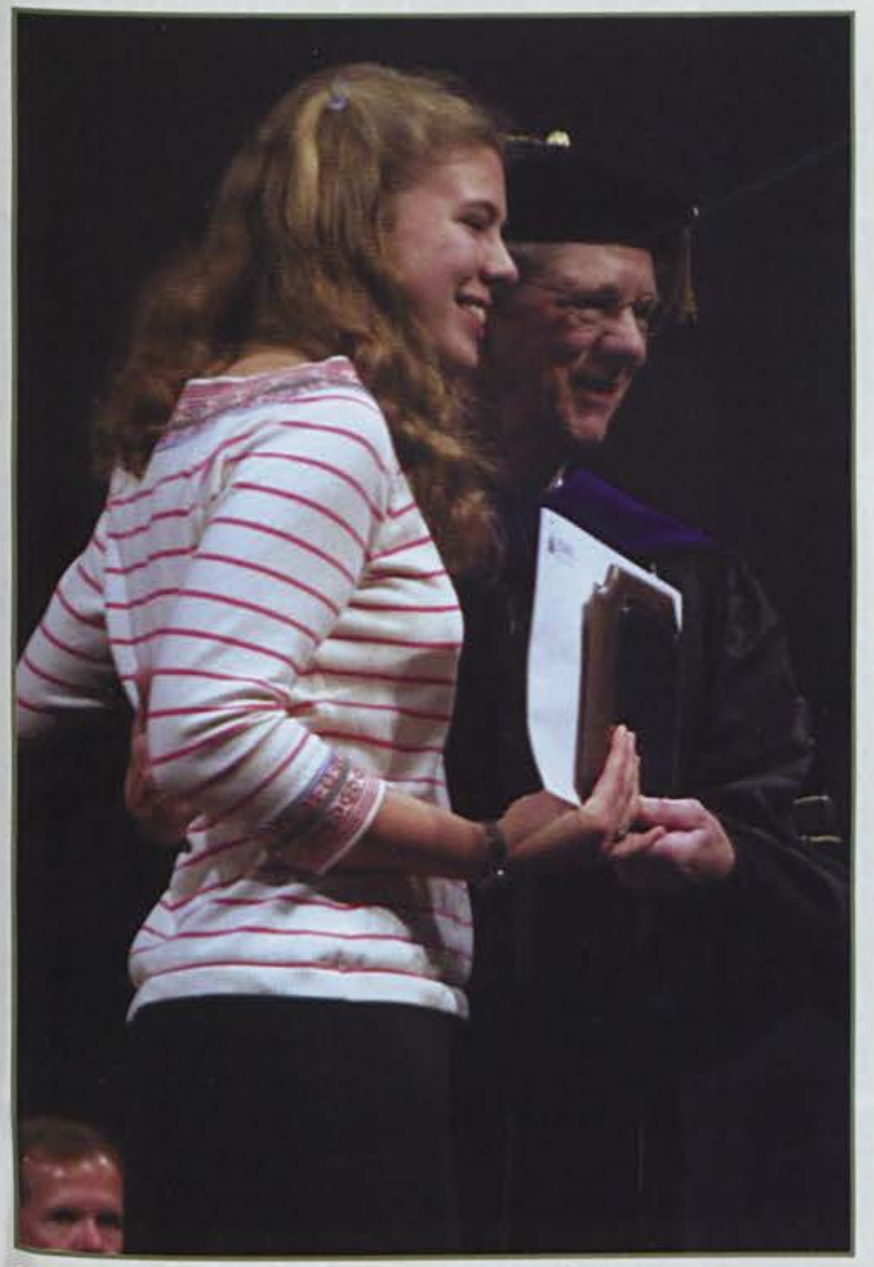

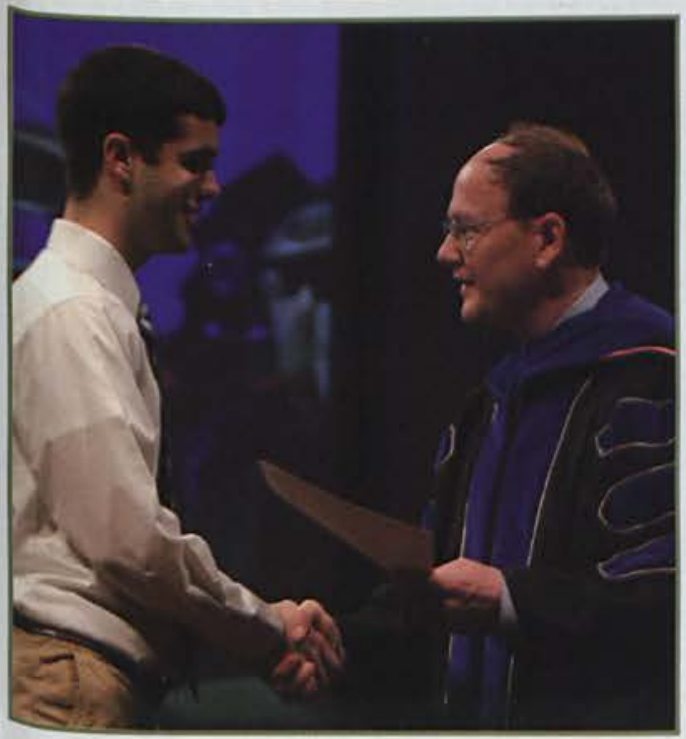

Eric Simonaire recieves his award from

Dr. Zavodney, the head of the Engineering Department.

As I sat in my pew, awaiting the announcement regarding the next honoree, I was astounded by the sheer amount of hard-working scholars that we have here at Cedarville. The names continued to be announced as student after student received recognition. What fantastic examples of Christian diligence and academic brilliance! Maybe I'll study for that Old Testament final a little bit longer than usual. . .

This year's academic Honors Chapel was the highlight of the year and the culmination of countless hours of hard work for students who excelled in their field of study. Everything from literature to engineering awards was handed out, as a respectable representation of diligence from each field of study came to humbly receive their award. Most awards involved generous grants from the university or special fund-raising groups that wished to advance the merits of deserving students.

Obviously, not every student was recognized in Honors Chapel, but for many observers, this special event was necessary. Sophomore Carolyn Ericsson realized how important Honors Chapel was to the humble students who deserved recognition. She said, "High academic achievement is not something you walk around bragging about to your friends, so it is good to see the people who work so hard get recognized and awarded for their extra effort." Sophomore Amanda Brumbaugh felt similarly and explained, "I felt inspired to

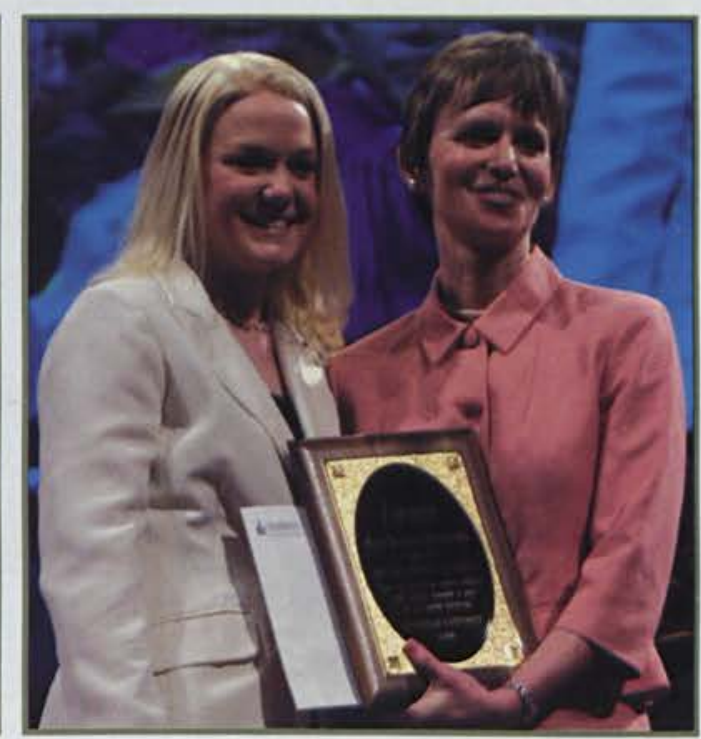

^ Megan Baker recieves the Ruby Jeremiah Scholarship, which honors excellence in Christian service and academics among married women. work harder and push for that higher GPA or better grade."

What it all came down to, however, was the One responsible for such academic talent and diligence. Junior Sarah Hall said, "We as Christians are supposed to be excellent in everything that we attempt, knowing that whatever we may achieve is for God's glory. For some students, their excellence lies in academics, and today, they were rightfully honored for that excellence." 


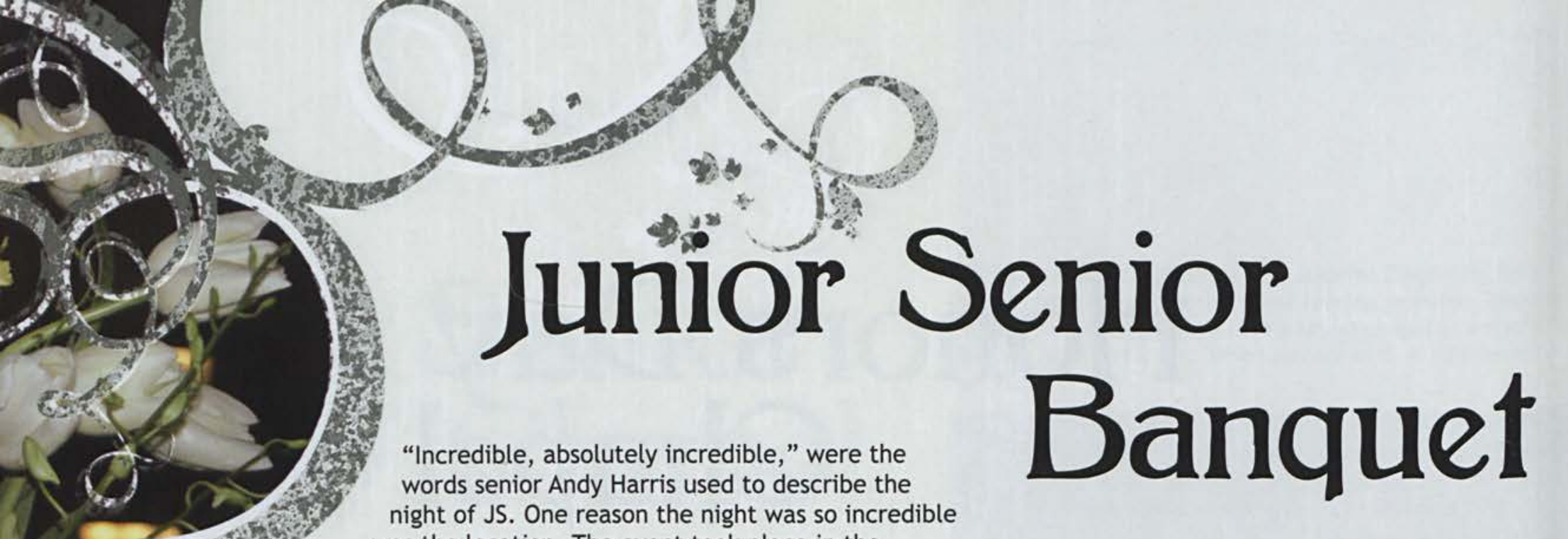

night of JS. One reason the night was so incredible was the location. The event took place in the Cincinnati Museum Center in downtown Cincinnati. The building itself is the refurbished train depot that was originally built in 1933 .

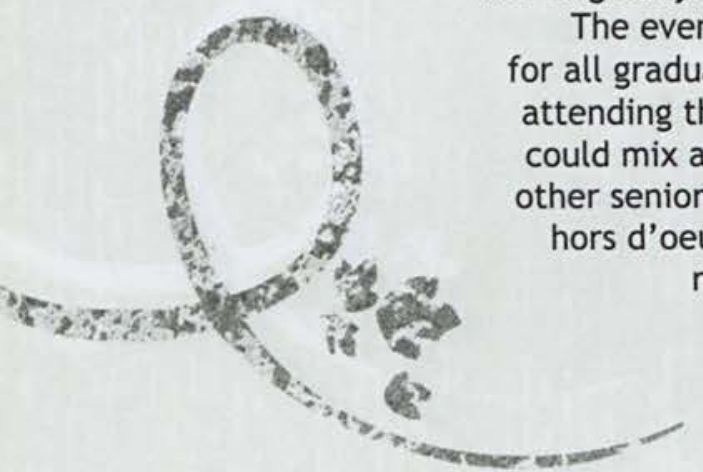

The evening began with a thirty minute reception

$\checkmark$ Seniors Amanda Hunter, Brigette Hunter, and Jessica Gigliotti sample punch beneath the grand rotunda. attending the event. Here they could mix and mingle with ther seniors and enjoy tasty hors d'oeuvres. After this reception, everyone came together in the central lobby of the museum for dinner. Dr. Dixon opened

the evening with prayer and a memorable goodbye to his last incoming class. During dinner we enjoyed a slideshow of pictures of the seniors from the past four years as well as a lively compilation of chapel clips and old SGA videos that reminded us of the fun times that we had in chapel. After Dr. Brown gave his closing remarks, the Cincinnati History Museum was opened for a private viewing.

Senior Kayla Smith said of the evening, "It was amazing to have the museum open for us. It gave us something to do and so many wonderful picture opportunities. The whole night was just incredible, and I had a marvelous time." After the beautiful evening, junior Stacy Alexander said, "I had so much fun, and the location was incredible. I loved the building and how it aided in the atmosphere of the night: grand and elegant."

The entire evening was a tribute to the graduating seniors, and from those who went, there was an overall consensus that it was a tribute well done. Everyone had an amazing time. And of course... who could argue with Dr. Dixon and his dandelions?
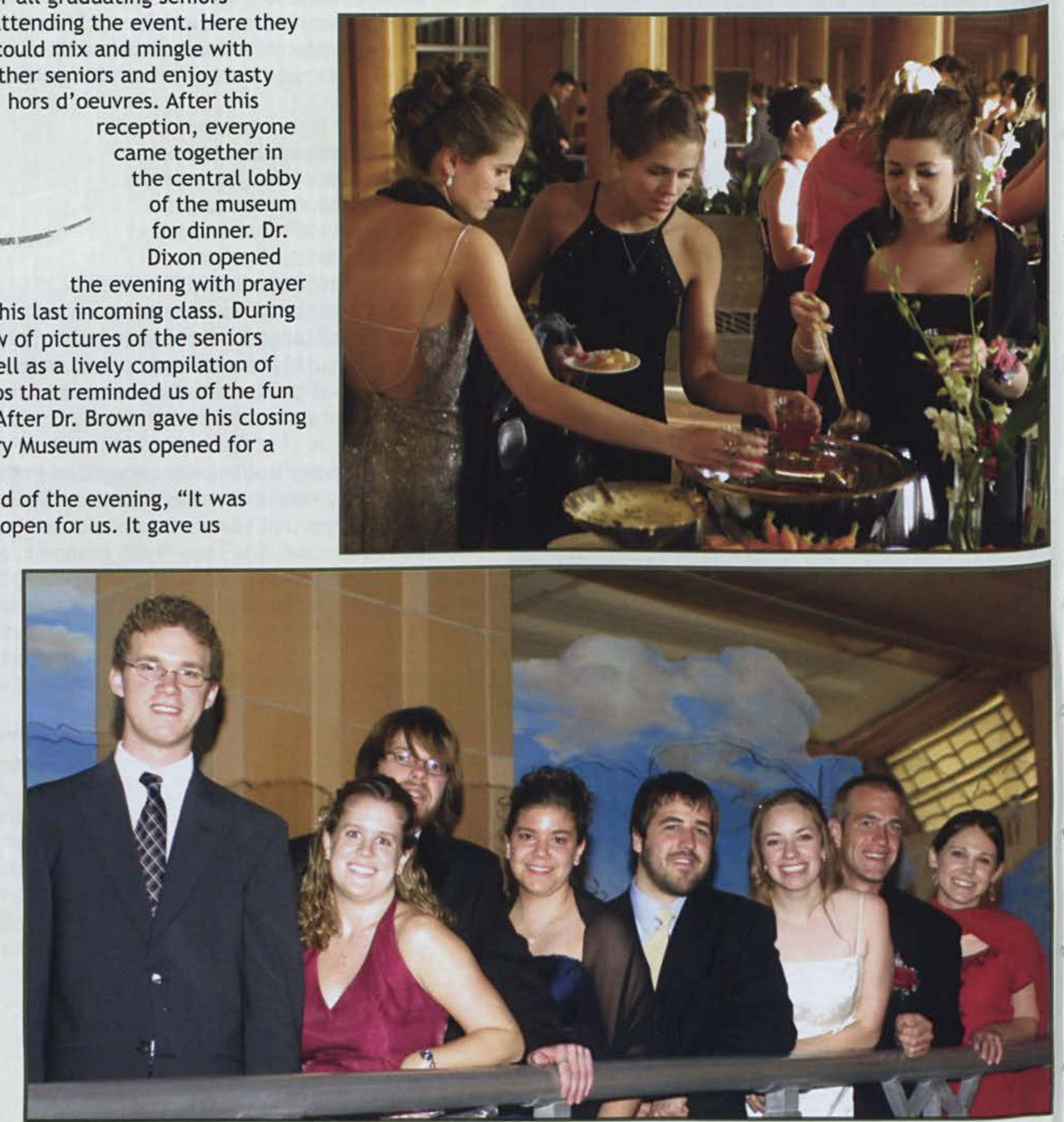

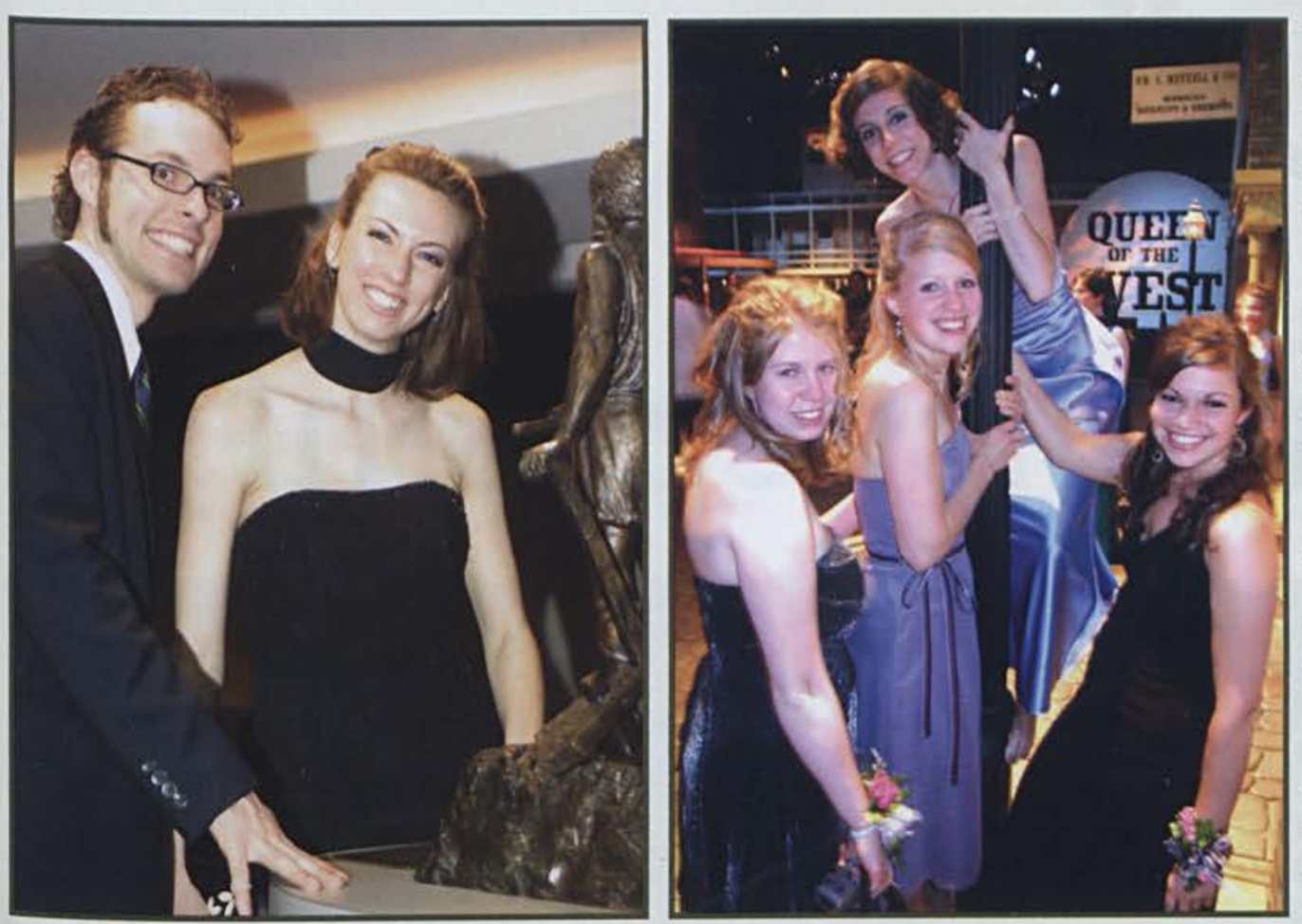

$\checkmark$ Beth Winner, Kate Miller, Rebekah Nettekoven, and Abbey Kauffman take a trip through time to enjoy the hayday of the American West. Rebekah explained, "We tried to enact 'Singin' in the Rain' but found it was rather difficult to spin around lamp posts in long dresses."

* David and Melissa Nickerson, seniors, admire a bronze statue during the museum's private showing. The history of the museum was definitely on display and, along with senior memories, was the showcase of the evening.

A Freshmen, sophomores, juniors, and seniors alike all crowd the floor beneath the grand rotunda to share in the wealth of memories made over the years. A slideshow of candids runs during the event.

$\checkmark$ Michelle Boudreau and Louis Yaklich enjoy their friendship as they wander through the exhibts on display.
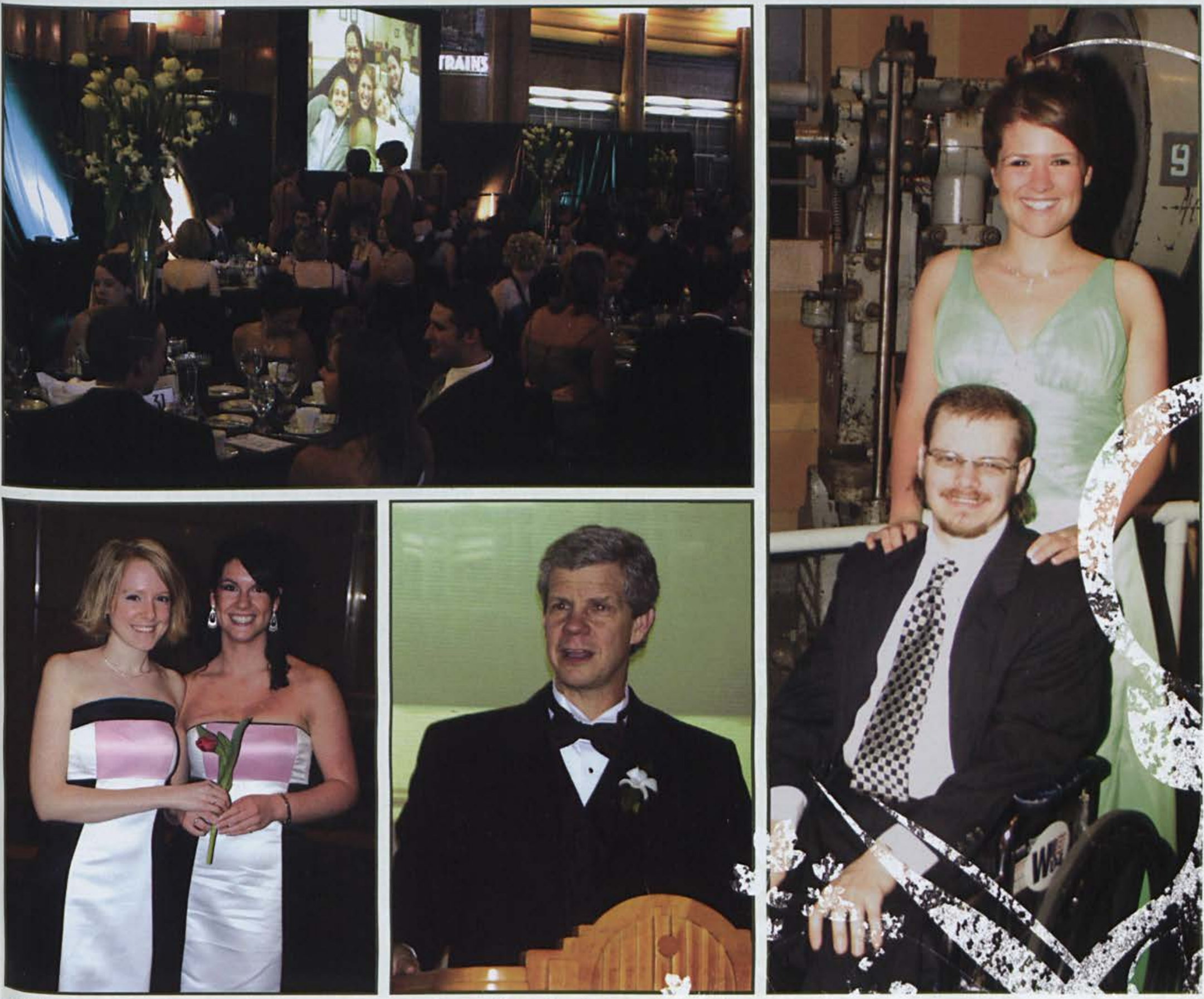

Kelsi Nussbaum and Karen Elliott share a moment at J/S. Karen thinks "going through the museum was a different and unique idea."

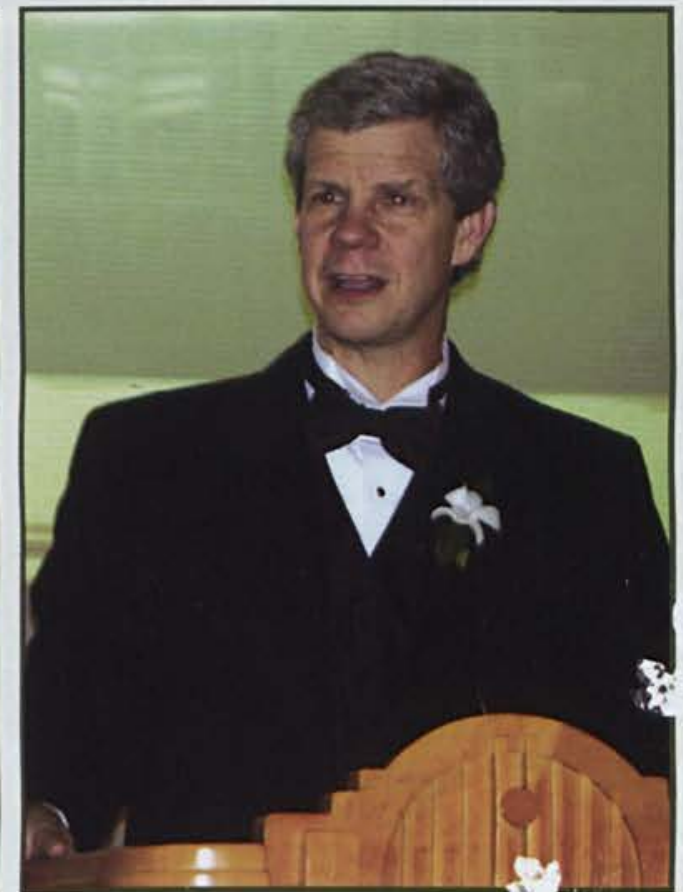

Dr. Brown shares his closing remarks with the seniors. 


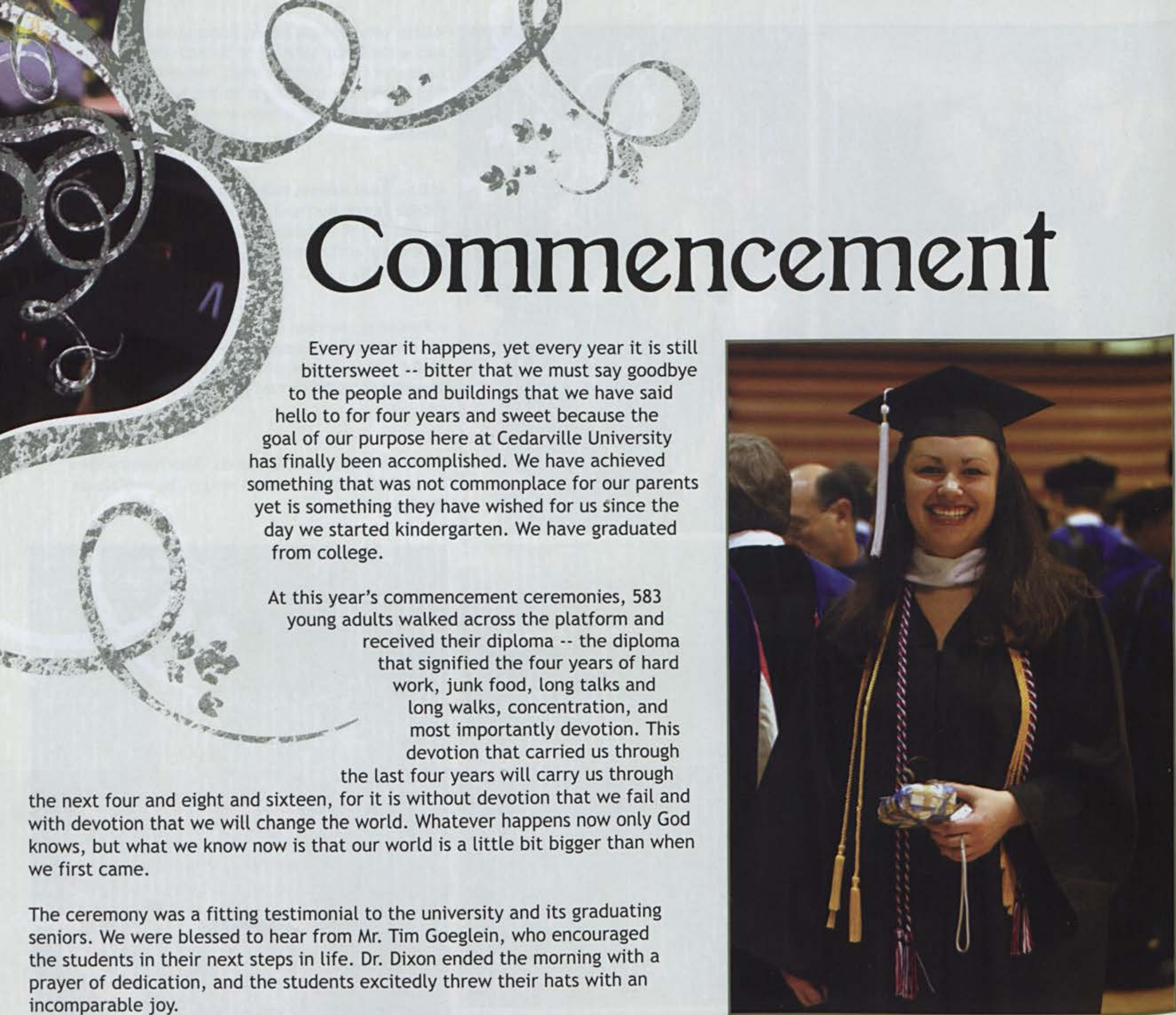

incomparable joy.

Following the graduation, the seniors, their families, and friends gathered across the campus to take pictures and say final goodbyes. As we cried and laughed, remembering the good times we had enjoyed, we hoped and prayed this would not be the last time we would see each other, and as I drove away, I knew that Cedarville was not just a college, but a part of my heart.

ॠAutumn West prepares for graduation. "All my friends were there, and we were celebrating the fact that we were done and trying to forget that being done also meant that we were leaving each other after four amazing years!" 262 Spring 8,8

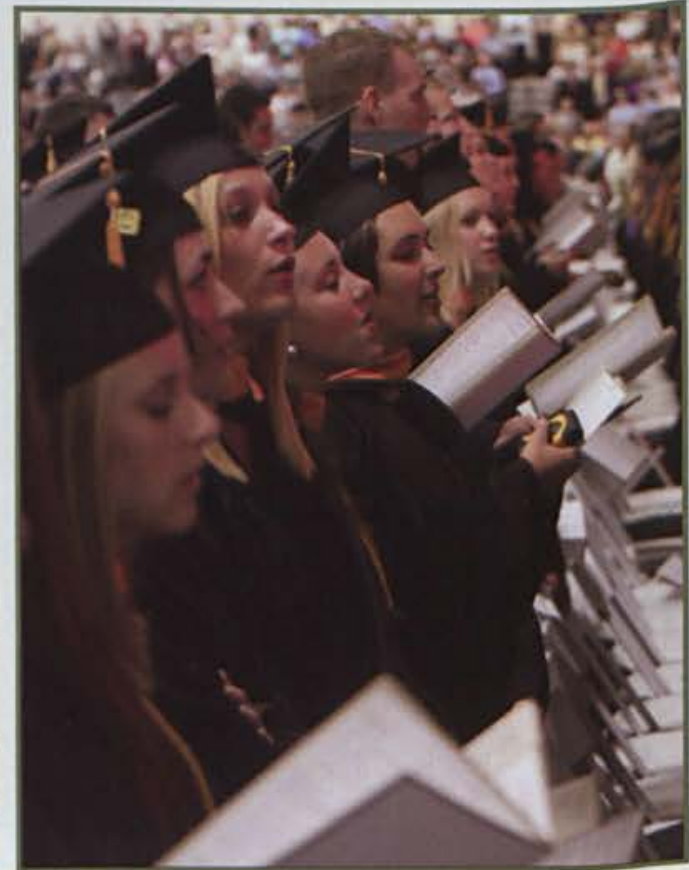

$\downarrow$ Seniors join the audience in singing their

class song: "Be Thou My Vision."

\section{$\mathrm{Br}$}

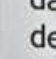

Every year it happens, yet every year it is still bittersweet -- bitter that we must say goodbye to the people and buildings that we have said goal of our purpose here at Cedarville University has finally been accomplished. We have achieved et is something they have wished for us since the day we started kindergarten. We have graduated this year's commencement ceremonies, 583 received their diploma .. the diplom long walks, concentration, and devotion that carried us through the last four years will carry us through

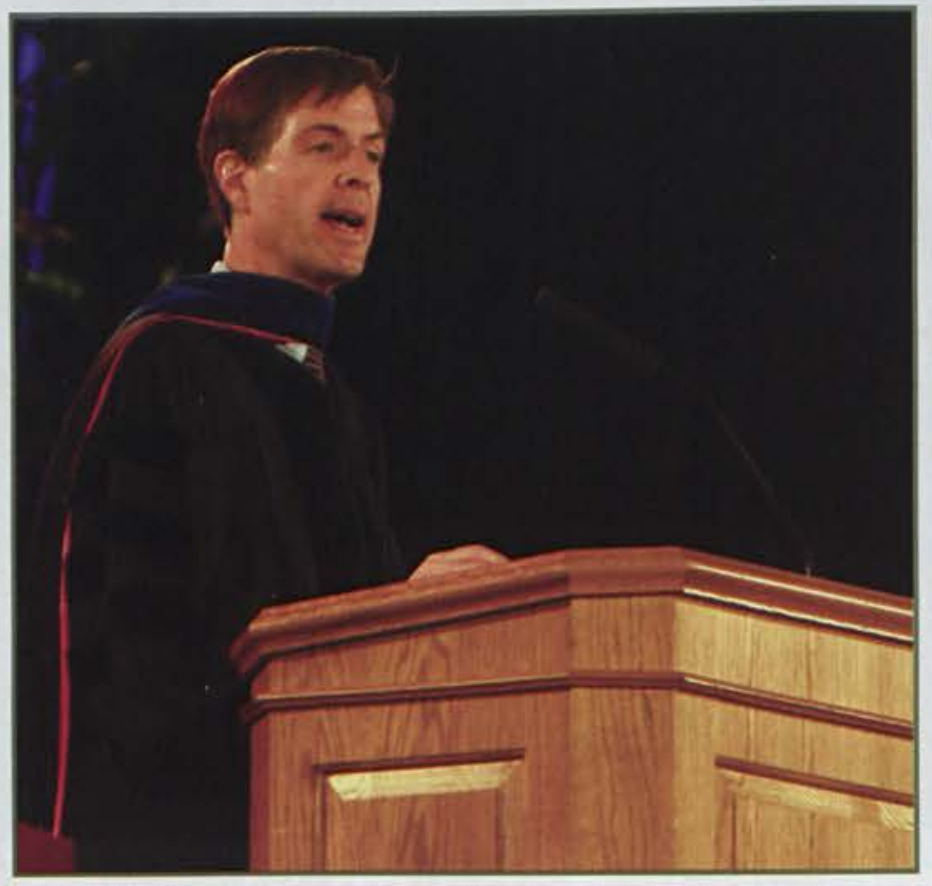

$\Delta$ Mr. Tim Goeglein, special assistant to the President of the United States and deputy director of Public Liaison, addresses the senior class. 
$\checkmark$ Josh Craig gratefully accepts his diploma from Dr. Brown. Like his friends, Josh has looked forward to this day for years and has worked hard to obtain his business degree.
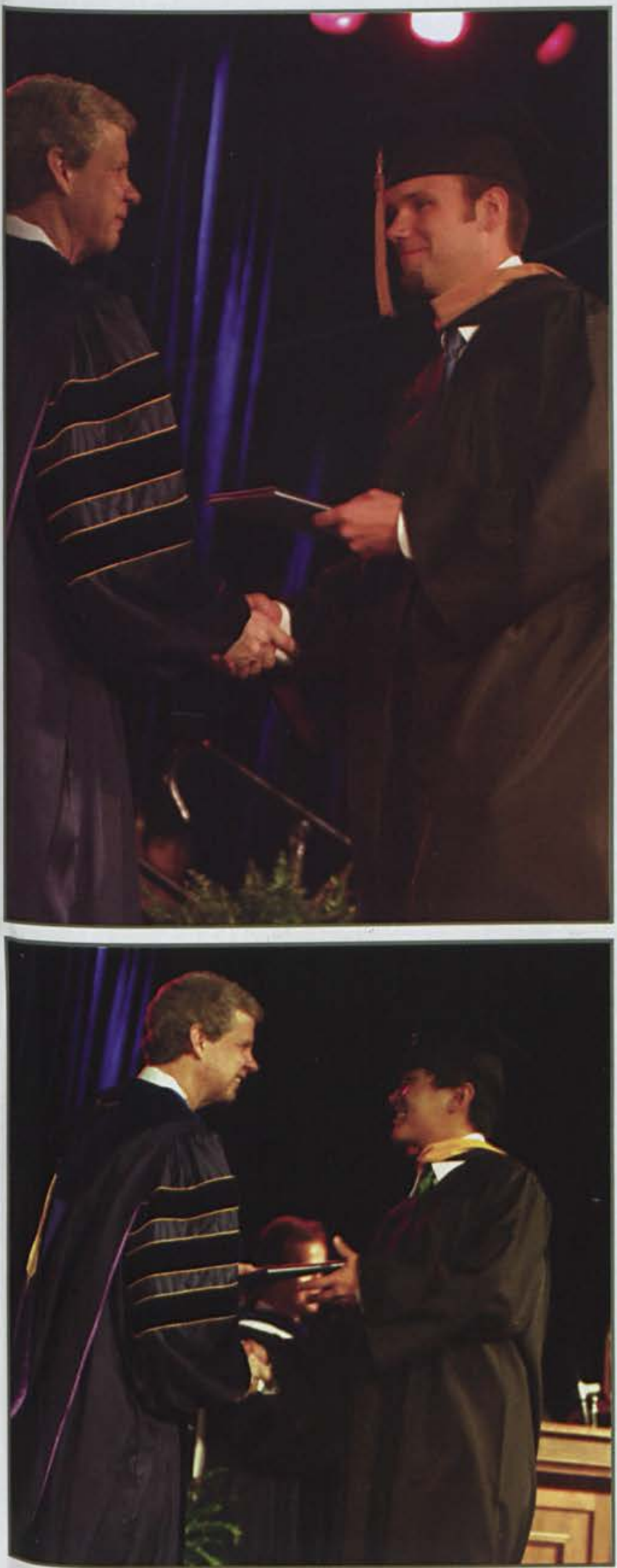

Alex Hollander accepts his diploma with pride from Dr. Brown.
₹ Joel Sigmon, Katie Rexford, Jeremy Motta, Andrea DeGuglielmo, Kurt Fraser, Matt Deakin, Rachel Petersen, Dan Gifford, Will Parmerlee, Dave Parmerlee, Brenton Moore, Suzanne Browning, James McClenahan, Rachel Verosky, Nate Johnson, and Brittany Rouse celebrate together. "It's really exciting to be able say good-bye to people who have been my friends all four years," said Rachel Petersen.

$\checkmark$ Emily Moser celebrates her graduation with family, friends, and her boyfriend, senior Tyler Anderson.
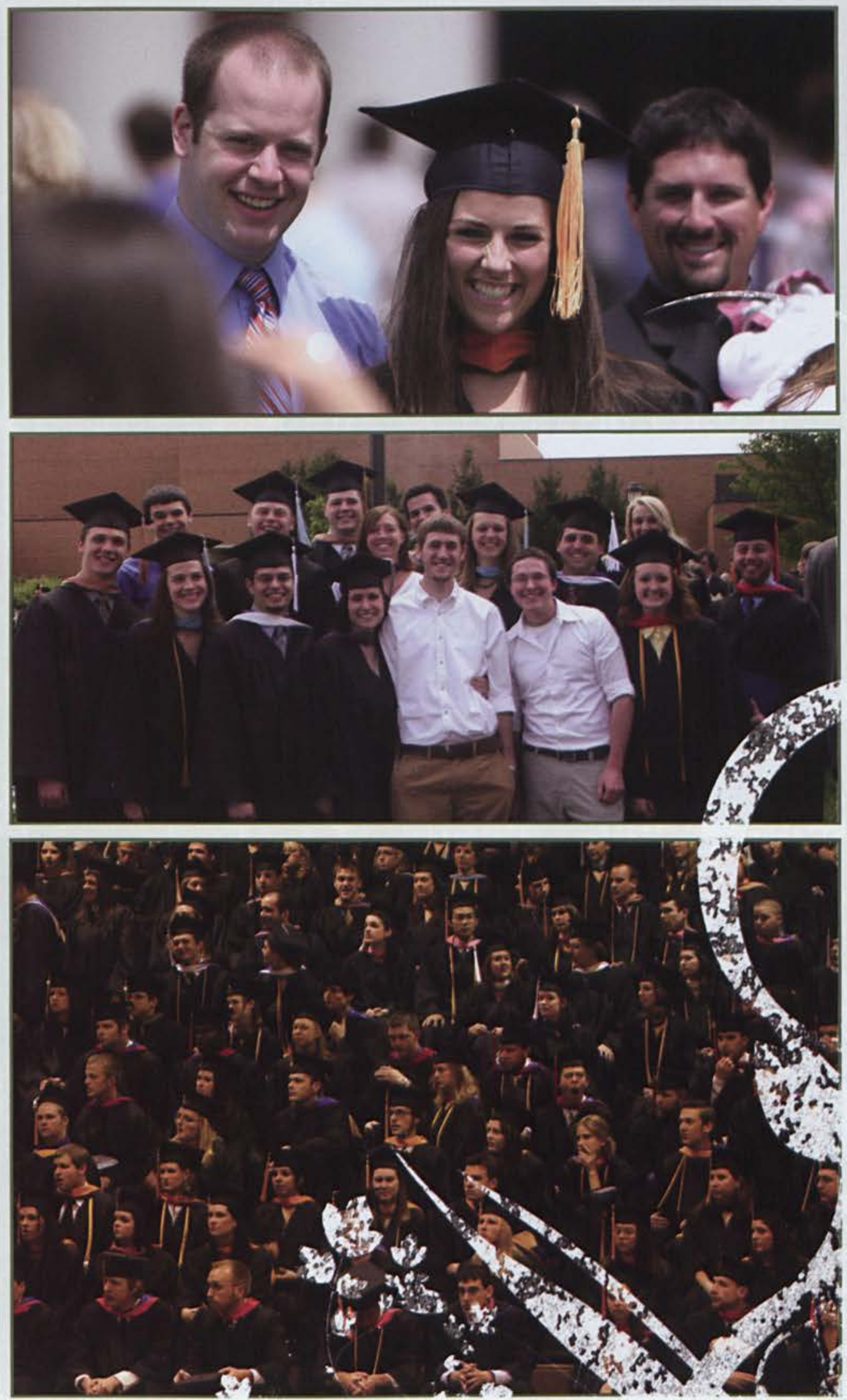

$\Lambda$ The Class of 2006 waits expectantly while they take in the words they have waited four long years to hear....at their graduation. 
$\checkmark$ Friends Ryan Bowen and Josh Flynn find it's only natural to celebrate their graduation together. In keeping with Cedarville's motto, Josh Flynn added, "We have enjoyed our time together and hope to remain friends for life."
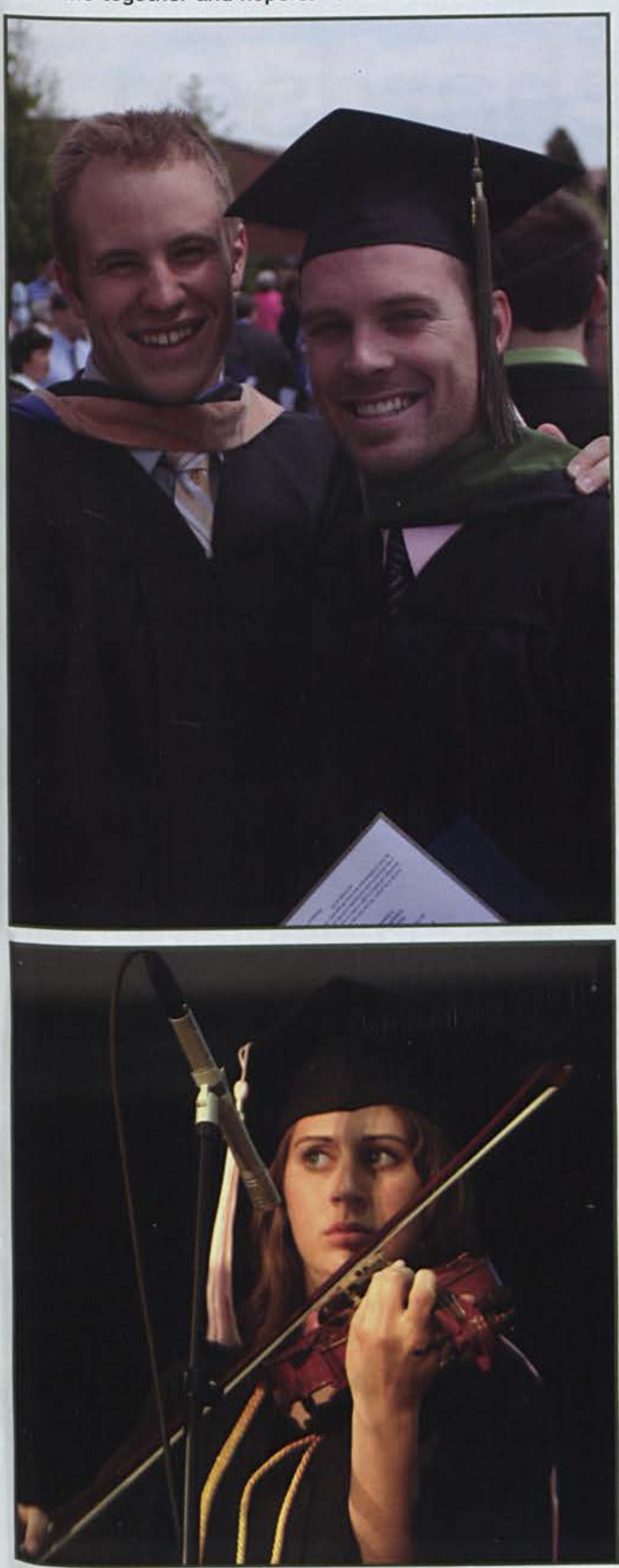

Rachel Talbott plays the violin for the seniors when they sing their class song: "Be Thou My Vision."
F Dustin Tursack, Jonathan Thomas, and Steve Turkovich thank professors Dr. Jeffrey Fawcett and Mr. John LeBlanc for their help in getting through four years in the business program.

$\checkmark$ "We did it!" Fiancés Joe Miller and Lana Terpstra gather immediately following graduation to celebrate with their parents.
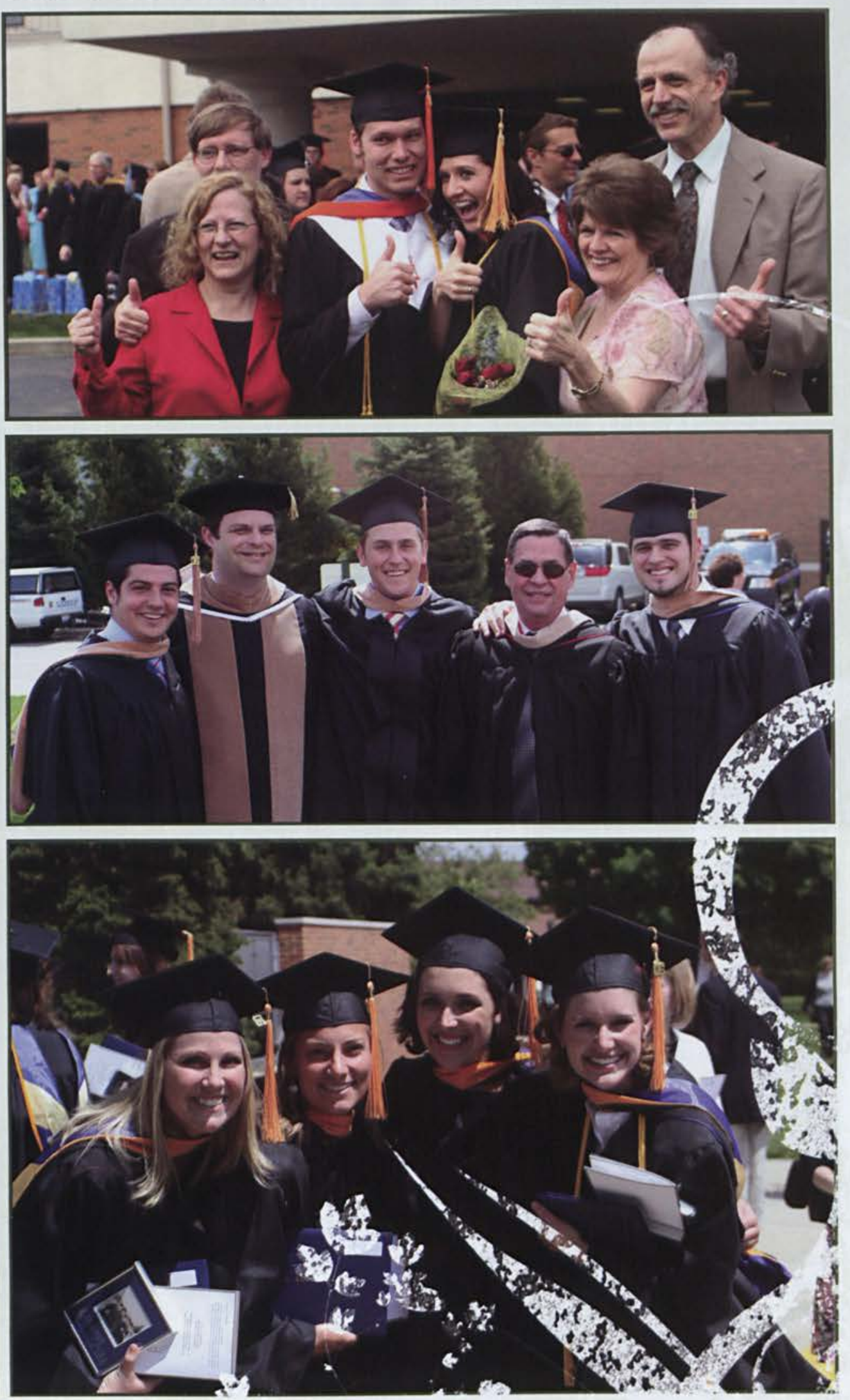

$\Delta$ Natalie Fox, Erin DiMeolo, Katie Krumeich, and Abigail Winburn, all fellow fifth year nursing majors lived together for their last year together. Natalie Fox said she "couldn't have asked for better friends to share my last year!"

Spring 265 

Charm is deceptive, and beauty is fleeting; but a woman who fears the Lord is to be praised. - Proverbs 31:30.
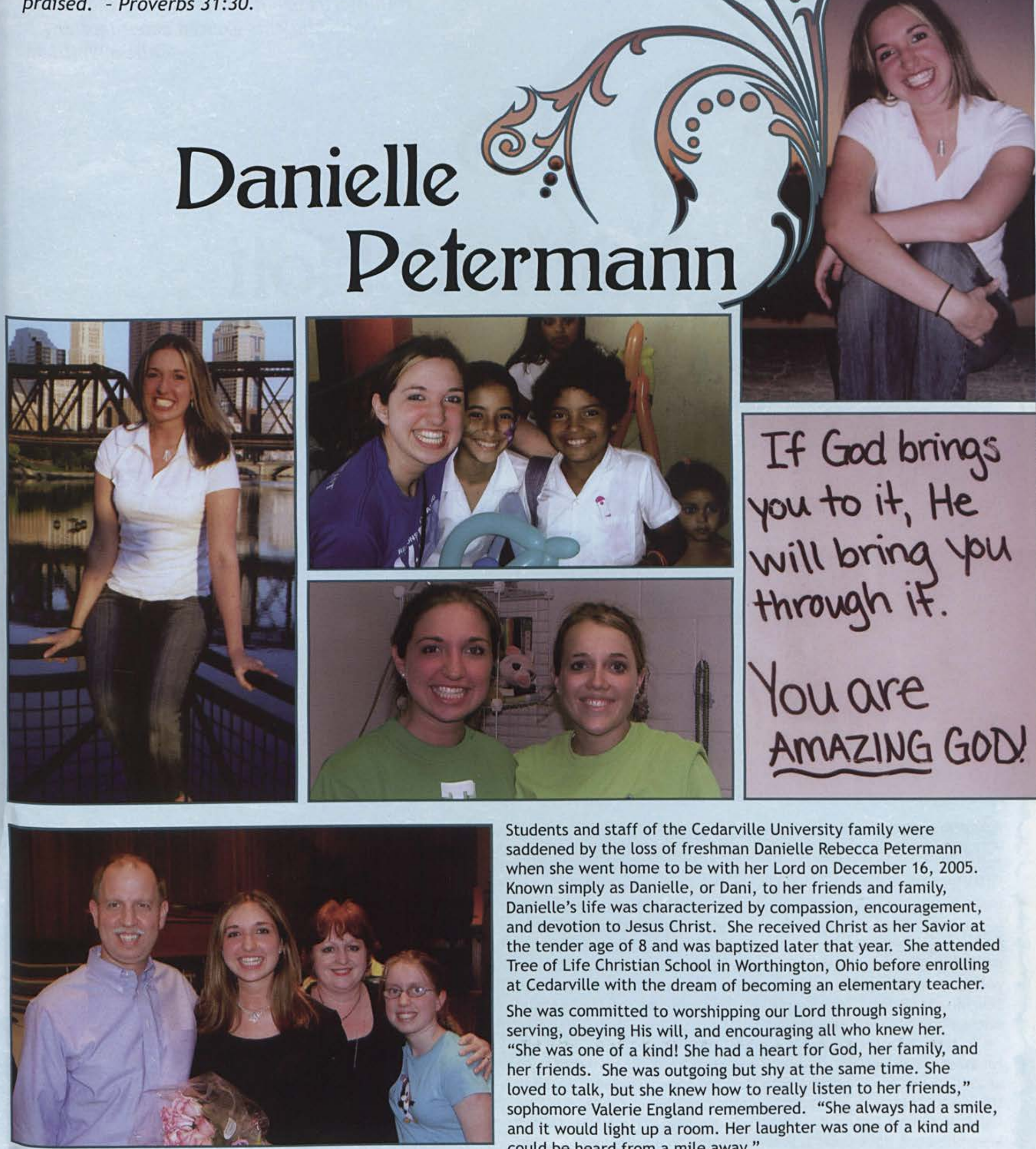

Students and staff of the Cedarville University family were saddened by the loss of freshman Danielle Rebecca Petermann when she went home to be with her Lord on December 16, 2005. Known simply as Danielle, or Dani, to her friends and family, Danielle's life was characterized by compassion, encouragement, and devotion to Jesus Christ. She received Christ as her Savior at the tender age of 8 and was baptized later that year. She attended Tree of Life Christian School in Worthington, Ohio before enrolling at Cedarville with the dream of becoming an elementary teacher.

She was committed to worshipping our Lord through signing, serving, obeying His will, and encouraging all who knew her. "She was one of a kind! She had a heart for God, her family, and her friends. She was outgoing but shy at the same time. She loved to talk, but she knew how to really listen to her friends," sophomore Valerie England remembered. "She always had a smile, and it would light up a room. Her laughter was one of a kind and could be heard from a mile away."

Though her light among us has grown dim, we carry the hope that it burns anew in the life everlasting where we will once again worship our Lord, together. 

Curtis "was a very unselfish man. Though his death is a sorrow to many, many more will be comforted by the memories of his love."
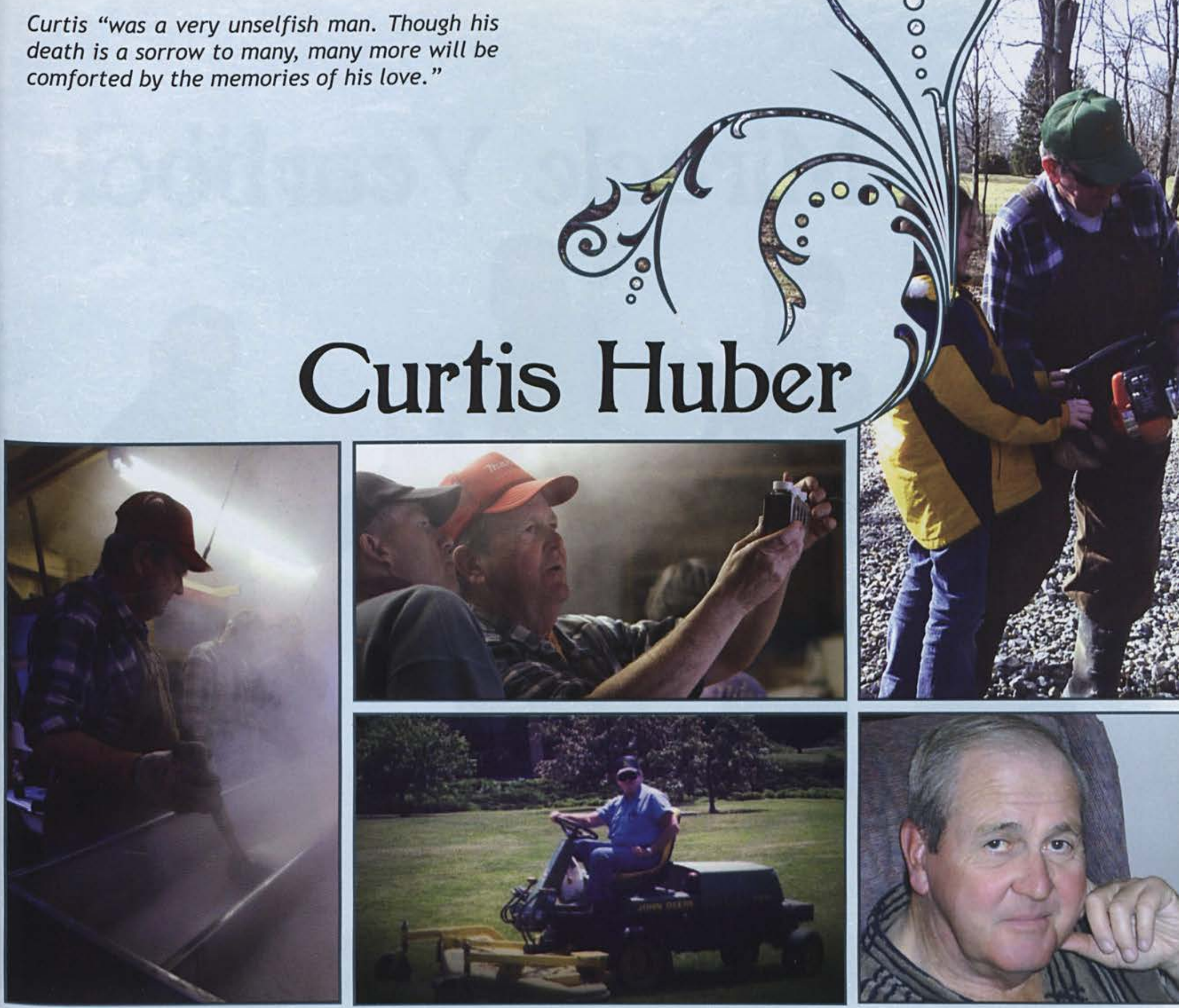

Curtis William Huber, who went to be with his Savior on July 9, 2005, was a full-time employee in the Grounds Department at Cedarville University. Curtis was husband to his high school

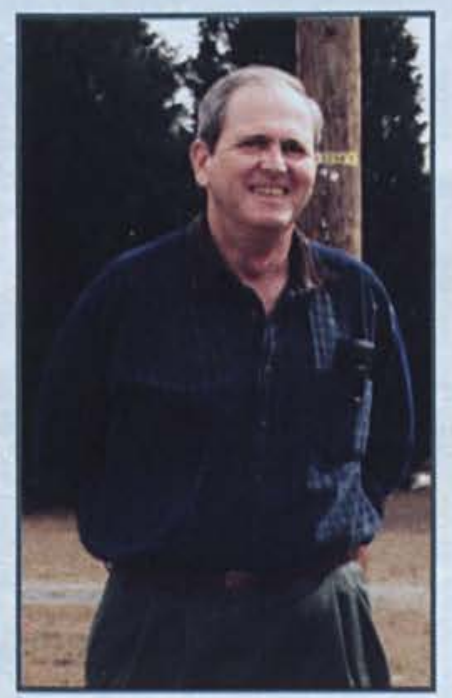

sweetheart Trish Huber, father to Terri Weiss and Cindy Weir, both Cedarville alums, and grandfather to Tirzah and Forest Weiss and Curtis and Jessica Maxson.

"Curt was honored to serve others, and he endeavored to go beyond what was asked of him. His famous words to those who would ask about the relationship he had with his wife and daughters were, 'strive to outlove one another,'" Trish Huber reminisced.

Terri (Huber) Weiss shared that, "after Curtis met his Savior in his early 20 s, he tried to be the friend you'd want to have lunch with, the coworker you looked forward to seeing on Mondays, the son who could make a mother smile from the inside out, the father you dreamed of, the husband you could only hope for, and the spiritual leader who set the pace and took pride in your accomplishments."

Curtis couldn't believe that he could come to work and pray with people. He also enjoyed working with the student employees. Samuel Hague, a coworker of Curt, said, "Curt was a simple guy who displayed to me a true servant's heart." When on campus, Curtis would cut grass, remove snow, and trim the trees. Nathanael Davis, a student who worked with Mr. Huber, reflected that Curtis "was a very unselfish man. Though his death is a sorrow to many, many more will be comforted by the memories of his love." 


\section{Miracle Yearbook}

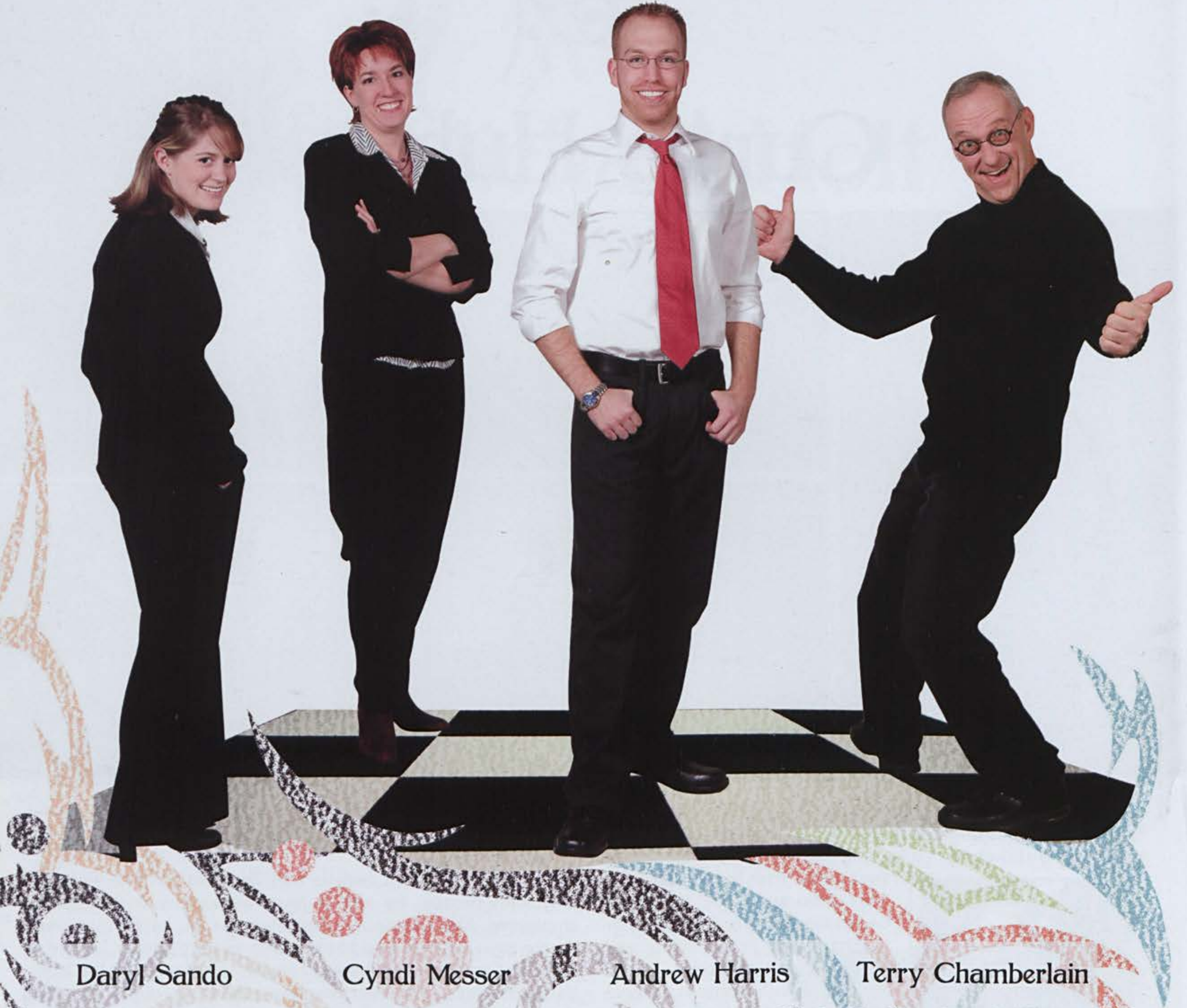

Position: Administrative Position: Faculty Ais Assistant Advisor Year: Junior Major: Global Economics \& Position: Editor-in-Chief Position: Faculty Assistant Professor of English

International Business 


\section{Editors}

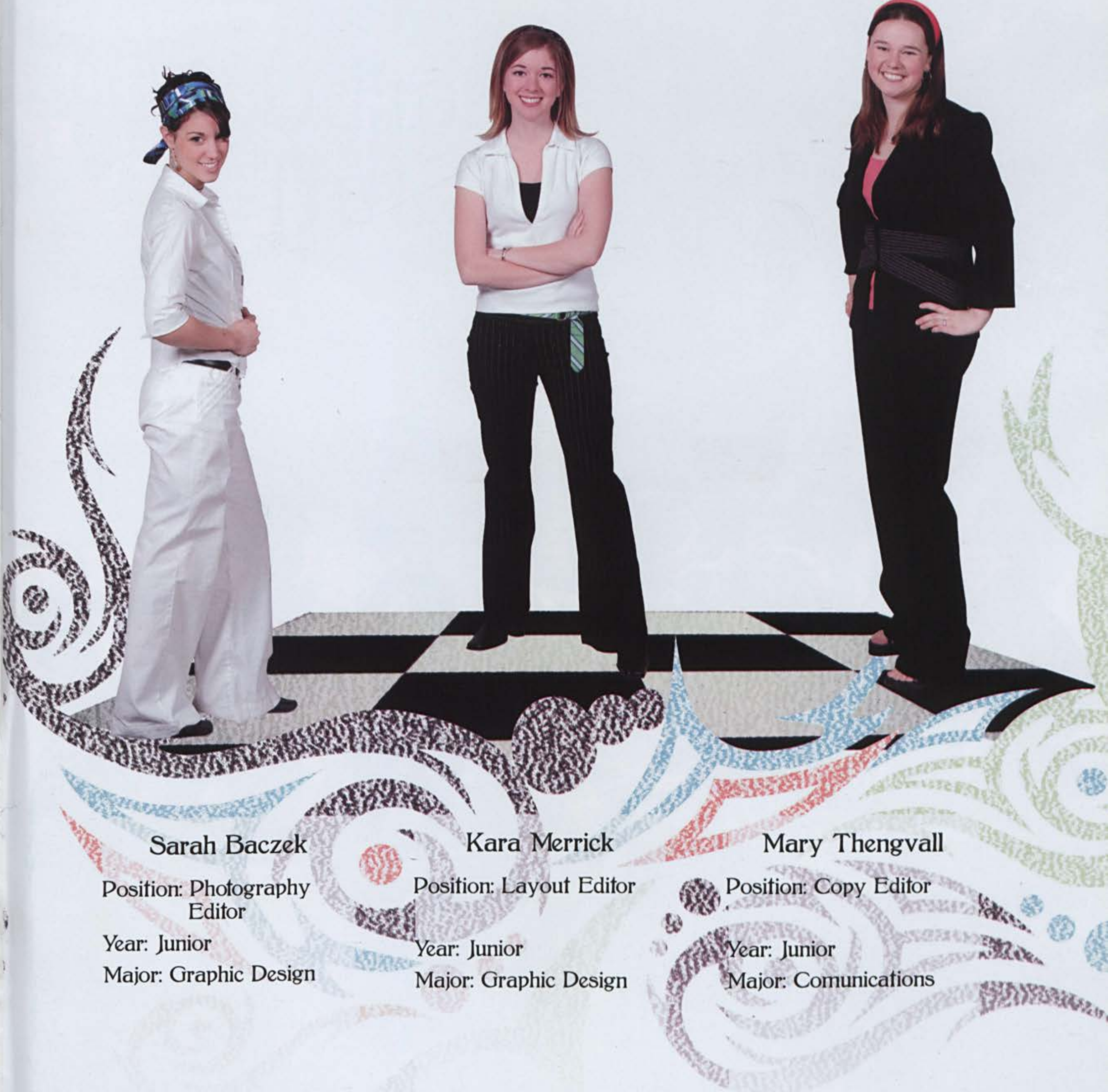



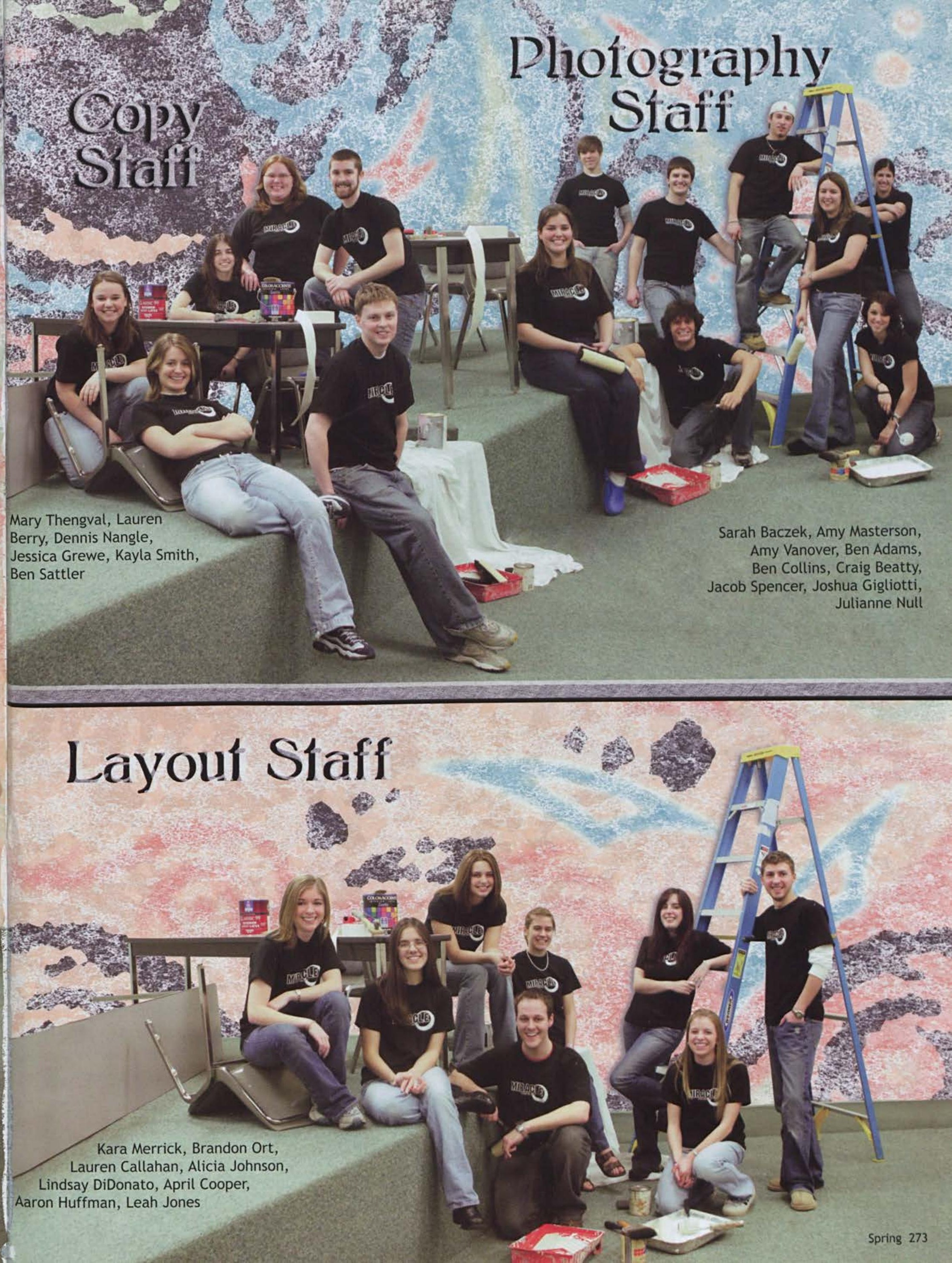


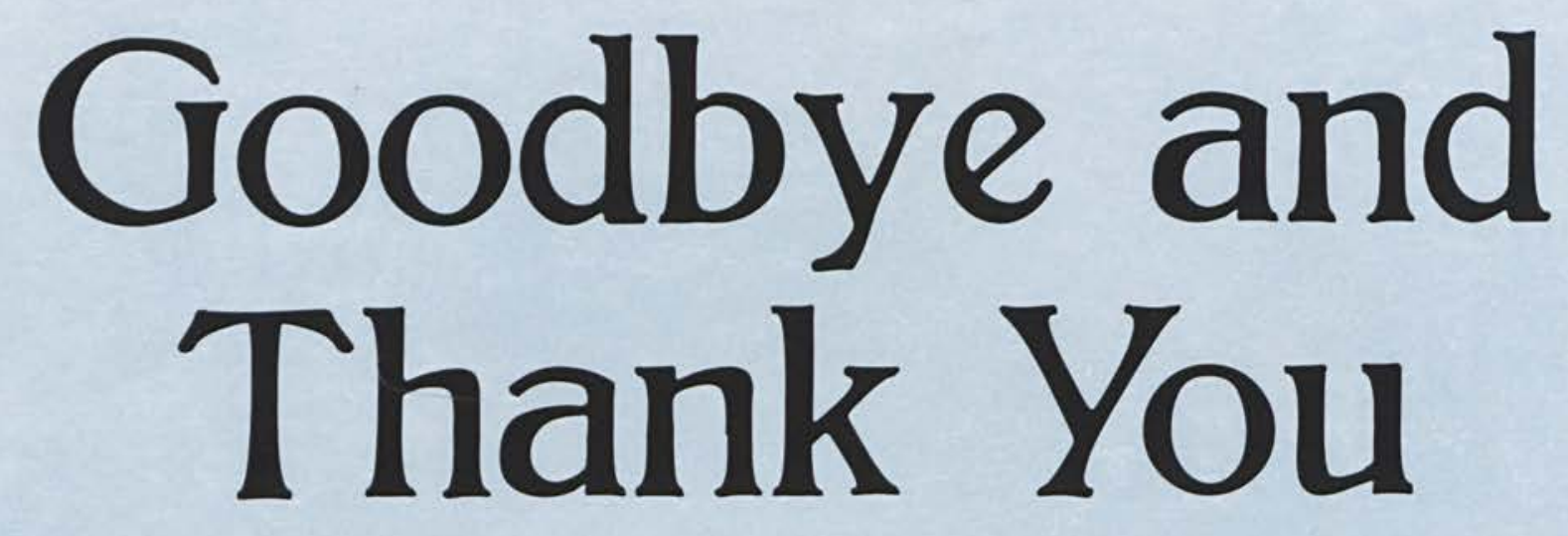

This past year has been one of hard work, dedication, and perseverance through a lot of difficulties. But despite all that we have come though, it was an amazing year filled with many unbelievable memories. One I am sure we will all not soon forget. This yearbook has been a big part of my year. Because of this, I would just like to say thank you to everyone involved with its production and those who have supported us through its creation. First, thank you to my Editors: Daryl, it has been a lot of fun getting to know you this past year. We would never have been able to accomplish as much as we did without your support in the background. I will miss most of all, the late nights and endless weekends we spent working together. Mary, thank you for all of your hard work and meticulousness. It was such a relief to know that the stories and captions were always there right when I needed them. I would never have to worry about you not coming through when I needed you. I have also enjoyed getting know you, you have become a good friend. Kara, one year was just not enough time to work with you. It has been a real privilege and honor to work side by side with you. I have learned a lot from you and I will miss those all-nighters in the office and the Cracker Barrel runs for breakfast afterwards. Sarah, it is the hardest to say goodbye to you. You have been someone I have counted on for the past three years. It is hard to find someone with so much dedication and loyalty. Thank you for all of your hard work and amazing talent. I will truly miss you as a coworker but more importantly as a close friend. I have enjoyed getting to know each and everyone of you this past year. It has been amazing to watch as you all have grown and learned so much. I feel completely confident in leaving the future of this organization in your hands. We have all become very close in such a short amount of time. If what I have seen in the past from each and every one of you is any indication of where you will all be in the future; then those futures will be bright and full of remarkable opportunities. To my staff: Thank you all for your hard work and devotion. This book is a compilation of the talent and vision of each and every one of you. I am proud to have worked with you all and I wish you all the very best. To my advisors: Terry, thank you for your insight and vision. You have truly been an invaluable resource. It has been a privilege to learn from you for the past three years. I will not soon forget what you have taught me. Cyndi, thank you for your constant support. It has been a privilege to have worked with you these past three years. Thank you for always encouraging us to explore new options and never accepting things for the way they are but for how we can make them better. I will never forget the friendship you have given me. To my friends, thank you to all of you for the encouragement and support you have given me. There are so many of you who have been there for me whenever I needed you. The memories we have shared will connect us for the rest of our lives no mater where we end up. Brant and Eric, thank you guys for always being there for me. I will greatly miss all of the nights spent talking and just hang out. I am still waiting for another camping trip with the two of you. Flynn, thank you for your friendship and guidance. I have learned a lot from you as my RA, Bible study leader, and friend. And to all of my friends, I have been truly blessed to know each and every one of you. There isn't enough time or pages in this book to recollect all of the incredible times we have had or thank you for everything you all have done for me. It is hard to suddenly move on after four years, but remember that I am only a phone call away and would love to hear how you all are doing. I miss you all and wish you all the best as you go out to follow the path that has been set before you. To my Family: you have always been behind me every step of the way. Thank you for listening to both my joys and frustrations over the past four years of my college career. I love you all very much. It is impossible to fail with a solid background of love and support that you have given me to lean on. And finally to the entire Cedarville Family, this is your book. As you take the time to look through its pages I pray that it will remind you of all that Cedarville University has given you; education, friendships, memories, and so much more. It is hard to say goodbye. I have enjoyed the past four years. However, it is time to move on. I don't know what is in store for me or where I will end up. But I do know that I will be taking with me the skills and support that I have gained along the way. Thank You and Goodbye.

Andrew J. Harris

Editor-in-Chief 


\section{Colophon}

Publisher

The 2006 Miracle was published by Jostens, Inc. It was printed at their plant located in State College, PA. Our Jostens representative was Tillie Billheimer. Our Plant representative hase been Crystal Gault.

Cover

The 2006 Miracle cover was a Lithocraft Pictorial cover, using photographs provided by Andrew Harris and Sarah Baczek and design provided by Andrew Harris and Kara Merrick. It was rounded, sewed, and bound. It was printed on High Gloss Litho and had a matte finish.

Paper Stock

The 2006 Miracle's 288 pages were printed on Glossy 80\# paper.

Color

The 2006 Miracle was printed with four-color process ink.

Design

The 2006 Miracle cover, opening, dedication, dividers, section layouts, and closing were designed by Kara Merrick and Andrew Harris with help from the layout staff.

Theme

The 2006 Miracle theme was selected by the editorial staff at the beginning of the school year. It was our hope to show the faces and to tell the stories that represent all of us here at Cedarville this year.

\section{Production}

The 2006 Miracle was produced on both IBM and Macintosh computers. Layouts were produced using Adobe Indesign CS. Photos were scanned using HP Precision Scan. Photos were cropped, resized, and touched up using Adobe Photoshop CS2. Text effects and titles were produced using Adobe Illustrator CS. Copy was typed into Microsoft Word and then imported into InDesign.

\section{Typography}

There were two fonts used in the printing of this book; Bolton and Trebuchet. The stories and sports scoreboards were size 10pt. The Captions and Class Picture Names were size 9pt. Page numbers were size 8pt. All of these were using the Trebuchet font. The titles and cover used Bolton. The size of the Titles on the dividers were $82 \mathrm{pt}$ and the titles on individual pages were $68.5 \mathrm{pt}$.

\section{Photography}

The pictures in the 2006 Miracle were mainly taken by Sarah Baczek and her photography staff. We also accepted student submissions for some candids. Our DaVor representative was Jim Rainey. Some of the sports pictures, team pictures, and other candids were provided by Scott Huck, Photographer for Cedarville University, Public Relations.

Inquiries

The 2006 Miracle may be contacted for additional information about its production at:

Miracle, Cedarville University

251 North Main Street

Cedarville, $\mathrm{OH} 45314$
(937) 766-4995

miracle@cedarville.edu 


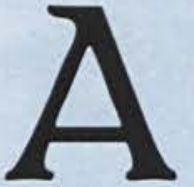

Dr. Bob Abbas 249 Rebekah Abbey 124 Isaac Abbott 60, 221 Timothy Abel 94 Joshua Abernathy 124 Heidi Abraham 60 Matthew Abraham 110, 215, 255

Venus Abrams 124 Allen Acker 60 Amanda Acker 94 Juliet Ackerman 60 Katherine Ackerman 60 Alyssa Adams 110 Benjamin Adams 272, 273

Rebekah Adams 124 Ryan Adams 60 Theodore Adams 110 Dr. Merlin Ager 8, 9, 47 Jonathan Agler 110 Ellen Ahern 94, 218 Scott Aker 94, 186 Mary Anne Alanoly 124 , 180

Jenna Albarano 124 , 247

Dannielle Albert 110 Brian Alburger 94 Lana Aldana 124 Karen Alenskis 16, 94 Emily Alexander 110 Sarah Alexander 60 Stacy Alexander 94, 155, 233

Allison Allen 60 Brietta Allen 21, 28, 124

Jennifer Allen 110 Kevin Allen 124 Travis Allen 192 Alicia Allerton 124 Andy Allgrim 176 Andrew Althouse 94 Dr. Irene Alyn 42, 259 Holly Amarante 124 Benjamin Amberg 60 Joy Ambuske 124 Bryan Amerine 94 Jenee Amstutz 60 Matthew Anderkin 60, 207

Sarah Andersen 124
Sarah

Andersen

107, 184

Benjamin Anderson

124, 211

Connie Anderson 39

Courtney Anderson 110

Dr. Anderson 215

Dr. Lori Anderson 51

Dr. Lyle Anderson 39,

214

Eric Anderson 188

Harry Anderson 46

Jillian Anderson 60,

256, 257, 258, 264

Kara Anderson 124

Karen Anderson 60

Melissa Anderson 110 ,

227

Rachel Anderson 28, 60

Tai Anderson 161

Tyler Anderson 19, 152,

207, 233, 263

Fran Andrews 53

Jamie Anfang 60

Michael Anfang 94, 162

Tammy Angelini 29, 94

John Anglea 33

Jessica Annable 94

Nathaniel Anthony 124

Amanda Anzalone 60

Cameron Arch 60, 236

Nicholas Arch 124

Daniel Archer 94

Kandis Armstrong 194,

195

Joshua Arndt 94

Andrew Arthur 124

Amy Ashinghurst 110

Aimee Auclair 163, 207

Hana Aultman 124

Dr. Jon Austin 46

Laura Austin 157

Wendy Austin 110, 147

Jeremy Auyer 166

Matthew Averbeck 94

Judy Awabdy 54

Jason Ayers 60

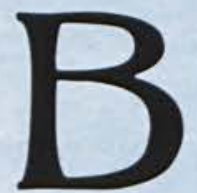

Amy Babbitt 110

Becky Bachman 110

Angela Bacon 110
Dr. Stanley Baczek 42, 56

Sarah Baczek 94, 271, 272, 273

Melany Baessler 219

Bryce Bahler 94, 222,

235

Megan Bailey 60

Ron Becker 34, 124 Dr. Milt Becknell 47

Mark Becknell 124

Lewis Beeler 61, 228

Melanie Befus 61

Joy Behlert 124

Kathryn Behlert 94 ,

149, 236

Sarah Behn 110

Katie Bell 124

Chad Bainbridge 110

Brian Baker 94

Dr. Lois Baker 42

Dr. Sue Baker 47

Dr. Wes Baker 36

Katharine Baker 153

Marisa Baker 124

Megan Baker 61, 259

Rebecca Baker 36

Michelle Baker-Karl 110

Crystal Baldwin 94,

206, 207

Dr. Dick Baldwin 46

Josh Bales 27, 160, 161

Daniel Ballard 110, 182

Sarah Banas 110

Jonathan Banks 110

Shanna Banks 94

Brittany Baranowski

124

Erin Baranski 61, 168,

169

Andrew Barber 110

John Bardakjy 110

Rachel Barnard 124

Brandon Barnes 61

Andrea Barnett 61

Jessica Barnette 61

Esther Barron 124

Katherine Barry 179

David Bartlett 21, 61

Erica Bartlett 94

Megan Bartoletti 61

Caleb Barton 124

Charity Barton 61

Julia Basford 223

Daniel Basham 94, 227

Jessica Bashore 124

Reuben Bassett 110

Dave Bastress 36

Grace Bates 94

Ivan Batista 61

Brittany Battaglia 124

Kendra Battaglia 61 ,

230,232

Lisa Battaglia 61

Aaron Bauer 94

Alison Bauer 61

Ashley Bauer 94

Dr. Donald Baumann 44

Dr. Eddie Baumann 47

Brendon Bayley 94

Sarah Bayne 61

Kassandra Beachy 61

Danielle Beal 94

Brittany Beals 178

Craig Beatty 272, 273

Melanie Beatty 94

Stacey Beaty 61, 222

Mary Becker 61

166

Ben Belleman 41, 166

Lee Belleman 52

Jessica Bellew 94

Daniel Benner 124

David Bennett 110

Kevin Bennett 124

Robert Bentley 124

Erin Benz 124

Justin Benz 61, 166

Matthew Beres 110

William Bernhard 34

Lauren Berry 94, 200,

272, 273

Eva Bertsche 124

Laura Besaw

Jeff Beste 15, 51

Nathan Bethel 110

Mark Betts 110

Michelle Bicking 62 ,

149, 224, 225

Jennifer Bidwell 125

Kathryn Biehl 110

Amy Bielek 54

Denver Bierman 161

Jordan Biga 110

Brandiann Biggar 62, 238

Brett Bigler 125, 186

Debra Birch 94

Virginia Bishop 62

Deb Bissett 48

Karis Bissett 62

Mickey Bitsko 188

Tyler Bixel 62

Andrew Black 17, 62

Jessica Black 62

John Black 110

Stephanie Black 110

Joshua Blackburn 94

169

Melody Blackburn 125

John Blake 110

Julie Bledsoe 62

Hugh Blocker 125

Dr. John Blodgett 34

205

Brant Bloem 93, 110

Helen Blumenstock 51

Alison Blythe 62

Rita Blythe 125

Jonathan Boche 95

Heidi Bochman 125
Dr. Bock 248

Cara Bodenmiller 62,

230

Bader Boland 125

Bashar Boland 95

Benjamin Bolander 95

Rebekah Bole 62

Jeff Bolender 41, 188

Stephen Boles 110 ,

150, 155

Karissa Bollenbacher

125

Kristi Beougher 94, 178 125

Jacob Bezeck 182, 183

Dr. James Bjornstad 37

Lisa Blackburn 94, 168,

Philip Blodgett 62, 150,

Stephanie Bollman 110

William Bolthouse 34

Jordana Bonardi 111

Renae Bonde 111

Omer Bonenberger 47

Matthew Bonin 62, 192

Jillian Boone 125

Tarah Boone 95, 149,

201

Nicole Boop 21, 29 ,

62, 227

Thomas Borck 111

Andrew Borton 62 ,

176, 177

Kristin Borton 51

Melissa Borton 27, 95

Jan Bosma 53

Jonathan Boston 62

Susan Bosworth 29, 95

Corbin Boucek 125

Michael Bouchard 62

Curtis Boudreau 19, 62

Michelle Boudreau 19,

95, 160, 200, 261

Jennifer Boulet 217 ,

233

Jeffrey Bowen 62, 265

Ryan Bowen 186

Crystal Bowers 62

Jesse Bowers 111

Melanie Bowers 125

Amanda Bowersox 111

Roland Bowersox 125

Brittney Bowman 95 
Elizabeth Burkett 95 Naomi Burks 95

Jade Burley 111, 184

Ashley Burnett 95, 155, 219

Brian Burns 19

Lindsey Burns 95

Sara Bursack 63

Philip Buskohl 63, 154

Siera Butler 125, 149

Anthony Butz 125, 180

Gregory Butz 95

Rhonda Byers 125

Melissa Byler 111

Christopher Byrum 125

Christopher Byus 95

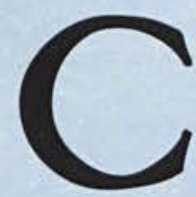

Jessica Cable 95

Benjamin Cady 95

Erin Cahilly 111

Emily Cain 111, 219

Dr. Scott Calhoun 38

Ryan Calkins 111

Lauren Callahan 63,

272, 273

Karen Callan 42

Sarah Calloway 126

Daniel Campbell 63,

170, 189

Fran Campbell 48

Julie Campbell 17, 95 ,

209, 218, 219

Seth Campbell 170

Rachel Capps 63

John Carey 126

Brian Carlson 95

Dr. Carolyn Carlson 42

Aaron Carpenter 63

Sue Carpenter 194

David Carr 161

Brandon Carrier 96

Abigail Carroll 126

Nikki Carroll 96, 148

Jeremiah Carsey 111

Ashley Carter 126

Evan Carter 111

Jace Carter 126

Rachel Carter 96

Thomas Cary 18

Tiffany Cary 96

Ian Casper 126

Katherine Cassity 63

Laura Castle 63, 235

Mark Caterinacci 221

Celeste Cato 126

Kristen Cato 199

Melody Cato 52

Linda Cave 42

Nate Cawood 156

Kyle Cayton 188

Stephanie Caywood 96,

174,160

Terry Chamberlain 39,

270, 272

Joshua Chamberlin 192

Matthew Chamberlin

126, 192

Micah Chambers 111

Christena Chandler 64

Jeremy Chandler 64

Joy Chandler 64

Steven Curtis Chapman

19
21, 43

Helen Chasnov 96

Rebecca Chasnov 64 ,

243

Sarah Cheatham 126

Justin Cheney

Evan Chiu 96, 205

So Mang Cho 111

Anne Christensen 96

Shauna Christensen 96

Heidi Christiansen 126

Alicia Christman 111,

148

Dr. Charon Christman

42, 56

Lindsey Christman 126

Hannah Christofer 64

Sarah Christofer 111

Susan Christoph 111

Linda Chrystal 48

Michael Cimba 96

Beth Cinson 64

Abby Clark 126

Amy Clark 126

Bev Clark 201

Daniel Clark 38

Elizabeth Clark 126

Jim Clark 41, 174

Krista Clark 111, 149

Krysten Clark 64

Matthew Clark 96, 170

Nathaniel Clark 96

Teresa Clark 41, 174

Sue Clarkson 200

Brian Clawson 111

Melissa Clawson 126

Jordan Clay 21, 126

Rachel Clayton 64

AJ Clemans 226

Andrew Clemans 96

Alexandra Clements

126

Bob Clements 36

Steven Clemons 96 ,

231, 257

Dr. Chuck Clevenger 39

Rhonda Clevenger 227

Sarah Clift 64, 231

Patrick Clinton 176

Jamie Clore 96

Joshua Clore 64

Elise Clowser 148

Alissa Clutcher 111,

146

Kaydrie Clymer 126,

190

Nicole Coakley 96

Rachel Coakley 109,

111

Ashley Coale 23, 180,

181

Jeremy Cobb 16, 64,

211

Joshua Cobb 64, 162,

207, 255

Stephen Cobucci 126,

166

Kimberly Cocca 17, 64

Timothy Cochran 126

John Cody 96

Joanna Coffelt 111

Kari Coffindaffer 126 ,

168

Jennifer Coffman 126

Michelle Cohoon 111

Danielle Colao 64, 205

Brandon Colas 64

Amanda Cole 111, 168

Esther Cole 111
Katherine Cole 64

Kraig Cole 126

Laura Coleman 126

Sarah Coleman 126

Janet Colgain 96, 209

Michael Colletto 64 ,

$162,163,207,255$

Brianne Collier 64

Amanda Collins 64, 214

Benjamin Collins 111,

272, 273

Christopher Collins 64

Emilynn Collins 126

Kristine Collins 96

TerryComers 38

Bethany Compton 111

Katherine Condit 111

Elisabeth Congdon 112

Daniel Congrove 65

Elizabeth Conkling 126

Laura Connolly 96, 234

Mary Connolly 126

Douglas Conway 96

Dr. Janet Conway 42,

219

Jeremy Conyers 96 ,

220

Aaron Cook 112

Abigail Cook 253

Dr. Jeff Cook 37

Margaret Cook 126

Matthew Cook 112

Natalie Cook 12

Robert Cook 112, 244

Shannon Cookson 96,

234

Gregory Cooley 112

246

Heather Coon 65

April Cooper 112, 272,

273

Michael Cooper 96

Daniel Copeland 112

Jessica Corbin 96, 224

Kristin Corder 65

Shelby Corley 112

Alexandre Costa 65

Andrew Costerisan 126,

253

Bethany Cottings 126

Jessica Coudriet 112

Christi Coulter 96, 236

Dr. Gregory Couser 37

Laura Cover 126

Benjamin Cowell 112

Kimberly Cowell 96 ,

148

Lauren Cowell 112

Amanda Cox 126

Gabriel Coyle 14, 112 ,

207

Hannah Cozad 147

Dr. Thomas Cragoe 37

Christina Craig 65

Joshua Craig 65, 262

Sara Craig 126

Lori Crain 96

Katrina Crandall 112

Scott Crawford 126

166

Abigail Craycraft 112,

208

Sarah Crichlow 127

Misheal Crocker 112,

220

Dr. April Crommett 41

Christina Cronshaw 17.

230

Chris Cross 40, 176,

178

Benjamin DeLarme 65

Morgan DelGrosso 65

Emily Delimpo 65, 178

Samuel DeLong 112

Terry Delong 53

Kara DeMustz 55

Marie Denio 65

Amy Dennis 127, 148,

207

Elizabeth Dennis 112

Heather DePree 65

Colleen Derry 168

Shannon DeRusha 13,

127

Dr. James DeVries 34

Merissa DeVries 112

Dr. Timothy Dewhurst

43

Gwendolyn Dewhurst

127

Tobias Dewhurst 112

Mike DiCuirci 15, 39 ,

Gabe Custer 49

Amanda Cyrus 127, 147

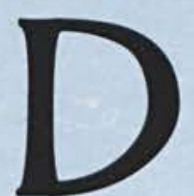

Stephanie Dager 65

Jonathan Daiello 96

Heather Daniel 112

Ruth Daniel 96

Cathy Daniels 96, 231

Stephen Danielsen 65

Andrea Dankert 96

Kelli Daugherty 224

Nicholas Davey 127

Priscilla David 127

Michelle Davidson 22,

96

Robert Davidson 127

Bethany Davies 127

Cynthia Davies 127

Richard Davies 112

Andrew Davis 127, 145

Brent Davis 166

Cynthia Davis 52

Ellen Davis 112

Jennifer Davis 146

Kenneth Davis 166, 167

Matthew Davis 112

Melissa Davis 65

Michelle Davis 112

Nathanael Davis 96

Danny Davison 188

Lisa Daxer 224

Daron Day 65, 207,

253, 255

Melanie Day 97

Matthew de Waal

Malefyt 127

Matthew Deakin 97, 
Lisa Engel 67

Jessica England 128

Thomas England 67 Valerie England 128 James Englemann 34 Kristen Ensminger 128 Sarah Ensslen 97, 190 Sara Eplin 67, 222, 231, 232

David Erlandson 67, 180

Joshua Erlandson 243

Caleb Ernst 113

Tiffany Erspamer 113

Rebecca Erwin 113

Pat Estepp 41, 176

Carol Estes 47

Dr. Dan Estes 37

Jeremy Estes 67

Pamela Eustace 113, 210

Adam Evans 128

Dr. Tony Evans 159

Jonathan Evans 113

Michelle Evington 202

Lacy Ewers 128

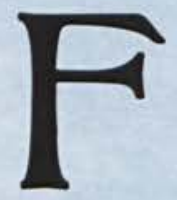

Lynsey Fabian 178

Dr. Joy Fagan 37

Siobhan Fagan 40

Sarah Fakan 67

Dea Fallin 128

Dr. Vicky Fang 43

Elizabeth Fannon 113

Megan Fansler 128

Katelyn Farrar 113, 227

Katherine Farrell 128

Chrissy Faulkner 55,

227

Amanda Fawcett 128

Dr. Jeffrey Fawcett 46

,155, 249, 256, 265

Tonya Fawcett 53

Kristie Febo 97

Jillian Feczer 128

Katherine Feiler 128

Kimberly Feiler 97

Michael Feiler 97

Sarah Feiler 113

Ann Felix 113, 168

Nicole Fenstermaker 67

Abigail Fenton 67, 149

Jenna Ferguson 113

Kristen Ferguson 128

Kyle Ferguson 67

Martha Ferguson 67
Annamarie Franke 128 Heidi Fraser 113, 214

Kurt Fraser 263

Laurie Fraser 68

Joshua Frederick 113

Luke Frederick 68

Richard Freed 128

Kathy Freese 41

Kathy Freese 194

Kaitlyn French 128

Rachel Freswick 98

Aaron Frey 21, 129

Dr. Darrin Frey 44

Johanna Frichtl 129

Nicholas Fridenmaker

200, 231

Gabriel Friend 68

Holly Friend 68

Michal Friend 98, 205

Sarah Friesen 68

Donyo Frisco 68, 246

Jeffrey Fulmer 51

David Fuls 98, 152

Christa Funke 129

Lynne Funtik 53

Kevin Furst 129, 182

Ryan Futrell 38

Elyse Fye 129, 243

Mallory Fisher 113 ,

204, 239

Tammy Fitzgerald 67

Steven Fladda 97

Drew Flamm 17

Jonathan Fleetwood

128

Adam Flenar 97

Dr. Dennis Flentge 44

Caleb Fletcher 97

Crystal Flippin 128

Pam Flippin 55

Bonnie Flora 128

Elizabeth Flow 128

Kari Flunker 174

Jennifer Flynn 113

198, 227

Joshua Flynn 67, 150, 265

Matthew Focht 128

Ann Foley 97

Jamie Foo 98, 224

Connie Ford 42

Timothy Ford 113

Mark Forsberg 68

Jessica Forsythe 180 ,

181, 233

Dustin Foster 68

Janna Foster 98

Kathryn Fowler 68, 149

Amanda Fox 68, 98

Jesse Fox 166

Matthew Fox 128, 188

189

Natalie Fox 68, 265

Nathan Fox 68

Jannette Foy 19,98 ,

227

Melanie Frageorgia 98

Dara Fraley 53

Timothy Frame 39

Dr. Dwayne Frank 47

Kirsten Gibbs 50, 91

Sarah Gibson 98, 168
Seth Gibson 207

Zachary Gibson 98

Daniel Gier 68, 205

Megan Gier 113

Daniel Gifford 98, 263

Rebekah Gifford 129

Benjamin Giglio 129

Jessica Gigliotti 68,

260

Joshua Gigliotti 123 ,

$129,150,272,273$

Bonnie Gilbert 68

Sarah Gilbert 69, 207

Shelley Gilbert 98

Sarah Gilchrist 44

Daniel Giles 98

Timothy Gill 69

Justin Gillett 69

Jacinda Gillette 113 ,

148

Kelsey Gilsdorf 129 ,

207

Kevin Gilsdorf 98

Elizabeth Gingrich 69

Cori Ginter 98

Earl Girouard 155

Jessica Gish 129

Rebecca Givens 69, 219

Jessica Glass 98

Ashley Gleichauf 129

Sarah Gneiser 180

Ashley Gochenaur 98 ,

107

Kathleen Godinez 69,

90,152

Monica Godinez 98, 231

Tim Goeglein 262

Peter Gohdes 98

Joshua Goheen 129

Adam Golden 113, 215

Robert Gole 129

Dr. Timothy Gombis 37

Catherine Gooch 98

Emilie Good 129

Audree Goodew 13,

$113,172,190$

Danielle Goodling 69 , 198

Terri Goodman 113

Whitney Gordon 129

Aaron Gosser 39

Emilie Gouch 129

Dr. David Gower 34

Michelle Graeff 129

Stephanie Graff 129 ,

190

David Graham 34

Jennifer Grant 129

Jennifer Grant 69

Elizabeth Graves 113

John Gray 114

Michelle Gray 114

Rachel Gray 69

Amy Gredy 129

Dr. John Gredy 33

Cory Green 149

Derrick Green 36

Joel Green 26, 98, 155 ,

157, 198
Katherine Green 69

Sean Green 98

Shawn Green 239

Amy Greenfield 129

Dr. Clark Greer 36

Kimberly Greer 114 ,

205

Carissa Greetham 174

Jacquelyn Greetham

69, 194, 195

Jedediah Gregory 98

Vanessa Grehl 129

Gabriel Greve 129

Joshua Greve 98, 153,

176

Jessica Grewe 114

161, 272, 273

Heather Grier 69, 231

Aubrey Gries 114

Jared Griest 114, 192

Joshua Griffith 129

Aaron Griggs 170, 188

Dr. Don Grigorenko 37

Lyndie Grigorenko 69

Gerald Grimson 69

Michael Groeber 129

Adam Groff 129, 152

Brian Groff 69, 152

Julie Grollimund 129

Sarah Grollimund 69

Dr. Bob Gromacki 37

Heather Grooms 178

Joshua Grosso 98

Shannon Grosso 114

Dr. Steve Gruber 47

Joshua Gruber 18, 155

Scott Gruber 69

Shavon Gruber 24, 25

David Grudda 114

Stephen Gruenbers

129,188

Christopher Guarnera

$15,98,248$

Roy Guenin 34

Kathleen Guenther 129

Jeffrey Guernsey 46 ,

249

Abigail Guiler 230

Amy-Hope Guisleman

37

Kevin Gulley 114, 233

Carrie Gumm 230, 232

Michael Gumprecht 98 ,

224, 225

Kyle Gunn 144, 180

Jessie Gustafson 69

Jennifer Guth 114

Justin Gutierrez 114 ,

$150,170,188,189,232$

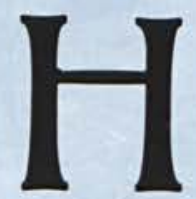

Lew Gibbs 50

Jonathan Haber 69 ,

89, 207

Elizabeth Hacking 70

Drew Hackney 114

Lauren Haegele 129

Amy Haetinger 114

Dr. Debby Haffey 36

Angela Hagensen 98

Ruthann Hager 98

Joseph Hague 70

Krista Haines 129

Bryan Haithcox 129

Jenna Hakala 130, 190

Paul Hale 114

Andrew Hall 130

Jessica Hall 70

Kevin Hall 70, 170 ,

171,188

Sarah Hall 99

Stacia Hall 70

Benjamin Halladay 70

Bradley Halladay 70

Debra Halsmith 17, 49,

233

Maria Hamann 130

Amanda Hamilton 130

John Hamilton 130

Nathan Hamilton 99

Kristin Hamminga 130 
Melissa Hartman 174 , 184

Timothy Hartman 70, $90,182,183,260$

Alison Hatch 12, 130

Ann Marie Haug 130

Jaime Hawkins 55

Jeremy Hawkins 25, 180

John Hawkins 237, 250

Taylor Hawkins 99

Nathan Hay 49

Anna Hayes 99

Erin Hayes 130

Heather Hayes 114

Sara Hayes 114

Hannah Haynes 114

Rachel Haynes 114

Elizabeth Hays 114

John Hays 34

Heather Heagy 114

Megan Heagy 70

Dr. Kevin Heath 38

Dr. Tim Heaton 47

Allison Heavner 168

Rachel Heffield 51

Beth Hegarty 130

Dr. Harwood Hegna 43,

221

Kristen Heiner 70, 235

Elizabeth Heinig 99

Josh Heinrich 53

Leslie Heiss 114

Anna Hejl 130

Daniel Hekel 99

Dr. Evan Hellwig 40

Kelly Hellwig 49

Dr. Larry Helmick 44

Angela Henker 99

Heather Hennessey 212

Lindsay Hennig 70

Dr. Nelson Henning 45

Neil Henning 188

Jessica Henry 130

Rebekah Henschel 70

Caleb Hensley 114

Matt Hensley 208

Sarah Henslin 99

Joseph Hepworth 224

Justin Herbert 70, 170 ,

171, 188

Joshua Herbolt 114

Rachel Herbolt 130

Brittany Herbst 114,

172, 173

Hannah Hermiz 224

Mark Hershey 99, 156

Alison Hess 99

Daniel Hess 130

John Hess 47

Ruth Hess 56

John Hessler 130

Kristin Hessler 99, 243

Kyle Hiestand 70

Kim Higginbothan 42,

219

Erin Higley 71, 253

Samuel Hilbert 71

Michelle Hildebrand

130

Stephanie Hilerio 114

Jonathan Hill 71

Kristy Hill 55

LeAnne Hill 51

Robert Hill 71, 152

Roger Hill 99

Keri Hilley 190

Keri Hilty 172

Benjamin Himsel 99

Hannah Hindle 130
Tricia Hine 99

Corrie Hines 227

Kristi Hines 114

Vicky Hines 55

Elizabeth Hinks 99

Joanna Hinks 71, 221,

264

Nathan Hinks 99

Angela Hinton 130

Jamie Hishmeh 71

Melanie Hitchcock 99 ,

149, 208

Melissa Hitchcock 130

Katlyn Hiteshew 29,

130

Lindsey Hlad 114

Seth Hobbes 130

Matthew Hobbs 71

Rachel Hobbs 114

Bethany Hochstaetter

71, 226, 227

Dave Hoecke 15

Dr. David Hoffeditz

29, 37

Chelsie Hoffman 114,

148

Crystal Hoffman 130

Joel Hoffman 99

Sarah Hoffman 130 ,

194

Mike Hogan 160

Amelia Hoganson 71

Shannah Hogue 38

Sarah Holden 71

Amy Holderby 54

Joseph Holecko 18

107, 148

Darren Holland 71

Alexander Hollander

71, 206, 207, 255, 263

Hannah Hollingsworth

130, 228

Matthew Hollis 114

Aaron Hollopeter 211

Nicole Hollopeter 131

Jada Holloway 114

Michael Holt 268

Karley Hood 224

Ashley Hoopes 71

Sara Hoover 99

Allison Hope 71

Rebecca Hornbrook

71, 152

Timothy Hornbrook 71

Maray Horning 131

William Horosz 71

Jacqueline Horst 71

Ragen Horst 115

Bridgett Horton 115

Kristen Horton 115

Sarah Hosan 131

Emily Hose 99

Derek Hostetter 115,

182

Matthew Houchin 192

Benjamin Houghton 99

Katherine Houk 115

Maxwell Hounshell 131

Craig Houser 115

Dale Houser 115, 205

Bethany Houts 148

Christina Hovestol 153

Angela Howard 131

Chelsea Howard 131.

194

Christie Howard 115

Mark Howard 71

Mary Howard 34

David Howdyshell 115

Chu-Yu Huang 43
Michael Hubenthal 99

Curtis Huber 269

Jessica Huber 131

Anthony Hubin 115

Timothy Hubler 192

Scott Huck 57

Lori Huckaby 178

John Hudson 71

Joshua Hueni 150

Stephanie Hueni 17,

72, 205, 232

George Huff 45

Virginia Huff 49

Eric Huffer 176

Aaron Huffman 99

272, 273

Greg Hughes 192

Julie Hull 99

Marcus Hull 131

Laura Humberson 72

Ashley Hume 99

John Hummitzsch 99,

149

William Humphrey 131

Phillip Hundley 131

Aftan Hunt 72, 232

Ashley Hunt 99

Hillary Hunt 72

Amanda Hunter 72, 260

Ashley Hunter 99, 107

Brigette Hunter 72, 260

Jennifer Huster 72

Todd Huster 72

Aaron Hutchinson 44

Dr. Tom Hutchinson 37

Mandy Hutchinson 13

50

Sarah Hyatt 115

Ryan Hyde 131, 166

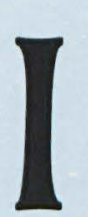

Chad Imgrund 205

Lindsey Ingram 131

Bryan Inskeep 153

Tiffany Inskeep 115

Dr. Cheryl Irish 47

Mark Irving 55

Meredith Irving 50

Jocely Izor 72, 89

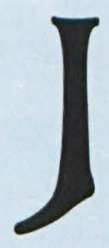

Brent Jackson 115

Brittany Jackson 115

Chad Jackson 55

Clarissa Jackson 131

Jennifer Jackson 131

Katherine Jackson 153

Kyle Jackson 19, 115

Nathanael Jackson 131

Tonisia Jackson 131,

231

Amy Jacobs 131

Dr. Jack Jacobs 34

Heather Jacobs 72

Nicole James 72, 168

Raymond James 23,

176

Frederick Jansohn 115 Carol Keller 100

Elaine Keller 49

Justin Keller 100, 107

204

Jessica Jantzen 72, 235

Daniel Jaquery 3, 99,

155

Phillip Jarvis 72

Mary Jeffcoate 99

Candace Jelinek 72

Benjamin Jenkins 131

Aaron Jenne 131

Mary Jennings 131

Sarah Jett 72

Nicole Jillich 131

Alicia Johnson 272, 273

Alyssa Johnson 99

Benjamin Johnson 72

Christopher Johnson

100,142

Courtney Johnson 100 , 234

Dr. Pam Johnson 40,

184

Dr. Sharon Johnson 46

Eric Johnson 100

Eric L. Johnson 44

Heather Johnson 72 ,

210

Holly Johnson 100, 149

Ian Johnson 131, 192

Jennifer Johnson 72

Jori Johnson 131

Joseph Johnson 72

Melissa Johnson 100

Nathan Johnson 72 ,

263

Nicole Johnson 115

Rachel Johnson 73

Sarah Johnson 73, 174

Steven Johnson 115,

227

Yuki Johnson 54

Laura Jolly 47

Allison Jones 131

Amber Jones 131

Andrew Jones 115

Bill Jones 57

Grace Jones 184

Kelsey Jones 73, 174

Lauren Jones 100

Leah Jones 6, 100,

$107,272,273$

Kimberly Jonker 115

Jesse Jordan 115

Amanda-Jo Joswig 100

Isaac Judd 16, 100, 215

Jonathan Juhl 115

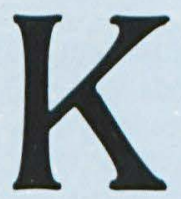

Claire Kaemmerling

115

Martha Kaercher 51

Heather Kahl 115

Darin Kaiser 73

Janelle Kamauf 73

Amanda Kapp 131

Abigail Kauffman 73 , 
Elliot Moore 102, 166 Jennifer Moore 134 Julie Moore 38

Kenneth Moore 117

Matt Moore 37

Stephanie Moore 117,

224, 225

Christopher Moran 77, 163

Julia Moran 233

Stan Moran 134

Kurt Moreland 37

Dr. Gerson Moreno-

Riano 249

Ashley Morgan 117

Danielle Morgan 117

Darrell Morgan 118

Jonathan Morgan 118

Jennifer Moriarty 118

Brody Morris 77

Dr. Dee Morris 41, 184

Hannah Morris 77

Phyllis Morris 52

James Morrow 118

Sara Morrow 77

Camille Morse 118, 222

Dr. John Mortensen 39 , 211

Emily Moser 77, 263

Ryan Mosher 134, 176

Jason Moss 25, 134 ,

243

Joseph Moss 118

Jeremy Motta 77, 263

Andi Mounts 53

Caleb Mounts 118

Trevor Mueller 77

Stephanie Mullenhour 78

David Mulligan 134 Laura Mullikin 134

Sarah Mumme 78

Aubrey Muncy 134

Monique Muncy 38

Katharine Mundy 134

Aubree Munson 194

Wesley Muntz 134

Dr. Murray Murdoch 45

Dr. George Murray 243

Bradley Muschott 134,

188, 189

Jamie Musho 134

Katybeth Muste 134

Andrew Myers 78

Jason Myers 102

Jennifer Myers 53

Justin Myers 118, 206

Kelly Myers 231

Leslie Myers 118

Leslie Myers 209

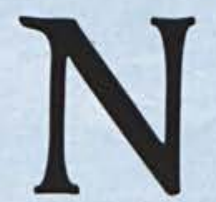

Dennis Nangle 272, 273

Phil Nanney 118

Brian Napp 102

Alissa Neal 78

Kristen Neal 135

Rebekah Nearhoof 102 ,

107, 228

Timothy Neetz 102

Brian Nelson 118

Emily Nelson 78

Kelsey Nelson 118, 220,

221
Megan Nelson 135

Benjamin Nesbitt 118,

223

Rebekah Nettekoven

$78,258,261$

Micaela Neuhart 135

Eric Neumann 102

Danielle New-Day 135

Allison Newfield 118

Jason Ney 78, 149

Sara Ney 135

Kathryn Nicholl 102, 199

David Nickerson 78 ,

261

Tiffany Nickum 102

Sarah Niedermayer 118

Amber Nielsen 78

Katie Niemi 135

Christin Nightingale

102

Daniel Nikitin 102, 155

Jonathan Niknam 118

Katie Nixon 78

Amberly Noble 102

Andrew Noble 78, 89,

192

Emily Noble 178

Garrett Noble 135

Rondi Noden 102, 107,

225, 226

Bryan Norman 118

Dr. Timothy Norman

43, 158

Nicole Normandeau

$135,148,228$

Ricky Normandeau 102

John Norton 166

Bayley Nosal 23, 178

Kelly Nowels 135, 145

Julianne Null 78, 90 ,

$147,198,242,272,273$

Lauren Nuss 103, 153,

$230,232,233$

Kelsi Nussbaum 78, 261

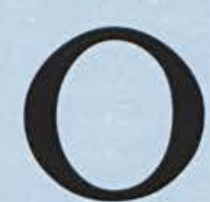

Daniel O'Callaghan

103, 236

Sara Oddis 78, 153

John O'Donnell 103

Hannah Officer 78

Joshua Ohms 118, 170,

188

Brendan Ojala 186

Erin O'Keefe 103, 190,

191

Gary Olin 49

Amanda Oliver 103

Joshua Oliver 78

Patrick Oliver 45

Holly Olmstead 103,

155

Laura Olmstead 103,

146

Lucy Olney 135,172 ,

190

April Olson 103

Krista Olson 16, 78

Toryn Olson 135, 190

Casey O'Neal 78, 146,

232

Elise O'Neal 146, 147,

190
Whitney Oosterhouse

118, 204

Paul Orchard 41, 170,

188

Paul Organ 118

Dave Ormsbee 33

Mary Orr 78

78, 236

Ashley Ort 79

Brandon Ort 107, 272,

273

Jared Osborn 103, 212

Melissa Osborn 79

Julie Otis 135

Devon Overby 17, 79

Matthew Owens 103 ,

192

Tyler Owens 135, 192

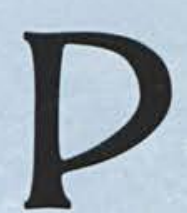

Anna Pabody 79

Charles Pagnard 39 ,

210, 211

Brynn Paine 103, 227,

235

Claire Palmer 103

Kaitlin Pankratz 103,

148

Michael Parido 103

Joshua Parker 79

Laura Parker 135

David Parmerlee 79,

263

Melissa Parmerlee 174

William Parmerlee 263

Rachel Parrill 43

Daniel Parrott 135, 222

Mike Parrott 38

Nicholas Parry 118

Lisa Partain 79

Erin Pasma 118

Neil Patnaude 103, 227

Randy Patten 35

Dr. Paige Patterson 35

Rebecca Patterson 79 ,

204

Rory Patterson 53

Erica Paugh 79, 174,

175

Donna Paulsen 54

Julianne Paulson 103 ,

148

Emily Peacock 103,

190, 191

Rebecca Peak 118

Geoffrey Peck 79

Israel Peck 79, 235

Cassandra Peletis 135

Katherine Pelon 118

Kristen Pelon 79

Karie Pepe 28, 135, 228

Michelle Percival 103

Joshua Perkins 79

Joshua Perrel 103

Kelly Perrel 118

Laura Perrien 103, 149

Rebecca Perry 135

Elizabeth Pesce 79,

247, 253

Danielle Petermann

267

Rachel Petersen 79 ,

157, 263

Sarah Petersen 118

David Peterson 118

Joel Peterson 135

Jared Petry 135

Kelsey Pfeifer 147

Tabitha Pfoutz 79

Joanna Pheasant 79

Paula Pheasant 118

Alissa Phillips 79

Andrea Phillips 135

Maribeth Phillips 103

Mark Phillips 135

Rebecca Phillips 103

Dr. Jim Phipps 37

Timothy Phipps 37

Kathleen Picard 118

Kaitlyn Picazo 118

Shannon Picazo 79, 90,

152, 260

Amanda Pierce 135

Timothy Piette 118

Sarah Piles 103

Hannah Pilgrim 200

Daniel Pinkham 135

Bryan Pittman 103,

$170,188,189$

Isaac Pittman 79, 180,

181, 258

Randall Plate 80,212 ,

221

Adam Ploeg 103, 150,

174

Christopher Plume 103,

151

Natalie Plunket 211

Karen Poch 103

David Podboy 80

Joseph Poelzer 103

Jonathan Polsdorfer

118

Laura Polston 135

Beth Porter 39, 215

Charmaine Porter 135

Courtney Potter 80

John Potter 51

Andrew Powell 235

Jennifer Powell 135

Mac Powell 160

Karen Power 38

Christopher Powers 135

Kathleen Powers 80 ,

235

Steve Powers 170

Jordan Pratt 103

Joshua Presley 103

Abigail Price 103, 168

Amy Price 135

Christen Price 13, 135

Ty Price 80

Lisa Prins 135

Ryan Prins 221

Kathleen Pritts 118

Mary Pritts 80

Michael Pritts 103, 155

Pamela Proctor 135

Daniel Prokop 103

Renee Provo 17

Robin Provo 17, 233

Alexander Pummell

80,192 


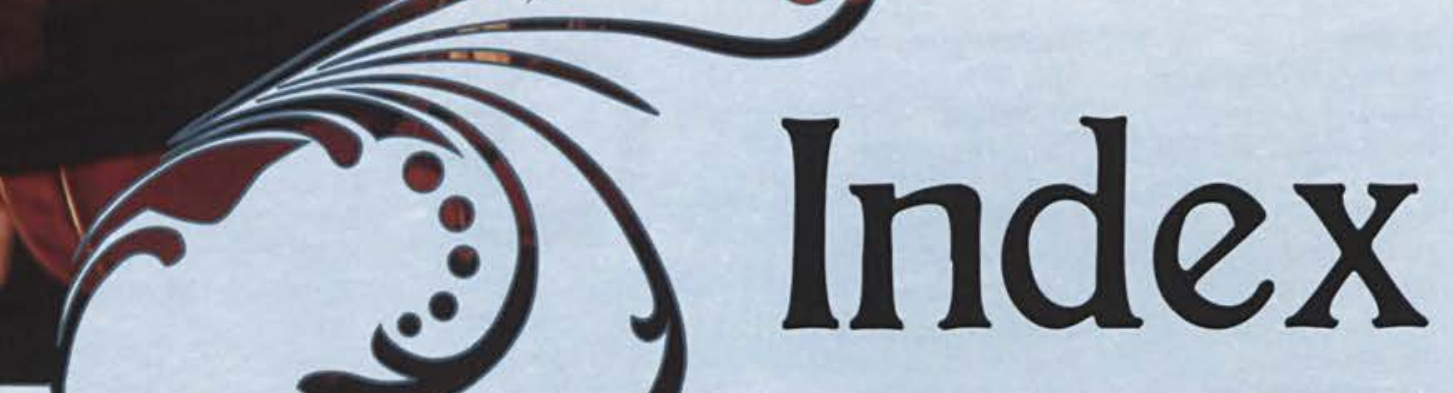

\section{7}

\section{Cristine}

Rozelle 104

Dr. Carl Ruby 33

Kristine Rucker

178,179

Dr. William Rudd 35

Stacie Rudd 136

Stephanie Rudd 81

Tyler Ruegsegger 119

Karen Ruhlman 104,

168

Mindy Russell 104, 157

Gary Rutrough 104, 150

Alisa Rutt 136, 184,

185,218

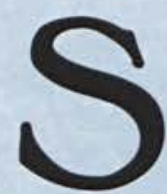

Kelsey Salisbury 104,

230

Dr. Susan Salladay 43

Brian Sallee 104, 228

Emily Sammons 136

Jessica Sammons 119

Aubrey Samuelsen 104

Ryan Samuelsen 136,

201

Julianne Sandberg 119

Angela Sanders 136

Stephanie Sanders 119

Emily Sandlund 136

Timothy Sandlund 104,

155, 233

Daryl Sando 104, 151,

231, 270, 272

Kaitlin Sands 119

Amy Sanford 119

Dr. Sam SanGregory 43

Erin SanGregory 136 ,

235

Nicole Santos 136, 190

Danah Sargent 149

Sarah Sargent 149

Benjamin Sattler 272,

273

Kaitlin Sattler 137

Marybeth Sattler 81

Joshua Saunders 137,

170,188

Katherine Sawdon 149

James Sawin 104, 188

Daniel Scarberry 26,

137

Michael Schaefer 137 , 188

Philip Schanely 81 Lorne Scharnberg 35 Anne Scheftic 119 Jessica Scheidt 104 Bradley Schellin 137

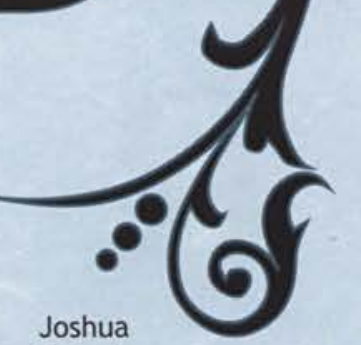

Schenk

104, 205

Terri Schenk 119, 253

Matthew Schermer 155

Dan Schetter 178

Ryan Schildroth 81

Rebecca Schillack 81,

226

Ryan Schiller 119

Jessica Schimmenti 119

Adam Schlappi 153,

186, 187

Jessica Schlener 137

Tiffany Schlueter 137,

146, 147

Peter Schmid 188

Robert Schmid 137

Dr. Dave Schmidt 46

Frances Schmidt 119

Jessica Schmidt 168 ,

169

Bradley Schmoyer 104

Hannah Schnake 81

Sara Schnepp 119

David Schoen 137

Shannon Schramm 81

Rebecca Schrank 104,

259

Erica Schroeder 194,

195

Karah Schroeder 104

Trevor Schuh 82

Marie Schumacher 104

Robert Schumacher 44

Tyler Schumacher 166

Steven Schutter 82

Janna Schwab 82, 209.

230, 232

Brittny Scott 104, 174

David Scott 82, 154

Dawn Scott 180

Jason Scott 104, 188,

189

Jessica Scott 137

Phill Scott 188

Tyler Scott 119

Zachary Scott 119

Kathleen Scull 104

Dianne Seals 53

Sarah Searles 163, 180

Bethany Seawell 120

Jennifer Secor 44

Lukas Seelye 207

Jordan Seibert 170,

188

Matthew Seibler 137,

180

Ashley Selegue 82

Lindsey Selegue 82

Tara Self 120
Daniel Servi 186

Pamela Seymour 17,

120, 230, 232, 233

Jason Shaeffer 105

Kimberly Shank 82

Laura Shank 82

Merilee Shank 174

Stephanie Shank 224, 226

Dr. David Sharp 43

Dr. Kimberly Sharp 43

Joy Shaughnessy 157

Dr. Annis Shaver 38

Libby Shearer 41

Lane Sheats 120

Jennifer Shelamer 82

Peter Shellabarger 105, 231, 257

Karlee Shelton 120

Juliana Shepherd 137

Melodie Sheppard 105

Nathan Sheppard 228,

237

Sarah Sherick 120

Matthew Sherman 137

Rachel Sherwood 137

Lindsey Shev 82

Elizabeth Shimer 82

Philip Shimer 105, 166

Rachel Shinabarger 137

Michael Shipper 82

Matthew Shiraki 17,

82, 224, 225, 236, 264

Dr. Keith Shomper 43

Rebekah Shomper 137

Olivia Short 137, 174

Ryan Short 23, 176,

177

Mikal Shorts 137

Dr. Jeff Shortt 43

Dr. Sandra Shortt 43

Amanda Shriber 82,

264

Benjamin Shroyer 82,

$170,171,188,189$

Jordan Shumaker 192 ,

193

Laura Shuneson 82

Cheryl Shupe 55

Bethany Sibbitt 105

Keith Sider 105, 207

Jordan Siefkes 137, 192

Jennifer Siegrist 120

Bethany Sierawski 218

Dr. Rebecca Sietman 37

Joel Sigmon 82, 263

Adrian Silaghi 24

Summer Joy Silaghi 24

Matt Silveira 188

Dr. John Silvius 44

John Simmons 224

Ryan Simmons 82

Ashley Simon 120

Jeffrey Simon 82

Trenton Simon 120, 152

Eric Simonaire 259

Jill Simons 105

Kara Simons 82

Tasha Simons 105
Adam Simpson 188

Brittany Simpson 172,

173

Katherine Simpson 83,

231

Nikole Simpson 83

Dr. Kevin Sims 45

Grace Sims 137

Paul Sims 120

Benjamin Sincock 137,

214

Megan Sjoquist 137

Andrew Skaggs 83

Jason Skelton 83, 156,

207

Hillary Skrabacz 153,

194

Jay Skrabacz 194, 195

Ray Slagle 41, 176

Elizabeth Slavish 83,

205

Cara Slaybaugh 105 ,

207

Rachel Slothower 83

Samantha Slusher 83

Stephanie Small 137

Brittany Smart 23, 120 ,

178, 179

Mallory Smart 137

Allison Smith 83, 89,

194

Amanda Smith 137

Amie Smith 137

Andrea Smith 83, 219,

231

Anna Smith 105

Ben Smith 52

Brandon Smith 105

Brett Smith 120

Candace Smith 105 ,

230

Cody Smith 83, 153

Dr. Galen Smith 46

Dr. Mark Smith 45

Dr. Sarah Smith 46

Jacob Smith 137, 144

Joel Smith 83, 188

Kayla Smith 83, 153,

233, 243, 272, 273

Kyle Smith 27, 137

Lydia Smith 137

Mark Smith 120

Matthew Smith 120

Michael Smith 253

Nathan Smith 83

Nathan Smith 83

Nathaniel Smith 120

Rebekah Smith 120 ,

227

Rhonda Smith 43

Ricky Smith 137

Roscoe Smith 54

Ryan Smith 137

Sarah Smith 83

Stephen Smith 21, 137

Timothy Smith 137

Travis Smith 137, 188

Joel Smoyer 120

Sarah Smoyer 138

Rachelle Snavely 120

Kara Sneed 138, 205

Luke Snider 19, 105,

144,221

David Snow 83

David Snow 192

Dana Snyder 105, 207

Wade Snyder 138

Rachel Solsky 83, 204

Nathaniel Sowder 120

Eric Spahr 105

Stacey Spain 138

Justin Spann 83

Dr. Mark Spencer 39

Ed Spencer 38, 232

Jacob Spencer 120 ,

144, 272, 273

Nathanael Spencer 83

Lacy Sposato 83

Benjamin Sprague 84

Jessica Squier 172

Paul Squires 105

Kettia St Paul 138

Andrew Staab 105

Jennifer Stackhouse

138

Robert Stahl 105, 149, 155 
Matthew Taylor 153

Rebekah Taylor 231

Paige Taylorr 153

Kelly Teague 138, 168

Laura Teague 180, 181, 253

Rachel Teat 105, 152, 233

Jaimie Teekell 105

Rachel Tellish 84

Daniel Tello 105, 220

Kathryn Temple 138

Susan Terkelsen 46

Lana Terpstra 84, 265

Judith TerreBlanche

105, 149, 199

Melody Terry 84, 218

Molly Thayer 85

Kelly Theiss 174

Mary Thengvall 105,

271, 272, 273

Mike Thigpen 38

Brandon Thomas 207

Ian Thomas 121

Jessica Thomas 105 ,

168

Jonathan Thomas 85 ,

233, 265

Michele Thomas 85

Mugabe Thomas 27, 85,

176, 177

Robert Thomas 35

Carrie Thompson 121

Christy Thompson 149

Cristy Thompson 231,

233

Crystal Thompson 106

Dr. Mark Thompson 243

Dr. Thomas Thompson

44

Katherine Thompson

$85,230,232$

Martha Thompson 106

Paula Thompson 152

Rachel Thompson 121,

174

Sara Thompson 85

Teah Thomson 85

Megan Thornburg 85

Kipp Tiger 85

Emily Tilton 138

Rachelle Tipton 85

Danielle Tisdale 85

Kristen Tisdale 106

Joseph Tobias 121

Joel Tomkinson 55

Stephen Tormey 138 ,

144

Joshua Townsend 247

Christopher Travis 139

Mallory Travis 139

Stacie Travis 106, 178

Dr. Ted Traylor 27

Matthew Trego 139

Robert Trennepohl 170,

188

Emily Trevino 17, 232

Andrew Tripp 106, 245

Kristin Troiano 121

Brent Trowbridge 139

Aubrey Trulsson 139

Conor Trulsson 139

Cherie Tryon 121, 209

Dr. Luke Tse 47, 57

Roleigh Tuazon 85

Katie Tucker 121

Stephanie Tucker 85

Kelly Tuinstra 172

Tim Tuinstra 44 mes Tullett 25, 85 , 162, 221, 254

Stephen Turkovich 85 , 265

Bethany Turner 106,

147, 199

Dustin Tursack 85, 265

Alan Tuttle 106, 221

Katherine Tuttle 139

Crystal Tuxhorn 85

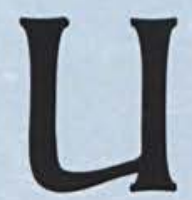

Seth Unruh 85

Mark Urman 106

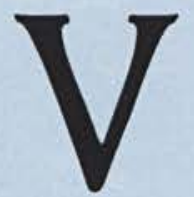

Amanda Valenti 85

Andrew Van Curan 85

John van der Wal 139

Emily Van Dette 16

Jennifer Van Dyke 106,

146

Scott Van Dyke 144

David Van Schepen 139

Emily Van Vliet 152

Megan Vance 86

Christopher Vande

Lune 106

Shandra VanderZouwen 121

Emily VanDette 250

William VanDyke 106

Matthew Vanlier 121

Emily VanMatre 139

Amy Vanover 272, 273

Valerie VanWingerden

106

Sarah VanWinkle 86

Kate Varney 86

Ashley Varvel 86

Donald Vasser 121

Alexander Vaughan 139

Kevin Vaughn 86, 212,

231

Timothy Vaught 121

Tyler Vawser 205

Sherri Veldhuizen 121

Mordecai Veldt 139

Richard Velilla 86

Maicol Venter 106, 176

Lynn Verlander 147

Timothy Vernon 106

Rachel Verosky 86

Elizabeth Verwys 106,

155, 184, 185

Sara Villanyi 121

Timothy Vincent 21,

139

Craig Vinson 106

Philip Vinson 139

Chad Viront 263

Andrew Vitaliti 139

Hannah Vitt 86

Carol Vollnogle 86

Benjamin Volpe 121

Kimberly Volpe 86

Timothy Voltz 139 Mehden 86

Amy Voris 43, 56

Angel Voris 248

Brad Voumard 49

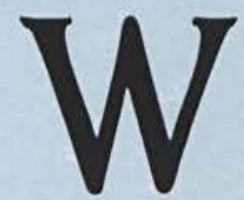

Benjamin Waddell 17,

$26,28,86,156,236$,

250

Sean Wadley 139

Lee Wadlington 139

Jessica J. Waggoner

86, 151, 206, 207

Jessica M. Waggoner 86

Joshua Waggoner 139

Diane Wagner 86

Grace Wagner 139

Jonathan Wagner 121

Hannah Wailes 169

Laura Walach 139

Athan Waldron 86

Andrea Walker 121,

194, 195

Catherine Walker 86

Chris Walker 22

Dick Walker 51, 226

Douglas Walker 121

Dr. Ron Walker 46

Grant Walker 23

Tricia Walker 53, 91

Elizabeth Walkley 139

Joel Wallen 106

Katharine Walter 86 , 168

Melissa Walter 87

Nathaniel Walter 87

Tyler Walter 139

Joshua Walther 121

Karah Walton 23, 178

Sharon Waltz 139

Samuel Wanderi 87

Jerica Ward 26, 139

Robert Ward 87

Stephen Ward 139

Katie Warndahl 106

Jana Warner 139

Dr. David Warren 35

Timothy Warren 227

Wesley Warriner 53

Nathaniel Washatka

106, 238

Jonathon Wasson 87 ,

252

Mallery Waters 87

Megan Waters 27, 121

Nathan Watkins 139

Krista Watson 87, 168,

169

Matthew Watson 106

Megan Watson 87

Christina Weathers 121

George Weber 168

Nathan Weeber 87, 207

Tricia Wegman 121

Cassie Weideman 121

Monica Weigel 87

Natalie Weil 21, 87

Daniel Weiser 139

Sarah Welch 16, 106,

155, 256

Anthony Weleski 87,

192

Jillian Weleski 87

Kendall Welker 121

Gina Wells 106

Justin Wells 139

Rebekah Wells 87

Heather Wenzel 121,

239

Allison West 19, 87 ,

230

Andrea West 87, 260

Autumn West 87, 262

Susan West 50

Susan West 149

Philip Westenbarger

192

Ashley Westfall 121

Nathan Wexler 121 ,

228

Jean Weyandt 44

Dr. Bert Wheeler 46

Katie Wheeler 149

Amy Whitacre 139

Jeffrey Whitacre 139

Scott Whitaker 87

Daniel White 121, 192

Ethan White 121

Sara White 139

Jeffrey Whited 121

Alicia Whitman 121

Dr. John Whitmore 44

Charis Whitney 87

Sarah Whitson 139

Dr. Hayes Wicker 35

Rebecca Widener 87

Garrett Wieronski 21

Christopher Wiest 139

Abigal Wight 140

Tyler Wightman 121

Joshua Wilcox 106

John Wildman 140

Benjamin Wiley 140 ,

170

Crystal Wiley 88,178 ,

179

Dr. Peg Wilfong 38

Matthew Wilhelm 140

Michelle Wilkey 88

Cindy Wilkins 227

Angela Wilkinson 88

Zachary Wilkinson 140

Katie Willaman 106

Jeremy Willetts 88

Joshua Willetts 122

Garrett Williams 122

Jordan Williams 88

Joy Williams 49

Karen Williams 122

Kimberly Williams 122 ,

204, 214

Michelle Williams 122

Nathan Williams 16 ,

88,219

Sara Williams 106

Rebekah Williamson

106, 151

Kristen Wills 106, 157

Amanda Wilson 122

Joan Wilson 55

Matthew Wilson 88

Micah Wilson 192

Paul Wilson 192, 193

Suzanne Wilson 140 ,

157

Timothy Wilson 88

Jordan Winar 106

Abigail Winburn 88, 


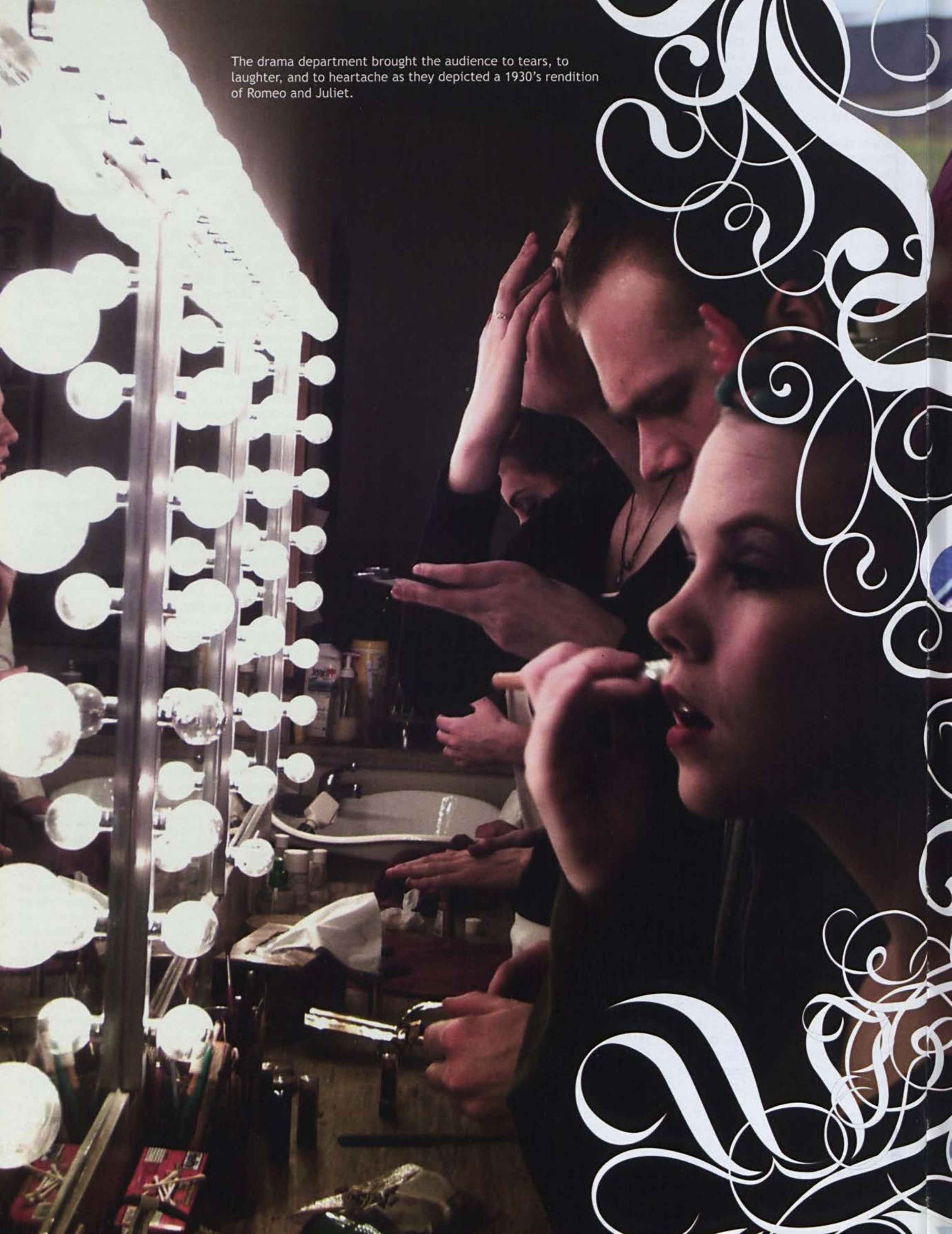




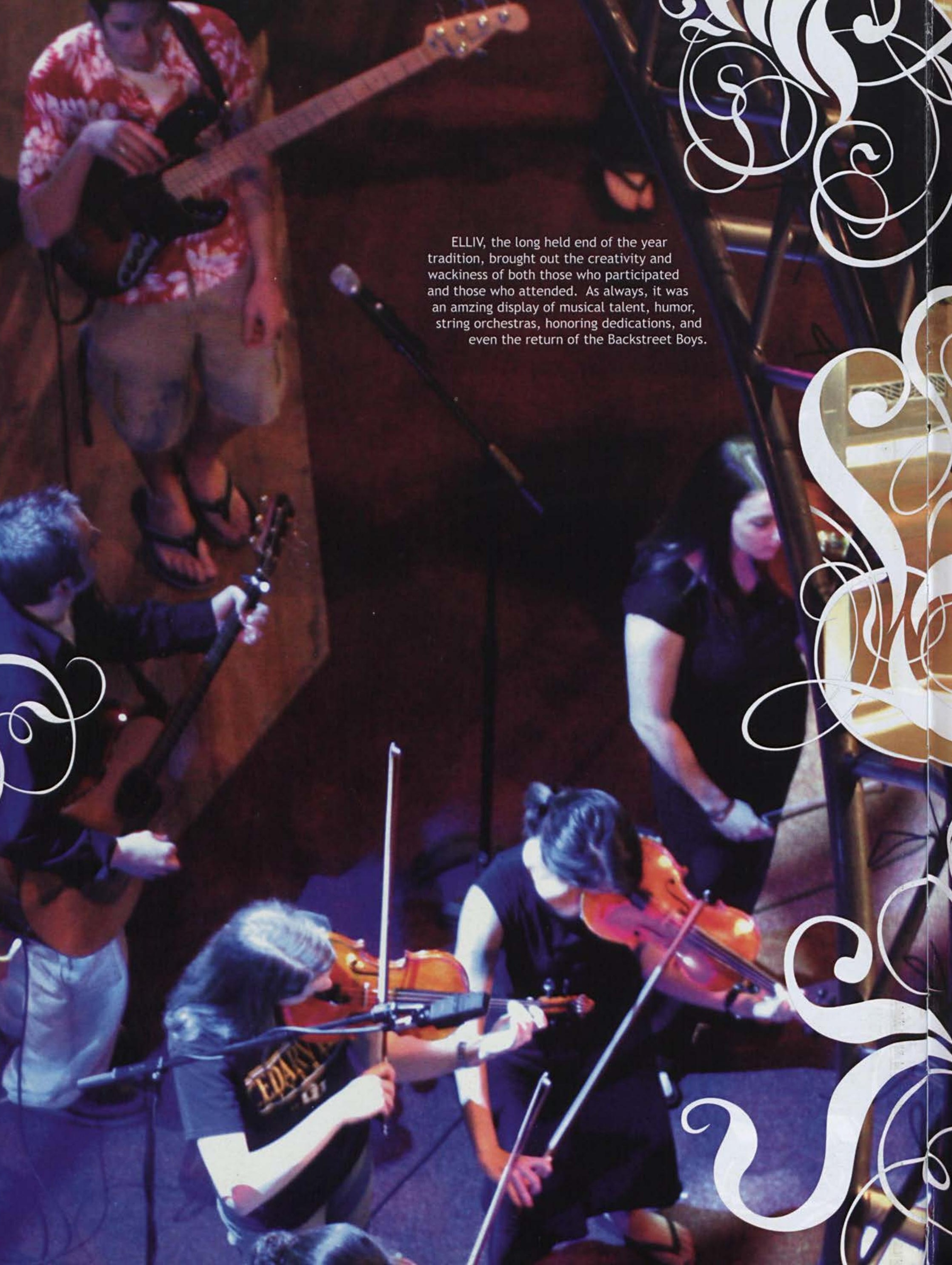




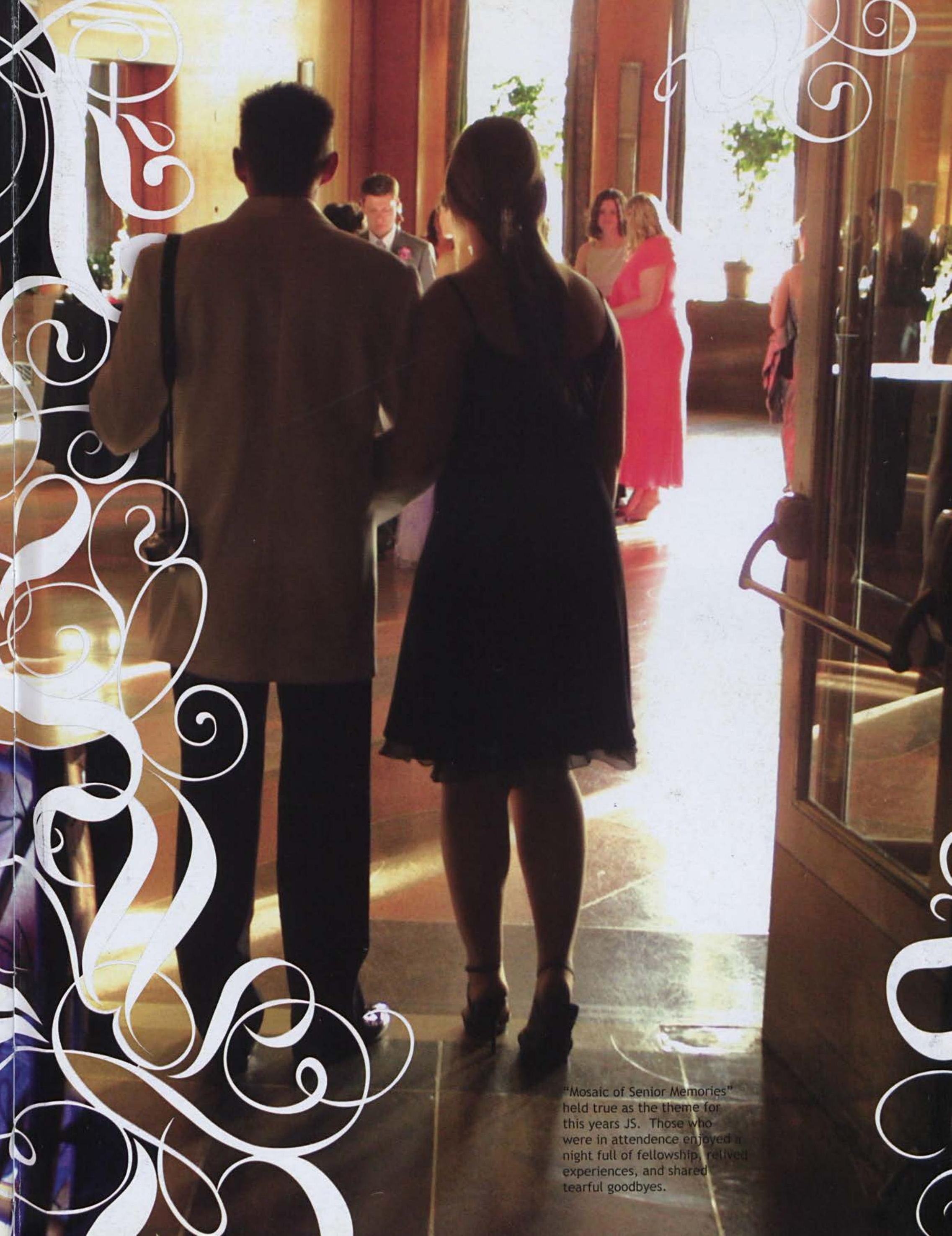




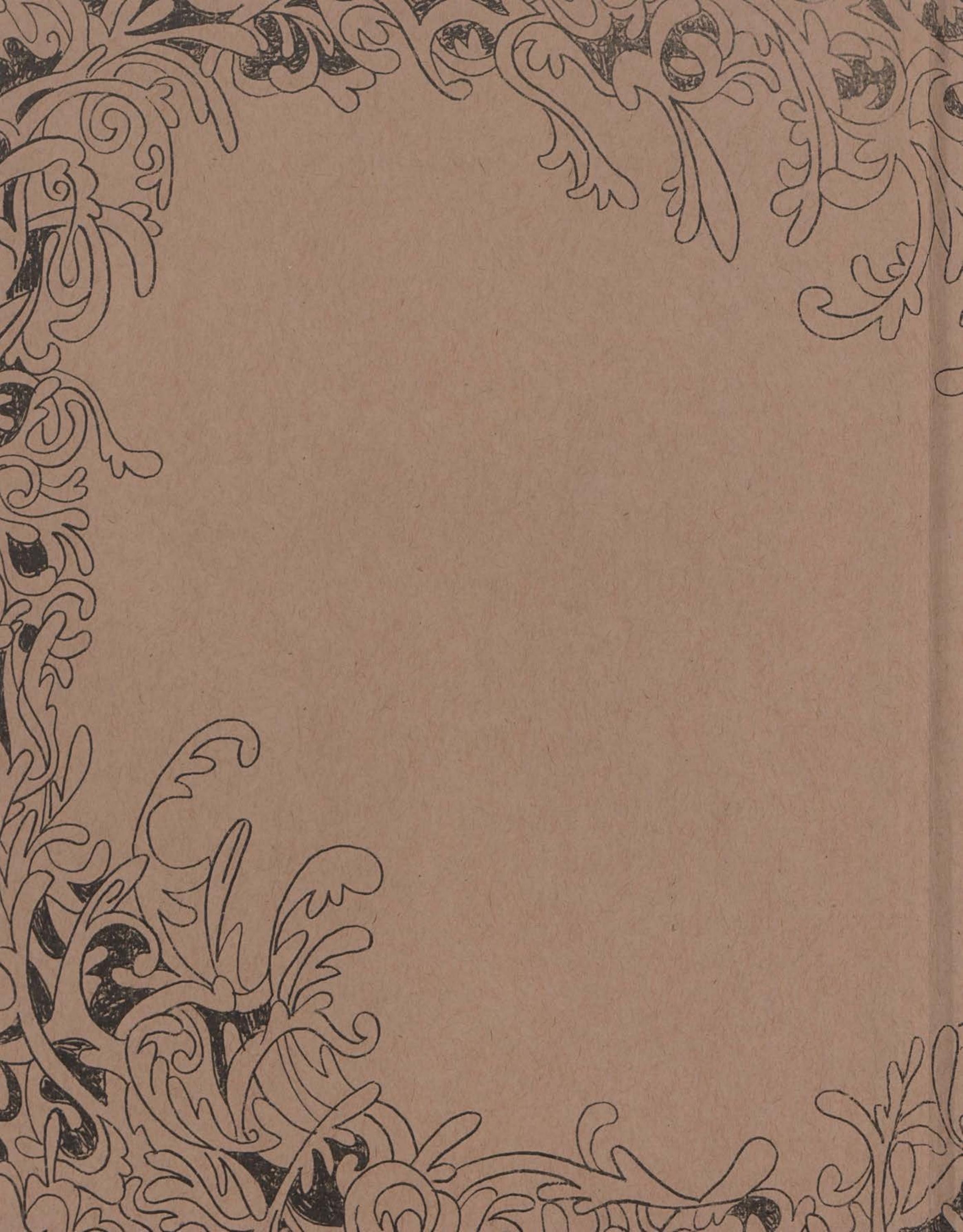




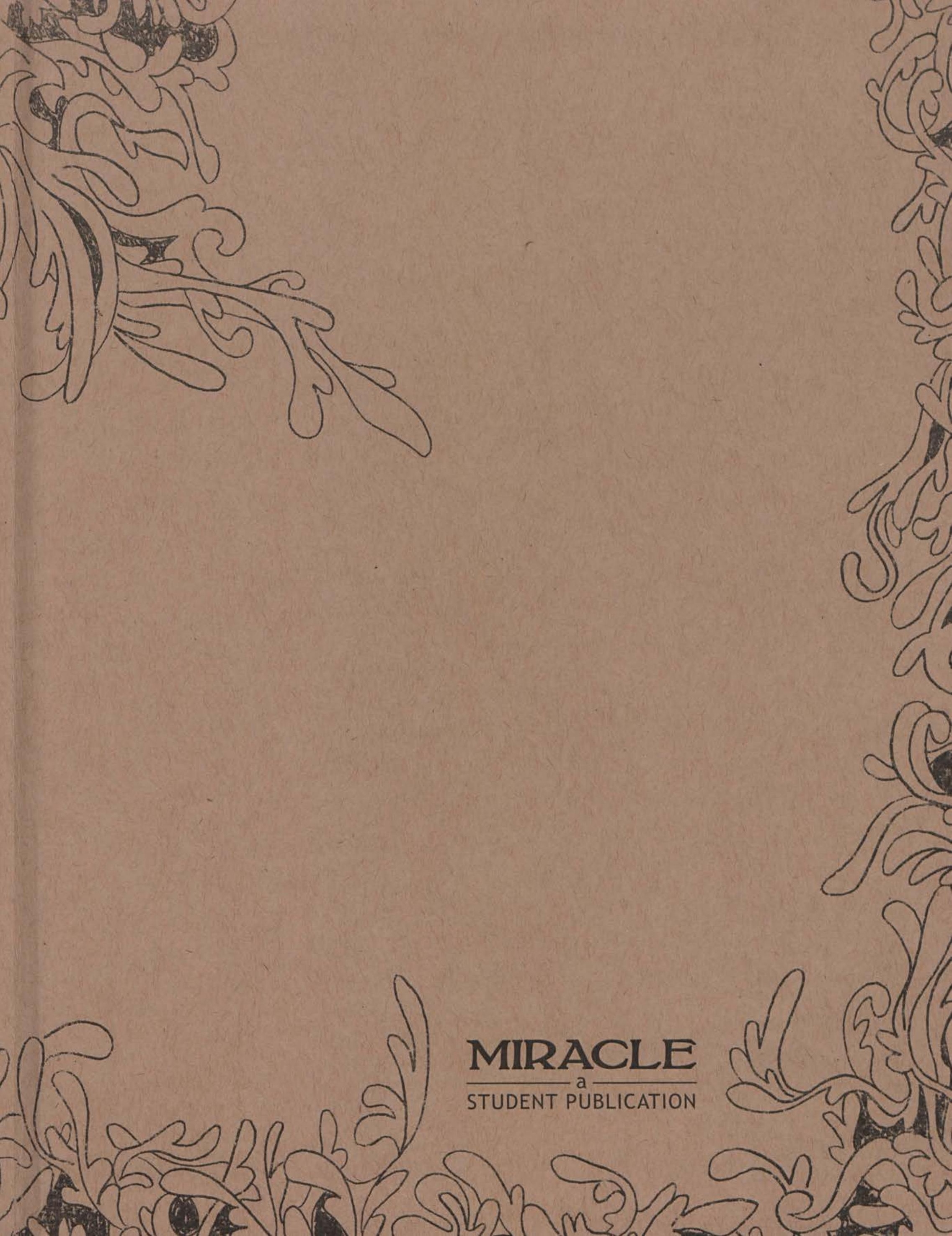




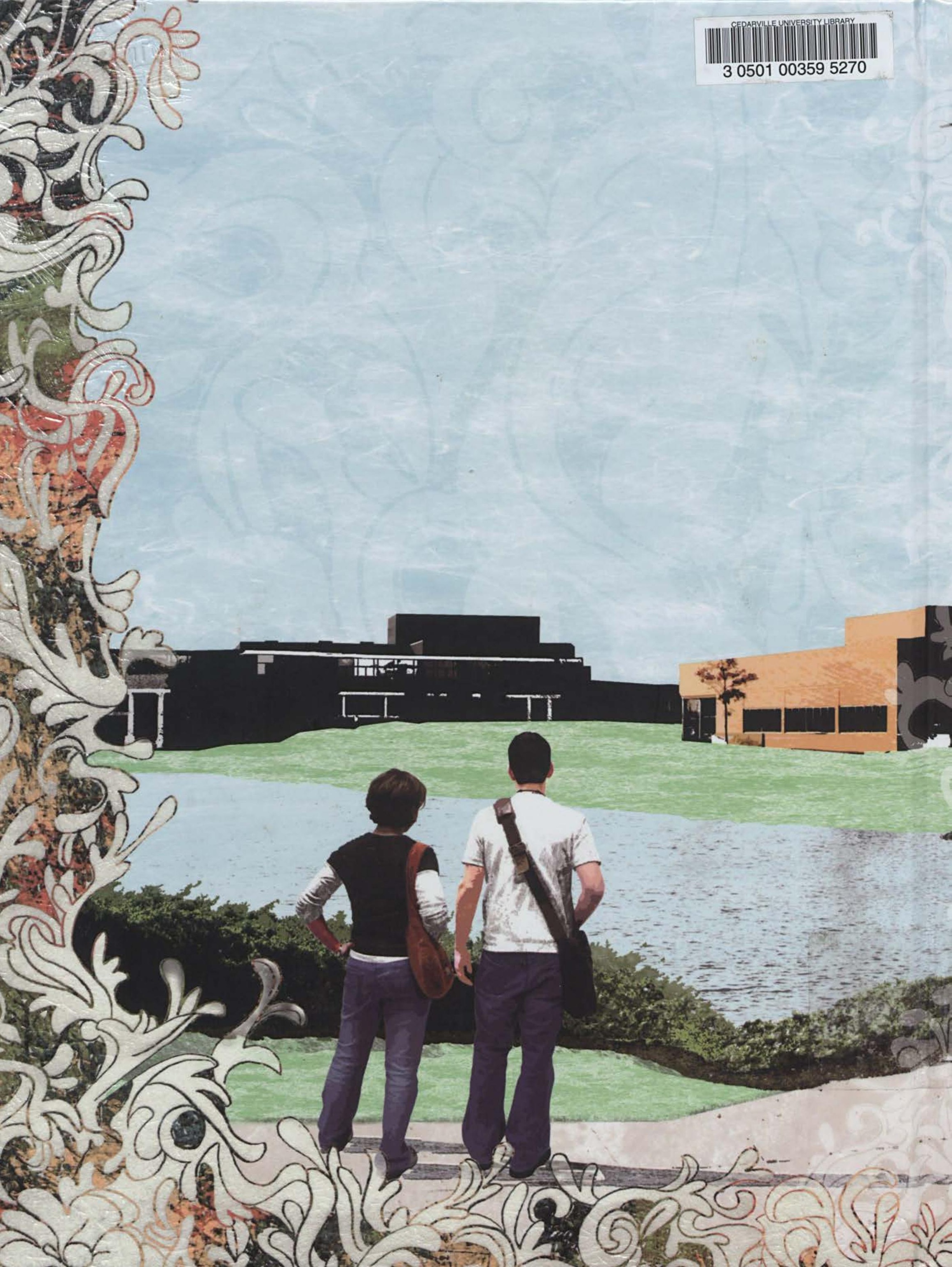

